\title{
Wettability and Imbibition: Microscopic Distribution of Wetting and its Consequences at the Core and Field Scales
}

\author{
Final Report
}

February 1, 1999 through January 31, 2003

Principal Investigators:

Dr. Jill S. Buckley

Dr. Norman R. Morrow

Co-Investigators:

Dr. Chris Palmer

Dr. Purnendu K. Dasgupta Contributors:

Shannon Bays

Tianguang Fan

Dr. Yu Li

Dr. David Lord

Zhengxin Tong

Dr. Jianxin Wang

Royan Wang

Dr. Xina Xie

Lei Yang

Dr. Z. Yang

PRRC, New Mexico Institute of Mining and Technology

Dept. of Chemical \& Petroleum Eng., University of Wyoming

formerly with Dept. of Chemistry, NMIMT

Dept. of Chemistry and Biochemistry, Texas Tech University

Undergraduate, Chemical Eng., NMIMT

Research Chemist, PRRC, NMIMT

Dept. of Chemical \& Petroleum Eng., University of Wyoming formerly with PRRC, NMIMT

PhD student, Chemical Eng., U. Wyo.

Sr. Research Associate, PRRC, NMIMT

MS student, Chemical Eng. U. Wyo.

formerly with U. Wyo.

MS student, Chemistry, NMIMT

Dept. of Chemistry and Biochemistry, TTU

Collaborators:

Dr. Geoff Mason

Dr. Doug Ruth

U. Loughborough

U. Manitoba

February 2003

DE-AC26-99BC15204

\author{
Submitted by: \\ New Mexico Petroleum Recovery Research Center \\ New Mexico Institute of Mining and Technology \\ 801 Leroy Place \\ Socorro NM 87801 \\ Department of Chemical and Petroleum Engineering \\ University of Wyoming \\ P.O. Box 3295 \\ Laramie, WY 82071-3295
}




\section{Disclaimer}

This report was prepared as an account of work sponsored by an agency of the United States Government. Neither the United States Government nor any agency thereof, nor any of their employees, makes any warranty, express or implied, or assumes any legal liability or responsibility for the accuracy, completeness, or usefulness of any information, apparatus, product, or process disclosed, or represents that its use would not infringe privately owned rights. Reference herein to any specific commercial product, process, or service by trade name, trademark, manufacturer, or otherwise does not necessarily constitute or imply its endorsement, recommendation, or favoring by the United States Government or any agency thereof. The views and opinions of authors expressed herein do not necessarily state or reflect those of the United States Government or any agency thereof. 


\title{
Project Objectives
}

The main objectives of this project are:

(1) to quantify the microscopic distribution of altered wetting that occurs in oil reservoirs as crude oil components adsorb on rock surfaces, and

(2) to apply quantitative descriptions of wetting at the microscopic level to interpretation of imbibition in mixed-wet porous media.

\begin{abstract}
The questions of reservoir wettability have been approached in this project from three directions. First, we have studied the properties of crude oils that contribute to wetting alteration in a reservoir. A database of more than 150 different crude oil samples has been established to facilitate examination of the relationships between crude oil chemical and physical properties and their influence on reservoir wetting. In the course of this work an improved SARA analysis technique was developed and major advances were made in understanding asphaltene stability including development of a thermodynamic Asphaltene Solubility Model (ASM) and empirical methods for predicting the onset of instability. The CO-Wet database is a resource that will be used to guide wettability research in the future.
\end{abstract}

The second approach is to study crude oil/brine/rock interactions on smooth surfaces. Contact angle measurements were made under controlled conditions on mica surfaces that had been exposed to many of the oils in the CO-Wet database. With this wealth of data, statistical tests can now be used to examine the relationships between crude oil properties and the tendencies of those oils to alter wetting. Traditionally, contact angles have been used as the primary wetting assessment tool on smooth surfaces. A new technique has been developed using an atomic forces microscope that adds a new dimension to our ability to characterize oil-treated surfaces.

Ultimately we aim to understand wetting in porous media, the focus of the third approach taken in this project. Using oils from the CO-Wet database, experimental advances have been made in scaling the rate of imbibition, a sensitive measure of core wetting. Application of the scaling group to mixed-wet systems has been demonstrated for a range of core conditions. Investigations of imbibition in gas/liquid systems provided the motivation for theoretical advances as well.

As a result of this project we have many new tools for studying wetting at microscopic and macroscopic scales and a library of well-characterized fluids for use in studies of crude oil/brine/rock interactions. 


\section{Table of Contents}

Wettability and Imbibition: Microscopic Distribution of Wetting and its Consequences at the

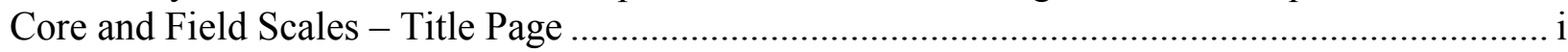

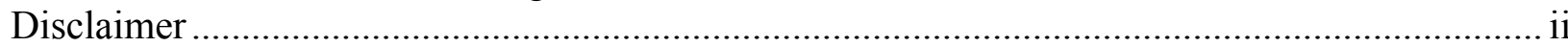

Project Objectives ................................................................................................ii

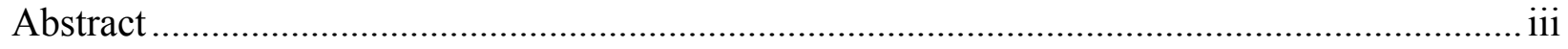

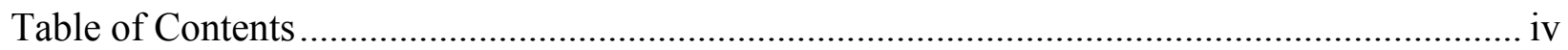

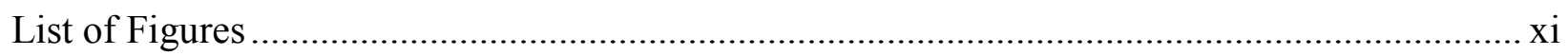

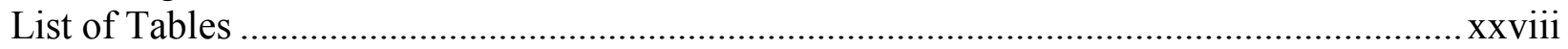

Executive Summary .............................................................................................. Xxxi

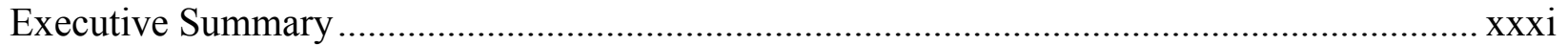

Part I-Crude Oil Characterization ........................................................................... 1

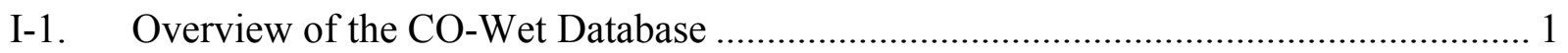

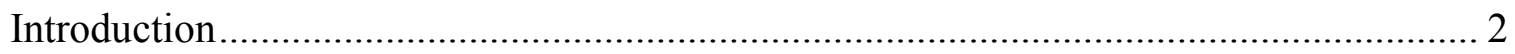

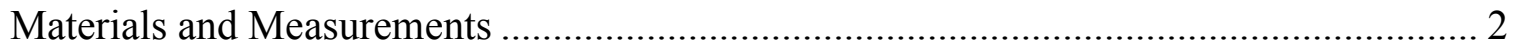

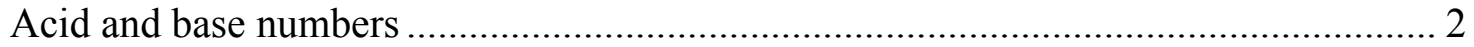

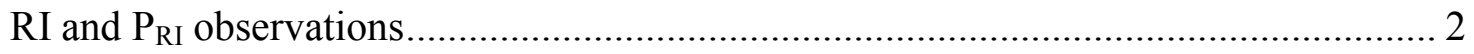

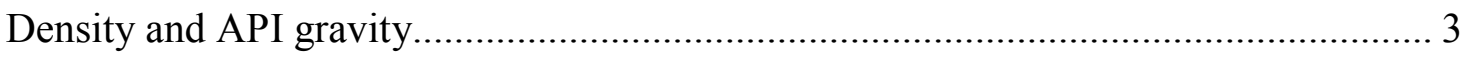

Database Design ..................................................................................................... 3

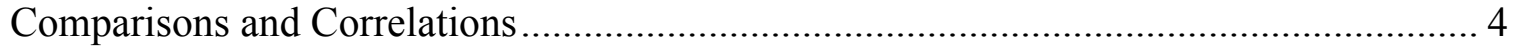

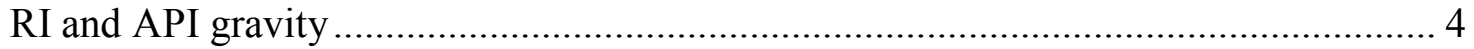

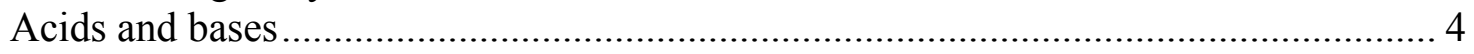

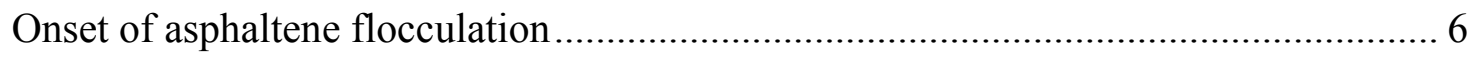

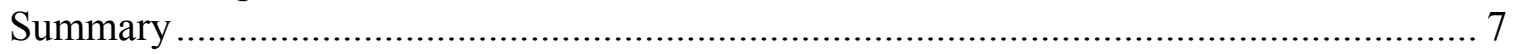

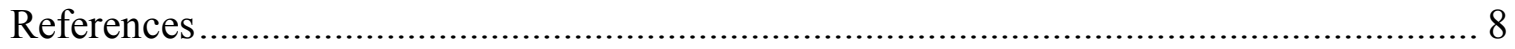

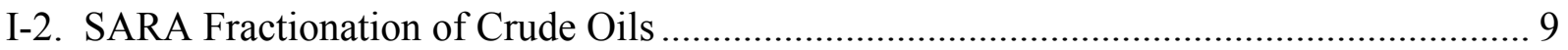

I-2.1. SARA Analysis of SQ-95 Crude Oil by the ASTM Method.................................. 9

Experimental Methods and Materials ......................................................................... 10

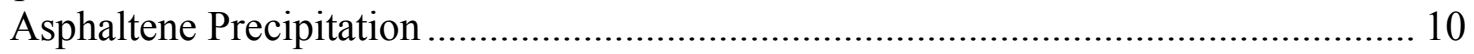

Saturate, Aromatic, and Resin Fractions................................................................. 10

Measurements of chemical and physical properties .................................................... 11

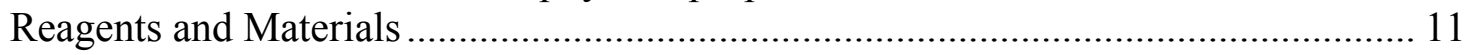

Azobenzene Activity Test for Attapulgus Clay …………........................................ 12

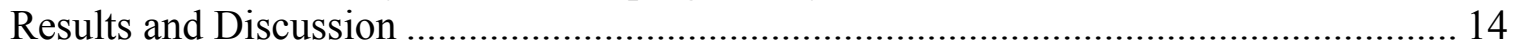

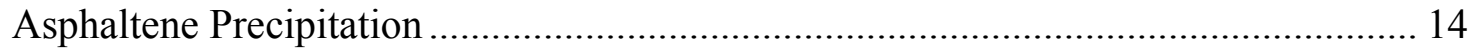

Column Separations ........................................................................................... 15

Properties of the SARA Fractions...................................................................... 15

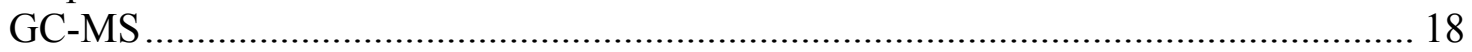

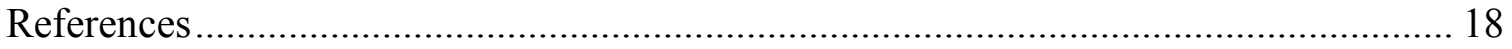

I-2.2. Evaluation of SARA Analysis Techniques.................................................. 22

Crude Oils and Hydrocarbon Group Separations …………………………………..... 22

SARA Fractions and Wettability Alteration............................................................... 22

Brief Review of SARA Fractionation Techniques ..................................................... 23

SARA Fractionation Problems ………………….................................................. 23

Experimental Techniques and Results........................................................................ 24 


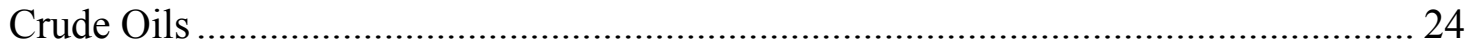

ASTM-Recommended Column Separation ............................................................ 25

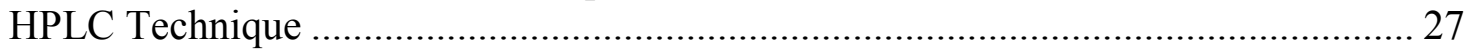

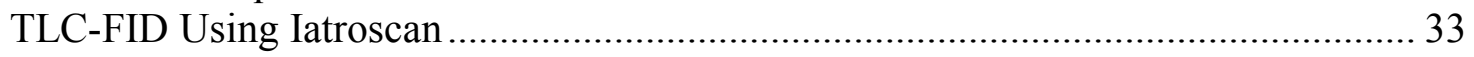

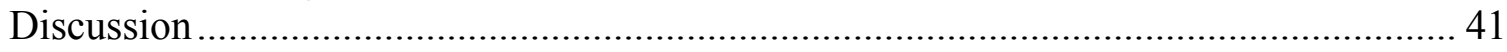

Comparison of Improved Techniques with ASTM Standard ..................................... 41

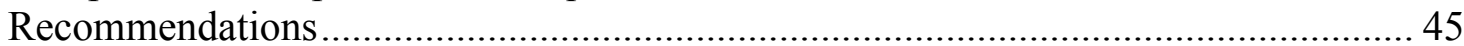

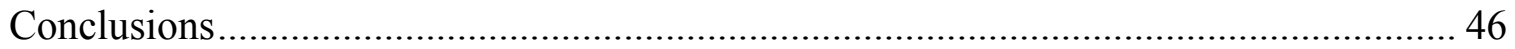

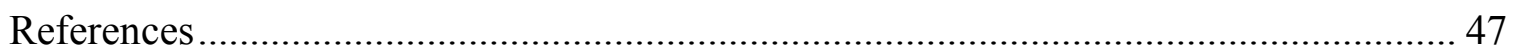

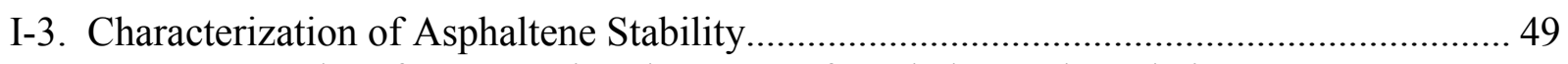

I-3.1. Procedure for Measuring the Onset of Asphaltene Flocculation .......................... 49

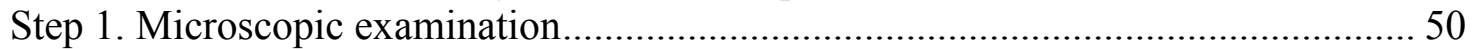

Step 2. Redispersion of asphaltene aggregates .......................................................... 51

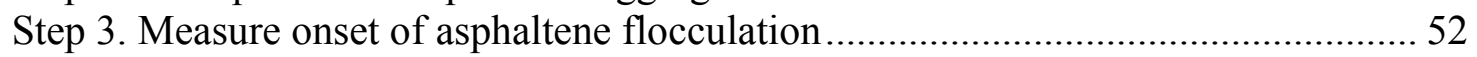

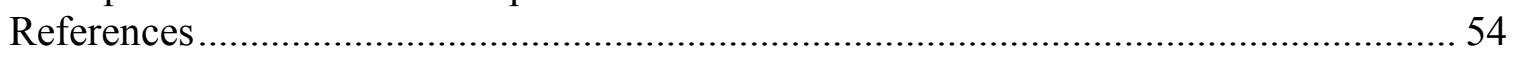

I-3.2. An Experimental Approach to Prediciton of Asphaltene Flocculation ................. 55

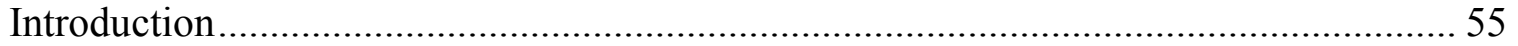

Solubility Tests of Onset of Asphaltene Flocculation .............................................. 55

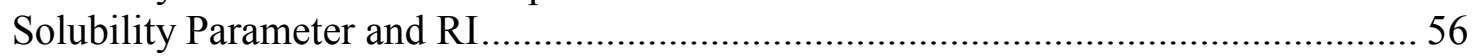

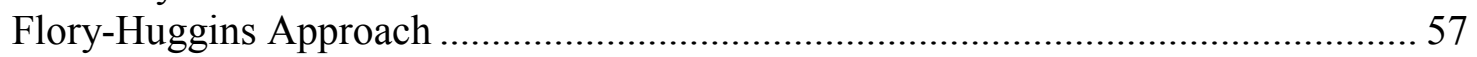

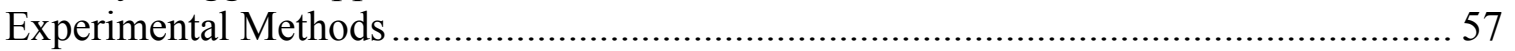

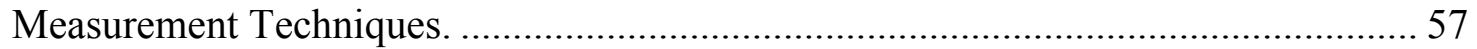

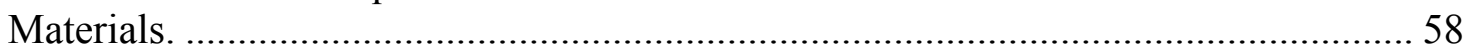

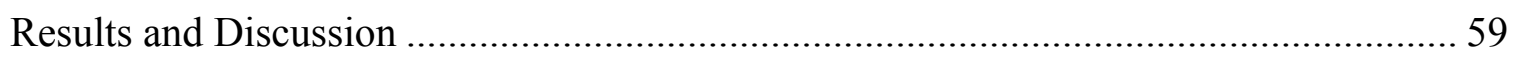

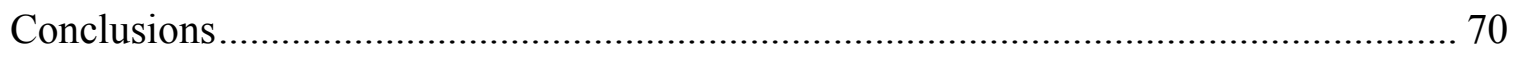

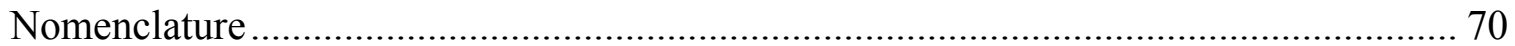

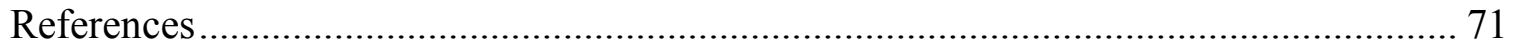

I-3.3. Thermodynamic Modeling of the Onset of Asphaltene Flocculation................... 73

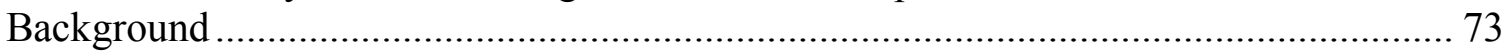

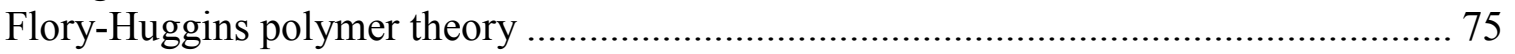

Chemical potential ................................................................................................ 79

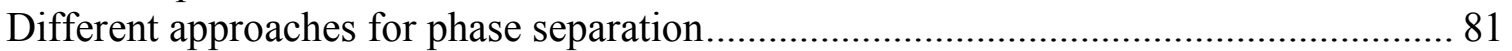

Approach assuming pure solid asphaltene phase at phase separation (Hirschberg

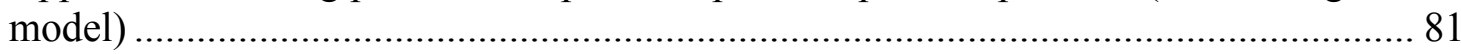

Approach assuming pure solvent phase at phase separation ...................................... 82

Proposed approach without approximations of phase compositions ............................ 83

Comparisons of these three different approaches ………........................................... 92

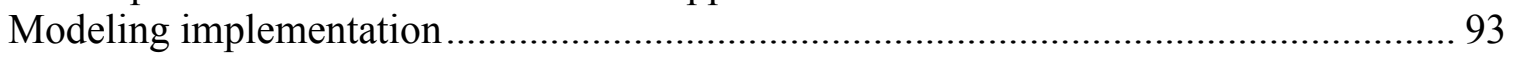

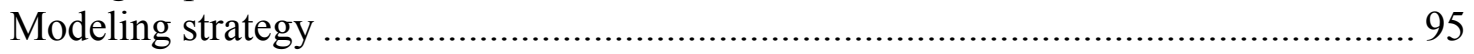

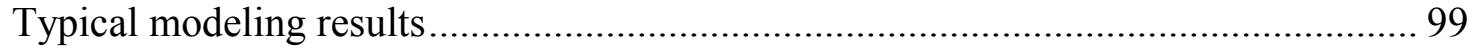

Comparison with other approaches in literature ………........................................... 102

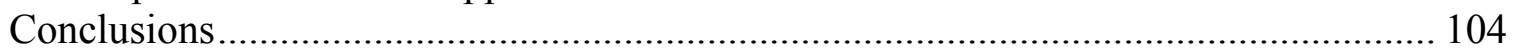

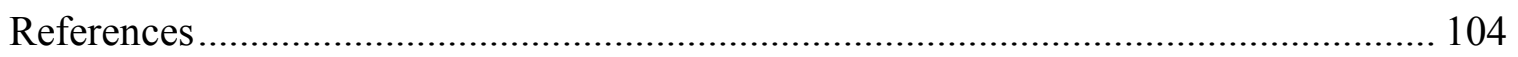

I-3.4. Asphaltene Stability in Other Crude Oil Fractions .............................................. 107

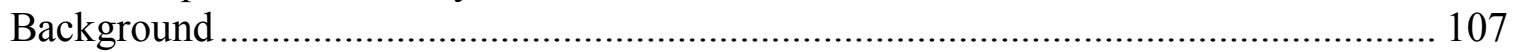

Experimental Materials and Procedures .................................................................... 108 


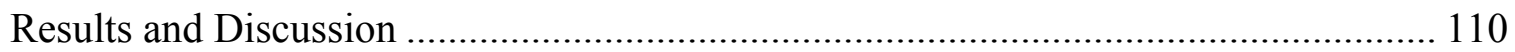

Effect of solvents on asphaltene stability in crude oil ........................................... 110

Onset of flocculation of asphaltene fractions in a solvent ........................................ 111

Effect of resins on asphaltene stability ................................................................. 112

Effect of mixing two crude oils on asphaltene stability............................................ 114

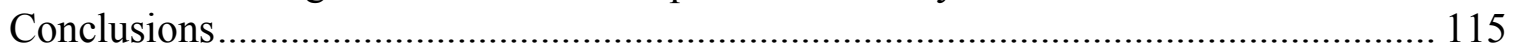

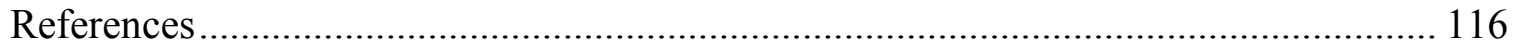

I-3.5. Simultaneous Measurement of RI and Asphaltene Onset Detection ................... 117

Materials and Methods....................................................................................... 117

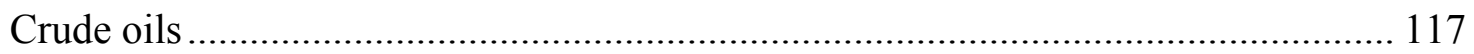

Development of RI measurement techniques ........................................................ 117

Detecting the onset of asphaltene flocculation ................................................... 122

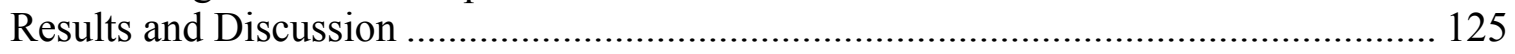

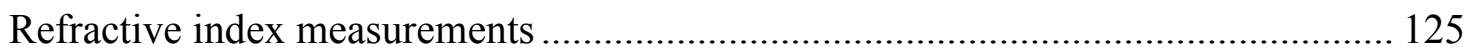

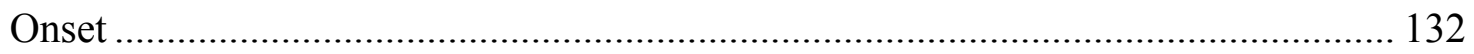

Correspondence between microscopic and light intensity onset measurements ........ 135

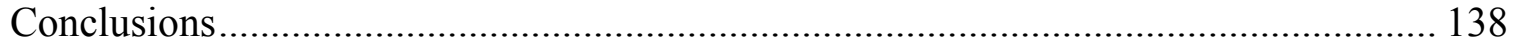

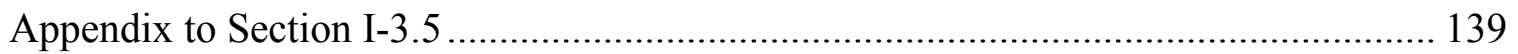

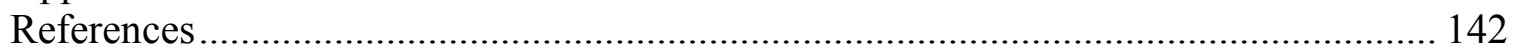

I-3.6. A Unified Approach to Asphaltene Stability Modeling …………………......... 144

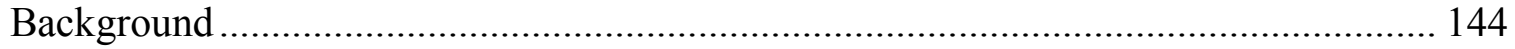

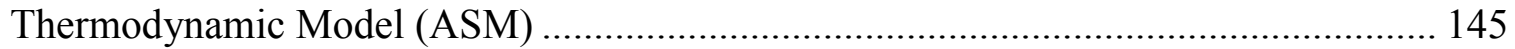

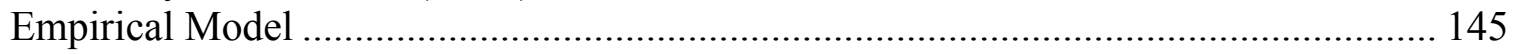

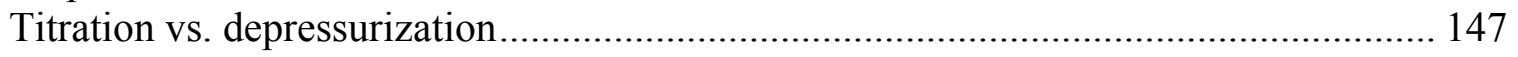

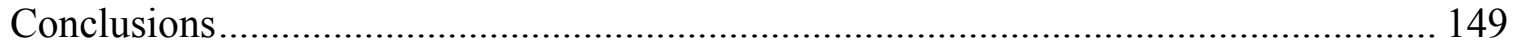

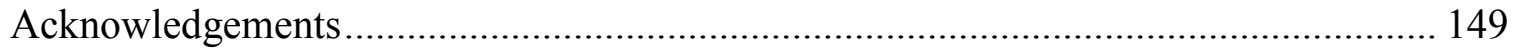

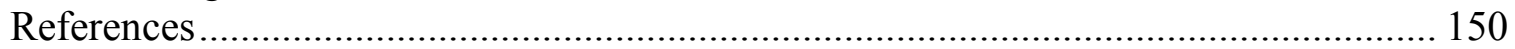

Part II — Studies of Wetting Alteration on Smooth Surfaces ...................................................... 151

II-1. Review of the Effective Wettability of Minerals Exposed to Crude Oil ................ 151

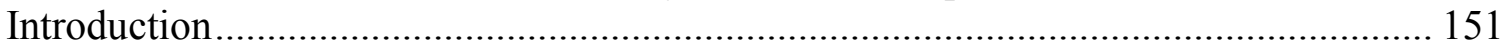

Review of Surface Studies of Effective Wetting ......................................................... 152

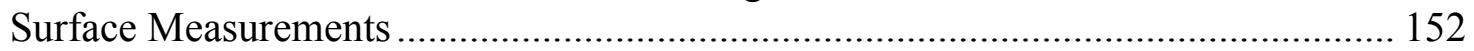

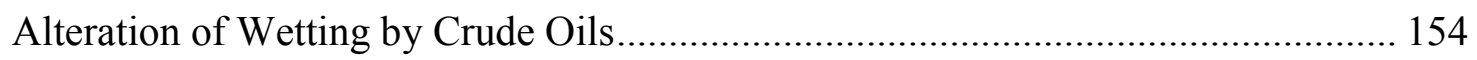

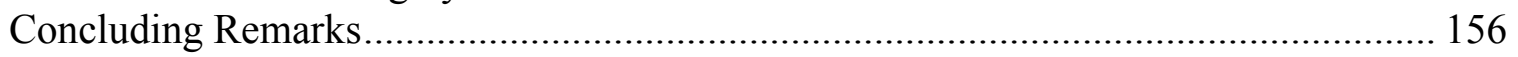

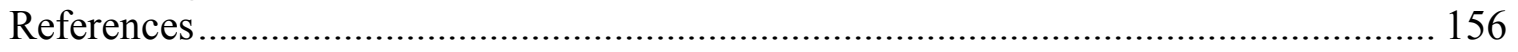

II-2. Contact Angles on Oil-Treated Surfaces .......................................................... 161

II-2.1. Effect of Crude Oil Composition on Wettability of Mica .................................. 161

Wetting Alteration by Crude Oils ..................................................................................... 161

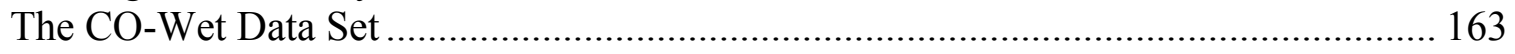

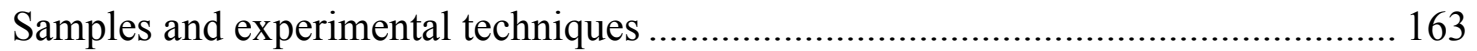

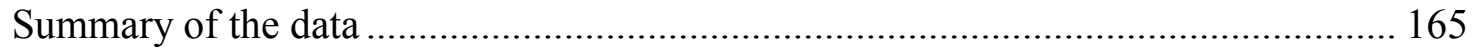

Statistical relationships among crude oil properties .................................................... 165

Relationships between Crude Oil Properties and Wettability Alteration ........................ 167

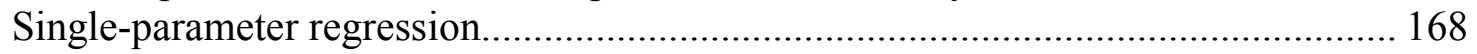

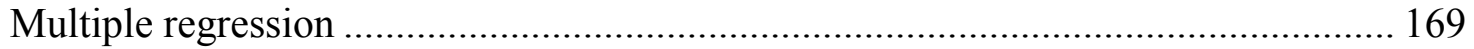

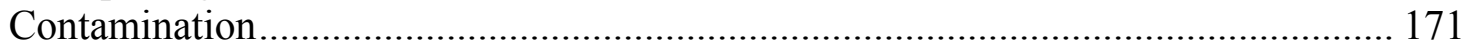




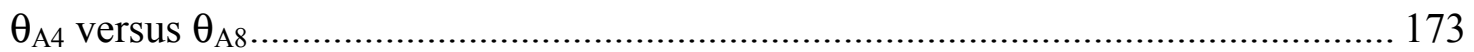

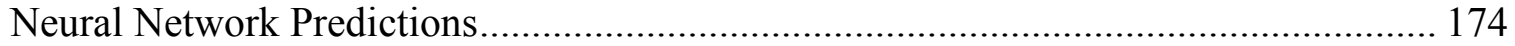

Summary and Conclusions …………………………........................................ 179

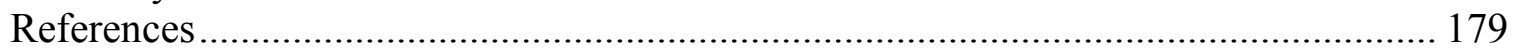

Appendix to Section II-2.1 ....................................................................................... 182

II-2.2. Contact Angle Hysteresis and the Stability of Wetting Changes Induced by

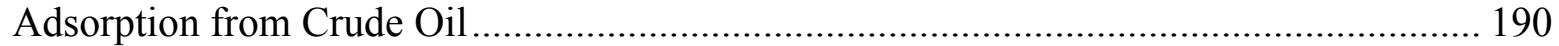

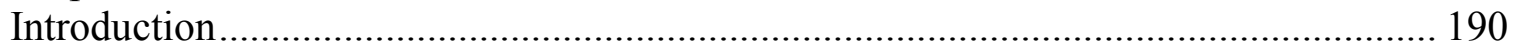

The Dynamic Wilhelmy Plate Method ....................................................................... 191

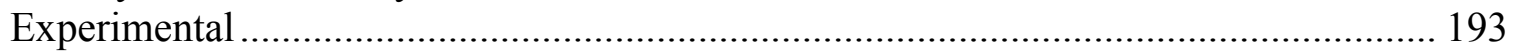

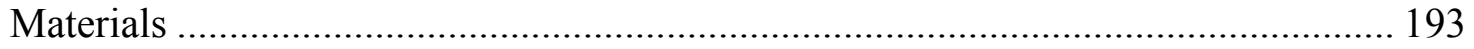

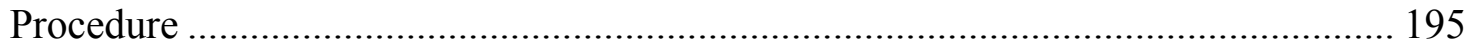

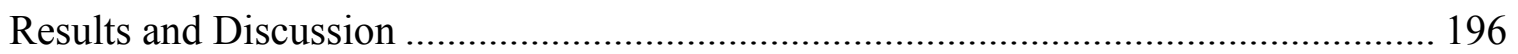

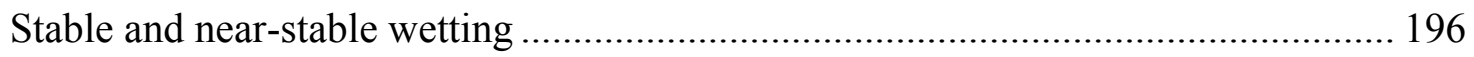

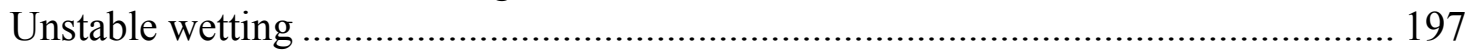

Contact angle hysteresis and crude oil properties .................................................... 199

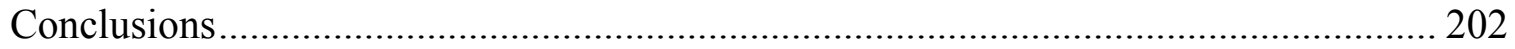

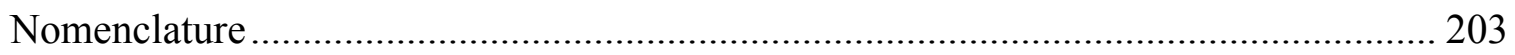

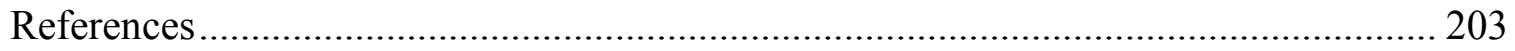

II-3. Atomic Force Microscopy of Oil-Treated Surfaces................................................. 206

II-3.1. A Methodology for Examining Crude Oil-Treated Surfaces with the Atomic Force

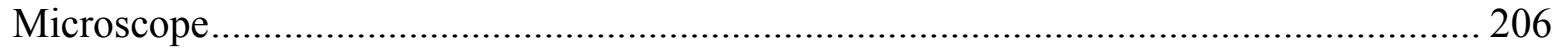

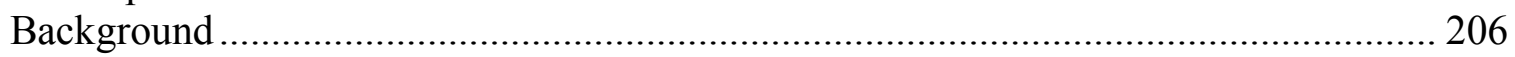

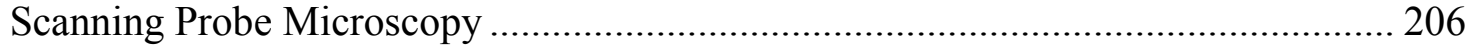

Prior Applications of AFM to Crude Oil Systems .................................................. 207

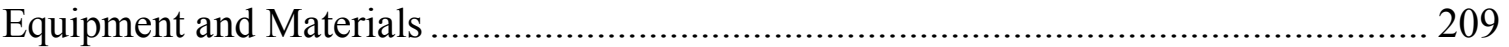

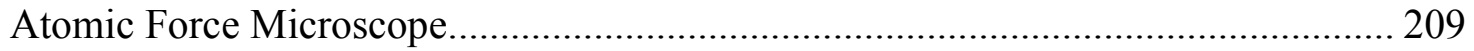

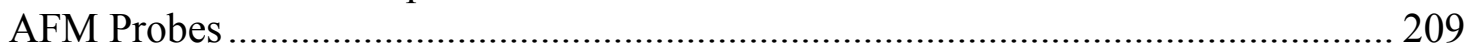

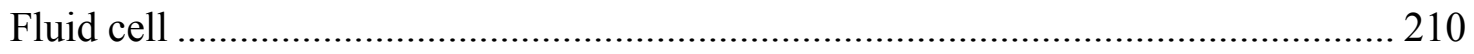

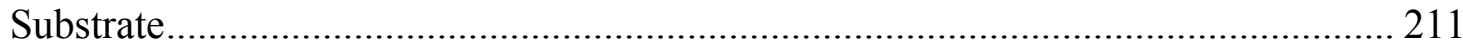

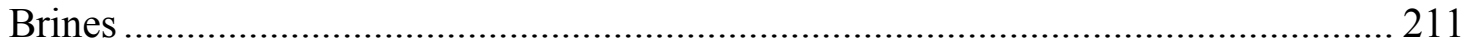

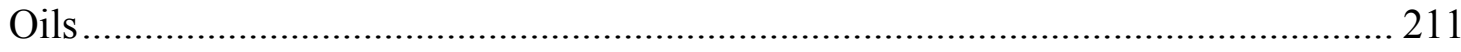

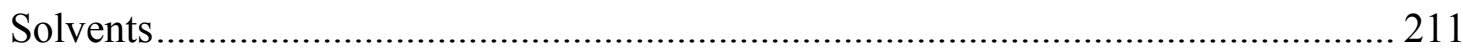

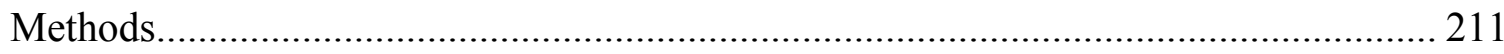

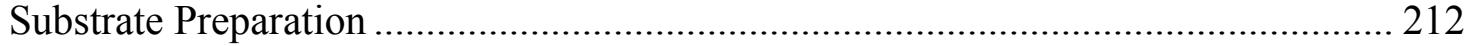

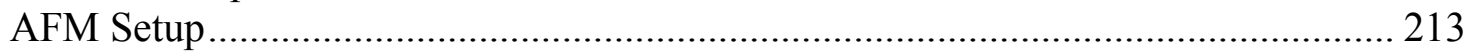

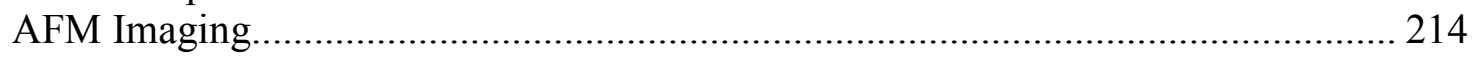

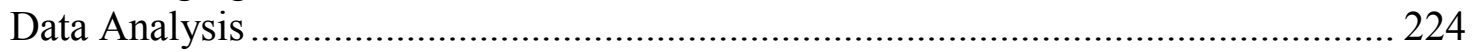

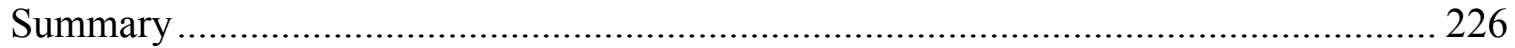

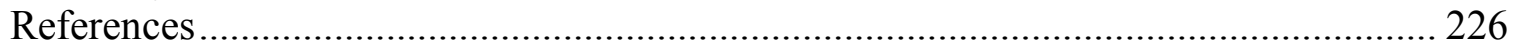

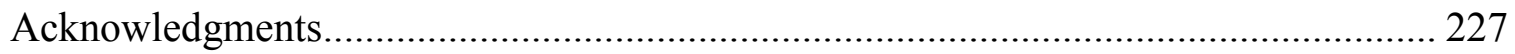

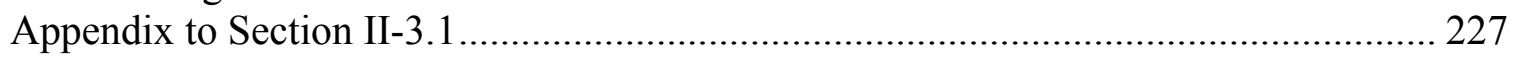

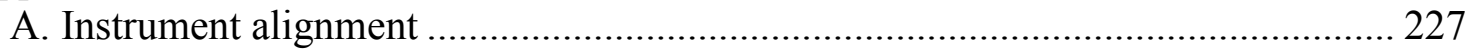

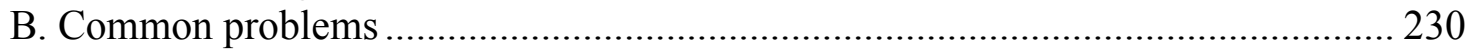

C. File management …………………............................................................. 233

II-3.2. Application of the AFM Technique to Mica Surfaces Exposed to Crude Oil .... 234 


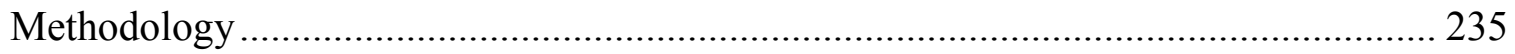

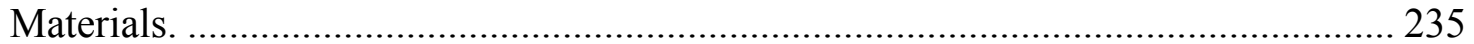

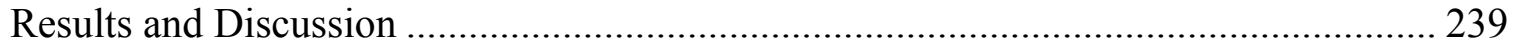

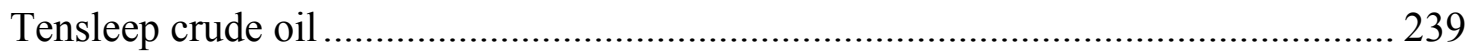

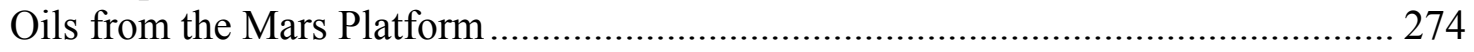

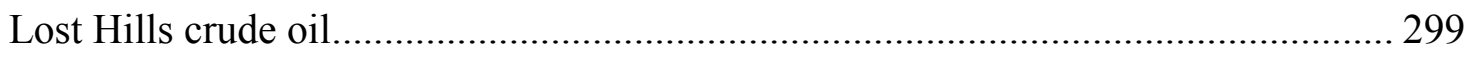

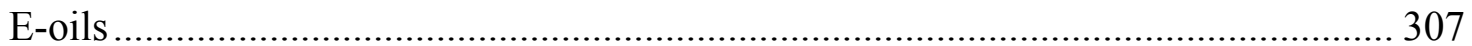

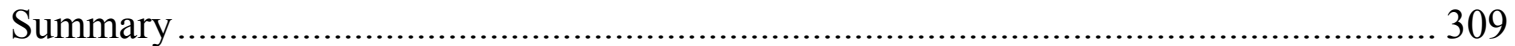

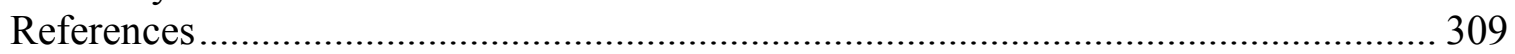

Part III—Rate of Imbibition and Assessment of Wetting in Porous Media ................................ 312

III-1. A Review of Recovery of Oil by Spontaneous Imbibition .................................... 312

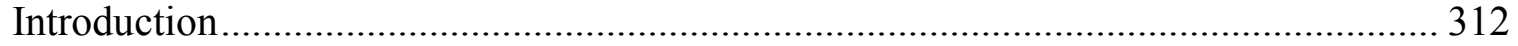

Imbibition into Completely Wetted Capillaries ............................................................... 313

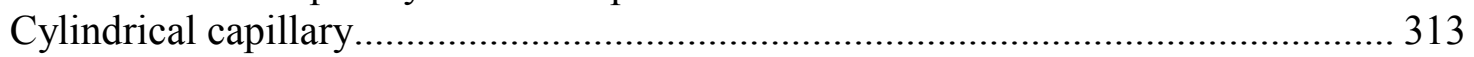

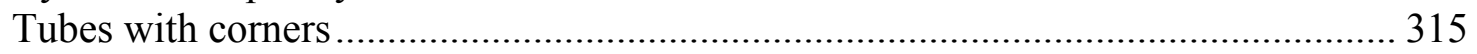

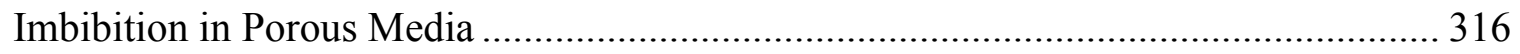

Capillary pressure versus saturation - pseudo quasistatic imbibition ......................... 316

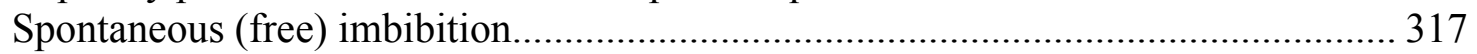

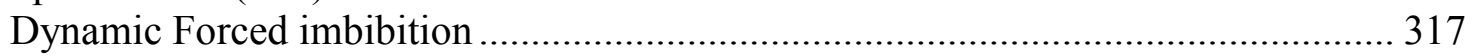

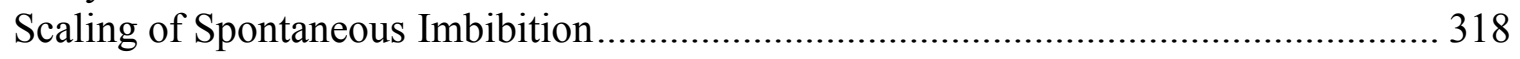

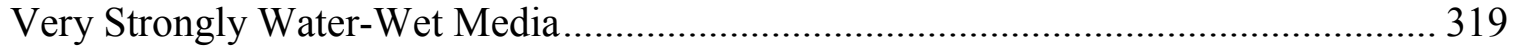

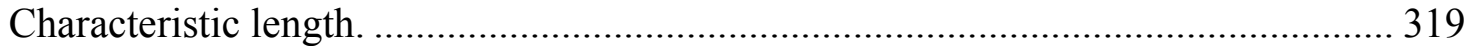

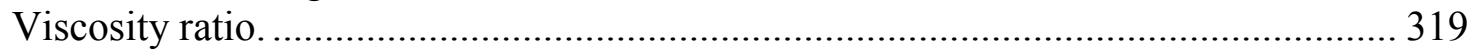

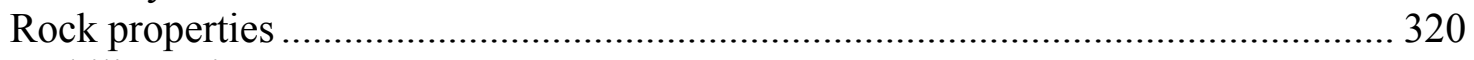

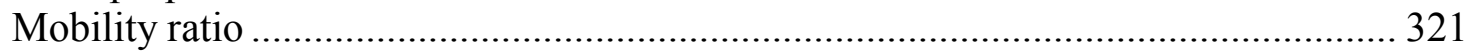

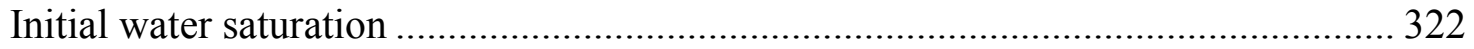

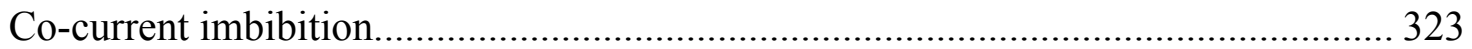

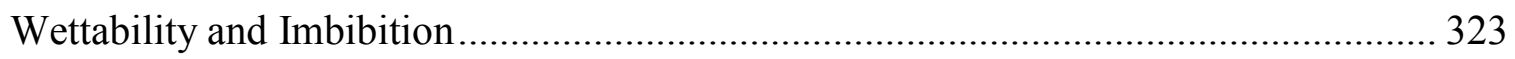

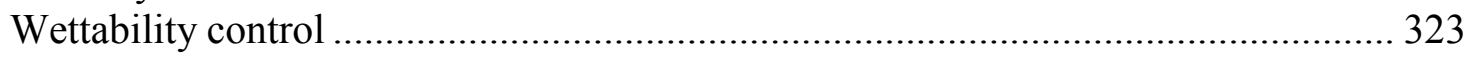

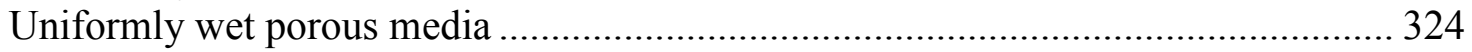

Mixed wettability .............................................................................................. 325

Large-Scale Laboratory Models and Imbibition Mechanisms....................................... 329

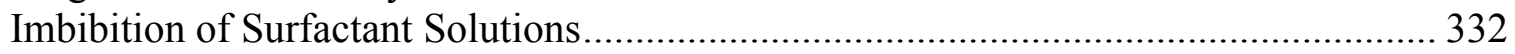

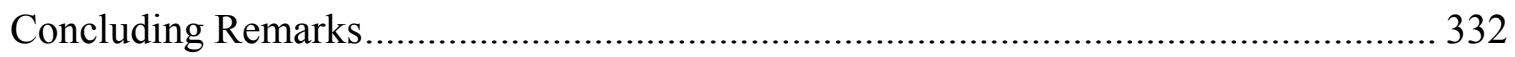

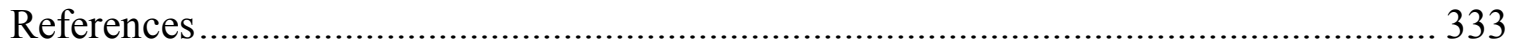

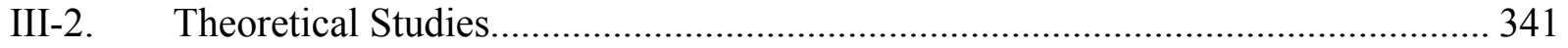

III-2.1. Simulation Study of Spontaneous Imbibition...................................................... 341

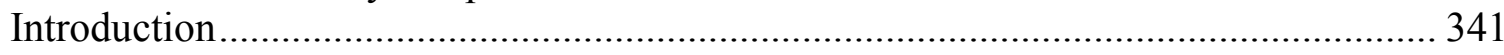

Formulation of the Numerical Simulator ...................................................................... 342

Test Parameter Specifications.................................................................................. 343

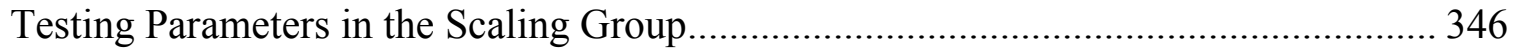

Testing Parameters Not Included in the Scaling Group................................................. 348

Capillary pressure …………………......................................................... 348

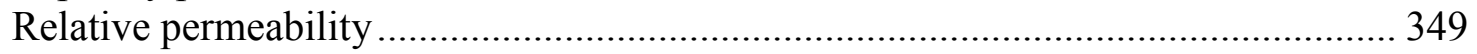

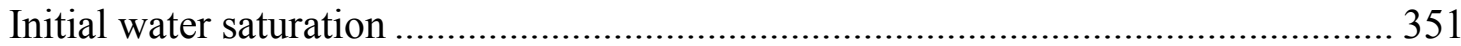

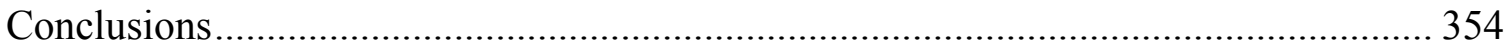




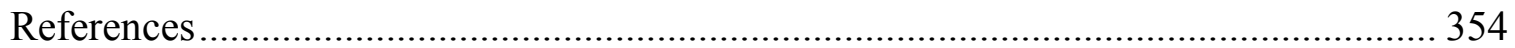

III-2.2. Similarity Solution for Linear Counter-Current Spontaneous Imbibition.............. 356

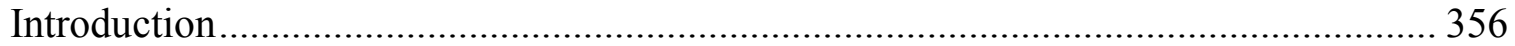

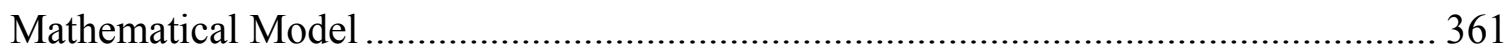

Governing flow equation for linear counter-current imbibition ............................. 362

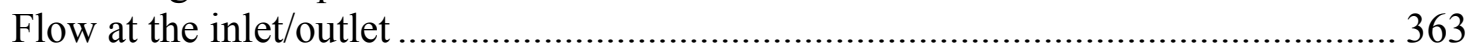

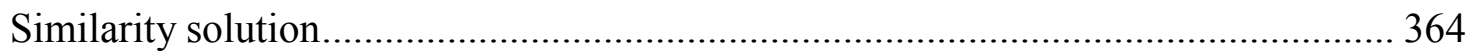

Comparison with the Buckley Leverett model of forced imbibition ......................... 365

Relationship between Swept Volume and Cumulative Imbibed Volume .................. 365

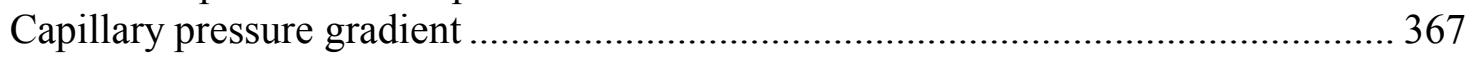

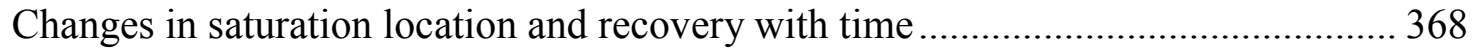

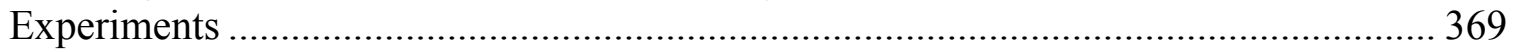

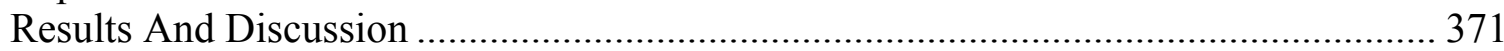

Test for similarity of the saturation profile during frontal flow............................... 372

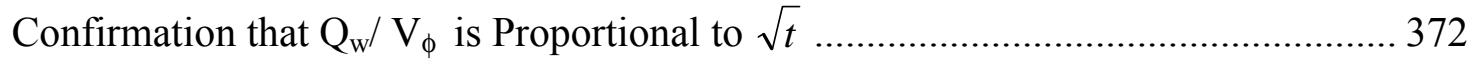

Recovery during Frontal Flow and Post Contact Flow.......................................... 373

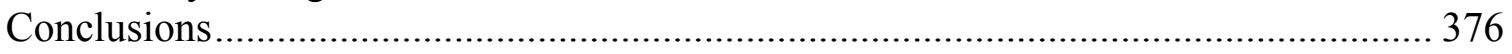

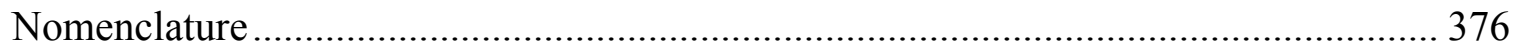

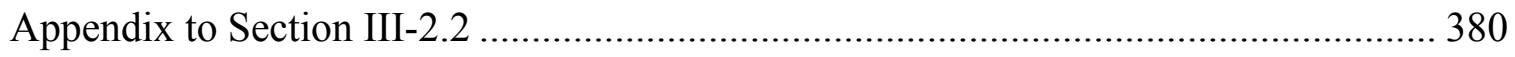

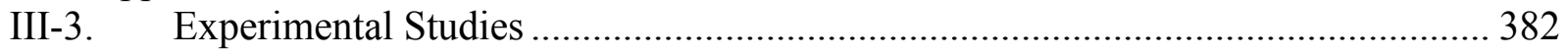

III-3.1. Test of Characteristic Length for Scaling Air/Oil/Rock Imbibition Rates (VSWW)

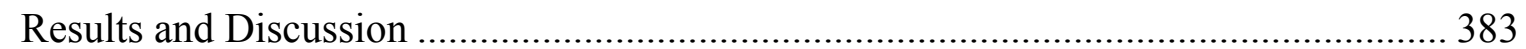

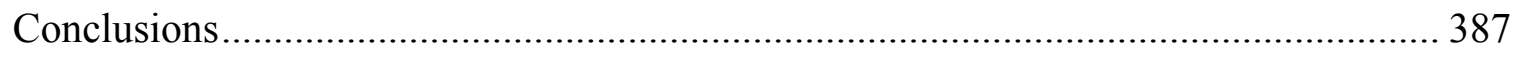

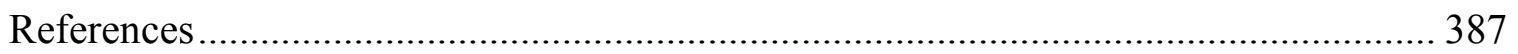

III-3.2. Oil Recovery by Spontaneous Imbibition from Weakly Water-Wet Rocks (MWX)

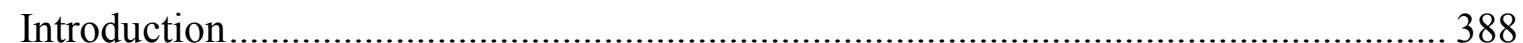

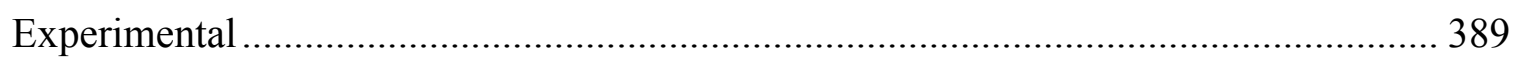

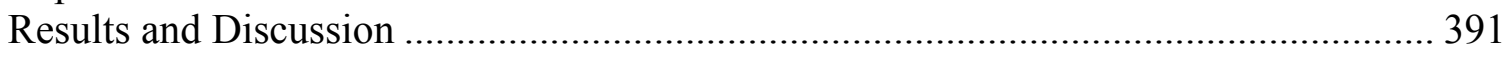

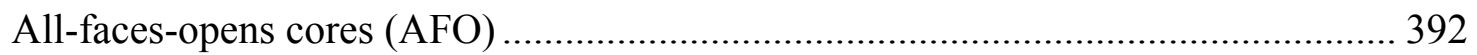

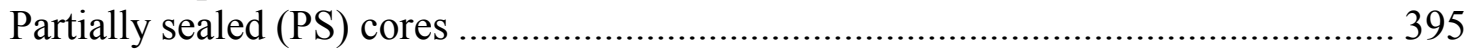

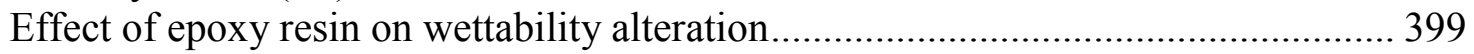

Scaling of wettability and gravity ..................................................................... 400

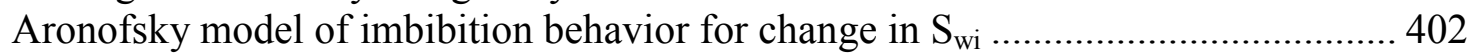

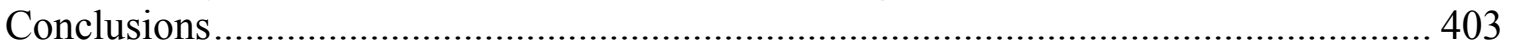

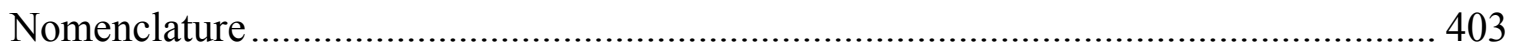

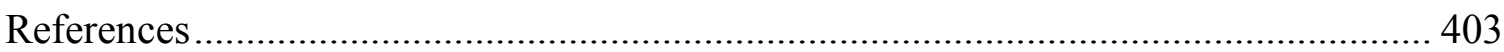

III-3.3. Scaling of Viscosity Ratio for Oil Recovery by Imbibition from Mixed-Wet Rocks

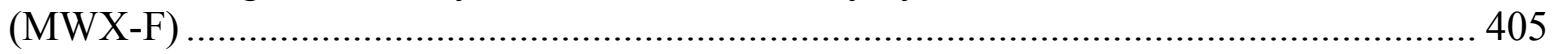

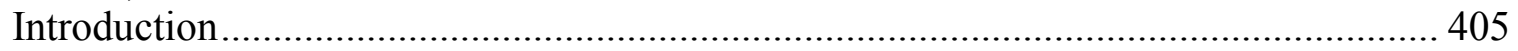

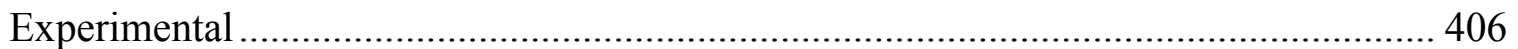

Results and Discussion ................................................................................. 408

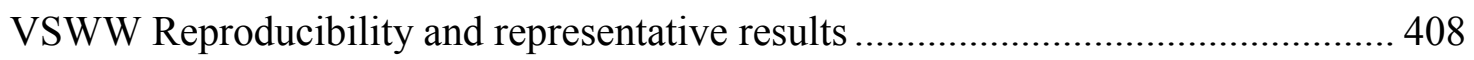

Wettability Alteration and Reproducibility of MXW spontaneous imbibition: ........ 410

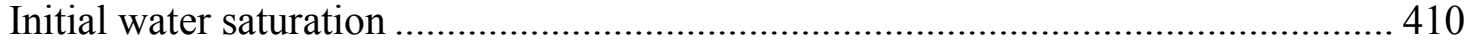




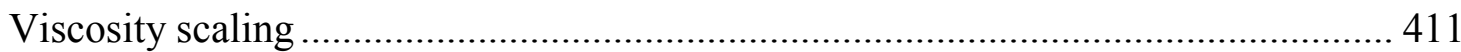

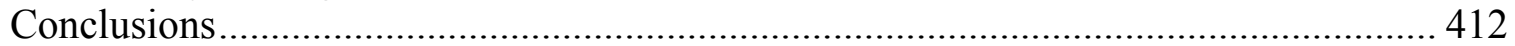

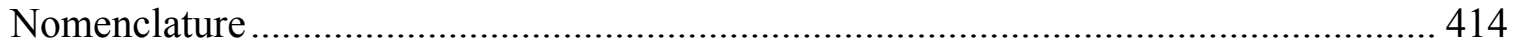

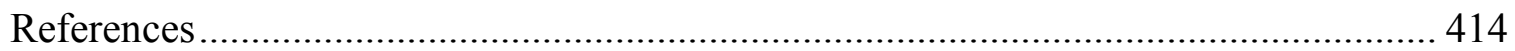

III-3.4. Spontaneous Imbibition for Mixed-Wettability States in Sandstones Induced by

Adsorption from Crude Oil (MWX-F) ....................................................................... 416

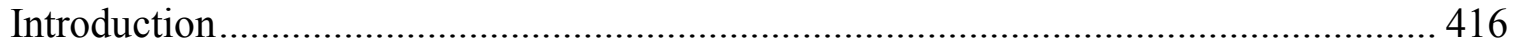

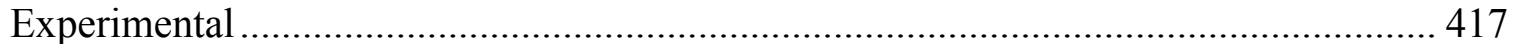

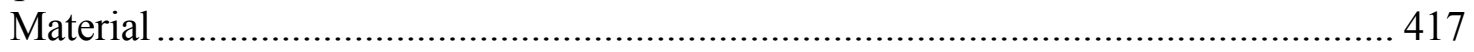

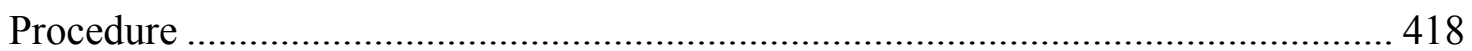

Results and Discussion .................................................................................... 420

The effect of solvent flush temperature and volume used to displace crude oil........ 420 Wettability change caused by multiple cycles of spontaneous imbibition and forced

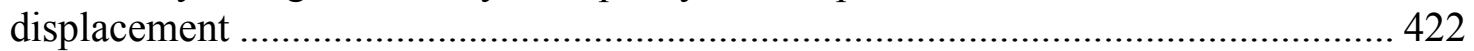

Stability of induced wetting states to flushing with solvent at ambient temperature . 425

Mixed wettability and initial water saturation ....................................................... 427

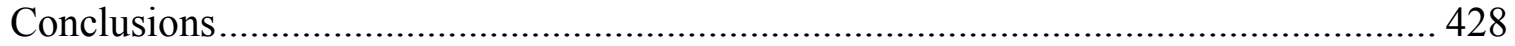

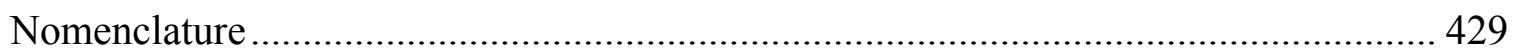

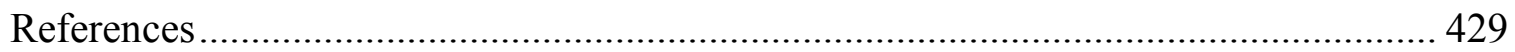

III-3.5. Crude Oil Composition and the Stability of Mixed Wettability in Sandstones

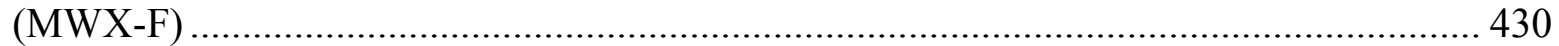

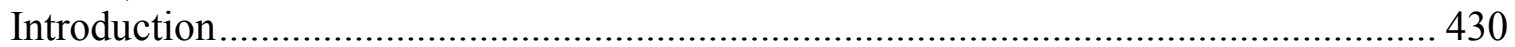

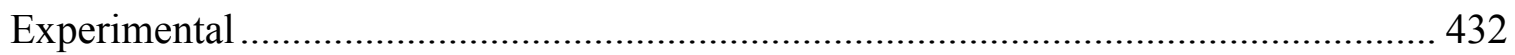

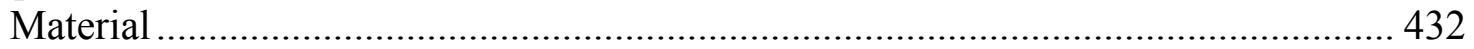

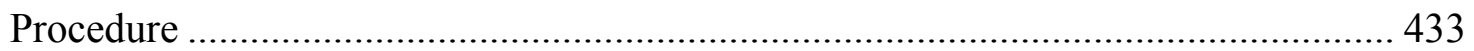

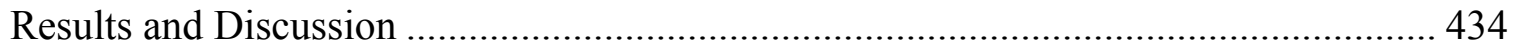

Core Samples Aged with Minnelusa Crude Oil.................................................. 435

Core Samples Aged with Gullfaks Crude Oil.......................................................... 437

Comparison of Spontaneous Imbibition with Contact Angles ..................................... 438

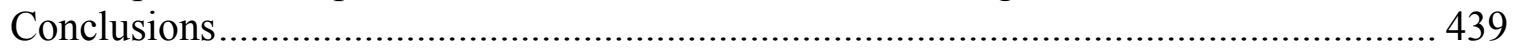

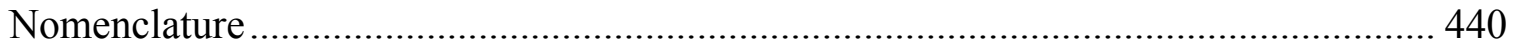

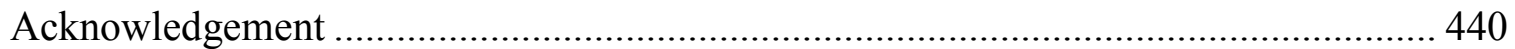

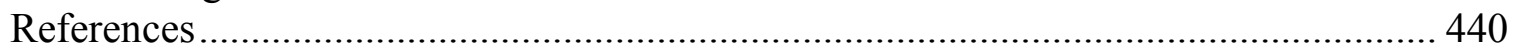




\section{List of Figures}

Figure I-1.1. Correlation between RI (sodium D line at $20^{\circ} \mathrm{C}$ ) and API gravity at $60^{\circ} \mathrm{F}$............. 4

Figure I-1.2. Acid number vs. API gravity at $60^{\circ} \mathrm{F}$ for 73 crude oil samples.............................. 5

Figure I-1.3. Base number vs. API gravity at $60^{\circ} \mathrm{F}$ for 73 crude oil samples............................... 5

Figure I-1.4. Acid number (A) vs. base number (B) for 73 samples from the CO-Wet database. Diagonals show constant ratios (B/A) of 10 and 1 . Most samples have at least as high a base number as acid number and many have base numbers that exceed their acid number by more than 10 times

\section{6}

Figure I-1.5. Asphaltene stability map for 53 crude oil samples from the CO-Wet database. Diagonals toward the upper left are less stable and those toward the lower right are more stable. (All measurements were made at $20^{\circ} \mathrm{C}$.). 7

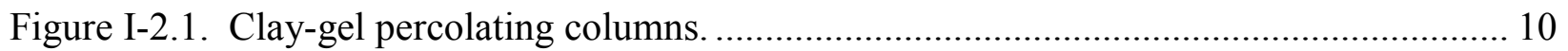

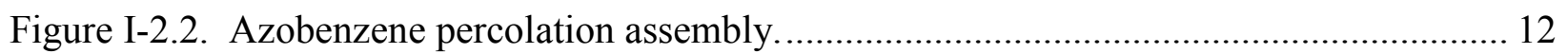

Figure I-2.3. Azobenzene absorbance standard curve. ……………………............................ 13

Figure I-2.4. Azobenzene activity test for clay............................................................... 14

Figure I-2.5. Comparison of fraction RI's for four separations................................................ 16

Figure I-2.6. Gas chromatogram and selected mass spectrum of light ends.............................. 19

Figure I-2.7. Gas chromatogram and selected mass spectrum of saturates. ............................... 20

Figure I-2.8. Gas chromatogram and selected mass spectrum of aromatics................................ 21

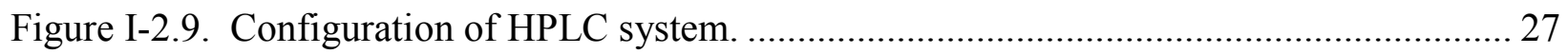

Figure I-2.10. Chromatograph of a mixture of $n$-decane, $n$-tridecane, $n$-pentadecane, and $n$ -

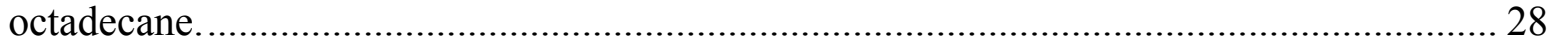

Figure I-2.11. Chromatograph of a mixture of tridecane and tetradecene.................................. 28

Figure I-2.12. Chromatograph of a mixture of toluene and diisopropylbenzene......................... 28

Figure I-2.13. Chromatographs of diisopropylbenzene and methylnaphthalene......................... 29

Figure I-2.14. Chromatographs of $n$-pentadecane, diisopropylbenzene, and methylnaphthalene. 29

Figure I-2.15. HPLC RI detector signal (area/mg) versus RI for hydrocarbons at $20^{\circ} \mathrm{C} \ldots \ldots \ldots . . .30$

Figure I-2.16. RI Values of Saturate, Aromatic and Oils versus Oils’ API Gravity at $20^{\circ} \mathrm{C} \ldots . . .30$

Figure I-2.17. Chromatographs of HPLC separation for the maltenes of crude oil C-R-00...... 32

Figure I-2.18. Weight loss $\%$ on plate without development. ............................................. 35

Figure I-2.19. Effects of spotting amount on weight loss................................................. 36

Figure I-2.20. Undeveloped spots of A-95. Numbers represent the amount of time elapsed after spotting and before the rod was scanned. 37 
Figure I-2.21. Comparison of SARA fractions determined by the ASTM method, by an improved HPLC method and by TLC-FID................................................................. 42

Figure I-2.22. Evaporative loss in TLC-FID measurements as a function of API gravity.......... 43

Figure I-2.23. Crude oils wt. loss in clay-gel method versus in TLC-FID method..................... 43

Figure I-2.24. If all of the volatile species are assumed to be saturates, the ASTM and HPLC methods produce comparable results; the TLC-FID estimates are significantly higher in

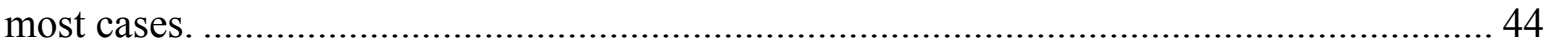

Figure I-2.25. Three estimates of the amount of resins in six crude oils................................... 45

Figure I-2.26. Three estimates of the amount of asphaltenes in six crude oils............................ 45

Figure I-3.1. Outline of the asphaltene onset determination scheme.......................................... 50

Figure I-3.2. Solubility parameter, $\delta$, of nonpolar molecules is a linear function of $F_{R I} \ldots \ldots \ldots \ldots . . .56$

Figure I-3.3. The volume of precipitant required to initiate flocculation of asphaltenes varies with oil and precipitant, but trends with precipitant chain length vary. ............................... 60

Figure I-3.4. The experimental observations in Fig. I-3.3 can be viewed in terms of the mixture $R I$ when flocculated asphaltenes are first observed $\left(P_{R I}\right)$ rather than the volume of precipitant added. $P_{R I}$ increases monotonically with chain length of the $n$-paraffin precipitating agent. Trends for seven crude oil samples are almost parallel. 61

Figure I-3.5. For a given crude oil, $P_{R I}$ is a linear function of $v_{p}{ }^{1 / 2}$, where $v_{p}$ is precipitant molar volume. Fits to these linear trends are summarized in Table I-3.3. (Chain length of the $n$ alkane precipitants is indicated on the top $\mathrm{x}$-axis.)

Figure I-3.6. $P_{R I}$ measured for Mars-Pink crude diluted with $\alpha$-MN or toluene. The precipitant is $n$-heptane. 62

Figure I-3.7. Flocculation of asphaltenes that have been separated from their crude oils and redissolved as $1 \%$ solutions in toluene. Trend of $P_{R I}$ with $v_{p}^{1 / 2}$ is linear, but slopes and values of $P_{R I}$ are different than those for the original oil samples. 63

Figure I-3.8. $P_{R I}$ is a linear function of precipitant volume fractions for three crude oils with mixtures of $n-\mathrm{C}_{9}$ and $n-\mathrm{C}_{15}$ as precipitant and for two crude oils with mixtures of $n-\mathrm{C}_{7}$ and $n-\mathrm{C}_{15}$ as precipitant. 64

Figure I-3.9. Branched alkanes follow a similar trend of $P_{R I}$ vs. $v_{p}^{1 / 2}$ to that established with $n$ alkanes. Also shown are the results of precipitation tests with the mixtures of two and three precipitants shown in Fig. I-3.8. 65

Figure I-3.10. Both $R I$ and $v_{p}$ change as $P$ decreases from the reservoir pressure $\left(P_{r e s}\right)$ to ambient conditions $\left(P_{a t m}\right)$. Below the bubblepoint $\left(P_{b}\right)$, oil composition is also changing. The arrows suggest the expected trends, although the details of the path through the $R I-v_{p}{ }^{1 / 2}$ diagram will be different for each oil. 66

Figure I-3.11. PVT data for calculation of $R I$ in Fig. I-3.12 (after McCain, 1990)..................... 68

Figure I-3.12. Example of $P_{R I}$ prediction. PVT data are for a $41.5^{\circ} \mathrm{API}$ oil at a reservoir temperature of $84^{\circ} \mathrm{C}$. The $P_{R I}$ vs $v_{p}{ }^{1 / 2}$ relationship used is that for A-93+ $\alpha$-MN (Fig. I-3.5) with a temperature adjustment of $-0.0008 R I$ units $/{ }^{\circ} \mathrm{C}$. Solution gas is estimated to be 
composed of methane, ethane, and propane with mole fractions of $0.5,0.4$, and 0.1 , respectively.

Figure I-3.13. Molar Gibbs free energy calculated from Flory-Huggins theory (Eq. I-3.27). $\mathrm{x}_{2}$ is the molar fraction of solute $\mathrm{A}$ in the mixture. The composite solvent contains $80 \%$ toluene and $20 \%$ n-heptane in volume. The solubility parameter and molar volume for each component is: 1) solute $\left.\mathrm{A}\left(\mathrm{v}_{\mathrm{a}}=160 \mathrm{ml} / \mathrm{mol}, \delta_{\mathrm{a}}=23 \mathrm{MPa}^{1 / 2}\right) ; 2\right)$ toluene $\left(\mathrm{v}_{\mathrm{s}}=106.3 \mathrm{ml} / \mathrm{mol}\right.$,

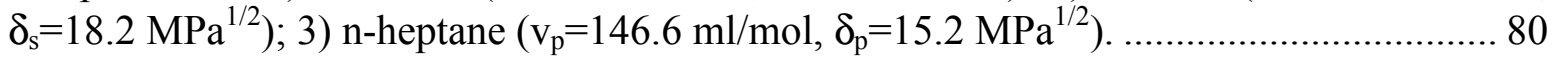

Figure I-3.14. Gibbs free energy varies with volume ratio of toluene to n-heptane in the

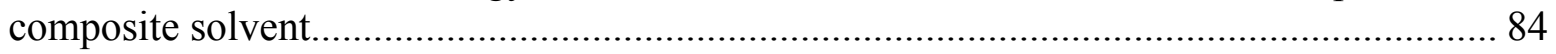

Figure I-3.15. For a Gibbs free energy curve with concave shape, the solution is stable against phase separation. Any potential phase separation would increase the overall Gibbs free energy and thus is not favored. 84

Figure I-3.16. For a Gibbs free energy curve with two local valleys, the mixture will separate into two coexisting phases if composition falls in the range between point $M_{I}$ and $M_{I I}$. At phase equilibrium, asphaltene molar fraction in each phase is given by point $\mathrm{M}_{\mathrm{I}}$ and $\mathrm{M}_{\mathrm{II}}$. 85

Figure I-3.17. Calculated lines for two-phase coexistence and inflection points (spinodal curve), for a mixture of n-C7 Mars-pink asphaltenes dissolved in toluene (1 wt \%) plus n-heptane.88

Figure I-3.18. The cross point (S) of asphaltene volume fraction line with two-phase coexistence line represents the minimum precipitant volume fraction before phase separation. The mixture will never enter absolutely unstable region in this example..... 89

Figure I-3.19. Asphaltene volume fraction in the mixture decreases linearly with addition of precipitant before it reaches a point $(\mathrm{S})$ where phase separation begins. After this point, asphaltene volume fraction in phase-I, $\phi_{\mathrm{a}, \mathrm{I}}$, decreases while asphaltene volume fraction in phase-II, $\phi_{\mathrm{a}, \mathrm{II}}$, increases. Note the significant difference between $\phi_{\mathrm{a}, \mathrm{I}}$ and $\phi_{\mathrm{a}, \mathrm{II}}$ at the phase separation point. 90

Figure I-3.20. Normalized asphaltene content in solution before phase separation, in phase I and phase II after phase separation as a function of precipitant volume fraction in the mixture. When the precipitant volume fraction is high enough, almost all asphaltenes are concentrated in phase II. 90

Figure I-3.21. A criterion to judge the visible "onset" point for asphaltene flocculation. The onset is the point when Gibbs free energy curve gives a flat inflection point. If more precipitant is added after this point, two local minima will emerge on Gibbs free energy curve. 91

Figure I-3.22. A schematic diagram to show the major differences among three different

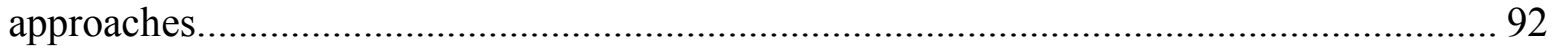

Figure I-3.23. Flow-chart for modeling algorithm....................................................... 95

Figure I-3.24. Comparison of asphaltene volume fraction in phase I, $\phi_{\mathrm{a}, \mathrm{I}}$, to the average asphaltene volume fraction in the overall mixture, $\phi_{\mathrm{a}}$, at the onset point for Mars-pink crude oil indicates that there is a limit on the combination of molar volume and solubility parameter that can be used to make physically realistic predictions. 97 
Figure I-3.25. Pairs of asphaltene molar volume, $\mathrm{v}_{\mathrm{a}}$, and solubility parameter, $\delta_{\mathrm{a}}$, that best match the predicted onset $\mathrm{P}_{\mathrm{RI}}$ to measured ones. Beyond this line, precipitant volume fraction is underestimated with respect to the onset condition, while under this line it is overestimated (Mars-pink crude oil)..... 98

Figure I-3.26. For Mars-pink crude oil, a good match for the calculated visible onset to measured aggregate onset is achieved when appropriate asphaltene properties are selected. Measured fine onset falls within the metastable region. 100

Figure I-3.27. Measured and predicted onset conditions for flocculation from crude oils induced by precipitants from n-pentane to n-pentadecane. Asphaltene molar volume $=2500 \mathrm{ml} / \mathrm{mol}$. Other model parameters are given in Table 2. 101

Figure I-3.28. Good matches can be obtained between calculated visible onsets and measured aggregate onsets for all $1 \%$ asphaltene solutions in toluene. Asphaltene molar volume is held constant, while the solubility parameter is adjusted. 102

Figure I-3.29. Comparisons of predicted results from three different approaches (Mars-pink crude oil, $\left.\mathrm{v}_{\mathrm{a}}=2500 \mathrm{ml} / \mathrm{mol}\right)$. 103

Figure I-3.30. Addition of solvent to SQ-95 crude oil can change the onset $\mathrm{P}_{\mathrm{RI}}$. For precipitation with n-heptane, shifts are greater with toluene than AMN. Lines are ASM calculations. 110

Figure I-3.31. $\mathrm{P}_{\mathrm{RI}}$ for $1 \%$ toluene solutions of asphaltenes separated from Lagrave and MarsPink crude oils. Stabilities of redissolved asphaltenes depend on the identity of the n-alkane used to separate them. $n-C_{5}$ asphaltenes are the most stable; $n-C_{15}$ asphaltene are least stable. Lines are linear fits to the data points. 112

Figure I-3.32. Addition of resin reduces $\mathrm{P}_{\mathrm{RI}}$, indicating that the asphaltenes are more stable. The effect of resin is similar to the effect of precipitating asphaltenes with broader spectrum of compositions. Lines are linear fits to the data points. 113

Figure I-3.33. Addition of $\mathrm{n}-\mathrm{C}_{5}$ SQ-95 resin to $1 \% \mathrm{n}-\mathrm{C}_{5}$ SQ-95 or Mars-Pink asphaltene solutions in toluene decreases onset $\mathrm{P}_{\mathrm{RI}}$. No critical resin concentration was found for any of the six n-alkane precipitants. 113

Figure I-3.34. $\mathrm{P}_{\mathrm{RI}}$ for a mixtures of two crude oils or their asphaltene solutions lies between the values of $\mathrm{P}_{\mathrm{RI}}$ for the two components. Lines are calculations from ASM solubility model.

Figure I-3.35. Absorptive reflective transmission through a glass window cell filled with oil. 118

Figure I-3.36. Emission spectrum of the $930 \mathrm{~nm}$ LED...................................................... 120

Figure I-3.37. Simple arrangement for testing reflection from the glass-liquid interface. ........ 121

Figure I-3.38. Schematic illustration of apparatus for measurement of reflected signal intensity. 122

Figure I-3.39. Schematic illustration of apparatus for measurement of precipitation onset..... 123

Figure I-3.40. Close-up view of knotted mixer.......................................................... 124

Figure I-3.41. Reflected light intensity data obtained with $\mathrm{NaCl}$ solutions at $891 \mathrm{~nm}$. 125 
Figure I-3.42. Fit to Eq. I-3.76 over a large RI range, using a glass window. Data go through a minimum at the RI of glass.

Figure I-3.43. Fit to Eq. I-3.76 over a large RI range, using a sapphire window..................... 127

Figure I-3.44. Calibration for $940 \mathrm{~nm}$ LED with RI measurements at $589 \mathrm{~nm}$ (Abbe refractometer). 128

Figure I-3.45. RI calculated from reflected light intensities for known compounds as a function of wavelength. 130

Figure I-3.46. Reflected light intensities for mixtures of toluene and E-1XR-00 crude oil, both of which have RI values of 1.4922, as measured at $660 \mathrm{~nm}$ by Abbe refractometer...... 130

Figure I-3.47. Absorbance of mixtures of toluene and E-1XR-00 crude oil at $660 \mathrm{~nm}$ (blue diamonds) and $910 \mathrm{~nm}$ (red circles).

Figure I-3.48. Absorbance of E-1XR-00 crude oil as a function of wavelength.

Figure I-3.49. Absorbance of mixtures of an $0.25 \%$ bromothymol blue solution in $0.1 M \mathrm{NaOH}$ with the $0.1 M \mathrm{NaOH}$ solution alone show a nonlinear decrease in absorbance. 132

Figure I-3.50. Absorbance is a linear function of volume fractions if no precipitation occurs in the mixture. 133

Figure I-3.51. Absorbance-composition plot for mixtures of SQ-95 oil with hexane at $940 \mathrm{~nm}$, with pump flow rate of $0.9569 \mathrm{~mL} / \mathrm{min}$. 133

Figure I-3.52. Reflection intensity for mixtures of E-1XD-00 crude oil and n-hexane, measured at $660 \mathrm{~nm}$, flow rate $=0.9569 \mathrm{ml} / \mathrm{min}$. 134

Figure I-3.53. Onset of precipitation is a function of residence time (higher flow rates correspond to shorter residence times). 135

Figure I-3.54. Reflected light intensity of mixtures of SQ-95 and n-hexane. Microscopic examination of the mixtures for the existence of flocculated asphaltenes show that reflected light intensity begins to fall before the first fine asphaltic particles can be seen. 136

Figure I-3.55. Reflected light intensity of mixtures of C-LH-99 and n-hexane. Microscopic examination of the mixtures for the existence of flocculated asphaltenes show that reflected light intensity begins to fall long before the first fine asphaltic particles can be seen. ...... 136

Figure I-3.56. Reflected light intensity of mixtures of S-Ven-39 and 1-MN with n-hexane. In this case, microscopic examination of the mixtures for the existence of flocculated asphaltenes reveals the presence of the first fine asphaltic particles before the reflected light intensity begins to fall, whereas aggregates can only be found well after the break in the intensity curve. 137

Figure I-3.57. Effect of adsorption is illustrated by decreasing reflected intensities in successive measurements with mixtures of SQ-95 and n-hexane. 138

Figure I-3.58. Reflected light measurement arrangement using a flow-through cell................ 139

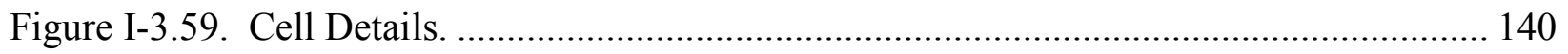

Figure I-3.60. Referenced LED reflected light detection configuration ..................................... 141 
Figure I-3.61. Two samples of oil from a single reservoir have very different asphaltene stability characteristics. ASM fits to experimental data measured at $20^{\circ} \mathrm{C}$ show that the asphaltene solubility parameters for C-T-01 and C-T-02 are 20.88 and $20.18(\mathrm{MPa})^{1 / 2}$, respectively, if the asphaltene molar volume is assumed to be $2500 \mathrm{ml} / \mathrm{mole}$. 146

Figure I-3.62. Linear fits to experimental data where $P_{R I}$ is plotted as a function of precipitant molar volume, $\mathrm{v}_{\mathrm{p}}{ }^{1 / 2}$, for the results shown in Fig. I-3.61. 146

Figure I-3.63. Onset characterization of a mixture of $1 \% n-C_{7}$ asphaltenes from Oil-R dissolved in toluene. The partial molar volume of methane is roughly in the range indicated and is a function of pressure, temperature, and composition.

Figure I-3.64. Experimental and predicted onset pressures for a series of mixtures of n-C7 asphaltenes from Oil-R, toluene, and methane. (Experimental data from Ting et al., 2002)

Figure I-3.65. Calculated values of live oil RI compared to predicted onset values of PRI show that asphaltenes from $\mathrm{C}-\mathrm{T}-01$ are likely to be unstable during depressurization whereas those from C-T-02 are likely to be stable. The fact that these two samples are from the same reservoir emphasizes the importance of sample quality in the assessment process. . 149

Figure II-2.1. Refractive index is closely related to oil density. 165

Figure II-2.2. Acid and base numbers tend to be higher for lower gravity oils, but are not closely correlated with gravity or density. 166

Figure II-2.3. Tests of crude oil wetting: (a) map of adhesion of oil under $\mathrm{NaCl}$ brines of varying $\mathrm{pH}$ and ionic composition and (b) contact angles between probe liquids (water and decane) on surfaces aged first in brine, then in crude oil..... 168

Figure II-2.4. Comparisons of measured water-advancing contact angles with those calculated from equations with the coefficients given in Table II-2.3. The $\theta_{\mathrm{A} 4}$ case respresents conditions selected to minimize water film stability; $\theta_{\mathrm{A} 8}$ values are measured for the case where stable water films are more likely.

171

Figure II-2.5. Comparisons of measured water-advancing contact angles with those calculated from equations with the coefficients given in Table II-2.4. Samples with more than $3 \mathrm{ppm}$ $\mathrm{Zn}$ were removed from the data set. 172

Figure II-2.6. The highest values of $\theta_{\mathrm{A} 4}$ tend to correlate weakly with lower values of $\theta_{\mathrm{A} 8}$. The trend is masked by including oils with $\mathrm{Zn}$ concentrations $>3 \mathrm{ppm}$ (a), but is more apparent when the $\mathrm{Zn}>3$ samples are removed from the data set (b). 174

Figure II-2.7. Architecture of a BP neural network with one input layer (3nodes), two hidden layers (5 nodes each), and one output layer (two nodes). 175

Figure II-2.8. Example of the "memorization" capability of a BP net. Using (nC7 asph\%, resins, $\mathrm{dRI}$, IEP) as the input vector, the trained BP net can efficiently "remember" each corresponding contact angle $\theta_{\mathrm{A} 4}$, i.e., the calculated $\theta_{\mathrm{A} 4}$ matches the measured $\theta_{\mathrm{A} 4}$ very well, even though the original data are quite scattering. Even better results could be obtained if more time and iterations were allowed. The net is composed of one input layer (4 nodes), one hidden layer (5 nodes), and one output layer (one node). 177 
Figure II-2.9. The best training results from BP neural network by using $90 \%$ of data set for training while the rest $10 \%$ data set for testing. Variables selected on the basis of multiple linear regression analyses: (a) $\theta_{\mathrm{A} 4}=\theta_{\mathrm{A} 4}$ (nC7 asph\%, resins, dRI, IEP). Total sample number $=49$. (b) $\theta_{\mathrm{A} 8}=\theta_{\mathrm{A} 8}(\mathrm{nC} 7 \mathrm{asph} \%, \mathrm{~B} / \mathrm{A})$. Total sample number $=58$. 178

Figure II-2.10. One realization of a network trained to predict $\theta_{\mathrm{A} 4}$ from percentages of $\mathrm{nC}_{7}$ asphaltenes and resins, RI, and acid and base numbers. 178

Figure II-2.11. Schematic force-distance diagrams and contact angles for multiple cycles. Advancing contact angles are indicated as solid symbols and receding angles as open symbols. Results for the plate that was initially in decane (loops around the path outlined by OSTMO) are indicated by square symbols; circles correspond to the plate that was initially in brine (loops around OMNPO). The first measured contact angle is indicated at the first half of a wetting cycle (receding for the plate that was initially in brine and advancing for the plate that was initially in decane). Consecutive advancing and receding angles follow the wetting cycle sequence. 192

Figure II-2.12. Plate treatment and Wilhelmy plate measurement of advancing and receding contact angles. 194

Figure II-2.13. Force-distance diagrams for plates prewetted in $0.1 \mathrm{M} \mathrm{NaCl}$ brine, then aged in A95 crude oil for 10 days at $80^{\circ} \mathrm{C}$. 196

Figure II-2.14. Stable contact angles. Clean plates were prewetted with $0.1 \mathrm{M} \mathrm{NaCl}$ brine, then aged in the designated crude oils at $74-80^{\circ} \mathrm{C}$. 197

Figure II-2.15. Example of wetting loops with advancing contact angles that decrease with each measurement cycle. Clean plates were prewetted with $0.1 \mathrm{M} \mathrm{NaCl}$ brine, then aged in SQ95 crude oil for 10 days at $75^{\circ} \mathrm{C}$. 198

Figure II-2.16. Example of wetting loops with unstable receding and advancing contact angles. Clean plates were prewetted with $0.1 \mathrm{M} \mathrm{NaCl}$ brine, then aged in Gullfaks-96 crude oil for 10 days at $75^{\circ} \mathrm{C}$. 198

Figure II-2.17. Contact angles that change with wetting cycles. Clean plates were prewetted with $0.1 \mathrm{M} \mathrm{NaCl}$ brine, then aged in designated crude oil at $74-80^{\circ} \mathrm{C}$ for 10 days. 200

Figure II-2.18. Contact angles on quartz plates treated with $0.1 \mathrm{M} \mathrm{NaCl}$ and Gullfaks-96 for 10 days at $75^{\circ} \mathrm{C}$. Similar results were obtained whether cycles were measured continuously or whether they were interrupted by periods during which the plates were soaked in brine (two hours after the first cycle, five hours after the third cycle). 201

Figure II-2.19. Acid and base numbers of crude oils, sorted in order of decreasing n-heptane asphaltene content. Note that the scales for acid and base number are different. Oil properties fall into two groups. Above the dashed line, asphaltene content is greater than $2 \%$ and amounts of either acids or bases or both are high. Below the line, amounts of asphaltene are less than $1.5 \%$ and amounts of acids and bases are lower. 201

Figure II-3.1. SEM micrographs of two common AFM tips. Images were obtained from the

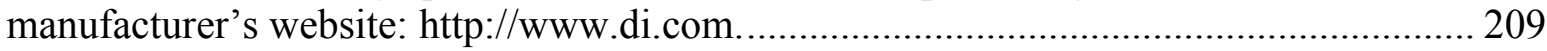

Figure II-3.2. Schematic of OTR4 probe mini-wafer with four $\mathrm{Si}_{3} \mathrm{~N}_{4}$ cantilevers. ................. 210 
Figure II-3.3. Sample vial used to age mica substrate in oil phase. 212

Figure II-3.4. Schematic of typical SPM installation.

213

Figure II-3.5. Cut-away view of tip, sample, and laser configuration. Laser must be manually aligned so that it reflects off the top of the cantilever and onto the photodetector in the AFM head. 214

Figure II-3.6. View of control monitor with suggested starting parameters for CM-AFM imaging of oil-treated mica surface in air, water, or decane imaging medium..... 215

Figure II-3.7. Force calibration plot which relates substrate z-position to cantilever deflection. Scanning force may be minimized by lowering the setpoint, an adjustable scanning parameter, to just above the "contact" point. 216

Figure II-3.8. Simultaneous height (left) and deflection (right) signals for AFM scan of mica exposed to Tensleep crude oil. Scan parameters were optimized for height signal. ........ 218

Figure II-3.9. Simultaneous height (left) and deflection (right) signals for AFM scan of mica exposed to Tensleep crude oil. Scan parameters were optimized for deflection signal. ... 219

Figure II-3.10. AFM scan of mica exposed to a $1 \mathrm{wt} . \%$ solution of Tensleep asphaltenes in toluene.

Figure II-3.11. AFM scan of mica exposed to a 1 wt.\% solution of Tensleep asphaltenes in toluene...... 220

Figure II-3.12. AFM scan of mica exposed to Tensleep crude for 1 day. ................................ 221

Figure II-3.13. AFM scan of mica exposed to Tensleep crude for 1 day. .............................. 222

Figure II-3.14. AFM scan of mica exposed to Mars Yellow crude for 99 days. ...................... 223

Figure II-3.15. AFM scan of mica exposed to Mars Yellow crude for 99 days. ...................... 223

Figure II-3.16. Section analysis of AFM height signal image of mica exposed to Mars Yellow crude for 99 days. The vertical distance between red cursors indicates a film thickness of about $19 \mathrm{~nm}$ in this section. 224

Figure II-3.17. Section analysis of AFM deflection signal image of mica exposed to Mars

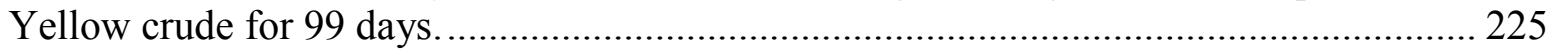

Figure II-3.18. AFM base, scanner, and head assembly .............................................. 228

Figure II-3.19. Side view of instrument alignment with fluid cell, cantilever/tip, mica sample, and imaging medium in place. 230

Figure II-3.20. Contact mode AFM deflection image of mica exposed to Mars Yellow crude for 1 day. Excessive tip-sample adhesion caused the poor image quality............................ 232

Figure II-3.21. Tapping mode AFM height image of mica exposed to Mars Yellow crude for 1 day. Excessive tip-sample adhesion caused the poor image quality ................................ 232

Figure II-3.22. AFM scan of clean mica....................................................................... 236

Figure II-3.23. AFM scan of $\{3.8,0.01\}$ acetate brine dried on mica, imaged under air......... 237

Figure II-3.24. AFM scan of $\{3.8,0.01\}$ acetate brine dried on mica, imaged under decane... 237 
Figure II-3.25. Optical microscope image of Tensleep crude. Asphaltene aggregates appear as dark particulate material while oil background color is light gray................................... 240

Figure II-3.26. AFM scan of cationic surfactant on mica at $2 \times \mathrm{CMC}$. Cylindrical surface micelles run diagonally in above scan. Molecule length of $\sim 2.3 \mathrm{~nm}$, corresponds to radii of cylinders. 240

Figure II-3.27. AFM scan of mica exposed to Tensleep crude. 241

Figure II-3.28. AFM scan of mica exposed to Tensleep crude. A zoom of the area indicated by the box is shown in Figure II-3.30. 242

Figure II-3.29. Section analysis of scan 08111204. The cursors were placed in order to determine the film thickness at the edge of a hole. The vertical distance between the cursor positions is $12.8 \mathrm{~nm}$ here. 243

Figure II-3.30. AFM scan of mica exposed to Tensleep crude. Zoom of area indicated in Figure II -3.28 . 244

Figure II-3.31. AFM scan of mica exposed to Tensleep crude. A zoom of the area indicated by the box is shown in Figure II-3.33. 245

Figure II-3.32. AFM scan of mica exposed to Tensleep crude. 245

Figure II-3.33. AFM scan of mica exposed to Tensleep crude. Zoom of area indicated in Figure II -3.31 246

Figure II-3.34. AFM scan of mica exposed to Tensleep crude. 247

Figure II-3.35. AFM scan of mica exposed to Tensleep crude 247

Figure II-3.36. AFM scan of mica exposed to Tensleep crude. Zoom of area indicated in Figure II -3.34 248

Figure II-3.37. Section analysis of scan 01121405 . The film thickness indicated by the vertical distance between cursors measures about $11 \mathrm{~nm}$. 248

Figure II-3.38. AFM scan of mica exposed to Tensleep crude. Surface immersed in decane for 28 minutes. 249

Figure II-3.39. AFM scan of mica exposed to Tensleep crude. Surface immersed in decane for 99 minutes. 250

Figure II-3.40. AFM scan of mica exposed to Tensleep crude. Zoom of area indicated in Figure II-3.39. 250

Figure II-3.41. AFM scan of mica exposed to Tensleep crude................................................. 251

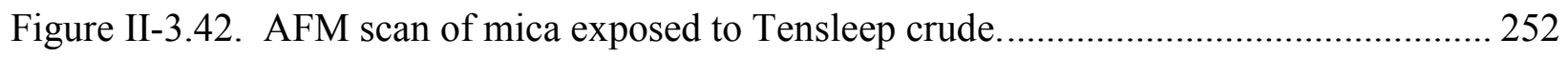

Figure II-3.43. AFM scan of mica exposed to Tensleep crude. Zoom of area indicated in Figure II -3.42 . 253

Figure II-3.44. AFM scan of mica exposed to Tensleep crude. Zooms of the areas indicated by the boxes are shown in Figures II-3.45 and II-3.46. 253

Figure II-3.45. AFM scan of mica exposed to Tensleep crude. Zoom of region in upper right corner of Figure II-3.44. 254 
Figure II-3.46. AFM scan of mica exposed to Tensleep crude. Zoom of area indicated in center of Figure II-3.44. 254

Figure II-3.47. AFM scan of mica exposed to Tensleep crude. Precipitated salt is indicated by the arrows. 255

Figure II-3.48. AFM scan of mica exposed to Tensleep crude. Imaging medium was changed from decane to decane+ toluene as indicated. While precipitated salt crystals retain their original size and shape, other structures (likely asphaltenes) swell upon addition of toluene, which is an effective asphaltene solvent. 256

Figure II-3.49. AFM scan of mica exposed to Tensleep crude. The $\mathrm{pH}=8,\left[\mathrm{Na}^{+}\right]=1.0 \mathrm{M}$ brine in this case provided a stable water film which prevented the oil phase from contacting the mica surface. 257

Figure II-3.50. AFM scan of mica exposed to Tensleep crude. A zoom of the area indicated in

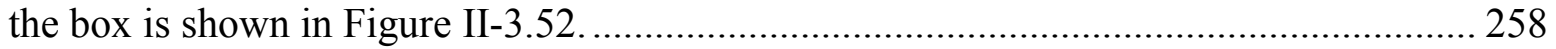

Figure II-3.51. AFM scan of mica exposed to Tensleep crude.............................................. 258

Figure II-3.52. AFM scan of mica exposed to Tensleep crude. Zoom of area indicated in Figure II -3.50 259

Figure II-3.53. AFM scan of mica exposed to Tensleep crude............................................... 260

Figure II-3.54. AFM scan of mica exposed to Tensleep crude................................................ 260

Figure II-3.55. Optical microscope image of Tensleep crude + 1-MN (65:35 vol./vol.). 1-MN effectively dispersed asphaltene aggregates such that they were not visible (compare with Figure II-3.25 which shows 100\% Tensleep crude)....................................................... 261

Figure II-3.56. AFM scan of mica exposed to Tensleep + 1-MN. Zoom of area in box is shown in Figure II-3.57. 262

Figure II-3.57. AFM scan of mica exposed to Tensleep + 1-MN. Zoom of area indicated in

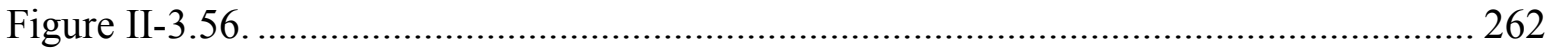

Figure II-3.58. AFM scan of mica exposed to Tensleep + 1-MN .......................................... 263

Figure II-3.59. AFM scan of mica exposed to Tensleep + 1-MN.......................................... 264

Figure II-3.60. AFM scan of mica exposed to Tensleep + 1-MN............................................. 265

Figure II-3.61. AFM scan of mica exposed to Tensleep + 1-MN............................................. 266

Figure II-3.62. AFM scan of mica exposed to Tensleep + 1-MN............................................. 266

Figure II-3.63. AFM scan of mica exposed to a $1 \mathrm{wt} . \%$ solution of Tensleep asphaltenes in

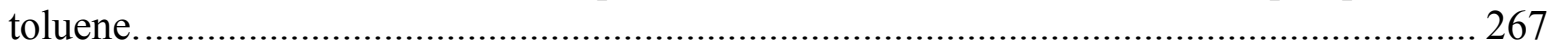

Figure II-3.64. AFM scan of mica exposed to a 1 wt.\% solution of Tensleep asphaltenes in toluene. A zoom of the area indicated in the box is shown in Figure II-3.65 ................... 268

Figure II-3.65. AFM scan of mica exposed to a 1 wt.\% solution of Tensleep asphaltenes in toluene. A zoom of the area indicated in the box is shown in Figure II-3.66..................... 268

Figure II-3.66. AFM scan of mica exposed to a $1 \mathrm{wt} . \%$ solution of Tensleep asphaltenes in toluene. Zoom of area indicated in Figure II-3.65. 269 
Figure II-3.67. AFM scan of mica exposed to a 1 wt.\% solution of Tensleep asphaltenes in toluene.

Figure II-3.68. AFM scan of mica exposed to a 1 wt.\% solution of Tensleep asphaltenes in toluene. 270

Figure II-3.69. AFM scan of mica exposed to a $1 \mathrm{wt} . \%$ solution of Tensleep asphaltenes in toluene.

Figure II-3.70. Surface coverage increases as aging time in Tensleep crude oil increases from 1 day to 25 days. 272

Figure II-3.71. Comparison of mica surfaces treated first with $\{3.8,0.01\}$ brine, then with Tensleep oil, a mixture of Tensleep and 1-MN, or a solution of Tensleep asphaltenes in toluene. Asphaltene aggregates are present only in the original oil. Incomplete films are formed by the mixture and asphaltene solution, even after 11 and 26 days of aging, respectively. 273

Figure II-3.72. Optical microscope image of Mars Yellow crude. Precipitated waxes appear as particulate material while oil background resembles a dark mustard color. 274

Figure II-3.73. TM-AFM scan of mica exposed to Mars Yellow crude. Tip-sample adhesion caused the poor resolution in this scan. 275

Figure II-3.74. AFM scan of mica exposed to Mars Yellow crude. Tip-sample adhesion caused

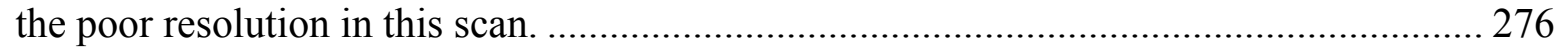

Figure II-3.75. AFM scan of mica exposed to Mars Yellow crude. ..................................... 277

Figure II-3.76. AFM scan of mica exposed to Mars Yellow crude. This figure represents the same scan area shown in Figure II-3.75. Note the blistering of the film upon addition of the brine imaging medium. 278

Figure II-3.77. AFM scan of mica exposed to Mars Yellow crude. ....................................... 279

Figure II-3.78. AFM scan of mica exposed to Mars Yellow crude. ........................................ 279

Figure II-3.79. AFM scan of mica exposed to Mars Yellow crude. A zoom of the area indicated by the box is shown in Figure II-3.80. 280

Figure II-3.80. AFM scan of mica exposed to Mars Yellow crude. Roughness of areas indicated by $0.4 \mu \mathrm{m}^{2}$ boxes measured: a) $1.64 \mathrm{~nm}$, b) $7.73 \mathrm{~nm}$, c) $1.708 \mathrm{~nm}$. 281

Figure II-3.81. AFM scan of mica exposed to Mars Yellow crude. A zoom of area indicated by the box is shown in Figure II-3.82 ........................................................................ 282

Figure II-3.82. AFM scan of mica exposed to Mars Yellow crude. ...................................... 282

Figure II-3.83. AFM scan of mica exposed to Mars Yellow crude. Roughness of areas indicated by $0.4 \mu \mathrm{m}^{2}$ boxes measured: a) $1.58 \mathrm{~nm}$, b) $7.44 \mathrm{~nm}$............................................. 283

Figure II-3.84. AFM scan of mica exposed to Mars Yellow crude. ...................................... 284

Figure II-3.85. AFM scan of mica exposed to Mars Pink crude. Mean roughness in the indicated

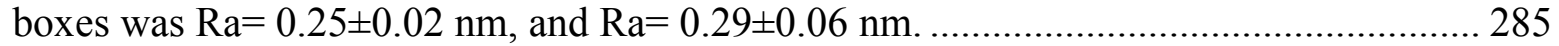

Figure II-3.86. AFM scan of mica exposed to Mars Pink crude............................................ 286 
Figure II-3.87. AFM scan of mica exposed to Mars Pink crude...... 286

Figure II-3.88. AFM scan of mica exposed to Mars Pink crude. A zoom of the area indicated by the box is shown in Figure II-3.89. 287

Figure II-3.89. AFM scan of mica exposed to Mars Pink crude. A zoom of the area indicated by the box is shown in Figure II-3.90 .......................................................................... 288

Figure II-3.90. AFM scan of mica exposed to Mars Pink crude............................................ 288

Figure II-3.91. AFM scan of mica exposed to Mars Pink crude. A zoom of the area indicated by

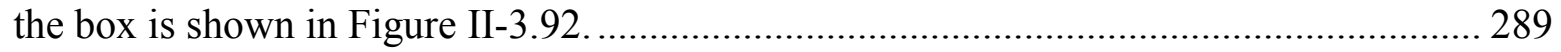

Figure II-3.92. AFM scan of mica exposed to Mars Pink crude............................................ 289

Figure II-3.93. AFM scan of mica exposed to Mars Pink crude. Mean roughness of five selected areas similar to box $a$ was $\mathrm{R}_{\mathrm{a}}=0.25 \pm 0.08 \mathrm{~nm}$................................................. 290

Figure II-3.94. AFM scan of mica exposed to Mars Pink crude........................................... 291

Figure II-3.95. AFM scan of mica exposed to Mars Pink crude............................................ 292

Figure II-3.96. AFM scan of mica exposed to Mars Pink crude.......................................... 292

Figure II-3.97. AFM scan of mica exposed to Mars Pink crude.............................................. 293

Figure II-3.98. AFM scan of mica exposed to Mars Pink crude. The roughness of the area

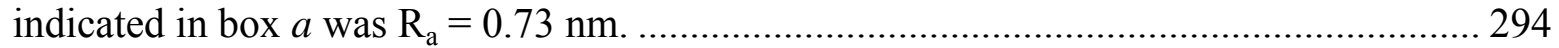

Figure II-3.99. AFM scan of mica exposed to Mars Pink crude. The roughness of the area indicated in box $a$ was $\mathrm{R}_{\mathrm{a}}=2.96 \mathrm{~nm}$. 295

Figure II-3.100. AFM scan of mica exposed to Mars Pink crude. A zoom of the area indicated

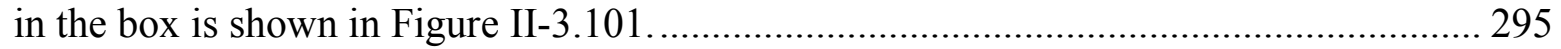

Figure II-3.101. AFM scan of mica exposed to Mars Pink crude.......................................... 296

Figure II-3.102. Contact angles on mica surfaces exposed to Lost Hills crude oil and mixtures of Lost Hills with n-heptane. 299

Figure II-3.103. AFM of mica surface aged in pH4 buffer (1 day) followed by Lost Hills crude oil (3 weeks). 300

Figure II-3.104. AFM of mica treated with a pH4 buffer and a 50:50 mixture of Lost Hills crude oil and n-heptane. Holes are developing as the area is scanned under decane. The coating thickness measurement was made on scan \# 04201134, a height image of the same area. 301

Figure II-3.105. AFM of mica treated with a pH4 buffer and a 26:74 mixture of Lost Hills crude oil and n-heptane imaged under water. 301

Figure II-3.106. Apparent coating thickness measured by section analysis using AFM height images in which some bare mica was exposed. 302

Figure II-3.107. AFM of mica treated with a pH4 buffer and a 26:74 mixture of Lost Hills crude oil and n-heptane imaged under decane. 303 
Figure II-3.108. Section analysis of mica treated with a pH4 buffer and a 26:74 mixture of Lost Hills crude oil and n-heptane imaged under air. The dark, elongated depressions do not reveal any bare mica surface. 303

Figure II-3.109. AFM of mica treated with a pH4 buffer and a 20:80 mixture of Lost Hills crude

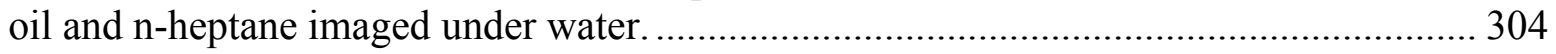

Figure II-3.110. Zoom of a feature in the upper left-hand quadrant of Fig. II-3.109 .............. 304

Figure II-3.111. AFM of mica treated with a pH4 buffer and a 30:70 mixture of Lost Hills crude oil and n-heptane imaged under air. The area shown is a portion of the surface on which a water drop dried.

305

Figure II-3.112. Edge of the dried water drop shown in Fig. II-3.111 where water drop and oil coating can both be seen. Note that the elongated features are associated only with the dried water drop. 306

Figure II-3.113. TEM image of platelets of octopus porphyrin (from Komatsu et al., 1996)... 307

Figure II-3.114. AFM of mica surface aged in pH4 buffer (1 day) followed by E-1XD-00 crude oil (3 weeks). 307

Figure II-3.115. Section analysis of a hole on a mica surface exposed to E-1XD-00 crude oil.308

Figure II-3.116. AFM of mica surface aged in pH4 buffer (1 day) followed by E-1XR-00 crude oil (3 weeks). 308

Figure II-3.117. Section analysis of a hole on a mica surface exposed to E-1XR-00 crude oil.309

Figure III-1.1. Growth of corner menisci by spontaneous imbibition (a) and maximum displacement attained before instability and snap-off for tubes of triangular cross section, (b) equilateral, (c) isosceles (height $=1 / 2$ base). 315

Figure III-1.2. Illustration of spontaneous and forced imbibition and drainage capillary pressure curves. 317

Figure III-1.3. Boundary conditions for core samples used by Mattax and Kyte [9] (a and b), Hamon and Vidal [12] (a to c) and Zhang et al [14] (a to d).... 320

Figure III-1.4. Normalized oil recovery versus dimensionless time for very strongly water-wet imbibition. 322

Figure III-1.5. Mixed wettability in a tube of triangular cross-section. 325

Figure III-1.6. Examples of change in spontaneous imbibition with change in time of aging with an asphaltic crude oil at $15 \%$ initial water saturation [1]. 326

Figure III-1.7. Normalized dimensionless time for $50 \%$ of ultimate recovery versus connate water saturation. Imbibition rate is highly sensitive to initial water saturation for mixed-wet conditions [58]. 328

Figure III-1.8. Dependence of mode of imbibition (frontal vs. global) on initial water saturation and wettability. [73]..... 331

Figure III-2.1. The upstream differencing scheme. 343 
Figure III-2.2. Relative permeabilities and capillary pressure obtained by matching the waterwet correlation with base case parameters...................................................................... 345

Figure III-2.3. Comparison of base-case simulation to the correlation. .................................... 345

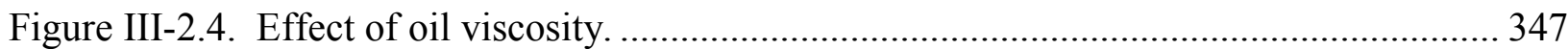

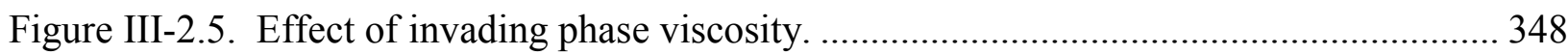

Figure III-2.6. Effect of the shape of $P c$ (with shape varies by varying $B$ ) ............................ 349

Figure III-2.7. Effect of varying the end-point relative permeability to water, $k_{r w e}$.................. 350

Figure III-2.8. Effect of varying the shape of the water relative permeability curve by varying $n_{w}$. 350

Figure III-2.9. Effect of varying the shape of the water relative permeability curve by varying $n_{o}$. .

Figure III-2.10. Effect of initial water saturation................................................................... 352

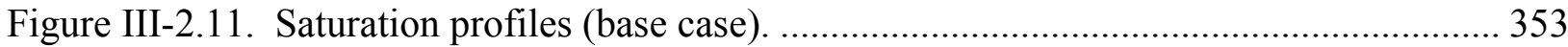

Figure III-2.12. Saturation profiles (10\% initial water saturation).......................................... 353

Figure III-2.13. Illustrations of piston-like and global displacement. The heavy solid line represents the saturation profile at an early time $\left(\mathrm{t}_{1}\right)$ and the heavy broken line at a later time $\left(t_{2}\right) . S_{w i}$ is the initial WP saturation and $\mathrm{S}_{\mathrm{nw}, \mathrm{r}}$ is the residual NWP saturation. both GD and PD are idealized. For the PD model, the nonwetting phase can leak through the piston. All displacement occurs during the frontal flow period. For the GD model, the gradient is zero and all displacement occurs during the post contact period. 358

Figure III-2.14. Ries and Cil COUSCI model. The capillary pressure gradient and the saturation gradient between the front and open face are assumed to be linear. 359

Figure III-2.15. Sketches of truncated and non-truncated saturation profiles. The heavy solid line corresponds to a saturation profile during the frontal flow period. The heavy broken line corresponds to the profile when the front contacts the no-flow boundary. The heavy dotted line shows a profile during the post contact flow period. Production by frontal flow is given by the area swept by the profile up to the time of contact of the front with the no flow boundary. Production during post contact flow period is given by the area under the curve minus the total frontal flow production. 360

Figure III-2.16. Equivalent conditions for linear counter-current spontaneous imbibition (COUCSI) given by cores with (a) one end open and (b) two ends open. 361

Figure III-2.17. Examples of increase in WP saturation with time for air/oil and oil/brine. In comparison of results, the increase in WP saturation at long times caused by dissolution of trapped air is neglected. The indicated plateau achieved at long times is used to define the maximum increase in WP saturation by DSI. 371

Figure III-2.18. Experimental results representative of Berea sandstone of $0.5 \mu \mathrm{m}^{2}$ permeability, for which the recovery behind the front is a constant fraction of the swept volume. For Berea sandstone with the same permeability, the fractional recoveries for oil/brine pairs are always less than for air/oil pairs by a constant proportion. 372 
Figure III-2.19. Recovery of NWP versus adjusted $\sqrt{t}$. Apart from the very small correction to $t$ for the development of flow at very early time, the rate of frontal advance for both air-oil and oil-water pairs is proportional to the square root of time until the front is at the boundary $(f a b)$. 373

Figure III-2.20. Saturation profiles before, at, and after contact of the saturation front with the no flow boundary. It is assumed that the saturation profile within the core developed as if the core length is infinite. Graphically, when the initial WP saturation, $S_{w i}$ is zero, the recovery for incompressible fluids $Q_{w} / V_{\phi}$ is equal to the ratio of the area under the saturation profile (if $S_{w i}$ is finite the related area is excluded) within the core length, $L$ to the total area, $L \times 1$.

Figure III-2.21. Recovery, $Q_{w} / V_{\phi}$, vs $\sqrt{t}$. Fractional recoveries corresponding to the profiles in Fig.8 are indicated. The frontal flow period part of the recovery curve (straight line A, on the $Q_{w} / V_{\phi} \sim \sqrt{t}$ plot), ends when the front reaches the no flow boundary $\left(t_{2}=t_{f a b}\right)$. Curve B is the post contact flow period part of recovery curve, which is the locus of the arrival of all saturation sections at the no flow boundary.For any section with $S_{w}$ greater than $S_{w, f}$, there exists a comparable linear relation between $Q_{w} * / V_{\phi}$. and $\sqrt{t}$, where $Q_{w} *$ is defined by the volume of imbibed WP left behind the section. Line $C$ is a plot of the increase in saturation with respect to the imbibed wetting phase behind the section with a saturation of $0.522 . .374$

Figure III-2.22. Relations between the saturation profile and recovery after time, $t_{f a b}$, at which the front reaches the no flow boundary. After $t_{f a b}$, the recovery curve is given by the locus of the average saturations, $Q_{w} * V_{\phi}$, corresponding to the value of $S_{w}$ at the no flow boundary. The relationships between arrival of saturation sections at the no flow boundary and recovery for arrival times $t_{f a b}$ and $t_{2}$ are indicated by the two connecting lines which link the figures. 375

Figure III-2.23. Pressure distribution and flow in the piston like COUCSI model (a) and in the non-piston like COUCSI model (b). $P_{n w}$ is the pressure in NWP. $P_{w}$ is the pressure in WP. $P_{c}$ is the capillary pressure. $P_{c 0}$ is the average capillary pressure at the open face associated with production of the NWP. $x_{S w}$ is distance from the open face to the front. 381

Figure III-3.1. Countercurrent imbibition into cores with varying boundary conditions.......... 382

Figure III-3.2. Imbibition of water into very strongly water-wet cylindrical cores. Boundary conditions are specified using the notation of Fig. III-3.1. Core lengths for these four samples were $\mathrm{L}_{\mathrm{AFO}}=1.3059 \mathrm{~cm} \mathrm{~L}_{\mathrm{TEC}}=1.3506 \mathrm{~cm}, \mathrm{~L}_{\mathrm{TEO}}=3.8700 \mathrm{~cm}$, and $\mathrm{L}_{\mathrm{OEO}}=4.998 \mathrm{~cm}$; displaced mineral oil had a viscosity of 37.82 cP. (after Zhang et al., 1995)................. 383

Figure III-3.3. Rate of imbibition of $163.2 \mathrm{cP}$ mineral oil into air-filled cylindrical cores of Berea sandstone. 384

Figure III-3.4. Rate of imbibition of $11.8 \mathrm{cP}$ mineral oil into air-filled cylindrical cores of Berea sandstone. 385

Figure III-3.5. Rate of imbibition of $68.1 \mathrm{cP}$ mineral oil into air-filled cylindrical cores of Berea sandstone. 385

Figure III-3.6. Rate of imbibition of $4.32 \mathrm{cP}$ Soltrol 220 mineral oil into air-filled cylindrical cores of Berea sandstone. 385 
Figure III-3.7. Schematic diagram illustrating vent tube inserted into core CW-9 with OEO

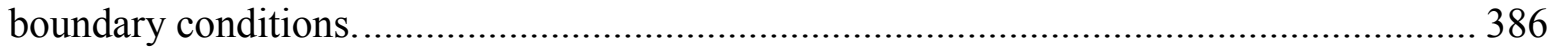

Figure III-3.8. Rate of imbibition of $163.2 \mathrm{cP}$ mineral oil into air-filled cylindrical cores of

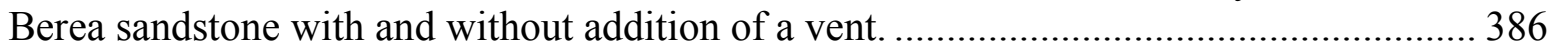

Figure III-3.9. Sensitivity of spontaneous imbibition to initial water saturation at weakly waterwet conditions for cores with all faces open............................................................... 392

Figure III-3.10. Application of the characteristic length to scaling imbibition data for weakly

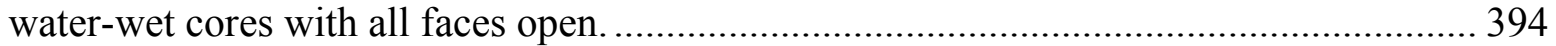

Figure III-3.11. Effect of initial water saturation on imbibition rate and oil recovery for weakly

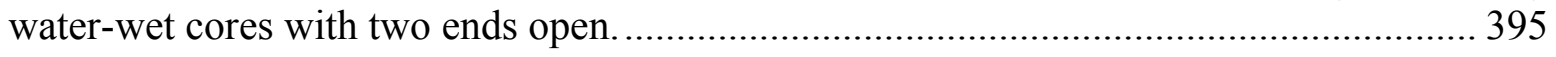

Figure III-3.12. Effect of initial water saturation on imbibition behavior for weakly water-wet

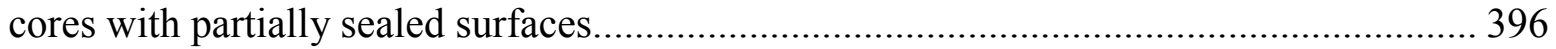

Figure III-3.13. Scaling of imbibition data for weakly water-wet cores with partially sealed

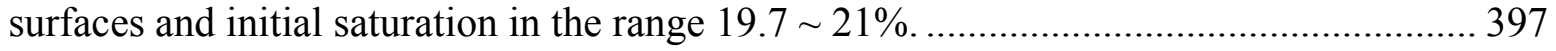

Figure III-3.14. Scaling of results for partially sealed weakly water-wet cores with partially sealed surfaces and with initial water saturation in the range of 17 to $18.5 \% \ldots \ldots \ldots \ldots \ldots \ldots . . . .398$

Figure III-3.15. The relationship between Swi and imbibition time for $50 \%$ oil recovery. Insert shows effect of epoxy resin on imbibition by AFO cores. .............................................. 399

Figure III-3.16. Effect of initial water saturation and change in wettability on imbibition time for

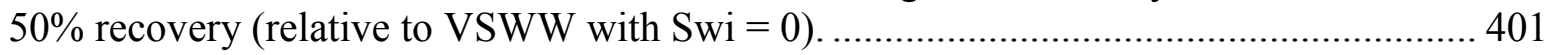

Figure III-3.17. Aronofsky model based on values of tD at 50\% recovery for AFO cores....... 402

Figure III-3.18. Comparison of recovery of mineral oil by spontaneous imbibition from Berea 90

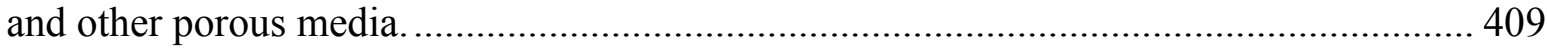

Figure III-3.19. Reproducibility of recovery of mineral oil by spontaneous imbibition........... 410

Figure III-3.20. Sensitivity of MXW-F imbibition to decalin flush volume. ........................... 410

Figure III-3.21. Effect of initial water saturation on oil recovery by spontaneous imbibition. . 411

Figure III-3.22. Recovery by imbibition from mixed-wet cores for different oil viscosities.... 413

Figure III-3.23. The effect of temperature of $5 \mathrm{PV}$ decalin flush on oil recovery.................... 421

Figure III-3.24. The effect of flush volume (PV) of decalin on oil recovery........................... 422

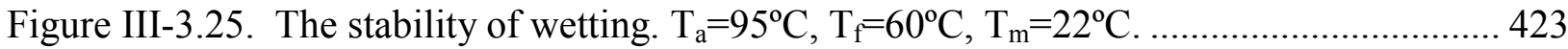

Figure III-3.26. Sequential imbibition curves for mixed-wettability with $\mu_{\mathrm{o}}$ of 3.8, 8.4, 18, 39.4, and $66 \mathrm{cp}$. Results for individual cores are indicated as follows: $\square 2 \mathrm{~B} 27, \bullet 1 \mathrm{~B} 32, \boldsymbol{\Delta} 1 \mathrm{~B} 33$,

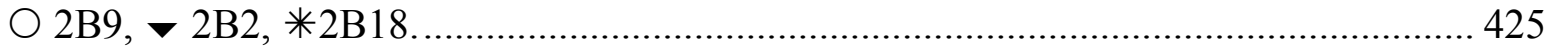

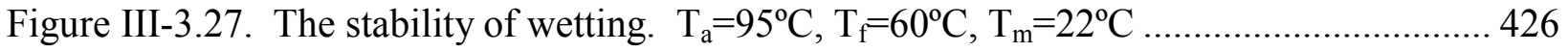

Figure III-3.28. Comparison of effect on spontaneous imbibition of initial water saturation at time of aging and reduction in initial water saturation after mixed wettability had been established. 428 
Figure III-3.29. Properties of crude oil and contact angles after deposition of organic films on smooth quartz surfaces (Xie, et al, 2002). Advancing $\left(\theta_{A}\right)$ and receding $\left(\theta_{R}\right)$ results shown in (b) and (c) obtained from asphaltic crude oil are characterized as reproducible and stable. Results in (d) obtained from non- asphaltic crude oil are reproducible but unstable. 431

Figure III-3.30. First imbibition recovery and scaled results for MXW-F (Minnelusa) cores. . 435

Figure III-3.31. Scaled subsequent spontaneous imbibition results of MXW-F (Minnelusa)

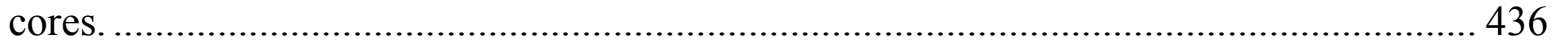

Figure III-3.32. The effect of Minnelusa oil re-aging on spontaneous imbibition. ................. 436

Figure III-3.33. Scaled first imbibition results for MXW-F (Gullfaks) cores. ....................... 437

Figure III-3.34. Sequential spontaneous imbibition tests for MXW-F (Gullfaks) cores.......... 438 


\section{List of Tables}

Table I-2.1. Absorbance of Azobenzene Standards ................................................................ 13

Table I-2.2. Azobenzene Mass Concentration in Effluent.............................................................. 14

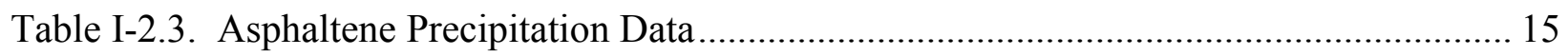

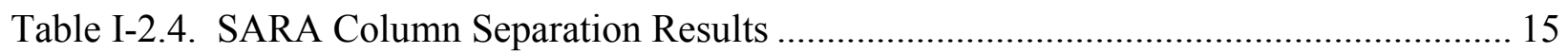

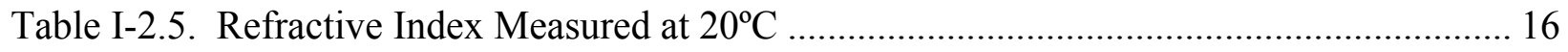

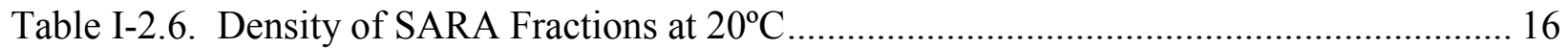

Table I-2.7. Comparison of Measured and Calculated RI for Maltenes ...................................... 17

Table I-2.8. Estimate of Asphaltene RI. .............................................................................. 17

Table I-2.9. Physical and Chemical Properties of Crude Oil Samples ......................................... 24

Table I-2.10. SARA Fractions by the ASTM Clay-Gel Adsorption Method ................................. 26

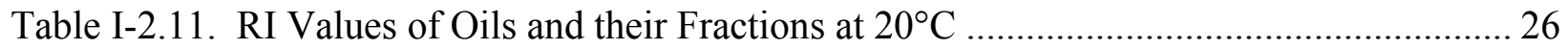

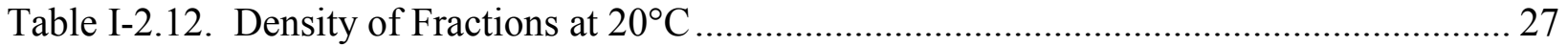

Table I-2.13. HPLC RI Detector Signal (area/mg) and RI of Hydrocarbons at $20^{\circ} \mathrm{C}$.................. 29

Table I-2.14. HPLC Detector Signals of Calibration Hydrocarbons ............................................. 31

Table I-2.15. Chromatographic Peak Areas of Maltene HPLC Separation .................................. 32

Table I-2.16. SARA Data (wt \%) from HPLC Method ............................................................... 33

Table I-2.17. Crude Oil Weight Loss as a Function of Areal Distribution.................................... 35

Table I-2.18. Effects of Spotting Amount on Weight Loss …………………………............... 36

Table I-2.19. Crude Oil Weight Loss on PTLC Plates after Solvent Developments.................... 36

Table I-2.20. Crude Oil TLC-FID Peak Area Comparison ......................................................... 37

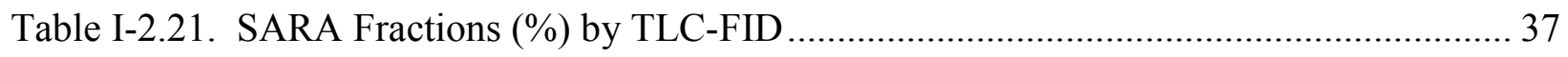

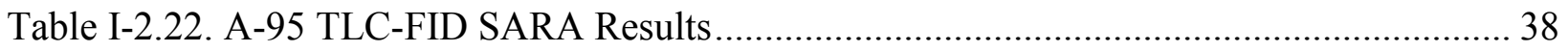

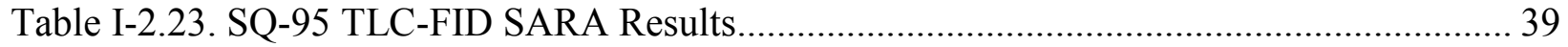

Table I-2.24. Tensleep-99 TLC-FID SARA Results .................................................................. 39

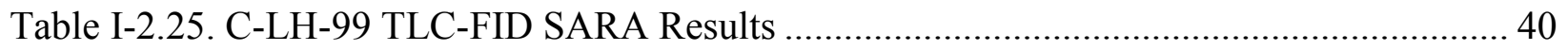

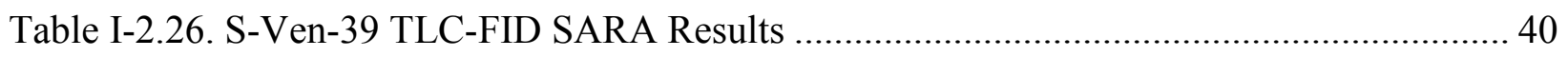

Table I-2.27. C-R-00 TLC-FID SARA Results ......................................................................... 41

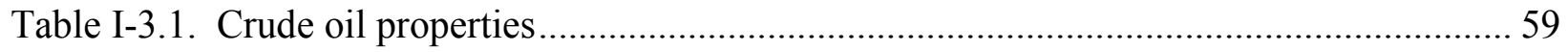

Table I-3.2. Properties of asphaltene precipitants and solvents ................................................... 59

Table I-3.3. Summary of least-squares fits to data in Fig. I-3.5 .................................................. 62 
Table I-3.4. Comparison of $n-\mathrm{C}_{7} P_{R I}$ values for crude oils and their $n$ - $\mathrm{C}_{7}$ asphaltenes in $1 \%$

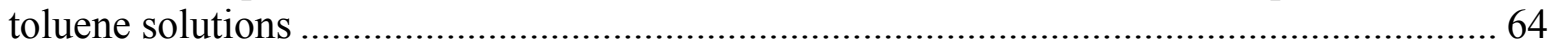

Table I-3.5. Onset of flocculation with branched paraffins ......................................................... 65

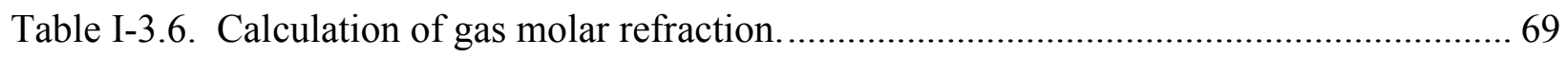

Table I-3.7. Comparisons for three different approaches based on Flory-Huggins polymer theory 94

Table I-3.8. Measured properties of crude oils and the adjusted properties for asphaltene at $20^{\circ} \mathrm{C}$

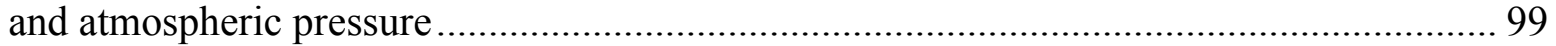

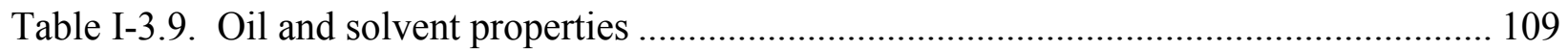

Table I-3.10. Amount of asphaltene separated with different precipitants............................ 111

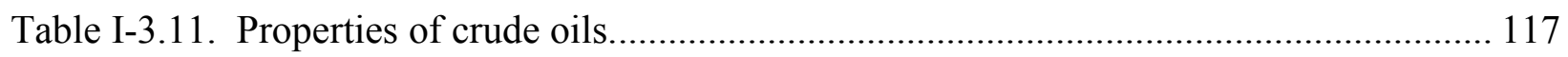

Table I-3.12. Fresnel fits to reflected light intensity data........................................................ 126

Table I-3.13. Comparisons of calculated RI to measured RI for crude oils .............................. 128

Table I-3.14. RI for mixtures of SQ-95 with toluene .......................................................... 129

Table II-2.1. Correlations between amount of asphaltene and other oil properties.................... 167

Table II-2.2. Single-parameter linear relationships between oil properties and wetting alteration. 169

Table II-2.3. Coefficients from multiple linear regression analyses of contact angle data. ...... 170

Table II-2.4. Coefficients for multiple regression analyses on the data set without high $\mathrm{Zn}$

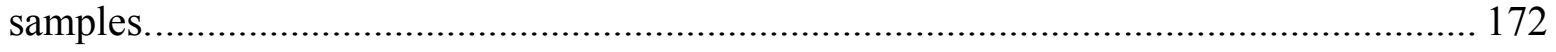

Table II-2.5. Crude Oil Physical and Chemical Properties..................................................... 182

Table II-2.6. Contact Angles on Oil-treated Mica Surfaces ...................................................... 188

Table II-2.7. Selected physical properties of crude oil samples ............................................... 195

Table II-3.1. Summary of published AFM studies of crude oil systems. .................................. 208

Table II-3.2. Olympus Oxide-Sharpened Silicon Nitride Probe Specifications ......................... 210

Table II-3.3. Physical and chemical properties of crude oils.................................................. 238

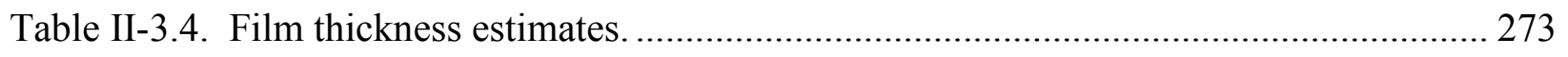

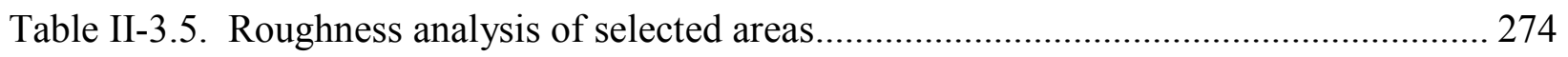

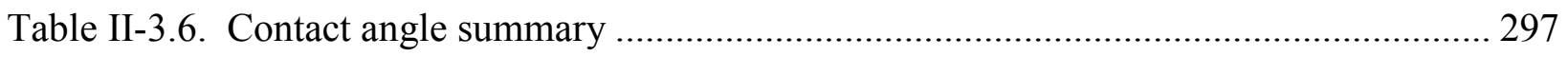

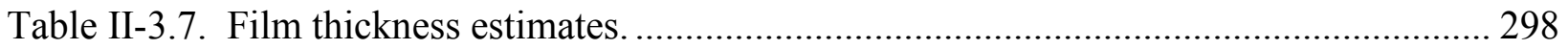

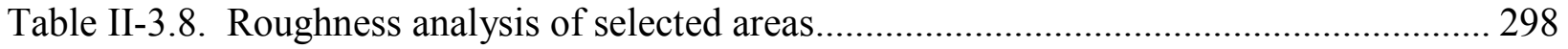

Table II-3.9. Conditions under which narrow, multi-armed features were observed. ................ 305

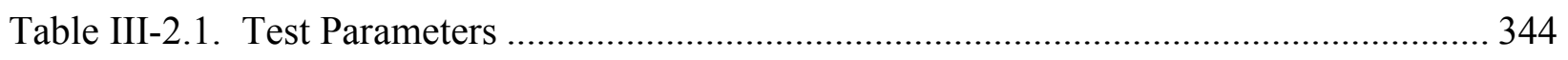

Table III-2.2 Core Dimensions and Rock and Fluid Properties $\left(\rho_{w}=1 \mathrm{~kg} / \mathrm{L}\right) \ldots \ldots \ldots \ldots \ldots \ldots \ldots \ldots \ldots \ldots . . . . . . . . . . . . .370$ 
Table III-3.1. Experimental conditions for a strongly oil-wet oil/air/rock system .................. 384

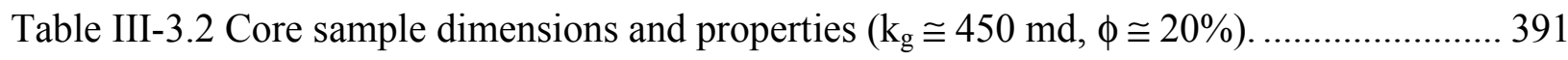

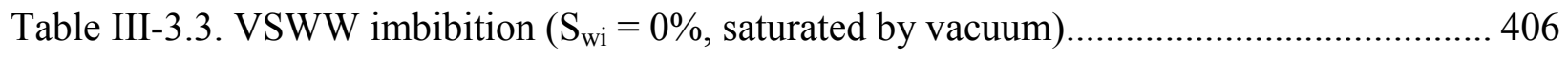

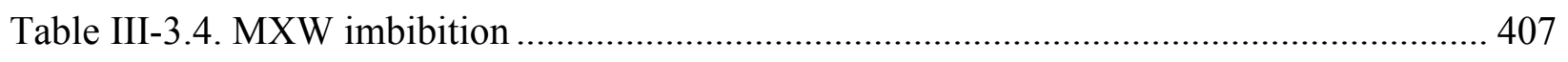

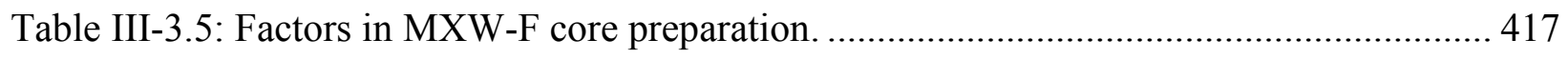

Table III-3.6. Physical properties of the cores and the displaced oil ..................................... 418

Table III-3.7. Conditions for cycles of imbibition and flushing ......................................... 420

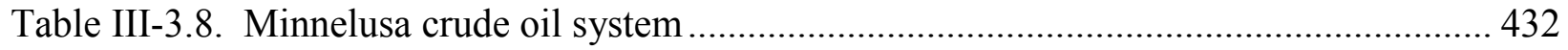

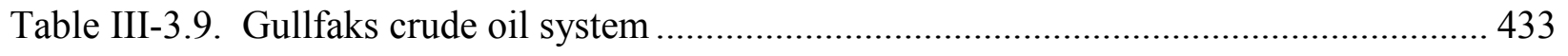

Table III-3.10. Selected properties of crude oil samples ................................................. 433

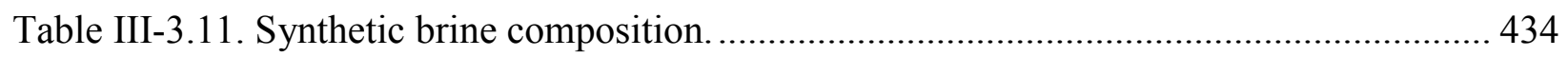




\section{Executive Summary}

Petroleum fluids are recovered from the pore space of rocks where they coexist with water. The equilibrium conditions between all of the components in an oil reservoir are disturbed first by penetration of producing wells and subsequently by invasion of fluids from natural or man-made sources or both. It is, therefore, not possible to study the surface state of an undisturbed oil reservoir. Recovered core samples are highly perturbed by changes in temperature, pressure, fluid saturations, and often by invasion of surface active materials from drilling muds. In this project we establish and study relevant wetting conditions using produced fluids in outcrop rock samples and on smooth mineral surfaces to learn about the factors that affect wetting and how wetting influences oil recovery.

The wetting condition in an oil reservoir reflects adsorption of organic compounds from the oil onto portions of the pore lining minerals in the presence of connate brine. Most of the oil is composed of hydrocarbons that have no affinity for high-energy mineral surfaces. Polar compounds are found mainly in the high molecular weight resin and asphaltene fractions. Their tendency to adsorb can vary with their environment in the oil phase and at the oil/water interface. Adsorption can be inferred from its effects on (1) oil/water contact angles on smooth surfaces or (2) the rate and extent of spontaneous imbibition of water or oil into porous media. Both of these techniques have been used extensively in this study. In addition we have developed new approaches to observing and understanding the effects of crude oils on reservoir wettability.

This report is the culmination of four years of work at three different universities. The work was divided into three principal tasks. The first task was to improve crude oil characterization techniques that are most directly applicable to reservoir wetting and to develop a database of characterized oil samples. The second task focussed on surface studies of wetting alteration and in task three, wetting alteration and characterization in cores was studied.

Work on the first task is reported in Part I, beginning with development of the CO-Wet database (§I-1., which now has data for more than 150 samples. The data accumulated include information specific to the tendancy of an oil to alter wetting - acid and base numbers (usually only an acid number is available), asphaltene stability measures, and SARA fraction data - as well as more general information - density, average molecular weight, and viscosity, for example. This wealth of data permits exploration of many issues regarding crude oil composition and its effect on reservoir wettability, as discussed further in Part II.

In the remainder of Part I, investigations of two important facets of crude oil chemistry are reported in detail. The first is the standard SARA analysis of saturates, aromatics, resins, and asphaltenes (§I-2.). The ASTM standard for these measurements is a lengthy column separation that requires a substantial volume of sample and large volumes of solvent. Industry practice often deviates from the standard procedure, with significant degradation of the quality of SARA data. We have developed, validated, and applied an improved HPLC technique that requires only small amounts of sample, much smaller volumes of solvents, and is much faster than the ASTM technique, without sacrificing the quality of the resulting data. 
In §I-3, an exciting new approach to evaluating asphaltene stability is presented Asphaltene stability, which influences the mechanism by which asphaltenes alter wettability, varies with composition, pressure, and temperature. Predicting instability from a small number of measurements has been the goal of a huge amount of research in labs worldwide. The key to solving the problem is the use of refractive index to assess solvent quality, including the critical conditions at the onset of instability, and relating the onset conditions to molar volume of the species causing asphaltenes to flocculate. Subsections of $\S \mathrm{I}-3$ explain our current procedure for observing and quantifying the onset of flocculation, present both an empirical approach and a thermodynamic model for using the data, show how solvents, resins, other crude oils, and asphaltenes themselves affect stability, developments in simultaneous observations of RI and instability, and finally present confirmation of predictions of methane-induced precipitation based on ambient conditions tests for a model fluid. Industry is beginning to recognize the utility of our data and to encourage service companies to provide our asphaltene stability evaluation tests on a routine basis. Predictions of asphaltene stability over a wide range of conditions will make it possible to anticipate changes in wetting that may accompany oil production by pressure depletion, gas injection, and a variety of IOR processes.

Part II begins with a review of recent studies of the crude oil/brine/rock interactions that alter wettability. In $\S$ II-2 two new contact angle studies are presented. In addition to the basic crude oil data, wettability alteration on smooth surfaces has been tested for more than 1000 sets of conditions (principal variables include crude oil, brine, solid surface, temperature, and asphaltene stability), all of which are included in the CO-Wet database (§II-2.1). The volume of data is sufficient to begin testing hypotheses about interaction mechanisms and crude oil chemistry using the CO-Wet data. While no one measured crude oil property correlates with wettability alteration, trends emerge when groups of variables are considered together. A neural network has been trained to predict contact angles on mica that was first treated with standard brines, an important first step toward predicting wetting properties for suites of oil, brine, and mineral surfaces. Contact angles with probe fluids on brine and oil-treated glass surfaces are used to study hysteresis between advancing and receeding conditions, persistence of wetting alteration (§II-2.2). Two extremes of behavior were observed. Asphaltic oils tended to produce stable wetting alteration. Changes in contact angles were small for up to 5 cycles in which a treated glass plate was repeatedly passed through a decane/brine interface. Waxy oils produced an initial change in wetting, but the contact angles were lower during the second cycle and sometimes continued to fall during successive cycles.

Oil treated mica surfaces were examined extensively with a newly developed atomic forces microscopy (AFM) technique ( $(\mathrm{II}-3)$. The images obtained by monitoring the deflection of the cantilever in contact mode were the more detailed than anything previously published. The first images were obtained of such surfaces in a liquid environment. Details of the methodology are given in §II-3.1 and applications to a variety of crude oils are presented in $\S I I-$ 3-2. Surface preparation mimics that for the contact angle tests. For the first time, the adsorbed and/or deposited material that produces wetting alteration can be observed directly, its persistence tested by varying the imaging force, and its thickness measured.

In cores contact angles are not well defined, although deposition similar to that seen in AFM images undoubtedly is a factor contributing to imbibition behavior and there are analogies 
between the smooth surface and core test protocols. Part III begins with a review of oil recovery by imbibition processes. Subsequent sections are divided into studies that are primarily theoretical examinations of spontaneous imbibition and experimental studies measuring imbibition rates under a wide variety of conditions and testing the scaling group developed for strongly wetted systems in mixed-wet cores. In the first theoretical approach, a commercial simulator was adapted to perform simulations of imbibition processes (§III-2.1). The simulator was used in a parametric study to test variables in the imbibition scaling group as well as other variables that affect the rate of imbibition. Over the range of variables for which data is available, the comparisons between predicted and measured responses were reasonable. Using the simulator, a wider range of values can be investigated, resulting in predictions of imbibition rates that have not yet been observed. In a second theoretical study (§III-2.2), an analytical solution for linear counter-current spontaneous imbibition, based on a similarity solution, has been developed. Model results are consistent with viscosity ratios that vary over seven orders of magnitude.

The experimental studies of spontaneous imbibition cover a broad range of conditions. In §III-3.1, the scaling group is tested for a strongly wetted oil/air system. Cores with similar geometry could be scaled, but results did not scale to the general correlation previously obtained for liquid/liquid imbibition. The remaining studies all examined cores that were rendered mixedwet by contact with brine and oil. The first of these studies (§III-3.2) demonstrated the dominant effect of initial water saturation on the extent of wetting alteration. Provided that cores with similar initial water saturations were compared, however, geometrical variations could be accounted for by previously developed scaling rules developed for strongly wetted systems. It is not so straightforward to test the effects of viscosity ratio in mixed-wet systems because changing oil viscosity can affect its tendency to alter wetting as well and cores with the same wetting are needed for the comparisons. To avoid uncertainties about wetting, cores were exposed to brine and crude oil, then flushed with decalin before further testing using a refined mineral oil. Using this technique, cores with equivalent wettability were tested with viscosity ratios from 3.8 to 180 and the results were satisfactorily correlated by the geometric mean of the viscosities. The strategy of preparing mixed-wet cores by contact with crude oil, but using mineral oil for subsequent tests was further examined in §III-3.4 and §III-3.5. Wettability states were generally stable after the first cycle of imbibition and drainage. As in the cycles of contact angle measurement, an asphaltic oil produced fairly stable wetting conditions, whereas a waxy oil did not.

As a result of this project, we now have a library of well-characterized oils that will be invaluable in future wettability projects. We have developed an improved method for measuring SARA fractions rapidly and reliably. Our method for evaluation of asphaltene stability enables predictions over a wide range of conditions from a few observations; it promises to become an industry standard. Imbibition scaling has been applied to mixed-wet systems, enabling the use of imbibition rate as a measure of wetting in porous media, and the theory of countercurrent imbibition has been advanced. 


\section{Part I-Crude Oil Characterization}

There are a variety of characteristics of a crude oil that predispose it to alter the wetting of solid surfaces with which it comes in contact. There are compounds with various polar substituents, whose chemical properties influence adsorption and thus wetting alteration. Some crude oil constituents, often referred to as asphaltenes, are incompatible with the oil's light ends. Changes in thermodynamic conditions can affect the tendency of these components to partition out of the bulk oil in preference to oil/water or oil/solid interfaces or to form a separate asphaltene-rich phase. Standard oil characterizations do not provide the information needed to assess all of the features of a crude oil that influence its ability to alter reservoir wetting. In this project we have defined a set of properties that we expect to influence crude oil interactions with solids in the presence of an aqueous phase. In the first section in Part I, the CO-Wet database set up to accumulate these oil properties for subsequent analysis is introduced and standard measurements are described.

In two areas, standard tests were either inadequate or unavailable. The first of these is the "standard" SARA fractionation in which oils are subdivided into their saturate, aromatic, resin, and asphaltene components. Because results of such tests vary widely with the details of the technique used to analyze the oil, we have reexamined the most popular techniques and developed an improved method that we recommend for standard practice. In the case of asphaltenes, an entirely new approach to characterization of asphaltene stability has been developed, as documented in the final section of Part 1.

\section{I-1. Overview of the CO-Wet Database}

Buckley, J.S. and Wang, J.X.: “Crude Oil and Asphaltene Characterization for Prediction of Wetting Alteration,” J. Pet. Sci. Eng, (2002) 33, 195-202.

Measures of a crude oil's polar functionality and asphaltene stability are key to predicting its wetting behavior. No publicly available data sources and few proprietary databases include all of the information necessary to characterize a crude oil with respect to its interactions with solid surfaces in the presence of an aqueous phase. At a minimum this information includes both acid and base numbers, which are related to polar and ionic interactions between oil components and charged mineral surfaces, and refractive index of both the oil and oil mixtures at the onset of asphaltene flocculation, which are related to asphaltene stability.

Data accumulated thus far illustrate a linear relationship between API gravity and refractive index that can be used to estimate refractive index from existing data. Acid and base numbers for many different crude oil samples show little evidence of correlation between the two, thus both numbers are needed for prediction of wetting alteration. Measurements of refractive index at the onset of precipitation with n-heptane are used to characterize asphaltene stability. 


\section{Introduction}

Crude oils have many components that, under the right conditions, can adsorb onto mineral surfaces and alter wetting in an oil reservoir or other subsurface setting. In previous work it has been shown that the mechanisms of interaction between crude oil components and rock surfaces differ depending on whether or not water is also present (Buckley et al., 1998c). Interactions with asphaltenes that are well dispersed can also be distinguished from those that occur near the onset of asphaltene flocculation (Buckley, 1998a, Al-Maamari and Buckley, 2000). Understanding these different mechanisms of crude oil/brine/rock interactions has guided the design of $\mathrm{CO}-\mathrm{Wet}$, a crude oil characterization database used to relate oil properties to wetting alteration tendencies.

Information about the chemical and physical properties of crude oils that has been generated by oil companies is usually unavailable because it is proprietary. There are some publicly available sources of crude oil data, but from the vantage point of wettability alteration, there are significant omissions in the information they provide. Acid numbers (ASTM D664-89) are often reported, but both acid and base numbers are needed to make reasonable conjectures about the likelihood of acid/base and other ionic interactions between oil and mineral surfaces in the presence of an aqueous phase. Base numbers measured using the standard ASTM procedure (ASTM D2896-88) are not sufficiently accurate to be of use. Improvements first suggested by Dubey and Doe (1993) are required, but properly measured base numbers are seldom available. Asphaltene characterization is usually based on ASTM D2007-80 in which the amounts of pentane or heptane insolubles are measured. Only recently has the importance of the stability of those asphaltenes, as opposed to their quantity, been recognized. Data quantifying asphaltene stability is unique to the CO-Wet database.

\section{Materials and Measurements}

Crude oil samples were obtained from many sources including major and independent oil companies, service companies, and other universities. Samples that are free of any treatment chemicals are always requested although the extent to which additive-free samples are provided probably varies.

\section{Acid and base numbers}

Standard non-aqueous potentiometric titration methods were used for both acid and base number determinations. Acid numbers are measured by the standard method (ASTM, 1989); the corresponding base numbers are measured by an adaptation of the standard method (ASTM, 1988) recommended by Dubey and Doe (1993).

\section{RI and $P_{R I}$ observations}

Refractive index (RI) was measured as a function of temperature using either an Abbe refractometer or an automated Index Instruments GPR 11-37 refractometer. Both instruments use the sodium D line (589nm) as a standard wavelength. The GPR 11-37 uses a critical angle technique that permits measurement of RI for opaque liquid samples. In most cases RI could be determined for neat crude oil. If not, RI was determined for a series of oil/toluene mixtures and the results were extrapolated to determine the value for the crude oil. A sample cell that can 
maintain a pressure up to $1.5 \mathrm{~atm}$ was used to limit evaporation during measurements with the automated refractometer.

The onset of asphaltene flocculation was observed microscopically one day after mixing oil and precipitants. Visible light and a maximum magnification of $320 \mathrm{X}$ were used in this work. RI was measured for the onset mixture, using the techniques described above. RI at the onset is denoted $\mathrm{P}_{\mathrm{RI}}$. In some cases, the dead crude samples contain precipitated asphaltenes. To these samples $\alpha$-methyl naphthalene, a good asphaltene solvent, was added to completely dissolve existing precipitated asphaltenes before onset measurements began.

\section{Density and API gravity}

Density was measured as a function of temperature using a digital Mettler/Paar DMA40 densitometer. API gravity was calculated from the density measurements, corrected to a temperature of $60^{\circ} \mathrm{F}$.

\section{Database Design}

Each oil sample is identified uniquely in a Microsoft Access relational database. Oils from the same source, but received at different times, are given distinct identifications since there are often significant differences between such samples.

Various measured oil properties are stored in special-purpose tables, related by their oil identifiers. The simplest data tables are collections of records, each of which includes an oil ID, value of the measured property, measurement variables (if any), and information to facilitate verification in original laboratory notes.

Tables store data including density, RI, and viscosity as functions of measurement temperature, onset conditions and amounts of asphaltene precipitated by different alkane precipitants, acid and base numbers, average molecular weight, and, whenever available, additional information such as SARA fractions, elemental analyses, and isoelectric points. From these, derived quantities such as API gravity, base/acid, $\mathrm{H} / \mathrm{C}, \mathrm{O} / \mathrm{C}, \mathrm{N} / \mathrm{C}$, and $\mathrm{S} / \mathrm{C}$ ratios can be calculated automatically. More complex tables record the results of adhesion and contact angle tests.

More than 150 oil samples are currently included in the database although for historical or practical reasons not all tests have been performed on every sample. Samples received and used before the current suite of oil properties was defined necessarily have incomplete characterization data. Often only small amounts of a particular sample were available, limiting the number of measurements that could be made. Data presented in this paper represent all the direct comparisons available at this time. 


\section{Comparisons and Correlations}

\section{RI and API gravity}

RI can be used as a measure of asphaltene stability (see Section 4.3). Often, however, the value of RI is not included in crude oil databases. Buckley et al. (1998b) estimated RI from a correlation with API gravity based on results for 11 oils. Since then, additional data have been accumulated, giving the improved correlation as shown in Fig. I-1.1. While direct measurement of RI is clearly preferable, estimation from the API gravity can be expected to give a reasonable approximation in cases where oil samples are no longer available for measurement.

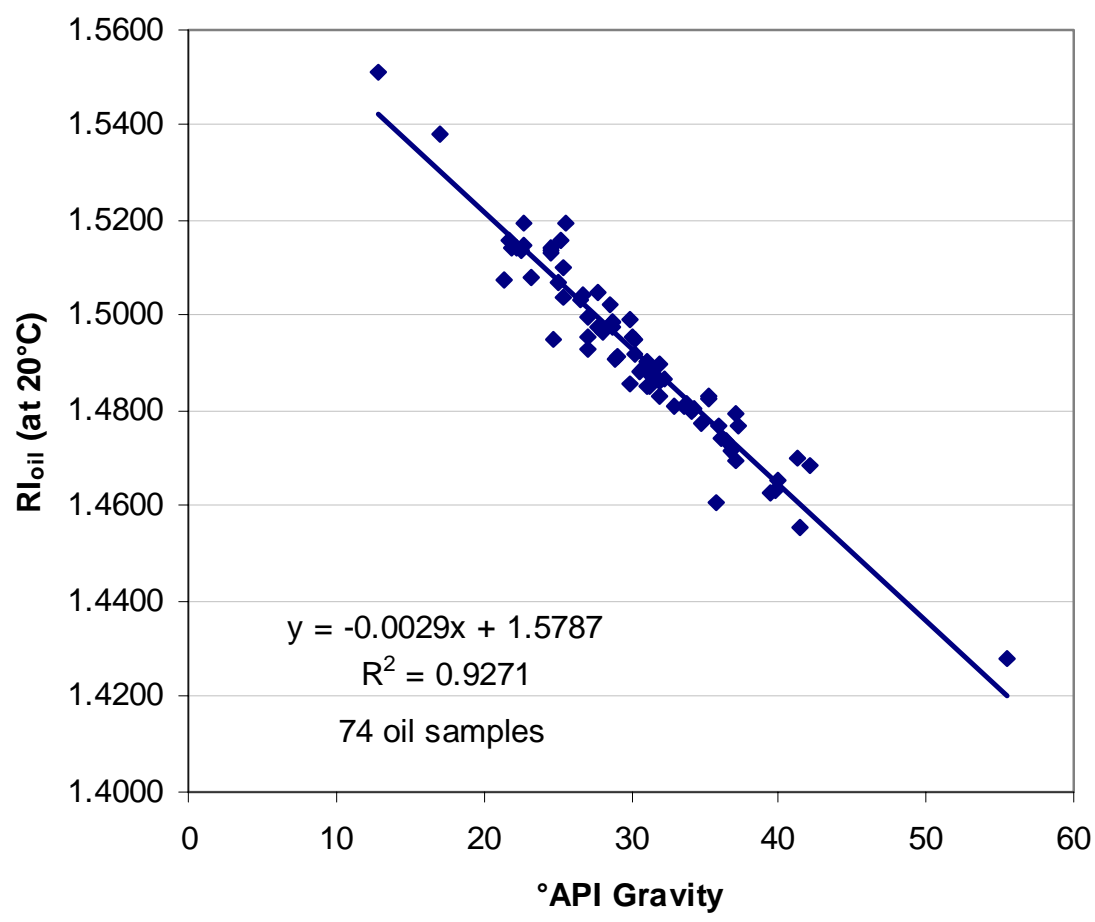

Figure I-1.1. Correlation between RI (sodium D line at $20^{\circ} \mathrm{C}$ ) and API gravity at $60^{\circ} \mathrm{F}$.

\section{Acids and bases}

Organic acids and bases can participate in the adsorption interactions that alter the wetting of mineral surfaces. The circumstances under which they do so have been discussed previously (Buckley et al., 1998c). Although a correlation between API gravity and base number (Manrique et al., 1997) has been reported, such a correlation may apply only to oils from related sources. The wide variety of oil samples in the CO-Wet database show only a broad trend of increasing acid and base number with decreasing API gravity, illustrated in Figs. I-1.2 and I-1.3, so that API gravity cannot be used to estimate acid or base number. 


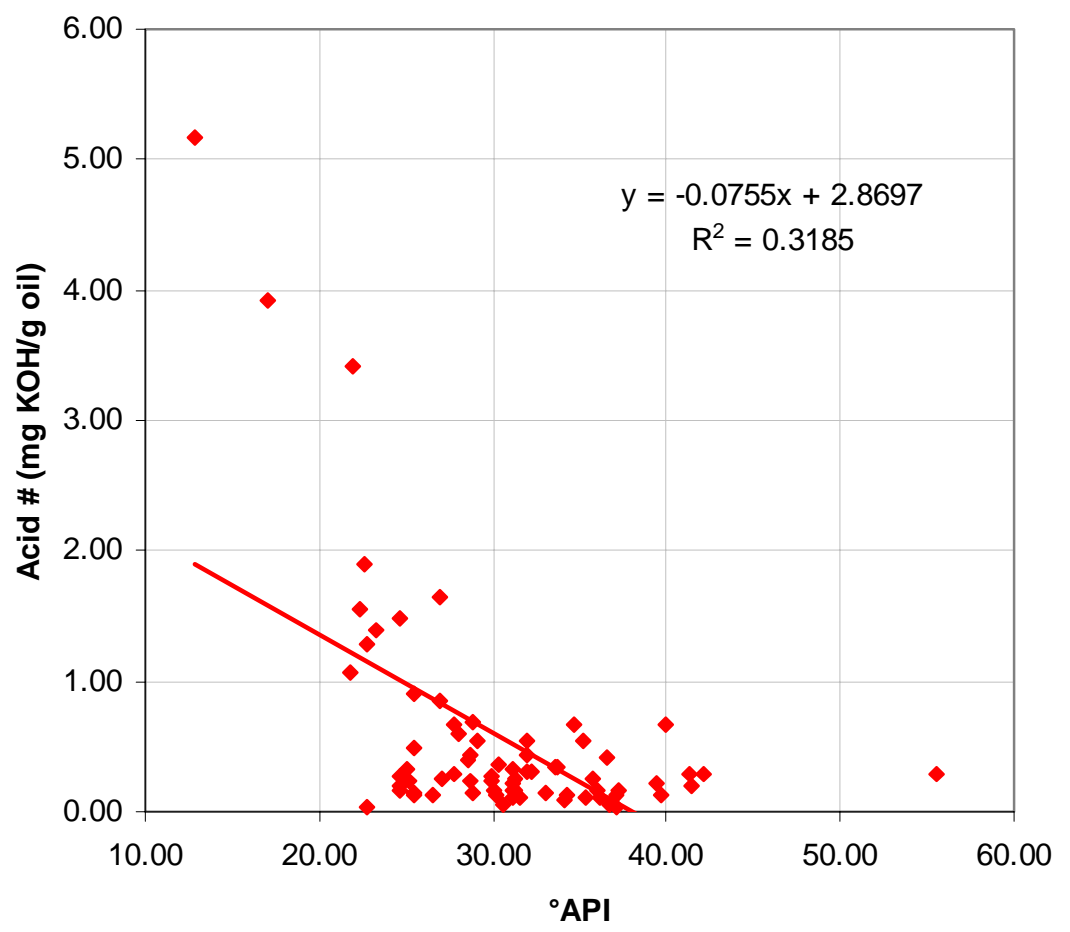

Figure I-1.2. Acid number vs. API gravity at $60^{\circ} \mathrm{F}$ for 73 crude oil samples.

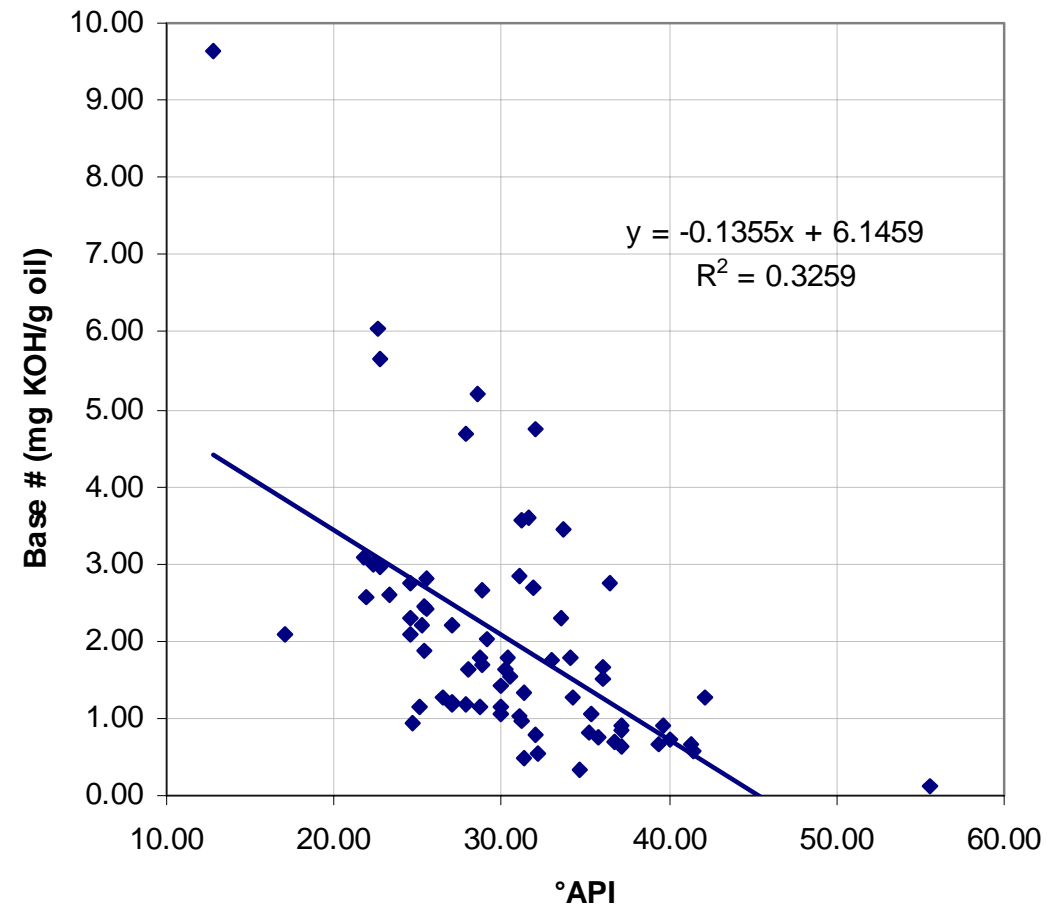

Figure I-1.3. Base number vs. API gravity at $60^{\circ} \mathrm{F}$ for 73 crude oil samples. 
Oils with an excess of bases over acids or acids over bases, as measured by their acid and base numbers, are expected to be those most likely to alter wetting by mechanisms that fall into the ionic interaction categories (e.g., acid/base and ion-binding). Figure I-1.4 demonstrates that, for a large number of crude oil samples from many geographic regions, these two quantities are poorly correlated. Most of the oil samples currently in the database have base numbers that are much higher than their acid numbers. Estimates of the ratios most likely to correspond to wetting alteration characteristics have yet to be established.

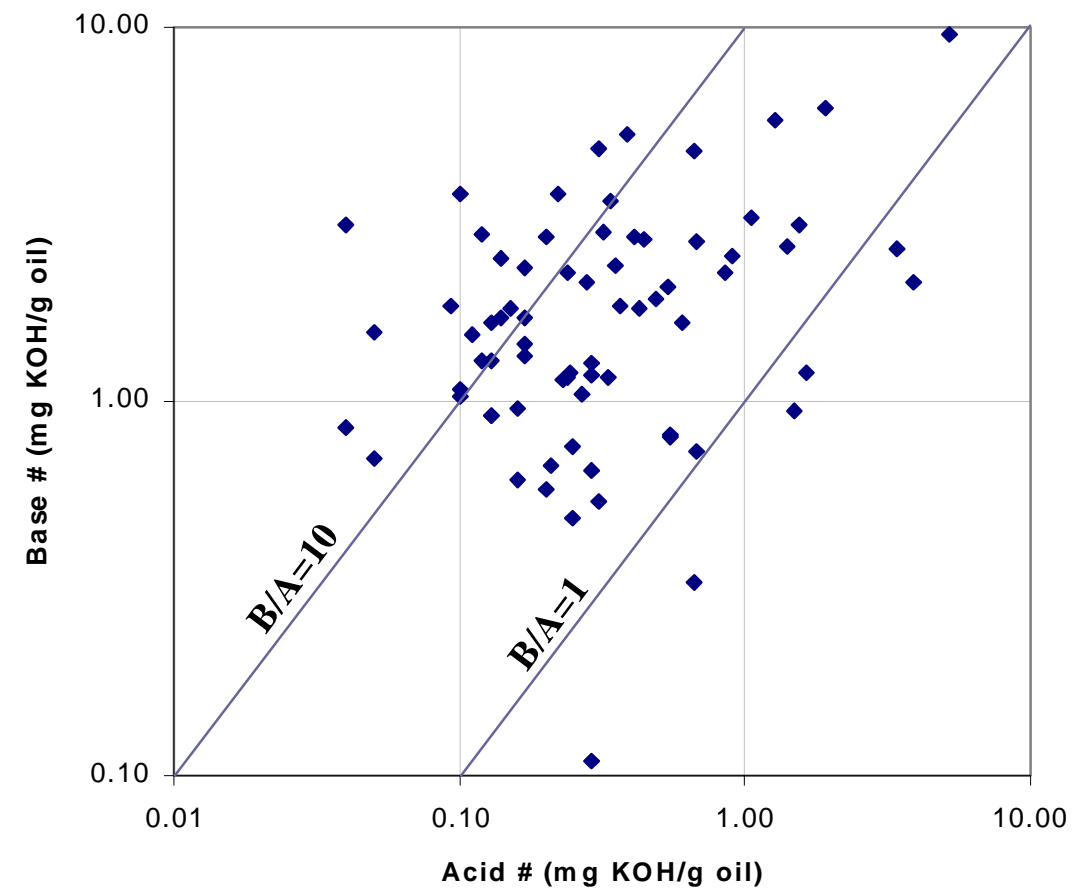

Figure I-1.4. Acid number (A) vs. base number (B) for 73 samples from the CO-Wet database. Diagonals show constant ratios (B/A) of 10 and 1 . Most samples have at least as high a base number as acid number and many have base numbers that exceed their acid number by more than 10 times.

Onset of asphaltene flocculation

A transition from ionic interactions to those dominated by surface precipitation of asphaltenes occurs near the onset of asphaltene flocculation (Al-Maamari and Buckley, 2000). Thus, evaluation of the likelihood of wetting changes must include a measure of the onset condition.

\section{ASPHALTENE STABILITY MAP}

The onset conditions can be quantified by measurements of the refractive index in mixtures of oil and precipitating agents when asphaltene aggregates can first be observed microscopically (Buckley et al., 1998b). RI at the onset, $\mathrm{P}_{\mathrm{RI}}$, is characteristic of each crude oil/precipitant pair. Figure I-1.5 shows the range of values of $\mathrm{RI}_{\mathrm{oil}}$ and $\mathrm{P}_{\mathrm{RI}}$ measured with nheptane for 53 crude oil samples. The abscissa, $\mathrm{RI}_{\text {oil }}$, gives an indication of how good a solvent 
the oil is for its asphaltenes. The ordinate, where $\mathrm{P}_{\mathrm{RI}}$ is plotted, is a measure of the stability or instability of asphaltenes from each oil. Together these numbers determine asphaltene stability to precipitation by $\mathrm{n}$-heptane. Diagonals represent constant values of the difference between $\mathrm{RI}_{\mathrm{oil}}$ and $\mathrm{P}_{\mathrm{RI}}$. Samples that fall into the same diagonal band have about the same stability.

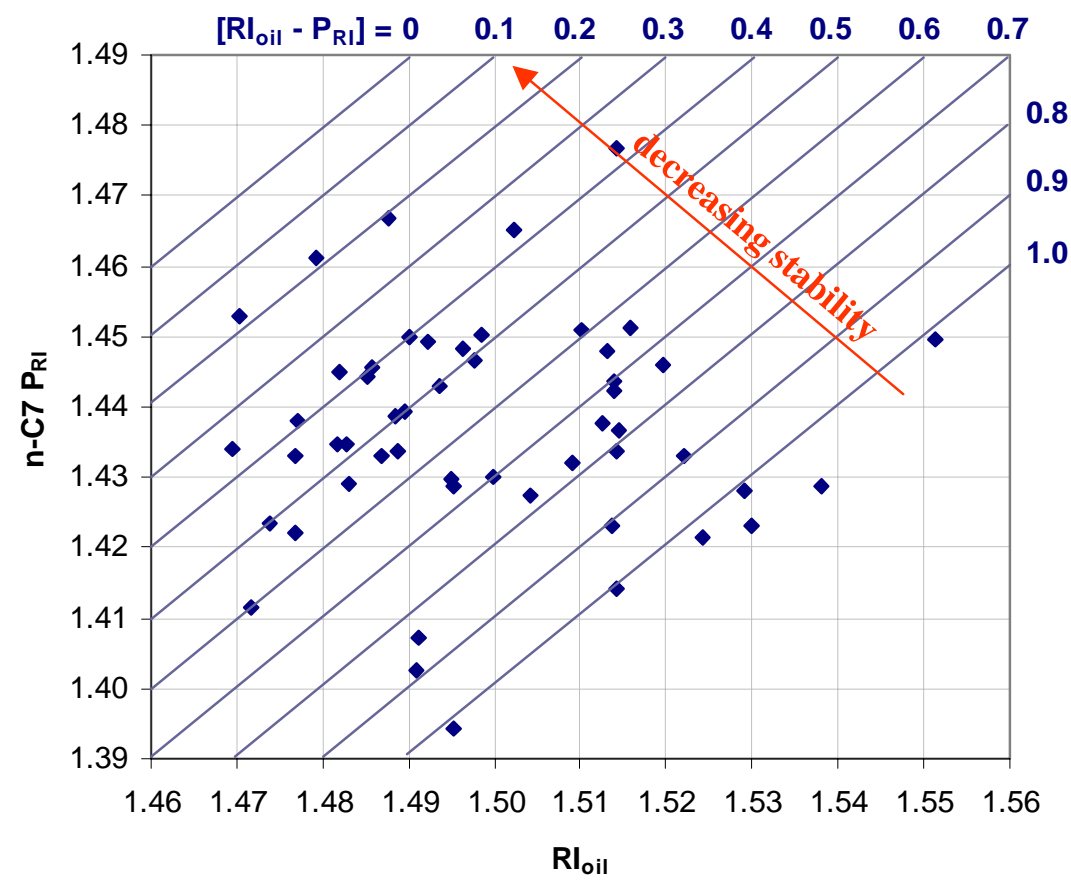

Figure I-1.5. Asphaltene stability map for 53 crude oil samples from the CO-Wet database. Diagonals toward the upper left are less stable and those toward the lower right are more stable. (All measurements were made at $20^{\circ} \mathrm{C}$.)

\section{Summary}

The data being accumulated will provide a broad range of well characterized oils whose wettability altering tendencies can be tested and related to parameters such as acid and base numbers and oil RI. It is clear that many oil samples are needed to avoid focusing on small differences that may be of little general significance. With data for more than 150 different samples, a library of characterized oils is being established from which samples for further wetting alteration tests can be selected systematically.

Applications of the CO-Wet data include establishment of a correlation between API gravity and RI, comparison of acid and base numbers, and development of a simple correlation between the onset of asphaltene flocculation and molar volume of precipitating agents.

The next major challenge is defining wetting tests. Since wetting alteration is influenced by brine and solid phases as well as the oil, tests must incorporate an adequate range of those components as well as oil chemistries. It should now be possible to establish limits on asphaltene stability in terms of mixture RI and onset $\mathrm{P}_{\mathrm{RI}}$ over which surface precipitation is expected. The limits of base/acid ratios for which oils are most active in ionic interaction 
mechanisms can also be tested. If there are important mechanisms and/or variables not yet captured in our crude oil characterizations, they should be apparent as deviations from these trends. These are among the issues that will be addressed in subsequent sections of this report.

\section{References}

Al-Maamari, R.S.H., and Buckley, J.S., 2000. Asphaltene precipitation and alteration of wetting: can wettability change during oil production? SPE 59292, SPE/DOE IOR Symp., Tulsa, 3-5 Apr.

ASTM D2007-80, 1980. Standard Test Method for Characteristic Groups in Rubber Extender and Processing Oils by the Clay-Gel Adsorption Chromatographic Method. ASTM.

ASTM D2896-88, 1988. Standard Test Method for Base Number of Petroleum Products by Potentiometric Perchloric Acid Titration. ASTM.

ASTM D664-89, 1989. Standard Test Method for Acid Number of Petroleum Products by Potentiometric Titration. ASTM.

Buckley, J.S., 1998a. Wetting alteration of solid surfaces by crude oils and their asphaltenes. Revue de l'Institut Français du Pétrol. 53(3): 303-312.

Buckley, J.S., Hirasaki, G.J., Liu, Y., Von Drasek, S., Wang, J.X., and Gill, B.S., 1998 b. Asphaltene precipitation and solvent properties of crude oils. Petrol. Sci. Tech. 16 (3\&4): 251285.

Buckley, J.S., Liu, Y., and Monsterleet, S., 1998c. Mechanisms of wetting alteration by crude oils. SPEJ, 3: 54-61.

Dubey, S.T. and Doe, P.H., 1993. Base number and wetting properties of crude oils. SPE Reservoir Engineering: 195-200.

Manrique, E.J., Mogollón, J.L., Linares, J.A., and Farrera, M., 1997. Basic nitrogen compounds in crude oils: effect on mineral dissolution during acid stimulation processes. SPE 37224, 1997 Internat. Oilfield Chem. Symp., Houston, 18-21 Feb. 


\section{I-2. SARA Fractionation of Crude Oils}

Despite wide use to characterize crude oils, SARA fraction tests are anything but standard. Different techniques are used in different laboratories and their results not comparable. Before generating SARA fraction data for the oils in our database, we undertook an exhaustive study of SARA methods, beginning with an examination of the ASTM-recommended method (Section I-2.1). With the results of that study to serve as a baseline, we compared other popular techniques including thin-layer chromatography and HPLC (Section I-2.2) before settling on an improved HPLC method.

\section{I-2.1. SARA Analysis of SQ-95 Crude Oil by the ASTM Method}

\section{T. Fan and J.S. Buckley, NM PRRC}

Separation of crude oils into saturate, aromatic, resin, and asphaltene (SARA) fractions is a widely used method of crude oil characterization. As part of the DOE Wettability and Imbibition Program, we are testing the use of SARA fraction data to improve crude oil characterization especially with respect to asphaltene stability. Stability of the asphaltene fraction is reduced as the amount of saturated hydrocarbons in an oil increases and is improved by increasing amounts of aromatics and resins in the oil. Since the tendency of an oil to alter wetting is influenced by asphaltene instability (e.g. Al-Maamari and Buckley, 1999), some measure of the state of the asphaltenes is an important part of crude oil characterization.

Refractive index (RI) has been shown to be a useful measure that is related to the state of asphaltenes in oil (Buckley et al., 1998; Buckley, 1999). If a crude oil sample is available, RI can readily be measured. If not, it may be possible to evaluate asphaltene stability from volumetric SARA fraction data. To establish whether SARA data can be used to reconstruct oil properties as they relate to asphaltene stability, oil and SARA fraction data will be compared.

The first step is to establish a repeatable SARA separation procedure, based on industry standard methods. Separation of crude oil into any number of fractions will involve some arbitrary decisions about dividing lines in what is really a continuum of molecular species. The only fraction that is chemically well defined is the saturate fraction. Aromatic, resin, and asphaltene fractions will include molecules with paraffinic side chains and varying degrees of aromaticity. The resins and asphaltenes encompass most of the heteroatomic species present in the oil and are separated mainly on the basis of the tendency of asphaltenes to flocculate in low molecular weight paraffins. In this report, a reproducible method of separating and collecting SARA fractions is documented. Physical properties of the fractions produced are reported and compared to the properties of the original oil sample. 


\section{Experimental Methods and Materials}

\section{Asphaltene Precipitation}

The first step in this experiment is to separate the asphaltenes from the remainder of the oil (maltenes). The column separation method is not directly applicable to oils with more than $0.1 \mathrm{wt} \%$ of pentane insolubles because of the difficulty or even impossibility of desorbing such species once they adsorb onto the separation columns.

Crude oil was thoroughly mixed with n-pentane at a weight ratio of 1:40. After 48 hours, the mixture was filtered through a funnel filter assembly (CAT 953805, KONTES GLASS) with a $0.22 \mu$ filter. Pentane was removed from the filtered liquid by evaporation. The asphaltenes and maltenes so produced were collected and weighed. Maltenes collected in this step were the starting mixture for the following SARA column separation. The lightest fraction of the crude oil, which evaporated with the pentane, was collected in a cold trap in some experiments. If not collected, the amount was calculated by difference.

Saturate, Aromatic, and Resin Fractions

The SARA separation process followed the ASTM method D 2007-93. Maltene samples, recovered from the asphaltene precipitation above, were used as the starting material for a twocolumn separation, as shown in Fig. I-2.1.

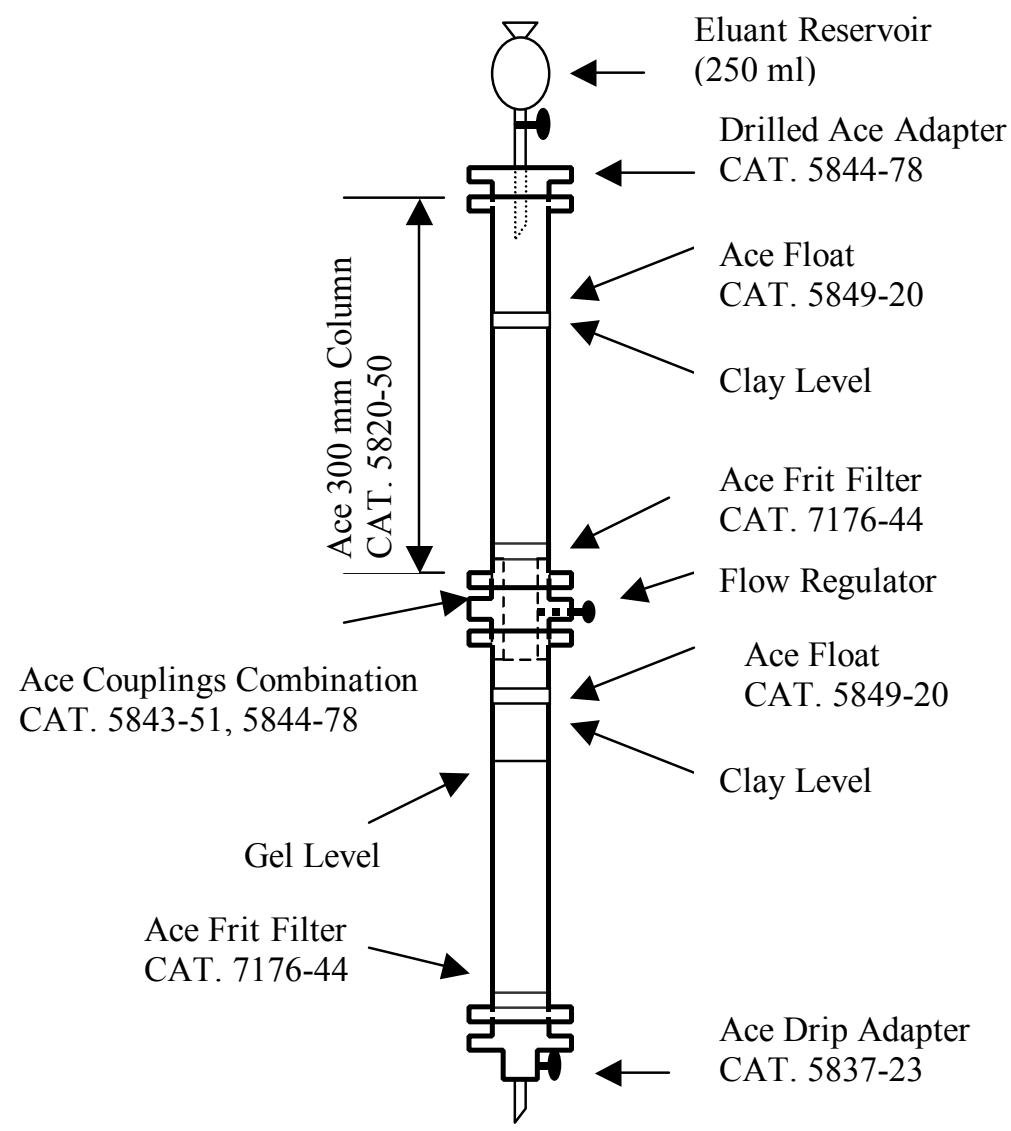

Figure I-2.1. Clay-gel percolating columns. 
The top column, through which a pentane solution of maltenes first passes, is packed with Attapulgite clay. Resins adsorb onto the clay, while saturates and aromatics are eluted and pass on to the lower column, packed with activated silica gel. The aromatic fraction adsorbs onto the silica gel, while the saturates elute and are collected. After evaporation of the pentane carrier, the weight of the saturate fraction can be determined. A mixture of toluene and acetone can be used to recover the resin fraction from the clay packing. The aromatics can be recovered by Soxhlet extraction of the silica gel in hot toluene, although quantitative recovery is not assured.

Initially, the column fractionating procedures in Part 8 of ASTM method D 2007 were strictly followed, but sample recovery from the columns was slightly less than $95 \%$. Two major adaptations were made in the final run that improved sample recovery. First, in order to distribute eluent uniformly over the cross section of the columns, two $50 \mathrm{~mm}$ porous floats (CAT. 5849-20, ACE GLASS) were placed on top of the clay and gel packing. Second, a flow regulator valve was drilled on the side of the Teflon Reducing Coupling (CAT 5843-51, Ace Glass), between the upper and lower sections of column (See Fig I-2.1), to allow adjustment of the pressure in the column. Adsorption of aromatics to silica gel is exothermic; temperatures as high as $80^{\circ} \mathrm{C}$ can be generated. Pressure increases to about 40 psi were observed as pentane vaporized, causing loss of sample due to leaks through the seals. Addition of a relief valve solved this problem and improved sample recovery.

\section{Measurements of chemical and physical properties}

Fractions were collected and weighed. For safety and convenience, a rotary evaporator in a water bath at $40^{\circ} \mathrm{C}$ replaced the hot plate recommended in ASTM D 2007. Additional measurements included density (Mettler/Paar DMA 40), RI (Index Instruments GPR 11-37), and gas chomatography coupled with mass spectrometry (VARIAN CHROMPACK, SATURN 2000 GC-MS). The GC-MS technique was used to examine the range of chemical structures in each fraction and to judge how successfully the various chemical species were separated.

\section{Reagents and Materials}

Acetone, HPLC Grade (99.5\%), Fisher Scientific.

Calcium Chloride, desiccant, 96+\%, Aldrich Chemical Co.

Azobenzene, 99\% (UV-VIS), ACROS ORGANICS

Pentane, HPLC Grade (99+\%), Aldrich Chemical Co.

Toluene, HPLC Grade (99.8\%), Fisher Scientific

Silica Gel, Grade 12, 28-200 mesh, Aldrich Chemical Co. (Activated for 4 hours at $190^{\circ} \mathrm{C}$ )

Clay Adsorbent, 30-60 mesh, Attapulgus.

SQ-95 crude oil from the Sulimar Queen field in Southeastern New Mexico 


\section{Azobenzene Activity Test for Attapulgus Clay}

\section{SUMMARY OF TEST METHOD}

Clay quality may be determined using the azobenzene equivalent test according to ASTM D2007-93. The azobenzene activity test measures the adsorptive characteristics of the clay. The requested azobenzene equivalent value should be 30 to 35. This test follows ASTM method D 2007 for measuring the adsorption activity of Attapulgus (Milwhite Co. Houston, Texas) clay with particle size 30 to 60 mesh. A solution of 1 mass\% azobenzene in isooctane is percolated through 20 grams of clay in a column as illustrated in Fig. I-2.2. The amount of liquid recovered when the concentration of azobenzene is half of the original concentration is a measure of the adsorption activity of the clay.

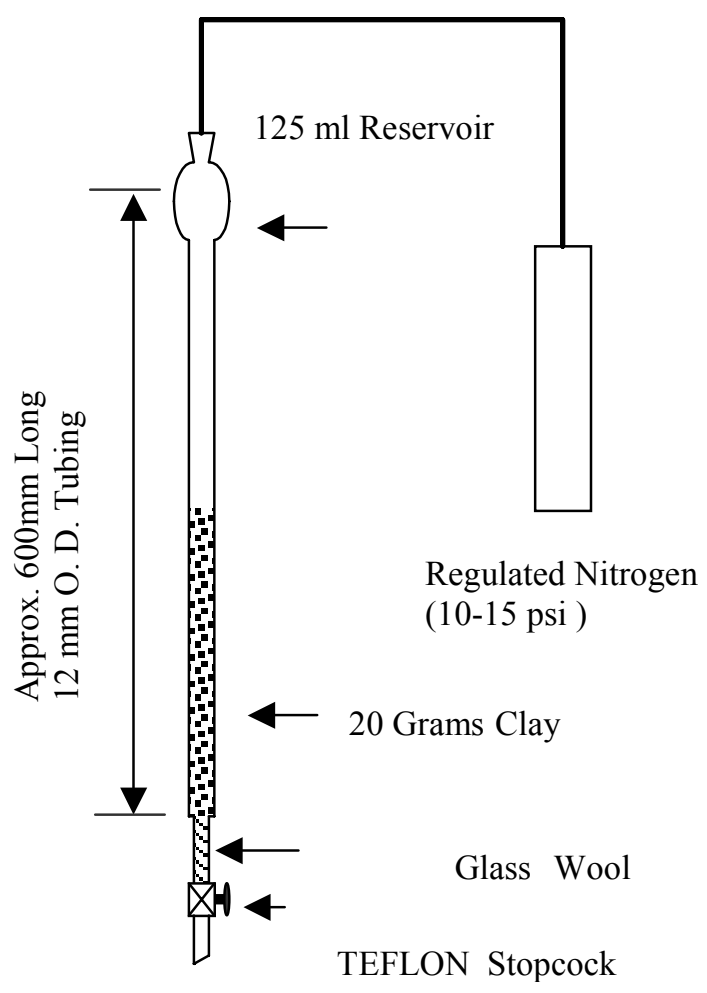

Figure I-2.2. Azobenzene percolation assembly.

REAGENTS

Azobenzene solution (1\% mass), prepared by dissolving $5 \pm 0.001 \mathrm{~g}$ of c.p. azobenzene in $445 \mathrm{~g}$ of isooctane. 


\section{PROCEDURES}

1) Insert a small piece of glass wool into the bottom of the glass column to prevent clay blocking. Weigh $20 \pm 0.001 \mathrm{~g}$ of clay sample and pour it into the column. Pack the clay in the column to a constant level by vibration. Add $100-115 \mathrm{ml}$ of $1 \%$ azobenzene solution to the column.

2) Collect the percolate in a $5 \mathrm{ml}$ graduated cylinder. Maintain the percolation rate at about 1 $\mathrm{ml} / \mathrm{min}$ by adjusting the stopcock valve or apply nitrogen pressure (10-15 psi).

3) After $25 \mathrm{ml}$ of effluent has passed through the column, collect six sequential samples of $2 \mathrm{ml}$ each. Measure the azobenzene concentration of these samples by comparing absorbance at $446 \mathrm{~mm}$ (MILTONROY SPECTROIC 20D) with absorbance of known concentrations of azobenzene in isooctane. The azobenzene standard absorption data is shown in Table I-2.1 and the calibrated standard curve is shown in Fig. I-2.3.

Table I-2.1. Absorbance of Azobenzene Standards

\begin{tabular}{|c|c|}
\hline $\begin{array}{c}\text { Azobenzene Concentration } \\
\left(\mathbf{1 0}^{-4} \mathbf{b y} \text { mass }\right)\end{array}$ & Absorbance \\
\hline 0 & 0 \\
\hline 1.0 & 0.100 \\
\hline 2.0 & 0.212 \\
\hline 3.0 & 0.304 \\
\hline
\end{tabular}

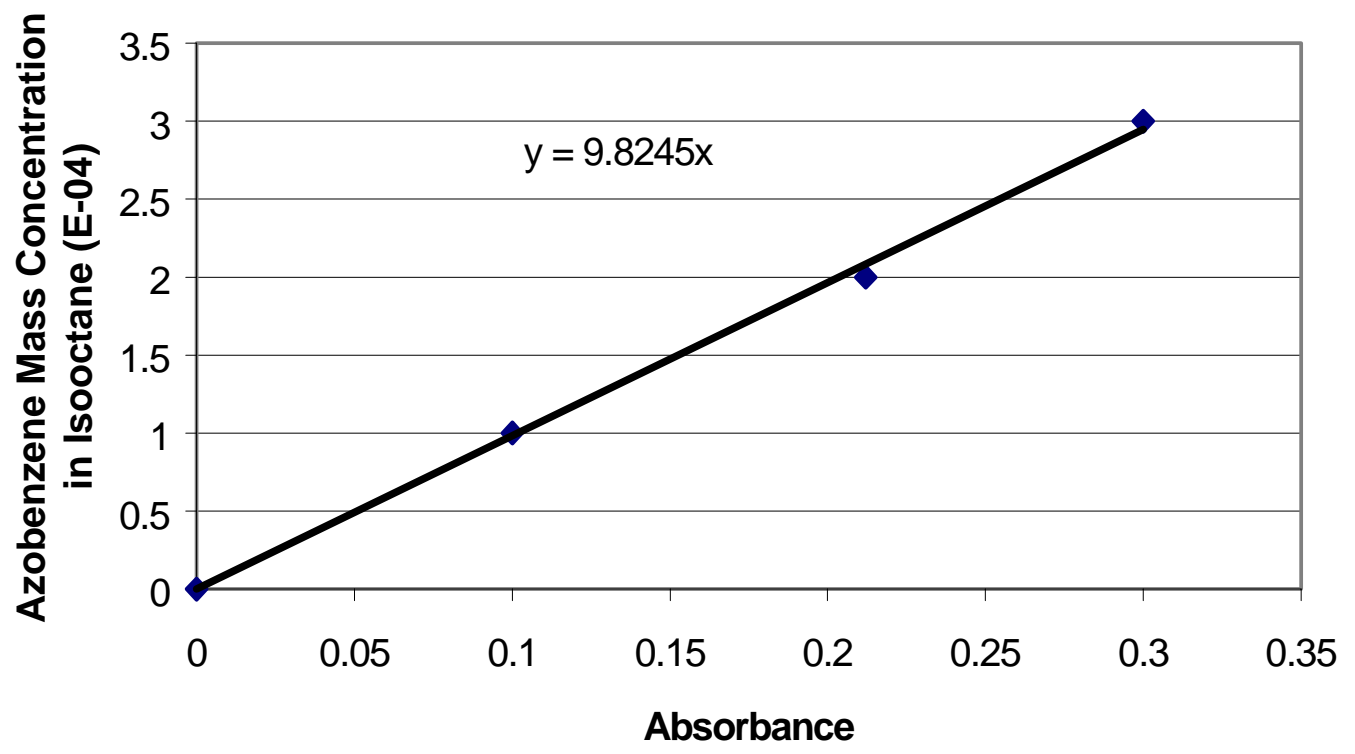

Figure I-2.3. Azobenzene absorbance standard curve.

4) Plot the effluent azobenzene concentration versus the effluent volume (Table I-2.2) as shown in Fig. I-2.4, and graphically determine the effluent volume at 0.5 mass $\%$ azobenzene concentration ( $50 \%$ of the starting solution concentration). The azobenzene equivalent of the 
Attapulgus clay is around $32 \mathrm{ml}$. This value meets the clay activity criterion stipulated in the ASTM method D 2007-93 which requires an azobenzene equivalence between 30 and $35 \mathrm{ml}$.

Table I-2.2. Azobenzene Mass Concentration in Effluent

\begin{tabular}{|c|c|}
\hline $\begin{array}{c}\text { Effluent Volume } \\
(\mathbf{m l})\end{array}$ & $\begin{array}{c}\text { Azobenzene Concentration } \\
\text { (mass \%) }\end{array}$ \\
\hline 26 & 0.33 \\
\hline 28 & 0.38 \\
\hline 30 & 0.48 \\
\hline 32 & 0.51 \\
\hline 34 & 0.55 \\
\hline 36 & 0.63 \\
\hline
\end{tabular}

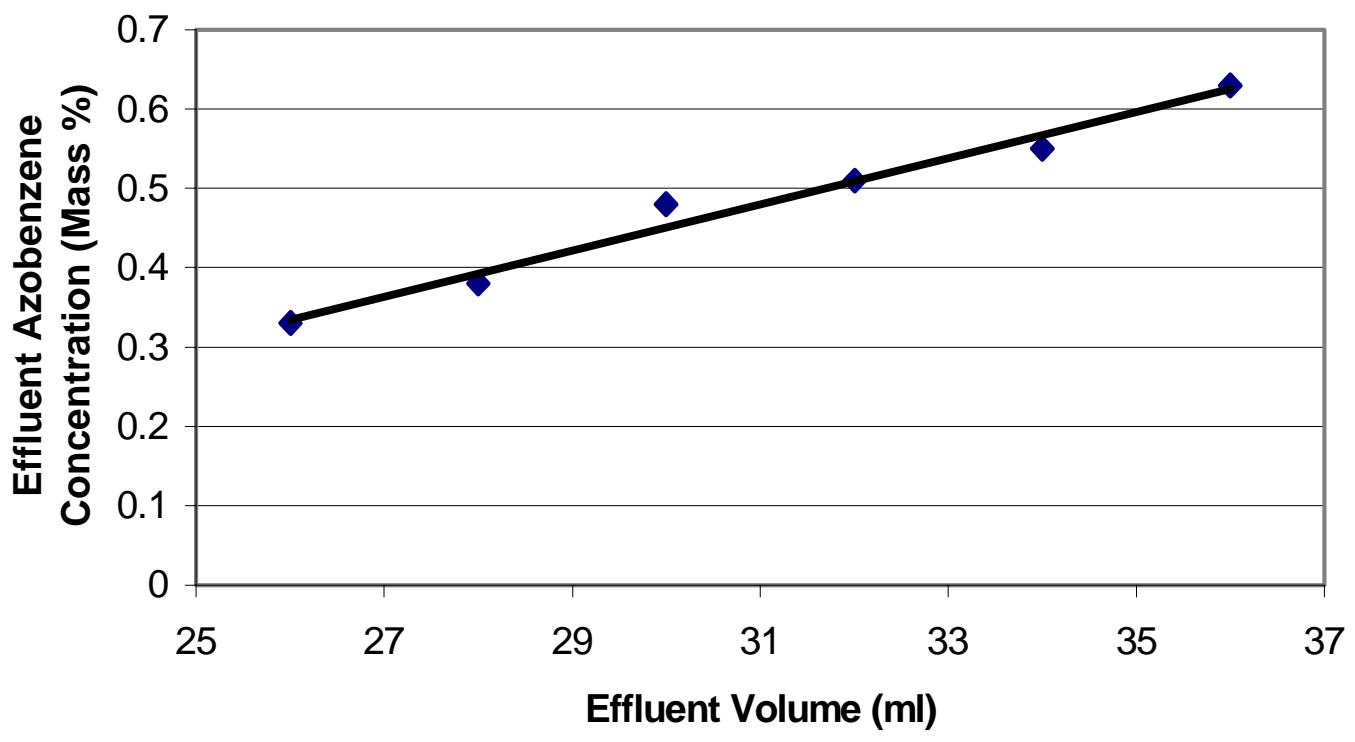

Figure I-2.4. Azobenzene activity test for clay.

\section{Results and Discussion}

\section{Asphaltene Precipitation}

Three asphaltene precipitation experiments are recorded in Table I-2.3. The SQ-95 crude oil samples used were all from the same storage bottle. During the weighing of crude oil, the balance readings changed continuously because of the evaporation of the light fraction unless the sample bottle was well sealed. The maltenes that were produced in runs \#1, \#2, and \#3 were mixed and used in the subsequent column separation processes. 
Table I-2.3. Asphaltene Precipitation Data

\begin{tabular}{|c|c|c|c|c|c|c|}
\hline Run \# & $\begin{array}{c}\text { Crude Oil } \\
\text { SQ-95 (g) }\end{array}$ & $\begin{array}{c}\text { Asphaltene } \\
(\mathbf{g})\end{array}$ & $\begin{array}{c}\text { Maltenes } \\
(\mathbf{g})\end{array}$ & $\begin{array}{c}\text { Asphaltene } \\
(\mathbf{w t} \%)\end{array}$ & $\begin{array}{c}\text { Maltenes } \\
(\mathbf{w t} \text { \%) }\end{array}$ & $\begin{array}{c}\text { Light Fraction } \\
(\mathbf{w t} \text { \%) }\end{array}$ \\
\hline 1 & 29.9076 & 1.3130 & 24.33 & 4.39 & 81.35 & 14.26 \\
\hline 2 & 32.2072 & 1.4036 & 25.98 & 4.36 & 80.67 & 14.97 \\
\hline 3 & 32.1323 & 1.3768 & 26.92 & 4.28 & 83.78 & 11.94 \\
\hline Average & & & & 4.34 & 81.93 & $13.73 *$ \\
STD & & & & 0.06 & 1.64 & 137 \\
\hline
\end{tabular}

* calculated by difference

\section{Column Separations}

Several samples of the mixture made up of all the maltenes produced in the asphaltene precipitation step were separated on the clay and silica gel columns. The first indication of successful separation is the colors of the SARA fractions: saturates are colorless, aromatics are yellow, and resins are black. Contamination, especially of the saturate fraction by aromatics, is readily observed.

Four replicate experiments are summarized in Table I-2.4. The first three runs were affected to some extent by loss of sample due to leakage; hence the recovery was less than the requisite $97 \%$ required by the ASTM procedure. Installation of a pressure relief valve before run \#4 appears to have improved the total recovery of sample, increasing the recovery from less than $95 \%$ in the first three runs to almost $98 \%$ in the final experiment. Other factors that may have affected recovery include percolation rate and head of the carrier fluids, which were adjusted manually to avoid formation of channels through the packing materials. Daytime room temperatures varied somewhat, from about $25^{\circ} \mathrm{C}$ to $28^{\circ} \mathrm{C}$, over the period of these experiments.

Table I-2.4. SARA Column Separation Results

\begin{tabular}{|c|c|c|c|c|c|c|c|c|}
\hline Run $(\#)$ & $\begin{array}{c}\text { Maltenes } \\
(\mathrm{g})\end{array}$ & $\begin{array}{c}\text { Saturate } \\
(\mathrm{g})\end{array}$ & $\begin{array}{c}\text { Saturate } \\
(\mathrm{wt} \%)\end{array}$ & $\begin{array}{c}\text { Aromatic } \\
(\mathrm{g})\end{array}$ & $\begin{array}{c}\text { Aromatic } \\
(\mathrm{wt} \%)\end{array}$ & $\begin{array}{c}\text { Resin } \\
(\mathrm{g})\end{array}$ & $\begin{array}{c}\text { Resin } \\
(\mathrm{wt} \%)\end{array}$ & $\begin{array}{c}\text { Mass Balance } \\
(\%)\end{array}$ \\
\hline 1 & 10.01 & 5.50 & 55.01 & 2.37 & 23.69 & 1.59 & 15.89 & 94.58 \\
\hline 2 & 11.01 & 6.61 & 60.06 & 2.04 & 18.54 & 1.78 & 16.17 & 94.77 \\
\hline 3 & 20.05 & 11.47 & 57.20 & 3.92 & 19.55 & 3.13 & 15.61 & 92.36 \\
\hline 4 & 20.03 & 11.70 & 58.40 & 4.66 & 23.26 & 3.24 & 16.17 & 97.80 \\
\hline Average & & & 57.67 & & 21.26 & & 15.96 & 94.91 \\
STD & & & 2.13 & & 2.60 & & 0.27 & 2.28 \\
\hline
\end{tabular}

\section{Properties of the SARA Fractions}

RI values were measured for each fraction, as summarized in Table I-2.5 and plotted in Fig. I-2.5. The distinction between saturates, aromatics, and resins is clear and reproducible, especially for the second, third, and fourth separation attempts. 
Table I-2.5. Refractive Index Measured at $20^{\circ} \mathrm{C}$

\begin{tabular}{|l|c|c|c|c|c|}
\hline & Run \#1 & Run \#2 & Run \#3 & Run \#4 & Mixture \\
\hline Light ends & & & & 1.3969 & \\
\hline Saturates & 1.4543 & 1.4414 & 1.4401 & 1.4389 & 1.4414 \\
\hline Aromatic & 1.5263 & 1.5402 & 1.5400 & 1.5296 & 1.5365 \\
\hline Resins & 1.5623 & 1.5790 & 1.5810 & 1.5805 & 1.5804 \\
\hline Maltenes & & & & & 1.4773 \\
\hline
\end{tabular}

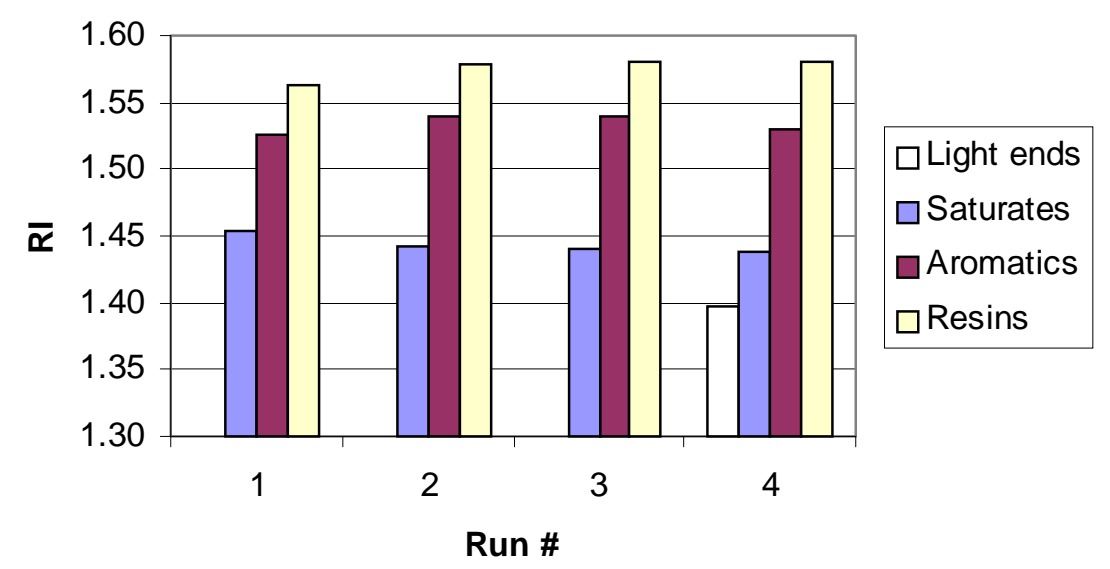

Figure I-2.5. Comparison of fraction RI's for four separations.

Saturates, aromatics, and resins from all four separations were mixed to provide enough of each fraction for further measurements. Densities of these mixtures are shown in Table I-2.6, where they are used to calculate volumetric fraction yields from the weights of each fraction recovered (Table I-2.4). In these calculations, the average weight percentages of saturates and resins are calculated from the weights of these fractions and of the original maltene sample weight. All of the under-recovery is attributed to aromatics that remain on the silica gel column. Even after six hours of extraction with hot toluene, the silica gel remained weakly yellow in color, indicating that some aromatics were still adsorbed. Since resins and saturates are easier to collect, the ASTM method D 2007 recommends calculating the weight percentage of aromatics by difference.

Table I-2.6. Density of SARA Fractions at $20^{\circ} \mathrm{C}$

\begin{tabular}{|c|c|c|c|c|c|}
\hline Fraction & Light ends & Saturate & Aromatics & Resin & Maltenes \\
\hline Density (g/ml) & 0.6892 & 0.7938 & 0.9361 & 0.9974 & 0.8559 \\
\hline Weight (\%) & & 57.67 & $26.37^{*}$ & 15.96 & 100 \\
\hline Volume (\%) & & 62.19 & 24.11 & 13.70 & 100 \\
\hline
\end{tabular}

* calculated by difference

If the volume-weighted average density is calculated from the numbers in Table 4 , the calculated value is $0.8560 \mathrm{~g} / \mathrm{ml}$, which agrees very well with the measured value of $0.8559 \mathrm{~g} / \mathrm{ml}$. This agreement supports the use of the higher value for the weight percent of aromatics that was 
calculated from mass balance, rather than the lower value for the amount of aromatics removed from the silica gel.

Mixture RI can also be calculated by a volume-weighted average, according to Equation $\mathrm{I}-2.1$.

$$
\frac{n^{2}-1}{n^{2}+2}=\sum_{i} f_{\text {volume }, i}\left(\frac{n_{i}^{2}-1}{n_{i}^{2}+2}\right)
$$

where $\mathrm{n}$ is the mixture RI, $n_{i}$ is the RI of component $\mathrm{i}$, and $f_{\text {volume }, i}$ is the volume fraction of component i. Each of the fraction-saturates, aromatics, resins - can be considered as one component of the maltenes. If this equation is true, it should also be used to estimate the RI of maltenes by knowing the RIs and volumetric amounts of its component saturates, aromatics, and resins. The test results in Table I-2.7 show that the accuracy of this equation is good for estimating RI from volumetric amounts of each fraction in maltenes.

Table I-2.7. Comparison of Measured and Calculated RI for Maltenes

\begin{tabular}{|c|c|c|c|c|}
\hline Fraction & Maltenes & Saturates & Aromatics & Resins \\
\hline$N$ (or RI) & 1.4773 & 1.4414 & 1.5365 & 1.5804 \\
\hline$f_{\text {volume }, i}$ & 1 & 0.6219 & 0.2411 & 0.1370 \\
\hline \multirow{2}{*}{$f_{\text {volume }, i}\left(\frac{n_{i}^{2}-1}{n_{i}^{2}+2}\right)$} & \multirow{2}{*}{0.2827} & 0.1638 & 0.0466 & 0.0749 \\
\cline { 3 - 5 } & & \multicolumn{3}{|c|}{$\sum=0.2853(n=1.482)$} \\
\hline
\end{tabular}

A similar calculation can be made using the RIs and densities of the original SQ-95 crude oil and its maltene and light fractions, as shown in Table I-2.8. There are two unknown quantities: density and RI of the asphaltenes. If a density of $1.2 \mathrm{~g} / \mathrm{ml}$ is assumed, the RI of the $n$-pentane asphaltenes can be estimated from Eq. 1 and the original RI of the oil. The resulting estimate for RI of the asphaltene fraction of 1.8 is somewhat higher than the values of 1.72 estimated for $n$-hexane asphaltenes from A-93 crude oil (Buckley, et al., 1998) and 1.67 reported by Christianson and Israelachvili (1986) for material adsorbed from crude oil onto mica surfaces.

Table I-2.8. Estimate of Asphaltene RI.

\begin{tabular}{|c|c|c|c|c|}
\hline Fraction & SQ-95 & Light ends & Maltenes & Asphaltenes $\left(\mathbf{n C}_{5}\right)$ \\
\hline Weight \% & 100 & 13.73 & 81.93 & 4.34 \\
\hline Density (g/ml) & 0.8351 & 0.6892 & 0.8459 & $1.2^{*}$ \\
\hline$n($ or RI) & 1.4750 & 1.3969 & 1.4773 & $1.8^{* *}$ \\
\hline$f_{\text {volume }, I}$ & 1.0000 & 0.1664 & 0.8088 & 0.0302 \\
\hline \multirow{2}{*}{$f_{\text {volume }, i}\left(\frac{n_{i}^{2}-1}{n_{i}^{2}+2}\right)$} & \multirow{2}{*}{0.2815} & 0.0401 & 0.2287 & 0.0129 \\
\cline { 3 - 5 } & & \multicolumn{4}{|c|}{$\Sigma=0.2816$} \\
\hline
\end{tabular}

*value used by Buckley et al. (1998); **estimate based on matching calculated and measured $\mathrm{RI}_{\mathrm{oil}}$ 
The saturate and aromatic and light fractions were analyzed by GC-MS. The GC chromatograms (Figs I-2.6 - I-2.8) help to show how thoroughly the various oil fractions were separated. In chromatograms of the saturate fraction, there are about 20 major representative peaks and the majority of the constituents elute within a $\mathrm{GC}$ column temperature range of $90^{\circ} \mathrm{C}$ to $270^{\circ} \mathrm{C}$. The mass spectra of the major peaks indicate that these compounds are alkanes. The aromatic fraction is a very complex mixture and gives a great number of peaks eluting from the $\mathrm{GC}$ column at a temperature range from $90^{\circ} \mathrm{C}$ to $300^{\circ} \mathrm{C}$. The mass spectra of these peaks indicate that these are mainly single-ring aromatic compounds with one to four substitutions on the ring. Naphthalene compounds are also identified in smaller amounts. Attempts to analyze the resin fraction by GC-MS failed due to low volatility of the sample components. No peaks were observed in the temperature range of $90^{\circ} \mathrm{C}$ to $320^{\circ} \mathrm{C}$, the maximum $\mathrm{GC}$ oven temperature.

The components of the lightest fraction mostly eluted at a column temperature of $35^{\circ} \mathrm{C}$, with minor components eluting at temperatures approaching $110^{\circ} \mathrm{C}$. Without cryogenic cooling, many of the components coelute at or near $t_{0}$, the retention time of an unretained component. Because of this, it is difficult to identify their structures. Spectra of higher boiling components that were well separated, as well as some earlier components, identified them as alkanes.

\section{References}

Al-Maamari, R. and Buckley, J.S.: "Effects of Crude Oil Solvent Properties on Wetting Alteration," Quarterly Report for the project "Evaluation of Reservoir Wettability and its Effect on Oil Recovery," DOE Cooperative Agreement No. DE-FC22-96ID13421, January 1, 1999 - March 31, 1999.

Buckley, J.S.: "Predicting the Onset of Asphaltene Precipitation from Refractive Index Measurements," Energy \& Fuels (1999) 13, No. 2, 328-332.

Buckley, J.S., Hirasaki, G.J., Liu, Y., Von Drasek, S., Wang, J.X., and Gill, B.S.: "Asphaltene Precipitation and Solvent Properties of Crude Oils," Petroleum Science and Technology (1998) 16, No. 3\&4, 251-285.

Christenson, H.K. and Israelachvili, J.N.: "Direct Measurements of Interactions and Viscosity of Crude Oils in Thin Films between Model Clay Surfaces," J. Colloid Interface Sci. (1987) 119, 194. 


\section{Chromatogram Plot}

Filo: c:lsaturnwsidatalinstallivolatiles 9-19-99001.sms $9 / 19 / 994: 22$ PM Operator: chris

Scan Rango: 1 - 1939 Time Rango: $0.00-23.98 \mathrm{~min}$
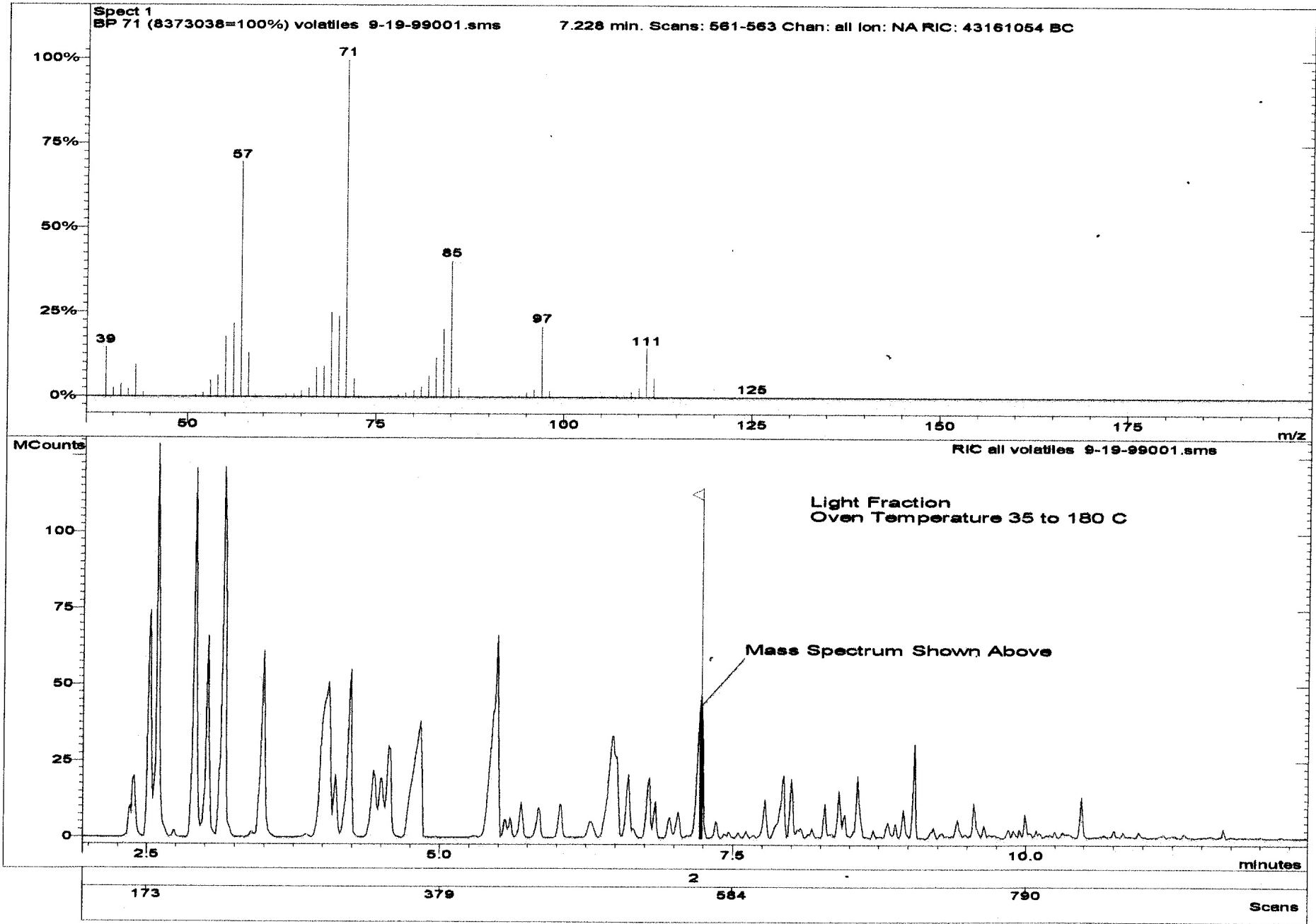

Figure I-2.6. Gas chromatogram and selected mass spectrum of light ends. 


\section{Chromatogram Plot}

File: c:lsaturnwsldatalinstallsaturates 5 9-12-99.sms
Samplo: saturates 5
Sample Notes:

Oporator: chris

Scan Range: 1 - 1577 Time Range: $0.00-19.98 \mathrm{~min}$.
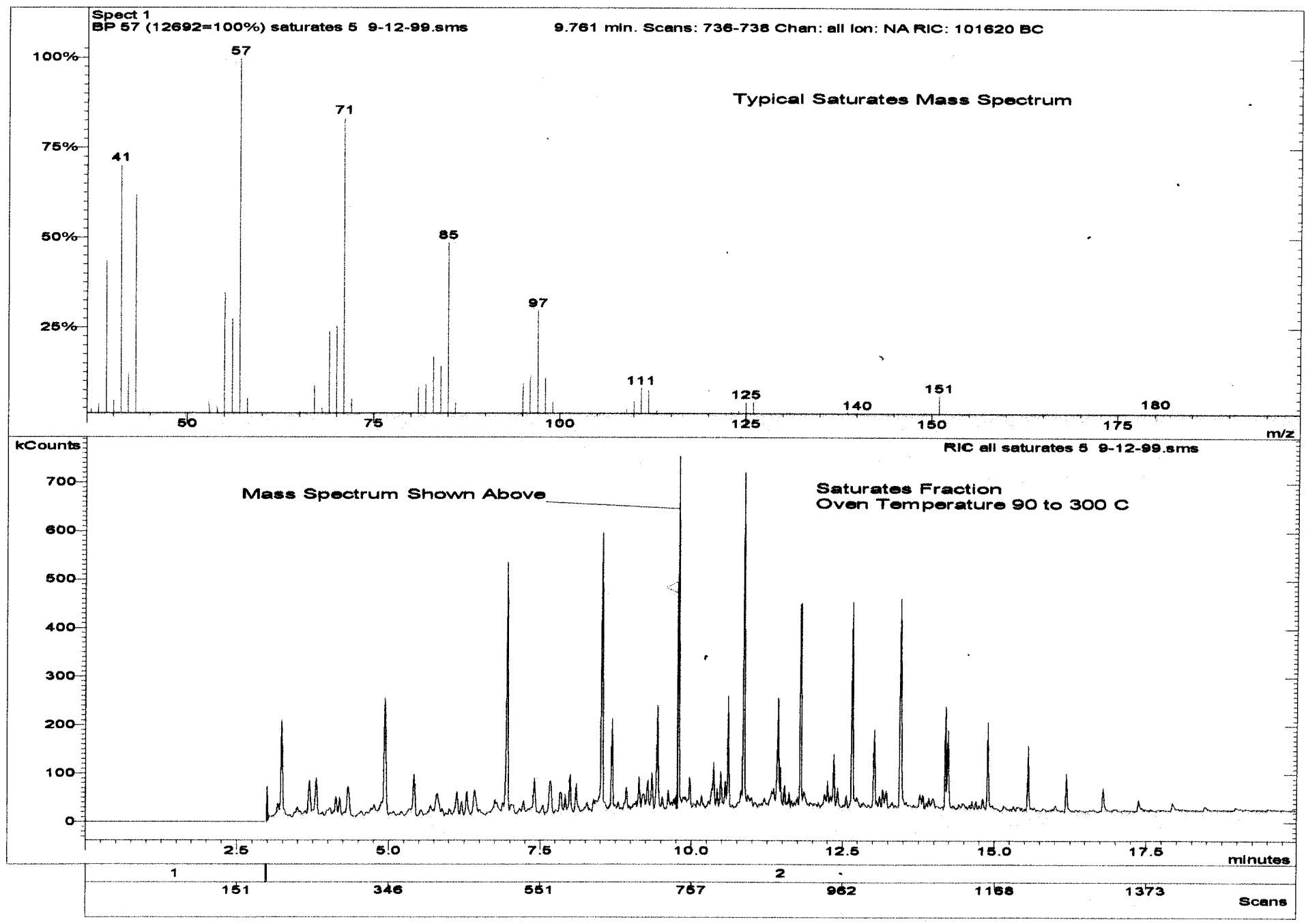

Figure I-2.7. Gas chromatogram and selected mass spectrum of saturates. 


\section{Overlaid Chromatogram Plots}

Plot 1: elsaturnwoldatavinstaipblank 3 9-17-00.m. RiC all

Plot Z: eil. Vdatalinstaillaromaties tout 9-19-99. $\mathrm{sm}$ s RiC ail

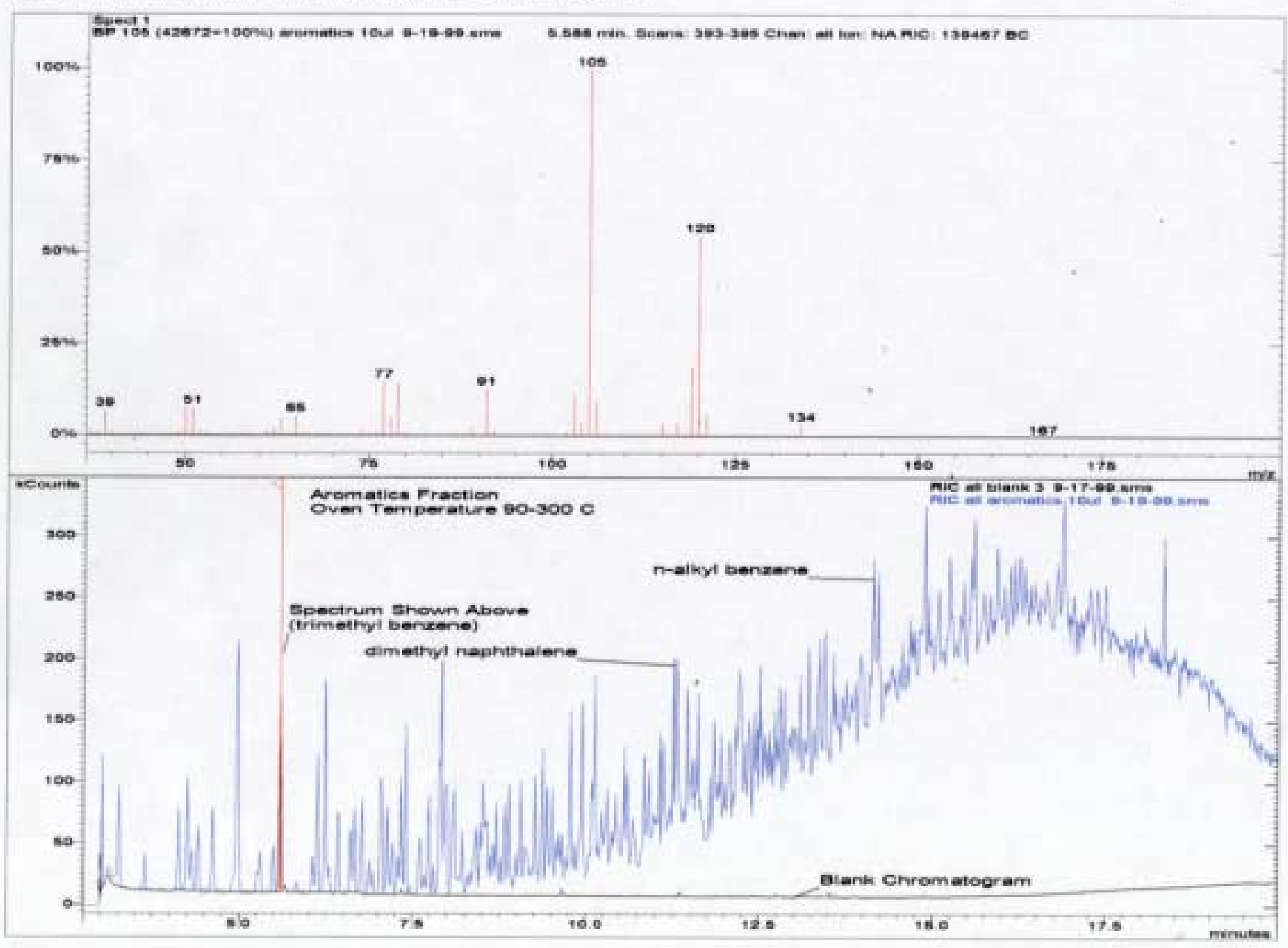

Figure I-2.8. Gas chromatogram and selected mass spectrum of aromatics. 


\section{I-2.2. Evaluation of SARA Analysis Techniques}

Fan, T. and Buckley, J.S.: "Rapid and Accurate SARA Analysis of Medium Gravity Crude Oils," accepted by Energy and Fuels, 2002.

\section{Crude Oils and Hydrocarbon Group Separations}

Crude oils are complex mixtures of many components, not all of which are miscible under all conditions of temperature, pressure, and composition. As temperature and pressure decrease during oil production, the lightest components can partition into a vapor phase, waxes can precipitate, and the heaviest, most polar components can form a separate, asphaltene-rich phase. Complete chemical characterization is not feasible, but characterization of related groups of oil components can be useful. How the oil is subdivided depends on the purpose of the characterization. For example, to predict gas phase evolution, the mole fractions of the lightest components need to be measured, with everything larger than some cutoff value (e.g., $\mathrm{C}_{7}+$ ) lumped into a single heavy pseudo-component. For the purposes of wettability alteration we need two types of fractionation: (1) acids, bases, and neutral species, and (2) saturate, aromatic, resin, and asphaltene fractions, often abbreviated as SARA.

In this report we focus on SARA separations, comparing existing techniques and presenting newly developed improvements that aim to make SARA analyses faster, more reproducible, and more representative for a wide range of crude oils. SARA fractions are of interest because most of the polar species that have the potential to alter wetting occur in either the resin or asphaltene fractions. It is also necessary to divide the lighter, non-polar materials into saturates and aromatics because asphaltenes are soluble in aromatics and insoluble in much of the saturate fraction. Separation of crude oil into any number of fractions involves some arbitrary decisions, however, about dividing lines in what is really a continuum of molecular species. The only fraction that is chemically well defined is the saturate fraction. Aromatic, resin, and asphaltene fractions will include molecules with paraffinic side chains and varying degrees of aromaticity. The resins and asphaltenes encompass most of the heteroatomic species present in the oil and are separated from each other mainly on the basis of the tendency of asphaltenes to flocculate in low molecular weight paraffins.

\section{SARA Fractions and Wettability Alteration}

Because asphaltenes are soluble in aromatics and resins, whereas they are insoluble in most saturates, SARA fraction data can help to characterize different crude oils with respect to asphaltene solvation and stability. The tendency of asphaltenes to alter wettability is probably greatest near the onset of flocculation (e.g. Al-Mamaari and Buckley, 1999). Thus a measure of asphaltene stability is necessary as part of the evaluation of the wettability altering propensity of a crude oil.

One way that we might be able to use SARA data to evaluate crude oils for their ability to solvate their asphaltenes is to estimate a refractive index (RI) for the oil from the SARA fractions. For the mostly non-polar species in a crude oil, RI is related to solubility parameter (Buckley et al., 1998; Buckley, 1999) and thus can be used to predict the onset of asphaltene flocculation (Wang, 2000; Buckley and Wang, 2001). If a crude oil sample is available, RI can 
readily be measured. If not, it may be possible to evaluate asphaltene stability from volumetric SARA fraction data.

\section{Brief Review of SARA Fractionation Techniques}

SARA analysis began with the work of Jewell et al. (1972). Three approaches have been widely used to separate crude oils and other hydrocarbon materials into SARA fractions. The first of these involves clay-gel adsorption chromatography and is summarized in ASTM D2007. Because this method requires large amounts of sample, is time consuming, and difficult to automate, a great deal of effort has been focused on development of improved methods of SARA fractionation.

The improved methods fall into two broad groups. In the first group are high pressure liquid chromatographic (HPLC) methods, first introduced by Suatoni and Swab (1975). Early HPLC techniques used silica or alumina columns to separate lighter petroleum fractions. The development in preparation of bonded phase HPLC columns-especially $\mathrm{NH}_{2}$-bonded materials - made it practical to separate heavier fractions of petroleum samples (Miller, 1982; Radke et al., 1984; Grizzle and Sabotny, 1986; Félix et al., 1987; Chaffin et al., 1996). Many combinations of solvent sequences with and without backflushing can be used to optimize separations of specific groups. In general, HPLC techniques are faster, more reproducible, and more readily automated than the ASTM column technique. In both cases, however, it is necessary to remove the asphaltene fraction before proceeding with the chromatography. Asphaltenes are either irreversible adsorbed or precipitated during the saturate elution step and quantitative recovery cannot be achieved (McLean and Kilpatrick, 1997).

An even more rapid separation can be made using thin layer chromatography (TLC) using quartz rods coated with sintered silica particles. Unlike column and HPLC techniques, asphaltenes need not be separated from other crude oil components before chromatographic analysis. A popular technology known as the Iatroscan that combines TLC with flame ionization detection (TLC-FID) was first applied by Suzuki (1972) to automate quantitative SARA separations, a method which has since been used extensively (e.g., Karlsen and Larter, 1991; Vela et al., 1998). Barman (1996) compared SARA analyses of heavy hydrocarbon distillates by both the clay-gel and TLC-FID methods. TLC-FID uses very small amounts of sample. SARA fractions in a crude oil sample are often well separated using established development procedures and quantitative results are obtained by the measurement of peak areas, assuming that each SARA fraction has an identical FID response factor.

\section{SARA Fractionation Problems}

Each of these techniques has disadvantages as well as advantages. No standard SARA fractionation has emerged. Comparisons of SARA fraction measurements by different techniques, usually from different laboratories, can show large differences. To some extent these differences might be real-caused by the use of different samples. In this work we will examine the same samples by different techniques to illuminate the strengths and weaknesses of each and to develop a standard method.

In a previous report (Fan et al., 1999), a reproducible method of separating and collecting SARA fractions by using the clay-gel absorption technique was documented. Physical properties 
of the fractions produced from a waxy oil were reported and compared to the properties of the original oil sample. Additional measurements made by the clay-gel method are reported here to serve as the baseline for evaluation of improved techniques. Disadvantages of the clay-gel absorption method include the consumption of large material requirements (sample, solvents, and adsorbents) and lengthy analysis time. Material balance indicates some loss of light ends, especially during the asphaltene precipitation step.

Gravimetric measurements have often been used for quantification of SARA fractions in crude oil HPLC analyses (Mckay and Latham, 1980; Grizzle and Sablotny, 1986). Because there were no standard materials that could be considered representative of the SARA fractions from a broad range of crude oils, calibration of methods other than gravimetric detection was a problem. Suatoni and Swab (1975) separated saturates and aromatics from a wide range of crude oils to establish calibration curves between RI and boiling point for these two groups. This method is limited by the need for sufficient quantities of separated saturates and aromatics and the requirement for boiling point information that may not be readily available.

The greatest problem with the TLC-FID method is excessive loss of low boiling compounds that may be either saturates or aromatics or, most likely, a mixture of the lowest molecular weight species in both groups. Weight loss of low boiling hydrocarbons can be expected to be larger in measurements by the TLC-FID method where rods are exposed to atmospheric conditions than by the clay-gel method in a sealed column assembly. Karlsen and Larter (1991) measured losses of approximately $50 \%$ of $n-C_{14}$ (b.p. $253.5^{\circ} \mathrm{C}$ ), whereas the loss of $n-\mathrm{C}_{16}$ (b.p. $286.8^{\circ} \mathrm{C}$ ) was negligible. Large losses of components that boil below about $250^{\circ} \mathrm{C}$ should therefore be expected. TLC-FID analysis results can be considered quantitative only for very heavy oil samples.

\section{Experimental Techniques and Results}

\section{Crude Oils}

Six medium gravity crude oils, varying in API gravity from 22.6 to $37.2^{\circ}$ were used in this study. Selected physical and chemical parameters are shown in Table I-2.9.

Table I-2.9. Physical and Chemical Properties of Crude Oil Samples

\begin{tabular}{|l|c|c|c|c|c|c|c|c|c|}
\hline \multicolumn{1}{|c|}{ Oil } & ${ }^{\circ} \mathbf{A P I}$ & $\begin{array}{c}\mathbf{M W} \\
(\mathbf{g} / \mathbf{m o l})\end{array}$ & $\begin{array}{c}\mathbf{R I} \\
\left(\mathbf{2 0}{ }^{\circ} \mathbf{C}\right)\end{array}$ & $\mathbf{P}_{\mathbf{R I}}$ & $\begin{array}{c}\mathbf{n C}_{\mathbf{7}} \mathbf{a s p h} \\
(\mathbf{\%})\end{array}$ & $\begin{array}{c}\mathbf{d e n s i t y} \\
\mathbf{a t ~ 2 0} \mathbf{C} \\
\left(\mathbf{g} / \mathbf{c m}^{\mathbf{3}}\right)\end{array}$ & $\begin{array}{c}\text { viscosity } \\
\mathbf{a t} \mathbf{2 0} \mathbf{C} \\
(\mathbf{c P})\end{array}$ & Acid\# & Base \# \\
\hline A-95 & 25.2 & 236 & 1.5128 & 1.4513 & 8.67 & 0.8956 & 41.20 & 0.24 & 2.20 \\
\hline C-LH-99 & 22.6 & 268 & 1.5137 & 1.4231 & 2.78 & 0.9161 & 89.57 & 1.90 & 6.05 \\
\hline C-R-00 & 31.3 & 235 & 1.4851 & 1.4444 & 1.88 & 0.8673 & 19.05 & 0.25 & 0.49 \\
\hline S-Ven-39 & 28.8 & 240 & 1.4976 & 1.4465 & 5.79 & 0.8795 & 29.80 & 0.14 & 1.68 \\
\hline SQ-95 & 37.2 & 213 & 1.4769 & 1.4223 & 1.30 & 0.8409 & 5.84 & 0.17 & 0.62 \\
\hline Tensleep-99 & 31.1 & 270 & 1.4906 & $\sim 1.44$ & 4.10 & 0.8685 & 18.65 & 0.10 & 1.03 \\
\hline
\end{tabular}




\section{ASTM-Recommended Column Separation}

Baseline data were produced using the ASTM recommended method for separation of saturates, aromatics, resins, and asphaltenes (ASTM D2007-93). In an earlier report (Fan et al., 1999) the use of this method was documented in detail.

\section{EXPERIMENTAL METHOD}

Asphaltene precipitation

Crude oil was thoroughly mixed with $n$-hexane at a weight ratio of 1:40. After 48 hours, the mixture was filtered through a funnel filter assembly (CAT 953805, KONTES GLASS) with a $0.22 \mu$ filter (Millipore cellulose nitrate). Hexane was removed from the filtered liquid by evaporation. The asphaltenes and maltenes so produced were collected and weighed. Maltenes collected in this step were the starting mixture for the subsequent column separation. The amount of volatile material that was lost during the hexane evaporation step was calculated by difference.

\section{Column separation}

Maltene samples recovered from the asphaltene precipitation step were used as the starting material for a two-column separation, as shown in Fig. I-2-1. The top column, through which the hexane solution of maltenes first passes, is packed with Attapulgite clay. Resins adsorb onto the clay, while saturates and aromatics are eluted and pass on to the lower column, packed with activated silica gel. The aromatic fraction adsorbs onto the silica gel, while the saturates elute and are collected. After evaporation of the hexane carrier, the weight of the saturate fraction can be determined. A 50:50 mixture of toluene and acetone is used to recover the resin fraction from the clay packing. The aromatics can be recovered by Soxhlet extraction of the silica gel in hot toluene, although quantitative recovery is not assured.

Adjustments to the column fractionating procedures in Part 8 of ASTM D2007 were required to improve sample recovery. Two $50 \mathrm{~mm}$ porous floats (CAT. 5849-20, ACE GLASS) were placed on top of the clay and gel packings to distribute eluent uniformly over the cross section of the columns,. A flow regulator valve was drilled on the side of the Teflon Reducing Coupling (CAT 5843-51, Ace Glass), between the upper and lower sections of column (See Fig I-2-1), to allow adjustment of the pressure in the column. Adsorption of aromatics to silica gel is exothermic; temperatures as high as $80^{\circ} \mathrm{C}$ can be generated. Pressure increases to about 40 psi were observed as hexane vaporized, causing loss of sample due to leaks through the seals. Addition of a relief valve solved this problem and improved sample recovery. Fractions were collected and weighed. For safety and convenience, a rotary evaporator in a water bath at $40^{\circ} \mathrm{C}$ replaced the hot plate recommended in ASTM D2007. Additional details are included in a previous report (Fan et al., 1999).

Materials used for the ASTM column separations included:

- $\quad$ acetone, HPLC grade (99.5\%), Fisher Scientific.

- calcium chloride, desiccant, 96+\%, Aldrich Chemical Co.

- azobenzene, 99\% (UV-VIS), ACROS ORGANICS 
- $n$-hexane, HPLC grade (99+\%), Aldrich Chemical Co.

- toluene, HPLC grade (99.8\%), Fisher Scientific

- silica gel, grade 12, 28-200 mesh, Aldrich Chemical Co. (Activated for 4 hours at $190^{\circ} \mathrm{C}$ )

- clay adsorbent, 30-60 mesh, Attapulgus. (Clay quality may be determined using the azobenzene equivalent test according to ASTM D2007-93. The azobenzene activity test measures the adsorptive characteristics of the clay. The azobenzene equivalent value should be 30 to 35. The clay test data is illustrated in Section I-2.1.)

\section{EXPERIMENTAL RESULTS}

SARA fractions measured by the ASTM method

The baseline ASTM results for six crude oils are given in Table I-2.10 for asphaltenes precipitated with and saturates eluted with $n$-hexane in all cases.

Table I-2.10. SARA Fractions by the ASTM Clay-Gel Adsorption Method

\begin{tabular}{|c|c|c|c|c|c|}
\hline Oil & Volatiles & Saturates & Aromatics & Resins & Asphaltenes \\
\hline A-95 & 6.7 & 46.2 & 19.7 & 18.6 & 8.8 \\
\hline C-LH-99 & 10.3 & 38.8 & 23.6 & 23.9 & 3.4 \\
\hline C-R-00 & 2.4 & 68.7 & 17.4 & 9.9 & 1.6 \\
\hline SQ-95 & 16.1 & 47.0 & 19.4 & 14.3 & 2.6 \\
\hline S-Ven-39 & 6.3 & 45.6 & 27.8 & 14.2 & 6.1 \\
\hline Tensleep-99 & 3.2 & 59.6 & 23.2 & 11.8 & 3.2 \\
\hline
\end{tabular}

Physical properties of SARA fractions

Quantities of SARA fractions collected using the ASTM separation procedure are sufficient to allow some measurements of their physical properties. Table I-2.11 lists RI of the six oil samples together with fraction RI values. All were measured at $20^{\circ} \mathrm{C}$ at the wavelength of the sodium D-line using an Index Instruments model GPR11-37 refractometer. In Table I-2.12, API gravity and fraction densities are summarized. Densities were measured at $20^{\circ} \mathrm{C}$ with a Mettler/Paar DMA40 densitometer. API gravity was calculated from the density of the oil, measured at $20^{\circ} \mathrm{C}$ and adjusted to $60^{\circ} \mathrm{F}$.

Table I-2.11. RI Values of Oils and their Fractions at $20^{\circ} \mathrm{C}$

\begin{tabular}{|l|c|c|c|c|}
\hline \multicolumn{1}{|c|}{ Oil } & $\mathbf{R I}_{\text {Oil }}$ & $\mathbf{R I}_{\text {Saturates }}$ & $\mathbf{R I}_{\text {Aromatics }}$ & $\mathbf{R I}_{\text {Resins }}$ \\
\hline A-95 & 1.5157 & 1.4711 & 1.5718 & 1.5680 \\
\hline C-LH-99 & 1.5136 & 1.4746 & 1.5541 & 1.5695 \\
\hline C-R-00 & 1.4851 & 1.4661 & 1.5498 & 1.5979 \\
\hline SQ-95 & 1.4769 & 1.4491 & 1.5375 & 1.5804 \\
\hline S-Ven-39 & 1.4976 & 1.4575 & 1.5502 & 1.5887 \\
\hline Tensleep-99 & 1.4906 & 1.4520 & 1.5458 & 1.5937 \\
\hline
\end{tabular}


Table I-2.12. Density of Fractions at $20^{\circ} \mathrm{C}$

\begin{tabular}{|l|c|c|c|c|}
\hline Oil ID & API Gravity & $\boldsymbol{\rho}_{\text {Saturates }}$ & $\boldsymbol{\rho}_{\text {Aromatics }}$ & $\boldsymbol{\rho}_{\text {Resins }}$ \\
\hline A-95 & 25.4 & 0.8536 & 0.9935 & $\mathrm{~nm}$ \\
\hline C-LH-99 & 22.5 & 0.8671 & 0.9755 & $\mathrm{~nm}$ \\
\hline C-R-00 & 31.1 & 0.8344 & 0.9671 & $\mathrm{~nm}$ \\
\hline SQ-95 & 36.2 & 0.7938 & 0.9361 & 0.9974 \\
\hline S-Ven-39 & 28.8 & 0.8270 & 0.9710 & $\mathrm{~nm}$ \\
\hline Tensleep-99 & 30.8 & 0.8161 & 0.9614 & $\mathrm{~nm}$ \\
\hline
\end{tabular}

\section{HPLC Technique}

\section{EXPERIMENTAL METHOD}

Liquid chromatographic system

The HPLC chromatographic separation system consisted of a Model 110A pump (Beckman), an R401 differential refractometer (Waters), a U6K universal injector (Waters), a Model 7040 high pressure switching valve (Rheodyne), and a Waters 486 UV detector (Millipore). Analog signals from the RI detector were interfaced to a Model 203 PeakSimple data acquisition system (SRI Instrument). Two $3.9 \times 300 \mathrm{~mm} \mu$ Bondapak $\mathrm{NH}_{2}$ columns (Waters) were used in series for chromatographic separation of crude oil. The UV detector was operated at a wavelength of $254 \mathrm{~nm}$ to monitor elution of each fraction. The configuration of the system is shown in Fig. I-2.9.

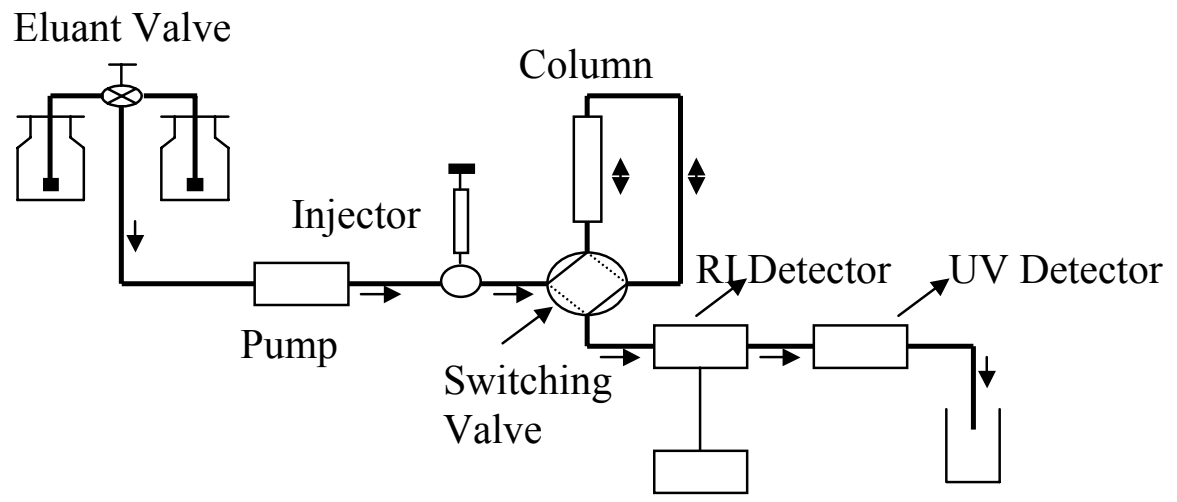

$\begin{array}{ll}\text { Data Processor } & \text { Fraction } \\ \text { Collector }\end{array}$

Figure I-2.9. Configuration of HPLC system.

Selectivity of the $\mathrm{NH}_{2}$-bonded column

Saturated and aromatic hydrocarbons were mixed in hexane to test the selectivity of the $\mathrm{NH}_{2}$-bonded HPLC columns. Mixtures of $\mathrm{C}_{10}$ ( $n$-decane), $\mathrm{C}_{13}$ ( $n$-tridecane), $\mathrm{C}_{15}$ ( $n$-pentadecane), and $\mathrm{C}_{18}$ (n-octadecane) eluted as a single peak, as shown in Fig. I-2.10. There was no differentiation between saturates with different hydrocarbon chain lengths. Similarly, hexane 
solutions of 1-tetradecene $\left(\mathrm{C}_{14}\right.$, one double bond $)$ and tridecane $\left(\mathrm{C}_{13}\right.$, saturated $)$ eluted as a single peak, as shown in Fig. I-2.11. Olefins, if any, could thus be expected to elute with the saturated hydrocarbons.

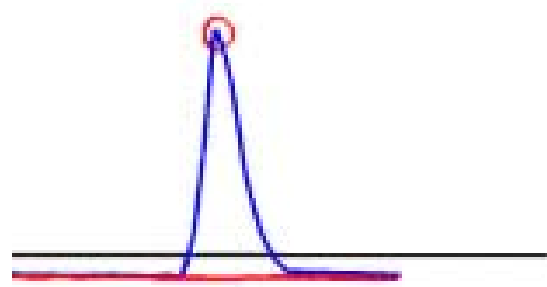

Figure I-2.10. Chromatograph of a mixture of $n$ decane, $n$-tridecane, $n$-pentadecane, and $n$-octadecane.

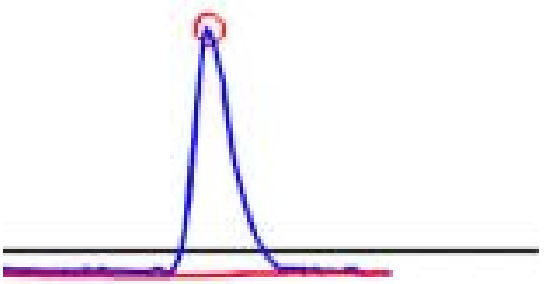

Figure I-2.11. Chromatograph of a mixture of tridecane and tetradecene.

When the hexane solutions of toluene (benzene- $\mathrm{CH}_{3}$ ) and 1,3-diisopropylbenzene (benzene- $\left[\mathrm{CH}\left(\mathrm{CH}_{3}\right)_{2}\right]_{2}$ ) were mixed, they eluted as a single peak (Fig. I-2.12), whereas the hexane solutions of 1,3-diisopropylbenzene (1-ring) and 1-methylnaphthalene (2-rings) eluted as two well-separated peaks (Fig. I-2.13). Based on their retention times, the first peak in Fig. I-2-6 was identified as diisopropylbenzene and the second one was 1-methylnaphthalene. Mixtures of the hexane solutions of 1,3-diisopropylbenzene, 1-methylnaphthalene, and $\mathrm{C}_{15}$ (n-pentadecane) eluted as three peaks (Fig. I-2.14). The first peak in the sequence Fig. I-2.14 is $n$-pentadecane, the second is diisopropylbenzene, and the third is 1-methylnaphthalene. Thus the $\mathrm{NH}_{2}$-bonded columns can discriminate between saturates, single-ring aromatics, and two-ring aromatics regardless of their carbon chain lengths or molecular weights.

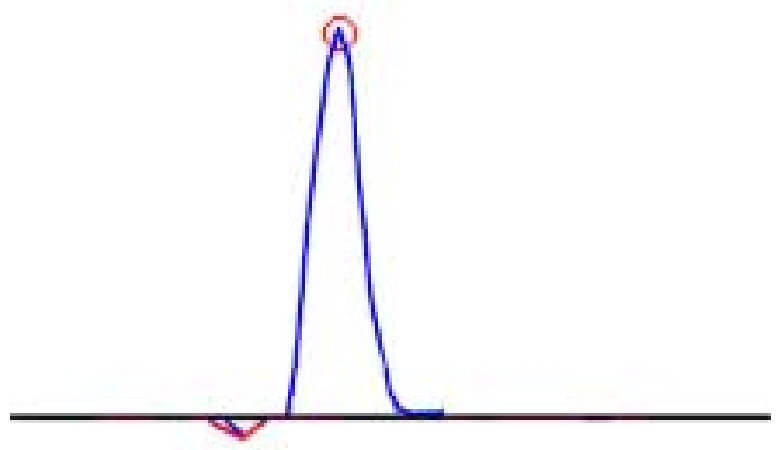

Figure I-2.12. Chromatograph of a mixture of toluene and diisopropylbenzene. 


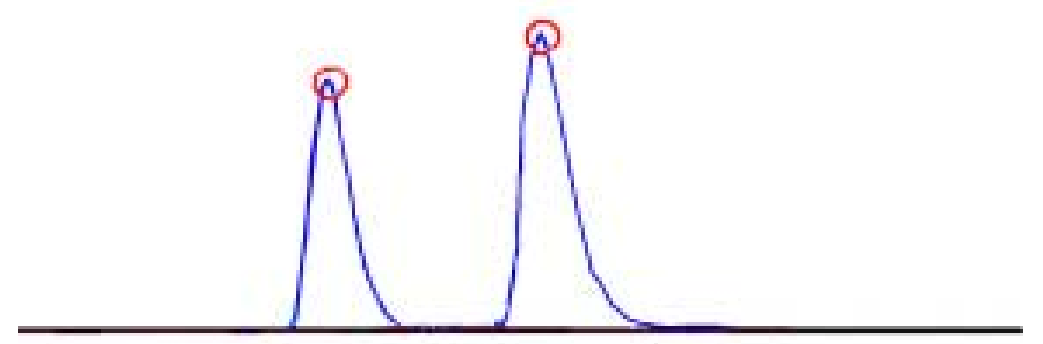

Figure I-2.13. Chromatographs of diisopropylbenzene and methylnaphthalene.

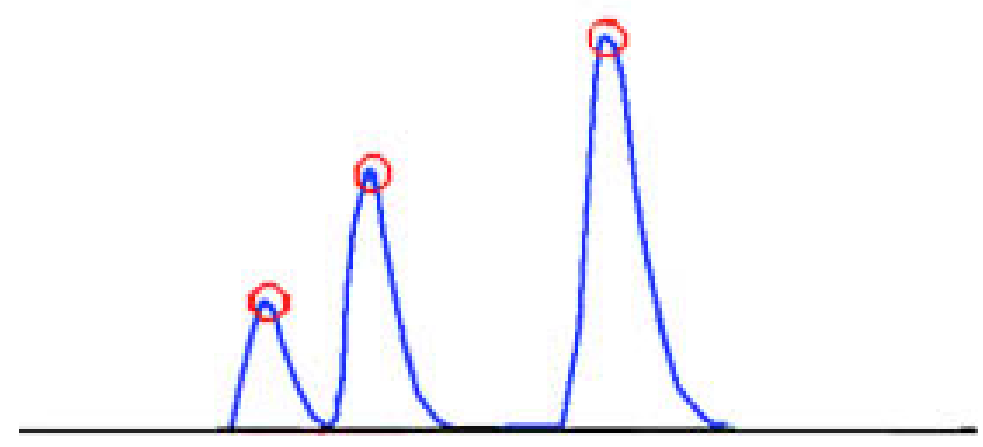

Figure I-2.14. Chromatographs of $\boldsymbol{n}$-pentadecane, diisopropylbenzene, and methylnaphthalene.

Calibrations for saturates and aromatics

RI detector responses to known amounts of several different hydrocarbons, both saturates and aromatics, are listed in Table I-2.13 and plotted in Fig. I-2.15. For constant conditions, the correlation between RI detector signal and RI of the same compound measured at $20^{\circ} \mathrm{C}$ at the wavelength of the sodium $\mathrm{D}$ line is linear. If an average RI of the components of each crude oil peak were known, the relationship in Fig. I-2.15 would provide a direct method of calibration to convert peak area to the amount of material that it represents. For crude oils, of course, these average values will vary from one sample to another and in general usually only the RI of the total sample is known.

Table I-2.13. HPLC RI Detector Signal (area/mg) and RI of Hydrocarbons at $20^{\circ} \mathrm{C}$

\begin{tabular}{|l|r|r|r|r|l|l|r|}
\hline Hydrocarbon & Decane & Tridecane & N-pentadecane & Toluene & $\begin{array}{l}\text { Qmethy- } \\
\text { naphthalene }\end{array}$ & \multicolumn{1}{l|}{$\begin{array}{l}\text { 1,3-diisopropyl- } \\
\text { benzene }\end{array}$} & $\begin{array}{l}\text { Tetramethyl- } \\
\text { benzene }\end{array}$ \\
\hline Rl & 1.411 & 1.425 & 1.4317 & 1.496 & 1.614 & 1.489 & 1.5187 \\
\hline Signal (area/mg) & 2711 & 3870 & 4204 & 7230 & 12517 & 7088 & 8198 \\
\hline
\end{tabular}




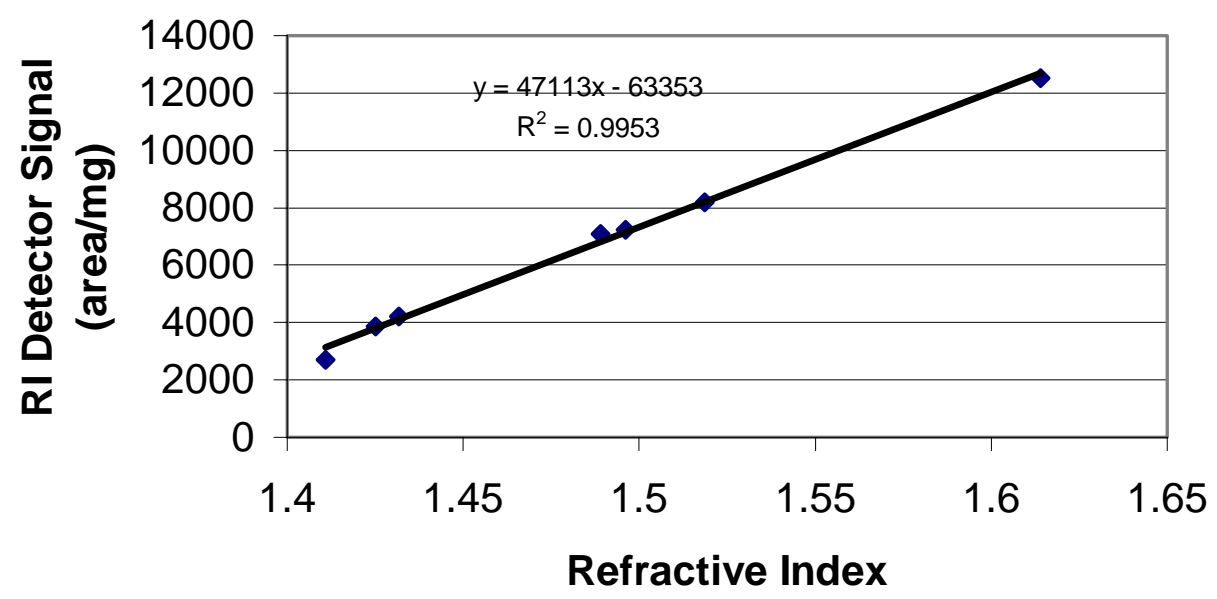

Figure I-2.15. HPLC RI detector signal (area/mg) versus RI for hydrocarbons at $20^{\circ} \mathrm{C}$.

For crude oils, of course, these average values will vary from one sample to another and in general only the RI of the total sample is known. We have, however, measured the RI of fractions of the oils in this study that were separated by the standard ASTM technique. These RI values for saturates and aromatics are plotted along with $\mathrm{RI}_{\text {oil }}$ in Fig. I-2.16 as a function of each oil's API gravity.

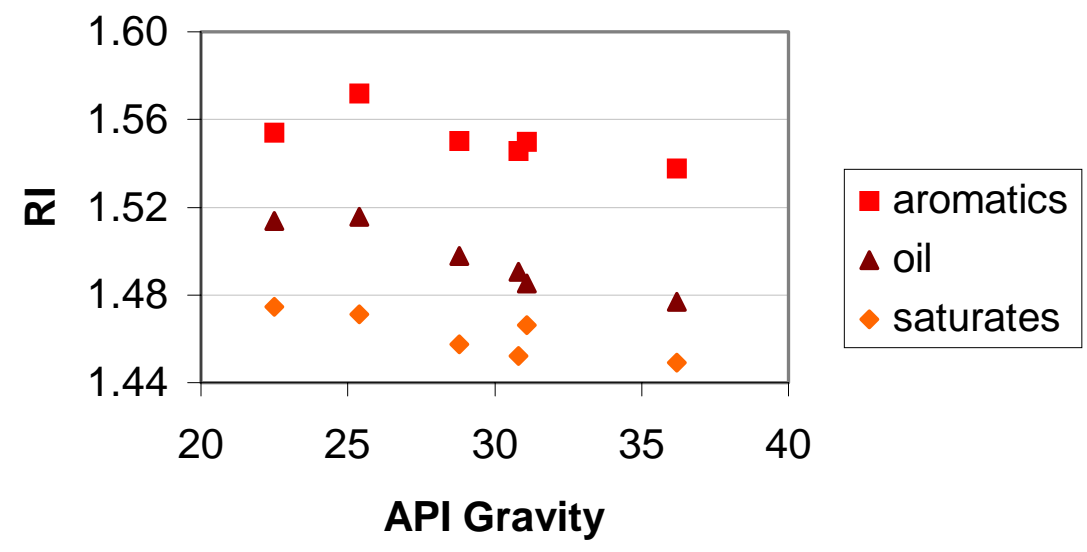

Figure I-2.16. RI Values of Saturate, Aromatic and Oils versus Oils’ API Gravity at $20^{\circ} \mathrm{C}$

Overall, there is a trend toward lower RI values of both the saturate and aromatic fractions as API gravity increases, but the ratio between them is fairly constant, falling in a narrow range from 1.05 to 1.07 . If this ratio can be taken as constant, then constant calibration factors can be used to apportion the total amount of saturates plus aromatics, which is found by the difference between the original amount of oil less the amounts of asphaltenes and resins, both of which are determined gravimetrically.

The RI values of the saturate and aromatic fractions cannot be used directly to calibrate the HPLC peaks because of the lost volatile compounds which are in the HPLC fractions but not 
in the ASTM fractions. The case of the aromatics is further complicated because they split into several peaks that depend on the number of fused aromatic rings. Instead standard compounds have been chosen; these are listed in Table I-2.14.

Table I-2.14. HPLC Detector Signals of Calibration Hydrocarbons

\begin{tabular}{|r|c|c|c|c|}
\hline & saturates & 1-ring aromatics & 2-ring aromatics & 3-ring aromatics \\
\hline Hydrocarbon & $n$-heptadecane & 1,3-diisopropylbenzene & $\alpha$-methylnaphthalene & phenanthrene \\
\hline RI (20 $\left.{ }^{\circ} \mathbf{C}\right)$ & 1.4369 & 1.4890 & 1.6150 & $2.0677^{*}$ \\
\hline Signal $(\mathbf{a r e a} / \mathbf{m g})$ & 4301 & 7088 & 12517 & 16022 \\
\hline
\end{tabular}

* estimated from peak area.

Mixtures of 2 to $3 \mathrm{wt} \%$ of these known hydrocarbons were prepared in hexane. These mixtures were eluted through the HPLC system with hexane at a flow rate of $1.5 \mathrm{ml} / \mathrm{min}$ and response factors were calculated. These response factors were used to calculate the amounts of the saturate and aromatic fractions for the crude oil samples separated by HPLC.

SARA fractions measured by HPLC

$1 \mathrm{ml}$ of crude oil was weighed and dissolved in hexane at a volume ratio of 1:40 in a 40 $\mathrm{ml}$ amber vial with open-top screw cap and Teflon/silicon septa (VWR Cat. No. 15900-012),. After 48 hours, clear maltene solution was withdrawn from the sample vial with a $3 \mathrm{ml}$ needle syringe through a $0.2 \mu$ PTFE syringe filter (NALGENT Cat. No. 187-1320). The withdrawn maltene solution was preserved in a $5 \mathrm{ml}$ crimp-top glass vial with an aluminum seal and PTFE/silicon septum for later HPLC column injection. The precipitated asphaltene in the Amber sample vial was subsequently filtered through a funnel filter assemble (CAT 953805, KONTES GLASS) with a $0.22 \mu$ filter (CORNING, Cat. No. 141128). The asphaltene so produced was collected and weighed after solvent evaporation.

The preserved maltene/hexane solution was then injected onto the HPLC columns for chromatographic separation. Several injections were made, each consisting of $0.5 \mathrm{ml}$ of maltene/hexane solution, using a gas tight HPLC syringe (HAMILTON, CAT No. 14815113). Saturate and aromatic separation was achieved by forward-flow elution through the column with hexane at a flow rate $1.5 \mathrm{ml} / \mathrm{min}$. The amounts of saturate and aromatic fractions were calculated from the calibration factors measured with known compounds.

Resins were absorbed near the column entrance and could not be eluted by hexane. A $10 \%(\mathrm{v} / \mathrm{v})$ dichloromethane/hexane eluant was used to backflush the columns. At least three aliquots of $0.5 \mathrm{ml}$ each of the maltene/hexane solution were injected to ensure that sufficient resins could be collected for accurate gravimetric determinations. The UV detector, connected in series with the RI detector, was used to monitor the eluent to ensure that all of the saturates and aromatics were completely eluted before the column was backflushed. Each injection cycle took about 10 to 15 minutes to completely elute all the saturates and aromatics, depending on the oil sample. Solvents were allowed to evaporate in the hood at room temperature, after which resin weight was recorded. 


\section{EXPERIMENTAL RESULTS}

Injection of $8.38 \mathrm{mg}$ of C-R-00 maltenes produced the typical chromatograph shown in Fig. I-2.17. From left to right, the four peaks represent saturates, 1-, 2-, and multi-ring aromatics, respectively. The RI detector signals (in units of dimensionless peak area) for the chromatographs of all six oil samples are listed in Table I-2.15. In most cases these are the average of at least three injections.

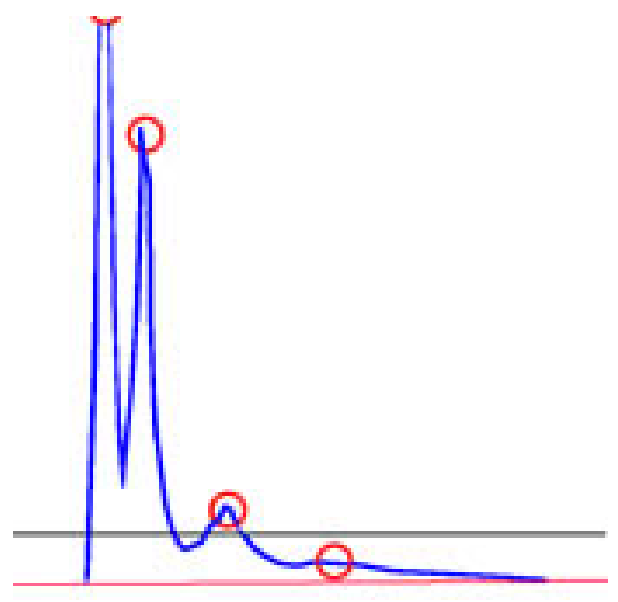

Figure I-2.17. Chromatographs of HPLC separation for the maltenes of crude oil C-R-00.

Table I-2.15. Chromatographic Peak Areas of Maltene HPLC Separation

\begin{tabular}{|l|c|c|c|c|c|}
\hline Oil & $\begin{array}{c}\text { maltene injected } \\
(\mathbf{m g})\end{array}$ & $\begin{array}{c}\text { saturates } \\
\text { (area) }\end{array}$ & $\begin{array}{c}\text { arom (1-ring) } \\
\text { (area) }\end{array}$ & $\begin{array}{c}\text { arom (2-ring) } \\
\text { (area) }\end{array}$ & $\begin{array}{c}\text { arom (multi-ring) } \\
\text { (area) }\end{array}$ \\
\hline A-95 & 8.81 & $11618 \pm 20$ & $5642 \pm 38$ & $3101 \pm 124$ & $558 \pm 144$ \\
\hline C-LH-99 & 4.19 & $7360 \pm 392$ & $4154 \pm 221$ & $2309 \pm 250$ & 0 \\
\hline C-R-00 & 8.38 & $11589 \pm 136$ & $5183 \pm 60$ & $2536 \pm 96$ & $1867 \pm 13$ \\
\hline SQ-95 & 5.50 & $10982 \pm 115$ & $3481 \pm 21$ & $1391 \pm 71$ & 0 \\
\hline S-VEN-39 & 7.75 & $11562 \pm 18$ & $8428 \pm 173$ & $4933 \pm 266$ & 0 \\
\hline Tensleep-99 & 4.84 & $8579 \pm 335$ & $3201 \pm 175$ & $1526 \pm 155$ & $438 \pm 62$ \\
\hline
\end{tabular}

The fraction of each sample that is asphaltene was determined in the asphaltene precipitation step. The concentration and total amount of maltene solution injected is known. From that amount, the resins - which are determined gravimetrically - can be subtracted. The remainder is apportioned between the saturate and aromatic peaks as described in the calibration section. The results of these and 16 other crude oil separations are summarized in Table I-2.16 as weight percentages of the original oil sample. 
Table I-2.16. SARA Data (wt \%) from HPLC Method

\begin{tabular}{|c|c|c|c|c|}
\hline Oil & Saturates & Aromatics & Resins & Asphaltenes \\
\hline A-95 & $55.36 \pm 0.11$ & $20.33 \pm 0.28$ & 15.47 & 8.84 \\
\hline C-LH-99 & $49.95 \pm 2.75$ & $22.49 \pm 1.12$ & 24.15 & 3.40 \\
\hline C-R-00 & $70.41 \pm 0.75$ & $20.77 \pm 0.38$ & 7.22 & 1.61 \\
\hline SQ-95 & $68.99 \pm 0.74$ & $16.48 \pm 0.18$ & $12.16 \pm 0.64$ & 2.60 \\
\hline S-VEN-39 & $52.78 \pm 0.09$ & $29.13 \pm 0.64$ & 12.04 & 6.06 \\
\hline Tensleep-99 & $67.34 \pm 2.72$ & $20.28 \pm 0.97$ & 9.13 & 3.24 \\
\hline W-Cl-01 & 53.24 & 22.28 & 20.98 & 3.50 \\
\hline W-Fo-01 & 61.25 & 28.97 & 9.01 & 0.68 \\
\hline W-Gu-01 & 68.83 & 22.78 & 7.10 & 1.28 \\
\hline W-Mac-01 & 60.35 & 16.44 & 20.03 & 3.17 \\
\hline W-Mau-01 & 49.99 & 21.59 & 18.38 & 10.05 \\
\hline W-Mi-01 & 75.46 & 18.44 & 5.73 & 0.38 \\
\hline W-Mu*-01 & 73.82 & 17.89 & 7.83 & 0.46 \\
\hline W-Pe-01 & 38.31 & 19.49 & 25.72 & 16.48 \\
\hline W-PM-01 & 58.45 & 24.96 & 14.21 & 2.37 \\
\hline W-Po*-01 & 66.86 & 20.09 & 12.27 & 0.77 \\
\hline W-PT*-01 & 63.08 & 23.54 & 12.66 & 0.72 \\
\hline W-Tr*-01 & 64.44 & 23.05 & 11.51 & 1.00 \\
\hline W-TU*-01 & 60.62 & 24.75 & 13.37 & 1.17 \\
\hline W-Up-01 & 55.28 & 26.34 & 13.99 & 4.40 \\
\hline W-Ur-01 & 53.45 & 26.58 & 17.86 & 2.11 \\
\hline W-Wy*-01 & 73.30 & 15.48 & 10.67 & 0.55 \\
\hline
\end{tabular}

\section{TLC-FID Using Iatroscan}

\section{EXPERIMENTAL METHOD}

\section{$T L C-F I D$}

An Iatroscan ${ }^{\circledR}$ MK-5 (Iatron Labs Inc., Tokyo), equipped with a flame ionization detector (FID), interfaced with a Model 203 PeakSimple data system (SRI Instrument), was used for rod scanning and quantification. This instrument is an improved model that has been shown to have better FID sensitivity and wider detector linear range than earlier models (Vela et al., 1995). Silica-coated quartz rods were used (Chromarod-SIII, Iatron Laboratories). The rods were 15.2$\mathrm{cm}$ long and $1.0-\mathrm{mm}$ in diameter with a uniform coating of $5.0-\mu \mathrm{m}$ silica gel particles (pore diameter $60 \AA$ ). The FID detector was operated with a pure grade hydrogen flow rate of 160 $\mathrm{ml} / \mathrm{min}$, an air flow rate of $2.0 \mathrm{~L} / \mathrm{min}$ supplied by a pump and a scan speed of $60 \mathrm{~s} / \mathrm{scan}$.

Crude oil samples were dissolved in HPLC grade dichloromethane (DCM) at about 20 $\mathrm{mg} / \mathrm{mL}$. Aliquots of about $0.5-1.0 \mu \mathrm{L}$ of this solution were spotted on freshly activated rods using a $1.0-\mu \mathrm{L}$ repeating syringe (Alltech Associate, Inc.). To prevent band spreading, the rods were spotted in a fume hood with air blowing continuously to enhance rapid solvent evaporation. About 10 to $20 \mu \mathrm{g}$ of sample were found by experience to optimize the signal-to-noise ratio and avoid sample overloading. 
The inside back wall of the development tank was lined with filter paper to help saturate the vapor phase with solvent. Immediately after spotting, each rod was developed in three steps. First, the rods were developed with HPLC grade hexane for $30 \mathrm{~min}$ - during which time the wetting front moved $10 \mathrm{~cm}$ from the origin - to separate colorless saturated hydrocarbons. After drying in air for about $3 \mathrm{~min}$, the second development step, exposure to HPLC grade toluene, was initiated. After $10 \mathrm{~min}$, the wetted front had moved $5 \mathrm{~cm}$ from the origin, separating yellow aromatic hydrocarbons. Rods were again dried in air for about 5 min, then developed with $\mathrm{DCM} /$ methanol (95:5 by volume) for 4 min to separate resins from asphaltenes. The entire procedure including sample spotting, solvent development steps, and scanning took about 90 $\min$.

\section{Evaporative losses}

During the processes of spotting, development, drying and scanning, ample opportunity exists for evaporation of lighter oil components. The small sample size makes weight loss difficult to assess accurately. Several methods have therefore been developed to measure the amount of evaporation that occurred with different oil samples. A gravimetric method was used in which a known amount of oil was spread on a clean, tared preparative thin layer chromatography (PTLC) plate. The PTLC plates used were Si250F with fluorescent indicator (J. T. Baker) with either a glass and aluminum base, coated with a uniform layer of $250 \mu \mathrm{m}$ silica gel (particle size $10-12 \mu \mathrm{m}$, pore diameter $60 \AA$ ), cut into $2 \times 5 \mathrm{~cm}, 2 \times 10 \mathrm{~cm}$ or $2 \times 15 \mathrm{~cm}$ pieces. Weight after $90 \mathrm{~min}$ was used to estimate the loss of weight for different oils. Losses during development could also be estimated by immediate scanning of duplicate rods with identical amounts of sample to those used in the SARA fractionation tests. Differences in peak areas with and without the development steps can be attributed to evaporation of light components during the development and drying steps.

\section{EXPERIMENTAL RESULTS}

Evaporative loss estimates

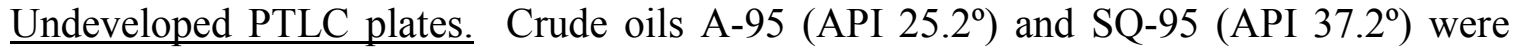
tested on undeveloped PTLC plates. One $\mathrm{ml}$ of a mixture of crude oil and DCM was spread over an area of a weighed PTLC plate using a 1.0-ml Pressure-Lok syringe (Precision Sampling Co.). After 90 minutes, the plates were reweighed to determine the loss of oil components. On the TLC rods, $1.0 \mu 1$ of sample typically covers an area of about $2 \mathrm{~mm}^{2}$. The equivalent areal coverage for a $1.0 \mathrm{ml}$ sample would be $20 \mathrm{~cm}^{2}$. As shown in Table I-217 and Fig. I-2.18, the evaporative losses are increased by spreading the sample over a greater area. The loss rate is greater for the lighter oil (SQ-95). 
Table I-2.17. Crude Oil Weight Loss as a Function of Areal Distribution

\begin{tabular}{|c|c|c|c|c|}
\hline Oil & $\begin{array}{c}\text { Concentration } \\
(\%)\end{array}$ & $\begin{array}{c}\text { Amount } \\
(\mathrm{ml})\end{array}$ & $\begin{array}{l}\text { Area } \\
\left(\mathrm{cm}^{2}\right)\end{array}$ & $\begin{array}{c}\text { Wt. Loss } \\
(\%)\end{array}$ \\
\hline \multirow{4}{*}{ A-95 } & \multirow{4}{*}{2.239} & \multirow{4}{*}{1.0} & 8 & 2.6 \\
\hline & & & 10 & 4.4 \\
\hline & & & 20 & 7.1 \\
\hline & & & 30 & 15.1 \\
\hline \multirow{4}{*}{ SQ-95 } & \multirow{4}{*}{2.187} & \multirow{4}{*}{1.0} & 8 & 5.5 \\
\hline & & & 10 & 6.2 \\
\hline & & & 20 & 12.9 \\
\hline & & & 30 & 20.0 \\
\hline
\end{tabular}

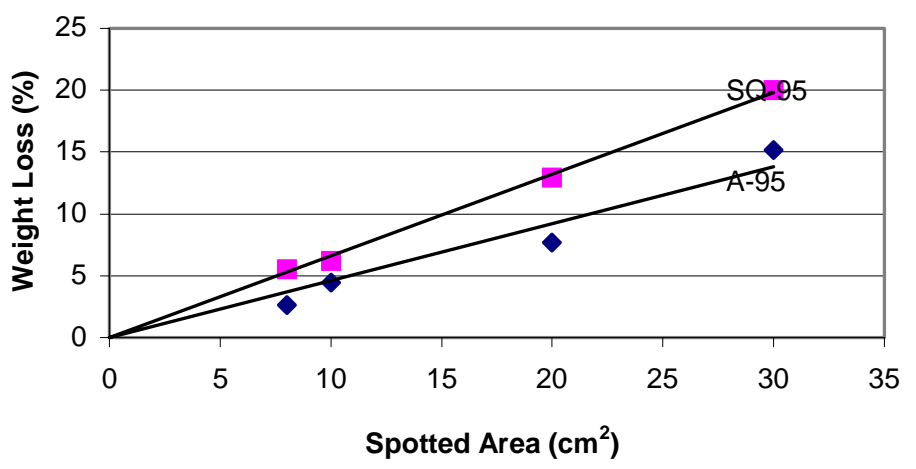

Figure I-2.18. Weight loss \% on plate without development.

The effect of spotting volume over equal spotting areas on weight loss was demonstrated with A-95 crude oil mixed with DCM. The results are summarized in Table I-2.18 and plotted in Fig. I-2.19. For volumes less than about $2.0 \mathrm{ml}$, weight loss over a $90 \mathrm{~min}$ period increased with volume. Above $2.0 \mathrm{ml}$, weight loss increased, but more slowly and approached a constant value of about $21 \%$. The PTLC plate has some absorption capacity limit. For the same area, smaller volumes are more efficiently absorbed, limiting the evaporation rate. As the sample volume increases, the absorption capacity of the plate is eventually exceeded and it is the area exposed that controls the evaporation rate. Accuracy of these tests was limited by: 1) limitations on measurement of sub-milligram sample amounts; 2) effects of ambient conditions of temperature, humidity, and convection on evaporation rates; 3) difficulties in distributing the sample uniformly. 
Table I-2.18. Effects of Spotting Amount on Weight Loss

\begin{tabular}{|c|c|c|c|c|}
\hline Oil & $\begin{array}{c}\text { Concentration } \\
(\%)\end{array}$ & $\begin{array}{l}\text { Area } \\
\left(\mathrm{cm}^{2}\right)\end{array}$ & $\begin{array}{c}\text { Amount } \\
(\mathrm{ml})\end{array}$ & $\begin{array}{c}\text { Wt. Loss } \\
(\%)\end{array}$ \\
\hline \multirow{6}{*}{ A-95 } & \multirow{6}{*}{2.239} & \multirow{6}{*}{20} & 0.5 & 2.2 \\
\hline & & & 1.0 & 9.3 \\
\hline & & & 2.0 & 15.4 \\
\hline & & & 3.5 & 21.3 \\
\hline & & & 4.0 & 21.6 \\
\hline & & & 4.8 & 21.1 \\
\hline
\end{tabular}

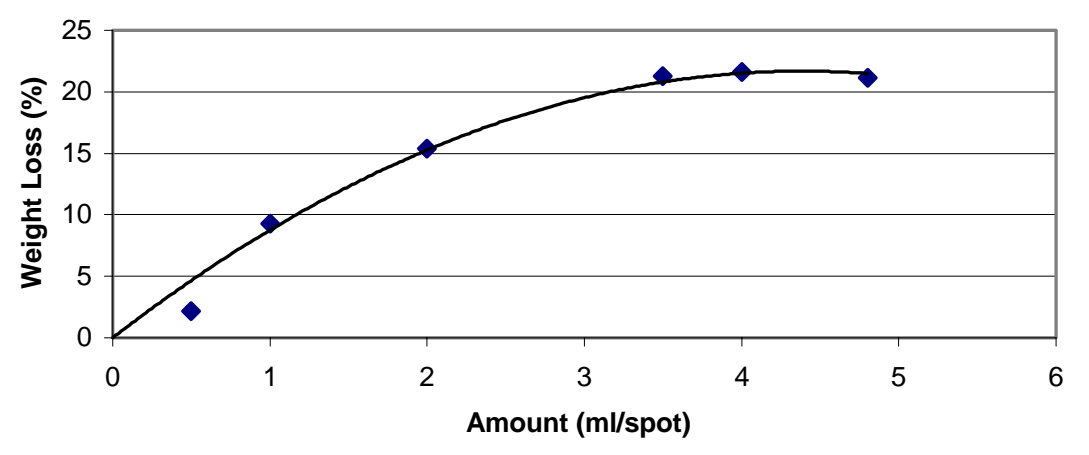

Figure I-2.19. Effects of spotting amount on weight loss.

PTLC plates with solvent development. The development process, during which components partition between stationary and mobile phases, can accelerate the evaporative loss of light compounds. Tests analogous to those described in the previous section, with the addition of all the solvent development steps, are summarized in Table I-2.19 for A-95 and SQ-95 crude oils. Preliminary tests indicated that neither the sample volume nor the area over which it was spread had any affect on evaporative losses from the developed plates.

Table I-2.19. Crude Oil Weight Loss on PTLC Plates after Solvent Developments

\begin{tabular}{|c|c|c|c|}
\hline Oil & $\begin{array}{c}\text { Concentration } \\
(\boldsymbol{\%})\end{array}$ & $\begin{array}{c}\text { Amount } \\
(\mathbf{m l})\end{array}$ & $\begin{array}{c}\text { Wt. Loss } \\
(\boldsymbol{\%})\end{array}$ \\
\hline A-95 & 2.239 & 1.0 & 20.9 \\
\hline SQ-95 & 2.187 & 1.0 & 31.4 \\
\hline
\end{tabular}

Scanning an undeveloped rod. If the total peak areas of all SARA fractions are summed, these values can be compared for the developed and undeveloped rods with identical amounts of sample. These areas, their ratio, and the calculated losses are shown for A-95 and SQ-95 crude oils in Table I-2.20. These losses are even higher than those measured by weight differences. Evidently, additional evaporation occurs when the rods are scanned. Such losses have been reported previously and have been attributed to volatilization and loss of compounds outside the active part of flame where the temperature on the rod surface can be as high as $200^{\circ} \mathrm{C}$ (Ranny, 1987). 
Table I-2.20. Crude Oil TLC-FID Peak Area Comparison

\begin{tabular}{|c|c|c|c|c|c|c|c|}
\hline Oil & $\begin{array}{c}\text { API } \\
\text { Gravity }\end{array}$ & $\begin{array}{c}\text { Concentration } \\
(\boldsymbol{\%})\end{array}$ & $\begin{array}{c}\text { Amount } \\
(\mathbf{m l})\end{array}$ & $\begin{array}{c}\text { Peak Area (A) } \\
\text { (not developed) }\end{array}$ & $\begin{array}{c}\text { Peak Area (B) } \\
\text { (developed) }\end{array}$ & $\begin{array}{c}\text { B/A } \\
(\boldsymbol{\%})\end{array}$ & $\begin{array}{c}\text { Loss } \\
(\mathbf{W t} \%)\end{array}$ \\
\hline A-95 & 25.2 & 2.165 & 0.6 & $58.0 \pm 1.8$ & $27.1 \pm 1.1$ & 46.7 & 53.3 \\
\hline SQ-95 & 37.2 & 2.070 & 0.6 & $58.8 \pm 1.3$ & $22.6 \pm 1.9$ & 38.5 & 61.5 \\
\hline
\end{tabular}

For a typical TLC-FID detector, Bharati et al. (1994) calculated that the average response factors for SARA fractions were $0.63,1.0,1.1$, and 2.0, respectively. Compounds that evaporate during spotting, development, and scanning are the lower boiling point, saturated and aromatic hydrocarbons. Thus their overall response factor should be less than 1.0. Using peak areas to estimate the amount of material evaporated should, if anything, underestimate the losses. The undeveloped rods were scanned shortly after spotting. Tests in which the rods were allowed to sit for varying amounts of time did not show any significant increases in the evaporative losses.

SARA fractions measured by TLC-FID

TLC-FID analyses of the six oil samples were repeated at least three times. Average results are summarized in Table I-2.21. The volatiles were determined by scanning undeveloped rods with identical amounts of sample applied in both the developed and undeveloped tests (Fig. I-2.20). The amount of volatiles were subtracted from the total and the remainder of the sample was assigned to the SARA fractions on the basis of peak area uncorrected for differences in response factors. Thus asphaltene are likely to be overestimated and saturates somewhat underestimated.

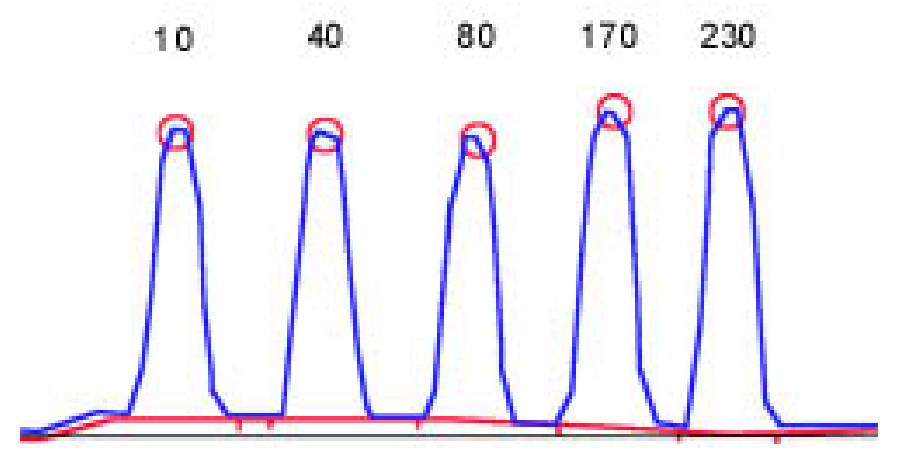

Figure I-2.20. Undeveloped spots of A-95. Numbers represent the amount of time elapsed after spotting and before the rod was scanned.

Table I-2.21. SARA Fractions (\%) by TLC-FID

\begin{tabular}{|c|c|c|c|c|c|}
\hline Oil & Volatiles & Saturates & Aromatics & Resins & Asphaltenes \\
\hline A-95 & 53.3 & 13.8 & 12.4 & 13.4 & 7.1 \\
\hline C-LH-99 & 54.3 & 12.5 & 15.3 & 13.5 & 4.4 \\
\hline C-R-00 & 37.5 & 38.0 & 12.1 & 9.2 & 3.2 \\
\hline SQ-95 & 61.5 & 17.3 & 10.2 & 9.1 & 1.9 \\
\hline S-VEN-39 & 47.5 & 17.2 & 10.5 & 14.5 & 10.4 \\
\hline Tensleep-99 & 39.6 & 26.5 & 18.8 & 8.1 & 6.9 \\
\hline
\end{tabular}


In the tables below (Tables I-2.22 to I-2.27), typical TLC-FID chromatograms are shown for each of the six crude oils in this study. The solvent development in each case was as described above. Elution order of the peaks from left to right is saturates, aromatics, resins, and asphaltenes. Weight percent of each fraction was calculated based on integrated peak areas. The horizontal integration baseline was drawn manually from the starting point of the first peak to the ending point of the last peak since the separation between SARA fractions was not clear cut and there was always some overlap between adjacent peaks. On a developed rod, a very weak yellow residue was found between the aromatics (yellow) and resins (black). A weak black residue was found between the resin band and the asphaltenes (black). On a TLC plate developed with a fluorescent indicator, weak residue signals were found between two neighboring bands when the plate was exposed to UV light at a wavelength of $254 \mathrm{~nm}$.

Table I-2.22. A-95 TLC-FID SARA Results

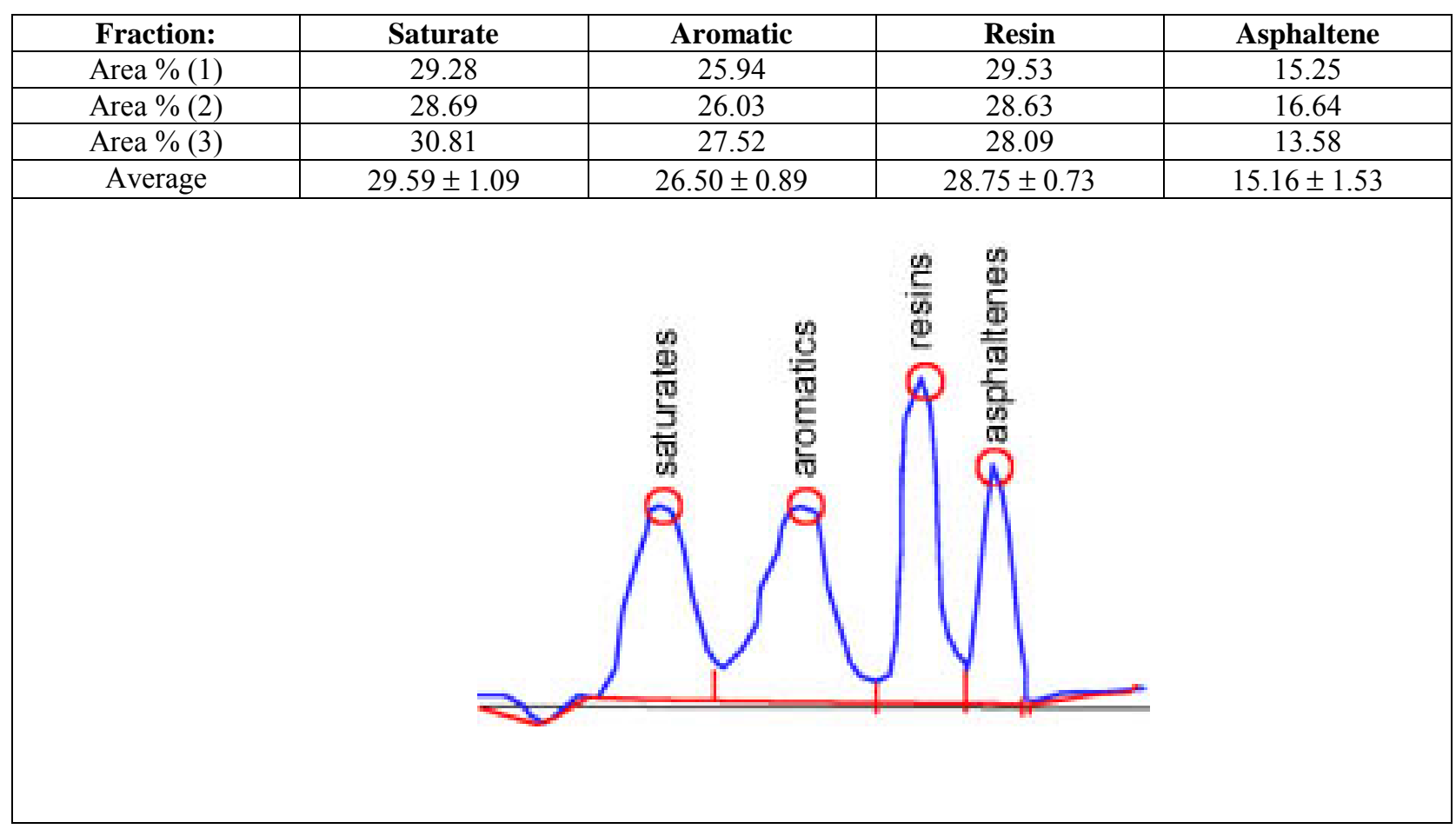


Table I-2.23. SQ-95 TLC-FID SARA Results

\begin{tabular}{|c|c|c|c|c|}
\hline Fraction: & Saturate & Aromatic & Resin & Asphaltene \\
\hline Area \% (1) & 43.62 & 24.53 & 22.98 & 5.52 \\
\hline Area \% (2) & 44.64 & 26.93 & 23.48 & 5.09 \\
\hline Area \% (3) & 46.58 & 27.88 & 24.45 & 4.44 \\
\hline Average & $44.95 \pm 1.50$ & $26.45 \pm 1.73$ & $23.64 \pm 1.73$ & $5.02 \pm 1.73$ \\
\hline
\end{tabular}

Table I-2.24. Tensleep-99 TLC-FID SARA Results

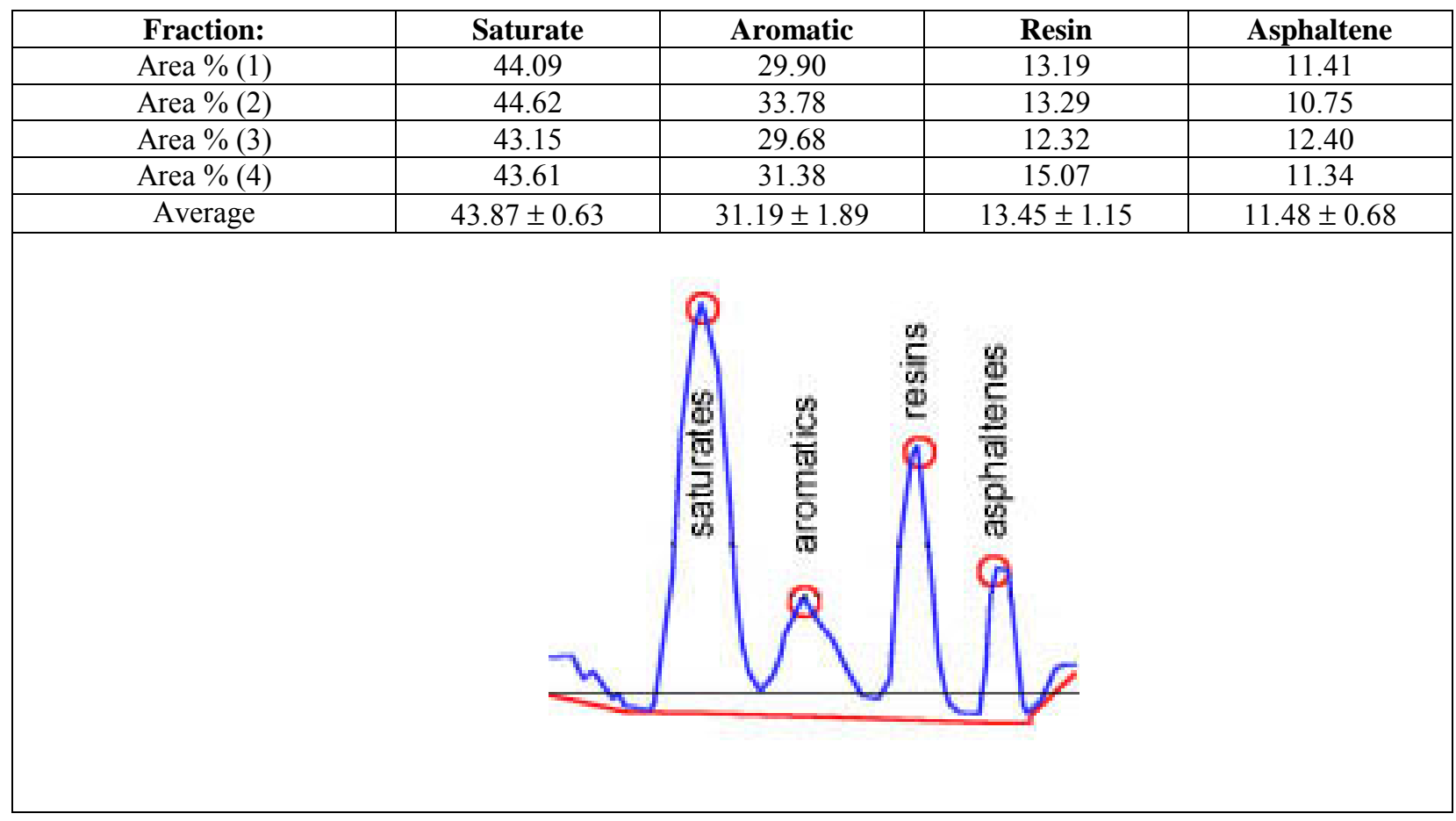


Table I-2.25. C-LH-99 TLC-FID SARA Results

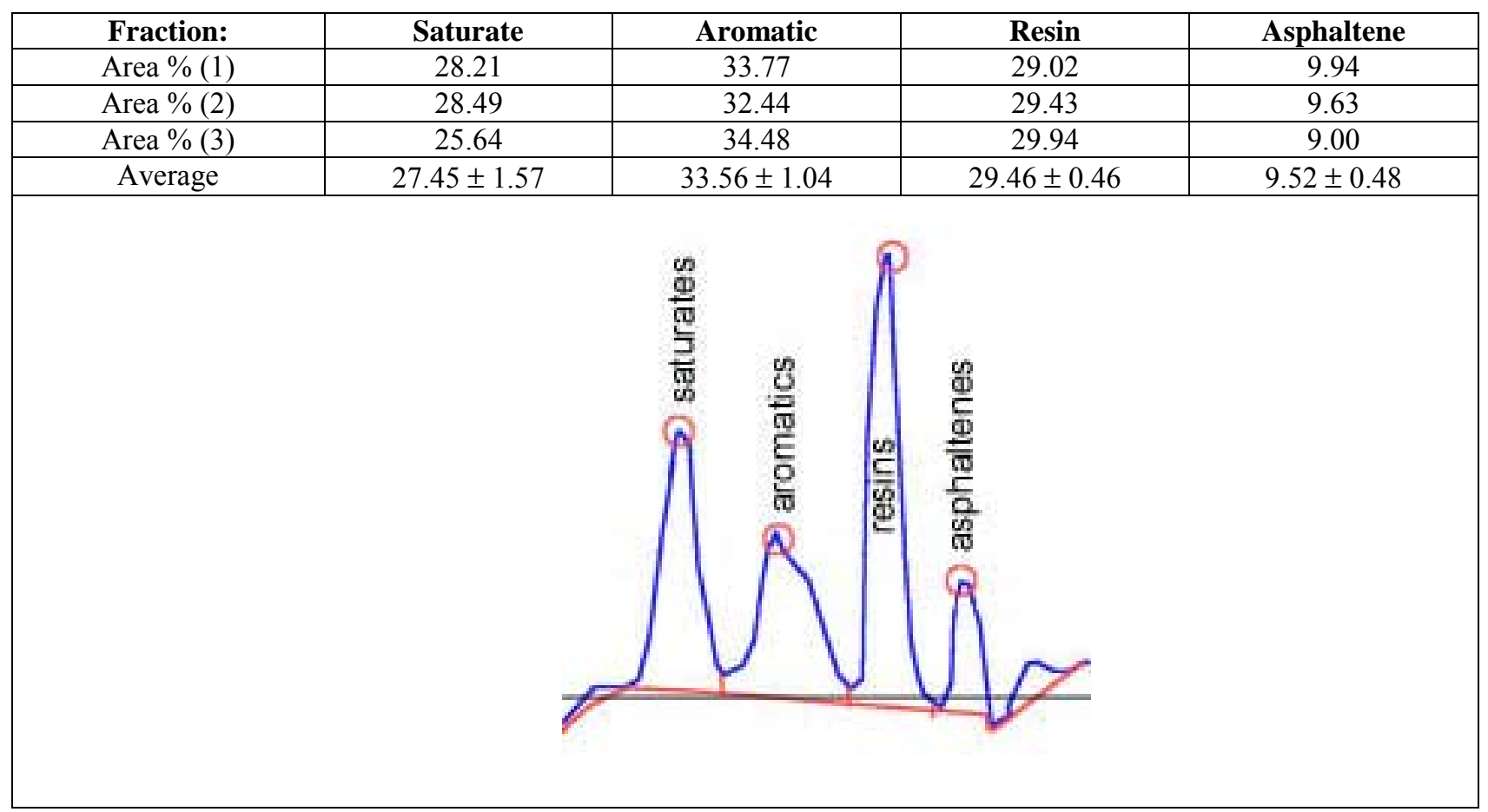

Table I-2.26. S-Ven-39 TLC-FID SARA Results

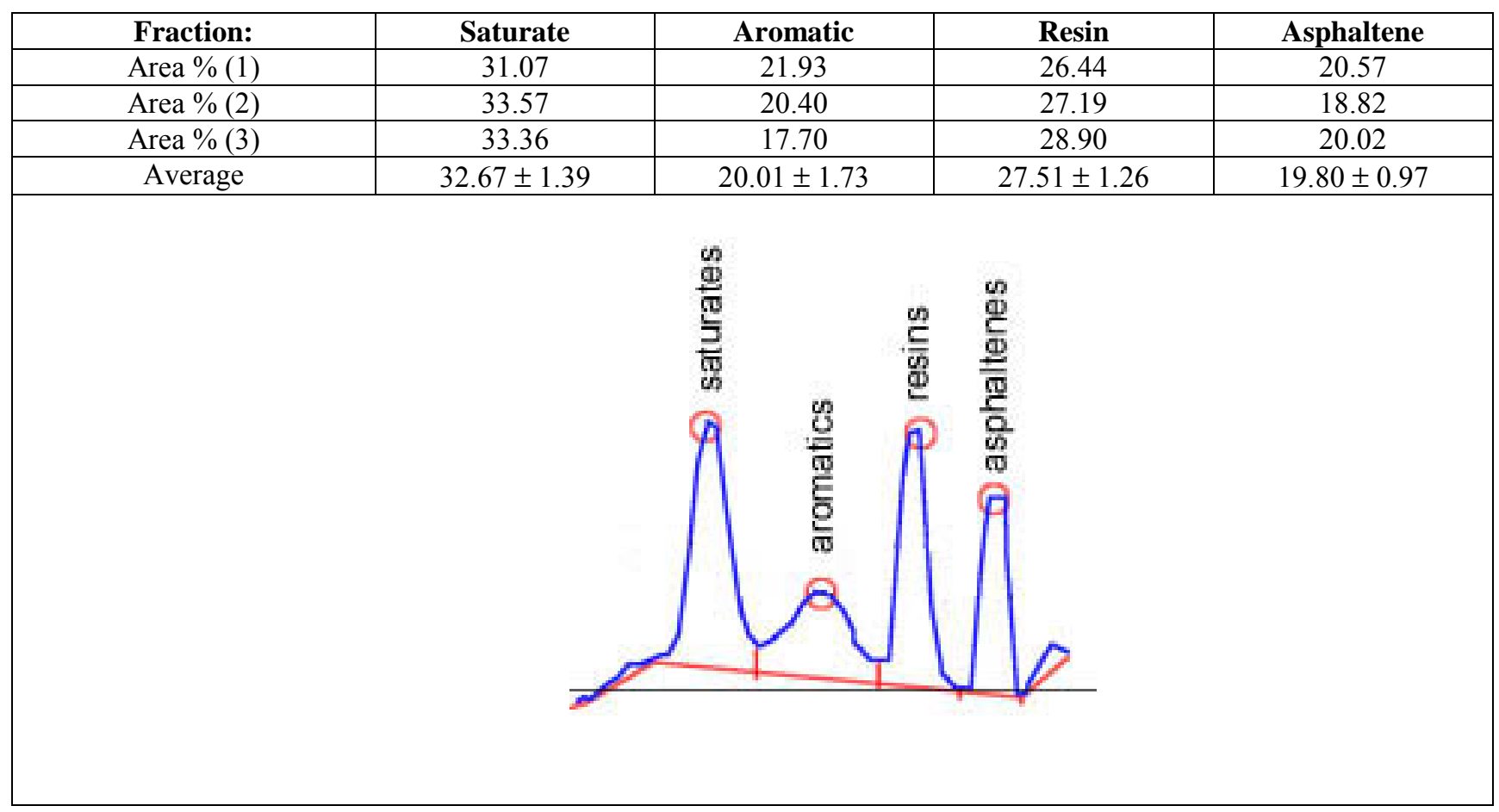


Table I-2.27. C-R-00 TLC-FID SARA Results

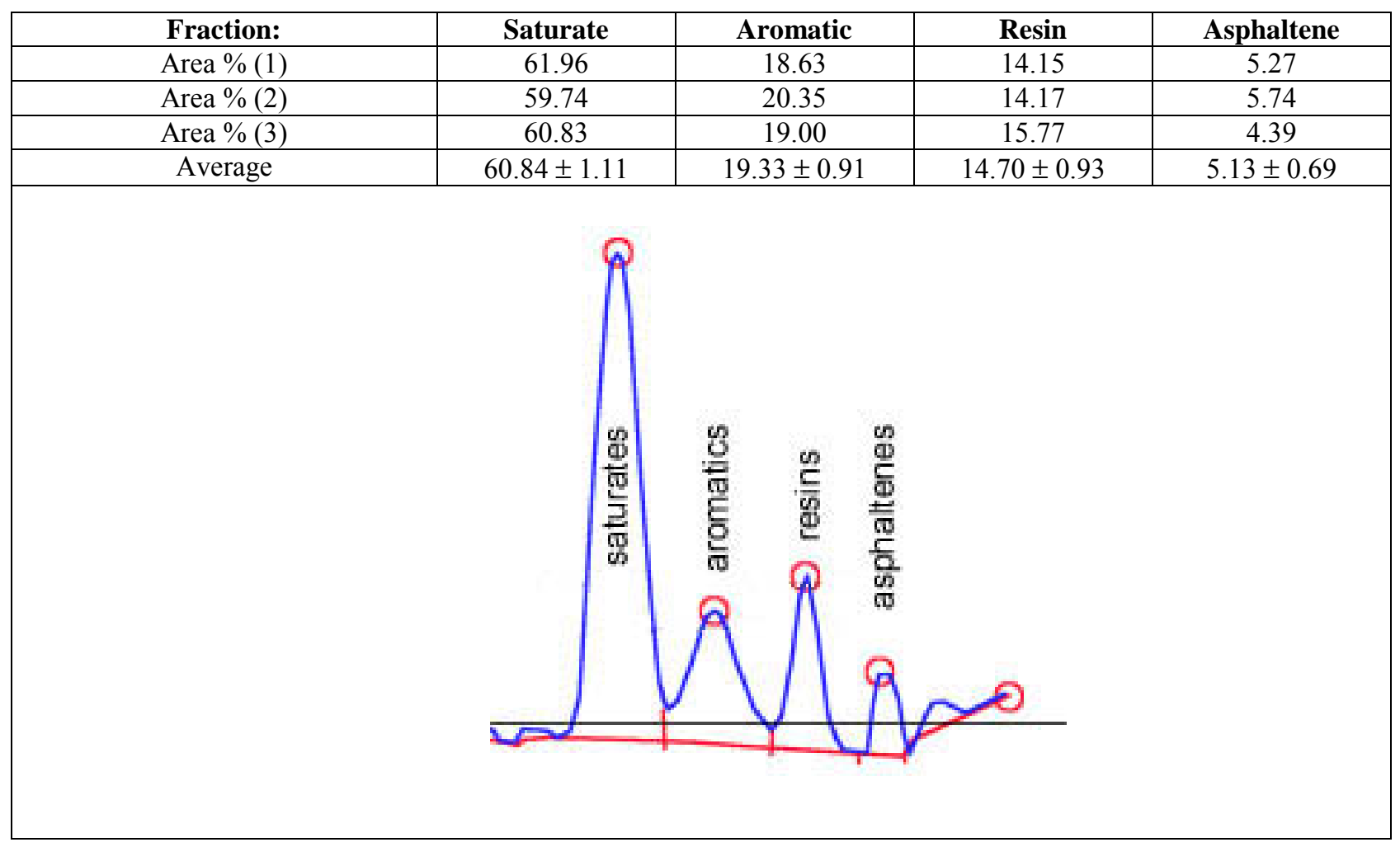

Discussion

Comparison of Improved Techniques with ASTM Standard

SARA FRACTION COMPARISONS

Sara fraction data for all six crude oils by the three methods discussed above are summarized in Figs. I-2.21a-f. Amounts of asphaltenes measured by precipitation with n-hexane are identical as the procedures for both the ASTM and HPLC methods are the same and they were measured only once. 

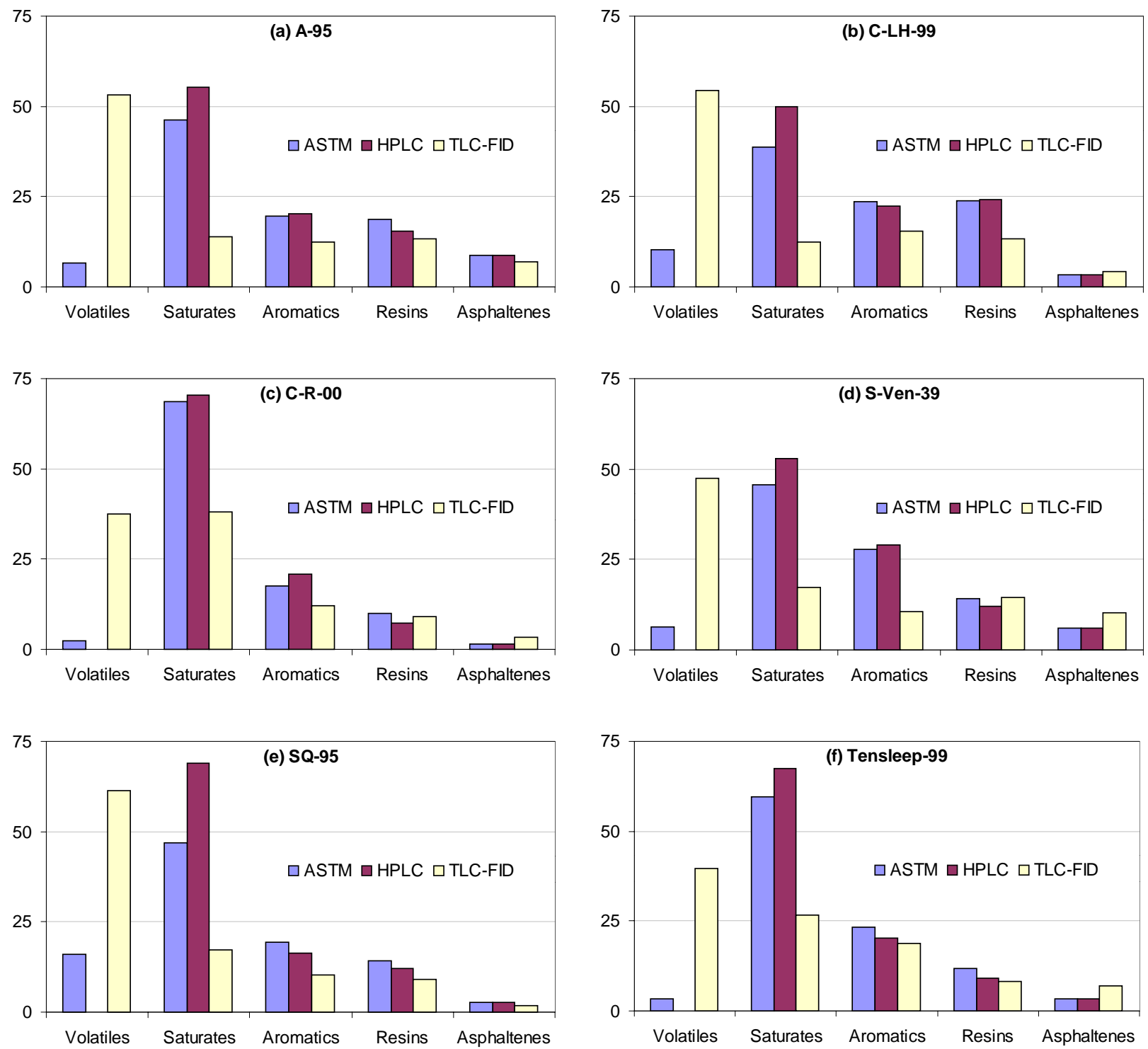

Figure I-2.21. Comparison of SARA fractions determined by the ASTM method, by an improved HPLC method and by TLC-FID.

EVAPORATIVE LOSSES

Examination of the comparisons in Fig. I-2.22 shows that the effects of evaporative losses are greatest for the TLC-FID method and that they are significant for all six oils. Smaller amounts of evaporation were found in the ASTM clay-gel column measurements. Karlson and Larter (1991) reported that evaporative losses in the TLC-FID technique correlated with API gravity for samples from different sources within a single reservoir. The results shown in Fig. I2.22 do not show such a correlation, perhaps because the samples were all from different reservoirs. Figure I-2.23 shows that the amount of oil lost to evaporation in the TLC-FID procedure correlates with the losses measured in the ASTM test. 


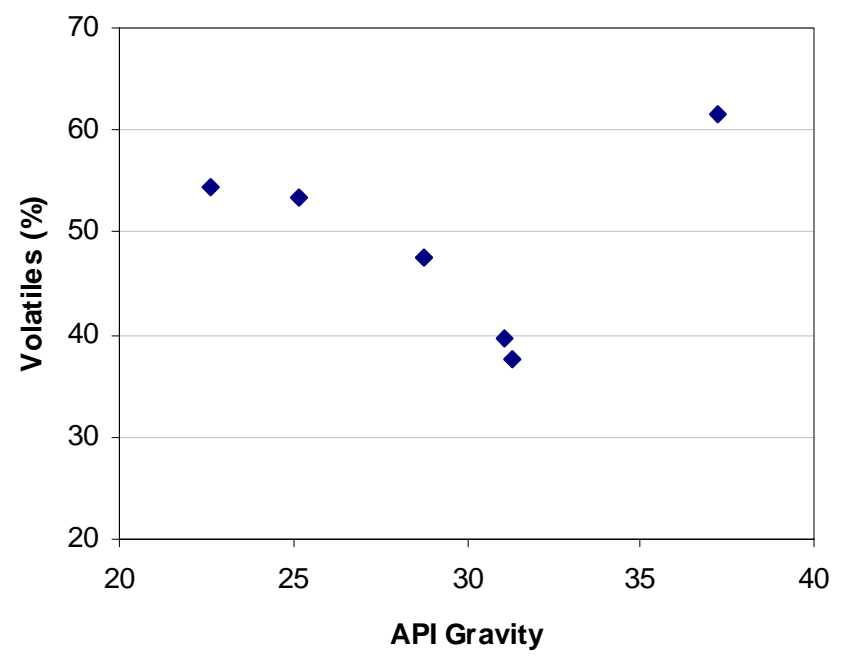

Figure I-2.22. Evaporative loss in TLC-FID measurements as a function of API gravity.

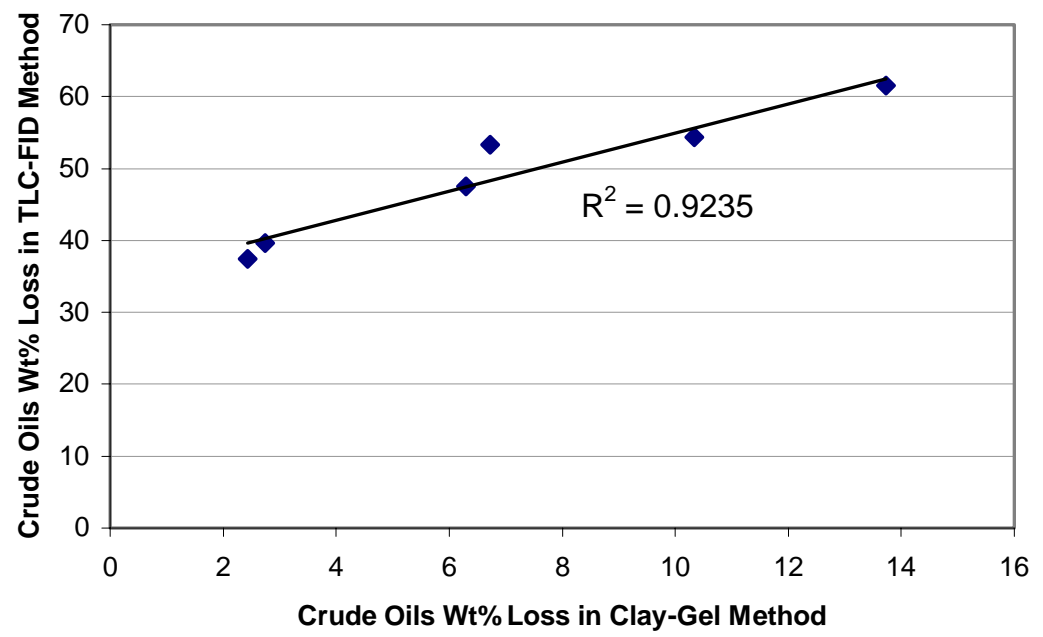

Figure I-2.23. Crude oils wt. loss in clay-gel method versus in TLC-FID method.

\section{COMPONENTS IN GROUPS SEPARATED BY DIFFERENT METHODS}

The loss of a volatile fraction complicates comparisons of the different methods since there are low boiling point species in both the saturate and aromatic categories that may have been lost. One solution is to lump saturates and aromatics so the total amount can be calculated by difference (McLean and Kilpatrick, 1997), but knowing the amounts of these two fractions is an important factor in discriminating between good and poor asphaltene solvents; that information is lost if these two categories are lumped together. 
In Fig. I-2.24 the sum of the volatile and saturate fractions is plotted for all three methods. Assuming that all of the ASTM volatiles are saturates gives estimates of the saturate fraction that are comparable to the HPLC measurements, where there were no evaporative losses. The same is not true, however, for the TLC-FID method where assigning all of the volatiles to the saturate fraction significantly over estimates the amount of saturates for four of the six oil samples as compared to the HPLC results. The differences between HPLC and TLC-FID saturate amounts for these six samples can be minimized by assigning $82 \%$ of the volatiles to the saturate fraction and the remainder to the aromatics, although six samples are too few from which to generalize.

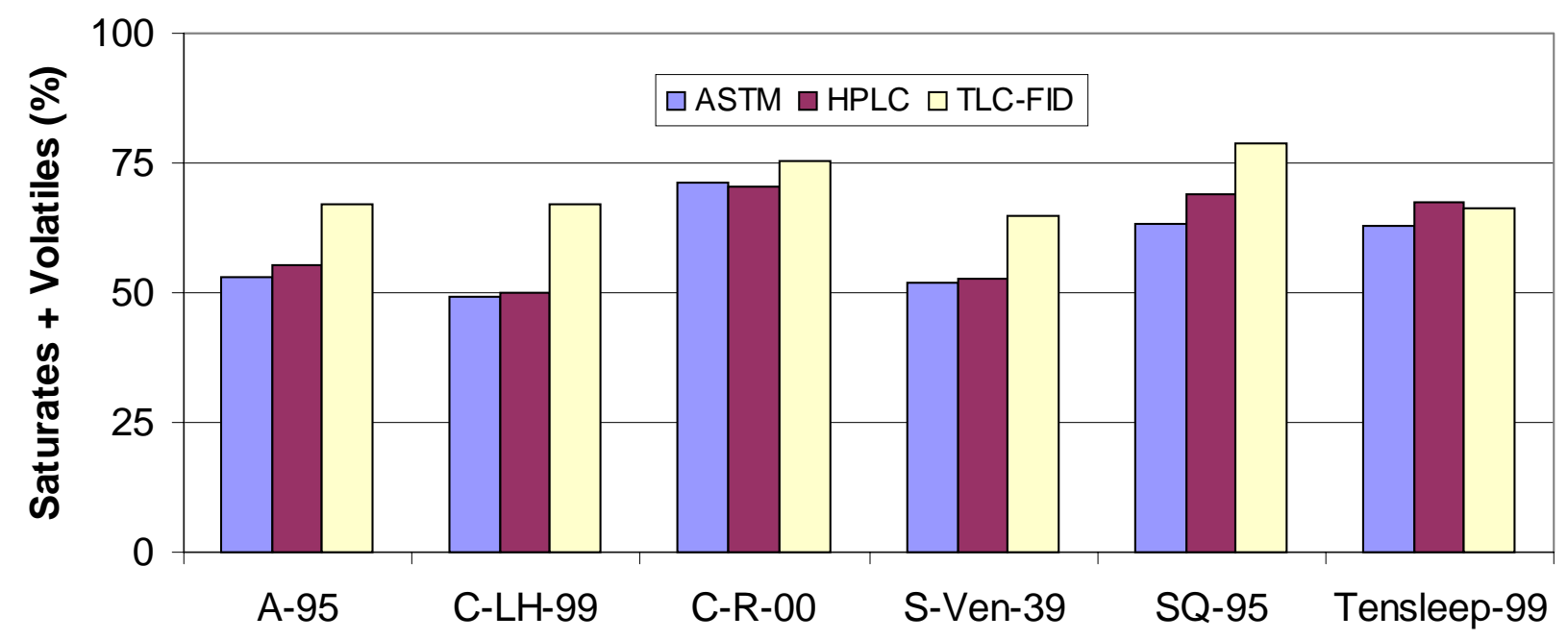

Figure I-2.24. If all of the volatile species are assumed to be saturates, the ASTM and HPLC methods produce comparable results; the TLC-FID estimates are significantly higher in most cases.

The fractions separated by the ASTM and HPLC techniques can be expected to be similar in composition since the asphaltenes are separated prior to chromatography and the remaining components are separated on the basis of polarity with a similar sequence of solvents. There are more significant differences between these two methods and the TLC-FID separation, especially in discrimination between resins and asphaltenes. The resins and asphaltenes together represent the heaviest and most polar species in an oil. Their amounts are not directly affected by evaporation. Note, however, that ignoring evaporative losses in the TLC-FID method would have produced much higher estimates of both resin and asphaltene fractions. Using higher response factors for resins and asphaltenes would also have the effect of raising estimates of these fractions by the TLC-FID method. It should also be mentioned that in the TLC-FID method the separation of resins from asphaltenes was achieved by elution with a mixture of DCM and methanol (95:5 vol\%). Even a small increase in methanol would greatly increase the amount appearing in resin fraction relative to the asphaltene fraction (Karlsen and Larter, 1991). Bharati et al. (1994) showed that asphaltenes separated by precipitation contain a significant proportion of components that chromatograph as resins by the TLC-FID method and that the maltenes included components that chromatograph as asphaltenes. Clearly these different techniques are far from equivalent. For example, the amount of resins in C-LH-99 is considerably higher by the ASTM and HPLC methods than by TLC-FID (Fig. I-2.25). The 
amounts of asphaltenes in S-Ven-39 and Tensleep-99 are considerably higher in the TLC-FID tests than in either of the others (Fig. I-2.26).

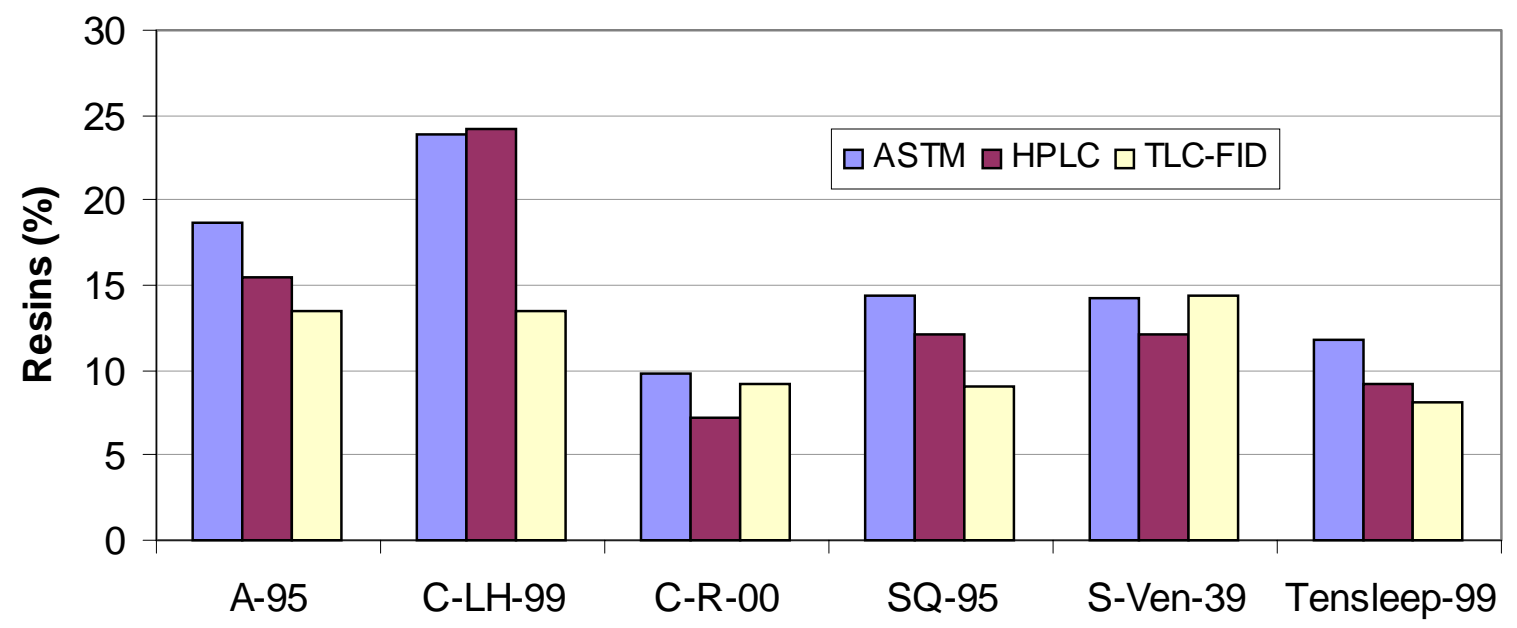

Figure I-2.25. Three estimates of the amount of resins in six crude oils.

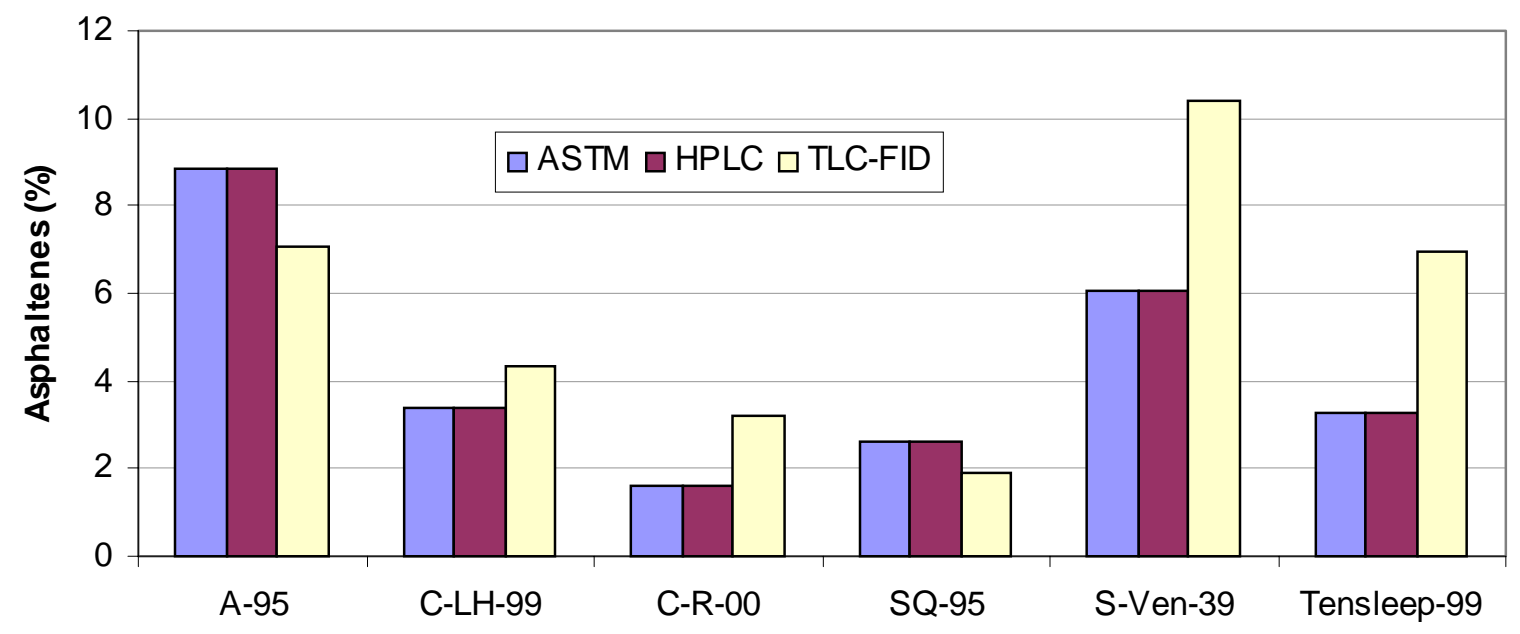

Figure I-2.26. Three estimates of the amount of asphaltenes in six crude oils.

\section{Recommendations}

The ideal fractionation procedure would be rapid, reproducible, and readily interpreted with respect to phase separation as well as oil/brine and oil/rock interactions. Of the three types of analyses tested, the ASTM method has the advantage of producing fractions in sufficient quantity to enable further tests to verify their chemical properties. Both the HPLC and TLC-FID techniques, as modified in the work reported here, are rapid and reproducible. What distinguishes between them is that the HPLC technique comes much closer than does the thin layer technique to reproducing the fractions recovered in the ASTM procedure. Too much is lost 
from the saturate and aromatic fractions in the TLC-FID procedure to give accurate estimates of those fractions except for quite heavy oil samples. Since the saturates are poor asphaltene solvents while the aromatics are good solvents for asphaltenes, assessment of the state of asphaltenes in a sample requires accurate measurements of these fractions. At the other end of the oil component spectrum, differentiation between insoluble polar species and soluble ones is best achieved by a preliminary asphaltene precipitation. The TLC-FID technique separates these components primarily on the basis of polarity and can lead to much different estimates of their relative amounts than the baseline values provided by the ASTM test.

As a standard method, therefore, we recommend an HPLC technique such as the improved method described in this work. The simple, reproducible procedure described here has advantages over previously published HPLC techniques in that saturate and aromatic fractions are quantified directly from peak areas (using representative hydrocarbons as calibration standards), eliminating the errors from evaporative loss of light saturate and aromatic components. With repeated injections of maltenes in hexane solution into the HPLC column sufficient resins can be collected for accurate gravimetric determination when the column is backflushed with a more polar solvent.

\section{Conclusions}

The ASTM-recommended method for separating SARA fractions can be used as a baseline to provide an objective method for developing and evaluating more convenient SARA separation methods.

An improved HPLC method has been devised to separate saturate, aromatic, and resin fractions from maltenes from which asphaltenes have already been precipitated. The key improvements are:

- Gravimetric quantification of the resin fraction, built up in the column over several injection cycles, eluted by backflushing.

- Saturates and aromatics (single and multiple rings) are quantified by difference, subtracting asphaltenes and resins from the total oil sample. Species are apportioned to the saturate and aromatic fractions from their peak areas using selected hydrocarbon standards that have been shown to give comparable results to the ASTM baseline data for the same set of oils. The inevitable evaporative losses that occur with solvent removal for standard gravimetric determinations are eliminated.

Careful tracking of the weight losses during development and FID scanning of TLC results have shown that the amount of material lost from medium gravity oils is excessive, making quantification of the saturate and aromatic fractions highly questionable. Further questions arise regarding the division between asphaltenes and resins, making it difficult to relate TLC-FID SARA fractions to the ASTM baseline data.

The best method for determining SARA fractions of crude oils is an HPLC technique that is rapid, reproducible, and requires only small sample volumes. The analyses so obtained are comparable to the ASTM method that is much more consumptive of time and materials. 


\section{References}

Al-Maamari, R.S.H. and Buckley, J.S.: "Asphaltene Precipitation and Alteration of Wetting: Can Wettability Change During Oil Production?" paper SPE 59292 at the 2000 SPE/DOE IOR Symposium, Tulsa, 3-5 Apr.

ASTM D2007-93: "Standard Test Method for Characteristic Groups in Rubber Extender and Processing Oils by the Clay-Gel Adsorption Chromatographic Method," ASTM (1993).

Barman, B.N.: "Hydrocarbon-Type Analysis of Base Oils and other Heavy Distillates by ThinLayer Chromatography with Flame-Ionization Detection and by the Clay-Gel Method," J.Chromatogr.Sci. (1996) Vol.34, pp.219-225.

Bharati, S., Røstum, G.A., and Løberg, R.: "Calibration and Standardization of Iatroscan (TLCFID) Using Standards Derived from Crude Oils," Org. Geochem. (1994) 22, 835-862.

Buckley, J.S., Hirasaki, G.J., Liu, Y., Von Drasek, S., Wang, J.X., and Gill, B.S.: "Asphaltene Precipitation and Solvent Properties of Crude Oils," Petroleum Sci. \& Tech. (1998) 16, No. $3 \& 4,251-285$.

Buckley, J.S.: "Predicting the Onset of Asphaltene Precipitation from Refractive Index Measurements," Energy \& Fuels (1999) 13, No. 2, 328-332.

Chaffin, J.M., Lin, M.S., Liu, M., Davison, R.R., Glover, C.J., and Bullin, J.A.: "The Use of HPLC to Determine the Saturate Content of Heavy Petroleum Products", J.Liq. Chrom. \& Rel. Technol. (1996), 19(10), 1669-1682.

Fan, T., Palmer, C., and Buckley, J.S.: "SARA Analysis of SQ-95 Crude Oil," PRRC Report 9934 (1999), PRRC, New Mexico Tech, Socorro, NM.

Félix, G., Thoumazeau, E.,Colin, J.M., and Vion, G.: "Hydrocarbon Groups Type Analysis of Petroleum Products by HPLC on Specific Stationary Phases", J. Liq. Chrom. (1987), 10(10), 2115-2132.

Grizzle, P.L. and Sablotny, D.M.: "Automated Liquid Chromatographic Compound Class Group-Type Separation of Crude Oils and Bitumens Using Chemically Bonded Aminosilane", Anal. Chem. (1986), Vol.58, NO.12, 2389-2396.

Jewell, D.M., Weber, J.H., Bucger, J.W., Plancher, H., Latham, D.R., Anal. Chem. (1972) 44, 1391-1395.

Karlsen, D.A. and Larter, S.R.: "Analysis of Petroleum Fraction by TLC-FID: Application to Petroleum Reservoir Description,”Org. Geochem. (1991) Vol.17, No.5, pp.603-617.

Mckay, J.F., and Latham, D.R.: "High Performance Liquid Chromatographic Separation of Olefin Saturate, and Aromatic Hydrocarbons in High-Boiling Distillates and Residues of Shale Oil”, Anal. Chem. (1980), 52, 1618-1621.

McLean, J.D. and Kilpatrick, P.K.: "Comparison of Precipitation and Extrography in the Fractionation of Crude Oil Residua,” Energy \& Fuels (1997) 11, 570-585.

Miller, R.: "Hydrocarbon Class Fractionation with Bonded-Phase Liquid Chromatography," Anal. Chem. (1982), 54, 1742-1746. 
Radke, M., Willsch, H., and Welte, D.H.: "Class Separation of Aromatic Compounds in Rock Extracts and Fossil Fuels by Liquid Chromatography”, Anal. Chem. (1984), 56, 2538-2546.

Ranny, M.: “Thin-layer Chromatography with Flame Ionization Detection.” (1987) Reidel, Dordrecht.

Suatoni, J.C. and Swab, R.E.: "Rapid Hydrocarbon Group-Type Analysis by High Performance Liquid Chromatography”, J. Chrom. Sci. (1975), Vol.13, 361-366.

Suzuki, Y: "Studies on Quantitative TLC by means of a Hydrogen FID. II Rapid Analysis of Fuel Oil Constituents," $21^{\text {st }}$ Annual Meeting of the Japan Society for Analytical Chemistry 47 (1972). (in Japanese)

Vela, J., Cebolla, V.L., Membrado, L., and Andres, J.M.: “Quantitative Hydrocarbons Group Type Analysis of Petroleum Hydroconversion Products Using an Improved TLC-FID System," J.Chromatogr.Sci. (1995) Vol.33, 417-424.

Wang, J.X.: "Predicting Asphaltene Flocculation in Crude Oils," PhD Thesis, NMIMT, Socorro, April, 2000.

Wang, J.X. and Buckley, J.S.: “A Two-Component Model of the Onset of Asphaltene Flocculation in Crude Oils," Energy \& Fuels (ASAP, 5 Jun 2001). 


\section{I-3. Characterization of Asphaltene Stability}

\section{I-3.1. Procedure for Measuring the Onset of Asphaltene Flocculation}

\section{J.X. Wang, NM PRRC}

Asphaltene stability is a function of composition, temperature and pressure. In the procedure outlined here, dead crude oil is titrated at ambient conditions with normal alkanes to find the first appearance of asphaltene aggregates. Microscopic aggregates are detected optically with transmitted light, setting an effective lower limit of about $0.5 \mu \mathrm{m}$ on the size that can be reliably discerned. For some oils, the first appearance of aggregates is readily distinguished from clear solutions with only slightly less alkane precipitant. In other cases fine particles appear before aggregates are observed and it is more difficult to define an onset condition. In the latter case, two onset conditions can be defined: (1) the conditions where fine asphaltic particles first appear or onset of fines, and (2) the conditions where those particles first begin to aggregate or onset of aggregates. Between these conditions is a more or less continuous increase in the amount of particulate material.

The solubility conditions in the dead oil sample and at the onset are quantified by refractive index (RI) measured at $20^{\circ} \mathrm{C}$ at the wavelength of the sodium-D line $(589 \mathrm{~nm})$. Quantities of interest include RI of the dead oil sample $\left(\mathrm{RI}_{\mathrm{oil}}\right)$ and $\mathrm{RI}$ of the mixture of oil and alkane at the onset of precipitation $\left(\mathrm{P}_{\mathrm{RI}}\right)$. In effect, $\mathrm{RI}$ is a surrogate for the solubility parameter $(\delta)$, used because it can be readily measured whereas $\delta$ must be estimated for complex fluids like crude oils. For nonpolar liquids including crude oils, $\delta$ can be calculated from RI using an empirical correlation.

Interference is often encountered from asphaltene aggregates and other particulates and emulsified matter that are present in the dead oil samples, as received. Methods for dealing with samples that contain emulsions and/or particulates are included in this procedure.

Asphaltene flocculation can be a slow process, especially near the onset point. In most titration techniques the precipitant is added fairly quickly, which probably leads to an overestimate of the amount of precipitant at the onset point and a corresponding overestimate of asphaltene stability. Experience has shown that one day of aging is the minimum amount of time needed to make a reliable judgment about the onset; longer times would be better, but are seldom practical.

The overall asphaltene onset characterization scheme is outlined in Fig. I-3.1 and discussed in detail in the sections that follow. 


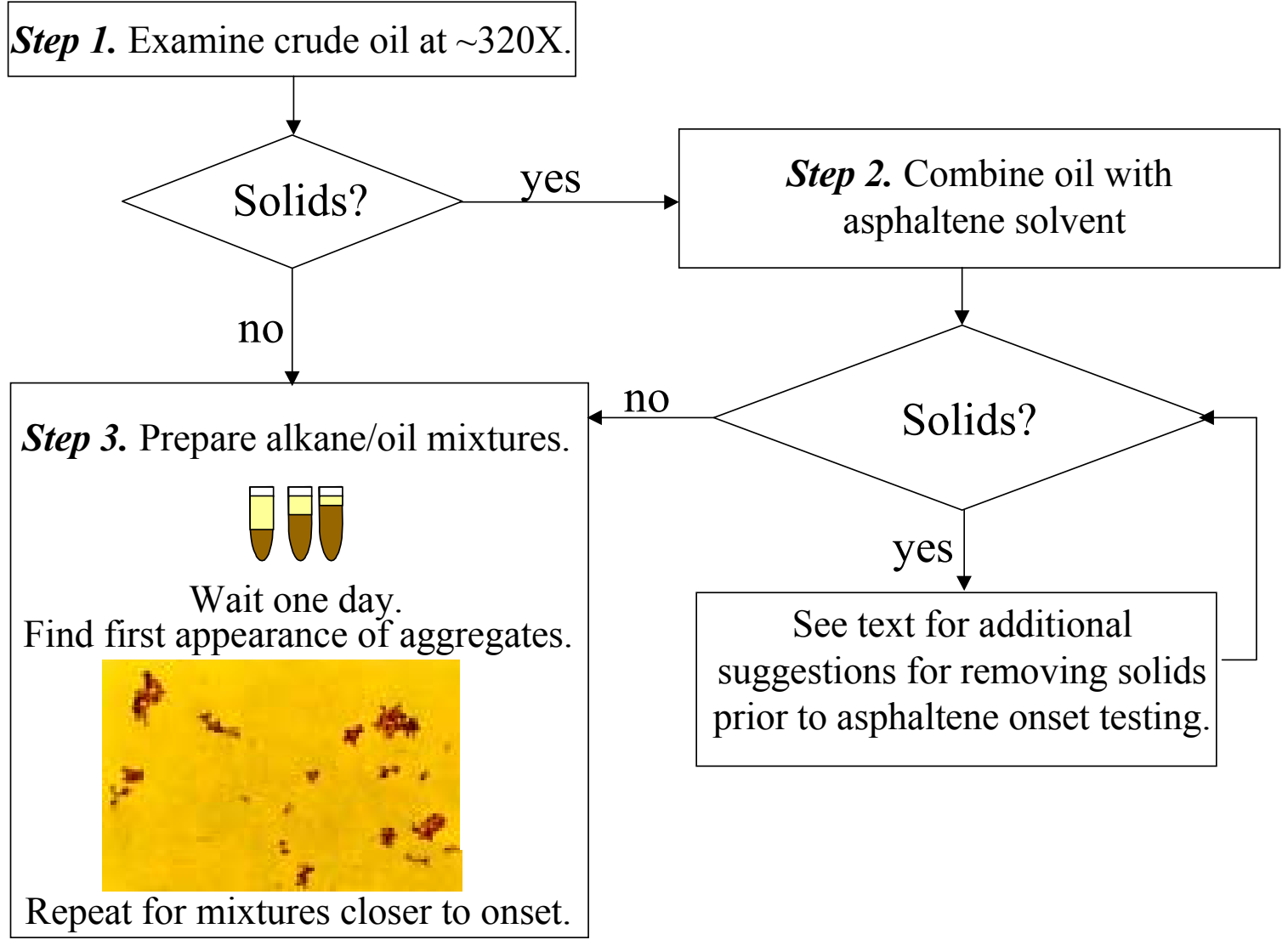

Figure I-3.1. Outline of the asphaltene onset determination scheme.

\section{Step 1. Microscopic examination}

A drop of dead crude oil is placed on a microscope slide, covered with a cover slip, and observed microscopically at a magnification of about 320X. If the sample is clear and free from any particles, no special pretreatment is required. Skip Step 2 and proceed to Step 3.

Often crude oils contain aggregated asphaltenes, wax crystals, emulsified water, and other unidentified solid particles. The aggregated asphaltenes may appear as large aggregates, small aggregates, or individual fines or particles. Asphaltenes can often be observed adhering to one another. They can be distinguished from wax crystals by observing between polarizing filters oriented at $90^{\circ}$ to one another. The crystalline waxes can pass polarized light and appear as bright spots whereas asphaltenes are amorphous and cannot be seen. Highly spherical objects are likely to be emulsified liquids. These oils cannot be used directly to find the onset point induced by adding n-alkanes since the existing aggregates or particles may obscure the onset observation. Strategies for removing sources of interference are outlined in Step 2. 


\section{Step 2. Redispersion of asphaltene aggregates}

Asphaltene aggregates, waxes, and other particulates, could be removed mechanically by filtration or centrifugation. A better strategy, however, is to redisperse the asphaltene aggregates by addition of a good asphaltene solvent. This option is preferable because all the oil components are retained. Aromatic compounds, such as toluene or 1-methylnaphthalene (1$\mathrm{MN}$ ), are good asphaltene solvents. 1-MN is the stronger solvent, but it is also more viscous than toluene so the time required to disperse the asphaltenes is longer. The value of $P_{R I}$ is shifted more by addition of toluene than by 1-MN. The value of $\mathrm{P}_{\mathrm{RI}}$ obtained from a 50:50 mixture of oil mixed with 1-MN is normally only slightly different from the value of $\mathrm{P}_{\mathrm{RI}}$ that would be measured for the crude oil without added solvent. If time and oil sample volume permit, however, a more accurate value of $\mathrm{P}_{\mathrm{RI}}$ can be obtained by testing two or more mixtures with different ratios of oil to 1-MN. $P_{R I}$ can then be evaluated as a function of the amount of added solvent and extrapolated to the value of $\mathrm{P}_{\mathrm{RI}}$ for oil with no added solvent.

For oil samples with asphaltene aggregates and/or other particulates or emulsions, the following steps are recommended:

2.1 Prepare a 1:1 mixture of oil with 1-MN. Stir the mixture for one to two days, checking for particles at $\sim 320$ X. If no asphaltenes are visible proceed to Step 2.5 .

2.2 If complete dispersion is not achieved, agitate the mixture in an ultrasonic water bath for several hours. If asphaltenes disappear, proceed to Step 2.5.

2.3 If asphaltene are still not completely dissolved, heat the mixtures to 60 to $70^{\circ} \mathrm{C}$ for several hours in sealed vials, taking care to prevent evaporation. If asphaltenes disappear, proceed to Step 2.5.

2.4 If none of these treatments redisperses the asphaltenes, add a known amount of $1-\mathrm{MN}$ to the mixture to further reduce oil:1-MN volume ratio and repeat the sequence starting at 2.1 above.

2.5 If waxes remain after asphaltenes are completely redispersed, heat the solution as described at 2.3 above. Usually this will be sufficient to dissolve the waxes, which may reappear, but slowly so that they do not interfere with the onset tests. If there are no emulsions, proceed to Step 3.

2.6 Emulsified water may segregate from the oil and collect as a sediment on the bottom of the container during steps 2.1 to 2.5 . If the sediment is gray or white in color, most asphaltenes that once may have collected at the oil/water interface have been redispersed. Proceed to Step 2.7. If the sediment is brown, some of the asphaltene fraction may remain associated with the emulsion interfaces. Add a large volume of distilled water to the oil/1-MN mixture. Agitate in an ultrasonic water bath for about 30 minutes. This washing step should help to break the original emulsion, releasing the asphaltenes. The mixture can then be separated as described in Step 2.7. 
2.7 If no emulsified droplets remain suspended, carefully separate the supernatant and proceed with Step 3. If emulsified droplets remain in suspension, centrifuge at 2000-3000 rpm for several hours in sealed centrifuge tubes. Separate the supernatant and proceed with Step 3. This step should also remove extraneous inorganic solids and extremely refractory organics (known as carbenes and carboids).

\section{Step 3. Measure onset of asphaltene flocculation}

The onset condition (or conditions-if both the onset of fines and onset of aggregates exist) can be determined to a reasonable tolerance by examination of mixtures of oil and precipitant that vary by $1 \%$ (by volume) in composition. If aggregates are observed in a mixture consisting of $50 \%$ oil (or oil/1-MN mixture) and 50\% heptane, but a mixture with $51 \%$ oil and $49 \%$ heptane is clear, then the 50:50 mixture would be considered to be the onset mixture.

The onset search can be refined in a series of steps. If oil is the limiting factor, fewer mixtures would be included in each step and the number of steps would be increased. The extreme example would be a search by the bisection method beginning with a 50:50 mixture of oil and precipitant, followed by either a $25: 75$ or 75:25 mixture depending on whether asphaltenes were observed in the first mixture. If there is plenty of oil, time can be saved by making more mixtures in each step. In the extreme case, 99 separate mixtures would be prepared in the first step if the $1 \%$ tolerance level were the target. Clearly this is impractical and the most efficient search strategy lies between the two extremes.

As a compromise between conserving oil and minimizing time, begin with a coarse screening, consisting of a series of seven widely space mixtures (oil:precipitant $=20: 80,30: 70$, $40: 60,50: 50,60: 40,70: 30,80: 20)$. Note that "oil" refers either to the original oil or the mixture of oil and solvent prepared in Step 2. Subsequent mixtures would be selected on the basis of the results of the coarse screening step. For precipitation with n-heptane, the onset is often found between 20:80 and 40:60, so oil might be conserved by beginning with mixtures in that range.

3.1 Prepare small glass vials. Clean glass vials ( $\sim 6 \mathrm{ml}$ is a convenient size), being careful to remove any traces of cleaning solution by flushing with plenty of distilled water. Contamination by trace amounts of surfactant can affect results. We soak vials in distilled water in an ultrasonic bath overnight. Dry thoroughly.

3.2 Add oil (or oil mixed with 1-MN) to vial. Accurately measure the amount of oil required into the sample vial. If the oil can be delivered by syringe, one strategy is to use a $1-\mathrm{ml}$ glass syringe with $0.01 \mathrm{ml}$ resolution. Parts of the syringe in contact with oil should be either glass or Teflon. If the oil cannot be delivered by syringe, the desired amount can be weighed into the vial and the volume calculated from the density. Larger volumes can be used to increase accuracy if sufficient oil is available.

3.3 Add precipitant to the oil. The required amount of n-heptane or other precipitant can be added to the vial using a similar syringe. Note: always add 
precipitant to oil (add slowly, with plenty of stirring to avoid high local concentrations of precipitant); never add oil to precipitant! Adding small volumes of oil to a large volume of precipitant will cause premature precipitation of the asphaltenes. While asphaltenes may, in theory, be stable in the final mixture, prematurely precipitated material may persist much more than the oneday period allotted for these tests.

3.4 Seal the vial immediately. A Teflon-lined aluminum cap works well. Precipitants and many of the components of the oil are volatile; evaporation will change the overall composition and invalidate the results of these tests. Shake the vial vigorously until the mixture appears homogeneous. Then allow the mixture to rest at ambient conditions for one day before observation.

3.5 Observe asphaltene flocculation. After aging for one day, a few drops of each mixture can be removed from the vial (disposable syringes are useful for this step), deposited on a glass microscope slide, covered with a cover slip, and observed immediately. It is helpful to prefocus the microscope using an old sample in which asphaltenes are obvious that is kept by the microscope for this purpose. Since evaporation begins when the sample is removed from the vial, it is important not to waste time during the actual observation. This is especially problematic with very light oils and highly volatile precipitants such as n-pentane and $\mathrm{n}$-hexane; the accuracy of tests may be questionable if rapid evaporation is a problem. The flocculated asphaltenes usually appear as flowing particles and/or aggregates.

3.6 Measure refractive index. An additional sample can be removed from each vial and used to measure RI. An automatic refractometer (Index Instruments, model GPR 11-37) is convenient for this purpose. Temperature should be maintained at $20^{\circ} \mathrm{C}$ with a circulating water bath. Transfer mixture to the prism surface of refractometer as quickly as possible; seal immediately with the specially designed cap to prevent evaporation. Temperature equilibration requires a few seconds. Record the initial stabilized RI reading. With time, the RI may drift for these mixtures as asphaltenes adsorb and/or deposit on the prism surface, so it is important to take the initial reading.

3.7 Testing for the onset with additional precipitants. Once the onset has been found with n-heptane, that result can be used to predict the onset with other alkanes and used to streamline the search strategy for any additional tests. A maximum variation of between 5 and $15 \%$ in the amount of precipitant required at onset has been found for $n$-alkanes from pentane to pentadecane. For more information on the relationship between alkane molecular size and the onset conditions, refer to the references below. 


\section{References}

Buckley, J.S., Hirasaki, G.J., Liu, Y., Von Drasek, S., Wang, J.X., and Gill, B.S.: "Asphaltene Precipitation and Solvent Properties of Crude Oils," Petroleum Science and Technology (1998) 16, No. 3\&4, 251-285.

Buckley, J.S. and Wang, J.X.: "Crude Oil and Asphaltene Characterization for Prediction of Wetting Alteration,” J. Pet. Sci. Eng, (2002) 33, 195-202.

Wang, J.X.: "Predicting Asphaltene Flocculation in Crude Oils," PhD Thesis, NMIMT, Socorro, April, 2000.

Wang, J.X. and Buckley, J.S.: "A Two-Component Solubility Model of the Onset of Asphaltene Flocculation in Crude Oils," Energy \& Fuels (2001) 15, 1004-1012.

Wang, J.X. and Buckley, J.S.: "An Experimental Approach to Prediction of Asphaltene Flocculation," paper SPE 64994 presented at the 2001 OCS, Houston, 13-16 Feb. 


\title{
I-3.2. An Experimental Approach to Prediciton of Asphaltene Flocculation
}

\author{
Wang, J.X. and Buckley, J.S.: “An Experimental Approach to Prediction of Asphaltene \\ Flocculation," paper SPE 64994 presented at the 2001 OCS, Houston, 13-16 Feb.
}

Many of the existing models that have been developed to predict the onset of asphaltene flocculation rely on approximations to a full thermodynamic treatment of asphaltene phase behavior and on parameters that are not available except by fitting HTHP data to the model. A much simpler approach has been developed based on experimental measurements of refractive index $(R I)$ at the onset of flocculation for precipitants representing a range of molecular sizes.

The onset of asphaltene flocculation is often reported in mixtures of oil, solvent, and asphaltene precipitants. In this work, direct measurements of $R I$ replace estimates of solubility parameters. $R I$ is readily measured and is a good indicator of the ability of the oil to maintain a stable dispersion of asphaltenes. Tests with stock tank oil samples have shown that there is a common trend relating $R I$ at the onset of instability to precipitant molecular size. This observation facilitates predictions of asphaltene instability for a wide range of conditions from as few as one or two onset determinations with precipitants of known molecular size. Results obtained at ambient conditions for stock tank oil can, in principle, be extended to reservoir conditions using standard PVT and compositional data, as demonstrated in this paper.

\section{Introduction}

Asphaltenes can cause problems in oilfield operations, oil transportation, and in petroleum refining operations. Despite extensive study, questions remain about how best to predict asphaltene instability under a wide variety of conditions from a limited number of experimental results.

\section{Solubility Tests of Onset of Asphaltene Flocculation}

Tests of asphaltene stability that have in common dilution of crude oil, asphalt, or bitumen with solvents have long been used to evaluate the stability of asphaltenes. Mertens (1960) refers to the spot test of asphaltic materials (AASHO T102-42) that was already a standard for the American Association of State Highway Officials in 1950. He appears to have been the first to suggest that stable and unstable conditions can be related to regular solution theory Hildebrand and Scott (1950). He calculated a critical solubility parameter at infinite dilution:

$$
\delta_{c}=\delta_{s}-p_{a}\left(\delta_{s}-\delta_{p}\right)
$$

where $\delta$ is the solubility parameter of critical mixture $(c)$, solvent $(S)$, or precipitant $(P)$. The dispersability of the least soluble asphaltenes, $p_{a}$, is related to the precipitant and solvent volumes $(V)$ by $p_{a}=V_{P} /\left(V_{P}+V_{S}\right)$ which, at infinite asphaltene dilution, is simply the volume fraction of precipitant.

A variety of combinations of solvent and precipitant volumes have been suggested to evaluate oil and asphalt samples (Heithaus, 1962; Waxman et al., 1980; Hotier and Robin, 1983; deBoer et al, 1995; Cimino et al., 1995). All reflect the appearance of flocculated asphaltenes in 
some critical mixture of solvent and precipitant. What is captured in each of these different approaches is the critical mixture composition at infinite dilution of the asphaltene component. Effectively, these methods evaluate the critical value of solubility parameter by using compounds for which solubility parameters are known, although they do not necessarily express their results in solubility parameter terms. Extrapolation from these results to conditions of practical importance fail for two important reasons: (1) flocculation at infinite dilution is not representative of flocculation from oil and (2) sufficiently accurate models are not available to describe the relationship between critical solubility conditions and physical properties of the precipitating medium.

\section{Solubility Parameter and RI}

We have shown previously that refractive index $(R I)$ is directly related to solubility parameter in solutions of nonpolar molecules and for crude oils, which are mixtures of mostly nonpolar hydrocarbons (Buckley et al., 1998; Buckley, 1999; Wang, 2000; Wang et al., 2000). Figure I-3.2 illustrates the linear relationship between $\delta$ and a simple function of $R I, F_{R I}$, for a series of paraffinic hydrocarbons from methane to eicosane and for aromatics including benzene, toluene, ethylbenzene, $o$-xylene, styrene, and $\alpha-\mathrm{MN} . F_{R I}$ is defined by:

$$
F_{R I}=\left(n^{2}-1\right) /\left(n^{2}+2\right)
$$

where $n$ is refractive index.

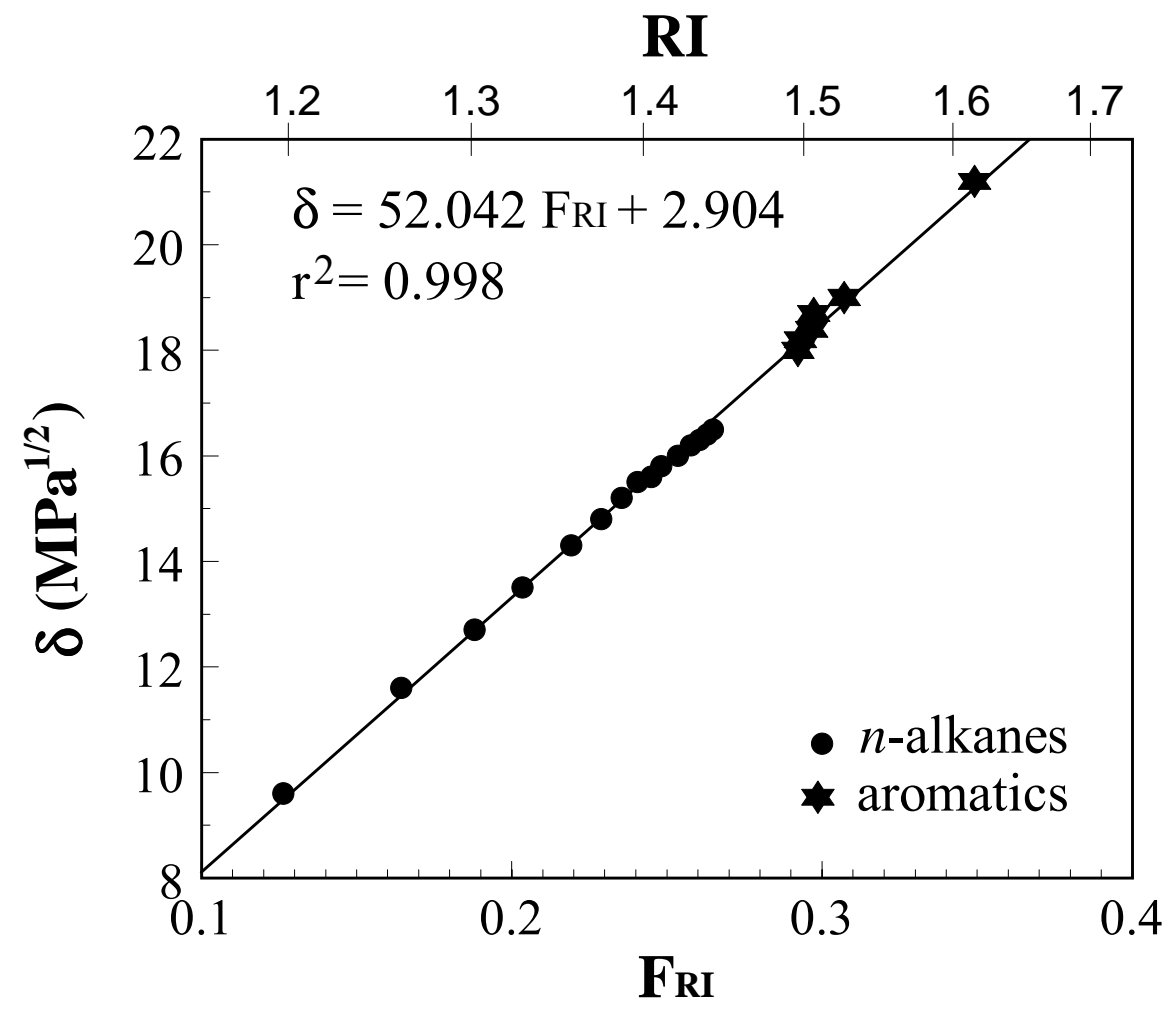

Figure I-3.2. Solubility parameter, $\delta$, of nonpolar molecules is a linear function of $F_{R I}$. 
Simple additivity rules relate volume-weighted component properties $\left(F_{R I, i}\right.$ and $\left.\delta_{i}\right)$ to the resulting mixture characteristics:

$$
\begin{aligned}
F_{R I, \text { mixture }} & =\sum \phi_{i} F_{R I, i} \\
\delta_{\text {mixture }} & =\sum \phi_{i} \delta_{i}
\end{aligned}
$$

where $\phi_{i}$ is the volume fraction of component $i$ in the mixture.

$R I$ measurements eliminate the guesswork about solubility parameters and the need to observe the onset at infinite dilution in order to quantify the critical solubility conditions.

\section{Flory-Huggins Approach}

Application of the Flory-Huggins approach (Flory, 1942; Huggins, 1941) to asphaltene solutions, was an important advance in description of asphaltene stability (Hirschberg et al., 1984). The Flory-Huggins interaction parameter $\chi$ is proportional to the enthalpy of mixing solutes and solvents of widely different sizes:

$$
\chi=\frac{v_{m}}{R T}\left(\delta_{a}-\delta_{m}\right)^{2}
$$

where the subscripts $a$ and $m$ refer to asphaltene and mixture (including all other oil components), respectively, $v$ is molar volume, $T$ is absolute temperature, and $R$ is the gas constant. Mixing is favored when the difference between $\delta_{a}$ and $\delta_{m}$ or the value of $v_{m}$ is minimized.

\section{Experimental Methods}

\section{Measurement Techniques.}

\section{RI MEASUREMENT}

An automatic refractometer (Index Instruments model GPR 11-37) was used to measure refractive index at constant temperature. The accuracy for RI measurement is \pm 0.00005 for RI values less than 1.45 , and \pm 0.0001 for RI values greater than 1.45. Most RI measurements were made with an SGP5 process cell, which could be sealed to minimize evaporation. Temperature was controlled with a water bath. The RI reading was allowed to stabilize for several seconds to allow the temperature to equilibrate, after which the RI value was recorded. Variations of RI after longer periods, especially for samples near or past the onset of flocculation, can occur because of asphaltene deposition on the prism surface.

\section{MOLECULAR WEIGHT AND MOLAR VOLUME}

Average molecular weights were determined from freezing point depression measurements using a Cryoscope 5009 from Precision Systems. Conversion to molar volumes required measurement of the density, which was obtained using a Mettler/Paar DMA 40 digital densitometer with a water-bath to control temperature. 


\section{ONSET OF ASPHALTENE FLOCCULATION}

The first appearance of particles that tend to aggregate has been defined as the onset of flocculation for this study. Aggregates may be preceded by individual particles that remain dispersed. In some cases, the first appearance of these fine particles occurred with significantly less added precipitant (as much as 10\% less). In other cases, there was no distinction between the first appearance of particles and aggregates.

The onset of asphaltene flocculation was determined for a series of n-alkanes, from npentane $\left(n-C_{5}\right)$ through $n$-pentadecane $\left(n-C_{15}\right)$. To obtain a first estimate of $\phi_{\text {oil,onset }}, n$-heptane $\left(n-C_{7}\right)$ was mixed with oil in varying ratios $\left(\phi_{\text {oil }}\right.$ from 0.2 up to 0.8 in increments of 0.1$)$. After aging for one day, asphaltene flocculation was observed microscopically at a magnification of 320X. The range of $\phi_{\text {oil,onset }}$ in which asphaltene aggregates were observed with $n-C_{7}$ as precipitant was then used as a guide to preparation of mixtures of oil with other chain-length precipitants. The range of $\phi_{\text {oil,onset }}$ for each precipitant was eventually narrowed to a volume fraction increment of 0.01 or $1 \%$ oil in the mixture.

The visual onset of flocculation represents an arbitrary point in the continuous growth of asphaltene aggregates, i.e., the size at which asphaltene aggregates become large enough to be distinguished with an optical microscope (about $0.5 \mu \mathrm{m}$ with the system currently in use). This threshold has practical implications especially for asphaltene plugging. At this size, the particles already contain thousands of asphaltene macromolecules.

\section{SEPARATION OF ASPHALTENES}

Asphaltene separation was based on the standard procedure recommended in ASTM D2007-80 (ASTM D2007-80). Mixtures of one part oil to 40 parts n-paraffin were allowed to age for two days before filtration through $0.22 \mu \mathrm{m}$ cellulose acetate membrane filters, followed by air drying and weighing.

When $n-C_{15}$ was used to precipitate asphaltenes, drying was extremely slow due to the low volatility of $n-C_{15}$. These samples were redissolved in $20 \mathrm{ml}$ of toluene, and asphaltenes were re-precipitated by adding $40: 1 \mathrm{n}-\mathrm{C}_{5}$ to the toluene solution.

\section{Materials.}

\section{CRUDE OILS}

Seven dead crude oil samples were tested in this study. Two were used as received. The other five samples contained some asphaltene aggregates or fine asphaltic particles. Those samples were diluted with enough $\alpha$-methylnaphthalene $(\alpha-M N)$ to disperse the existing aggregates before further tests to determine the onset of precipitation. Amounts of added $\alpha-\mathrm{MN}$ are indicated in Table I-3.1, along with other oil properties. 
Table I-3.1. Crude oil properties

\begin{tabular}{|l|c|c|c|c|}
\hline Oil & $n_{D}{ }^{20} *$ & $\begin{array}{c}\phi_{\alpha-M N} \\
(\mathrm{vol} \text { frac })\end{array}$ & $\begin{array}{c}n-\mathrm{C}_{7} \mathrm{asph} \\
(\mathrm{wt} \%)^{*}\end{array}$ & $\begin{array}{c}\text { mol wt } \\
(\mathrm{g} / \mathrm{mol})\end{array}$ \\
\hline A-93 & 1.5220 & 0.3 & 6.86 & 256 \\
\hline A-95 & 1.5159 & 0.3 & 8.67 & 271 \\
\hline Lagrave & 1.4832 & 0.3 & 6.69 & 188 \\
\hline Lost Hills & 1.5137 & 0.0 & 2.78 & 265 \\
\hline Mars-Pink & 1.5380 & 0.0 & 4.40 & 305 \\
\hline Moutray & 1.4827 & 0.2 & 1.29 & 242 \\
\hline Tensleep & 1.4880 & 0.35 & 3.20 & 275 \\
\hline
\end{tabular}

\section{SOLVENTS AND PRECIPITANTS}

Normal paraffins from $\mathrm{n}-\mathrm{C}_{5}$ through $\mathrm{n}-\mathrm{C}_{15}$ were used to initiate asphaltene flocculation. Two aromatic solvents, toluene and $\alpha-M N$, were used in selected tests. Properties of precipitants and solvents are summarized in Table I-3.2.

Table I-3.2. Properties of asphaltene precipitants and solvents

\begin{tabular}{|c|c|c|c|}
\hline hydrocarbon & $\begin{array}{c}\text { molar } \\
\text { volume } \\
(\mathrm{ml} / \mathrm{mol})\end{array}$ & $n_{D}{ }^{20}$ & $\begin{array}{c}\delta \\
(\mathrm{MPa}) \\
1 / 2\end{array}$ \\
\hline$n$-pentane & 116.1 & 1.3574 & 14.3 \\
\hline$n$-hexane & 131.6 & 1.3751 & 14.8 \\
\hline$n$-heptane & 147.5 & 1.3873 & 15.2 \\
\hline$n$-octane & 163.5 & 1.3966 & 15.5 \\
\hline$n$-nonane & 179.7 & 1.4050 & 15.6 \\
\hline$n$-decane & 211.2 & 1.4112 & 15.8 \\
\hline$n$-undecane & 228.6 & 1.4165 & 15.9 \\
\hline$n$-dodecane & 243.7 & 1.4212 & 16.0 \\
\hline$n$-tridecane & 261.3 & 1.4251 & 16.1 \\
\hline$n$-tetradecane & 276.4 & 1.4290 & 16.2 \\
\hline$n$-pentadecane & 294.1 & 1.4314 & 16.25 \\
\hline toluene & 106.3 & 1.4964 & 18.2 \\
\hline $\begin{array}{c}\alpha \text {-methyl- } \\
\text { naphthalene }\end{array}$ & 139.1 & 1.6156 & 21.2 \\
\hline
\end{tabular}

\section{Results and Discussion}

The amount of $n$-paraffin added to one $\mathrm{ml}$ of oil at the onset of flocculation varies with both crude oil and precipitant as shown in Fig. I-3.3. Similar results, showing a maximum in the amount of precipitant for octane or nonane, have been reported previously for dilute solutions of oil (Hotier and Robin, 1983; Cimino et al., 1995). To what extent these results reflect 
differences in stability between asphaltenes from different oils cannot be evaluated without knowing more about the solvent properties of the non-asphaltenic portions of these crude oil samples.

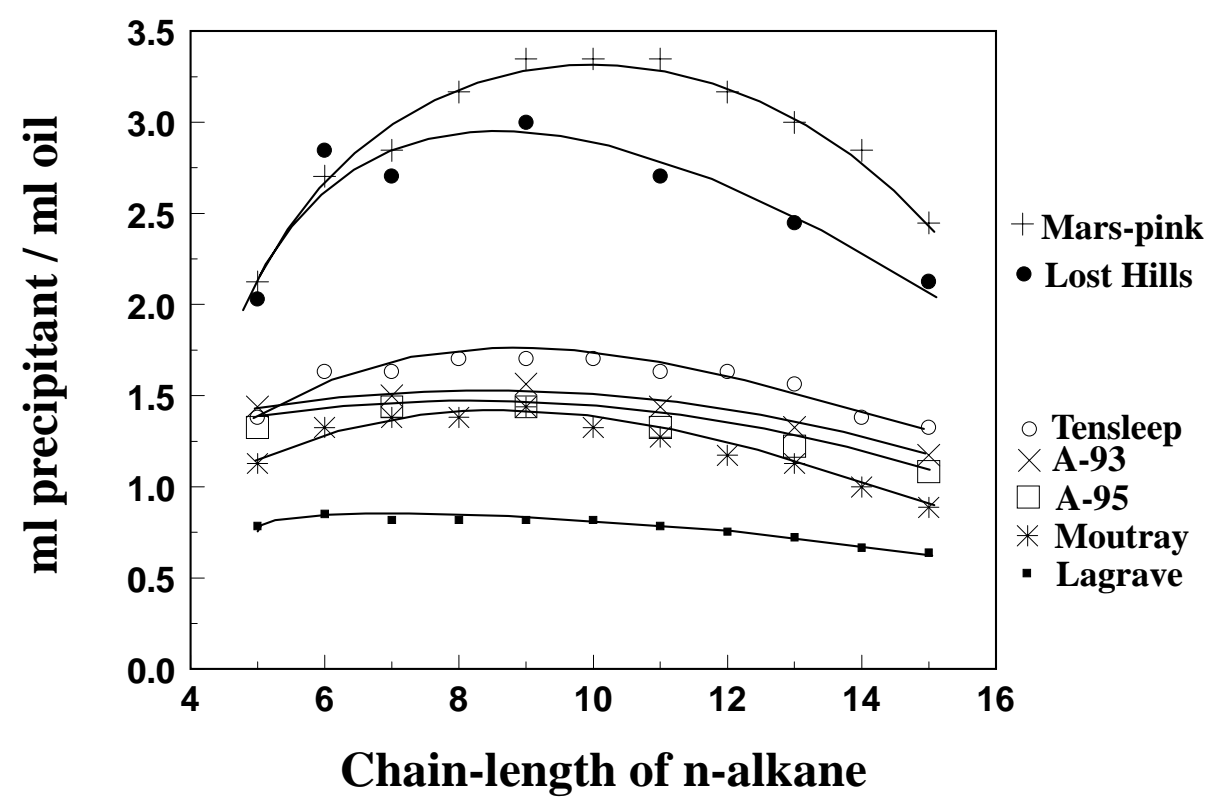

Figure I-3.3. The volume of precipitant required to initiate flocculation of asphaltenes varies with oil and precipitant, but trends with precipitant chain length vary.

\section{$P_{R I}$ FOR CRUDE OILS}

The same onset mixtures of crude oil (or oil plus $\alpha$-MN) and each alkane can be characterized by their $R I$, as shown in Fig. I-3.4, where the $R I$ at the onset is denoted by the symbol $P_{R I}$. The pattern that emerges shows a remarkably consistent increase in mixture $R I$ at the onset with increasing molecular size of the precipitating alkane. While there are differences in magnitude of $P_{R I}$ as a function of paraffin chain length among the seven oils, all have nearly the same slope. In this plot, the differences in magnitude do reflect differences in stability of asphaltenes in these different oils.

\section{CORRELATION TO PRECIPITANT PROPERTIES}

Chain length is a measure of molecular size that can be used to compare the precipitants in this study since they are all members of an homologous series, the $n$-paraffins. However, there are other, more physically meaningful measures that we could choose. Referring back to Eq. I-3.5, the Flory Huggins interaction parameter is related to the molar volume of the mixture and the square of the difference between solubility parameters. We therefore select the square root of molar volume of precipitant, $v_{p}{ }^{1 / 2}$, to represent the range of precipitant sizes and replot the data from Fig. I-3.4 in Fig. I-3.5, which shows that $P_{R I}$ is linearly related to $v_{p}{ }^{1 / 2}$ for all the oil samples in this study. Least squares fits, all with $r^{2}$ greater than 0.99 , are summarized in Table I3.3 . 


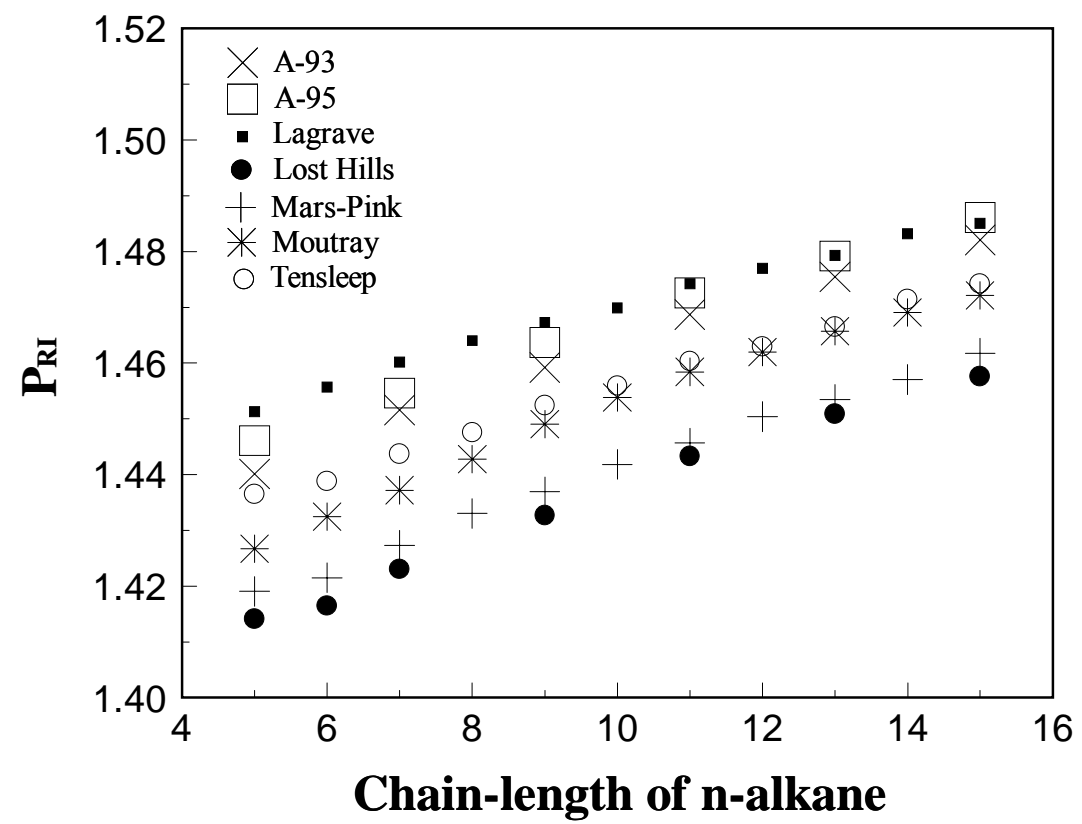

Figure I-3.4. The experimental observations in Fig. I-3.3 can be viewed in terms of the mixture $R I$ when flocculated asphaltenes are first observed $\left(P_{R I}\right)$ rather than the volume of precipitant added. $P_{R I}$ increases monotonically with chain length of the $n$-paraffin precipitating agent. Trends for seven crude oil samples are almost parallel.

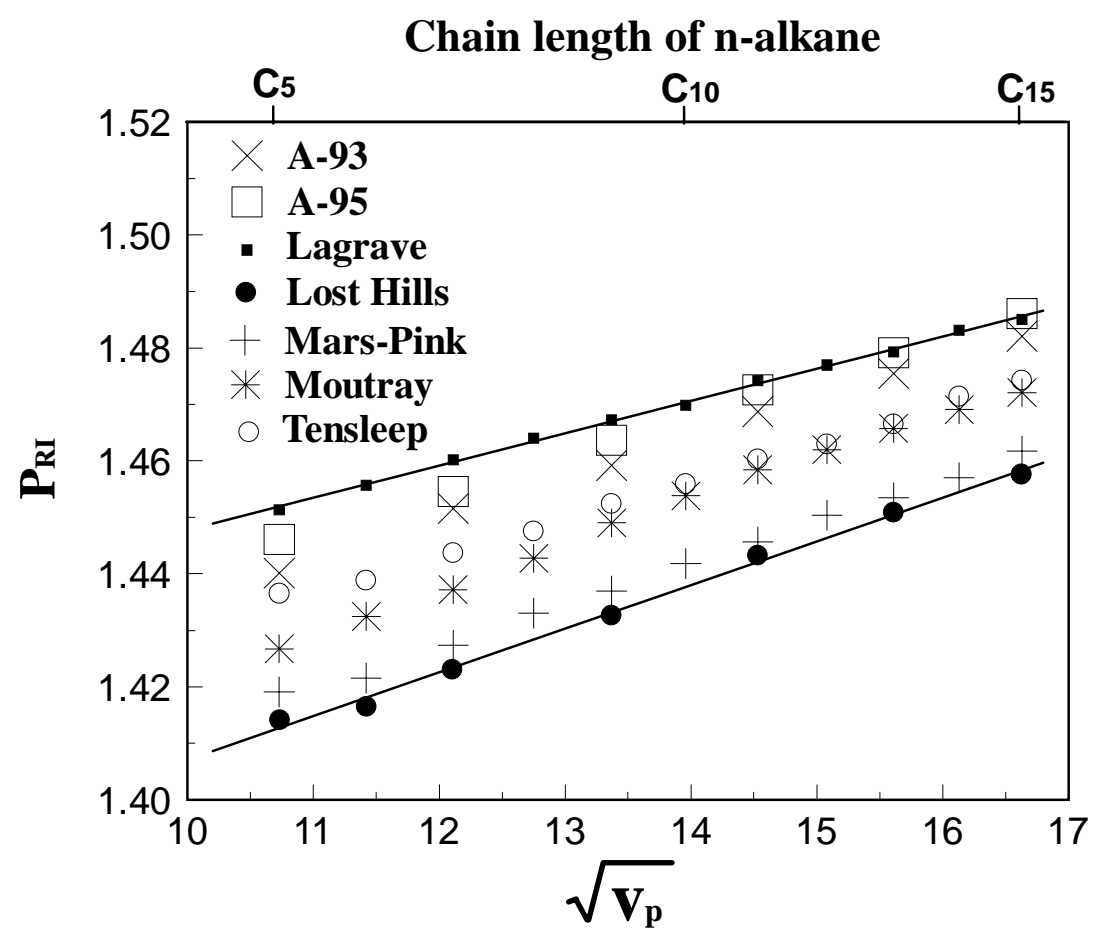

Figure I-3.5. For a given crude oil, $P_{R I}$ is a linear function of $v_{p}{ }^{1 / 2}$, where $v_{p}$ is precipitant molar volume. Fits to these linear trends are summarized in Table I-3.3. (Chain length of the $n$-alkane precipitants is indicated on the top $x$-axis.) 
Table I-3.3. Summary of least-squares fits to data in Fig. I-3.5

\begin{tabular}{|l|c|c|c|}
\hline \multicolumn{1}{|c|}{ Oil } & intercept & slope & $r^{2}$ \\
\hline A-93 & 1.3649 & 0.0071 & 0.998 \\
\hline A-95 & 1.3721 & 0.0069 & 0.998 \\
\hline Lagrave & 1.3906 & 0.0057 & 0.998 \\
\hline Lost Hills & 1.3298 & 0.0077 & 0.996 \\
\hline Mars-Pink & 1.3386 & 0.0074 & 0.998 \\
\hline Moutray & 1.3429 & 0.0079 & 0.996 \\
\hline Tensleep & 1.3644 & 0.0055 & 0.997 \\
\hline
\end{tabular}

\section{EFFECT OF SOLVENT ADDITION ON ONSET OF FLOCCULATION}

The effect of adding solvents, a common practice in heptane titrations for determining the onset of asphaltene flocculation, is illustrated in Fig. I-3.6 for Mars-Pink crude oil. RI at the onset changes little with addition of even $80 \% \alpha$-MN, but addition of large amounts of toluene systematically shift $P_{R I}$ to lower values, i.e., asphaltenes are more stable in the solution diluted with toluene than in the original oil.

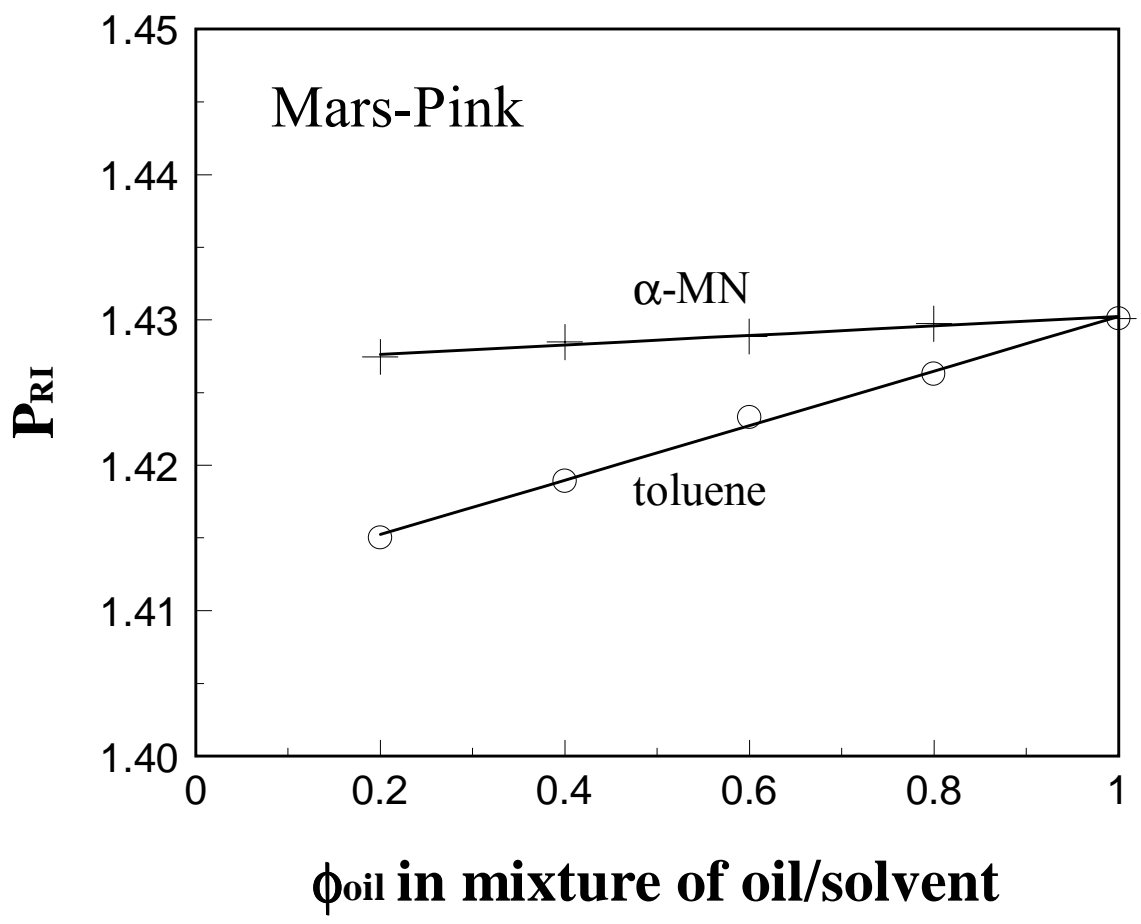

Figure I-3.6. $P_{R I}$ measured for Mars-Pink crude diluted with $\alpha$-MN or toluene. The precipitant is $n$-heptane.

As discussed in the Introduction, large dilutions of oil or bitumen in solvent have often been used for onset tests. Advantages are that the solutions are much lighter in color and the constant ratio of solvent to precipitant at the onset can be used as to estimate a critical solubility condition for the onset of asphaltene flocculation. The assumption is that the onset is not 
affected by dilution, but, as suggested by the toluene solution results in Fig. I-3.6, this may not be true. To test the effect of high levels of dilution, asphaltenes were prepared from each of six crude oils, using a 40:1 ratio of $n-C_{7}$ to oil. The asphaltenes were used to make $1 \%$ solutions in toluene. The results of onset tests starting with these solutions of asphaltenes in toluene are summarized in Fig. I-3.7.

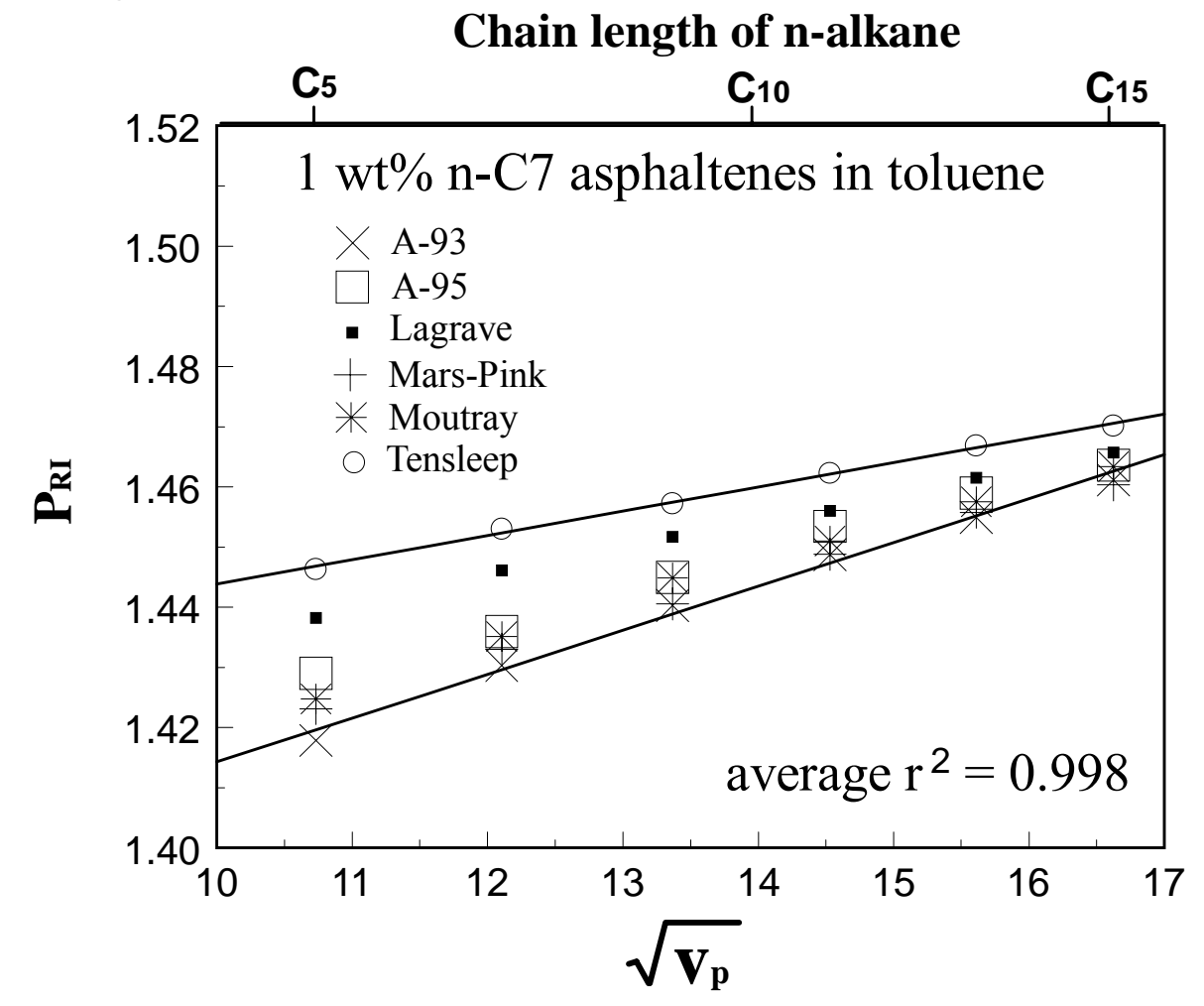

Figure I-3.7. Flocculation of asphaltenes that have been separated from their crude oils and redissolved as $1 \%$ solutions in toluene. Trend of $P_{R I}$ with $v_{p}{ }^{1 / 2}$ is linear, but slopes and values of $P_{R I}$ are different than those for the original oil samples.

Comparing conditions for onset from crude oils (Fig. I-3.5) with those at infinite dilution (Fig. I-3.7) shows that there can be a considerable difference between the two. The comparison should be focused on the onset induced by $n-C_{7}$, since the composition of the asphaltenes varies with precipitant and thus asphaltene stability to other precipitants may be affected by the selection of only the $n-\mathrm{C}_{7}$ asphaltenes in this test. Table I-3.4 summarizes the $n-\mathrm{C}_{7}$ onsets for the two cases. Differences between onset conditions in the crude oil and at infinite dilution in toluene can exceed $0.02 R I$ units, equivalent to more that $0.5 \mathrm{MPa}^{1 / 2}$ in solubility parameter. Mars-Pink and Tensleep asphaltenes were more stable in their original oil than they were in toluene solutions, perhaps reflecting some stabilization by the resin fraction. However, in most cases the asphaltenes dispersed in toluene were more stable than they were in the original oil. 
Table I-3.4. Comparison of $n-\mathrm{C}_{7} P_{R I}$ values for crude oils and their $n$ - $\mathrm{C}_{7}$ asphaltenes in $1 \%$ toluene solutions

\begin{tabular}{|c|c|c|c|}
\hline \multirow{2}{*}{ Oil } & \multicolumn{2}{|c|}{$n-\mathrm{C}_{7} P_{R I}$} & \multirow{2}{*}{$\begin{array}{c}\text { difference in } \\
\delta\left(\mathrm{MPa}^{1 / 2}\right)\end{array}$} \\
\hline & oil & toluene soln. & \\
\hline A-93 & 1.4516 & 1.4303 & 0.576 \\
\hline A-95 & 1.4546 & 1.4359 & 0.504 \\
\hline Lagrave & 1.4602 & 1.4462 & 0.378 \\
\hline Mars-Pink & 1.4274 & 1.4330 & -0.155 \\
\hline Moutray & 1.4372 & 1.4351 & 0.057 \\
\hline Tensleep & 1.4438 & 1.4530 & -0.248 \\
\hline
\end{tabular}

\section{EFFeCt OF PReCIPITANT Mixtures ON $P_{R I}$}

The linear empirical relationship between $P_{R I}$ and $v_{p}^{1 / 2}$ suggests that conditions of practical interest can be predicted from simple laboratory measurements without the necessity of using infinite dilution to characterize solubility parameters at the onset. As a first step toward generalizing these results, we examine the effect of mixtures of precipitants on $P_{R I}$. As shown in Fig. I-3.8, mixing $n$-alkanes of different chain lengths results in onset conditions that are intermediate between the two. A simple linear interpolation gives the onset $P_{R I}$ if the volume fractions of precipitants are known.

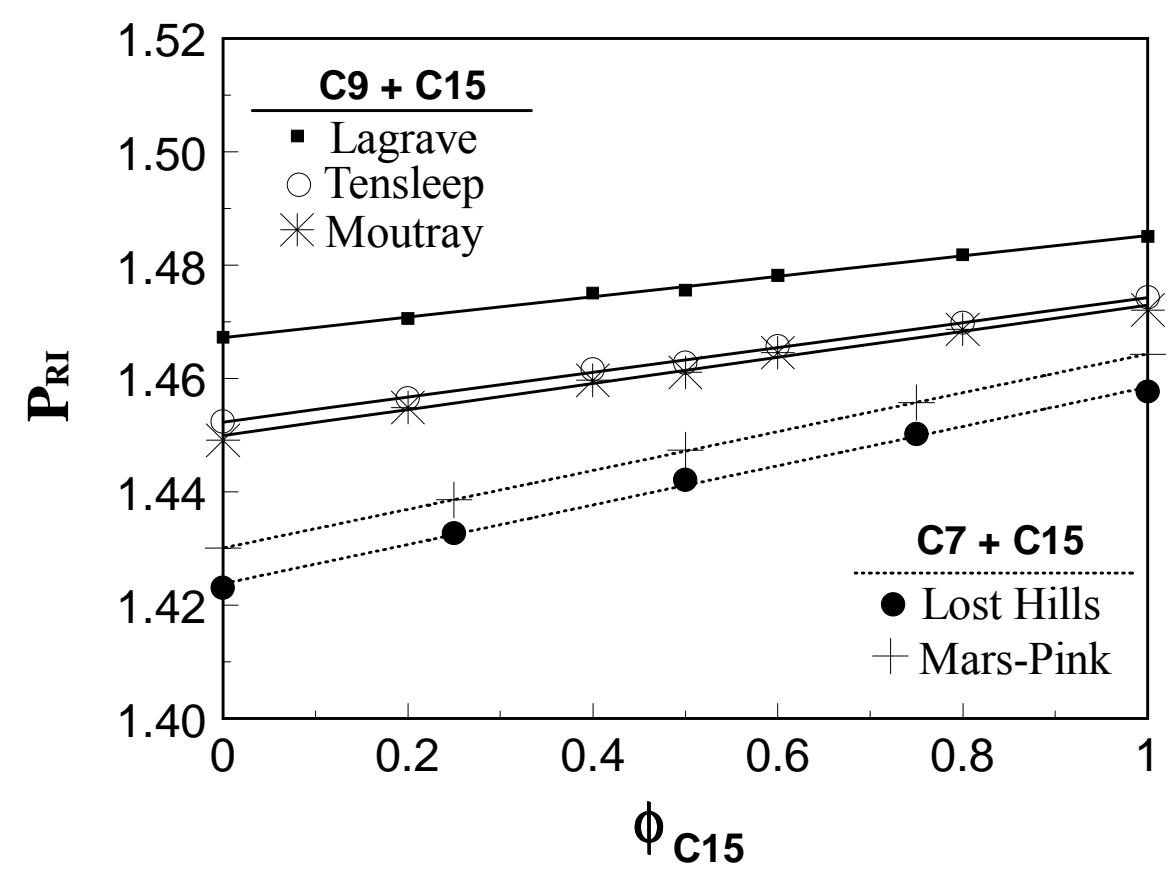

Figure I-3.8. $P_{R I}$ is a linear function of precipitant volume fractions for three crude oils with mixtures of $n$-C $\boldsymbol{C}_{9}$ and $n-\mathrm{C}_{15}$ as precipitant and for two crude oils with mixtures of $n-\mathrm{C}_{7}$ and $n-\mathrm{C}_{15}$ as precipitant. 


\section{EFFECT OF PRECIPITANT BRANCHING ON $P_{R I}$}

Precipitants that exist in crude oil are, of course, not exclusively linear alkanes. Many branched paraffinic compounds are included in the saturate fraction. A few such compounds have been tested (Table I-3.5). A comparison of the onset conditions for branched compounds with $n$-alkanes is shown in Fig. I-3.9 for Mar-Pink crude oil. In the $\mathrm{C}_{6}-\mathrm{C}_{8}$ range, asphaltenes appear to be slightly less stable to the branched compounds and somewhat more stable to precipitation induced by squalane, $\mathrm{C}_{30} \mathrm{H}_{62}$, although differences in the kinetics of flocculation that might influence these results have not yet been tested.

Table I-3.5. Onset of flocculation with branched paraffins

\begin{tabular}{|c|c|c|c|c|}
\hline \multirow{2}{*}{ Precipitant } & \multirow{2}{*}{$\begin{array}{c}v_{p} \\
(\mathrm{ml} / \mathrm{mol})\end{array}$} & \multicolumn{3}{|c|}{$P_{R I}$} \\
\cline { 3 - 5 } & 132.0 & 1.4248 & 1.4301 & \\
\hline iso-hexane & 132.0 & Mars-Pink & Tensleep \\
\hline $\begin{array}{c}\text { 2,2 dimethyl } \\
\text { butane }\end{array}$ & 132.8 & 1.4224 & 1.4348 & \\
\hline iso-octane & 165.1 & 1.4348 & 1.4430 & \\
\hline squalane & 522.0 & 1.4874 & 1.5018 & 1.5048 \\
\hline
\end{tabular}

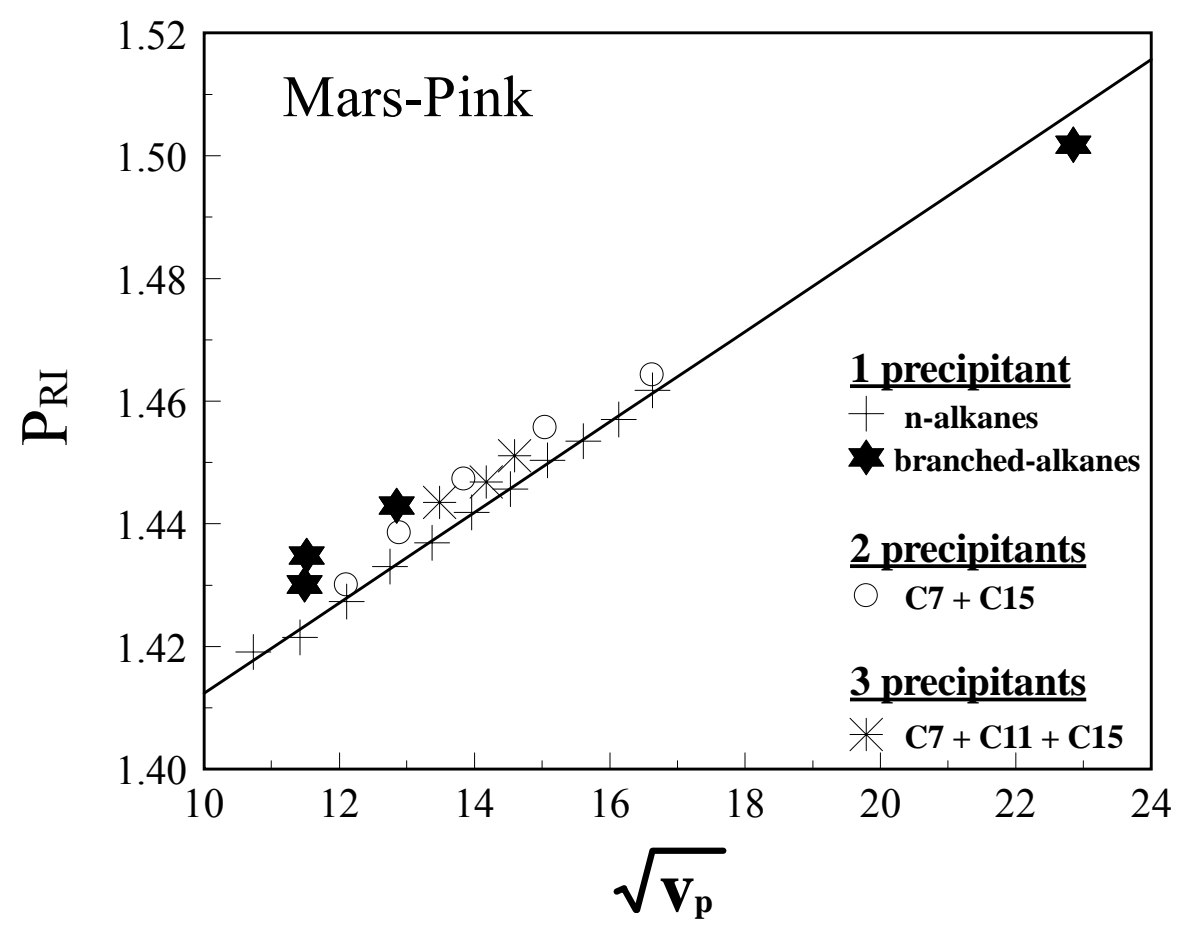

Figure I-3.9. Branched alkanes follow a similar trend of $P_{R I}$ vs. $v_{p}^{1 / 2}$ to that established with $n$-alkanes. Also shown are the results of precipitation tests with the mixtures of two and three precipitants shown in Fig. I-3.8. 


\section{EFFECTS OF PRESSURE}

The effects of changing pressure on the physical properties that influence the onset of asphaltene flocculation depend on whether the system is above or below the bubblepoint pressure $\left(P_{b}\right)$. Above $P_{b}$, decreasing pressure decreases density and $R I$ while increasing the volume fraction occupied by the lightest paraffinic compounds that can destabilize the asphaltenes. Below $P_{b}$, compositional changes also influence asphaltene stability. The heavier phase containing the asphaltenes increases in $R I$ as the light ends separate into the gas phase. Possible changes in asphaltene stability are illustrated schematically in Fig. I-3.10. Starting at the reservoir pressure, $P_{\text {res }}, R I$ decreases and $v_{p}{ }^{1 / 2}$ increases with decreasing pressure. The potential exists for the mixture to cross the $P_{R I}$ line, initiating asphaltene flocculation. As suggested in this diagram, separation of the light ends should shift the heavier phase back into the stable region as the pressure continues to decrease below $P_{b}$. Further loss of intermediate compounds will increase both $R I$ and $v_{p}$. Depending on the shape of the $R I$ vs. $v_{p}{ }^{1 / 2}$ curve, the heaviest, least soluble asphaltenes may be soluble in the stock-tank oil or the mixture may cross again into the insoluble region.

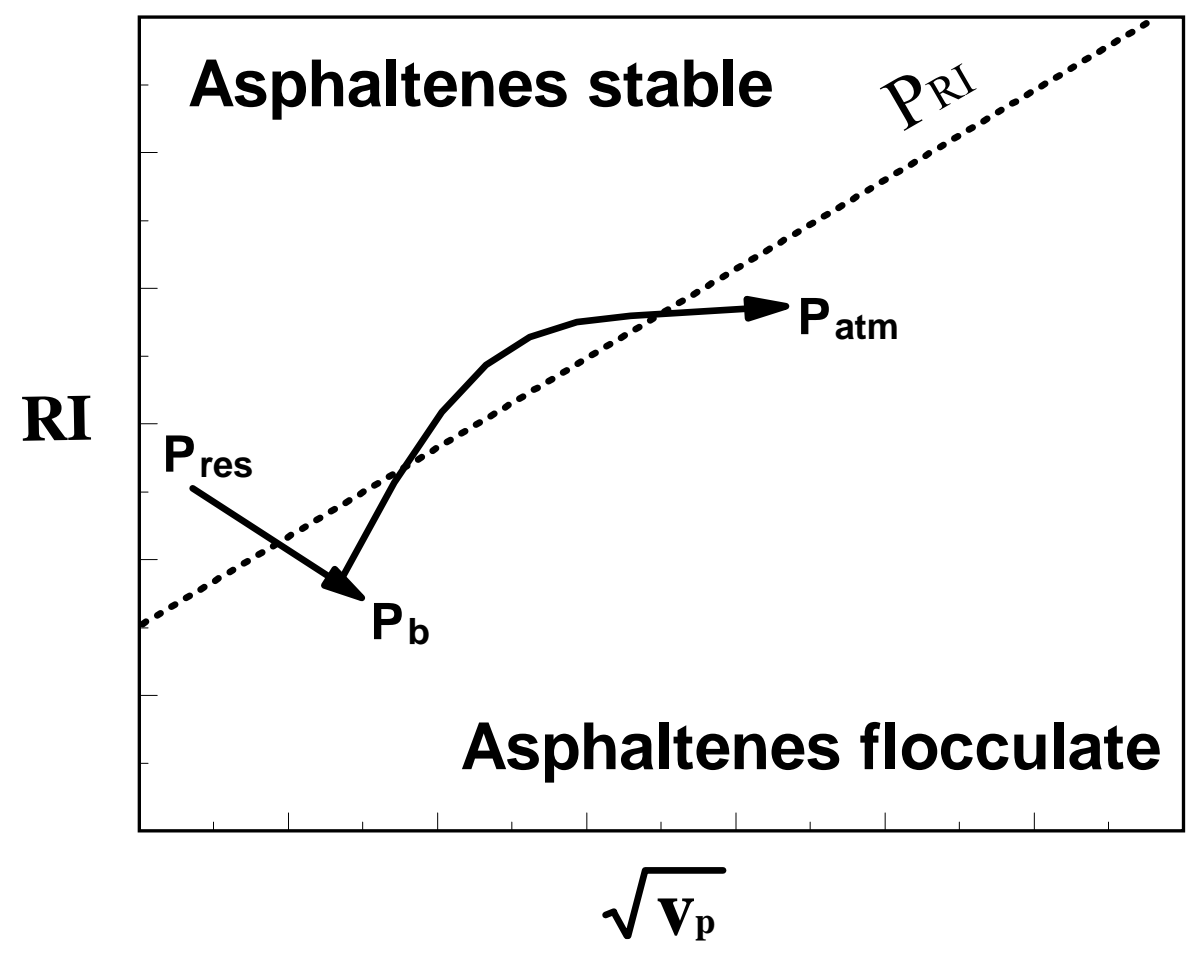

Figure I-3.10. Both $R I$ and $v_{p}$ change as $P$ decreases from the reservoir pressure $\left(P_{r e s}\right)$ to ambient conditions $\left(P_{a t m}\right)$. Below the bubblepoint $\left(P_{b}\right)$, oil composition is also changing. The arrows suggest the expected trends, although the details of the path through the $R I-v_{p}^{1 / 2}$ diagram will be different for each oil.

\section{EFFECTS OF TEMPERATURE}

Changing temperature not only changes solution properties (e.g., $R I, v_{p}$, and $\phi_{p}$ ), asphaltene aggregation is affected as well (e.g., Espinat and Ravey, 1993). Changes in $P_{R I}$ on the order of $-0.0008 R I$ units per ${ }^{\circ} \mathrm{C}$ have been estimated for A-93 crude oil with precipitants from $n$ - 
$\mathrm{C}_{5}$ to $n-\mathrm{C}_{7}$ over the temperature range from about 30 to $60^{\circ} \mathrm{C}$ (Wang et al., 2000), but direct measurements have yet to be made.

\section{PREDiction of Flocculation OnSET at Reservoir Conditions}

Predicting asphaltene stability at reservoir conditions from measurements of the onset of asphaltene flocculation at ambient conditions requires several steps:

- evaluation of oil properties as a function of $T$ and $P$,

- temperature correction for $P_{R I}$, and

- calculation of an appropriate value of $v_{p}$.

Oil properties can be calculated directly from PVT data (Buckley et al., 1998) using equation A-1:

$$
\left(\frac{n^{2}-1}{n^{2}+2}\right)_{\text {live-oil }}=\frac{1}{B_{o}}\left(\frac{n^{2}-1}{n^{2}+2}\right)_{\text {STO }}^{o}+K \frac{R_{s}}{B_{o}} \sum_{i=1}^{m} x_{i} R_{i}
$$

where $K=7.52 \times 10^{-6}$, converts from scf $/ \mathrm{bbl}$ to $\mathrm{mol} / \mathrm{ml}$, STO $=$ stock tank oil, superscript ${ }^{\circ}$ indicates standard conditions $\left(1 \mathrm{~atm}, 60^{\circ} \mathrm{F}\right)$, and other symbols are as defined in the Nomenclature.

\section{CALCULATION OF $V_{P}$}

The thermal expansion per STB of stock tank oil from room temperature to reservoir temperature is $\left(B_{o}(1)-1\right)$, where $B_{o}(1)$ is $B_{o}$ at $P=P_{o}=1 \mathrm{~atm}$. We assume this volume change is independent of pressure.

The volume change due to compression can also be estimated from PVT data, as shown in the following two steps.

1. For most $n$-alkanes and aromatics, calculated density from STRAPP (1990) shows a linear increase with pressure, with density compressibility, $d \rho / d P$, about $4 \times 10^{-6} \mathrm{~g} / \mathrm{ml} / \mathrm{psi}$. If we assume that crude oil has the similar behavior, the density at pressure $P$ equals to

$$
\rho=\rho_{o}+\left(P-P_{o}\right) \times d \rho / d P
$$

where $\rho_{o}$ is density at pressure $P_{o}$. For $1 \mathrm{STB}$ of stock tank oil, the volume at reservoir pressure, without considering the thermal expansion, is

$$
V=\rho_{o} / \rho
$$


Now, assume that the volume of dissolved gas at reservoir temperature and pressure is $V_{\text {gas }}$; the total volume for 1 STB stock tank oil at reservoir condition (including gas) is given by:

$$
B_{o}(P)=\rho_{o} / \rho+\left(B_{o}(1)-1\right)+V_{\text {gas }}
$$

which can be rearranged to give:

$$
V_{g a s}=B_{o}(P)-\frac{\rho_{o}}{\rho_{o}+\left(P-P_{o}\right) d \rho / d P}-\left(B_{o}(1)-1\right)
$$

2. Since one barrel (STB or RB) is equal to $1.5890 \times 10^{5} \mathrm{ml}$, and one scf is equal to 1.1957 mole of gas, the approximate molar volume of the dissolved gas at reservoir condition is

$$
v(\mathrm{ml} / \mathrm{mol})=\frac{V_{\text {gas }} \times 1.5890 \times 10^{5}}{R_{s} \times 1.1957}
$$

Above the bubblepoint, dissolved gas is assumed to be the precipitant. Hence, the value of $v_{p}$ is that given by Eq. I-3.11.

To correct for shifts in $P_{R I}$ with changing temperature, we assume that the entire $P_{R I}$ vs. $v_{p}{ }^{1 / 2}$ curve is shifted by $-0.0008 R I$ units per ${ }^{\circ} \mathrm{C}$. Finally, a value of $v_{p}$ is needed. If we assume that the dissolved gas species are the main precipitating agents above the bubblepoint and that other paraffinic oil components can be neglected, $v_{p}$ can be calculated from PVT data.

To illustrate the predictions produced by this method, we use some example PVT data for a $41.5^{\circ} \mathrm{API}$ oil (McCain, 1990). The sample data for a $41.5^{\circ} \mathrm{API}$ oil that were used to calculate $R I$ are shown in Fig. I-3.11. Calculation of $R_{i}$, the molar refraction of dissolved gas phase, is shown in Table I-3.6. The solution gas is assumed to be a mixture of methane, ethane, and propane.

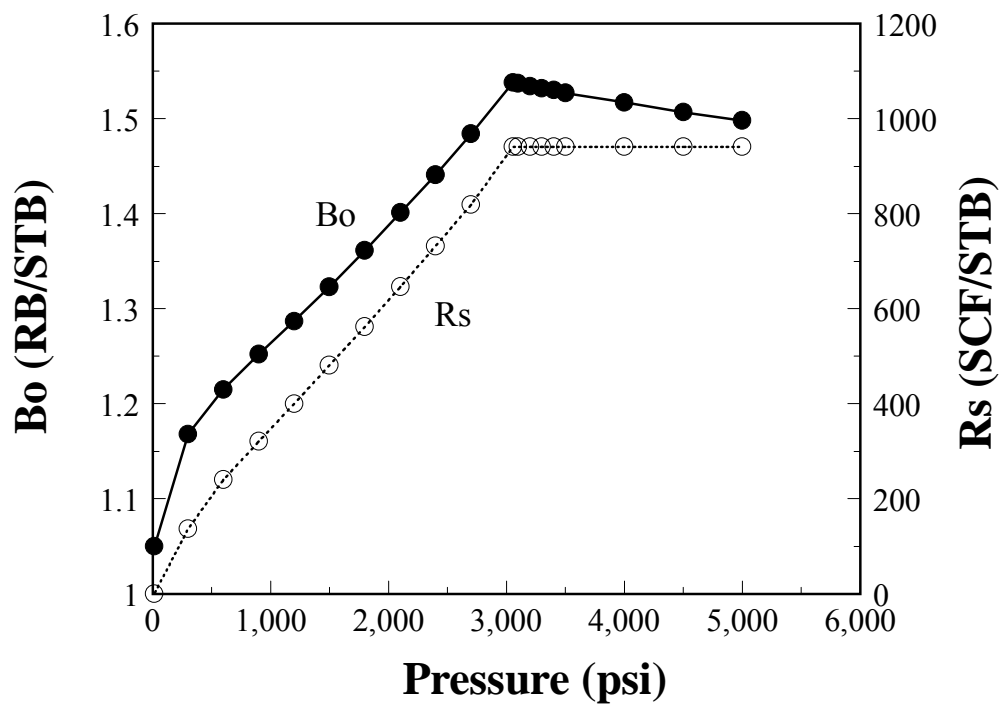

Figure I-3.11. PVT data for calculation of $R I$ in Fig. I-3.12 (after McCain, 1990). 
Table I-3.6. Calculation of gas molar refraction.

\begin{tabular}{|c|c|c|c|}
\hline Component & $\begin{array}{c}R_{i} \\
(\mathrm{ml} / \mathrm{mol})\end{array}$ & $\begin{array}{c}x_{i} \\
(\mathrm{~mol} . \mathrm{Frac} .)\end{array}$ & $\begin{array}{c}x_{i} R_{i} \\
(\mathrm{ml} / \mathrm{mol})\end{array}$ \\
\hline $\mathrm{C} 1$ & 6.70 & 0.5 & 3.35 \\
\hline $\mathrm{C} 2$ & 11.35 & 0.4 & 4.54 \\
\hline $\mathrm{C} 3$ & 16.00 & 0.1 & 1.60 \\
\hline Total & & & 9.49 \\
\hline
\end{tabular}

The $P_{R I}$ curve measured for A-93, adjusted to a reservoir temperature of $184^{\circ} \mathrm{F}\left(84^{\circ} \mathrm{C}\right)$ is used in this illustration. As shown in Fig. I-3.12, asphaltene flocculation is predicted to occur near the bubblepoint, where the oil $R I$ falls below the calculated $P_{R I}$ curve. Below the bubblepoint pressure, estimation of $v_{p}$ would require additional compositional information about both the solution gas and precipitants in the oil. Thus we do not extend this prediction to the pressure where asphaltenes would be redispersed.

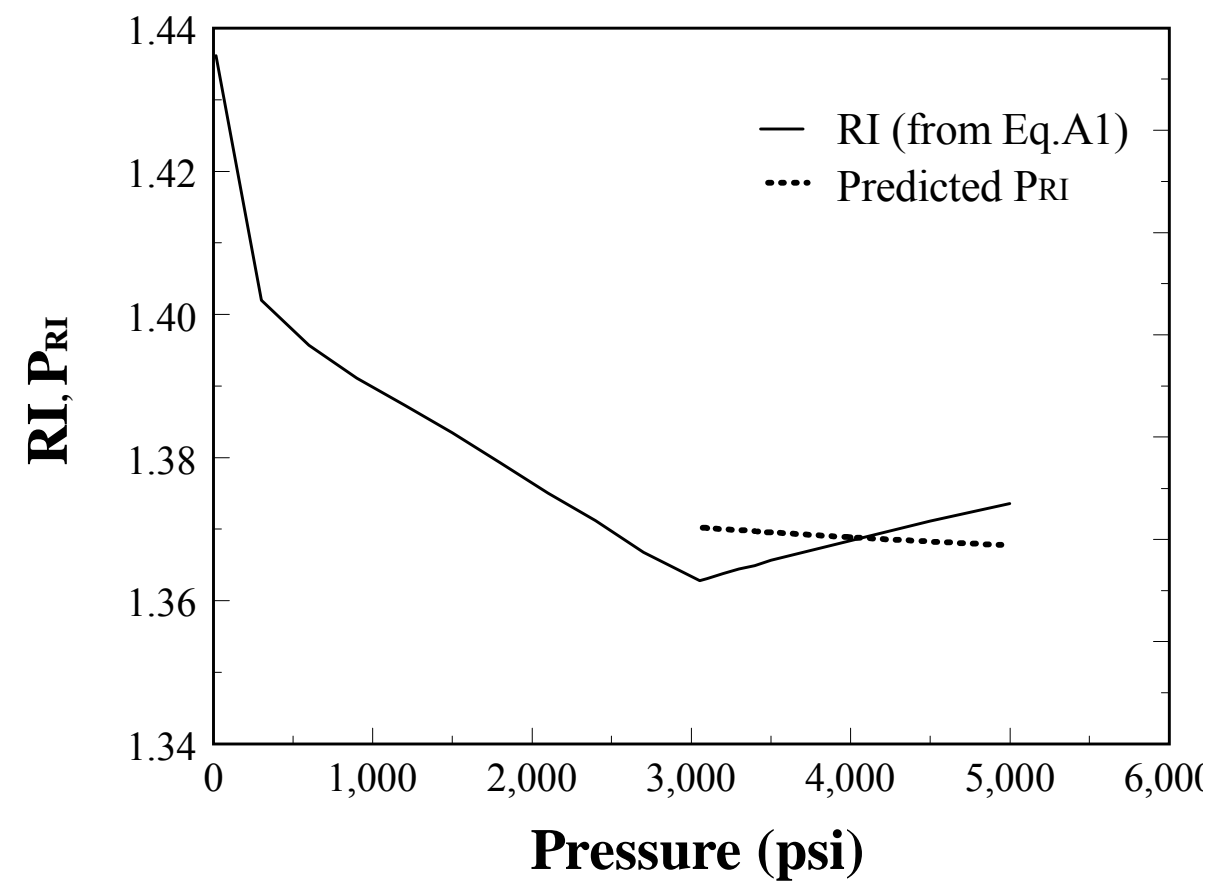

Figure I-3.12. Example of $P_{R I}$ prediction. PVT data are for a $41.5^{\circ} \mathrm{API}$ oil at a reservoir temperature of $84^{\circ} \mathrm{C}$. The $P_{R I}$ vs $v_{p}^{1 / 2}$ relationship used is that for A-93+ $\alpha$-MN (Fig. I-3.5) with a temperature adjustment of $-0.0008 R I$ units $/{ }^{\circ} \mathrm{C}$. Solution gas is estimated to be composed of methane, ethane, and propane with mole fractions of $0.5,0.4$, and 0.1 , respectively.

\section{VALIDATION OF PREDICTIONS}

Before this method can be applied to solve asphaltene problems at reservoir conditions, some additional information and validation are needed. To validate the predictions, comparisons between live-oil tests and ambient onset measurements are needed for the same fluids. The assumption that $P_{R I}$ is independent of pressure and the dependence of $P_{R I}$ on temperature both need to be tested. Predictions below the bubblepoint will require additional compositional 
information. The assumption that dissolved gas dominates $v_{p}$ will not be valid as larger components in the saturate fraction become important precipitants and thus predictions below the bubblepoint are beyond the scope of this simple empirical approach. A theoretical model that will permit more complicated predictions has been developed and tested with the experimental data presented here. ${ }^{10,18}$

\section{Conclusions}

- A systematic set of visual observations of the onset of asphaltene flocculation has shown that there is a very simple relationship between onset mixture properties and the size of the paraffinic precipitant.

- Onset mixture solubility properties can be quantified by mixture refractive index, which is linearly related to square root of the precipitant molar volume.

- The same relationship has been found to apply to different crude oils although the $R I$ at which asphaltenes become unstable varies from one crude oil to another. Thus, precipitant molar volume can be used to predict the onset of flocculation for a wide range of precipitating conditions from even a single heptane titration experiment.

- $\quad$ Trends established for $n$-alkane precipitants are predictive of flocculation caused by mixtures of $n$-alkanes and by branched alkane precipitants.

- Onset solubility properties determined at infinite dilution of asphaltene are not the same as those in the original crude oil.

- Reasonable predictions can be made of flocculation at reservoir conditions although the validity of these predictions remains to be tested.

\section{Nomenclature}

$$
\begin{aligned}
B_{o}= & \text { formation volume factor, } \mathrm{RB} / \mathrm{STB} \\
n= & \text { refractive index }(R I), \text { dimensionless } \\
n_{D}{ }^{20}= & R I \text { at sodium-D line at } 20^{\circ} \mathrm{C} \\
P= & \text { pressure, psia } \\
P_{R I}= & R I \text { of the mixture of oil/solvent } / \text { precipitant } \\
& \text { at onset of asphaltene flocculation } \\
r= & \text { correlation coefficient, dimensionless } \\
R= & \text { Universal gas constant, } 8.31441 \mathrm{~J} / \mathrm{K} / \mathrm{mol} \\
R I= & \text { refractive index, dimensionless } \\
R_{i}= & \text { molar refraction of component } i, \mathrm{ml} / \mathrm{mol} \\
R_{S}= & \text { gas/oil ratio, scf } / \mathrm{STB} \\
T= & \text { absolute temperature, } \mathrm{K} \\
v= & \text { molar volume, ml } / \mathrm{mol} \\
V= & \text { volume, ml } \\
x= & \text { mole fraction, dimensionless }
\end{aligned}
$$




$$
\begin{aligned}
\alpha-M N= & \alpha \text {-methylnaphthalene } \\
\delta= & \text { solubility parameter, } \mathrm{MPa}^{1 / 2} \\
\chi= & \text { Flory-Huggins interaction parameter, } \\
& \text { dimensionless } \\
\phi= & \text { volume fraction, dimensionless } \\
\rho= & \text { density, } \mathrm{g} / \mathrm{ml}
\end{aligned}
$$

Subscripts

$$
\begin{aligned}
a & =\text { asphaltene } \\
\text { atm } & =\text { atmospheric } \\
b & =\text { bubblepoint } \\
i & =\text { ith component } \\
m & =\text { the rest of the mixture rather than asphaltenes } \\
p & =\text { precipitant } \\
r e s & =\text { reservoir }
\end{aligned}
$$

\section{References}

ASTM D2007-80: "Standard Test Method for Characteristic Groups in Rubber Extender and Processing Oils by the Clay-Gel Adsorption Chromatographic Method," ASTM (1980).

Buckley, J.S.: "Predicting the Onset of Asphaltene Precipitation from Refractive Index Measurements," Energy \& Fuels (1999) 13, No. 2, 328-332.

Buckley, J.S., Hirasaki, G.J., Liu, Y., Von Drasek, S., Wang, J.X., and Gill, B.S.: "Asphaltene Precipitation and Solvent Properties of Crude Oils," Petroleum Science and Technology (1998) 16, No. 3\&4, 251-285.

Cimino, R., Correra, S., Del Bianco, A., and Lockhart, T.P.: "Solubility and Phase Behavior of Asphaltenes in Hydrocarbon Media," Asphaltenes: Fundamentals and Applications, E.Y. Sheu and O.C. Mullins (eds.), NY: Plenum Press (1995) 97-130.

de Boer, R.B., Leerlooyer, K., Eigner, M.R.P., and van Bergen, A.R.D.: "Screening of Crude Oils for Asphalt Precipitation: Theory, Practice, and the Selection of Inhibitors," SPE PF (Feb. 1995) 55-61.

Espinat, D. and Ravey, J.C.: "Colloidal Structure of Asphaltene Solutions and Heavy-Oil Fractions Studied by Small-Angle Neutron and X-Ray Scattering," paper SPE 25187 presented at the 1993 SPE International Symposium on Oilfield Chemistry, New Orleans, March 2-5.

Flory, P.J.: “Thermodynamics of High Polymer Solutions,” J. Chem. Phys., 10, 51, 1942.

Heithaus, J.J.: "Measurement and Significance of Asphaltene Peptization," J. Inst. of Petroleum (Feb. 1962) 48 No. 458, 45.

Hildebrand, J.H. and Scott, R.L.: Solubility of Non-Electrolytes, 3rd ed., Reinhold, New York (1950).

Hirschberg, A., deJong, L.N.J., Schipper, B.A., and Meijer, J.G.: "Influence of Temperature and Pressure on Asphaltene Flocculation," SPEJ (June 1984) 283-293. 
Hotier, G. and Robin, M.: "Effects of different diluents on heavy oil products: measurement, interpretation, and a forecast of asphaltene flocculation," Revue de l'IFP (1983) 38, 101.

Huggins, M.L.: "Solutions of Long Chain Compounds," J. Chem. Phys., 9, 440, 1941.

McCain, W.D., Jr.: The Properties of Petroleum Fluids, $2^{\text {nd }}$ ed., PennWell Books, Tulsa, 1990, pg 243.

Mertens, E.W.: "Predicting Weatherability of Coating Grade Asphalts from Asphaltene Characteristics," ASTM Bulletin (Dec. 1960) 40-44 (TP 218-222).

STRAPP, software version 1.0, distributed by NIST, Gaithersberg MD 20899 (1990).

Wang, J.X.: "Predicting Asphaltene Flocculation in Crude Oils," PhD Thesis, NMIMT, Socorro, April, 2000.

Wang, J.X. and Buckley, J.S.: "Improved Modeling of the Onset of Asphaltene Flocculation," $2^{\text {nd }}$ International Conf. on Petrol. and Gas Phase Behavior and Fouling, Copenhagen, 27-31 August, 2000.

Wang, J.X., Brower, K.R., and Buckley, J.S.: "Advances in Observation of Asphaltene Destabilization," paper SPE 50745 presented at the 1999 International Oilfield Chemistry Symp. Houston, 16-19 Feb. (SPEJ,2000, in press)

Waxman, M.H., Deeds, C.T., and Closmann, P.J.: "Thermal Alterations of Asphaltenes in Peace River Tars," paper SPE 9510 presented at the 1980 SPE Annual Fall Technical Conference and Exhibition, Dallas, Sept. 21-24. 


\section{I-3.3. Thermodynamic Modeling of the Onset of Asphaltene Flocculation}

Wang, J.X. and Buckley, J.S.: "A Two-Component Solubility Model of the Onset of Asphaltene Flocculation in Crude Oils,” Energy \& Fuels (2001) 15, 1004-1012.

Wettability altering tendencies of crude oils and especially of their asphaltenes can vary with asphaltene stability. Predicting instability over a wide range of conditions from a few laboratory measurements requires physically-based models.

Thermodynamic models based on Flory-Huggins adaptation of regular solution theory are widely used to predict the onset of asphaltene flocculation. In current applications, solubility parameters of asphaltene and of the remainder of the oil are either treated as fitting parameters or inferred from experiments with asphaltenes in known solvents. Mathematical solutions are simplified by assuming that either the flocculating asphaltene (Hirschberg et al., 1984) or the remaining oil (Cimino et al., 1995) is a pure phase. While these assumptions simplify the calculations, neither is consistent with experimental evidence.

In this report we present a model rigorously derived from Flory-Huggins polymer theory, without any arbitrary assumptions about the compositions of the two phases formed when the asphaltenes separate from the oil. Solubility parameters are obtained-via a simple correlation-from measured values of refractive index of the original oil and of oil-alkane mixtures at the onset of asphaltene flocculation. The full Flory-Huggins model has been tested with an extensive series of experimental measurements of asphaltene flocculation from different crude oils mixed with alkanes ranging in molecular size from propane to pentadecane. Comparisons to the simplified solutions demonstrate the improved predictions of onset conditions available using the full solution.

\section{Background}

Problems associated with asphaltene flocculation are being encountered more and more frequently as oil production moves towards more challenging zones and as improved oil recovery methods are increasingly used. It is widely recognized that flocculation and deposition of asphaltenes can cause plugging in a reservoir formation, fouling of downhole production equipment and refinery facilities, coating of pipes, and deposition in storage facilities. In addition, asphaltenes are implicated in contributing to wetting alteration in the reservoir by directly depositing on mineral surfaces, stabilizing of water-in-oil emulsions by enhancing the rigid films at water/oil interfaces, and poisoning catalysts during refinery processes.

Cleanup of deposited asphaltenes in the field may necessitate well shut-in and loss of oil production. Large amounts of organic solvents may be required, and in some cases solvent treatments may not be effective. Therefore, preventing asphaltene flocculation is preferable to cleanup, from both operational and economic viewpoints.

Asphaltene flocculation can be induced by changes in temperature, pressure and composition that reduce the stability of asphaltenes in crude oils. Although experimental 
determination of the full set of thermodynamic conditions at which asphaltenes begin to flocculate might be desirable for each specific crude oil, the cost of such detailed characterization is prohibitive. An alternative is to obtain a minimum amount of laboratory data and incorporate these measurements into a thermodynamic model to predict the onset conditions.

At present, most modeling in the literature employs many adjustable parameters that cannot be determined experimentally. Improvements in physical description and ultimately in the ability to model and predict the onset of asphaltene flocculation are imperative. From a practical point of view, what we need is a simple model that enables prediction of phase behavior of asphaltenes in crude oil to the required accuracy with only a minimum of input data that are easily obtained from experiments.

There are two fundamentally distinct physical models, lyophilic and lyophobic, that lead to two broad categories of modeling approaches for asphaltene phase behavior in crude oil. The first group assumes that asphaltene in oil is solvated (lyophilic) and that flocculation occurs if the maximum solubility is lower than asphaltene concentration in solution. The solubility is assessed by minimizing the Gibbs free energy change upon mixing which can be calculated from a solution theory such as the Flory-Huggins polymer theory. The first thermodynamic model proposed to describe asphaltene phase behavior in solution, published by Hirschberg et al. in 1984, was such a lyophilic model. Asphaltene solution is treated as a two-component system, with asphaltene as one component and everything else, including resins, as the other component. Since then numerous applications and adaptations of varying degrees of complexity have been reported (e.g. Burke et al., 1990; Novosad and Costain, 1990; Nor-Azian and Adewumi, 1993; MacMillan et al., 1995; Cimino et al., 1995; Dahir et al., 1996; Yang et al., 1999).

The second group adopts the long-standing assumption that asphaltenes are peptized by resin molecules and it is this resin-asphaltene structure that is suspended in solution. This lyophobic hypothesis led to a thermodynamic-colloidal model proposed by Leontaritis and Mansoori $(1987,1989)$. In this model, resin is critical in providing steric stabilization for asphaltenes. Flocculation occurs if the chemical potential of resin in solution is lower than some critical value. The usefulness of this model is limited because the critical chemical potential for resin cannot be measured and because the underlying physical assumptions of this model have never been verified.

There are other approaches to modeling asphaltene stability. Nghiem et al. (1993, 1997, 1998) divide the $\mathrm{C}_{31}{ }^{+}$heavy ends of crude oil into nonprecipitating and precipitating pseudocomponents. An equation-of-state is applied to calculate the phase behavior of the whole system. It is assumed that the nonprecipitating and the precipitating pseudo-components have different interaction coefficients with the light compounds. This model can be used to interpolate between observations of phase behavior if sufficient data exists, but it depends on interaction coefficients that cannot be independently verified.

In 1998, Pan and Firoozabadi proposed a micellization model to calculate asphaltene aggregation in oil. Asphaltene molecules are assumed to self-associate to form a liquid-like micellar core with resin molecules adsorbed onto the micellar surface to form a solvated shell. The Gibbs free energy change for micellization is calculated based on mean-field theory and by 
assigning some specific interaction energy to asphaltene-asphaltene, and asphaltene-resin molecule pairs. The phase equilibrium is calculated by minimizing the Gibbs free energy, which involves 14 variables including the postulated micelle structure parameters.

In this work, we focus on the lyophilic view. It is the simplest explanation of asphaltene phase behavior and is based on parameters whose physical significance is clear even when their values cannot be measured directly. Existing thermodynamic models based on Flory-Huggins polymer theory will be summarized. The asphaltene solution is treated as composed of two pseudo-components: asphaltene and the remainder of the solution, where the remainder includes the deasphalted crude oil (maltenes) plus added precipitant and/or solvent. Two different approaches from the literature that use different phase separation criteria will be evaluated and a new approach will be proposed.

\section{Flory-Huggins polymer theory}

Considering a binary mixture of solvent (1) with solute (2), the change of Gibbs free energy upon mixing at constant pressure is equal to

$$
\Delta \mathrm{G}_{\text {mix }}=\Delta \mathrm{H}_{\text {mix }}-\mathrm{T} \Delta \mathrm{S}_{\text {mix }}
$$

where $\Delta \mathrm{H}_{\text {mix }}$ is the enthalpy change, $\Delta \mathrm{S}_{\text {mix }}$ is the combinatorial entropy change, and $\mathrm{T}$ is absolute temperature. For mixing to occur, it is required that

$$
\Delta \mathrm{G}_{\text {mix }}<0
$$

The combinatorial entropy change of ideal mixing for small, equal-sized molecules is a function of compositions (Barton, 1991, Chapter 2; Tompa, 1956)

$$
\Delta \mathrm{S}_{\text {mix }}=-\mathrm{R}\left(\mathrm{n}_{1} \ln \mathrm{x}_{1}+\mathrm{n}_{2} \ln \mathrm{x}_{2}\right)
$$

where $\mathrm{R}$ is universal gas constant, $\mathrm{n}$ and $\mathrm{x}$ are number of moles and molar fraction, respectively, and subscripts 1 and 2 represent solvent and solute, respectively. For a binary system, we have

$$
\mathrm{x}_{1}=\frac{\mathrm{n}_{1}}{\mathrm{n}_{1}+\mathrm{n}_{2}}
$$




$$
\mathrm{x}_{2}=\frac{\mathrm{n}_{2}}{\mathrm{n}_{1}+\mathrm{n}_{2}}
$$

and

$$
\mathrm{x}_{1}+\mathrm{x}_{2}=1
$$

Note that molar fraction $\mathrm{x}<1$, thus $\ln \mathrm{x}<0$, and $\Delta \mathrm{S}_{\text {mix }}$ is positive. This indicates that combinatorial entropy always increases in the mixture and thus favors mixing.

For mixing of compounds with large differences in molecular sizes, however, equation (I3.14) overestimates the combinatorial entropy change since the probability of intermolecular combinations under such conditions is dramatically reduced. Flory (1942) and Huggins (1941) derived the entropy change for large, linear polymer molecules dissolving in a solvent composed of small molecules with the following assumptions:

- Polymer molecules are mono-dispersed in solution;

- Each solvent molecule or segment of polymer molecule occupies a lattice site in a solution lattice network where all the sites have identical volume;

- The successive segments of a polymer molecule must occupy adjacent sites.

Details of the derivation can be found in Tompa (1956). The combinatorial entropy change under such assumptions is given by

$$
\Delta \mathrm{S}_{\text {mix }}=-\mathrm{R}\left(\mathrm{n}_{1} \ln \phi_{1}+\mathrm{n}_{2} \ln \phi_{2}\right)
$$

where $\phi_{1}, \phi_{2}$ are volume fraction of solvent and solute, respectively. For ideal mixing,

$$
\begin{gathered}
\phi_{1}=\frac{\mathrm{n}_{1} \mathrm{v}_{1}}{\mathrm{n}_{1} \mathrm{v}_{1}+\mathrm{n}_{2} \mathrm{v}_{2}} \\
\phi_{2}=\frac{\mathrm{n}_{2} \mathrm{v}_{2}}{\mathrm{n}_{1} \mathrm{v}_{1}+\mathrm{n}_{2} \mathrm{v}_{2}}
\end{gathered}
$$


with

$$
\phi_{1}+\phi_{2}=1
$$

$\mathrm{v}_{1}$ and $\mathrm{v}_{2}$ are molar volumes for solvent and solute, respectively. For equal-sized molecules, $\mathrm{v}_{1}=$ $\mathrm{v}_{2}$ thus $\phi=\mathrm{x}$, and equation (I-3.18) reduces to equation (I-3.14).

It has been shown that equation (I-3.18) does not always provide satisfactory results for large non-polymer molecules, such as long-chain surfactant and plasticizer molecules (Shinoda and Hildebrand, 1957). Another limitation of Flory-Huggins theory is its inadequacy in accounting for the effect of varying temperature. When temperature increases, the free volume of each lattice site occupied by solvent molecule increases markedly, while the volume of a lattice site occupied by each polymer segment may change only slightly due to the restriction of adjacent segments on the same polymer chain (Hunter, 1991). This temperature effect would reduce the entropy between solvent molecules and polymer molecules especially near the critical point.

The excess enthalpy change in the mixture for ideal mixing of a regular solution is governed by the van Laar-Hildebrand-Scatchard equation (Barton, 1991, Section 4.2)

$$
\Delta \mathrm{H}_{\text {mix }}=\left(\mathrm{n}_{1} \mathrm{v}_{1}+\mathrm{n}_{2} \mathrm{v}_{2}\right)\left(\delta_{1}-\delta_{2}\right)^{2} \phi_{1} \phi_{2}
$$

where $\delta_{1}$ and $\delta_{2}$ are solubility parameters for solvent and solute, respectively. From (I-3.19) and (I-3.20), equation (I-3.22) can be rewritten as

$$
\Delta \mathrm{H}_{\text {mix }}=\mathrm{n}_{1} \mathrm{v}_{1} \phi_{2}\left(\delta_{2}-\delta_{1}\right)^{2}
$$

or

$$
\Delta \mathrm{H}_{\text {mix }}=\mathrm{n}_{2} \mathrm{v}_{2} \phi_{1}\left(\delta_{2}-\delta_{1}\right)^{2}
$$

Considering that for a dilute solution, molar fraction of solute, $\mathrm{x}_{2}$, is very small, and the molar volume for solute, $\mathrm{v}_{2}$, is usually not conveniently obtainable, especially for large, complex compounds such as polymers or asphaltenes, the expression in (I-3.23) is more practical. Using RT as the energy unit to rewrite equation (I-3.23), we get the common form of $\Delta \mathrm{H}_{\text {mix }}$ as

$$
\Delta \mathrm{H}_{\text {mix }}=\mathrm{RT} \chi \mathrm{n}_{1} \phi_{2}
$$


where $\chi$ is the Flory-Huggins interaction parameter between solvent and solute, defined as

$$
\chi=\frac{\mathrm{v}_{1}}{\mathrm{RT}}\left(\delta_{2}-\delta_{1}\right)^{2}
$$

It should be emphasized that by this definition $\chi$ corresponds to the enthalpy expression in equation (I-3.25). Another definition of $\chi$ is also available in the literature, with $\mathrm{v}_{1}$ displaced by $\mathrm{v}_{2}$.

Applying (I-3.18) and (I-3.25) to (I-3.11) results in the well-known Flory-Huggins equation for polymer solutions

$$
\Delta \mathrm{G}_{\text {mix }}=\mathrm{RT}\left(\mathrm{n}_{1} \ln \phi_{1}+\mathrm{n}_{2} \ln \phi_{2}+\mathrm{n}_{1} \phi_{2} \chi\right)
$$

The first two terms in equation (I-3.27) represent the Gibbs free energy change resulting from the positive combinatorial entropy of mixing, which always make a negative contribution to $\Delta \mathrm{G}_{\mathrm{mix}}$, promoting the mixing process. The third term arises from the enthalpy change upon mixing, which is always positive. The overall Gibbs free energy change for mixing is determined by these two opposite contributions. To satisfy $\Delta \mathrm{G}_{\mathrm{mix}}<0$, the entropic term must be higher than the enthalpic term.

Although $\chi$ was originally introduced as a purely enthalpic term, results in the literature based on laboratory measurements showed that $\chi$ is actually the sum of an entropic part, $\chi_{\mathrm{S}}$, and an enthalpic part, $\chi_{\mathrm{H}}$,

$$
\chi=\chi_{\mathrm{S}}+\chi_{\mathrm{H}}
$$

where $\chi_{\mathrm{H}}$ is described by equation (I-3.26). The entropic part $\chi_{\mathrm{S}}$ never goes to zero; instead, $\chi_{\mathrm{S}}$ is found to be almost constant with a value of 0.34 for most nonpolar or slightly polar molecular interactions (Barton, 1991, section 13.5). The Gibbs free energy based on this consideration should therefore be modified

$$
\Delta \mathrm{G}_{\text {mix }}=\mathrm{RT}\left[\mathrm{n}_{1} \ln \phi_{1}+\mathrm{n}_{2} \ln \phi_{2}+\mathrm{n}_{1} \phi_{2}\left(\chi_{\mathrm{H}}+\chi_{\mathrm{S}}\right)\right]
$$




\section{Chemical potential}

We will first adhere to the general form of Gibbs free energy change expressed in equation (16) without considering the split of $\chi$ into $\chi_{\mathrm{S}}$ and $\chi_{\mathrm{H}}$ to derive the chemical potentials. The chemical potentials for both solvent and solute in the mixture are the partial molar Gibbs free energy with respect to each number of moles at constant temperature, $\mathrm{T}$, and pressure, $\mathrm{P}$

$$
\begin{aligned}
& \Delta \mu_{1}=\mu_{1}-\mu_{1}^{0}=\left(\frac{\partial \Delta \mathrm{G}_{\text {mix }}}{\partial \mathrm{n}_{1}}\right)_{\mathrm{P}, \mathrm{T}, \mathrm{n}_{2}} \\
& \Delta \mu_{2}=\mu_{2}-\mu_{2}^{0}=\left(\frac{\partial \Delta \mathrm{G}_{\text {mix }}}{\partial \mathrm{n}_{2}}\right)_{\mathrm{P}, \mathrm{T}, \mathrm{n}_{1}}
\end{aligned}
$$

where $\mu$ and $\mu^{0}$ are chemical potentials in the mixture and at the reference state (pure phase), respectively. $\Delta \mu$ is therefore the chemical potential in the mixture relative to the reference state. Subscripts 1 and 2 represent solvent and solute. Differentiating equation (I-3.27) and taking into account (I-3.19), (I-3.20) and (I-3.26) results in

$$
\begin{aligned}
& \Delta \mu_{1}=\mathrm{RT}\left[\ln \phi_{1}+\phi_{2}\left(1-\frac{\mathrm{v}_{1}}{\mathrm{v}_{2}}\right)+\phi_{2}^{2} \frac{\mathrm{v}_{1}}{\mathrm{RT}}\left(\delta_{2}-\delta_{1}\right)^{2}\right] \\
& \Delta \mu_{2}=\mathrm{RT}\left[\ln \phi_{2}+\phi_{1}\left(1-\frac{\mathrm{v}_{2}}{\mathrm{v}_{1}}\right)+\phi_{1}^{2} \frac{\mathrm{v}_{2}}{\mathrm{RT}}\left(\delta_{2}-\delta_{1}\right)^{2}\right]
\end{aligned}
$$

To demonstrate how Gibbs free energy and chemical potentials vary with compositions in the mixture, we now consider one mole of a binary system of a solute A mixing with a mixture of solvents. The solute A has a solubility parameter very similar to asphaltene $\left(23 \mathrm{MPa}^{1 / 2}\right)$, but with much lower molar volume $(160 \mathrm{ml} / \mathrm{mol})$. The solvent is composed of $80 \mathrm{vol} \%$ toluene and 20 vol \% n-heptane. Now let the volume ratio of toluene to n-heptane be held constant and vary the molar fraction of solute, $\mathrm{x}_{2}$, in the mixture, the corresponding Gibbs free energy, $\Delta \mathrm{G}_{\mathrm{mix}}$, as a function of $\mathrm{x}_{2}$ can be calculated from equation (16), as shown in Fig. I-3.13. 


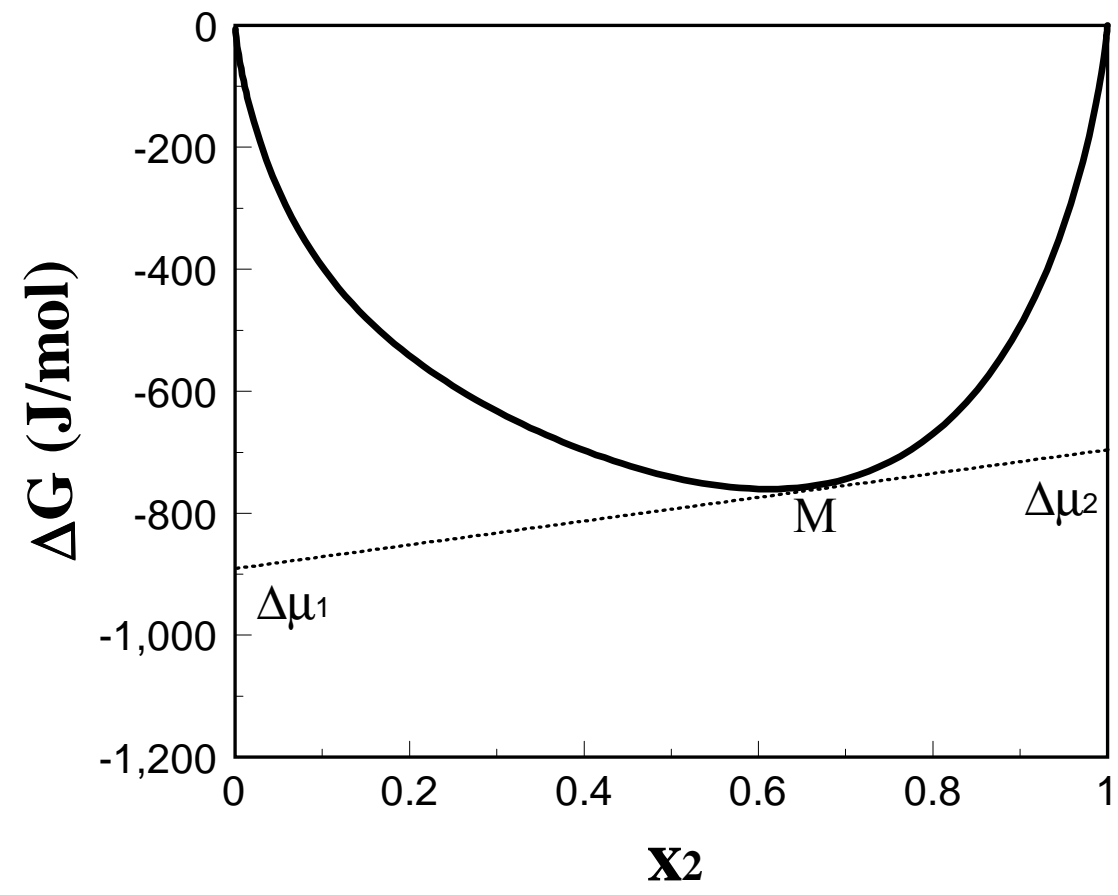

Figure I-3.13. Molar Gibbs free energy calculated from Flory-Huggins theory (Eq. I-3.27). $\mathbf{x}_{2}$ is the molar fraction of solute $A$ in the mixture. The composite solvent contains $80 \%$ toluene and $20 \%$ n-heptane in volume. The solubility parameter and molar volume for each component is: 1) solute $A\left(v_{a}=160\right.$ $\left.\left.\mathrm{ml} / \mathrm{mol}, \delta_{\mathrm{a}}=23 \mathrm{MPa}^{1 / 2}\right) ; 2\right)$ toluene $\left.\left(\mathrm{v}_{\mathrm{s}}=106.3 \mathrm{ml} / \mathrm{mol}, \delta_{\mathrm{s}}=18.2 \mathrm{MPa}^{1 / 2}\right) ; 3\right) \mathrm{n}$-heptane $\left(\mathrm{v}_{\mathrm{p}}=146.6 \mathrm{ml} / \mathrm{mol}\right.$, $\left.\delta_{\mathrm{p}}=15.2 \mathrm{MPa}^{1 / 2}\right)$.

It is obvious from equations (I-3.30) and (I-3.31) that for such a binary system under constant pressure and temperature, molar $\Delta \mathrm{G}_{\text {mix }}$ can be expressed as

$$
\Delta \mathrm{G}_{\text {mix }}=\mathrm{x}_{1} \Delta \mu_{1}+\mathrm{x}_{2} \Delta \mu_{2}
$$

where subscript 1 represents composite solvent of toluene with n-heptane, and subscript 2 represents solute A. Since $\mathrm{x}_{1}=1-\mathrm{x}_{2}$, equation (I-3.34) is equivalent to

$$
\Delta \mathrm{G}_{\text {mix }}=\Delta \mu_{1}+\mathrm{x}_{2}\left(\Delta \mu_{2}-\Delta \mu_{1}\right)
$$

For a given composition $\mathrm{x}_{2}$ (point $\mathrm{M}$ in Fig. I-3.13), Eq. (I-3.35) represents a tangent line through point $\mathrm{M}$. The slope of the tangent line is $\left(\Delta \mu_{2}-\Delta \mu_{1}\right)$, and the intercepts of the tangent line with $\mathrm{x}_{2}=0$ and $\mathrm{x}_{2}=1$ correspond to $\Delta \mu_{1}$ and $\Delta \mu_{2}$, respectively, as shown in Fig. I-3.13. 


\section{Different approaches for phase separation}

Approach assuming pure solid asphaltene phase at phase separation (Hirschberg model)

In Hirschberg's approach (Hirschberg, et al., 1984), some asphaltenes are assumed to nucleate as a pure solid phase (phase II) at phase separation, while leaving the remainder in solution (phase I). In other words, the chemical potential of the nucleated asphaltene equals the chemical potential of solid asphaltene (reference state)

$$
\mu_{2}^{\mathrm{II}}=\mu_{2}^{0}
$$

or

$$
\Delta \mu_{2}^{\mathrm{II}}=0
$$

Since the chemical potentials for asphaltene in phase I and phase II must be equal to each other at phase equilibrium, the chemical potential of asphaltene in phase I must also be equal to zero, i.e.,

$$
\Delta \mu_{2}^{\mathrm{I}}=\Delta \mu_{2}^{\mathrm{II}}=0
$$

Applying equation (I-3.33) to (I-3.38) and noting that $\phi_{1}{ }^{\mathrm{I}}=1-\phi_{2}{ }^{\mathrm{I}}$, we get

$$
\ln \phi_{2}^{\mathrm{I}}+\left(1-\phi_{2}^{\mathrm{I}}\right)\left(1-\frac{\mathrm{v}_{2}}{\mathrm{v}_{1}}\right)+\left(1-\phi_{2}^{\mathrm{I}}\right)^{2} \frac{\mathrm{v}_{2}}{\mathrm{RT}}\left(\delta_{2}-\delta_{1}\right)^{2}=0
$$

where $\phi_{2}{ }^{\mathrm{I}}$ is the volume fraction of asphaltene in phase I, which at the onset point is equal to asphaltene concentration in the solution. For a given asphaltene solution, either in crude oil or organic solvent, $\phi_{2}{ }^{\mathrm{I}}, \mathrm{v}_{1}$ and $\delta_{1}$ vary with the addition of asphaltene precipitant. Equation (I-3.39) is a nonlinear, implicit equation for the amount of precipitant needed to trigger the onset of asphaltene precipitation. Numerical methods can be employed to solve this equation, provided that the molar volume $v_{2}$ and solubility parameter $\delta_{2}$ for asphaltene are known.

A simplification of equation (I-3.39) can be made if we take into account the fact that for most asphaltene solutions $\phi_{2}{ }^{\mathrm{I}}<<1$. Equation (I-3.39) is then approximated to 


$$
\ln \phi_{2}^{\mathrm{I}}+\left(1-\frac{\mathrm{v}_{2}}{\mathrm{v}_{1}}\right)+\frac{\mathrm{v}_{2}}{\mathrm{RT}}\left(\delta_{2}-\delta_{1}\right)^{2}=0
$$

or

$$
\phi_{2}^{\mathrm{I}}=\exp \left[-1+\frac{\mathrm{v}_{2}}{\mathrm{v}_{1}}-\frac{\mathrm{v}_{2}}{\mathrm{RT}}\left(\delta_{2}-\delta_{1}\right)^{2}\right]
$$

This is the result given by Hirschberg et al. (1984). The same assumption, $\phi_{2}{ }^{\mathrm{I}}<<1$ can also be found in a model proposed by Nor-Azian and Adewumi (1993). The error introduced by this simplification would be significant if asphaltene content in the mixture is not low.

\section{Approach assuming pure solvent phase at phase separation}

In a second approach (Cimino et al., 1995), a pure solvent phase (phase I), rather than a pure asphaltene solid phase, is assumed to nucleate at phase separation, while asphaltenes concentrate in an asphaltene-rich phase (phase II) that also contains solvent. The chemical potential in phase I is equal to the chemical potential of pure solvent

$$
\mu_{1}^{\mathrm{I}}=\mu_{1}^{0}
$$

or

$$
\Delta \mu_{1}^{\mathrm{I}}=0
$$

Since the chemical potentials for solvent (or solvent mixture) in phase I and phase II must equal to each other at phase equilibrium, it is required that

$$
\Delta \mu_{1}^{\mathrm{II}}=\Delta \mu_{1}^{\mathrm{I}}=0
$$

Applying equation (I-3.32) to (I-3.44) and noting that $\phi_{1}{ }^{\mathrm{II}}=1-\phi_{2}{ }^{\mathrm{II}}$, we get

$$
\ln \left(1-\phi_{2}^{\mathrm{II}}\right)+\left(1-\frac{\mathrm{v}_{1}}{\mathrm{v}_{2}}\right) \phi_{2}^{\mathrm{II}}+\left(\phi_{2}^{\mathrm{II}}\right)^{2} \frac{\mathrm{v}_{1}}{\mathrm{RT}}\left(\delta_{2}-\delta_{1}\right)^{2}=0
$$

where $\phi_{2}{ }^{\text {II }}$ is the volume fraction of asphaltene in phase II with respect to the total volume of phase II. Experimentally there is no way to evaluate $\phi_{2}{ }^{\mathrm{II}}$, making it an inherently adjustable 
parameter. A fairly constant value of 0.8 for $\phi_{2}{ }^{\mathrm{II}}$ for most asphaltene solutions was reported by Cimino et al. (1995) based on simulations. Once again solvent properties, $v_{1}$ and $\delta_{1}$, vary with the amount of asphaltene precipitant added. Numerical solution of equation (34) will give the amount of precipitant at the onset, provided that a reasonable value of $\phi_{2}{ }^{\mathrm{II}}$ is assumed.

\section{Proposed approach without approximations of phase compositions}

The simplifying assumptions made in both these models (equations I-3.37 and I-3.43) are not mathematically necessary. Consider a binary system with an asphaltene-poor phase (I) and an asphaltene-rich phase (II), which form at phase separation. At equilibrium, the chemical potentials for either asphaltene or composite solvent must equal each other in both phases, i.e.,

$$
\begin{gathered}
\Delta \mu_{1}^{\mathrm{I}}=\Delta \mu_{1}^{\mathrm{II}} \\
\Delta \mu_{2}^{\mathrm{I}}=\Delta \mu_{2}^{\mathrm{II}}
\end{gathered}
$$

Applying equations (I-3.32) and (I-3.33) to (I-3.46) and (I-3.47), and noting that $\phi_{1}=1-\phi_{2}$ for both phase I and phase II, we have

$$
\begin{aligned}
& \ln \left(1-\phi_{2}^{\mathrm{I}}\right)+\left(1-\frac{1}{\mathrm{~m}}\right) \phi_{2}^{\mathrm{I}}+\chi\left(\phi_{2}^{\mathrm{I}}\right)^{2}=\ln \left(1-\phi_{2}^{\mathrm{II}}\right)+\left(1-\frac{1}{\mathrm{~m}}\right) \phi_{2}^{\mathrm{II}}+\chi\left(\phi_{2}^{\mathrm{II}}\right)^{2} \\
& \ln \phi_{2}^{\mathrm{I}}+\left(1-\phi_{2}^{\mathrm{I}}\right)(1-\mathrm{m})+\left(1-\phi_{2}^{\mathrm{I}}\right)^{2} \mathrm{~m} \chi=\ln \phi_{2}^{\mathrm{II}}+\left(1-\phi_{2}^{\mathrm{II}}\right)(1-\mathrm{m})+\left(1-\phi_{2}^{\mathrm{II}}\right)^{2} \mathrm{~m} \chi
\end{aligned}
$$

where

$$
\mathrm{m}=\frac{\mathrm{v}_{2}}{\mathrm{v}_{1}}
$$

is the ratio of molar volumes for asphaltene to composite solvent. $\phi_{2}{ }^{\mathrm{I}}$ and $\phi_{2}{ }^{\mathrm{II}}$ are asphaltene volume fractions in phase I and phase II, respectively. For a given mixture, $v_{1}, v_{2}$, and $\delta_{1}, \delta_{2}$ are known, thus Eqs. I-3.48 and I-3.49 can be solved simultaneously to get $\phi_{2}{ }^{\mathrm{I}}$ and $\phi_{2}{ }^{\mathrm{II}}$ at phase equilibrium. Once $\phi_{2}{ }^{\mathrm{I}}$ and $\phi_{2}{ }^{\mathrm{II}}$ are obtained, the percentage of the asphaltene precipitated (in phase II) over the total amount of asphaltene in mixture can easily be calculated.

\section{COEXISTING PHASES}

Note that equations (I-3.48) and (I-3.49) can be solved for $\phi_{2}{ }^{\mathrm{I}}$ and $\phi_{2}{ }^{\mathrm{II}}$ only when phase separation occurs. Mathematically, this is because the molar volume, $\mathrm{v}_{1}$, and solubility 
parameter, $\delta_{1}$, for the composite solvent, and thus $m$ and $\chi$, are functions of the volume fraction of precipitant, $\phi_{\mathrm{p}}$, in the mixture. Whether or not equations (I-3.48) and (I-3.49) can be solved is determined by the shape of the Gibbs free energy curve, which varies with $\phi_{\mathrm{p}}$ as shown in Fig. I3.14 .

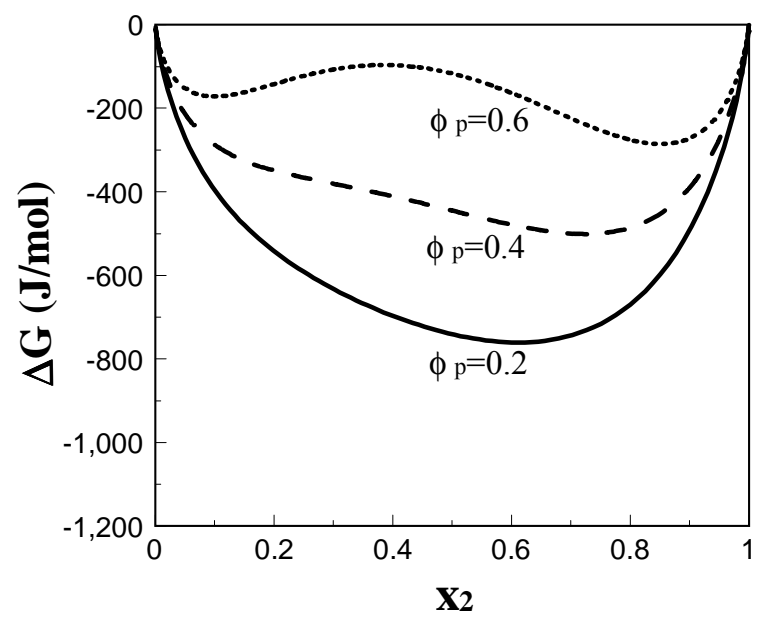

Figure I-3.14. Gibbs free energy varies with volume ratio of toluene to n-heptane in the composite solvent.

For a low volume fraction of precipitant, the Gibbs free energy curve is concave everywhere (Fig. I-3.15). For a given composition (point M), the Gibbs free energy is equal to the length of segment MN. If phase separation occurs, the two resulting phases are represented by points $M_{I}$ and $M_{I I}$. The corresponding Gibbs free energy for each phase is the length of segments $M_{I} N_{I}$ and $\mathrm{M}_{\mathrm{II}} \mathrm{N}_{\mathrm{II}}$, respectively. A simple calculation of mass conservation shows that the overall Gibbs free energy for the system, after phase separation, is equal to the length of segment ON. Since ON is always shorter than MN for a concave curve, the total Gibbs free energy would increase after separating $\mathrm{M}$ into $\mathrm{M}_{\mathrm{I}}$ and $\mathrm{M}_{\mathrm{II}}$. Thus, for a concave Gibbs free energy curve, any phase separation is energetically unfavorable and cannot occur anywhere.

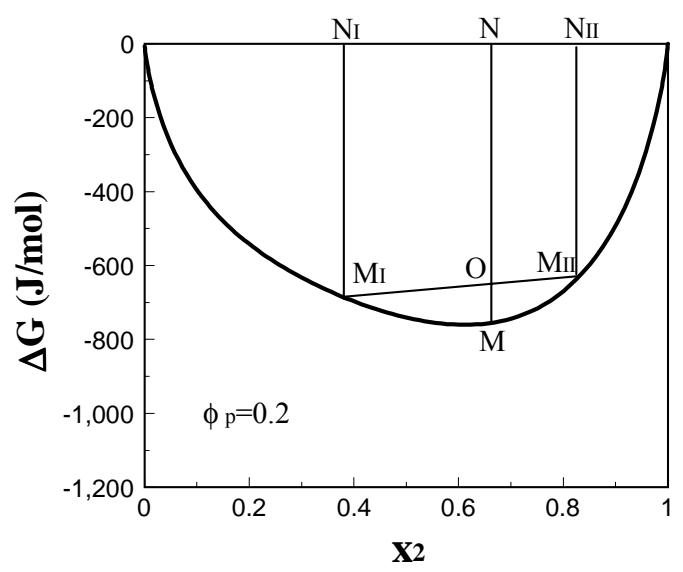

Figure I-3.15. For a Gibbs free energy curve with concave shape, the solution is stable against phase separation. Any potential phase separation would increase the overall Gibbs free energy and thus is not favored. 
When the precipitant volume fraction in a mixture is high enough, two local valleys emerge in the Gibbs free energy curve (Fig. I-3.16). A double tangent line to both minima can be drawn to give two points $\mathrm{M}_{\mathrm{I}}$ and $\mathrm{M}_{\mathrm{II}}$, which represent asphaltene molar fractions in coexisting phase I and phase II, respectively. Correspondingly, Eqs. I-3.48 and I-3.49 have solutions to $\phi_{2}{ }^{\mathrm{I}}$ and $\phi_{2}{ }^{\mathrm{II}}$. The overall Gibbs free energy after separation (segment ON in Fig. I-3.16) is lower than that before separation (segment MN). Any composition that falls in the range between $M_{I}$ and $M_{I I}$ can separate into phase I $\left(\mathrm{M}_{\mathrm{I}}\right)$ and phase II $\left(\mathrm{M}_{\mathrm{II}}\right)$ since the total energy is reduced. Note that $\mathrm{M}_{\mathrm{I}}$ and $\mathrm{M}_{\mathrm{II}}$ are the only stable compositions for the separated phase since only they meet the requirement for equivalent chemical potentials for both asphaltene and composite solvent in phase I and phase II.

\section{METASTABLE REGION AND ABSOLUTELY UNSTABLE REGION}

There exist two inflection points on the Gibbs free energy curve, indicated as $\mathrm{U}_{\mathrm{I}}$ and $\mathrm{U}_{\mathrm{II}}$ on Fig. I-3.16. The curves between $\mathrm{M}_{\mathrm{I}}$ and $\mathrm{U}_{\mathrm{I}}$ or between $\mathrm{M}_{\mathrm{II}}$ and $\mathrm{U}_{\mathrm{II}}$ are concave. Any phase separations into neighbor concentrations are not favored as discussed above in Fig. I-3.15, although phase separation into $\mathrm{M}_{\mathrm{I}}$ and $\mathrm{M}_{\mathrm{II}}$ is energetically favored. These two concentration ranges are metastable regions. For compositions that fall into these regions, phase separation into $\mathrm{M}_{\mathrm{I}}$ and $\mathrm{M}_{\mathrm{II}}$ is possible, but might be very slow. On the other hand, the curve bounded by points $\mathrm{U}_{\mathrm{I}}$ and $\mathrm{U}_{\mathrm{II}}$ is convex and thus represents an absolutely unstable region. Any composition that falls in this region will separate immediately into $\mathrm{M}_{\mathrm{I}}$ and $\mathrm{M}_{\mathrm{II}}$.

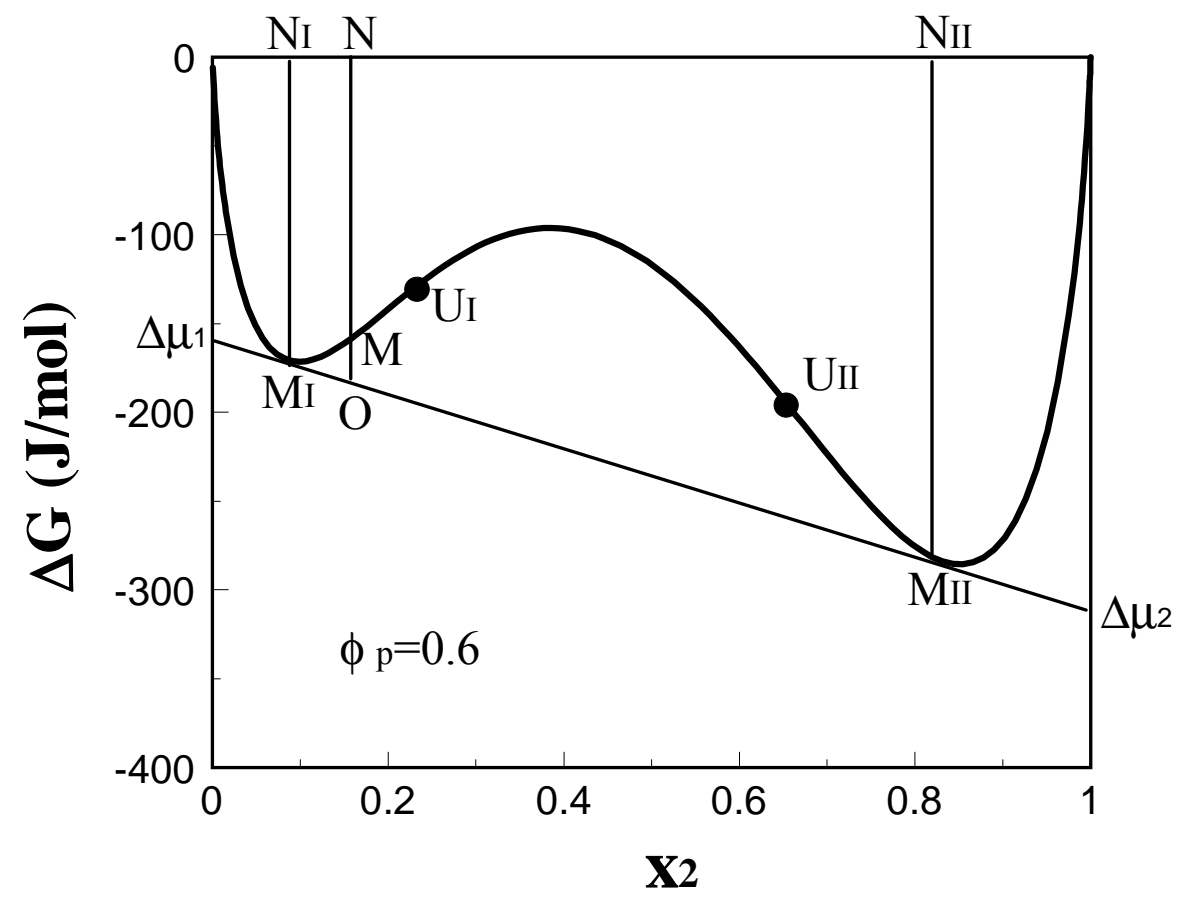

Figure I-3.16. For a Gibbs free energy curve with two local valleys, the mixture will separate into two coexisting phases if composition falls in the range between point $M_{I}$ and $M_{I I}$. At phase equilibrium, asphaltene molar fraction in each phase is given by point $M_{I}$ and $M_{I I}$.

The points at which asphaltenes become absolutely unstable can be obtained as follows. The inflection points $\mathrm{U}_{\mathrm{I}}$ and $\mathrm{U}_{\mathrm{II}}$ correspond to 


$$
\left(\frac{\partial^{2} \Delta G_{\text {mix }}}{\partial x_{2}^{2}}\right)_{P, T}=0
$$

From (I-3.35), this is equivalent to

$$
\frac{\partial \Delta \mu_{2}}{\partial x_{2}}-\frac{\partial \Delta \mu_{1}}{\partial x_{2}}=0
$$

According to the Gibbs-Duhem equation (Tompa, 1956), at constant $\mathrm{P}$ and $\mathrm{T}, \Delta \mu_{1}$ and $\Delta \mu_{2}$ satisfy

$$
\mathrm{x}_{1} \mathrm{~d}\left(\Delta \mu_{1}\right)+\mathrm{x}_{2} \mathrm{~d}\left(\Delta \mu_{2}\right)=0
$$

where $\mathrm{d}\left(\Delta \mu_{1}\right)$ and $\mathrm{d}\left(\Delta \mu_{2}\right)$ represent infinitesimal change of $\Delta \mu_{1}$ and $\Delta \mu_{2}$. Differentiating (I-3.53) with respect to $\mathrm{x}_{2}$ gives

$$
\mathrm{x}_{1} \frac{\partial \Delta \mu_{1}}{\partial \mathrm{x}_{2}}+\mathrm{x}_{2} \frac{\partial \Delta \mu_{2}}{\partial \mathrm{x}_{2}}=0
$$

Combining (I-3.52) and (I-3.54) and noting $\mathrm{x}_{1}+\mathrm{x}_{2}=1$, gives

$$
\frac{\partial \Delta \mu_{1}}{\partial x_{2}}=\frac{\partial \Delta \mu_{2}}{\partial x_{2}}=0
$$

Differentiating (I-3.33) with respect to $\mathrm{x}_{2}$ and using (I-3.19) to (I-3.21), equation (I-3.55) gives

$$
\frac{\partial \Delta \mu_{2}}{\partial x_{2}}=\frac{\mathrm{RTv}_{1} \mathrm{v}_{2}}{\left[\mathrm{v}_{1}+\mathrm{x}_{2}\left(\mathrm{v}_{2}-\mathrm{v}_{1}\right)\right]^{2}}\left[\frac{1}{\phi_{2}}-(1-\mathrm{m})-2 \mathrm{~m} \chi\left(1-\phi_{2}\right)\right]=0
$$

Since $v_{1}$ and $v_{2}$ are not zero, this requires 


$$
\frac{1}{\phi_{2}}-(1-\mathrm{m})-2 \mathrm{~m} \chi\left(1-\phi_{2}\right)=0
$$

or

$$
2 \mathrm{~m} \chi \phi_{2, \mathrm{U}}^{2}+(\mathrm{m}-1-2 \mathrm{~m} \chi) \phi_{2, \mathrm{U}}+1=0
$$

where $\phi_{2, \mathrm{U}}$ denotes the volume fraction of asphaltene in the mixture corresponding to the inflection points bounding the absolutely unstable region. Solutions to the quadratic equation (I3.58) will give two inflection points, $\phi_{2, \mathrm{U}}^{\mathrm{I}}$ and $\phi_{2, \mathrm{U}}^{\mathrm{II}}$.

\section{ASPHALTENE STABILITY}

Now let us summarize the stability of the binary mixture with respect to phase separation.

- If the asphaltene volume fraction in the mixture, $\phi_{2}$, is lower than asphaltene volume fraction in phase I, $\phi_{2}{ }^{\mathrm{I}}$, as calculated from equations (I-3.48) and (I-3.49), any phase separation is impossible and the solution is stable.

- If $\phi_{2}$ falls in the region between $\phi_{2}{ }^{\mathrm{I}}$ and $\phi_{2, \mathrm{U}}{ }^{\mathrm{I}}$, the solution is metastable. In this region, flocculation could occur, but it might be slow.

- If $\phi_{2}$ falls in the region bounded by $\phi_{2, U}{ }^{I}$ and $\phi_{2, U}{ }^{I I}$, the solution is absolutely unstable. Asphaltenes will flocculate rapidly.

As an example, both the two-phase coexistence line and the inflection point line, or spinodal line (Sanchez, 1987), were calculated for an actual asphaltene solution from equations (I-3.48)-( I-3.49) and equation (I-3.58), respectively, as shown in Fig. I-3.17. The toluene solution was prepared by dissolving $1 \mathrm{wt} \% \mathrm{n}$-heptane asphaltenes, separated from Mars-pink crude oil. Precipitant n-heptane was then added to the solution gradually. Both the calculated and measured onset results will be compared later in this report. Asphaltene volume fractions in the mixture, $\phi_{2}$, at both two-phase coexisting points and inflection points are plotted as a function of nheptane volume fraction, $\phi_{\mathrm{p}}$. The area bounded by these two lines is the metastable region, while the area to the right of the inflection-point line is the absolutely unstable region. 


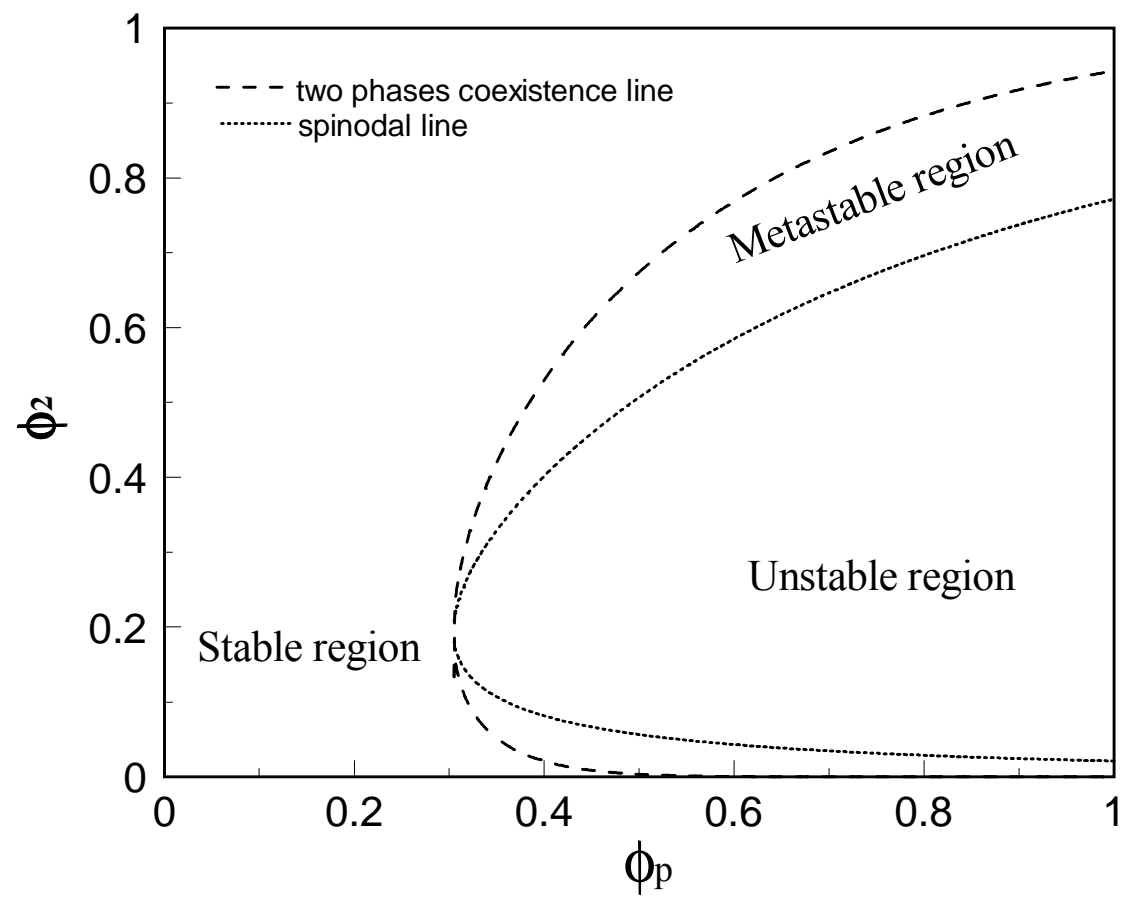

Figure I-3.17. Calculated lines for two-phase coexistence and inflection points (spinodal curve), for a mixture of $n-C 7$ Mars-pink asphaltenes dissolved in toluene $(1 \mathrm{wt} \%)$ plus $n$-heptane.

If the asphaltene volume fraction in a mixture as a function of $\phi_{\mathrm{p}}$ is added in the same plot (shown on an expanded scale in Fig. I-3.18), we can see that the earliest stage at which phase separation could occur is the point $\mathrm{S}$ where the asphaltene volume fraction line crosses the twophase coexistence line. The precipitant volume fraction corresponding to this point can be calculated by setting asphaltene volume fraction, $\phi_{2}$, equal to the asphaltene volume fraction in phase I, $\phi_{2}{ }^{\mathrm{I}}$

$$
\phi_{2}=\phi_{2}^{\mathrm{I}}
$$

Since both $\phi_{2}$ and $\phi_{2}{ }^{\mathrm{I}}$ are functions of $\phi_{\mathrm{p}}$, equation (I-3.59), combined with (I-3.48) and (I-3.49), can be used to solve for the minimum $\phi_{\mathrm{p}}$ at which the mixture enters the metastable region. 


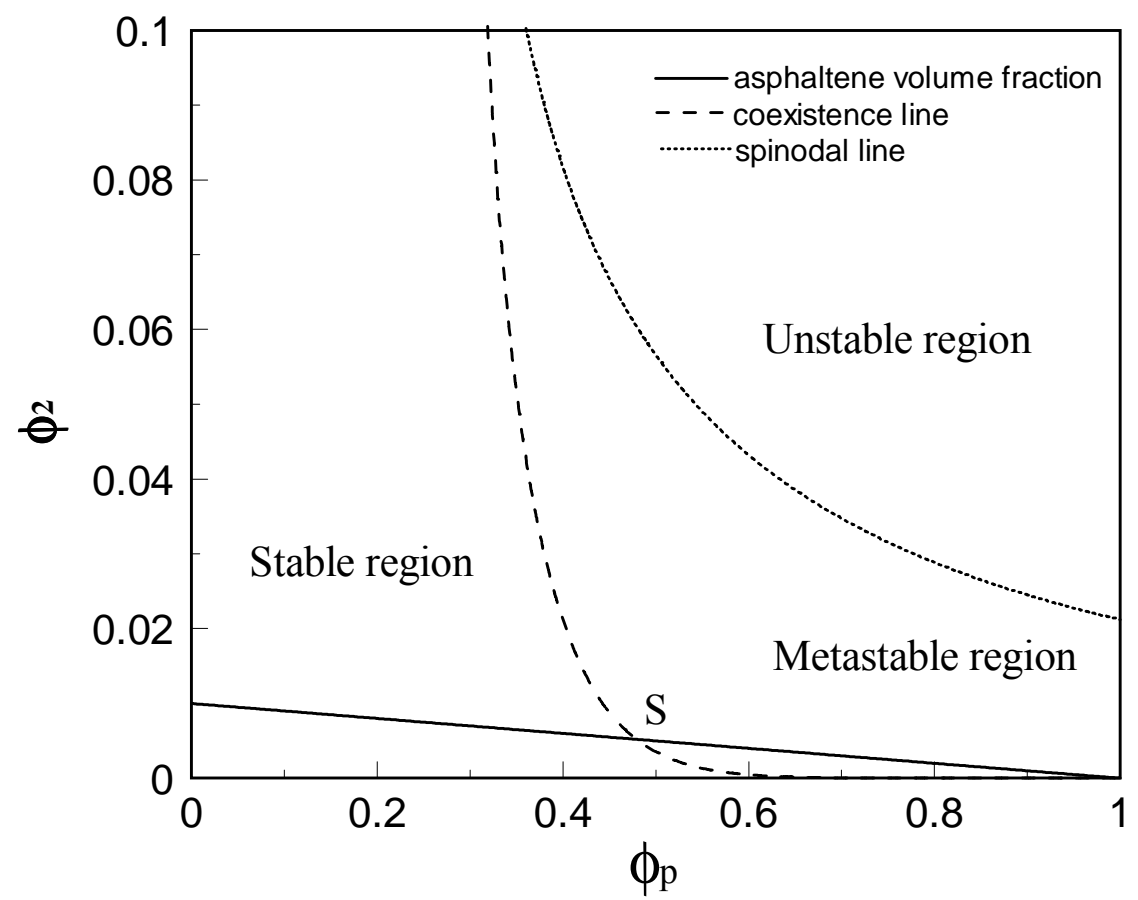

Figure I-3.18. The cross point (S) of asphaltene volume fraction line with two-phase coexistence line represents the minimum precipitant volume fraction before phase separation. The mixture will never enter absolutely unstable region in this example.

On the other hand, whether or not the mixture could enter the absolutely unstable region can be found mathematically by setting asphaltene volume fraction, $\phi_{2}$, equal to the asphaltene volume fraction in phase I corresponding to the first inflection point, $\phi_{2, \mathrm{U}}^{\mathrm{I}}$,

$$
\phi_{2}=\phi_{2, \mathrm{U}}^{\mathrm{I}}
$$

Since both $\phi_{2}$ and $\phi_{2, \mathrm{U}}^{\mathrm{I}}$ are functions of $\phi_{\mathrm{p}}$, if there is any solution to the combination of equation (I-3.60) and (I-3.58), the solution $\phi_{\mathrm{p}}$ is the precipitant volume fraction at which the mixture enters the unstable region. Otherwise it indicates no cross-point between asphaltene volume fraction line and spinodal line, as is the case shown in Fig. I-3.18; the mixture will never enter the absolutely unstable region and no catastrophic flocculation will occur.

\section{ONSET CRITERIA}

Asphaltene volume fraction before phase separation, $\phi_{2}$, and in phase I, $\phi_{2}{ }^{\mathrm{I}}$, and phase II, $\phi_{2}{ }^{\mathrm{II}}$, after phase separation are calculated as described above and plotted in Fig. I-3.19. At the beginning, $\phi_{2}$ linearly decreases with increase in the volume fraction of precipitant, $\phi_{\mathrm{p}}$, until it reaches the phase separation point S. At this point, two phases, one asphaltene-poor phase (I) and the other asphaltene-rich phase (II), begin to separate. However, most of asphaltenes still remains in phase I and the asphaltene volume fraction in phase II is so small that it might not be 
observable (see Fig. I-3.20). As more and more precipitant is added, asphaltene volume fraction in phase I decreases while in phase II increases. When $\phi_{\mathrm{p}}$ is higher than a certain value (about 0.7 in this case), most of asphaltenes are concentrated in phase II, leaving the asphaltene in phase I essentially zero.

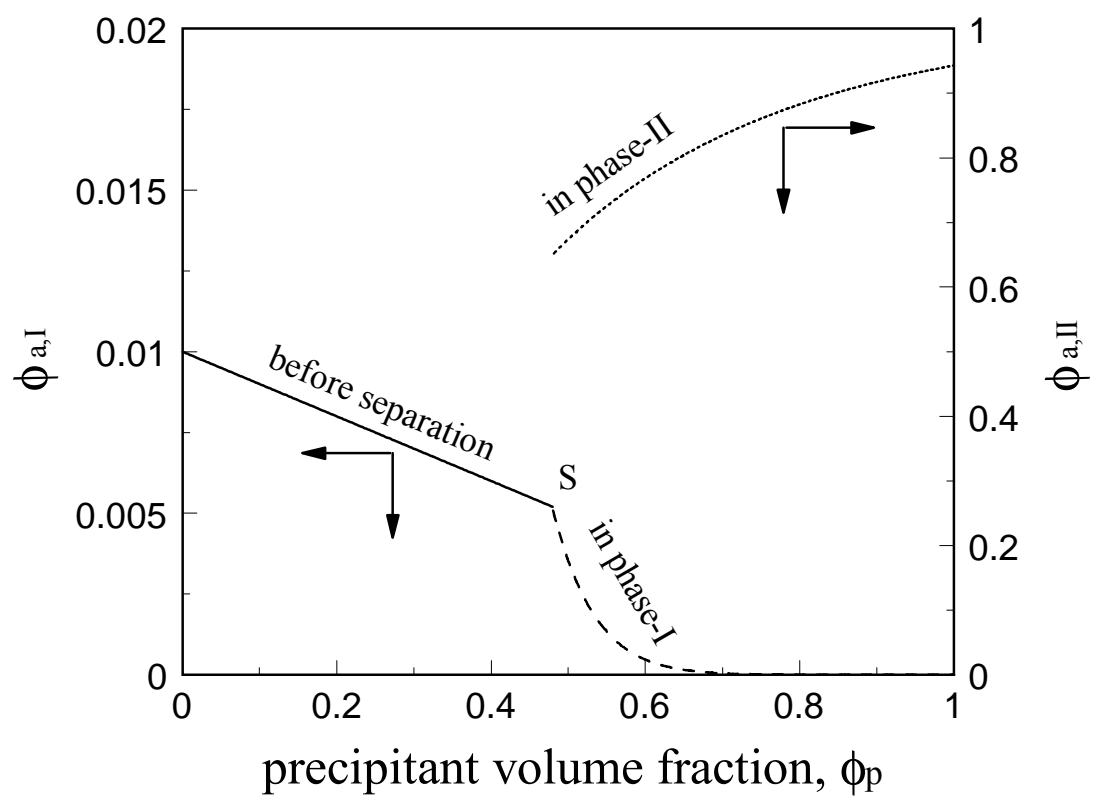

Figure I-3.19. Asphaltene volume fraction in the mixture decreases linearly with addition of precipitant before it reaches a point $(S)$ where phase separation begins. After this point, asphaltene volume fraction in phase-I, $\phi_{\mathrm{a}, \mathrm{I}}$, decreases while asphaltene volume fraction in phase-II, $\phi_{\mathrm{a}, \mathrm{II}}$, increases. Note the significant difference between $\phi_{\mathrm{a}, \mathrm{I}}$ and $\phi_{\mathrm{a}, \mathrm{II}}$ at the phase separation point.

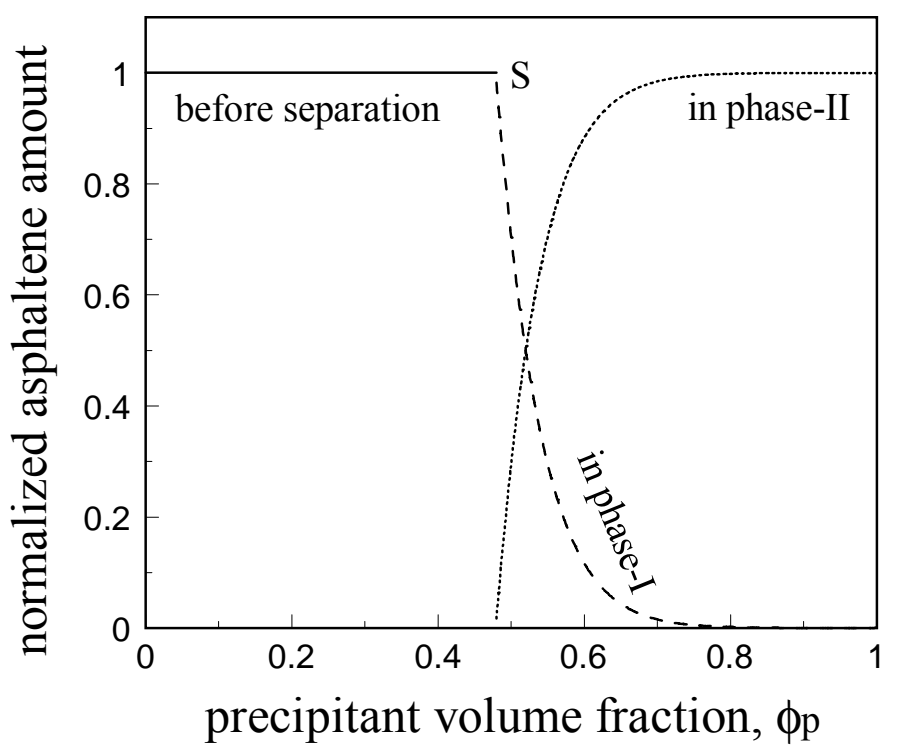

Figure I-3.20. Normalized asphaltene content in solution before phase separation, in phase I and phase II after phase separation as a function of precipitant volume fraction in the mixture. When the precipitant volume fraction is high enough, almost all asphaltenes are concentrated in phase II. 
The question now is how much asphaltene must separate into phase II before it becomes visible under the microscope? The size of asphaltene aggregates must be increasing as more and more asphaltenes are concentrated in phase II with addition of precipitant. However, the minimum volume fraction of asphaltene in phase II at which point the size, as well as the population, of aggregates begins to emerge under microscope is not well defined. Criteria to relate the visible "onset" point to the calculated phase separation are needed.

As shown in Fig. I-3.21, there can exist an inflection point at the low asphaltene concentration end on the Gibbs free energy curve at which the tangent line through this point is horizontal. We will denote this point as flat inflection point. As more precipitant is added, two local minima will emerge (see Fig. I-3.14). When this occurs, any potential phase separations into phase I and phase II should be relatively stable since an energy barrier between phase I and phase II exists (see Fig. I-3.16). Conditions that result in a flat inflection point may therefore be equated with the point at which asphaltene flocculation becomes visible. The validity of this criterion will be tested later in this report.

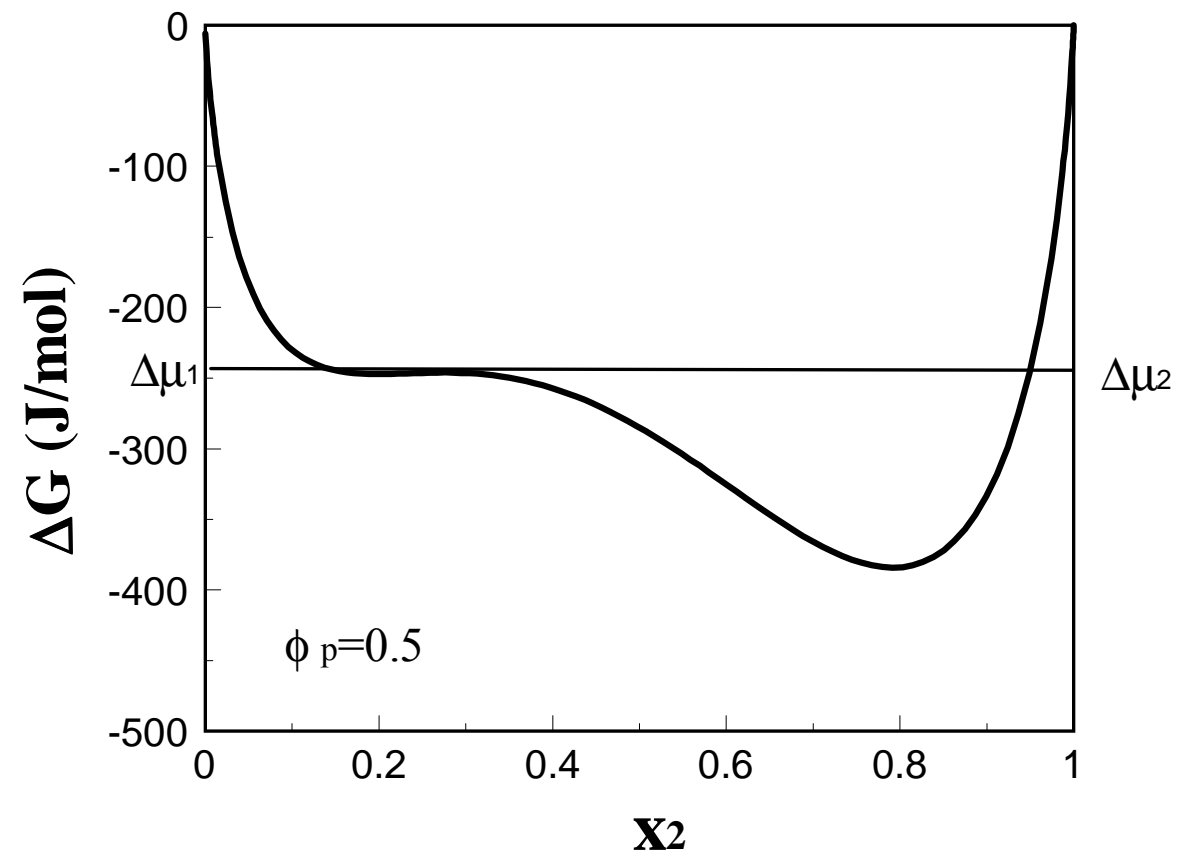

Figure I-3.21. A criterion to judge the visible "onset" point for asphaltene flocculation. The onset is the point when Gibbs free energy curve gives a flat inflection point. If more precipitant is added after this point, two local minima will emerge on Gibbs free energy curve.

Mathematically, this flat inflection point can be found as follows. The flat tangent line intercepts $\mathrm{x}_{2}=0$ and $\mathrm{x}_{2}=1$ (Fig. I-3.21) resulting in equal chemical potentials for solvent and asphaltene, i.e.,

$$
\Delta \mu_{1}=\Delta \mu_{2}
$$


It should be emphasized at this point that $\Delta \mu_{1}$ and $\Delta \mu_{2}$ in equation (I-3.61) do not represent the chemical potentials for solvent and asphaltene at phase equilibrium; the later ones are still described by equation (I-3.46) and (I-3.47). From (I-3.32) and (I-3.33), equation (I-3.61) is equivalent to

$$
\ln \left(1-\phi_{2}\right)+\left(1-\frac{1}{\mathrm{~m}}\right) \phi_{2}+\chi\left(\phi_{2}\right)^{2}=\ln \phi_{2}+(1-\mathrm{m})\left(1-\phi_{2}\right)+\mathrm{m} \chi\left(1-\phi_{2}\right)^{2}
$$

Solving equation (I-3.62) by a numerical method will give the volume fraction of precipitant that corresponds to the flat inflection point, and thus the onset condition.

\section{Comparisons of these three different approaches}

The major differences among these three approaches are the calculations of compositions in phase I and phase II at phase equilibrium and the criterion for onset conditions. A schematic diagram is shown on Fig. I-3.22 to demonstrate the characteristics for these three approaches.

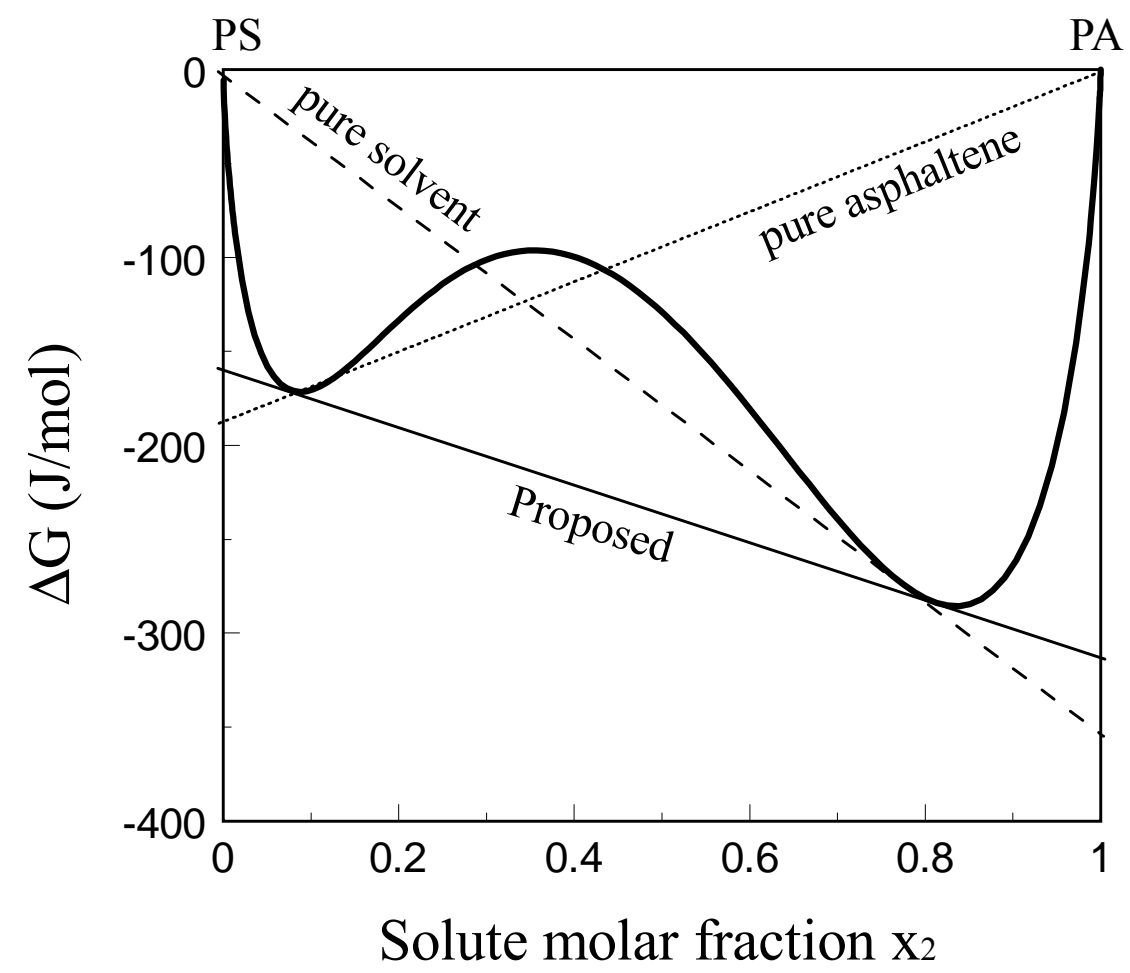

Figure I-3.22. A schematic diagram to show the major differences among three different approaches.

In the new approach proposed above, asphaltene volume fractions in phase I, $\phi_{2, \mathrm{I}}$, and phase II, $\phi_{2, \text { II }}$, at phase equilibrium are obtained by a mutual tangent line to two minima on Gibbs free energy curve. The onset criterion is set when the first inflection point at low $\mathrm{x}_{2}$ end has a flat tangent line. 
In the pure asphaltene approach (Hirschberg et al., 1984), phase II is assumed to contain pure asphaltene, represented by point PA $\left(\phi_{2, I I}=1\right.$ thus $\left.x_{2}=1\right)$. The volume fraction of asphaltene in phase I, $\phi_{2, \mathrm{I}}$, is determined by the tangent point drawn from PA to the Gibbs free energy curve. This obviously introduces some errors. Furthermore, the onset is assumed to occur when calculated $\phi_{2, \mathrm{I}}$ is equal to asphaltene solubility in the mixture. This is equivalent to the metastable point as discussed above. We can therefore expect to obtain a modeled onset condition similar to the metastable boundary condition, which strongly depends on original asphaltene content of the oil.

In the pure solvent approach (Cimino et al., 1995), phase I is assumed to contain pure solvent, represented by point PS $\left(\phi_{2, \mathrm{I}}=1\right.$ thus $\left.\mathrm{x}_{2}=0\right)$. Although this assumption might help to simplify the phase equilibrium calculation, it implies that all the asphaltenes will be separated into phase II once flocculation occurs, in contrast to what is normally observed for asphaltenes in solutions. Asphaltene volume fraction in phase II, $\phi_{2, \text { II }}$, is obtained by the tangent point drawn from PS to Gibbs free energy curve. This, as well, introduces some errors. The onset is the point when the shape of the Gibbs free energy curve will result in an empirical constant value (0.8) for $\phi_{2, \mathrm{II}}$.

A comparison of these three distinct approaches is summarized in Table I-3.7.

\section{Modeling implementation}

The principles described above have been implemented with Visual Basic as a macro inside Microsoft Office Excel. The model obtains input data from the spreadsheet, solves for the onset conditions, then shows the results as a table and a chart in the same spreadsheet. Three solutions are obtained for each set of parameters: the minimum precipitant volume fraction for any potential flocculation (the metastable boundary), the precipitant volume fraction corresponding to the visible "onset" condition, and the point of absolute instability. The first two points can be

found as long as appropriate input parameters are provided, while the third point might or might not exist (see Fig. I-3.18). 
Table I-3.7. Comparisons for three different approaches based on Flory-Huggins polymer theory

\begin{tabular}{|c|c|c|c|c|}
\hline Characteristics & \multicolumn{2}{|c|}{ Pure asphaltene approach } & Pure solvent approach & Proposed approach \\
\hline $\begin{array}{l}\text { SIGNIFICANT } \\
\text { ASSUMPTIONS }\end{array}$ & \multicolumn{2}{|c|}{$\begin{array}{l}\text { PURE SOLID ASPHALTENE PHASE } \\
\text { NUCLEATING AT PHASE } \\
\text { SEPARATION }\end{array}$} & $\begin{array}{l}\text { PURE SOLVENT PHASE } \\
\text { NUCLEATING AT PHASE } \\
\text { SEPARATION, WHILE } \\
\text { ASPHALTENE FORMS AN } \\
\text { ASPHALTENE-RICH PHASE }\end{array}$ & $\begin{array}{l}\text { PHASE SEPARATION CREATES } \\
\text { ASPHALTENE-RICH AND } \\
\text { ASPHALTENE-POOR PHASES }\end{array}$ \\
\hline Input parameters & \multicolumn{2}{|c|}{$\begin{array}{l}\text { molar volumes of } \\
\text { - solvent } \\
\text { - asphaltene } \\
\text { solubility parameters of } \\
\text { - solvent } \\
\text { - asphaltene } \\
\text { asphaltene content } \\
\text { temperature }\end{array}$} & $\begin{array}{l}\text { molar volumes of } \\
\text { - solvent } \\
\text { - asphaltene } \\
\text { solubility parameters of } \\
\text { - solvent } \\
\text { - asphaltene } \\
\text { temperature } \\
\text { asphaltene volume fraction } \\
\text { in asphaltene-rich phase } \\
\text { (adjustable) }\end{array}$ & $\begin{array}{l}\text { molar volumes of } \\
\text { - solvent } \\
\text { - asphaltene } \\
\text { solubility parameters of } \\
\text { - solvent } \\
\text { - asphaltene } \\
\text { asphaltene content } \\
\text { temperature }\end{array}$ \\
\hline Onset criteria & \multicolumn{2}{|c|}{$\begin{array}{l}\text { At onset point, asphaltene } \\
\text { volume fraction in asphaltene- } \\
\text { poor phase is equal to original } \\
\text { asphaltene content }\end{array}$} & $\begin{array}{l}\text { Onset is determined by } \\
\text { setting a constant asphaltene } \\
\text { concentration in asphaltene- } \\
\text { rich phase (about } 0.8 \text { ) }\end{array}$ & $\begin{array}{l}\text { Onset is determined from } \\
\text { the flat inflection point on } \\
\text { Gibbs free energy curve }\end{array}$ \\
\hline $\begin{array}{l}\text { Sensitivity of } \\
\text { calculated onset } \\
\text { on asphaltene } \\
\text { properties }\end{array}$ & \multicolumn{2}{|c|}{$\begin{array}{l}\text { - original content: very sensitive } \\
\text { - molar volume: sensitive } \\
\text { - solubility parameter: very } \\
\text { sensitive }\end{array}$} & $\begin{array}{l}\text { - original content: not } \\
\text { affected } \\
\text { - molar volume: not } \\
\text { sensitive } \\
\text { - solubility parameter: very } \\
\text { sensitive }\end{array}$ & $\begin{array}{l}\text { - original content: not } \\
\text { sensitive } \\
\text { - molar volume: not } \\
\text { sensitive } \\
\text { - solubility parameter: very } \\
\text { sensitive }\end{array}$ \\
\hline \multirow{2}{*}{\multicolumn{2}{|c|}{$\begin{array}{l}\text { MATCHES BETWEEN } \\
\text { SIMULATED AND MEASURED } \\
\text { ONSETS } \\
\text { CRUDE OIL: } \\
\text { ASPHALTENE } \\
\text { SOLUTIONS IN TOLUENE: }\end{array}$}} & & & \\
\hline & & NOT GOOD & $\begin{array}{l}\text { NOT GOOD } \\
\text { GOOD }\end{array}$ & GOOD \\
\hline SOLUTI & $\begin{array}{l}\text { ASPHALTENE } \\
\text { S IN 1-MN*: }\end{array}$ & NOT GOOD & NOT GOOD & NOT GOOD \\
\hline
\end{tabular}

* $1-\mathrm{MN}$ is 1 -methylnaphthalene 
Modeling strategy

A schematic diagram in Fig. I-3.23 shows the modeling process. Details of the strategy used to obtain solutions and to ensure that they are physically meaningful are discussed below.

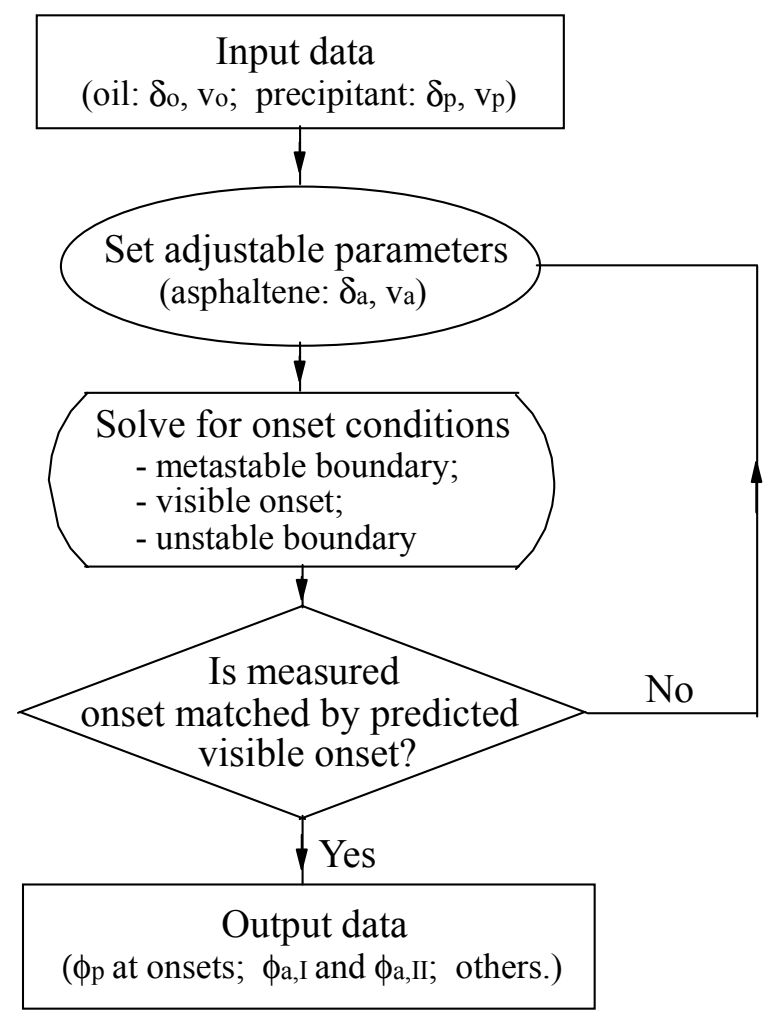

Figure I-3.23. Flow-chart for modeling algorithm.

INPUT DATA

The model is designed to be capable of simultaneously solving for onset conditions for a given oil induced by a variety of precipitants. Input data provided by the user include the molar volumes and solubility parameters for solvent, precipitant and asphaltene, along with asphaltene content in the original oil. The sources for the input data are as follows:

\section{Solubility parameters:}

- $\quad$ pure substances: obtained from CRC handbook (Barton, 1991);

- crude oils: interpreted from measured RI based on $\delta-F_{R I}$ correlation for non-polar substances (Buckley et al., 1998);

- asphaltenes: adjustable parameter; 


\section{Molar volumes:}

- pure substances: calculated from measured density and molecular weight;

- crude oils: calculated from measured density and number-average molecular weight, measured by the freezing-point-depression technique;

- asphaltenes: adjustable parameter.

Asphaltene content:

The $\mathrm{n}-\mathrm{C} 7$ asphaltene content is used as the input to the model. The volume fraction of asphaltene in the solution is calculated by dividing asphaltene content $(\mathrm{g} / 100 \mathrm{ml})$ by its density. A moderate asphaltene density of $1.15 \mathrm{~g} / \mathrm{ml}$ has been adopted for all the asphaltenes studied here. This value is about the average of most asphaltene densities, i.e., $1.1-1.2 \mathrm{~g} / \mathrm{ml}$. It will be demonstrated later that predicted onset conditions are quite insensitive to both asphaltene content and density. Thus errors resulting from these approximations should be insignificant.

\section{OUTPUT DATA}

The major outputs from modeling include precipitant volume fractions at the metastable boundary, at the visible onset, and at the point of instability (if it exists). $\mathrm{P}_{\mathrm{RI}}$ of mixtures at these points is calculated based on the volume fractions and RI for each component, and compared graphically with measured $\mathrm{P}_{\mathrm{RI}}$. At the same time, the volume fractions of asphaltene in the asphaltene-poor phase (phase I), asphaltene-rich phase (phase II) and in the mixture are calculated. The Flory-Huggins interaction parameter, $\chi$, and change in Gibbs free energy at the onset are also calculated.

\section{ADJUSTABLE PARAMETERS}

In principle, this thermodynamic model of asphaltene solubility, based on Flory-Huggins' theory, requires no adjustable parameters. It is a fully predictive model, assuming the properties of asphaltene, solvent, and precipitant, including molar volumes and solubility parameters, can be provided. However, there is considerable uncertainty about both the molar volume and solubility parameter of asphaltene, so that these properties are more appropriately treated as adjustable parameters. Whether the average molar volumes and average solubility parameters used for both crude oil and asphaltene adequately describe the interactions among various components in these complex mixtures remains an open question.

Increasing either the solubility parameter or molar volume of asphaltene increases the predicted $P_{R I}$ value. At least two measured onset points are required to adjust these two unknown parameters. Additional limitations are necessary to ensure that the parameters selected have physical meaning and that they give physically realistic predictions of onset conditions. 
Reasonable values for the asphaltene solubility parameter should be in the range of 18-24 $\mathrm{MPa}^{1 / 2}$; and molar volume should be in the range of $1000-10000 \mathrm{ml} / \mathrm{mol}$.

\section{Establishing a lower limit of asphaltene molar volume}

An example of the limits of physically reasonable predictions is given in Fig. I-3.24. For Mars-pink crude oil, the model was used to match measurements of $\mathrm{P}_{\mathrm{RI}}$ with precipitants ranging from nC5 up to nC15. A family of solutions, consisting of pairs of values of asphaltene molar volume and solubility parameter, can be obtained. For each of these solutions, the asphaltene volume fractions in phase I, $\phi_{\mathrm{a}, \mathrm{I}}$, can be calculated, as shown in Fig. I-3.24. Also shown is the asphaltene volume fraction in the mixture at onset point, $\phi_{\mathrm{a}}$.

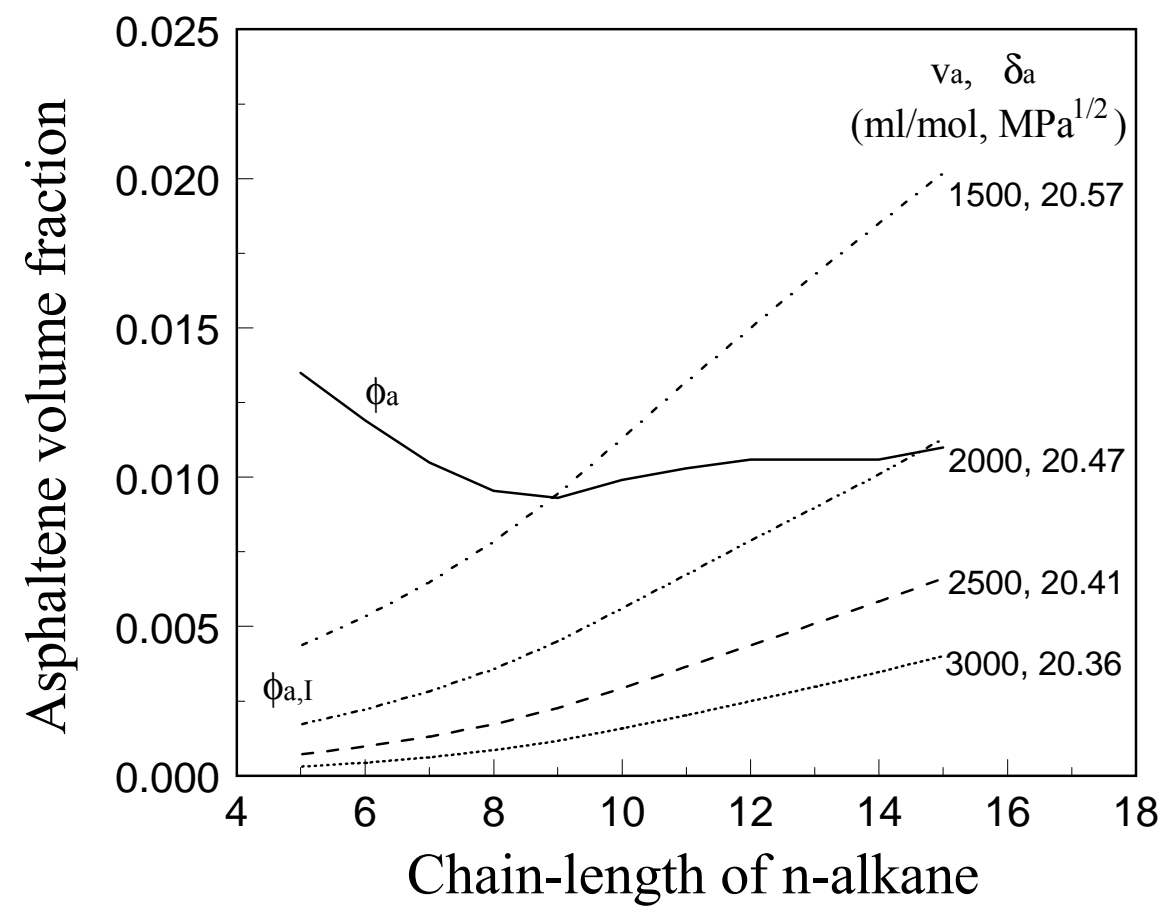

Figure I-3.24. Comparison of asphaltene volume fraction in phase $I, \phi_{a, I}$, to the average asphaltene volume fraction in the overall mixture, $\phi_{\mathbf{a}}$, at the onset point for Mars-pink crude oil indicates that there is a limit on the combination of molar volume and solubility parameter that can be used to make physically realistic predictions.

As can be seen, $\phi_{\mathrm{a}, \mathrm{I}}$ increases with decreasing asphaltene molar volume. When asphaltene molar volume is adjusted to lower than $2000 \mathrm{ml} / \mathrm{mol}, \phi_{\mathrm{a}, \mathrm{I}}$ becomes higher than $\phi_{\mathrm{a}}$ for some precipitants. This is obviously impossible because mass conservation requires that asphaltene content remaining in the solution (phase I) must be lower than the overall asphaltene content in the mixture, setting limits on asphaltene molar volume. Correspondingly, asphaltene solubility parameter can not exceed $20.47 \mathrm{MPa}^{1 / 2}$ in this case. 
Lower limit of asphaltene solubility parameter

A family of solutions, each consisting of a value of asphaltene molar volume $\left(\mathrm{v}_{\mathrm{a}}\right)$ and solubility parameter $\left(\delta_{\mathrm{a}}\right)$, all of which produce matches to measured $\mathrm{P}_{\mathrm{RI}}$ for Mars-pink crude oil, are shown in Fig. I-3.25. As discussed above, asphaltene molar volume for Mars-pink crude oil cannot be lower than $2000 \mathrm{ml} / \mathrm{mol}$. With increasing molar volume, solubility parameter must be reduced. When molar volume is set higher than approximately $10,000 \mathrm{ml} / \mathrm{mol}$, the modeling results become very insensitive to asphaltene molar volume. The curve levels off at the higher molar volume end, corresponding to a lower limit of the asphaltene solubility parameter of about $20.07 \mathrm{MPa}^{1 / 2}$.

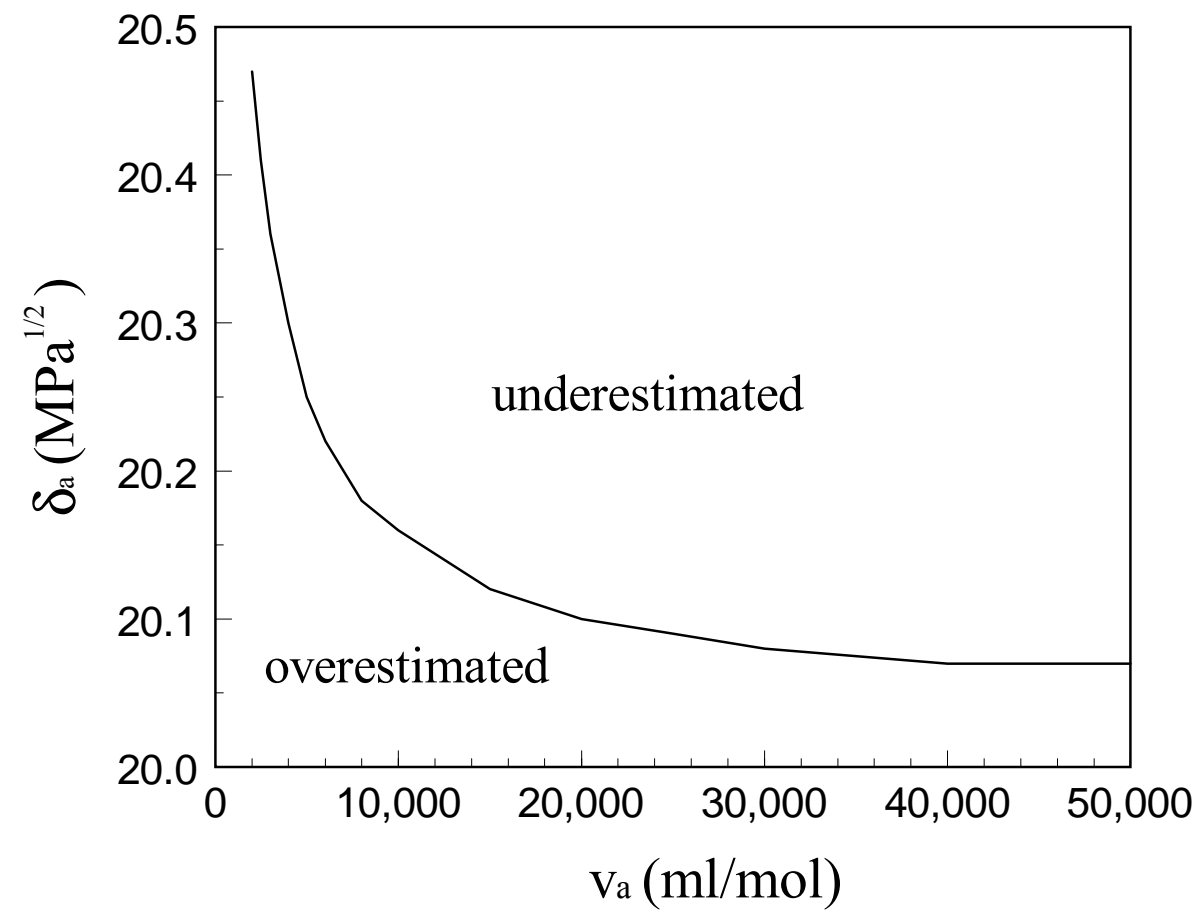

Figure I-3.25. Pairs of asphaltene molar volume, $v_{a}$, and solubility parameter, $\delta_{a}$, that best match the predicted onset $P_{R I}$ to measured ones. Beyond this line, precipitant volume fraction is underestimated with respect to the onset condition, while under this line it is overestimated (Marspink crude oil).

Adjusting asphaltene properties

Based on the above observations, a strategy for adjusting asphaltene properties can be outlined:

1. Use the same molar volume and solubility parameter of asphaltene for all different n-alkane precipitants for a given crude oil or asphaltene solution.

2. Starting from a low value of asphaltene molar volume, adjust the asphaltene solubility parameter until a good match to the measured onset $\mathrm{P}_{\mathrm{RI}}$ is achieved. Calculate the asphaltene 
volume fraction in phase I and compare it to the average asphaltene volume fraction in the mixture. If the former is higher than the later, the given asphaltene molar volume cannot be accepted. Adjust the asphaltene molar volume to a higher value and repeat tests of solubility parameter values until another match is obtained.

3. Repeat this process until a good match to $P_{R I}$ with conservation of mass is attained.

4. Apply the molar volume and solubility parameter values to asphaltenes in other tests with the same oil or same asphaltene. Satisfactory results should be obtained with little or no further adjustments in the solubility parameter and molar volume. If not, repeat the whole process until asphaltene properties are found that adequately fit all of the available experimental data.

\section{Typical modeling results}

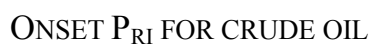

In a series of tests with six crude oils, the onset of asphaltene flocculation was determined by addition of the n-alkanes from pentane to pentadecane. Table I-3.8 summarizes the physical properties of these crude oils. Four of the oil samples originally contained asphaltene aggregates. 1-Methylnaphthalene was mixed with those oils to disperse the aggregates in order to avoid ambiguity during onset observations. Addition of 1-methylnaphthalene should affect onset $\mathrm{P}_{\mathrm{RI}}$ only slightly (Buckley et al., 1998; Wang, 2000). The onset of flocculation was observed microscopically one day after mixing crude oil with precipitant and is reported in terms of the RI of the onset mixture $\left(\mathrm{P}_{\mathrm{RI}}\right)$. RI was measured using an Index Instruments GPR 11-37 refractometer.

Table I-3.8. Measured properties of crude oils and the adjusted properties for asphaltene at $20^{\circ} \mathrm{C}$ and atmospheric pressure

\begin{tabular}{|l|c|c|c|c|c|c|c|}
\hline Crude oil & $\begin{array}{c}\text { n-C7 asph. } \\
(\mathrm{wt} \%)\end{array}$ & RI & $\rho(\mathrm{g} / \mathrm{ml})$ & $\mathrm{v}(\mathrm{ml} / \mathrm{mol})$ & $\begin{array}{c}\text { 1-MN } \\
\text { added (\%) }\end{array}$ & $\begin{array}{c}\delta_{\mathrm{a}} \\
\left(\begin{array}{c}\text { (rude oil) } \\
\left(\mathrm{MPa}^{1 / 2}\right)\end{array}\right.\end{array}$ & $\begin{array}{c}\delta_{\mathrm{a}} \\
\left(\begin{array}{c}\text { asphaltene }) \\
\left(\mathrm{MPa}^{1 / 2}\right)\end{array}\right.\end{array}$ \\
\hline A-93 & 6.87 & 1.5220 & 0.8911 & 287 & 30 & 20.60 & 21.15 \\
\hline A-95 & 8.67 & 1.5159 & 0.8879 & 305 & 30 & 20.80 & 21.40 \\
\hline Lagrave & 6.69 & 1.4832 & 0.8136 & 231 & 30 & 21.28 & 21.64 \\
\hline Lost Hills & 2.78 & 1.5137 & 0.9173 & 289 & 0 & 20.32 & - \\
\hline Mars-pink & 4.40 & 1.5380 & 0.8805 & 319 & 0 & 20.41 & 21.25 \\
\hline Tensleep & 3.20 & 1.4880 & 0.8685 & 317 & 35 & 20.92 & 21.90 \\
\hline
\end{tabular}

Typical results are shown in Fig. I-3.26 for onsets associated with Mars-pink crude oil. Two experimental observations are presented: the first appearance of fine particles and the appearance of asphaltene aggregates. With asphaltene properties set to $\mathrm{v}_{\mathrm{a}}=2500 \mathrm{ml} / \mathrm{mol}$ and $\delta_{\mathrm{a}}=20.41$ $\mathrm{MPa}^{1 / 2}$, the calculated values for the visible onset criteria match the experimentally measured aggregate onset values of $\mathrm{P}_{\mathrm{RI}}$. The predicted metastable boundary occurs at much higher RI values, suggesting that there is the potential for flocculation well before the asphaltenes become 
visible. The region between the metastable boundary and the visible onset line represents a zone within which asphaltenes might be unstable with respect to flocculation, while asphaltenes will be completely stable above the metastable boundary. The measured onset for fine particles is much lower than this metastable boundary. The difference between the metastable boundary and visible onset lines decreases with increasing chain-length of n-alkane precipitants, as does the difference between fine and aggregate onsets. Note that while the magnitudes of the predicted $\mathrm{P}_{\mathrm{RI}}$ values could be varied by choosing different values of $\mathrm{v}_{\mathrm{a}}$ and $\delta_{\mathrm{a}}$, the slope of the line depends primarily on the onset criterion. Thus, we cannot find another set of asphaltene parameters that will match, for example, the metastable boundary to experimentally observed onset values of $\mathrm{P}_{\mathrm{RI}}$ because the slope of the predicted line is wrong.

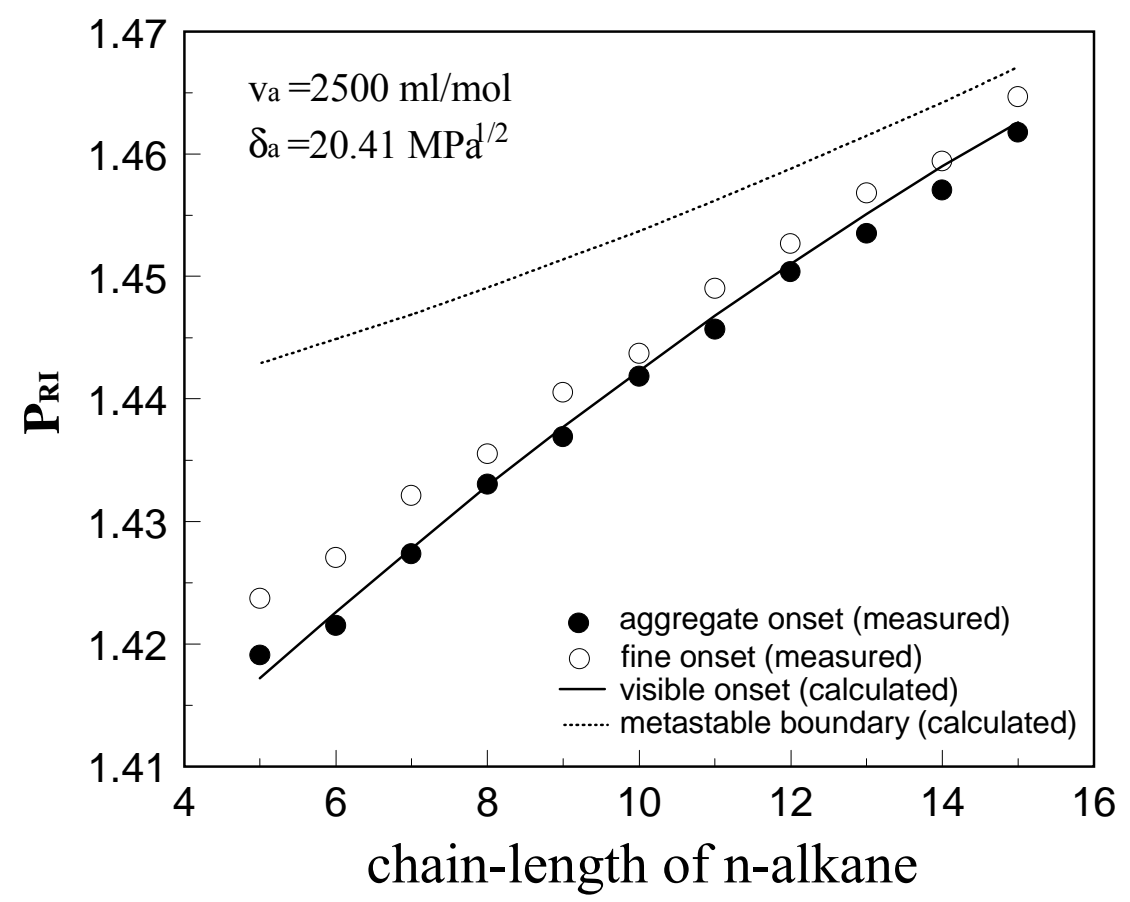

Figure I-3.26. For Mars-pink crude oil, a good match for the calculated visible onset to measured aggregate onset is achieved when appropriate asphaltene properties are selected. Measured fine onset falls within the metastable region.

Measured $\mathrm{P}_{\mathrm{RI}}$ for onsets of asphaltene aggregates for all six crude oils are summarized in Fig. I-3.27. Using fixed properties for the crude oils and precipitants, the asphaltene properties (molar volume $\mathrm{v}_{\mathrm{a}}$ and solubility parameter $\delta_{\mathrm{a}}$ ) were adjusted to match predicted values of $\mathrm{P}_{\mathrm{RI}}$ to the measured ones. The best matches for each oil are shown in Fig. I-3.27. A constant value of $v_{a}$ of $2500 \mathrm{ml} / \mathrm{mol}$ was used for all these calculations. Good matches were obtained with $\delta_{a}$ values between 20.32 and $21.28 \mathrm{MPa}^{1 / 2}$ (Table I-3.8), consistent with values reported in the literature (e.g., Hirschberg et al., 1984; Burke et al., 1988). 


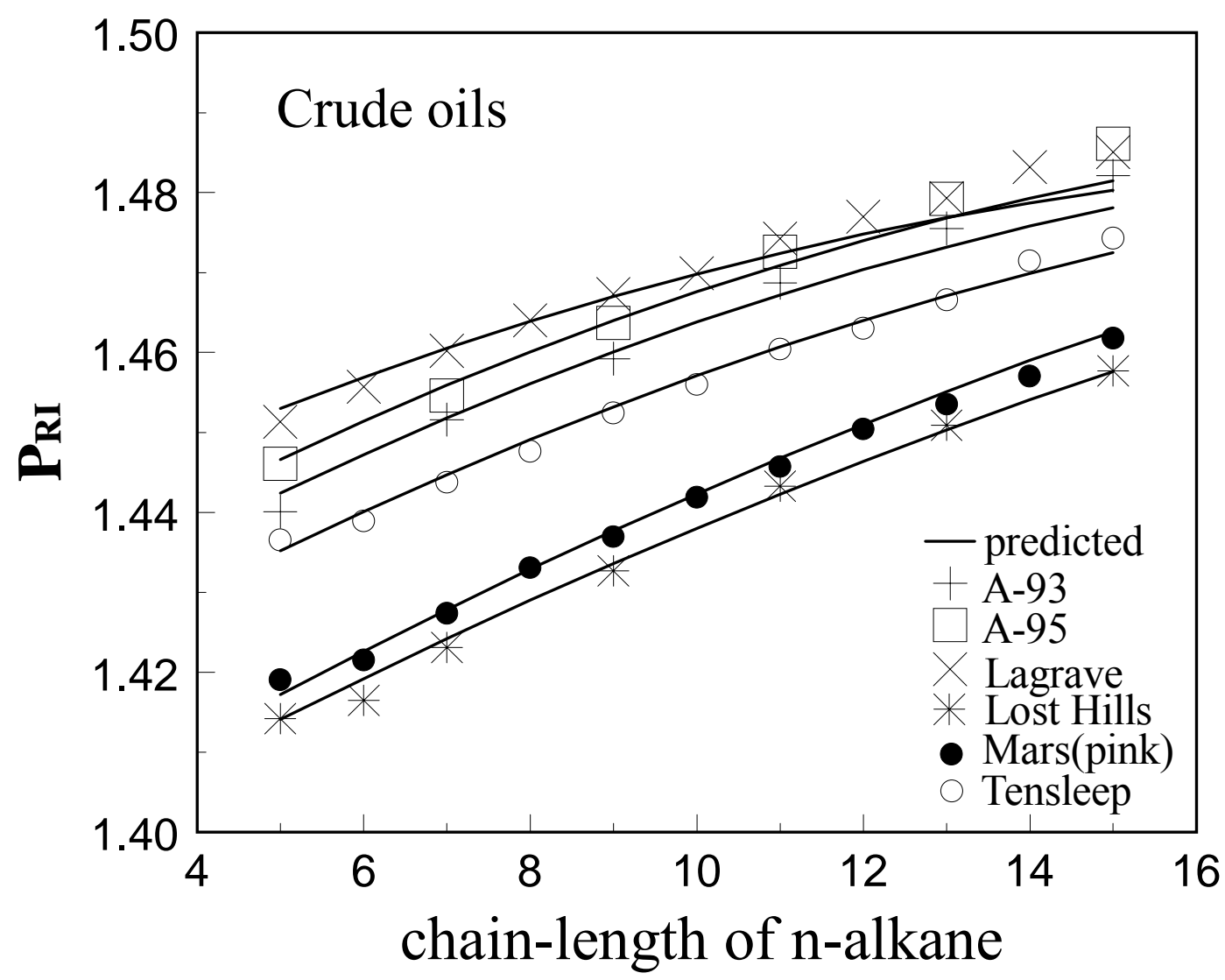

Figure I-3.27. Measured and predicted onset conditions for flocculation from crude oils induced by precipitants from n-pentane to n-pentadecane. Asphaltene molar volume $=2500 \mathrm{ml} / \mathrm{mol}$. Other model parameters are given in Table 2.

\section{ONSET FOR N-HEPTANE ASPHALTENES IN TOLUENE}

Asphaltenes were separated from five of the crude oils by addition of an excess of n-heptane (ASTM, 1980). These asphaltenes were redissolved in toluene at $1 \mathrm{wt} \%$. Onset measurements were repeated for these mixtures of asphaltenes in toluene; $\mathrm{P}_{\mathrm{RI}}$ values are shown in Fig. I-3.28. Using the same constant value of $\mathrm{v}_{\mathrm{a}}(2500 \mathrm{ml} / \mathrm{mol})$, excellent matches were obtained between calculated $\mathrm{P}_{\mathrm{RI}}$ and the measured values (Fig. I-3.28) with $\delta_{\mathrm{a}}$ varying from 21.15 to $21.90 \mathrm{MPa}^{1 / 2}$ (Table I-3.8). The fits gave values of solubility parameters for the $n$-heptane asphaltenes that were consistently higher than those for the whole crude oil asphaltenes. In some cases, e.g., Mars-pink, onsets from the asphaltene solutions occur under conditions that are similar to those of the original oil, but in many cases there are substantial differences. 


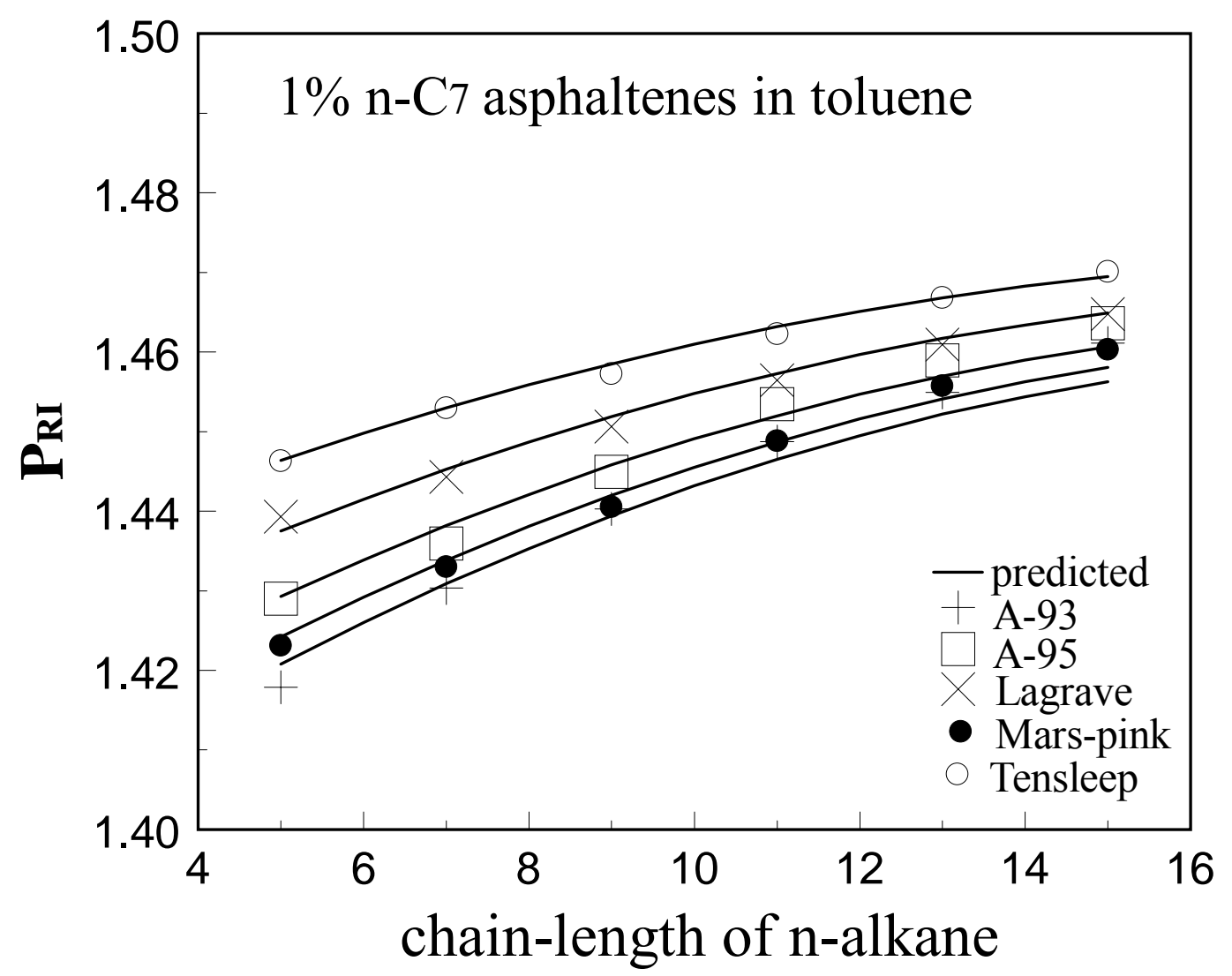

Figure I-3.28. Good matches can be obtained between calculated visible onsets and measured aggregate onsets for all 1\% asphaltene solutions in toluene. Asphaltene molar volume is held constant, while the solubility parameter is adjusted.

\section{Comparison with other approaches in literature}

Both the pure asphaltene approach (Hirschberg et al., 1984) and the pure solvent approach (Cimino et al., 1995) have been used to match the onset conditions for asphaltene flocculation from Mars-pink, for comparison with the results from the approach developed here, as shown in Fig. I-3.29. Asphaltene molar volume was set to $2500 \mathrm{ml} / \mathrm{mol}$, while the asphaltene solubility parameter was adjusted to get the best matches that each model could achieve. To facilitate comparison, all the predicted onset $\mathrm{P}_{\mathrm{RI}}$ curves were forced to match the experimental data for the lowest carbon-number precipitants.

As discussed above, the pure asphaltene approach sets the onset as the point when asphaltene content equals calculated asphaltene solubility. This to some extent is analogous to the metastable boundary point discussed above. Results in Fig. I-3.29 show that the onsets calculated by the pure-asphaltene approach and the metastable boundary line from the full FloryHuggins approach have similar slopes that deviate significantly from the measured onset $\mathrm{P}_{\mathrm{RI}}$. 


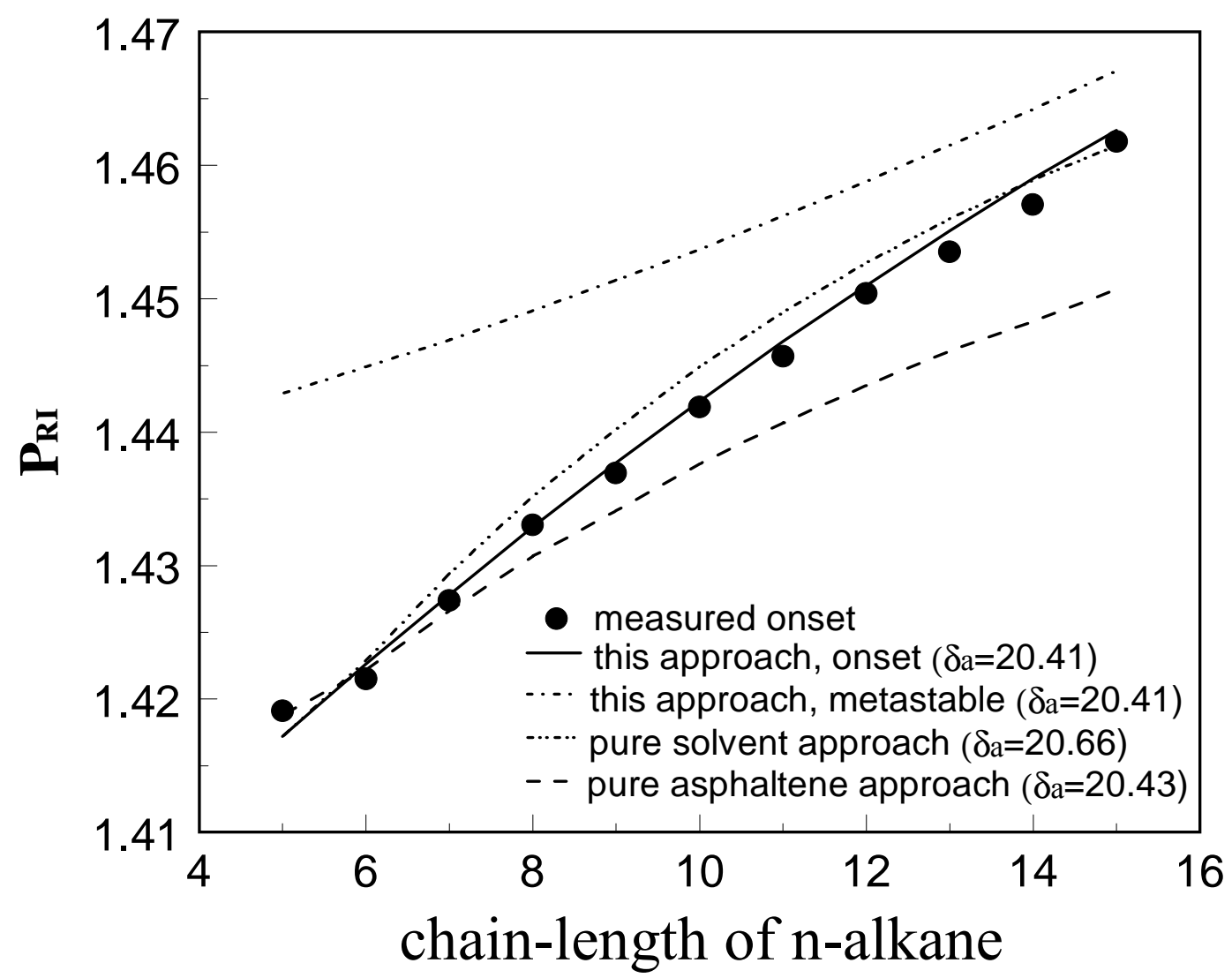

Figure I-3.29. Comparisons of predicted results from three different approaches (Mars-pink crude oil, $\mathbf{v}_{\mathrm{a}}=\mathbf{2 5 0 0} \mathrm{ml} / \mathbf{m o l}$ ).

On the other hand, the pure solvent approach uses a constant value (0.8) of asphaltene volume fraction in the asphaltene-rich phase (phase II) as the onset criteria. This arbitrary value unnecessarily restricts the prediction of the onset conditions. In the example shown in Fig. I3.29 , it produces almost as good a fit to the data, but it cannot be used to predict the amount of asphaltene that will precipitate, an extension which may be possible with the full Flory-Huggins model.

It has been observed from many calculations with this new model that asphaltene volume fraction in phase I, $\phi_{\mathrm{a}, \mathrm{I}}$, frequently is about $10^{-3}$ near the onset point, but never goes to zero, as assumed in the pure solvent approach. Asphaltene volume fraction in phase II at onset point, $\phi_{\mathrm{a}, \mathrm{II}}$, is found to be around 0.71-0.75 for crude oils and for most asphaltene solutions. $\phi_{\mathrm{a}, \mathrm{II}}$ increases slightly as the precipitant changes from $\mathrm{n}-\mathrm{C} 5$ to $\mathrm{n}-\mathrm{C} 15$. This differs from the constant value of $\phi_{a, I I}(0.8)$ assumed in pure solvent approach. The assumption made in the pure asphaltene approach - that a solid (100\%) asphaltene is nucleated at the onset - is even less consistent with observations. 


\section{Conclusions}

1. A thermodynamic model has been rigorously derived from Flory-Huggins polymer theory, without any arbitrary assumptions about the compositions of the two phases formed when the asphaltenes separate from the oil. In principle, this model is fully predictive once parameters are established for asphaltene properties.

2. Numerical solutions of this model result in three points with respect to asphaltene stability: the metastable boundary at which potential asphaltene flocculation might occur, the visible onset point where asphaltenes can be observed, and the absolute instability point at which immediate flocculation will occur. There is a transition zone between the potential flocculation point and the visible onset point. Asphaltene will be free from flocculation if solution conditions are in the region beyond the metastable boundary.

3. There exists a lower limit for asphaltene molar volume below which no realistic prediction for onset of asphaltene flocculation could be obtained. Too high a value of asphaltene molar volume, on the other hand, will result in a wider transition zone between metastable and visible onset conditions.

4. Predicted visible onset conditions match measured aggregate onset conditions quite well for crude oils, and even better for asphaltene solutions in toluene.

5. The full Flory-Huggins model is better able to match experimental observation than are existing models that make simplifying assumptions about compositions of either the asphaltene or non-asphaltene phase.

\section{References}

Barton, Allan F.M.: CRC Handbook of Solubility Parameters and Other Cohesion Parameters, $2^{\text {nd }}$ ed. CRC Press, Boca Raton (1991).

Buckley, J.S., Hirasaki, G.J., Liu, Y., Von Drasek, S., Wang, J.X., and Gill, B.S.: “Asphaltene Precipitation and Solvent Properties of Crude Oils," Petroleum Science and Technology (1998) 16, No. 3\&4, 251-285.

Burke, N.E., Hobbs, R.D., and Kashou, S.F.: "Measurement and Modeling of Asphaltene Precipitation,” JPT (1990) 1440-1446.

Chang, C-L, and Fogler, H.S.: "Asphaltene Stabilization in Alkyl Solvents Using Oil-Soluble Amphiphiles," paper SPE 25185, presented at the SPE International Symposium on Oilfield Chemistry held in New Orleans, LA, USA, March 2-5, 1993. 
Cimino, R., Correra, S., Del Bianco, A., and Lockhart, T.P.: "Solubility and Phase Behavior of Asphaltenes in Hydrocarbon Media," Asphaltenes: Fundamentals and Applications, E.Y. Sheu and O.C. Mullins (eds.), NY: Plenum Press (1995) 97-130.

Dahir, B., Nematry, M., and Sahimi, M.: “Asphalt Flocculation and Deposition: I. The Onset of Precipitation,”AIChE J. (Jan., 1996) 42, 10.

Flory, P.J.: "Thermodynamics of High Polymer Solutions,” J. Chem. Phys., 10, 51, 1942.

Hirschberg, A., deJong, L.N.J., Schipper, B.A., and Meijer, J.G.: "Influence of Temperature and Pressure on Asphaltene Flocculation,” paper SPE 11202, SPEJ, 283-293, June, 1984.

Huggins, M.L.: "Solutions of Long Chain Compounds,” J. Chem. Phys., 9, 440, 1941.

Hunter, R.J.: Foundations of Colloid Science, Vol. I, Clarendon Press, Oxford, New York, 1991.

Leontaritis, K.J., and Mansoori, G.A.: "Asphaltene Flocculation During Oil Production and Processing: A Thermodynamic Colloidal Model," paper SPE 16258, presented at the SPE International Symposium on Oilfield Chemistry held in San Antonio, Texas, 4-5 Feb., 1987.

Leontaritis, K.J.: "Asphaltene Deposition: A Comprehensive Description of Problem Manifestations, and Modeling Approaches," paper SPE 18892, 1989 SPE Production Operation Symposium, SPE, Richardson, TX.

MacMillan, D.J., Tackett, J.E., Jessee, M.A. and Monger-McClure, T.G., A unified approach to asphaltene precipitation: Laboratory measurement and modeling, JPT(1995) 47, 788-795

Nghiem, L.X., Hassam, M.S., and Nutakki, R.: "Efficient Modeling of Asphaltene Precipitation," paper SPE 26642, presented at $68^{\text {th }}$ Annual Technical Conference and Exhibition of the Society of Petroleum Engineers hold in Houston, Texas, 3-6 Oct., 1993.

Nghiem, L.X., and Coombe, D.A.: "Modeling Asphaltene Precipitation During Primary Depletion," paper SPE 36106, SPEJ, vol.2, Jun. 1997.

Nghiem, L.X., Coombe, D.A., and Farouq Ali, S.M.: "Compositional Simulation of Asphaltene Deposition and Plugging," paper SPE 48996, presented at the 1998 SPE Annual Technical Conference and Exhibition held in New Orleans, Louisiana, 27-30, Sept., 1998.

Nor-Azian, N., and Adewumi, M.A.,: "Development of Asphaltene Phase Equilibria Predictive Model," paper SPE 26905, presented at the 1993 Eastern Regional Conference \& Exhibition held in Pittsburgh, PA, USA., 2-4 November, 1993.

Novosad, Z., and Costain, T.G.: "Experimental and Modeling Studies of Asphaltene Equilibria for a Reservoir Under $\mathrm{CO}_{2}$ Injection," paper SPE 20530, presented at the $65^{\text {th }}$ Annual Technical Conference and Exhibition held in New Orleans, LA, Sept. 23-26, 1990.

Pan, H. and Firoozabadi, A.: "A Thermodynamic Micellization Model for Asphaltene Precipitation: Part I: Micellar Size and Growth,” paper SPE 36741, presented at the 1996 ATCE, Denver, 6-9 Oct.

Shinoda, K. and Hildebrand, J.H.: "The Solubility and Entropy of Solution of Iodine in Octamethylcyclotetrasiloxane and Tetraethoxysiloxane,” J. Phys. Chem., 61, 789, 1957.

Tompa, H.: Polymer Solutions, Academic Press Inc. Publishers, New York, 1956. 
Wang, J.X.: "Predicting Asphaltene Flocculation in Crude Oils," PhD dissertation, New Mexico Institute of Mining \& Technology, April, 2000.

Weast, R.C.: CRC Handbook of Chemistry and Physics, $68^{\text {th }}$ ed. (1987) CRC Press, Inc.

Yang, Z., Ma, C-F, Lin, X-S, Yang, J-T, Guo, T-M: "Experimental and Modeling Studies on the Asphaltene Precipitation in Degassed and Gas-Injected Reservoir Oils," Fluid Phase Equilibria, 157 (1999) 143-158. 


\section{I-3.4. Asphaltene Stability in Other Crude Oil Fractions}

Wang, J.X. and Buckley, J.S.: “Asphaltene Stability in Other Crude Oil Fractions," paper 49a presented at the 2002 Spring AIChE Symposium, New Orleans, 10-14 Mar.

Changes in oil composition are unavoidable during production, transportation, and processing of crude oils. Questions about the effects of these changes on asphaltene stability have been addressed in this systematic study of different crude oils, asphaltene fractions, resins, and a range of hydrocarbon solvents and precipitants. The refractive index of onset mixtures is used to quantify asphaltene stability and the results are analyzed with an asphaltene solubility model.

The results show that working with highly diluted oil samples or with isolated fractions of asphaltenes can lead to inaccurate predictions of the onset of asphaltene flocculation in their original crude oil. Toluene acts as an ideal solvent, but $\alpha$-methylnaphthalene appears to interact with asphaltenes in solution. From a given crude oil, asphaltene fractions separated with higher molecular weight paraffins are more unstable than those separated by lower molecular weight precipitants. When resins are added, the changes in stability are continuous; there is no critical amount of resin that imparts stability. Instead, resins appear to be part of the continuum of species in which asphaltenes represent one end of the spectrum. Stability of asphaltenes in blended oils is intermediate between the two oils in the mixture.

\section{Background}

Crude oils are complex mixtures of hydrocarbons and other compounds. Often these components are divided, somewhat arbitrarily, into saturates, aromatics, resins, and asphaltenes. Asphaltenes are, by definition, insoluble in the lower molecular weight saturates and are soluble in toluene. Other interactions among these fractions have been the subject of a great deal of speculation, but many questions remain.

A widely used approach to modeling asphaltene stability is based on regular solution theory, as adapted by the Flory-Huggins approach to account for large size differences between solute and solvent (Hirschberg, 1984). The onset condition can be quantified by the solubility parameter $(\delta)$ of mixtures at the onset of asphaltene flocculation. In previous studies we have shown that the refractive index (RI) of a solution can be measured in place of $\delta$ as an indicator of asphaltene stability (Buckley et al., 1998). RI and $\delta$ can be interconverted by taking advantage of a simple empirical correlation between $\mathrm{F}_{\mathrm{RI}}$ and $\delta$ that is generally applicable to all nonpolar hydrocarbons at standard conditions:

$$
\delta=52.042 F_{R I}+2.904
$$

where $\mathrm{F}_{\mathrm{RI}}$ is defined as:

$$
F_{R I}=\left(R I^{2}-1\right) /\left(R I^{2}+2\right)
$$


The main advantage of this approach lies in the fact that RI is readily measured whereas solubility parameters must be estimated for complex mixtures. Both $\delta$ and $F_{R I}$ follow simple additivity rules, with volume fraction weighting of each component (or pseudo-component) in a mixture, so that

$$
F_{R I}(\text { mixture })=\sum_{i} \phi_{i} F_{R I}(i)
$$

where $\phi_{i}$ is the volume fraction of component $i$. Further, it has been shown that onset conditions, as characterized by RI of the solution at onset (denoted as $\mathrm{P}_{\mathrm{RI}}$ ), follow a simple, linearly increasing trend with the molar volume of the precipitating agent raised to the $1 / 2$ power $\left(\mathrm{v}_{\mathrm{p}}{ }^{1 / 2}\right)$ (Wang and Buckley, 2001a).

Estimation of the onset solubility parameter by diluting oil with toluene, titrating with heptane, then using the amounts of these known compounds to calculate the solubility parameter at the onset is widely practiced (e.g., Cimino et al., 1995 and references cited therein), but the implicit assumption - that infinite dilution has no effect on the critical solubility conditions - has never been verified. $\mathrm{P}_{\mathrm{RI}}$ measurements can be used to investigate the effects of solvents on the stability of asphaltenes.

A major topic of debate has been the role that the resin fraction plays in stabilizing asphaltene. The view that resins "peptize" asphaltenes by forming a coating around them has widely been assumed throughout the asphaltene literature. A critical resin concentration has been suggested as the basis for quantifying asphaltene stability, based on this view of specific interactions between the asphaltene and resin fractions (Leontaritis and Mansoori, 1987). Models of asphaltenes and resins as inverse micelles are based on similar assumptions (Andersen and Birdi, 1991; Carnahan et al., 1993). A contrary view holds that specific interactions between asphaltenes and resins are not required to explain asphaltene stability (Cimino et al., 1995), that resins are primarily good asphaltene solvents, and that non-polar van der Waals forces are primarily responsible for flocculation of asphaltenes (Buckley et al., 1998). Beyond the questions of stabilization mechanisms, which are important with regard to physically meaningful modeling, there are practical concerns about the possibility that two crude oils, both of which are stable, might together be unstable. If specific asphaltene-resin interactions account for asphaltene stability, resins from one oil might be ineffective as stabilizing agents for asphaltenes from another oil and mixing of oils might well have unexpected results. These are all questions that can be investigated by measurements of the RI at the onset of asphaltene flocculation.

\section{Experimental Materials and Procedures}

Crude Oils. Depressurized crude oil samples from a number of different sources were tested in this study. Properties of these oils are summarized in Table 1. Tensleep crude oil contained aggregated asphaltenes that would have interfered with onset tests. This oil was diluted with 35 vol \% of $\alpha$-methylnaphthalene (AMN) and stirred for two days to dissolve preexisting asphaltenes. Tensleep properties in Table I-3.9 are given for the oil before addition of AMN 
Table I-3.9. Oil and solvent properties

\begin{tabular}{|c|c|c|c|c|c|}
\hline Oil ID & API gravity & $\begin{array}{c}\text { n- } C_{5} \text { asphaltene } \\
(w t \%)\end{array}$ & $\begin{array}{c}\text { MW } \\
(\mathrm{g} / \mathrm{mol})\end{array}$ & $\begin{array}{c}\rho @{ }^{\rho} @ 20^{\circ} \mathrm{C} \\
(\mathrm{g} / \mathrm{ml})\end{array}$ & $\mathbf{R I} @ 2^{\circ} \mathbf{C}$ \\
\hline C-HD-01 & 20.7 & 4.8 & 346 & 0.9264 & 1.5220 \\
\hline C-HL-01 & 29.2 & 3.7 & 259 & 0.8771 & 1.4920 \\
\hline Lagrave & 41.3 & 7.5 & 231 & 0.8136 & 1.4832 \\
\hline Lost Hills & 22.6 & 3.4 & 265 & 0.9173 & 1.5142 \\
\hline Mars-Pink & 16.5 & 6.2 & 305 & 0.9514 & 1.5388 \\
\hline SQ-95 & 37.2 & 4.3 & 213 & 0.8517 & 1.4801 \\
\hline SQ-95 n- $\mathrm{C}_{5}$ resins & 9.9 & not applicable & 231 & 0.9974 & 1.5804 \\
\hline Tensleep & 31.1 & 4.0 & 275 & 0.8685 & 1.4880 \\
\hline toluene & 31.1 & not applicable & 92 & 0.8670 & 1.4964 \\
\hline AMN & 6.4 & not applicable & 142 & 1.0225 & 1.6156 \\
\hline
\end{tabular}

Solvents and Precipitants. Normal paraffins from $\mathrm{n}-\mathrm{C}_{5}$ through $\mathrm{n}-\mathrm{C}_{15}$ were used as precipitants to initiate asphaltene flocculation. Two aromatics, toluene and AMN, were used as asphaltene solvents.

Onset of Asphaltene Flocculation. The onset of asphaltene flocculation was determined for a series of $n$-alkanes, from $n$-pentane $\left(n-C_{5}\right)$ through $n$-pentadecane $\left(n-C_{15}\right)$. For each oil $/ n$ alkane pair, a series of oil and precipitant mixtures were prepared in 1-vol \% increments in small glass vials sealed with Teflon-lined aluminum caps. The mixtures were examined microscopically after 24 hours of equilibration at a magnification of 320X. The onset is defined as the mixture with the minimum amount of precipitant in which aggregated asphaltene particles appear. $\mathrm{P}_{\mathrm{RI}}$ is the RI of that mixture. Temperature was maintained at $20^{\circ} \mathrm{C}$ for all onset tests in this study.

Refractive Index Measurement. RI was measured with an automatic refractometer (Index Instruments model GPR 11-37) at $20^{\circ} \mathrm{C}$. The sample chamber was sealed to prevent evaporation. Accuracy is $\pm 0.00005 \mathrm{RI}$ units for RI values less than 1.45 , and \pm 0.0001 for RI values greater than 1.45 .

Separation of Asphaltenes. Asphaltene separation followed the standard procedure recommended in ASTM D2007-93. Mixtures of one part oil to 40 parts n-paraffin were allowed to age for two days before filtration through $0.22 \mu \mathrm{m}$ cellulose acetate membrane filters, followed by air drying and weighing. When $\mathrm{n}-\mathrm{C}_{15}$ was used to precipitate asphaltenes, drying was extremely slow due to the low volatility of $n-C_{15}$. These samples were redissolved in $20 \mathrm{ml}$ of toluene, and asphaltenes were re-precipitated by adding $40: 1 \mathrm{n}-\mathrm{C}_{5}$ to the toluene solution.

Separation of Resins. Resins were recovered from a column separation of oil deasphalted with n-pentane as specified in ASTM D2007-93.

Molecular Weight and Molar Volume. Average molecular weights were determined from freezing point depression measurements using a Cryoscope 5009 from Precision Systems. Conversion to molar volumes required measurement of the density, which was obtained using a Mettler/Paar DMA 40 digital densitometer with temperature maintained at $20^{\circ} \mathrm{C}$. 


\section{Results and Discussion}

Effect of solvents on asphaltene stability in crude oil

Mixtures of SQ-95 crude oil were prepared with 20, 40, 60, and 80 vol \% toluene and AMN. The mixtures were well stirred and allowed to equilibrate for two days, after which the onset of asphaltene flocculation was determined for each of these mixtures with a series of nalkanes, as shown in Fig. I-3.30. Calculations using an asphaltene solubility model known as ASM (Wang and Buckley, 2001b) are shown as solid lines. The model parameters are asphaltene molar volume $\left(\mathrm{v}_{\mathrm{a}}\right)$ and the asphaltene solubility parameter $\left(\delta_{\mathrm{a}}\right)$. These parameters are not independent; a family of solutions can be found if both are varied. If $\mathrm{v}_{\mathrm{a}}$ is set arbitrarily at $2500 \mathrm{ml} / \mathrm{mol}$, however, values of $\delta_{\mathrm{a}}$ can be obtained that are comparable on a relative scale. Using the data for toluene solutions (Fig. 1a), a value of $20.22 \mathrm{MPa}^{1 / 2}$ was determined for $\delta_{\mathrm{a}}$.

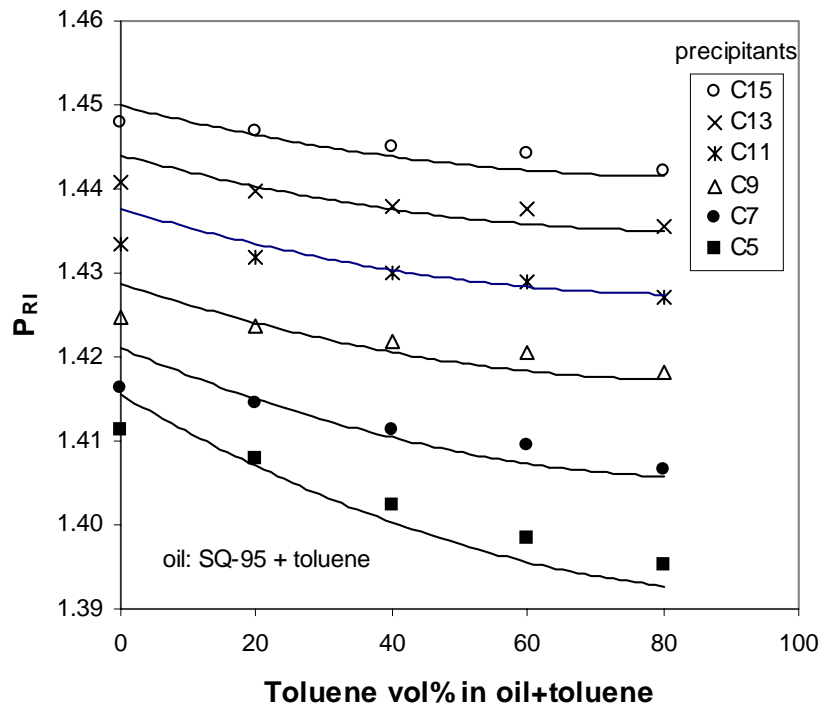

(a) Addition of toluene reduces $\mathrm{P}_{\mathrm{RI}}$ for SQ-95 crude oil in accordance with ASM.

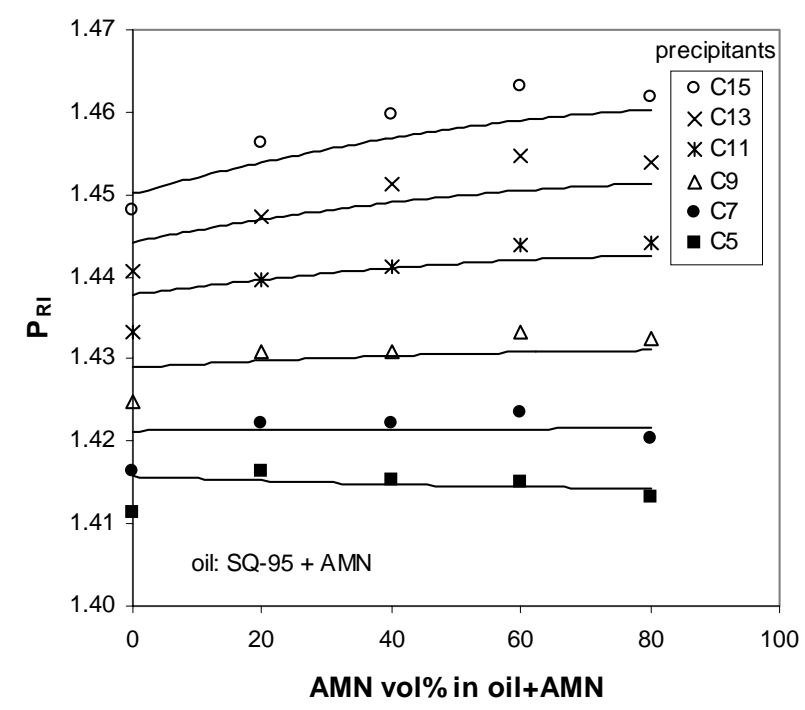

(b) Addition of AMN affects $\mathrm{P}_{\mathrm{RI}}$ of SQ-95 as if it had a much higher than expected molar volume.

Figure I-3.30. Addition of solvent to SQ-95 crude oil can change the onset $\mathbf{P}_{\mathrm{RI}}$. For precipitation with nheptane, shifts are greater with toluene than AMN. Lines are ASM calculations.

As reported previously (Wang and Buckley, 2001b), applying the asphaltene parameters determined in the toluene experiments to the onsets from AMN solutions consistently produces predictions of much lower $\mathrm{P}_{\mathrm{RI}}$ values than observed. The results can be explained (as shown in Fig. I-3.30b) if AMN interacts with the asphaltenes in a way that toluene does not. The calculations shown as solid lines in Fig. I-3.30b were made using asphaltene parameters determined from the toluene experiments and assuming that effective molar volume of AMN $\left(\mathrm{v}_{\mathrm{AMN}}\right)$ is not $139 \mathrm{ml} / \mathrm{mol}$, the value measured for pure $\mathrm{AMN}$, but is much higher, on the order of $500 \mathrm{ml} / \mathrm{mol}$. Asphaltenes exist as aggregates, even in good solvents (Espinat and Ravey, 1993). If AMN can participate in the aggregate stacking, its effective molar volume in solutions 
containing asphaltene might be quite different than in ideal solutions. Onset observations with AMN certainly suggest that this is the case. The data are also consistent with a value of $\delta_{\mathrm{AMN}}$ of about $19 \mathrm{MPa}^{1 / 2}$, but it is not clear why the solubility parameter of AMN should be so low. Attempts to match the AMN solution data by altering the values of the asphaltene parameters were completely unsuccessful.

Agreement between model and experiment improves with increasing percentage of solvent. One explanation may be that SQ-95 is a waxy oil that is below its wax appearance temperature at room conditions. Aromatic solvents affect the solubility of waxes as well as asphaltenes. If waxes dissolve upon addition of toluene, they affect the overall oil composition.

It is important to note that at high dilution with toluene, the onset solution properties are significantly different than those obtained for the whole oil. Thus evaluations of asphaltene stability based on experiments at high dilution with toluene would be more optimistic than warranted by the actual condition of asphaltenes in oil.

\section{Onset of flocculation of asphaltene fractions in a solvent}

Asphaltenes were separated from Lagrave and Mars-Pink crude oils using n- $\mathrm{C}_{5}, \mathrm{n}-\mathrm{C}_{7}, \mathrm{n}-$ $\mathrm{C}_{9}$, and $\mathrm{n}-\mathrm{C}_{15}$ as precipitants. The amounts of asphaltene obtained are summarized in Table I3.10; as expected from the experience of others (e.g., Mitchell and Speight, 1973), the amount decreased as the molecular size of the precipitant increased.

Table I-3.10. Amount of asphaltene separated with different precipitants

\begin{tabular}{|c|c|c|c|c|c|c|c|c|}
\hline \multirow{2}{*}{ Oil } & \multicolumn{4}{|c|}{ wt \% asphaltene precipitated by } & \multicolumn{3}{c|}{ RI of 1\% asphaltene solution in toluene } \\
\cline { 2 - 9 } & $\mathbf{n - C _ { 5 }}$ & $\mathbf{n - C _ { \mathbf { 7 } }}$ & $\mathbf{n}-\mathbf{C}_{\mathbf{9}}$ & $\mathbf{n}-\mathbf{C}_{\mathbf{1 5}}$ & $\mathbf{n}-\mathbf{C}_{\mathbf{5}}$ & $\mathbf{n}_{\mathbf{7}} \mathbf{C}_{\mathbf{7}}$ & $\mathbf{n}-\mathbf{C}_{\mathbf{9}}$ & $\mathbf{n}-\mathbf{C}_{\mathbf{1 5}}$ \\
\hline Lagrave & 7.48 & 6.69 & 5.45 & 5.09 & 1.4981 & 1.4986 & 1.4979 & 1.4983 \\
\hline Mars-Pink & 6.20 & 4.40 & 3.05 & 2.94 & 1.4979 & 1.4981 & 1.4979 & 1.4980 \\
\hline
\end{tabular}

Solutions were prepared by dissolving 1 gram of each asphaltene fraction in $100 \mathrm{ml}$ toluene. The mixtures were free from particulates two days after mixing. Values of RI for these asphaltene solutions averaged $1.4982 \pm 0.0003$ for Lagrave and $1.4980 \pm 0.0001$ for Mars-Pink. Results from onset measurements are shown in Fig. I-3.31 where $\mathrm{P}_{\mathrm{RI}}$ is plotted as a function of $\mathrm{v}_{\mathrm{p}}{ }^{1 / 2}$ of $\mathrm{n}$-alkane precipitants.

Although the trends of $\mathrm{P}_{\mathrm{RI}}$ increasing with the chain-length of precipitant are similar for the various asphaltene fractions from these two oils, a significant drop in $\mathrm{P}_{\mathrm{RI}}$ is observed between the $\mathrm{n}-\mathrm{C}_{15}$ asphaltene solution and the $\mathrm{n}-\mathrm{C}_{5}$ asphaltenes that is much larger than the minor differences in RI of the initial asphaltene solutions. Asphaltenes separated with $\mathrm{n}-\mathrm{C}_{5}$ are consistently more stable than those separated with $n-C_{15}$. The $n-C_{5}$ fraction contains all of the unstable components from the $\mathrm{n}-\mathrm{C}_{15}$ asphaltenes, but the fact that it also contains a broader distribution of asphaltic material appears to stabilize the least stable components. 


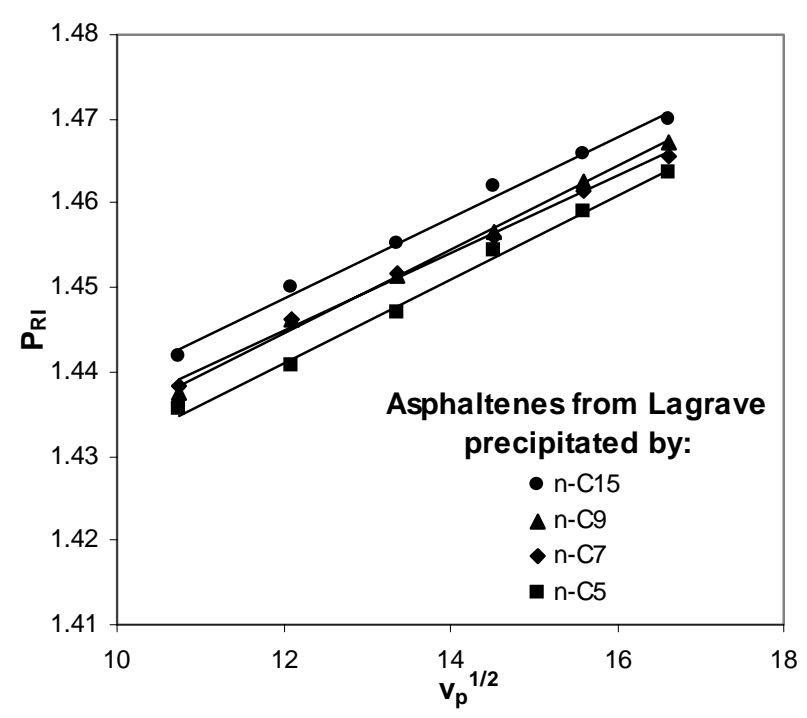

(a) Lagrave asphaltenes.

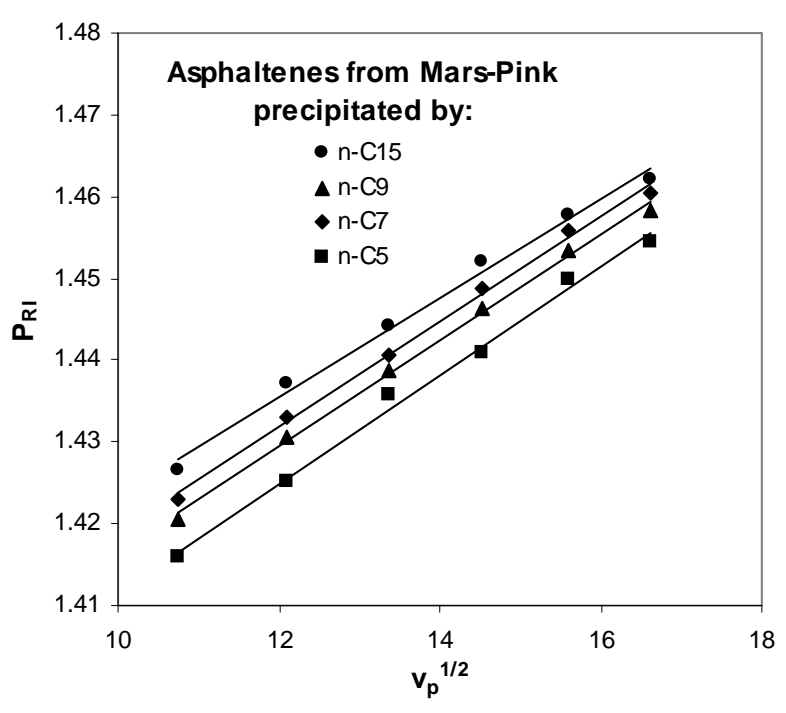

(b) Mars-Pink asphaltenes.

Figure I-3.31. $P_{R I}$ for $1 \%$ toluene solutions of asphaltenes separated from Lagrave and Mars-Pink crude oils. Stabilities of redissolved asphaltenes depend on the identity of the n-alkane used to separate them. $n-C_{5}$ asphaltenes are the most stable; $n-C_{15}$ asphaltene are least stable. Lines are linear fits to the data points.

\section{Effect of resins on asphaltene stability}

Addition of resins to asphaltene fractions increases their stability as shown in Figs. I-3.32 and I3.33. In the experiments shown in Fig. 3a, resins separated from SQ-95 crude oil were added to the toluene solutions of the $n-C_{5}$ and $n-C_{15}$ asphaltenes from Mars-Pink crude oil to give solutions that contained $1 \%$ asphaltene and $1 \%$ resin. The onset experiments were repeated using $\mathrm{n}-\mathrm{C}_{5}, \mathrm{n}-\mathrm{C}_{7}, \mathrm{n}-\mathrm{C}_{9}, \mathrm{n}-\mathrm{C}_{11}, \mathrm{n}-\mathrm{C}_{13}$ and $\mathrm{n}-\mathrm{C}_{15}$ as precipitants. The change in asphaltene stability is similar to that attributed above to broadening the distribution of components in the asphaltene fraction. It is useful to recall at this point that the distinction between asphaltenes and resins is an artificial one. Only a portion of the asphaltic material is separated from crude oil with any given precipitant (Long, 1981). Lower molecular weight precipitants separate a larger fraction of asphaltene while the larger precipitants only separate the heaviest, most polar and/or aromatic asphaltenes. Standardizing on n-pentane or n-heptane helps to reduce imprecision in discussions of asphaltenes, but these are imposed standards that have no chemical significance. Figure $3 \mathrm{~b}$ shows that the onset trends for a $1 \%$ toluene solution of its $n-C_{9}$ asphaltenes and for the toluene solution with $1 \% \mathrm{n}-\mathrm{C}_{15}$ Mars-Pink asphaltenes and 1\% SQ-95 resins are indistinguishable from that for the original Mars-Pink crude oil sample. It is not generally true that $\mathrm{P}_{\mathrm{RI}}$ values for an asphaltene fraction match those from the crude oil. In fact they can be quite different. Precipitation from Lagrave oil occurs at significantly higher values of $P_{R I}$ than measured for any of the fractions shown in Fig. 2a (Wang and Buckley, 2001b). 


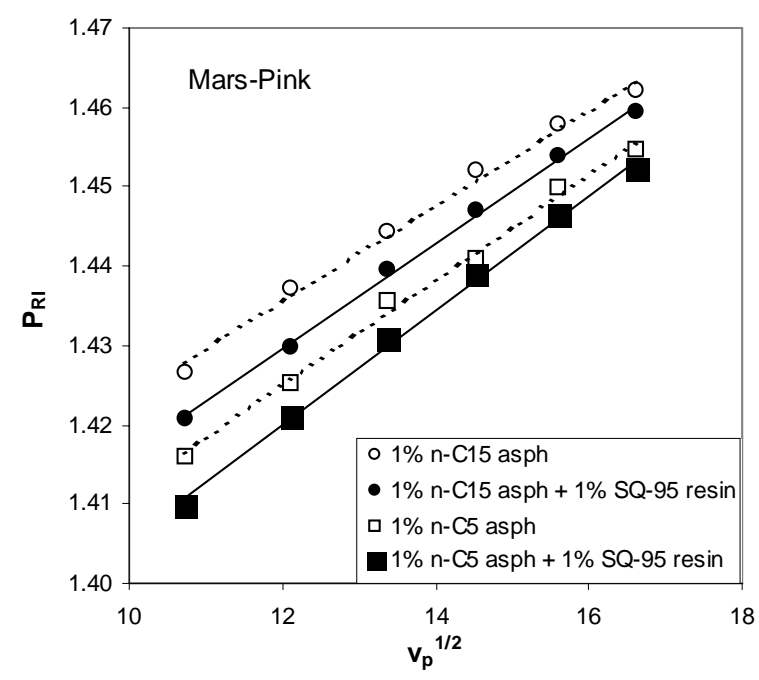

(a)Addition of SQ-95 resin to $n-C_{5}$ and $n-C_{15}$ asphaltenes from Mars-Pink.

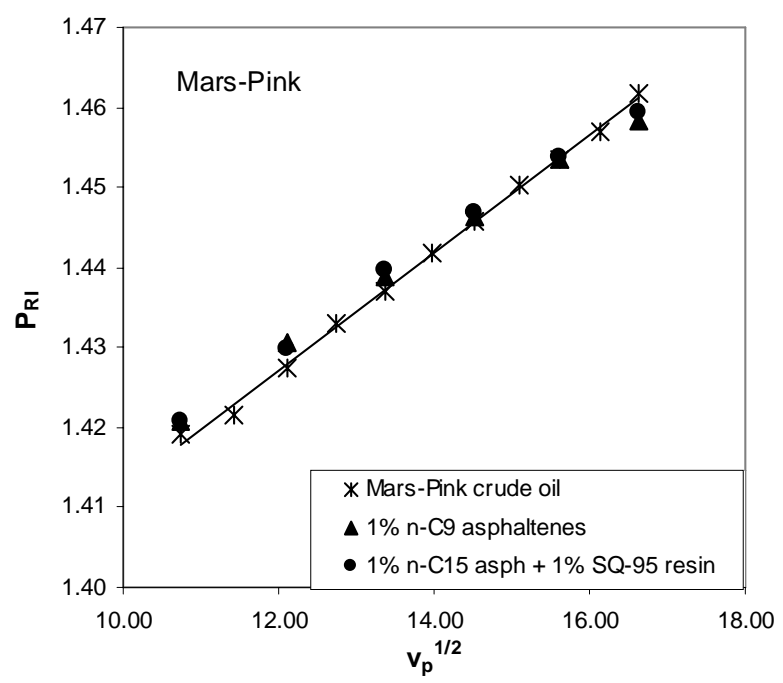

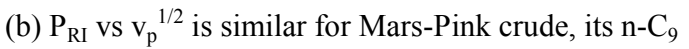
asphaltenes, and Mars-Pink $n-\mathrm{C}_{15}$ asphaltenes with SQ-95 resins.

Figure I-3.32. Addition of resin reduces $P_{R I}$, indicating that the asphaltenes are more stable. The effect of resin is similar to the effect of precipitating asphaltenes with broader spectrum of compositions. Lines are linear fits to the data points.

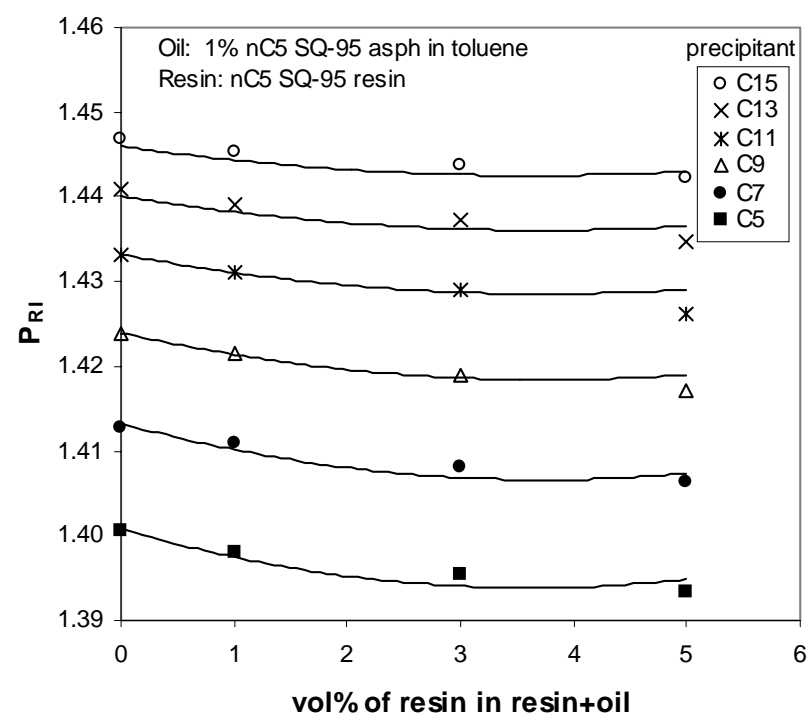

(a) Onset $\mathrm{P}_{\mathrm{RI}}$ for $1 \% \mathrm{n}-\mathrm{C}_{5}$ SQ-95 asphaltenes in toluene with addition of 1 to $5 \% n-C_{5}$ SQ-95 resins. Lines are ASM calculations.

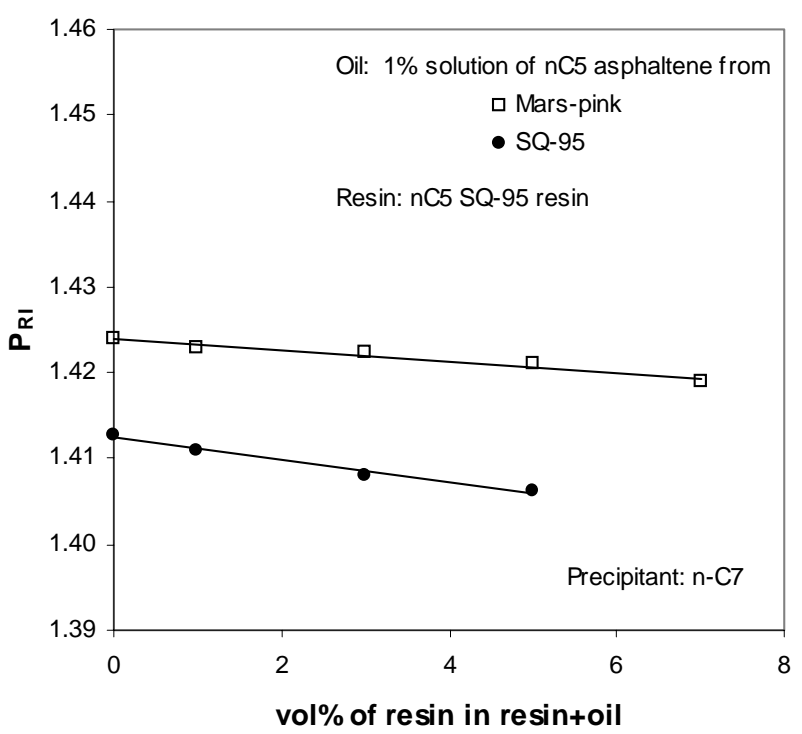

(b) Comparison of the effect of SQ-95 resin on the onset of $n-\mathrm{C}_{5}$ asphaltenes from Mars-Pink and SQ-95 crude oils. Lines are linear fits to data points.

Figure I-3.33. Addition of $n-C_{5}$ SQ-95 resin to $1 \%$ n- $C_{5}$ SQ-95 or Mars-Pink asphaltene solutions in toluene decreases onset $P_{\mathrm{RI}}$. No critical resin concentration was found for any of the six $n$-alkane precipitants. 
It might be argued that adding resins from one oil to asphaltenes from another is not a fair test of the special interactions postulated between resins and asphaltenes. These interactions might be highly specific so that the resins from one crude oil only peptize asphaltenes from the same crude oil. To test the effect of resins on asphaltenes from the same source, a $1 \%$ toluene solution of $\mathrm{n}^{-\mathrm{C}_{5}} \mathrm{SQ}-95$ asphaltenes was prepared. SQ-95 resins were added to the solution at concentrations varying from $1 \%$ to $5 \%$ (the original $n-C_{5}$ resin to $n-C_{5}$ asphaltene ratio in SQ-95 crude oil is approximately $3: 1$ ). The mixtures were thoroughly stirred and allowed to equilibrate for two days. Onsets were measured with each mixture using n- $\mathrm{C}_{5}, \mathrm{n}-\mathrm{C}_{7}, \mathrm{n}-\mathrm{C}_{9}, \mathrm{n}-\mathrm{C}_{11}, \mathrm{n}-\mathrm{C}_{13}$ and $n-\mathrm{C}_{15}$ as the precipitants. Results are shown in Fig. I-3.33a. For all the precipitants tested, $\mathrm{P}_{\mathrm{RI}}$ decreases continuously with addition of resins. In no case is there a sharp change, as might be expected if there were some critical concentration of resins at which the mechanism of asphaltene stabilization changed. If the resins are considered just as additional solvents, the amount of stabilization predicted would be imperceptible. If they are treated as asphaltenes, however, ASM calculations give the same trends as the experimental observations. Similar tests with $\mathrm{n}^{-} \mathrm{C}_{5}$ asphaltenes from Mars-Pink gave the results as shown in Fig. I-3.33b; addition of the SQ-95 resins has a similar effect on asphaltene stability whether the asphaltenes were also from SQ-95 or from a completely different oil.

\section{Effect of mixing two crude oils on asphaltene stability}

Finally, there is the question of whether the components from one oil can destabilize those in another. Certainly it should be possible to construct mixtures of light paraffinic oils and heavy asphaltic ones where components from the light oil reduce the mixture solubility parameter to the extent that asphaltenes from the second oil precipitate. The question is whether destabilization occurs beyond that which can be accounted for by the solubility model or should stability of mixtures be predictable from the characteristics of the two oils. To test the stability of mixed oils, a mixture of 50\% Lost Hills crude oil with 50\% Tensleep/AMN (65:35) was prepared and $\mathrm{P}_{\mathrm{RI}}$ was measured with $\mathrm{n}-\mathrm{C}_{7}$. $\mathrm{P}_{\mathrm{RI}}$ of the mixture corresponded to the calculated ASM values, as shown in Fig. I-3.34a. Similar results, also shown in Fig. I-3.34a, were obtained when $1 \%$ toluene solutions of $n-C_{7}$ asphaltenes from Tensleep and from Lost Hills were mixed. A second pair of crude oil designated C-HD-01 and C-HL-01 show the same behavior (Fig. I3.34b). No unexpected increase (or decrease) in stability resulted from mixing of either the oils or their asphaltenes. Note however that the amount of asphaltene precipitated is not expected to be the average of the amounts from the two different oils and could well exceed the amount obtained from either oil alone since paraffinic constitutents of one oil may destabilize a broader range of material from the other oil. 


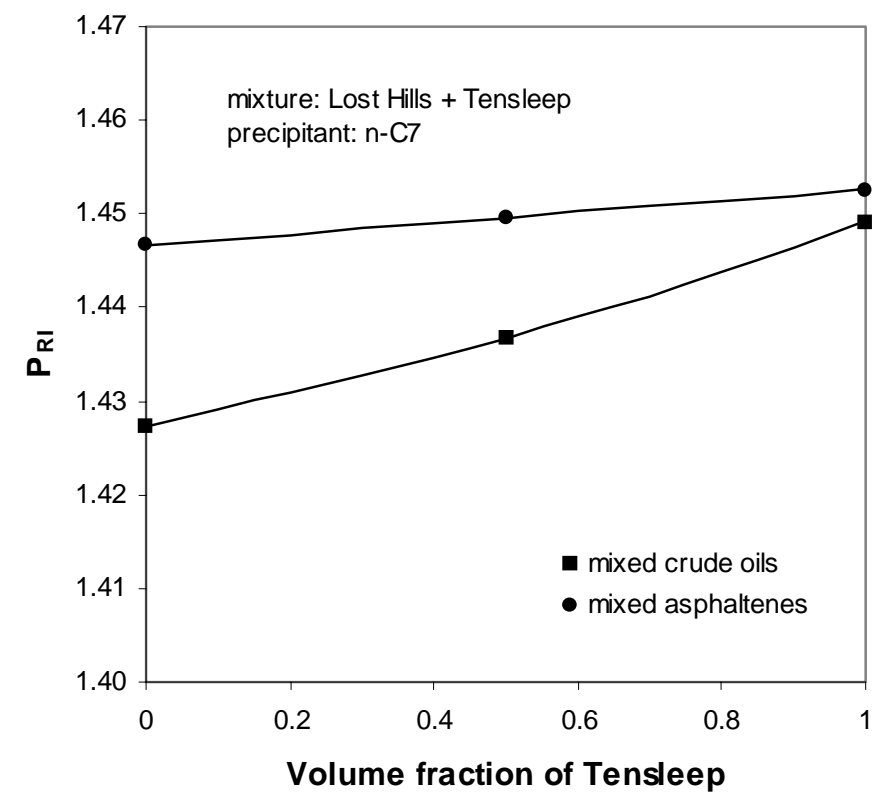

(a) Lost Hills and Tensleep.

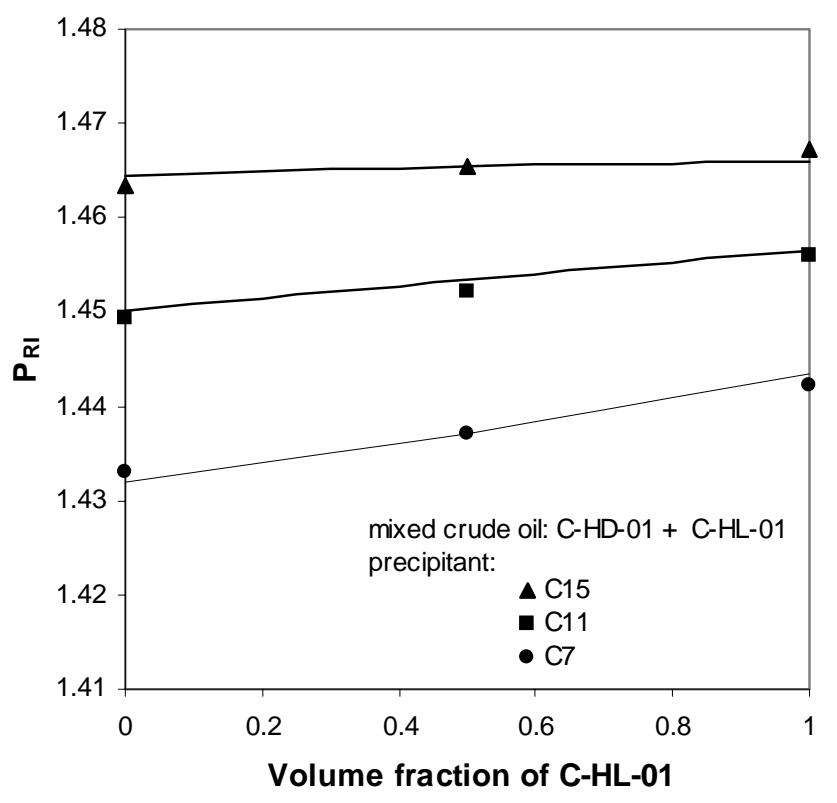

(b) C-HD-01 and C-HL-01

Figure I-3.34. $P_{R I}$ for a mixtures of two crude oils or their asphaltene solutions lies between the values of $\mathbf{P}_{\mathrm{RI}}$ for the two components. Lines are calculations from ASM solubility model.

\section{Conclusions}

- Toluene acts as an ideal solvent in mixtures with crude oils and asphaltenes. Addition of toluene has predictable effects on the onset of asphaltene flocculation in accordance with the ASM solubility model of asphaltene phase behavior.

- Critical solubility parameters evaluated from dilute solutions of oil may be in error because of the effect of toluene on the onset of asphaltene flocculation.

- The influence of AMN on asphaltene flocculation suggests that mixing is not ideal. Smaller changes in $\mathrm{P}_{\mathrm{RI}}$ with addition of AMN make it a better choice when addition of some solvent is required.

- The stability of the least stable asphaltenes is influenced by the distribution of other components in the asphaltene fraction. Broader distributions, i.e. asphaltenes precipitated by lower molecular weight paraffins, are more stable than narrowly distributed fractions precipitated by higher molecular weight paraffins.

- The role of resins in stabilizing a given asphaltene appears to be analogous to broadening the distribution of asphaltene components. Their effect is greater than can be accounted for by changes in solvent properties, but no evidence of specific interactions between asphaltenes and resins has been found in this study. 


\section{References}

Andersen, S.I. and Birdi, K.S.: "Aggregation of Asphaltenes as Determined by Calorimetry," J. Colloid and Interface Sci. (1991) 142, No. 2, 497-502.

ASTM D2007-93: "Standard Test Method for Characteristic Groups in Rubber Extender and Processing Oils by the Clay-Gel Adsorption Chromatographic Method," ASTM (1993).

Buckley, J.S., Hirasaki, G.J., Liu, Y., Von Drasek, S., Wang, J.X., and Gill, B.S.: "Asphaltene Precipitation and Solvent Properties of Crude Oils," Petroleum Science and Technology (1998) 16, No. 3\&4, 251-285.

Carnahan, N.F., Quintero, L., Pfund, D.M., Fulton, J.L., Smith R.D., Capel, M., and Leontaritis, K.: "A Small Angle X-Ray Scattering Study of the Effect of Pressure on the Aggregation of Asphaltene Fractions in Petroleum Fluids under Near-Critical Solvent Conditions," Langmuir (1993) 9, No. 8, 2035-2044.

Cimino, R., Correra, S., Del Bianco, A., and Lockhart, T.P.: "Solubility and Phase Behavior of Asphaltenes in Hydrocarbon Media," Asphaltenes: Fundamentals and Applications, E.Y. Sheu and O.C. Mullins (eds.), NY: Plenum Press (1995) 97-130.

Espinat, D. and Ravey, J.C.: "Colloidal Structure of Asphaltene Solutions and Heavy-Oil Fractions Studied by Small-Angle Neutron and X-Ray Scattering," paper SPE 25187 presented at the 1993 SPE International Symposium on Oilfield Chemistry, New Orleans, March 2-5.

Hirschberg, A., deJong, L.N.J., Schipper, B.A., and Meijer, J.G.: "Influence of Temperature and Pressure on Asphaltene Flocculation," SPEJ (June 1984) 283-293.

Leontaritis, K.J. and Mansoori, G.A.: "Asphaltene Flocculation During Oil Production and Processing: A Thermodynamic - Colloidal Model," paper SPE 16258 presented at the 1987 SPE Internat. Symp. on Oilfield Chem., San Antonio, 4-6 Feb.

Long, R.B.: "The Concept of Asphaltenes," Chemistry of Asphaltenes, J.W. Bunger and N.C. Li (eds.), ACS, Washington, DC (1981) 17-27.

Mitchell, D.L. and Speight, J.G.: "The Solubility of Asphaltenes in Hydrocarbon Solvents, "Fuel (1973) 52, 149-152.

Wang, J.X., and Buckley, J.S.: "An Experimental Approach to Prediction of Asphaltene Flocculation," paper SPE 64994, presented at the SPE International Symposium on Oilfield Chemistry, Houston, 13-16 Feb., 2001a.

Wang, J.X. and Buckley, J.S.: "A Two-Component Model of the Onset of Asphaltene Flocculation in Crude Oils," Energy \& Fuels (2001b) 15, 1004-1012. 


\title{
I-3.5. Simultaneous Measurement of RI and Asphaltene Onset Detection
}

\author{
Z. Yang and P.K. Dasgupta, Chemistry Dept. TTU, J.X. Wang and J.S. Buckley, NM PRRC
}

Light passing through crude oil or reflecting from crude oil interfaces can potentially provide information relevant to identifying and predicting the onset of asphaltene flocculation. In particular, two pieces of information are needed. A number of researchers have used scattering techniques to identify the first appearance of particles, presumed to be asphaltenes (Fuhr et al., 1991). Coupling that to information quantifying solvent quality would provide data for solubility-based models that can then predict the range of conditions of temperature, pressure, and composition over which asphaltenes are in stable dispersion, are metastable, or are unstable. In nonpolar environments, refractive index (RI) can be used as a measure of the solubility parameter needed for such models (Buckley et al., 1998; Wang, 2000).

The aim of this work was to design, test, and validate at ambient conditions methods to observe RI and absorbance changes in fluid mixtures simultaneously that would have the potential of being extended to observations at elevated temperatures and pressures.

\section{Materials and Methods}

\section{Crude oils} I-3.11.

Properties of the crude oils that have been used in these studies are summarized in Table

Table I-3.11. Properties of crude oils.

\begin{tabular}{|l|c|c|c|c|c|c|}
\hline \multicolumn{1}{|c|}{ oil } & $\begin{array}{c}\mathrm{RI}_{589} \\
(\mathrm{Na} \mathrm{D-line} \\
\text { at 20 }\end{array}$ & $\mathrm{nC}_{7} \mathrm{P}_{\mathrm{RI}}$ & $\phi_{\text {oil }}$ at $\mathrm{nC}_{7}$ onset & $\begin{array}{c}\% \mathrm{nC}_{7} \\
\text { asphaltenes }\end{array}$ & $\begin{array}{c}\rho \text { at } 20^{\circ} \mathrm{C} \\
(\mathrm{g} / \mathrm{ml})\end{array}$ & $\begin{array}{c}\mu \text { at } 20^{\circ} \mathrm{C} \\
(\mathrm{mPa} . \mathrm{s})\end{array}$ \\
\hline A-95 & 1.5159 & 1.4546 & 0.52 & 8.7 & 0.8956 & 41.2 \\
\hline C-LH-99 & 1.5132 & 1.4231 & 0.27 & 2.8 & 0.9173 & 89.6 \\
\hline E-1XR-00 & 1.4906 & 1.4071 & 0.18 & 0.3 & 0.8781 & 20.5 \\
\hline Gullfaks-96 & 1.4930 & not measurable & --- & 0.4 & 0.8827 & 15.8 \\
\hline SQ-95 & 1.4787 & 1.4175 & 0.32 & 1.3 & 0.8467 & 5.8 \\
\hline Tensleep & 1.4877 & 1.4438 & 0.38 & 3.2 & 0.8684 & 18.7 \\
\hline
\end{tabular}

Development of RI measurement techniques

APPROACH 1: TRANSMISSION LOSS COMPENSATION

Figure I-3.35 shows a cell of short path length $(l)$ with incident light intensity on the cell, $\mathrm{I}_{0}$. A fraction $\mathrm{f}$ is immediately lost at the glass-air interface due to reflection. According to Fresnel's law, this loss is solely dependent on the refractive indices of glass and air at the operative wavelength. This loss is independent of the nature of the oil. The intensity of the light 
incident at the glass/oil interface is therefore $\mathrm{I}_{0}(1-\mathrm{f})$. The fraction of the light lost at the glassoil interface, $\mathrm{f}_{1}$, is dependent on the refractive indices of the glass and the oil:

$$
\mathrm{f}_{1}=\left(\frac{\mathrm{n}_{\text {glass }}-\mathrm{n}_{\text {oil }}}{\mathrm{n}_{\text {glass }}+\mathrm{n}_{\text {oil }}}\right)^{2}
$$

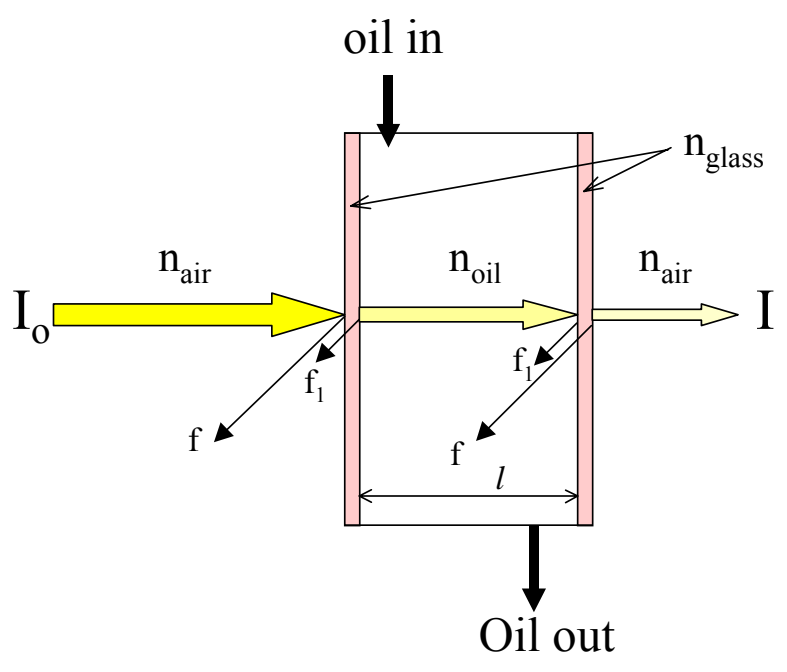

Figure I-3.35. Absorptive reflective transmission through a glass window cell filled with oil.

The rest of the light, $\mathrm{I}_{0}(1-\mathrm{f})\left(1-\mathrm{f}_{1}\right)$, proceeds through the cell and is attenuated according to Beer's law during its passage through the oil. When it reaches the second oil-glass interface, it has an intensity equal to $I_{0}(1-f)\left(1-f_{1}\right) 10^{-A}$ where $A$ is the Beer's law absorbance of the oil. At the oil-glass interface another fraction $\mathrm{f}_{1}$ is lost. Finally, at the glass-air interface another fraction $\mathrm{f}$ is lost. Thus the light intensity I exiting the cell is given by:

$$
I=I_{0}(1-f)^{2}\left(1-f_{1}\right)^{2} 10^{-A}
$$

The overall observed absorbance, $\mathrm{A}_{\mathrm{obsd}}$, is thus given by:

$$
A_{\text {obsd }}=-\log \left(I / I_{0}\right)=-2 \log (1-f)-2 \log \left(1-f_{1}\right)+A
$$

Of the three terms that contribute to $\mathrm{A}_{\text {obsd }}$, the first two are due to reflective losses and the third one is due to the absorption of light in oil. The first term is independent of the nature of the liquid in the cell and can be considered as a constant.

Consider now a second cell, identical in all respects except that the cell path length is $2 l$, twice that of the first cell. An identical light attenuation experiment in this case results in an observed absorbance of $\mathrm{A}_{\text {obsd }}$ where

$$
\mathrm{A}_{\text {obsd }}^{\prime}=-2 \log (1-\mathrm{f})-2 \log \left(1-\mathrm{f}_{1}\right)+2 \mathrm{~A}
$$


Note that only the Beer's law loss term changes. One can thus readily calculate A as

$$
\mathrm{A}=\mathrm{A}_{\text {obsd }}^{\prime}-\mathrm{A}_{\mathrm{obsd}}
$$

Putting this in Eq. I-3.68, and setting the constant term $2 \log (1-\mathrm{f})$ equal to $\mathrm{K}$, we obtain:

$$
2 \log \left(1-\mathrm{f}_{1}\right)=\mathrm{A}_{\text {obsd }}^{\prime}-2 \mathrm{~A}_{\text {obsd }}-\mathrm{K}
$$

This results in:

$$
1-\mathrm{f}_{1}=10^{\left(\mathrm{A}^{\prime}{ }_{\text {obsd }}-2 \mathrm{~A}_{\text {obsd }}-\mathrm{K}\right) / 2}
$$

Combining Eqs. I-3.66 and I-3.72,

$$
1-\left(\frac{\mathrm{n}_{\text {glass }}-\mathrm{n}_{\text {oil }}}{n_{\text {glass }}+n_{\text {oil }}}\right)^{2}=10^{\left(\mathrm{A}_{\text {obsd }}^{\prime}-2 \mathrm{~A}_{\text {obsd }}-\mathrm{K}\right) / 2}
$$

This can be transformed to:

$$
\frac{4 \mathrm{n}_{\text {glass }} \mathrm{n}_{\text {oil }}}{\left(\mathrm{n}_{\text {glass }}+\mathrm{n}_{\text {oil }}\right)^{2}}=10^{\left(\mathrm{A}_{\text {obsd }}^{\prime}-2 \mathrm{~A}_{\text {obsd }}-\mathrm{K}\right) / 2}
$$

or

$$
2 \log \left(\mathrm{n}_{\text {glass }} \mathrm{n}_{\text {oil }}\right)-4 \log \left(\mathrm{n}_{\text {glass }}+\mathrm{n}_{\text {oil }}\right)=\mathrm{A}_{\text {obsd }}^{\prime}-2 \mathrm{~A}_{\text {obsd }}-\mathrm{K}^{\prime}
$$

where $K^{\prime}=K+2 \log 4$. Since $A_{o b s d}$ and $A^{\prime}{ }_{\text {obsd }}$ can be measured directly, the system can be calibrated with at least two fluids of known RI to obtain the values of $\mathrm{K}^{\prime}$ and of $\mathrm{n}_{\text {glass }}$ at the probe wavelength. If $n_{\text {glass }}$ is known, only a single calibration that could easily be made with air or water in the cell is necessary.

It would also appear from the above considerations that it is desirable to reduce the absorptive loss as much as possible (otherwise the measurement becomes dependent on the difference between two large numbers). For this reason, we chose a light emitting diode (LED) emitting at around $\sim 930 \mathrm{~nm}$ as the light source. Eq. I-3.66 clearly indicates that the reflective loss increases as the difference in RI between the test substance and the glass increases. For this reason we built:

(a) two identical cells with sapphire windows (RI 1.7), one with $1 \mathrm{~mm}$ and another with $2 \mathrm{~mm}$ path length; 
(b) two fiber optic based absorbance detectors with $930 \mathrm{~nm}$ light sources and a measured noise level of $10^{-5}$ absorbance units.

Initially these cells were tested with a yellow LED emitting at $589 \mathrm{~nm}$, close to the Na Dline. When calibrated with air and a high RI liquid such as $\mathrm{CCl}_{4}$, they produced RI values for many other liquids in good agreement with literature values.

RI values for only a few liquids are available at wavelengths other than the Na D-line. The only data we are aware of are due to Schiebener et al. (1990) for water and McCay (1996) for aqueous solutions of $\mathrm{NH}_{4} \mathrm{Cl}$. These data were used to calibrate the cells at $930 \mathrm{~nm}$. We then prepared various dilutions in toluene of a light colored crude and a dark crude and measured their absorbances to calculate the apparent refractive indices for these various dilutions using the above calibration results.

With our experimental system, the above relationship held up to a volume fraction of 0.8 for the light crude (20\% toluene) and failed even at low concentrations for the dark crude. Analysis of the data showed that as the absorbance, A, approaches 1, the reflective loss becomes much smaller in proportion and major errors are introduced. We considered replacing the LED with an even longer wavelength emitter at $1300 \mathrm{~nm}$ or $1500 \mathrm{~nm}$ to reduce the absorptive loss, but the bandwidth of LEDs increases with increasing wavelength. The absorption by the oil is not flat across the finite emission span of the LED, introducing further errors in the absorbance correction mode. For example, the emission spectrum of a $930 \mathrm{~nm}$ LED is shown in Figure I3.36. It has a full width at half maximum (FWHM) of $40 \mathrm{~nm}$. Even wider FWHM can be expected for $1300 \mathrm{~nm}$ and $1500 \mathrm{~nm}$ LEDs.

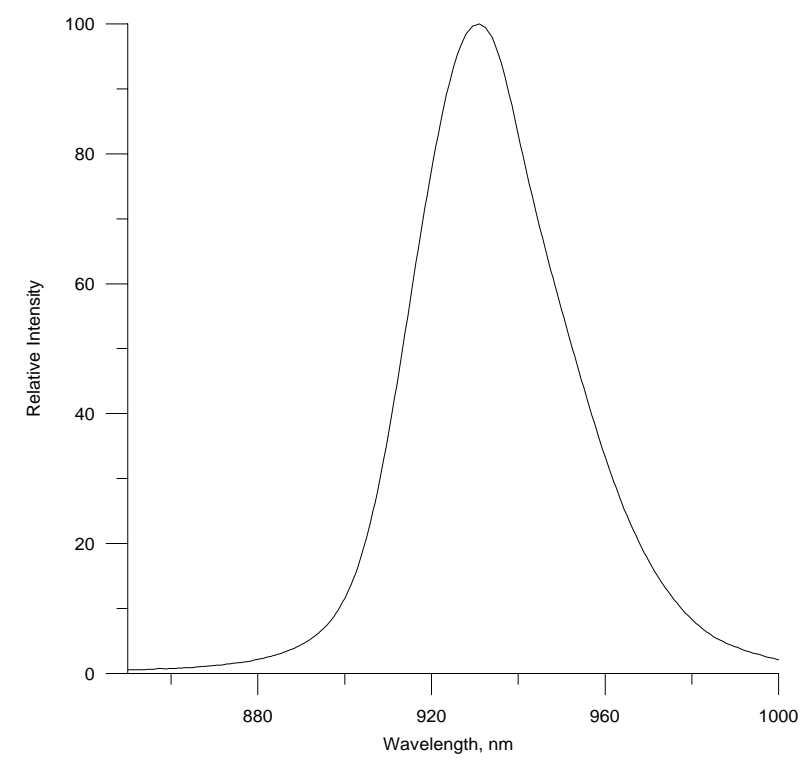

Figure I-3.36. Emission spectrum of the $930 \mathrm{~nm}$ LED.

We considered using a communication-type diode laser emitting at $1300-1500 \mathrm{~nm}$ as the light source to reduce the bandwidth, but this would be offset by the lower intensity and stability 
of a laser compared to an LED. We therefore opted to abandon this approach and chose an alternative measurement, described in the following section, that in principle does not require an absorption correction.

\section{APPROACH-2: REFLECTED LIGHT}

This strategy involves measurement of the light reflected from the glass-liquid interface, as illustrated in Fig. I-3.37, with a constant light intensity incident on the system. A randomized bifurcated glass fiber optic bundle is cemented to the window with UV-cure refractive index matching adhesive. An LED driven by constant current sends light down to the glass-liquid interface along one leg of the glass fiber bundle and the reflected light goes to a silicon photodiode detector (BPW 34, Siemens) through the other leg.

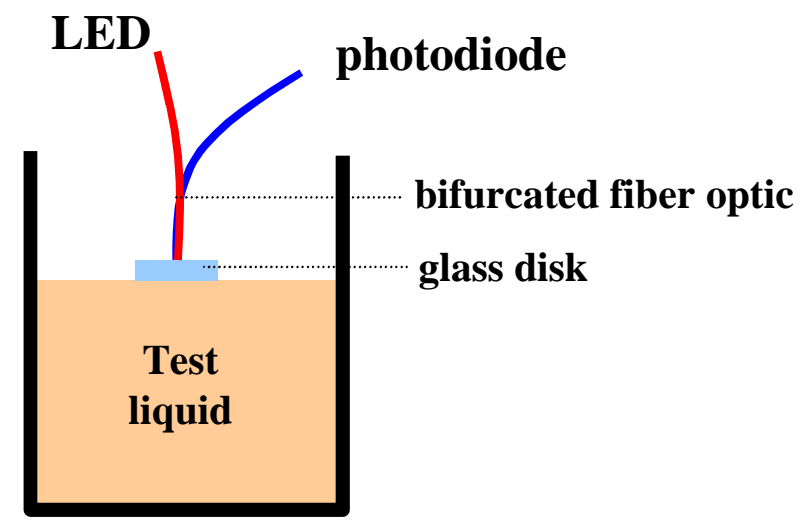

Figure I-3.37. Simple arrangement for testing reflection from the glass-liquid interface.

The photocurrent is converted to voltage using a linear $\mathrm{i} \rightarrow \mathrm{V}$ converter/amplifier (Keithley model 427). The amplifier output was recorded at $1 \mathrm{~Hz}$ on a laptop PC using DAS WIZARD software (Computerboards Inc.) that runs as a subprogram in Microsoft Excel. The reflected light intensity measured by the detector is the sum of a constant amount of light reflected by the glass/adhesive/sapphire interface(s) and a variable component from the glassliquid interface that is governed by Eq. I-3.66. Thus the total light intensity measured by the photodiode is:

$$
\mathrm{I}_{\mathrm{r}}=\mathrm{K}+\mathrm{I}_{0}\left(\frac{\mathrm{n}_{\text {glass }}-\mathrm{n}_{\text {oil }}}{\mathrm{n}_{\text {glass }}+\mathrm{n}_{\text {oil }}}\right)^{2}
$$

There are two unknowns in Eq. I-3.76, $\mathrm{I}_{0}$ and $\mathrm{K}_{\mathrm{r}}$, requiring two calibrations to determine their values. A third calibration may be needed to provide a value for $\mathrm{n}_{\text {glass, }}$, if this is not known $a$ priori.

Based on this principle, a series of designs were tested to find an optimal configuration; the present configuration for measuring the intensity of light reflected is illustrated in Fig. I-3.38 below. LEDs of different wavelengths were tested. 


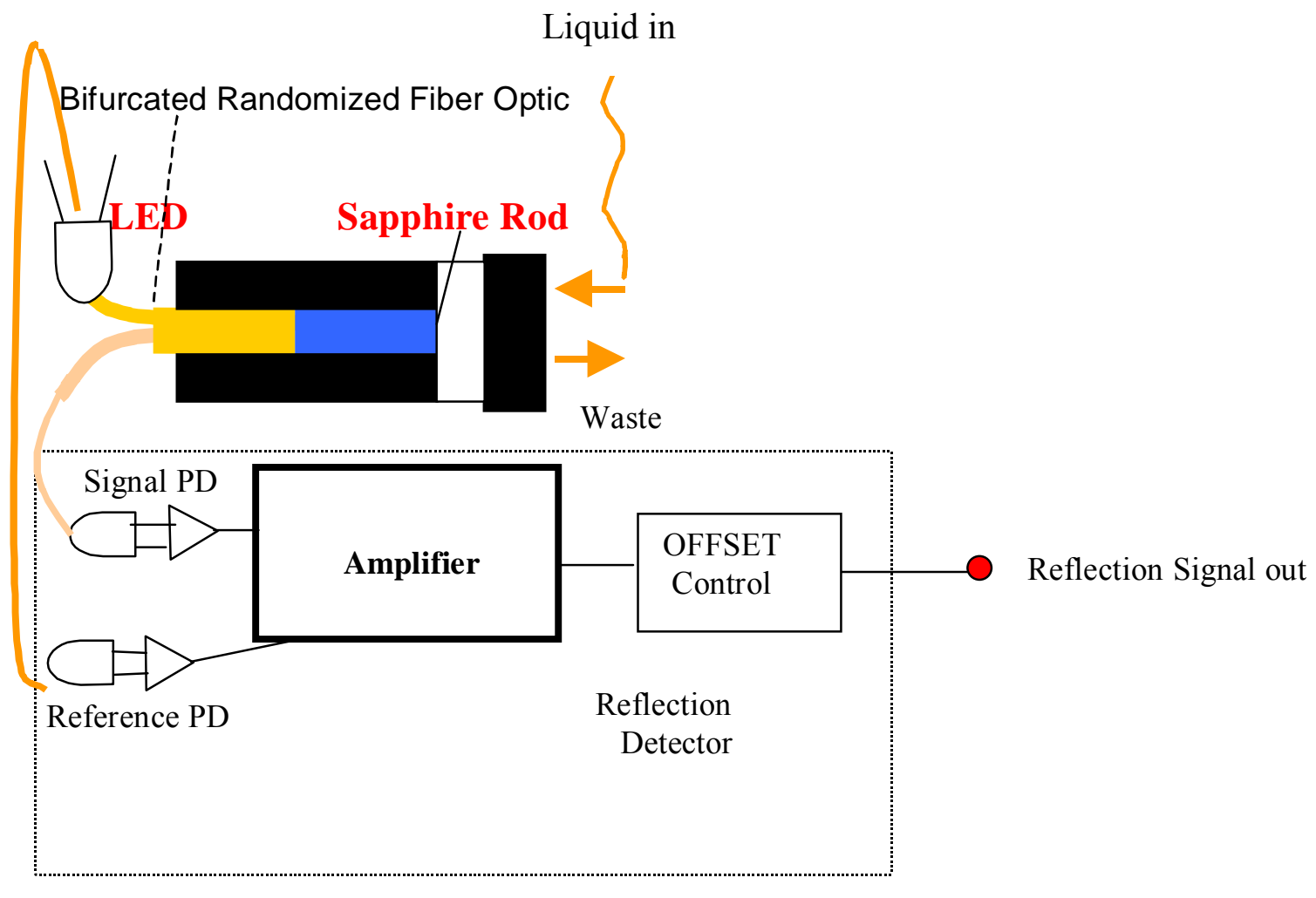

Figure I-3.38. Schematic illustration of apparatus for measurement of reflected signal intensity.

\section{Detecting the onset of asphaltene flocculation}

To apply the measurement of apparent absorbance to detection of the onset of asphaltene flocculation, we made the following hypotheses:

(1) The oil will have a much greater absorbance relative to a diluent like hexane at any given probe wavelength practically accessible to us. Even if any specific absorption by the oil did not exist, there will be a continuous change in the apparent absorbance of an oil-hexane mixture due to the continuous change in RI of the mixture as a function of composition (and hence a change in the reflective light loss in any practical flow cell system). There should be some discontinuity in an apparent absorbance - composition plot at the onset of precipitation because of the sudden presence of particles that are large relative to the probe wavelength and thus cause scattering. In other words, the onset of precipitation should be discernible in an absorbance-composition graph. 
(2) Asphaltene precipitation may be incipient to a system of given composition. In a mixture of given composition, it may be kinetic rather than thermodynamic factors that govern the observed onset of precipitation. Researchers in this area are well aware that how quickly asphaltene precipitation is actually observed depends on the extent to which the nonpolar solvent content exceeds that at which precipitation just begins to occur under equilibrium conditions. If we are able to mix crude oil and hexane and measure at what composition the onset of precipitation occurs as a function of the time between mixing and measurement, this will produce interesting and valuable kinetic information. If the data are amenable, extrapolation can be made to infinite reaction time that will predict the composition at which asphaltene precipitation will become thermodynamically incipient. Extrapolation to zero residence time, on the other hand will indicate the composition at which asphaltene precipitation must occur immediately. In some ways, these measurements may be of more practical value since it is not thermodynamic possibility but the actual precipitation event that is of interest (despite the greater stability of the latter, diamond does not turn into graphite!).

The experimental system is shown schematically in Fig. I-3.39. A variable speed peristaltic pump (Rainin Dynamax), indicated in Fig. 5 by P, using Pharmed or Viton tubing was used to pump the oil. A 48000 step stepper motor-driven syringe pump, equipped with a $25 \mathrm{~mL}$ capacity glass/Teflon syringe (Kloehn model 50300), was used for pumping the hexane because the high volatility of hexane tends to cause bubble problems in a peristaltic pump. The two streams were actively mixed in a microliter-volume magnetically stirred mixer designed and built in-house. The mixed stream proceeded through a knotted coil (Fig. I-3.40, see Waiz et al., 2001) composed of $\sim 50 \mathrm{~cm}$ length of a $\sim 1.2 \mathrm{~mm}$ i.d. PTFE tube containing $10 x 15 \mathrm{~mm}$ elliptical knots.

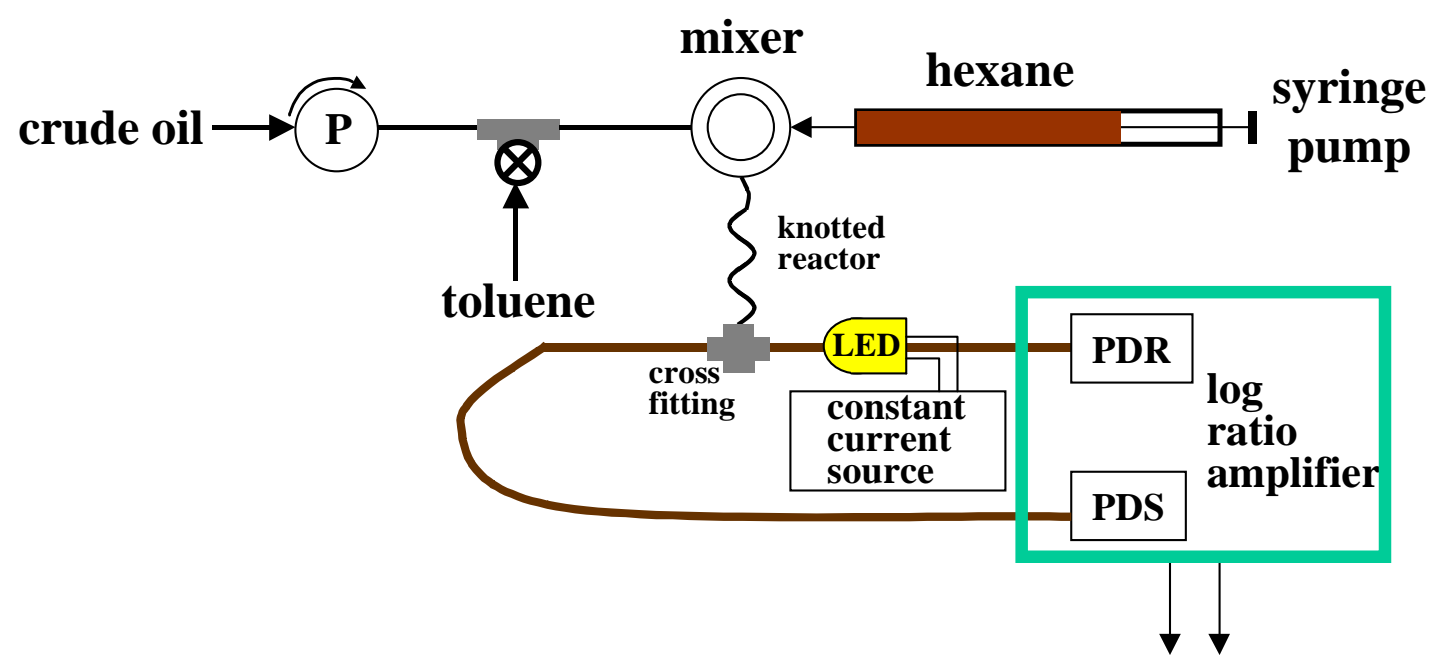

Figure I-3.39. Schematic illustration of apparatus for measurement of precipitation onset 


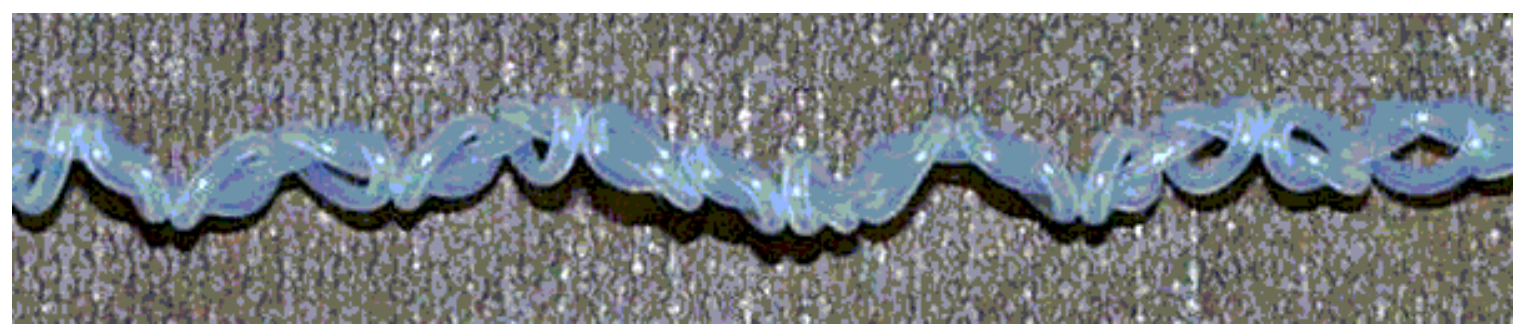

Figure I-3.40. Close-up view of knotted mixer

The oil line has a tee port, which normally remains closed. When opened, toluene can be injected with a syringe through this port to clean any precipitated material out of the system. The effluent from the reaction coil proceeds through the optical cell which is essentially of a cross configuration, and is finally collected in a waste container. Two very large core (diameter = $1300 \mu \mathrm{m})$ silica optical fibers are affixed opposite each other inside the optical cell with a spacing of $\sim 500 \mu \mathrm{m}$, which constitutes the optical path length. Liquid flows through the cell in the direction perpendicular to the optical path. One optical fiber is connected to a high output LED, with its emission centered at $940 \mathrm{~nm}$, driven by a $50 \mathrm{~mA}$ constant current source. The second fiber terminates in a silicon photodiode (signal detector, PDS, Siemens BPW 34). A third separate optical fiber connects the LED directly to a reference photodetector (PDR), which is identical to the signal photodetector (PDS). The two photocurrents constitute the inputs to a log ratio amplifier (Burr-Brown 100JP) and the output of the amplifier (zero offsettable) is the absorbance ( $5 \mathrm{~V} /$ absorbance unit). The absorbance signal was recorded at $1 \mathrm{~Hz}$ on a laptop PC using DAS Wizard software (Computerboards Inc.) that runs as a subprogram in Microsoft Excel.

During each experiment, the hexane flow rate was held constant. The peristaltic pump speed can be adjusted to change the volume ratio of oil to hexane. At present this is done manually, but rate adjustment could easily be carried out under automated PC control. We typically start at a 70:30 v/v ratio of oil:hexane. There is no precipitation at this ratio for the crude oils currently under examination. The absorbance output is allowed to stabilize for 5-30 min, depending on flow rate, with faster stabilization at faster flow rates. Data are collected for about $10 \mathrm{~min}$, from which mean and standard derivation are calculated. The fraction of oil in the mixture is decreased in small steps of about $1-5$ vol\% per step to about $20 \%$, allowing the system to stabilize and recording data at each step. Until precipitation occurs, the observed absorbance value of the oil/hexane mixture decreases monotonically with decreasing oil:hexane ratio. There is a change in this slope, or even a reversal in slope direction after asphaltenes begin to precipitate. 


\section{Results and Discussion}

Refractive index measurements

Initial experiments were conducted in a dark room to ensure no other light influenced the experiment. The fiber assembly was lowered to the liquid (contained in a non-reflecting container) so that the glass surface was in contact with the liquid.

RI OF NACL SOLUTIONS.

Different concentrations of $\mathrm{NaCl}$ solution were studied. With the $550 \mathrm{~nm}$ LED, the reflected light intensity showed very little dependence on $\mathrm{NaCl}$ concentration, probably because the source intensity was too low. With the $660 \mathrm{~nm}$ and the $891 \mathrm{~nm}$ LEDs, the reflected light intensity changed significantly with the concentration of $\mathrm{NaCl}$. The fits to Eq. I-3.76 were very good, as shown in Fig. I-3.41 for the data at $891 \mathrm{~nm}$. Using these solutions, however, only a limited range of RI values could be studied (equivalent to 1.3331 to 1.3684 measured at the $\mathrm{Na}$ D-line). The best fits to these and subsequent measurements are summarized in Table I-3.12.

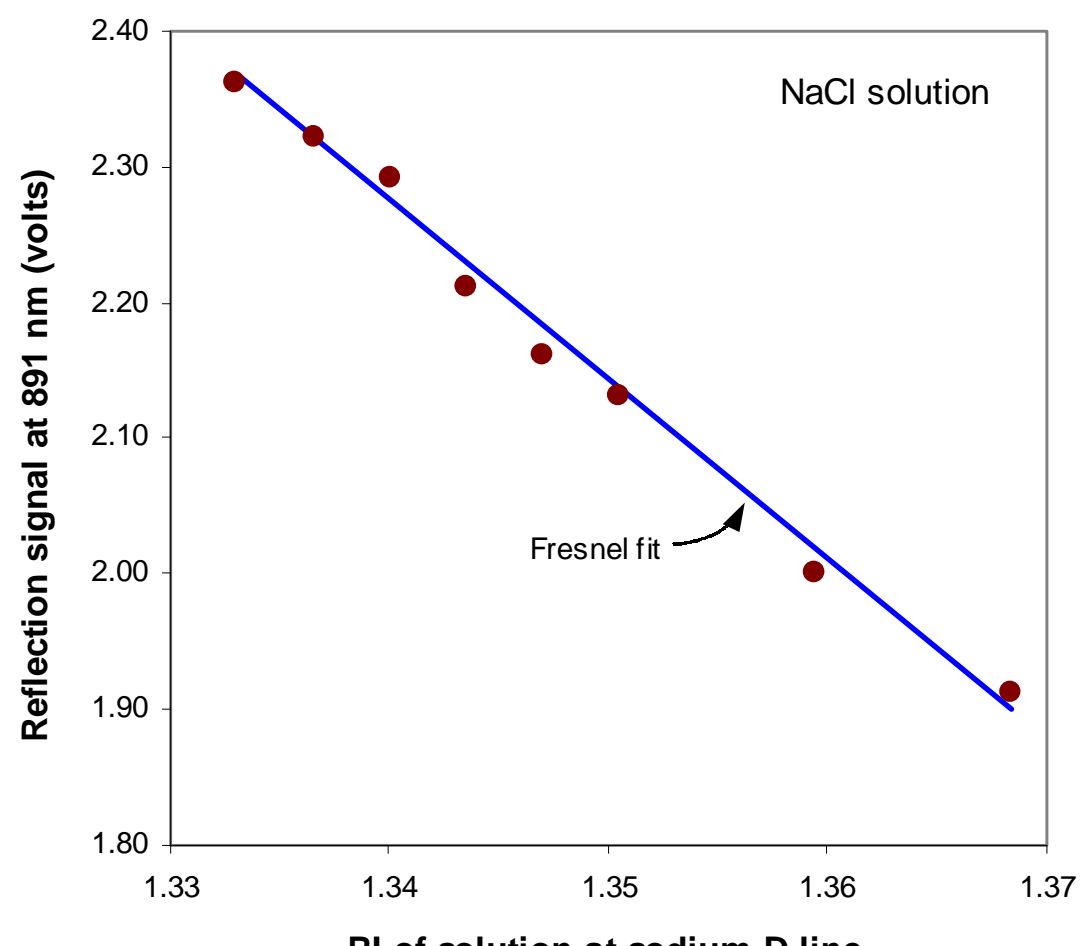

Figure I-3.41. Reflected light intensity data obtained with $\mathrm{NaCl}$ solutions at 891 nm. 
Table I-3.12. Fresnel fits to reflected light intensity data.

\begin{tabular}{|lccccccc|}
\hline \multicolumn{1}{|c}{ liquid media } & $\begin{array}{c}\text { window } \\
\text { material }\end{array}$ & $\begin{array}{c}\text { wavelength } \\
(\mathbf{n m})\end{array}$ & $\mathbf{K}$ & $\begin{array}{c}\text { Io } \\
(\mathbf{v o l t s})\end{array}$ & $\mathbf{n}_{\text {window }}$ & $\mathbf{r}^{\mathbf{2}}$ & Fig \# \\
\hline $\mathrm{NH}_{4} \mathrm{Cl}$ solutions & Glass & 891 & 0.5120 & 199.9 & 1.617 & 0.9993 & $\mathrm{I}-3.41$ \\
& & 660 & 0.6378 & 200.0 & 1.602 & 0.9991 & \\
\hline organic liquids & Glass & 891 & 0.1035 & 467.7 & 1.4932 & 0.9951 & $1-3.42$ \\
& & 660 & 0.1231 & 650.7 & 1.4937 & 0.9966 & $1-3.42$ \\
\hline organic liquids & Sapphire & 910 & 0.6323 & 211.8 & 1.678 & 0.9984 & $1-3.43$ \\
& & 905 & 0.3785 & 231.3 & 1.769 & 0.9995 & \\
& & 891 & 1.486 & 447.1 & 1.771 & 0.9995 & \\
\hline miscellaneous & Sapphire & 660 & 3.912 & 886.9 & 1.640 & 0.9968 & $1-3.44$ \\
\hline
\end{tabular}

\section{RI OF KNOWN LIQUIDS}

To further examine the applicability of Eq. I-3.76, we tested acetone, ethanol, hexane, propanol, methyl isobutyl ketone (MIBK), triethylamine, dioxane, cyclohexane, carbon tetrachloride, glycerol, triethanolamine, toluene, benzyl alcohol, benzaldehyde, benzoyl chloride and carbon disulfide with LEDs emitting with center wavelengths of $550 \mathrm{~nm}, 585 \mathrm{~nm}, 660 \mathrm{~nm}$, $891 \mathrm{~nm}, 905 \mathrm{~nm}$ and $910 \mathrm{~nm}$. Both glass and sapphire windows were tested. Again, the $550 \mathrm{~nm}$ and $580 \mathrm{~nm}$ emitters did not produce meaningful results, probably due to poor source intensity. In contrast, the $660 \mathrm{~nm}, 891 \mathrm{~nm}, 905 \mathrm{~nm}$ and $910 \mathrm{~nm}$ emitters all showed good fits to Eq. I-3.76. Fig. I-3.42 shows the results with a glass window at 660 and $891 \mathrm{~nm}$ and Fig. I-3.43 illustrates the measurements with the $910 \mathrm{~nm}$ LED and a sapphire window. Numerical fits are included in Table I-3.12.

According to these fits, the RI values of sapphire are 1.640 at $660 \mathrm{~nm}, 1.771$ at $891 \mathrm{~nm}$, 1.769 at $905 \mathrm{~nm}$ and 1.768 at $910 \mathrm{~nm}$. This is in agreement with literature values for the RI of sapphire at different wavelengths (Levi, 1980). In another test, a $940 \mathrm{~nm}$ LED was used to measure the reflected light intensities for $\mathrm{H}_{2} \mathrm{O}, \mathrm{C}_{2} \mathrm{H}_{5} \mathrm{OH}, 25 \% \mathrm{NH}_{4} \mathrm{Cl}$, n-hexane, toluene, and $\mathrm{CS}_{2}$. Results are shown in Fig. I-3.44. These results support the use of this measuring system. However, it still remains to be shown that optically dense oil samples can be measured. 


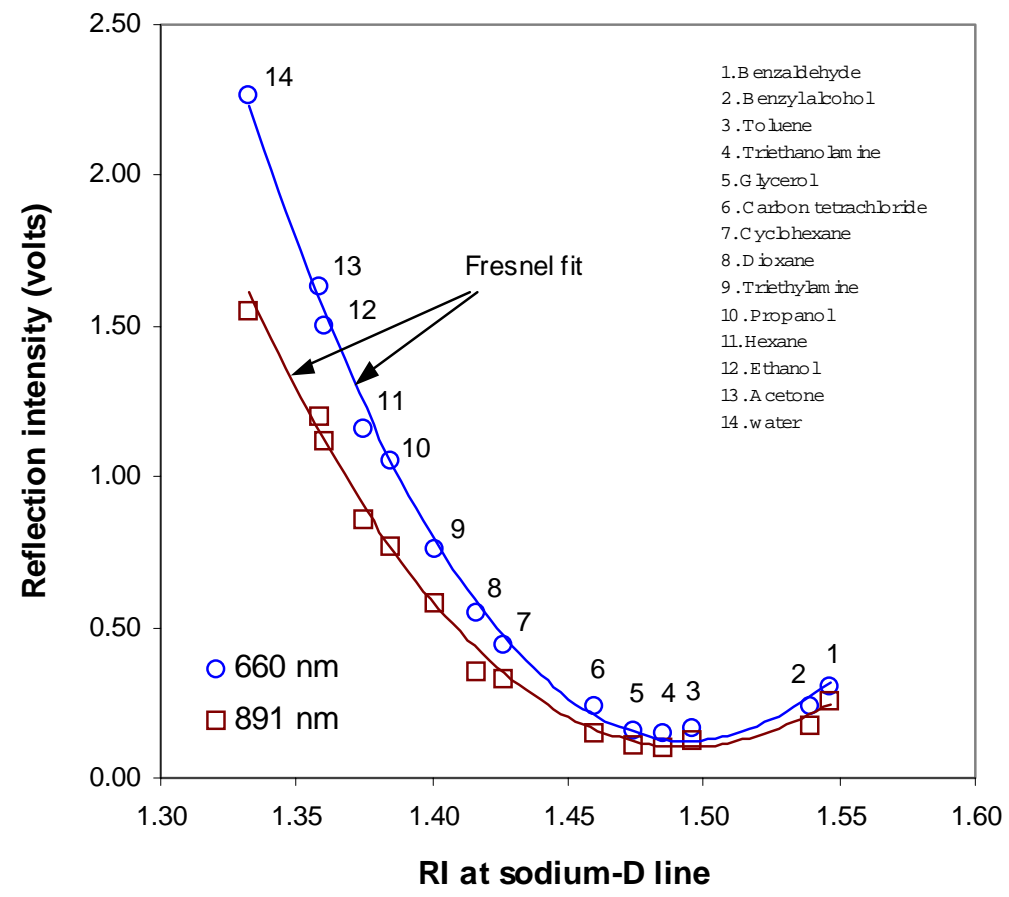

Figure I-3.42. Fit to Eq. I-3.76 over a large RI range, using a glass window. Data go through a minimum at the RI of glass.

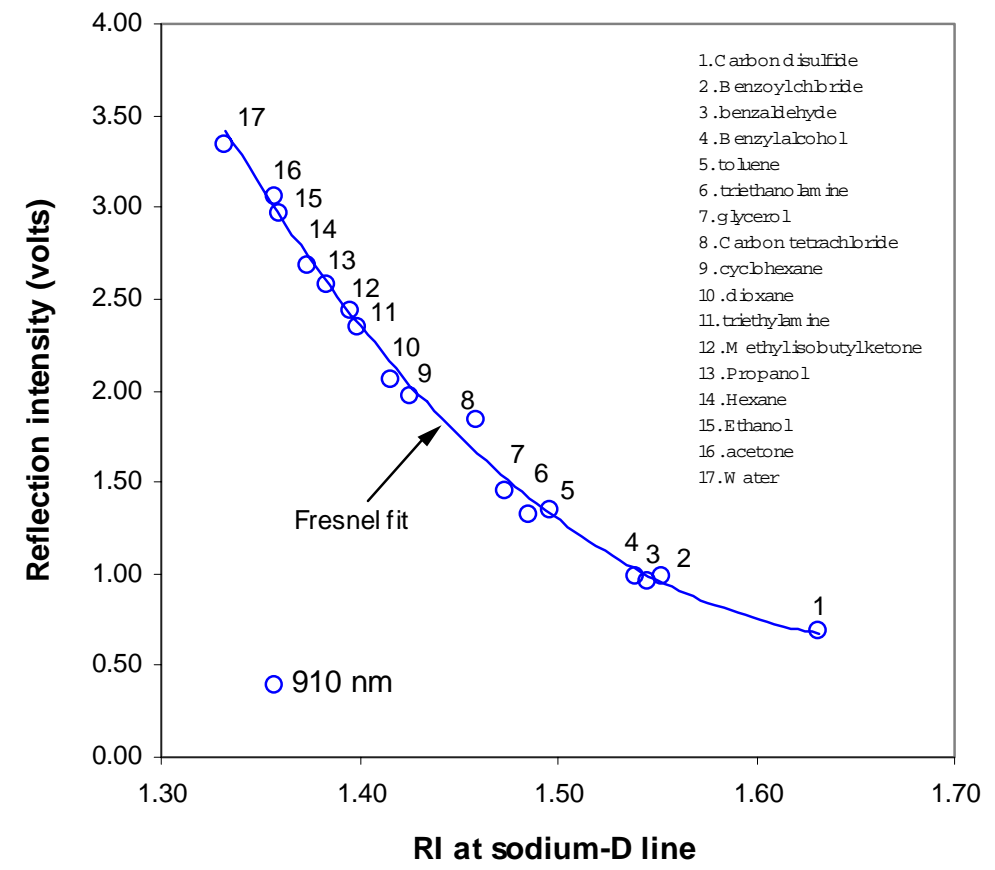

Figure I-3.43. Fit to Eq. I-3.76 over a large RI range, using a sapphire window. 


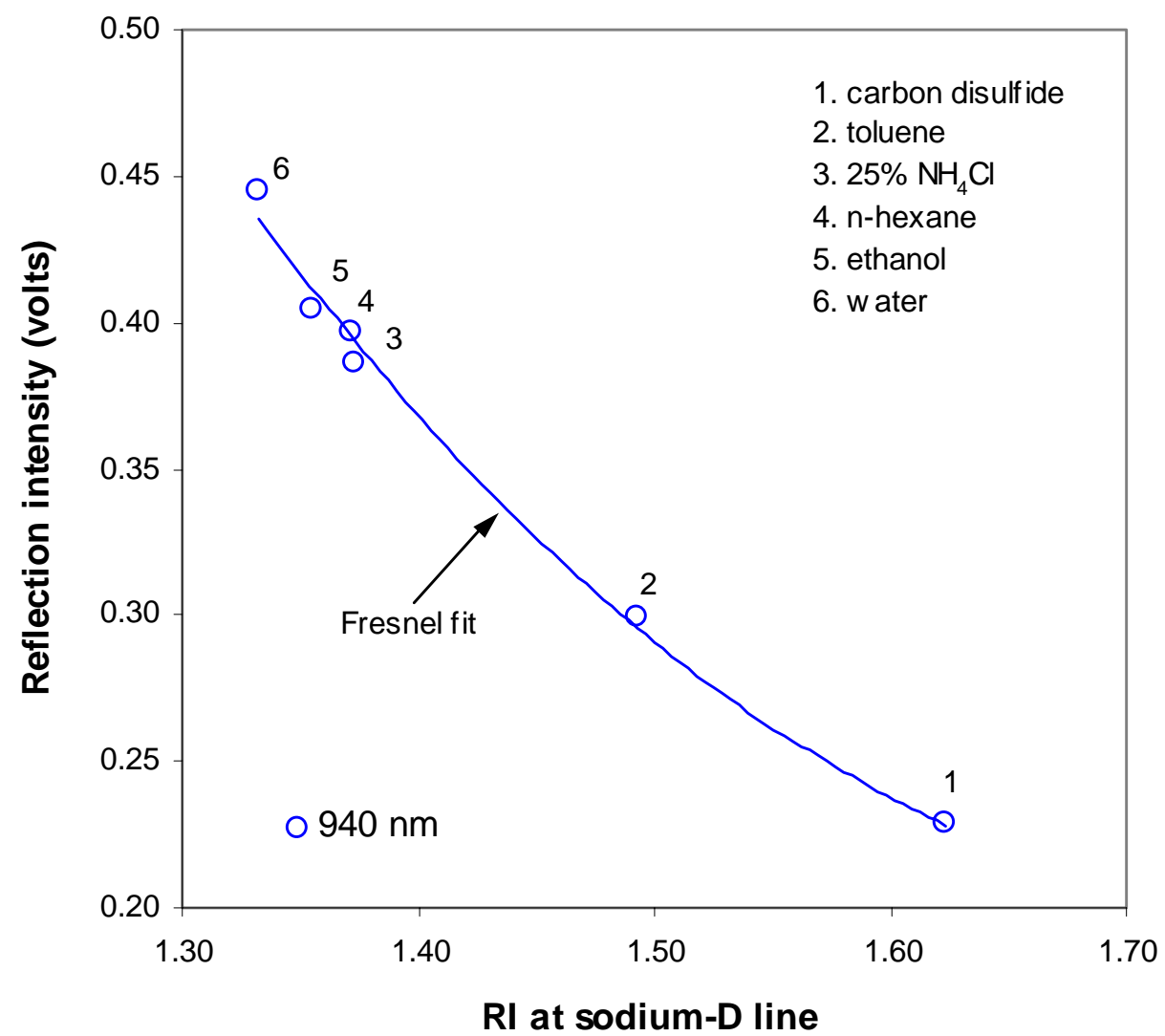

Figure I-3.44. Calibration for $940 \mathrm{~nm}$ LED with RI measurements at $589 \mathrm{~nm}$ (Abbe refractometer).

RI OF CRUDE OILS AND CRUDE OIL/TOLUENE MIXTURES

Reflected light intensities were measured at $940 \mathrm{~nm}$ for six crude oils under the same conditions as those established for known liquids. These intensities were then used to calculate RI values from the calibration curves shown in Fig. I-3.44. Measured intensities, calculated RI values, and comparisons to $\mathrm{RI}_{589}$, measured at the sodium-D line with a refractometer, are summarized in Table I-3.13.

Table I-3.13. Comparisons of calculated RI to measured RI for crude oils

\begin{tabular}{|c|c|c|c|}
\hline Crude oil & $\begin{array}{c}\mathbf{I}_{\mathbf{r}, 940} \\
(\mathbf{m v})\end{array}$ & $\mathbf{R I}_{\text {calc }}$ & $\boldsymbol{\Delta} \mathbf{R I}=\mathbf{R I}_{\text {calc }}-\mathbf{R I}_{\mathbf{5 8 9}}$ \\
\hline A-95 & 0.2261 & 1.6293 & 0.1134 \\
\hline C-LH-99 & 0.2364 & 1.6031 & 0.0899 \\
\hline E-1XR-00 & 0.2823 & 1.5138 & 0.0233 \\
\hline Gullfaks-96 & 0.2734 & 1.5287 & 0.0357 \\
\hline sQ-95 & 0.2870 & 1.5063 & 0.0275 \\
\hline $\begin{array}{c}\text { Tensleep + 1-MN* } \\
\text { (50:50) }\end{array}$ & 0.2060 & 1.6949 & 0.1445 \\
\hline
\end{tabular}

*1-MN = 1-methyl naphthalene 
Measured light intensities for mixtures of SQ-95 crude oil and toluene are listed in Table I-3.14. For comparison, RI can be calculated from the volume fractions of the mixture components using Eq. I-3.77 (Synovec and Yeung, 1983):

$$
\frac{\mathrm{n}_{\text {mix }}^{2}-1}{\mathrm{n}_{\text {mix }}^{2}+2}=\sum_{\mathrm{i}}\left(\phi_{\text {volume, } \mathrm{i}} \frac{\mathrm{n}_{\mathrm{i}}^{2}-1}{\mathrm{n}_{\mathrm{i}}^{2}+2}\right)
$$

where $n_{\text {mix }}$ is the RI value of the mixture, $n_{i}$ is the RI value of component $i$, and $\phi_{\text {volume, } i}$ is the volume fraction of component i. and considering oil as a single component (Buckley et al, 1998). The RI of the oil is given in Table 1 and RI of toluene at $20^{\circ} \mathrm{C}$ is 1.4962 . Differences between the values calculated from Eq. I-3.77 and those based on the reflected light intensity measurements at $940 \mathrm{~nm}$ are significant. Why they are so different remains to be explained, but clearly the errors listed in both Table I-3.13 and I-3.14 indicate that we cannot assume that reflected intensities can be converted directly to RI values for crude oils. However, it is to be noted that the Fresnel fit values appear to vary from the calculated values in a monotonic manner: With the exception of the datum at $80 \%$ SQ-95 that can be statistically identified as an outlier, the Fresnel-fit values can be related to the calculated values with a second order polynomial with an $\mathrm{r}^{2}$ value of 0.9547 .

Table I-3.14. RI for mixtures of SQ-95 with toluene

\begin{tabular}{|c|c|c|c|c|c|}
\hline $\begin{array}{c}\text { SQ-95 } \\
(\mathbf{v o l} \%)\end{array}$ & $\begin{array}{c}\text { Toluene } \\
(\mathbf{v o l} \%)\end{array}$ & $\begin{array}{c}\mathbf{I}_{\mathbf{r}, 940} \\
(\mathbf{m v})\end{array}$ & $\begin{array}{c}\mathbf{R I}_{\text {fit }} \\
(\mathbf{F r e s n e l})\end{array}$ & $\begin{array}{c}\mathbf{R I}_{\text {calc. }} \\
(\text { volume fractions })\end{array}$ & $\boldsymbol{\Delta R I}$ \\
\hline 100 & 0 & 0.2832 & 1.5124 & 1.4787 & 0.0336 \\
\hline 90 & 10 & 0.2838 & 1.5114 & 1.4805 & 0.0309 \\
\hline 80 & 20 & 0.2886 & 1.5038 & 1.4822 & 0.0215 \\
\hline 70 & 30 & 0.2842 & 1.5107 & 1.4839 & 0.0268 \\
\hline 60 & 40 & 0.2862 & 1.5075 & 1.4857 & 0.0219 \\
\hline 50 & 50 & 0.2897 & 1.5020 & 1.4874 & 0.0146 \\
\hline 40 & 60 & 0.2913 & 1.4995 & 1.4892 & 0.0104 \\
\hline 30 & 70 & 0.2921 & 1.4983 & 1.4909 & 0.0074 \\
\hline 20 & 80 & 0.2930 & 1.4969 & 1.4927 & 0.0043 \\
\hline 10 & 90 & 0.2942 & 1.4951 & 1.4944 & 0.0007 \\
\hline 0 & 100 & 0.2978 & 1.4897 & 1.4962 & -0.0065 \\
\hline
\end{tabular}

\section{WAVELENGTH DEPENDENCE}

One possibility is that RI is wavelength dependent. Some tests with known compounds show, however, that RI values measured by this technique are constant or decrease very slightly with increasing wavelength (Fig. I-3.45). 


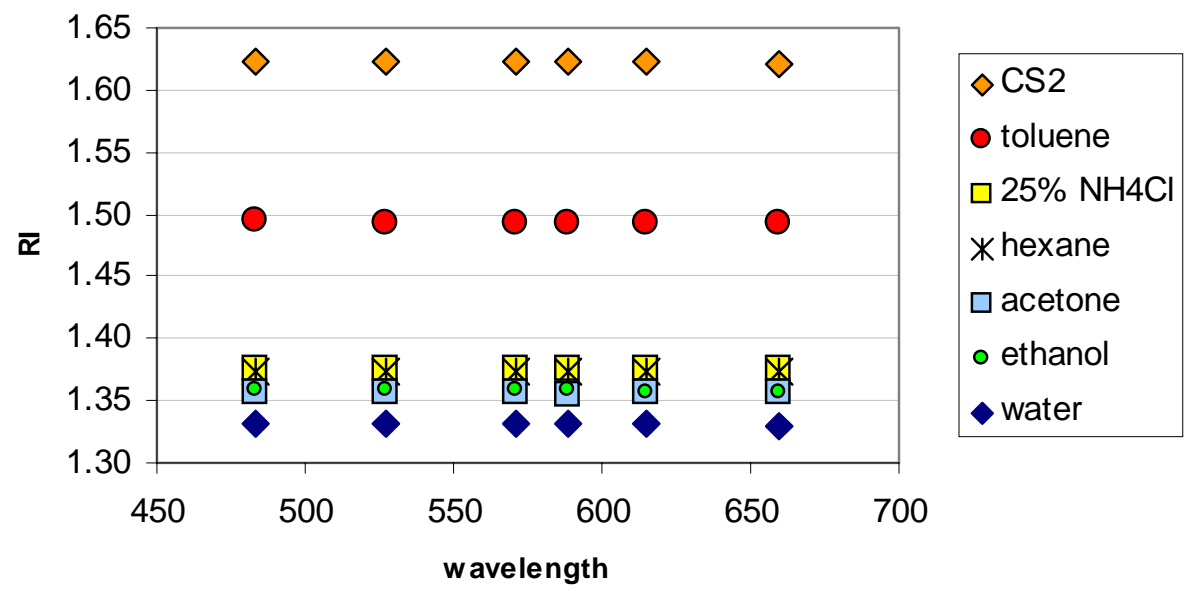

Figure I-3.45. RI calculated from reflected light intensities for known compounds as a function of wavelength.

Some of the components of crude oils may show greater wavelength dependence than these small molecules, but it seems unlikely that change in RI with wavelength alone can account for the high values calculated from the reflected light intensities.

\section{EFFECT OF ABSORBANCE}

Abbe refractometer measurements of RI at $660 \mathrm{~nm}$ gave very similar values for toluene and E-1XR-00 crude oil (1.4922). As shown in Fig. I-3.46, however, $\mathrm{I}_{\mathrm{r}}$ of mixtures of toluene and E-1XR-00 is not constant, nor do mixtures vary linearly (although $\mathrm{I}_{\mathrm{r}}$ does change monotonically), although absorbance of similar mixtures is quite linear (Fig. I-3.47). The absorbance measured for the neat crude oil agrees well with measurements by a conventional diode array spectrometer (Hewlett Packard 8453) using a $0.1 \mathrm{~mm}$ path-length cell (see Fig. I3.48).

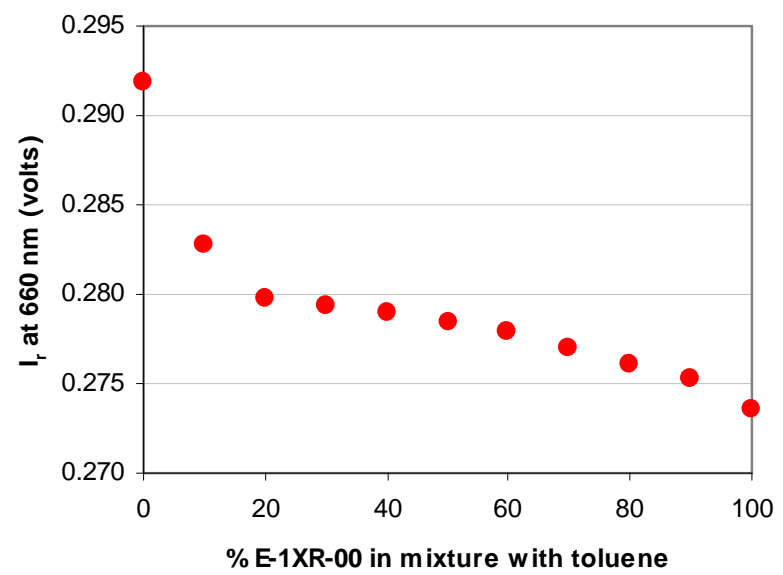

Figure I-3.46. Reflected light intensities for mixtures of toluene and E-1XR-00 crude oil, both of which have RI values of 1.4922, as measured at $660 \mathrm{~nm}$ by Abbe refractometer. 


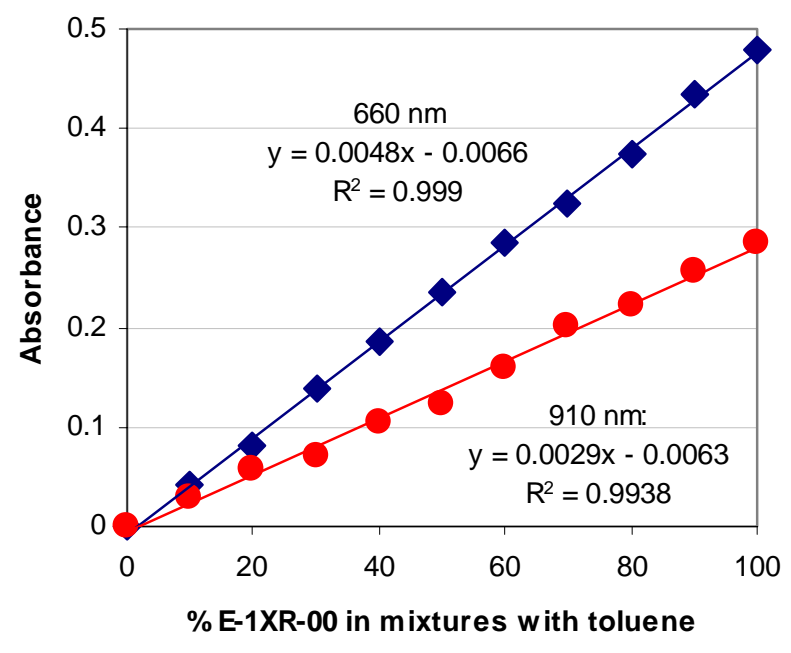

Figure I-3.47. Absorbance of mixtures of toluene and E-1XR-00 crude oil at $660 \mathrm{~nm}$ (blue diamonds) and 910 nm (red circles).

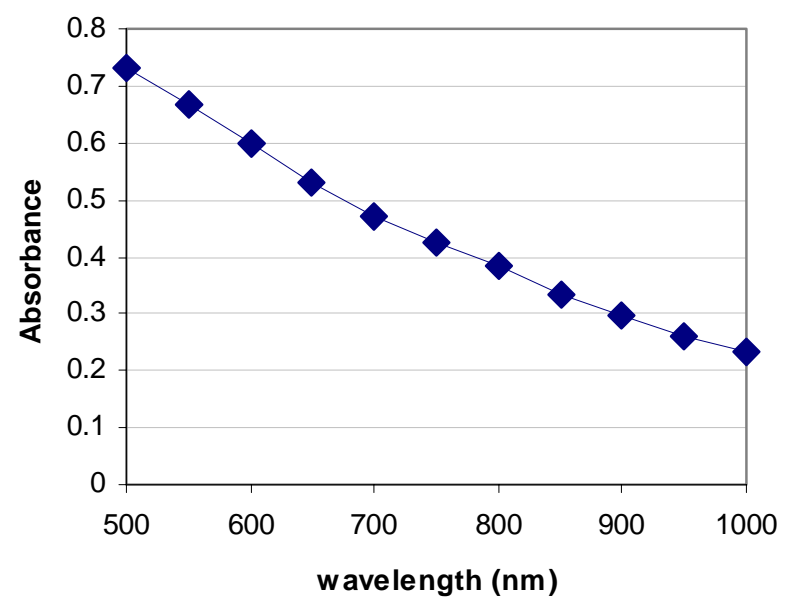

Figure I-3.48. Absorbance of E-1XR-00 crude oil as a function of wavelength.

To test whether this behavior is unique to the oil-toluene mixture or generic to a system where absorbance is changing, we made a solution of $0.25 \%$ bromothymol blue (BTB) in $0.1 M$ $\mathrm{NaOH}$. With $0.1 \mathrm{M} \mathrm{NaOH}$ set as the reference zero absorbance level, this solution has an absorbance at $660 \mathrm{~nm}$ identical to that of the E-1XD-00 crude oil, with toluene set as the zero absorbance liquid. The reflectance signal at $660 \mathrm{~nm}$ was then measured for various mixtures of the above two solutions (BTB in $\mathrm{NaOH}$ ) and $\mathrm{NaOH}$. A similar nonlinear drop in $\mathrm{I}_{\mathrm{r}}$ was observed, as shown in Fig. I-3.49, showing that the observations are not unique to the oil-toluene mixtures. 


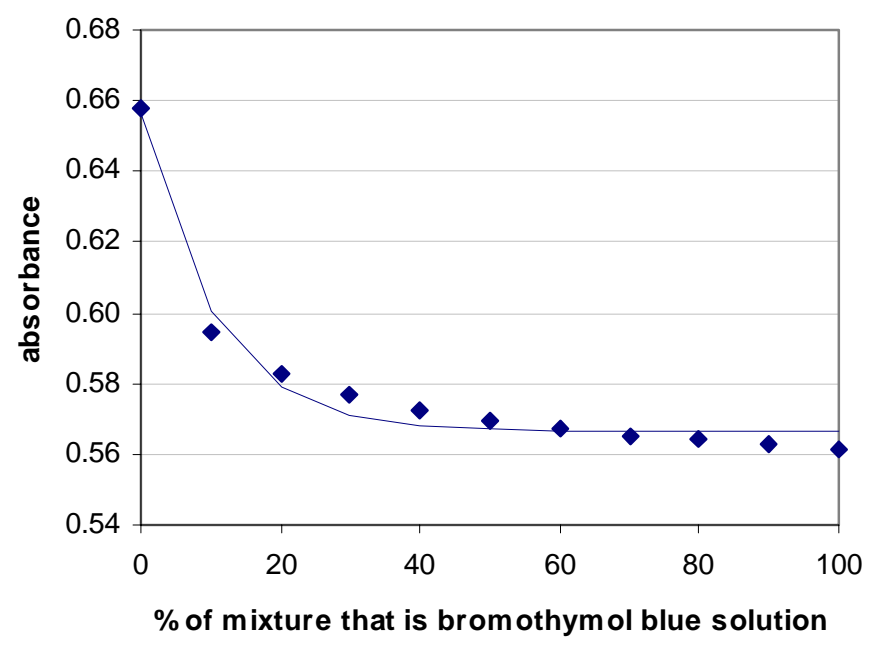

Figure I-3.49. Absorbance of mixtures of an $0.25 \%$ bromothymol blue solution in $0.1 M \mathrm{NaOH}$ with the $0.1 M$ $\mathrm{NaOH}$ solution alone show a nonlinear decrease in absorbance.

Although the intention is to measure purely the reflected light intensity from the interface, the measurement may be compromised by a number of factors that vary with the composition of the oil. Particles present in the oil may act as specular reflectors and can contribute to the observed $\mathrm{I}_{\mathrm{r}}$ value. Any light that penetrates through the liquid and then is reflected back from the container walls and is transmitted through the liquid and enters the sapphire rod will also contribute to the observed $\mathrm{I}_{\mathrm{r}}$ value. The latter factor appears to be more important. Even in containers of significant size $(30 \mathrm{~mL}$ beakers $)$ containing the test liquid and the probe dipped therein, very recent darkroom experiments show that walls and bottom of the beaker wrapped in shiny $\mathrm{Al}$ foil produce a higher Ir value than when it is wrapped in black tape. Moreover, the differences in the $I_{r}$ values are much more apparent when the more transmitting toluene is present. When the experiment is conducted in a tall test tube (with poorly reflecting walls and the bottom, which is the primary reflecting surface, far away) there is very little difference in reflection intensity between toluene and E-1XR-00 oil.

\section{EFFECT OF ADSORPTION}

A third potential source of error in the reflected signal is adsorption of crude oil components on the glass or sapphire surface. Decreases in signal strength that suggest adsorption have been documented in systems with crude oil mixed with n-hexane that will be discussed in a later section of this report.

\section{Onset}

For mixtures of two liquids, the absorbance should be a linear function of volume fractions unless precipitation occurs. Fig. I-3.50 shows measured absorbance for mixtures of SQ-95/toluene and C-LH-99/toluene at $940 \mathrm{~nm}$, both of which are linear. Deviations from linearity provide an indication of the onset of precipitation, as shown in Fig. I-3.51 for mixtures of SQ-95 oil mixed with hexane. 


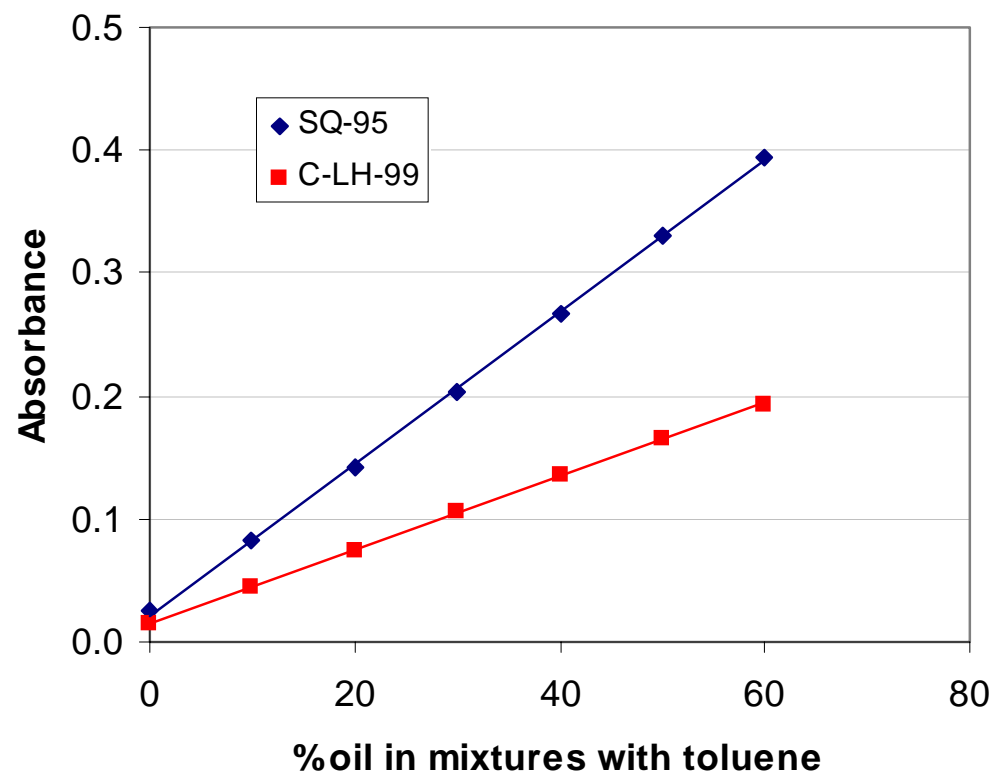

Figure I-3.50. Absorbance is a linear function of volume fractions if no precipitation occurs in the mixture.

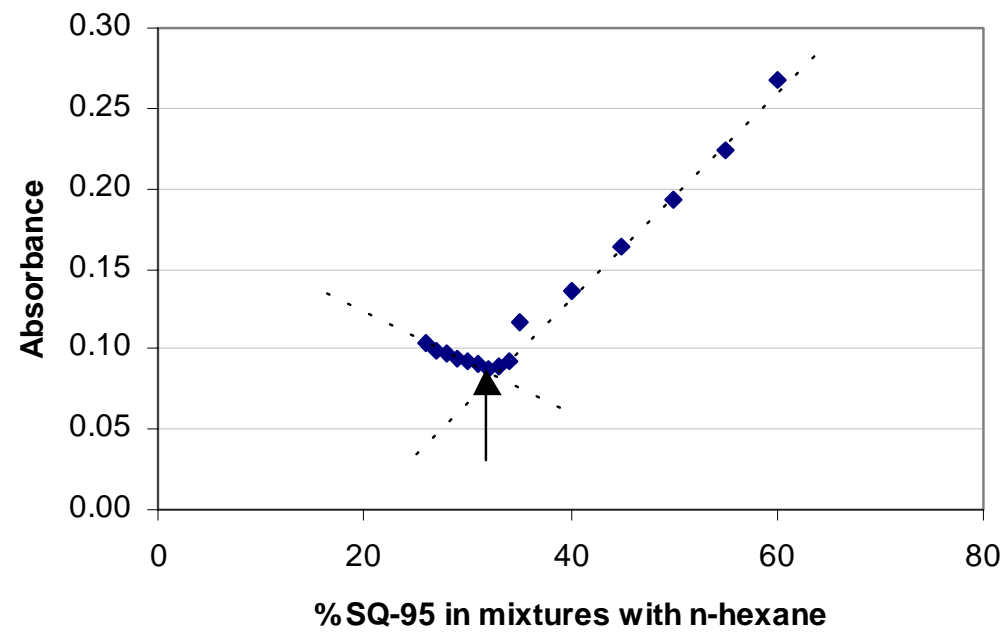

Figure I-3.51. Absorbance-composition plot for mixtures of SQ-95 oil with hexane at $940 \mathrm{~nm}$, with pump flow rate of $0.9569 \mathrm{~mL} / \mathrm{min}$.

Two possible interpretations of the data to estimate the onset of precipitation are suggested in Fig. I-3.51. The intersection of the dashed lines that fit the data above and below the onset point give an estimate of about $32.6 \%$ whereas the minimum point (indicated by the arrow) occurs at about $31.8 \%$ crude oil. It is not expected that these two points will always be as close as they are in this example. 
Also used is a third definition, the point where the data begin to diverge from linearity above the onset condition. This condition is illustrated in Fig. I-3.52 by reflected intensity data for E-1XR-00 and hexane mixtures measured at $660 \mathrm{~nm}$. Note that the deviation from linearity produces lower values of $\mathrm{I}_{\mathrm{r}}$ whereas the results presented in Fig. I-3.46 might suggest that they should deviate to higher values of $\mathrm{I}_{\mathrm{r}}$. Similar results are seen from absorbance studies. It is essential to determine which features of the curves represent the onset that can be visually determined before this method can be put into more routine service.

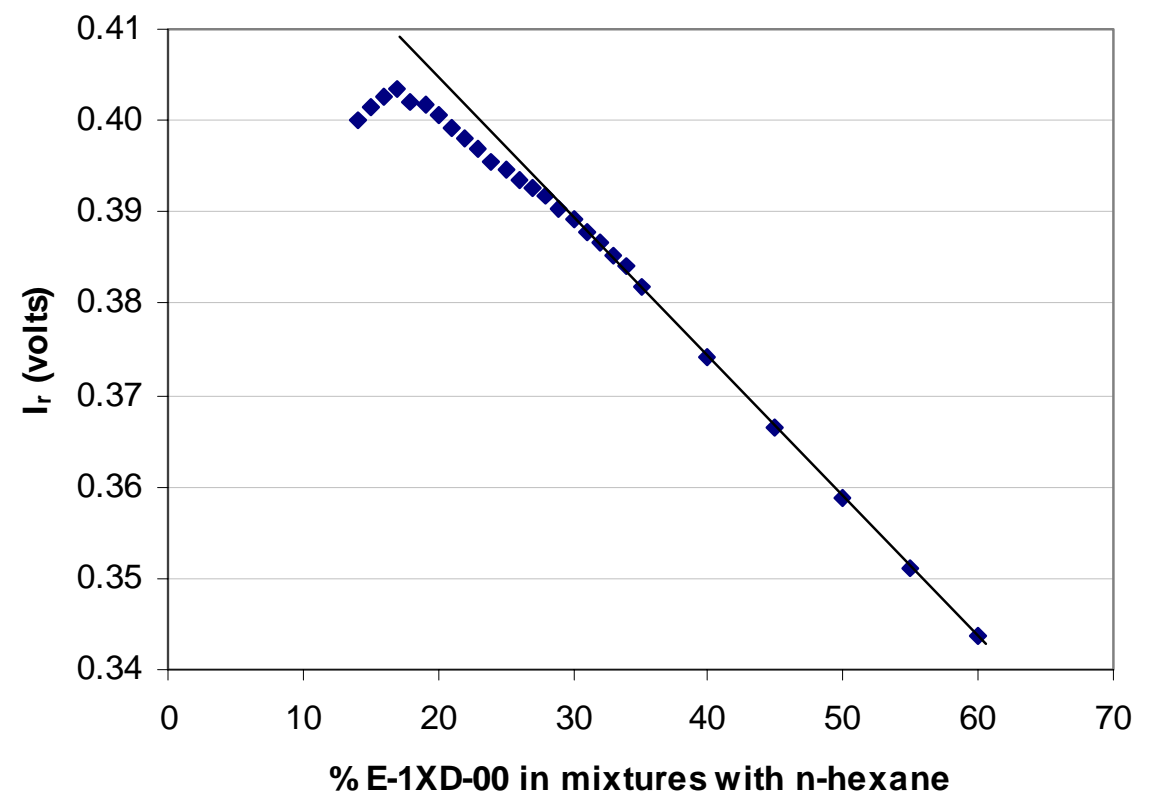

Figure I-3.52. Reflection intensity for mixtures of E-1XD-00 crude oil and n-hexane, measured at $660 \mathrm{~nm}$, flow rate $=0.9569 \mathrm{ml} / \mathrm{min}$.

The syringe pump flow rate (for hexane) can be varied so that the composition at the onset of precipitation is determined for different flow rates, which correspond to different residence/reaction times. As the residence time is decreased (i.e., flow rate is increased), higher volume fractions of hexane are required to trigger precipitation. This is consistent with previous observations that the kinetics of formation of asphaltene aggregates is slow, especially near the onset point. Precipitation onset compositions were obtained from the intersections of linear trends at different flow rates for all three oils, and are presented in Fig. I-3.53 as a function of residence time. Over the span of short residence times investigated, estimates of the amount of hexane required to initiate precipitation all increase with decreasing residence times. It is likely that longer residence times are needed to approach equilibrium conditions. 


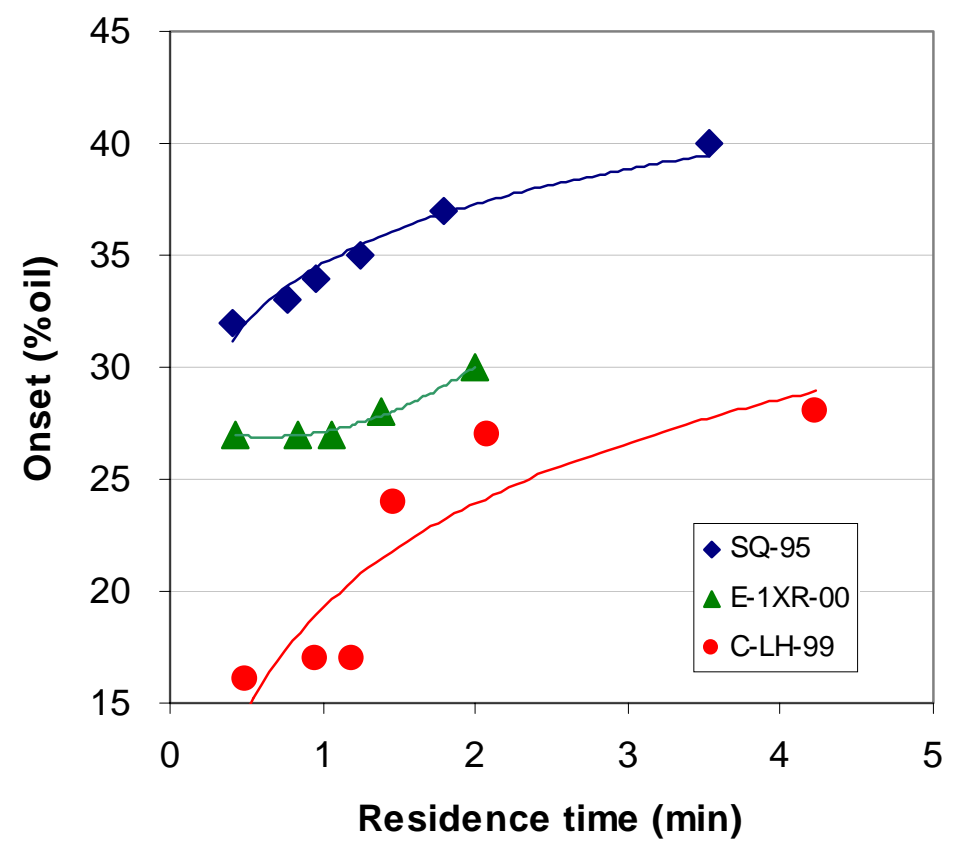

Figure I-3.53. Onset of precipitation is a function of residence time (higher flow rates correspond to shorter residence times).

Correspondence between microscopic and light intensity onset measurements

Onsets based on reflected light intensity show differences from microscopic observations that may in part be due to equilibration times that are much shorter than in the batch tests with the same oils. Additional uncertainty results from oil volume fractions that are not measured, but are calculated from the pump rates. Because of the difference in viscosity between the crude oils and hexane, the actual volume fractions may differ from those reported here. In a final set of tests, microscopic observations were made of the oil/hexane mixtures from which a sample was removed in line with the cell in which reflected light intensity was measured. The following three plots (Figs. I-3.54 - I-3.56) show the results of reflected light intensity measurements in tests that began with the flow of oil and a small amount of hexane through the cell. Intensity increased as more hexane was added by increasing the hexane pump flow rate. The first microscopic detection of fine asphaltic particles is marked as the "fine onset" and the first asphaltene aggregates are indicated as the "aggregate onset" in each case. 


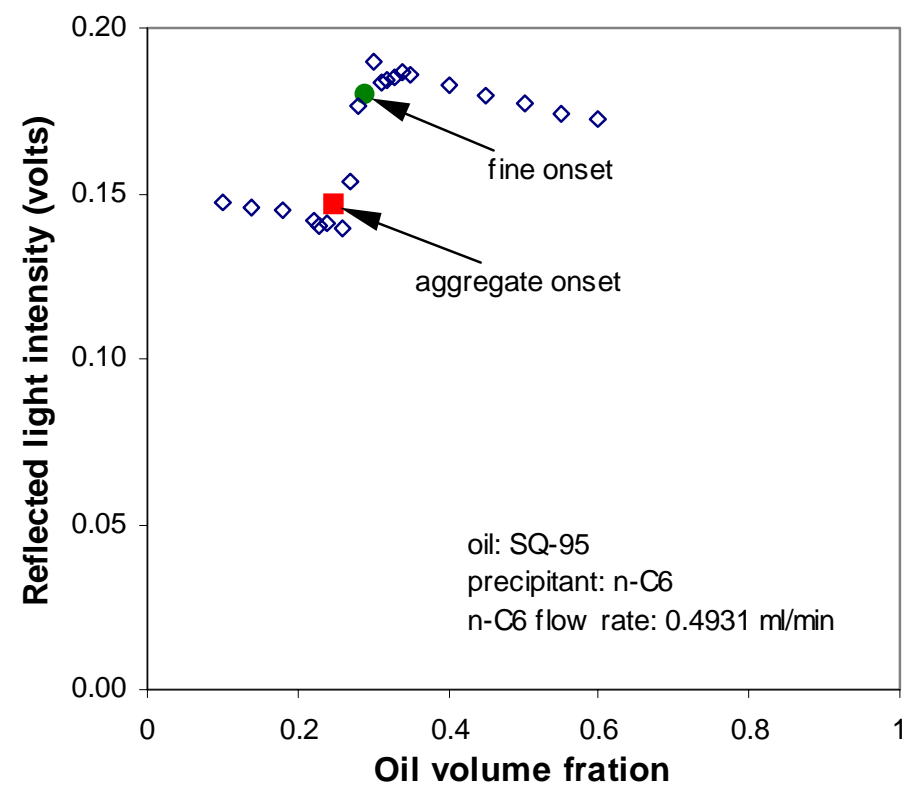

Figure I-3.54. Reflected light intensity of mixtures of SQ-95 and n-hexane. Microscopic examination of the mixtures for the existence of flocculated asphaltenes show that reflected light intensity begins to fall before the first fine asphaltic particles can be seen.

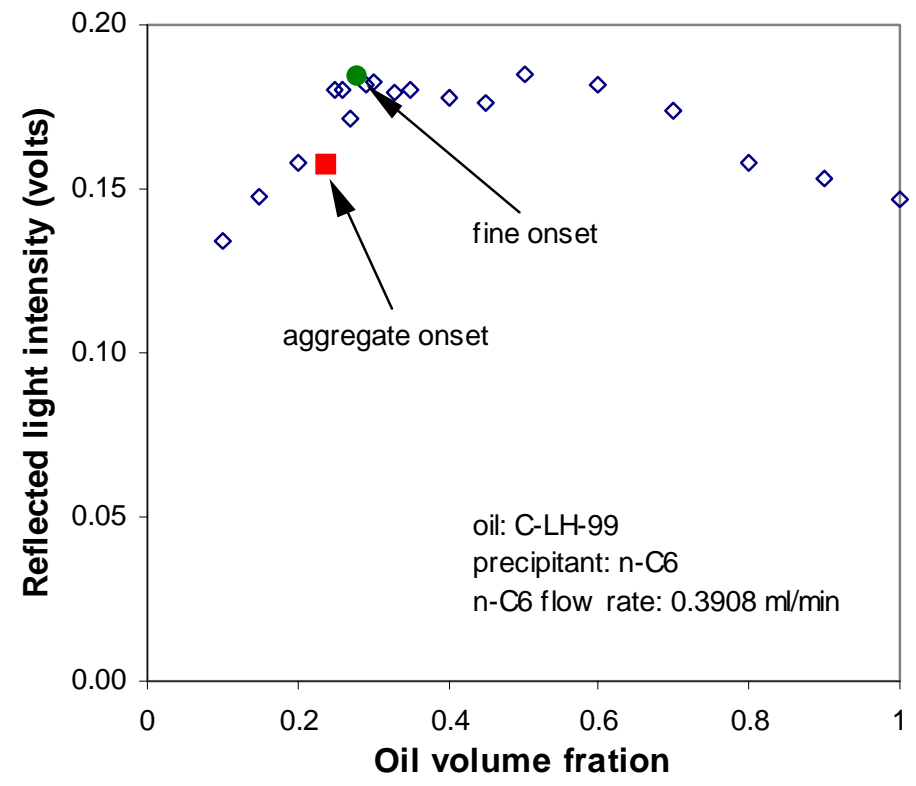

Figure I-3.55. Reflected light intensity of mixtures of C-LH-99 and n-hexane. Microscopic examination of the mixtures for the existence of flocculated asphaltenes show that reflected light intensity begins to fall long before the first fine asphaltic particles can be seen. 


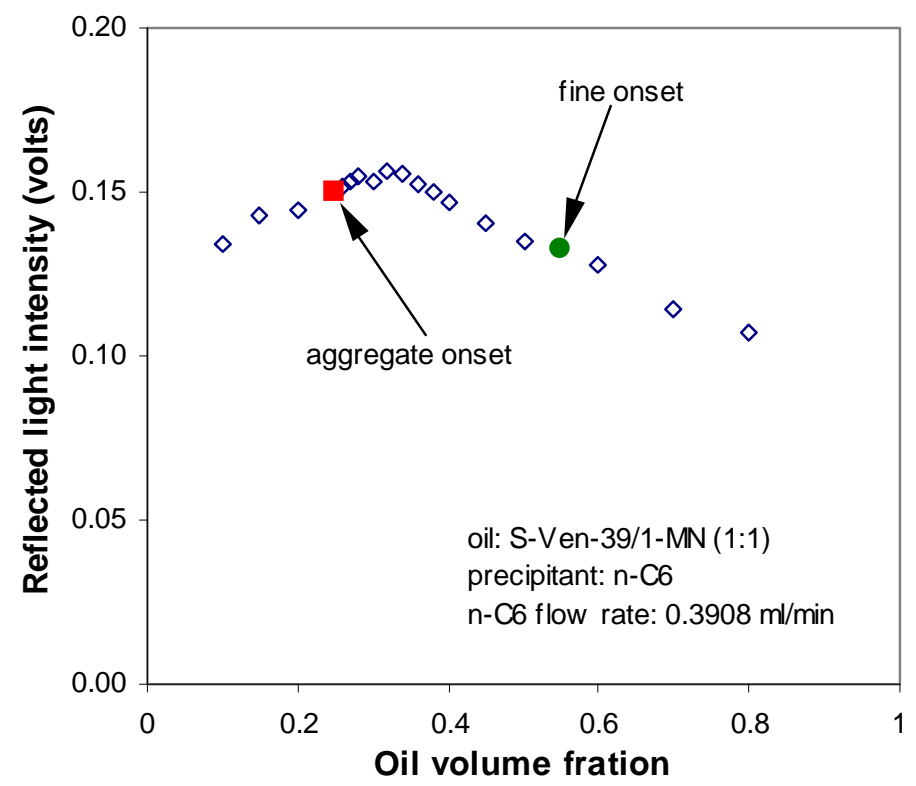

Figure I-3.56. Reflected light intensity of mixtures of S-Ven-39 and 1-MN with n-hexane. In this case, microscopic examination of the mixtures for the existence of flocculated asphaltenes reveals the presence of the first fine asphaltic particles before the reflected light intensity begins to fall, whereas aggregates can only be found well after the break in the intensity curve.

In all cases the intensity curve deviates from linearity at oil volume fractions well above the onset of asphaltene aggregate formation determined by microscopic observations. In two cases the break in the curve occurs even before fine particles can be detected. While it is possible that the reflected light intensity is simply more sensitive to the formation of flocculated asphaltene, a more likely explanation for these results is an increase in adsorption of asphaltic material on the cell windows, causing a decrease in the reflected light intensity. In previous studies we have observed increased wetting alteration by oils destabilized by addition of heptane to near-onset conditions (Al-Maamari and Buckley, 2000) that suggests an increased tendency of asphaltic material to adsorb as the onset of flocculation is approached.

The effects of adsorption of crude oil components on the cell window are demonstrated in the tests shown in Fig. I-3.57 where the results of a series of replicate experiments, conducted without cleaning the cell between runs, are shown. A run begins with a mixture of $60 \%$ SQ-95 and $40 \% \mathrm{n}$-hexane, a mixture in which the asphaltenes are stable. The concentration of $\mathrm{n}$-hexane was increased steadily and changes in reflected intensity were monitored. The first run is \#1 in Fig. I-3.57. Eliminating precipitated asphaltenes from the system for subsequent runs was best achieved by switching to an $80 \%$ oil solution and increasing the flow rate to flush the system for 30 minutes. Successive runs were initiated by again increasing the concentration of hexane, producing the data for runs $\# 2, \# 3$, and $\# 4$. In each case, the break point in the curve remains the same, but the reflected intensity of the whole curve decreases. Adsorption on the window is a likely explanation for the change in magnitude. Adsorption is especially a problem with a sapphire window. Other materials may have less propensity to accumulate crude oil components. Adsorption adds to the uncertainty in RI estimates and the break point in the reflected light 
intensity curve may indicate a near-onset condition where adsorption increases, rather than the first appearance of aggregates.

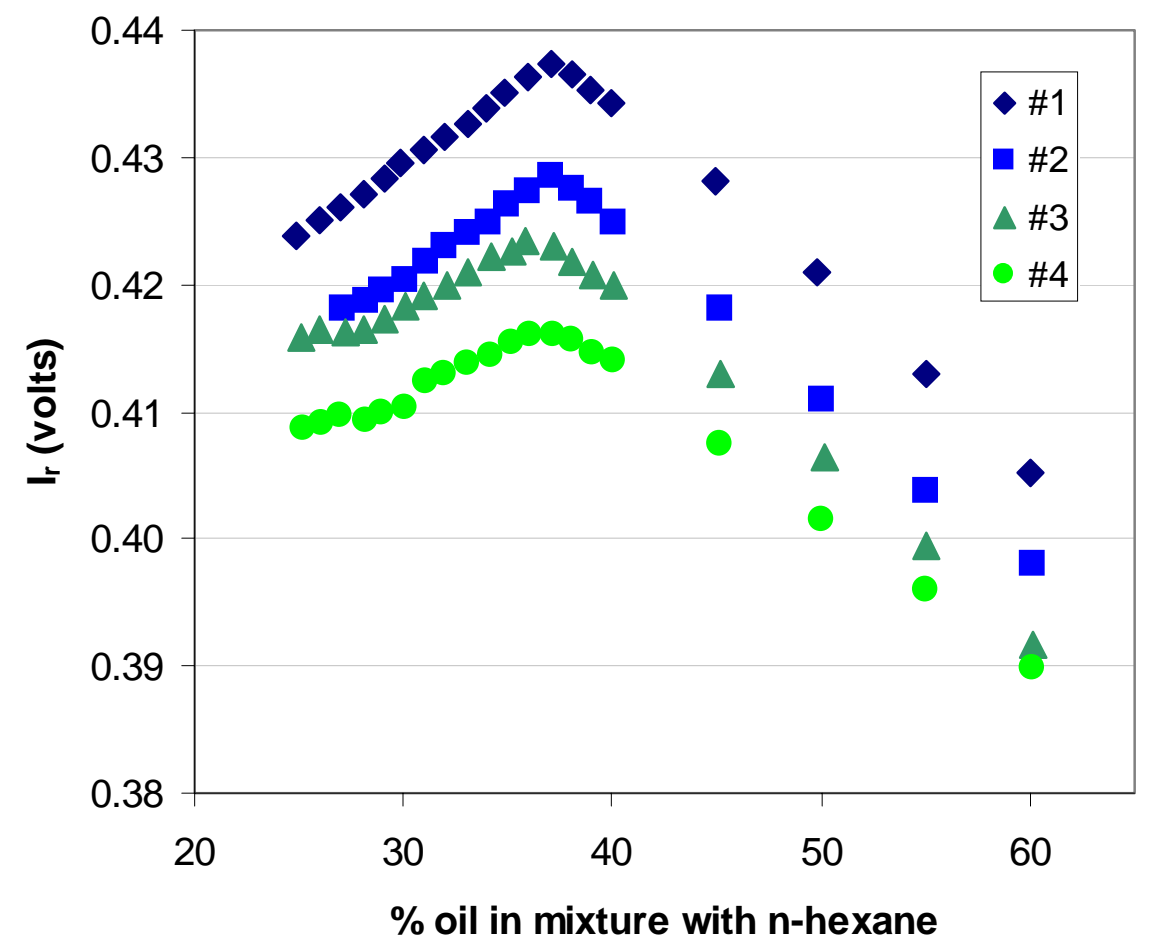

Figure I-3.57. Effect of adsorption is illustrated by decreasing reflected intensities in successive measurements with mixtures of SQ-95 and n-hexane.

\section{Conclusions}

- A system to measure reflected intensity has been developed and shown to produce consistent estimates of RI of known compounds.

- Absorbance measurements show linearity of absorbance for mixtures of crude oil with toluene.

- A minimum in absorbance (or a maximum in reflected intensity) indicates formation of asphaltene precipitate.

- Increasing residence time shifts the onset of asphaltene precipitation to lower amounts of hexane precipitant, consistent with the slow kinetics of asphaltene formation.

- Difficulties in measuring RI of crude oils and mixtures with hydrocarbon diluents, including high levels of absorbance, possible wavelength dependence, and adsorption of crude oil components on the cell window, have been identified. 


\section{Appendix to Section I-3.5}

\section{FIXED GEOMETRY REFLECTANCE INSTRUMENT}

Reproducibility of the measurement system was compromised by having a fiber optic bundle that had to be raised and lowered into the test fluid, especially since it is known that minor bends in such fiber bundles can affect the degree of cross talk between individual fibers. In addition, fluid evaporation from an open container also compromises compositional accuracy in mixed systems. A flow-through cell was therefore constructed. This is shown schematically in Fig. I-3.58. A machine drawing is shown in Fig. I-3.59. In this configuration, a confined small volume cell with in and out flow passages is equipped with a sapphire window. A randomized bifurcated glass fiber optic bundle is cemented to the window with UV-cure refractive index matching adhesive. Pumped fluid is in contact with the sapphire window and flows past it. Provision was made to imbed a Platinum RTD within the cell body and to wrap the cell (1 inch in diameter) with a siliconized flexible heater, such that the cell could be maintained at any desired temperature.

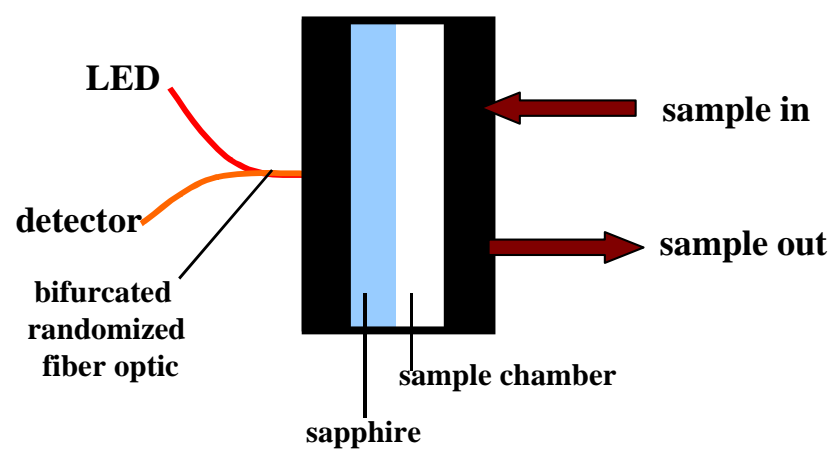

Figure I-3.58. Reflected light measurement arrangement using a flow-through cell.

A $910 \mathrm{~nm}$ LED was used in all experiments. The interior surface of the cell was covered in black epoxy paint to eliminate reflected light. The cell was wrapped with thermal insulation. The test samples were pumped by a peristaltic pump (Dynamax Model RP-1, Rainin Inc.) equipped with Viton pump tubing. The electronics, including the LED, were left turned on. Before testing, the test liquid was degassed by ultrasonication for $10 \mathrm{~min}$. The liquid was pumped into the measurement cell at a flow rate of $0.25 \mathrm{~mL} / \mathrm{min}$. The pump was stopped during the measurement to avoid pump pulsation noise.

The reflected signal intensity data were collected at a frequency of $0.1 \mathrm{~Hz}$. The average and standard deviation of each measurement were evaluated from $1 \mathrm{hr}$ of data sampling. Each sample was measured 6 times. As standards, we used the RI values listed for $\mathrm{H}_{2} \mathrm{O}$ and $25 \%$ $\mathrm{NH}_{4} \mathrm{Cl}$ solutions at the probe wavelength (Schiebener et al., 1990; McCay et al., 1996). 


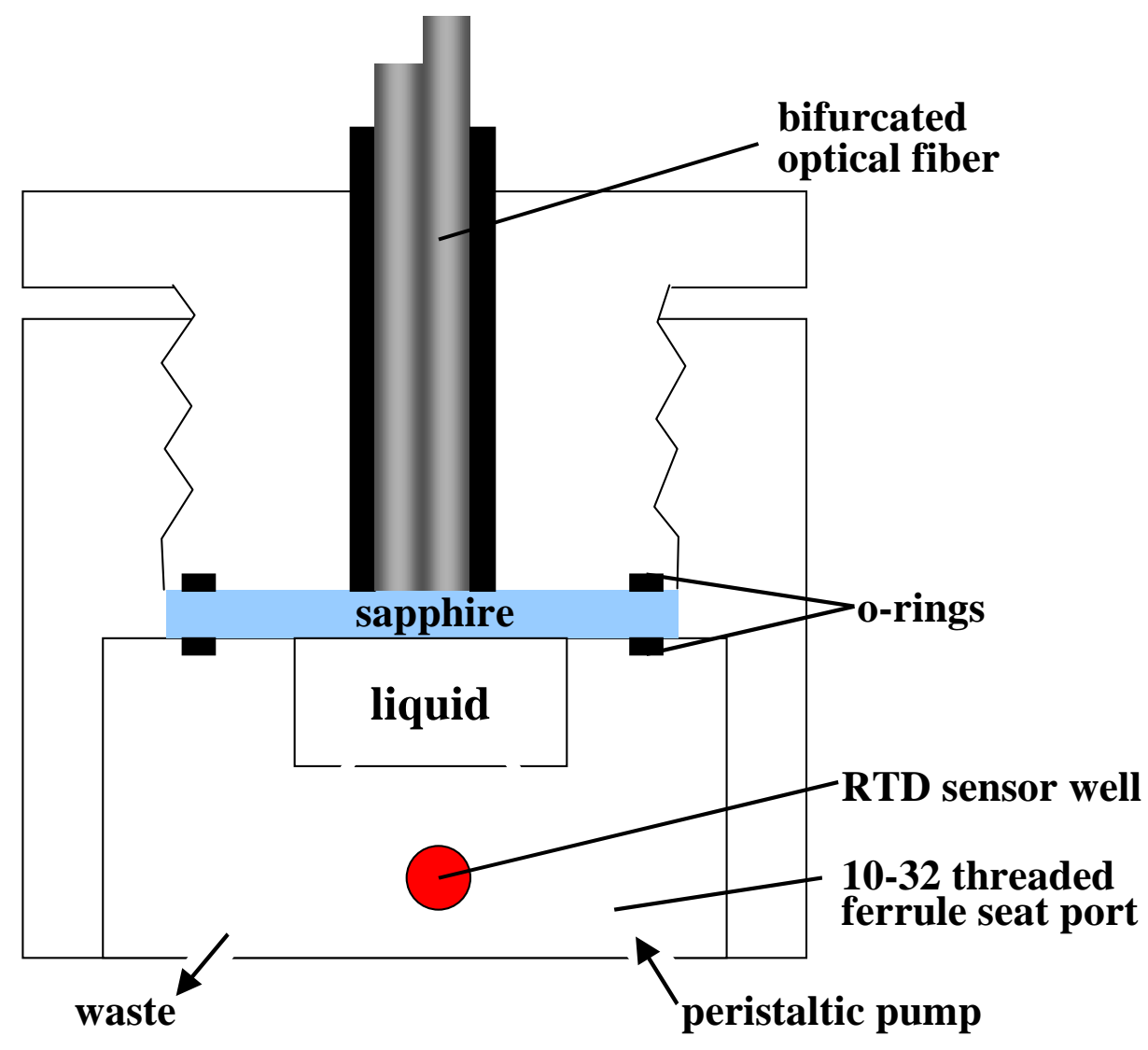

Figure I-3.59. Cell Details.

There were some initial problems. The sapphire windows were thin and several of them cracked during initial assembly. Also in the beginning, the interior surface was painted with flat black epoxy paint to eliminate internal reflection. Initially, the cell was tested with $\mathrm{H}_{2} \mathrm{O}, 25 \%$ $\mathrm{NH}_{4} \mathrm{Cl}$ solution and hexane/oil mixtures, these data showed a good fit to Eq. I-3.76.

Continued experiments with toluene and toluene/oil mixtures led, however, to detachment of the paint layer. When the problem was diagnosed, the cell disassembled and sent to a custom anodizing company. The interior has been anodized black.

The temperature control circuit in this design does not control proportionally and on/off surges affect the low level current signal from the detector. This can be taken care of in future designs. In addition, the absorbance detectors originally built for this instrument can be used to measure the reflected light intensity as illustrated in Fig. I-3.60. 


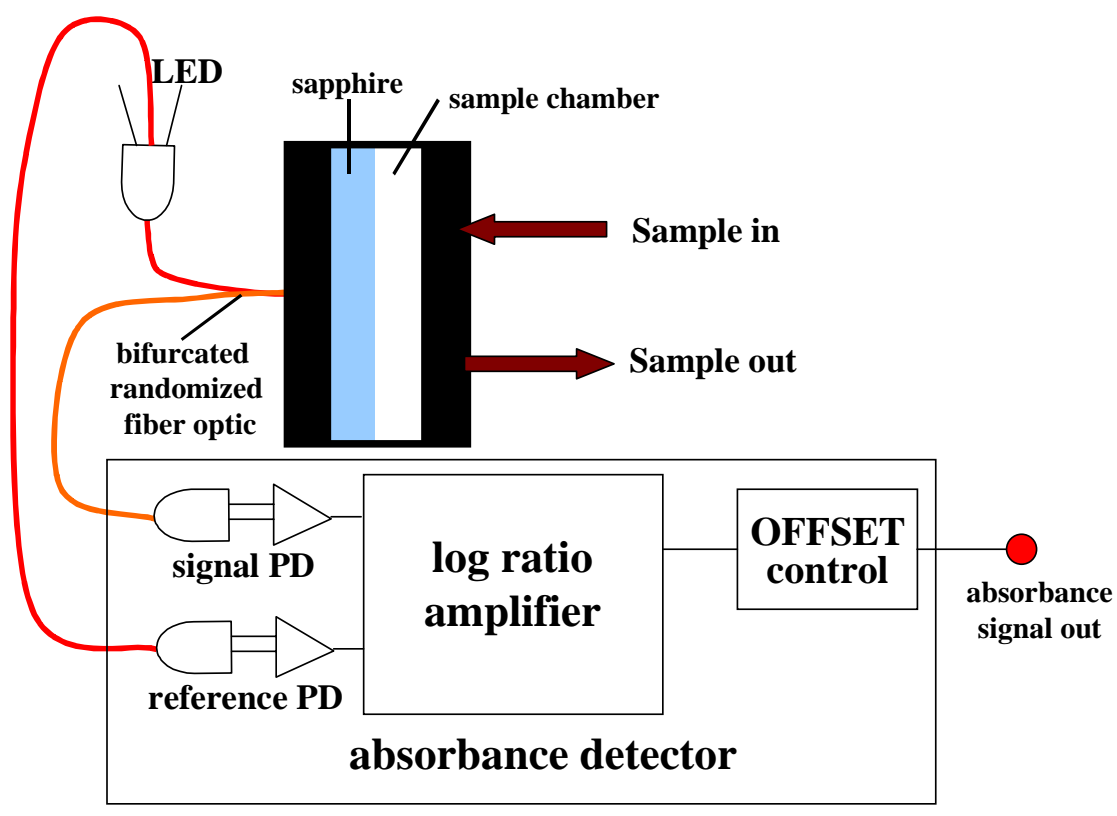

Figure I-3.60. Referenced LED reflected light detection configuration

This configuration facilitates data interpretation and reduces the number of parameters to be fitted, above and beyond providing a reference measurement of the LED. The light intensity generated by the LED is $\mathrm{I}_{0}$ and some fixed fraction $f_{R}$ of this, $f_{R} \mathrm{I}_{0}$, is measured by the reference detector. Some fixed fraction $f_{1}$, amounting to a signal proportional of $f_{1} \mathrm{I}_{0}$, is reflected back prior to reaching the sapphire internal cell interface. Then the primary reflection of interface occurs and if air $(\mathrm{RI}=1)$ is in the cell, this reflected signal is equal to $\left(1-f_{l}\right) \mathrm{I}_{0}\left[\left(\mathrm{n}_{\text {sapph }}-1\right) /\left(\mathrm{n}_{\text {sapph }}+1\right)\right]^{2}$.

Thus

$$
\begin{gathered}
\text { Total } \mathrm{I}_{\text {ref }}=\mathrm{f}_{\mathrm{R}} \mathrm{I}_{0} \\
\text { Total } \mathrm{I}_{\text {refl }}=\mathrm{f}_{1} \mathrm{I}_{0}+\left(1-\mathrm{f}_{1}\right) \mathrm{I}_{0}\left(\frac{\mathrm{n}_{\text {sapph }}-1}{\mathrm{n}_{\text {sapph }}+1}\right)^{2}=\mathrm{I}_{0} \frac{4 \mathrm{f}_{1} \mathrm{n}_{\text {sapph }}+\left(\mathrm{n}_{\text {sapph }}-1\right)^{2}}{\left(\mathrm{n}_{\text {sapph }}+1\right)^{2}}
\end{gathered}
$$

The absorbance output $\mathrm{A}$ is $\log \left[\left(\right.\right.$ Total $\left.\mathrm{I}_{\text {reff }}\right) /\left(\right.$ Total $\left.\left.\mathrm{I}_{\mathrm{ref}}\right)\right]$ plus a constant that is dictated by the offset control. With air in the cell, the overall output is set to zero so that

$$
\mathrm{A}_{\text {air }}=0=\log \left(\frac{\text { total } \mathrm{I}_{\text {refl }}}{\text { total }_{\text {ref }}}\right)+\mathrm{K}=\log \left(\frac{4 \mathrm{f}_{1} \mathrm{n}_{\text {sapph }}+\left(\mathrm{n}_{\text {sapph }}-1\right)^{2}}{\mathrm{f}_{\mathrm{R}}\left(\mathrm{n}_{\text {sapph }}+1\right)^{2}}\right)+\mathrm{K}
$$

where $\mathrm{K}$ is the offset constant. When the second calibrant, e.g., $\mathrm{NH}_{4} \mathrm{Cl}$ solution, is put in the cell, without changing the offset control, the absorbance $\mathrm{A}$ is measured (since $\mathrm{RI}$ of this will be closer to $\mathrm{n}_{\text {sapph }}$, less light will be reflected and A' will be positive). This will be given as: 


$$
A^{\prime}=\log \left(\frac{4 f_{1} n_{\text {sapph }}+\left(n_{\text {sapph }}-n_{\mathrm{NH}_{4} \mathrm{Cl}}\right)^{2}}{\mathrm{f}_{\mathrm{R}}\left(\mathrm{n}_{\text {sapph }}+\mathrm{n}_{\mathrm{NH}_{4} \mathrm{Cl}}\right)^{2}}\right)+\mathrm{K}
$$

Subtracting Eq. I-3.80 from Eq. I-3.81 and rearranging yields:

$$
10^{\mathrm{A}^{\prime}}=\frac{\left[4 \mathrm{f}_{1} \mathrm{n}_{\text {sapph }}+\left(\mathrm{n}_{\text {sapph }}-\mathrm{n}_{\mathrm{NH}_{4} \mathrm{Cl}}\right)^{2}\right]\left(\mathrm{n}_{\text {sapph }}-1\right)^{2}}{\left[4 \mathrm{f}_{1} \mathrm{n}_{\text {sapph }}+\left(\mathrm{n}_{\text {sapph }}-1\right)^{2}\right]\left(\mathrm{n}_{\text {sapph }}+\mathrm{n}_{\mathrm{NH}_{4} \mathrm{Cl}}\right)^{2}}
$$

The only unknown in Eq. I-3.82 is $f_{1}$ and this is now fully calibrated. The exact configuration was not used however, because of excessive breakage of sapphire windows. It can be profitably used with a thicker window.

\section{References}

Al-Maamari, R.S.H. and Buckley, J.S.: "Asphaltene Precipitation and Alteration of Wetting: Can Wettability Change During Oil Production?" paper SPE 59292 at the 2000 SPE/DOE IOR Symposium, Tulsa, 3-5 Apr.

Buckley, J. S., Prediction the onset of asphaltene precipitation from refractive index measurements, Energy and Fuels (1999) 13:328-332

Buckley, J.S., Hirasaki, G.J., Liu, Y., Von Drasek, S., Wang, J.X. and Gill, B.S., , Asphaltene precipitation and solvent properties of crude oil, Petroleum Science and Technology (1998) 16:251-285

Buckley, J.S., Liu, Y., Xie, X.N and Morrow, N.R., 1997, Asphaltenes and crude oil wetting the effect of oil composition, SPEJ, (June):107-119

Dasgupta, P. K., Bellamy, H. S., Liu, H., Lopez, J. L., Loree, E. L., Morris, K., Petersen K., and Mir, K. A.: "Light Emitting Diode Based Flow-Through Optical Absorption Detectors," Talanta (1993) 40, 53-74.

Fuhr, B.J., Cathrea, C., Coates, L., Kalra, H., and Majeed, A.I., Properties of asphaltenes from a waxy crude, Fuel (1991) 70, 1293-1297.

Levi, L. In Applied Optics, - A guide to optical system Design, Volume 2, Wiley, NY, 1980. pp. $1-29$.

Liu, H. and Dasgupta, P. K., Dual-wavelength Photometry With Light Emitting Diodes. Compensation of Refractive Index and Turbidity Effects in Flow-Injection Analysis, Anal. Chim. Acta (1994) 289, 347-353.

MacMillan, D.J., Tackett, J.E., Jessee, M.A. and Monger-McClure, T.G., A unified approach to asphaltene precipitation: Laboratory measurement and modeling, JPT(1995) 47, 788-795

McCay, M. H. Semak, V.V., Hopkins, J.A. and Blaha, B. 1996, Refractive index of $\mathrm{NH}_{4} \mathrm{Cl}_{-} \mathrm{H}_{2} \mathrm{O}$ as a function of wavelength: The effect of temperature and concentration, Applied Optics, 35: 3715-3718. 
Munk, M.N., 1983, Refractive index detectors, In: Liquid Chromatography Detectors, Ed. T.M. Vickrey, Marcel Dekker, Inc., pp.165-204

Schiebener. P., Straub, J., Levelt Sengers, J.M.H. and Gallagher, J.S., 1990, Refractive Index of Water and Steam as Function of Wavelength, Temperature and Density. Journal of Physical and Chemical Reference Data. 19(3):677-715

Synovec, R.E. and Yeung, E.S.: "Quantitative Analysis without Analyte Identification by Refractive Index Detection," Anal. Chem. (1983) 55, 1599-1603.

Takhar, S., Ravenscroft, P.D. and Nicoll, D.C.A., 1995, Prediction of asphaltene deposition during production - Model description and experimental details, European Formation Damage Conf., The Hague, Netherlands, 15-16 May.

Waiz, S.; Cedillo, B. M.; Jambunathan, S.; Hohnholt, S. G.; and Dasgupta, P. K. "Dispersion in Open Tubular Reactors of Various Geometries." Anal. Chim. Acta 428, 163-171 (2001)

Wang, J.X., 2000, Predicting asphaltene flocculation in crude oils, Ph.D. Dissertation, New Mexico Institute of Mining and Technology, Socorro, NM. 


\section{I-3.6. A Unified Approach to Asphaltene Stability Modeling}

\section{Buckley, J.S. and Wang, J.X.: “A Unified Approach to Asphaltene Stability Modeling,"}

Invited Paper, HOD-2002, Puerto Vallarta, 17-21 Nov. 2002.

Asphaltenes can flocculate under many different conditions. Two commonly considered examples are the asphaltenes produced by titration with $n$-alkanes and those that appear during depressurization of live crude oils, but there are many other situations of practical interest such as mixing of two or more different crude oil streams, gas lift, and gas injection processes where the potential for asphaltene problems must be evaluated with minimal amounts of sample on very tight development schedules.

Solubility models provide the key to predicting the onset of asphaltene instability as a function of composition and pressure. As few as two or three onset titrations with different $n$ alkanes at ambient conditions may be required. PVT and compositional data can be used to extend predictions from stock-tank conditions to the live oil. Refractive index-either measured or calculated - is related to solubility parameter for nonpolar species and thus can be used to provide a quantitative description of solubility conditions with respect to asphaltenes.

\section{Background}

Just a decade ago, Trbovich and King (1991) listed 11 different causes of asphaltene deposition $\left(\mathrm{CO}_{2}\right.$, rich gas, $\mathrm{pH}$ shift, mixing of crude streams, incompatible organic chemicals, stimulation, shear, pressure drop, streaming potential, temperature drop, and charged, bare metal surfaces). Acidizing may increase asphaltene problems through chemical reactions. Streaming potential, $\mathrm{pH}$-dependent phenomena generally, and charged bare metal surfaces may affect deposition mechanisms, rather than asphaltene stability per se. The remaining phenomena are usually subdivided into pressure, temperature, and composition effects. In particular, the effects of isothermal depressurization are often distinguished from those observed in ambient conditions solvent titration experiments. Models of asphaltene stability that depend on specific interactions between "asphaltenes" and "resins" for explaining the solvent titration phenomena have been pervasive throughout the literature on asphaltenes, but such models cannot explain asphaltene instability during isothermal depressurization.

An alternative approach to modeling asphaltene stability, based on the Flory-Huggins theory of polymer solubility, was introduced by Hirschberg et al. (1984). deBoer et al. (1995) showed how to apply the Hirschberg model to predict the likelihood of asphaltene deposition problems as a function of pressure and crude oil properties (especially density). Cimino et al. (1995) reported on a thermodynamic model that improved on the ability to predict the pressure onset of asphaltene instability from solvent titration data. Predictions of onset pressures remained inaccurate, mainly because there was no direct measure of crude oil solubility parameter available; many researchers continued to treat pressure depletion and solvent titration as different phenomena. 
Recent advances in fluid characterization and onset observations include recognition that refractive index (RI) provides a direct measure of solvent properties for non-polar species (Buckley et al., 1998). It is no longer necessary to estimate solubility parameters from dilute mixtures of oil in known solvents or to use other estimation techniques. Because the oil solubility characteristics are included explicitly in the asphaltene analysis, changes in stability that occur upon addition of good solvents, such as toluene, can also be investigated (Wang and Buckley, 2002). It is no longer necessary to make unverified assumptions about the effect (or lack thereof) of toluene dilution on asphaltene stability. Solubility models have been improved by reconsidering the criteria used to define the onset (Wang and Buckley, 2001a). Since it is now possible to make live-oil onset observations, they can be used to test predictions (Hammami, et al., 2000). Validated models can be applied to prediction of asphaltene stability for a wide range of conditions of temperature, pressure, and composition.

\section{Thermodynamic Model (ASM)}

Solubility models have succeeded in providing guidelines regarding the potential for asphaltene instability (deBoer et al., 1995), but have been less successful in predicting onset pressures, leading some to conclude that the mechanisms responsible for asphaltene instability during depressurization were different than those operating in experiments where asphaltenes formed in response to addition of heptane or pentane. A careful study of asphaltene flocculation induced by a series of n-alkanes from pentane to pentadecane (Wang and Buckley, 2001a) has shown (1) that the Hirschberg formulation cannot fit asphaltene onset conditions even over that limited range and (2) that the Hirschberg approach, modified with regard to simplifying assumptions and onset criteria, can explain experimental observations. The resulting Asphaltene Solubility Model (ASM), like the model proposed by Hirschberg et al. (1984), is based on FloryHuggins theory and treats the oil as a mixture of only two components (asphaltene is one component and all other compounds in the oil, including added solvents and anti-solvents are the second component). Predictions based on the ASM model are compared in Fig. I-3.61 with measured onsets for two crude oil samples with n-heptane, n-undecane, and n-pentadecane as precipitants. In both cases the asphaltene molar volume is set at $2500 \mathrm{ml} / \mathrm{mole}$. The only adjustable parameter is the asphaltene solubility parameter $\left(\delta_{\mathrm{a}}\right)$. Although these two samples are from the same reservoir, they are clearly different with respect to the asphaltene solubility parameters and thus to the stability of their asphaltenes.

\section{Empirical Model}

Because of the opposing contributions of solubility parameter and oil molar volume to asphaltene stability, ASM predicts that $\mathrm{P}_{\mathrm{RI}}$ will decrease with decreasing precipitant molar volume. In fact, as shown by the dashed lines in Fig. I-3.62, a linear relationship is often

observed between $P_{R I}$ and $v_{p}{ }^{1 / 2}$ where $v_{p}$ is the molar volume of the precipitant or flocculating agent (Buckley and Wang, 2002). The empirical model, which like ASM emphasizes asphaltene solubility and the solution properties of the oil, is useful in many situations where insufficient information is available for a complete ASM fit to experimental results. 


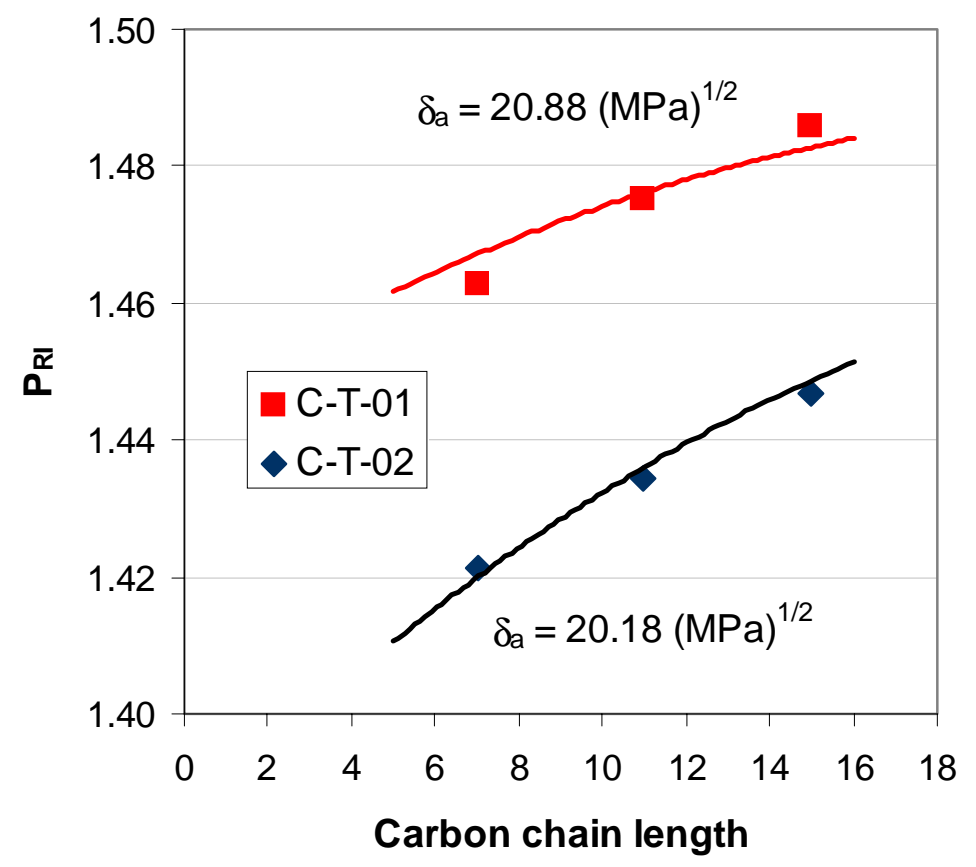

Figure I-3.61. Two samples of oil from a single reservoir have very different asphaltene stability characteristics. ASM fits to experimental data measured at $20^{\circ} \mathrm{C}$ show that the asphaltene solubility parameters for C-T-01 and C-T-02 are 20.88 and $20.18(\mathrm{MPa})^{1 / 2}$, respectively, if the asphaltene molar volume is assumed to be $2500 \mathrm{ml} / \mathrm{mole}$.

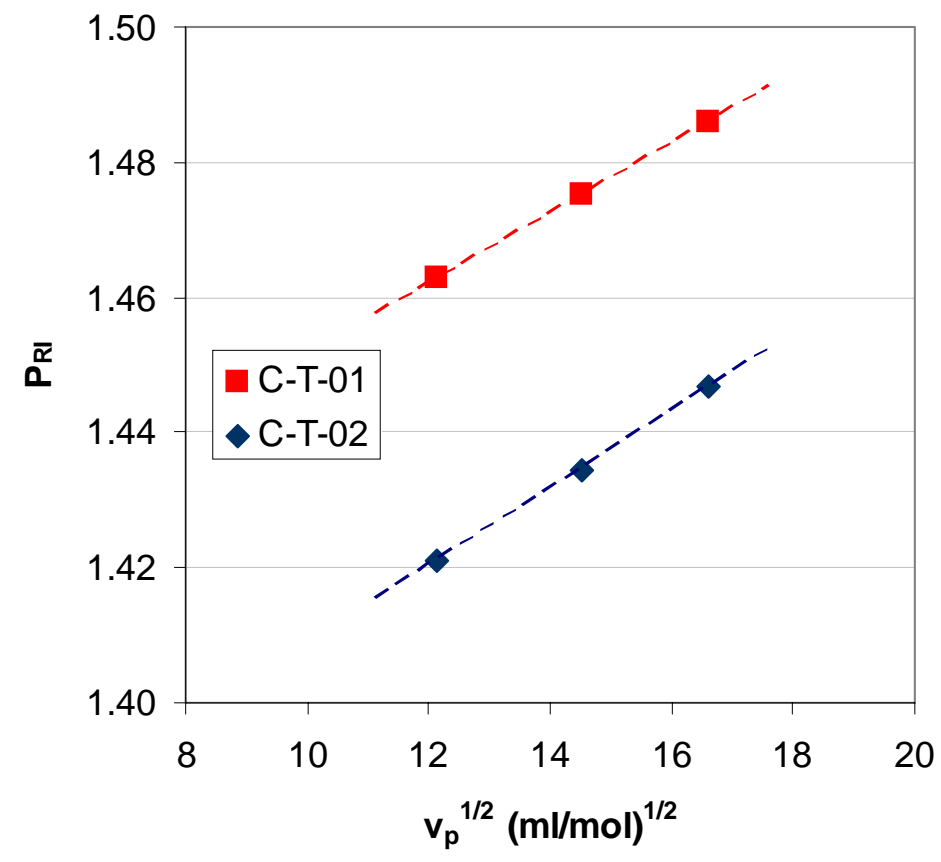

Figure I-3.62. Linear fits to experimental data where $\mathbf{P}_{\mathrm{RI}}$ is plotted as a function of precipitant molar volume, $v_{p}{ }^{1 / 2}$, for the results shown in Fig. I-3.61. 


\section{Titration vs. depressurization}

One application of the information shown in Fig. I-3.62 is prediction of onset conditions for precipitants other than those measured in the titration experiment. Interpolation to predict the onsets for normal alkanes between heptane and pentadecane is extremely accurate. Extrapolation is harder to test, but potentially much more interesting.

In a live oil, asphaltene precipitation is caused by incompatibility of asphaltenes with methane and other light, saturated alkane components. Wang and Buckley (2001b) describe in detail how to extrapolate the results of liquid alkane titration experiments to estimate the onset pressure during depressurization of a live oil. A key assumption is that the extrapolation follows the linear trend established by titrations with liquid alkanes. That assumption has been tested using the depressurization onset results of Ting et al. (2002) with a model oil, composed of asphaltenes dissolved in toluene to which varying amounts of methane were added. Figure I3.63 shows the liquid titration results that were extrapolated to the partial molar volume of methane in the asphaltene solution. The experimental and predicted results are shown in Fig. I3.64. Onset pressures are extremely dependent on the amount of methane dissolved in the model oil. The matches to exact onset pressures show some deviation, but the trend of onset pressure with the amount of dissolved methane is quite accurately predicted. This result confirms that methane precipitates asphaltenes by the same mechanism as does heptane and other liquid alkanes.

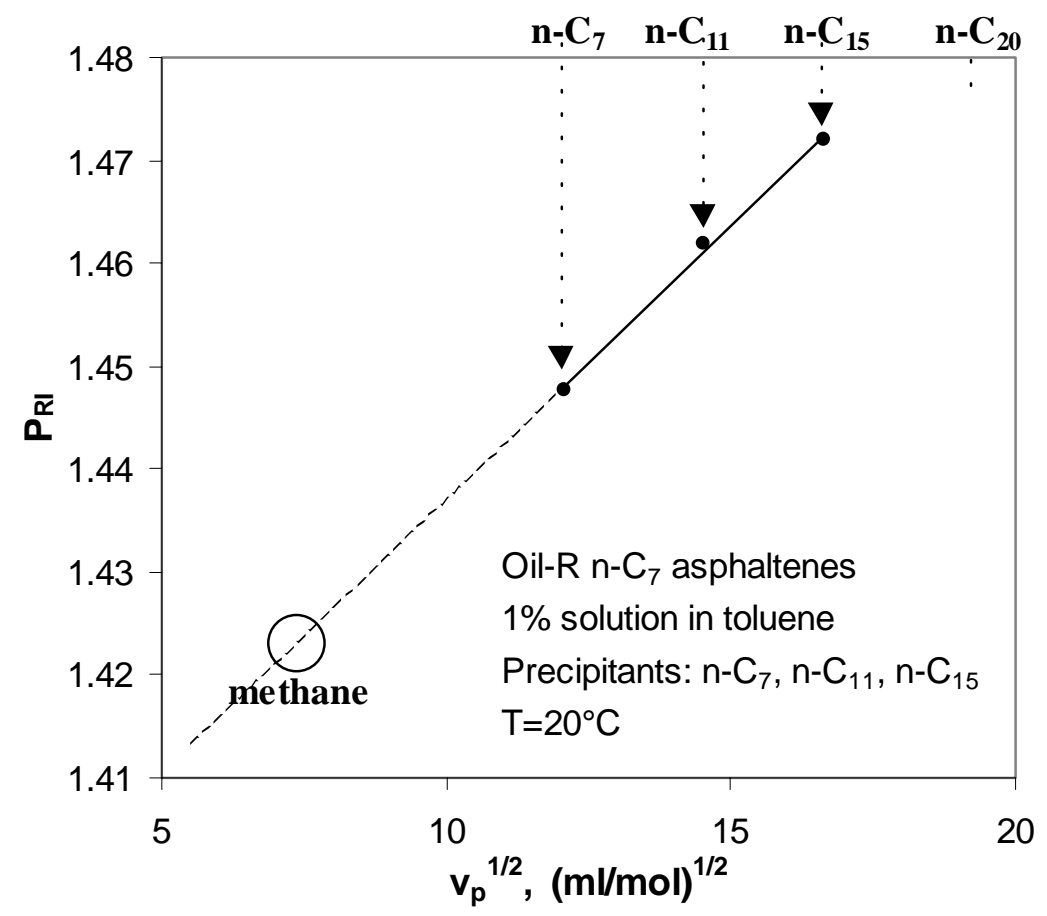

Figure I-3.63. Onset characterization of a mixture of $1 \% \mathrm{n}-\mathrm{C}_{7}$ asphaltenes from Oil-R dissolved in toluene. The partial molar volume of methane is roughly in the range indicated and is a function of pressure, temperature, and composition. 


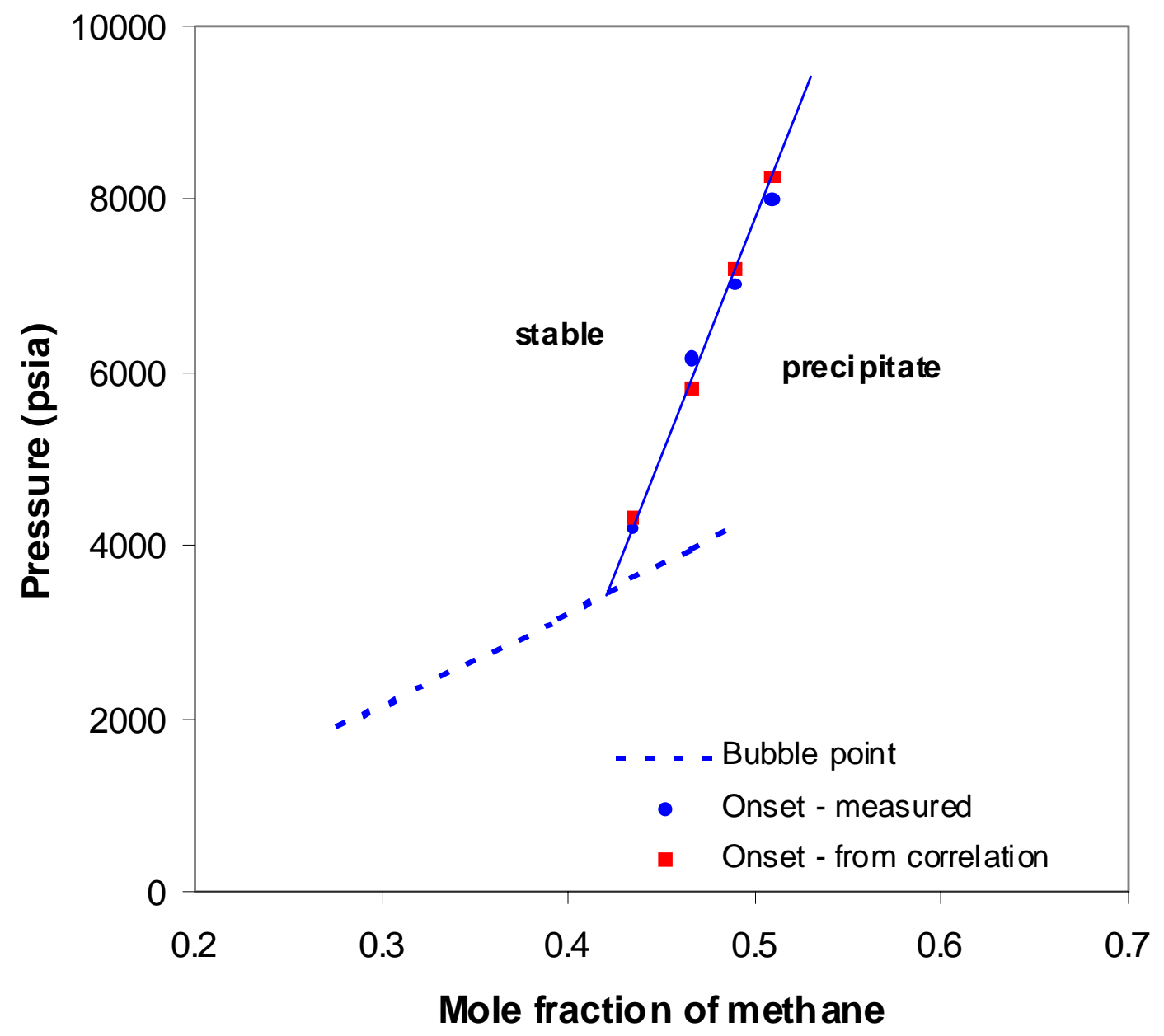

Figure I-3.64. Experimental and predicted onset pressures for a series of mixtures of n-C7 asphaltenes from Oil-R, toluene, and methane. (Experimental data from Ting et al., 2002)

Predictions of onset conditions for the two C-T crude oils are shown in Fig. I-3.65. For $\mathrm{C}-\mathrm{T}-01, \mathrm{RI}$ of the live crude falls below $\mathrm{P}_{\mathrm{RI}}$ during depressurization. The onset of asphaltene flocculation should occur near that crossover pressure of 8500 psi. RI decreases during depressurization of C-T-02, but the bubble point is reached before RI falls below $\mathrm{P}_{\mathrm{RI}}$. No asphaltene precipitation should occur during depressurization of C-T-02. Validation of crude oil predictions is in progress and will be the subject of future presentations. 


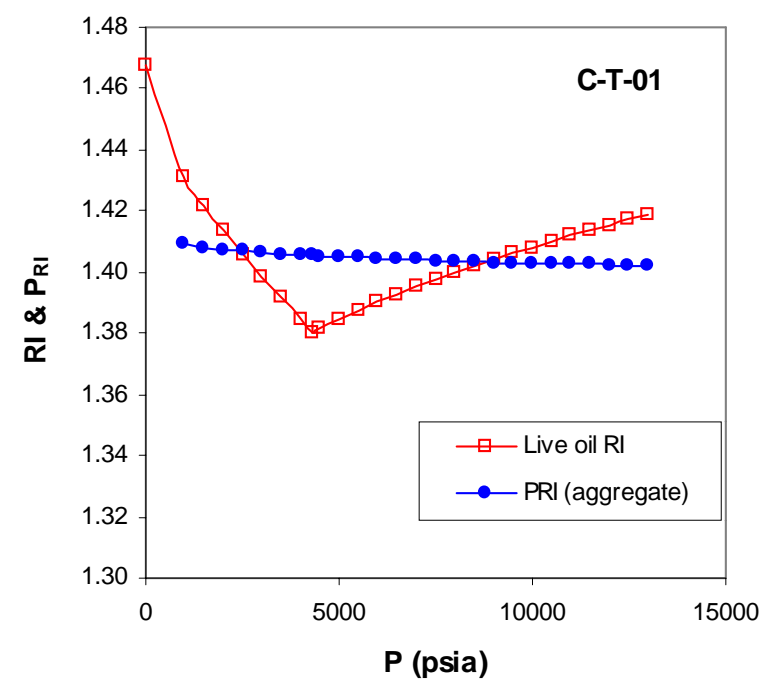

(a) Depressurization of C-T-01 will produce asphaltenes below about 8500 psi,

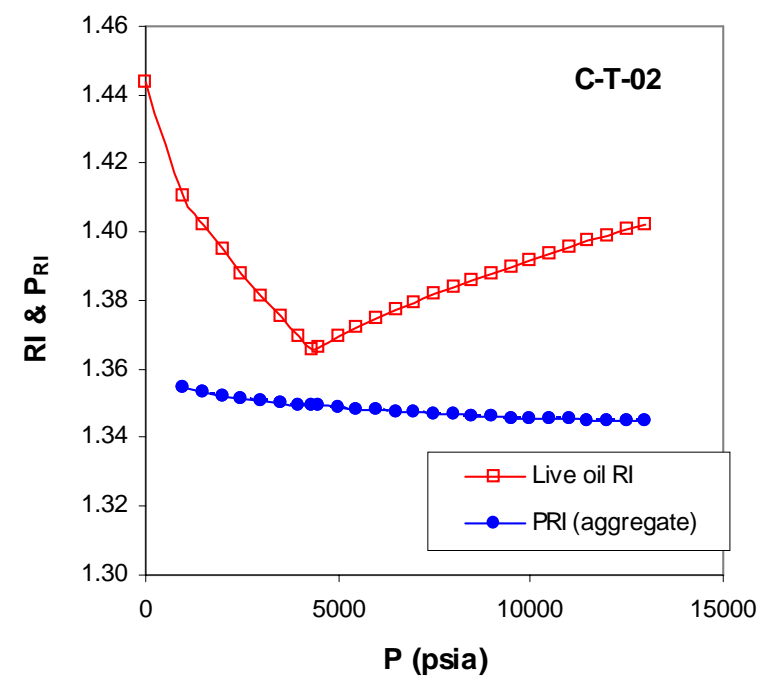

(b) C-T-02 is stable throughout depressurization.

Figure I-3.65. Calculated values of live oil RI compared to predicted onset values of PRI show that asphaltenes from C-T-01 are likely to be unstable during depressurization whereas those from CT-02 are likely to be stable. The fact that these two samples are from the same reservoir emphasizes the importance of sample quality in the assessment process.

\section{Conclusions}

The onset of asphaltene flocculation is a solubility phenomenon. It can be modeled themodynamically using the Flory-Huggins approach as proposed by Hirschberg, if the oil and onset conditions are correctly and independently assessed by measurements of refractive index and if the correct onset criterion is established as in the ASM model.

Flocculation during depressurization occurs because the solubility conditions change just as flocculation occurs when liquid alkanes are added to an oil. The onset pressure can be predicted from liquid alkane titration experiments if PVT and compositional data are available for the same samples. The accuracy of predictions has been demonstrated for a model oil system consisting of asphaltenes dissolved in toluene and methane. Tests of predictions for crude oils are in progress.

\section{Acknowledgements}

Crude oils and PVT data were provided by ChevronTexaco. The authors are grateful to David Ting, George Hirasaki, and Walter Chapman of Rice University for access to their experimental data. 


\section{References}

Buckley, J.S., Hirasaki, G.J., Liu, Y., Von Drasek, S., Wang, J.X., and Gill, B.S.: "Asphaltene Precipitation and Solvent Properties of Crude Oils," Petroleum Science and Technology (1998) 16, No. 3\&4, 251-285.

Buckley, J.S. and Wang, J.X.: "Crude Oil and Asphaltene Characterization for Prediction of Wetting Alteration,” J. Pet. Sci. Eng, (2002) 33, 195-202.

Cimino, R., Correra, S., Del Bianco, A., and Lockhart, T.P.: "Solubility and Phase Behavior of Asphaltenes in Hydrocarbon Media," Asphaltenes: Fundamentals and Applications, E.Y. Sheu and O.C. Mullins (eds.), NY: Plenu Press (1995) 97-130.

deBoer, R.B., Leerlooyer, K., Eigner, M.R.P., and van Bergen, A.R.D.: "Screening of Crude Oils for Asphalt Precipitation: Theory, Practice, and the Selection of Inhibitors," SPE PF (Feb. 1995) 55-61.

Hammami, A., Phelps, C.H., Monger-McClure, T., and Little, T.M.: "Asphaltene Precipitation from Live Oils: An Experimental Investigation of the Onset Conditions and Reversibility," Energy \& Fuels (2000) 14, 14-18.

Hirschberg, A., deJong, L.N.J., Schipper, B.A., and Meijer, J.G.: "Influence of Temperature and Pressure on Asphaltene Flocculation," SPEJ (June 1984) 283-293.

Ting, P.D., Chapman, W., Hirasaki, G.: "Asphaltene Phase Behavior: Experiment and Modeling using the SAFT Equation of State," paper 49b presented at the 2002 Spring AIChE Symposium, New Orleans, 10-14 Mar.

Trbovich, M.G. and King, G.E.: "Asphaltene Deposit Removal: Long-Lasting Treatment With a Co-Solvent," paper SPE 21038 presented at the 1991 SPE Int. Symp. on Oilfield Chemistry, Anaheim, Feb. 20-22.

Wang, J.X. and Buckley, J.S.: "A Two-Component Solubility Model of the Onset of Asphaltene Flocculation in Crude Oils," Energy \& Fuels (2001a) 15, 1004-1012.

Wang, J.X. and Buckley, J.S.: "An Experimental Approach to Prediction of Asphaltene Flocculation," (2001b) paper SPE 64994 presented at the SPE OCS Symp., Houston, 13-16 Feb.

Wang, J.X. and Buckley, J.S.: “Asphaltene Stability in Other Crude Oil Fractions,” paper 49a presented at the 2002 Spring AIChE Symposium, New Orleans, 10-14 Mar. 


\section{Part II-Studies of Wetting Alteration on Smooth Surfaces}

Smooth mineral surfaces provide opportunities to study the complex interactions among the many factors involved in wetting alteration by crude oils, interactions that may be too complex to be amenable to being studied directly in porous media. Part II of this report is devoted to studies of wetting alteration of mineral surfaces exposed to crude oil. In section II-1, the state of the art is reviewed. Section II-2 is devoted to new studies of contact angles between probe liquids on oil-treated surfaces. The study described in section II-2.1 brings to bear the power of the CO-Wet database described in Part I (section I-1). Crude oil properties are correlated with contact angles measurements on mica surfaces after exposure to standard aqueous solutions and to the same set of crude oils. In section II-2.2, contact angle measurements by the Wilhelmy plate technique on silica glass surfaces are used to explore the persistence of altered wetting and hysteresis between advancing and receeding conditions on oiltreated surfaces. Finally, development of a new AFM technique is reported in section II-3. Details of the methodology appear in section II-3.1 and applications to a variety of crude oils is presented in section II-3.2.

\section{II-1. Review of the Effective Wettability of Minerals Exposed to Crude Oil \\ Buckley, J.S.: "Effective Wettability of Minerals Exposed to Crude Oil," Current Opinion in Colloid \& Interface Sci. (2001) 6, 191-196.}

This review is focused on the macroscopic phenomena that can be used to assess effective wetting, especially the use of contact angles to quantify wetting conditions in the presence of brine and crude oil or on surfaces that have previously been exposed to brine and crude oil. Reservoir wettability has long been a puzzle. Wetting is almost certainly changed during all but the most careful core recovery processes and there is no guarantee that it can be preserved or recreated in the lab. Thus a great deal of effort has been directed at trying to understand reservoir wetting at a more fundamental level. The most important recent advances have demonstrated that there are multiple ways that crude oil components can adsorb to alter effective wetting, especially when an aqueous phase is also present.

\section{Introduction}

When Anderson published his six-part review of the wettability literature in 1986 and 1987 [1-6], his combined lists of references amounted to hundreds of citations. One section of Anderson's review was devoted to wettability measurement [2]; even the apparently simple question of how to measure wettability had no straightforward answer. It was understood in general that adsorption of crude oil components could alter the apparent wettability of rocks exposed to crude oil from their original strongly water-wet condition to some other wettability state. What wettability condition should be expected for a particular combination of oil, brine, and rock, however, was less well understood. 
In a well-defined system, the contact angle at which two fluid phases meet a smooth solid surface is the macroscopic indication of wetting preference that depends upon solid-fluid and fluid-fluid interfacial energies. It is the boundary condition for interfaces between those two fluids in a porous medium composed of that solid. Wetting thus controls fluid distribution and the capillary pressure between immiscible phases in a porous medium.

A system comprised of brine, crude oil, and rock, however, is not well defined. Each of the three phases is a complex mixture. Although contact angles are often measured in such nonideal systems, both the measurements themselves and their interpretation can be problematic, as discussed by Morrow [7] in his 1990 review of reservoir wettability.

Consider a smooth mica surface and a drop of crude oil formed at a microburette tip, both submerged in an aqueous salt solution. When the captive oil drop first contacts the surface, it adopts a shape that reflects the original wetting preference of the solid for water. Water in this case is receding and the water-receding contact angle $\theta_{R}$, measured through the water phase, is low and does not change with time. If, however, the drop volume is decreased, several phenomena can be observed. The extreme cases are (1) the water re-advances at nearly the same contact angle $\left(\theta_{\mathrm{A}} \approx \theta_{\mathrm{R}}\right)$ or $(2)$ the three phase contact line is pinned at its greatest extent and the contact angle increases continuously $\left(\theta_{\mathrm{A}}>\theta_{\mathrm{R}}\right)$. This extreme hysteresis is evidence that the surface under the drop has changed because of its contact with the crude oil. Many different approaches to measuring the extent to which the effective wetting of mineral surfaces is altered by contact with crude oil have been pursued. Techniques aimed at quantifying the extent of wetting alteration on smooth surfaces and what those techniques have taught us about the nature of crude oil/brine/rock interactions that control reservoir wettability are the main subjects of this review.

\section{Review of Surface Studies of Effective Wetting}

\section{Surface Measurements}

CONTACT ANGLES

Crude oil, brine, and initially water-wet surfaces

Despite the difficulties outlined above, measurements of contact angles between crude oils (and oil derivatives or related fluids such as coal tar and creosote) and brines on initially water-wet surfaces are made by the captive or sessile drop [8-13] and dynamic Wilhelmy plate [14-17] techniques. Captive or sessile drops sample a small area of the surface and angles are the measured quantity, whereas the Wilhelmy plate produces an average adhesion tension $(\gamma$ $\cos \theta)$ for the circumference of the test plate from which an average contact angle can be calculated if interfacial tension $\gamma$ is known.

Tests of adhesion that distinguish between the two extreme cases of an oil drop not adhering $\left(\theta_{\mathrm{A}} \approx \theta_{\mathrm{R}}\right)$ or adhering $\left(\theta_{\mathrm{A}}>>\theta_{\mathrm{R}}\right)$ to a clean mineral surface as a function of brine composition are also common [10,18-20]. Adhesion tests typically show a $\mathrm{pH}$ dependenceevidence of the importance of DLVO interactions between a charged solid surface and charged oil/water interface - that is best defined at ionic strengths of $1 \mathrm{M}$ or less in solutions of monovalent salt species. For negatively charged silicate minerals, adhesion tends to occur at 
more acidic values of $\mathrm{pH}(\mathrm{pH} 4-6)$ where oil drops can have a net positive charge and nonadhesion occurs at more basic conditions (pH8-10) where both oil drop and surface are negatively charged and the intervening water film is stable $[18,21,22]$. Outside a $\mathrm{pH}$ range of 410 , surfactant extraction and consequent low interfacial tensions complicate the wetting picture. High salinity and even low concentrations of divalent or multivalent ions can also lead to results that cannot be readily explained simply by DLVO forces. Only one report of a direct measurement of surface forces in the presence of crude oil has ever appeared [23].

Most often these tests are conducted using 'dead' oil samples (from which the components that are gases at $1 \mathrm{~atm}$ have been removed) at ambient conditions of temperature and pressure.

There are many differences between reservoir and ambient conditions including pressure, temperature, composition of the oil, and state of the oil's asphaltene components. There have been a few attempts to assess crude oil wetting properties at conditions that approach reservoir conditions using variations of the captive drop arrangement [24,25] but problems of pinning and rigid film formation can occur at elevated temperatures and pressures as well and the results to date are contradictory. Hjelmeland and Larrando [24] found for one oil that conditions become more water-wet. Rao [25] suggests that, in general, calcite becomes more water-wet and quartz more oil-wet as temperature is increased, but the evidence is limited. Some intermediate conditions of temperature have also been examined [17,26-28] with contradictory results so that questions regarding the influence of pressure and especially temperature on wetting properties of crude oils must be regarded as unresolved.

Only in cases where selected crude oil components are dissolved in a solvent can adsorption isotherms be measured, since some measure of concentration in the equilibrated fluid is required and contact angles can be measured only if the surrounding fluid is transparent. Nearly all such studies have explored oil/solid interactions in the absence of water [29-31].

\section{Crude oil-treated surfaces}

Some of the problems of measurements with crude oils can be circumvented if exposure to crude oil is separated from the evaluation process. Surfaces can be exposed to brine and oil under a wide range of conditions. The resulting wetting conditions can then be stabilized for subsequent evaluation by removal of the crude oil.

To evaluate the effective wetting in an oil reservoir, it is not sufficient to observe the interactions between crude oil and rock under dry conditions. Brine is always present in oil reservoirs and the influence of brine must also be considered. Liu and Buckley [32] showed how that could be done by pre-equilibrating a surface with brine, then contacting with oil while still wet. After exposure to oil for some period of time ranging from minutes to months, the crude oil can be removed by gentle rinsing with a solvent such as toluene that does not cause destabilization of asphaltenes. Probe fluids or surface analytical techniques can then be used to explore the effect of adsorbed material. Unlike the dry surfaces treated with oil, the resulting wetting conditions produced can vary from strongly water-wet to strongly oil-wet and almost anything in between, a surprise to many who assumed that the presence of water would always shield solid from oil. Results depend on the nature of the solid, components in the oil, and on the composition of the intervening brine [10,17,20,32,33]. Recently, this technique has been used to measure surface forces between oil-exposed and clean mica in an aqueous environment [34]. 
The results showed long-range, polymer-like interactions that did not affect approaching surfaces, but appeared when the surfaces were moved apart after contact.

\section{OTHER SURFACE TECHNIQUES}

A variety of other surface techniques have been tested for their ability to measure properties that relate to empirical tests of wetting in porous media. XPS can quantify the amount of specific elements as a function of position on the surface [35-37]. Funk et al. [38] used FTIR to follow adsorption of polar functional groups on a carbonate surface. Pyrolysis techniques, adapted from source rock evaluation procedures, quantify the amount of material adsorbed, but do not necessary correlate well with wetting changes [39].

\section{Alteration of Wetting by Crude Oils}

There are many components in any crude oil; detailed chemical evaluations tend to produce unmanageable amounts of information. Test such as those reviewed above have helped to identify the most relevant information with respect to wetting alteration tendencies.

\section{ADSORPTION OF CRUDE OIL COMPONENTS}

\section{Acid/base oil chemistry}

Cuiec [40] pointed out that some mineral surfaces (such as silicates) are acidic whereas others (e.g., carbonates) are basic so that different materials should be expected to adsorb from a crude oil to these different mineral types. Contact angles have been used to characterize interactions with known compounds including adsorption of organic acids on calcite $[41,42]$ and an organic base on quartz [43].

Depending on brine composition, crude oil/water interfaces can be either negatively or positively charged, reflecting the presence of both acidic and basic ionizable functional groups [18]. The methods available for quantifying acidic and basic components in oil are not very precise, but improvements in the standard non-aqueous potentiometric titration to obtain base number [44] and recognition that acid and base numbers should be considered together have improved oil characterization. Oils with either a high ratio of bases to acids or the converse, a high ratio of acids to bases, have recognizable tendencies to alter wetting [45].

\section{Asphaltenes and other crude oil fractions}

It is often assumed that the material that absorbs most strongly from a crude oil is that in its asphaltene fraction. The asphaltenes - any material in the oil that is not soluble in a designated alkane (usually n-pentane or n-heptane) that is soluble in toluene-include the highest molecular weight, most polar components in a crude oil. González and Moreira [30,31] compared dry surfaces exposed to solutions of asphaltenes and resins (polar species that are soluble in n-pentane) and found greater changes in wetting on the asphaltene-treated surfaces. However, comparisons of glass surfaces treated either with crude oils or with their asphaltenes in toluene solution, in which brine effects were considered, showed that significantly more oil-wet conditions could be produced by the whole oil than by treatment with just the asphaltene fraction [46].

Adsorption of asphaltenes is influenced by the tendency of those species to aggregate, forming larger, colloidal-sized entities in the oil. Extent of aggregation varies with solvent quality. As the asphaltenes approach phase separation, their tendency to partition to either 
oil/water or oil/solid interfaces can be expected to increase. Compared to treatment with an oil in which the asphaltenes are in stable dispersion, more oil-wet surfaces have been produced on mica that was exposed first to an aqueous salt solution of known $\mathrm{pH}$ and ionic composition, then to oil to which an amount of asphaltene precipitant slightly less than what would be required to initiate phase separation was added [47].

Precise formulae to predict the balance of different types of interactions between suites of crude oil components and solid surfaces are not yet available, but more general assessments about which oils are good or poor solvents and which have an excess of either acidic or basic species provide a framework for understanding the complex interactions by which crude oils alter the wetting of mineral surfaces.

\section{HYSTERESIS BETWEEN WATER-RECEDING AND WATER-ADVANCING CONDITIONS}

The hysteresis between initial contact of crude oil with a clean, water-wet mineral surface and subsequent re-advance of water over the oil-exposed surface can be accounted for by adsorption that occurs during the measurement. Removing the crude oil eliminates further adsorption, but hysteresis - between advancing and receding probe fluids — can be extensive and repeated through multiple displacement cycles [48].

Although the probe fluids are initially pure, either phase may extract material from the surface. Even more likely, however, is that the hysteresis is related to the failure of the surface to fit the assumptions that it is smooth, rigid, and chemically homogeneous. Oil-treated surfaces are probably none of these.

Artificially prepared surfaces with areas of high and low contact angles, uniformly or randomly distributed have been used to test the Cassie equation [49] that relates contact angles to the fractions of area covered by materials with different properties [50-52]. Yang et al. [10] envision such partially covered surfaces with areas to which components from the oil adsorb and other areas of exposed high-energy mineral surface. They hypothesize that, depending on the extent of coverage, bridges of oil may form between rough, oil-wet patches that make the surface appear more oil-wet to an advancing water interface than to a receding one. Average roughness of surfaces treated with two crude oils and their mixtures with n-heptane was estimated from AFM images and a positive logarithmic correlation between roughness and advancing angle was found for one of the oils [10].

Detailed studies on self-assembled monolayers (SAMs) of hexadecanethiol and mercaptohexadecanoic acid on gold have been prepared to investigate deviations from the Cassie equation [53]. Hysteresis between advancing and receding conditions was greatest not for surfaces with alternating strips of high and low contact-angle material, but for smooth gold surfaces covered with a uniform monolayer that terminated in acid groups, perhaps due to a surface reconstruction process [54]. The extent of hysteresis on some oil-treated surfaces is higher still and may reflect a combination of chemical heterogeneity, roughness, and surface reconstruction. While hysteresis between imbibition (water saturation increasing) and drainage (water saturation decreasing) is well recognized in oil displacement, extreme differences between wetting conditions due to contact angle hysteresis are not included in any current models of recovery processes. 


\section{DEVELOPMENT OF MIXED WETTING}

It is now generally agreed that Salathiel-type mixed-wetting is probably the most common reservoir condition [55]. This is a situation in which wetting is altered-by adsorption of crude oil components - on some surfaces, but the original, more water-wet condition is preserved on others. Mixed wetting might occur if wetting alteration were dictated by the geologic distribution of the rock-forming and pore-lining minerals, but Salathiel's contribution was to suggest that wetting alteration can be dictated by the areas of contact between oil and rock. Kaminsky and Radke [56] demonstrated that strongly adsorbing species would convert all surfaces to oil-wet conditions over much less than geologic time. Since oil reservoirs do not seem to be universally oil-wet, it appears that the combination of weakly adsorbing crude oil species in contact with rock surfaces where water films are unstable and water-wet surfaces protected from contact with oil by bulk water yields the non-uniform effective wetting situation that is referred to as mixed-wet.

Models of triangular tubes [57,58], star-shaped pores [59] and networks of pores with a distribution of contact angles [60-62] are progressing toward the ability to incorporate a mechanistic understanding of wetting alteration into predictions of displacement of oil.

\section{Concluding Remarks}

During the period covered by this review, progress has been made in understanding the interactions that can occur between crude oil components and mineral surfaces in the presence of an aqueous solution. Contact angle measurements using probe fluids can be used to assess wetting after prolonged exposure of mineral surfaces to brine and oil. More sophisticated techniques have been evaluated, but have not yet been shown to provide evidence specifically about the material that most influences effective wetting and thus displacement properties.

Translating this understanding into a knowledge base that can be used to define wetting for newly discovered reservoirs or to predict changes in wetting during production or in the course of a contamination event remains as a goal for the future. Modeling tools are being developed that can incorporate mixed wetting and a range of contact angles, but questions remain. The effect of temperature on those interactions that govern effective reservoir wettability are complex and must be better understood. Specific interactions involving multivalent ions must also be addressed. The time available for interactions to occur is very long and approach to equilibrium conditions is probably very slow. The possibility that laboratory time scales are insufficient to approach reservoir conditions must also be considered.

\section{References}

1. Anderson WG: Wettability literature survey - part 1: rock/oil/brine interactions and the effects of core handling on wettability. J Petrol Tech 1986, 38:1125-1144.

2. Anderson WG: Wettability literature survey-part 2: wettability measurement. J Petrol Tech 1986, 38:1246-1262. 
3. Anderson WG: Wettability literature survey - part 3: the effects of wettability on the electrical properties of porous media. J Petrol Tech 1986, 38:1371-1378.

4. Anderson WG: Wettability literature survey—part 4: effects of wettability on capillary pressure. J Petrol Tech 1987, 39:1283-1300.

5. Anderson WG: Wettability literature survey_part 5: the effects of wettability on relative permeability. J Petrol Tech 1987, 39:1453-1468.

6. Anderson WG: Wettability literature survey-part 6: the effects of wettability on waterflooding. J Petrol Tech 1987, 39:1605-1621.

7. Morrow NR: Wettability and its effect on oil recovery, J Petrol Tech 1990, 42:1476-1484.

8. Doe PH: Salinity dependence in the wetting of silica by oils. In Proc. $3^{\text {rd }}$ International Symposium on Evaluation of Reservoir Wettability and Its Effect on Oil Recovery, Laramie: Univ of Wyoming; 1996:15-20.

9. Israelachvili J, Drummond C: Fundamental studies of the oil-surface-water interactions and its relationship to wettability. In Proc 5th Internat Symp on Reservoir Wettability and Its Effect on Oil Recovery, Trondheim, 22-24 June, 1998.

10. Yang SY, Hirasaki GJ, Basu S, Vaidya R: Mechanisms for contact angle hysteresis and advancing contact angles. J Petrol Sci Eng 1999, 24:63-73.

11. Skauge A, Standal S, Boe SO, Skauge T, Blokhus AM: Effects of organic acids and bases, and oil composition on wettability. SPE 56673 In Proc SPE ATCE, Houston, 3-6 Oct 1999.

12. Barranco FT, Jr, Dawson HE: Influence of aqueous $\mathrm{pH}$ on the interfacial properties of coal tar. Env Sci \& Tech 1999, 33:1598-1603.

13. Powers SE, Anckner WH, Seacord TF: Wettability of NAPL-contaminated sands. $J$. Environ. Eng. 1996, 122:889-896.

14. Andersen MA, Thomas DC, Teeters DC: A new formation wettability test: the dynamic Wilhelmy plate wettability technique. SPE/DOE 17368 In Proc SPE/DOE EOR Symp, Tulsa, 17-20 Apr 1988.

15. Mennella A, Morrow NR, Xie X: Application of the dynamic Wilhelmy plate to identification of slippage at a liquid-liquid-solid three-phase line of contact, J Petrol Sci Eng 1995, 13:179-192.

16. Xie X, Morrow NR: Contact angles on quartz induced by adsorption of heteropolar hydrocarbons. J Adhesion Sci Technol 1999, 13:1119-1135.

17. Xie X, Morrow NR, Buckley JS: Crude oil/brine contact angles on quartz glass. SCA-9712 In Proc SCA Internat Symp, Calgary, 8-10 Sept, 1997.

18. Buckley JS, Takamura K, Morrow NR: Influence of Electrical Surface Charges on the Wetting Properties of Crude Oils, SPE Reservoir Eng 1989, 4:332-340.

19. Skauge A, Fosse B: A Study of the Adhesion, Interfacial Tensions, and Contact Angles for a Brine, Quartz, Crude Oil System. In Proc. $3^{\text {rd }}$ International Symposium on Evaluation of Reservoir Wettability and Its Effect on Oil Recovery, Laramie: Univ of Wyoming; 1996:39-42.

20. Liu L, Buckley JS: Alteration of wetting of mica surfaces. J Petrol Sci Eng 1999, 24:75-83. 
21. Hirasaki GJ: Wettability: fundamentals and surface forces, SPE Formation Eval 1991, 6:217-226.

22. Ward AD, Ottewill RH, Hazlett RD: An investigation into the stability of aqueous films separating hydrocarbon drops from quartz surfaces. J Petrol Sci Eng 2000, 24:213-220.

23. Christenson HK, Israelachvili JN: Direct measurements of interactions and viscosity of crude oils in thin films between model clay surfaces. J Colloid Interface Sci 1987, 119:194.

24. Hjelmeland O, Larrondo LE: Experimental investigation of the effects of temperature, pressure, and crude oil composition on interfacial properties, SPE Reservoir Eng 1986, 1:321328.

25. Rao DN: Wettability effects in thermal recovery operations. SPE Reservoir Eng Eval 1999, 2:420-430.

26. Hjelmeland $\mathrm{O}$, Selle OM, Rueslåtten $\mathrm{H}$ : Wetting and interfacial properties of North Sea reservoir fluids/rocks systems. In Proc IEA Workshop, Collaborative Project on EOR, Hannover, West Germany, Sept 16-17, 1986.

27. Sayyouh MH, Hemeida AM, Al-Blehed MS, Desouky SM: Role of polar compounds in crude oils on rock wettability. J Petrol Sci Eng 1991, 6:225-233.

28. Ghoniem SAA: The alteration of the wettability of some Egyptian crudes by salt addition. Erdöl und Kohle-Erdgas-Petrochemie 1988, 41:265-368.

29. Gloton MP, Turmine M, Mayaffre A, Letellier P, Toulhoat H: Study of asphaltenes adsorption on mineral surfaces by contact angle measurements: kinetics of wettability changes. In Physical Chemistry of Colloids and Interfaces in Oil Production. Edited by Toulhoat H, Lecourtier J. Paris: 1992:89-96.

30. González G, Moreira MBC: The wettability of mineral surfaces containing adsorbed asphaltenes. Colloids and Surfaces 1991, 58:293-302.

31. González G, Moreira MBC: Adsorption of asphaltenes and resins on various minerals. In Asphalt and Asphaltenes. Edited by Yen, TF. Elsevier; 1994:219.

32. Liu Y, Buckley JS: Evolution of wetting alteration by adsorption from crude oil. SPE Formation Eval 1997, 12:5-11.

33. Akhlaq MS, Kessel D, Dornow W: Separation and chemical characterization of wetting crude oil compounds. J Colloid Interface Sci 1996, 180:309-314.

34. Drummond C, Israelachvili, JN: Surface forces and wettability. In Proc. $6^{\text {th }}$ Internat Symp on Reservoir Wettability, Socorro, 27-28 Sep 2000.

35. Quet C, Chenevière P, Glotin G, Mourrel M: Pore surface chemistry and wettability. In Physical Chemistry of Colloids and Interfaces in Oil Production. Edited by Toulhoat H, Lecourtier J. Paris: 1992:81-88.

36. Mitchell AG, Hazell LB, Webb KJ: Wettability determination: pore surface analysis. SPE 20505 In Proc SPE ATCE. New Orleans, 23-26 Sept 1990.

37. Durand C, Beccat P: Use of XPS for reservoir sandstone wettability evaluation. application to kaolinite and illite. J Petrol Sci Eng 1998, 20:259-265. 
38. Funk JJ, Dahlstrom PL, Haltmar WC, Perdue JM: Technique for observing oil-wetting of carbonate surfaces using FT-IR. SCA-9512 In Proc of SCA Internat Symp. San Francisco, 1214 Sept 1995.

39. Wolcott JM, Groves FR, Jr, Trujillo DE, Lee HG: Investigation of crude-oil/mineral interactions: factors influencing wettability alteration. SPE Adv Tech Ser 1993, 1:117-126.

40. Cuiec L: Restoration of the natural state of core samples. SPE 5634 In Proc SPE ATCE, Dallas, 28 Sept - 1 Oct 1975.

41. Thomas MM, Clouse JA, Longo JM: Adsorption of organic compounds on carbonate minerals 1. model compounds and their influence on mineral wettability. Chem. Geol. 1993, 109:201-213.

42. Legens C, Palermo T, Toulhoat H, Fafet A, Koutsoukos P: Carbonate rock wettability changes induced by organic compound adsorption. J Petrol Sci Eng 1998, 20:277-282.

43. Lord DL, Demond AH, Hayes KF: Effects of organic base chemistry on interfacial tension, wettability, and capillary pressure in multiphase subsurface waste systems, Transp in Porous Media 2000, 38:79-92.

44. Dubey ST, Doe PH: Base number and wetting properties of crude oils. SPE Reservoir Eng 1993 8:195-200.

45. Buckley JS, Liu Y, Monsterleet S: Mechanisms of wetting alteration by crude oils. SPE J 1998, 3:54-61.

46. Buckley JS, Liu Y, Xie X, Morrow NR: Asphaltenes and crude oil wetting-the effect of oil composition. SPE J 1997, 2:107-119.

47. Al-Maamari RSH, Buckley JS: Asphaltene precipitation and alteration of wetting: can wettability change during oil production? SPE 59292 In Proc SPE/DOE IOR Symp. Tulsa, 3-5 Apr 2000.

48. Xie X, Morrow NR, Buckley JS: Contact angle hysteresis and the stability of wetting changes induced by adsorption from crude oil. In Proc. $6^{\text {th }}$ Internat Symp on Reservoir Wettability, Socorro, 27-28 Sep 2000.

49. Cassie ABD: Contact angles. Discussions Faraday Soc 1948, 3:11-16.

50. Paterson A, Robin M, Fermigier M, Jenffer P, and Hulin JP: Effect of density and spatial distribution of wettability heterogeneities on contact angle, J Petrol Sci Eng 1998, 20:127-132.

51. Robin M, Paterson A, Cuiec L, Yang C: Effects of chemical heterogeneity and roughness on contact angle hysteresis: experimental study and modeling, SPE 37291 In Proc SPE OCS, Houston, 18-21 Feb 1997.

52. Robin M, Koci, X: Wettability heterogeneities on planar minerals: application to reservoir rocks. In Proc. $3^{\text {rd }}$ International Symposium on Evaluation of Reservoir Wettability and Its Effect on Oil Recovery, Laramie: Univ of Wyoming; 1996:47-53.

53. Drelich J, Wilbur JL, Miller JD, Whitesides GM: Contact angles for liquid drops at a model heterogeneous surface consisting of alternating and parallel hydrophobic/hydrophilic strips. Langmuir 1996, 12:1913-1922. 
54. Kacker, N, Kumar SK, Allara DL: Wetting-induced reconstruction in molecular surfaces. Langmuir 1997, 13:6366-6369.

55. Salathiel RA: Oil recovery by surface film drainage in mixed-wettability rocks. J Petrol Tech 1973, vol:1216-24.

56. Kaminsky R, Radke CJ: Asphaltenes, water films, and wettability reversal. SPE J 1997, 2:485-493.

57. Mason G, Morrow NR: Capillary behavior of a perfectly wetting liquid in irregular triangular tubes. J Colloid Interface Sci 1991, 141:262-274.

58. Ma S, Mason G, Morrow NR: Effect of contact angle on drainage and imbibition in regular polygonal tubes. Colloids and Surfaces A 1996, 117:273-291.

59. Kovscek AR, Wong H, Radke CJ: A pore-level scenario for the development of mixed wettability in oil reservoirs. AIChE J 1993, 39:1072-1085.

60. Dixit AB, McDougall SR, Sorbie KS, Buckley JS: Pore scale modelling of wettability effects and their influence on oil recovery. SPE Reservoir Eng Eval 1999, 2:25-36.

61. Hui MH, Blunt MJ: Effects of wettability on three-phase flow in porous media. J Phys Chem B 2000, 104:3833-3845.

62. Øren PE, Bakke S, Arntzen OJ: Extending predictive capabilities to network models. SPE J 1998, 3:324-336. 


\title{
II-2. Contact Angles on Oil-Treated Surfaces
}

\section{II-2.1. Effect of Crude Oil Composition on Wettability of Mica}

\author{
L. Yang, J.X. Wang, T. Fan, and J.S. Buckley
}

Over the course of this project, a large volume of data has been acquired for nearly two hundred crude oil samples. These data include chemical and physical properties as well as measures of the tendency of these oils to alter the wetting of mineral surfaces. In this report, statistical tools are employed to examine the database for relationships between oil properties and alteration of wetting of mica surfaces.

The data are first examined for relationships between crude oil properties. Some trends reported for small data sets can be shown to be general (e.g., RI vs. API gravity or density) whereas other trends broaden as additional samples are examined.

Wetting alteration is not simply a function of crude oil chemistry. Stable water films can prevent interactions between oil and solid. Water films that are not stable can influence interactions between oil components and solid surfaces beyond simply providing access to the surface. Components of the aqueous phase can participate in the oil/solid interactions as well. Two sets of conditions, one designed to promote stable water films and another selected to minimized water-film stability, have been chosen for tests of wetting alteration. Only monovalent $\mathrm{Na}^{+}$and $\mathrm{Cl}^{-}$were added to buffered aqueous solutions, to avoid complex interactions with divalent and multivalent ionic species.

No single oil property correlates significantly with wetting alteration under either of the two standard conditions. In combination, however, trends do emerge. The amount of asphaltene is the most significant variable in all cases, although the effect of asphaltenes can be to increase or to decrease the extent of wetting alteration, depending on water-film stability. Beyond amount of asphaltene, other variables are shown to influence wetting alteration in consistent ways, but the combinations of variables are different for the water film stable and unstable conditions. Nonlinear analyses produce even stronger relationships.

\section{Wetting Alteration by Crude Oils}

Material that is adsorbed or deposited on mineral surfaces from a crude oil can change the wetting preferences of those surfaces. The effect of any crude oil on the wetting of solid surfaces with which it comes in contact can vary widely, depending on factors that include the surface chemistry of the solid, the presence or absence of an aqueous phase, composition of the aqueous phase if it is present, temperature, contact time, and the crude oil's composition.

INTERACTION MECHANISMS.

Several mechanisms of interactions between crude oils, brines and mineral surfaces have been identified (Buckley et al., 1998a). These include ionic interactions that occur between oil components and mineral surfaces that are influenced by brine strength and composition and 
surface precipitation that can dominate when asphaltenes are close to the onset of flocculation. (Al-Maamari and Buckley, 2000). Ionic interactions can be further subdivided into simple acidbase interactions that can be tested using brines with monovalent ions at controlled values of $\mathrm{pH}$ and more complex interactions that can occur when brines contain ions of higher valence. Reservoir brines nearly always contain divalent and trivalent ions and can vary widely in composition. It might be argued that synthetic reservoir brines, specific to each reservoir, would be appropriate for use in wettability testing. There are several reasons why that is not practical for a general screening of crude oils. Reservoir brine data is not always available. Moreover, crude oil/brine/rock (COBR) interactions involving divalent (or multivalent) ions are much slower than the simple acid/base interactions and can be specific to each oil so that results cannot be generalized.

When acid/base interactions dominate, no wetting change is observed if there is a stable water film between oil and solid. This situation occurs when there is sufficient electrostatic repulsion between a negatively charged oil/water interface and the negatively charged mica surface. For some basic oils a stable water film has been observed when the aqueous phase is buffered at $\mathrm{pH} 8$ with an ionic strength equivalent to $1 M \mathrm{NaCl}$. An unstable water film often results from the use of an aqueous phase buffered at $\mathrm{pH} 4$ with a low salt concentration $(0.01 \mathrm{M}$ $\mathrm{NaCl}$ in this study) because the basic oil/brine interface is positively charged and the mica is negatively charged. In this situation oil components can adsorb, altering the wetting of the mica surface. It is not possible to select a single set of two aqueous solutions that produce one stable and one unstable situation with all oils since water-film stability also depends on the charge at the oil/water interface. For screening purposes, however, these two conditions should tend to bracket the extremes of wettability alteration for many crude oils.

\section{MINERAL SURFACE.}

Studies in core samples of reservoir and outcrop rocks are complicated by the details of pore geometry, topology, and surface chemistry. In order to isolate the influence of oil composition on the interactions between crude oil components and mineral surfaces in the presence of an aqueous phase, we examine the wetting of smooth, homogeneous surfaces. Muscovite mica is an ideal model surface because it is molecularly smooth, is well characterized, has a fixed surface charge, and is easily cleaved to produce clean surfaces (Liu and Buckley, 1999). Since it is negatively charged above $\mathrm{pH} \mathrm{2,} \mathrm{mica} \mathrm{is} \mathrm{representative} \mathrm{of} \mathrm{many} \mathrm{silicate} \mathrm{and}$ aluminosilicate minerals, including quartz and illite, that are the main constituents of a sandstone reservoir.

\section{TEMPERATURE EFFECTS.}

The effects of temperature on COBR interactions is as yet poorly understood. There are reports that COBR systems become more water-wet as temperature increases (Hjelmeland and Larrondo, 1986; Tang and Morrow, 1997). Not all of the evidence confirms this trend, however (Hjelmeland et al., 1986). Aging at an elevated temperature followed by wetting tests at ambient conditions may produce artifacts due to wax phase transition (Buckley et al., 1997; Xie et al., 2002). To avoid uncertainties introduced by changes in temperature, all aging and testing in this project were conducted at ambient conditions of temperature and pressure. 


\section{WETTING TESTS.}

A two-step procedure was used to alter wetting (Liu and Buckley, 1997). Clean mica was first equilibrated with one of two buffer solutions ( $\mathrm{pH} 4$ or $\mathrm{pH} 8$ ). The lower $\mathrm{pH}$ buffer has been shown to produce unstable water films with A-93 crude oil whereas the water film produced by the $\mathrm{pH} 8$ buffer is stable with that oil. Although these conditions are not expected to be general, they were chosen as standards in an attempt to probe the extremes of wetting alteration by each crude oil. The $\mathrm{pH} 4$ buffer had a molar concentration of chloride ions of $0.01 \mathrm{M}$; the concentration of $\mathrm{Cl}^{-}$in the $\mathrm{pH} 8$ buffer was $1 \mathrm{M}$.

After 24 hours the mica samples were removed from the buffer and drained by contacting an edge to absorbent paper. The pre-wetted mica samples were then immersed in crude oil where they remained for 21 days. Previous studies have established this time period as sufficient equilibration time, even when initial water is present. Samples were removed from the crude oil, rinsed with toluene (a good asphaltene solvent that avoids precipitation of asphaltenes during the rinsing step), then immersed in decane (a poor asphaltene solvent in which the oil-treated surfaces remain unchanged over the duration of contact angle measurements). Contact angles between water and decane, measured by the captive drop technique (Gaudin, 1963) quantify the extent of wetting change. The angles are measured quasi-statically after water has been either advanced or receded.

\section{CRUDE OIL PROPERTIES.}

Crude oil properties have been selected that are likely related to asphaltene stability and to the acidic and basic properties of the oil. The suite of tests has evolved over the duration of this and previous projects, hence not all tests were made for all oil samples. SARA analysis measures the amount of asphaltene as well as the amounts of asphaltene solvents (aromatics and resins) and asphaltene precipitants (saturates), which relate to asphaltene stability. Another measure of asphaltene stability is the difference in refractive index (RI) between the dead oil and the onset mixture of oil and n-heptane (Buckley et al., 1998). Asphaltenes and resins contain the most polar oil components, thus the amounts of these fractions is related to oil polarity. The amounts of both n-hexane and n-heptane asphaltenes were measured for many oil samples. Acid number and base number measure the total amounts of acidic and basic species in the oil, some fraction of which is interfacially active. Trace metals, especially Ni and V, can be stabilized by naturally occurring porphyrins, another interfacially active group of compounds. Standard physical measurements of density (and API gravity), average molecular weight, and viscosity complete the crude oil characterizations.

\section{The CO-Wet Data Set}

\section{Samples and experimental techniques}

Crude oil samples were obtained from many sources including major and independent oil companies, service companies, and other universities. Samples that are free of any treatment chemicals are always requested although the extent to which additive-free samples are provided is uncertain. 


\section{ACID AND BASE NUMBERS.}

Standard non-aqueous potentiometric titration methods were used for both acid and base number determinations. Acid numbers are measured by the standard method (ASTM, 1989); the corresponding base numbers are measured by an adaptation of the standard method (ASTM, 1988) recommended by Dubey and Doe (1993).

\section{ISOELECTRIC POINT.}

The isoelectric point of each oil sample was measured by preparing an emulsion of oil in buffered $0.01 \mathrm{M} \mathrm{NaCl}$ brine. Zeta potential was measured with a Delsa $440 \mathrm{SX}$.

\section{RI AND $P_{\text {RI }}$ OBSERVATIONS.}

Refractive index (RI) was measured as a function of temperature using either an Abbe refractometer or an automated Index Instruments GPR 11-37 refractometer. Both instruments use the sodium D line $(589 \mathrm{~nm})$ as a standard wavelength. The GPR 11-37 uses a critical angle technique that permits measurement of RI for opaque liquid samples. In most cases RI could be determined for neat crude oil. If not, RI was determined for a series of oil/toluene mixtures and the results were extrapolated to determine the value for the crude oil. A sample cell that can maintain a pressure up to $1.5 \mathrm{~atm}$ was used to limit evaporation during measurements with the automated refractometer.

The onset of asphaltene flocculation was observed microscopically one day after mixing oil and precipitants. Visible light and a maximum magnification of $320 \mathrm{X}$ were used in this work. RI was measured for the onset mixture, using the techniques described above. RI at the onset is denoted $\mathrm{P}_{\mathrm{RI}}$. In some cases, the dead crude samples contain precipitated asphaltenes. To these samples $\alpha$-methyl naphthalene, a good asphaltene solvent, was added to completely dissolve existing precipitated asphaltenes before onset measurements began.

\section{DENSITY AND API GRAVITY.}

Density was measured as a function of temperature using a digital Mettler/Paar DMA40 densitometer. API gravity was calculated from the density measurements, corrected to a temperature of $60^{\circ} \mathrm{F}$.

\section{VISCOSITY.}

Kinetic viscosity was measured at $20^{\circ} \mathrm{C}$ with Cannon-Fenske viscometers and converted to absolute viscosity using the measured values of density.

\section{SARA ANALYSIS.}

The saturate, aromatic, resin, and asphaltene fractions of a crude oil are particularly relevant to evaluation of the oil as a solvent for its asphaltenes. Unfortunately, few if any labs use the standard ASTM procedure, which is time consuming and requires significant volumes of sample and solvents. Instead, each lab uses its own modified set of procedures. Those based on HPLC analysis of deasphalted oil are likely to be reasonably comparable. Thin layer 
chromatographic techniques are less comparable, especially for medium or high gravity oils. Details of the HPLC technique used in this study have recently appeared, along with a comparison to other SARA analysis techniques (Fan et al., 2002).

\section{TRACE METALS.}

The trace metal analyses were carried out according to ASTM D 5863-95. Method A-in which oil samples are decomposed and digested for further analyses-was used. About 20 grams of sample were digested and finally dissolved and diluted to $100 \mathrm{ml}$ with nitric acid $(5 \%$ $\mathrm{V} / \mathrm{V})$. A blank, identically treated sample containing only the reagents was also tested. Aluminum nitrate was used as suppressant for the analysis of vanadium. Nickel and iron were analyzed by using flame AA, and zinc was analyzed by using ICP-mass spectrometry.

\section{Summary of the data}

Crude oil properties are provided for a total of 192 samples in Appendix Table II-2.5. Varying amounts of sample affect the number of tests to which each oil can be subjected. Many more oils have been tested to compare their chemical and physical properties than have been tested for their tendency to alter the wetting of mica surfaces (Appendix, Table II-2.6).

\section{Statistical relationships among crude oil properties}

Many of the properties reported in Appendix Table II-2.5 are related. For example, we have shown previously (Buckley and Wang, 2001) that API gravity and RI are linearly related. Figure II-2.1a shows that the values for 139 samples are well correlated. A positive slope is found when RI is compared to density (Fig. II-2.1b), both measured at $20^{\circ} \mathrm{C}$.

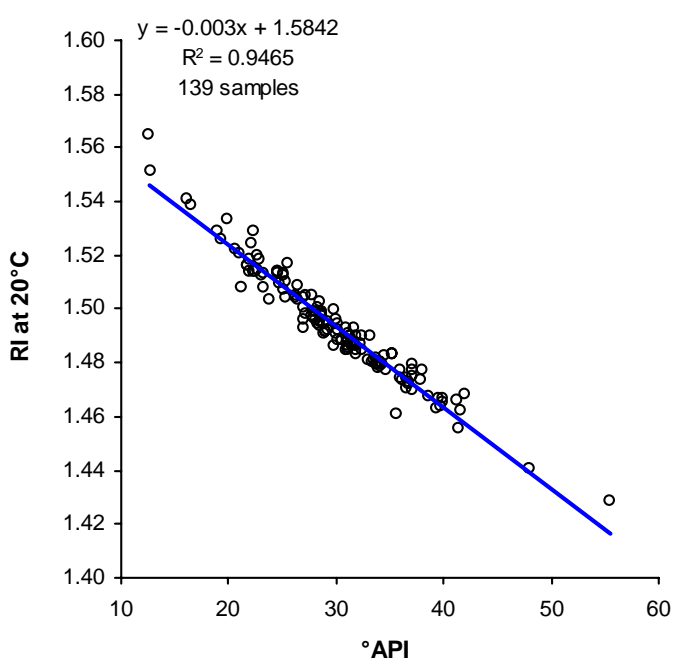

(a) $\mathrm{RI}$ at $20^{\circ} \mathrm{C}$ vs. API gravity (defined at $60^{\circ} \mathrm{F}$ )

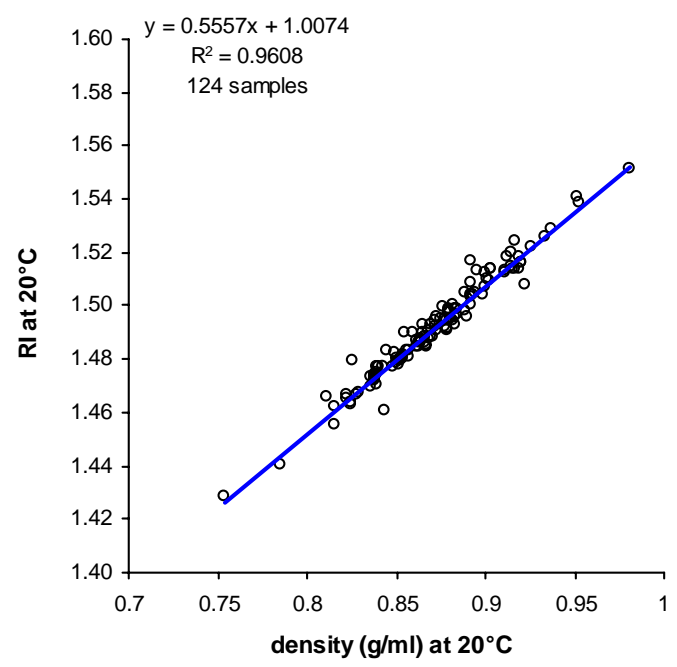

(b) $\mathrm{RI}$ at $20^{\circ} \mathrm{C}$ vs. density at $20^{\circ} \mathrm{C}$

Figure II-2.1. Refractive index is closely related to oil density. 
Trends between oil properties are sometimes reported on the basis of only a few samples. For example, Manrique et al. (1997) reported base number decreasing with increasing API gravity. As shown in Fig. II-2.2, both acid and base numbers are broadly higher for lower gravity oils, but neither is closely correlated with API gravity.

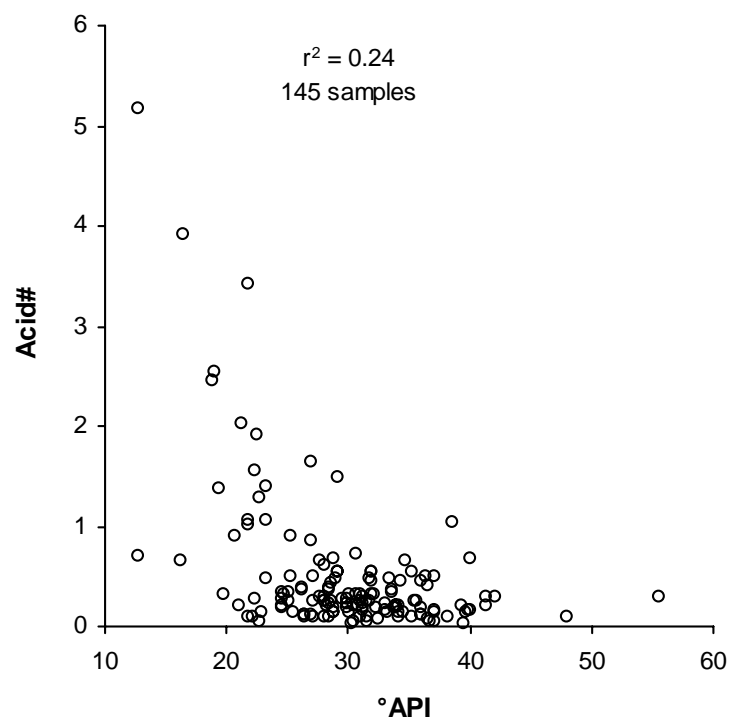

(a) acid number vs API gravity

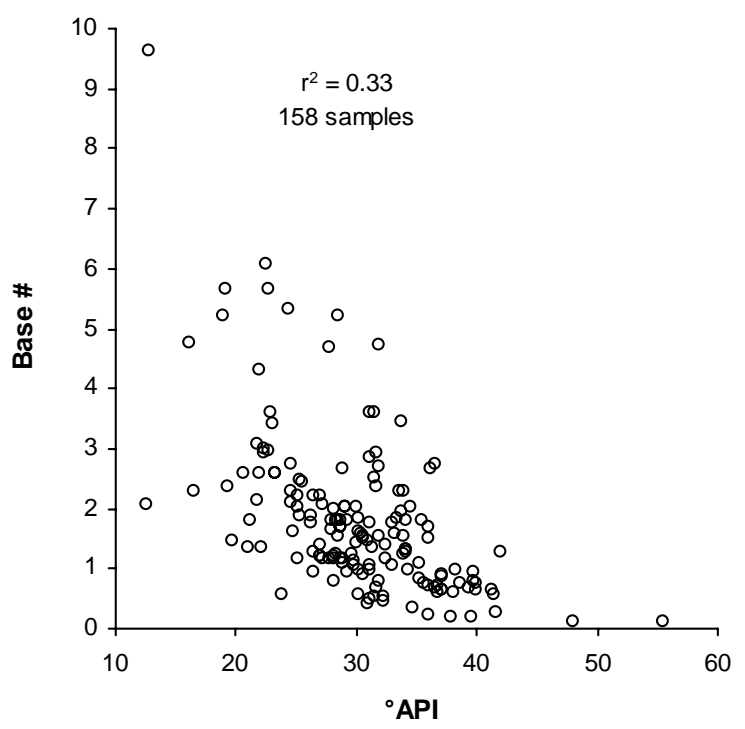

(b) base number vs API gravity

Figure II-2.2. Acid and base numbers tend to be higher for lower gravity oils, but are not closely correlated with gravity or density.

Yang et al. (2001) used statistical analyses to show relationships among crude oil properties, many of which are related to the amount of asphaltene. Their database consisted of 25 oils, one of which was excluded on the basis of high acid and base numbers and anomalous adhesion behavior. They saw weak correlations between the amount of asphaltenes in an oil and its RI, API gravity, and acid number. A stronger correlation was found between amount of asphaltenes and the base number. The published results are compared with correlations calculated for the much larger number of oil samples in this work in Table II-2.1. Weak correlations are found between the amount of $n-C_{7}$ asphaltenes and API gravity, average molecular weight, RI, density, base number, and the amounts of resins, Ni and V. No correlation with acid number or the amounts of aromatics, Fe, or $\mathrm{Zn}$ was found. Stronger trends appeared between $n-C_{7}$ asphaltenes and the amount of saturates (negatively correlated since asphaltenes have limited solubility in saturated hydrocarbons) and the $\mathrm{P}_{\mathrm{RI}}$ (the $\mathrm{RI}$ at which asphaltene aggregates appear in n-heptane titration experiments). Differences between the present results and those of Yang et al. are probably related mainly to the number and identity of crude oils in the two databases, although differences in measurement techniques could also be a factor. None of the oil properties in our database has a correlation coefficient above 0.5 with the amount of $n$ C7 asphaltenes in their crude oils. In particular, base number appears to be much less wellcorrelated and acid number uncorrelated with $\mathrm{n}-\mathrm{C}_{7}$ asphaltenes. Both acid and base numbers are better correlated to the resin fraction $\left(\mathrm{R}^{2}=0.34\right.$ and 0.57 , respectively) in our data set, suggesting that differences in procedures for separation of asphaltene and resin may account for the 
differences in these observations. Although it has been suggested that acid and base number are correlated to one another (Skauge et al., 1999), in this data set the value of $\mathrm{R}^{2}$ is 0.25 and if Cymric-12, a particularly heavy, polar oil, is removed the value of $\mathrm{R}^{2}$ drops to only 0.12 .

Table II-2.1. Correlations between amount of asphaltene and other oil properties.

\begin{tabular}{|c|c|c|c|c|}
\hline \multirow[b]{2}{*}{ measured oil properties } & \multicolumn{2}{|c|}{ linear correlation to $\% \mathrm{n}-\mathrm{C}_{7}$ asphaltenes } & \multicolumn{2}{|c|}{ Yang et al. (2001) } \\
\hline & $\mathbf{n}$ & $\mathbf{R}^{2}$ & $\mathrm{n}$ & $\mathbf{R}^{2}$ \\
\hline${ }^{\circ} \mathrm{API}$ & 109 & 0.32 & 21 & 0.10 \\
\hline $\operatorname{avg~MW}$ & 109 & 0.28 & & \\
\hline $\mathrm{RI}\left(\right.$ at $\left.20^{\circ} \mathrm{C}\right)$ & 107 & 0.38 & 21 & 0.17 \\
\hline $\mathrm{P}_{\mathrm{RI}}\left(\right.$ at $\left.20^{\circ} \mathrm{C}\right)$ & 79 & 0.43 & & \\
\hline density $\left(\mathrm{g} / \mathrm{ml}\right.$ at $\left.20^{\circ} \mathrm{C}\right)$ & 107 & 0.32 & & \\
\hline viscosity (cP at $\left.20^{\circ} \mathrm{C}\right)$ & 69 & 0.07 & & \\
\hline saturates (\%) & 85 & 0.45 & & \\
\hline aromatics (\%) & 85 & 0.00 & & \\
\hline $\mathrm{n}-\mathrm{C}_{6}$ resins $(\%)$ & 85 & 0.21 & & \\
\hline $\mathrm{n}-\mathrm{C}_{6}$ asphaltenes (\%) & 85 & 0.95 & & \\
\hline acid \# (mg KOH/g oil) & 101 & 0.00 & 24 & 0.13 \\
\hline base \# (mg KOH/g oil) & 110 & 0.14 & 23 & 0.54 \\
\hline IEP (pH units) & 70 & 0.04 & & \\
\hline $\mathrm{Fe}(\mathrm{ppm})$ & 64 & 0.05 & & \\
\hline $\mathrm{Ni} \quad(p p m)$ & 64 & 0.25 & & \\
\hline V (ppm) & 63 & 0.14 & & \\
\hline Zn (ppm) & 65 & 0.05 & & \\
\hline
\end{tabular}

\section{Relationships between Crude Oil Properties and Wettability Alteration}

Given the impact of brine composition on the tendency of a given oil to alter the wetting of a particular mineral surface, a test that explores a region of aqueous phase compositions is needed. Yang et al. (2001) used "adhesion maps" of adhesion tendency as a function of $\mathrm{pH}$ and ionic strength (Buckley et al., 1989) as the basis of a quantitative measure of wetting alteration. The fraction of the map over which adhesion occurs was used as a quantitative measure of the tendency of an oil to alter wetting. An adhesion map for A-93 crude oil from Prudhoe Bay is shown in Fig. II-2.3a. (Buffer $\mathrm{pH}$ is controlled by varying amounts of acetic acid and acetate or by sodium phosphates, additional adjustments to ionic strength are made by adding $\mathrm{NaCl}$.) At each brine condition, a piece of mica is submerged in brine and a drop of crude oil is pressed against the surface, trapped between mica and the glass tip of a microburette. After a brief contact time ( 2 minutes is typical), the oil is drawn slowly back into the burette. Adhesion and non-adhesion refer to oil drops that remain on the surface. In the example shown, the fraction of the map that is in the adhesive region is less than one-half, although the exact number chosen to represent this oil would depend on the exact range of conditions included in the map. Fig. II$2.3 \mathrm{~b}$ shows contact angles measured on surfaces exposed to the same brine compositions, then to A-93 crude oil for varying lengths of time. In this example the surface was glass and the temperature was $80^{\circ} \mathrm{C}$. Decane/water contact angles on the treated surfaces are reported after 
bulk crude oil has been removed by rinsing with a minimal amount of toluene. Up to 10 days of aging in oil can be required before constant levels of contact angles are reached.

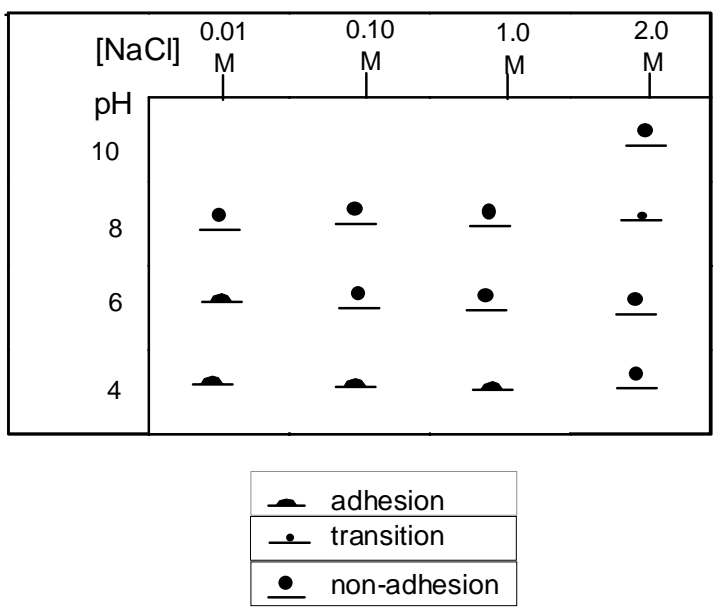

(a) Adhesion of A-93 on mica (after Liu and Buckley, 1999)

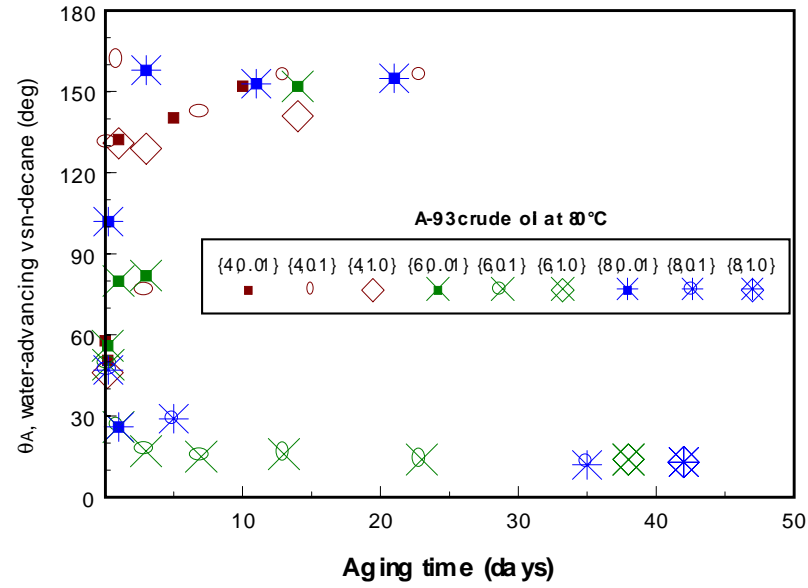

(b) Decane/water contact angles for water advancing on glass surfaces after exposure to A-93 crude oil (after Buckley et al., 1998a)

Figure II-2.3. Tests of crude oil wetting: (a) map of adhesion of oil under $\mathrm{NaCl}$ brines of varying $\mathrm{pH}$ and ionic composition and (b) contact angles between probe liquids (water and decane) on surfaces aged first in brine, then in crude oil.

Adhesion occurs when the water film that initially separates oil and mineral surface is unstable. For A-93, contact angles for water advancing against decane are about $150^{\circ}$ for the buffers that produce adhesion. Buffers that produce a stable water film tend to show much lower contact angles on the surfaces aged for three weeks. The differences between angles on mica treated at $25^{\circ} \mathrm{C}$ were not as extreme, but the same trend was observed as that shown for glass surfaces aged at $80^{\circ} \mathrm{C}$.

For A-93, therefore, it appears that stable water films are produced by higher $\mathrm{pH}$, higher ionic strength buffers, whereas with lower $\mathrm{pH}$, lower ionic strength solutions, the water films are not stable. Based on these observations, two buffer compositions $(\mathrm{pH} 4, \mathrm{I}=0.01 \mathrm{M}$ and $\mathrm{pH} 8$, $\mathrm{I}=1 \mathrm{M}$ ) were chosen as standards for subsequent tests with other crude oils. In the discussion that follows, these will be identified by $\mathrm{pH}$. The intention was that the $\mathrm{pH} 4$ buffer should maximize the likelihood of water film instability, resulting in high contact angles, whereas the pH8 buffer should maximize water film stability and produce lower contact angles. By choosing these two formulations, a range of controlled conditions could be explored.

\section{Single-parameter regression}

Since there are numerous mechanisms by which wetting can be altered, it is unlikely that any single oil parameter will closely correlate with wetting alteration. In Table II-2.2, regression of each measured oil property is shown with decane/water contact angles for mica surfaces pretreated with either $\mathrm{pH} 4\left(\theta_{\mathrm{A} 4}\right)$ or $\mathrm{pH} 8\left(\theta_{\mathrm{A} 8}\right)$ buffer, followed by aging in crude oil for about 
three weeks. The angles reported are for water advancing over decane-covered surfaces, hence the subscript A to distinguish from water-receding angles that are typically quite a bit lower. The number of crude oils for which data were available is indicated by $n$.

Table II-2.2. Single-parameter linear relationships between oil properties and wetting alteration.

\begin{tabular}{|c|c|c|c|c|}
\hline \multirow[b]{2}{*}{ measured oil properties } & \multicolumn{2}{|c|}{$\boldsymbol{\theta}_{\mathrm{A} 4}$} & \multicolumn{2}{|c|}{$\theta_{\mathrm{A} 8}$} \\
\hline & $\mathbf{n}$ & $\mathbf{R}^{2}$ & $\mathbf{n}$ & $\mathbf{R}^{2}$ \\
\hline${ }^{\circ} \mathrm{API}$ & 78 & 0.02 & 76 & 0.01 \\
\hline avg MW & 78 & 0.03 & 76 & 0.02 \\
\hline $\mathrm{RI}\left(\right.$ at $\left.20^{\circ} \mathrm{C}\right)$ & 78 & 0.01 & 76 & 0.03 \\
\hline $\mathrm{P}_{\mathrm{RI}}\left(\right.$ at $\left.20^{\circ} \mathrm{C}\right)$ & 72 & 0.07 & 69 & 0.09 \\
\hline density $\left(\mathrm{g} / \mathrm{ml}\right.$ at $\left.20^{\circ} \mathrm{C}\right)$ & 77 & 0.02 & 74 & 0.01 \\
\hline viscosity $\left(\mathrm{cP}\right.$ at $\left.20^{\circ} \mathrm{C}\right)$ & 65 & 0.06 & 65 & 0.00 \\
\hline saturates $(\%)$ & 77 & 0.01 & 75 & 0.06 \\
\hline aromatics (\%) & 77 & 0.01 & 75 & 0.00 \\
\hline $\mathrm{n}-\mathrm{C}_{6}$ resins (\%) & 77 & 0.10 & 75 & 0.00 \\
\hline $\mathrm{n}-\mathrm{C}_{6}$ asphaltenes (\%) & 77 & 0.02 & 75 & 0.24 \\
\hline $\mathrm{n}-\mathrm{C}_{7}$ asphaltenes (\%) & 76 & 0.03 & 73 & 0.25 \\
\hline acid \# (mg KOH/g oil) & 70 & 0.09 & 69 & 0.00 \\
\hline base \# (mg KOH/g oil) & 78 & 0.06 & 76 & 0.00 \\
\hline IEP (pH units) & 64 & 0.00 & 61 & 0.00 \\
\hline $\mathrm{Fe}(\mathrm{ppm})$ & 61 & 0.10 & 60 & 0.03 \\
\hline $\mathrm{Ni}(\mathrm{ppm})$ & 61 & 0.00 & 60 & 0.07 \\
\hline $\mathrm{V}(\mathrm{ppm})$ & 60 & 0.11 & 59 & 0.12 \\
\hline Zn (ppm) & 62 & 0.08 & 61 & 0.19 \\
\hline \multicolumn{5}{|l|}{ derived oil characteristics } \\
\hline$\Delta \mathrm{RI}=\mathrm{RI}-\mathrm{P}_{\mathrm{RI}}$ & 72 & 0.12 & 69 & 0.02 \\
\hline base \# / acid \# & 70 & 0.00 & 69 & 0.08 \\
\hline
\end{tabular}

As expected, none of the regressions produced strong correlations between oil properties and wetting alteration. Slightly better correlations could be obtained by relaxing the requirement that the relationship be linear (Yang, 2002), but the improvements were not sufficiently dramatic to warrant the additional complexity, especially when we move on to multiple regression statistics.

\section{Multiple regression}

Many linear combinations of two or more parameters have been tested. The purpose is not to find complicated equations that exactly match the data, nor is it to predict the outcome of future measurements. The main objective is to establish whether there are trends that link any of the measured oil properties, or parameters derived from the measurements, with observations of wetting alteration.

A survey of variables began with those that had the highest correlations in single parameter fits, but even variables that showed no correlation were included in the multiple regression tests. Subdivision of the data set into high and low asphaltene content oils was tested 
(Yang, 2002). In some cases the fits improve, but the numbers of data points are reduced and the reliability of the statistics can be questioned on the basis of small sample size.

Two correlations have been derived from the data set, one for water-advancing angles on surfaces pretreated with $\mathrm{pH} 4$ buffer and another for water-advancing angles for mica pretreated with $\mathrm{pH} 8$ buffer. Note that only samples for which all the correlation parameters are available are included in these relationships. Exclusion of samples on the basis of incomplete data may have the unintended consequence of excluding data from consideration, but the minimum number of oil samples on which the resulting relationships are based is 46 , a significant number of samples. No deliberate pruning of the data was permitted.

The resulting coefficients are summarized in Table II-2.3. In Fig. II-2.4 the measured values of $\theta_{\mathrm{A} 4}$ (Fig. II-2.4a) and $\theta_{\mathrm{A} 8}$ (Fig. II-2.4b) are compared to the corresponding values calculated from these relationships.

Table II-2.3. Coefficients from multiple linear regression analyses of contact angle data.

\begin{tabular}{|r|cc|cc|}
\hline & \multicolumn{2}{|c|}{$\boldsymbol{\theta}_{\mathbf{A} 4}$} & \multicolumn{2}{c|}{$\boldsymbol{\theta}_{\mathbf{A} 8}$} \\
\hline Term & Coefficient & $\mathbf{p}$ & Coefficient & $\mathbf{p}$ \\
\hline Intercept & 86.4 & 0.0629 & 45.1 & $<0.0001$ \\
$\mathbf{n C 7}$ asph (\%) & 8.21 & $<0.0001$ & -2.58 & 0.0001 \\
$\mathbf{Z n}$ & 1.33 & 0.0017 & 0.441 & 0.0185 \\
$\mathbf{B} / \mathbf{A}$ & & & -0.434 & 0.0498 \\
Resins (\%) & -3.33 & 0.0065 & & \\
$\mathbf{R I}-\mathbf{P}$ & -280 & 0.2858 & & \\
IEP & 6.33 & 0.4270 & & \\
\hline
\end{tabular}

The signs of the coefficients in Table II-2.3 show whether a variable tends to increase or decrease contact angles. It is interesting to note that the amount of $n-C_{7}$ asphaltenes is the most significant variable in both cases, but its influence on $\theta_{\mathrm{A} 8}$ is opposite to its effect on $\theta_{\mathrm{A} 4}$. The level of significance can be judged from the p-values in column 3, which represent the probability of observing this set of outcomes if the null hypothesis is true, i.e., of finding an apparent correlation when there is no real correlation. Thus, lower values of $p$ represent greater significance. Resins as well as asphaltenes contribute to the contact angles measured on mica pretreated with $\mathrm{pH} 4$ buffer, but increased resins decrease the contact angle whereas increased asphaltenes increase it. Less significant are the contributions of RI - $\mathrm{P}_{\mathrm{RI}}$, a measure of asphaltene stability, and IEP, the $\mathrm{pH}$ at which drops of crude oil have no net charge. It is worth noting, however, that these two measures do improve the correlation. They may have reduced significant because we impose linearity on the relationships. IEP is measured in $\mathrm{pH}$ units, which are logarithmic, and asphaltene stability has been shown to have a very non-linear effect on wetting (Al-Maamari and Buckley, 2000). 


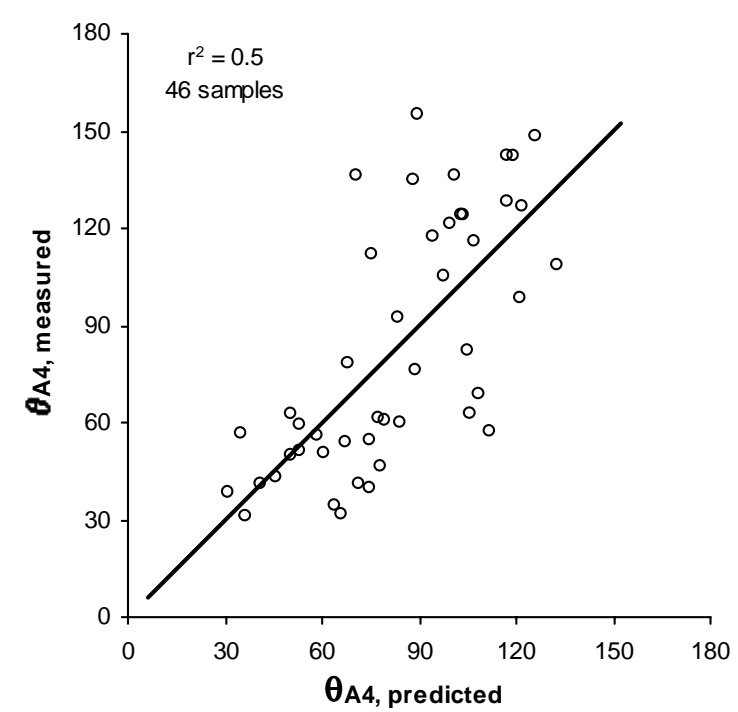

(a) less stable water films

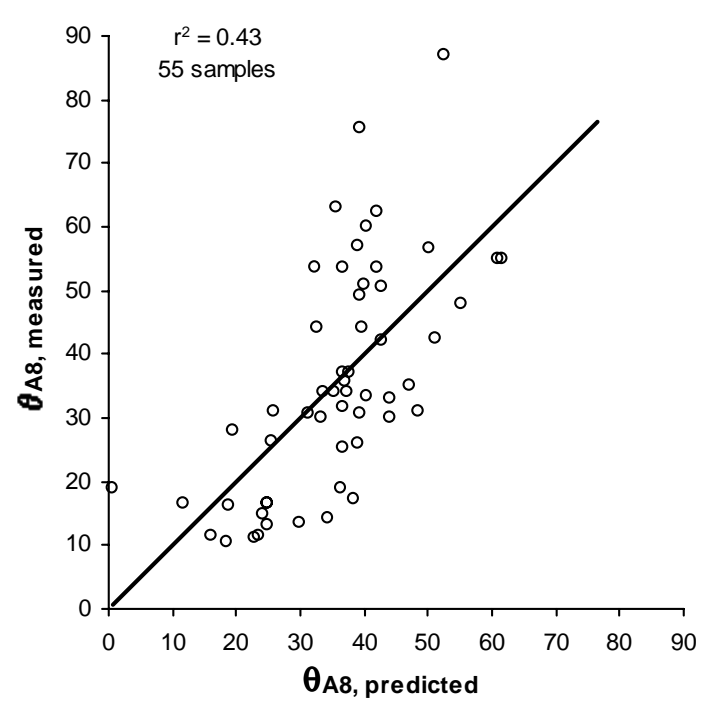

(b) more stable water films

Figure II-2.4. Comparisons of measured water-advancing contact angles with those calculated from equations with the coefficients given in Table II-2.3. The $\boldsymbol{\theta}_{\mathrm{A} 4}$ case respresents conditions selected to minimize water film stability; $\boldsymbol{\theta}_{\mathrm{A} 8}$ values are measured for the case where stable water films are more likely.

Acid number and base number are broad characterizations that may or may not highlight the important surface-active species in crude oils. It is encouraging, therefore to see that the base\#/acid\# ratio $(\mathrm{B} / \mathrm{A})$ appears in the $\theta_{\mathrm{A} 8}$ correlation and that oils with higher base numbers have lower values of $\theta_{\mathrm{A} 8}$. This confirms the hypothesis, developed with A-93 - a high basenumber oil - that water films are stabilized for the high $\mathrm{pH}$ buffer. The mechanism of interaction between higher acid number oils and mica surfaces requires some additional consideration. For $\theta_{\mathrm{A} 4}$, the contribution of surface charge may be represented by the correlation with IEP. Increasing basicity should increase IEP (Buckley, 1996) and strengthen the acid/base interactions by which basic crude oil components alter wetting when the intervening water film is unstable, consistent with the observed positive correlation with IEP.

\section{Contamination}

A disturbing aspect of both correlations is the significance of zinc. Zinc is not expected to be a natural component of crude oils except at very low levels. Speight (1991) estimates natural occurrence at only 0.5 to $1 \mathrm{ppm}$, whereas levels measured in some oil samples were as high as $40 \mathrm{ppm}$. Zinc is used in some drilling fluids and its presence at elevated concentrations might indicate that surface-active drilling fluid contaminants are also present and are affecting wettability alteration tests. Some of the samples with elevated zinc concentrations were provided in galvanized steel containers although not all such samples had high zinc levels, so it is difficult to say whether the containers might have been the source of zinc, rather than drilling fluid contamination. Whether zinc is an indicator of the presence of other contaminants or an active participant in interactions that bind crude oil components to the surface is not known. 
If all oil samples with more than $3 \mathrm{ppm}$ of $\mathrm{Zn}$ are removed from the data set, the correlations summarized in Table II-2.4 are obtained. The comparisons between measured and calculated contact angles are shown in Fig. II-2.5.

Table II-2.4. Coefficients for multiple regression analyses on the data set without high $\mathrm{Zn}$ samples.

\begin{tabular}{|r|rr|cl|}
\hline & $\boldsymbol{\theta}_{\mathbf{A} 4}$ & \multicolumn{2}{|c|}{$\boldsymbol{\theta}_{\mathbf{A} 8}$} \\
\hline Term & Coefficient & $\mathbf{p}$ & Coefficient & $\mathbf{p}$ \\
Intercept & 127 & $<0.0001$ & 45.4 & $<0.0001$ \\
nC7 asph (\%) & 5.87 & 0.0002 & -2.82 & $<0.0001$ \\
Resins (\%) & -2.41 & 0.0200 & & \\
B/A & & & -0.432 & 0.0268 \\
RI - P Pl & -465 & 0.0635 & & \\
IEP & -0.500 & 0.9278 & & \\
\hline
\end{tabular}

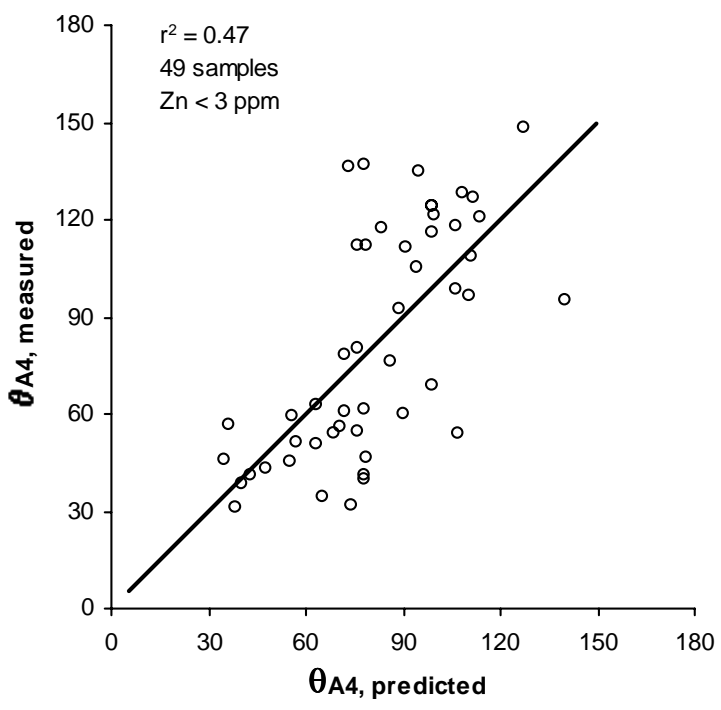

(a) less stable water films

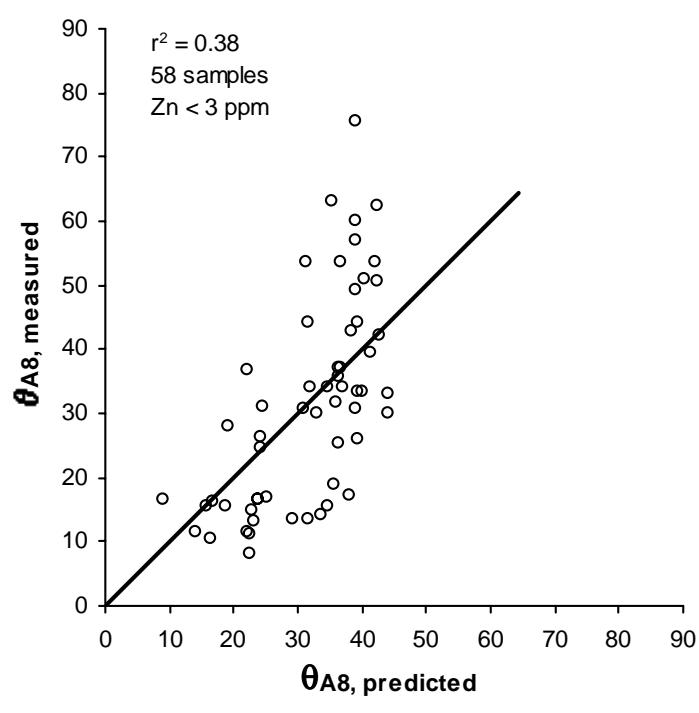

(b) more stable water films

Figure II-2.5. Comparisons of measured water-advancing contact angles with those calculated from equations with the coefficients given in Table II-2.4. Samples with more than $3 \mathrm{ppm} \mathrm{Zn}$ were removed from the data set.

Removing the high-Zn samples (and removing $\mathrm{Zn}$ concentration as a parameter from the correlations) changes the coefficients, but only in one case is the sign of the coefficient changed. IEP, the least significant variable probably should be dropped from the correlation for the $\mathrm{pH} 4$ advancing angles. Other relationships are similar to those calculated previously. Note that the significance of RI - $\mathrm{P}_{\mathrm{RI}}$ is increased in the $\theta_{\mathrm{A} 4}$ correlation. Values of $\mathrm{R}^{2}$ are lower than those that were calculated with $\mathrm{Zn}$ as a parameter, which is not surprising since there is one less fitting parameter in each case. A better comparison is between the fits for the same sets of parameters 
to the data with and without the high $\mathrm{Zn}$ samples. When the high $\mathrm{Zn}$ samples are included, but $\mathrm{Zn}$ is not a fitting parameter, the values of $\mathrm{R}^{2}$ are 0.31 and 0.37 for $\theta_{\mathrm{A} 4}$ and $\theta_{\mathrm{A} 8}$ respectively. Removing the high $\mathrm{Zn}$ samples makes a much bigger difference in the case of $\theta_{\mathrm{A} 4}$ (from an $\mathrm{R}^{2}$ of 0.31 to 0.47$)$ than for $\theta_{\mathrm{A} 8}\left(\mathrm{R}^{2}\right.$ changes only from 0.37 to 0.38$)$. Note that other combinations of variables were tested as in the initial fitting process, not only those that previously produced the best fits.

Contamination of oil samples is an important issue, especially in studies of wettability where small amounts of surface active material that may well escape detection by any realistic analytical techniques can have a major impact on wettability. It is possible that samples other than those containing elevated concentrations of zinc may be contaminated from sources that do not include any telltale zinc. It is reassuring that changes in the correlating parameters and trends were minor when the highest $\mathrm{Zn}$ containing samples were removed from the data set. Sources of the crude oils in this data set are reasonably diverse, both geographically and in terms of the companies that have provided samples. In future, we will be able to check sample groups against this established baseline of data and identify anomalous wetting behavior fairly quickly.

\section{$\theta_{A 4}$ versus $\theta_{A 8}$}

Alteration of wetting by crude oils can be thought of as a two-step process. Since water initially occupies the pore space in the rock that is invaded by oil, the first step in the wettability alteration process occurs when the water phase becomes a thin film that is either stable or unstable in the presence of oil, depending on DLVO and other surface forces. If the water film is stable, the rock surface remains water-wet. If the water film is unstable, further interactions between oil and solid are enabled. The standard conditions chosen for the tests of wetting alteration are intended to focus on these two different steps in the process.

When the $\mathrm{pH} 8,1 \mathrm{M} \mathrm{NaCl}$ solution is used to precondition the mica surface, we are testing the hypothesis that water films are stable. Clearly that is not always the case. Water-advancing angles greater than about $30^{\circ}$ suggest either that water films are not stable or that oils contain components that can adsorb through the water phase. More than half the oils are in this group, including all of the high $\mathrm{Zn}$ oils. However, none of the water-advancing angles exceeds $90^{\circ}$.

Using the $\mathrm{pH} 4,0.01 \mathrm{M} \mathrm{NaCl}$ buffer to pretreat mica intentionally biases the results toward unstable water film conditions. In these cases, we compare oils on the basis of their tendency to leave an organic coating on the exposed mica surface, such as those shown in AFM studies (Lord and Buckley, 2002; Buckley and Lord, 2002). None of the water advancing angles measured under these conditions were less than $30^{\circ}$. The range of $\theta_{\mathrm{A} 4}$ measured so far is from 31 to $155^{\circ}$.

High asphaltene content correlated with higher values of $\theta_{\mathrm{A} 4}$, suggesting that the asphaltenes are involved, as expected, in the wetting alteration of exposed mica surfaces. Values of $\theta_{\mathrm{A} 8}$, however are negatively correlated with amount of asphaltene, which means that asphaltenes also have a role to play in stabilizing water films. In other words, oils with higher amounts of asphaltenes tend to conform to the pattern of stable and unstable water films first shown for A-93 crude oil (Ling and Buckley, 1999). The extent of wetting alteration by oils 
with fewer asphaltenes, as measured by contact angles between water and decane on mica surfaces after aging in crude oil, is less in the region where unstable water films are expected and more where we expect stable water films. In fact there is a weak relationship (with a negative slope) between $\theta_{\mathrm{A} 4}$ and $\theta_{\mathrm{A} 8}$ that is only apparent when the high $\mathrm{Zn}$ samples are removed from the data set (Fig. II-2.6).

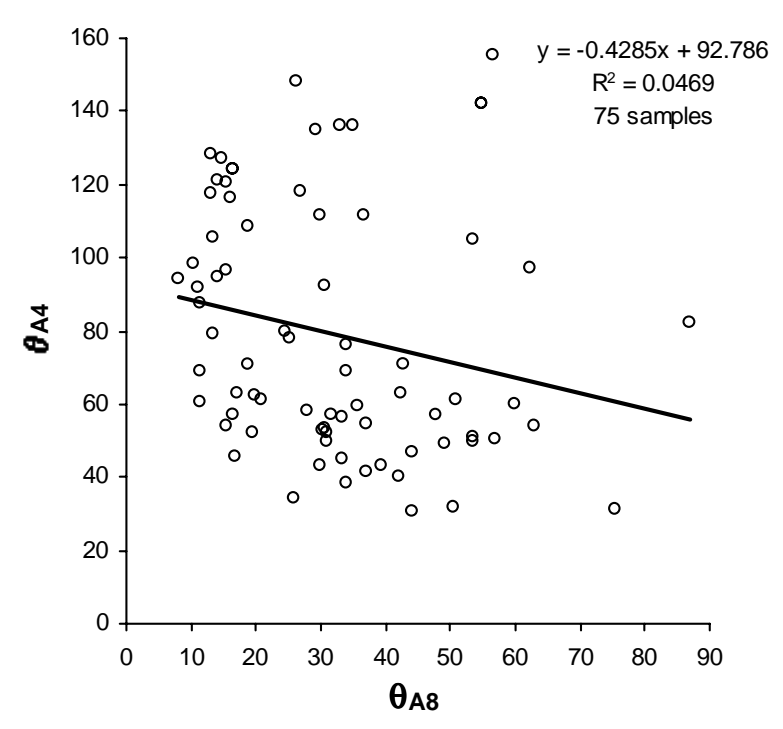

(a) all oil samples

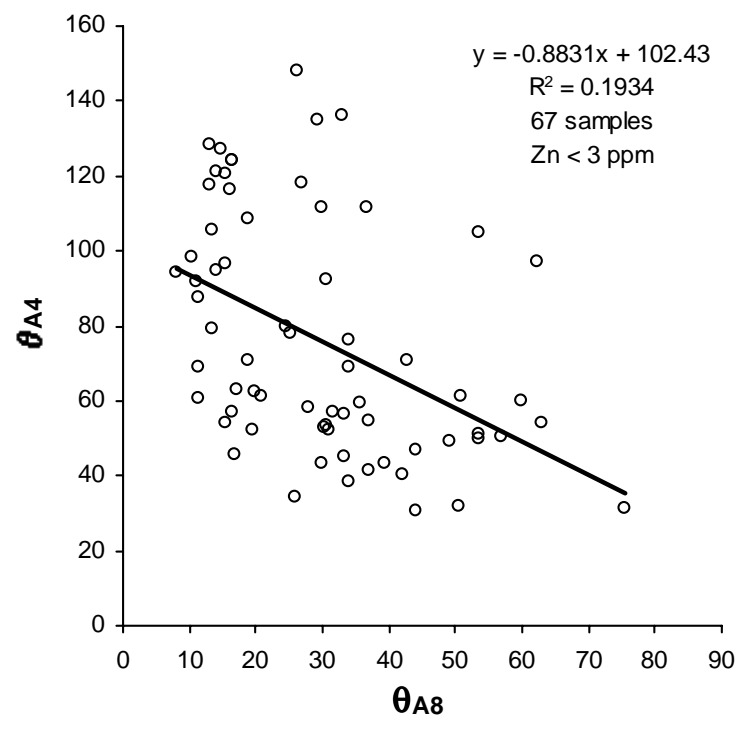

(b) oil samples with less than $3 \mathrm{ppm} \mathrm{Zn}$

Figure II-2.6. The highest values of $\theta_{\mathrm{A} 4}$ tend to correlate weakly with lower values of $\theta_{\mathrm{A} 8}$. The trend is masked by including oils with $\mathrm{Zn}$ concentrations $>3 \mathrm{ppm}$ (a), but is more apparent when the $\mathrm{Zn}>$ 3 samples are removed from the data set $(b)$.

\section{Neural Network Predictions}

The multivariable regression performed above was based on the assumption that contact angle is a linear function of each variable considered. Although this can simplify the problem, it may overlook non-linear correlations. In this section, we employ an artificial neural network (ANN) to investigate whether correlations can be improved by taking into account the potential non-linear correlations.

Since its first introduction approximately 50 years ago, ANN has been widely used in many logic-based digital computation areas to simulate human brain function, such as pattern recognition and pattern association. Application of ANN is most suitable in those areas where the relationships between input and output are so complex that any simple, analytical correlation is unobtainable. There are many kinds of neural networks available for different purposes, but generally they are all composed of an input layer, an output layer, and one or more intermediate (hidden) layers with various structures. An ANN can "learn" patterns by "training" with or without a "teacher." The information obtained from training is stored in the matrix, or so-called 
"weight," between input layer and output layer. The knowledge learned can then be used to generalize new data or recognize new patterns.

Among all the varying architectures, the back-propagation (BP) neural network is the most accurate and popular one developed so far. Figure II-2.7 shows the architecture of a BP neural network with one input layer ( 3 nodes), two hidden layers (5 nodes each), and one output layer (2 nodes). The nodes in the input layer form the input vector $\overrightarrow{\mathbf{x}}$. Each connection line between two nodes constitutes one of the elements of the weight matrix A. The nodes in the output layers form the output vector $\overrightarrow{\mathbf{b}}$. The idea of using BP, as well as other ANN nets, is based on the analogy that most mathematical problems, no matter how complex they are, can be expressed as a linear equation:

$$
\mathbf{A} \overrightarrow{\mathbf{x}}=\overrightarrow{\mathbf{b}}
$$

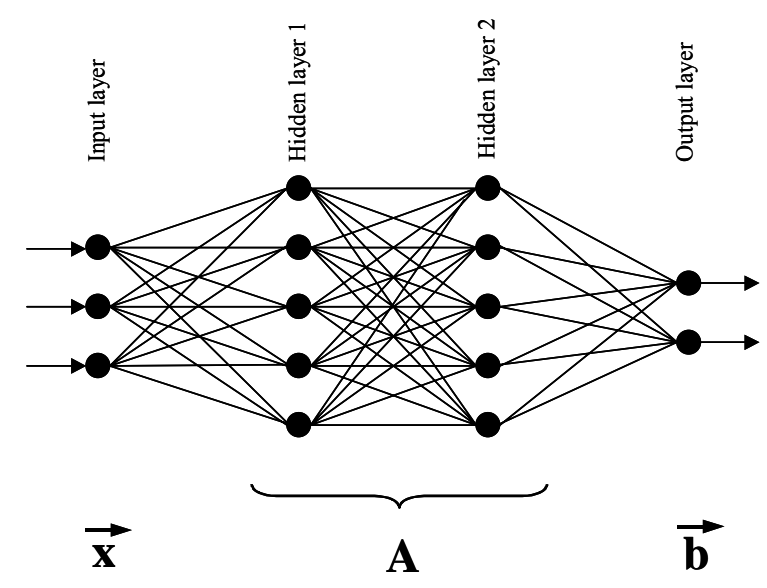

Figure II-2.7. Architecture of a BP neural network with one input layer (3nodes), two hidden layers (5 nodes each), and one output layer (two nodes).

The principle of training a BP net is as follows (Fausett, 1994):

- Initialize a weight A;

- Computer the output vector $\overrightarrow{\mathbf{b}^{\mathbf{1}}}$ using input vector $\overrightarrow{\mathbf{x}}$ and given weight $\mathrm{A}$, and compare it with target vector $\overrightarrow{\mathbf{b}}$. The error of $\left(\overrightarrow{\mathbf{b}^{\prime}}-\overrightarrow{\mathbf{b}}\right)$ is then back propagated to adjust weight $A$ following the gradient in the direction in which the error $\left(\overrightarrow{\mathbf{b}^{\mathbf{1}}}-\overrightarrow{\mathbf{b}}\right)$ decreases most rapidly;

- Repeat step b) until an error tolerance for $\left(\overrightarrow{\mathbf{b}^{\prime}}-\overrightarrow{\mathbf{b}}\right)$ is reached. 
Theoretically, the error of $\left(\overrightarrow{\mathbf{b}^{\prime}}-\overrightarrow{\mathbf{b}}\right)$ could be reduced to an infinitesimal value if enough iterations were allowed. In other words, a BP net can precisely "remember" the correspondence between input vector $\overrightarrow{\mathbf{x}}$ and output vector $\overrightarrow{\mathbf{b}}$ simply because it can re-generate $\overrightarrow{\mathbf{b}}$ from $\overrightarrow{\mathbf{x}}$, or vise versa. This application is very useful in some areas such as data compression. However, when a BP net is used as a "memory", it loses the ability to generalize, i.e., the capability to predict a new output vector $\overrightarrow{\mathbf{b}}$ from a new input vector $\overrightarrow{\mathbf{x}}$ using the knowledge it leaned from training. Therefore, if one would like to find out the physical link between input and output, rather than to record them, one should avoid over-training. This can be achieved by the following steps:

- Randomly select a subset from the whole data $\operatorname{set}(\overrightarrow{\mathbf{x}}, \overrightarrow{\mathbf{b}})$ as the testing data set;

- Use the rest of the data set as the training set to train the BP net;

- At each iteration step, use the updated weight $\mathrm{A}$ to calculate the output from input vector of the testing data set, and compare it with target of testing data set;

- If the residual of $\left(\overrightarrow{\mathbf{b}^{\prime}}-\overrightarrow{\mathbf{b}}\right)$ for testing data is decreasing, continue step c. If $\left(\overrightarrow{\mathbf{b}^{\prime}}-\overrightarrow{\mathbf{b}}\right)$ does not decrease a local or global minimum has been reached. If, in subsequent steps $\left(\overrightarrow{\mathbf{b}^{\prime}}-\overrightarrow{\mathbf{b}}\right)$ increases sharply, the minimum probably represents a global minimum. Training should stop at this point.

We have designed and programmed a BP net with options of using either one hidden layer or two hidden layers. Theoretically, BP with one hidden layer is sufficient to perform all the functions that can be performed by BP with multiple hidden layers. Increasing the number of hidden layer increases the complexity of program coding, but in some cases a BP with multiple hidden layer can speed up the convergence.

Based on the analysis described in the previous section, we choose $\mathrm{n}-\mathrm{C} 7 \mathrm{asph} \%$, resins, $\mathrm{dRI}$, and IEP as the major influencing factors for contact angle $\theta_{\mathrm{A} 4}$, while the factors for $\theta_{\mathrm{A} 8}$ are $\mathrm{n}-\mathrm{C} 7 \mathrm{asph} \%$ and $\mathrm{B} / \mathrm{A}$ only. Correspondingly, the input vector and output vector for $\theta_{\mathrm{A} 4}$ net are:

$$
\begin{aligned}
\overrightarrow{\mathbf{x}} & =(\mathrm{nC} 7 \text { asph\%, resins, dRI, IEP }) \\
\overrightarrow{\mathbf{b}} & =\left(\boldsymbol{\theta}_{\mathrm{A} 4}\right)
\end{aligned}
$$

while for $\theta_{\mathrm{A} 8}$ net:

$$
\begin{aligned}
& \overrightarrow{\mathbf{x}}=(\mathrm{nC7} \operatorname{asph} \%, \mathrm{~B} / \mathrm{A}) \\
& \overrightarrow{\mathbf{b}}=\left(\theta_{\mathrm{A} 8}\right)
\end{aligned}
$$

Figure II-2.8 shows an example of over-training in which all the 49 data samples from $\theta_{\mathrm{A} 4}$ data set are used to train the $\theta_{\mathrm{A} 4}$ net. The measured $\theta_{\mathrm{A} 4}$ data are fairly well "memorized", 
with the price of losing the generality of the net. Thus the trained net is useless for predictive purposes.

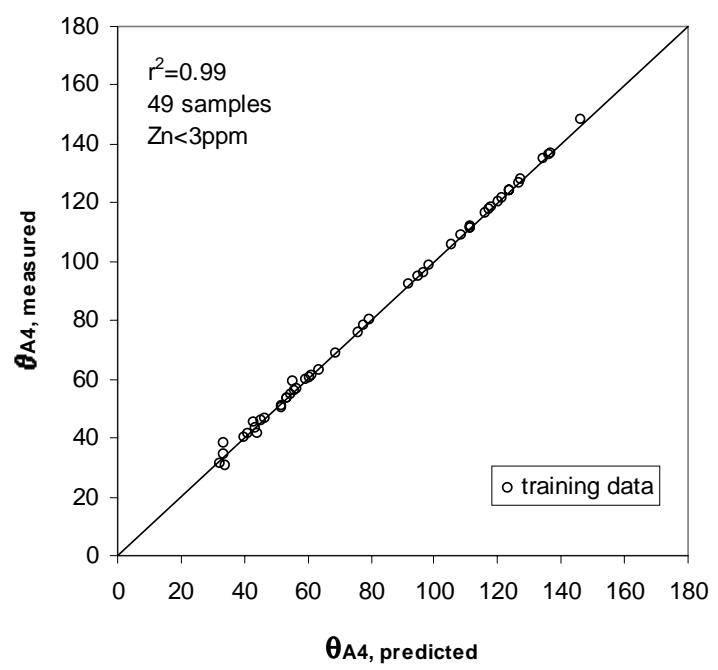

Figure II-2.8. Example of the "memorization" capability of a BP net. Using (nC7 asph\%, resins, dRI, IEP) as the input vector, the trained BP net can efficiently "remember" each corresponding contact angle $\theta_{\mathrm{A} 4}$, i.e., the calculated $\theta_{\mathrm{A} 4}$ matches the measured $\theta_{\mathrm{A} 4}$ very well, even though the original data are quite scattering. Even better results could be obtained if more time and iterations were allowed. The net is composed of one input layer (4 nodes), one hidden layer ( 5 nodes), and one output layer (one node).

By applying the scheme of simultaneous training-and-testing as described above, more general and physically meaningful results were obtained, as shown in Fig. II-2.9. Since the available data set is relatively small, we used $90 \%$ of the whole data as the training data, reserving $10 \%$ as the testing data. Correlations for both $\theta_{\mathrm{A} 4}$ and $\theta_{\mathrm{A} 8}$ cases are significantly improved. The correlation coefficient $r^{2}$ reaches 0.78 for $\theta_{\mathrm{A} 4}$ compared to 0.47 for the linear regression, while that for $\theta_{\mathrm{A} 8}$ is increased to 0.49 compared to 0.38 suggesting that non-linear relationships do exist between contact angles and selected physical properties.

It should be emphasized at this point that the results shown in Fig. II-2.9 are not unique. Different patterns can be obtained that meet the minimum error residual criteria, depending on the random selection of testing data. In most cases, however, the correlation coefficients from different simulations are quite similar. Thus from a practical point of view, the training results are sufficiently accurate and can be used to predict the contact angle for unknown oil samples. The prediction is very simple and straightforward; the user provides the physical properties of an oil to the input layer; BP net will calculate the corresponding output (the contact angle) using the trained, stored weight matrix. The accuracy could be further improved with additional experimental data. 


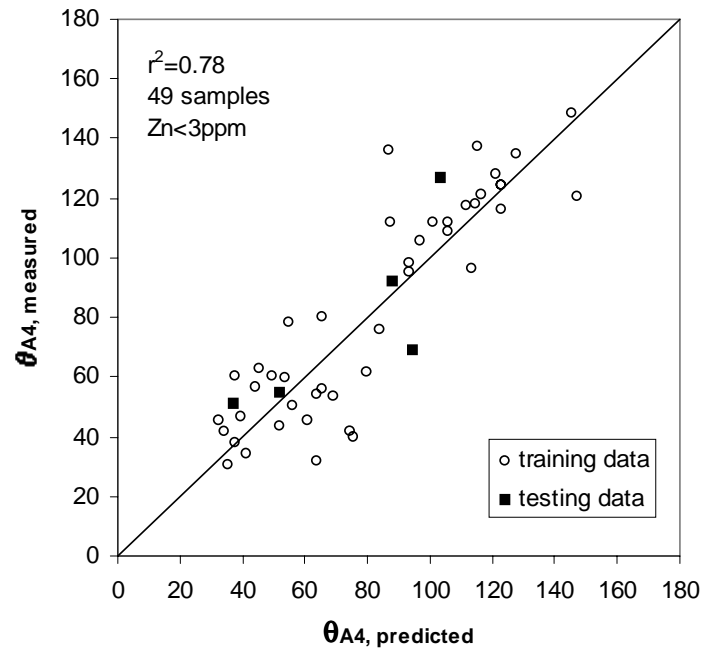

(a) less stable water films

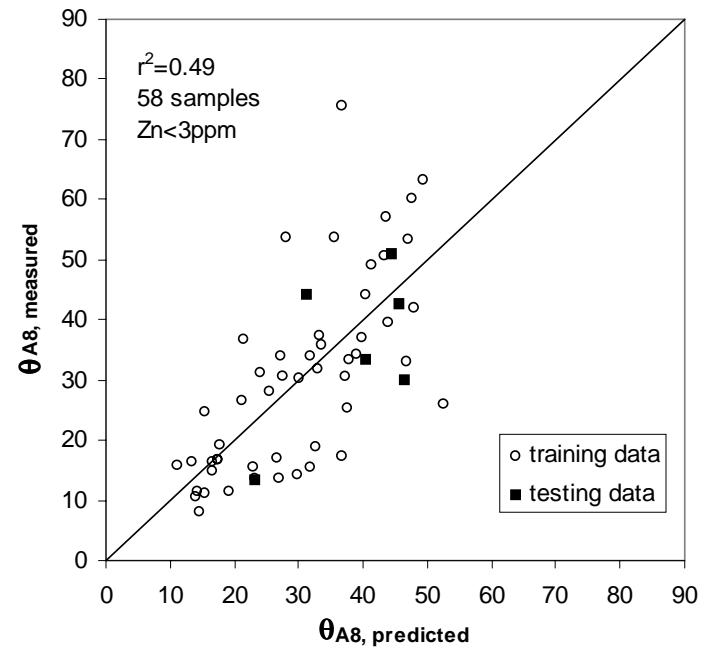

(b) more stable water films

Figure II-2.9. The best training results from BP neural network by using $90 \%$ of data set for training while the rest $10 \%$ data set for testing. Variables selected on the basis of multiple linear regression analyses: (a) $\theta_{\mathrm{A} 4}=\theta_{\mathrm{A} 4}\left(\mathrm{nC7}\right.$ asph\%, resins, dRI, IEP). Total sample number $=49$. (b) $\theta_{\mathrm{A} 8}=\theta_{\mathrm{A} 8}(\mathrm{nC} 7$ $\operatorname{asph} \%, \mathrm{~B} / \mathrm{A})$. Total sample number $=58$.

A drawback of using a neural network is that it cannot provide an analytical form of the correlations between input and output, thus no insight into physical significance can be obtained directly from the neural network, although information might be forthcoming from studies in which one or more properties are added to or removed from the input layer. The change in training results would be an indicator of the significance of those properties. For example, using as input the percentages of $\mathrm{nC}_{7}$ asphaltenes and resins, the values of RI, acid number, and base number, the training and predictions of $\theta_{\mathrm{A} 4}$ shown in Fig. II-2.10 were obtained.

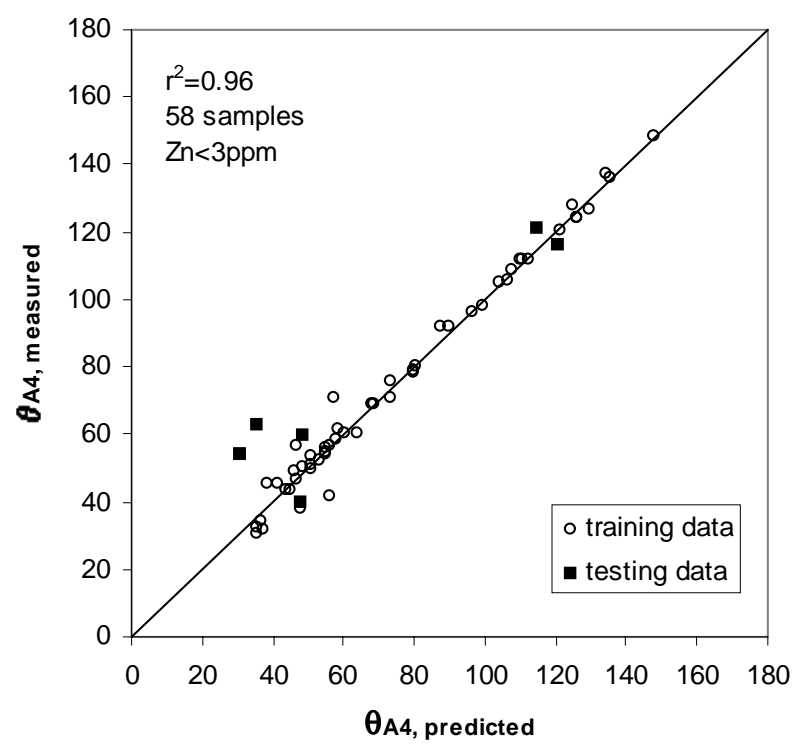

Figure II-2.10. One realization of a network trained to predict $\theta_{\mathrm{A} 4}$ from percentages of $\mathrm{nC}_{7}$ asphaltenes and resins, RI, and acid and base numbers. 


\section{Summary and Conclusions}

It should be clearly understood that this study does not provide a means of evaluating the wettability of a specific reservoir. Connate brine composition, saturation and distribution, live oil composition, pore lining minerals, temperature, and pressure are all important factors in establishing reservoir wetting, none of which are duplicated in this study. What we have done is establish a baseline for further wettability studies by measuring contact angles for reproducible surfaces treated at standard conditions. A library of well-characterized oils is now available for future experimental investigations. Crude oils can be compared on the basis of their chemical properties as well as their propensity to alter wetting.

Although the trends exhibit considerable scatter, the tendency to alter wetting at standard conditions can be related to measurable oil properties. The amount of asphaltene is found to be a key parameter in all cases, although higher concentrations of asphaltenes can produce either higher or lower contact angles, depending on other factors.

The effect of asphaltene concentration can be partially masked by elevated levels of resins, which has the opposite effect on contact angles to that of the asphaltenes when water films are unstable. At lower levels of significance, asphaltene stability and oil polarity are also shown to be important parameters.

Statistical analyses that consider only one oil property are insufficient to find relationships because many properties influence wetting alteration either in cooperation or competition with one another. Relationships can be seen in multiple regression analyses, even when linearity is imposed.

Artificial neural networks are ideal for handling the complex, nonlinear relationships between oil properties and wetting changes. A preliminary study has shown that oil properties can be used to predict contact angles with reasonable certainty for both the stable and unstable water film conditions. Additional testing is needed to optimize the input parameter set.

Some samples in the data set appear to be contaminated. High levels of $\mathrm{Zn}$, well above the $0.5-1 \mathrm{ppm}$ expected to occur naturally, have been measured in 13 oil samples. When those samples were removed from the data set, statistical measures of multiple regression of oil properties and contact angles were improved, especially for the unstable water-film case.

\section{References}

Al-Maamari, R.S.H. and Buckley, J.S.: "Asphaltene Precipitation and Alteration of Wetting: Can Wettability Change During Oil Production?" paper SPE 59292 at the 2000 SPE/DOE IOR Symposium, Tulsa, 3-5 Apr.

ASTM D2007-80, 1980. Standard Test Method for Characteristic Groups in Rubber Extender and Processing Oils by the Clay-Gel Adsorption Chromatographic Method. ASTM.

ASTM D2896-88, 1988. Standard Test Method for Base Number of Petroleum Products by Potentiometric Perchloric Acid Titration. ASTM. 
ASTM D664-89, 1989. Standard Test Method for Acid Number of Petroleum Products by Potentiometric Titration. ASTM.

ASTM D 5863-95, 1995. Standard Test Methods for Determination of Nickel, Vanadium, Iron, and Sodium in Crude Oils and Residual Fuels by Flame Atomic Absorption Spectrometry. ASTM

Buckley, J.S.: "Chemistry of the Crude Oil/Brine Interface," Proc. $3^{\text {rd }}$ International Symposium on Evaluation of Reservoir Wettability and Its Effect on Oil Recovery, Univ. of Wyoming, Laramie (1996) 33-38.

Buckley, J.S.: "Effective Wettability of Minerals Exposed to Crude Oil," Current Opinion in Colloid \& Interface Sci. (2001) 6, 191-196.

Buckley, J.S., Hirasaki, G.J., Liu, Y., Von Drasek, S., Wang, J.X., and Gill, B.S.: "Asphaltene Precipitation and Solvent Properties of Crude Oils," Petroleum Science and Technology (1998) 16, No. 3\&4, 251-285.

Buckley, J.S., Liu, Y., and Monsterleet, S.: "Mechanisms of Wetting Alteration by Crude Oils," SPEJ (Mar. 1998a) 3, 54-61.

Buckley J.S. and Lord. D.L.: "Wettability and morphology of mica surfaces after exposure to crude oil," $7^{\text {th }}$ Internat. Symp. on Reservoir Wettability, Freycinet, Tasmania, 12-14 Mar. 2002. (submitted to JPSE)

Buckley, J.S., Takamura, K., and Morrow, N.R.: "Influence of Electrical Surface Charges on the Wetting Properties of Crude Oils," SPERE (August 1989) 4, 332-340.

Buckley, J.S. and Wang, J.X.: "Crude Oil and Asphaltene Characterization for Prediction of Wetting Alteration,” J. Pet. Sci. Eng, (2002) 33, 195-202.

Buckley, J.S., Wang, J.X., Wu, Y.D., and Thomas, C.P.: "Wettability and Waterflooding of the Sulimar Queen Reservoir," presented to the International Energy Agency, Copenhagen, September, 1997.

Dubey, S.T. and Doe, P.H., 1993. Base number and wetting properties of crude oils. SPE Reservoir Engineering: 195-200.

Fan, T., Wang, J.X., and Buckley, J.S.: "Evaluating Crude Oils by SARA Analysis," paper SPE 75228 presented at the 2002 SPE/DOE IOR Symposium, Tulsa, 15-17 Apr.

Fausett, L.: Fundamentals of Neural Networks: Architectures, Algorithms, and Applications. Prentice Hall, 1994, ISBN 0-13-334186-0.

Gaudin, A.M., Witt, A.F., and Decker, T.G.:" Contact angle Hysteresis - Principles and Application of Measurement Methods," Trans.,AIME (March 1963) 107-112.

Hjelmeland, O. and Larrondo, L.E.: "Experimental Investigation of the Effects of Temperature, Pressure, and Crude Oil Composition on Interfacial Properties," SPERE (July 1986) 1 321328.

Hjelmeland, O., Selle, O.M., and Rueslåtten, H.: "Wetting and Interfacial Properties of North Sea Reservoir Fluids/Rocks Systems," presented at the 1986 IEA Workshop, Collaborative Project on EOR, Hannover, West Germany, Sept. 16-17. 
Liu, L. and Buckley, J.S.: “Alteration of Wetting of Mica Surfaces," J. Pet. Sci. Eng. (1999) 24, 75-83.

Liu, Y. and Buckley, J.S.: "Evolution of Wetting Alteration by Adsorption from Crude Oil," SPEFE (Mar. 1997) 12, 5-11.

Lord, D.L. and Buckley, J.S.: "An AFM Study of Morphological Features that Affect Wetting at Crude Oil-Water-Mica Interfaces," Colloids and Surfaces A (2002) 206, 531-546.

Manrique, E.J., Mogollón, J.L., Linares, J.A., and Farrera, M., 1997. Basic nitrogen compounds in crude oils: effect on mineral dissolution during acid stimulation processes. SPE 37224, 1997 Internat. Oilfield Chem. Symp., Houston, 18-21 Feb.

Skauge, A., Standal, S., Boe, S.O., Skauge, T., and Blokhus, A.M.: "Effects of Organic Acids and Bases, and Oil Composition on Wettability," paper SPE 56673 presented at the 1999 ATCE, Houston, 3-6 Oct.

Speight, J.G.: The Chemistry and Technology of Petroleum, 2nd ed., M. Dekker, New York (1991), $760 \mathrm{pp}$.

Tang, G.Q. and Morrow, N.R.: "Salinity, Temperature, Oil Composition, and Oil Recovery by Waterflooding," SPE RE (Nov. 1997) 269-276.

Yang, L.: "Statistical Analysis of Wetting Alteration and Oil Properties," MS Thesis, New Mexico Tech, Socorro, NM, 2002.

Yang, S.Y., Hirasaki , G.J., Basu, S., and Vaidya, R.N.: "Statistical Analysis on Parameters that Affect Wetting for the Crude Oil/Brine/Mica System," J. Petrol. Sci. Eng. (2002) 33, 203-215.

Xie, X., Morrow, N.R., and Buckley, J.S.: "Contact Angle Hysteresis and the Stability of Wetting Changes Induced by Adsorption from Crude Oil," J. Petrol. Sci. Eng. (2002) 33, 147159. 


\section{Appendix to Section II-2.1}

Table II-2.5. Crude Oil Physical and Chemical Properties

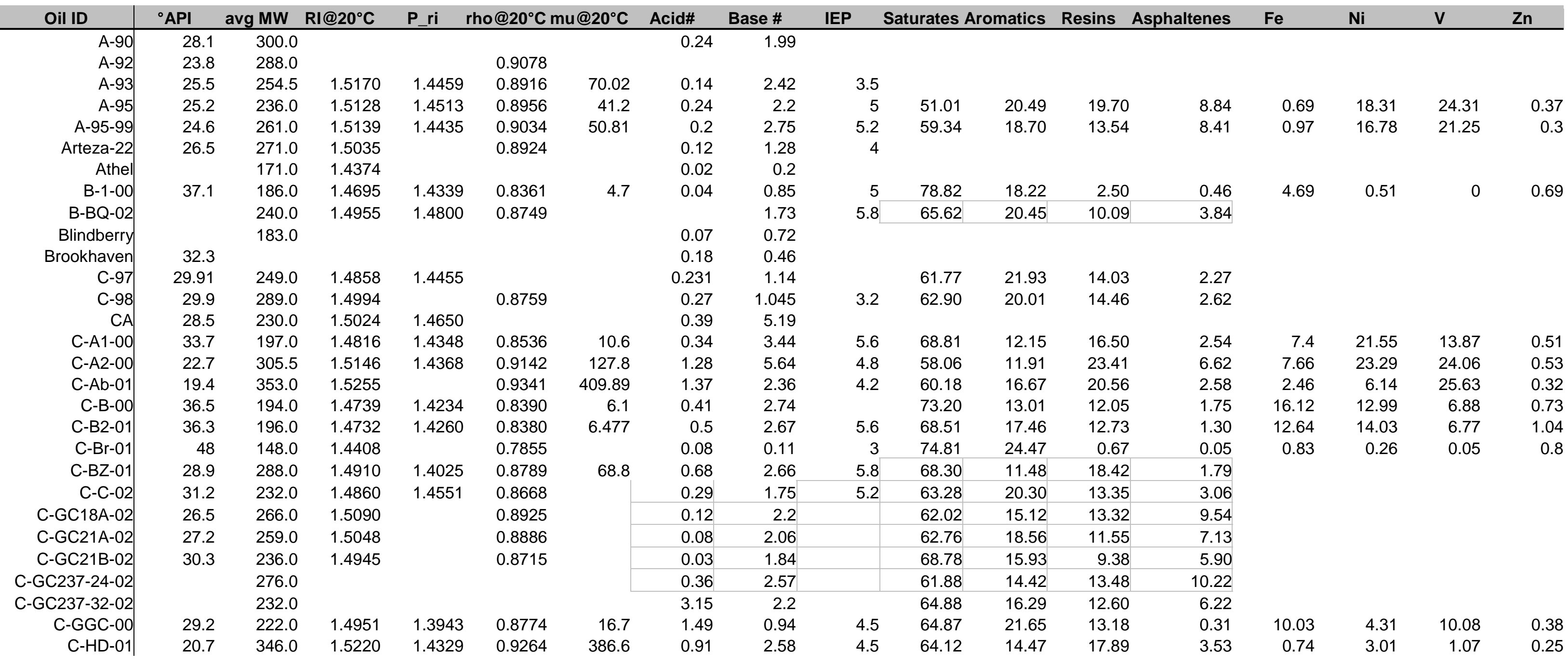




\begin{tabular}{|c|c|c|c|c|c|c|c|c|c|c|c|c|c|c|c|c|c|}
\hline Oil ID & ${ }^{\circ} \mathrm{API}$ & avg MW & $\mathrm{RI} @ 20^{\circ} \mathrm{C}$ & P_ri & rho@20 $\mathrm{C} \mathrm{m}$ & $@ 20^{\circ} \mathrm{C}$ & Acid\# & Base \# & IEP & Saturates & Aromatics & Resins & Asphaltenes & $\mathrm{Fe}$ & $\mathrm{Ni}$ & V & $\mathrm{Zn}$ \\
\hline C-HL-01 & 29.2 & 259.0 & 1.4920 & 1.4421 & 0.8771 & & & 1.8 & 4.6 & 67.30 & 14.93 & 15.14 & 2.64 & 4.12 & 2.67 & 1.24 & 1.13 \\
\hline C-HM-01 & 21.9 & 304.0 & 1.5187 & & 0.9192 & & 0.08 & 4.31 & 4.5 & 58.06 & 13.63 & 18.92 & 9.40 & 1.24 & 4.37 & 1.2 & 0.44 \\
\hline C-HM2-01 & 23 & 306.0 & 1.5186 & 1.4461 & 0.9126 & & 0.13 & 3.59 & & 49.75 & 18.54 & 21.21 & 10.49 & & & & \\
\hline C-K-01 & 18.9 & 319.0 & 1.5287 & 1.4320 & 0.9374 & 396 & 2.44 & 5.19 & 4 & 52.83 & 19.03 & 24.84 & 3.30 & 6.7 & 24.41 & 21.6 & 0.39 \\
\hline C-Kb-02 & 19.1 & & & & 0.9365 & & 2.55 & 5.66 & 5 & & & & & 7.07 & 26.28 & 19.25 & 0.58 \\
\hline C-L-01 & 34 & 216.0 & 1.4779 & 1.4260 & 0.8515 & 19.1 & 0.21 & 2.3 & 5.2 & 66.25 & 16.71 & 13.16 & 3.89 & 2.88 & 7.73 & 1.32 & 1.95 \\
\hline C-Lb-01 & 31.6 & 241.0 & 1.4858 & & 0.8644 & & 0.05 & 2.5 & 5.6 & 72.04 & 14.90 & 10.89 & 2.20 & 3.13 & 9.9 & 1.76 & 0.61 \\
\hline C-LH-99 & 22.6 & 268.3 & 1.5137 & 1.4231 & 0.9161 & 89.57 & 1.9 & 6.05 & 5.7 & 49.45 & 21.54 & 25.61 & 3.40 & 15.1 & 32.1 & 10.7 & 0.1 \\
\hline C-M103-02 & 31.7 & 243.0 & 1.4877 & & 0.8640 & & 0.48 & 2.37 & 6.3 & 66.28 & 17.04 & 13.85 & 2.83 & & & & \\
\hline C-M115-02 & 31.7 & 237.0 & 1.4871 & & 0.8641 & & 0.25 & 2.93 & 5.7 & 65.95 & 17.19 & 14.50 & 2.36 & & & & \\
\hline C-M122-02 & 34.5 & 213.0 & 1.4825 & & 0.8496 & & 0.13 & 2.02 & 4.9 & 67.48 & 19.46 & 12.05 & 1.00 & & & & \\
\hline C-P-01 & 31.9 & 236.0 & 1.4845 & 1.4358 & 0.8631 & & 0.54 & 1.55 & & 63.53 & 16.67 & 15.98 & 3.82 & & & & \\
\hline C-R-00 & 31.1 & 235.0 & 1.4851 & 1.4444 & 0.8673 & 19.05 & 0.25 & 0.49 & 4.9 & 70.62 & 16.34 & 11.40 & 1.61 & 0.74 & 0.4 & 0.3 & 0.22 \\
\hline C-R-01 & 31 & 254.0 & 1.4846 & 1.4446 & 0.8679 & & & 0.4 & 5 & 70.61 & 14.96 & 12.85 & 1.57 & 0.9 & 0.4 & 0.45 & 0.81 \\
\hline CS & 25.1 & 325.0 & 1.5070 & & 0.9003 & 81.14 & 0.33 & 1.16 & 4.8 & 68.23 & 18.40 & 12.86 & 0.52 & & & & \\
\hline C-T-01 & 26.2 & 280.0 & 1.5047 & 1.4628 & 0.8943 & & 0.38 & 1.87 & 3.3 & 60.55 & 17.01 & 11.61 & 10.83 & 2.06 & 13.34 & 18.2 & 2.19 \\
\hline C-T-02 & 34.2 & 214.0 & 1.4784 & 1.4211 & 0.8511 & & & 1.3 & 4 & 61.06 & 22.77 & 14.55 & 1.61 & & & & \\
\hline C-T1-00 & 31.6 & 210.0 & 1.4868 & 1.4332 & 0.8635 & 21.6 & 0.1 & 3.6 & 6.5 & 63.44 & 16.46 & 17.43 & 2.67 & 4.64 & 20.16 & 10.18 & 2.41 \\
\hline CV-P & 12.5 & 420.0 & & & 0.9780 & & & & & & & & & & & & \\
\hline Cymric-12 & 12.8 & 373.0 & 1.5510 & 1.4496 & 0.9805 & & 5.16 & 9.63 & 5.1 & 42.71 & 17.75 & 33.92 & 5.61 & & & & \\
\hline Cymric-28 & 27 & 212.0 & 1.5006 & 1.4301 & 0.8921 & 18.39 & 0.85 & 2.2 & 5.2 & 54.61 & 23.72 & 19.06 & 2.61 & & & & \\
\hline Dagang & 27.8 & 466.0 & 1.5051 & & & & 0.66 & 4.67 & & & & & & & & & \\
\hline DS-P-01 & 30.6 & 241.0 & 1.4881 & & 0.8698 & 16.47 & 0.31 & 1.51 & 5.2 & 69.68 & 16.72 & 13.03 & 0.57 & 1.68 & 5.46 & 0.65 & 0.24 \\
\hline$E-1 \times C O-01$ & 33.8 & 218.0 & 1.4795 & 1.4163 & 0.8532 & 9.3 & 0.18 & 1.93 & 5.4 & 71.49 & 18.05 & 9.73 & 0.74 & 1.38 & 2.1 & 0.48 & 19.15 \\
\hline E-1XD-00 & 22.3 & 287.0 & 1.5141 & 1.4336 & 0.9165 & 137.4 & 1.56 & 2.98 & 5 & 64.09 & 18.46 & 15.29 & 2.16 & 4.24 & 4.17 & 0.98 & 30.51 \\
\hline E-1XFR-01 & 40 & 179.0 & 1.4667 & 1.4039 & 0.8223 & 3.68 & 0.16 & 0.65 & 4.6 & 70.56 & 21.54 & 7.64 & 0.27 & 0.57 & 0.1 & 0.72 & 0.05 \\
\hline E-1XO-00 & 21.9 & 264.0 & 1.5142 & 1.4142 & 0.9193 & 15.19 & 3.42 & 2.57 & 5.1 & 57.77 & 22.12 & 19.37 & 0.74 & 1.52 & 3.98 & 0.67 & 13.31 \\
\hline E-1XR-00 & 29.1 & 218.0 & 1.4912 & 1.4071 & 0.8781 & 20.52 & 0.54 & 2.02 & 5.1 & 71.63 & 17.56 & 10.40 & 0.41 & & & & 42.5 \\
\hline E-1XR-00 & 29.1 & 218.0 & 1.4912 & 1.4071 & 0.8781 & 20.52 & 0.54 & 2.02 & 5.1 & 71.63 & 17.56 & 10.40 & 0.41 & 1.36 & 3.4 & 0.82 & 41 \\
\hline E-2XR-00 & 25.4 & 235.0 & 1.5040 & 1.4274 & 0.8986 & 46.66 & 0.91 & 2.46 & 5.3 & 65.66 & 18.37 & 14.85 & 1.12 & 3.14 & 3.61 & 0.77 & 22.12 \\
\hline E-8XFR-01 & 38.6 & 189.0 & 1.4676 & 1.4020 & 0.8290 & 4.662 & 1.03 & 0.74 & 4.7 & 70.86 & 19.69 & 9.09 & 0.36 & 0.58 & 0.27 & 1.15 & 0.63 \\
\hline E-BL-00 & 31.3 & 213.0 & 1.4896 & 1.4395 & 0.8651 & 23.37 & 0.17 & 1.33 & 5.4 & 66.34 & 21.17 & 9.45 & 3.00 & 0.47 & 6.14 & 10.11 & 2.81 \\
\hline Edop & 42.1 & 201.0 & 1.4684 & & & & 0.29 & 1.27 & & 75.70 & 16.01 & 7.93 & 0.36 & & & & \\
\hline
\end{tabular}




\begin{tabular}{|c|c|c|c|c|c|c|c|c|c|c|c|c|c|c|c|c|c|}
\hline Oil ID & ${ }^{\circ} \mathrm{API}$ & avg MW & $\mathrm{RI} @ 20^{\circ} \mathrm{C}$ & P_ri & rho@20 $\mathrm{C} \mathrm{m}$ & $\mathrm{u} @ 20^{\circ} \mathrm{C}$ & Acid\# & Base \# & IEP & Saturates & Aromatics & Resins & Asphaltenes & $\mathrm{Fe}$ & $\mathrm{Ni}$ & V & $\mathrm{Zn}$ \\
\hline EMSU & 32 & 212.0 & 1.4830 & 1.4290 & 0.8560 & & 0.55 & 0.8 & 4.3 & 65.96 & 21.97 & 10.68 & $\begin{array}{l}3.38 \\
31\end{array}$ & & & & \\
\hline E-S1XCA-01 & 23.2 & 298.0 & 1.5123 & 1.4626 & 0.9115 & 80.6 & 0.48 & 3.42 & 4.4 & 60.69 & 19.07 & 18.27 & 1.96 & 2.5 & 3.43 & 0.56 & 36.31 \\
\hline E-S1XG-01 & 33.3 & 237.0 & 1.4899 & 1.4681 & 0.8554 & 9.6 & 0.14 & 1.57 & 5 & 68.71 & 19.82 & 11.03 & 0.43 & 1.5 & 3.56 & 0.65 & 3.31 \\
\hline E-S1XL-01 & 33.5 & 220.0 & 1.4800 & 1.4088 & 0.8543 & 10.3 & 0.48 & 1.83 & 5.1 & 71.45 & 19.32 & 8.90 & 0.33 & 4.35 & 2.41 & 0.64 & 29.52 \\
\hline E-S3XR-01 & 30 & 251.0 & 1.4907 & 1.4238 & 0.8732 & 19.8 & 0.23 & 2.03 & 5.2 & 68.47 & 16.35 & 14.54 & 0.64 & 2.26 & 2.59 & 0.53 & 0.65 \\
\hline GOM(1)-00 & 26.3 & 271.0 & 1.5043 & 1.4342 & 0.8929 & 43 & 0.35 & 1.75 & 3.8 & 54.69 & 20.16 & 20.65 & 4.49 & & & & \\
\hline GOM(1)-01 & 24.8 & 284.0 & 1.5092 & 1.4463 & 0.9017 & 66.84 & 0.32 & 1.62 & 3 & 54.49 & 18.74 & 20.38 & 6.38 & & & & \\
\hline GOM(2)-00 & 21.2 & 287.0 & 1.5075 & 1.4504 & 0.9228 & 122.31 & 2.02 & 1.79 & 3.4 & 55.60 & 15.41 & 19.07 & 9.92 & & & & \\
\hline Gullfaks-96 & 27.1 & 245.0 & 1.4930 & & 0.8827 & 15.75 & 0.244 & 1.19 & 4.1 & 63.34 & 25.49 & 10.93 & 0.24 & 0.35 & 1.42 & 1.06 & 0.17 \\
\hline JR-1 & & 308.0 & 1.5220 & 1.4330 & & & 1.951 & 2.05 & & & & & & & & & \\
\hline JR-2 & & 275.0 & 1.5090 & 1.4320 & & & 0.563 & 1.92 & & & & & & & & & \\
\hline JR-3 & & & 1.5300 & 1.4230 & & & 2.073 & 2.05 & & & & & & & & & \\
\hline Lagrave & 41.3 & 168.0 & 1.4659 & 1.4530 & 0.8109 & & 0.29 & 0.65 & 3.4 & 59.37 & 24.89 & 10.24 & 5.50 & & & & \\
\hline Lagrave-97 & 37.1 & 188.0 & 1.4793 & 1.4613 & 0.8264 & 5.88 & 0.13 & 0.91 & 4.4 & 64.10 & 18.30 & 10.90 & 6.70 & 0.45 & 6.52 & 3.27 & 0.21 \\
\hline Leduc & & 184.0 & & & & & & & & & & & & & & & \\
\hline Maljamar & 36 & 230.0 & & & 0.8542 & & 0.12 & 0.72 & & & & & & & & & \\
\hline Mars-97 & 28.5 & 258.0 & 1.4952 & 1.4298 & 0.8804 & 24.7 & 0.368 & 1.79 & 3.8 & 57.20 & 22.50 & 17.28 & 3.02 & 3.27 & 10.55 & 20.81 & 0.63 \\
\hline Mars-P & 16.5 & 309.0 & 1.5384 & 1.4262 & 0.9524 & 481.1 & 3.92 & 2.295 & 4.3 & 38.43 & 29.76 & 25.81 & 5.99 & 4.3 & 17.55 & 25.35 & 0.6 \\
\hline Minnelusa-02 & 16 & 264.0 & 1.5123 & & 0.9000 & 167.8 & & 2.01 & 4.1 & 57.99 & 20.20 & 13.93 & 7.88 & & & & \\
\hline Moutray-1 & 35.2 & 239.0 & 1.4833 & 1.4346 & 0.8576 & & 0.55 & 0.81 & 3.7 & 66.64 & 19.61 & 12.33 & 1.42 & & & & \\
\hline Moutray-2 & 30.6 & 311.0 & & & & & 0.72 & 0.9 & & & & & & & & & \\
\hline MY1-02 & 27.2 & 256.0 & 1.4979 & & 0.8887 & & 0.5 & 1.17 & 3.2 & 64.02 & 24.59 & 9.67 & 1.71 & & & & \\
\hline MY2-02 & 28.9 & 239.0 & 1.4941 & & 0.8789 & & 0.17 & 1.16 & 3.7 & 62.52 & 23.72 & 12.68 & 1.08 & & & & \\
\hline MY3-02 & 28.2 & 245.0 & 1.4955 & & 0.8830 & & 0.2 & 1.17 & 3.3 & 61.86 & 24.83 & 12.22 & 1.09 & & & & \\
\hline MY4-02 & 28.4 & 244.0 & 1.4943 & & 0.8818 & & 0.22 & 1.23 & 3.4 & 65.28 & 23.69 & 9.94 & 1.10 & & & & \\
\hline NBU-1 & & 193.0 & & & & & 0.14 & 0.56 & & & & & & & & & \\
\hline NE-224-55-A & 35.3 & 182.0 & 1.4832 & & 0.8455 & & 0.1 & 1.07 & & & & & & & & & \\
\hline NE-224-55-B & 40 & 179.0 & 1.4655 & & 0.8222 & & 0.67 & 0.74 & & & & & & & & & \\
\hline NE-224-55-C & 34.7 & 217.0 & 1.4773 & & 0.8482 & & 0.66 & 0.33 & & & & & & & & & \\
\hline NE-224-55-D & 32.2 & 217.0 & 1.4870 & & 0.8613 & & 0.31 & 0.54 & & & & & & & & & \\
\hline NE-224-55-E & 22.7 & 285.0 & 1.5195 & & 0.9143 & & 0.04 & 2.95 & & & & & & & & & \\
\hline NE-224-55-F & 28.7 & 201.0 & 1.4985 & & 0.8802 & & 0.24 & 1.16 & & & & & & & & & \\
\hline
\end{tabular}




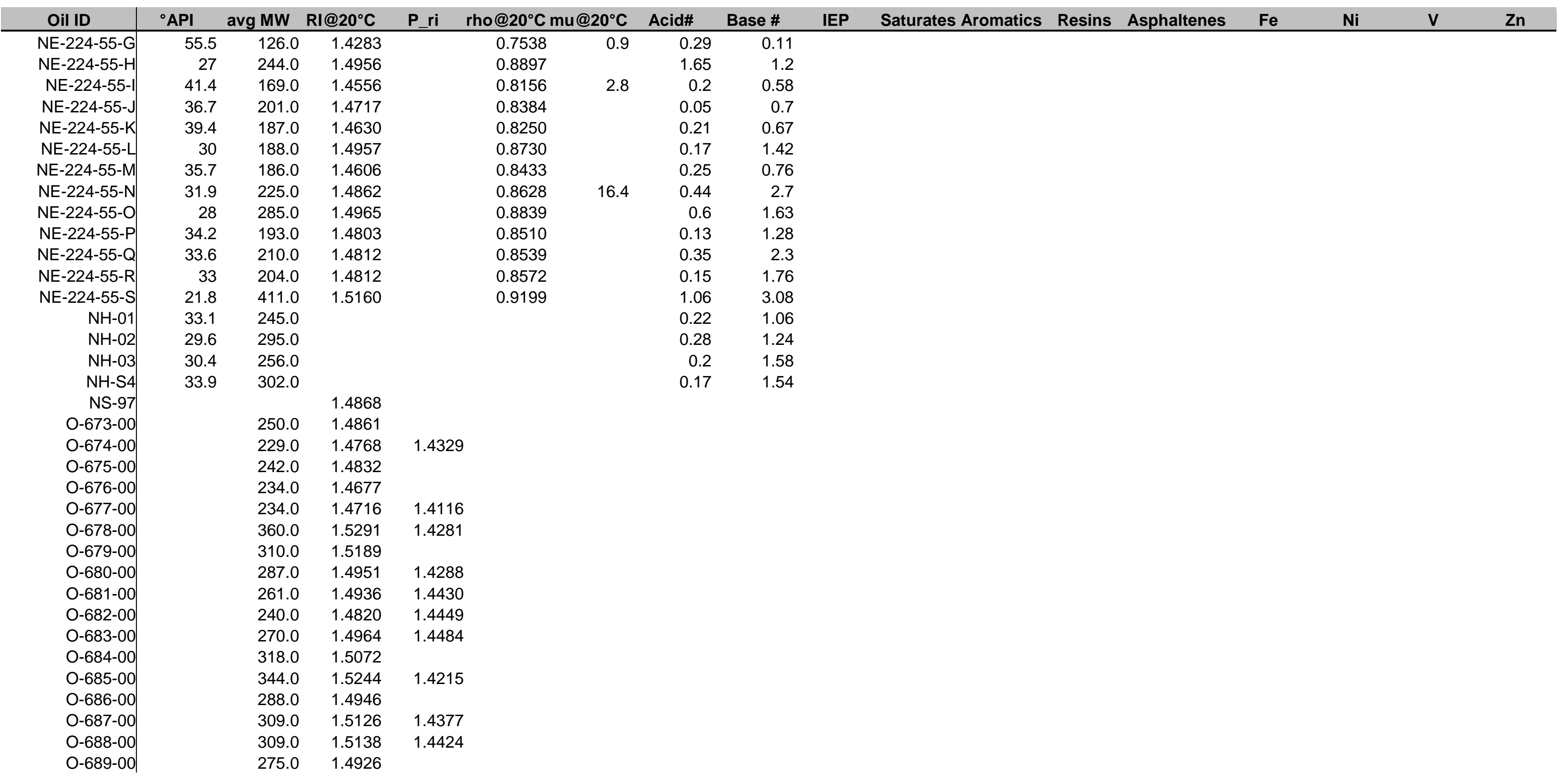




\begin{tabular}{|c|c|c|c|c|c|c|c|c|c|c|c|c|c|c|c|c|c|}
\hline Oil ID & ${ }^{\circ} \mathrm{API}$ & avg MW & $\mathrm{RI} @ 20^{\circ} \mathrm{C}$ & P_ri & rho@20 $\mathrm{C}$ mu & @20ㄷ & Acid\# & Base \# & IEP & Saturates & Aromatics & Resins & Asphaltenes & $\mathrm{Fe}$ & $\mathrm{Ni}$ & V & $\mathrm{Zn}$ \\
\hline OS034X & 39.8 & & & & 0.8300 & & 0.16 & 0.8 & & & & & & & & & \\
\hline P-E-00 & 36.1 & 209.0 & 1.4745 & 1.3929 & 0.8383 & 6.91 & 0.11 & 1.5 & 4.6 & 72.58 & 18.39 & 8.74 & 0.29 & 0.62 & 1.53 & 0.27 & 0.25 \\
\hline Petroglyph & & 333.0 & & & & & 0.11 & 1.55 & & & & & & & & & \\
\hline P-VE-00 & 30.55 & 242.2 & 1.4885 & 1.4388 & 0.8659 & 16.3 & 0.05 & 1.54 & & 66.76 & 17.18 & 11.58 & 4.49 & 0.54 & 12.23 & 102.8 & 0.3 \\
\hline SA051X & 28.1 & & & & 0.8973 & & 0.25 & 0.8 & & & & & & & & & \\
\hline S-A1-01 & 28.1 & 268.0 & 1.4986 & 1.4447 & 0.8834 & & 0.3 & 1.2 & & 60.73 & 17.06 & 14.04 & 8.17 & & & & \\
\hline S-A2-01 & 36.6 & 217.0 & 1.4705 & & 0.8389 & & 0.06 & 0.68 & & 75.63 & 15.79 & 7.47 & 1.09 & & & & \\
\hline S-A3-01 & 28 & 259.0 & 1.4990 & 1.4299 & 0.8838 & & 0.08 & 1.79 & 3.9 & 57.96 & 20.14 & 16.72 & 5.17 & & & & \\
\hline S-A4-01 & 36.8 & 207.0 & 1.4724 & 1.4365 & 0.8378 & & & 0.59 & 4 & 78.03 & 18.67 & 2.44 & 0.86 & & & & \\
\hline S-A6-01 & 28.4 & 267.0 & 1.5005 & 1.4495 & 0.8817 & & 0.08 & 1.78 & 5.3 & 60.19 & 19.68 & 12.96 & 7.16 & & & & \\
\hline Schuricht & 24.6 & 319.0 & 1.5130 & 1.4480 & & & 0.28 & 2.09 & & 59.98 & 19.16 & 17.41 & 3.46 & & & & \\
\hline Smackover & 19.8 & 353.0 & & & & & 0.325 & 1.445 & & & & & & & & & \\
\hline Spraberry-1 & 31.1 & & 1.4850 & & & & 0.32 & 2.83 & & & & & & & & & \\
\hline SQ-94 & 36.1 & 261.0 & & & 0.8648 & & 0.45 & 0.24 & & & & & & & & & \\
\hline SQ-95 & 37.2 & 213.0 & 1.4769 & 1.4166 & 0.8409 & 5.84 & 0.165 & 0.62 & 3.8 & 65.21 & 18.32 & 13.86 & 2.60 & 4.06 & 1.35 & 1.9 & 0.5 \\
\hline ST-86 & 29 & & & & & & 0.48 & 1.07 & & & & & & & & & \\
\hline ST-87 & 27.8 & 252.0 & 1.4977 & & & & 0.29 & 1.17 & & & & & & & & & \\
\hline ST-88 & 31.5 & & & & & & 0.24 & 0.53 & & & & & & & & & \\
\hline Steen-NM-1 & 22.3 & 279.0 & 1.5288 & 1.4683 & & & 0.26 & 2.93 & & 55.97 & 18.88 & 13.74 & 11.41 & & & & \\
\hline Steen-NM-2 & 28.6 & 265.0 & 1.4939 & 1.4620 & & & & 1.53 & & 66.75 & 19.04 & 12.22 & 1.98 & & & & \\
\hline Steen-OLEOD & 20 & 325.0 & 1.5331 & 1.4262 & & & & & & & & & & & & & \\
\hline Steen-TR-000 & 12.7 & 499.0 & 1.5644 & 1.4476 & & & 0.69 & 2.06 & & & & & & & & & \\
\hline Steen-TR-210 & 21 & 427.0 & 1.5205 & 1.4370 & & & 0.2 & 1.34 & & & & & & & & & \\
\hline Steen-TR-253 & 23.8 & 372.0 & 1.5033 & 1.4268 & & & & 0.55 & & & & & & & & & \\
\hline S-Ven-39 & 28.8 & 240.0 & 1.4976 & 1.4465 & 0.8795 & 29.8 & 0.14 & 1.68 & 5 & 51.09 & 28.30 & 14.51 & 6.06 & 0.81 & 23.33 & & 0.31 \\
\hline S-Ven-39 & 28.8 & 240.0 & 1.4976 & 1.4465 & 0.8795 & 29.8 & 0.14 & 1.68 & 5 & 51.09 & 28.30 & 14.51 & 6.06 & 0.86 & 18.81 & 187.37 & 0.23 \\
\hline S-Ven-40 & 30.2 & 226.0 & 1.4922 & 1.4492 & 0.8714 & 23.7 & 0.13 & 1.62 & 5.5 & 64.78 & 17.80 & 11.35 & 6.08 & 1.54 & 22.97 & 199.23 & 0.37 \\
\hline S-Ven-41 & 28.7 & 243.0 & 1.4985 & 1.4504 & 0.8802 & 33.7 & 0.43 & 1.78 & 5.3 & 60.03 & 18.31 & 14.48 & 7.17 & 0.43 & 21.28 & 208.36 & 0.19 \\
\hline Tensleep & 31.2 & 271.0 & 1.4877 & 1.4438 & 0.8684 & 21.57 & 0.16 & 0.96 & 4.8 & 69.32 & 16.01 & 9.38 & 5.29 & 0 & 0.58 & 2.2 & 0.16 \\
\hline Tensleep-99 & 31.1 & 270.0 & 1.4906 & 1.4428 & 0.8685 & 18.65 & 0.1 & 1.03 & 5.3 & 64.02 & 19.84 & 12.90 & 3.24 & 1.09 & 2.34 & 15.96 & 0.2 \\
\hline UBergen-1 & 34.1 & & 1.4802 & & & & 0.094 & 1.786 & & & & & & & & & \\
\hline UBergen-2 & 38.2 & & & & & & 0.087 & 0.97 & & & & & & & & & \\
\hline UBergen-3 & 35.4 & & & & & & 0.237 & 1.81 & & & & & & & & & \\
\hline
\end{tabular}




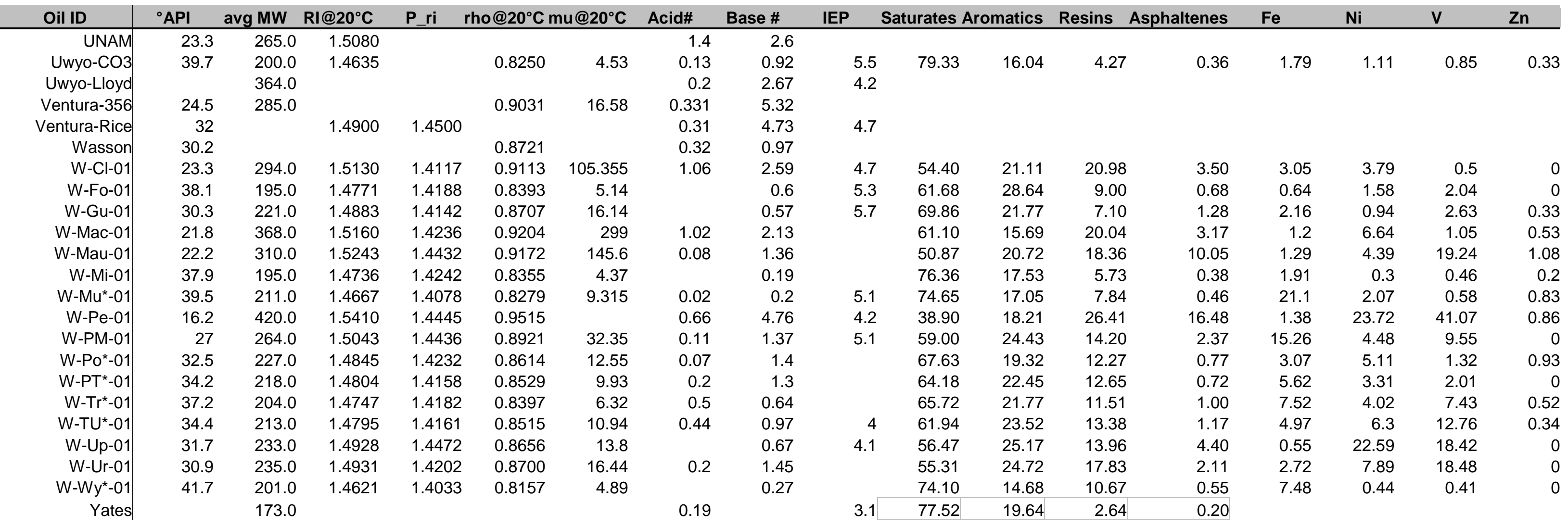


Table II-2.6. Contact Angles on Oil-treated Mica Surfaces

\begin{tabular}{|c|c|c|c|c|}
\hline Oil ID & A4 & R4 & A8 & R8 \\
\hline A-93 & 94 & 38 & 8 & 8 \\
\hline A-95 & 116 & 63 & 16 & 10 \\
\hline$A-95-99$ & 98 & 43 & 11 & 9 \\
\hline C-A1-00 & 78 & 46 & 25 & 14 \\
\hline C-A2-00 & 51 & 25 & 54 & 22 \\
\hline C-B-00 & 49 & 23 & 49 & 18 \\
\hline C-B2-01 & 47 & 20 & 44 & 12 \\
\hline C-GGC-00 & 43 & 21 & 30 & 15 \\
\hline C-HD-01 & 59 & 28 & 36 & 15 \\
\hline C-HL-01 & 41 & 19 & & \\
\hline C-K-01 & 38 & 21 & 34 & 14 \\
\hline C-L-01 & 55 & 24 & 37 & 15 \\
\hline C-Lb-01 & 58 & 31 & 28 & 13 \\
\hline C-LH-99 & 57 & 23 & 32 & 12 \\
\hline C-R-00 & 92 & 47 & 31 & 14 \\
\hline $\mathrm{CS}$ & 43 & 23 & 40 & 15 \\
\hline C-T1-00 & 60 & 29 & 11 & 9 \\
\hline C-T2-00 & 54 & 29 & 31 & 15 \\
\hline Cymric-12 & 46 & 24 & 17 & \\
\hline Cymric-28 & 45 & 17 & 33 & 15 \\
\hline DS-P-01 & 53 & 27 & 30 & 14 \\
\hline$E-1 X C O-01$ & 136 & 61 & 35 & 16 \\
\hline E-1XD-00 & 63 & 28 & 43 & 18 \\
\hline E-1XFR-01 & 40 & 15 & 42 & 12 \\
\hline E-1XO-00 & 50 & 14 & 31 & 17 \\
\hline$E-1 X R-00$ & 142 & 53 & 55 & 23 \\
\hline$E-1 X R-00$ & 142 & 53 & 55 & 23 \\
\hline E-2XR-00 & 155 & 16 & 57 & 28 \\
\hline E-8XFR-01 & 136 & 61 & 33 & 6 \\
\hline E-BL-00 & 69 & 18 & 34 & 21 \\
\hline Edop & & & 37 & 16 \\
\hline EMSU & 137 & 88 & & \\
\hline E-S1XCA-01 & 82 & 25 & 87 & 24 \\
\hline E-S1XG-01 & 60 & 30 & 60 & 16 \\
\hline E-S1XL-01 & 57 & 17 & 48 & 14 \\
\hline E-S3XR-01 & 50 & 25 & 57 & 22 \\
\hline GOM(1)-00 & 62 & 24 & 20 & 9 \\
\hline GOM(1)-01 & 112 & 45 & 37 & 24 \\
\hline GOM(2)-00 & 54 & 26 & 15 & 7 \\
\hline Gullfaks-96 & 105 & 48 & 53 & 18 \\
\hline Lagrave & 120 & 57 & 15 & 9 \\
\hline Lagrave-97 & 148 & 118 & 26 & 13 \\
\hline Mars-97 & 63 & 26 & 17 & 8 \\
\hline Mars-P & 31 & 13 & 44 & 23 \\
\hline Mars-TC & 52 & 25 & 31 & 20 \\
\hline Minnelusa & 69 & 40 & 12 & 7 \\
\hline Moutray-1 & 112 & 43 & & \\
\hline
\end{tabular}




\begin{tabular}{r|rrrr} 
Oil ID & A4 & R4 & A8 & R8 \\
\hline P-E-00 & 34 & 18 & 26 & \\
P-VE-00 & 92 & 42 & 11 & 7 \\
S-A2-01 & 71 & 34 & 43 & 23 \\
S-A3-01 & 80 & 42 & 25 & 14 \\
S-A4-01 & 118 & 63 & 27 & 15 \\
S-A5-01 & 95 & 50 & 14 & 8 \\
S-A6-01 & 96 & 53 & 16 & 8 \\
Schuricht & 79 & 39 & 14 & 9 \\
SQ-95 & 56 & 31 & 33 & 17 \\
S-Ven-39 & 124 & 52 & 17 & 10 \\
S-Ven-39 & 124 & 52 & 17 & 10 \\
S-Ven-40 & 127 & 63 & 15 & 10 \\
S-Ven-41 & 128 & 55 & 13 & 9 \\
Tensleep & 121 & 69 & 14 & 12 \\
Tensleep-99 & 105 & 55 & 14 & 8 \\
Uwo-CO3 & 97 & 35 & 62 & 34 \\
W-Cl-01 & 41 & 21 & 37 & 21 \\
W-Fo-01 & 61 & 31 & 21 & 14 \\
W-Gu-01 & 117 & 60 & 13 & 13 \\
W-Mac-01 & 71 & 33 & 19 & 14 \\
W-Mau-01 & 57 & 25 & 16 & 13 \\
W-Mi-01 & 88 & 45 & 11 & 9 \\
W-Mu*-01 & 76 & 41 & 34 & 17 \\
W-Pe-01 & 109 & 58 & 19 & 14 \\
W-PM-01 & 112 & 60 & 30 & 12 \\
W-Po*-01 & 54 & 19 & 63 & 28 \\
W-PT*-01 & 61 & 30 & 51 & 22 \\
W-Tr*01 & 32 & 15 & 51 & 22 \\
W-TU*-01 & 31 & 14 & 76 & 38 \\
W-Up-01 & 135 & 71 & 29 & 30 \\
W-Ur-01 & 50 & 27 & 54 & 22 \\
W-Wy*-01 & 52 & 9 & 19 & 13
\end{tabular}




\section{II-2.2. Contact Angle Hysteresis and the Stability of Wetting Changes Induced by Adsorption from Crude Oil}

Xie, X., Morrow, N.R., and Buckley, J.S.: "Contact Angle Hysteresis and the Stability of Wetting Changes Induced by Adsorption from Crude Oil," J. Petrol. Sci. Eng. (2002) 33, 147-159.

The dynamic Wilhelmy plate technique was used to investigate contact angle hysteresis and the stability of wetting changes induced on quartz surfaces by adsorption from crude oil. Some degree of contact angle hysteresis was always observed on surfaces that were first equilibrated with brine, then exposed to crude oil at elevated temperature. The extent to which hysteresis persisted through repeated cycles of advancing and receding of the probe fluids was different for different crude oil compositions. Regardless of whether or not the surface was dried, and whether the measurements began with the surface in brine or decane, quartz surfaces aged in asphaltic crude oils having more basic than acidic character showed large and reproducible contact angle hysteresis. For quartz surfaces aged in less asphaltic crude oils that are below their wax appearance temperatures at room conditions, receding contact angles were usually reproduced but advancing angles tended to decrease with each wetting cycle.

\section{Introduction}

Contact angles are commonly used to measure the wetting properties of a solid surface with respect to two immiscible fluids. Young's equation provides a definition of contact angle in terms of a surface energy or force balance that interrelates the interfacial tensions of the three phases.

$$
\sigma_{o s}=\sigma_{w s}+\sigma_{o w} \cos \theta_{o w}
$$

The contact angle is a thermodynamic property and provides an unambiguous definition of wetting if it is single-valued with respect to movement of the fluid/fluid interface. Zisman (1964) has summarized a large body of air/liquid contact angle data measured on polymeric solids and on adsorbed organic monolayers laid down by the Langmuir-Blodgett technique on metal substrates. Contact angles were usually reproducible to within one to two degrees and were essentially independent of the direction of motion of the fluid/fluid interface.

In many studies of wetting, hysteresis is observed between contact angles, depending on whether the interface has advanced or receded. Surface roughness on a polymeric solid has been shown to cause systematic differences between the intrinsic contact angle $\theta_{E}$ and the observed receding and advancing angles, $\theta_{R}$ and $\theta_{A}$ respectively (Morrow, 1975). Heterogeneity of surface wetting properties, often as a result of contamination, is also a widely recognized cause of hysteresis (Israelachvili, 1991). Deliberate generation of heterogeneous surfaces of selected geometry has been shown to result in predictable hysteresis in the contact angle (Robin and Koci, 1996). Hysteresis related to interface velocity has also been reported (Rose and Heins, 1962). 
The wetting behavior induced by adsorption of crude oil components onto rock surfaces initially covered with brine has a dominant effect on the efficiency of oil recovery from hydrocarbon reservoirs. Understanding the crude oil-brine-rock interactions that determine wetting behavior and oil recovery is a formidable task because many of the variables are only broadly defined. Smooth mineral surfaces, such as quartz and mica, are widely used in laboratory studies as models of negatively charged sandstone surfaces. Many attempts have been made to develop an understanding of wetting and oil recovery by using compounds that mimic the wetting properties induced by adsorption from crude oil; however, the wetting properties of crude oil depend on the complex chemistry of the oil as a whole. Even components isolated from crude oil, such as asphaltenes, will not mimic their adsorption behavior as it occurs from solution in the parent crude oil (Buckley et al., 1997a). Many studies of wetting have been focused on the wetting properties of the adsorbed layer. The mechanisms of wetting alteration of solid surfaces by crude oil and the wetting properties of the adsorbed layers have been extensively investigated (Buckley et al., 1997a; Akhlaq et al., 1996; Buckley et al., 1998b; Yang et al., 1998).

Adsorption from crude oils onto high-energy surfaces often results in surfaces that exhibit contact angle hysteresis. Receding contact angles will be operative when water is displaced by oil, whereas advancing conditions will apply in displacement of oil by water as occurs in oil recovery by waterflooding. In the present study, we examine the wetting behavior of quartz surfaces after adsorption from crude oils of varying composition, with attention to the magnitude and stability of contact angle hysteresis.

\section{The Dynamic Wilhelmy Plate Method}

Originally used as a means of measuring air-liquid surface tension, the dynamic Wilhelmy plate technique was modified to monitor the behavior of the oil/brine/solid threephase-line of contact by recording the change in force after the solid surface meets the liquidliquid interface (Teeters et al., 1988; Xie, 1996; Xie and Morrow, 1998, 1999). The forces acting on the plate are interfacial and buoyancy forces. The general equation is:

$$
F=p \sigma_{o w} \cos \theta_{o w}+F_{B}
$$

where $F=$ the force acting on the plate, $\mathrm{mN}$

$F_{B}=$ the buoyancy force, $\mathrm{mN}$

$p=$ the perimeter of the plate/liquid contact line, $\mathrm{m}$

$\sigma_{\text {ow }}=$ the oil/brine interfacial tension, $\mathrm{mN} / \mathrm{m}$

$\theta=$ contact angle, ${ }^{\circ}$ 


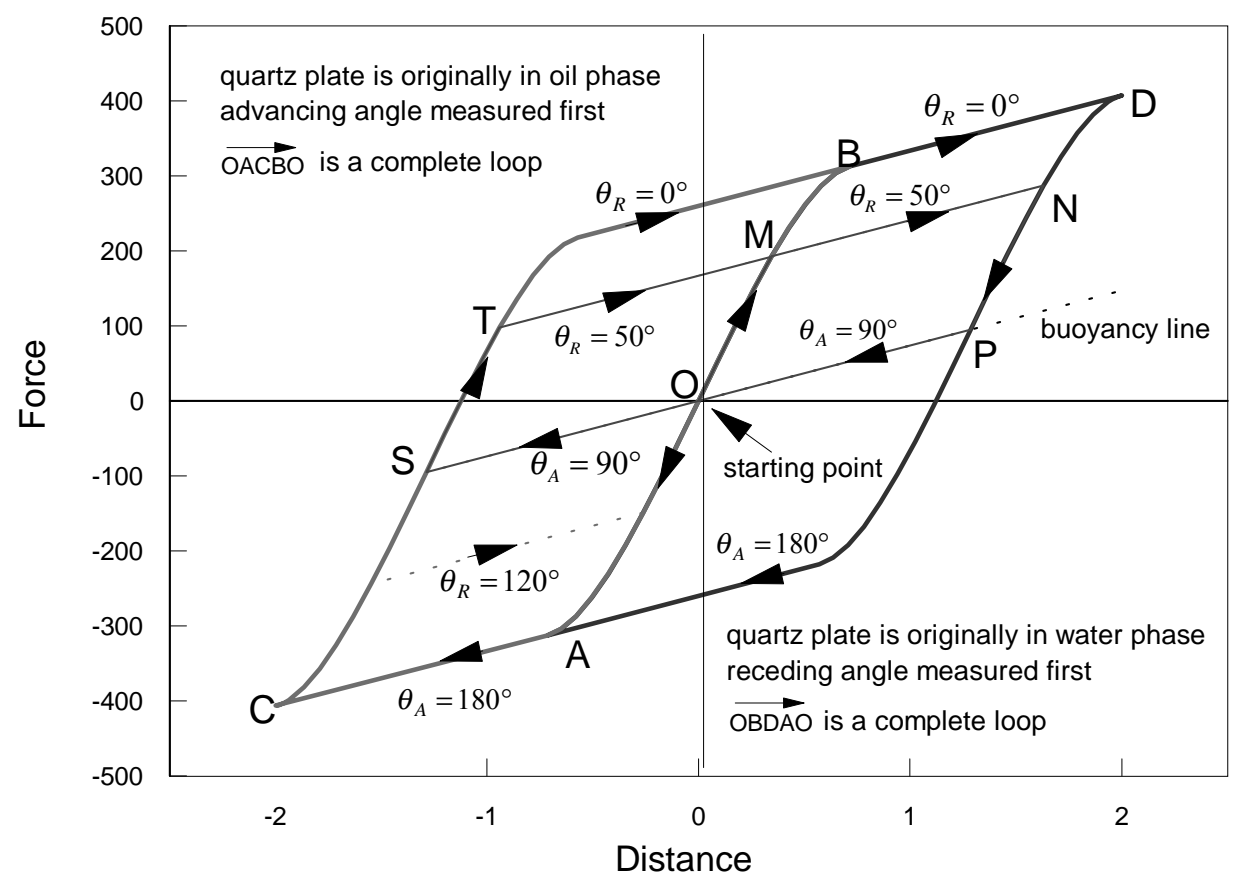

(a) Theoretical force-distance diagrams

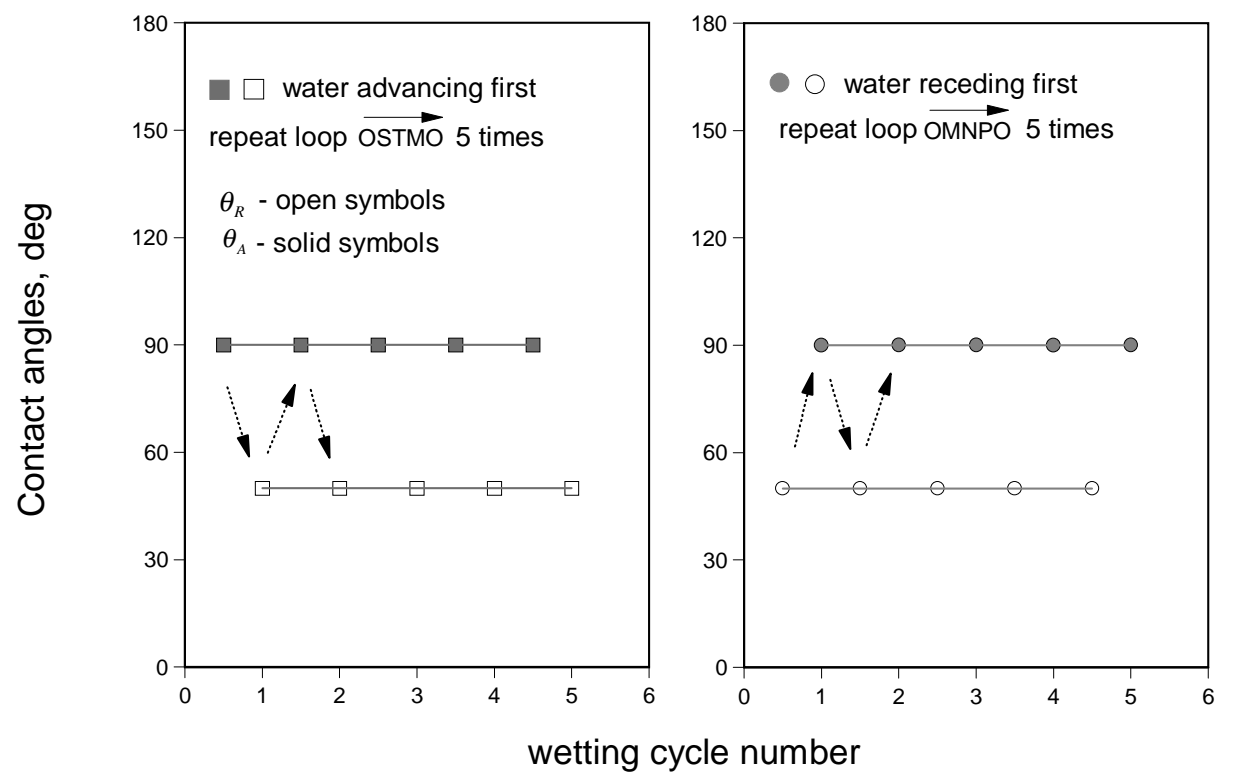

(b) Contact angles vs. wetting cycles

Figure II-2.11. Schematic force-distance diagrams and contact angles for multiple cycles. Advancing contact angles are indicated as solid symbols and receding angles as open symbols. Results for the plate that was initially in decane (loops around the path outlined by OSTMO) are indicated by square symbols; circles correspond to the plate that was initially in brine (loops around OMNPO). The first measured contact angle is indicated at the first half of a wetting cycle (receding for the plate that was initially in brine and advancing for the plate that was initially in decane). Consecutive advancing and receding angles follow the wetting cycle sequence. 
Figure II-2.11 illustrates the expected forms of a variety of force-distance loops calculated for the indicated advancing and receding contact angles. Upward travel of the plate is indicated by positive changes in distance; negative changes in distance correspond to downward movement, with zero defined as the position of the oil-water interface. Thus, wetting cycles bounded by OACBO would be measured for a plate initially immersed in the oil phase as it travels downwards through the oil-water interface into the water below under water-advancing conditions $\left(\theta_{\mathrm{A}}\right)$, then up again into the oil with water receding $\left(\theta_{\mathrm{R}}\right)$. The wetting cycles within the boundary defined by OBDAO apply to plates initially immersed in water. For the calculated force-distance loops shown in Fig. II-2.11(a), the following ideal conditions apply:

a) the segments of constant slope (e.g., OS and TM for a plate initially suspended in oil or $\mathrm{MN}$ and PO for an identical plate suspended initially in water) correspond to the change in buoyancy of the quartz plate as it passes from one phase to the other;

b) the three-phase line of contact is pinned during transitions between advancing and receding contact angles;

c) advancing and receding angles are constant and independent of position on the plate;

d) interfacial tension is constant.

Details of application of the Wilhelmy plate method are provided elsewhere (Mennella and Morrow, 1995; Xie, 1996; Xie and Morrow, 1998, 1999). An advantage of the plate method is that repeated wetting cycles can be measured under carefully controlled mechanical conditions of direction and velocity. Receding and advancing contact angles, averaged around the perimeter, can be measured over a substantial length of the plate. The wetting states presented by the adsorbed layer that result from passing through the interface from one phase to the other can be monitored for multiple wetting cycles.

In this study, primary, secondary, tertiary and subsequent receding and advancing contact angles were determined for a plate initially immersed in decane and for a duplicate plate initially immersed in brine. The method of presentation of contact angle data for such consecutive wetting cycles is shown in Fig. II-2.11(b) for the ideal conditions listed above for two hypothetical plates, each with $\theta_{\mathrm{R}}=50^{\circ}$ and $\theta_{\mathrm{A}}=90^{\circ}$.

\section{Experimental}

\section{Materials}

Clean quartz plates were used as the solid substrate. A quartz rod was fused at one end of each plate to facilitate attachment to the Wilhelmy plate micro-balance. The quartz plates were cleaned before use; perfect wetting by water against pure alkane was used as the criterion for cleanliness. The cleaning procedure (Mennella et al., 1995; Xie, 1996) is shown in Fig. II-2.12. 


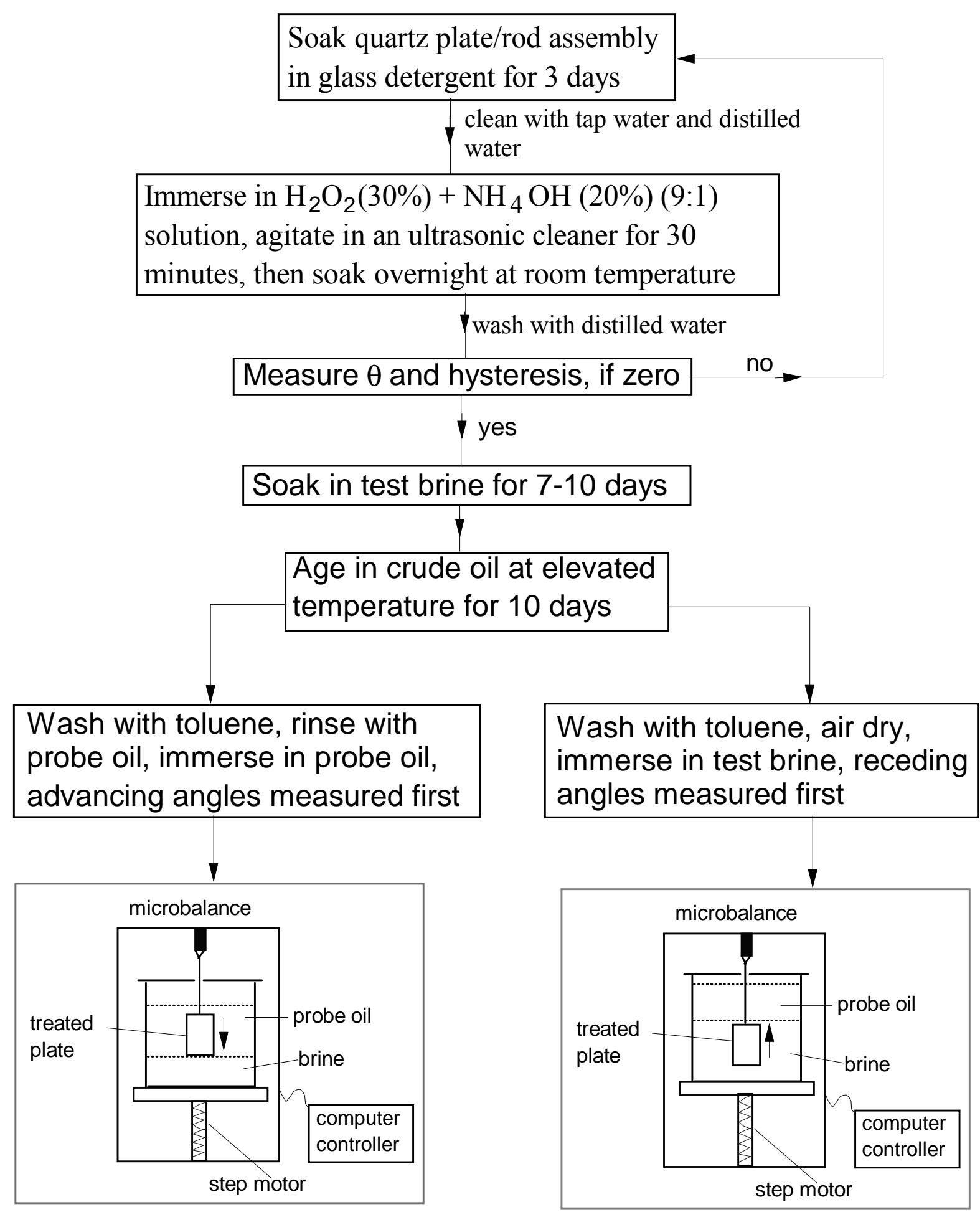

Figure II-2.12. Plate treatment and Wilhelmy plate measurement of advancing and receding contact angles.

Samples of ten different crude oils were used to treat quartz surfaces. The oils ranged in composition from asphaltic to paraffinic. The asphaltene contents and other properties of the 
crude oils including their refractive indices (RI) are listed in Table II-2.7. $\mathrm{P}_{\mathrm{RI}}$ is the RI at which n-heptane asphaltenes are first observed (Buckley et al., 1998a). As the difference between RI and $\mathrm{P}_{\mathrm{RI}}$ decreases, dramatic increases in contact angles were observed that can be attributed to surface precipitation of asphaltenes (Al-Maamari and Buckley, 2000). Note that none of the oil samples in the group used in this study is sufficiently close to the onset of precipitation for such interactions to influence this study. Surfaces were aged in crude oil for 10 days at temperatures between 74 and $80^{\circ} \mathrm{C}$, as shown in Table II-2.7. All the crude oils were filtered through Whatman \#541 filter paper to remove particles larger than about 20 to $25 \mu \mathrm{m}$ before use.

Table II-2.7. Selected physical properties of crude oil samples

\begin{tabular}{|l|c|c|c|c|c|c|c|c|c|}
\hline Crude oil & ${ }^{\circ} \mathrm{API}$ & $\begin{array}{c}\mu_{\mathrm{o}} @ \\
25^{\circ} \mathrm{C}, \\
\mathrm{cp}\end{array}$ & $\begin{array}{c}\mathrm{nC}_{7^{-}} \\
\mathrm{asph} \\
\mathrm{wt} \%\end{array}$ & $\begin{array}{c}\mathrm{RI} @ \\
20^{\circ} \mathrm{C}\end{array}$ & $\mathrm{P}_{\mathrm{RI}}$ & $\begin{array}{c}\text { Acid } \\
\#\end{array}$ & $\begin{array}{c}\text { Base } \\
\#\end{array}$ & $\begin{array}{c}\mathrm{B} / \mathrm{A} \\
{ }^{*}\end{array}$ & $\begin{array}{c}\text { Aging } \\
\mathrm{T}_{\mathrm{a}},{ }^{\circ} \mathrm{C}\end{array}$ \\
\hline A95 (Prudhoe Bay) & 25.2 & 33.8 & 8.67 & 1.5157 & 1.4498 & 0.24 & 2.20 & 9.2 & 80 \\
\hline CS & 25.1 & 61.4 & 0.78 & 1.5070 & $\mathrm{nd}^{*}$ & 0.33 & 1.16 & 3.5 & 75 \\
\hline DaGang & 27.8 & solid & 6.30 & 1.5051 & $\mathrm{~nm}^{*}$ & 0.66 & 4.67 & 7.1 & 75 \\
\hline Gullfaks-96 & 27.1 & 12.3 & 0.40 & 1.4930 & $\mathrm{nd}$ & 0.24 & 1.19 & 5.0 & 75 \\
\hline KC & 39.7 & 4.01 & $\mathrm{nd}$ & 1.4635 & $\mathrm{nd}$ & 0.13 & 0.92 & 7.1 & 74 \\
\hline Lost Hills & 22.6 & 70.0 & 2.78 & 1.5137 & 1.4231 & 1.90 & 6.05 & 3.2 & 75 \\
\hline Mars-Pink & 17.0 & 341.6 & 4.77 & 1.5382 & 1.4285 & 3.92 & 2.30 & 0.6 & 75 \\
\hline Minnelusa & 24.6 & 43.6 & 8.98 & 1.5142 & 1.4766 & 0.17 & 2.29 & $\begin{array}{c}13 . \\
5\end{array}$ & 74 \\
\hline P-E-00 & 36.1 & 7.5 & 0.04 & 1.4745 & $\mathrm{nd}$ & 0.11 & 1.50 & $\begin{array}{c}13 . \\
6\end{array}$ & 75 \\
\hline $\begin{array}{l}\text { Sulimar Queen } \\
\text { SQ-95) }\end{array}$ & 37.2 & 5.1 & 1.30 & 1.4769 & 1.4218 & 0.17 & 0.62 & 3.6 & 75 \\
\hline
\end{tabular}

* $\mathrm{B} / \mathrm{A}=$ base\#/acid $\# ; \mathrm{nd}=$ not detectable; $\mathrm{nm}=$ not measured

Contact angles were measured with normal decane as the probe oil. The rational for this approach was discussed in detail by Liu and Buckley (1997). Decane was purified by flow through silica and alumina gel columns before use. The interfacial tension between decane and the test brine $(0.1 \mathrm{M} \mathrm{NaCl}$ solution) is $48.31 \mathrm{mN} / \mathrm{m}$. The slowest available plate speed, 0.6 $\mathrm{mm} / \mathrm{min}$, was selected.

\section{Procedure}

The clean quartz plates were soaked in brine for about one week, then drained but not dried. The plates were submerged in crude oil for an aging time, $t_{\mathrm{a}}$, at an elevated temperature, $\mathrm{T}_{\mathrm{a}}$, which in most cases was $75^{\circ} \mathrm{C}$. After aging, excess crude oil was removed from the plate by rinsing with toluene. Choice of wash solvent relative to the solvency of the crude oil has been discussed in detail elsewhere (Liu and Buckley, 1997).

Comparisons were made between measurements for the plates initially immersed in brine or in the probe oil. Contact angles are reported with respect to the aqueous phase. To obtain 
receding angles first, the plates were dried, then immersed in the test brine; the probe oil was then carefully poured onto the brine. For initial measurement of water-advancing angles, after removing excess crude oil with toluene, plates were washed with the probe oil (decane) and never allowed to dry at any stage of preparation. The plate was then positioned in the decane layer (see Fig. II-2.12). The force-distance diagrams were measured at ambient conditions. Full details of the experimental procedure and data acquisition are available elsewhere (Xie, 1996).

\section{Results and Discussion}

Alteration of wettability by adsorption from crude oil was measured for ten crude oils. In some cases the force vs. distance plots followed nearly ideal paths with advancing and receding contact angles reproduced almost exactly in successive cycles, whereas others showed changes in contact angle from one cycle to the next and varying degrees of departure from the buoyancy line.

\section{Stable and near-stable wetting}

Results for A95 crude oil, shown in Fig. II-2.13, are close to ideal behavior. The experimental data match the buoyancy line and the hysteresis loops are reproduced from one cycle to the next. Advancing contact angles are virtually independent of the initial condition and the differences between the receding contact angles are minor. Thus, air-drying to remove toluene (plate first in water) and removal of toluene by washing with decane (plate first in decane) gave essentially the same results. The observed stable wetting behavior was unaffected by soaking one of the plates overnight in the brine phase before the fourth wetting cycle.

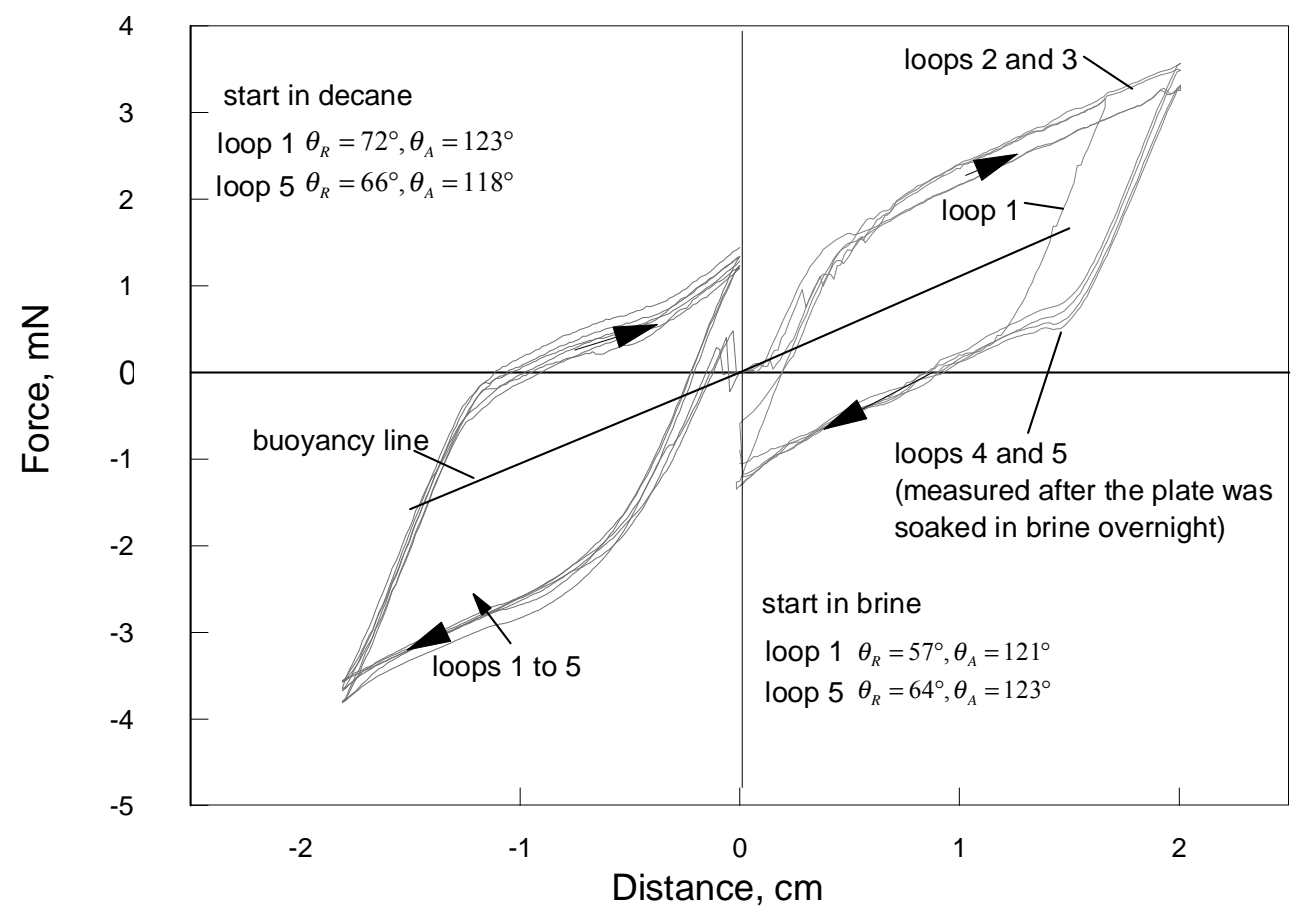

Figure II-2.13. Force-distance diagrams for plates prewetted in $0.1 \mathrm{M} \mathrm{NaCl}$ brine, then aged in $\mathrm{A95}$ crude oil for 10 days at $80^{\circ} \mathrm{C}$. 
Contact angles derived from the force-distance curves of Fig. II-2.13 for A95 crude oil are presented in Fig. II-2.14a. Advancing angles are almost identical for the two data sets and receding angles are close and converge. For the Lost Hills crude oil (Fig. II-2.14b), the contact angles decrease slightly with each wetting cycle, with the largest change being for advancing contact angles for the plate initially in brine. The contact angles were essentially stable for DaGang crude oil (Fig. II-2.14c). In some cases, the plate that began in brine had higher advancing angles than the one that began in decane (e.g., Lost Hills); in others it was the opposite (e.g., DaGang). No tendency for one treatment to produce consistently higher contact angles than the other could be seen, in agreement with previous studies by the captive drop technique (Buckley and Morrow, 1992). In wetting cycles for plates treated with Minnelusa crude oil starting either in brine or decane, both advancing and receding angles were very closely reproduced (Fig. II-2.14d). Comparing the first and fifth cycle results for plates that began in brine for all four oils included in Fig. II-2.14, variation in $\theta_{\mathrm{R}}$ was $7^{\circ}$ or less. Values of $\theta_{\mathrm{A}}$ agreed to within $4^{\circ}$. Considering the complexity of the crude oil/brine/rock interactions, the stability of hysteresis exhibited by these surfaces is remarkable.
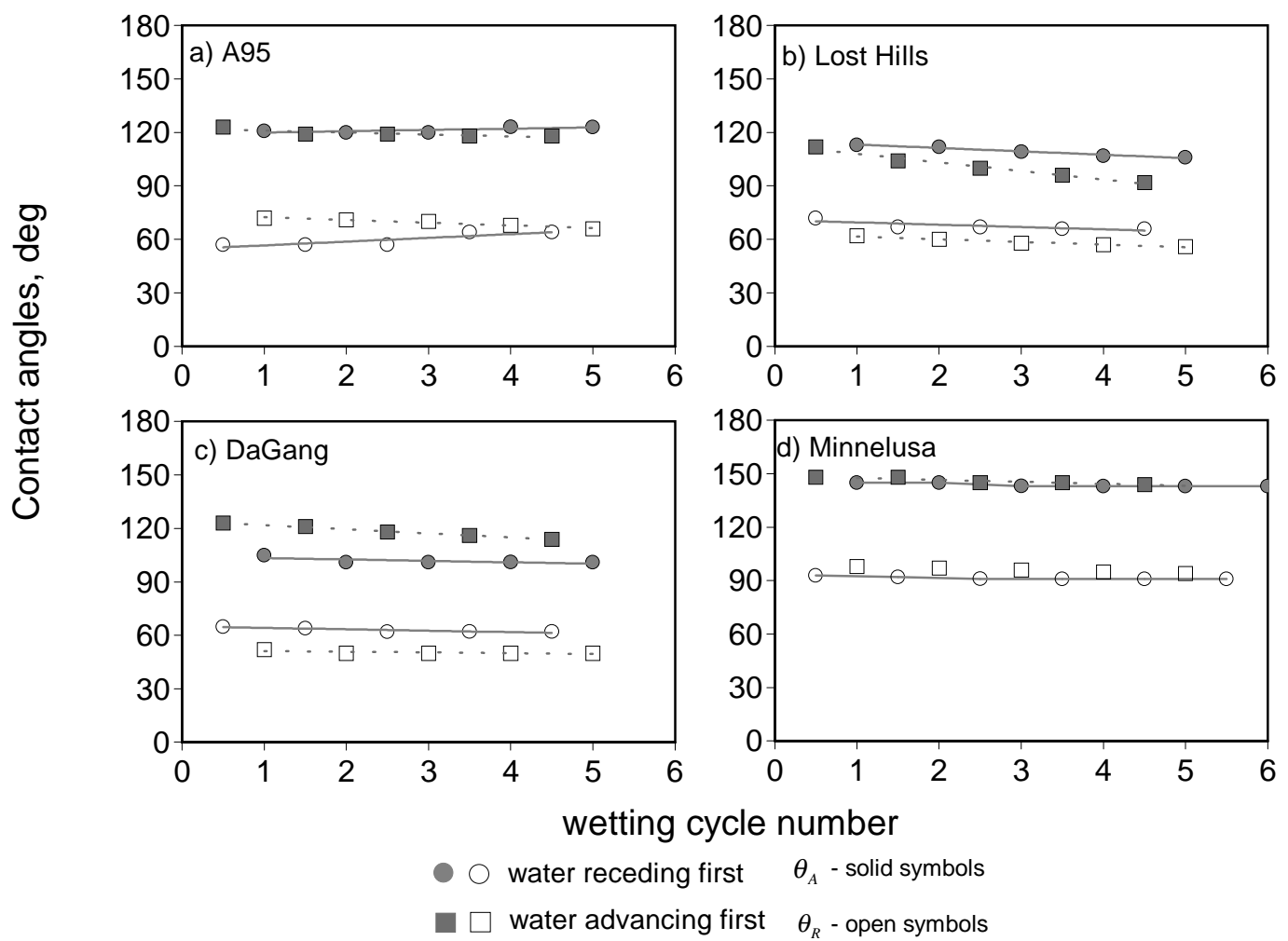

Figure II-2.14. Stable contact angles. Clean plates were prewetted with $0.1 \mathrm{M} \mathrm{NaCl}$ brine, then aged in the designated crude oils at $74-80^{\circ} \mathrm{C}$.

\section{Unstable wetting}

Unstable wetting was observed for six of the ten crude oils. Five of these had receding contact angles that were fairly constant, while advancing angles showed a systematic decrease with each cycle. An example set of force-distance relationships is shown in Fig. II-2.15 for plates treated with Sulimar Queen crude oil. Since only one of the angles is changing, interfacial 
tension can be assumed to remain constant. One oil, Gullfaks-96, showed a systematic decrease in both advancing and receding angles (see Fig. II-2.16).

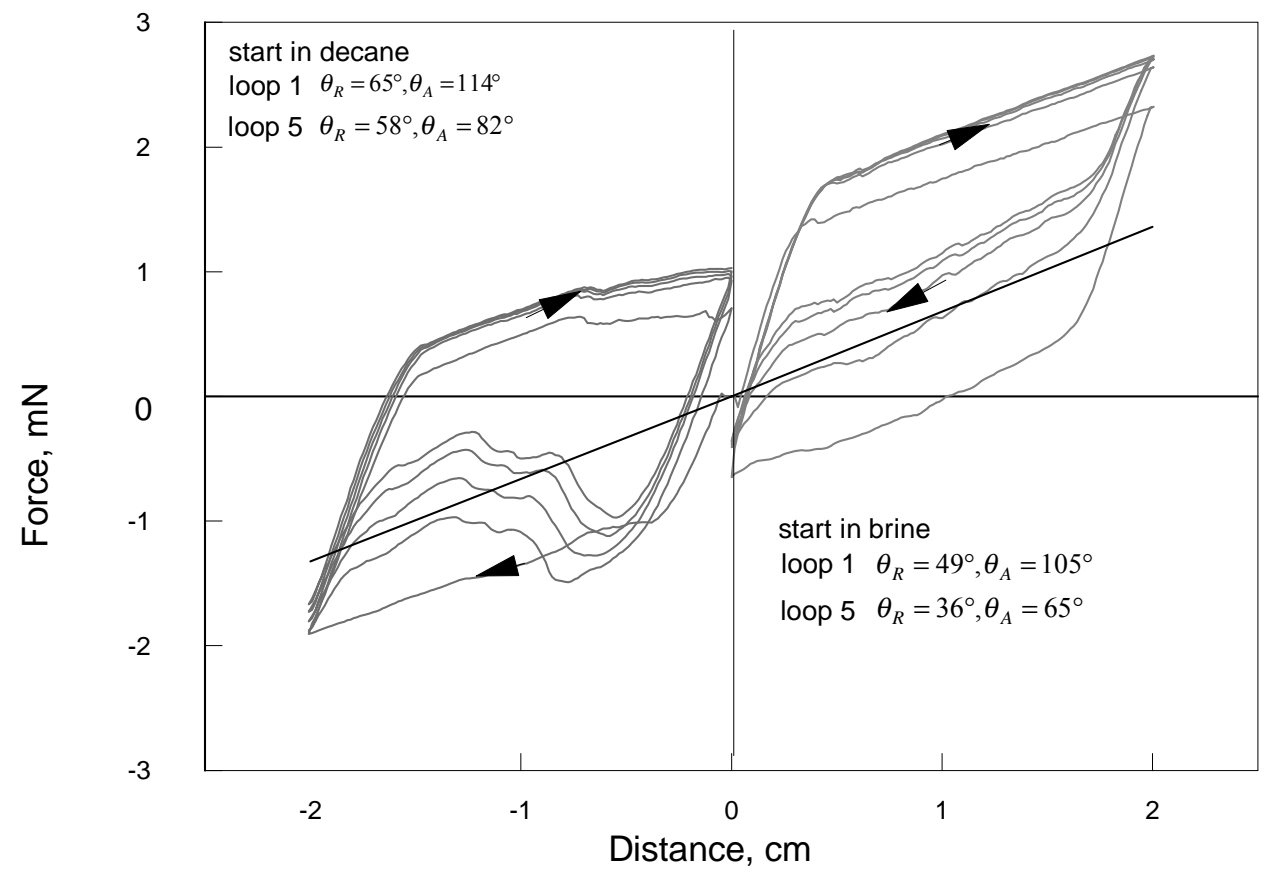

Figure II-2.15. Example of wetting loops with advancing contact angles that decrease with each measurement cycle. Clean plates were prewetted with $0.1 \mathrm{M}$ NaCl brine, then aged in SQ-95 crude oil for 10 days at $75^{\circ} \mathrm{C}$.

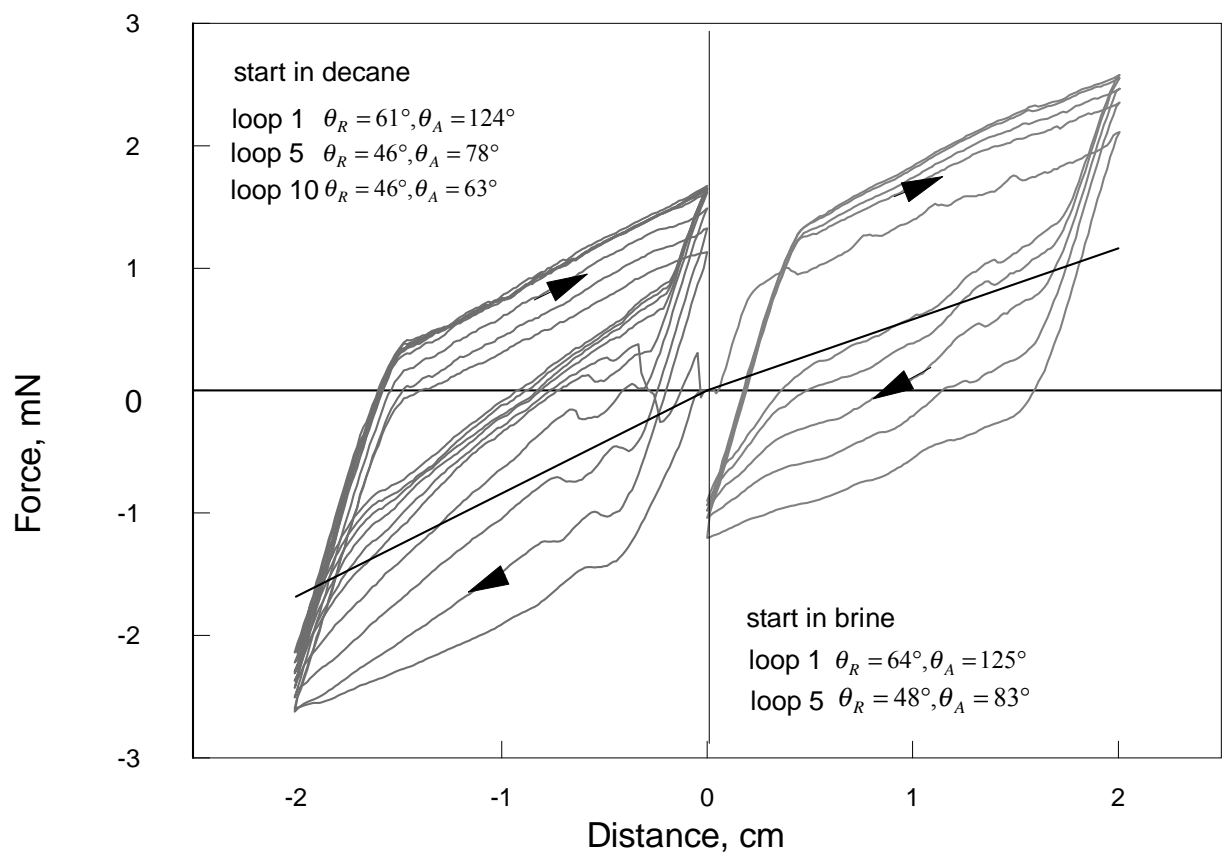

Figure II-2.16. Example of wetting loops with unstable receding and advancing contact angles. Clean plates were prewetted with $0.1 \mathrm{M} \mathrm{NaCl}$ brine, then aged in Gullfaks-96 crude oil for 10 days at $75^{\circ} \mathrm{C}$. 
Data for all the cases where wetting was judged to be unstable to some extent are summarized in Fig. II-2.17. The larger differences between advancing-first and receding-first results tend to correspond to data similar to that shown for Sulimar Queen (Fig. II-2.15), where there were deviations from linearity and from the expected buoyancy line. There was uncertainty in the contact angles, on the order of $\pm 2-5^{\circ}$, calculated for the plate treated with Sulimar Queen, starting in decane, and for both plates treated with KC and Mars-Pink crude oils, because only parts of the loops matched the calculated buoyancy lines. Mars-Pink (Fig. II-2.17b) is included with the unstable cases, but it is different from some of the other oils in that it shows a significant drop in advancing angle after the first cycle and little change thereafter.

A higher uncertainty $\left( \pm 3-10^{\circ}\right)$ is associated with the Gullfaks results. These loops have linear portions that do not match the buoyancy line. Nevertheless, the trends toward increasingly water-wet advancing angles were clear. This may result from the length of time that the surface is immersed in either the brine or the decane, or the number of transitions through the interface. As a test of the effect of soaking in brine, interrupted cycles were measured for plates treated with Gullfaks 96 and immersed first in brine. The estimated contact angles shown in Fig. II-2.18 for plates that were allowed to soak in brine for two to five hours between selected cycles are very similar to those for continuous measurement taken from Fig. II-2.17f. Thus, it appears that passage of the surface through the three-phase-line of contact is the important factor in the transition to water wetness.

\section{Contact angle hysteresis and crude oil properties}

Chemical properties of the crude oils used in this study are summarized in Table II-2.7. As shown in Fig. II-2.19, the oil samples fall into two groups: one group with n-heptane asphaltene contents higher than $2.8 \%$ and another with asphaltene amounts from zero to $1.3 \%$. Four of the five oils in the higher asphaltene group gave contact angles that were stable through repeated wetting cycles (Fig. II-2.14). All of the oils in the group with lower amounts of asphaltene produced surfaces with unstable wetting (Fig. II-2.17).

To the extent that asphaltenes represent some of the most polar components in crude oils, it is to be expected that oils with significant amounts of asphaltenes will, in general, have greater potential for interactions with high-energy surfaces. The exception, Mars-Pink, illustrates another important factor, however. Because the brine is a solution of monovalent salts, it is the oils with higher base numbers and lower acid numbers (high $\mathrm{B} / \mathrm{A}$ ratio) that interact most strongly with the negatively charged quartz surfaces. With another brine, the results could be quite different. The presence of asphaltenes and other polar crude oil components may be regarded as a necessary but not a sufficient condition for stable wetting alteration and highhysteresis surfaces. 

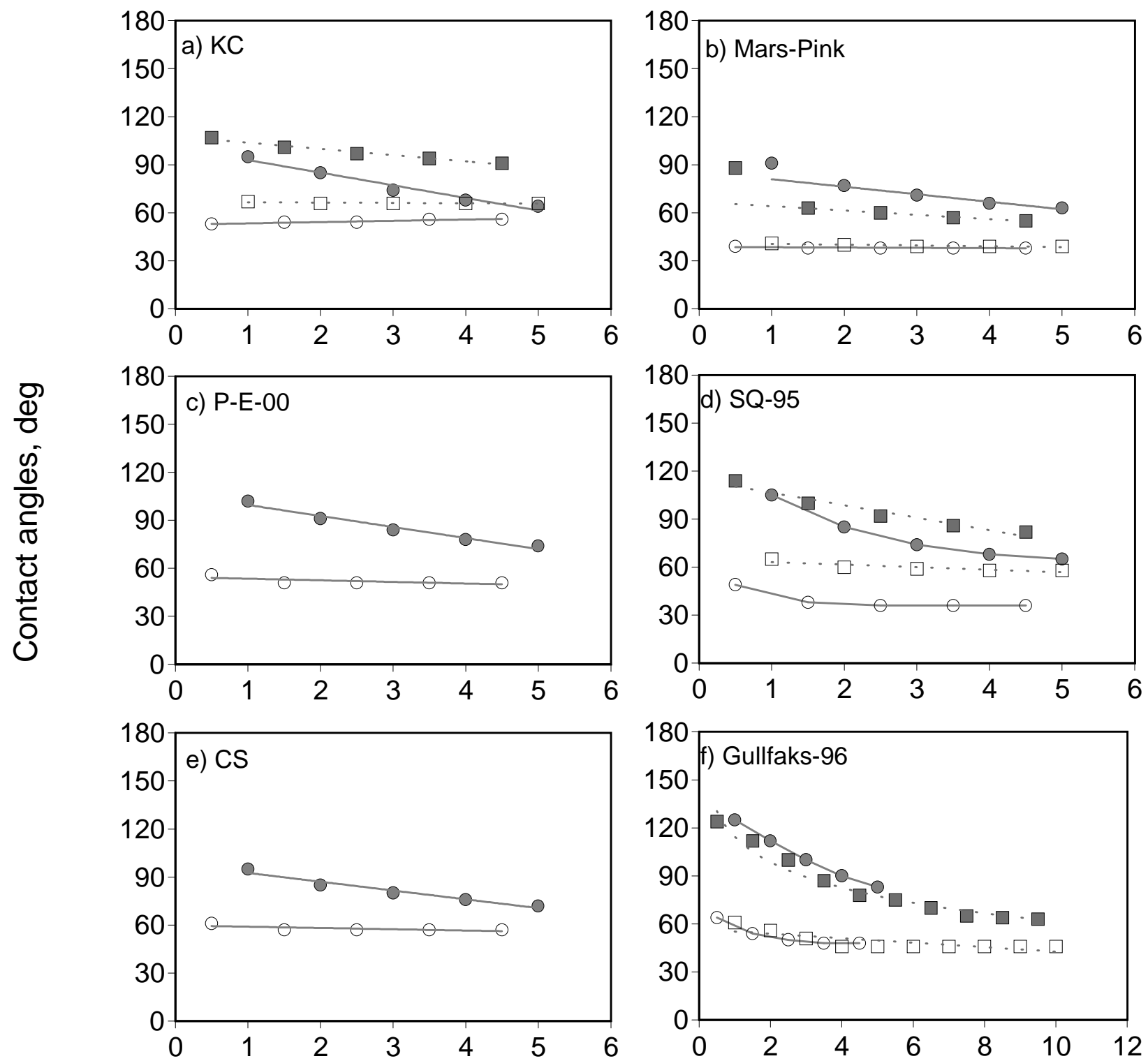

wetting cycle number

$\bigcirc$ water receding first $\quad \theta_{A}$ - solid symbols
$\square$ water advancing first $\quad \theta_{R}$ - open symbols

Figure II-2.17. Contact angles that change with wetting cycles. Clean plates were prewetted with $0.1 \mathrm{M}$ NaCl brine, then aged in designated crude oil at $74-80^{\circ} \mathrm{C}$ for 10 days. 


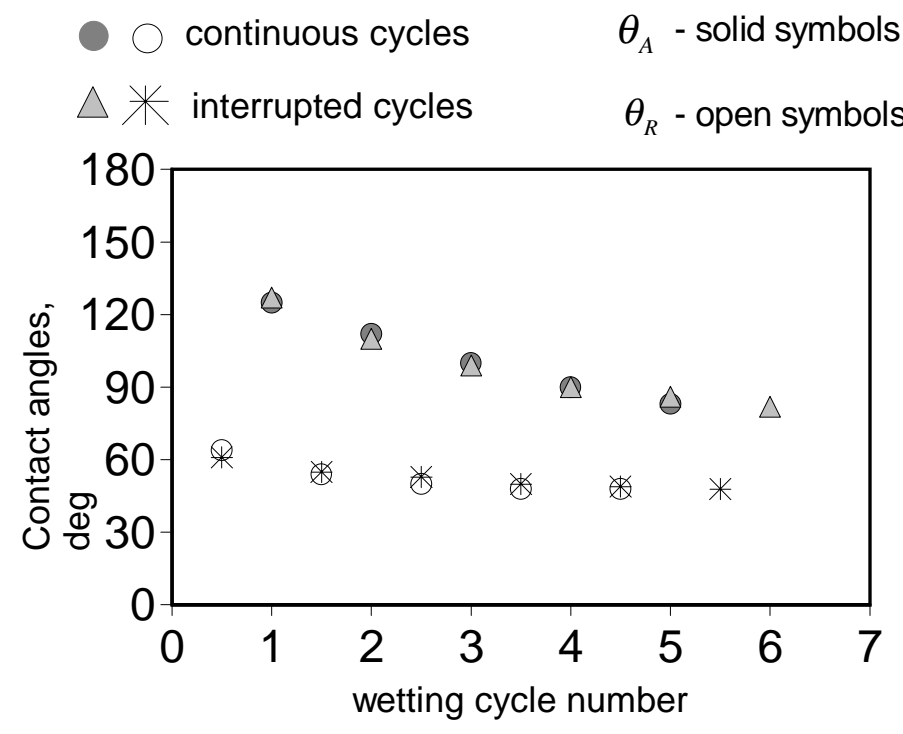

Figure II-2.18. Contact angles on quartz plates treated with $0.1 \mathrm{M} \mathrm{NaCl}$ and Gullfaks-96 for 10 days at $75^{\circ} \mathrm{C}$. Similar results were obtained whether cycles were measured continuously or whether they were interrupted by periods during which the plates were soaked in brine (two hours after the first cycle, five hours after the third cycle).

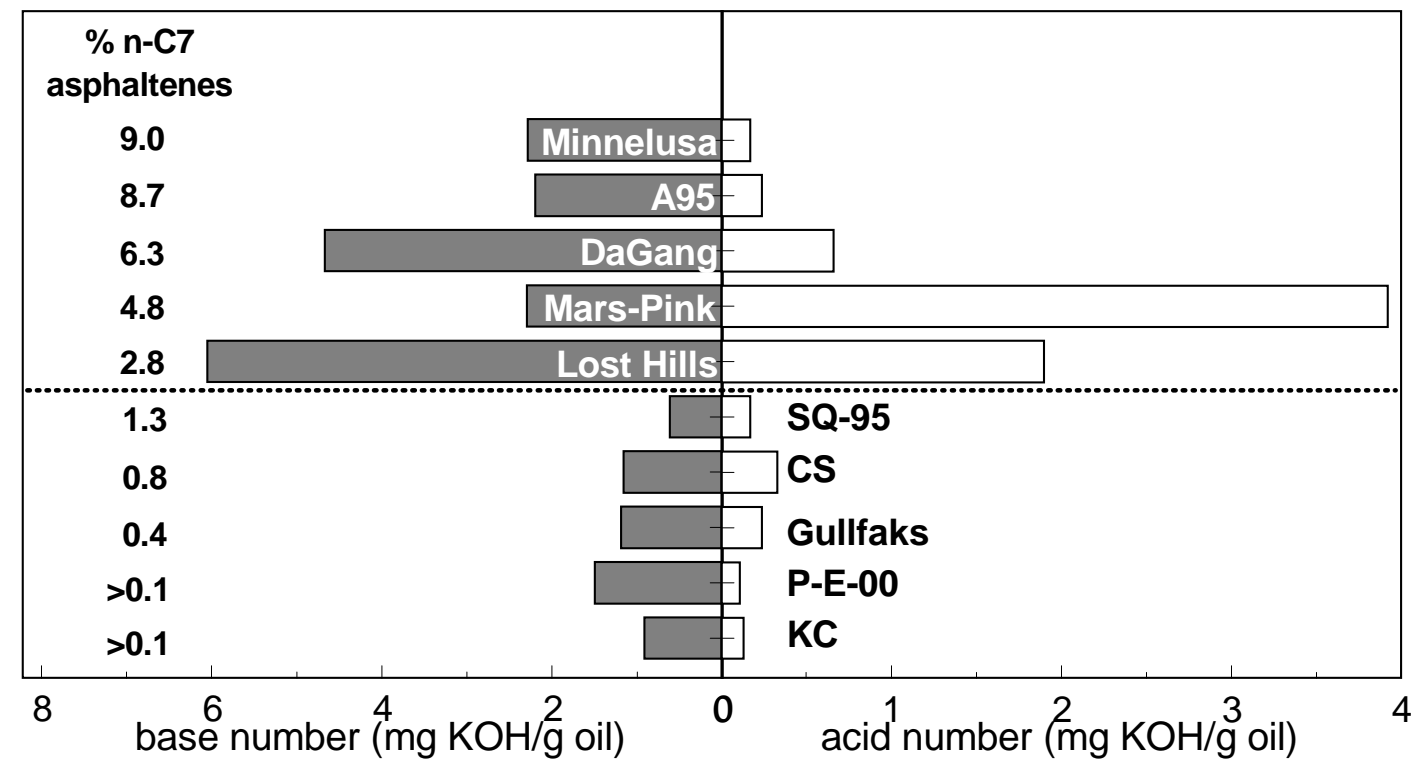

Figure II-2.19. Acid and base numbers of crude oils, sorted in order of decreasing n-heptane asphaltene content. Note that the scales for acid and base number are different. Oil properties fall into two groups. Above the dashed line, asphaltene content is greater than $2 \%$ and amounts of either acids or bases or both are high. Below the line, amounts of asphaltene are less than $1.5 \%$ and amounts of acids and bases are lower. 
It is likely that the use of temperatures greater than $75^{\circ} \mathrm{C}$ for aging the quartz plates in oil, followed by rinsing with room temperature with toluene, contributes to the initially high wateradvancing angles on many of the surfaces treated with the less asphaltic oils. Typical wax appearance temperatures (WAT) of stock tank oils are in the range of $30-50^{\circ} \mathrm{C}$ (e.g., MongerMcClure, et al., 1999), so in this procedure, waxes would first be melted, then solidified. Previous work with Sulimar Queen, a waxy oil (Buckley et al., 1997b) showed that wetting was altered to a greater extent if the temperature was initially maintained above the WAT, then allowed to fall below it than for surfaces aged at either the higher or lower temperature. DaGang is a waxy crude as well, as evidenced by the fact that at room temperature it is below its pour point. Other oils in this study for which waxes can be observed microscopically at room temperature include CS, Gullfaks, KC, and P-E-00. Thus all of the lower asphaltene oils that give unstable wetting are below their wax appearance temperature at room conditions. A possible explanation for the unstable conditions is an initial deposition as the temperature drops, followed by removal of the wax, which is not strongly adsorbed, during successive wetting cycles.

In a reservoir, wetting changes due to adsorption of polar components would be enhanced by the long exposure times, high temperatures, and presence of multivalent ions in the connate brine. Wax deposition might also affect wetting in near-well regions where temperature gradients are introduced, for example, by injection of cold fluids or cooling of injected steam. The links between crude oil composition and stability of wetting alteration support earlier observations about the roles of acidic and basic components. Measurements of contact angles over repeated wetting cycles may even provide a new diagnostic tool for distinguishing between wetting conditions as they are likely to exist in the reservoir at discovery and alteration in wetting that can be anticipated during specific stages of the production history of an oil reservoir.

In reservoirs with asphaltic oils, pronounced hysteresis between water-receding and water-advancing contact angles can be expected. This contact angle hysteresis contributes to the hysteresis in constitutive properties (capillary pressure and relative permeability). The physical explanation for persistent hysteresis of this magnitude remains to be explored.

\section{Conclusions}

1) Hysteresis between water-advancing and water-receding conditions existed for quartz surfaces exposed to brine and ten different crude oils, as measured by the dynamic Wilhelmy plate technique. In most cases the receding contact angles were found to be stable during repeated wetting cycles with decane and brine probe fluids. Differences between the surfaces of quartz plates that were dried and immersed in water at the start of the measurement and those that were not dried and began in decane were minor.

2) During the first measurement cycle, advancing contact angles ranged from intermediate to fairly oil-wet. In subsequent cycles, hysteresis was stable on some of the oil-treated surfaces and unstable on others. In the unstable cases, surfaces appeared to be more water-wet in subsequent measurements. 
3) In general, the advancing angles on quartz surfaces treated with more asphaltic crude oils were more stable, whereas those treated with low-asphaltene content oils were less stable.

4) Stable wetting appeared to be related to adsorption of positively charged oil species. MarsPink, an asphaltic oil with a high acid number compared to its base number, did not produce stable wetting. All other asphaltic oils that had high base numbers and base-to-acid ratios produced stable wetting conditions with advancing angles that were 40 to $70^{\circ}$ higher than the corresponding receding angles. Such wetting conditions have significant implications for oil recovery processes.

5) Oils that are above their wax appearance temperature during the aging step and below it during measurement may deposit wax that is removed by successive measurement cycles.

\section{Nomenclature}

A - acid number (mg KOH/g oil)

$\mathrm{B}$ - base number (mg KOH/g oil)

$F$ - the force acting on the plate, $\mathrm{mN}$

$F_{B}$ - the buoyancy force, $\mathrm{mN}$

$p$ - the perimeter of the plate/liquid contact line, $\mathrm{m}$

$\mathrm{P}_{\mathrm{RI}}$ - refractive index at the onset of asphaltene precipitation

RI - refractive index

WAT - wax appearance temperature

$\sigma_{o s}$ - the oil $/$ solid interfacial tension, $\mathrm{mN} / \mathrm{m}$

$\sigma_{w s}$ - the water/solid interfacial tension, $\mathrm{mN} / \mathrm{m}$

$\sigma_{o w}$ - the oil $/$ water interfacial tension, $\mathrm{mN} / \mathrm{m}$

$\theta_{A}$ - water advancing contact angle, ${ }^{\circ}$

$\theta_{R}$ - water receding contact angle, ${ }^{\circ}$

\section{References}

Akhlaq, M.S., Kessel, D. and Dornow, W., 1996. Separation and characterization of wetting crude oil compounds. J. Colloid Interface Sci., 180: 309-314.

Al-Maamari, R.S.H. and Buckley, J.S., 2000. Asphaltene precipitation and alteration of wetting: can wettability change during oil production? SPE 59292, 2000 SPE/DOE IOR Symp., Tulsa, 3-5 April.

Buckley, J.S., Liu, Y., Xie, X. and Morrow, N.R., 1997. Asphaltene and crude oil wetting - the effect of oil composition. SPEJ, June: 107-119.

Buckley, J.S., Hirasaki, G.J., Liu, Y., Von Drasek, S., Wang, J.X., and Gill, B.S., 1998a. Asphaltene precipitation and solvent properties of crude oils. Petr. Sci. and Technol. 16(3\&4): 251-285.

Buckley, J.S., Liu, Y. and Monsterleet, S., 1998b. Mechanisms of wetting alteration by crude oils. SPEJ (March): 54-61. 
Buckley, J.S. and Morrow, N.R., 1992. An overview of crude oil adhesion phenomena. In: Toulhoat, H. and Lecourtier, J, Eds., Physical Chemistry of Colloids and Interfaces in Oil Production. Éditions Technip, Paris, 39-45.

Buckley, J.S., Wang, J.X., Wu, Y.D., and Thomas, C.P., 1997b. Wettability and waterflooding of the Sulimar Queen Reservoir. Presented to the International Energy Agency, Copenhagen, September.

Israelachvili, J.N., 1991. Intermolecular and Surface Forces, 2nd Ed. Academic Press, San Diego

Liu, L. and Buckley, J.S., 2000. Alteration of wetting of mica surfaces. J. Pet. Sci. Eng., 24: 7583.

Liu, Y. and Buckley, J.S., 1997. Evolution of wetting alteration by adsorption from crude oil. SPE Formation Evaluation (March): 5-11.

Mennella, A. and Morrow, N.R., 1995. Point-by-point method of determining contact angles from dynamic Wilhelmy plate data for oil/brine/solid systems. J. Colloid and Interface Sci., 172: 48-55.

Mennella, A., Morrow, N.R. and Xie, X., 1995. Application of the dynamic Wilhelmy plate to identification of slippage at a liquid/liquid/solid three-phase line of contact. J. Petr. Sci. and Eng., 13: 179-192.

Monger-McClure, T.G., Tackett, J.E., and Merrill, L.S., 1999. Comparisons of cloud point measurement and paraffin prediction methods. SPE Production and Facilities, 14: 4-16.

Morrow, N.R., 1975. The effects of surface roughness on contact angle with special reference to petroleum recovery. J. Can. Pet. Tech. (Oct.-Dec.): 1-12.

Morrow N.R., Tang G., Valat M. and Xie X., 1998. Prospects of improved oil recovery related to wettability and brine composition. J. Pet. Sci. Eng. 20(June): 267-276.

Robin, M. and Koci, X., 1996. Wettability heterogeneities on planar minerals: application to reservoir rocks. Proceedings of the 3rd International Symposium on Reservoir Wettability and Its Effect on Oil Recovery, N.R. Morrow (Ed), University of Wyoming, Laramie, WY: 47-53.

Rose, W. and Heins, R.W., 1962. Moving interfaces and contact angle rate-dependency. J. Colloid Sci., 17: 39-48.

Teeters, D.C., Wilson, J.F., Andersen, M.A. and Thomas, D.C., 1988. A dynamic Wilhelmy plate technique used for wettability evaluation of crude oils. J. Colloid and Interface Sci., 126(2): 641-644.

Xie, X., 1996. Application of the dynamic Wilhelmy plate technique to investigation of oil/brine/quartz wetting alteration by adsorption from crude oil. Ph.D thesis, Laramie, University of Wyoming.

Xie, X. and Morrow, N.R., 1998. Wetting of quartz by oleic/aqueous liquids and adsorption from crude oil. Colloids and Surfaces, A: Physicochemical and Engineering Aspects, 138: 97-108.

Xie, X., Morrow, N.R., 1999. Contact angles on quartz induced by adsorption of heteropolar hydrocarbons. J. Adhesion Sci. Technol., 13(10): 1119-1135.

Yang, S.Y., Hirasaki, G.J., Basu, S. and Vaidya, R., 1999. Mechanisms for contact angle hysteresis and advancing contact angles. J. Pet. Sci. Eng. 24(December): 63-73. 
Zisman, W.A., 1964. Relation of the equilibrium contact angle to liquid and solid constitution. Chapter 1 in: Contact Angle, Wettability, and Adhesion, Adv. in Chem. Ser. 43, F.M. Fowkes, Symposium Chairman, Washington, D.C., ACS: 1-51. 


\section{II-3. Atomic Force Microscopy of Oil-Treated Surfaces}

\section{II-3.1. A Methodology for Examining Crude Oil-Treated Surfaces with the Atomic Force Microscope}

Lord, D.L. and Buckley, J.S.: "An AFM Study of Morphological Features that Affect

Wetting at Crude Oil-Water-Mica Interfaces," Colloids and Surfaces A (2002) 206, 531-546.

\section{Background}

This report describes methods by which the atomic force microscope (AFM) was applied to the study of surface interactions in crude oil systems. One of the many engineering challenges encountered during the production and distribution of crude oils is the accumulation of asphaltic and waxy solids on reservoir rocks and production equipment. In the reservoir, accumulation of organic material on rock surfaces changes the effective wettability of the rock, affecting fluid displacement processes. Outside the reservoir, accumulation of these materials can lead ultimately to losses in production efficiency and potentially high costs for restoration. An understanding of the basic mechanisms driving the accumulation of these materials onto solid surfaces is necessary in order to effectively combat their deleterious effects on crude oil production.

The AFM is a laboratory instrument capable of resolving micro- and nanoscale surface features in air and liquid environments. When properly configured, the AFM has the potential to observe closely the processes of adsorption and deposition on solid surfaces, potentially a useful tool for understanding the fundamental steps leading to asphaltene and wax deposition and plugging. Challenges in implementing AFM analysis are numerous, however. Crude oil systems are chemically complex and dynamic, making it difficult to isolate cause-effect relationships. Also, the AFM is a challenging instrument to master from an operational standpoint. Moreover, there is currently no standard methodology for AFM analysis of crude oil systems. Thus, in order for the value of AFM to be realized as an analytical tool in the study of crude oil surface interactions, method development and demonstration of its utility in model systems is an essential first step. The objective of this report is therefore to outline the recent advances in an ongoing effort by the authors to apply AFM to help understand the mechanisms controlling adsorption and deposition processes in crude oil systems.

\section{Scanning Probe Microscopy}

AFM is a scanning technique which falls under a more general classification of surface analyses called scanning probe microscopy (SPM), in which a sharp probe is raster scanned across a sample surface while tip-sample interactions are monitored. For most applications, SPM generates an approximate topographic image of very small areas, ranging from as small as $10 \mathrm{~nm} \times 10 \mathrm{~nm}$ up to $100 \mu \mathrm{m} \times 100 \mu \mathrm{m}$. Typical lateral resolution of a commercial SPM is about $2 \mathrm{~nm}$, and depth of focus is about $100 \mathrm{~nm}$. The two other most commonly-used instruments for examining surface morphology, the optical microscope $(\mathrm{OM})$ and the scanning electron microscope (SEM), have a maximum resolution of about $1 \mu \mathrm{m}$ (Campbell, 1993) and 5 $\mathrm{nm}$, respectively. While SEM resolution is comparable to that of SPM, samples must typically be freeze-dried, coated, and imaged in a vacuum. Environmental SEM allows imaging without 
the requirement for a high vacuum, but the environment must be a vapor, not a liquid. Most SPM applications, however, allow for imaging under conditions much closer to the native sample environment. This is particularly useful in applications to crude oil systems because surface morphology is expected to be a function of the properties of the fluid contacting the surface. AFM can be operated in gaseous, aqueous, and oleic media, allowing for a more representative picture of the surface under reservoir conditions than does a freeze-dried sample under vacuum. Changes in either the aqueous or oleic media can influence their interactions with the materials adsorbed on the surface. For example, some crude oil components that are insoluble in decane are soluble in toluene, so addition of toluene to a decane imaging medium can differentiate imaged features on the basis of their solubility.

\section{Prior Applications of AFM to Crude Oil Systems}

Current literature contains only a few studies in which AFM was utilized to analyze surfaces exposed to crude oils. The first record of direct imaging of oil-derived components with the AFM was given by Toulhoat et al. (1994). Asphaltenes isolated from crude oils were adsorbed from toluene solutions onto dry mica and imaged under air. Images were captured in contact mode (CM-AFM) using $\mathrm{Si}_{3} \mathrm{~N}_{4}$ cantilevers with an approximate spring constant of $k=$ $0.12 \mathrm{~N} / \mathrm{m}$. Scans ranging from $8 \mathrm{~nm} \times 8 \mathrm{~nm}$ to $150 \mu \mathrm{m} \times 150 \mu \mathrm{m}$ were reported. Some nanoscale features were resolved in the $8 \mathrm{~nm}$ scans, hypothesized by the authors to represent condensed and peri-substituted aromatic rings on adsorbed asphaltenes, although identification of features at this scale is highly uncertain. Scans at the largest scale revealed surface aggregates which had the rough appearance of isolated snowflakes, with the size of aggregates increasing with immersion times ranging from 30 seconds to 180 seconds. While some aggregates had lateral dimensions approaching $15 \mu \mathrm{m}$, their heights were limited to $20 \mathrm{~nm}$ or so.

Buckley et al. (1997) employed the AFM to measure the height of features on dry and wet glass surfaces resulting from exposure to crude oil. Scans were obtained under air using the tapping mode (TM-AFM) technique. The authors reported an "apparent height" parameter which measured the maximum difference between highest and lowest points on a selected $1 \mu^{2}$ area on a given sample. AFM images were not shown in the manuscript. Changes in treatment schemes resulted in increases in feature height on the glass. For example, the apparent height on clean, dry glass was about $4 \mathrm{~nm}$. Exposing the dry glass to crude oil and rinsing with toluene to remove the bulk oil caused adsorption of material which had an apparent height of $60 \mathrm{~nm}$. Soaking the glass with brine for one week prior to exposing it to oil increased the apparent height of features to $80 \mathrm{~nm}$. Adding an asphaltene precipitant to the crude oil prior to aging the glass resulted in a three-fold increase in apparent height to $250 \mathrm{~nm}$. While this preliminary study confirmed that the AFM was capable of detecting surface features likely important to wettability changes on glass surfaces exposed to oil, the resolution of the scans did not allow for any mechanistic explanation.

Yang et al. (1999) employed the AFM to investigate surface features on mica resulting from controlling aging time in crude oil, brine $\mathrm{pH}$, presence or absence of brine, and solvent quality of crude for its asphaltenes. Scanning was conducted in CM-AFM under air and surface roughness was used to distinguish between adsorbed material and clean mica. Distinctions in surface features were evident for changes in aging time from 1 to 7 days in oil, with more complete surface coverage after longer time. Also evident was the effect of raising the $\mathrm{pH}$ of the 
brine above the wetting transition $\mathrm{pH}$ for the particular oil/brine/mica system. Whereas the system which promoted adhesion of an oil drop to the mica showed conspicuous surface features and significant coverage, the system which preserved the original hydrophilic state was characterized by relatively clean mica.

The most recently published crude oil AFM study (Ese et al., 2000) examined LangmuirBlodgett films of asphaltenes and resins deposited on mica. Scans were obtained using TMAFM in air. Varying the proportion of asphaltene to resin content resulted in changes in film structure. The authors reported a more "open" film structure with increasing resin fraction. Also, larger individual structural units were observed when both asphaltene and resins were present as opposed to pure films of either.

The only other previous AFM applications to the study of oil/brine/solid interactions have focused on force measurements in air rather than producing surface images (e.g., Basu and Sharma, 1999).

The four AFM imaging studies described above are summarized in Table II-3.1. In all cases, the surfaces were imaged under air. While scanning in air is a logical starting point for method development, it does not represent the likely configuration of fluids in an oil reservoir where water and oil are both present and preferentially contact the solid surfaces over the gas phase. Choice of substrate was mica in three cases and glass in one. Mica has the advantage over glass in AFM work in that it provides a molecularly smooth, reproducible template. TMAFM was used in two cases, primarily because it was reported in manufacturer literature to impose lower lateral imaging forces than CM-AFM, an important consideration when imaging soft organic coatings. Several examples of successful applications of CM-AFM to soft samples are available in the literature (Manne et al., 1994; Manne and Gaub, 1995), so any advantages of TM-AFM over CM-AFM are currently unclear for this type of work. Probe selection is guided largely by the scanning mode and imaging medium, and selection criteria are discussed in detail in the section on AFM Probes. In general, TM-AFM in air requires a stiff Si cantilever, while CM-AFM requires a more pliable material like $\mathrm{Si}_{3} \mathrm{~N}_{4}$. Probe choices in Table II-3.1. reflect these general requirements.

Table II-3.1. Summary of published AFM studies of crude oil systems.

\begin{tabular}{|c|c|c|c|c|c|}
\hline Study & Oil & Substrate & $\begin{array}{l}\text { Imaging } \\
\text { Medium }\end{array}$ & $\begin{array}{l}\text { Scanning } \\
\text { Mode }\end{array}$ & Probe \\
\hline $\begin{array}{c}\text { Toulhoat et al. } \\
\text { (1994) }\end{array}$ & $\begin{array}{l}\text { Asphaltenes in toluene } \\
(0.15-10 \mathrm{mg} / \mathrm{L})\end{array}$ & Mica & Air & $\mathrm{CM}^{-\mathrm{AFM}^{\mathrm{a}}}$ & $\begin{array}{c}\mathrm{Si}_{3} \mathrm{~N}_{4} \\
k \cong 0.12 \mathrm{~N} / \mathrm{m}\end{array}$ \\
\hline $\begin{array}{c}\text { Buckley et al. } \\
\text { (1997) }\end{array}$ & $\begin{array}{c}\text { Crude } \\
\text { Crude }+ \text { isooctane } \\
\end{array}$ & Glass & Air & TM-AFM $^{b}$ & $\mathrm{Si}$ \\
\hline $\begin{array}{l}\text { Yang et al. } \\
\text { (1999) }\end{array}$ & $\begin{array}{c}\text { Crude } \\
\text { Crude + heptane }\end{array}$ & Mica & Air & CM-AFM & $\begin{array}{c}\mathrm{Si}_{3} \mathrm{~N}_{4} \\
k \cong 0.04 \mathrm{~N} / \mathrm{m}\end{array}$ \\
\hline $\begin{array}{l}\text { Ese } \text { et al. } \\
\quad(2000)\end{array}$ & $\begin{array}{l}\text { Resin }+ \text { asphaltene, L-B } \\
\text { deposition from water }\end{array}$ & Mica & Air & TM-AFM & $\mathrm{Si}$ \\
\hline
\end{tabular}




\section{Equipment and Materials}

\section{Atomic Force Microscope}

The microscope used for this work was a Nanoscope MultimodeTM Atomic Force Microscope driven by a Nanoscope IIIa controller, software version 4.31r7 (Digital Instruments, Santa Barbara, CA). The primary scanner was the "E" model, which has a maximum horizontal scanning range of about $15 \mu \mathrm{m} \times 15 \mu \mathrm{m}$. A second scanner, the " $\mathrm{A}$ " model, suitable for scans less than $1 \mu \mathrm{m} \times 1 \mu \mathrm{m}$, was available, though it was used infrequently because many of the features observed in crude oil systems had horizontal dimensions larger than $1 \mu \mathrm{m}$. The microscope was mounted on a vibration isolation table.

\section{AFM Probes}

The probe in scanning probe microscopy is analogous to the stylus on an analog phonograph. A probe is constructed by mounting a sharp tip on a compliant cantilever. The tip is then raster scanned over the sample surface. Subtle force interactions between the tip and sample cause the cantilever to deflect, which generates a force map of the scanned area. This force map is generally interpreted as a topographic map of the sample surface.

Selection of probes for AFM work is guided by the scanning mode, imaging medium, and the mechanical properties of the sample. Two primary classes of probes are used for most AFM applications: (1) silicon nitride v-shaped cantilevers with pyramidal tips, and (2) etched silicon wafer single beam cantilevers with pyramidal tips. SEM micrographs of each style are shown in Figure II-3.1.

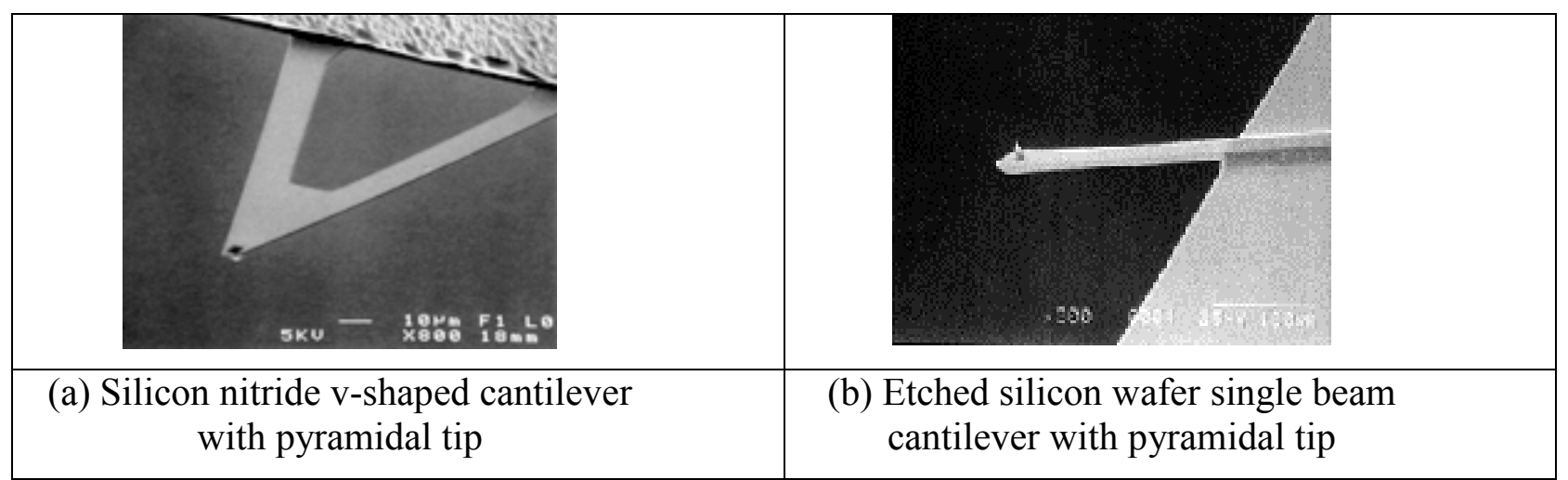

Figure II-3.1. SEM micrographs of two common AFM tips. Images were obtained from the manufacturer's website: http://www.di.com.

CM-AFM requires a relatively weak cantilever in order to minimize damage to the sample surface while scanning. Typical spring constants for contact mode tips fall in the range $k$ $=0.02-0.75 \mathrm{~N} / \mathrm{m}$. Conversely, TM-AFM requires a relatively stiff tip with a high resonant frequency to facilitate tapping mode imaging. Typical spring constants range from $k=20-100$ $\mathrm{N} / \mathrm{m}$ for tips designed for tapping in air. Tapping in liquids does not require such a high spring 
constant, and probe manufacturers recommend using the silicon nitride CM-AFM tips for liquid tapping applications.

The probes found to be most effective for the current work were Olympus OxideSharpened Silicon Nitride probes (Model OTR4, Digital Instruments, Santa Barbara, CA). These particular probes contained 4 cantilevers per mini-wafer as shown in Figure II-3.2. In practice, only two cantilevers were used on any mini-wafer, and the wafer was discarded at the end of each imaging session. Probe specifications are given in Table II-3.2 according to literature provided by the manufacturer. Selecting the particular cantilever for a given sample was often a trial-and-error process. The long cantilever was sometimes problematic because its ultra-low spring constant allowed for vibrational instabilities and considerable "noise" in some scanning situations. In cases when the long cantilever was not producing clean images, the short cantilever was used instead. Comparison of image features for samples which allowed acceptable scans with both short and long cantilevers showed no discernable differences as a function of cantilever length.

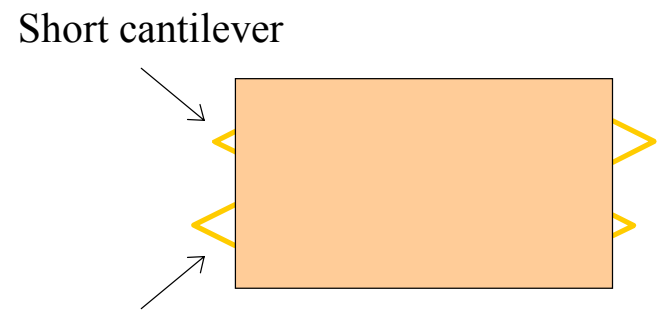

Long cantilever

Figure II-3.2. Schematic of OTR4 probe mini-wafer with four $\mathrm{Si}_{3} \mathrm{~N}_{4}$ cantilevers.

Table II-3.2. Olympus Oxide-Sharpened Silicon Nitride Probe Specifications

\begin{tabular}{|c|c|c|}
\hline Parameter & Short cantilever & Long Cantilever \\
\hline Spring constant $(\mathrm{N} / \mathrm{m})$ & 0.09 & 0.02 \\
\hline Resonant Frequency $(\mathrm{kHz})$ & $13-88$ & $13-88$ \\
\hline Nominal tip radius $(\mathrm{nm})$ & $\leq 20$ & $\leq 20$ \\
\hline Cantilever length $(\mu \mathrm{m})$ & 100 & 200 \\
\hline Cantilever thickness $(\mathrm{nm})$ & 400 & 400 \\
\hline
\end{tabular}

\section{Fluid cell}

The AFM was equipped with a "fluid cell" (TappingMode Fluid Cell, Digital Instruments, Santa Barbara, CA) designed to execute TM-AFM and CM-AFM scans in air or liquids. Extending the capability of the AFM to image under liquids is important for examining surface phenomena in crude-oil/brine/rock systems because oil and brine are the dominant phases contacting rock surfaces in reservoir systems, where a gas phase, if present, is probably the least wetting of the three fluid phases. 


\section{Substrate}

The substrate used in all measurements was freshly cleaved Muscovite mica (S\&J Trading, Inc., Glen Oaks, NY). For the AFM imaging, $12.7 \mathrm{~mm}$ diameter discs were punched from a sheet and cleaved by pressing tape to the top and bottom surfaces of the sample, slowly peeling the tape away to expose a fresh cleavage plane. After cleaving, samples were handled at the edges with a pair of tweezers and promptly immersed in brine solution.

\section{Brines}

Ultrapure water was used to prepare all solutions, and was obtained by passing distilled, deionized water through Milli-Q cartridge filters (Millipore, Bedford, MA), and distilling once more in an all-glass system prior to use. Acidic brine was prepared by adding sodium acetate trihydrate $\left(\mathrm{NaC}_{2} \mathrm{H}_{3} \mathrm{O}_{2} \bullet 3 \mathrm{H}_{2} \mathrm{O}\right)$ (Fisher Scientific, Fair Lawn, NJ), acetic acid $\left(\mathrm{HC}_{2} \mathrm{H}_{3} \mathrm{O}_{2}\right)$, and sodium chloride $(\mathrm{NaCl})$ in measured amounts to obtain a $\mathrm{pH}=3.8,\left[\mathrm{Na}^{+}\right]=0.01 \mathrm{M}$ solution. Basic brine was prepared by adding dibasic sodium phosphate 7-hydrate $\left(\mathrm{Na}_{2} \mathrm{HPO}_{4} \bullet 7 \mathrm{H}_{2} \mathrm{O}\right)(\mathrm{J} . \mathrm{T}$. Baker, Phillipsburg, $\mathrm{NJ}$ ), monobasic sodium phosphate $\left(\mathrm{NaH}_{2} \mathrm{PO}_{4}\right)$ (Aldrich Chemical Company, Milwaukee, WI), and $\mathrm{NaCl}$ in measured amounts to obtain a $\mathrm{pH}=8.0,\left[\mathrm{Na}^{+}\right]=1.0 \mathrm{M}$ solution. A shorthand notation indicates brine $\mathrm{pH}$ and sodium ion concentrations as $\left\{\mathrm{pH},\left[\mathrm{Na}^{+}\right]\right\}$.

\section{Oils}

Crude oils were obtained from a number of sources. While no systematic methodology was employed to prepare the oil for AFM testing, all oils were subjected to standardized analyses which included measurements of API gravity, refractive index, SARA fractions, density, viscosity, acid \#, base \#, and molecular weight. Oils were also examined for contact angle and adhesion characteristics on mica after Liu and Buckley (1997) and Liu and Buckley (1999). This suite of parameters allowed for oils to be categorized in ways which could be potentially important for linking chemical mechanisms with wettability change and film formation or adsorption characteristics visible from AFM observations.

\section{Solvents}

All solvents used for glassware cleaning, including isopropyl alcohol, acetone, toluene, hydrochloric acid, and ammonium hydroxide, were 99\%+ grade (Aldrich Chemical Company, Milwaukee, WI) and used as received from the manufacturer.

\section{Methods}

The methodology detailed here is designed to assist the AFM user in imaging crude oiltreated surfaces. While some basic AFM procedures are addressed here, it is essential that the AFM user recognizes that this report covers a special application of AFM technique, and is not a substitute for the basic user instructions. The new AFM user is strongly encouraged to study documentation provided by the instrument manufacturer (e.g., Scanning Probe Microscopy 
Training Notebook, 1998; On-Line Publications, 2000) in order to learn how to operate the apparatus safely and effectively before proceeding with the procedures discussed here.

\section{Substrate Preparation}

EXPOSURE TO BRINE AND OIL

Freshly cleaved mica samples were first soaked in brine solution for at least 24 hours at room temperature $\left(23 \pm 4^{\circ} \mathrm{C}\right)$. Samples were removed from brine, touched to filter paper or a Kimwipe to remove bulk water, and placed in the oil phase. The oil phase was contained in a 6$\mathrm{mL}$ crimp-top vial with a Teflon stand on the bottom to hold the mica sample vertically in order to prevent any deposition on the mica surface due to gravity-driven settling of suspended particles (Figure II-3.3). Samples were aged in oil for 1 to 26 days at room temperature and atmospheric pressure.

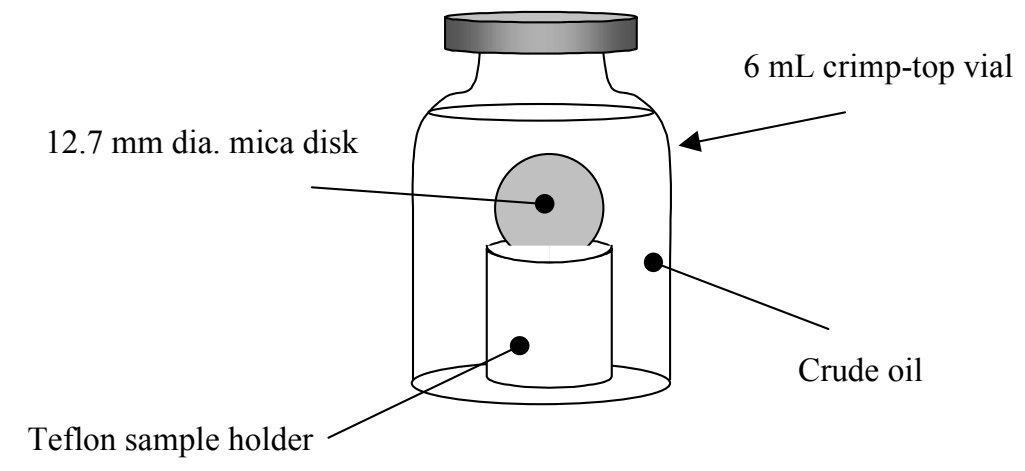

Figure II-3.3. Sample vial used to age mica substrate in oil phase.

After aging in oil for a specified time period, the mica substrate was removed and rinsed by dipping the sample in a toluene bath for about one second, removing the sample to allow the oil to drain off, and repeating the procedure until no visible oil adhered to the mica. This process usually entailed 5 to 7 cycles over a period of about 15 seconds. About $0.3 \mathrm{~mL}$ of clean toluene was then rinsed over the sample from a syringe. The mica was then air-dried for about 5 minutes and subsequently used in experiments.

\section{SUBSTRATE MOUNTING}

Atomic force microscopy experiments required that the mica disk be fixed to a $15 \mathrm{~mm}$ diameter stainless steel puck prior to imaging. The standard adhesive tabs provided with the AFM were not effective for imaging under liquids because the adhesive appeared to loosen when exposed to water. Instead, a small drop of $3 \mathrm{M}$ Super Glue Gel seemed to provide sufficient adhesive power and resistance to dissolution in water or decane such that it was adopted for this work. 


\section{AFM Setup}

\section{SYSTEM SCHEMATIC}

A schematic of the typical SPM installation is given in Figure II-3.4. The user controls scanning from the workstation and can view the real-time microscope output on the display monitor while adjusting imaging parameters on the control monitor. The controller takes instructions from the workstation and sends them to the AFM base/scanner which execute the scanning. Vibration isolation can be an important factor in high-resolution imaging. Some installations have an optional setup in which a cement disk is suspended by three bungee cords attached to a tripod. Determining the optimal vibration isolation technique for a specific experiment may require some trial-and error.

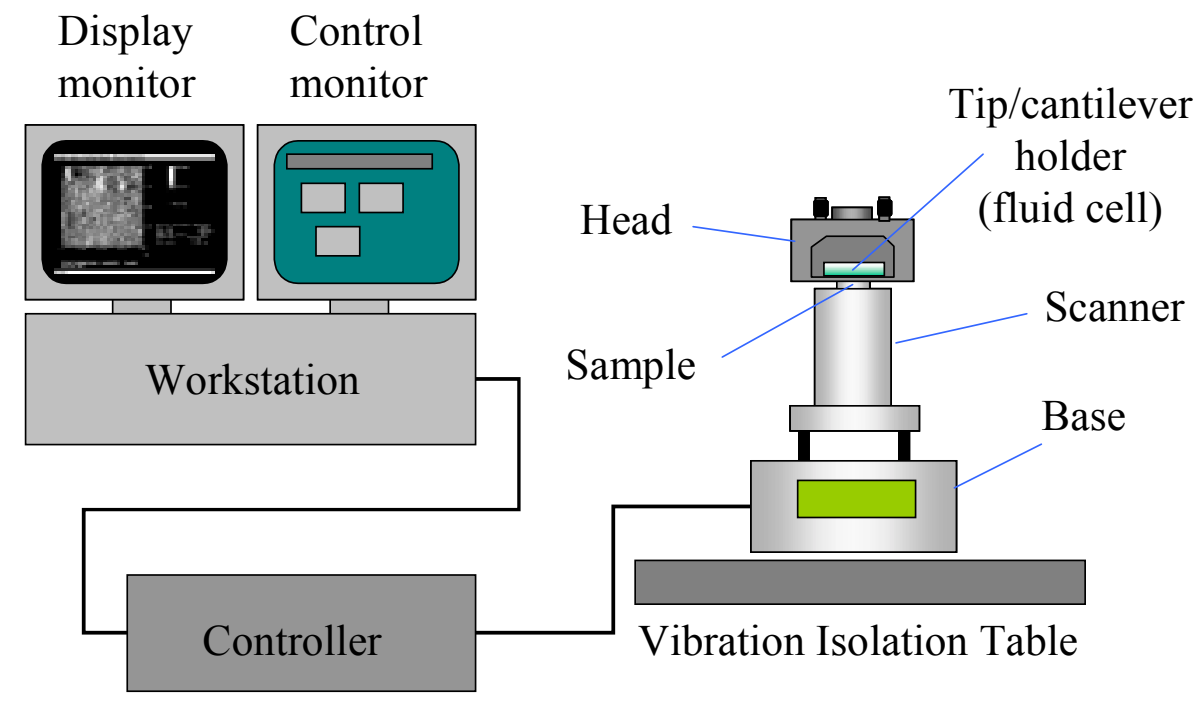

Figure II-3.4. Schematic of typical SPM installation.

\section{EQUIPMENT SELECTION}

The workstation, controller, base, and head are usually fixed for any given installation. The user must select the scanner and tip/cantilever holder. The most important factor controlling scanner selection is the maximum lateral scan size inherent to each scanner. This information is usually marked on the side of the scanner tube. The tip/cantilever holder determines what scanning modes are possible (TM-AFM, CM-AFM) and which imaging media may be used (air, liquids). For scanning in liquids, the fluid cell is required. For tapping in fluids, the tapping fluid cell is required. The tapping fluid cell is capable of operating in CM-AFM and TM-AFM in air or liquid, so the fluid tapping cell is the only cantilever/tip holder required to execute the techniques discussed in this report.

\section{INSTRUMENT ALIGNMENT}

Proper alignment of tip, sample, and laser are critical to successful AFM imaging. The process is tedious and difficult to monitor given the small size of the components. With some 
practice, these steps can be completed efficiently in a few minutes, though many new users struggle in their first few attempts. A simplified cut-away view of the configuration of tip, sample, and laser are given in Figure II-3.5. A step-by-step procedure for instrument alignment is given in Appendix II-3.A.

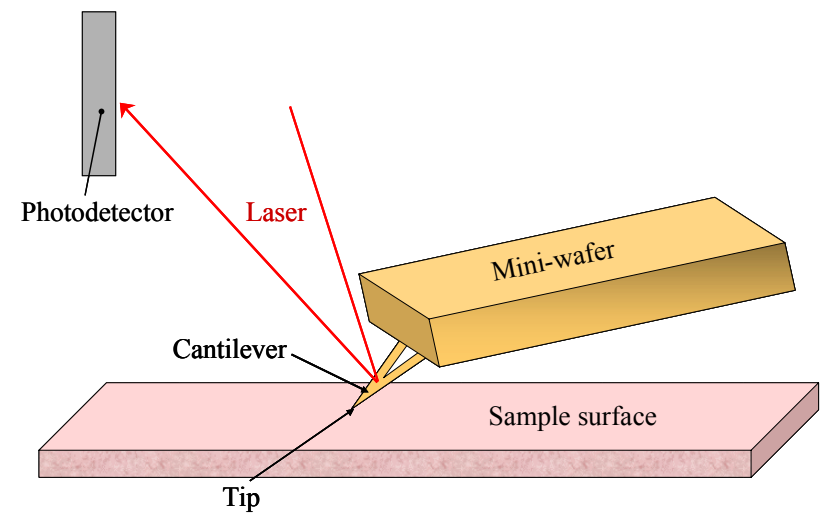

Figure II-3.5. Cut-away view of tip, sample, and laser configuration. Laser must be manually aligned so that it reflects off the top of the cantilever and onto the photodetector in the AFM head.

\section{AFM Imaging}

Once the manual alignment steps are completed, control is transferred to the PC workstation in order to set imaging parameters and execute scanning.

\section{STARTING PARAMETERS}

General guidelines for imaging parameters are suggested here in order to get started, although experience and trial-and-error will govern optimal settings for a particular experiment. Developing an understanding of the effects of these real-time settings on image quality is crucial to successful AFM imaging, but the learning process can be slow and tedious, and perseverance is required.

The control monitor screen should have at least four windows showing for proper execution of scanning, as shown in Figure II-3.6. The four primary parameters which control image quality are scan rate, integral gain, proportional gain, and setpoint. In general, slower scan rates acquire better images, though higher scan rates allow a faster survey of the sample. The gain settings affect the tip/surface tracking and are discussed in more detail under Height and Deflection Signals, and also in the literature provided by the instrument manufacturer. 


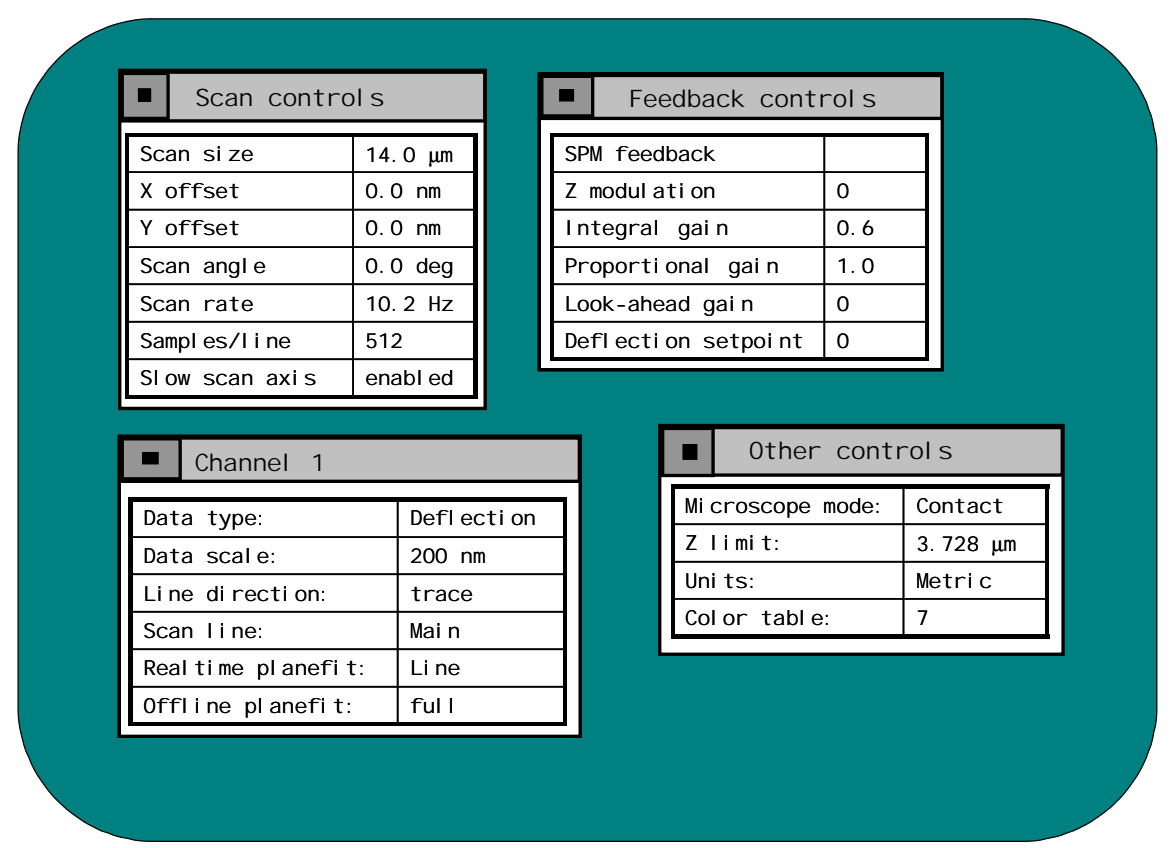

Figure II-3.6. View of control monitor with suggested starting parameters for CM-AFM imaging of oiltreated mica surface in air, water, or decane imaging medium.

CM-AFM scanning may be then executed by the following steps:

1. Engage tip.

2. Minimize scanning force by switching to the Force Calibration signal window (as described in the section on the Force Calibration Plot below).

3. Resume scanning while optimizing scan parameters (scan rate, integral gain, proportional gain, deflection setpoint) to obtain best possible image.

Common problems encountered during AFM scanning are addressed in Appendix II-3.B.

\section{ForCE CALIBRATION PLOT}

CM-AFM scanning of soft, adsorbed materials requires a very low contact force between tip and sample in order to avoid sample scarring. This must be balanced against the need for a sufficient scanning force to effect tip deflection which generates the topographic AFM data. Monitoring the Force Calibration Plot allows some control over the contact force while scanning. The Force Calibration Plot, an example of which is shown in Figure II-3.7, can be viewed on the display monitor as a signal window. 


\section{Force Calibration Plot}

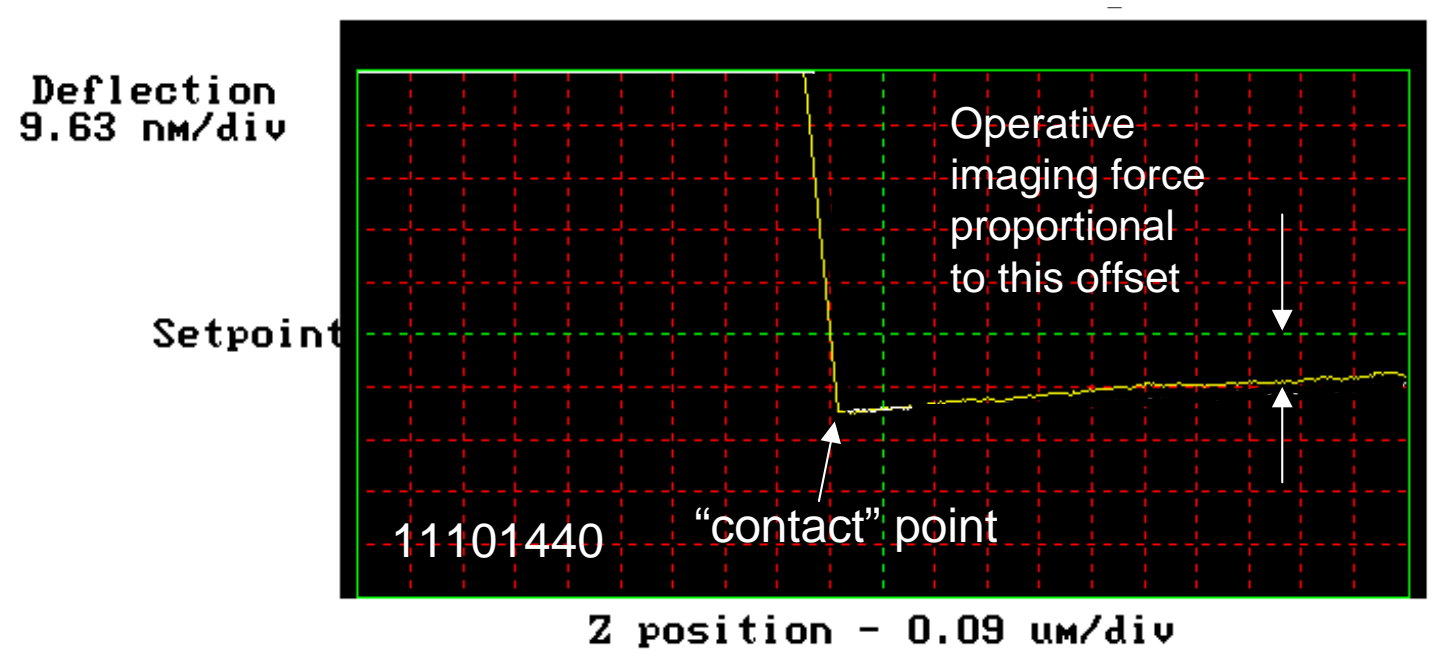

Figure II-3.7. Force calibration plot which relates substrate z-position to cantilever deflection. Scanning force may be minimized by lowering the setpoint, an adjustable scanning parameter, to just above the "contact" point.

The z-position of the sample surface (x-axis in Figure II-3.7) is plotted versus the deflection of the cantilever (y-axis in Figure II-3.7) while the horizontal position of the probe and substrate are fixed. Figure II-3.7 can be interpreted by following the curve from right-to-left as the surface approaches the tip. Since the position of the cantilever holder is always fixed, relative movement between substrate and cantilever is effected by movement of the of the substrate. A long-range attractive force between the tip and sample is evident as the tip deflects downward about $10 \mathrm{~nm}$ as the surface moves upward. Once the tip and sample are separated by a nm or so, the curve bends sharply upward, indicating "contact" in which any upward movement by the sample is matched by an equal deflection of the tip. Due to the mechanical spring property of the cantilever, the further it deflects upward, the greater the force acting to cause the deflection, and thus the greater the probability of mechanically disrupting soft surface features. The objective when imaging soft surfaces is to set the imaging setpoint such that the imaging force is just above that necessary to deflect the tip sufficiently to record surface features without damaging the surface. The setpoint is indicated on the Force Calibration Curve, and designates the voltage signal from the feedback control system that the scan controller attempts to match. The setpoint is selected by the user. The operative scanning force is proportional to the offset between the essentially horizontal portion of the extending curve and the imaging setpoint. In Figure II-3.7, the feedback control system would attempt to maintain a constant tip deflection of about $15 \mathrm{~nm}$ in order to maintain contact while imaging. 


\section{HEIGHT AND DEFLECTION SIGNALS}

The two primary signals monitored during CM-AFM scanning are the "height" and "deflection" signals. The height signal represents the z-position of the sample surface during a scan, while the deflection signal represents the deflection of the cantilever relative to an arbitrary baseline. These signals can be monitored simultaneously for each scan. It is customary to report the height signal as the approximation to sample topography.

In AFM scanning, the sample is translated in the $x-y$ direction while the probe remains stationary. While the surface moves in the $x-y$ plane, force interactions between the tip and sample cause the cantilever to deflect from its baseline position. A feedback loop in the AFM controller attempts to maintain a constant cantilever deflection by changing the z-position of the sample stage as the tip encounters features on the surface which cause it to deflect. For example, if the tip were to encounter a hump which caused the cantilever to deflect upward, the feedback controller would move the sample z-position downward until the tip deflection was at baseline level again.

In reality, the feedback system cannot achieve zero cantilever deflection and thus create a perfect height signal reproduction of the sample topography. The deflection signal may be interpreted as the "error" between the height signal and the actual surface topography. The magnitude of the deflection can be controlled by the gain settings in the feedback system. Large gain settings initiate a rapid response from the feedback system so that the error is minimized and the height signal is maximized. This technique is sometimes referred to as "constant deflection" scanning. Alternatively, small gain settings lower the sensitivity of the feedback system, resulting in a minimized height signal and maximized deflection signal. This technique is sometimes referred to as "constant height" scanning. Both signals can provide useful images, and a direct comparison of height signal and deflection signal images for identical scan areas illustrates the capabilities of each technique for resolving surface features.

\section{Simultaneous Signal Capture}

The AFM has the capability to display and capture both height and deflection signals for the same scan. Figure II-3.8 shows such simultaneously-acquired signals for a representative oiltreated mica surface. Scan parameters are listed in the table next to the figure caption. Int. Gain denotes the integral gain setting, and Prop. Gain denotes the proportional gain setting. The gain settings in this case were relatively high $(\geq 5)$, optimized for height signal imaging. Gains are usually raised until the onset of instability in the feedback system, at which point they are backed down slightly for a stable imaging signal. Feature edges are somewhat "blurred" in the height image in Figure II-3.8, and some horizontal streaking is evident. The deflection image, by comparison, is flatter than the height image, attesting to the feedback system's efforts to adjust the sample z-position to maintain a near-constant cantilever deflection. 


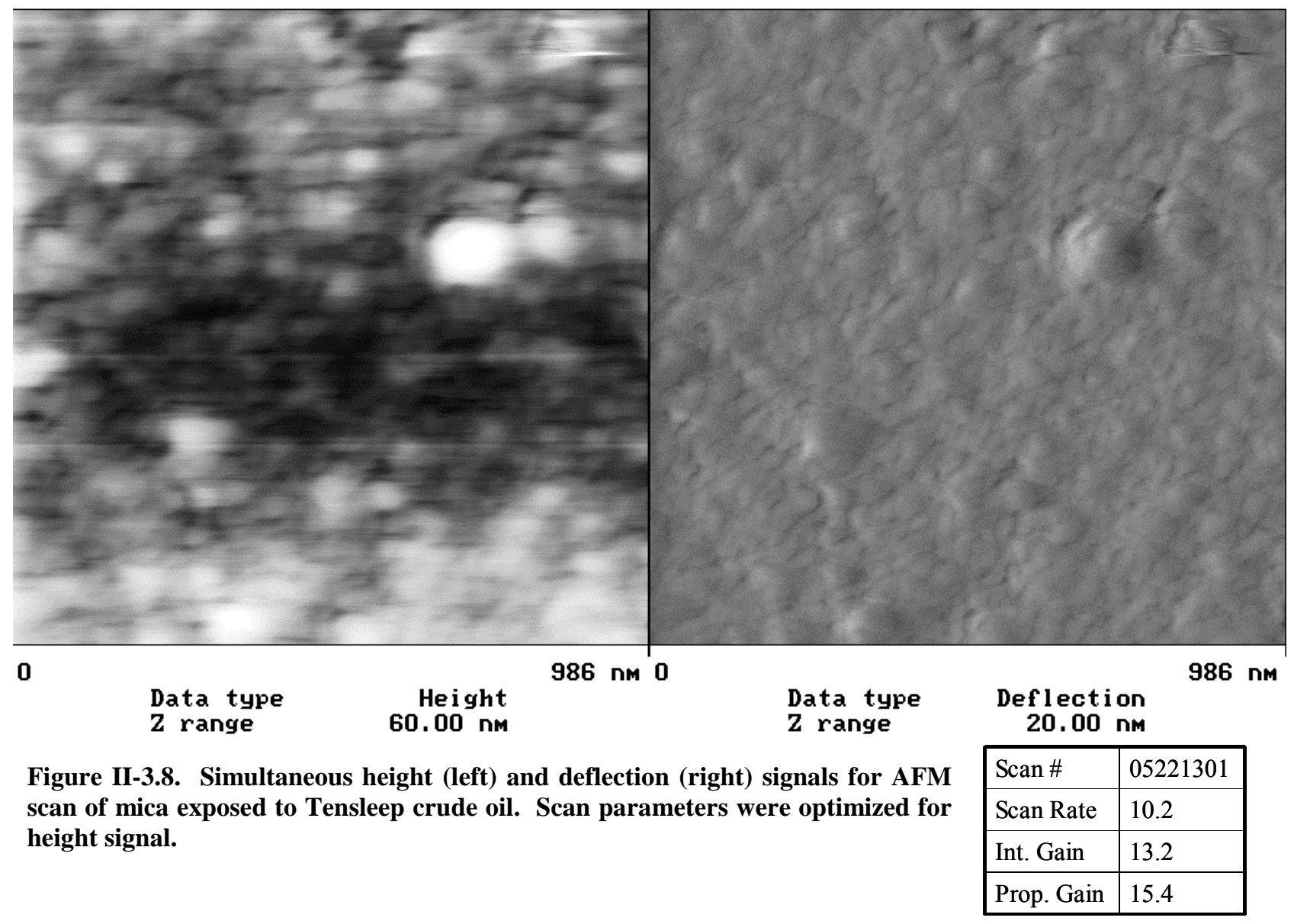

Figure II-3.9 shows simultaneously-acquired height and deflection signals for the same scan area shown in Figure II-3.8, but with gain settings much lower $(\leq 2.0)$. The deflection signal is much better-resolved here than in Figure II-3.8, while the height signal becomes streaked and essentially featureless. This occurs because the feedback system is impeded by the low gain settings, resulting in minimal height-signal sensitivity to features on the surface. Cantilever deflection, however, is maximized, so a deflection signal image gives a reasonable representation of the surface topography. 


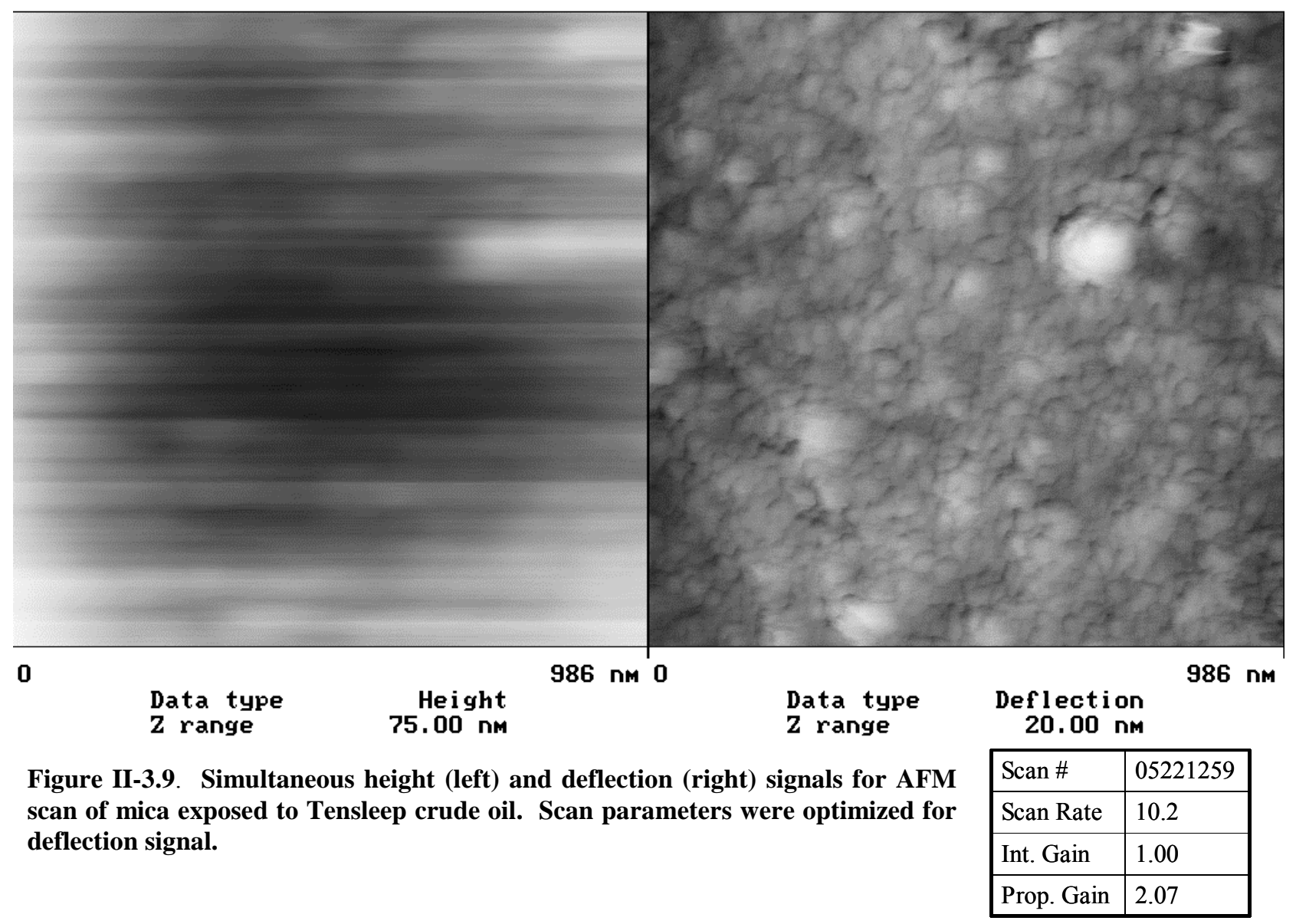

\section{Separate Signal Capture}

While simultaneous signal capture is an instructive academic exercise, it becomes unnecessarily cumbersome to handle twice the quantity of data that is typically used during offline analysis. It is more efficient to monitor and capture just one signal at a time with the scan parameters optimized to the particular signal of interest. Following are several examples of wellresolved images captured separately, with a discussion of the useful features of each image.

Sample 1114. Figures II-3.10 and II-3.11 represent scans on a mica substrate exposed to a $1.0 \mathrm{wt} \%$ solution of asphaltenes in toluene for 26 days. Imaging parameters for the scans are given in the table accompanying each figure. Image resolution in both cases is comparable. The primary observable difference is the presence of "shadows" on the deflection image. These result from the feedback system slowly correcting the z-position of the substrate after a significant change in cantilever deflection. Deflection images tend to be most useful for relatively flat samples with short, closely-spaced features. Alternatively, height images tend to be more useful on samples which exhibit tall, scattered features or steps. This sample appears to be well-characterized by both signals. 


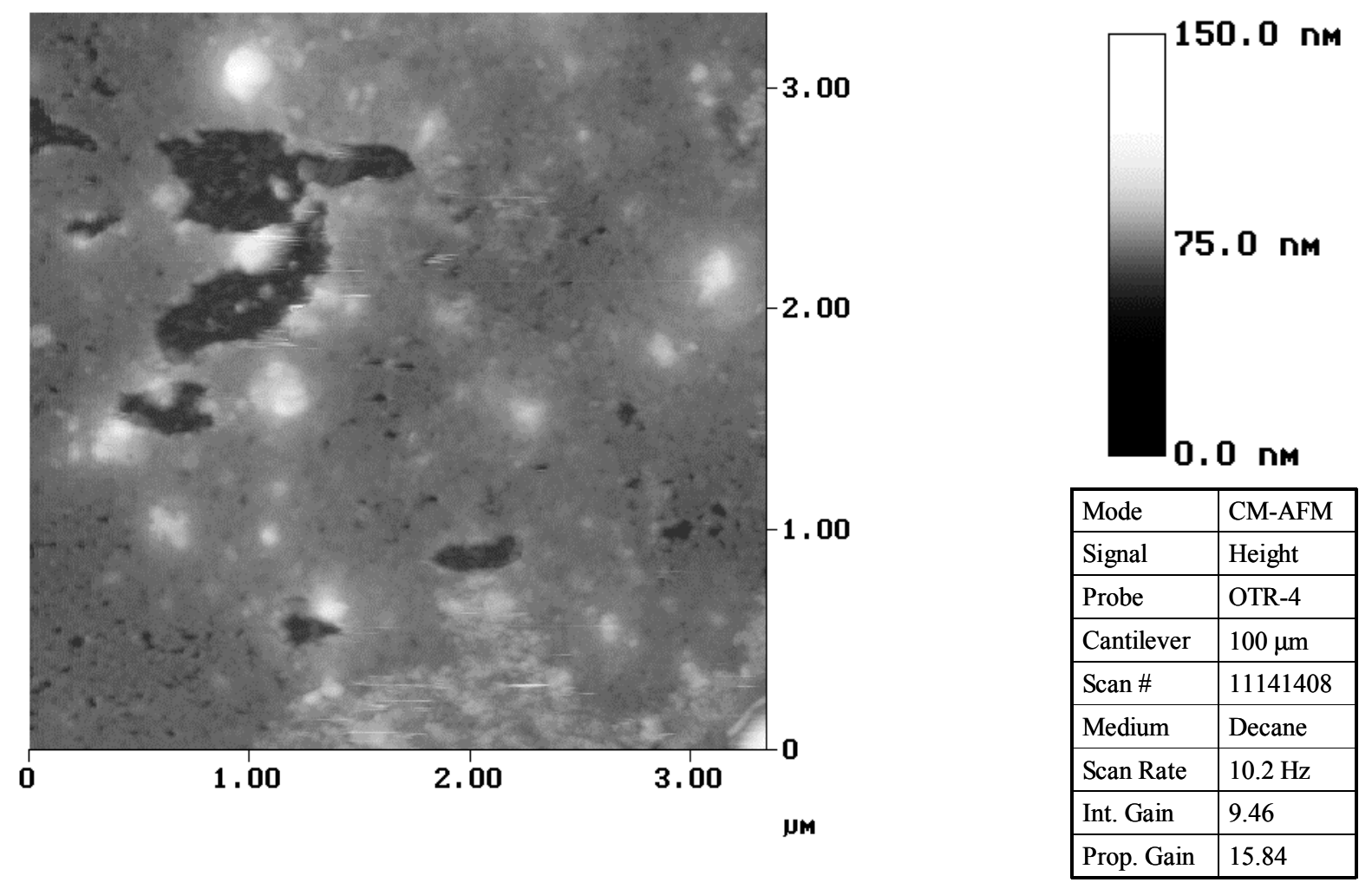

Figure II-3.10. AFM scan of mica exposed to a 1 wt.\% solution of Tensleep asphaltenes in toluene.
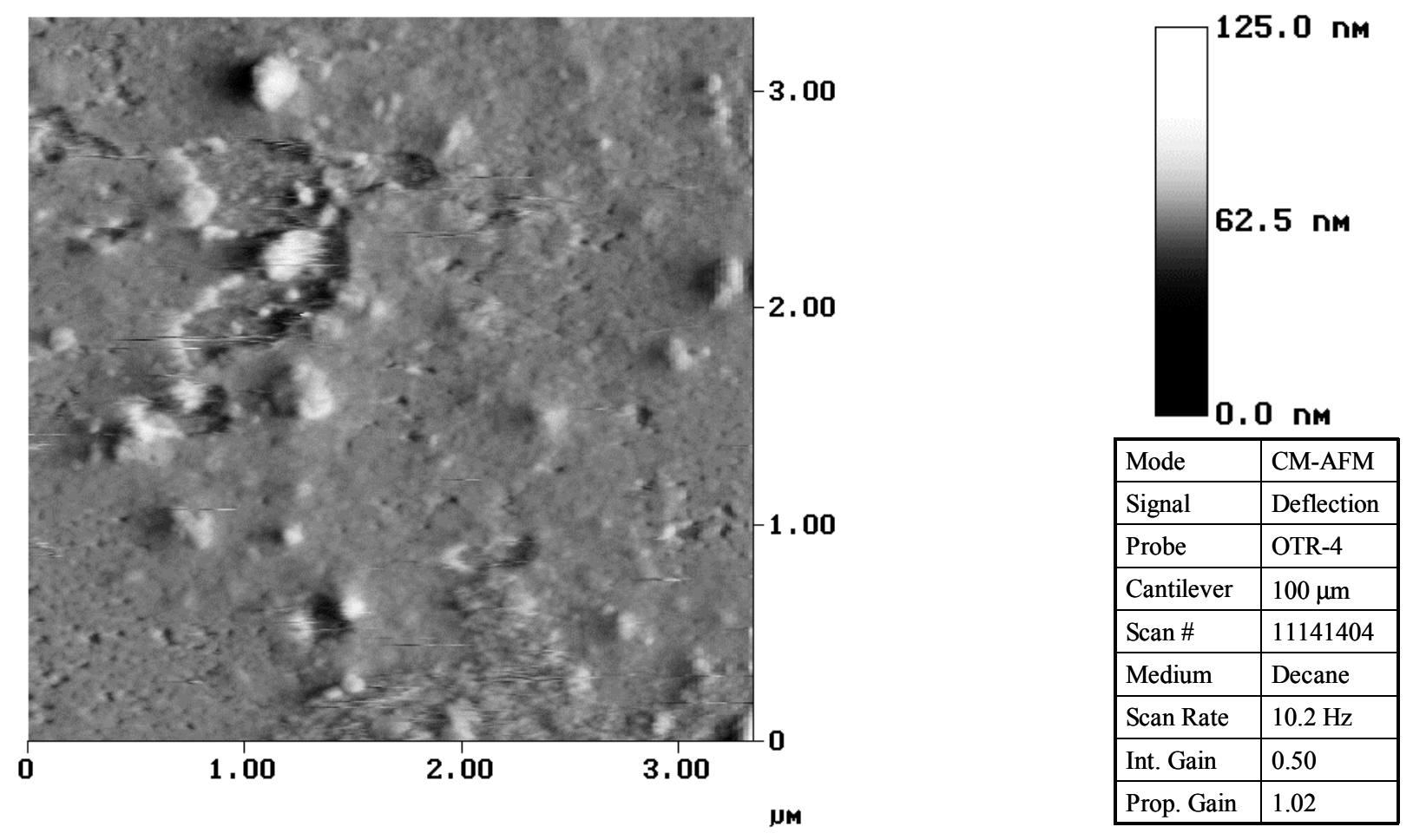

Figure II-3.11. AFM scan of mica exposed to a $1 \mathrm{wt}$ \% solution of Tensleep asphaltenes in toluene. 
Sample 0112. Holes in surface films provide an opportunity to estimate the film thickness, provided the substrate is clearly visible at the bottom of the hole. Height signal images are more appropriate for film thickness approximation because the deflection signal always tends toward zero as the feedback system attempts to achieve zero deflection. Large holes therefore look different according to which signal is used. Figures II-3.12 and II-3.13 illustrate this from a mica sample exposed to Tensleep crude for 1 day. The large black area in the height signal image (Figure II-3.12) is a hole in the surface film. The consistent low elevation at the bottom of the hole is represented by the black color on the grayscale. The hole is also conspicuous in the deflection image (Figure II-3.13), though it is characterized by distinct edges and some smooth areas visible in the base of the hole. Film depth in this case could be estimated by conducting an offline analysis of the height signal image. This procedure is discussed in more detail in the section on Data Analysis below.
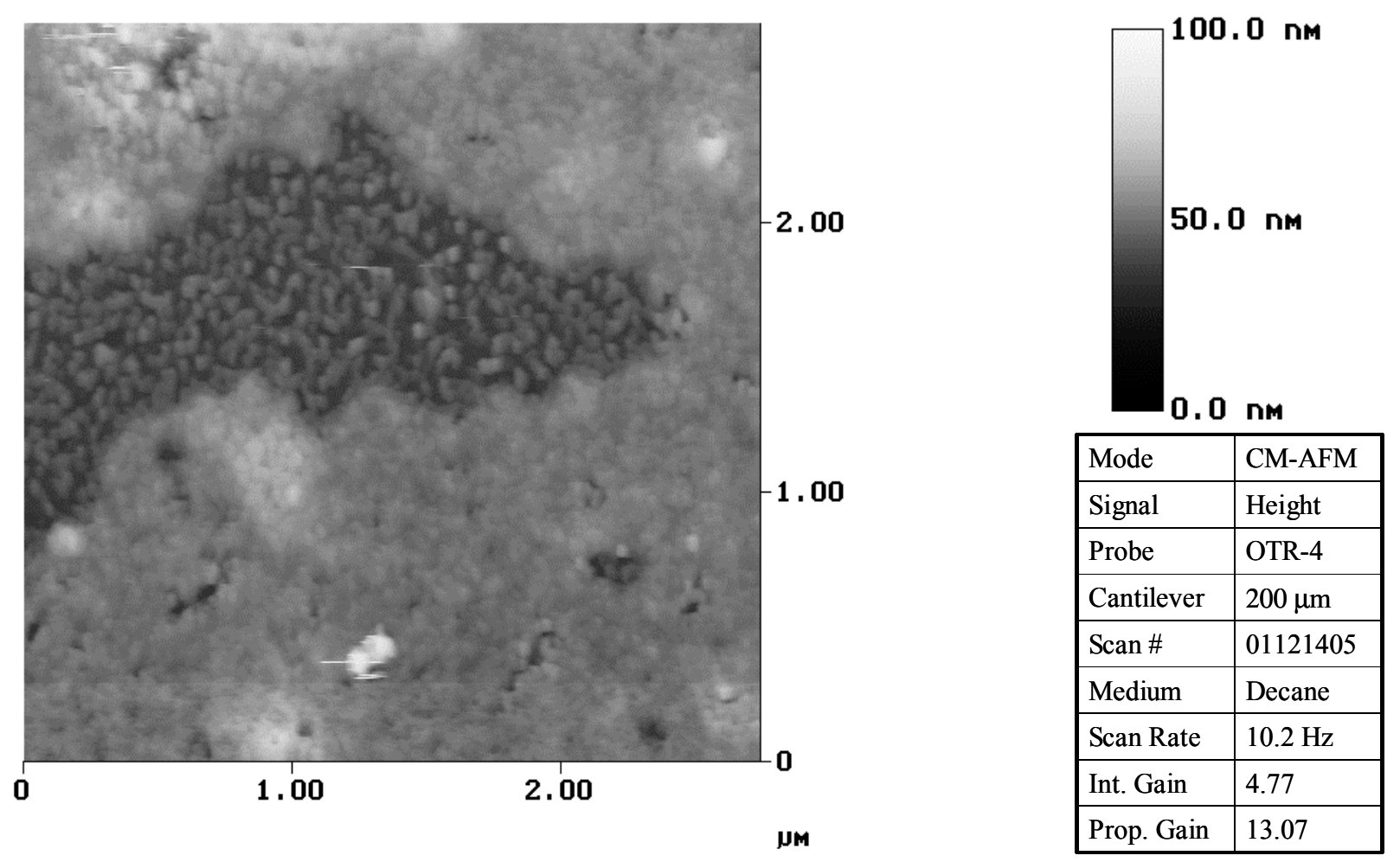

Figure II-3.12. AFM scan of mica exposed to Tensleep crude for 1 day. 

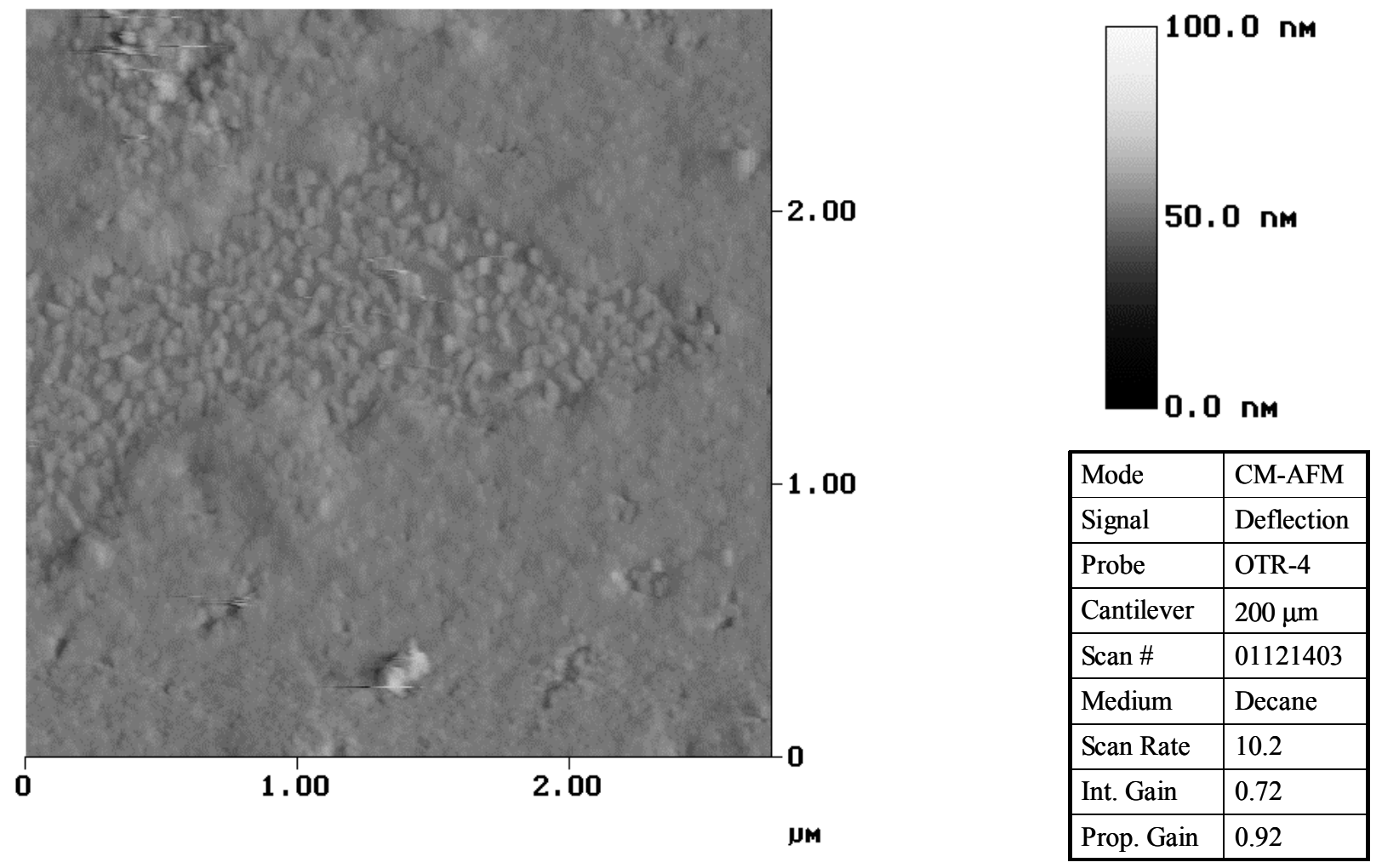

Figure II-3.13. AFM scan of mica exposed to Tensleep crude for 1 day.

Sample 0209. While sample scarring of soft organic films during scanning is usually an undesirable reality of SPM, it can be used for estimating film thickness and comparing film texture to substrate texture if done in a controlled manner. Since relative magnitude of contact force is controlled by the user through the Setpoint parameter, scanning force may be increased or decreased arbitrarily. Typically, the Setpoint is adjusted in order to minimize contact force. Should the user wish to scrape through the soft film down to the substrate, simply increasing the Setpoint value will usually drive the tip down to the hard surface. A small scan area is usually selected so the scarred block may be viewed from a wider perspective.

Figures II-3.14 and II-3.15 illustrate the result of creating a $3 \mu \mathrm{m}$ scar in a film formed by exposing mica to Mars Yellow crude oil for 99 days. Immediately prior to capturing these images, the surface film was continuous with no visible holes. The scan area was reduced to 3 $\mu \mathrm{m} \times 3 \mu \mathrm{m}$, and the Setpoint was increased from about $-2.0 \mathrm{~V}$ to about $8.0 \mathrm{~V}$. Once the small scan was completed, the Setpoint was returned to about $-2.0 \mathrm{~V}$, and the scan area was increased to $9 \mu \mathrm{m} \times 9 \mu \mathrm{m}$. The result is a distinct rectangular hole in the film, with the smooth substrate visible at the bottom of the hole. An offline section analysis was then used to estimate the thickness of this film. 


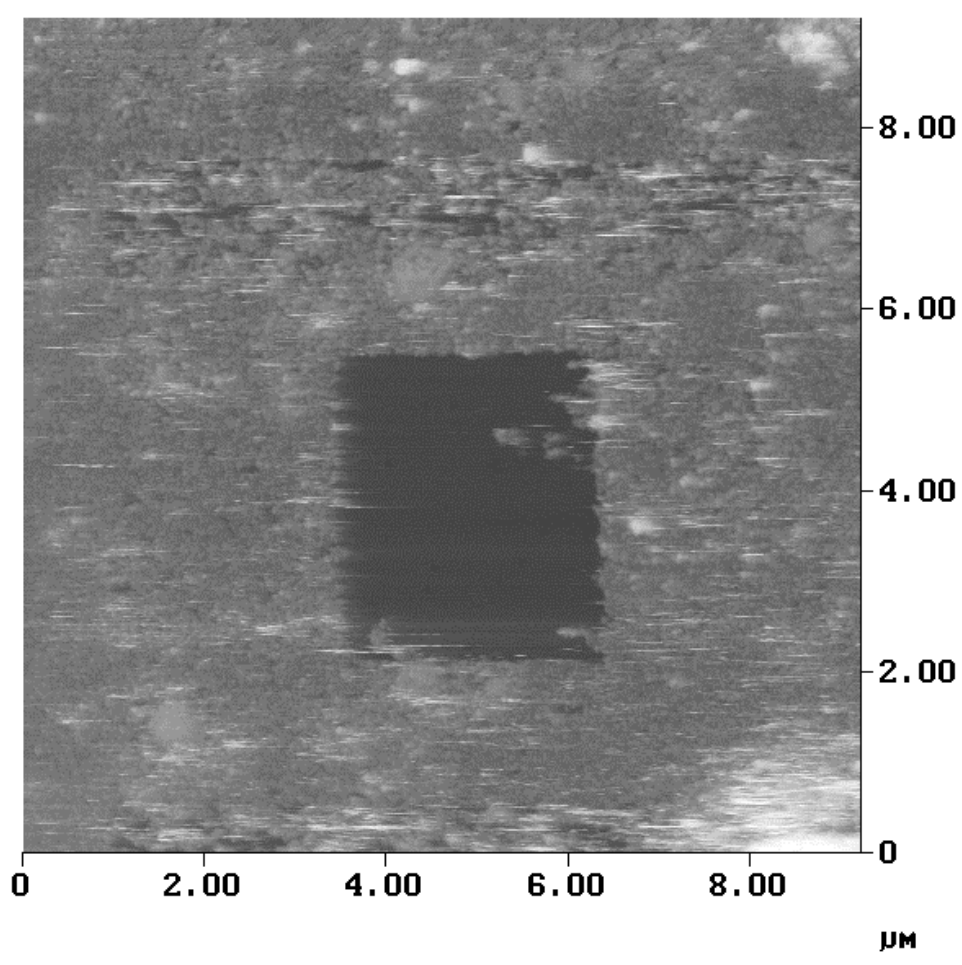

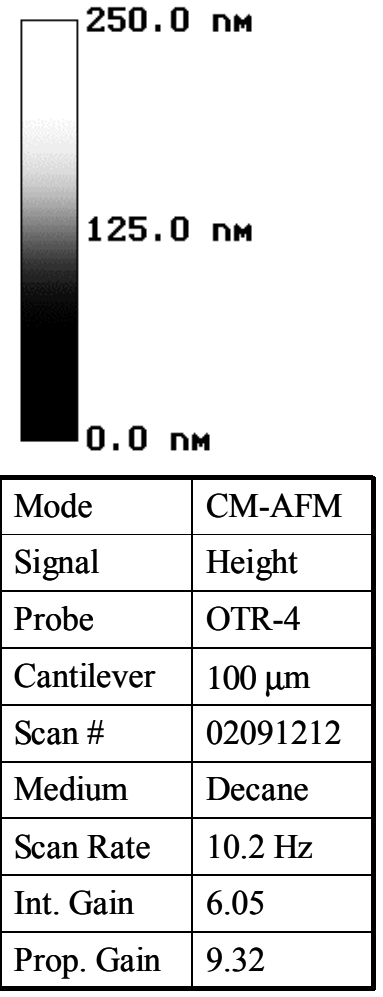

Figure II-3.14. AFM scan of mica exposed to Mars Yellow crude for 99 days.

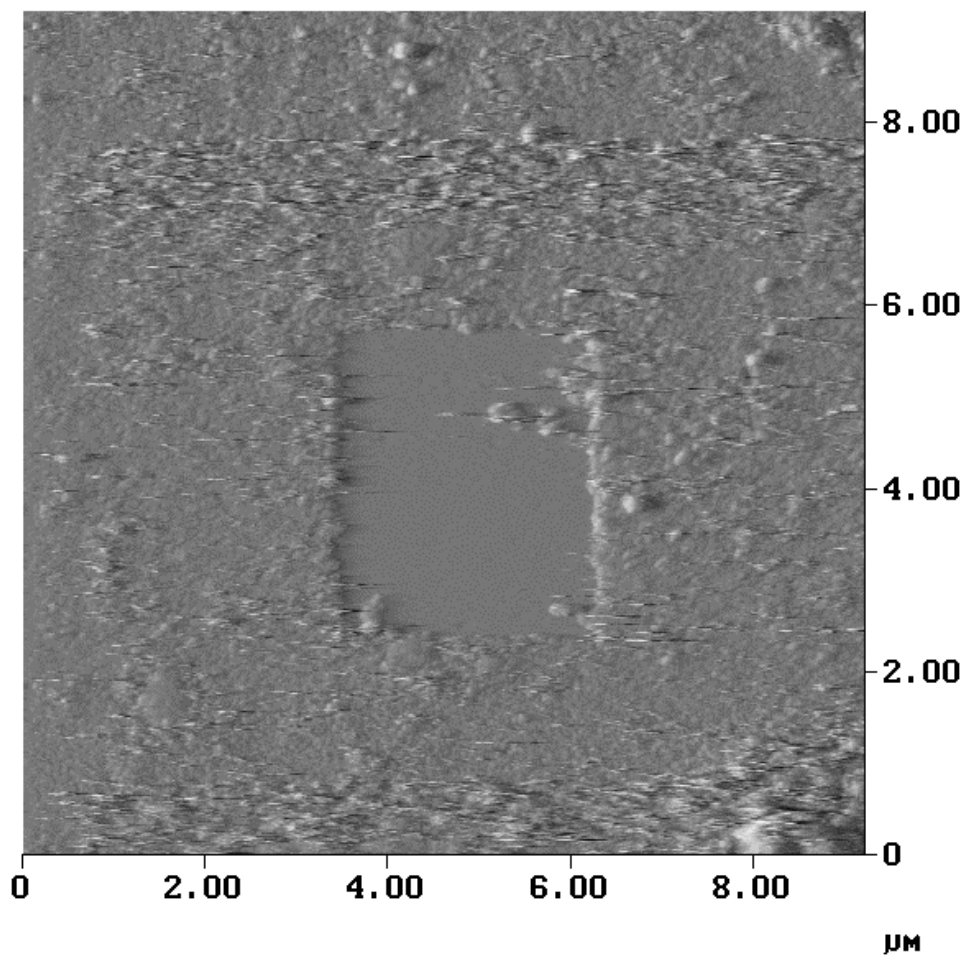

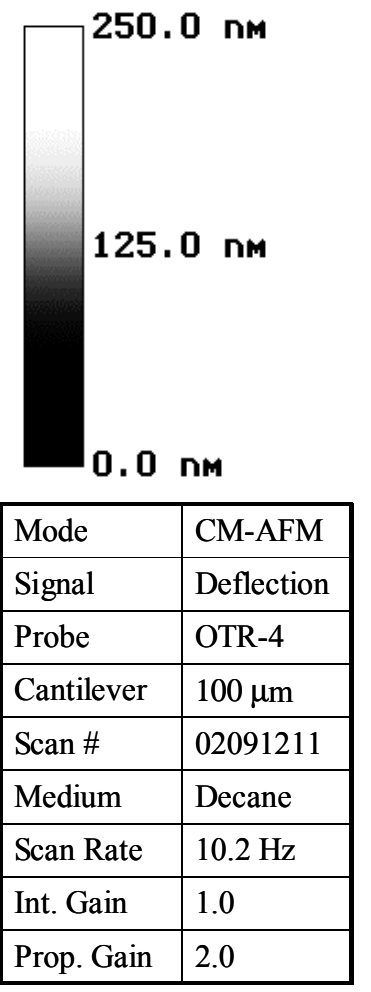

Figure II-3.15. AFM scan of mica exposed to Mars Yellow crude for 99 days. 


\section{Data Analysis}

\section{SECTION ANALYSIS}

The offline Section Analysis tool is useful for determining feature heights on crude oil AFM samples. Height signal images are more applicable to section analyses than deflection signal images. Figures II-3.16 and II-3.17 illustrate the use of section analysis in this context. The image under analysis is shown in the lower left corner of the Section Analysis screen. The user is prompted to draw a line on the image in order to denote the section of interest. The profile of the section appears on a plot in the upper left corner of the screen, with the horizontal axis representing the position along the section, and the vertical axis representing the corresponding signal value. In the case of a height image, the signal represents the z-position of the sample. In the case of a deflection image, the signal represents the deflection of the cantilever. The sections in Figures II-3.16 and II-3.17 are in nearly identical locations, as shown on the images in the lower left corner of each screen, though their profiles look decidedly different. Scan direction in both cases was from right to left. While the height profile traced a (1) downward slope, (2) a plateau, (3) a flat basin, and then (4) another plateau, the deflection signal traced (1) a flat region, followed by (2) a high spike and decay, (3) a low spike and recovery, and finally (4) a high spike and decay. The height and deflection images were captured separately in this case, so gains were optimized accordingly. The deflection signal shows a distinct tendency to trend toward zero, which is consistent with the design of the feedback system. The height signal, however, stepped up or down and did not tend toward any particular value.

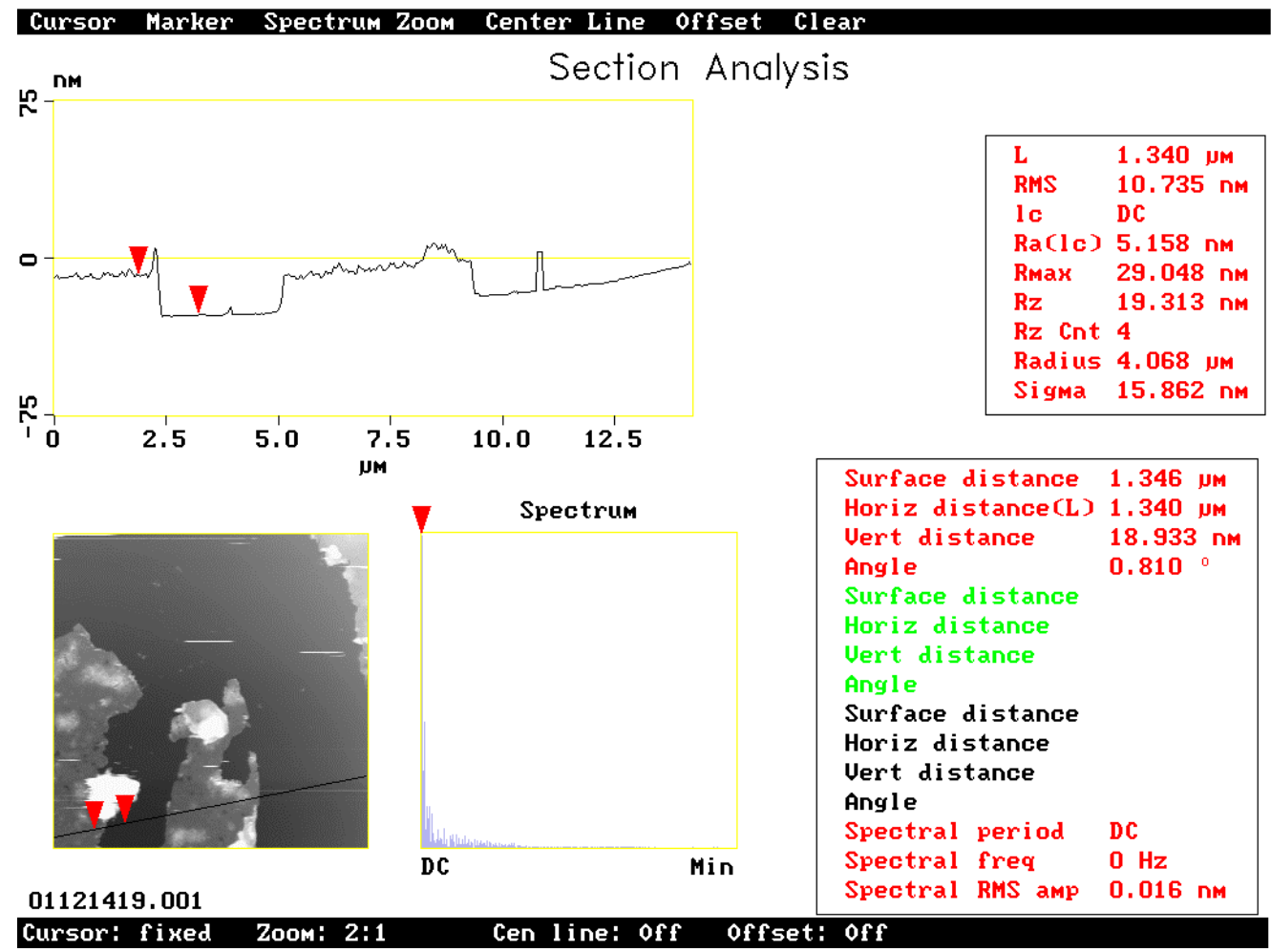

Figure II-3.16. Section analysis of AFM height signal image of mica exposed to Mars Yellow crude for 99 days. The vertical distance between red cursors indicates a film thickness of about $19 \mathbf{~ n m}$ in this section. 


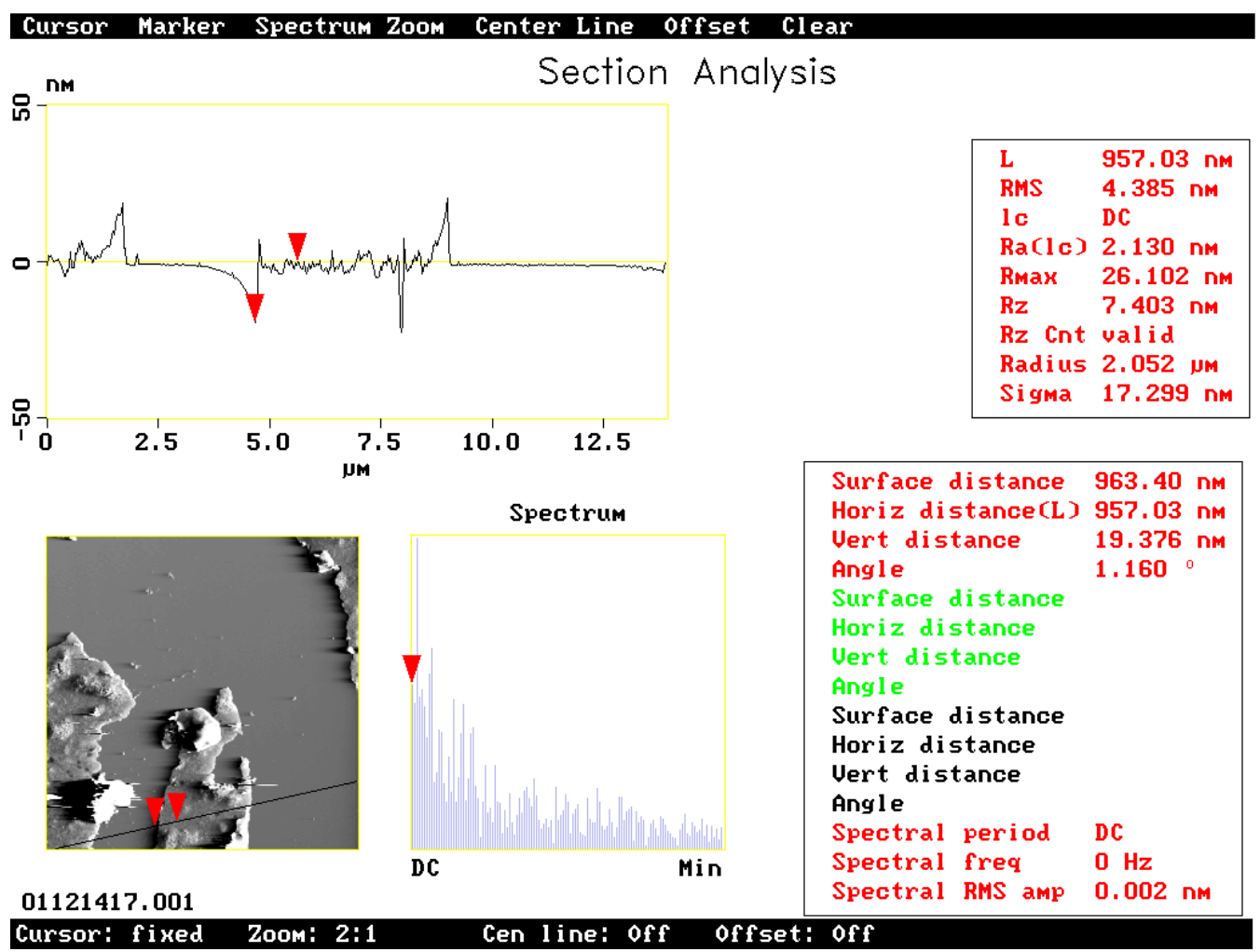

Figure II-3.17. Section analysis of AFM deflection signal image of mica exposed to Mars Yellow crude for 99 days.

Film thickness may be estimated from a section analysis by placing the red cursors at arbitrary points along a section, and measuring the elevation of the plateau relative to that of the basin. The extremely smooth texture of the basin in Figures II-3.16 and II-3.17 suggest that this is probably the mica substrate. The height signal image is more amenable to this type of analysis than the deflection signal image because the deflection signal is always trending toward zero after a step. Deflection images may prove useful in some cases if the cursors are placed such that they measure the height of the "spike." These techniques were used in the above figures to obtain relatively well-matched estimates of $\delta \cong 19 \mathrm{~nm}$ for both cases.

\section{ROUGHNESS ANALYSIS.}

The surface roughness of AFM samples maybe quantified according to the mean roughness $\left(\mathrm{R}_{\mathrm{a}}\right)$ calculation:

$$
R_{a}=\frac{\sum_{i=1}^{N}\left|h_{i}-\bar{h}\right|}{N}
$$

where $\mathrm{h}$ denotes elevation of the data point, and $\mathrm{N}$ is the number of points on the image. Any arbitrary rectangular area on an AFM image may be analyzed for the mean roughness parameter. Some features exhibit a characteristic roughness range, and the roughness parameter may be used as an indicator of the type of surface material visible in a given region of an AFM image. For 
example, analyses of the smooth substrate mica by Yang et al. (1999) and $\mathrm{R}_{\mathrm{a}}<0.01 \mathrm{~nm}$ (see section II-3.2). Given the wide variety of features found on surfaces exposed to crude oils, the roughness calculation can be useful in distinguishing substrate material from smooth film features.

FILE MANAGEMENT

Offline analyses are performed using data files created during the imaging session. Details of data file management are discussed in Appendix II-3.C.

\section{Summary}

This report describes a methodology by which mica was exposed to crude oil and then analyzed with the atomic force microscope (AFM) for surface film morphology. This methodology is distinguished from prior published attempts to analyze crude-oil systems with AFM because (1) the treated surface was imaged under a series of media including air, brine, decane, and toluene, and (2) the image resolution allowed for identification of structures which ranged in scale from $20 \mathrm{~nm}$ up to several microns. Using liquids as imaging media instead of air creates an imaging environment more representative of reservoir conditions. Also, changing imaging media allows for some features to be distinguished by virtue of their solubility and resulting morphology in various media. For example, ionic salts deposited on the substrate and evident in air will readily dissolve in water, leaving bare patches. Similarly, large polar asphaltic structures will swell in a polar organic solvent such as toluene relative to its appearance in a nonpolar solvent such as decane.

The development of the AFM technique for analyzing crude oil-treated surfaces adds another tool to a collection of techniques which are currently employed to determine the complex mechanisms that affect wetting phenomena in crude oil-brine-rock systems. The AFM data are particularly useful when analyzed in conjunction with a suite of other properties including crude oil asphaltene content and relative stability of its asphaltenes, crude oil acidity and basicity, brine $\mathrm{pH}$ and salinity, and substrate surface chemistry. Assessed individually, these properties cannot reliably determine the causes behind wettability changes. Assessed collectively, however, these measurements can give a reasonable explanation of the important mechanisms operative in a given system.

\section{References}

Basu, S.; Sharma, M.M.: "Investigating the Role of Crude-Oil Components on Wettability Alteration Using Atomic Force Microscopy," SPEJ (September, 1999) 235-241.

Buckley, J.S., Liu, Y., Xie, X., and Morrow, N.R.: "Asphaltenes and Crude Oil Wetting-The Effect of Oil Composition," SPEJ (June, 1997) 107-119.

Campbell, N.A.: Biology, $3^{\text {rd }}$ Ed., Benjamin/Cummings Publishing, Redwood City (1993). 
Ese, M.-H., Sjoblom, J., Djuve, J., Pugh, R. "An Atomic Force Microscopy Study of Asphaltenes on Mica Surfaces. Influence of Added Resins and Demulsifiers," Colloid Polym. Sci. (2000) 278, 532-538.

Liu, L.; Buckley, J.S. "Evolution of Wetting Alteration by Adsorption from Crude Oil," SPE Formation Eval. (March, 1997), 5-11.

Liu, L.; Buckley, J.S. “Alteration of Wetting of Mica Surfaces,” J. Pet. Sci. Eng. (1999), 24, 7583.

Manne, S, Cleveland, J.P., Gaub, H.E., Stucky, G.D., and Hansma, P.K.: "Direct Visualization of Surfactant Hemimicelles by Force Microscopy of the Electrical Double-Layer," Langmuir (1994) 10, 4409-4413.

Manne, S., Gaub, H.E.: "Molecular Organization of Surfactants at Solid-Liquid Interfaces," Science (December 1995), 270, 1480-1482.

On-Line Publications CD-ROM, v.2 Technical Publications, Digital Instruments, Santa Barbara, CA (2000).

Scanning Probe Microscopy Training Notebook, Digital Instruments, Santa Barbara, CA (1998).

Toulhoat, H., Prayer, C., and Rouquet, G.: "Characterization by atomic force microscopy of adsorbed asphaltenes," Colloids and Surfaces A (1994) 91, 267-283.

Yang, S.-Y., Hirasaki, G.J., Basu, S., and Vaidya, R.: "Mechanisms for Contact Angle Hysteresis and Advancing Contact Angles," J. Pet. Sci. Eng. (1999) 24, 63-73.

\section{Acknowledgments}

The authors wish to thank Sandia National Labs for access to the atomic force microscope, and Jim McLemore for assistance in developing the sample preparation techniques.

\section{Appendix to Section II-3.1}

\section{A. Instrument alignment}

Following are step-by-step instructions for aligning the tip, sample, and laser in preparation for CM-AFM imaging of a crude oil-treated sample under a liquid environment. It is strongly recommended that the user refer to the manufacturer's detailed instructions for setup and operation of the instrument and practice setup and imaging in air before attempting the same in liquid.

1. Select a new AFM probe from storage box and mount it in fluid cell (cantilever/tip holder). Make sure to seat the probe squarely in the cutout and secure it with the spring clip. The cantilevers are extremely delicate. If the probe is touched or dropped on end, the cantilevers will be damaged and the probe must be replaced. If in doubt, replace the probe.

2. Place sample (glued to stainless steel puck) on magnetic stage on top of scanner tube. 
3. Move the selector lever on the scanner base to AFM to select CM-AFM (see Figure II-3.18).

4. In the Other Controls panel on the control monitor, set the AFM Mode to Contact.

5. Lower the sample relative to the head so it is flush with bottom inner surface of the head. Some scanners have a manual screw on the bottom of the scanner tube which raises and lowers the sample. In the absence of a manual screw, use the lever on the right side of the scanner base which reads Up and Down (Figure II-3.18). Watch closely when using the Up and Down lever, because these markings refer to the relative movement of the probe, not the sample.

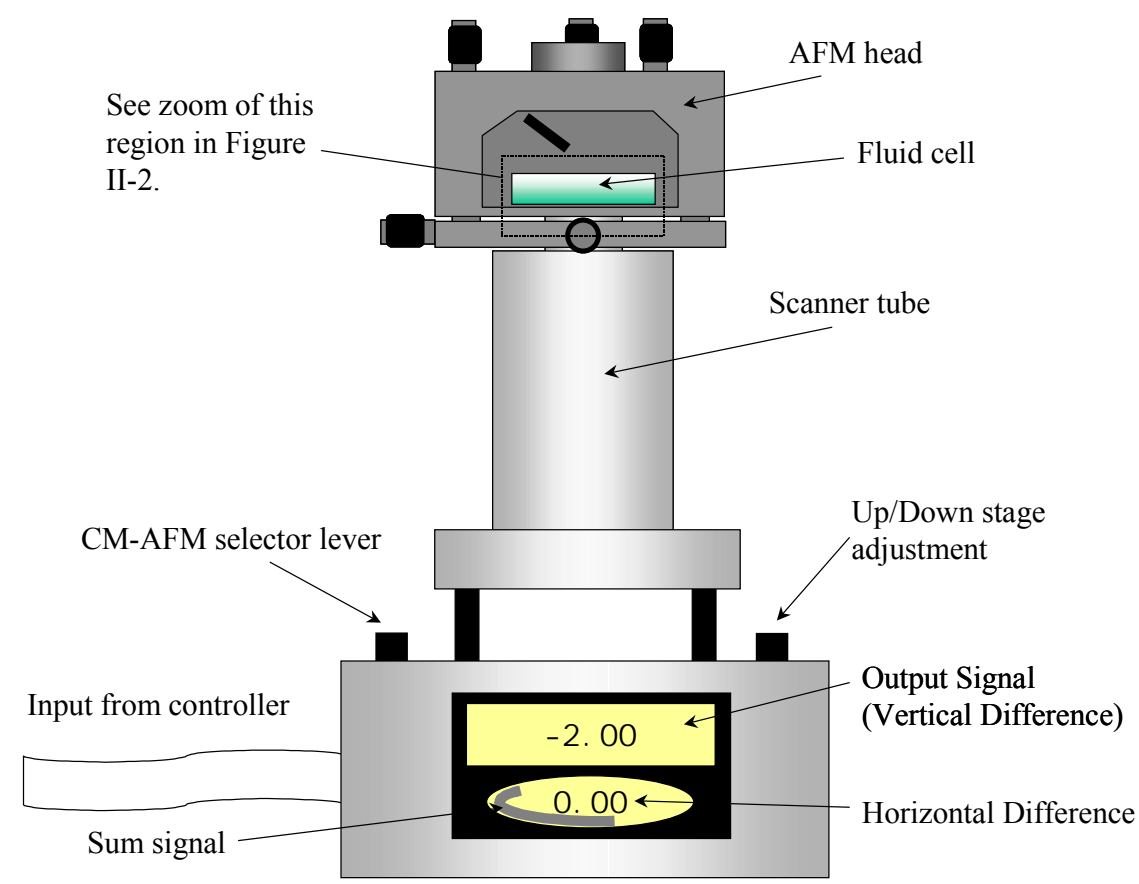

Figure II-3.18. AFM base, scanner, and head assembly

6. Insert the fluid cell and tighten thumb screw to secure. Be sure that there is sufficient clearance between the cantilever and the sample surface so the cantilever is not crushed.

7. Looking from above in the binocular optical microscope, find the cantilevers on the end of the mini-wafer.

8. Align the red laser spot so it is on the end of the desired cantilever.

9. Use a sliver of white paper to locate the laser as it reflects off the tip but before it hits the mirror. If the light is diffuse, realign the laser in small increments to create a sharp, horizontal rectangle.

10. Adjust the angle of the mirror and laser position to obtain the largest sum signal possible on the lower digital readout. 
11. Adjust the Horizontal Difference signal close to 0.0 volts by turning the screw on the back left side of the head. This screw adjusts the horizontal position of the photodetector.

12. Adjust AFM Output signal to about -2.0 volts by turning the screw on the top left side of the head. This screw adjusts the vertical position of the photodetector.

13. Position the monocle (attached to the flexible arm and movable base) so that the field of view captures the cantilever and sample surface. For a reflective surface, three red dots should be visible, corresponding to the tip, the surface, and a reflection of the tip. Because a mica sample has many layers, additional reflections may appear below these three red spots. Note that the image through the monocular scope is not inverted.

14. Raise the sample surface with the Up/Down switch until the cantilever and sample are separated by about $10-20 \mu \mathrm{m}$. The top three red spots will approach each other, but the manual approach should be stopped before they converge. At this point, the tip can be engaged from the options on the control monitor and imaging in air can commence.

15. To image in a liquid, loosen the thumb screw and carefully remove the fluid cell. Place 1 small droplet of imaging medium (water, decane) on sample and another on the fluid cell/probe assembly.

16. Re-position fluid cell in AFM and assure that the two droplets of imaging medium touch and form a bridge, trapped by capillary forces, between the fluid cell and the sample surface (see Figure II-3.19).

17. The sum signal will go to zero with the new imaging medium. Adjust the mirror position to achieve the maximum sum signal again.

18. Adjust the horizontal position of the photodiode to obtain a Horizontal Difference signal close to 0.0 volts.

19. Adjust the Vertical Difference to about -2.0 volts.

20. Watch the vertical deflection signal. It will probably drift for 10-15 minutes as the temperature stabilizes and thermal stresses on the cantilever are relaxed.

21 . The system is now ready to engage the tip and start imaging in liquid. 


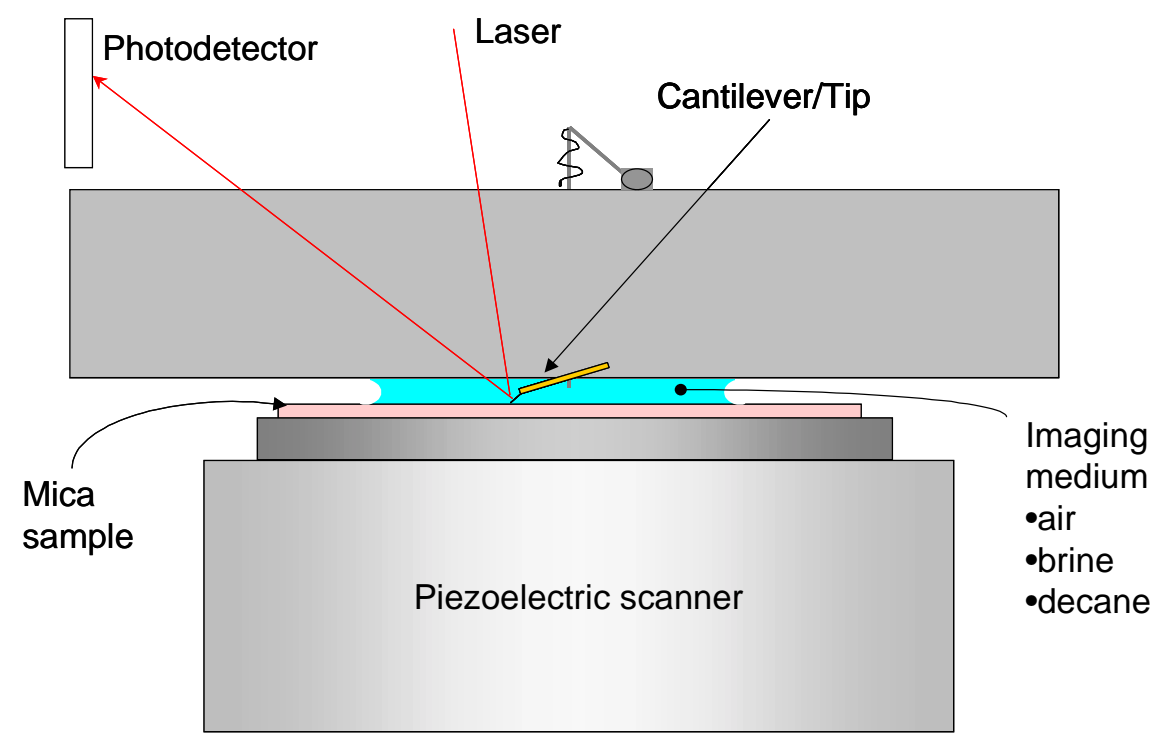

Figure II-3.19. Side view of instrument alignment with fluid cell, cantilever/tip, mica sample, and imaging medium in place.

\section{B. Common problems}

Atomic Force Microscopy requires a considerable degree of patience and diligence in order to obtain quality images. Recognizing common problems is an important step in learning the AFM technique, and will help overcome many frustrating hours of unproductive trial-anderror. The most comprehensive resource for identifying common problems is the manufacturer literature. In particular, the section "Typical Image Artifacts" in the Scanning Probe Microscopy Training Notebook (Digital Instruments, 1998) provides concise but helpful clues to sorting out real image features from the multitude of artifacts which appear in AFM images. The suggestions listed here relate to problems that are common to imaging crude oil samples, and not necessarily addressed in the manufacturer literature.

\section{CANTILEVER DRIFT}

Instrument alignment calls for careful alignment of tip, laser, and sample. Once aligned, the configuration is not necessarily static. Thermal drift will cause the cantilever to deflect slowly, and the contact AFM output signal value (Vertical Difference) will drift as a result. This is especially apparent when a liquid is inserted as the imaging medium. The immediate result of adding a new imaging fluid is a change in the refractive property of the fluid, which diverts the laser orientation instantaneously. This can be corrected by adjusting the mirror to realign the laser with the photodetector. The drift phenomenon will follow once the laser has been re-aligned, and should slow exponentially with time. Usually the drift has slowed sufficiently after about 10 minutes that imaging can commence. 


\section{LOOSE SAMPLE ADHESIVE}

Occasionally the substrate comes loose from the stainless steel puck due to failure of the adhesive. This is easy to detect and fix prior to placing the sample in the AFM, but can be subtle and confusing if it happens while imaging. Typically, when the adhesive fails, image features disappear, and the sample appears to have a considerable bow. The only way to fix this problem is to disengage the tip, remove and dry the sample, and re-attach it to the stainless steel puck with new adhesive. Touching the edge of the sample with tweezers and watching its position closely with respect to the stainless puck can indicate when the sample is loose.

The manufacturer provides adhesive tabs which work well to fix dry samples to the stainless puck. Imaging in liquids, however, can expose the adhesive to solvents which tend to loosen the sample. A better alternative for crude oil systems is $3 \mathrm{M}$ Super Glue Gel, which is inexpensive and readily available at any hardware store.

\section{STICKY SAMPLE SURFACE}

Not all crude oil samples may be imaged with the AFM according to the methods described here. One problematic scenario occurs when the organic film deposited on the substrate causes excessive tip-sample adhesion. Several samples exhibiting this behavior were encountered during the method development, and images were difficult or impossible to obtain. One example image is shown in Figure II-20, depicting a mica slide exposed to Mars Yellow crude for 1 day. A Force Calibration Plot image (not shown) indicated tip-sample adhesion throughout the entire z-range of the plot. This implies that the tip was "stuck" to the surface and would not snap off. The considerable horizontal streaking and lack of feature resolution in Figure II-20 bears this out. One potential strategy for overcoming such sticky surfaces is to try TM-AFM to get the tip out of the adhesive layer, but as the scan in Figure II-3.21 shows, little information could be drawn from this alternative technique for the sample shown. 


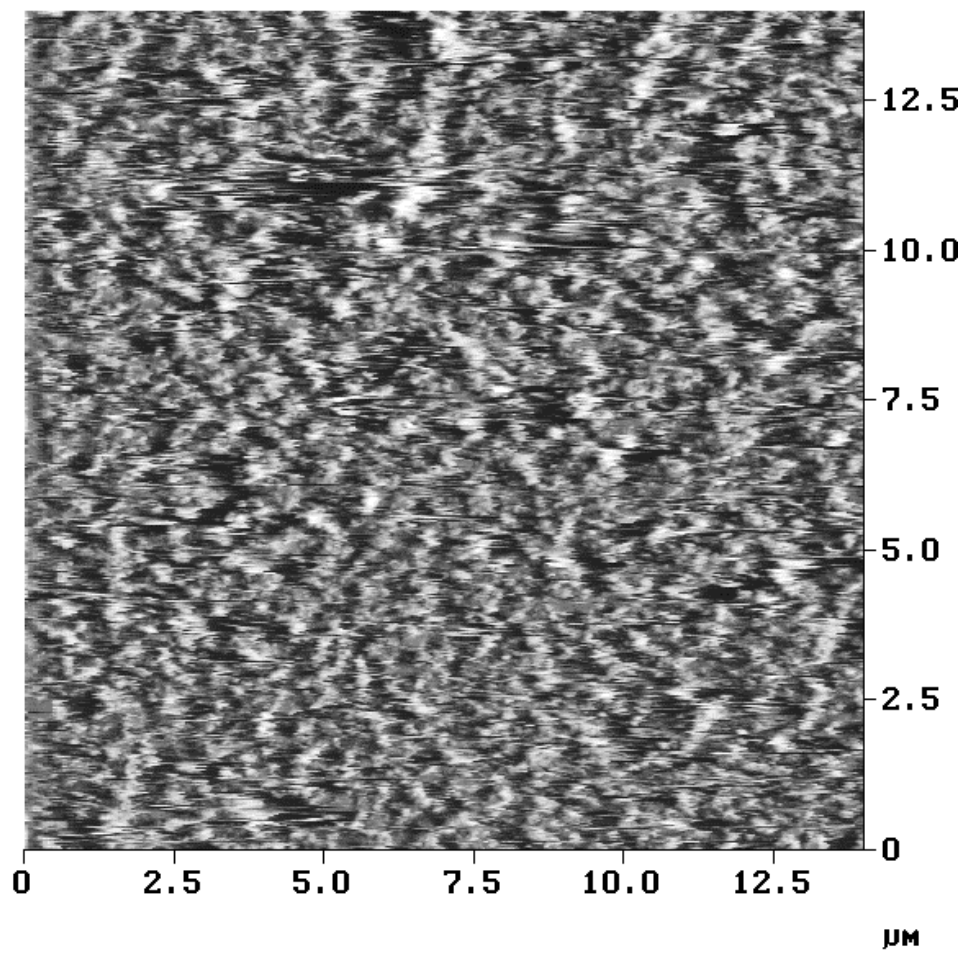

\begin{tabular}{|l|l|}
\hline & 00.0 nm \\
250. $\mathbf{~ n m ~}$ \\
\hline
\end{tabular}

Figure II-3.20. Contact mode AFM deflection image of mica exposed to Mars Yellow crude for 1 day. Excessive tip-sample adhesion caused the poor image quality.

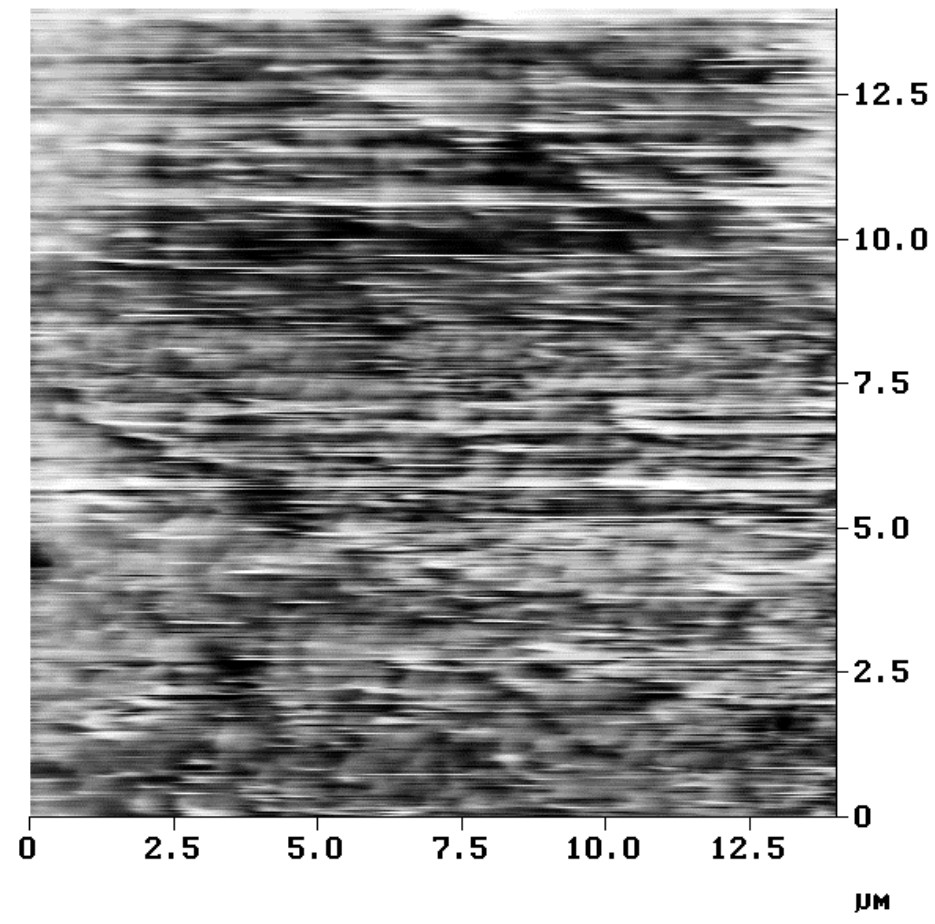

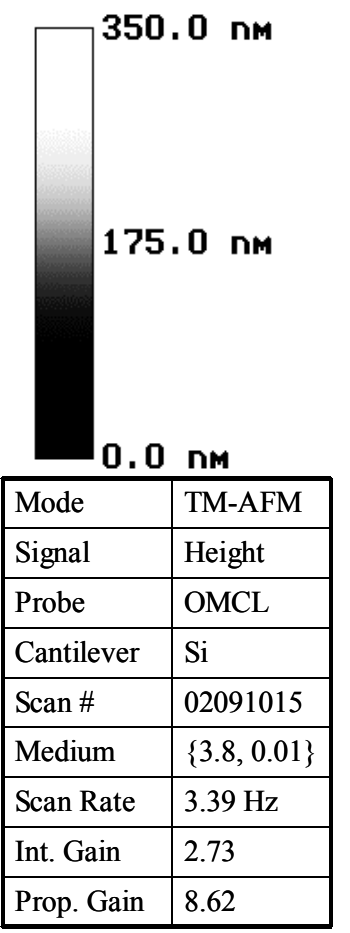

Figure II-3.21. Tapping mode AFM height image of mica exposed to Mars Yellow crude for 1 day. Excessive tip-sample adhesion caused the poor image quality. 


\section{CANTILEVER CORROSION}

The acidic acetate brine used throughout the method development described here was found to react with the reflective gold coating on the back of the $\mathrm{Si}_{3} \mathrm{~N}_{4}$ cantilevers after 20 or more minutes of immersion. For this reason, the aqueous imaging medium was changed to ultrapure water in more recent experiments. Decane and toluene did not appear to have any adverse effects on the AFM apparatus.

\section{File management}

AFM data are saved in raw data files (filename.001) which only the AFM software will readily recognize and open. Data files include date/timestamp information, scan parameters, and the height and/or deflection signal image(s). Raw files for a given scan vary in size from 0.5-1.0 MB depending on whether 1 (separate signal capture) or 2 (simultaneous signal capture) images were captured. Typical AFM installations require that the user bring some form of media to transfer data from the AFM controller hard drive to a remote storage location for long-term storage. Most installations have a $100 \mathrm{MB}$ ZIP drive available, so bringing two $100 \mathrm{MB}$ ZIP disks to the lab should cover data transfer and storage needs for any given day of imaging.

AFM data are typically viewed and presented as 2-D images. Since only the AFM controller software will recognize the raw data files, separate image files must be created if the images are to be viewed or exported to another application. The AFM software offers an option to create Tagged Image File Format (TIFF) files which all current graphics applications will recognize. TIFF files of AFM scans average about $0.77 \mathrm{MB}$ in size, so the actual disk space requirements for raw images plus TIFF files averages from 1.3-2.6 MB per scan.

Offline AFM analysis software for NT operating systems is available for Digital Instruments Nanoscope IIIa installations from the manufacturer. The offline software is very useful because (1) it allows for image analysis at a remote workstation at any time after the data are captured, and (2) reduces user instrument time because the data only need to be captured and transferred to the appropriate storage media instead of captured, analyzed, and then transferred. Users interested in obtaining the offline software must contact the manufacturer of the AFM directly. 


\section{II-3.2. Application of the AFM Technique to Mica Surfaces Exposed to Crude Oil}

Buckley, J.S. and Lord, D.L.: "Wettability and morphology of mica surfaces after exposure to crude oil," $7^{\text {th }}$ Internat. Symp. on Reservoir Wettability, Freycinet, Tasmania, 12-14 Mar. 2002. (accepted for publication in J.Petrol.Sci.Eng., 2003)

Although the porous rocks through which oil must flow to reach recovery wells are mainly formed of minerals with high surface energies, models of flow that assume strongly water-wet conditions do not predict either the rate or extent of oil production. The opposite extreme, assuming strongly oil-wet conditions, is usually not any more predictive. In fact, a more complex mixed wetting situation, first envisioned by Salathiel (1973), where bicontinuous pathways of preferential water-wetting and oil-wetting coexist, is now considered likely in many, if not most, oil reservoirs (Cuiec, 1991).

Diagnosis of complex wetting scenarios that involve an aqueous phase, one or more hydrocarbon phases and heterogeneous solid surfaces is limited by the tools available. The resolution of most imaging techniques (e.g., x-ray, gamma ray, or NMR) does not yet distinguish subpore-scale fluid distributions. Static images of uniformly wetted pores have been produced by solidifying fluid phases in place, then polishing to inspect two-dimensional sections (McKellar and Wardlaw, 1988) or dissolving the rock matrix to recover three-dimensional casts of the non-wetting phase (Chatzis et al., 1983). Neither of these techniques has been adapted to mixed-wet conditions, however. In very small samples, environmental SEM has been used to show differences in wetting tendencies of pore surfaces (Gauchet et al., 1993; Robin et al., 1999), but the evidence is limited to images of the solid and fluids in contact with a vapor phase.

In the absence of in situ measurement techniques, wetting is often inferred from experimental measurements of pressures and flow velocities of immiscible fluids as they flow through the pore spaces of a sample of rock. The information gained from such experiments is, however, insufficient to define uniquely the complexities of mixed-wetting, as demonstrated by a relatively simple network model that incorporates mixed-wetting (Dixit et al., 1999).

An alternate approach has been to study the interactions between the components of crude oil/brine/rock ensembles outside the confines of a porous medium. XPS shows differences in surface coverage, but direct links to other measures of wetting remain to be demonstrated (Mitchell et al., 1990; Durand and Beccat, 1998). Contact angles between crude oils and aqueous solutions have been measured on smooth mineral surfaces (Treiber et al., 1972; Hjelmeland and Larrondo, 1986). However, pinning of the three-phase line of contact often interferes with water-advancing measurements (Buckley et al., 1989; Morrow, 1990). To circumvent pinning, contact angles between pure fluids (e.g., decane and water) have been used to probe wetting conditions after exposure of smooth mineral surfaces to crude oil (Liu and Buckley, 1997; Yang and Hirasaki, 1999).

Contact angle tests on oil-treated surfaces help to delineate the conditions under which components from crude oil interact with mineral surfaces to alter their surface properties (Buckley et al., 1998). The simplest interactions are between basic components in the oil and an acidic surface or the inverse, acidic oil components adsorbing on a basic surface. Depending on $\mathrm{pH}$ and ionic strength of solutions of monovalent salts, acid/base interactions can be enhanced or prevented. Mica surfaces are negatively charged above a $\mathrm{pH}$ of about 2, whereas the isoelectric 
point of most crude oils is around $\mathrm{pH} 5$ or 6 . Pretreating mica with a low ionic strength $\mathrm{NaCl}$ solution with $\mathrm{pH}$ adjusted to about 4 should promote acid/base interactions. Higher $\mathrm{pH}$ and somewhat higher ionic strength can stabilize a thin film of water that prevents components from the oil from adsorbing on a mica surface. Changing the stability of asphaltenes, by changing temperature, pressure, or oil composition, can alter the tendency of aggregates of these large molecules to adsorb (Al-Maamari and Buckley, 2000). Other specific interactions that are likely to be important in reservoir situations have been recognized, but are not investigated in this study.

Contact angles are measured using small drop volumes. Nevertheless, the technique is inherently macroscopic. Surfaces exposed to crude oil are likely to be quite heterogeneous on the scale sampled by a single drop. A more detailed understanding of interactions between oil components and pore-lining minerals requires examination of the surfaces at a resolution beyond that provided by contact angles. Atomic Forces Microscopy (AFM) has shown that there is material on surfaces after exposure to solutions of asphaltenes (Toulhoat, 1994) or to crude oil (Buckley, et al., 1997; Yang and Hirasaki, 1999), but little differentiation of that material has previously been obtained in either tapping or contact mode in air.

Optimization of the contact mode technique and imaging oil-treated mica in aqueous and hydrocarbon environments can produce detailed images of surface morphology that correlate with contact angles between probe fluids on those surfaces and help to explain the crude oil/brine/rock interactions that determine wetting conditions in oil reservoirs.

\section{Methodology}

The methodology, described in detail in the previous section, is a combination of established and novel methods by which the wetting characteristics of crude oil-treated surfaces were examined. Of particular interest here is the application of atomic force microscopy to characterization of surface films and aggregates formed on the mica surface as a result of exposure to crude oils. The atomic force microscope has the capacity to resolve nanometer-scale surface features in a liquid environment, potentially a very useful tool for determining the mechanisms behind wettability changes in crude oil-rock-brine systems.

\section{Materials.}

\section{MICA SUBSTRATE}

The substrate used in all measurements was freshly cleaved Muscovite mica (S\&J Trading, Inc., Glen Oaks, NY). For the AFM imaging, $12.7 \mathrm{~mm}$ diameter discs were punched from a sheet. For contact angle samples, $11 \mathrm{~mm} \times 30 \mathrm{~mm}$ mica plates were cut with scissors. Cut plates or discs were cleaved by pressing tape to the top and bottom surfaces of the sample, slowly peeling the tape away to expose a fresh cleavage plane. Samples were handled at the edges with a pair of tweezers and promptly immersed in brine solution.

A sample of the clean mica substrate was imaged under brine in order to establish the starting point prior to exposure to crude oil (Figure II-3.22). No surface features are visible at the resolution shown, consistent with prior observations (Yang et al., 1999) for an identical 
surface. A roughness analysis of a $5 \mu \mathrm{m} \times 5 \mu \mathrm{m}$ area in the middle of Figure II-3.22 indicated a mean roughness of $R_{a}=0.16 \mathrm{~nm}$, about twice the value reported by Yang et al. (1999). The likely cause of this difference stems from the imaging technique used to capture the respective images. The deflection signal shown here tracks a regular oscillation of the tip during scanning. While the magnitude of oscillation is negligible compared to features that will be shown later, those oscillations are on the same order as the resident roughness of the mica surface. When reporting height signal as in Yang et al. (1999), small tip oscillations are not registered because the height signal represents the z-position of the substrate, not the tip.



Figure II-3.22. AFM scan of clean mica.

\section{BRINE SOLUTIONS}

Ultrapure water was used to prepare all solutions, and was obtained by passing distilled, deionized water through Milli-Q cartridge filters (Millipore, Bedford, MA), and distilling once more prior to use. Acidic brine was prepared by adding sodium acetate trihydrate $\left(\mathrm{NaC}_{2} \mathrm{H}_{3} \mathrm{O}_{2} \bullet 3 \mathrm{H}_{2} \mathrm{O}\right)$ (Fisher Scientific, Fair Lawn, NJ), acetic acid $\left(\mathrm{HC}_{2} \mathrm{H}_{3} \mathrm{O}_{2}\right)$, and sodium chloride $(\mathrm{NaCl})$ in measured amounts to obtain a $\mathrm{pH}=3.8,\left[\mathrm{Na}^{+}\right]=0.01 \mathrm{M}$ solution. Basic brine was prepared by adding dibasic sodium phosphate 7-hydrate $\left(\mathrm{Na}_{2} \mathrm{HPO}_{4} \bullet 7 \mathrm{H}_{2} \mathrm{O}\right)$ (J.T. Baker, Phillipsbug, NJ), monobasic sodium phosphate $\left(\mathrm{NaH}_{2} \mathrm{PO}_{4}\right)$ (Aldrich Chemical Company, Milwaukee, WI), and $\mathrm{NaCl}$ in measured amounts to obtain a $\mathrm{pH}=8.0,\left[\mathrm{Na}^{+}\right]=1.0 \mathrm{M}$ solution. A shorthand notation indicates brine $\mathrm{pH}$ and sodium ion concentrations as $\left\{\mathrm{pH},\left[\mathrm{Na}^{+}\right]\right\}$.

A $\mathrm{pH}=3.8,\left(\mathrm{Na}^{+}\right)=0.01 \mathrm{M}$ solution of acetate brine was dried on the clean mica surface in order to examine the features formed upon precipitation of salts. The sample was imaged under both air (Figure II-3.23) and decane (Figure II-3.24) at various locations and showed no discernable differences according to imaging medium. The salt precipitate structures took two 
basic forms: (1) needles, and (2) smooth patches. The needles were typically 200-500 nm wide and longer than $14 \mu \mathrm{m}$. The patchy material did not have any characteristic dimension, though its roughness measured from $R_{a}=2.5-4.0 \mathrm{~nm}$.

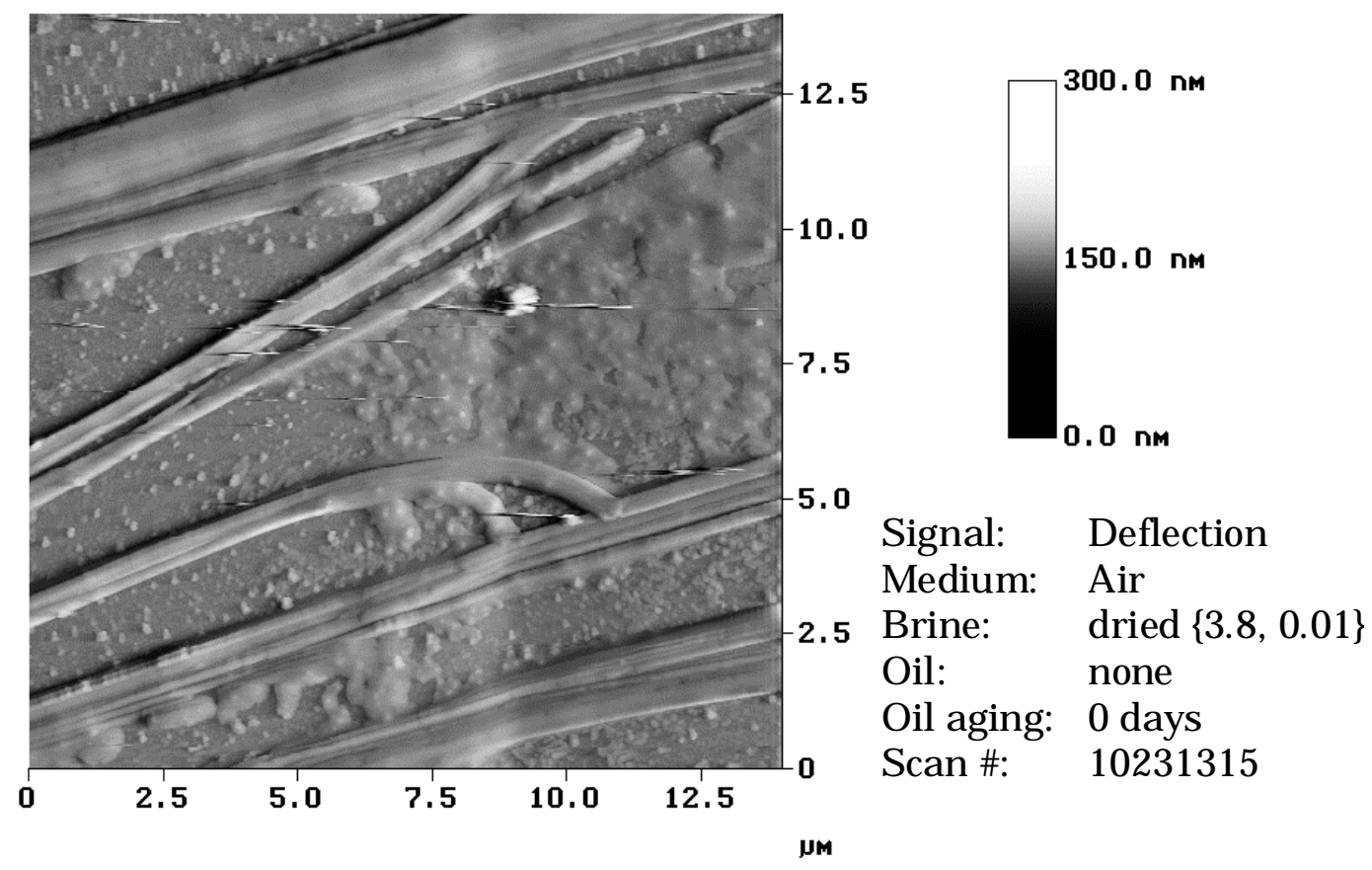

Figure II-3.23. AFM scan of $\{3.8,0.01\}$ acetate brine dried on mica, imaged under air.

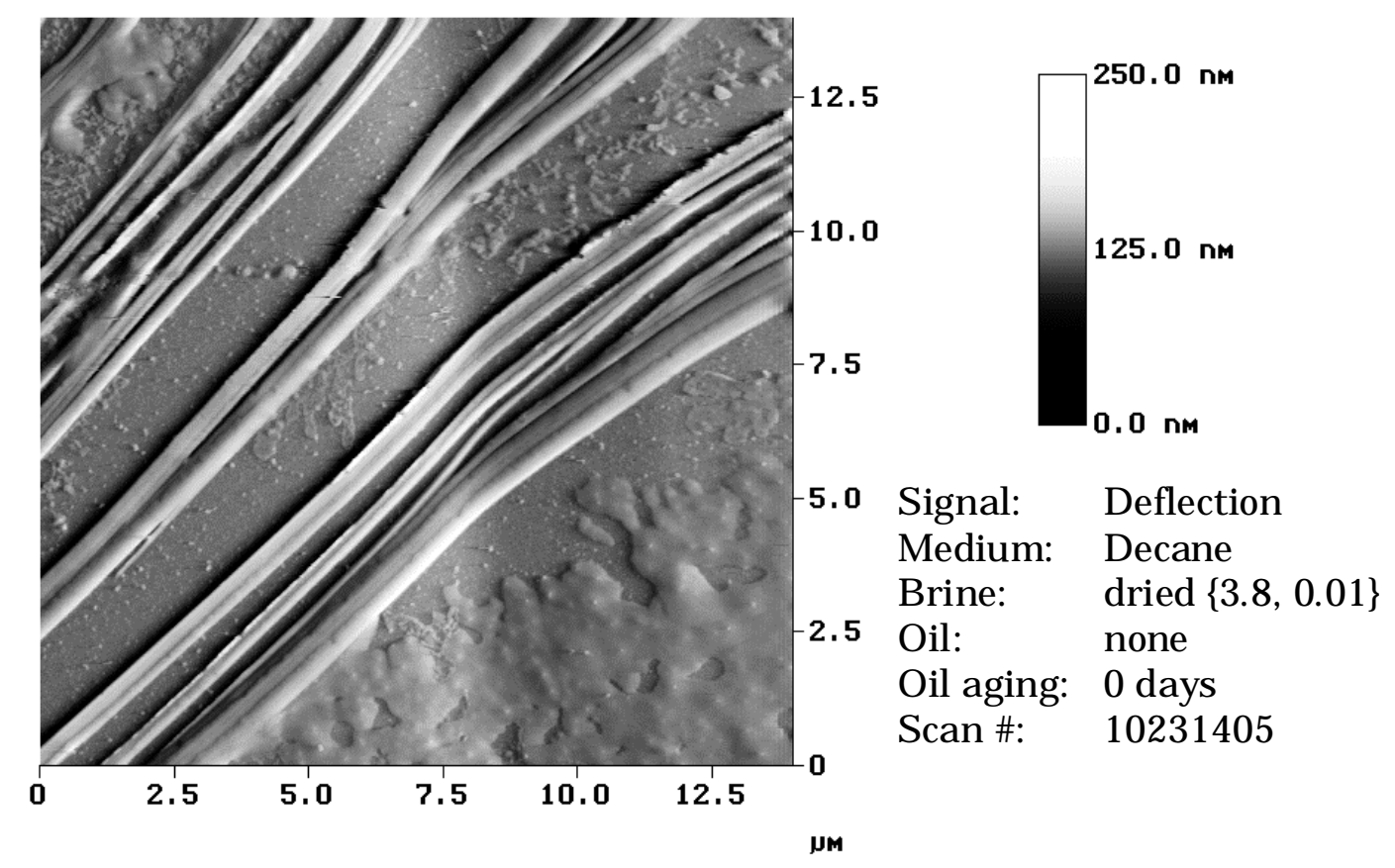

Figure II-3.24. AFM scan of $\{3.8,0.01\}$ acetate brine dried on mica, imaged under decane. 


\section{CRUDE OILS}

Some physical and chemical properties of the laboratory sample of the crude oils used in this study are summarized in Table II-3.3. Crude oil from the Tensleep formation, Wyoming, was provided by the University of Wyoming. Microscopic inspection showed that the sample contained aggregates of asphaltene and some emulsified water.

Table II-3.3. Physical and chemical properties of crude oils

\begin{tabular}{|c|c|c|c|c|c|c|}
\hline & Tensleep & Mars Y & Mars P & Lost Hills & E-1XD & E-1XR \\
\hline Gravity ( $\left.{ }^{\circ} \mathbf{A P I}\right)$ & 31.2 & 30.3 & 16.5 & 22.6 & 22.3 & 29.1 \\
\hline $\mathrm{RI}_{\text {oil }}\left(\text { at } 20^{\circ} \mathrm{C}\right)^{\mathrm{a}}$ & 1.4876 & 1.4952 & 1.5384 & 1.5137 & 1.5141 & 1.4912 \\
\hline$P_{\mathrm{RI}}(\mathrm{nC} 7)^{\mathrm{b}}$ & 1.467 & 1.4298 & 1.4288 & 1.4231 & 1.4336 & 1.4071 \\
\hline asphaltenes $(\% \mathrm{nC} 7)^{\mathrm{c}}$ & 3.2 & 1.86 & 4.77 & 2.78 & 2.54 & 0.27 \\
\hline$\rho\left(\right.$ at $\left.20^{\circ} \mathrm{C}, \mathrm{g} / \mathrm{ml}\right)$ & 0.8682 & 0.8804 & 0.9524 & 0.9161 & 0.9165 & 0.8781 \\
\hline$\mu\left(\right.$ at $\left.20^{\circ} \mathrm{C}, \mathrm{mPa} s\right)$ & 14 & 25 & 481 & 90 & 137 & 21 \\
\hline Acid \# (mg KOH/g oil) ${ }^{d}$ & 0.16 & 0.37 & 3.92 & 1.90 & 1.56 & 0.54 \\
\hline Base \# (mg KOH/g oil $)^{\mathrm{e}}$ & 0.96 & 1.79 & 2.3 & 6.05 & 2.98 & 2.02 \\
\hline Base\# / Acid\# & 6 & 4.8 & 0.6 & 3.2 & 1.9 & 3.7 \\
\hline $\mathrm{MW}(\mathrm{g} / \mathrm{mol})^{\mathrm{f}}$ & 271 & 258 & 309 & 268 & 287 & 218 \\
\hline
\end{tabular}

${ }^{a}$ Index Instruments (GPR11-70) measured at the wavelength of the sodium D line.

${ }^{\mathrm{b}}$.RI at first appearance of microscopically visible asphaltene aggregates.

${ }^{\mathrm{c}}$.Precipitated by 40 parts n-heptane to 1 part oil (ASTM D2007-80).

d.ASTM D664-89.

'.ASTM D2896-88, as modified by Dubey and Doe (1993).

${ }^{\mathrm{f}}$.By freezing point depression (Precision Systems Cryoscope 5009).

Compared to other crude oils, Tensleep is a poor solvent for its asphaltene fraction. Refractive index (RI) of the oil is low and the onset RI $\left(\mathrm{P}_{\mathrm{RI}}\right)$ for precipitation induced by addition of $n$-heptane is quite high. The difference between the two is only $0.02 \mathrm{RI}$ units. Tensleep has a fairly high ratio of base number to acid number; adsorption of crude oil components can be expected on acidic mica surfaces under conditions of low $\mathrm{pH}$ and low ionic strength where the mica is negatively charged whereas the oil/water interface has a net positive charge.

\section{HYDROCARBONS}

Toluene and 1-methylnaphpthalene (1-MN) used to disperse asphaltenes in the crude oil were HPLC grade and used as received. Decane used as the probe fluid in contact angle measurements and imaging medium in AFM experiments was purified by passing HPLC grade material through dual-packed columns of activated silica gel and alumina.

SOLVENTS

All solvents used for glassware cleaning, including isopropyl alcohol, acetone, toluene, hydrochloric acid, and ammonium hydroxide, were 99\%+ grade (Aldrich Chemical Company, Milwaukee, WI) and used as received from the manufacturer. 
SURFACE PREPARATION AND AFM TECHNIQUES

The surface preparation and AFM techniques were those described in detail in Section II3.1 above.

\section{CONTACT ANGLE BY CAPTIVE DROP METHOD}

All contact angles were measured using a contact angle goniometer (Gaetner Scientific, Chicago, IL) by the captive drop method (Gaudin et al., 1963). A glass microburet was used to form and then withdraw a water droplet against a mica surface immersed in decane. Unless otherwise noted, the water phase was a brine identical to that used to initially condition the mica surface. Contact angles measured through the water or brine phase are reported. The wateradvancing angle $\left(\theta_{\mathrm{A}}\right)$ is measured with a newly expanded water drop, whereas the water-receding angle $\left(\theta_{R}\right)$ is measured as the water drop is withdrawn. If the three-phase contact line pins, the water receding angle can be measured with a drop of decane expanding over a similarly treated mica surface immersed in water or brine. Typically, a minimum of 5 different areas are tested on each mica sample.

\section{Results and Discussion}

Experimental results are organized according to surface treatment protocol. The top-level parameter is the composition of the oil phase. For each oil-phase composition, results are subcategorized by exposure time in oil.

The atomic force micrographs shown here represent either the deflection signal or the height signal as indicated on the figure legend. The magnitude of the signal is designated by grayscale. Also on the figure legend, medium denotes imaging medium, brine indicates the brine composition that was used to condition the mica prior to exposure to oil, oil denotes oil phase, oil aging corresponds to the amount of time that the mica was aged in the oil, and scan \# is a date/timestamp which uniquely identifies each scan.

\section{Tensleep crude oil}

Tensleep crude oil contains asphaltene aggregates visible under an optical microscope (Figure II-3.25). The oil background color is light gray, while the asphaltenes appear as the brown, particulate material clustered together. Maximum resolution of the optical microscope is around $1 \mu \mathrm{m}$ (Campbell, 1993), so asphaltene aggregates smaller than this are not discernable. When observing such images in the context of wetting behavior, it is important to recognize that the aggregates in the optical image are suspended in the oil phase, and do not represent material which is selectively sorbed to the surface of the microscope slide. Alternatively, AFM images depict structures formed on the surface of the imaging substrate resulting from the surface treatment procedure. If asphaltene aggregates do appear in AFM images, they must first adsorb to the substrate with sufficient adhesive force so that they survive rinsing and the scanning process detects their presence without displacing them. 


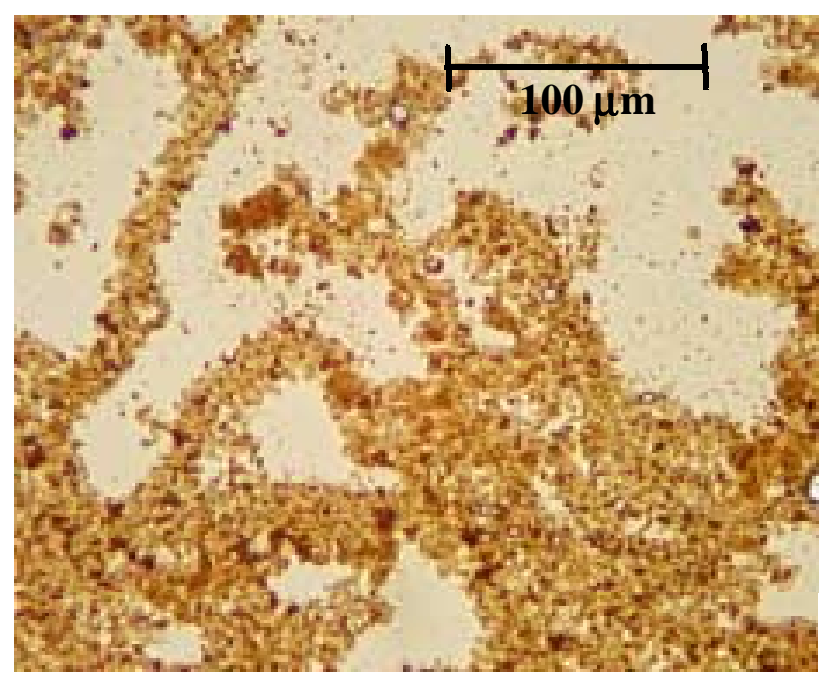

Figure II-3.25. Optical microscope image of Tensleep crude. Asphaltene aggregates appear as dark particulate material while oil background color is light gray.

Features detected by the AFM, which appear as the result of surface treatment procedures, need not originate from solid aggregate structures already existing in solution. Substances such as surfactant molecules may adsorb from solution to form surface micelles or aggregate structures. For example, exposing a clean mica disk to $7 \mathrm{mM}$ myristyltrimethylammonium bromide $\left(\mathrm{CH}_{3}\left(\mathrm{CH}_{2}\right)_{13} \mathrm{~N}\left(\mathrm{CH}_{3}\right)_{3} \mathrm{Br}\right)$ for an hour induced the formation of surface hemicylinders visible as meandering stripes shown in Figure II-3.26. The diameter of the stripes correspond to roughly twice the length of the surfactant molecule $(2.3 \mathrm{~nm})$ in this case. Several published studies (Manne et al., 1994; Manne and Gaub, 1995; Jaschke et al., 1997) support these observations. Since crude oils contain numerous surface-active constituents, surface aggregates are expected to form on mineral surface exposed to oil independently from the asphaltenes.

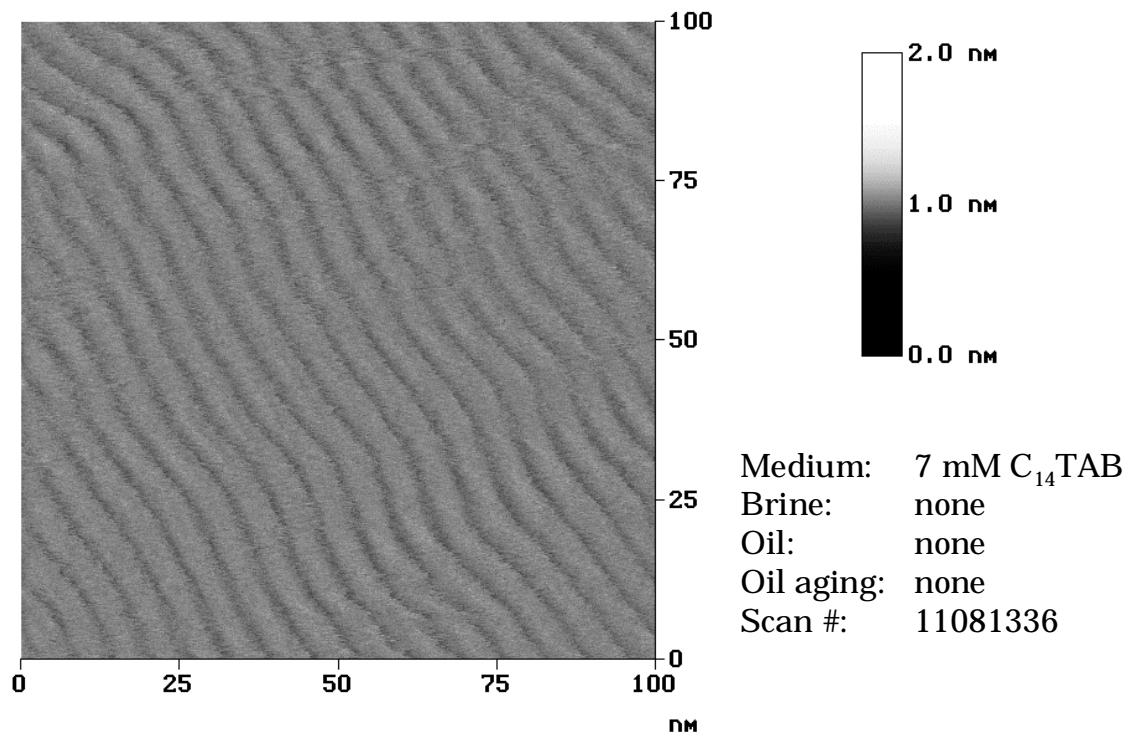

Figure II-3.26. AFM scan of cationic surfactant on mica at $2 \times$ CMC. Cylindrical surface micelles run diagonally in above scan. Molecule length of $\sim 2.3 \mathrm{~nm}$, corresponds to radii of cylinders. 


\section{TENSLEEP CRUDE}

Aging time: 1 day

Mica exposed to $\{3.8,0.01\}$ brine for 1 day and then Tensleep crude for 1 day exhibited decane-water contact angles of $\theta_{\mathrm{A}}=130^{\circ}$ for water-advancing conditions, and $\theta_{\mathrm{R}}=61^{\circ}$ for water-receding conditions. Both angles indicate a significant decrease in hydrophilicity compared to clean mica, which is strongly water-wet. Two important factors are likely responsible for such changes in wettability. First, the Tensleep crude is a poor solvent for its asphaltenes, indicated by the presence of visible aggregates under the optical microscope (Figure II-3.25). These aggregates tend to concentrate at surfaces, leading to decreases in water-wetness (Buckley et al., 1998). Also, the low ionic strength, low $\mathrm{pH}$ brine on the mica surface forms an unstable brine film, which likely collapsed and allowed the oil phase, rich in precipitated asphaltenes, to contact the mica.

Brine imaging medium. Aging the mica substrate in Tensleep crude oil clearly resulted in the formation of surface features (Figures II-3.27 and II-3.28) distinct from the clean mica case (Figure II-3.22). Surface coverage on sample 0811 shown in Figure II-3.27 is characterized by a film perforated with regularly-spaced and roughly circular holes measuring about 0.25 to 1 $\mu \mathrm{m}$ in diameter.

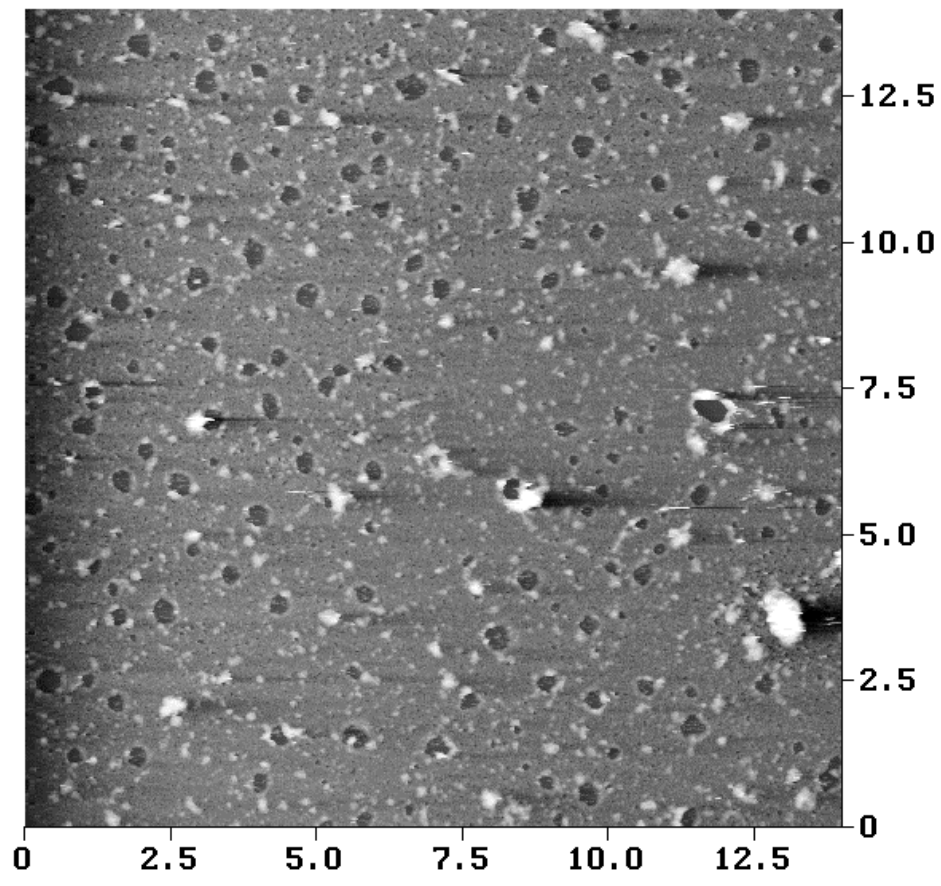

JM

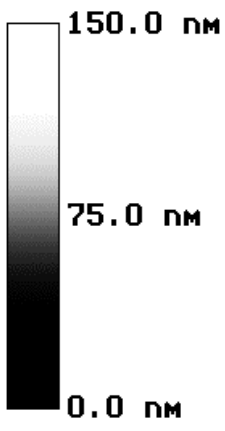

Signal: Deflection

Medium: $\quad\{3.8,0.01\}$

Brine: $\quad\{3.8,0.01\}$

Oil: $\quad$ Tensleep

Oil aging: 1 day

Scan \#: 08111204

Figure II-3.27. AFM scan of mica exposed to Tensleep crude. 


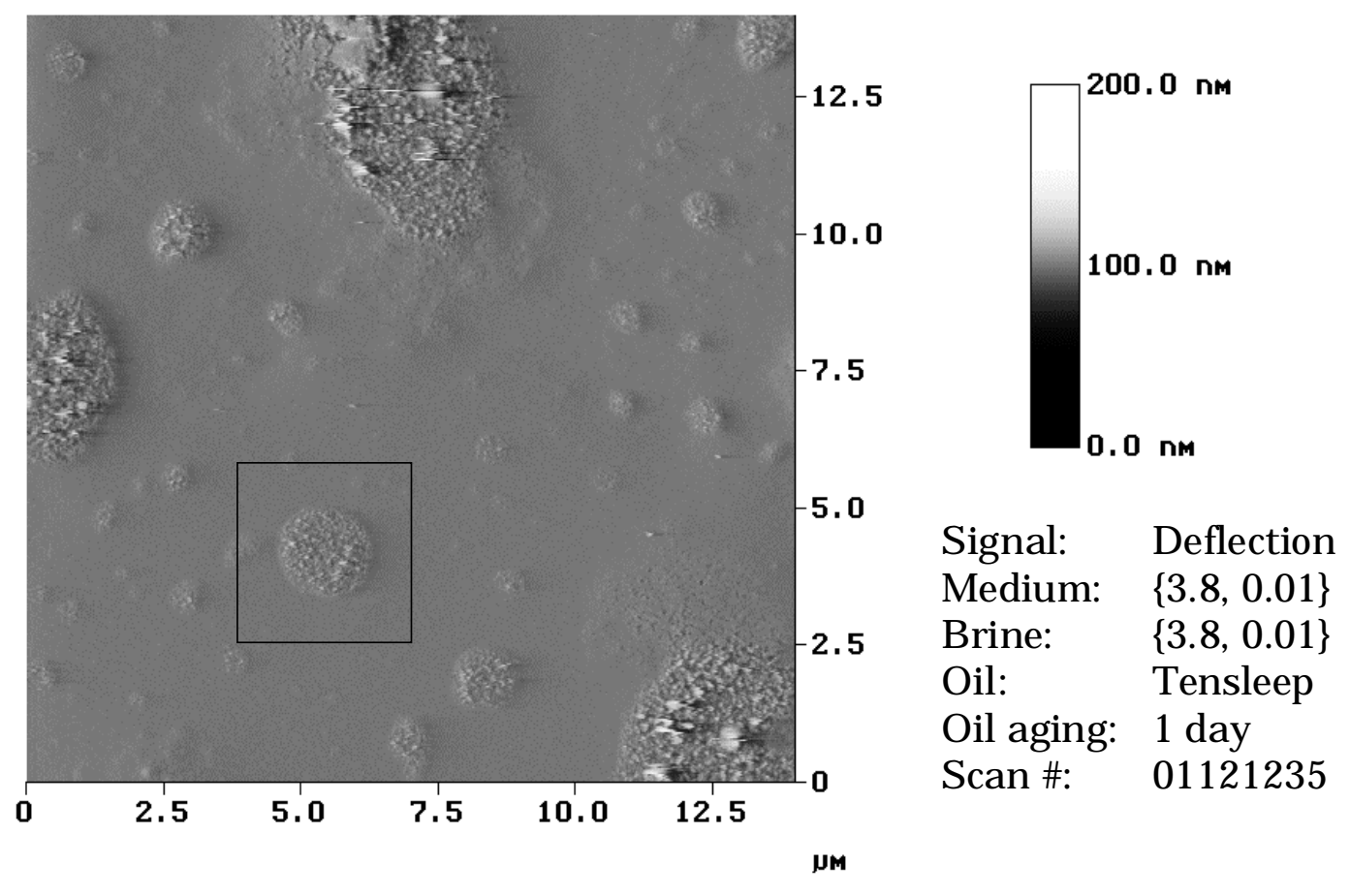

Figure II-3.28. AFM scan of mica exposed to Tensleep crude. A zoom of the area indicated by the box is shown in Figure II-3.30.

The use of the term "hole" to describe interruptions in what appears to be a continuous film of adsorbed material is in part figurative. In some cases, including sample 0811, the material at the base of the holes appears, by qualitative inspection, to be mica, or mica with small, isolated deposits of material which resembles some of the smallest discernible structural units of the film material. If bare mica is exposed, we can infer that the :hole" is topographically lower that the surrounding film. There will also be cases, however, where the "hole" is an interruption in the film that is completely filled with another material. In such cases, the real difference is not in height, but in interactions with the tip and what are described as "holes" might actually be accumulations of material with lower tip-surface forces than the surrounding material.

The approximate depth of the film at the edge of the holes was determined by performing a section analysis (Figure II-3.29) of the scan in Figure II-3.27 . The section analysis is an offline function that plots the height or deflection signal from an AFM scan along an arbitrary line chosen by the user. The section analyzed in Figure II-3.29 is indicated by the horizontal black line on the grayscale inset image. The line plot at the top of Figure II-3.29 represents the deflection signal along the section. The two triangular cursors may be moved along the section, and parameters such as surface distance, horizontal distance, and vertical distance between the cursors are given in the table in the lower right corner. For the particular section shown, one cursor was placed in a low region of a representative hole in Figure II-3.27, and the other cursor was placed on the film that appears to cover most of the sample surface. The vertical distance in this particular case was $12.8 \mathrm{~nm}$, as indicated in the table in Figure II-3.29. Ten such film depths 
were surveyed in various holes on Figure II-3.27 using the above technique, resulting in a mean value of $\delta=12.7 \mathrm{~nm}$, with a standard deviation of $\sigma=1.0 \mathrm{~nm}$.

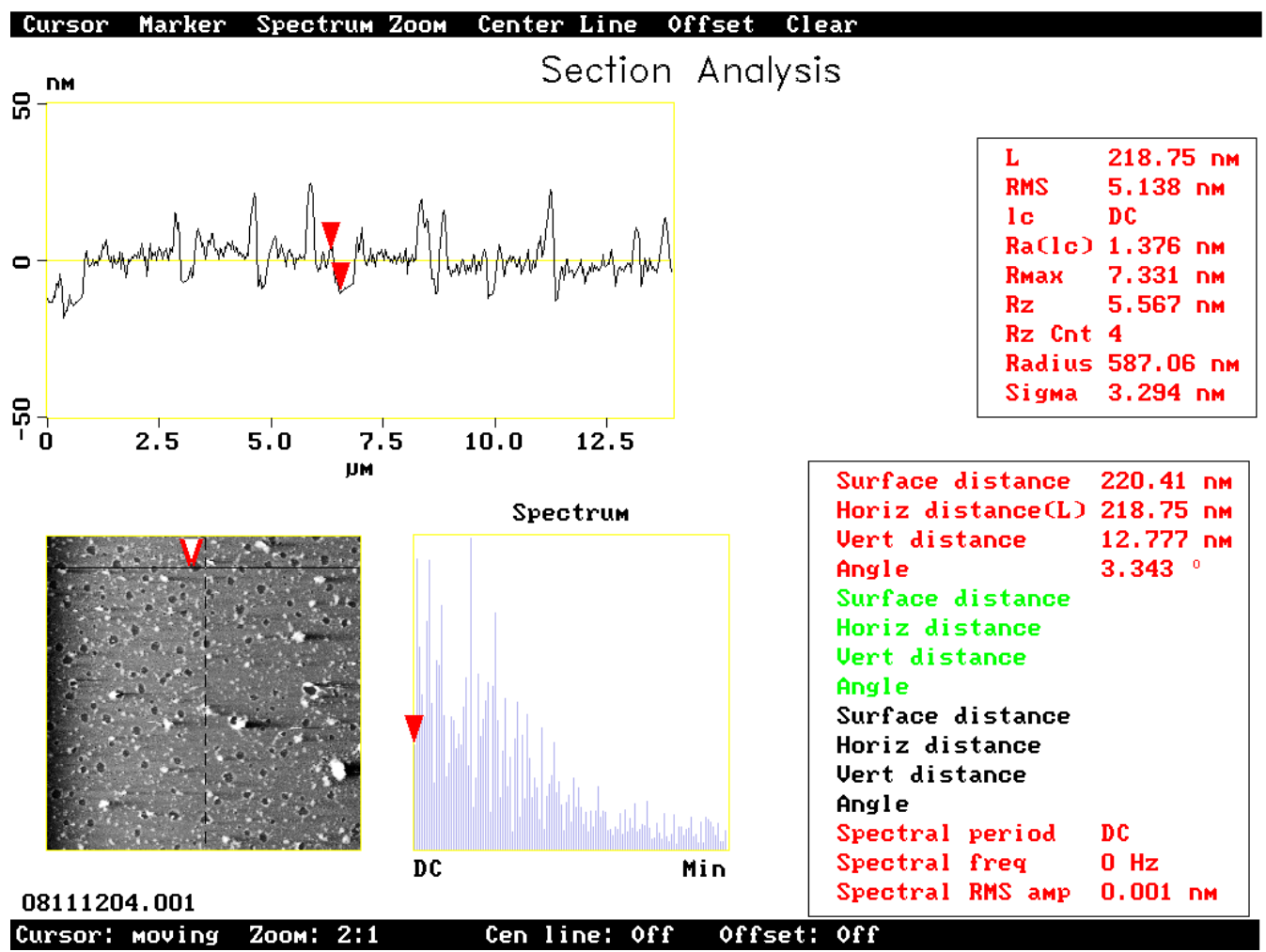

Figure II-3.29. Section analysis of scan 08111204 . The cursors were placed in order to determine the film thickness at the edge of a hole. The vertical distance between the cursor positions is $12.8 \mathrm{~nm}$ here.

Two hypotheses regarding the origin of these holes, both relating to particular steps in the surface treatment protocol, can be considered. The first possibility is that small water droplets shielded the mica from sorption of oil-soluble components. The mica in this system was immersed in brine and therefore coated with a continuous water film prior to exposure to the crude oil. Upon immersion in the oil, however, the water film collapsed and allowed surface precipitation of asphaltenes to proceed, evidenced by a marked increase in contact angle. It is possible that the collapse of the water film left behind discreet droplets, which, in turn, left holes in the surface film. A second hypothesis is that asphaltene aggregates were deposited on the surface and shielded the mica from the bulk oil. The toluene wash that follows exposure to oil may have rinsed away asphaltenes that were deposited by settling, but not sorbed, leaving a bare spot. The film material that remains on the surface is likely a collection of polar compounds, some of which may also be components of the asphaltene fraction (i.e., not soluble in an excess of heptane). Resins may also contribute to the adsorbed layer. All of these components have significant solubility in toluene, but because they are adsorbed on the mica surface they are not removed by the brief exposure to toluene during the rinsing step.

Some scans on sample 0112, such as that in Figure II-3.28 and the corresponding zoom in Figure II-3.30, have a fairly continuous film without distinct holes. Rough regions, several of which are conspicuously circular, range in diameter from approximately 1-5 $\mu \mathrm{m}$. The circular shape of these features suggest that these may have originated at the phase boundary between fluids - presumably water and oil. Material that was either hydrophilic and suspended in the 
water phase or amphipathic such that it collected at the oil-water boundary would mark the presence of water droplets in this manner. Also possible is that the water phase was eventually dissolved into the oil phase, and oil-insoluble substances such as the ionic salts that comprise the brine were precipitated on the substrate.

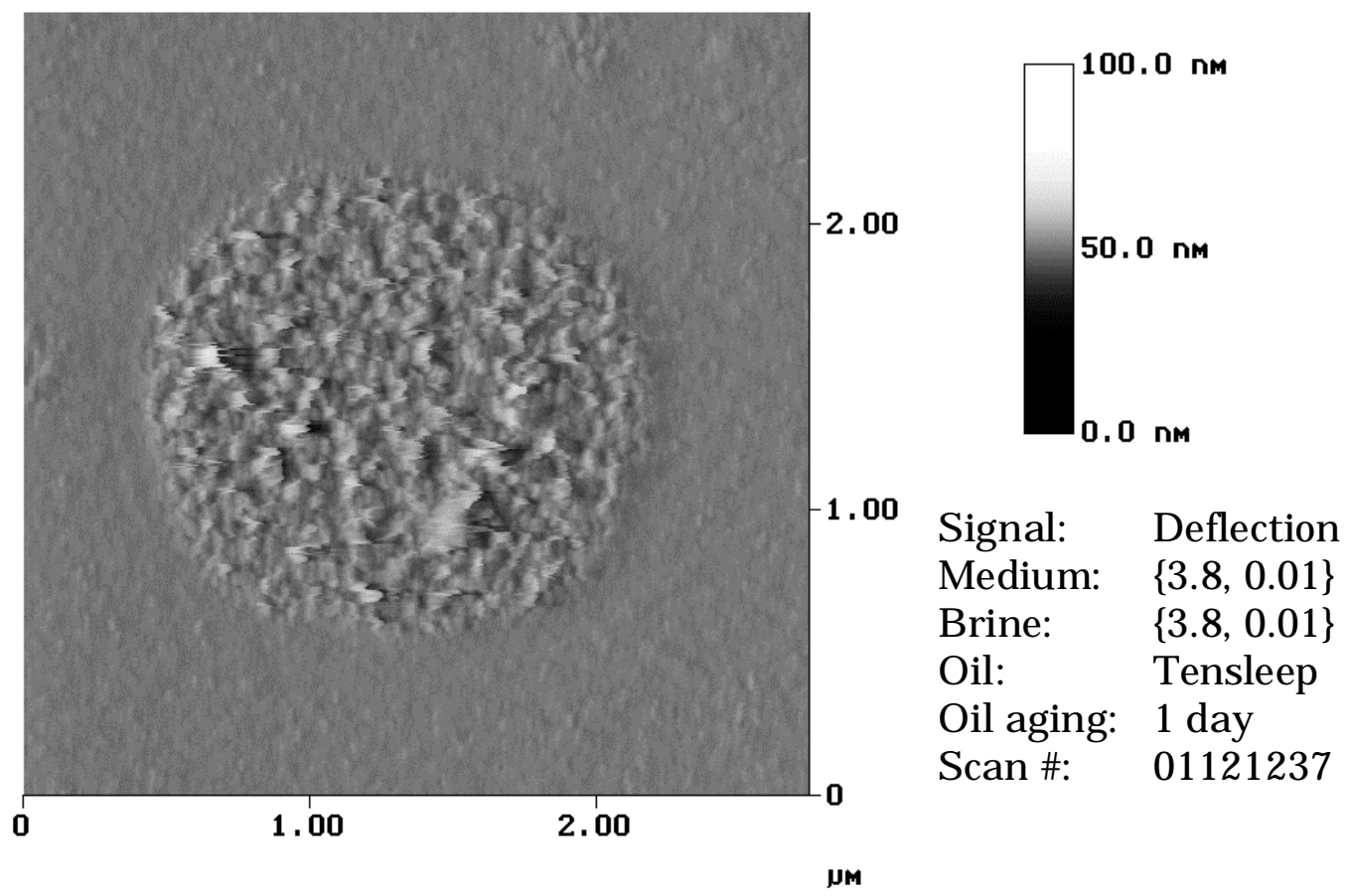

Figure II-3.30. AFM scan of mica exposed to Tensleep crude. Zoom of area indicated in Figure II-3.28.

Decane imaging medium. Replacing the $\{3.8,0.01\}$ brine with decane (Figures II-3.31, II-3.32) did not effect any immediate changes to the film structure. A zoom (Figure II-3.33) in the lower left corner of Figure II-3.31 shows that some of the holes contain sorbed material, though the thickness of this material is less than that of the bulk film. It is possible that water droplets shielded the surface here from adsorption, limiting the extent of film thickness in these spots. 


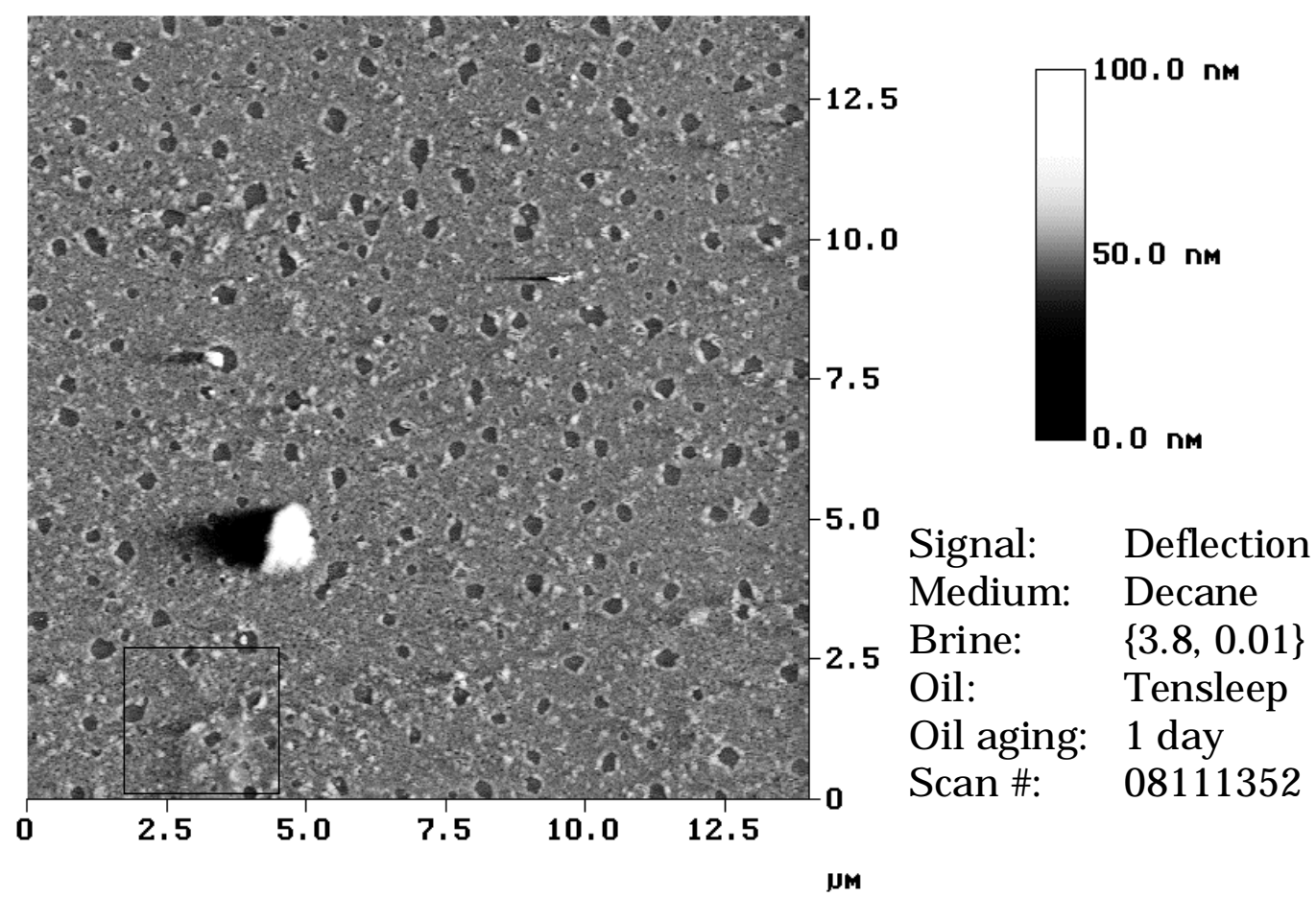

Figure II-3.31. AFM scan of mica exposed to Tensleep crude. A zoom of the area indicated by the box is shown in Figure II-3.33.

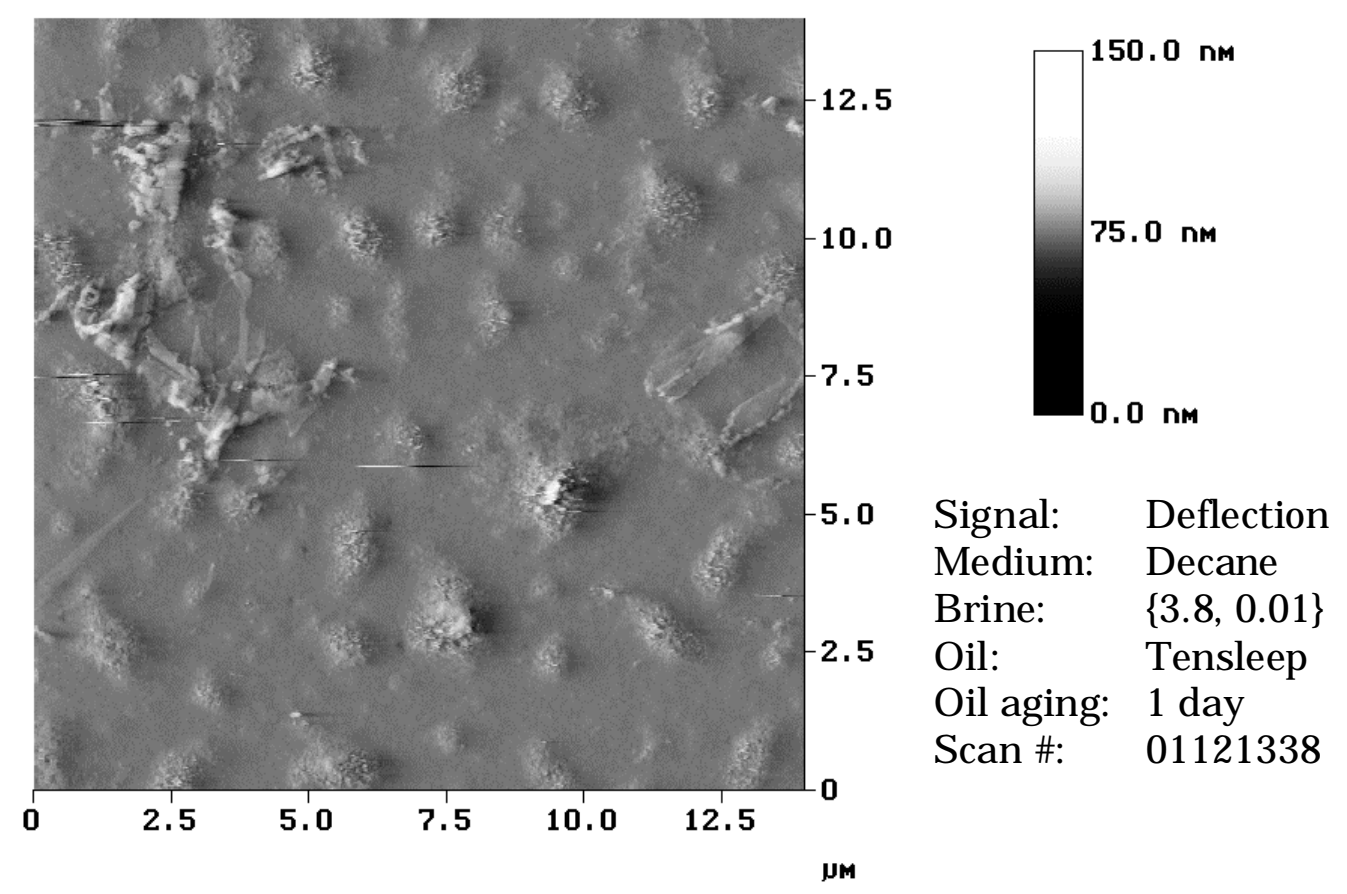

Figure II-3.32. AFM scan of mica exposed to Tensleep crude. 


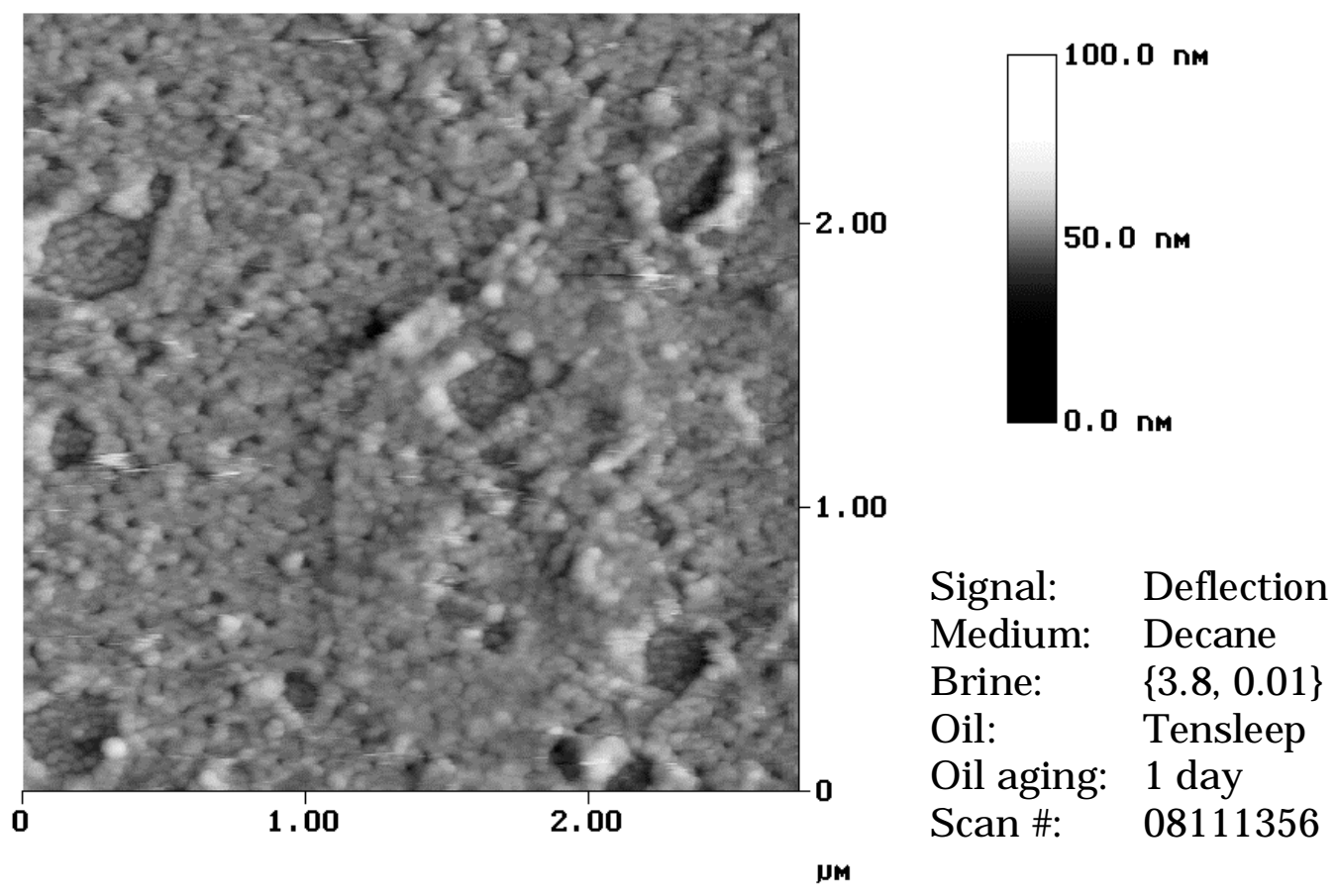

Figure II-3.33. AFM scan of mica exposed to Tensleep crude. Zoom of area indicated in Figure II-3.31.

A survey of several scan areas on sample 0112 revealed some nonuniformities in film structure. For example, Figure II-3.34 showed nearly complete coverage near the top of the scan, while scattered holes ranging in linear dimensions of $100 \mathrm{~nm}$ up to $5 \mu \mathrm{m}$ were evident in the lower portion of the scan. Also, the entire scan in Figure II-3.35 was dominated by holes in variety of shapes, with some significantly elongated while others were roughly circular. A zoom (Figure II-3.36) and section (Figure II-3.37) of the largest, elongated hole in Figure II-3.34 shows spotty sorption at the bottom of the hole. A sampling of ten different depth measurements around the perimeter of this hole yielded a mean film thickness at the rim of $\delta=13.3 \mathrm{~nm}$, with $\sigma$ $=1.6 \mathrm{~nm}$. This corresponds quite closely with the film thickness of $\delta=12.7 \pm 1.0 \mathrm{~nm}$, measured for the other 1-day sample (Figure II-3.29). Though the shapes of the holes seem to differ widely, the film which comprises the bulk of coverage on the surface appears to exhibit a relatively constant thickness. 


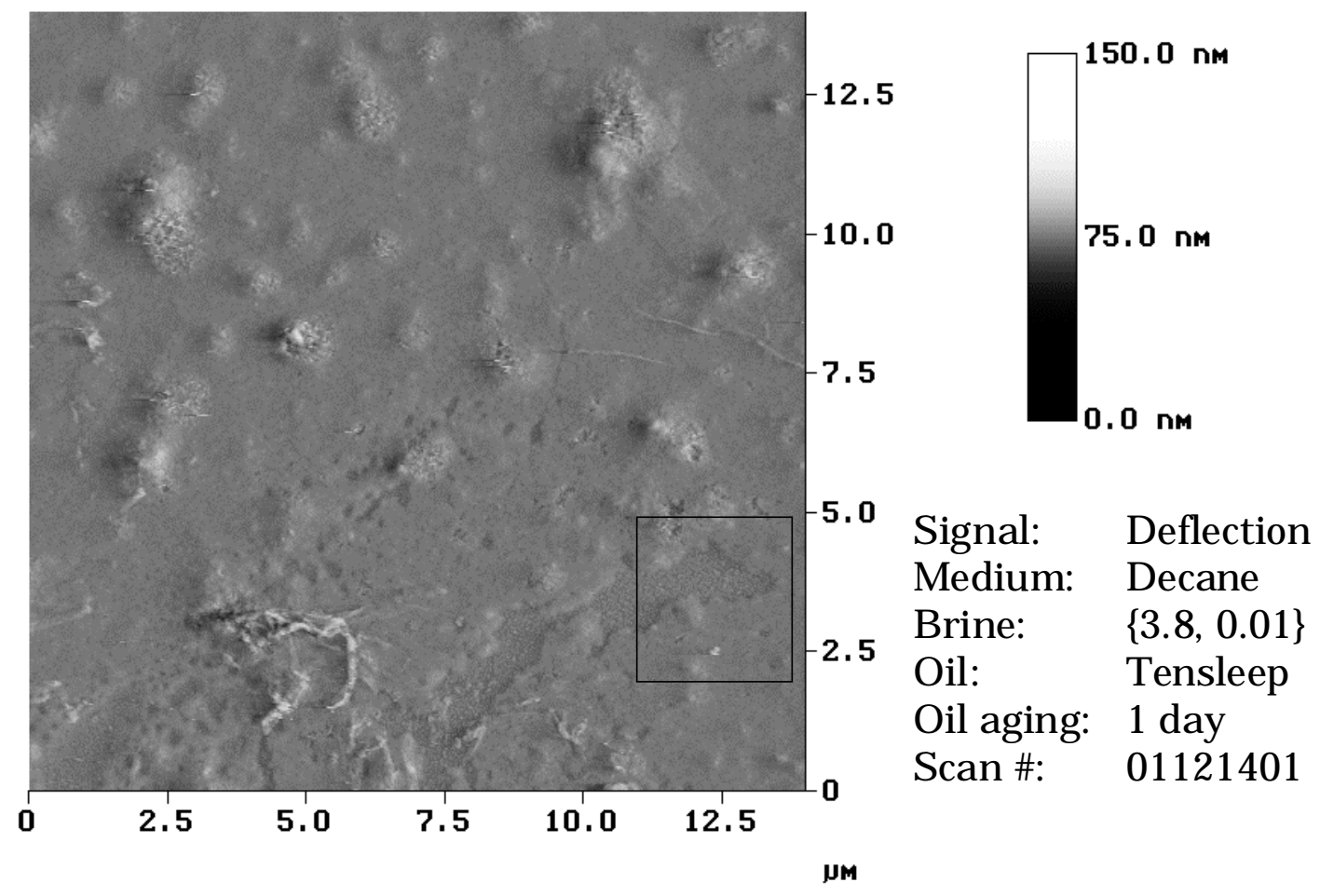

Figure II-3.34. AFM scan of mica exposed to Tensleep crude.

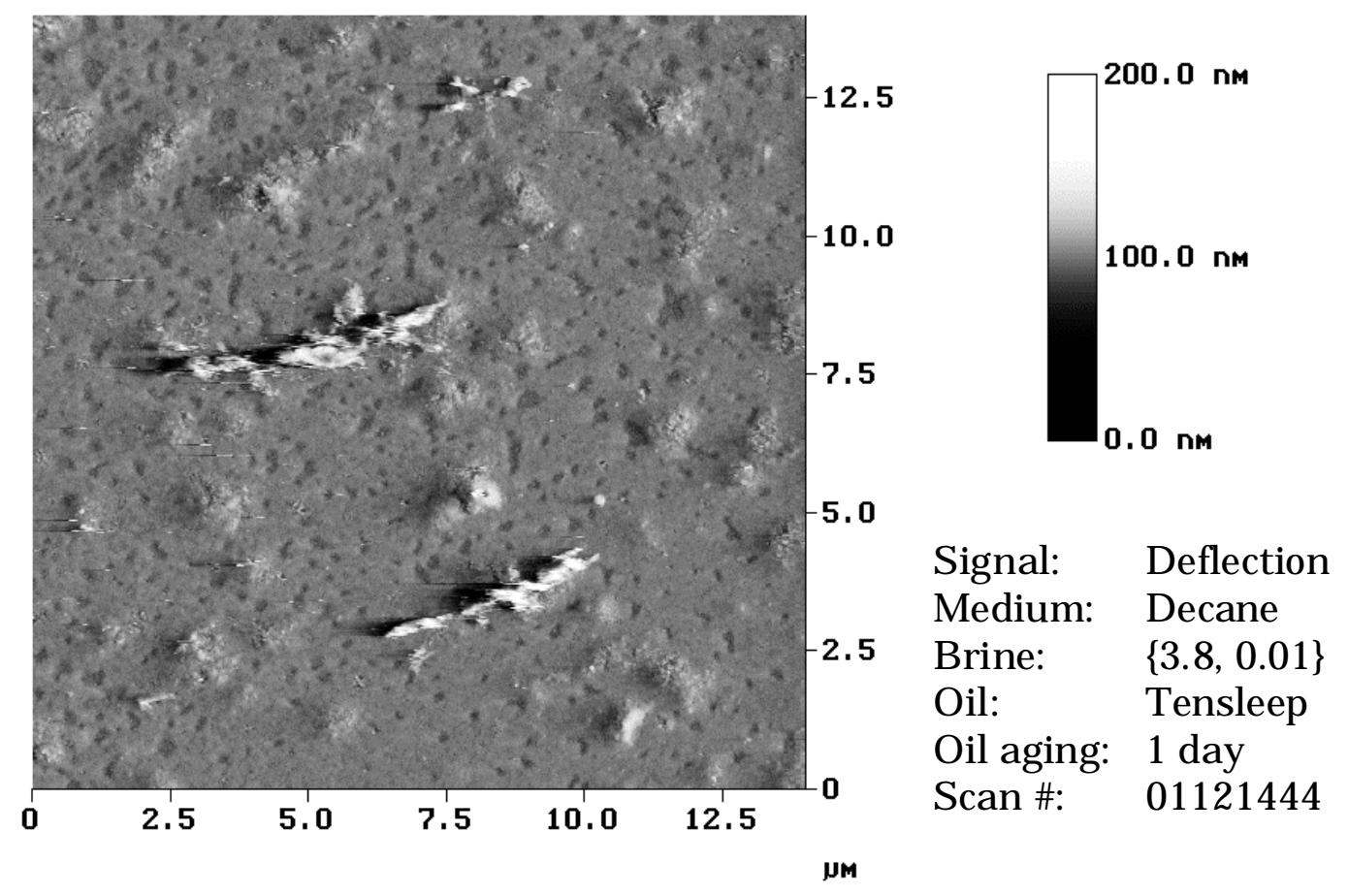

Figure II-3.35. AFM scan of mica exposed to Tensleep crude. 


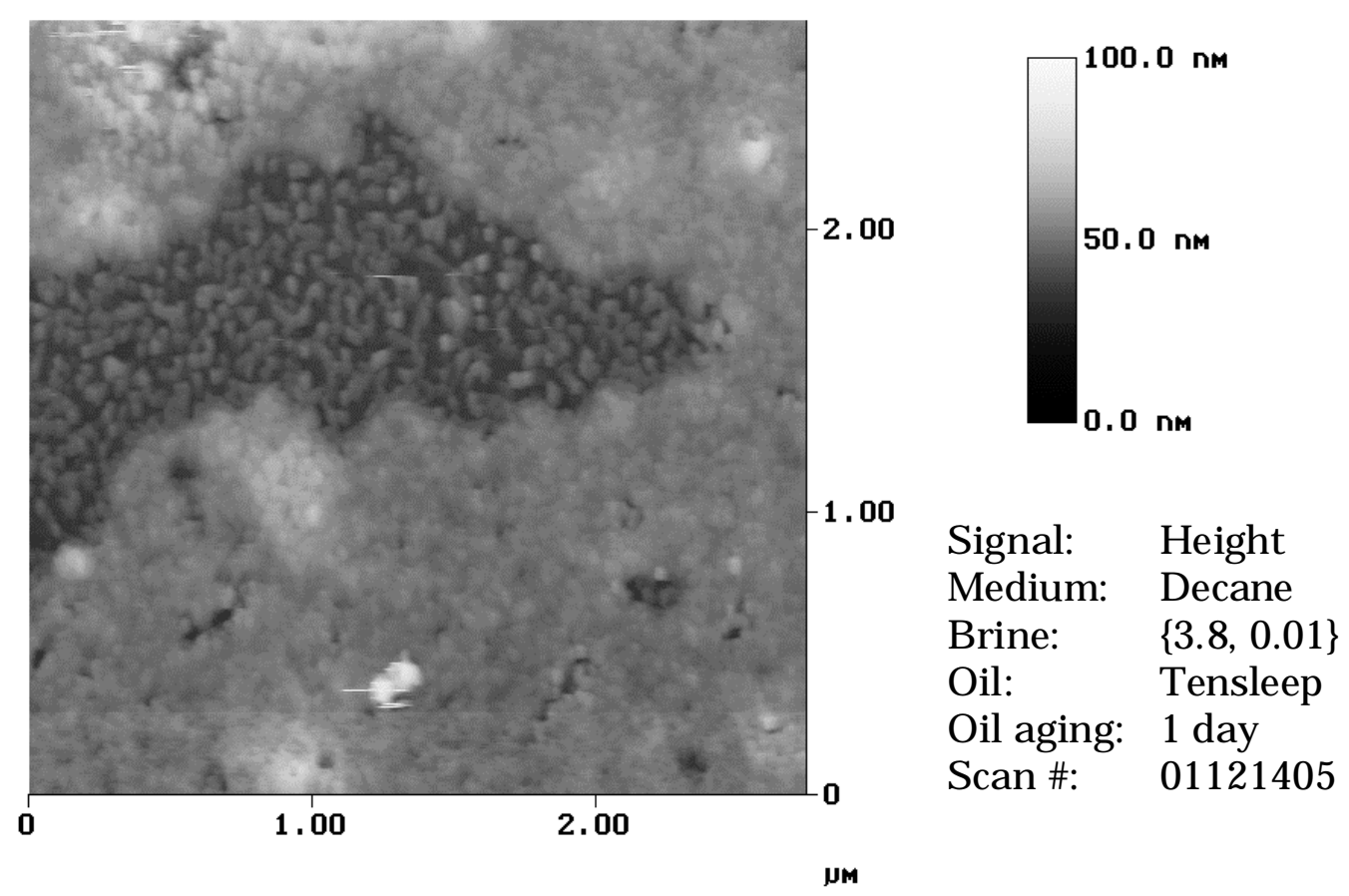

Figure II-3.36. AFM scan of mica exposed to Tensleep crude. Zoom of area indicated in Figure II-3.34.
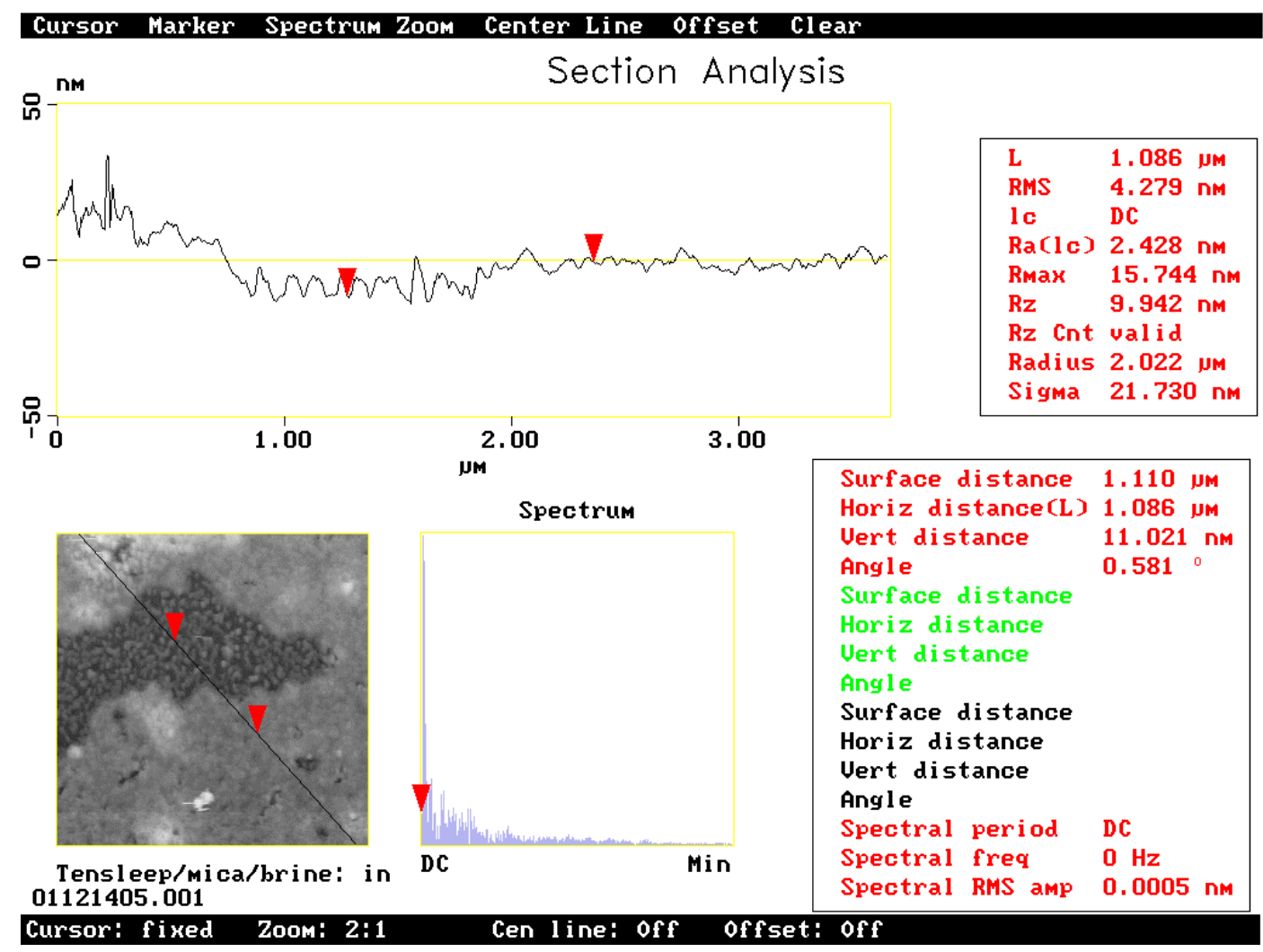

Figure II-3.37. Section analysis of scan 01121405 . The film thickness indicated by the vertical distance between cursors measures about $11 \mathrm{~nm}$. 
A time series of images was captured on sample 0811 in order to monitor the effect of aging the sample for about two hours in decane. Decane an effective solvent for some crude oil components, but it precipitates asphaltenes. To the extent that asphaltenes form the surface film, decane would not be expected to dissolve it significantly. Figures II-3.38 and II-3.39 show identical regions of a sample aged under decane for 28 minutes and 99 minutes, respectively. Features appear to remain unchanged during this time period. A zoom (Figure II-3.40) of a region of Figure II-3.39 shows a network-like coverage with bare mica visible in the holes.

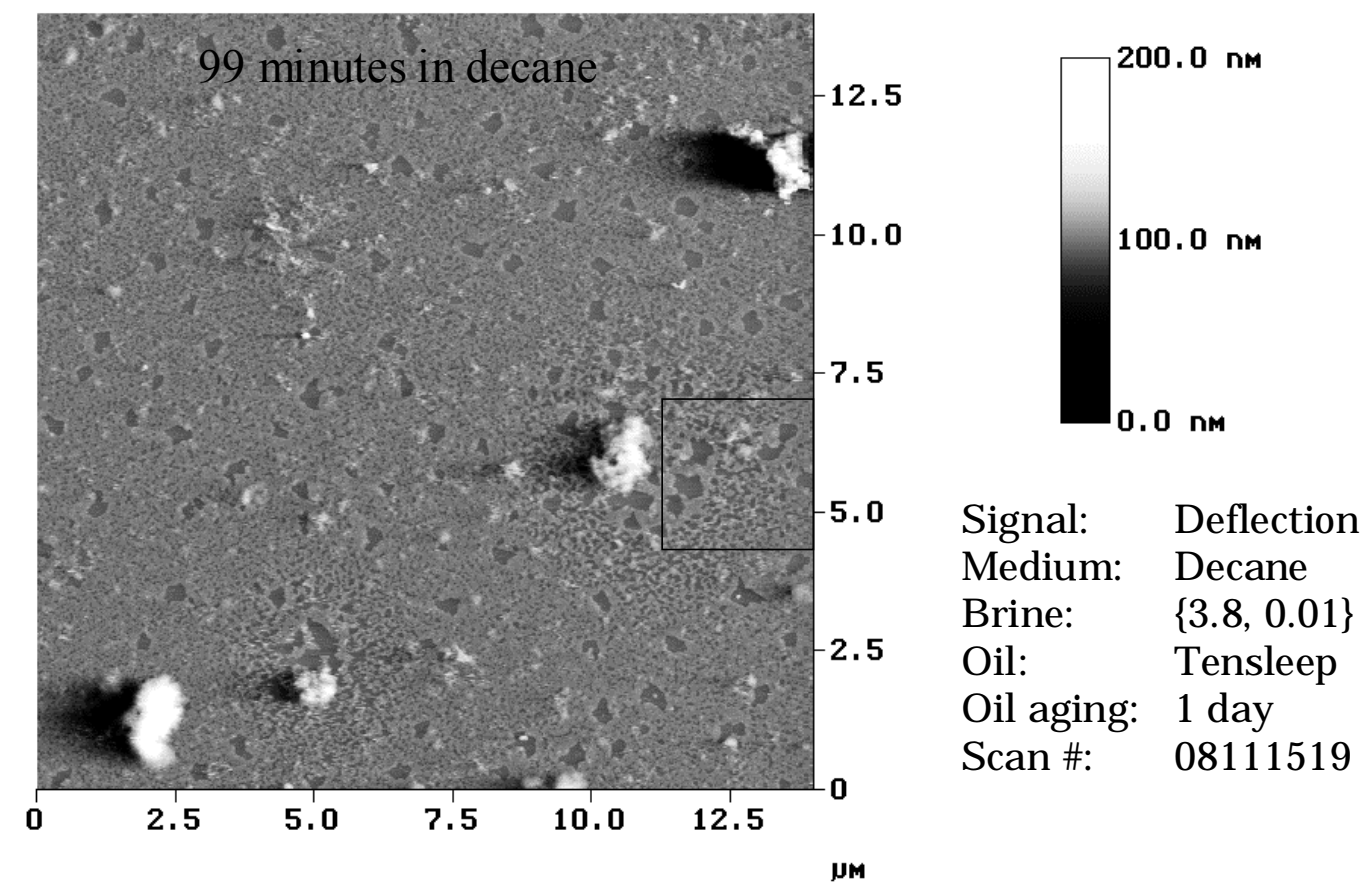

Figure II-3.38. AFM scan of mica exposed to Tensleep crude. Surface immersed in decane for 28 minutes. 


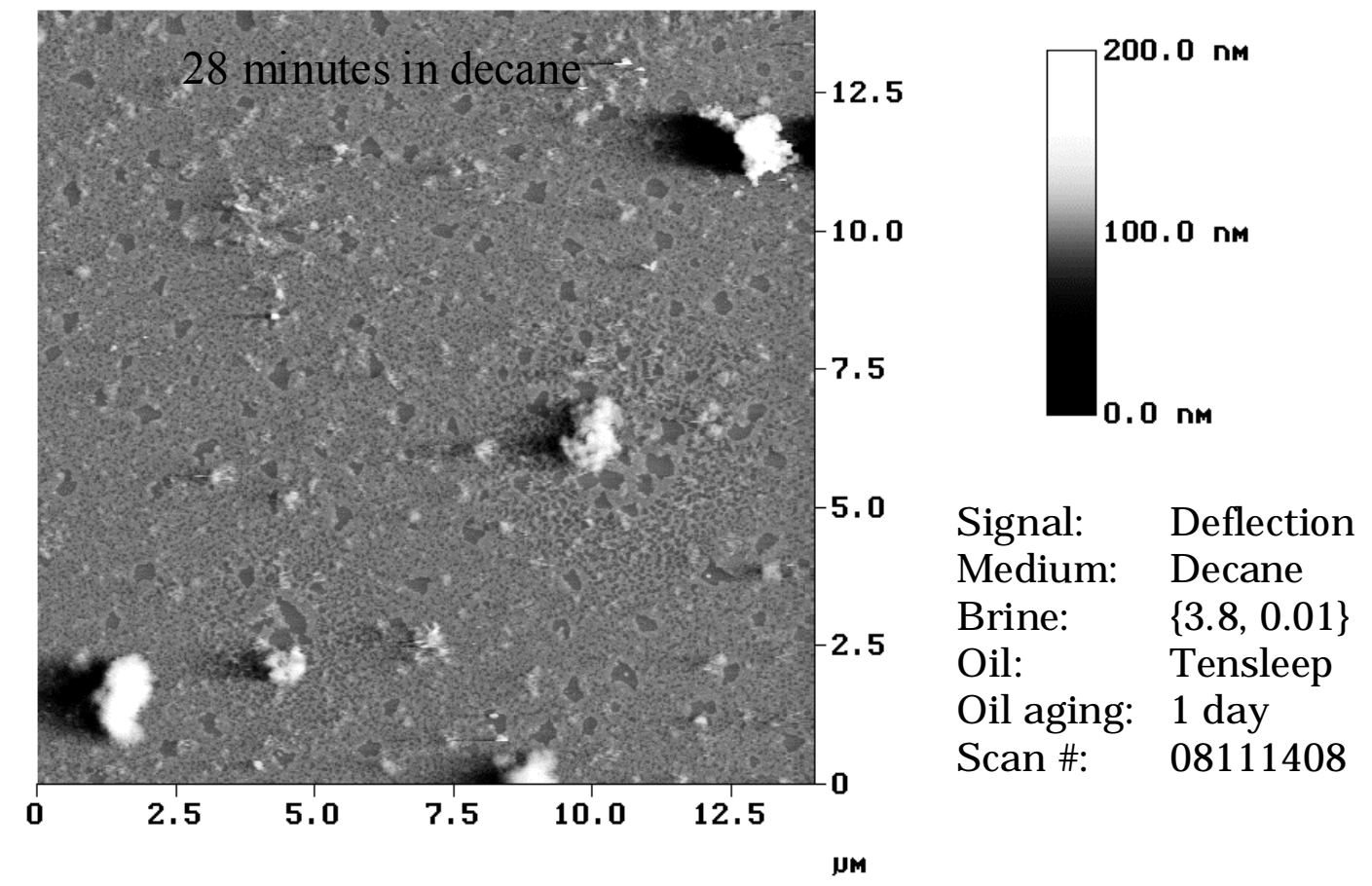

Figure II-3.39. AFM scan of mica exposed to Tensleep crude. Surface immersed in decane for 99 minutes.

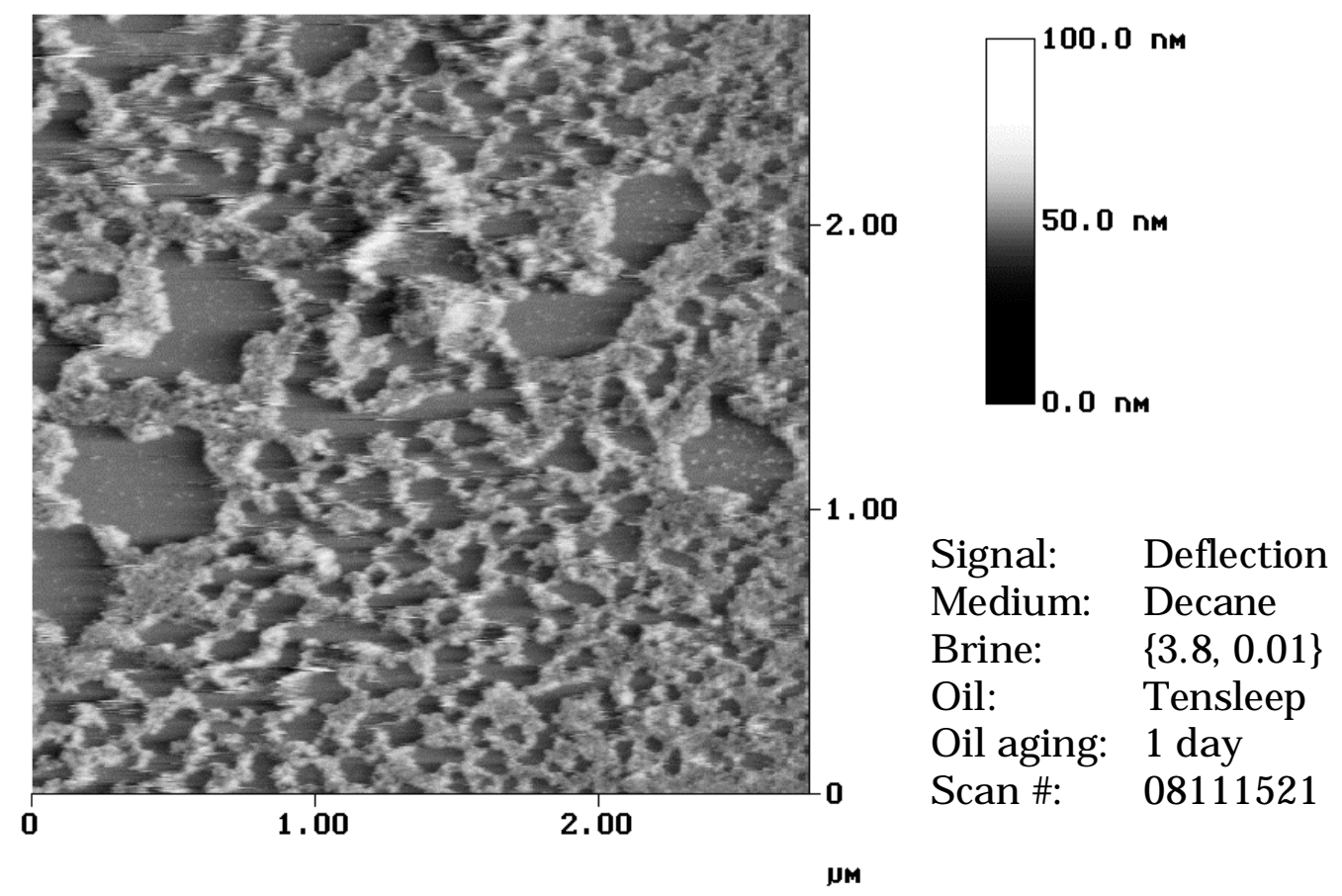

Figure II-3.40. AFM scan of mica exposed to Tensleep crude. Zoom of area indicated in Figure II-3.39. 
Aging time: 11 days

Brine imaging medium. Aging the mica for 1 day in $\{3.8 .0 .01\}$ and for 11 days in Tensleep crude resulted in the formation of a fairly continuous surface film (Figure II-3.41) with none of the holes down to bare mica that were observed in the 1-day sample (Figure II-3.27). The few holes that were present appeared to contain clusters of rounded objects with a characteristic diameter of about $50 \mathrm{~nm}$. A roughness analysis of a representative selection of several holes yielded a mean value of $8.4 \mathrm{~nm}$. The film material immediately adjacent to the holes was visibly smoother and contained structural units too small to resolve, and had a mean roughness value of $3.1 \mathrm{~nm}$. The presence of raised edges around the perimeters of the holes, coupled with the abrupt transition in texture suggest that the smooth film may actually overlay rougher features, with the holes occurring in areas where adsorption of film material was inhibited by the coarser objects already in place on the surface.

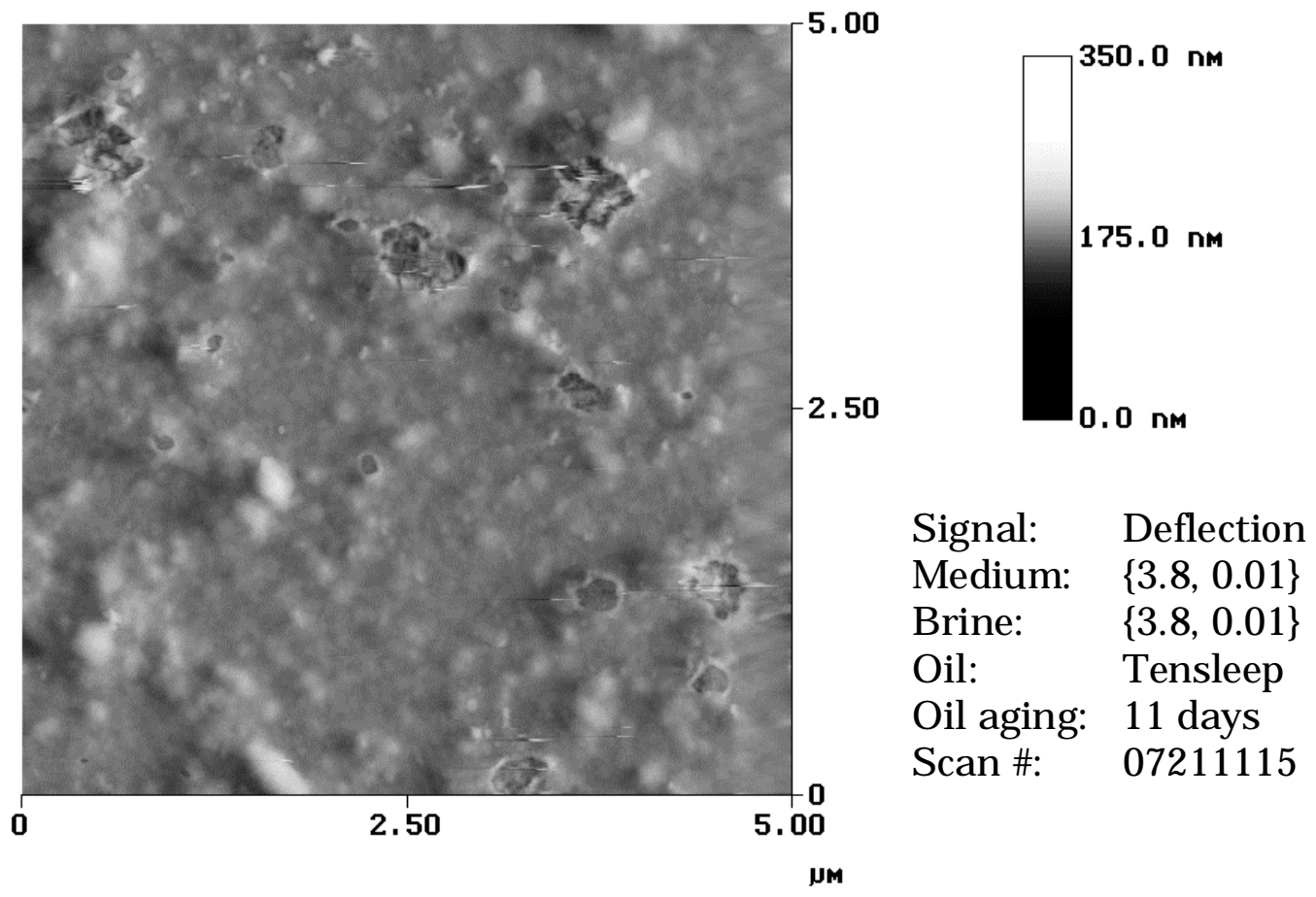

Figure II-3.41. AFM scan of mica exposed to Tensleep crude.

One working hypothesis is that the rough, clustered objects visible through the holes in the 11-day sample may be asphaltene aggregates, while the smoother film material may be a densely-packed layer of surfactants, such as organic acids and bases native to the crude oil. The initial presence water on the mica may have limited interactions between the crude oil and the solid surface within the first 24 hours, resulting in the perforated film observed in the 1-day sample. The 1-day film was discontinuous and a considerable amount of the mica surface was still visible. After 11 days, the mica surface was entirely covered, and the few holes visible revealed underlying material with a texture very different from the film itself or the mica. While the 10 intervening days likely allowed the original water film to be dispersed, it also apparently provided sufficient time for the fine-textured film to form. The rough, clustered objects appear to be "cemented" to the surface by the film. 
Decane imaging medium. Replacing brine with decane did not result in any marked changes to the film structure, as evidenced in Figure II-3.42. The dominant features are still a smooth film overlaying a rough-textured material, which is visible through holes in the film. A zoom (Figure II-3.43) of one of the holes shows structural units of which the rougher material is comprised. The units appear to be round with a diameter of roughly $50 \mathrm{~nm}$. One deep, roughly circular hole which revealed mica was observed in Figure II-3.44. This might be the result of the presence of a water droplet which shielded the mica from direct interactions with the oil. A zoom inside the hole (Figure II-3.45) shows a substrate, most likely mica, with a roughness of $0.41 \mathrm{~nm}$. Figure II-3.46, which is a $5 \mu \mathrm{m}$ zoom of the center of Figure II-3.44, illustrates the more typical topography of this sample in which mica is not necessarily exposed in the holes.

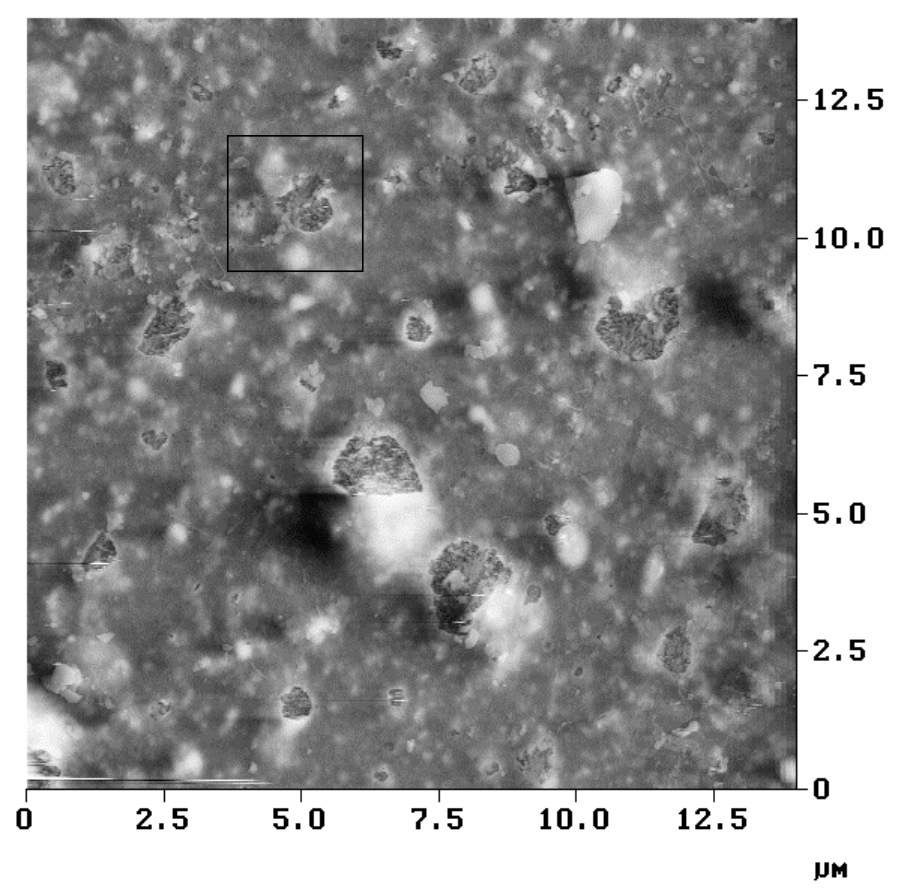

Figure II-3.42. AFM scan of mica exposed to Tensleep crude.

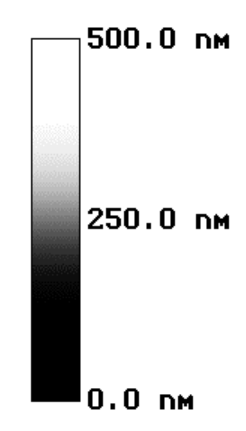

Signal: Deflection

Medium: Decane

Brine: $\quad\{3.8,0.01\}$

Oil: $\quad$ Tensleep

Oil aging: 11 days

Scan \#: 07211405 

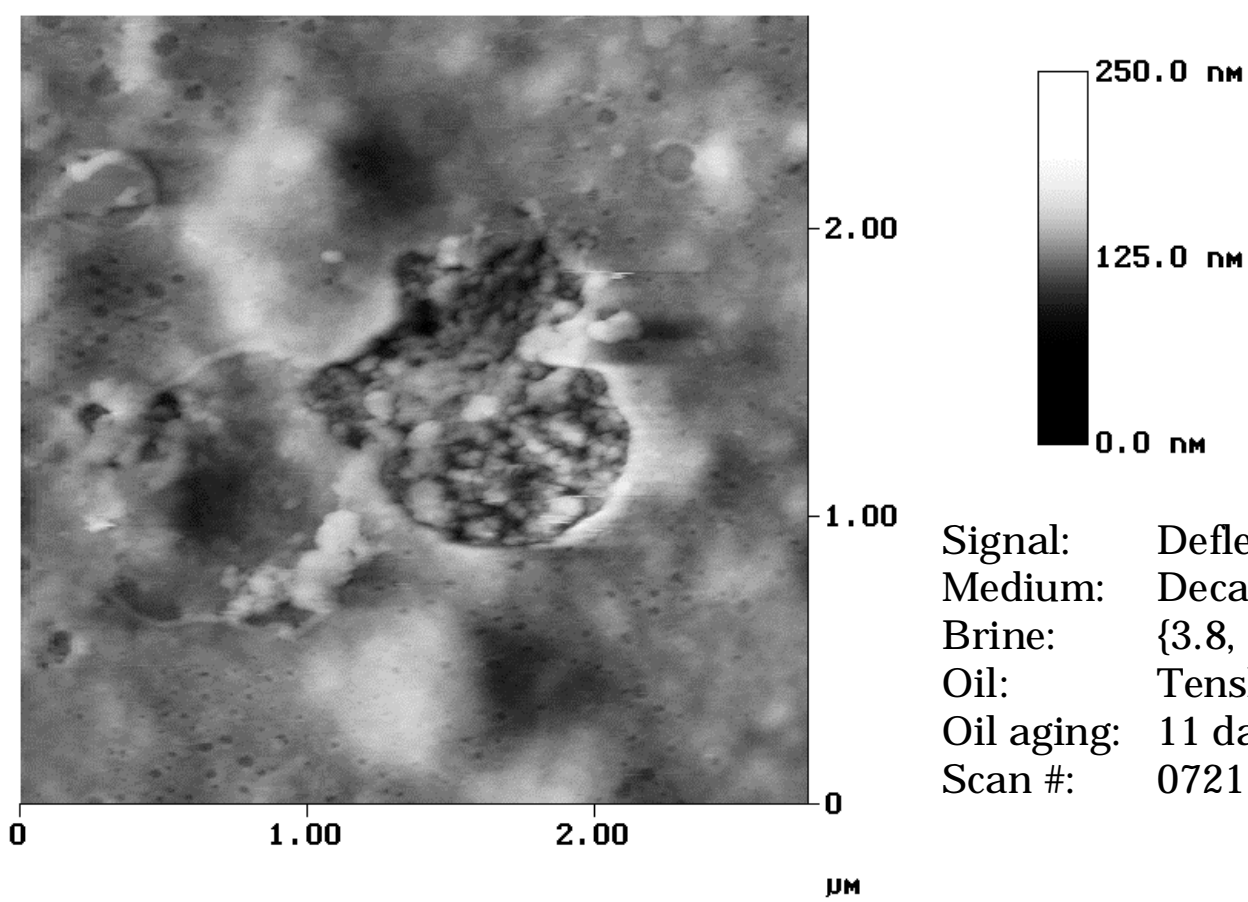

Signal: Deflection

Medium: Decane

Brine: $\quad\{3.8,0.01\}$

Oil: $\quad$ Tensleep

Oil aging: 11 days

Scan \#: 07211411

JM

Figure II-3.43. AFM scan of mica exposed to Tensleep crude. Zoom of area indicated in Figure II-3.42.
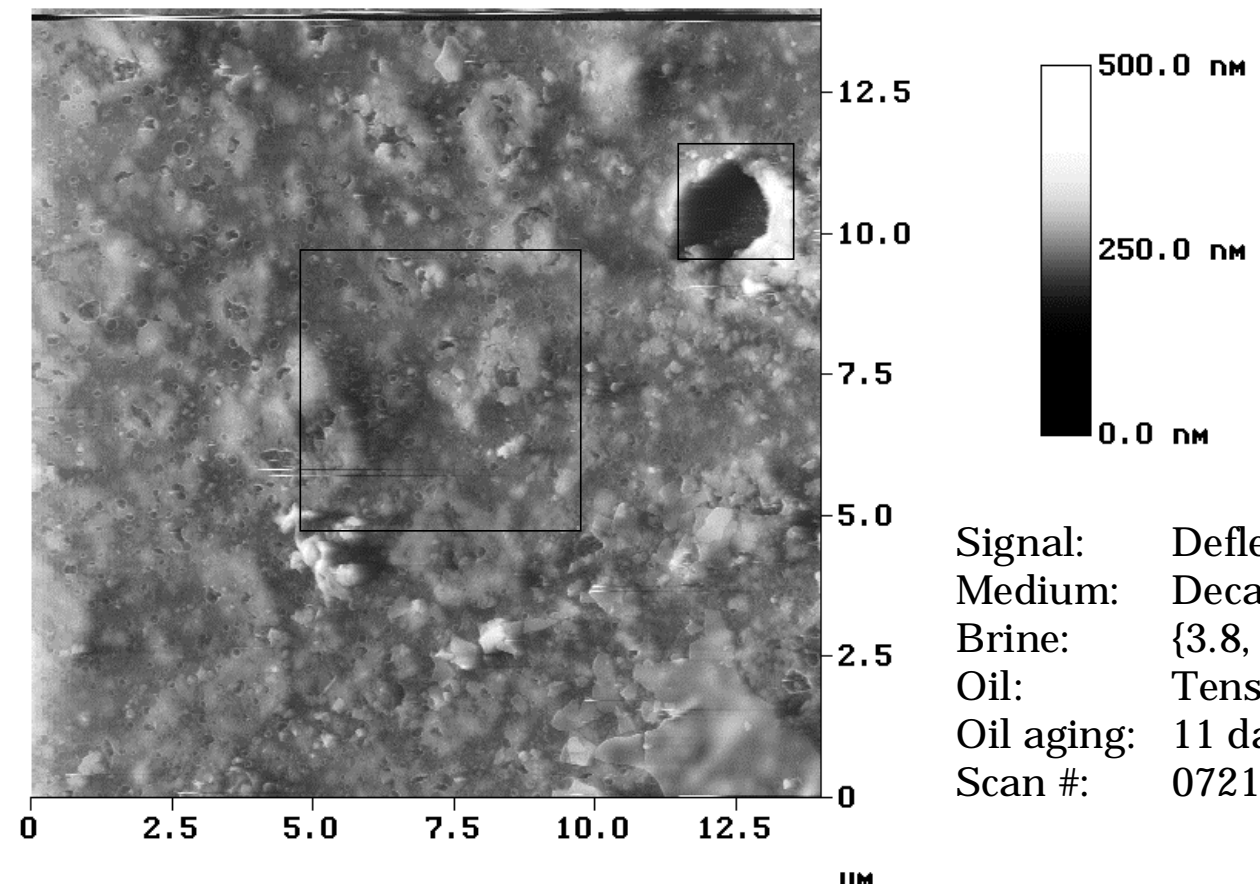

5.0

Signal: Deflection

Medium: Decane

Brine: $\quad\{3.8,0.01\}$

Oil: $\quad$ Tensleep

Oil aging: 11 days

Scan \#: 07211437

Figure II-3.44. AFM scan of mica exposed to Tensleep crude. Zooms of the areas indicated by the boxes are shown in Figures II-3.45 and II-3.46. 


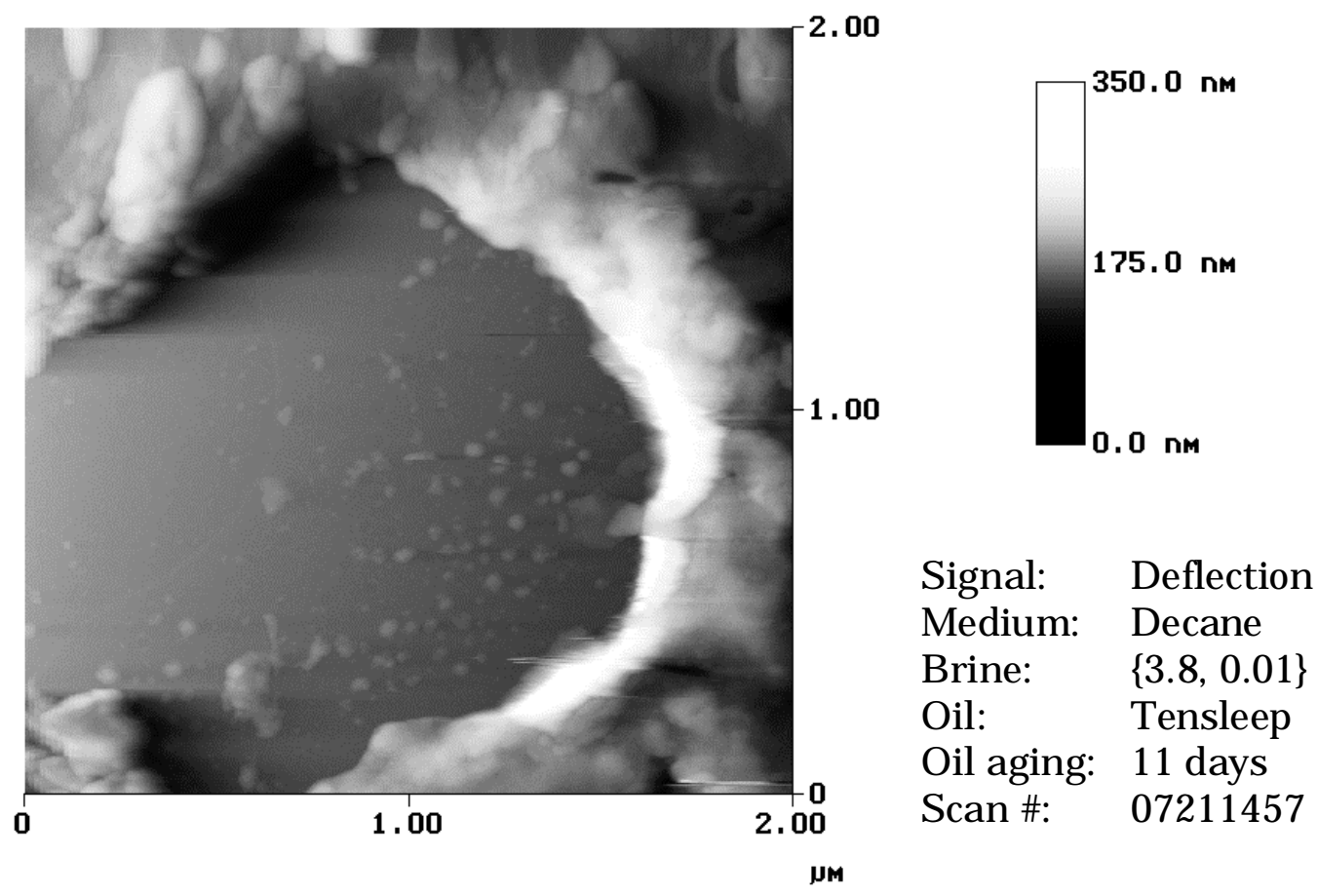

Figure II-3.45. AFM scan of mica exposed to Tensleep crude. Zoom of region in upper right corner of Figure II-3.44.

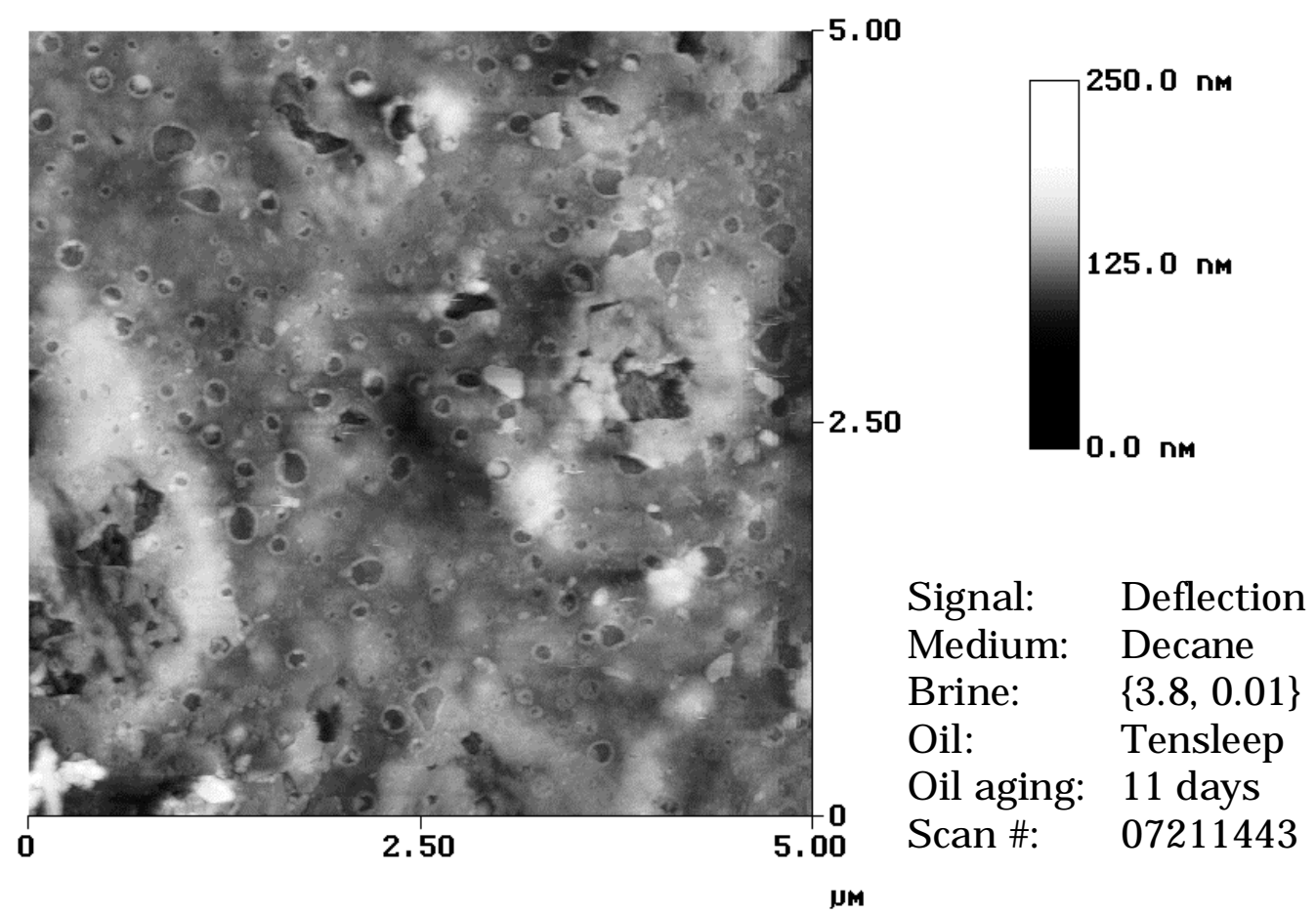

Figure II-3.46. AFM scan of mica exposed to Tensleep crude. Zoom of area indicated in center of Figure II3.44. 
Certain regions of the 0721 sample show evidence of salt precipitation resulting from prior use of brine as the imaging medium. In order to remove the brine droplet from the surface of the mica without moving the sample relative to the microscope, a piece of filter paper was touched to the droplet and capillary forces drew most of the drop volume into the paper. After about 30 seconds, a droplet of decane was placed on the mica surface where the brine droplet had been. This process likely left a thin film of brine that dried, leaving behind a small amount of precipitated salt. Some of the ensuing images captured under decane show salt deposits that appear as flat, smooth-textured material with sharp edges. Arrows in Figure II-3.47 indicate several such deposits, which resemble broken eggshells scattered on the surface. None of the needles observed in the dried brine sample (Figure II-3.23 and II-3.24) were encountered on the oil-treated surface. The salt deposits were stable during imaging, and not susceptible to disturbance by tip-sample interactions.
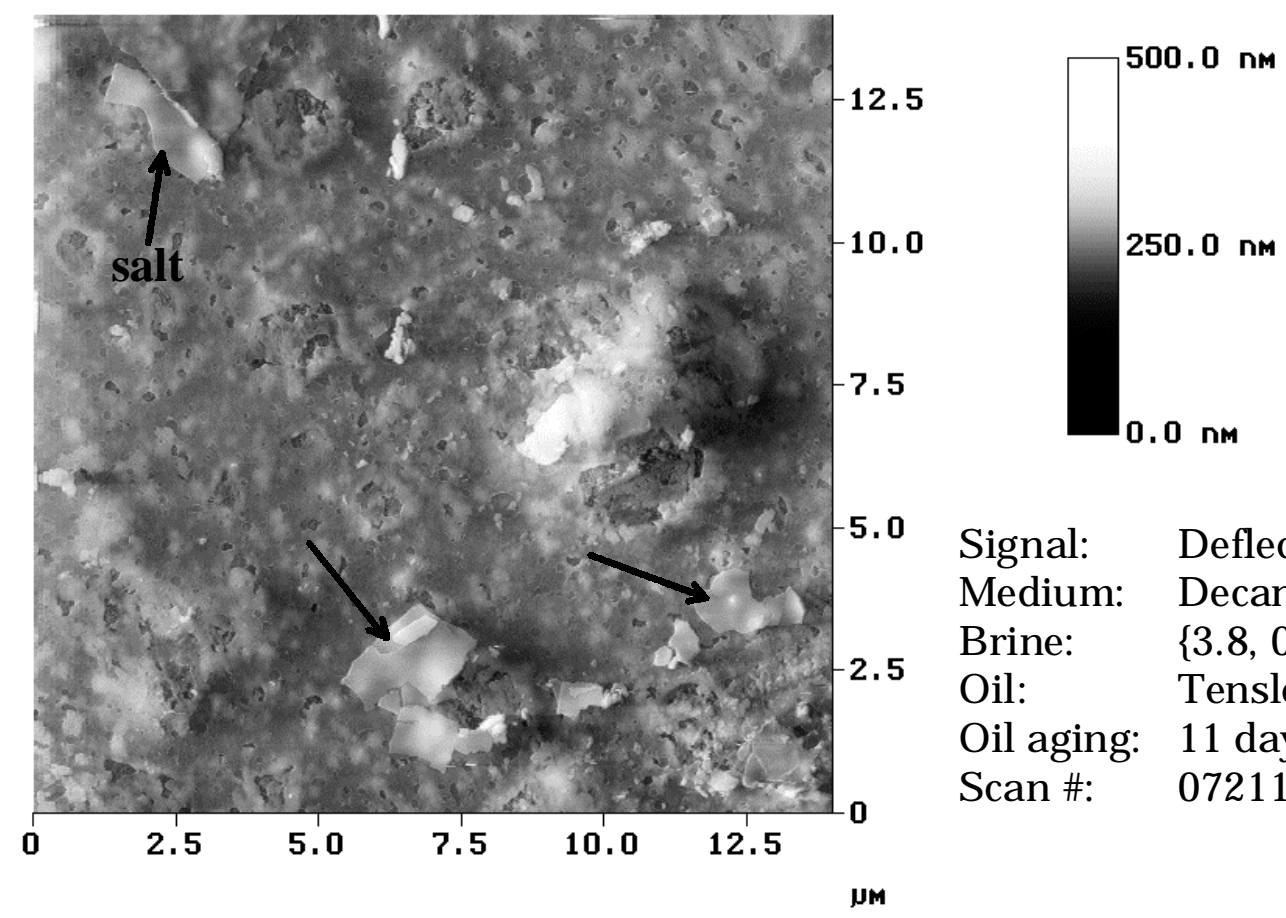

Signal: Deflection

Medium: Decane

Brine: $\quad\{3.8,0.01\}$

Oil: $\quad$ Tensleep

Oil aging: 11 days

Scan \#: 07211616

Figure II-3.47. AFM scan of mica exposed to Tensleep crude. Precipitated salt is indicated by the arrows.

Decane + toluene imaging medium. Adding toluene to the decane imaging medium produced a solution with a higher capability to dissolve asphaltic material. Changes in surface features resulting from the addition of toluene may be evaluated by examining Figure II-3.48, which shows the same region imaged under decane and decane + toluene. The salt deposits can be used as point of reference, as their size and shape do not change. Certain features appear to swell with the toluene. In particular, the rough regions that were visible through holes in the surface film underwent marked increases in the z-dimension. This behavior is consistent with the expected response of asphaltene aggregates to an increasingly effective solvent solution. Components of the smooth surface film, however, do not seem to respond so markedly to the toluene. The material which is presumed to constitute the film, surface-active polar compounds such as organic acids and bases that assembled densely at the mica-oil interface during the aging 
period in oil, should also be soluble in the decane-toluene mixture. The fact that it is not dissolving perceptibly on the timescale of these observations helps to distinguish between material that is adsorbed and that which is simply deposited.
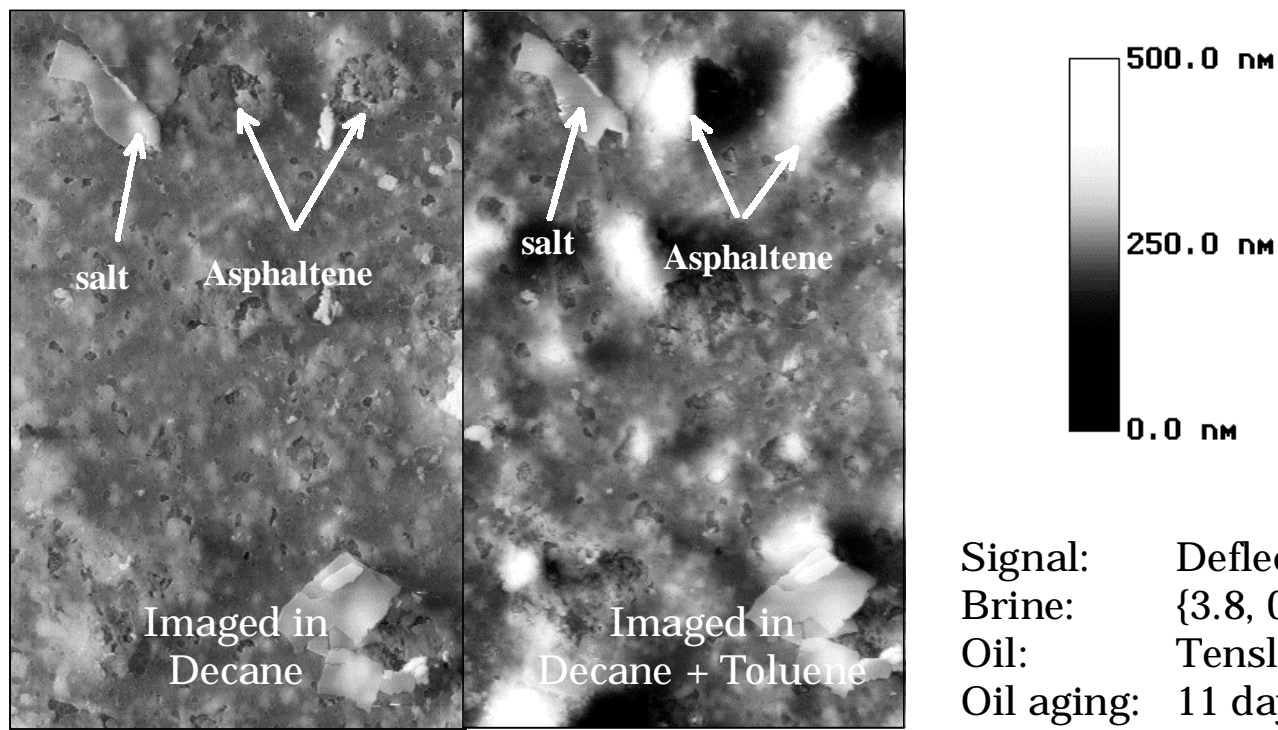

$\begin{array}{ll}\text { Signal: } & \text { Deflection } \\ \text { Brine: } & \{3.8,0.01\} \\ \text { Oil: } & \text { Tensleep } \\ \text { Oil aging: } & \text { 11 days }\end{array}$

Figure II-3.48. AFM scan of mica exposed to Tensleep crude. Imaging medium was changed from decane to decane+ toluene as indicated. While precipitated salt crystals retain their original size and shape, other structures (likely asphaltenes) swell upon addition of toluene, which is an effective asphaltene solvent.

Aging time: 14 days

Brine film stability is a critical factor controlling adsorption of crude oil components and consequent wettability alteration of mineral surfaces (Buckley et al., 1998). The sensitivity of these phenomena to brine $\mathrm{pH}$ and ionic strength were demonstrated here by exposing mica to $\{8.0,1.0\}$ brine for 1 day, followed by 14 days' exposure to Tensleep crude. Brine film stability (and consequent resistance to wettability change) is usually observed for aqueous solutions with higher $\mathrm{pH}$ and ionic strength although the boundaries between stable and unstable water films are influence by other factors, including oil composition.

Brine imaging medium. Mica exposed to $\{8.0,1.0\}$ brine for 1 day, and Tensleep crude for 14 days, exhibited no detectable sorption according to AFM scans (Figure II-3.49). This observation is consistent with the expectation that the high-pH, high-ionic strength brine would provide a stable barrier between the oil and substrate, therefore preserving the original surface properties of the mica (compare Figures II-3.22 and II-3.49). A roughness analysis of a $5 \mu \mathrm{m} \times$ $5 \mu \mathrm{m}$ area in the middle of Figure II-3.49 indicated a mean roughness of $\mathrm{R}_{\mathrm{a}}=0.05 \mathrm{~nm}$. This value is close to the value of $R_{a}=0.08 \mathrm{~nm}$ reported in the literature by Yang et al. (1999), though less than the roughness of $\mathrm{R}_{\mathrm{a}}=0.16 \mathrm{~nm}$ measured on clean mica in Figure II-3.22. For comparison, the lowest mean roughness of film material deposited on the mica measured in this report thus far is $3.1 \mathrm{~nm}$, two orders of magnitude higher. It is therefore unlikely that the smooth texture observed on sample 1026 is a result of film formation. Rather, it most closely resembles clean mica. 


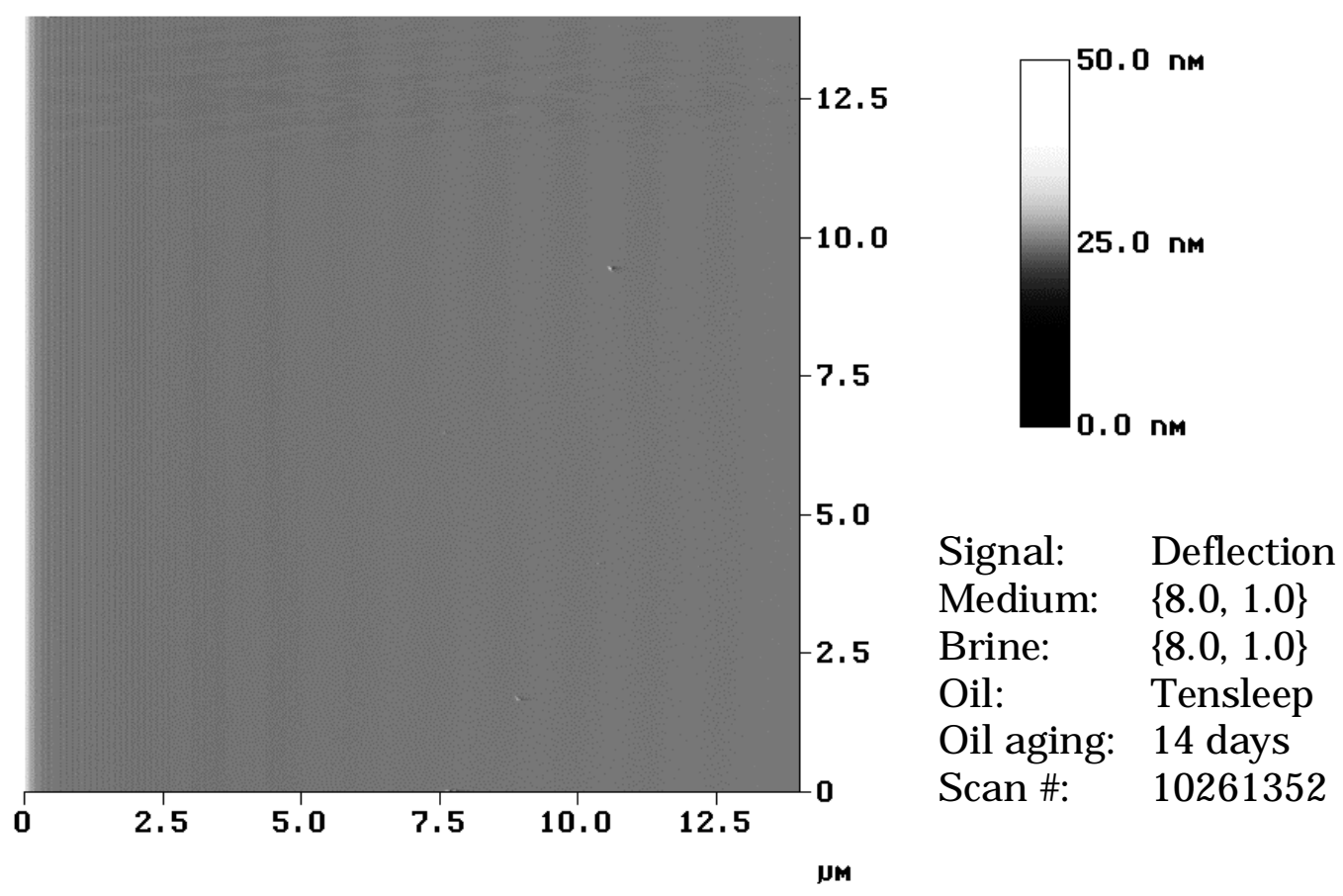

Figure II-3.49. AFM scan of mica exposed to Tensleep crude. The $\mathrm{pH}=8,\left[\mathrm{Na}^{+}\right]=1.0 \mathrm{M}$ brine in this case provided a stable water film which prevented the oil phase from contacting the mica surface.

Aging time: 25 days

Decane imaging medium. Mica samples aged 25 days in Tensleep crude exhibited relatively uniform and complete coverage with no bare mica evident (Figures II-3.50 and II3.51). Coverage appears to progress with the passage of time from 1 day in oil (e.g. Figure II3.27 ) to 25 days in oil, with the holes filling in and features exhibiting a more uniform elevation. A zoom of one of the oval-shaped rimmed features in Figure II-3.50 is shown in Figure II-3.52. Rounded structural units with an average diameter of about $50 \mathrm{~nm}$ appear to dominate all of the features evident, including the mounded structure in the center of the zoom as well as the flatter regions which cover most of the surface. 

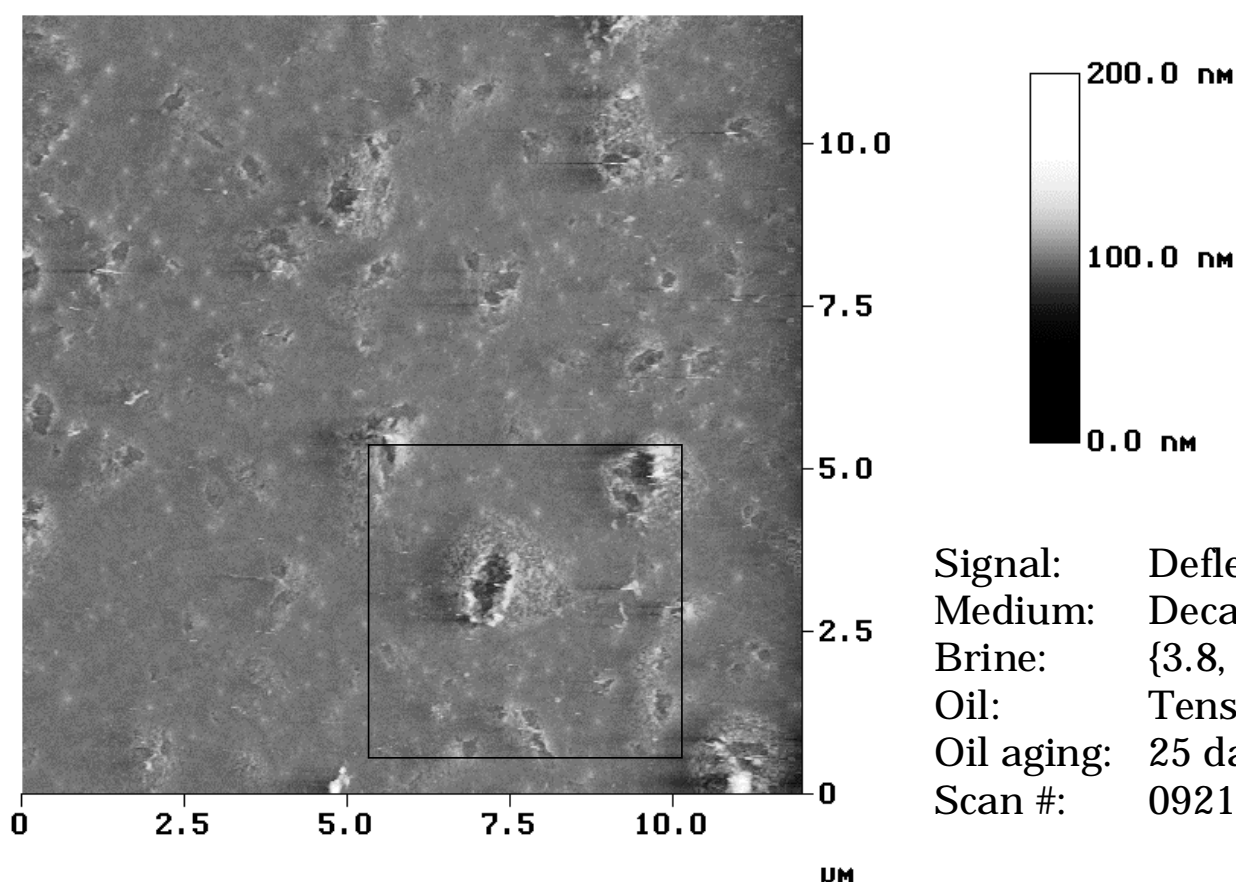

Signal: Deflection

Medium: Decane

Brine: $\quad\{3.8,0.01\}$

Oil: $\quad$ Tensleep

Oil aging: 25 days

Scan \#: 09211245

Figure II-3.50. AFM scan of mica exposed to Tensleep crude. A zoom of the area indicated in the box is shown in Figure II-3.52.

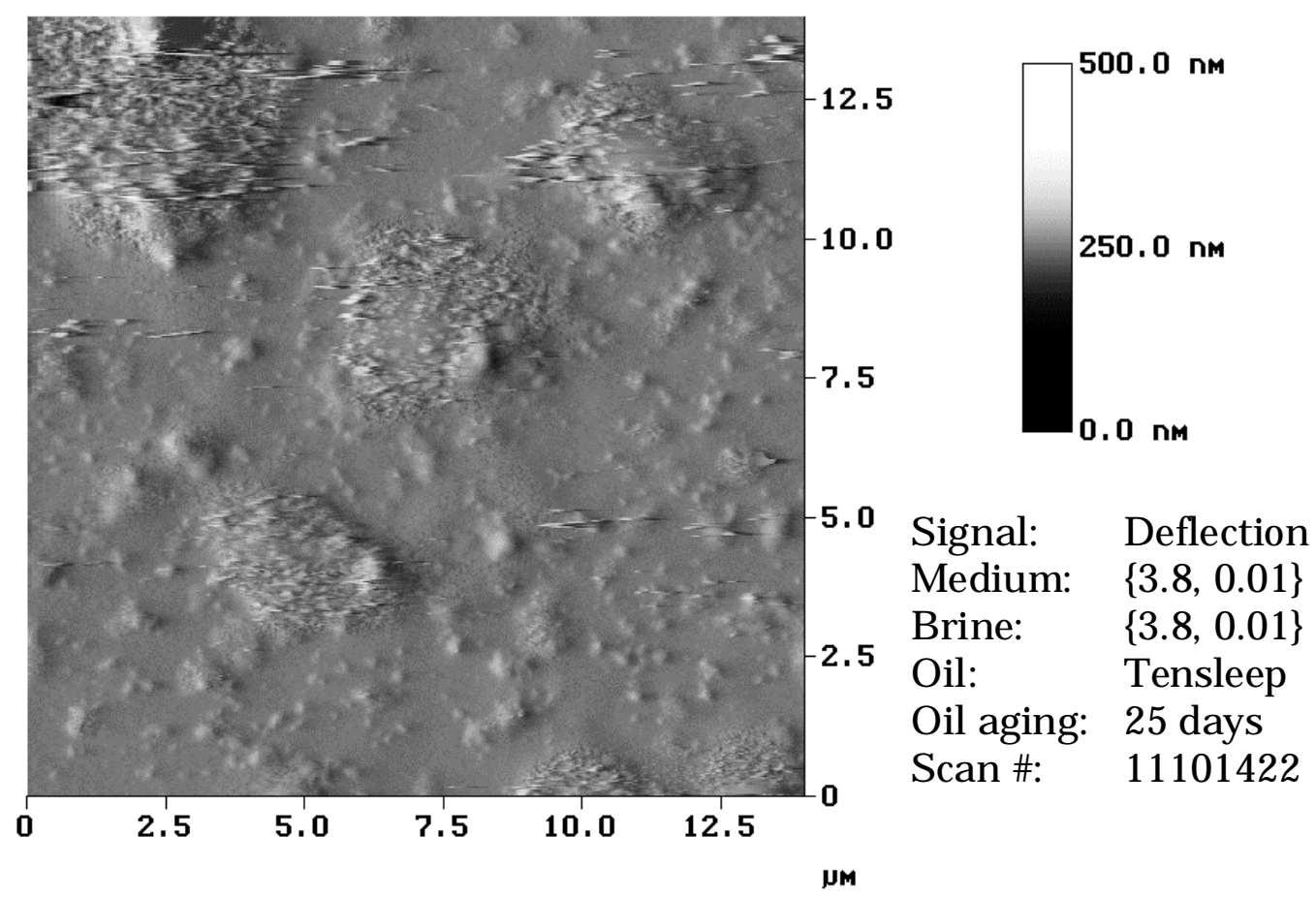

Figure II-3.51. AFM scan of mica exposed to Tensleep crude. 

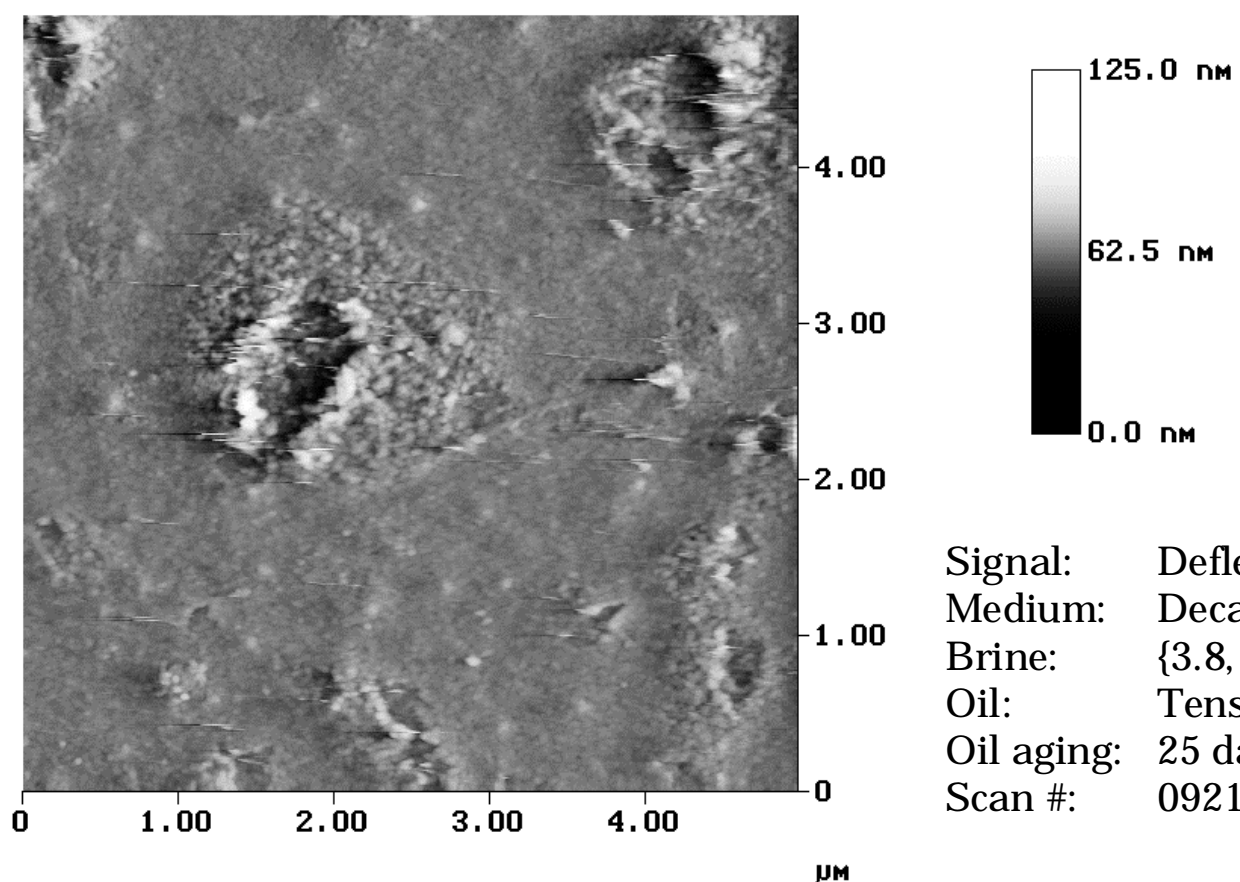

Signal: Deflection

Medium: Decane

Brine: $\quad\{3.8,0.01\}$

Oil: $\quad$ Tensleep

Oil aging: 25 days

Scan \#: 09211257

Figure II-3.52. AFM scan of mica exposed to Tensleep crude. Zoom of area indicated in Figure II-3.50.

1-MN imaging medium. Replacing decane with 1-MN yielded no apparent change in surface features (Figure II-3.53) after approximately 25 minutes exposure to 1-MN. The scanning force was increased by an order of magnitude to create a $3 \mu \mathrm{m} \times 3 \mu \mathrm{m}$ hole in the film down to the substrate (Figure II-3.54). A section analysis of this hole indicated that the depth of the film in this region was about $15 \mathrm{~nm}$, similar to the depth of $13.3 \pm 1.6 \mathrm{~nm}$ measured for a mica sample exposed to Tensleep oil for 1 day (Figure II-3.29). According to these two depth measurements, the net thickness of the film does not appear to increase with passage of time from 1 to 25 days exposure to Tensleep. 


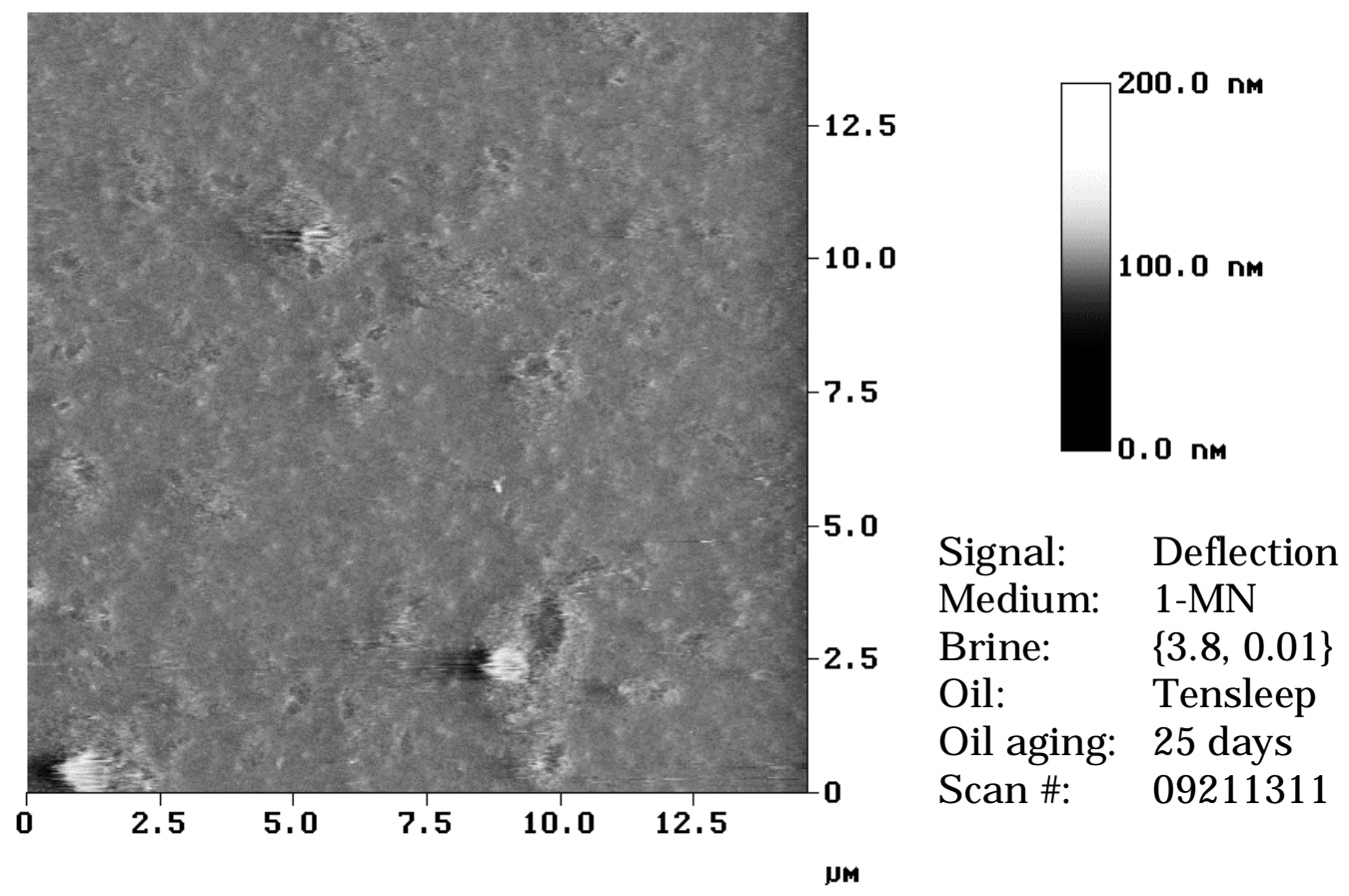

Figure II-3.53. AFM scan of mica exposed to Tensleep crude.

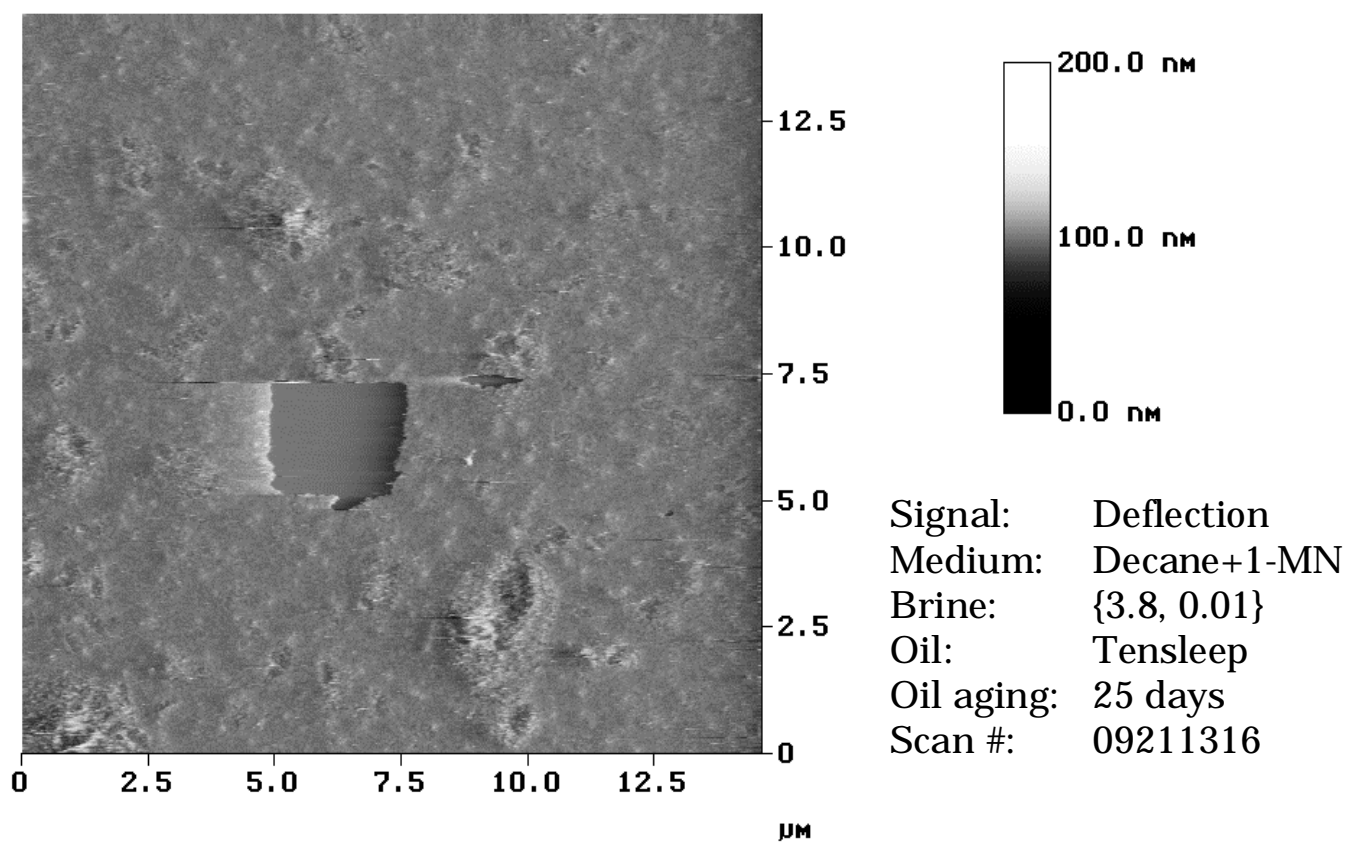

Figure II-3.54. AFM scan of mica exposed to Tensleep crude. 
TENSLEEP + 1-METHYLNAPHTHALENE

After addition of the asphaltene solvent 1-methylnaphthalene to the Tensleep crude (35:65 vol/vol), asphaltene aggregates were no longer visible under the optical microscope (Figure II-3.55). Although aggregates are not visible by optical means (compare Figures II-3.25 and II-3.55), the mass of asphaltic material in the oil remains unchanged.

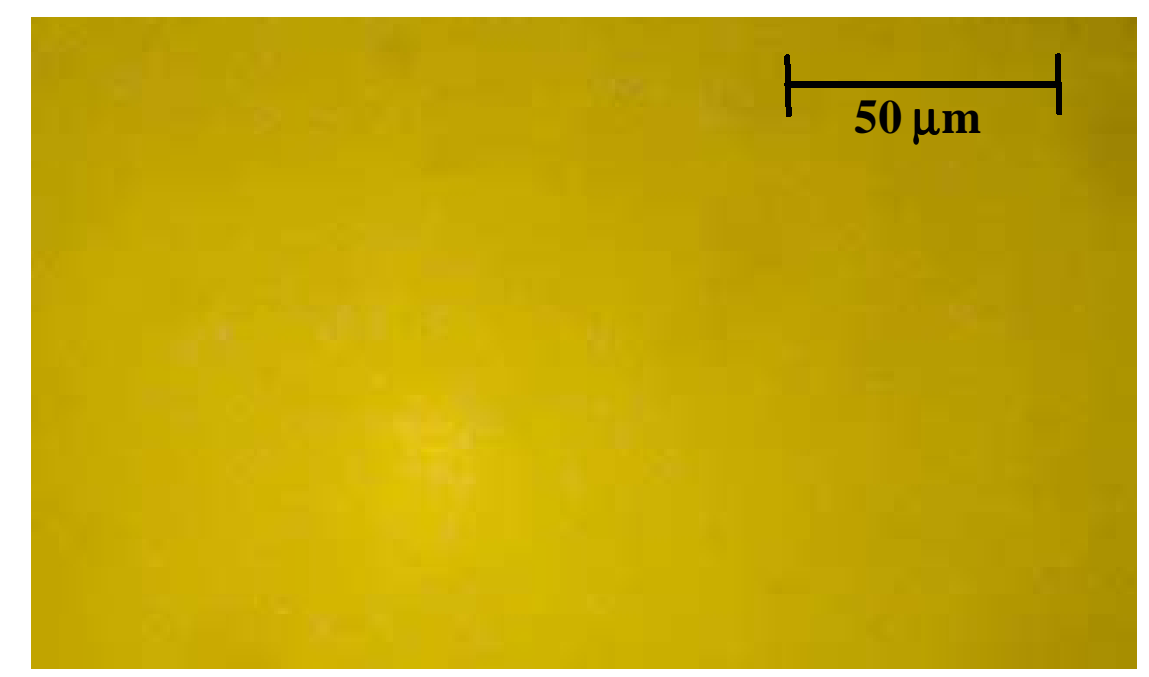

Figure II-3.55. Optical microscope image of Tensleep crude + 1-MN (65:35 vol./vol.). 1-MN effectively dispersed asphaltene aggregates such that they were not visible (compare with Figure II-3.25 which shows $100 \%$ Tensleep crude).

Decane-water contact angles on mica exposed first to $\{3.8,0.01\}$ brine for 1 day and then to the Tensleep $+1-\mathrm{MN}$ solution for 3 weeks indicated a surface with intermediate wetting, with $\theta_{\mathrm{A}}=69^{\circ} \pm 17^{\circ}$ for water-advancing, and $\theta_{\mathrm{R}}=37^{\circ} \pm 21^{\circ}$ for water-receding conditions (AlMaamari and Buckley, 2000). These angles are markedly lower than those measured for mica exposed to the Tensleep crude for 1 day, at $\theta_{\mathrm{A}}=130^{\circ}$, and $\theta_{\mathrm{R}}=61^{\circ}$. The solvent quality of the $1-\mathrm{MN}$ therefore apparently reduced the net surface activity of the Tensleep asphaltenes at the mica surface.

\section{Aging time: 1 day}

Air imaging medium. Mica exposed to the Tensleep+1-MN solution for 1 day and imaged with the AFM under air (Figure II-3.56) showed a thin coating with only a few features larger than $100 \mathrm{~nm}$ in diameter. The absence of features on the order of $1 \mu \mathrm{m}$ or larger is consistent with the observations in optical images of Tensleep+1-MN in which no large aggregates were detected. One circular feature is visible near the top center of Figure II-3.56 is captured in a zoom in Figure II-3.57. The circular shape of both this feature and a similar feature along the left side of Figure II-3.56 suggest that these may have originated at the phase boundary between fluids - presumably water and oil. The mica surface was originally completely covered by a water film, and as oil displaced the water, it is likely that discreet droplets of water were left behind. Material that was either hydrophilic and suspended in the water phase or amphipathic and concentrated at the oil-water boundary would be deposited on the surface in this manner as the water was dried or dissolved into the oil phase. 


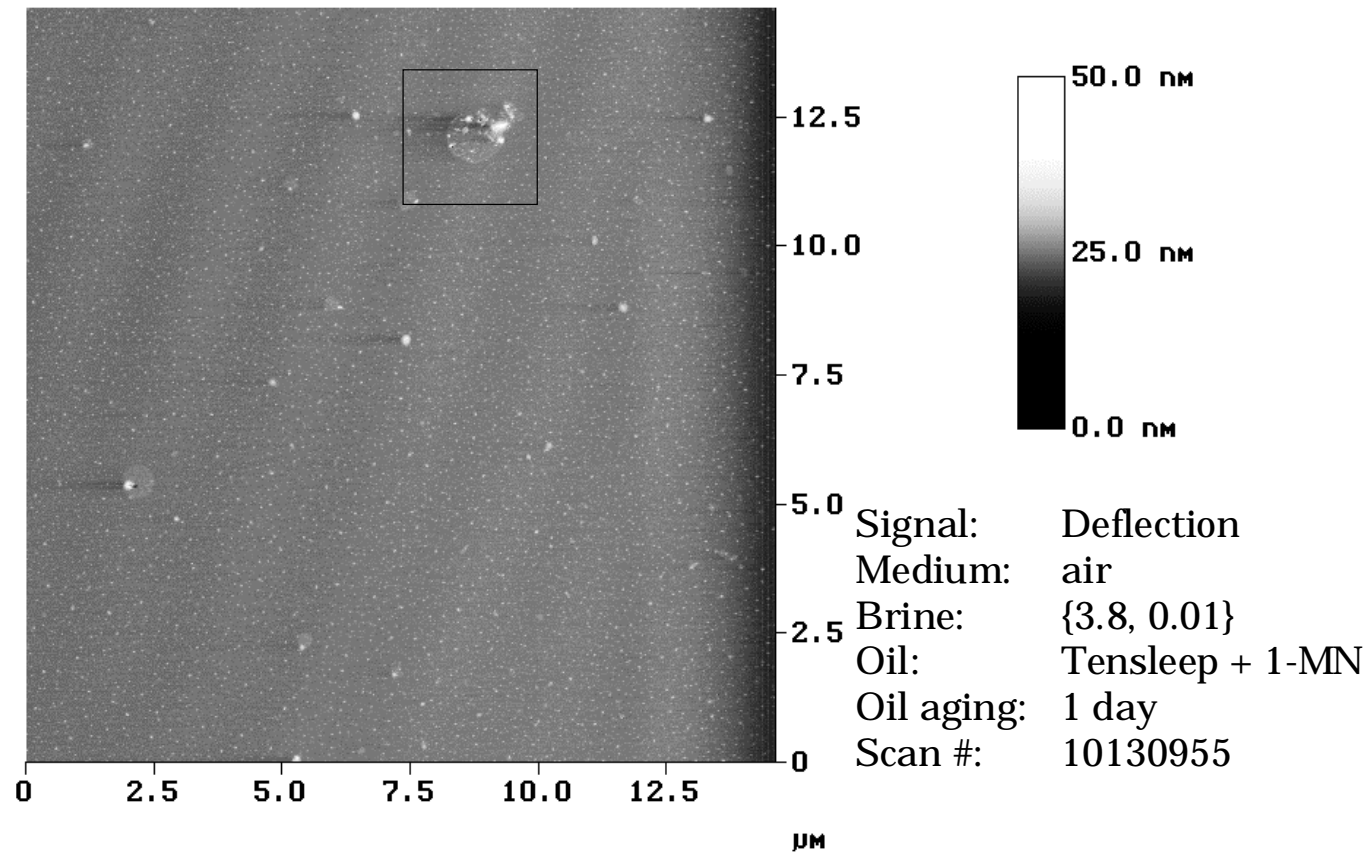

Figure II-3.56. AFM scan of mica exposed to Tensleep + 1-MN. Zoom of area in box is shown in Figure II3.57.

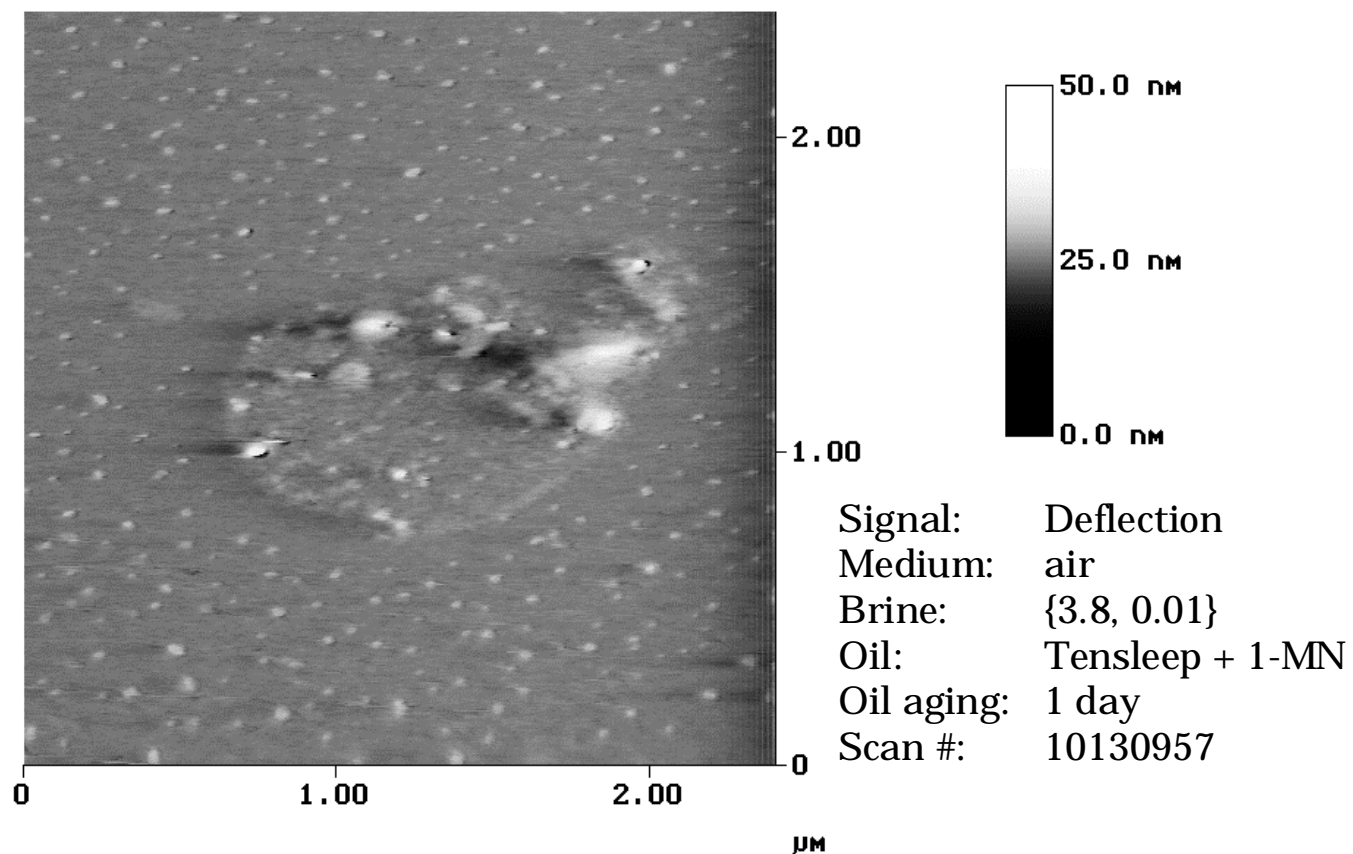

Figure II-3.57. AFM scan of mica exposed to Tensleep + 1-MN. Zoom of area indicated in Figure II-3.56.

Brine imaging medium. Imaging under brine (Figure II-3.58) over the same scan area as Figure II-3.56 caused some scarring of the surface film, indicated by the poorly resolved 
horizontal band in the top half of the scan. The features that remain look similar to those under air. Repeated scans over the same region swept away the surface features initially observed, despite efforts to minimize the contact force between tip and sample. The structures formed on mica after 1 day in Tensleep+1-MN were thus quite easily removed by mechanical agitation from the AFM tip, and less robust than the features formed in Tensleep without MN.

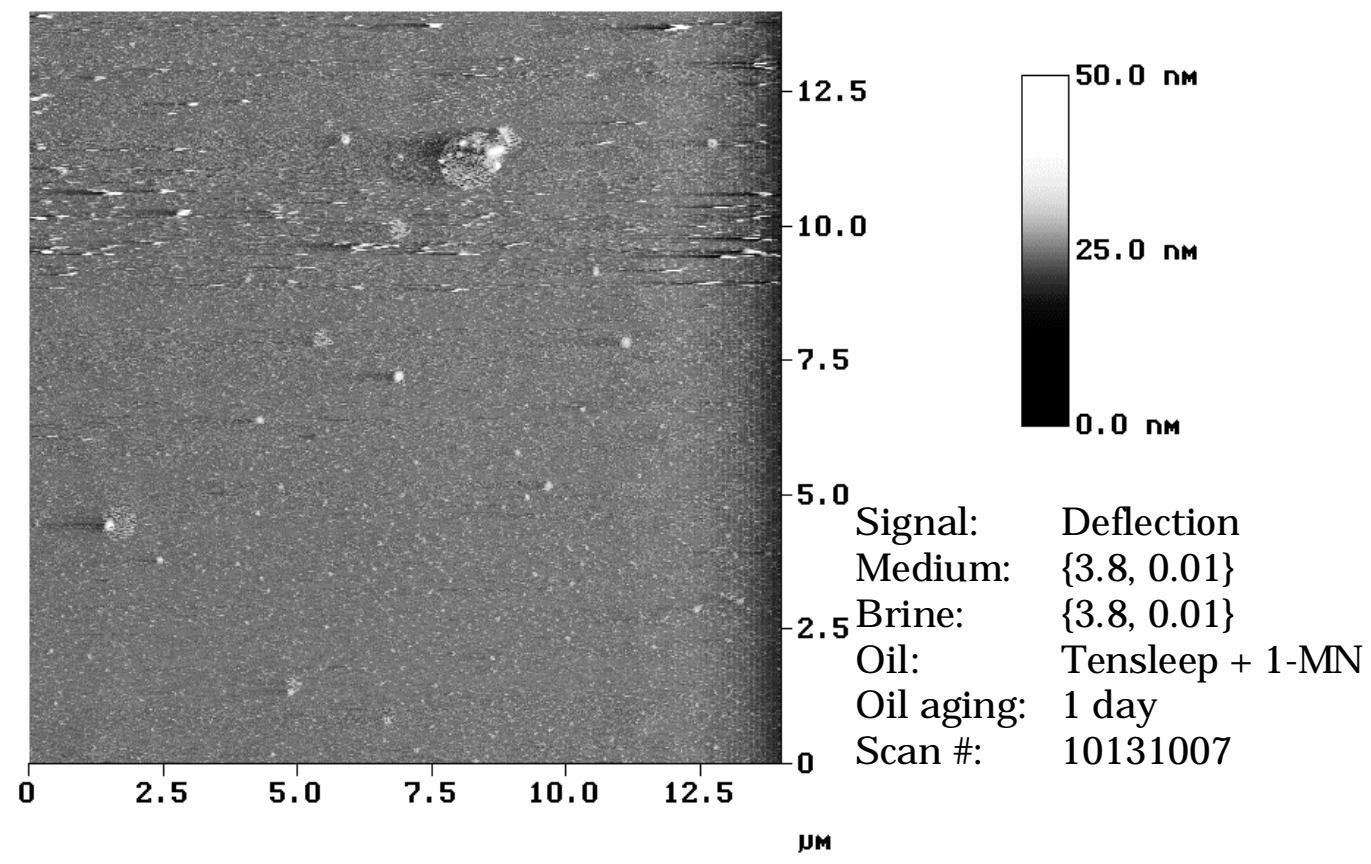

Figure II-3.58. AFM scan of mica exposed to Tensleep + 1-MN. 
Decane Imaging medium. No images of this sample were captured under decane.

Aging time: 11 days

Air imaging medium. Wet mica exposed to Tensleep+1-MN for 11 days exhibited some surface features, though coverage was inconsistent. (Figure II-3.59) Outlines of circular features were evident, suggesting that many discreet water droplets were scattered on the surface once the original water film collapsed. The tip sample-interactions were unstable in air, and dominated by long range attractive forces between the tip and sample which deflected the tip toward the surface during scanning. Image quality is difficult to optimize under such conditions. The apparent smooth texture of the surface is a result of imaging with attractive force as opposed to the more conventional repulsive force. The feature in the lower right corner of Figure II-3.59 which has an arc-shaped perimeter is likely precipitated salt from a brine droplet which originally coated the mica.

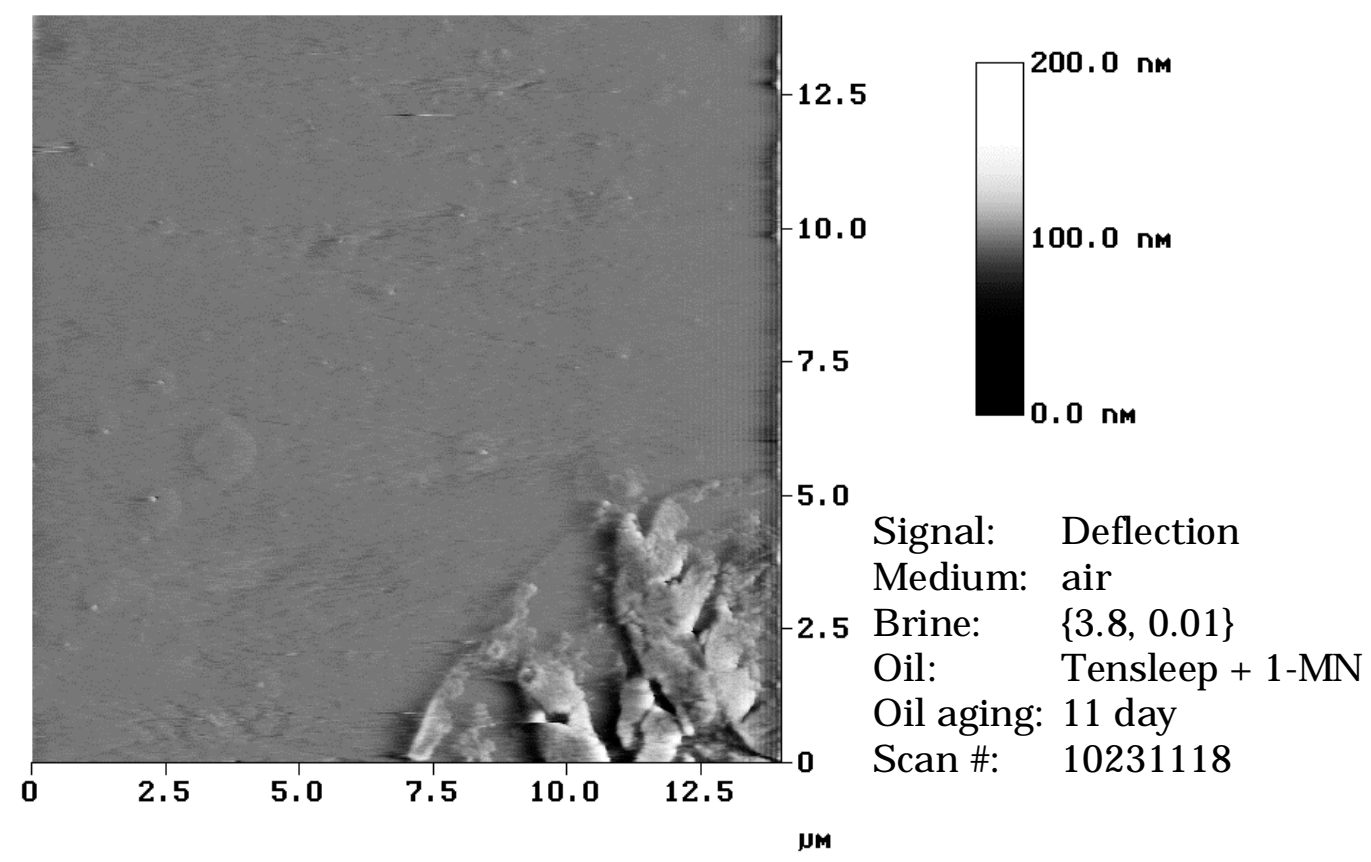

Figure II-3.59. AFM scan of mica exposed to Tensleep + 1-MN. 
Brine imaging medium. Adding brine (Figure II-3.60) apparently dissolved the salt which was the lower right corner of Figure II-3.59 revealing bare mica where the salt was. Material remaining on the surface, likely surfactants deposited from the oil solution, traced the outline of the salty deposit. Some of the vague circular features from the air image are also better-resolved in the brine medium. The balance of material on the surface traces the outlines of what would appear to have been brine droplets.

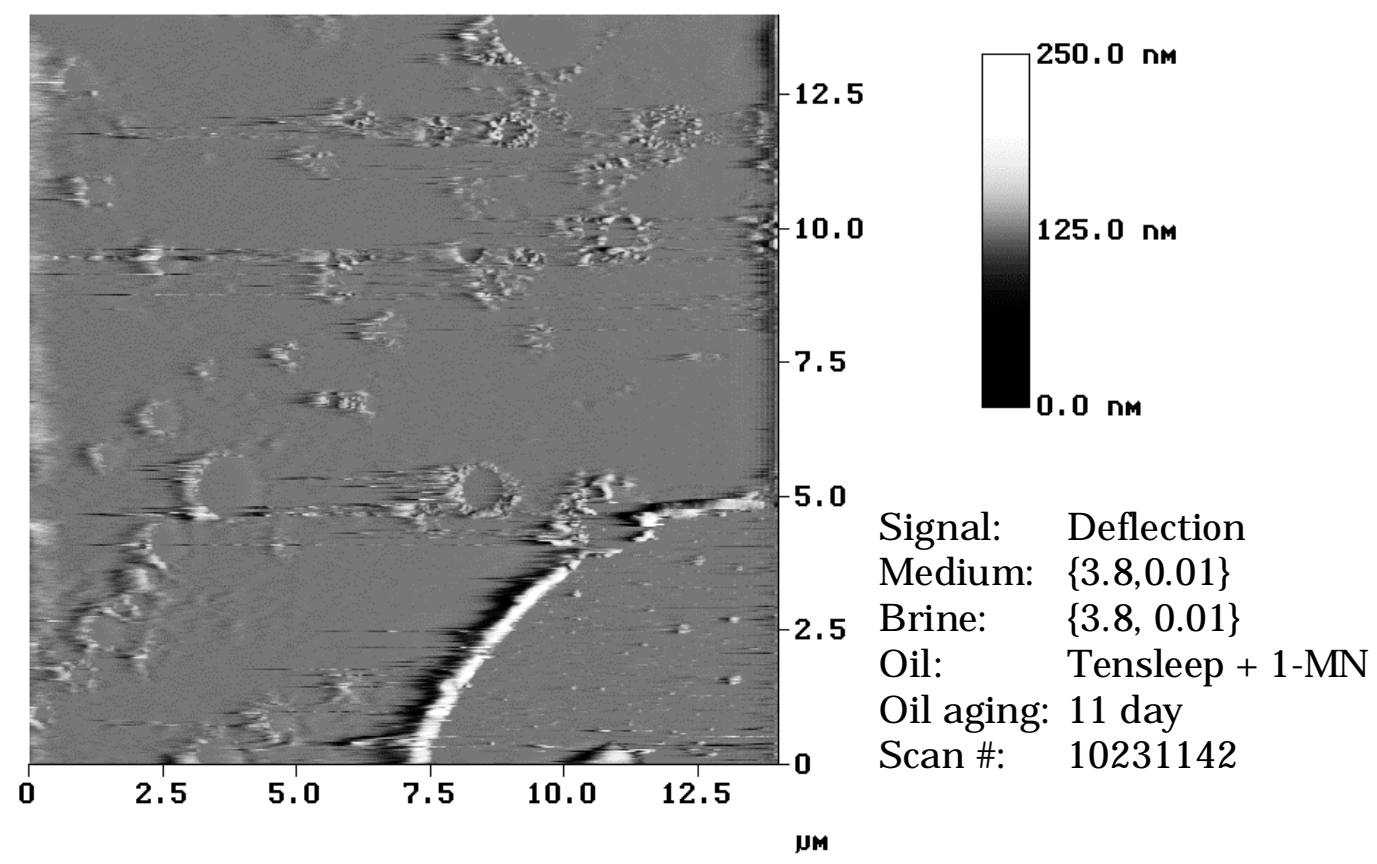

Figure II-3.60. AFM scan of mica exposed to Tensleep + 1-MN.

Decane imaging medium. The distribution of material on the surface looks similar in decane to that in brine, as shown in Figure II-3.61. Circular features are preserved and areas that appear to be mica are in evidence; in general images appear to have greater contrast in decane than in brine. Both are quite different from scans in air (compare Figure II-3.61 with II-3.60 and II-3.59). Figure II-3.62 is a zoom of features on a different area of the surface. 


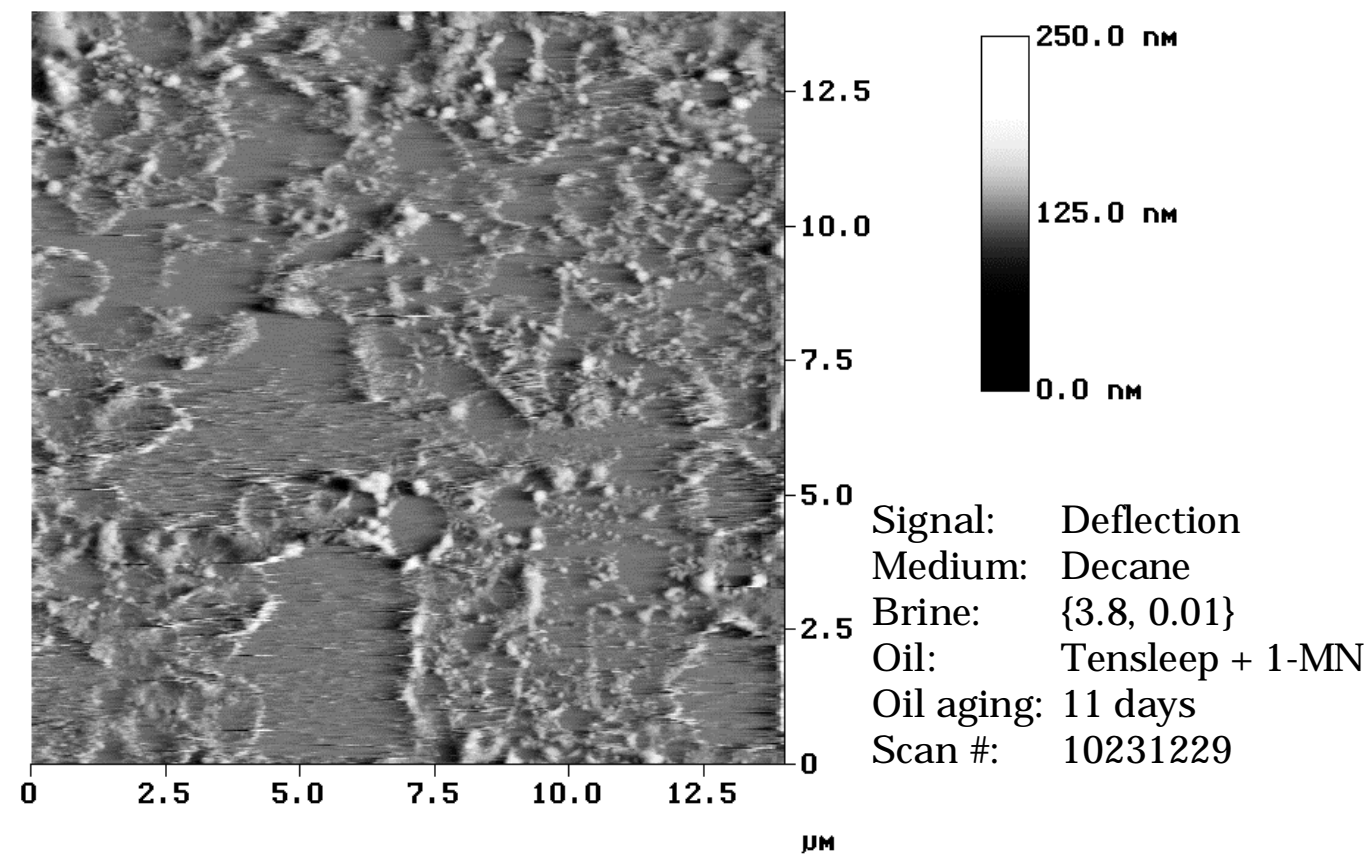

Figure II-3.61. AFM scan of mica exposed to Tensleep + 1-MN.

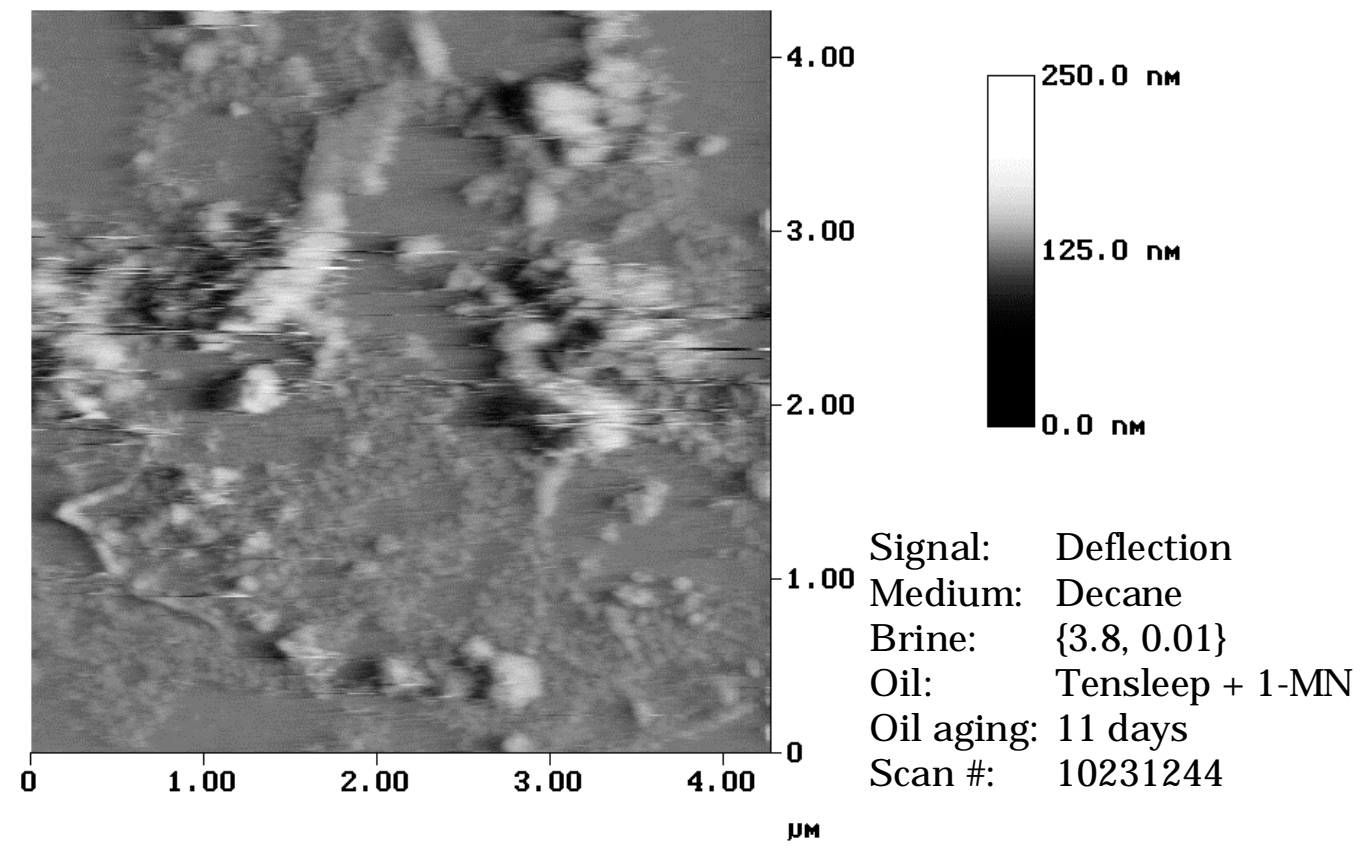

Figure II-3.62. AFM scan of mica exposed to Tensleep + 1-MN. 


\section{TENSLEEP ASPHALTENES IN TOLUENE}

Aging time: 26 days

Air imaging medium. Asphaltenes separated from Tensleep crude by mixing with nheptane and re-dissolved in toluene in a $1 \mathrm{wt} \%$ solution appeared to assemble on wet mica in a thin coating after 26 days of exposure time (Figure II-3.63). Coverage was not continuous, with bare mica visible in numerous scattered holes. None of the micron-sized rough features observed in the prior Tensleep systems and hypothesized to be aggregated asphaltenes were present. Since toluene is an effective asphaltene solvent, minimal sorption to the surface was expected, and no large aggregates were observed in either optical microscope images or the AFM scans.
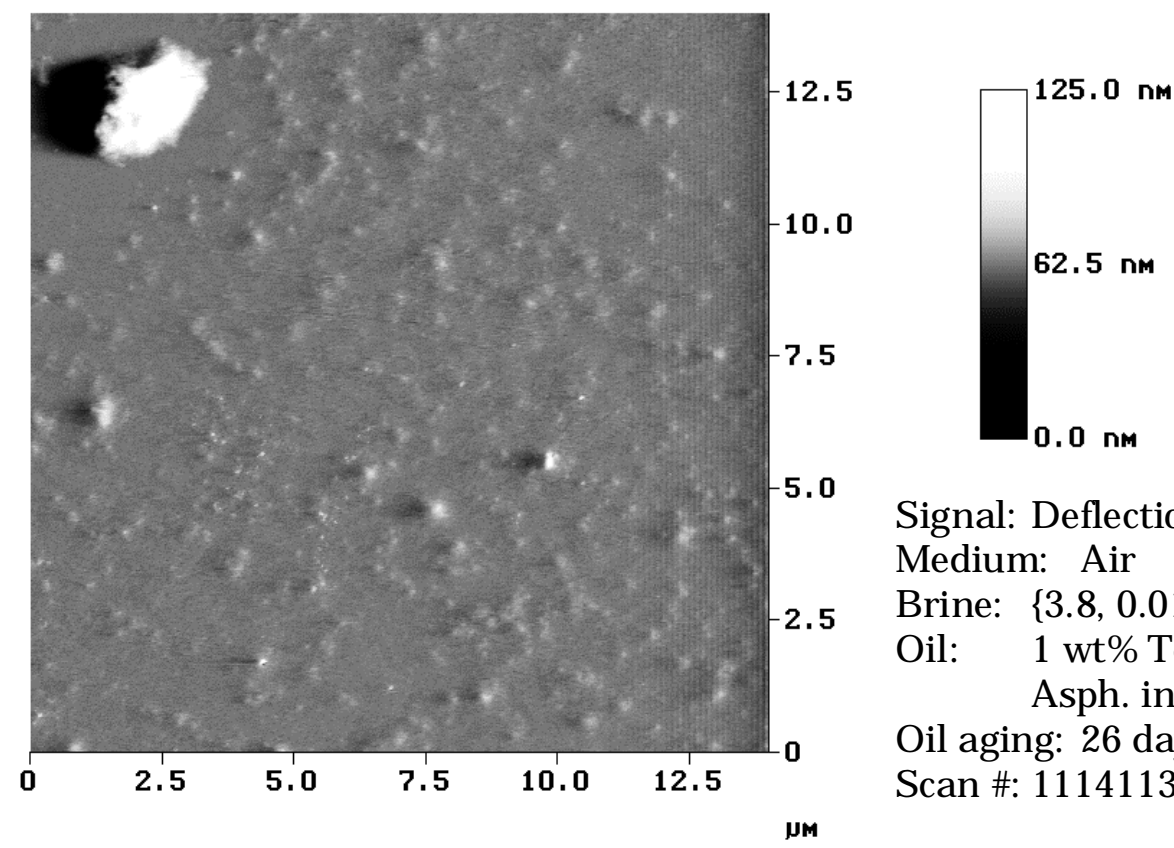

Signal: Deflection

Medium: Air

Brine: $\{3.8,0.01\}$

Oil: $\quad 1 \mathrm{wt} \%$ Tensleep

Asph. in Tol.

Oil aging: 26 days

Scan \#: 11141139

Figure II-3.63. AFM scan of mica exposed to a $1 \mathrm{wt}$ \% solution of Tensleep asphaltenes in toluene.

Brine imaging medium. The scan under brine shown in Figure II-3.64 is similar to the scans in air. A zoom (Figure II-3.65) of an oval-shaped feature just above the center in Figure II3.64 shows a consistent texture between the raised area and the rest of the film. While the film may overlay some larger object in this region, the exterior does not indicate that the object comprises a different material. The very smooth mica substrate also appears through some of the holes around the perimeter of the scan. A $300 \mathrm{~nm}$ zoom on top of the oval-shaped object (Figure II-3.66) reveals round structural units measuring about $20 \mathrm{~nm}$ in diameter. These units appear somewhat smaller than the units observed for the untreated crude Tensleep systems (see Figures II-3.43, II-3.52). 

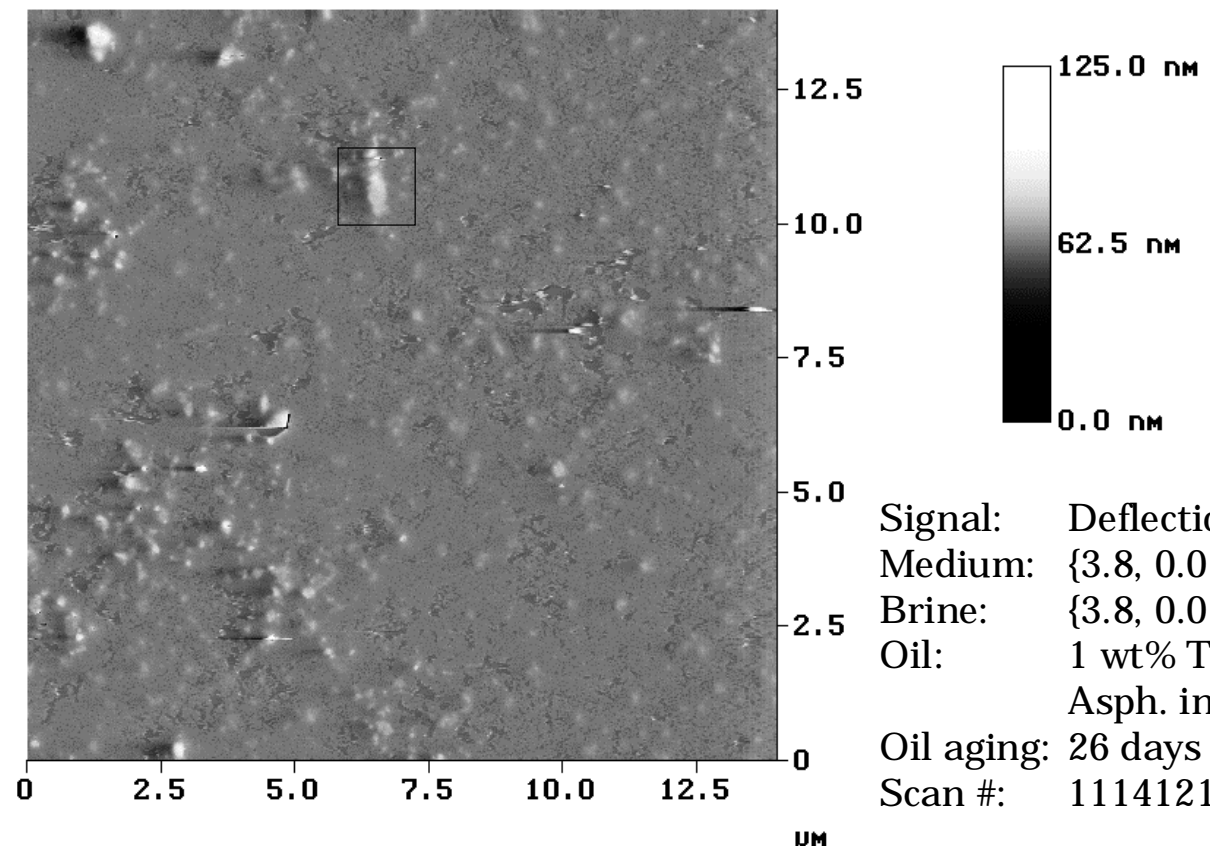

5.0

Signal: Deflection

Medium: $\{3.8,0.01\}$

Brine: $\quad\{3.8,0.01\}$

Oil: $\quad 1 \mathrm{wt} \%$ Tensleep

Asph. in Tol.

Oil aging: 26 days

Scan \#: 11141218

Figure II-3.64. AFM scan of mica exposed to a $1 \mathrm{wt} \%$ solution of Tensleep asphaltenes in toluene. A zoom of the area indicated in the box is shown in Figure II-3.65.
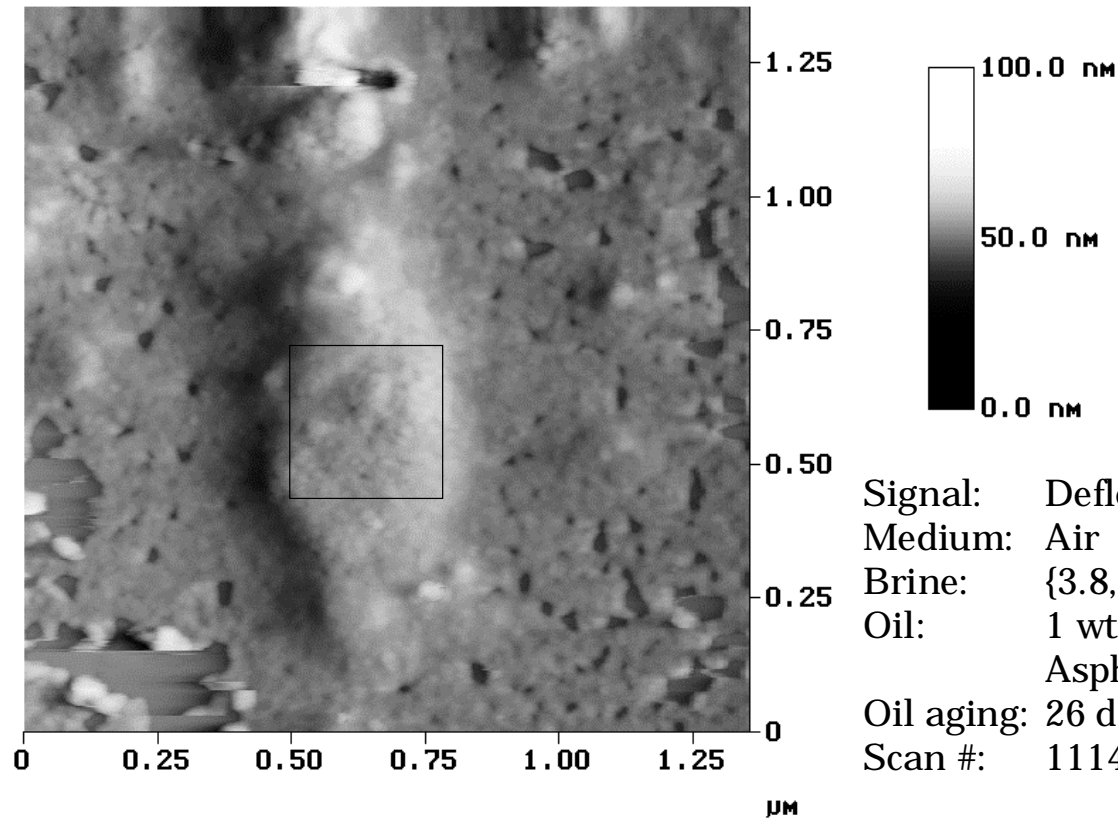

Signal: Deflection

Medium: Air

Brine: $\quad\{3.8,0.01\}$

Oil: $\quad 1 \mathrm{wt} \%$ Tensleep

Asph. in Tol.

Oil aging: 26 days

Scan \#: 11141220

Figure II-3.65. AFM scan of mica exposed to a $1 \mathrm{wt}$ \% solution of Tensleep asphaltenes in toluene. A zoom of the area indicated in the box is shown in Figure II-3.66. 


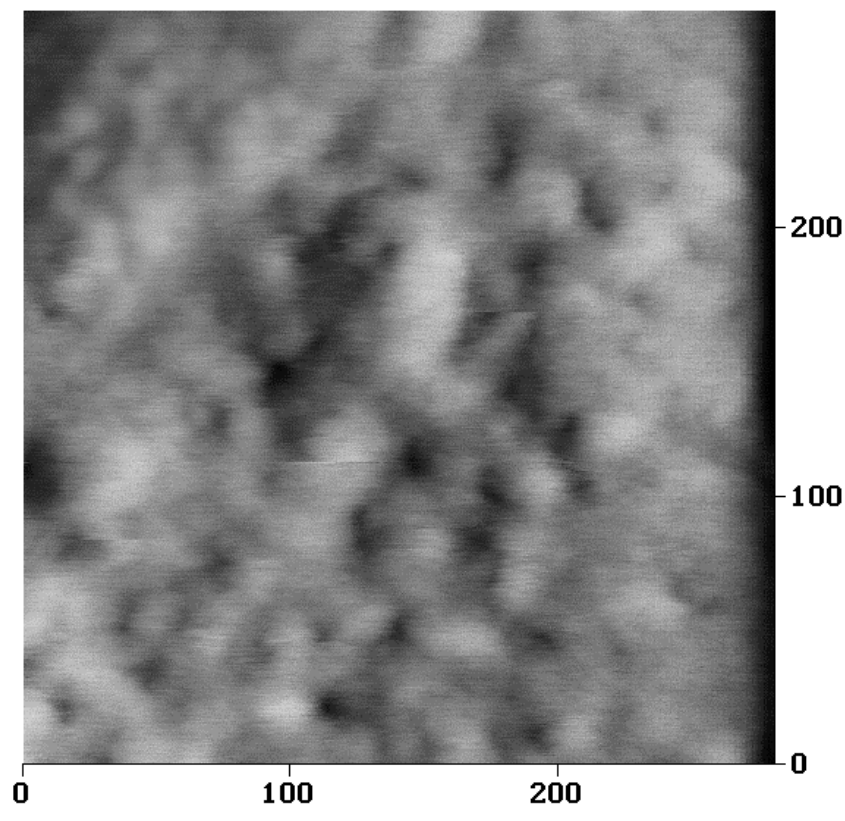

nM

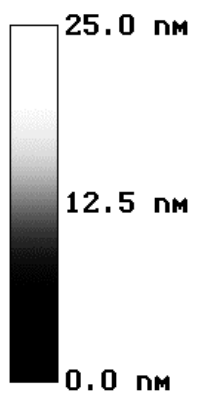

Signal: Deflection

Medium: Air

Brine: $\quad\{3.8,0.01\}$

Oil: $\quad 1$ wt\% Tensleep Asph. in Tol.

Oil aging: 26 days

Scan \#: 11141222

Figure II-3.66. AFM scan of mica exposed to a $1 \mathrm{wt}$ \% solution of Tensleep asphaltenes in toluene. Zoom of area indicated in Figure II-3.65.

Decane imaging medium. Imaging under decane (Figure II-3.67) yields results similar to those observed under air (Figure II-3.63) and brine (Figure II-3.64). A film covers most of the surface, with mica visible in small, scattered holes. A $4 \mu \mathrm{m}$ zoom (Figure II-3.68) indicates no discernable differences in texture between the raised features and the film, suggesting that the film may actually be overlaying material which was deposited on the surface either from the brine, or from the toluene solution early in the sample aging process. Several holes on the order of $1 \mu \mathrm{m}$ in diameter were found in the scan depicted in Figure II-3.69, and analyzed by section analysis for film thickness. The mean thickness according to a survey of 10 measurements was $\delta$ $=18.5 \mathrm{~nm}$, with $\sigma=4.3$. Though the mean thickness measured here is slightly larger that the thickness measured for mica exposed to Tensleep crude, the standard deviation here was sufficiently large that there is no definitive difference. 


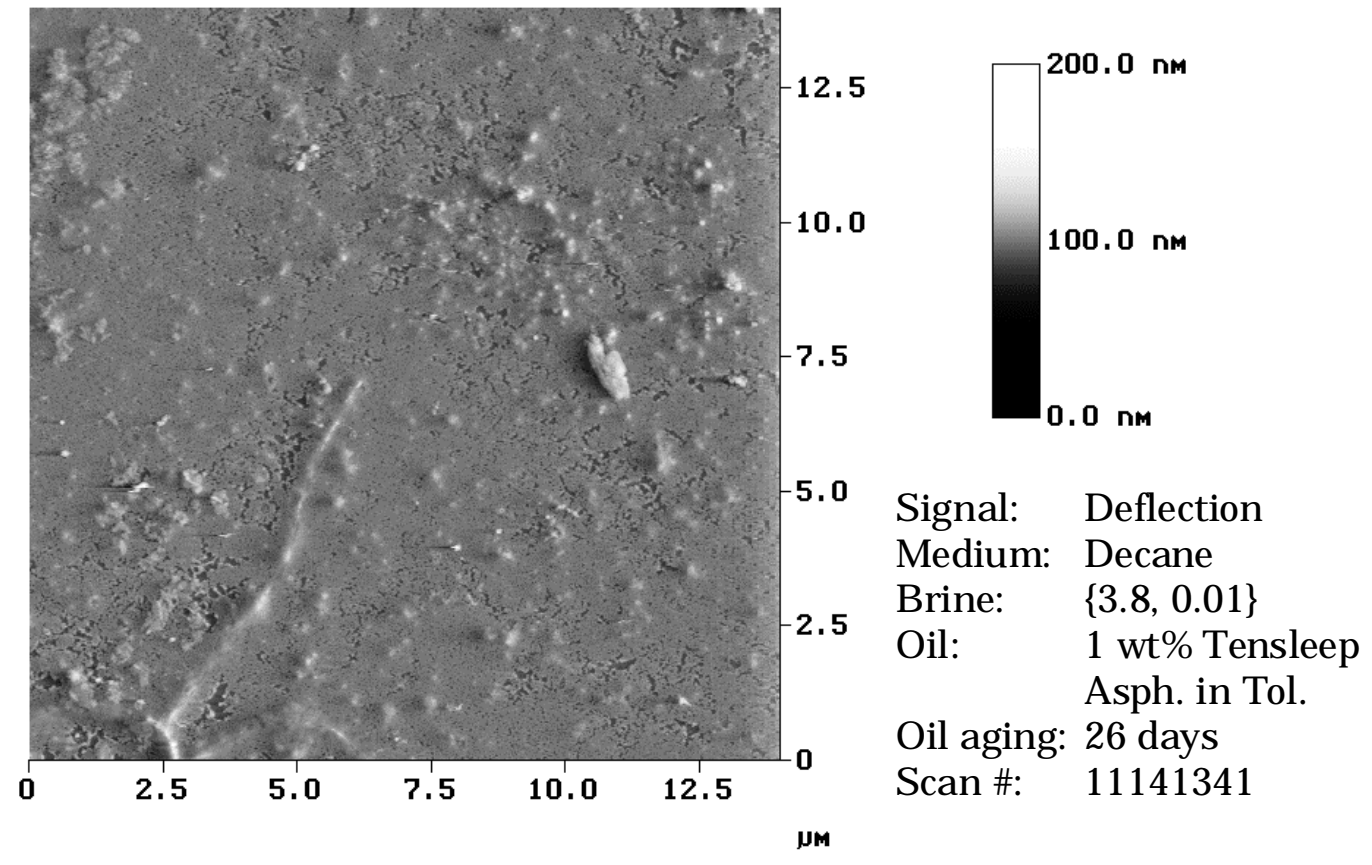

Figure II-3.67. AFM scan of mica exposed to a $1 \mathrm{wt}$ \% solution of Tensleep asphaltenes in toluene.

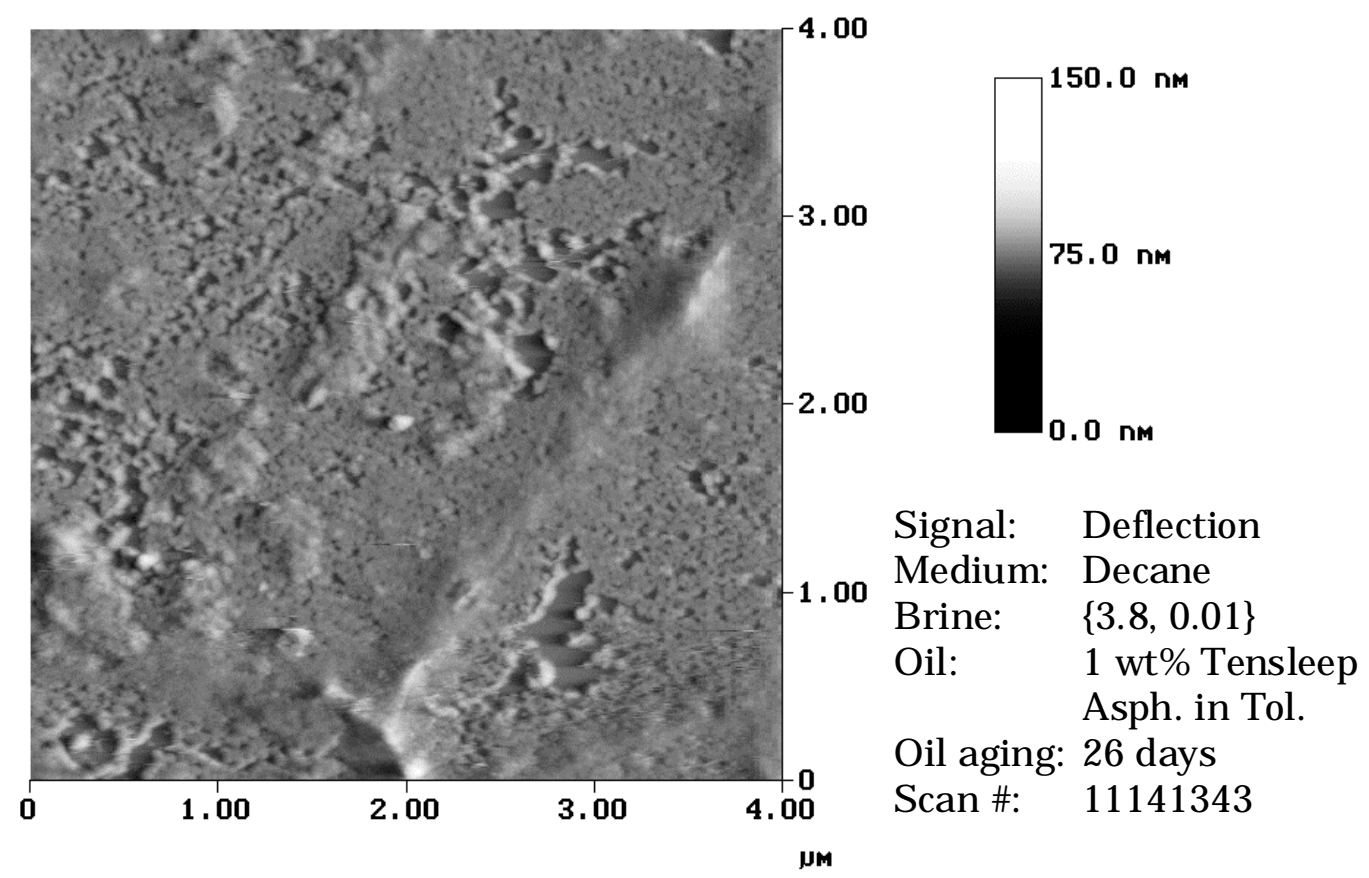

Figure II-3.68. AFM scan of mica exposed to a $1 \mathrm{wt}$.\% solution of Tensleep asphaltenes in toluene. 


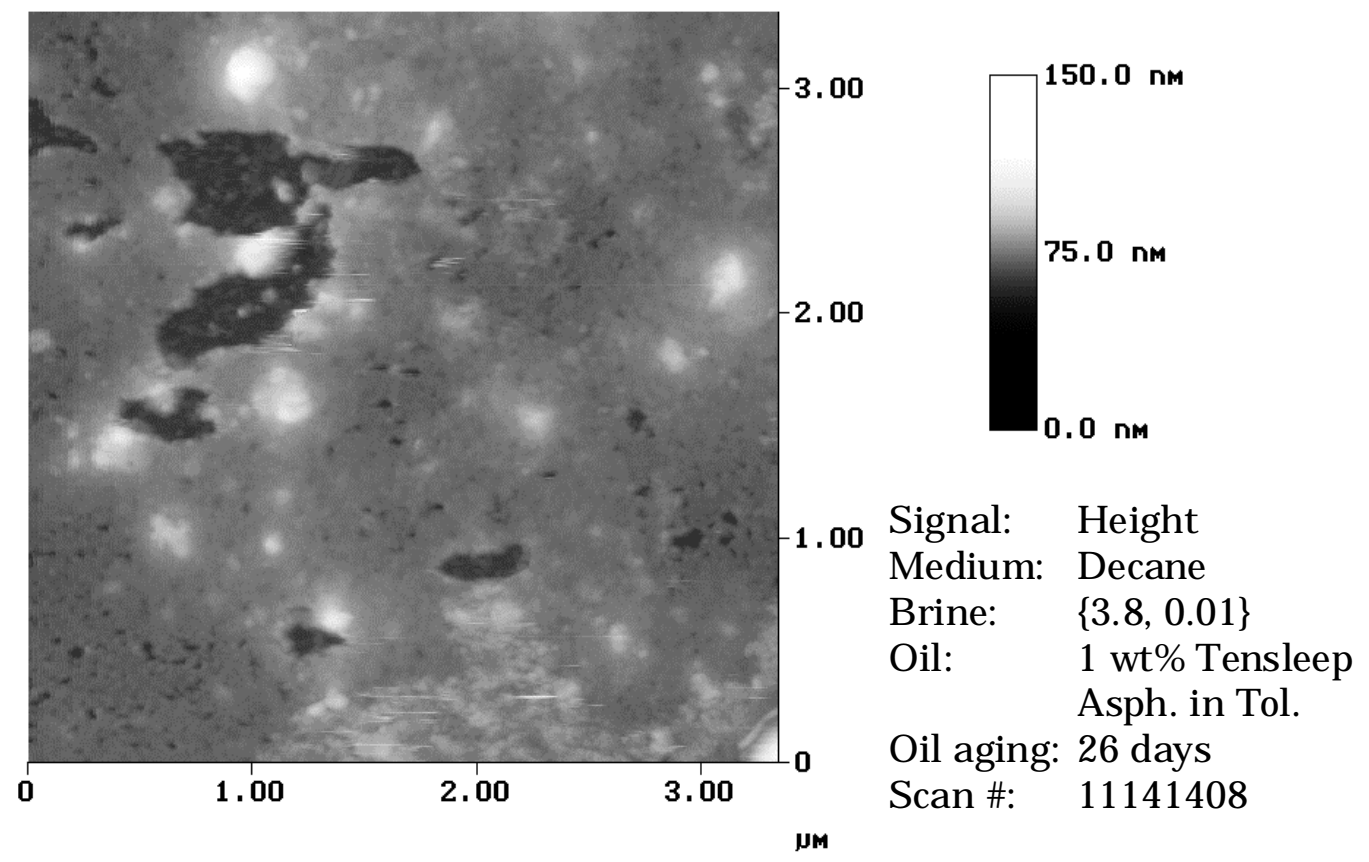

Figure II-3.69. AFM scan of mica exposed to a $1 \mathrm{wt} \%$ solution of Tensleep asphaltenes in toluene.

\section{TENSLEEP SUMMARY}

Imaging the surfaces that are produced when mica is exposed first to an aqueous phase and then a crude oil is a challenge. The organic material is soft and coverage can be extremely heterogeneous. Remnants of water drops and salt may persist. In this work imaging parameters have been selected to minimize imaging force in contact mode, reducing damage to the surface and producing details of the material on the surface that have never before been available. We have begun the task of interpretation of this wealth of information, keeping in mind that these are force maps and what appear to be high spots may represent either an accumulation of one material or a region occupied by a different material with stronger surface-tip interactions. We must also emphasize that in selecting images for this report, we have tried to choose not only those with minimal noise and interesting features, but images that are as representative of a majority of the sites sampled on any given surface. However each $14 \mu \mathrm{m} \times 14 \mu \mathrm{m}$ scan represents only about $0.00015 \%$ of the surface. More than six thousand such scans would be required to sample even $1 \%$ of the total area. Typically we are able to image fewer than ten different areas during one scanning session.

There may be artifacts due to sample preparation. Drying brine on clean mica surfaces showed at least two forms of salt, one that forms long linear features and another, smoother form. Both of these forms can be identified in images of oil-treated surfaces.

An important contribution of this work is the use of multiple imaging fluids. In some cases exactly the same view can be seen under brine and decane. Air and water are widely used, but imaging in decane is unique to this study. Air is the least suitable imaging medium for investigation of oil-treated surfaces for two reasons: (1) the imaging forces required are significantly higher in air than in either water or oil; and (2) air is not representative of 
conditions in an oil reservoir where water and oil are both present and the gas phase, if there is one, is the least wetting fluid and thus the least likely to contact the surface. Images in brine and decane were comparable and sometimes quite different from those in air (e.g., Figures II-3.59 II-3.61).

Contact angles on surfaces aged in crude oil often change slowly as aging time is increased. Figure II-3.70 shows a comparison of images from Figures II-3.31, II-3.42, and II3.50. There appears to be an overall increase in surface coverage that should correspond to increasingly oil-wet conditions and higher contact angles with aging time in oil. Contact angles corresponding to the surface treatments will be measured for comparison.
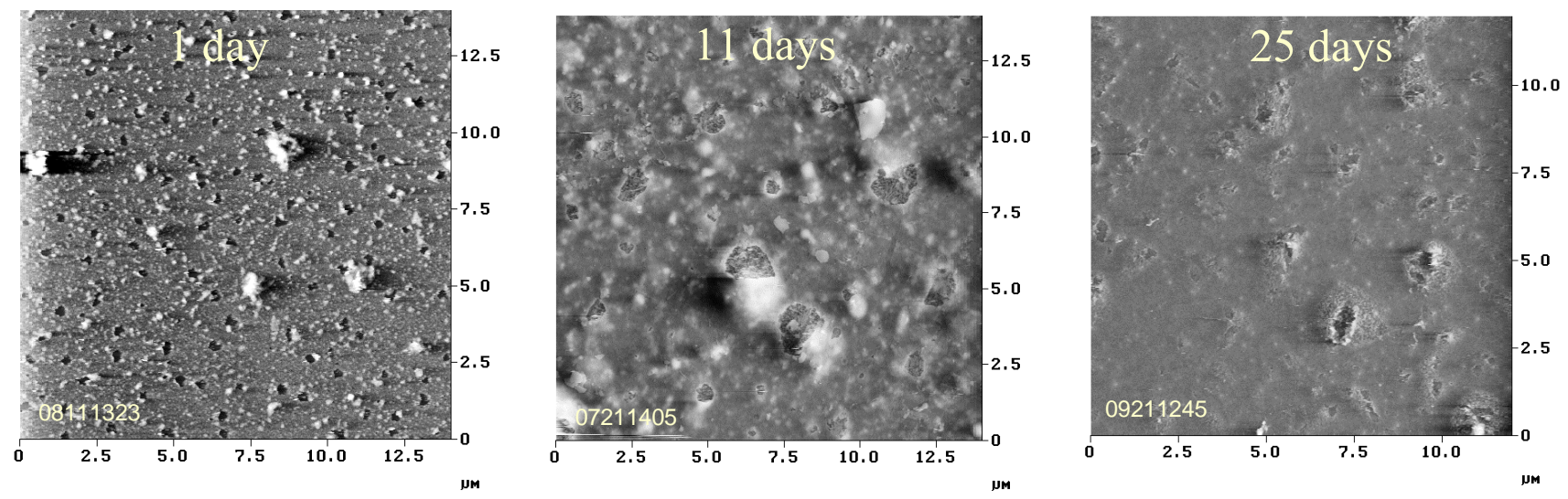

Figure II-3.70. Surface coverage increases as aging time in Tensleep crude oil increases from 1 day to 25 days.

Contact angle tests have also shown that surfaces exposed to crude oil fractions are not comparable to those resulting from exposure to the original oil (e.g., Buckley et al., 1997). Comparisons of AFM images for mica surfaces exposed first to $\{3.8,0.01\}$ brine, followed by Tensleep oil, Tensleep plus 1-MN, or $1 \mathrm{wt} \%$ of Tensleep asphaltenes in toluene for 11 - 26 days, demonstrate that the most complete film is formed from the original oil. Areas that appear to be bare mica are evident on surfaces exposed either to the mixture or the asphaltene solution, but a continuous film, interrupted only by regions that are covered with the coarser-grained material, is formed on mica aged in Tensleep oil.

As discussed above, the identity of the coarse-grained material is suspected to be asphaltene aggregate. The aggregates are not invariably present when samples were aged for only one day (Figure II-3.70), but appear, after longer aging, to be covered by other material that is gradually cementing them in place. These features are absent from the mixture of Tensleep and $1-\mathrm{MN}$ and from the surface contacted with the mixture. They are also absent from the 1\% asphaltene solution in toluene and from the surface contacted with the asphaltene solution (Figure II-3.71). Clearly something adsorbed from the asphaltene solutions and just as clearly that material must be asphaltic since toluene is the only other component of the solution and toluene alone does not adsorb on mica. The film forming material in all cases may contain asphaltene, but flocculated asphaltenes are found only when they are sufficiently covered by other materials to hold them on the surface, suggesting that they are deposited, but not adsorbed. 
When decane is the imaging fluid, solvent quality with respect to asphaltenes can be adjusted by addition of toluene or other asphaltene solvents to provide additional information about the solubility of materials deposited on the surface, as illustrated in Figure II-3.48. It is interesting to note that the coarse-grained, flocculated asphaltene features that are deposited rather than adsorbed begin to swell, but the adsorbed material, which should also be soluble in toluene, remains fixed on the surface.

\section{Tensleep \\ 11 days}

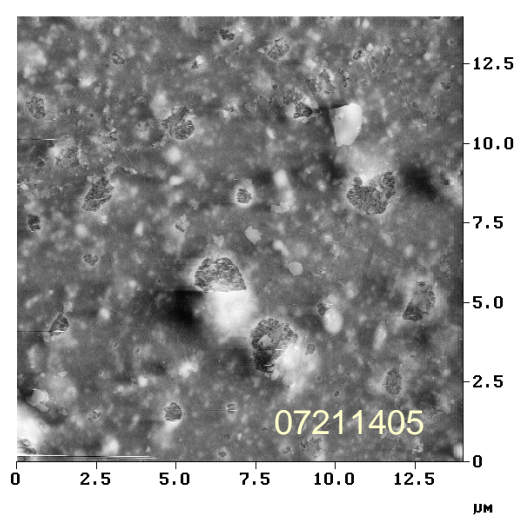

Tensleep $+1-\mathrm{MN}$ 11 days

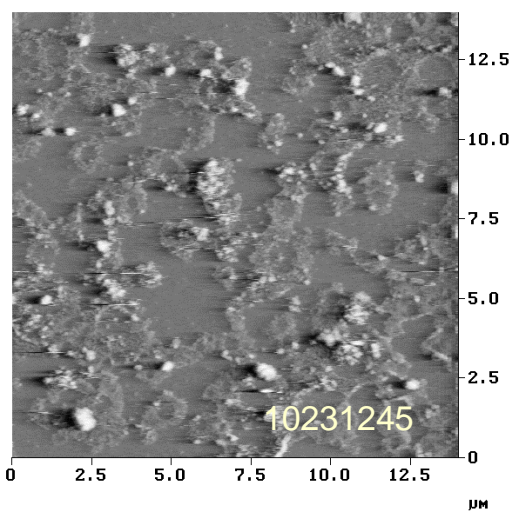

$1 \mathrm{wt} \%$ Tensleep asphaltenes in toluene 26 days

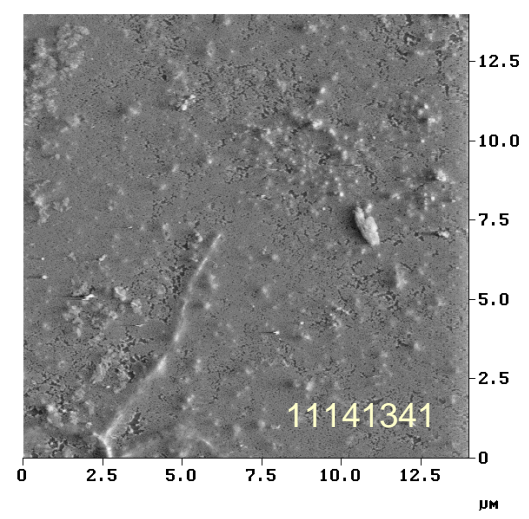

Figure II-3.71. Comparison of mica surfaces treated first with $\{3.8,0.01\}$ brine, then with Tensleep oil, a mixture of Tensleep and 1-MN, or a solution of Tensleep asphaltenes in toluene. Asphaltene aggregates are present only in the original oil. Incomplete films are formed by the mixture and asphaltene solution, even after 11 and 26 days of aging, respectively.

Film thickness cannot always be reliably estimated. Selected cases where bare mica appears to be visible so that film thickness can be estimated are summarized in Table II-3.4. Roughness of the surface is a quantitative measure that can help distinguish between films that are smooth in appearance and bare mica patches. The roughness analyses are summarized in Table II-3.5. Mica is at least an order of magnitude less rough than even the smoothest films of salt. The coarse-grained asphaltenes are the roughest areas measured.

Table II-3.4. Film thickness estimates.

\begin{tabular}{|c|l|l|l|}
\hline Scan \# & Fig. \# & \multicolumn{1}{|c|}{ Sample Preparation } & \multicolumn{1}{|c|}{\begin{tabular}{c}
\multicolumn{1}{|c|}{} \\
$(\mathbf{n m})$
\end{tabular}} \\
\hline 01121401 & 3.13 & $\{3.8,0.01\}$ brine, Tensleep, 1 day, imaged in decane & $13.3 \pm 1.6$ \\
\hline 08111204 & 3.8 & $\{3.8,0.01\}$ brine, Tensleep, 1 day, imaged in brine & $12.7 \pm 1.0$ \\
\hline 09211316 & 3.33 & $\begin{array}{l}\{3.8,0.01\} \text { brine, Tensleep, 25 days, imaged in decane } \\
+1-\mathrm{MN}\end{array}$ & 15 \\
\hline 11141343 & 3.47 & $\begin{array}{l}\{3.8,0.01\} \text { brine, 1\% Tensleep asphaltenes in toluene, } \\
\text { 25 days, imaged in decane }\end{array}$ & $18.5 \pm 4.3$ \\
\hline
\end{tabular}


Table II-3.5. Roughness analysis of selected areas.

\begin{tabular}{|c|l|l|l|l|}
\hline Scan \# & Fig. \# & \multicolumn{1}{|c|}{ Sample Preparation } & Area $(\boldsymbol{\mu m})^{\mathbf{2}}$ & $\mathbf{R}_{\mathbf{a}}(\mathbf{n m})$ \\
\hline 07260858 & 3.1 & Clean mica & 25 & 0.16 \\
\hline 10231315 & 3.2 & $\begin{array}{l}\text { Salt dried on mica imaged in air } \\
\text { Salt dried on mica imaged in decane }\end{array}$ & 0.2 & $2.5-4.0$ \\
\hline 0231405 & 3.3 & $\begin{array}{l}\{3.8,0.01\} \text { brine, Tensleep, 11 days, imaged in } \\
\text { brine } \\
\text { smooth film } \\
\text { holes in film filled with coarser-grained } \\
\text { material }\end{array}$ & $\begin{array}{l}0.25 \\
0.1\end{array}$ & 3.1 \\
\hline 07211457 & 3.24 & $\begin{array}{l}\{3.8,0.01\} \text { brine, Tensleep, 11 days, imaged in } \\
\text { decane }\end{array}$ & 0.1 & 0.41 \\
\hline 10261352 & 3.28 & $\begin{array}{l}\{8,1\} \text { brine, Tensleep, 11 days, imaged in } \\
\text { brine }\end{array}$ & 25 & 0.05 \\
\hline
\end{tabular}

Over the course of this study, the AFM scanning parameters have been optimized, artifacts have been identified, and a sequence of fluids identified that can maximize the amount of useful information, both qualitative and quantitative, obtained from these experiments. Comparisons with contact angle tests on similarly treated surfaces are in progress. The next step is to extend this AFM study to crude oils with varying physical and chemical properties.

\section{Oils from the Mars Platform}

\section{MARS-YELLOW CRUDE}

Mars Yellow crude examined under the optical microscope contained some particles that may be aggregated asphaltenes and wax crystals that are evident in Figure II-3.72.

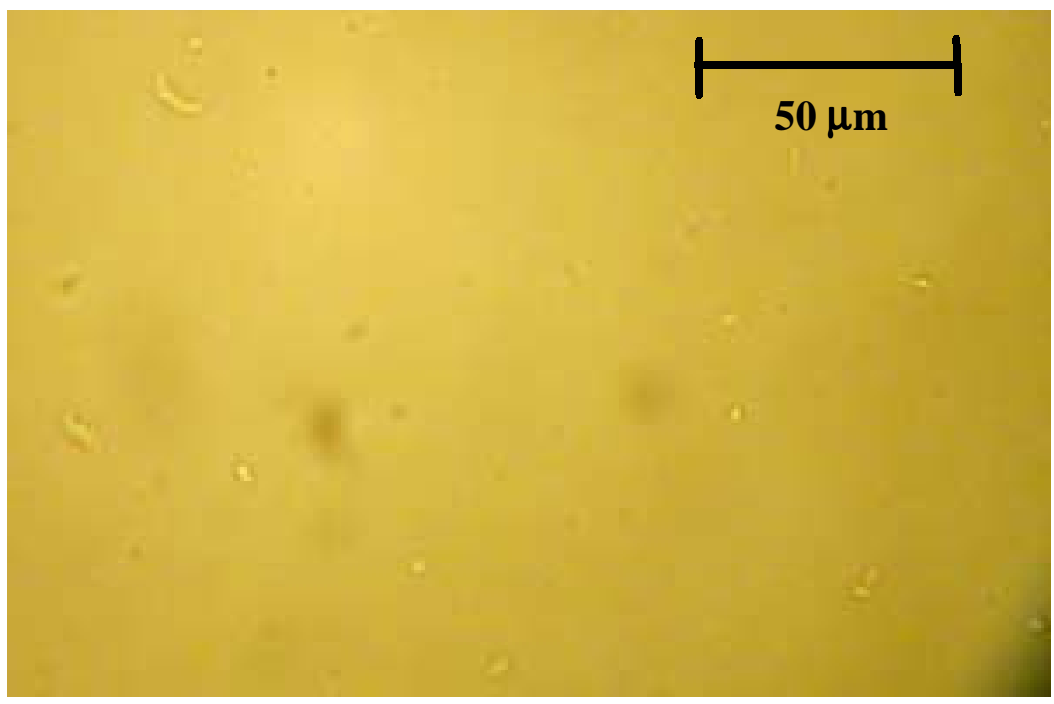

Figure II-3.72. Optical microscope image of Mars Yellow crude. Precipitated waxes appear as particulate material while oil background resembles a dark mustard color. 
Aging time: 1 day

Decane-water contact angles measured on mica plates treated with $\{3.8,0.01\}$ brine for 1 day and Mars Yellow for 1 day indicated a weakly water-wet to neutrally-wet system, with values of $\theta_{A}=81^{\circ} \pm 16^{\circ}$, and $\theta_{R}=38^{\circ} \pm 8^{\circ}$. All contact angles values were measured through the water phase. (A summary of all contact angle values measured for the Mars oil systems is given at the end of this section in Table II-3.6.)

Brine imaging medium. AFM scans of mica treated with $\{3.8,0.01\}$ brine for 1 day and Mars Yellow for 1 day were indeterminate. Tip-sample adhesion caused poor image resolution. Force calibration curves observed for this system indicated that there was no snap-off, implying that the tip was "stuck" to the sample surface. This behavior was likely caused by a soft, adhesive layer of material on the mica surface which prevented the tip from resolving the distinct surface features observed in prior systems. Tapping mode AFM (TM-AFM), a scanning technique in which the scanning tip is oscillated perpendicularly to the surface, was attempted on this sample (Figure II-3.73) to see if the oscillatory motion could overcome the adhesive forces to obtain a useful image. The considerable feature "smearing" evident in this scan indicates continued tip-sample adhesion despite the tip oscillation. Image resolution in this system was not sufficient for meaningful analysis.

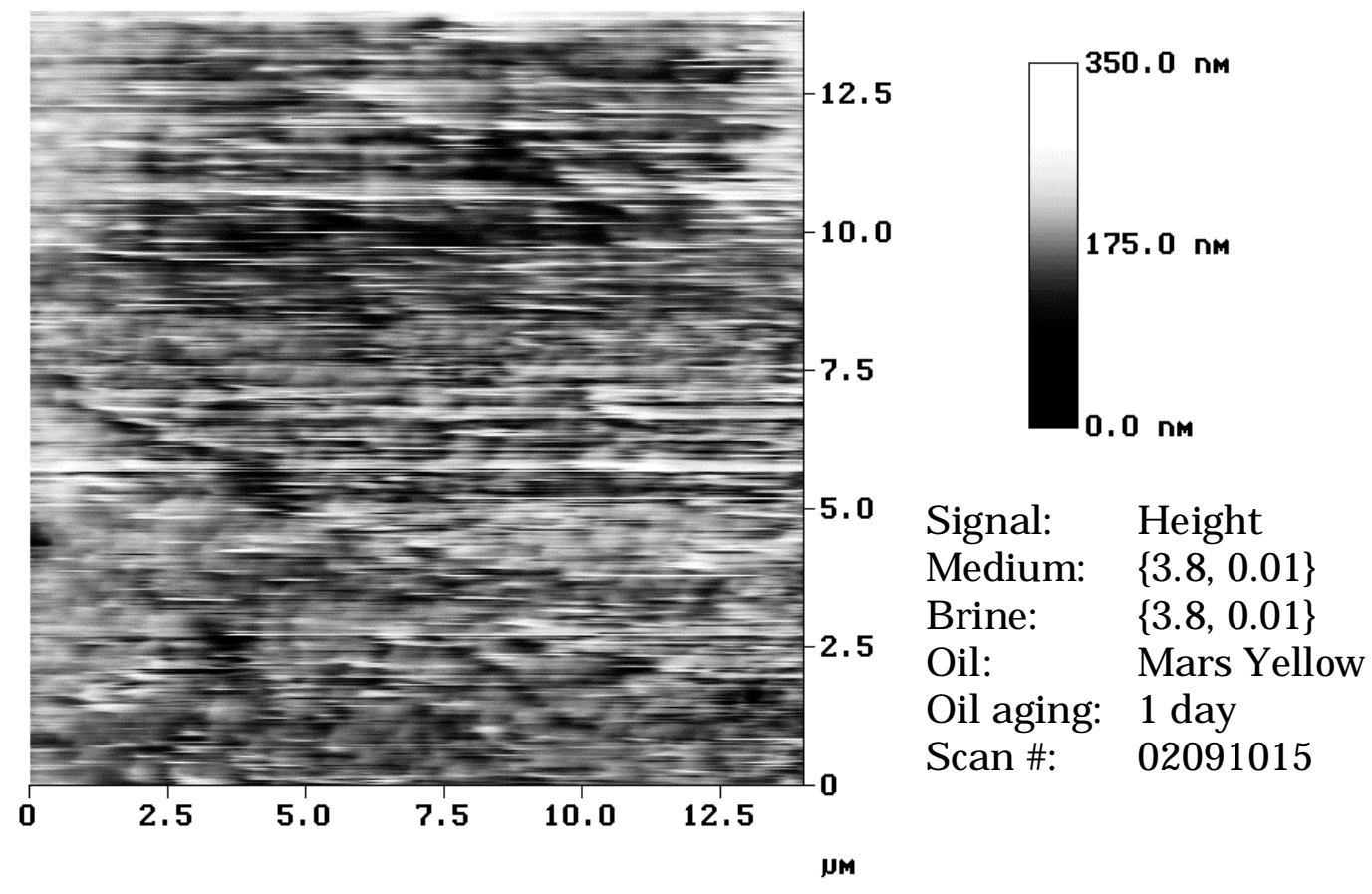

Figure II-3.73. TM-AFM scan of mica exposed to Mars Yellow crude. Tip-sample adhesion caused the poor resolution in this scan.

Decane imaging medium. Tip-sample adhesion was similarly problematic under decane. The contact mode AFM (CM-AFM) scan shown in Figure II-3.74 fails to provide sufficient resolution for analysis. 


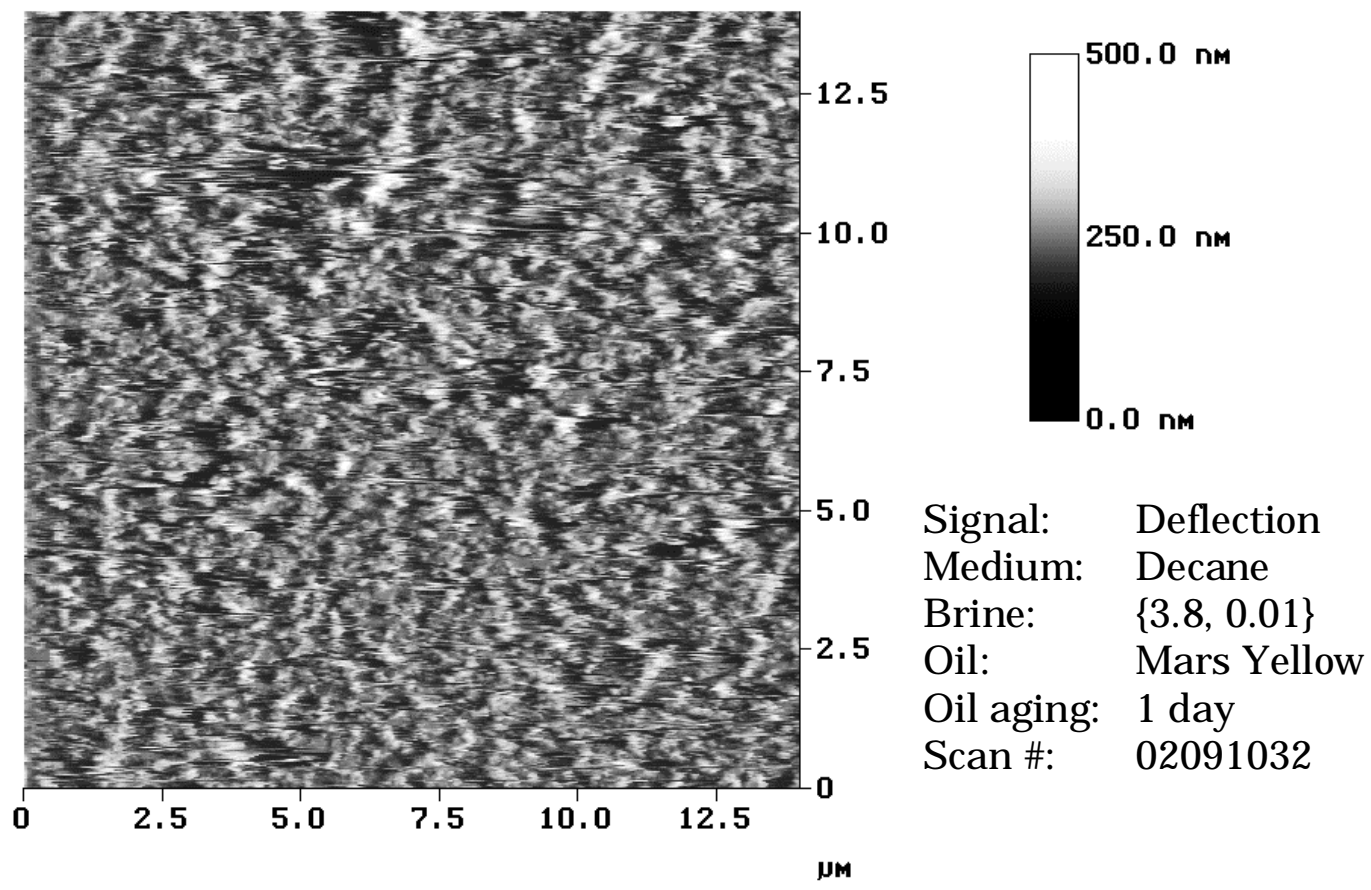

Figure II-3.74. AFM scan of mica exposed to Mars Yellow crude. Tip-sample adhesion caused the poor resolution in this scan.

Aging time: 21 days

Contact angles measured on the mica after 1 day exposure to $\{3.8,0.01\}$ brine followed by 21 days exposure to Mars Yellow crude were weakly water-wet, with $\theta_{\mathrm{A}}=67^{\circ} \pm 18^{\circ}$, and $\theta_{\mathrm{R}}$ $=25^{\circ} \pm 6$. While some asphaltene aggregates were visible in Mars Yellow, the wettability was altered to a relatively small extent. Attempts by Al-Maamari (2000) to induce wettability change on mica by precipitating Mars Yellow asphaltenes with heptane yielded only weakly water-wet conditions, evidenced by angles measuring $\theta_{A}=82^{\circ} \pm 6^{\circ}$, and $\theta_{R}=37^{\circ} \pm 8^{\circ}$ for mica aged 21 days in the onset mixture of Mars-Yellow and n-heptane. Therefore, it appears that the presence 
of precipitated asphaltenes does not necessarily lead to wettability reversal on mica pretreated with an acidic brine.

Air imaging medium. Despite the relatively low contact angles measured for this system, a considerable amount of material appeared to adsorb to the mica surface according to AFM scans in air (Figure II-3.75). Complete coverage of the mica surface is observed, with rough spots analogous to the features hypothesized to be asphaltenes in the Tensleep systems. Since aggregated asphaltenes and waxes were observed under the optical microscope for this oil, deposition of aggregates on the mica is plausible.

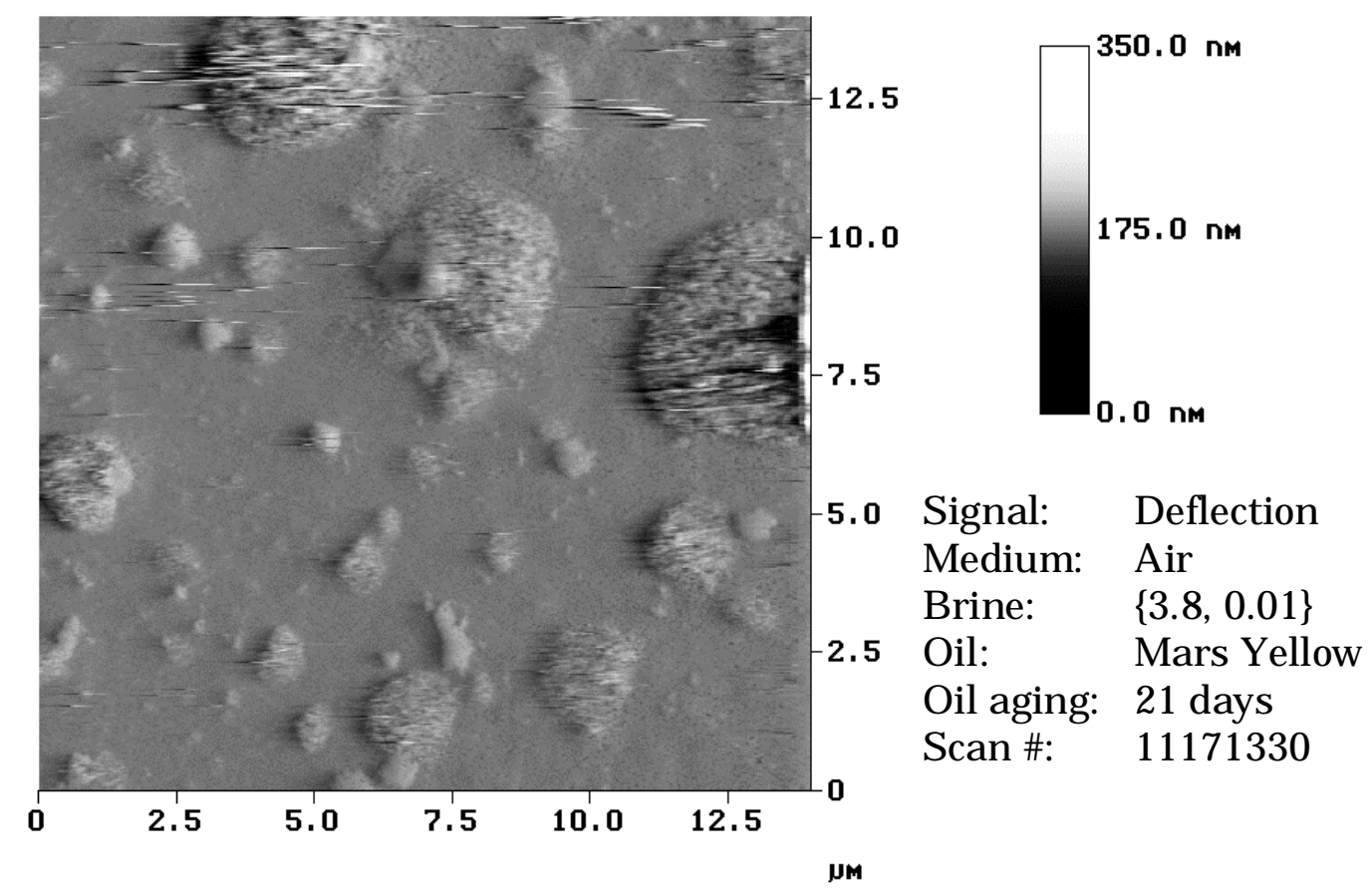

Figure II-3.75. AFM scan of mica exposed to Mars Yellow crude.

Brine imaging medium. Placing a $\{3.8,0.01\}$ brine droplet onto the dry sample surface induced blistering of the film, illustrated in Figure II-3.76, which shows the same scan area as Figure II-3.75. The blisters ranged from less than $1 \mu \mathrm{m}$ up to $5 \mu \mathrm{m}$ or more in diameter. A roughness analyses of two selected $0.4 \mathrm{~nm}^{2}$ regions of the smooth film gave a value of $R_{a}=1.8 \pm$ $0.3 \mathrm{~nm}$. Roughness analyses of the visibly coarser material yielded a value of $R_{a}=7.5 \pm 0.3 \mathrm{~nm}$. One blister along the left edge of Figure II-3.76 apparently tore open during scanning, exposing mica $\left(R_{a}=0.04\right)$ underneath. The fact that the film may be loosened from the substrate by water could have considerable implications on wetting characteristics of the solid. 


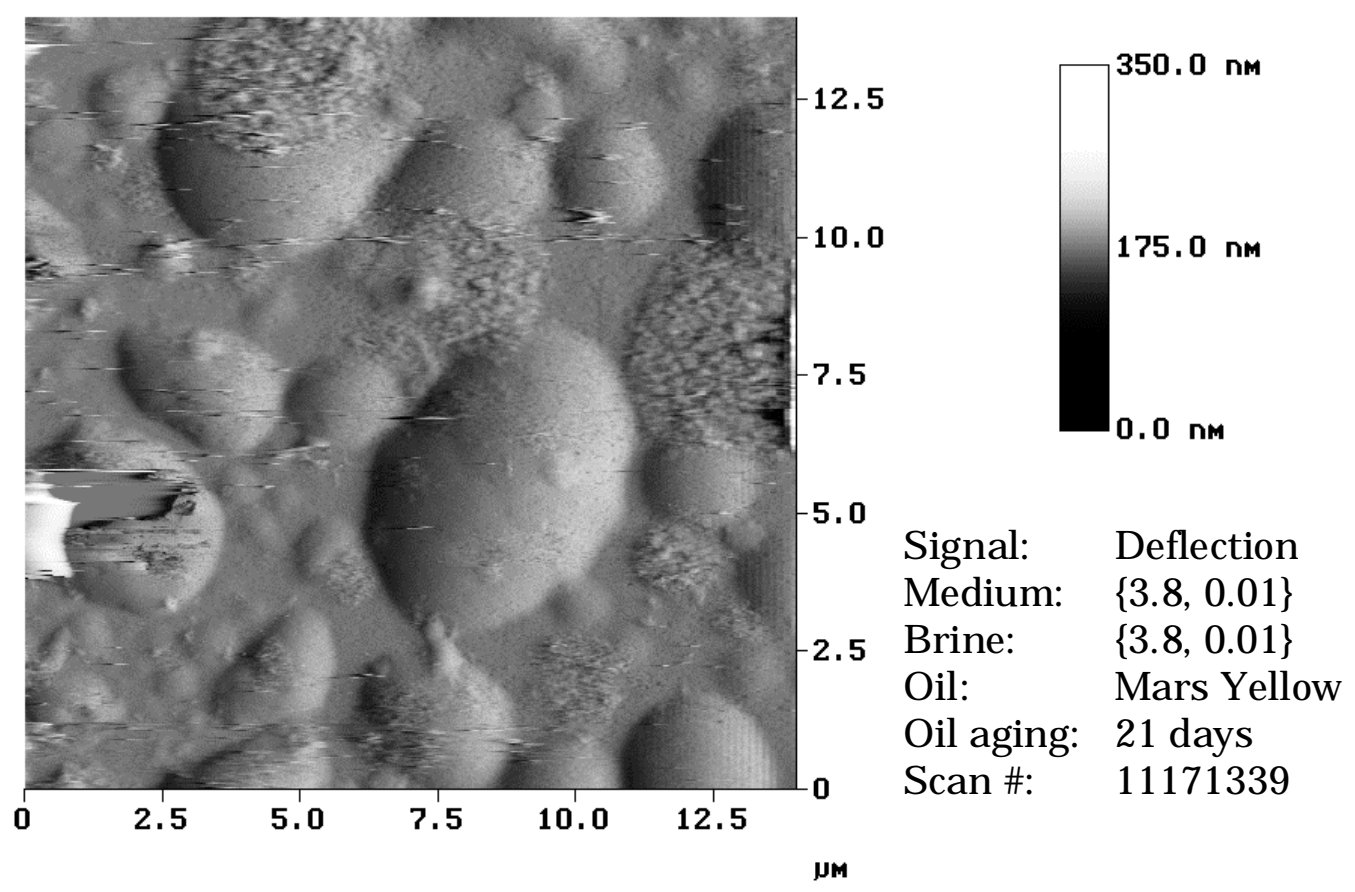

Figure II-3.76. AFM scan of mica exposed to Mars Yellow crude. This figure represents the same scan area shown in Figure II-3.75. Note the blistering of the film upon addition of the brine imaging medium.

Mars Yellow has been observed to form rigid films at the brine-oil interface (AlMaamari, 2000). Since the mica was enveloped in a film of brine prior to exposure to the oil, a static oil-brine interface was formed while the sample aged in oil, providing conditions for formation of a rigid film very close to the mica surface. This phenomenon may have played a role in forming the skin-like material which retained its continuity despite local detachment from the substrate. While many scan areas demonstrated the blistering effect (e.g., Figures II-3.76, II3.77), the surface was not entirely blistered (Figure II-3.78). 


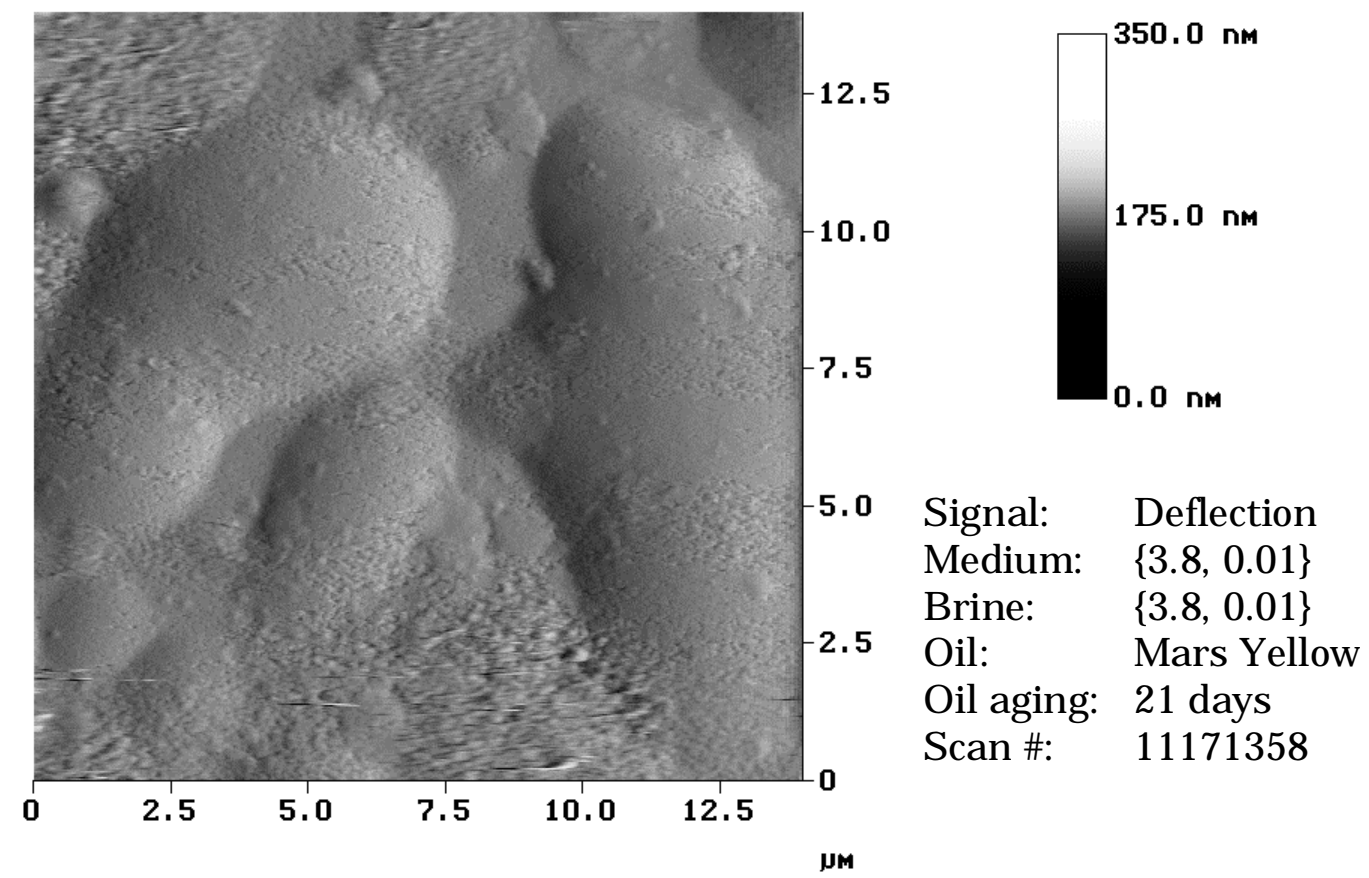

Figure II-3.77. AFM scan of mica exposed to Mars Yellow crude.
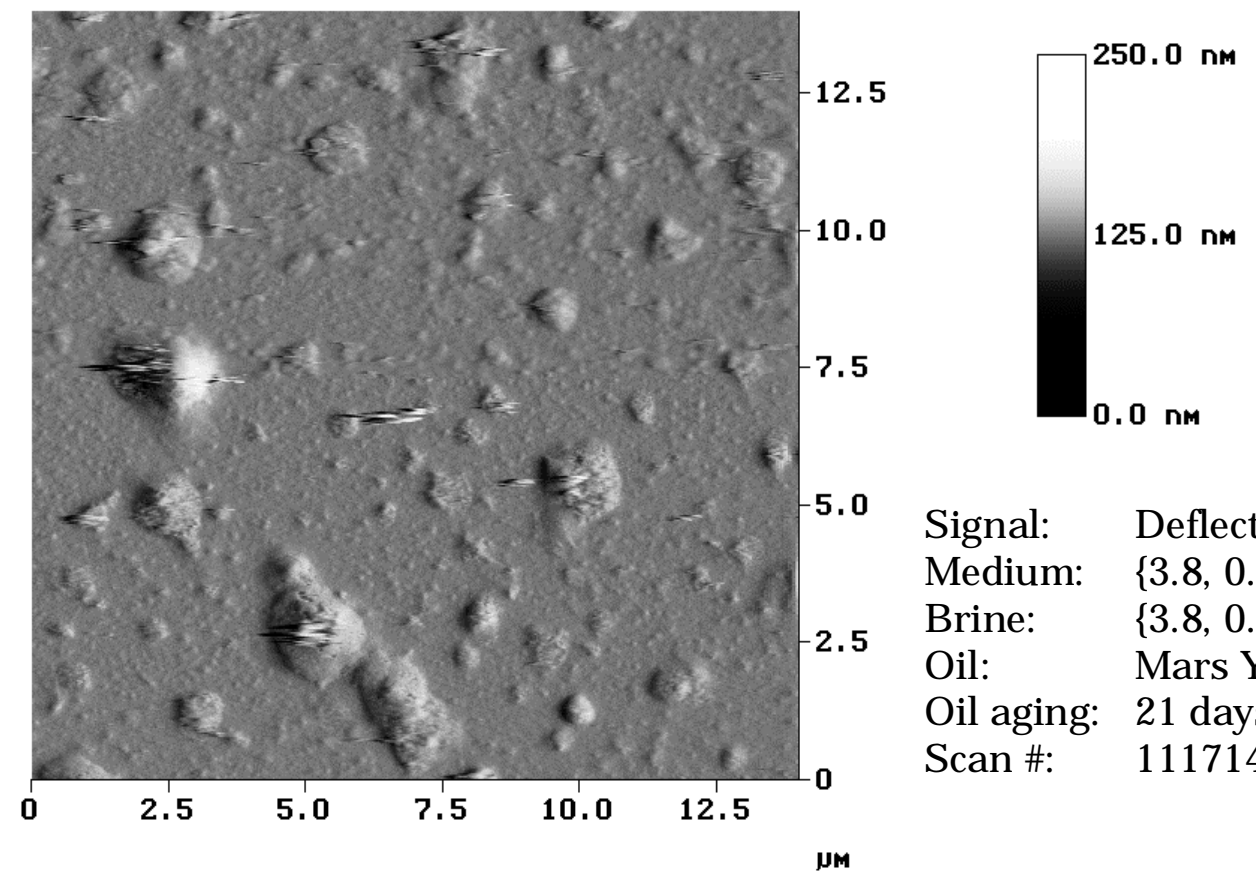

Signal: Deflection

Medium: $\quad\{3.8,0.01\}$

Brine: $\quad\{3.8,0.01\}$

Oil: $\quad$ Mars Yellow

Oil aging: 21 days

Scan \#: 11171418

Figure II-3.78. AFM scan of mica exposed to Mars Yellow crude. 
Decane imaging medium. The blistering effect was not observed when imaging under decane (Figure II-3.79). Rather, surface features resembled those imaged under air. Mica coverage was complete, and characterized by a coating of relatively uniform thickness and texture. Integrated into this coating were numerous asphaltene-like structures. Some of these rough features appeared to be covered by the coating, while others were exposed. A $3.5 \mu \mathrm{m}$ zoom of one rough feature (Figure II-3.80) shows fine detail of the structural units.

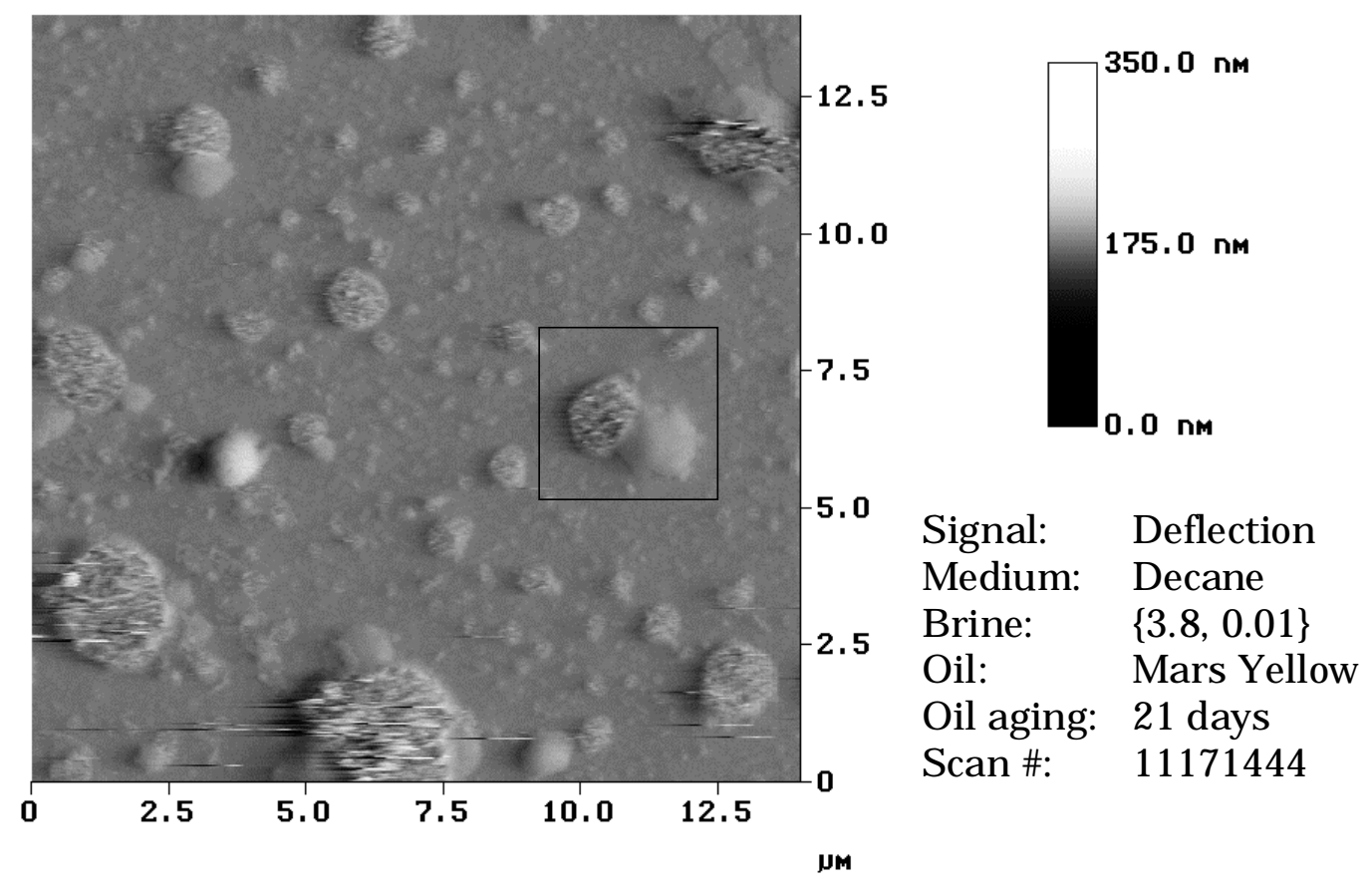

Figure II-3.79. AFM scan of mica exposed to Mars Yellow crude. A zoom of the area indicated by the box is shown in Figure II-3.80. 


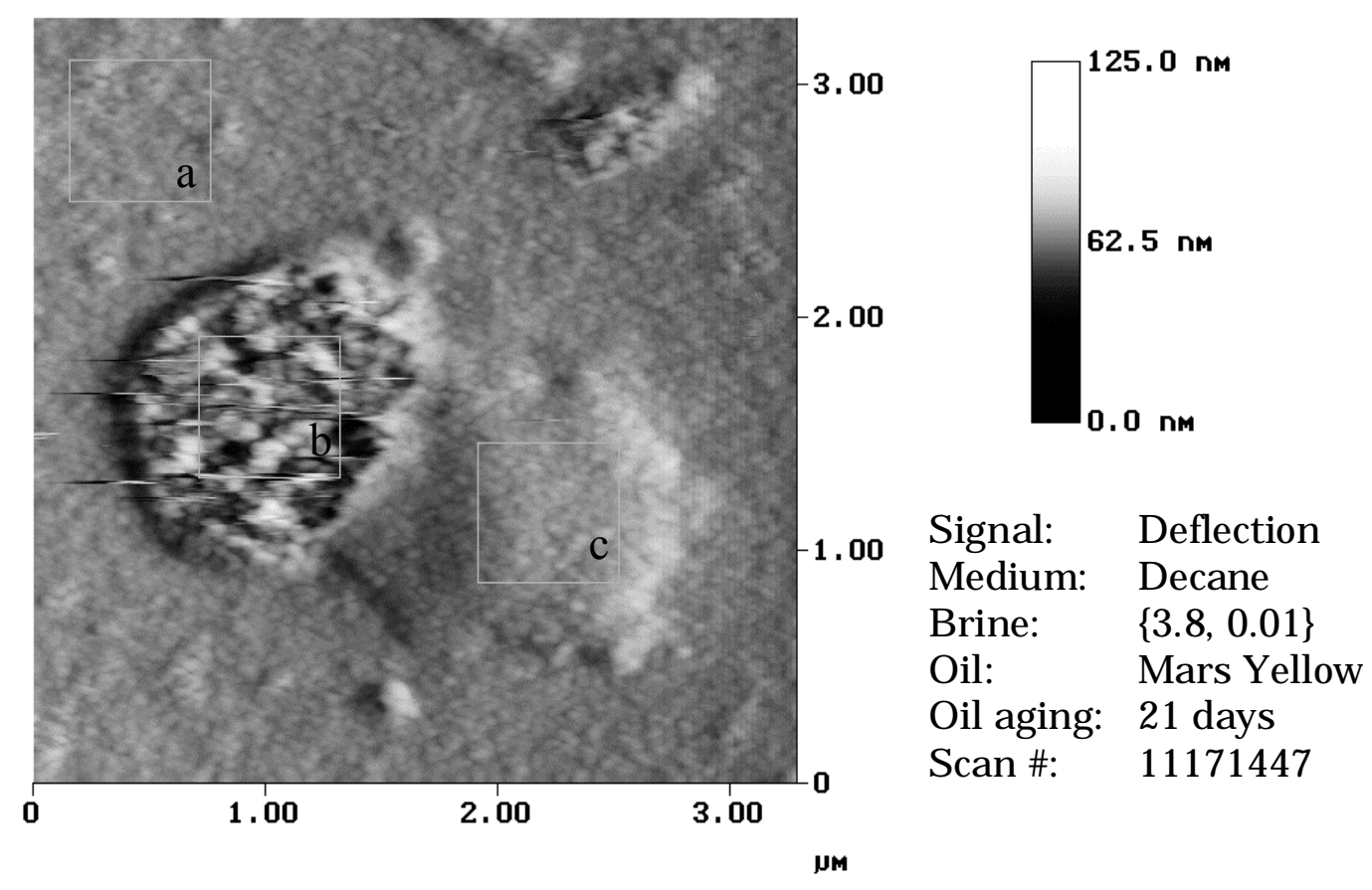

Figure II-3.80. AFM scan of mica exposed to Mars Yellow crude. Roughness of areas indicated by $0.4 \mu \mathrm{m}^{2}$ boxes measured: a) $1.64 \mathrm{~nm}$, b) $7.73 \mathrm{~nm}$, c) $1.708 \mathrm{~nm}$.

Roughness values for three boxed areas in Figure II-3.80 measured $(a) \mathrm{R}_{\mathrm{a}}=1.64 \mathrm{~nm},(b)$ $\mathrm{R}_{\mathrm{a}}=7.73 \mathrm{~nm}$, and $(c) \mathrm{R}_{\mathrm{a}}=1.71 \mathrm{~nm}$. Box $a$ covers a representative region of the uniform coating most prominent on the substrate. Box $b$ encompasses the asphaltene/wax feature, and the roughness value here $\left(\mathrm{R}_{\mathrm{a}}=7.73 \mathrm{~nm}\right)$ compares closely with the roughness of asphaltene structures identified in Figure II-3.76 and the Tensleep system $\left(\mathrm{R}_{\mathrm{a}}=8.4 \mathrm{~nm}\right.$, Table II-3.5). Box $c$, placed over the dome-shaped feature which resembles an asphaltene/wax feature covered by the surface film, has a roughness which matches that of box $a$. The identity of the material which lies under the dome feature is not known, although the material on top of the dome appears to be the same as that which comprises the dominant coating on the substrate.

Decane+toluene imaging medium. Some of the decane imaging medium was replaced with toluene so the new imaging medium was composed of roughly half decane and half toluene (Figures II-3.81 and II-3.82). For the same $350 \mathrm{~nm}$ height scale, features under decane + toluene (Figure II-3.81) appear to be taller than under decane alone (Figure II-3.79). This could be evidence of asphaltene swelling upon exposure to toluene, a phenomenon also observed in the Tensleep system. Also evident were several depressions or concave features that appeared to have lost some material upon addition of toluene. One such feature indicated in the box in Figure II-3.81 is captured in a $3 \mu \mathrm{m}$ zoom in Figure II-3.82. It is possible that the swelling of asphaltene aggregates caused some of them to dislodge from the surface, leaving a footprint behind. 


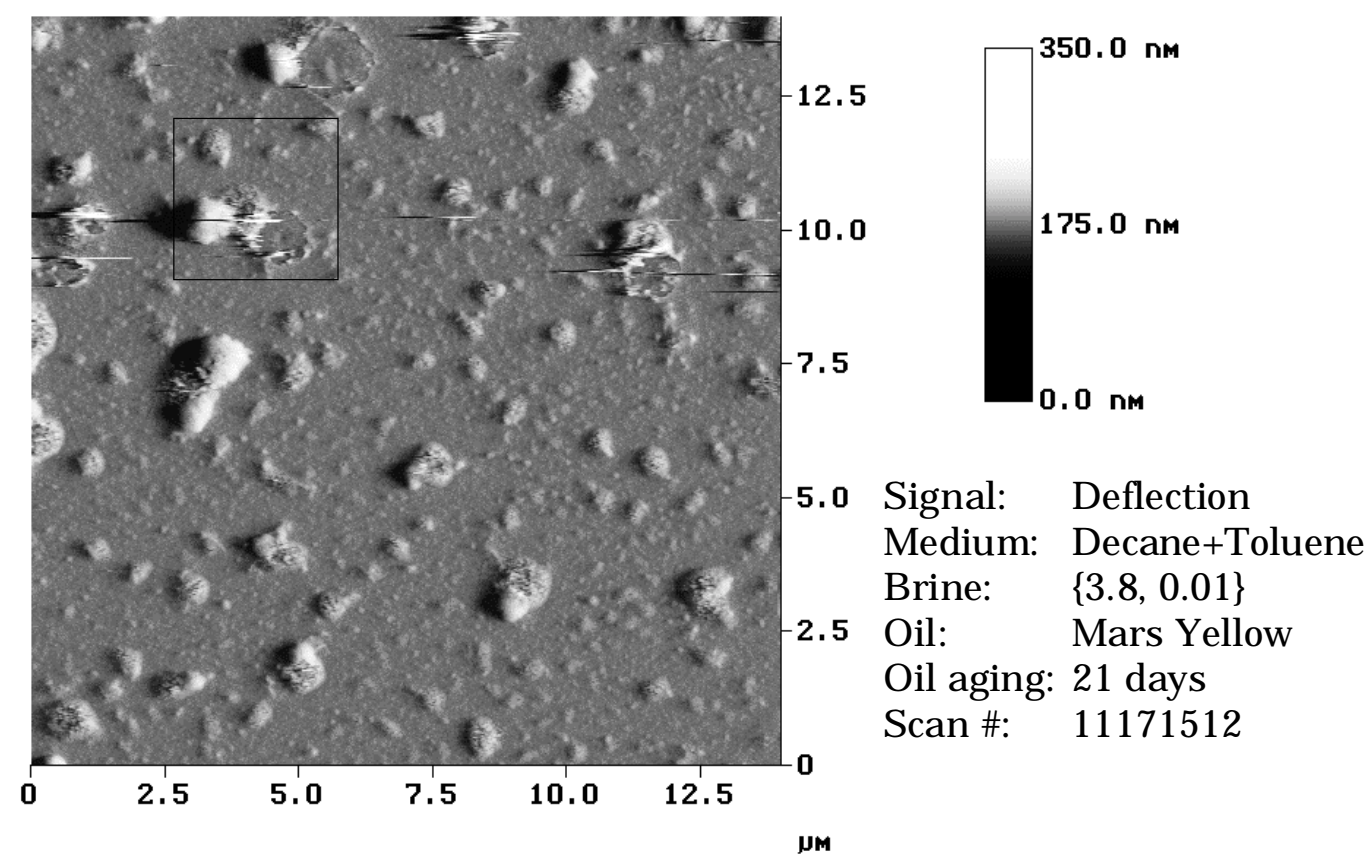

Figure II-3.81. AFM scan of mica exposed to Mars Yellow crude. A zoom of area indicated by the box is shown in Figure II-3.82.

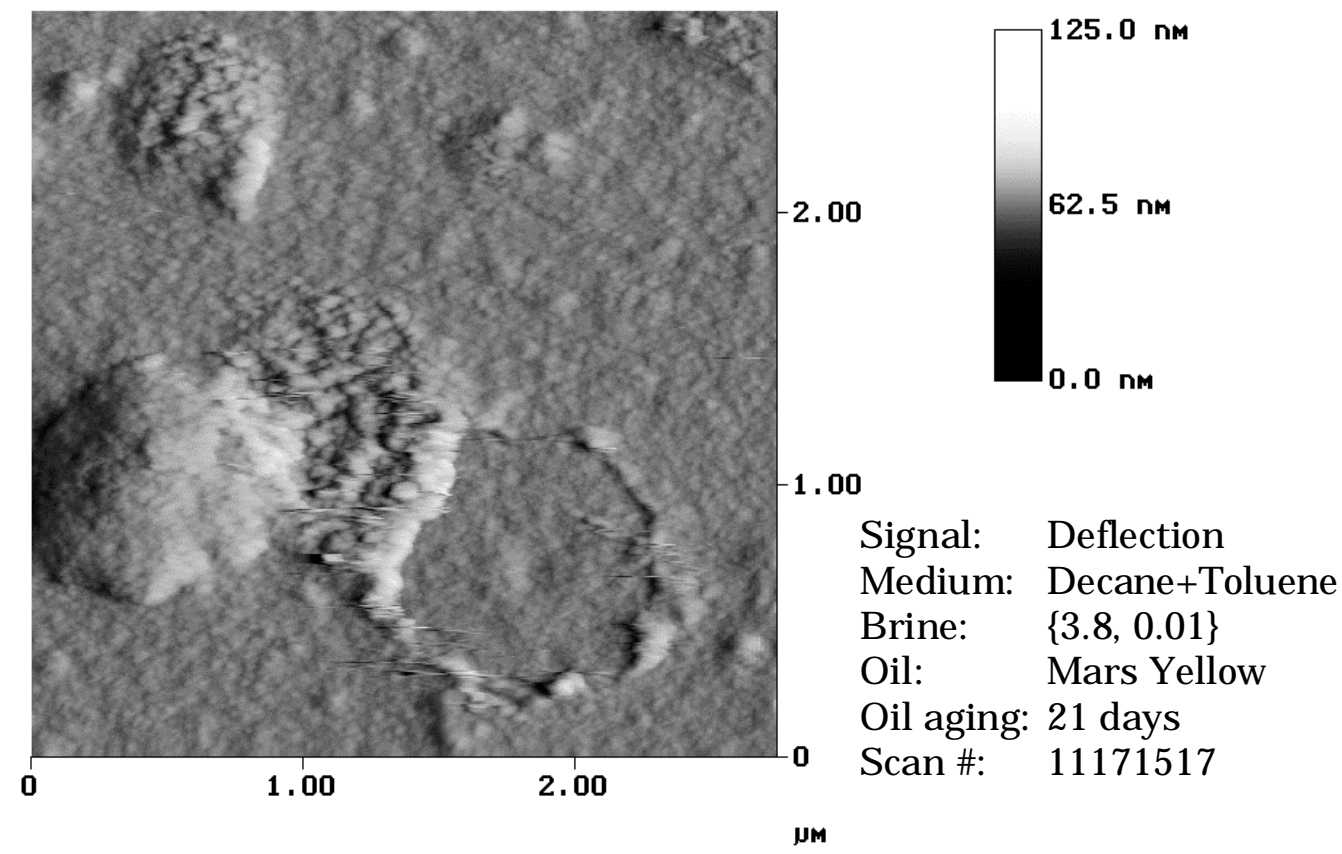

Figure II-3.82. AFM scan of mica exposed to Mars Yellow crude. 
Aging time: 99 days

Decane imaging medium. AFM scans on a mica sample aged for 1 day in $\{3.8,0.01)$ and 99 days in Mars Yellow are shown in Figures II-3.83 and II-3.84. Tip-sample adhesion in air and brine resulted in poor image quality, so images were obtained only in decane. Complete surface coverage is observed in Figure II-3.83, as expected after long exposure to oil. The roughness of two specific regions are shown on Figure II-3.83. Box $a\left(\mathrm{R}_{\mathrm{a}}=1.6 \mathrm{~nm}\right)$ samples a portion of the smooth film covering most of the substrate, while box $b\left(\mathrm{R}_{\mathrm{a}}=7.4 \mathrm{~nm}\right)$ is located over a structure suspected to be an adsorbed asphaltene or wax particle. These values compare closely with the roughness of analogous structures measured on the 21-day sample, where the smooth film roughness was $R_{a}=1.6 \mathrm{~nm}$, and the asphaltene roughness was $R_{a}=7.7 \mathrm{~nm}$. Roughness of the smooth film measured in three random locations on Figure II- 3.83 yielded $R_{a}=$ $1.4 \pm 0.1 \mathrm{~nm}$, while roughness over three asphaltene structures was $R_{a}=6.3 \pm 1.3 \mathrm{~nm}$.
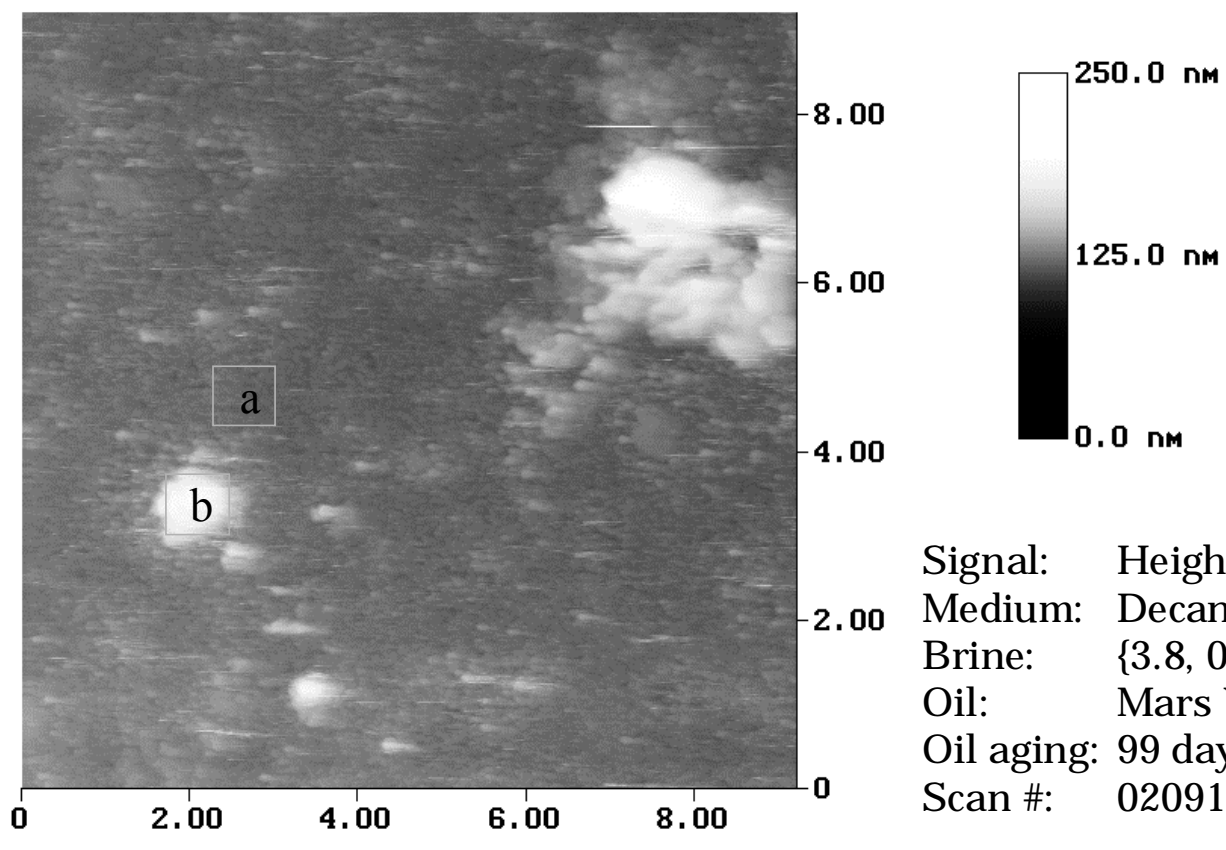

Signal: Height

Medium: Decane

Brine: $\quad\{3.8,0.01\}$

Oil: $\quad$ Mars Yellow

Oil aging: 99 days

Scan \#: 02091221

JM

Figure II-3.83. AFM scan of mica exposed to Mars Yellow crude. Roughness of areas indicated by $0.4 \mu \mathrm{m}^{2}$ boxes measured: a) $1.58 \mathrm{~nm}$, b) $7.44 \mathrm{~nm}$. 


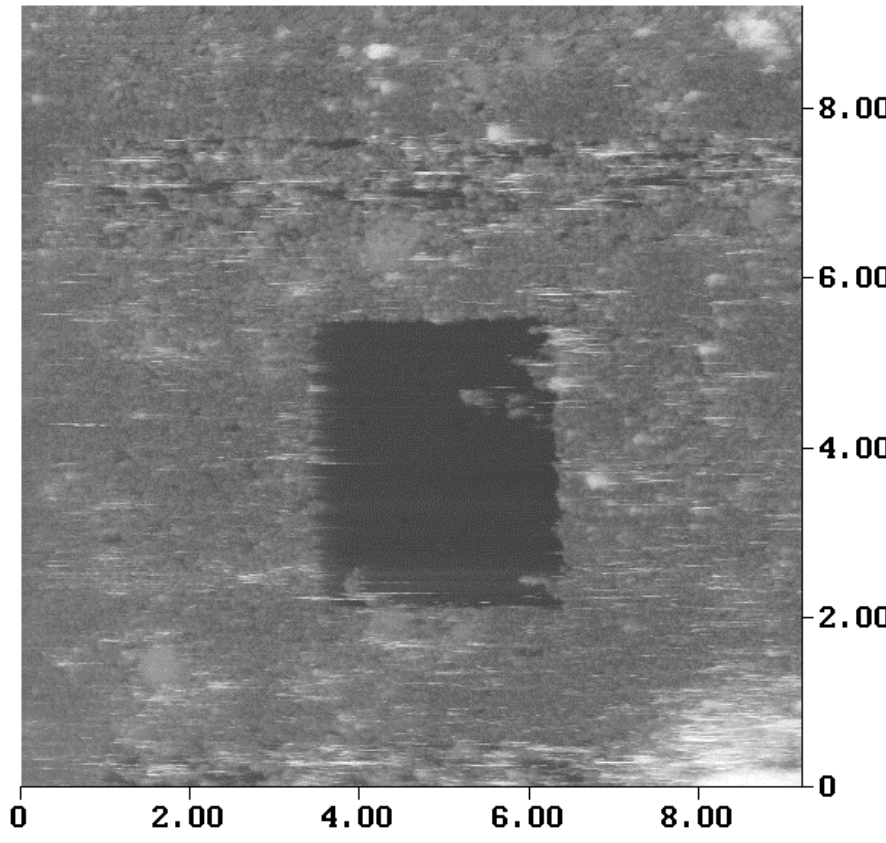

JM

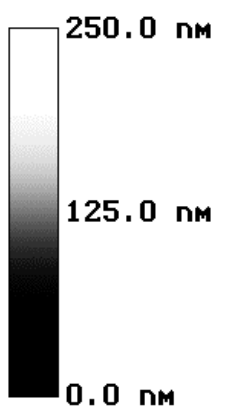

Signal: Height

Medium: Decane

Brine: $\quad\{3.8,0.01\}$

Oil: $\quad$ Mars Yellow

Oil aging: 99 days

Scan \#: 02091212

Figure II-3.84. AFM scan of mica exposed to Mars Yellow crude.

Film thickness on this sample was estimated by first increasing imaging force in order to sweep the surface clean of adsorbed material in a $3 \mu \mathrm{m} \times 3 \mu \mathrm{m}$ square, and then conducting a section analysis to obtain a representative film height relative to the substrate surface. Figure II3.84 shows the scan used for this analysis, with the $3 \mu \mathrm{m} \times 3 \mu \mathrm{m}$ square of swept substrate $\left(\mathrm{R}_{\mathrm{a}}=\right.$ $0.4 \mathrm{~nm}$ ) evident in the middle of the scan. A sampling of ten height difference measurements over four sections yielded a mean film thickness of $\delta=11.9 \mathrm{~nm}$, with $\sigma=1.8 \mathrm{~nm}$. So far in this study, the mean thickness of organic films adsorbed to mica due to exposure to crude oil under acidic brine conditions was uniform across all samples, independent of crude oil type (Tensleep crude, Mars Yellow crude) or substrate exposure time in oil (1-99 days).

\section{MARS-PINK CRUDE}

Mars Pink crude examined under the optical microscope revealed disperse, spherical, micron-sized colloidal structures which did not closely resemble asphaltenes or waxes. Asphaltenes and waxes often assume irregular geometric shapes and cluster together in solution. The colloids were hypothesized to be a water-in-oil emulsion, stabilized by natural surfactants in the oil and small, hydrophilic particulates.

Aging time: 1 day

Contact angles measured on mica exposed to $\{3.8,0.01\}$ brine for 1 day and Mars Pink for 1 day indicated that the mica remained strongly water-wet. The mean advancing angle measured $\theta_{\mathrm{A}}=18^{\circ} \pm 7^{\circ}$ while the mean receding angle measured $\theta_{\mathrm{R}}=6^{\circ} \pm 1^{\circ}$.

Air imaging medium. AFM scans of mica exposed to Mars Pink crude indicated sparse surface coverage, with some material deposited on the surface in clumps and strings, though 
much of the surface appeared to be clean (Figure II-3.85). Roughness measurements of five selected areas, indicated by the light gray boxes, yielded a mean $\mathrm{R}_{\mathrm{a}}=0.25 \mathrm{~nm}$, with $\sigma=0.03 \mathrm{~nm}$. The roughness of the surface enclosed by the circular features, indicated by the blue-gray boxes, was almost identical, with $\mathrm{R}_{\mathrm{a}}=0.29 \pm 0.06 \mathrm{~nm}$. This was several times higher than the roughness of clean mica, $0.05-0.16 \mathrm{~nm}$, calculated from Tensleep scans. Though rougher than clean mica, the surface exposed to Mars Pink was at least an order of magnitude smoother than mica exposed to Tensleep or Mars Yellow, where the surface film roughness ranged from 1.6-8.4 $\mathrm{nm}$.

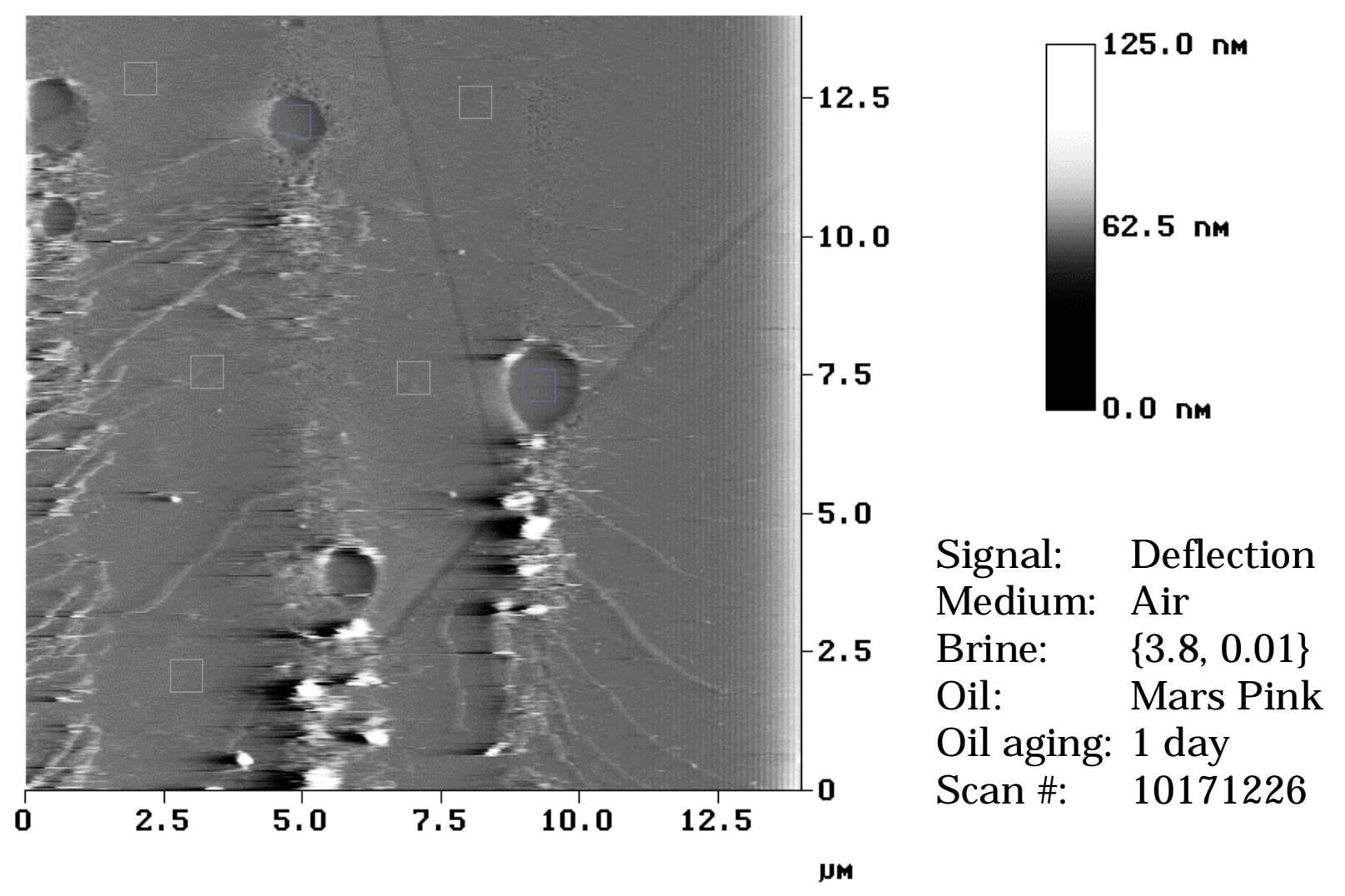

Figure II-3.85. AFM scan of mica exposed to Mars Pink crude. Mean roughness in the indicated boxes was $\mathrm{Ra}=0.25 \pm 0.02 \mathrm{~nm}$, and $\mathrm{Ra}=0.29 \pm 0.06 \mathrm{~nm}$.

Brine imaging medium. Placing a droplet of $\{3.8,0.01\}$ brine on the sample surface resulted in a marked change in surface features, illustrated in the two AFM scans in Figures II3.86 and II-3.87. Isolated and uniformly-dispersed mounds of material were clearly visible in each area scanned, whereas no such features were observed under air. The dimensions of the mounds in Figure II-3.87 measured about 25-70 nm in height, and approximately 250-360 $\mathrm{nm}$ in diameter. The $\mathrm{x}$-shaped scratch in the mica substrate visible under air in Figure II-3.85 is also visible in Figure II-3.86, although the scan direction was rotated by 90 degrees. Comparison of the these two figures reveals that the scan position, as well as the substrate, remain the same, while the surface features transform from amorphous clusters and threads to uniformlydistributed mounds. The low contact angles of $\left(\theta_{\mathrm{A}}=19^{\circ}\right.$ and $\left.\theta_{\mathrm{R}}=6^{\circ}\right)$ and relatively clean appearance of this surface are consistent with the expectation that a stable water film prevents 
adsorption of organic oil components from this acidic oil so that the mica surface remains hydrophilic.
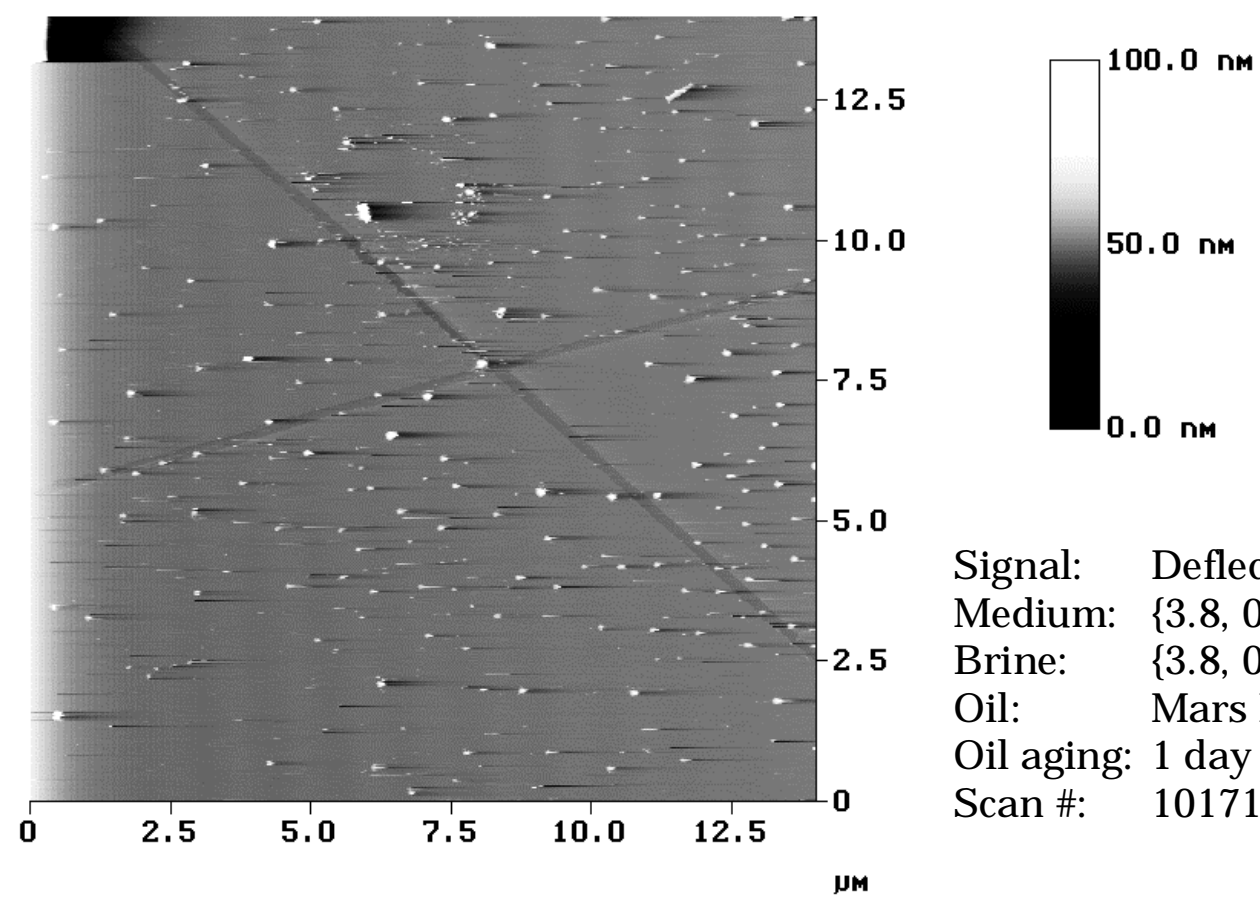

Signal: Deflection

Medium: $\{3.8,0.01\}$

Brine: $\quad\{3.8,0.01\}$

Oil: $\quad$ Mars Pink

Oil aging: 1 day

Scan \#: 10171304

Figure II-3.86. AFM scan of mica exposed to Mars Pink crude.
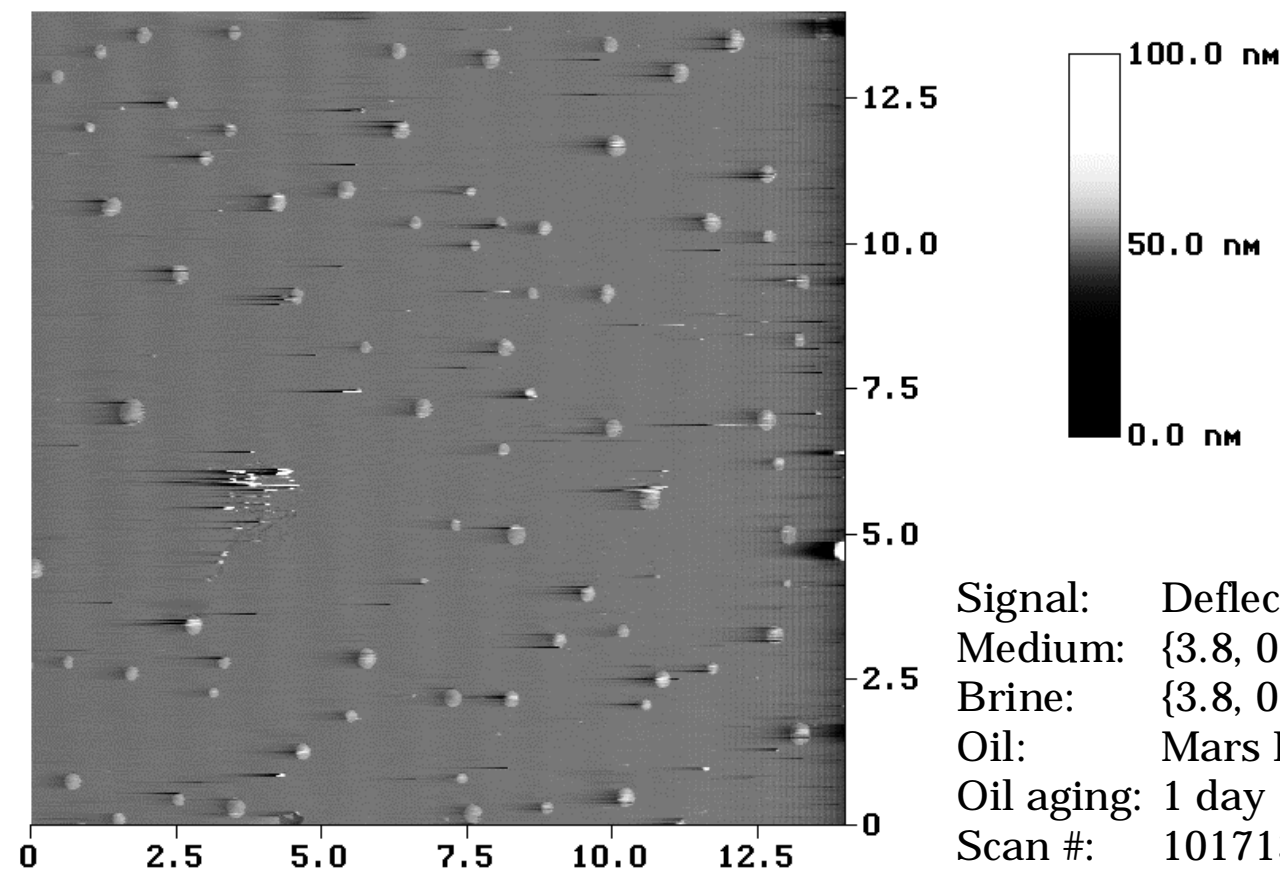

Signal: Deflection

Medium: $\{3.8,0.01\}$

Brine: $\quad\{3.8,0.01\}$

Oil: $\quad$ Mars Pink

Oil aging: 1 day

Scan \#: 10171320

JM

Figure II-3.87. AFM scan of mica exposed to Mars Pink crude. 
Decane imaging medium. Drying the surface and inserting a decane droplet as the imaging medium yielded yet another transformation in surface features. The scan shown in Figure II-3.88 contains four circular deposits, with an average diameter of $1.5 \mu \mathrm{m}$. The material here looks as if it was concentrated in drying water droplets on the surface. Other material is also distributed on the surface with very thin coverage over about $50 \%$ of the scan area. A zoom of the circular deposit (Figure II-3.89) near the middle of Figure II-3.88 reveals a distinct, constant radius, as well as what appears to be a wedge-shaped split, indicated by the arrow. This split likely resulted from mechanical stresses along the perimeter of the deposit as the water volume decreased underneath. A $500 \mathrm{~nm}$ zoom on top of the circular deposit (Figure II-3.90) resolves rounded structural units which measure $20-40 \mathrm{~nm}$ in diameter. Other scan areas such as the that shown in Figure II-3.91 portray similar features. In this case, a zoom (Figure II-3.92) of one feature near the center of the scan shows a deposit which has the familiar circular shape with a split, though the lower right portion of the deposit is broken apart and strewn over the surface. Individual units measure about $50 \mathrm{~nm}$ in diameter.
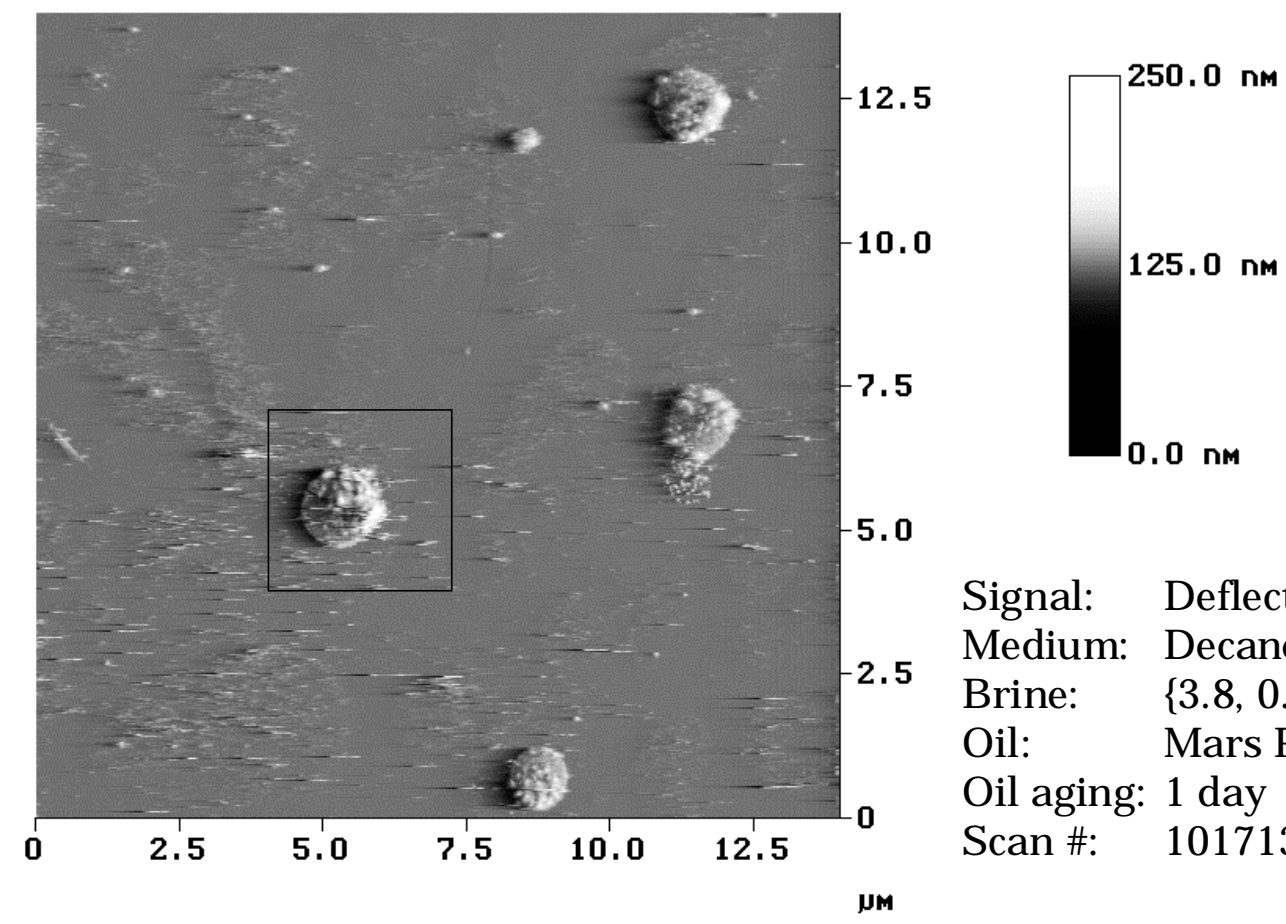

Signal: Deflection

Medium: Decane

Brine: $\quad\{3.8,0.01\}$

Oil: $\quad$ Mars Pink

Oil aging: 1 day

Scan \#: 10171343

Figure II-3.88. AFM scan of mica exposed to Mars Pink crude. A zoom of the area indicated by the box is shown in Figure II-3.89. 


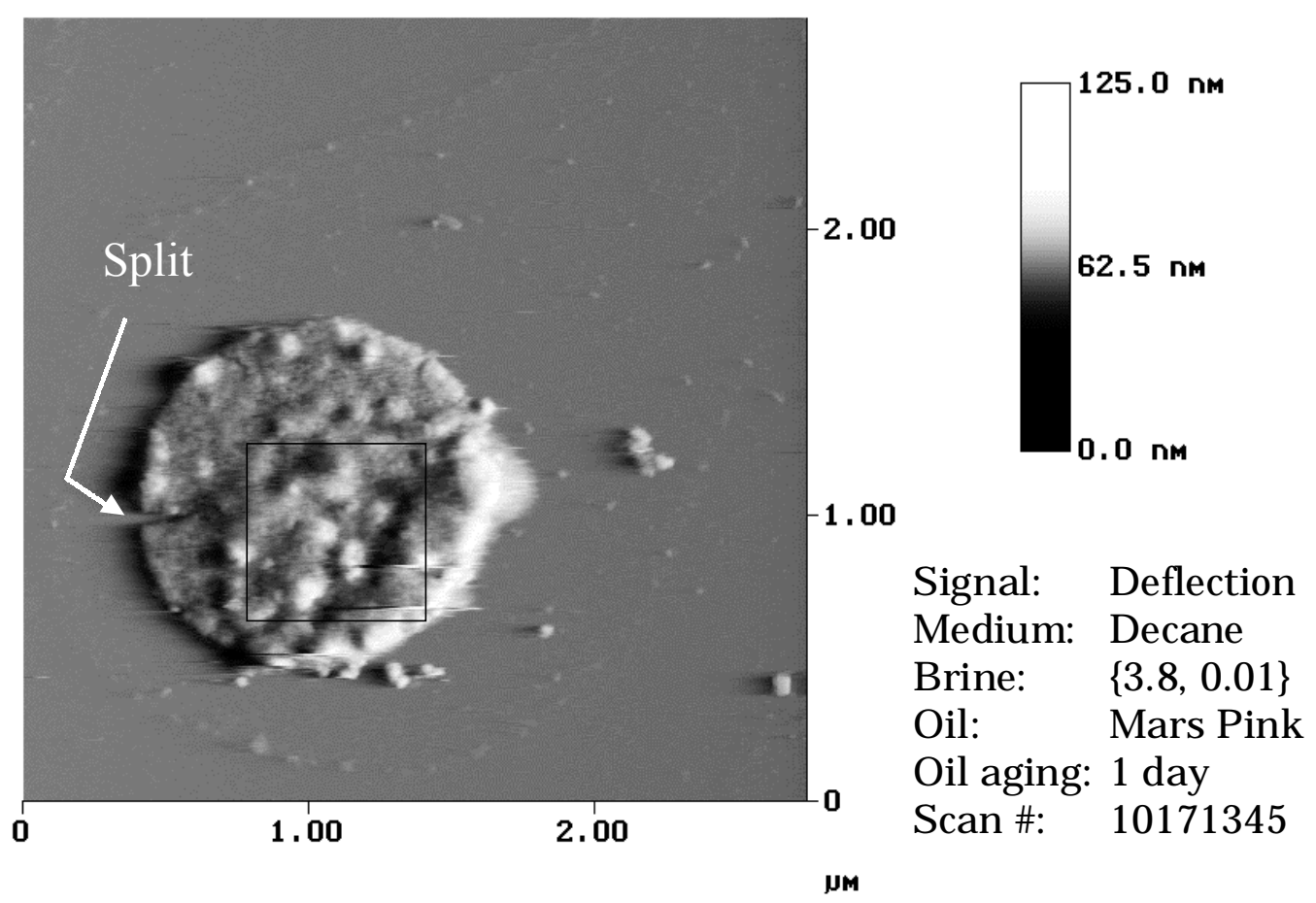

Figure II-3.89. AFM scan of mica exposed to Mars Pink crude. A zoom of the area indicated by the box is shown in Figure II-3.90.

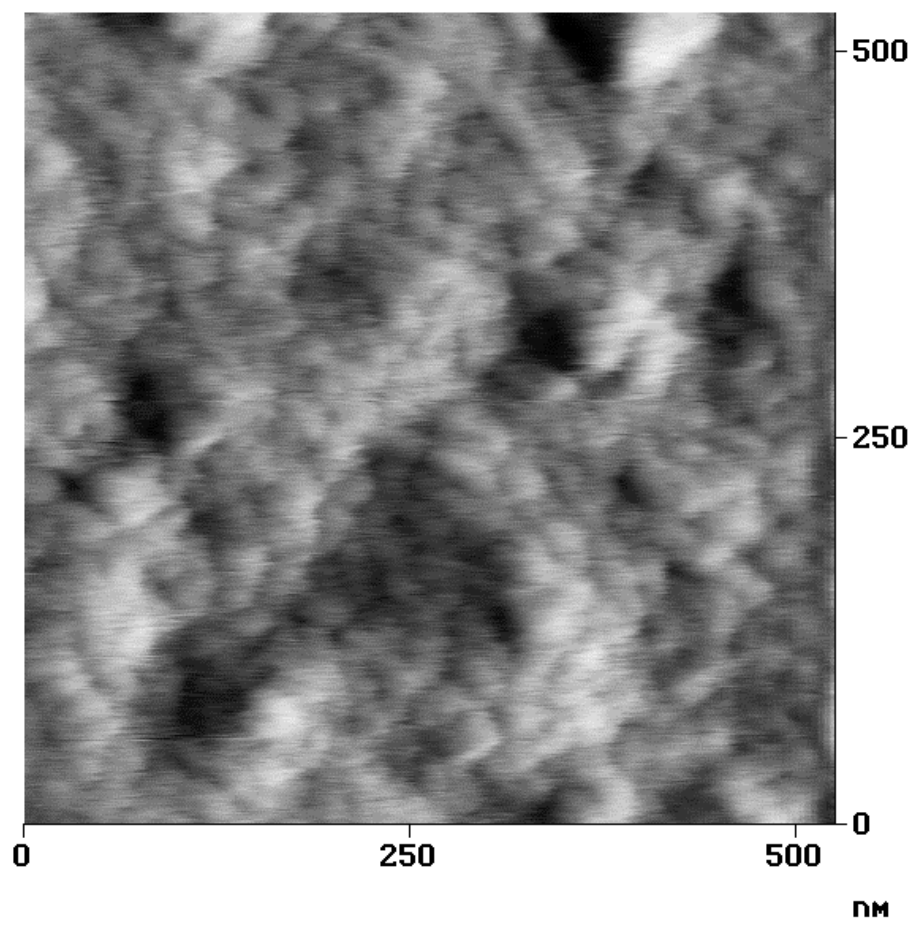

500

250

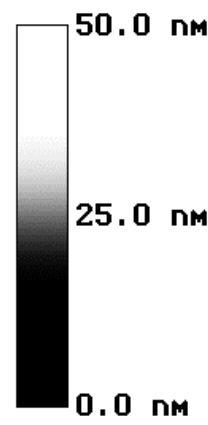

Signal: Deflection

Medium: Decane

Brine: $\quad\{3.8,0.01\}$

Oil: $\quad$ Mars Pink

Oil aging: 1 day

Scan \#: 10171347

Figure II-3.90. AFM scan of mica exposed to Mars Pink crude. 


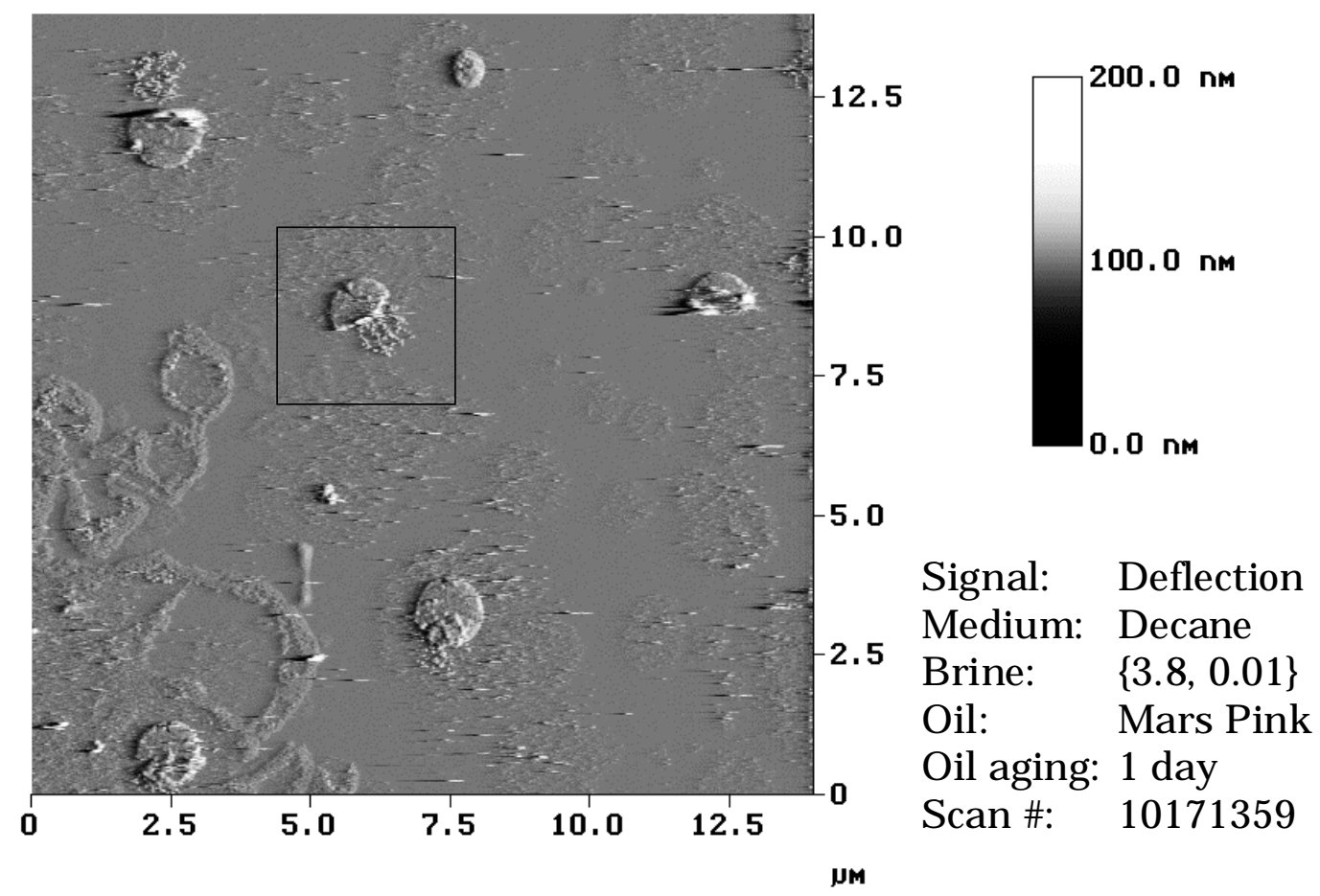

Figure II-3.91. AFM scan of mica exposed to Mars Pink crude. A zoom of the area indicated by the box is shown in Figure II-3.92.
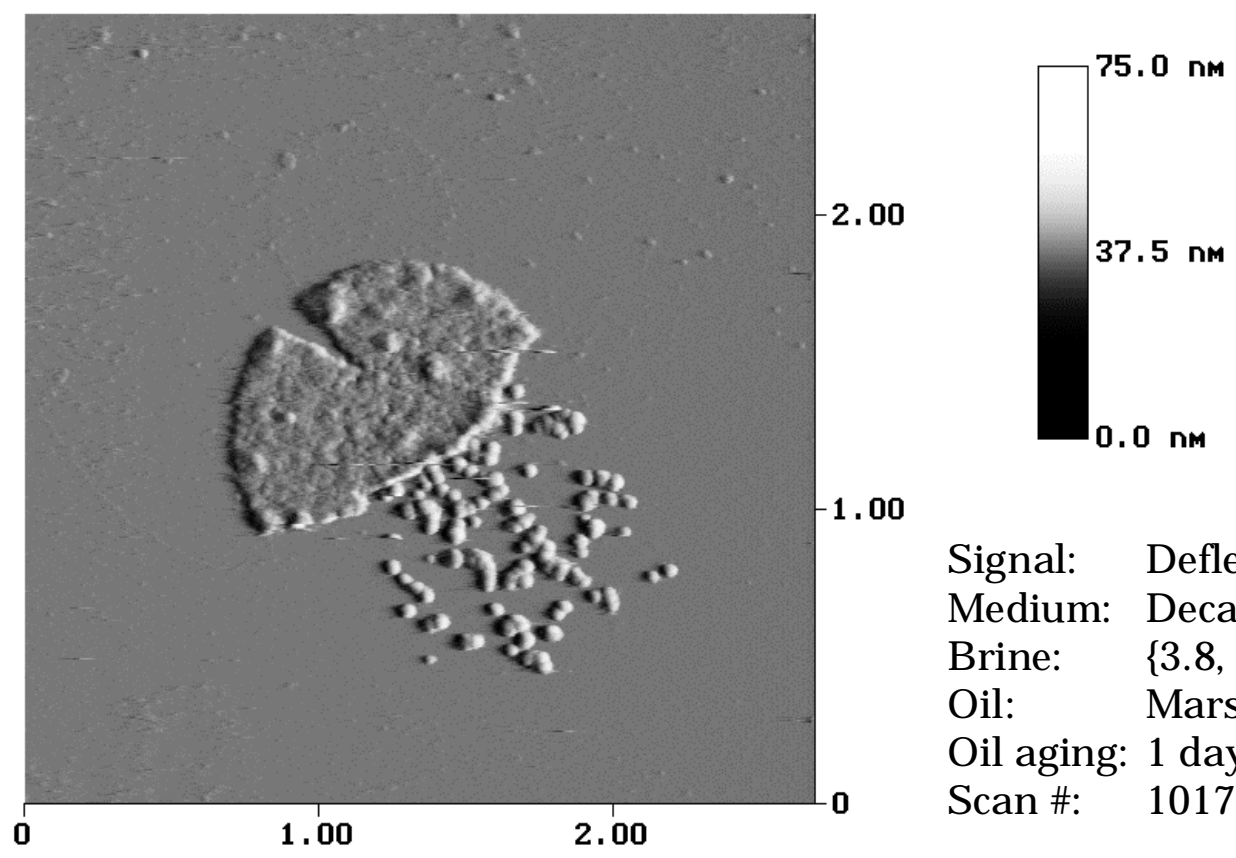

1.00

Signal: Deflection

Medium: Decane

Brine: $\quad\{3.8,0.01\}$

Oil: $\quad$ Mars Pink

Oil aging: 1 day

Scan \#: 10171402

JM

Figure II-3.92. AFM scan of mica exposed to Mars Pink crude. 
Aging time: 21 days

Aging mica for 1 day in $\{3.8,0.01\}$ and 21 days in Mars Pink yielded very similar contact angle results to the samples aged just 1 day in oil. Strongly water-wet conditions prevailed, with water-advancing contact angle measuring $\theta_{\mathrm{A}}=27^{\circ} \pm 8^{\circ}$, and water-receding contact angle measuring $\theta_{\mathrm{R}}=10^{\circ} \pm 3^{\circ}$.

Air imaging medium. The surface imaged under air appeared primarily clean (Figure II3.93). Several isolated circular deposits measuring about $2.5 \mu \mathrm{m}$ in diameter and $60-90 \mathrm{~nm}$ tall were present, though they covered only $5 \%$ of the scan area. The mean roughness of five selected regions in scan 11280951 revealed a fairly smooth surface, with $R_{a}=0.25 \pm 0.08 \mathrm{~nm}$, almost identical to the roughness measured on samples aged 1 day in Mars Pink.
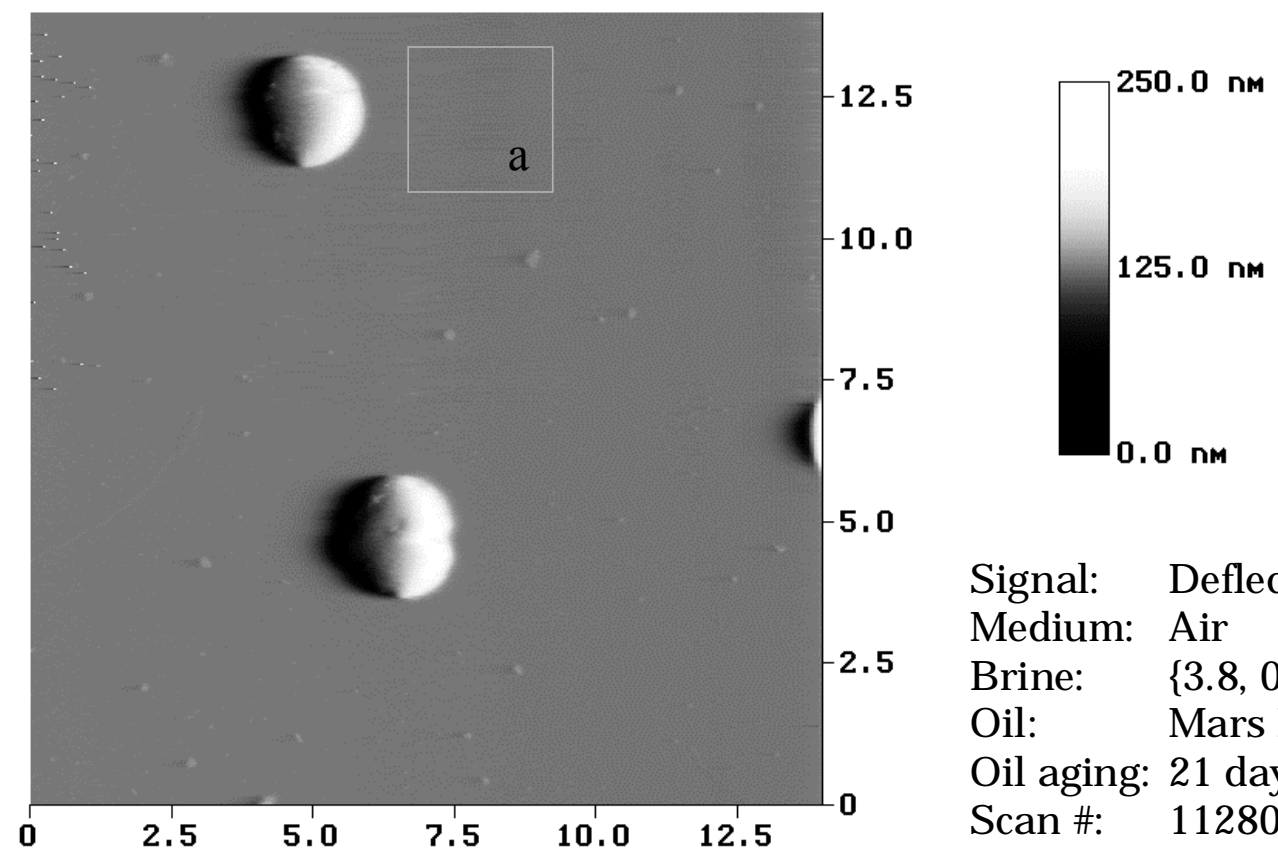

5.0

Signal: Deflection

Medium: Air

Brine: $\quad\{3.8,0.01\}$

Oil: $\quad$ Mars Pink

Oil aging: 21 days

Scan \#: 11280951

JM

Figure II-3.93. AFM scan of mica exposed to Mars Pink crude. Mean roughness of five selected areas similar to box $a$ was $R_{a}=0.25 \pm 0.08 \mathrm{~nm}$.

Brine imaging medium. Adding brine to the dry surface broke the micron-scale deposits into smaller, evenly distributed mounds $200-400 \mathrm{~nm}$ in diameter (Figure II-3.94), a sequence identical to that observed for mica slides exposed to Mars Pink for 1 day. AFM observations of minimal surface coverage under air and brine are consistent with the low contact angles measured for this surface treatment. 


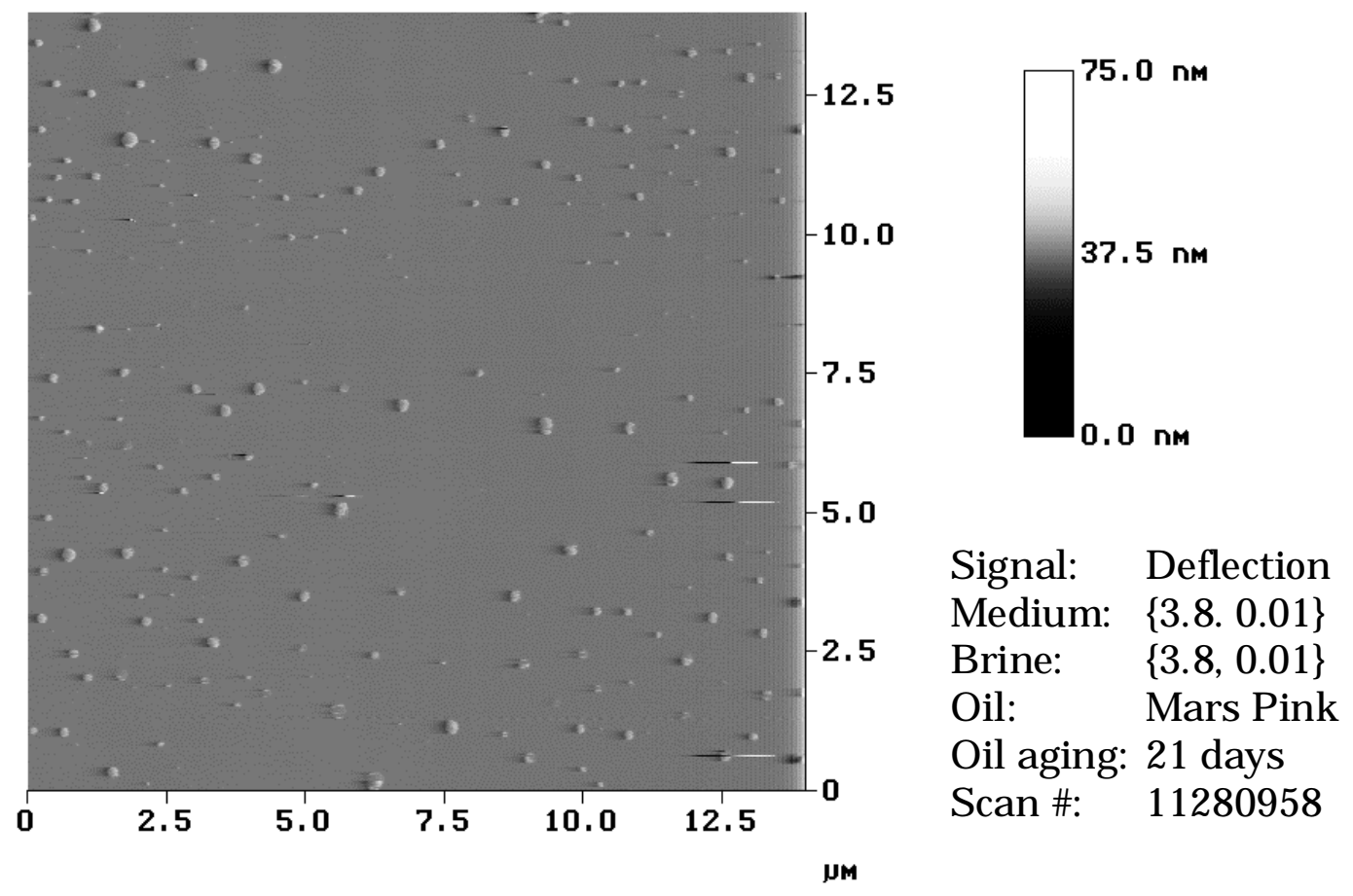

Figure II-3.94. AFM scan of mica exposed to Mars Pink crude.

Decane imaging medium. Removing the brine and replacing it with decane (Figures II3.95 - II-3.97) caused collection of the evenly-distributed mounds into roughly circular deposits again, suggesting that the material was deposited in drying water droplets on the surface. This sequence is again identical to that observed for the samples exposed to Mars Pink for 1 day. 


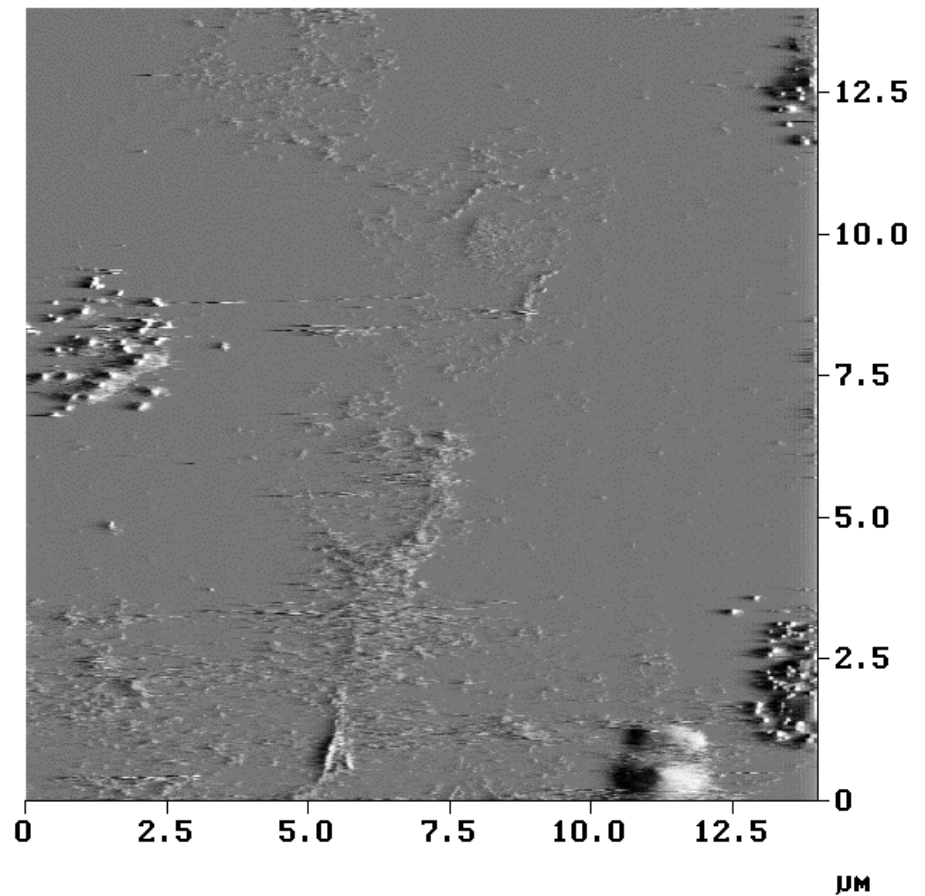

Figure II-3.95. AFM scan of mica exposed to Mars Pink crude.

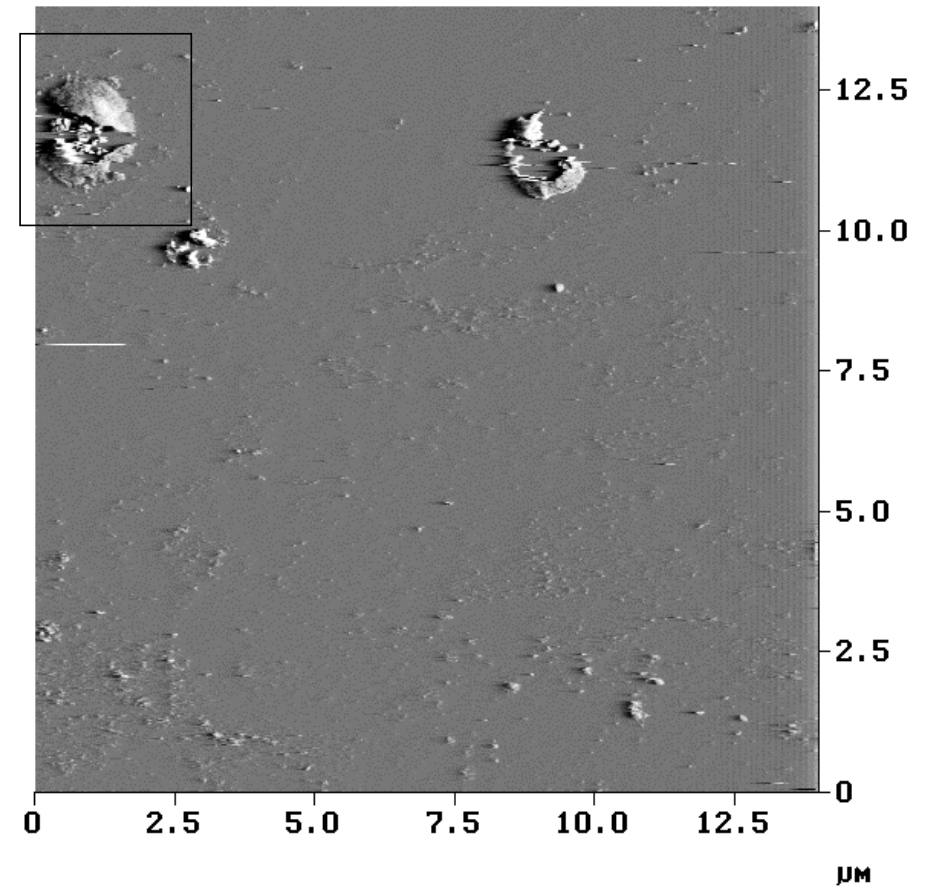

Figure II-3.96. AFM scan of mica exposed to Mars Pink crude.

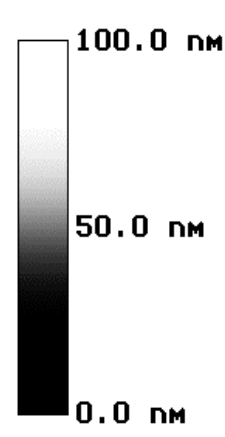

Signal: Deflection Medium: Decane Brine: $\quad\{3.8,0.01\}$ Oil: $\quad$ Mars Pink Oil aging: 21 days Scan \#: 11281046

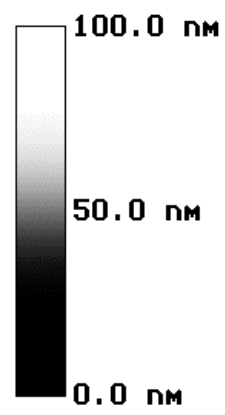

Signal: Deflection Medium: Decane Brine: $\quad\{3.8,0.01\}$ Oil: $\quad$ Mars Pink Oil aging: 21 days Scan \#: 11281136 


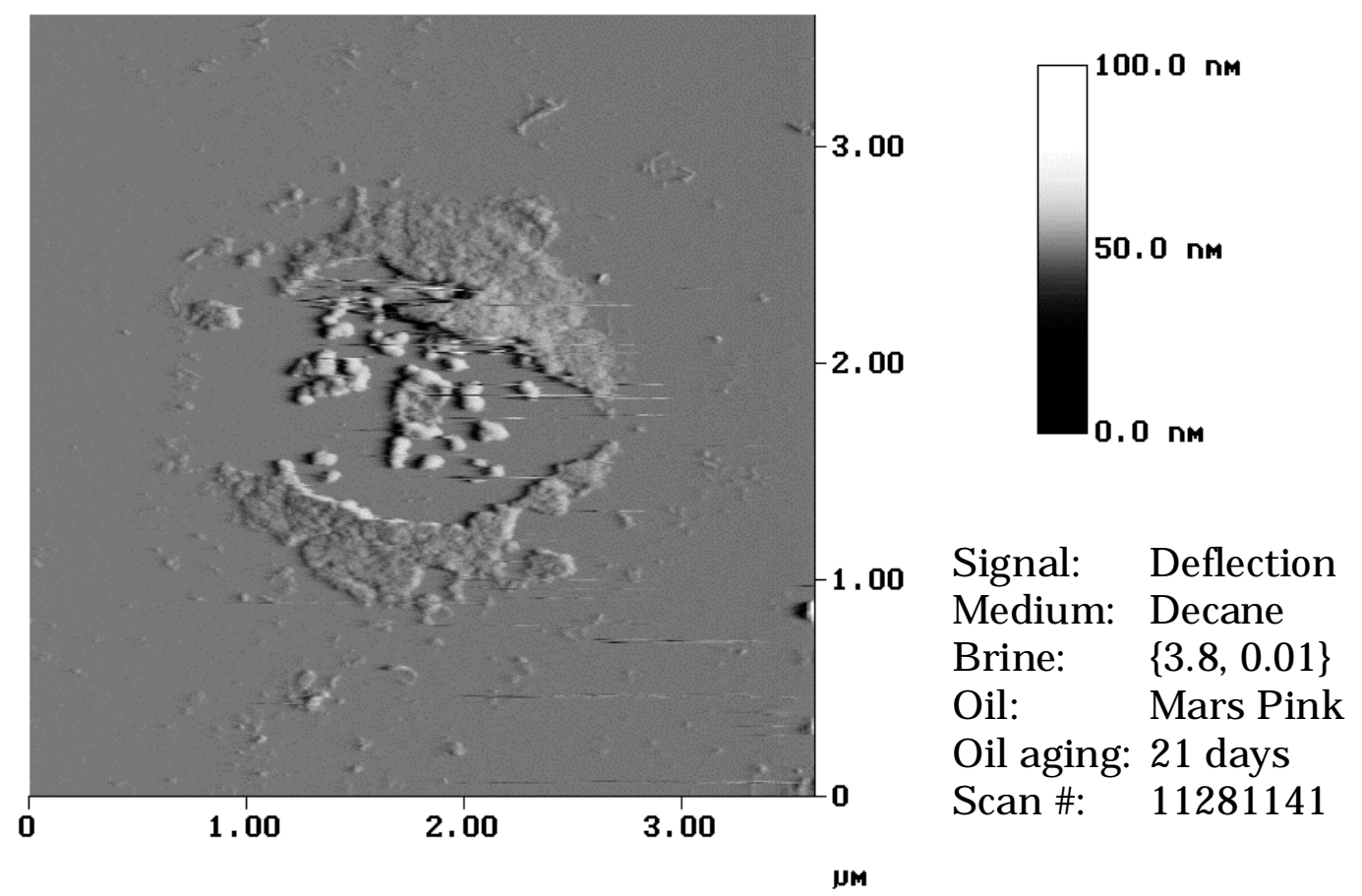

Figure II-3.97. AFM scan of mica exposed to Mars Pink crude. 
Aging time: 105 days

Decane imaging medium. AFM scans of mica exposed to $\{3.8,0.01\}$ for 1 day and Mars Pink crude for 105 days showed sparse surface coverage (Figure II-3.98), a consistent observation on for mica regardless of aging time in this particular crude. Surface features were more numerous after 105 days than after 1 or 25 days, though most of the mica was still relatively clean. The roughness of a $9 \mu \mathrm{m}^{2}$ area in the upper left corner of scan 02091349 was $R_{a}$ $=0.73 \mathrm{~nm}$.
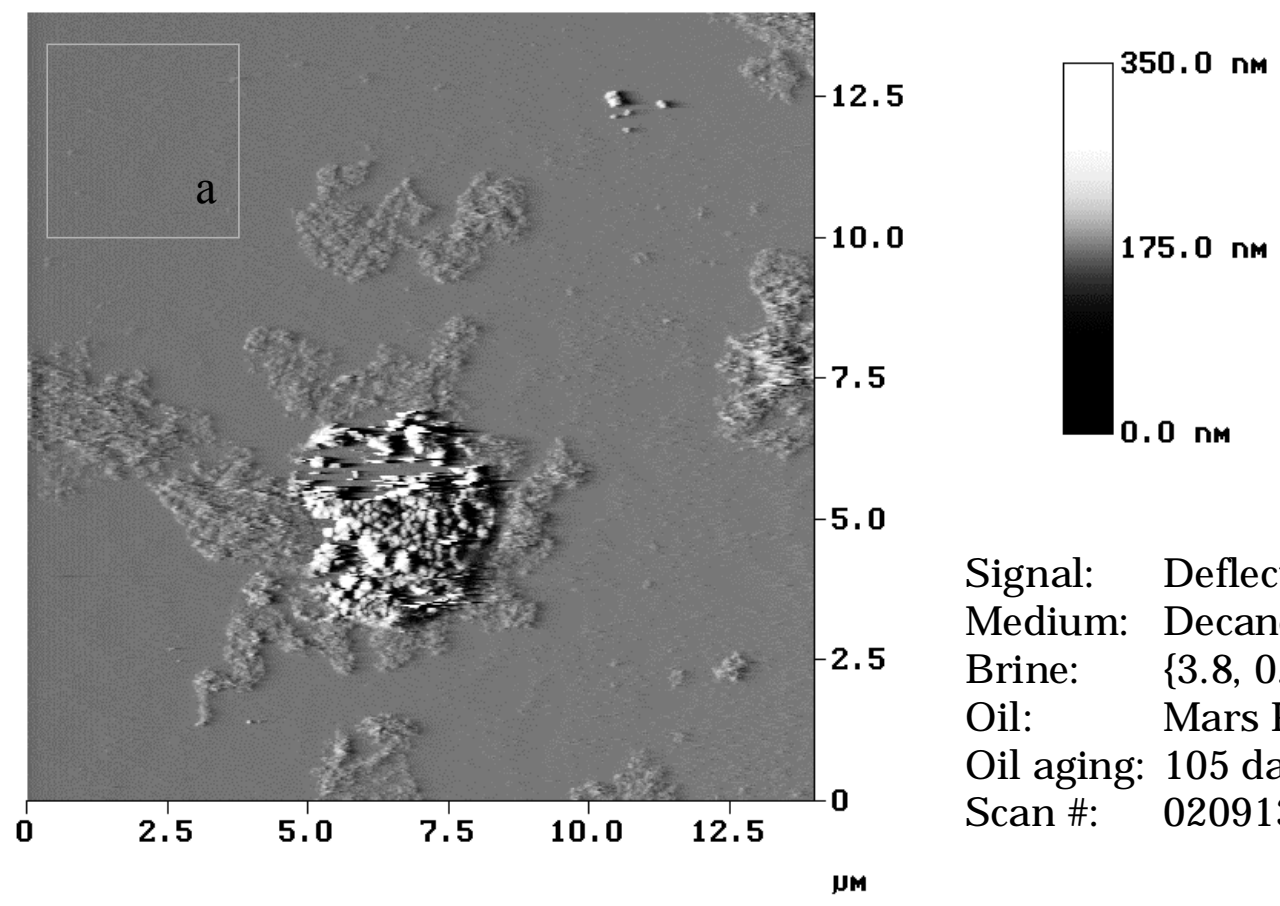

Signal: Deflection

Medium: Decane

Brine: $\quad\{3.8,0.01\}$

Oil: $\quad$ Mars Pink

Oil aging: 105 days

Scan \#: 02091349

Figure II-3.98. AFM scan of mica exposed to Mars Pink crude. The roughness of the area indicated in box $a$ was $R_{a}=0.73 \mathbf{~ n m}$.

Figure II-3.99 shows approximately $40 \%$ surface area coverage by a collection of features near the center and upper left quadrant of the scan. A section analysis indicated that the height of this population varied considerably, and no representative film thickness value could be determined. The roughness of a $5.5 \mu \mathrm{m} \times 3.1 \mu \mathrm{m}$ area indicated by box $a$, measured $\mathrm{R}_{\mathrm{a}}=2.96$ $\mathrm{nm}$. Another scan shown in Figure II-3.100 shows scattered features which cover approximately $20 \%$ of the scan area. A $3 \mu \mathrm{m}$ zoom (Figure II-3.101) of a feature near the center of Figure II3.100 resolves structural units which are approximately $50 \mathrm{~nm}$ diameter. 


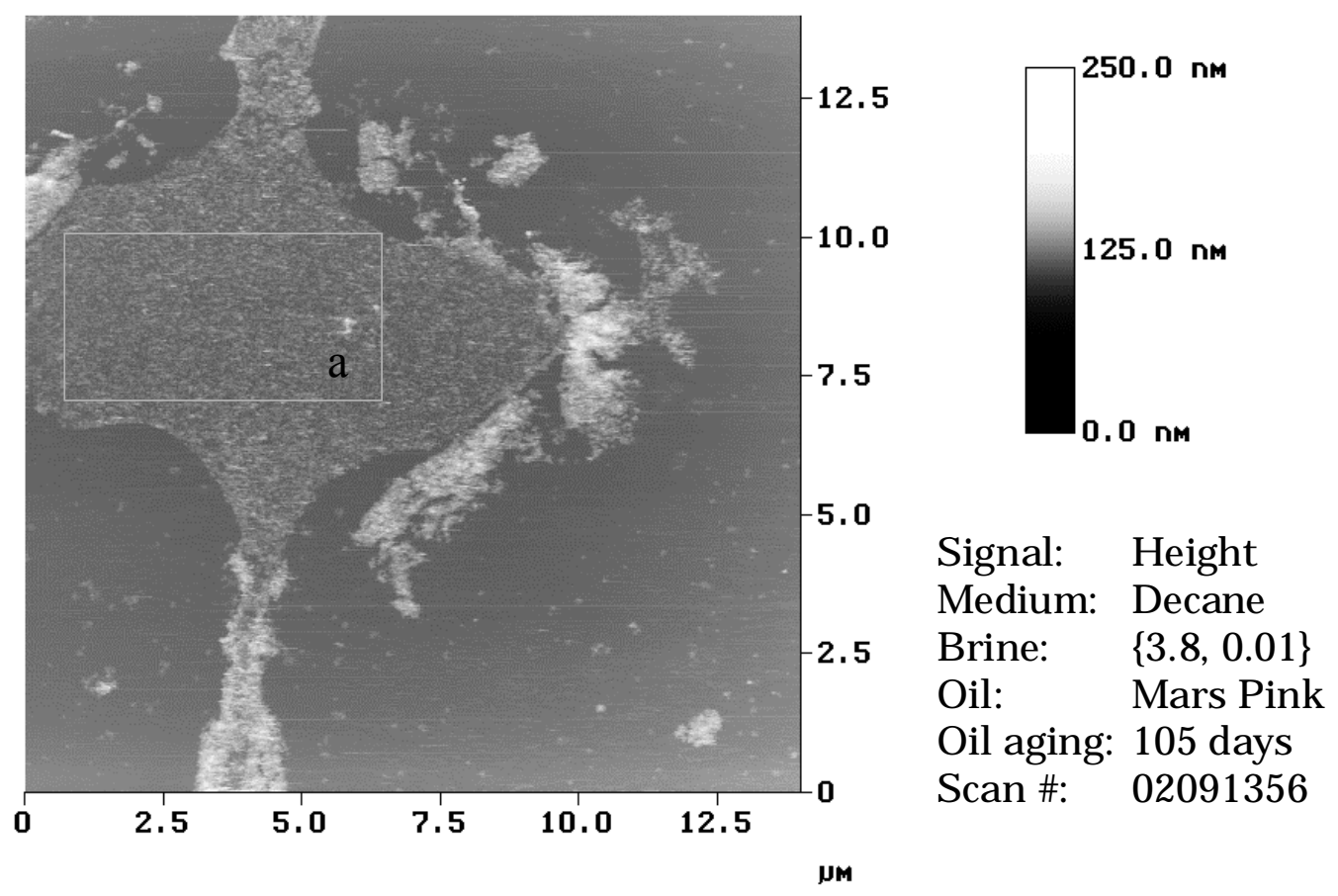

Figure II-3.99. AFM scan of mica exposed to Mars Pink crude. The roughness of the area indicated in box $a$ was $R_{a}=2.96 \mathrm{~nm}$.

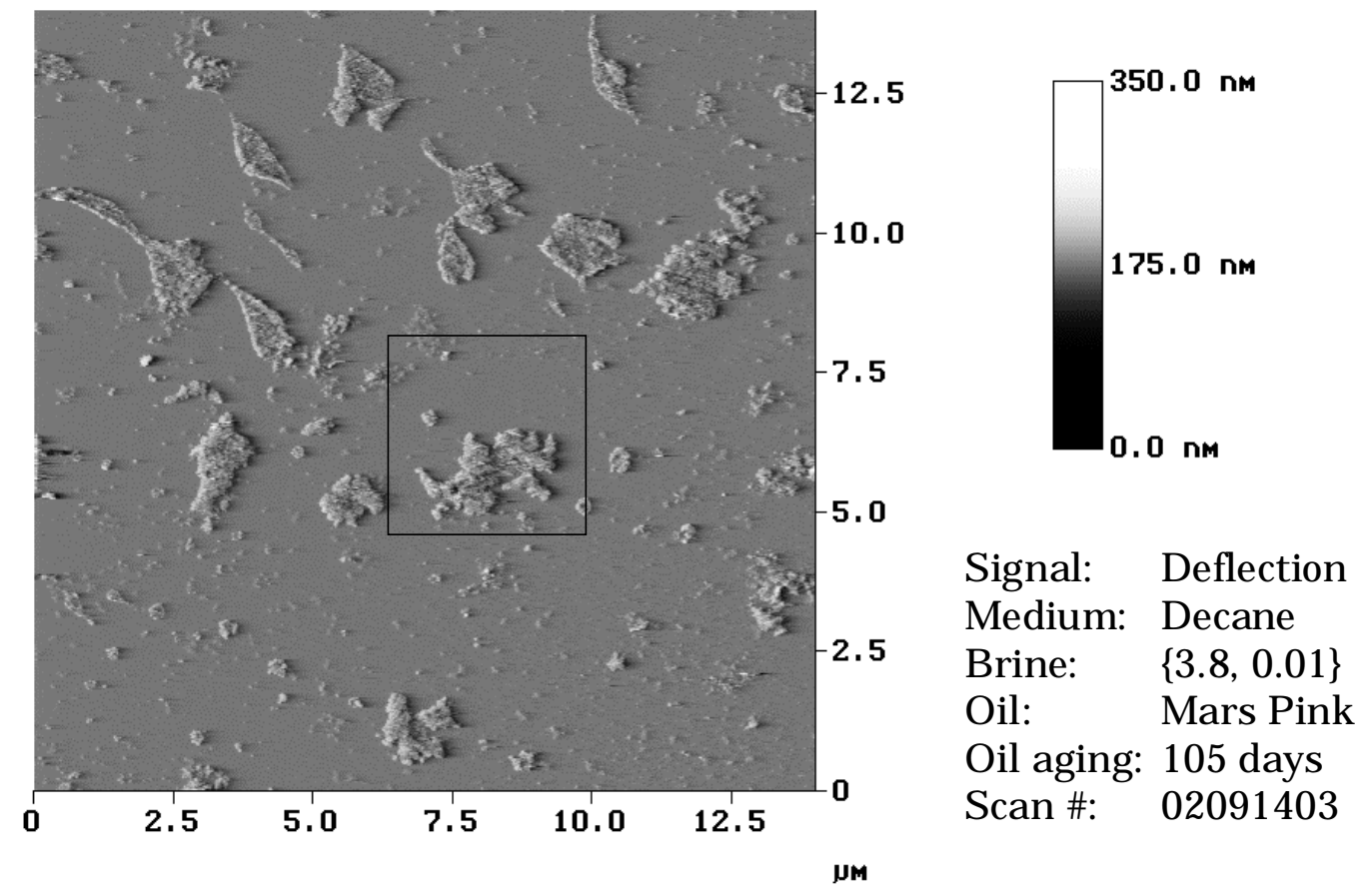

Figure II-3.100. AFM scan of mica exposed to Mars Pink crude. A zoom of the area indicated in the box is shown in Figure II-3.101. 

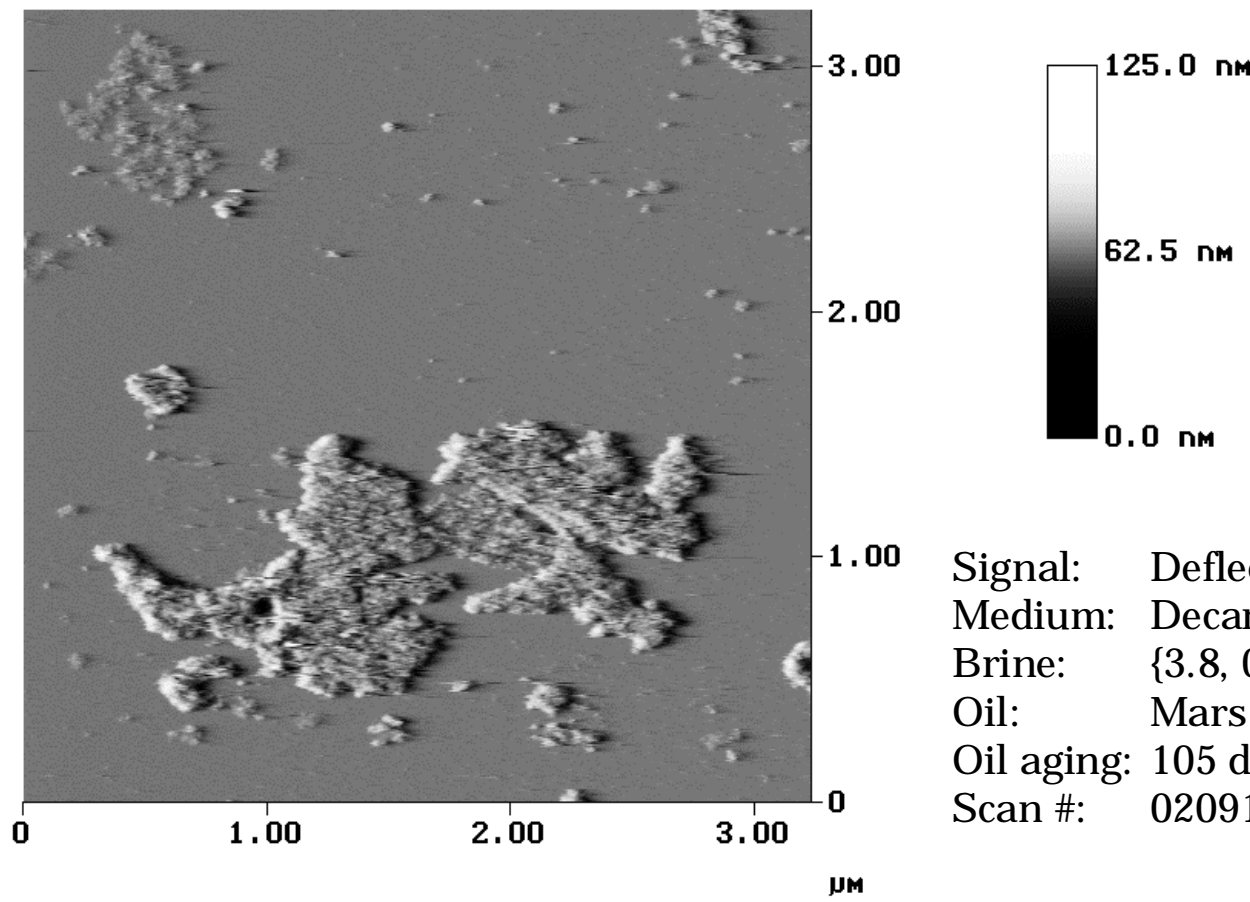

Signal: Deflection

Medium: Decane

Brine: $\quad\{3.8,0.01\}$

Oil: $\quad$ Mars Pink

Oil aging: 105 days

Scan \#: 02091405

Figure II-3.101. AFM scan of mica exposed to Mars Pink crude.

\section{DISCUSSION OF MARS OILS}

The two oils examined here, Mars Yellow and Mars Pink, exhibited markedly different adsorption and wetting properties on mica according to atomic force microscope and contact angle analyses. Mars Yellow, an oil with a base number to acid number ratio of 4.8, and aggregated asphaltenes and waxes visible in the optical microscope, was more surface-active than Mars Pink, with base\#/acid\# $=0.6$, and no visible asphaltenes or waxes. As summarized in Table II-3.6, mica treated with Mars Yellow under acidic conditions was weakly water-wet to neutrally wet after 1 day exposure, while Mars Pink remained strongly water-wet even after 21 days in oil. The Mars Yellow was likely more effective at changing wettability for two reasons. First, the organic bases in the oil can be positively-charged at the oil-water interface in an acidic aqueous environment $(\mathrm{pH}=3.8)$. This interface, located in close proximity to the negativelycharged mica, favors collapse of the water film on the mica, allowing direct interaction between the oil and solid interface. Positively-charged bases and asphaltene aggregates then proceed to collect on the mica, leading to formation of a neutrally-wet surface film and net reduction in hydrophilicity of the surface. The organic acid content in Mars Pink leads to a net negative charge at the oil-water interface, stabilizing the water film on the mica, which serves as a barrier to direct contact between oil and substrate. Furthermore, since the asphaltenes were dissolved in Mars Pink, they would not accumulate at the phase boundaries as they do when they are precipitated. The net result was little sorption of Mars Pink components to the mica surface, and preservation of hydrophilic conditions. 
Table II-3.6. Contact angle summary

\begin{tabular}{|l|l|l|l|l|}
\hline Oil & Brine & Days in oil & $\boldsymbol{\theta}_{\mathbf{A}}(\mathbf{d e g})$ & $\boldsymbol{\theta}_{\mathbf{R}}(\mathbf{d e g})$ \\
\hline Mars Yellow & $\{3.8,0.01\}$ & 1 & $81 \pm 16$ & $38 \pm 8$ \\
\hline & $\{3.8,0.01\}$ & 21 & $67 \pm 18$ & $25 \pm 6$ \\
\hline Mars Pink & $\{3.8,0.01\}$ & 1 & $18 \pm 7$ & $6 \pm 1$ \\
\hline & $\{3.8,0.01\}$ & 21 & $27 \pm 8$ & $10 \pm 3$ \\
\hline
\end{tabular}

Imaging fluids.

Reservoir rocks are normally exposed to brine and oil rather than air. Therefore, the morphology of oily surface films under liquids is thought to be more representative of in-situ conditions than on dry surfaces. Imaging in a sequence of fluids brought about some notable changes in surface features for both Mars oils.

Mars Yellow: 1-day sample. Imaging under air revealed a continuous smooth film with aggregated asphaltenes integrated into the film (Figure II-3.75). Addition of brine, however, led to blistering of the film (Figures II-3.76 and II-3.77) in many areas, though the entire surface was not blistered (Figure II-3.78). The local detachment of the film upon addition of water could have important implications on wettability. Although exact comparisons are not available, waterfloods of Berea sandstone cores containing a synthetic sea water brine and Mars-Yellow oil appeared to be water-wet even after 21 days of aging (Al-Maamari, 2000).

Upon washing the brine away and replacing it with decane (Figure II-3.79), the surface appeared the same as it did under air. Adding toluene to the decane induced subtle changes (Figure II3.81), including an increase in height of some of the asphaltene/wax structures, and in some cases, voids in the film which look like an asphaltene/wax particle was actually removed (Figure II-3.82). The behavior of the surface features under the decane + toluene medium suggests that asphaltene and/or wax surface attachment mechanisms may be related to the presence of natural surfactants in the oil. When toluene was added, some of the particles looked as if they broke away and may have been originally cemented in place by the oil's surfactants.

Mars Pink: 1-day and 21-day samples. Surfaces treated with an acidic aqueous phase followed by Mars-Pink crude oil remained strongly water-wet (Table II-3.6), consistent with micromodel tests with the same fluids (Al-Maamari, 2000). AFM imaging in a sequence of air, brine, and decane induced changes in the shape and distribution of surface features. Under air, features were organized into clumps and strings over an otherwise essentially bare mica surface (Figures II-3.85and II-3.93). With the addition of brine, the features reorganized into isolated and uniformly dispersed sub-micron scale mounds (Figures II-3.86, II-3.87, II-3.94). Replacing brine with decane yielded yet another reorganization into conspicuously round, flat, micron-scale deposits resembling pancakes (Figures II-3.88 - II-3.92, II-3.97). Optical microscopy of the crude revealed emulsified water, likely stabilized by natural surfactants in the oil. We speculate that the emulsified water is the source of the hydrophilic particulates which might be clays (from the formation or drilling fluids) or other inorganic material that is insoluble in both water and oil. 


\section{Film thickness}

For samples featuring a stable surface coating, film thickness estimates were attempted from AFM scans. The sample exposed to Mars Yellow for 99 days provided the best opportunity for a thickness estimate, with the resulting value shown in Table II-3.7. Tensleep samples exhibited a nearly identical film thickness, regardless of aging time or oil type. Such close correspondence among thickness measurements suggests that the film does not actually grow deeper over time. Instead, holes in the film may gradually fill, and some features may become smoother, leading to a more homogeneous coating and more hydrophobic surface.

Table II-3.7. Film thickness estimates.

\begin{tabular}{|c|c|c|c|}
\hline Scan \# & Fig. \# & Sample Preparation & Film thickness (nm) \\
\hline 02091212 & III-13 & $\{3.8,0.01\}$ brine, Mars Yellow 99 days, imaged in decane & $11.9 \pm 1.8$ \\
\hline
\end{tabular}

\section{Roughness measurements}

A first-order attempt to classify surface features in AFM scans yielded three types of commonly observed structures: clean mica, smooth film, and isolated rough features. Each type of structure exhibited a characteristic roughness, with the results for the Mars oils summarized in Table II-3.8. For example, typical $\mathrm{R}_{\mathrm{a}}$ values for mica measured less than $1 \mathrm{~nm}$, while values for the smooth film measured from 1-3 nm, and rough features measured 7-8 nm. Roughness calculations were useful in that they indicated that similar-looking features in different scans had a quantifiable property in common.

Table II-3.8. Roughness analysis of selected areas.

\begin{tabular}{|c|c|c|c|c|}
\hline Scan \# & Fig. \# & Sample Preparation & Area $(\mu \mathrm{m})^{2}$ & $\mathbf{R}_{\mathrm{a}}(\mathbf{n m})$ \\
\hline 11171330 & II-3.75 & $\begin{array}{c}\{3.8,0.01\} \text { brine, Mars Yellow, } 21 \text { days imaged in brine } \\
\text { - mica exposed through hole in film } \\
- \text { smooth film } \\
\text { - coarse material (asphaltene) }\end{array}$ & $\begin{array}{l}0.4 \\
0.4 \\
0.4\end{array}$ & $\begin{array}{c}0.04 \\
1.8 \pm 0.3 \\
7.5 \pm 0.3\end{array}$ \\
\hline 11171447 & II-3.80 & $\begin{array}{c}\{3.8,0.01\} \text { brine, Mars Yellow, } 21 \text { days imaged in decane } \\
- \text { smooth film } \\
\text { - coarse material (asphaltene) } \\
\text { - mound }\end{array}$ & $\begin{array}{l}0.4 \\
0.4 \\
0.4\end{array}$ & $\begin{array}{l}1.6 \\
7.7 \\
1.7\end{array}$ \\
\hline 02091221 & II-3.83 & $\begin{array}{c}\{3.8,0.01\} \text { brine, Mars Yellow, } 99 \text { days imaged in decane } \\
\text { - smooth film } \\
\text { - coarse material (asphaltene) }\end{array}$ & $\begin{array}{l}0.4 \\
0.4\end{array}$ & $\begin{array}{l}1.4 \pm 0.1 \\
6.3 \pm 1.3\end{array}$ \\
\hline 10171226 & II-3.85 & $\begin{array}{c}\{3.8,0.01\} \text { brine, Mars Pink, } 1 \text { day imaged in air } \\
\text { - substrate surface } \\
\text { - in hole }\end{array}$ & $\begin{array}{l}0.4 \\
0.4\end{array}$ & $\begin{array}{l}0.25 \pm 0.03 \\
0.29 \pm 0.06\end{array}$ \\
\hline 11280951 & II-3.93 & $\begin{array}{c}\{3.8,0.01\} \text { brine, Mars Pink, } 21 \text { days imaged in air } \\
\text { - substrate surface }\end{array}$ & 6.25 & $0.25 \pm 0.08$ \\
\hline 02091349 & II-3.98 & $\begin{array}{c}\{3.8,0.01\} \text { brine, Mars Pink, } 105 \text { days imaged in decane } \\
\text { - substrate surface }\end{array}$ & 9.0 & 0.73 \\
\hline 02091356 & II-3.99 & $\begin{array}{c}\{3.8,0.01\} \text { brine, Mars Pink, } 105 \text { days imaged in decane } \\
\text { - patch of rough features }\end{array}$ & 17.05 & 2.96 \\
\hline
\end{tabular}




\section{Lost Hills crude oil}

\section{CONTACT ANGLES}

A medium to heavy oil from the Lost Hills field produces fairly water wet contact angles on mica as shown in Fig. II-3.102. Contact angles were measured on mica surfaces exposed first to $\mathrm{pH} 4$ buffer, then to the crude oil or mixtures of oil and n-heptane. Mica was aged in the oil mixtures for three weeks at ambient conditions, rinsed briefly with toluene. Contact angles were measured with water and decane as the probe fluids.

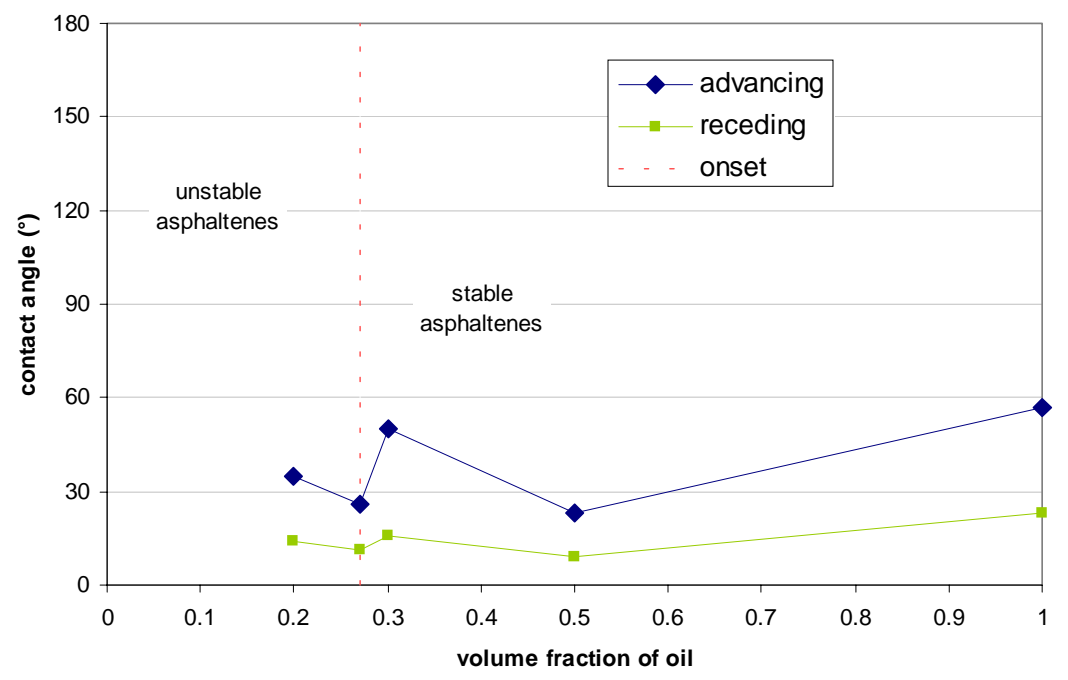

Figure II-3.102. Contact angles on mica surfaces exposed to Lost Hills crude oil and mixtures of Lost Hills with n-heptane.

Since none of the advancing angles exceeds $60^{\circ}$, it is impossible to judge on the basis of contact angles whether the trend observed for other oils (Al-Maamari and Buckley, 2000) of increasing oil-wetness near the onset of asphaltene instability plays any role in these results. There is a small spike in mixtures with an oil volume fraction of 0.3 , but the uncertainty in the contact angle measurements can be as high as plus or minus $10^{\circ}$, so the difference between any two values is probably not significant.

\section{AFM OF LOST HILLS CRUDE}

AFM images for similarly treated surfaces provide additional insight into changes on the surface that occur while it is being aged in crude oil. Roughness analyses of the surface under air clearly show that a coating was deposited by the neat crude oil, but all of the material was removed during the first scan under water leaving bare mica (Fig. II-3.103) and no estimate of thickness could be made. 


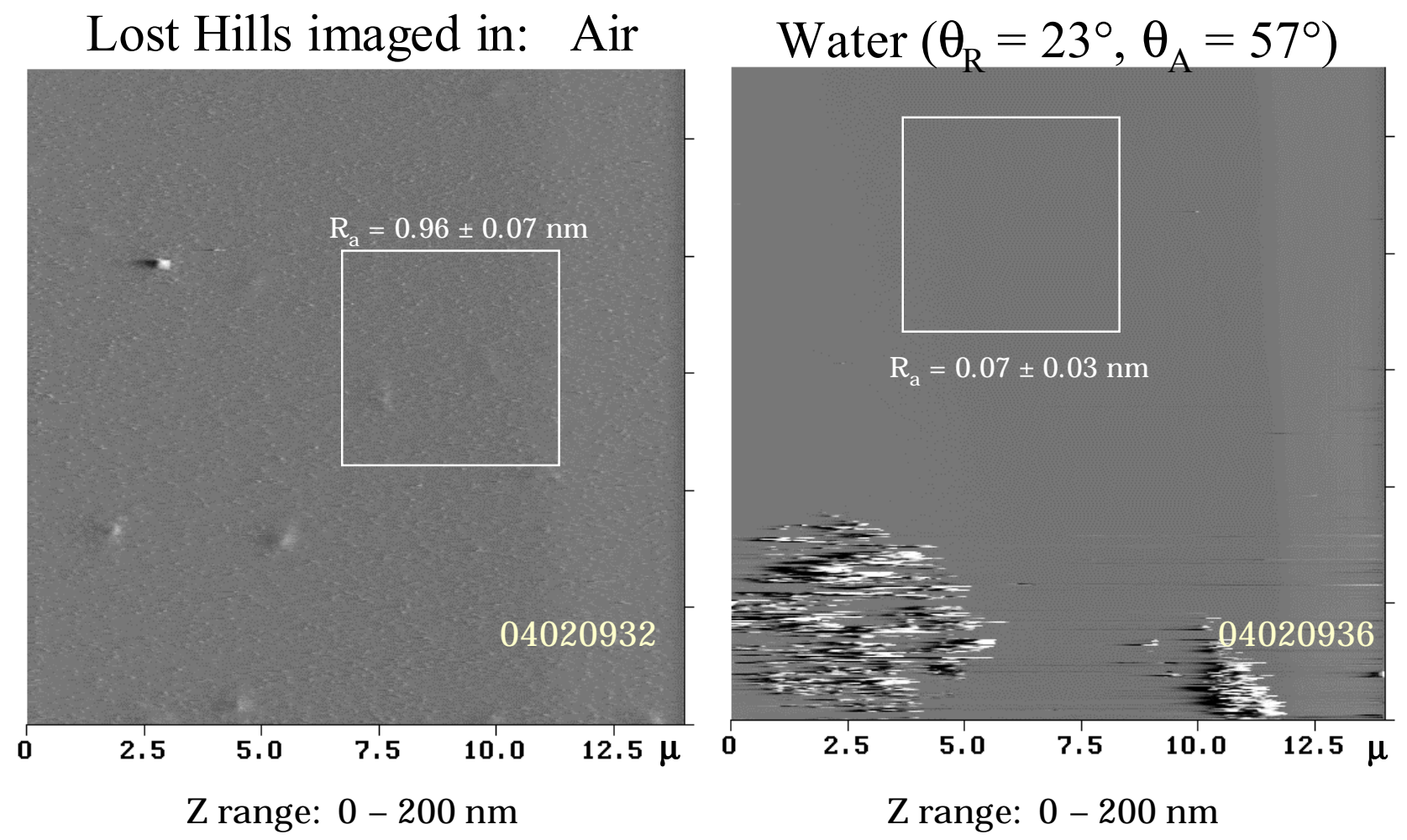

Figure II-3.103. AFM of mica surface aged in pH4 buffer (1 day) followed by Lost Hills crude oil (3 weeks).

\section{AFM OF LOST HILLS MIXTURES WITH N-HEPTANE}

Addition of $n$-heptane produced surfaces that were more resistant to removal during scanning. An example of a surface on mica aged in a 50:50 mixture of Lost Hills and n-heptane, imaged under decane, is shown in Fig. II-3.104. Holes that developed during the scanning process (as shown in Fig. II-3.104) and holes that were deliberately scraped both gave values of the coating thickness of less than $10 \mathrm{~nm}$. 


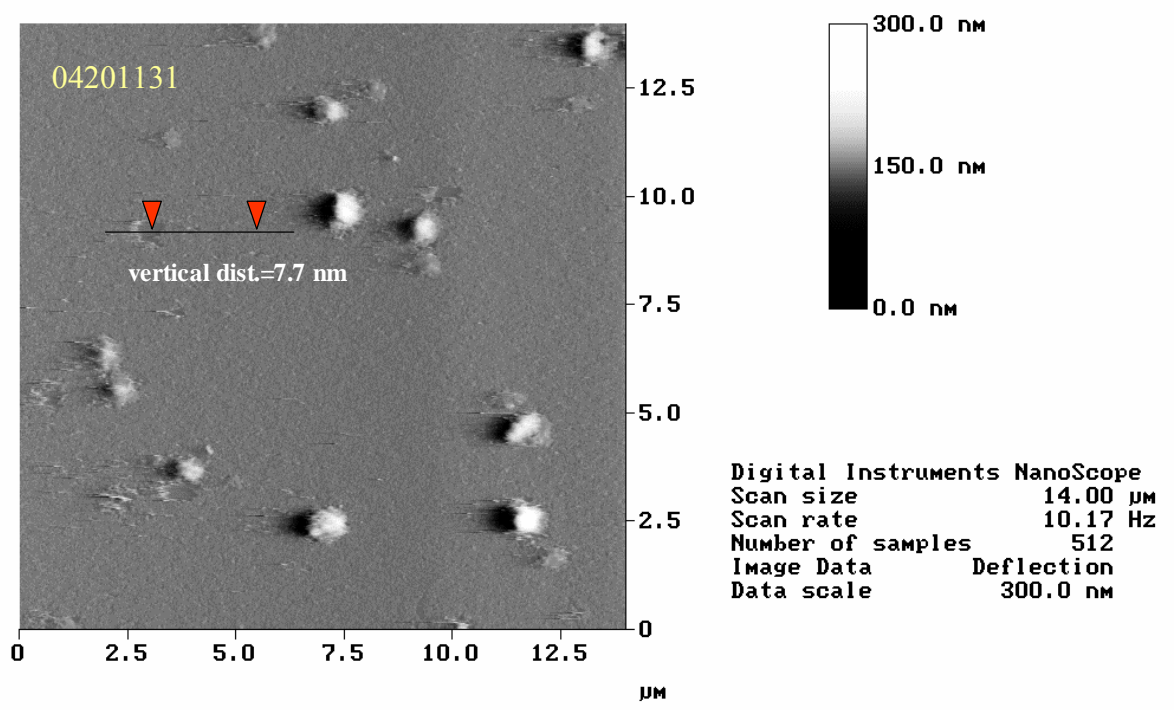

Figure II-3.104. AFM of mica treated with a pH4 buffer and a 50:50 mixture of Lost Hills crude oil and nheptane. Holes are developing as the area is scanned under decane. The coating thickness measurement was made on scan \# 04201134 , a height image of the same area.

Still more decane was added to the Lost Hills oil (26:74 oil:heptane) to produce the coating shown in Fig. II-3.105, which blistered near some large holes in the organic coating under water, as observed previously with Mars-Yellow (Figs. II-3.76 and 77). The blistering effect demonstrates that the organic coating can have more lateral integrity than adhesion to the mica surface.
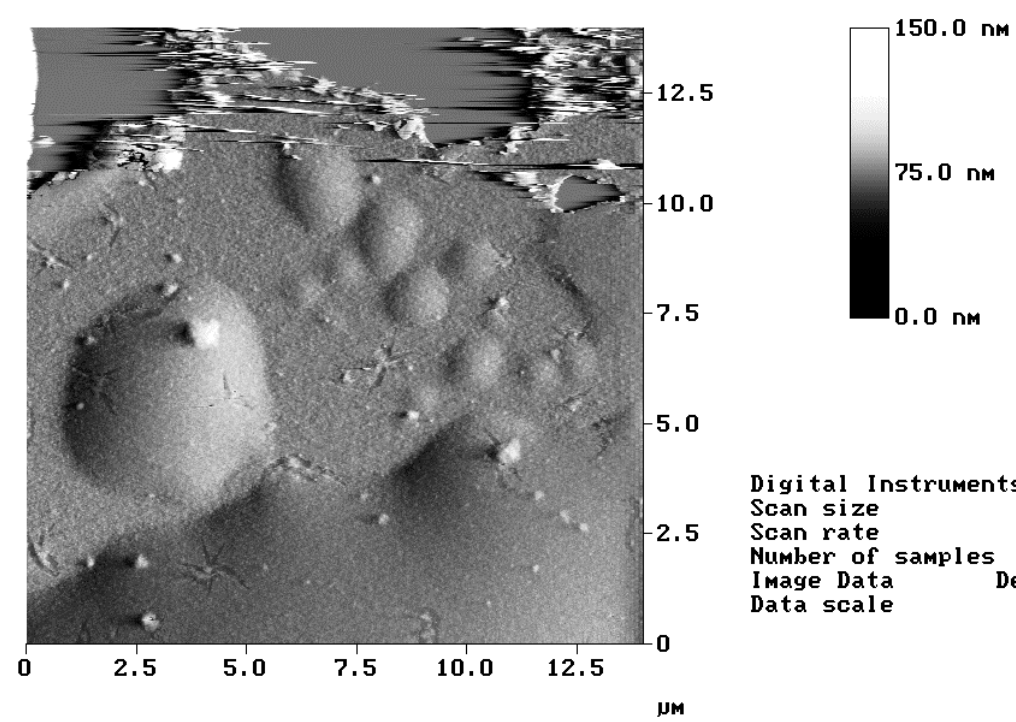

Digital Instruments NanoScope

Scan size $14.00 \mathrm{jm}$

Scan rate $10.17 \mathrm{~Hz}$

Number of samples $\mathbf{5 1 2}$

Image Data Deflection

Data scale $150.0 \mathrm{~nm}$

Figure II-3.105. AFM of mica treated with a pH4 buffer and a 26:74 mixture of Lost Hills crude oil and nheptane imaged under water.

Section analysis of different images shows how coating thickness varied with the amount of n-heptane in the crude oil/heptane mixtures (Fig. II-3.106). The onset condition at 27\% oil 
and $73 \%$ heptane provides a reference point with respect to asphaltene stability. The thickest coatings were measured on surfaces exposed to near-onset conditions. This observation is consistent with previous contact angle observations showing that mica surfaces become more oilwet when exposed to near onset mixtures of oil and heptane (Al-Maamari and Buckley, 2000). It appears that there is a maximum in the amount of material deposited near the onset of asphaltene instability, although formation of that deposit does not correlate with high contact angles.

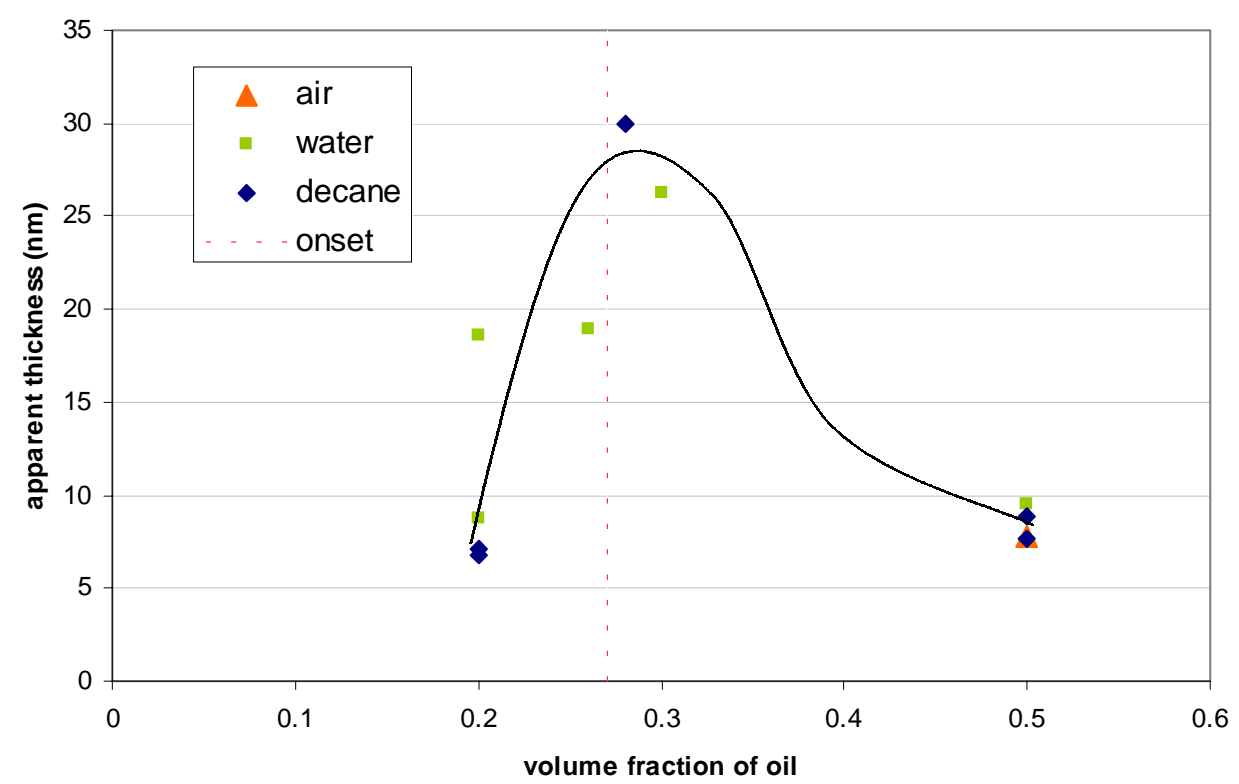

Figure II-3.106. Apparent coating thickness measured by section analysis using AFM height images in which some bare mica was exposed.

\section{UNUSUAL SURFACE FEATURES}

The surface shown above (Fig. II-3.105) was produced under conditions where asphaltenes are unstable. Another area of the same surface is shown imaged under decane in Fig. II-3.107. Note the dark, elongated markings. Narrow features of about $2 \mu \mathrm{m}$ length appear in many of the images produced from Lost Hills oil mixtures that are unstable with respect to asphaltenes. Fig. II-3.108 shows a zoom on one of these markings. Section analysis shows that what appears to be a hole or depression does not reveal the smooth surface indicative of bare mica. These features so far are unique to surfaces treated with Lost Hills crude mixed with heptane; nothing like them has been observed with any of the other oils or combinations of oil and solvents or asphaltene precipitant tested previously. In Figs. II-3.107 and 108, the features appear to be depressions. On other surfaces, they appear as elevated features, as shown in Fig. II-3.109 for a surface aged in a 20:80 mixture of Lost Hills oil and heptane, well into the region of asphaltene stability. Figure II-3.110 shows a zoom of a feature in the upper left-hand quadrant of Fig. II-3.109. 


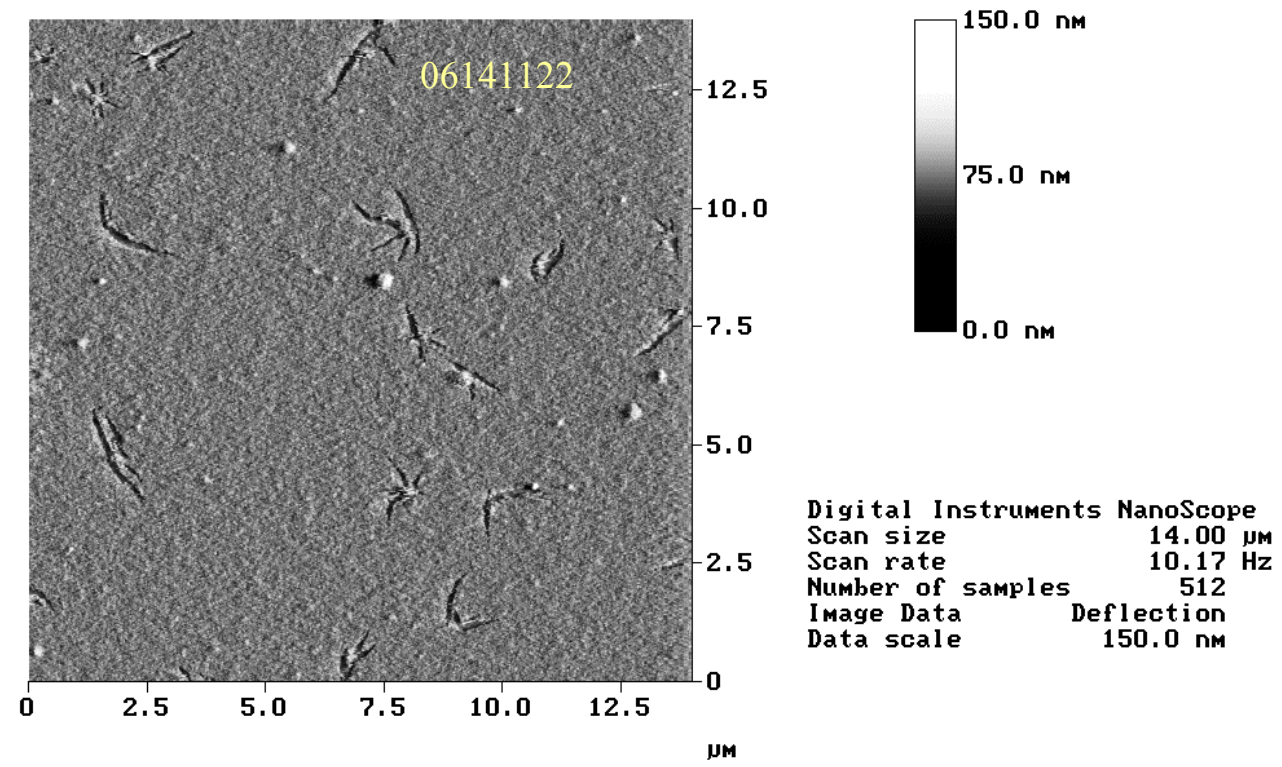

Figure II-3.107. AFM of mica treated with a pH4 buffer and a 26:74 mixture of Lost Hills crude oil and nheptane imaged under decane.

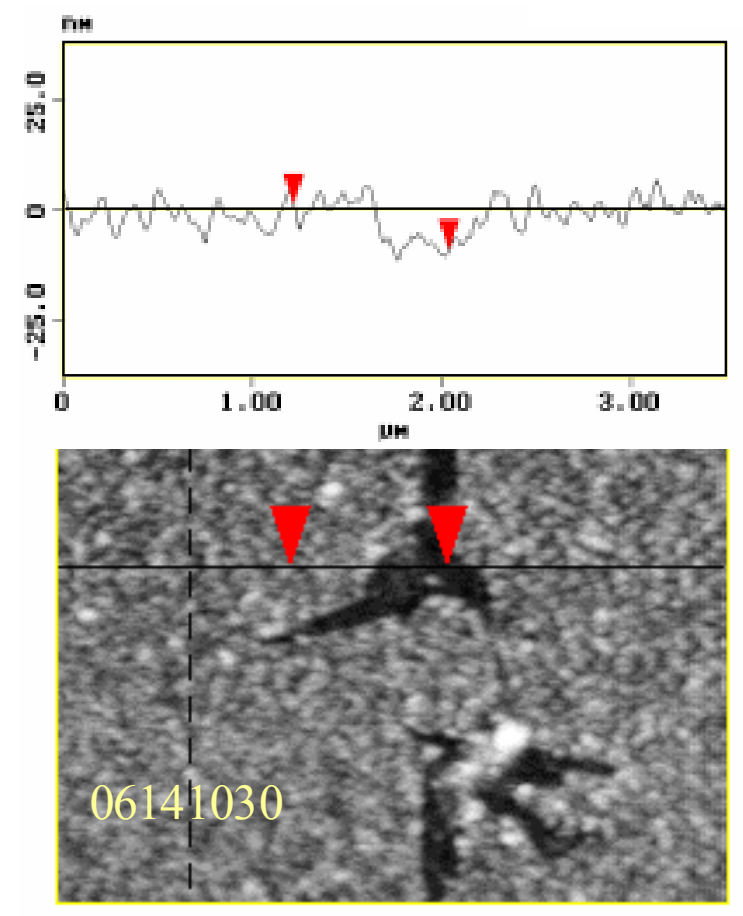

Figure II-3.108. Section analysis of mica treated with a pH4 buffer and a 26:74 mixture of Lost Hills crude oil and n-heptane imaged under air. The dark, elongated depressions do not reveal any bare mica surface. 


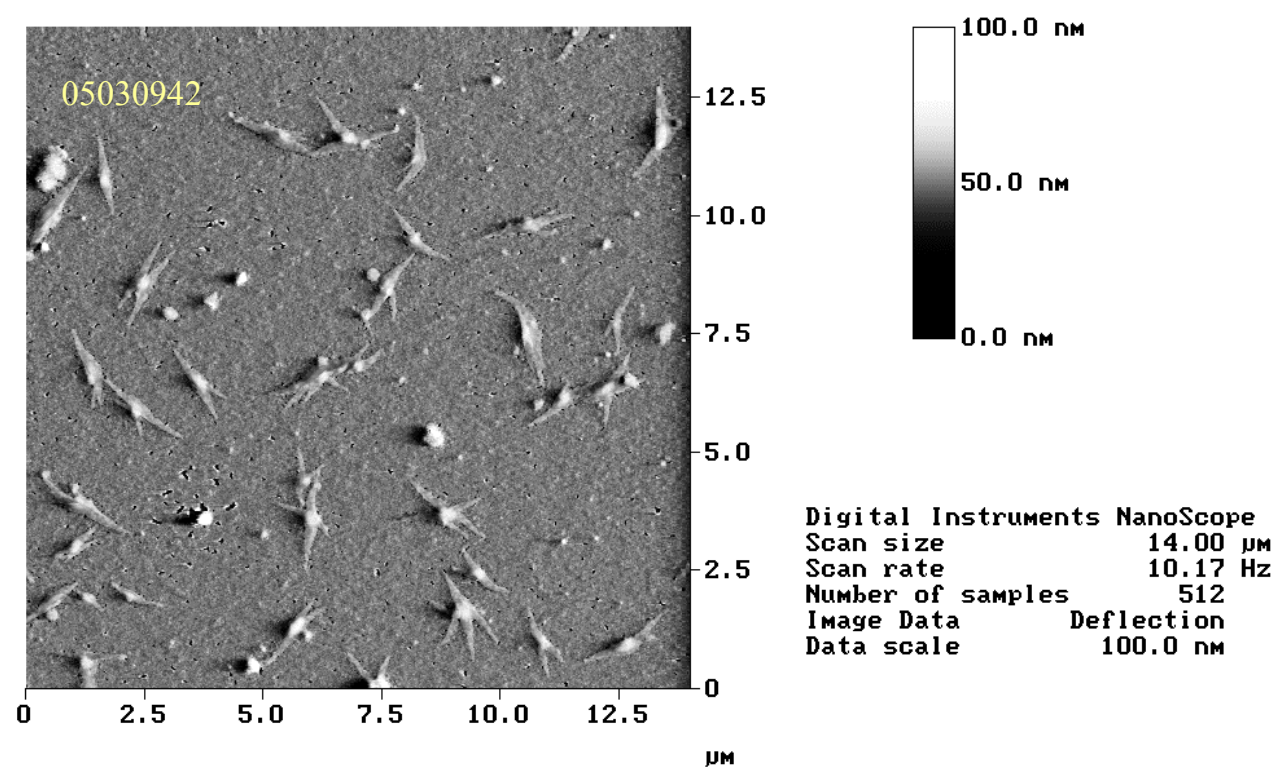

Figure II-3.109. AFM of mica treated with a pH4 buffer and a 20:80 mixture of Lost Hills crude oil and nheptane imaged under water.

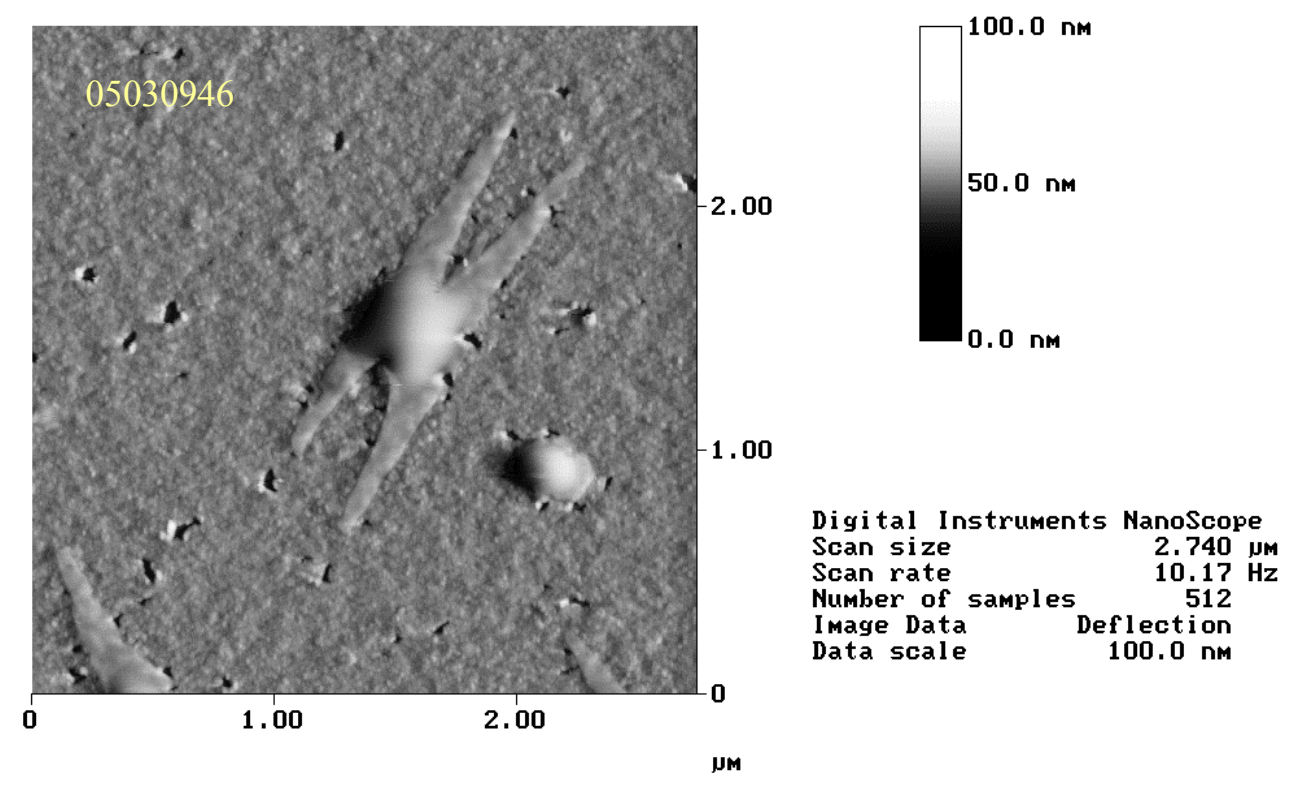

Figure II-3.110. Zoom of a feature in the upper left-hand quadrant of Fig. II-3.109.

The shape and appearance of these features does not suggest that they themselves are asphaltenes, although they do become associated with the organic coating as additional heptane is added to the oil. Some other observations can be made about these features. On some surfaces they appear in one imaging medium and not in others. Table II-3.9 summarizes the conditions under which these multi-armed features appear. Another observation made during the course of these experiments is the appearance of a drop of water or brine on the surfaces of mica samples removed from the oil/heptane mixtures. Often the last water drop could not be removed 
by rinsing with toluene and could be seen to dry on the surface, near the center of the sample. In one case, images were obtained of the area on which the water drop dried (Fig. II-3.111) and of the interface between dried water drop and oil-coated surface (Fig. II-3.112).

Table II-3.9. Conditions under which narrow, multi-armed features were observed.

\begin{tabular}{|c|c|c|c|}
\hline vol. fraction oil & Imaging Fluid & elevated/depressed & comments \\
\hline \multirow{2}{*}{0.3} & water & depressed or elevated & $\begin{array}{c}\text { associated with dried } \\
\text { water drop only }\end{array}$ \\
\hline \multirow{4}{*}{0.28} & air & depressed & very obvious \\
\cline { 2 - 4 } & water & & hard to see \\
\cline { 2 - 4 } & decane & depressed & very obvious \\
\hline \multirow{4}{*}{0.26} & air & depressed & very obvious \\
\cline { 2 - 4 } & water & depressed & very obvious \\
\cline { 2 - 4 } & decane+toluene & depressed & very obvious \\
\hline \multirow{4}{*}{0.2} & air & & barely visible \\
\cline { 2 - 4 } & water & elevated & very obvious \\
\cline { 2 - 4 } & decane & & invisible \\
\hline
\end{tabular}
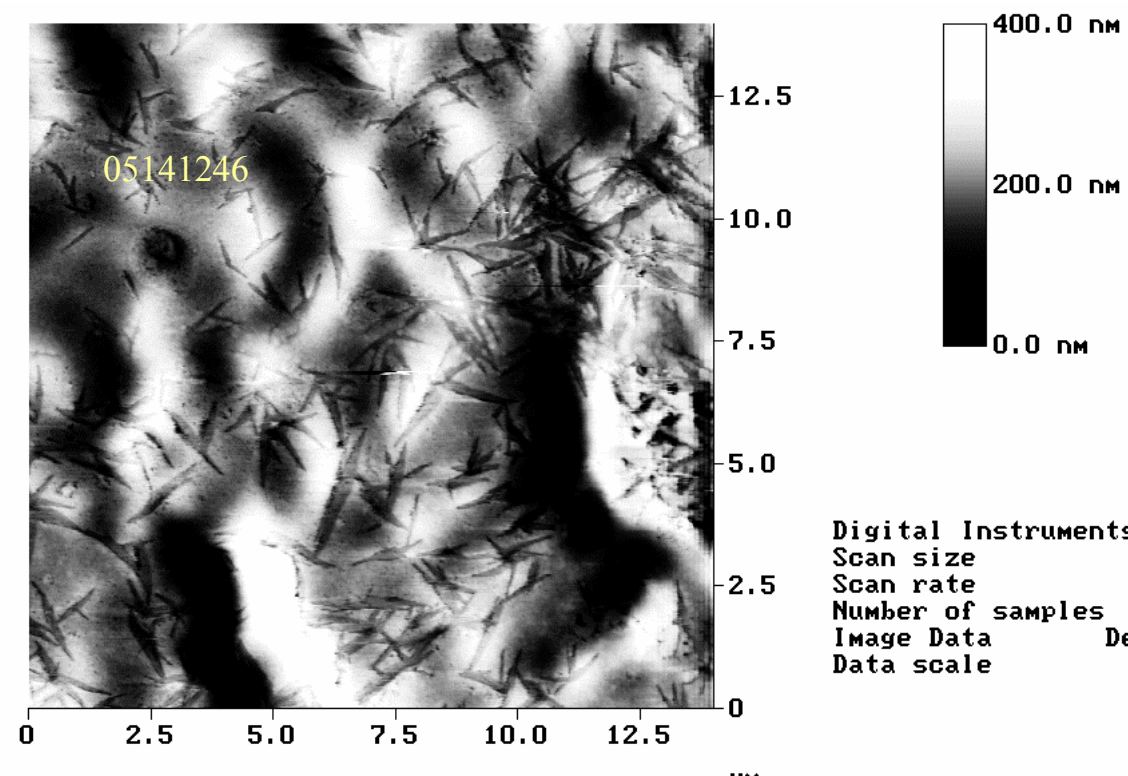

5.0

Digital Instruments NanoScope

Scan size $14.00 \mathrm{um}$

Scan rate $10.17 \mathrm{~Hz}$

Number of samples $\mathbf{5 1 2}$

Image Data Deflection

JM

Figure II-3.111. AFM of mica treated with a pH4 buffer and a 30:70 mixture of Lost Hills crude oil and nheptane imaged under air. The area shown is a portion of the surface on which a water drop dried. 

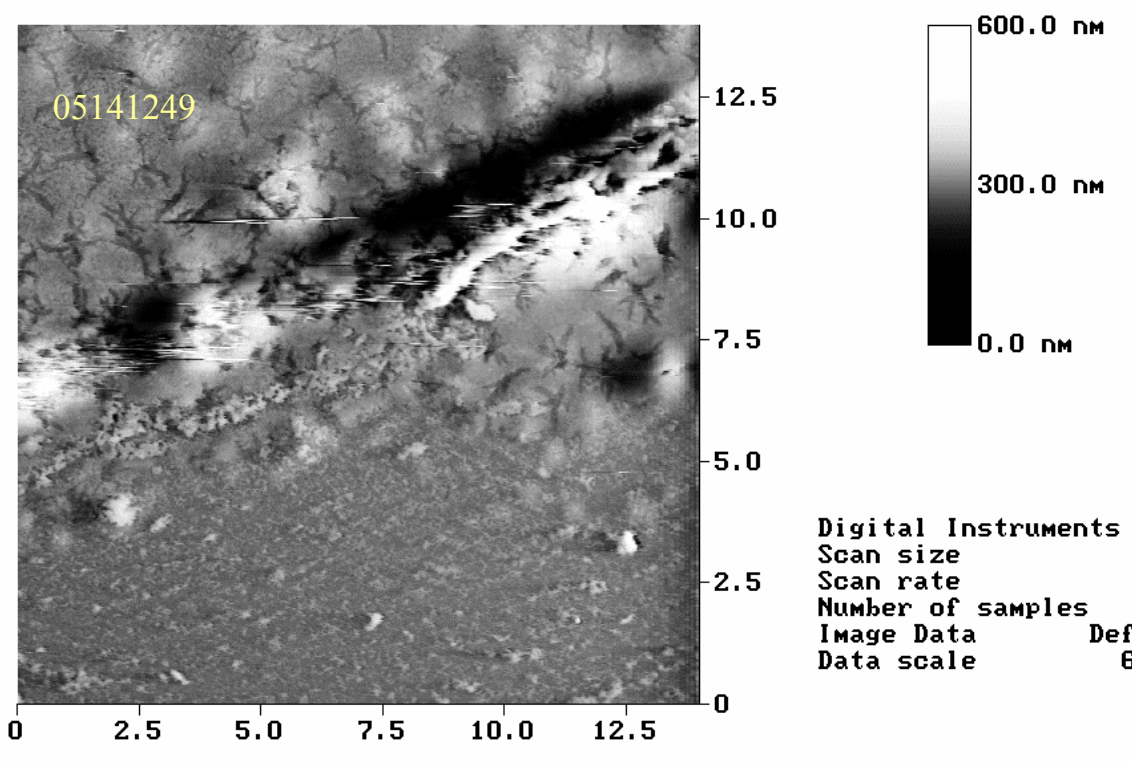

5.0

Digital Instruments NanoScope

Scan size $14.00 \mathrm{JM}$

Scan rate $10.17 \mathrm{~Hz}$

Number of samples $\mathbf{5 1 2}$

Image Data Deflection

Data scale $\quad 600.0 \mathrm{~nm}$

Figure II-3.112. Edge of the dried water drop shown in Fig. II-3.111 where water drop and oil coating can both be seen. Note that the elongated features are associated only with the dried water drop.

Microscopic examination of the Lost Hills oil shows that, like many oil samples, it contains emulsified water droplets. Addition of heptane often breaks such emulsions. Thus the water drop clinging to the oil-treated mica may be water that coalesced during the aging period. The only other source of water is the $\mathrm{pH} 4$ buffer which was originally on the mica surface. Oil treated surfaces sometimes show circular remnants that may be caused by water drying under the oil coating, but with no other oil has a drop of water been observed on top of the oil film.

There are numerous reasons why an emulsion should be stable. In some cases contaminants from drilling or treatment chemicals can contribute to emulsion stability. Unstable asphaltenes can also be a factor although in this case, the emulsion appears to be less stable as One possibility for emulsion stabilization is the presence of porphyrins (c.f., Dodd et al., 1952; Dunning et al., 1953). The Lost Hills sample has moderate levels of iron (15 ppm), nickel (32 $\mathrm{ppm})$ and vanadium $(11 \mathrm{ppm})$, any of which could be associated with porphyrins. It is interesting to compare a recently published TEM image of platelets formed from octopus porphyrin, showing structures with multiple arms, with the images shown above. There are obvious differences of scale and technique; nevertheless, the similarities are striking. It seems possible, although not yet proven, that the presence of polar porphyrins might account for the wetting conditions that are more water-wet than expected on surfaces exposed to Lost Hills oil.

Whether the features appear as depressed or elevated might depend on whether they can be dislodged from the surface by the tip, but an alternative explanation should also be considered. It is possible that the features are charged, especially under water. If they have the same charge as the tip, they will add repulsive forces and appear as an elevated portion of the surface. If they have the opposite charge with repect to the tip there will be an added attractractive force, pulling the tip downward. The pzc of the silicon nitride tip is at a $\mathrm{pH}$ of 6 so small changes in $\mathrm{pH}$ is the unbuffered aqueous phase could reverse its charge. 


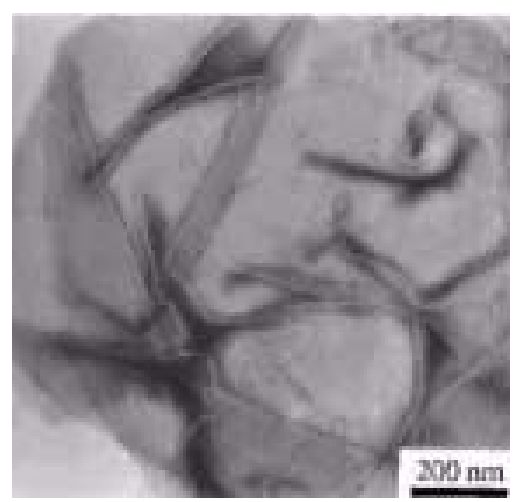

Figure II-3.113. TEM image of platelets of octopus porphyrin (from Komatsu et al., 1996).

E-oils

E-1XD-00

Mica surfaces were exposed to an oil from an unidentified reservoir, designated as E1XD-00, that gives preferentially water-wet conditions in core tests, producing the surface shown in Fig. II-3.114. Images under air, water, and decane were virtually indistinguishable. A hole scraped in the organic coating (Fig. II-3.115) showed the apparent thickness to be about $23 \mathrm{~nm}$.
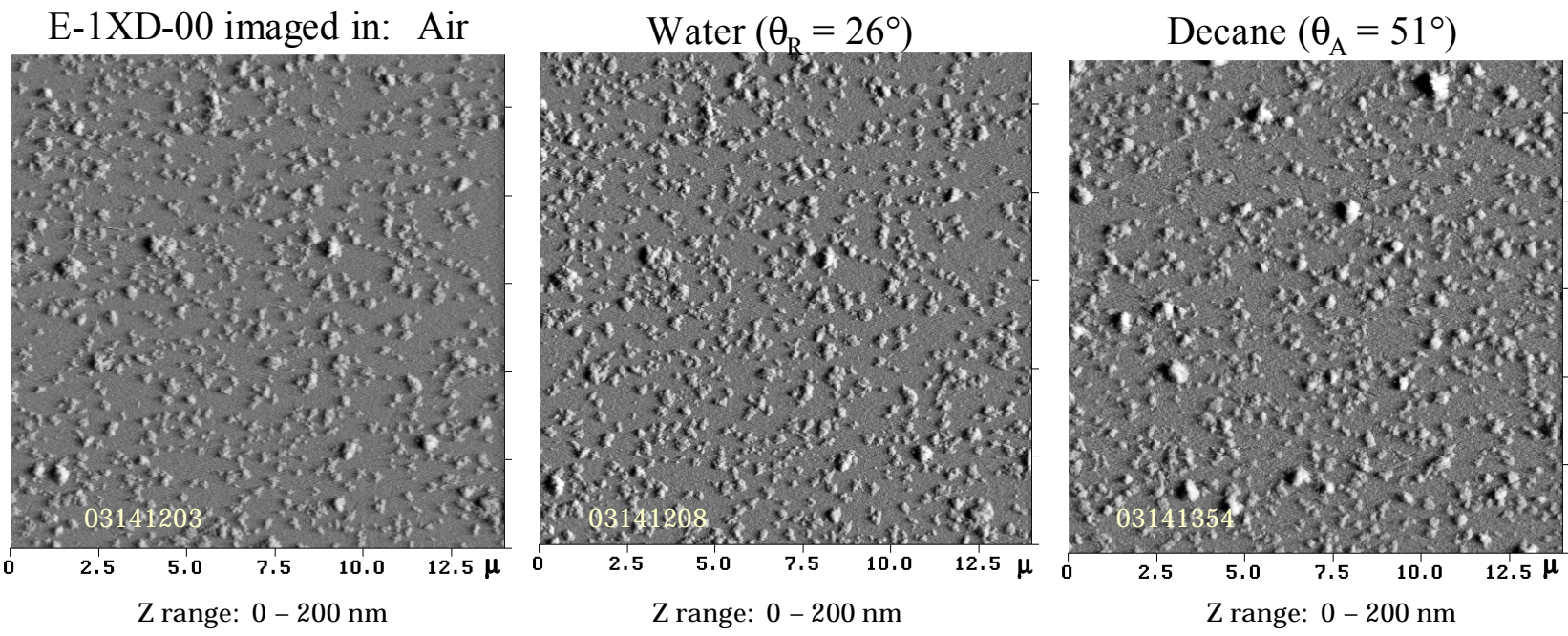

Figure II-3.114. AFM of mica surface aged in pH4 buffer (1 day) followed by E-1XD-00 crude oil (3 weeks). 


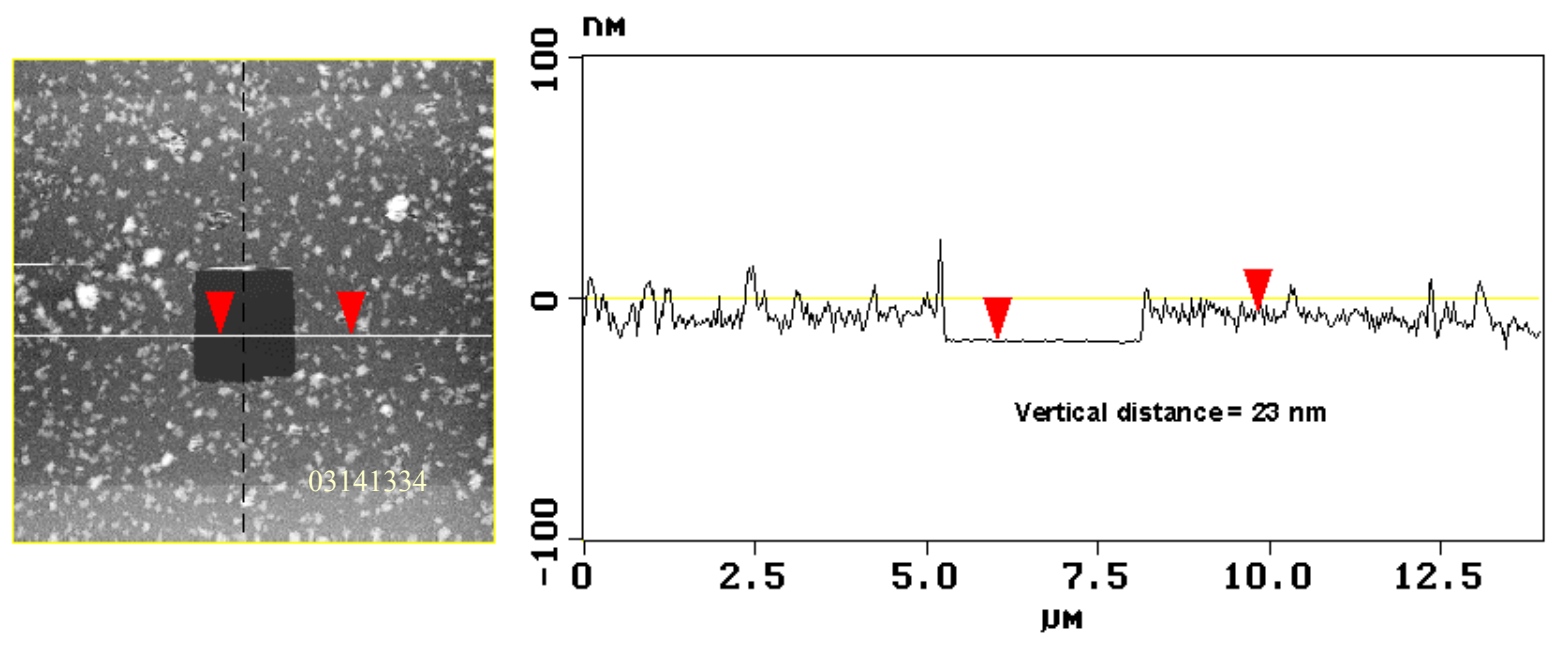

Figure II-3.115. Section analysis of a hole on a mica surface exposed to E-1XD-00 crude oil.

\section{E-1XR-00}

Results of exposure to a companion oil sample (E-1XR-00) that produces more oil wet conditions in core tests is shown in Fig. II-3.116. The coating thickness of about $42 \mathrm{~nm}$ produced by this oil sample (Fig. II-3.117) was the largest value observed in any of the crude oil tests during the course of this project, corresponding to high water/decane advancing contact angles on similarly treated surfaces.
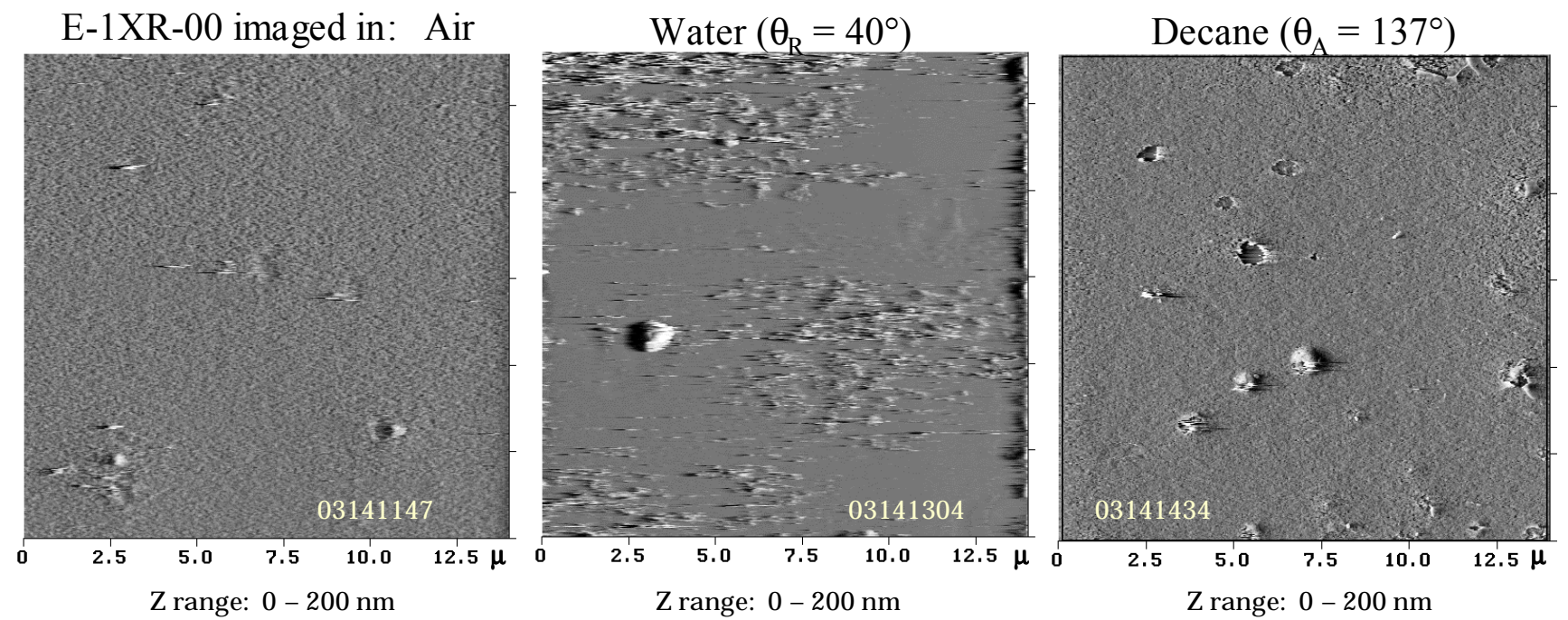

Figure II-3.116. AFM of mica surface aged in pH4 buffer ( 1 day) followed by E-1XR-00 crude oil (3 weeks). 

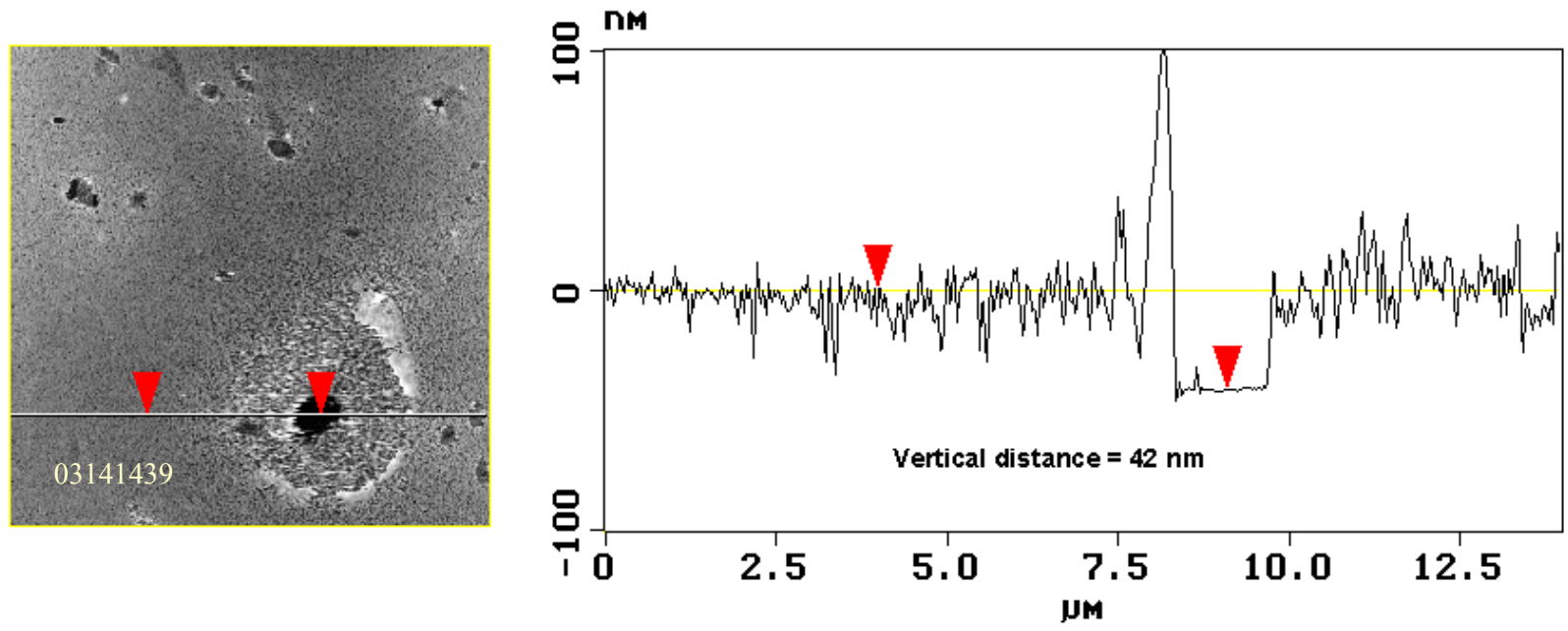

Figure II-3.117. Section analysis of a hole on a mica surface exposed to E-1XR-00 crude oil.

\section{Summary}

In the presence of a stable water film, AFM images confirm that exposure to crude oil does not result in any perceptible organic deposition on mica surfaces.

Weakly water-wet surfaces had coatings that could readily be removed by increased imaging forces and by immersion in water. Coatings produced by exposure to Mars-Yellow had more lateral integrity than those deposited by Lost Hills crude oil. This difference was not reflected in contact angles, which were quite similar. The amount of material deposited by a crude oil may not be a good indicator of the extent of wetting alteration. The strength of interactions with the surface in the presence of water is an important factor.

AFM images show that water-advancing angles are highest for oils that produce the thickest coatings that are resistant to disruption by the AFM tip. However, even apparently continuous organic coatings are not completely hydrophobic. Whether this is because the coatings are not as continuous as they appear or because the organic material is not completely hydrophobic is an important question that remains to be resolved.

\section{References}

Al-Maamari, R, Buckley, J.: "Asphaltene Precipitation and Alteration of Wetting: Can Wettability Change During Oil Production?" paper SPE 59292 presented at the 2000 SPE/DOE Improved Oil Recovery Symposium, Tulsa, OK, 3-5 April.

Al-Maamari, R.: Effect of Asphaltene Destabilization on Wettability, Ph.D. Dissertation, New Mexico Institute of Mining and Technology, Socorro, NM (2000). 
Buckley, J.S., Liu, Y., and Monsterleet, S.: "Mechanisms of Wetting Alteration by Crude Oils," SPEJ (Mar. 1998) 3, 54-61.

Buckley, J.S., Liu, Y., Xie, X., and Morrow, N.R.: "Asphaltenes and Crude Oil Wetting-The Effect of Oil Composition," SPEJ (June, 1997) 107-119.

Buckley, J.S., Takamura, K., and Morrow, N.R.: "Influence of Electrical Surface Charges on the Wetting Properties of Crude Oils," SPEFE (August 1989) 332-340.

Campbell, N.A.: Biology, $3^{\text {rd }}$ Ed., Benjamin/Cummings Publishing, Redwood City (1993).

Chatzis, I., Morrow, N.R., and Lim, H.T.: "Magnitude and Detailed Structure of Residual Oil Saturation," SPEJ, 311-326 (April, 1983).

Cuiec, L.: "Evaluation of Reservoir Wettability and Its Effects on Oil Recovery," in Interfacial Phenomena in Oil Recovery, N.R. Morrow, ed., Marcel Dekker, Inc., New York City (1991), 319-375.

Dixit, A.B., McDougall, S.R., Sorbie, K.S., and Buckley, J.S.: "Pore Scale Modelling of Wettability Effects and their Influence on Oil Recovery," SPEREE (Feb. 1999) 2, 25-36.

Dodd, C.G., Moore, J.W., and Denekas, M.O.: "Metalliferous Substances Adsorbed at Crude Petroleum-Water Interfaces," Ind.\& Eng. Chem. (Nov. 1952) 44, No. 11, 2585-90.

Dubey, S.T. and Doe, P.H.: "Base Number and Wetting Properties of Crude Oils," SPERE (Aug. 1993) 195-200.

Ducker, W.A.; Grant, L.M.: "Effect of Substrate Hydrophobicity on Surfactant SurfaceAggregate Geometry," J. Phys. Chem. Lett. (1996) 100, 11507-11511.

Dunning, H.N., Moore, J. W., and Denekas, M. D.: Interfacial Activities and Porphyrin Contents of Petroleum Extracts," Ind \& Eng. Chem. (Aug. 1953) 45, No. 8, 1759-65.

Durand, C. and Beccat, P.: "Use of XPS for Reservoir Sandstone Wettability Evaluation. Application to Kaolinite and Illite," J. Pet. Sci. Eng. (1998) 20, 259-265.

Ese, M.-H., Sjoblom, J., Djuve, J., Pugh, R. "An Atomic Force Microscopy Study of Asphaltenes on Mica Surfaces. Influence of Added Resins and Demilsifiers," Colloid Polym. Sci. (2000) 278, 532-538.

Gauchet, R., Chenevier, P., and Tricart, J.-P.: "Visualization of Rock Samples in Their Natural State Using Environmental Scanning Electron Microscope," paper SPE 26620 presented at the 1993 ATCE, Houston, 3-6 Oct.

Hjelmeland, O. and Larrondo, L.E.: "Experimental Investigation of the Effects of Temperature, Pressure, and Crude Oil Composition on Interfacial Properties," SPERE (July 1986) 321-328.

Jaschke, M, Butt, H., Gaub, H, Manne, S.: "Surfactant Aggregates at a Metal Surfaces," Langmuir (1997), 13, 1381-1384.

Komatsu, T., Yamada, K., Tsuchida, E., Siggel, U.,Bottcher, C., and Fuhrhop, J.-H.: "Micellar Fibers of Octopus Porphyrin. Photoinduced Electron Transfer Reactions in Aqueous Media," Langmuir (1996) 12, 6242-6249.

Liu, Y. and Buckley, J.S.: "Evolution of Wetting Alteration by Adsorption from Crude Oil," SPEFE (Mar. 1997) 5-11. 
Manne, S, Cleveland, J.P., Gaub, H.E., Stucky, G.D., and Hansma, P.K.: "Direct Visualization of Surfactant Hemimicelles by Force Microscopy of the Electrical Double-Layer," Langmuir (1994) 10, 4409-4413.

Manne, S., Gaub, H.E.: "Molecular Organization of Surfactants at Solid-Liquid Interfaces," Science (December 1995), 270, 1480-1482.

McKellar, M. and Wardlaw, N.C.: "A Method of Viewing "Water" and "Oil" Distribution in Native-State and Restored-State Reservoir Core," AAPG Bull. (June, 1988) 72, 765-771.

Mitchell, A.G., Hazell, L.B., and Webb, K.J.: "Wettability Determination: Pore Surface Analysis," paper SPE 20505 presented at the 1990 ATCE, New Orleans, 23-26 Sept.

Morrow, N.R.: "Wettability and Its Effect on Oil Recovery," JPT (Dec. 1990) 1476-1484.

Robin, M., Combes, R., and Rosenberg, E.: "Cryo-SEM and ESEM: New techniques to Investigate Phase Interactions within Reservoir Rocks," paper SPE 56829 presented at the 1999 ATCE, Houston, 3-6 Oct.

Salathiel, R.A.: "Oil Recovery by Surface Film Drainage in Mixed-Wettability Rocks," J. Pet. Tech. (Oct. 1973) 1216-24.

Toulhoat, H., Prayer, C., and Rouquet, G.: "Characterization by atomic force microscopy of adsorbed asphaltenes," Colloids and Surfaces A (1994) 91, 267-283.

Treiber, L.E., Archer, D.L., and Owens, W.W.: "A Laboratory Evaluation of the Wettability of Fifty Oil-Producing Reservoirs," SPEJ (Dec. 1972) 531-540.

Yang, S.-Y., Hirasaki, G.J., Basu, S., and Vaidya, R.: "Mechanisms for Contact Angle Hysteresis and Advancing Contact Angles," J. Pet. Sci. Eng. (1999) 24, 63-73. 


\section{Part III-Rate of Imbibition and Assessment of Wetting in Porous Media}

Ultimately reservoir wettability questions must be resolved in porous media. The approach in this study, the use the rate of imbibition as the primary measure of wetting in rocks that have been exposed to brine and crude oil, is the focus of Part III. Oil recovery by spontaneous imbibition is reviewed in section III-1. Section III-2 focuses on theoretical developments. A commercial simulator was adapted to study spontaneous imbibition, as reported in section III-2.1. The simulator was used to examine factors included in imbibition scaling groups and other factors that may influence the rate of spontaneous imbibition. Another theoretical approach to linear counter-current imbibition using a similarity solution is explained in section III-2.2. Experimental work on a range of systems is reported in section III-3. Studies include an examination of the critical length for scaling experiments in liquid/gas, very strongly wetted systems (section III-3.1), scaling of imbibition rate in mixed-wet systems, and several studies of scaling and other issues with probe fluids in cores that were made mixed-wet by contact with crude oil.

\section{III-1. A Review of Recovery of Oil by Spontaneous Imbibition}

\section{Morrow, N.R. and Mason, G.: "Recovery of Oil by Spontaneous Imbibition,” Current Opinion in} Colloid and Interface Sci. (2001) 6, 321-337.

Spontaneous imbibition is of particular importance to oil recovery from fractured reservoirs. There has been a surge in the growth of technical literature over the past five years. This review is centered on developments in scaling of laboratory imbibition data. Results for variation in interfacial tension, wetting and nonwetting phase viscosity, sample size, shape and boundary conditions, and initial wetting phase saturation have been correlated for a variety of strongly water-wet rocks as plots of normalized oil recovery versus dimensionless time. Correlations have been tested for weakly water-wet conditions induced by adsorption from crude oil. In situ fluid saturation measurements have been used to distinguish between modes of imbibition that range from frontal to global displacement. Research on surfactant-enhanced imbibition has advanced from laboratory to field tests.

\section{Introduction}

Spontaneous imbibition is the process by which a wetting fluid is drawn into a porous medium by capillary action. Imbibition is important in numerous every day activities. For example the use of cloth or paper towels to dry dishes or clean-up spilt liquid, or the transfer of ink or paint to a porous material such as paper. Imbibition is driven by surface energy, through the action of capillary pressure. Capillary pressure is equal to the product of interfacial tension, $\sigma$, and the curvature, $\mathrm{C}$, of the interface. The magnitude of the curvature during imbibition depends on surface forces in combination with pore geometry. If the curvature is concave with respect to the phase which occupies the porous media, and the pore-scale interface boundary 
conditions allow the interface to advance, the resulting displacement is spontaneous. The contact angle at the three-phase line of contact and interface shape can be influenced by dynamic effects that result from spontaneous motion of the interface. A large proportion of the literature on spontaneous imbibition over the past fifty years has been related to recovery of crude oil from subterranean reservoirs. A tabulated summary of experimental studies of imbibition up to 1992 is given by Zhou et al.[1]. Many research groups are currently active in various aspects of wetting and imbibition. Topics range from molecular modeling of interface movement to studies of field scale reservoir performance. This article reviews background and recent developments with emphasis on laboratory studies of spontaneous imbibition.

The role of imbibition has been recognized in numerous types of recovery processes including waterflooding of heterogeneous reservoirs, thermal recovery by steam injection through imbibition of condensed water, and alternate injection of water and gas. Recovery of oil by spontaneous imbibition of brine into reservoir rock is of special importance in fractured reservoirs, particularly when the rock has low permeability. Many fractured reservoirs exhibit prolific initial oil production from the fractures, but this can be short lived. Under water injection or aquifer drive, subsequent recovery of oil from the rock matrix, if any, is mainly dependent on spontaneous imbibition of water, a relatively slow process. For both oil and gas reservoirs, an increasing proportion of reserves are held by very low permeability rocks. Large volumes of oil have been recovered from North Sea chalk reservoirs and much attention has been paid to reservoir wettability and its effect on imbibition. Oil bearing low-permeability fractured limestones and diatomaceous and siliceous shale formations are also examples of large potential reserves for which imbibition may be a viable recovery mechanism.

The rate of recovery and the extent to which oil is displaced from the matrix are key issues in the evaluation and engineering of fractured reservoirs. The recovery depends on crude oil/brine/rock (COBR) interactions, which depend on wetting and two-phase flow, and is governed by the interwoven complexities of the chemistry and physical properties of all three components, and the fracture geometry and pore structure of the rock. The scope of these complexities will be illustrated by examples that range from imbibition into single tubes to observations on COBR combinations.

\section{Imbibition into Completely Wetted Capillaries}

\section{Cylindrical capillary}

Washburn [2] provided an analysis of imbibition into a capillary tube, which demonstrates the basic competition between capillary driving force and viscous resistance for

flow that is clearly co-current. Results were reported for spontaneous displacement of air by mercury.

Consider a capillary tube of radius, $r$, containing a meniscus of a perfectly wetting liquid. The capillary pressure, $\mathrm{P}_{\mathrm{c}}$, is equal to 


$$
P_{c}=\frac{2 \sigma}{r}
$$

For laminar flow in a capillary tube, the Poisenille equation relates the length, $\mathrm{L}$, and the radius of the tube, $r$, to the viscosity, $\mu$, mean velocity, $v$, and the pressure drop, $\Delta \mathrm{P}$, of the fluid.

$$
\frac{\Delta P}{L}=\frac{8 \mu \nu}{r^{2}}
$$

If a capillary tube is being invaded by a fluid of viscosity, $\mu$ displacing a gas with negligible viscosity, then the driving pressure is Pc and the viscous resistance is proportional to the invaded length, so combining Eq. III-III-1.1 and Eq. III-III-1.2 gives:

$$
v=\frac{2 \sigma}{r} \frac{1}{L} \frac{r^{2}}{8 \mu}=\frac{\sigma r}{4 \mu L}
$$

But the velocity, $\mathrm{v}$, is $\mathrm{dL} / \mathrm{dt}$ and so, on integration, we get

$$
L^{2}=\frac{\sigma r t}{2 \mu}
$$

If the tube length is $\mathrm{L}_{\max }$ then the fractional length filled, $\mathrm{L} / \mathrm{L}_{\max }$ is

$$
\left(\frac{L}{L_{\max }}\right)^{2}=\frac{\sigma r t}{2 \mu L_{\max }^{2}}
$$

If the receding fluids have identical viscosities, then the mean velocity of the advancing interface becomes constant and:

$$
\frac{L}{L_{\max }}=\frac{\sigma r}{4 \mu L_{\max }^{2}} t
$$

If the invading fluid has zero viscosity and the receding fluid has viscosity $\mu$, then

$$
1-\left(1-\frac{L}{L_{\max }}\right)^{2}=\frac{\sigma r t}{2 \mu L_{\max }^{2}}
$$

and the interface velocity accelerates as the zero viscosity fluid invades.

The behavior for any ratio in the viscosities of the invading and receding phases falls between the two extremes given by Eq. III-III-1.5 and Eq. III-III-1.7. In all cases except where the two viscosities are exactly equal, the square of the fraction filled varies linearly with $\sigma, \mathrm{r}$ and $\mathrm{t}$ and 
inversely with $L_{\max }^{2}$. Thus, imbibition into a tube will be scaled by a dimenionless time defined by

$$
t_{D t}=\frac{\sigma r t}{f\left(\mu_{w}, \mu_{o}\right) L_{\max }^{2}}
$$

where $f\left(\mu_{w}, \mu_{o}\right)$ is a function with the dimensions of viscosity. Results for imbibition into completely wetted cylindrical tubes will be correlated by a plot of the fraction of the tube filled by invading liquid, $\mathrm{L} / \mathrm{L}_{\max }$, versus $\mathrm{t}_{\mathrm{Dt}}$.

\section{Tubes with corners}

Imbibition into tubes with corners such as with the cross- section of an equilateral triangle presents a number of variations on the basic case of the cylinder. If the tube is completely wetted and the supply of liquid is limited so that the displacement is essentially quasi-static, the interface will grow from the corners as three arc menisci that occupy the whole length of the tube (Fig. III-III-1.1a) [3]. When the arc menisci touch (Fig. III-1.1b), there is spontaneous redistribution of the liquid and loss of surface free energy [4]. For equilateraltriangle cross-section, the meniscus curvature rises to the drainage displacement curvature and $60.5 \%$ of the nonwetting phase initially in the tube is trapped. The displacement efficiency increases from a minimum of $39.5 \%$ towards $100 \%$ as the pore aspect ratio (base to height of the triangular cross-section) increases [3]. For the cross section of an isosceles triangle with the height equal to half the base (Fig. III-1.1c), the trapped saturation decreases to $30.03 \%$.

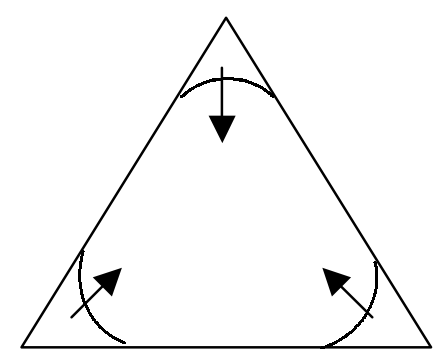

(a) corner filling equilateral triangle

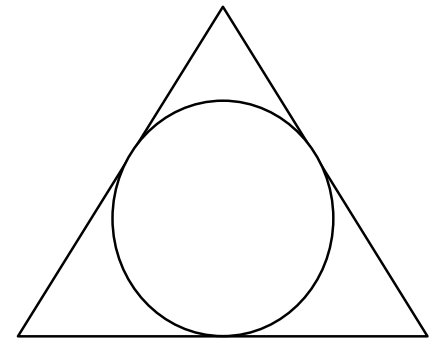

(b) $39.5 \%$ displacement efficiency

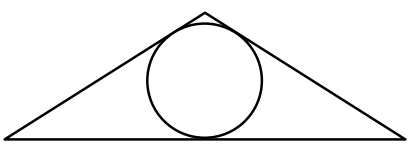

(c) $70 \%$ displacement efficiency

Figure III-1.1. Growth of corner menisci by spontaneous imbibition (a) and maximum displacement attained before instability and snap-off for tubes of triangular cross section, (b) equilateral, (c) isosceles (height $=1 / 2$ base).

If one end of the tube is sealed, the displacement is clearly countercurrent. If there is no restriction in the supply of liquid, the imbibition process is referred to as free. The rate of imbibition will depend on the liquid viscosities and tube shape but there will be no change in the final recovery. Dong and Chatzis [5] report on the fluid dynamics of advance of liquid along tubes with corners initially filled with a fluid of zero viscosity. 


\section{Imbibition in Porous Media}

In many two-phase displacement situations in porous media, such as in measurement of waterflood recovery curves and relative permeabilities to the flowing phases, it may not be possible to identify one phase unambiguously as the wetting phase. Many problems in the semantics of wetting and imbibition in oil recovery can be avoided if imbibition is defined as increase in percentage of pore space saturation, $S_{w i}$, of the aqueous phase, hereafter usually referred to as water, but also brine, surfactant, or polymer solution. It is then convenient to distinguish three types of imbibition, which are: pseudo-quasistatic imbibition, spontaneous imbibition and dynamic forced imbibition.

\section{Capillary pressure versus saturation - pseudo quasistatic imbibition}

Capillary pressures in porous media are measured with respect to fraction of pore space occupied by the wetting liquid. The uptake of liquid by spontaneous imbibition (usually preceded by capillary pressure drainage measurements from $100 \%$ initial water saturation) is measured for a series of reductions in capillary pressure. This process can be described as pseudo-quasistatic, at least to the extent that the capillary pressure curve does not show obvious dependence on the intermediate number of data points. However, numerous forms of capillary instability are inevitably involved in the imbibition process such as meniscus overlap (as for triangular tubes) or the spontaneous filling of pore bodies [6]. There is hysteresis in drainage-imbibition relationships because of pore structure, wetting, and capillary trapping. Spontaneous redistribution of fluids during the imbibition process results in loss of surface free energy.

Leverett [7] used a parallel bundle of tubes of equal radius, $r$, to define a microscopic radius in terms of permeability, $k$, and porosity, $\phi$.

$$
r=\sqrt{\frac{8 k}{\phi}}
$$

Capillary pressure data is scaled by the Leverett radius divided by the interfacial tension. The overall shape of the $\mathrm{P}_{c}$ drainage curve provides an apparent pore size distribution. Attention has also been give to interpretation of the shape of imbibition capillary pressure curves in terms of pore structure [8].

When the sample is oil-wet, water must be forced into the sample in order to displace oil. This process corresponds to forced imbibition and surface free energy is increased. For recovery of crude oil, it is often observed that water partially displaces oil by spontaneous imbibition, but further increase in water saturation can only be achieved with change in sign and increase in the applied capillary pressure. This type of measurement will be referred to as psuedo-quasistatic forced imbibition. The spontaneous and forced parts of a capillary imbibition curve are indicated in Fig. III-1.2. 


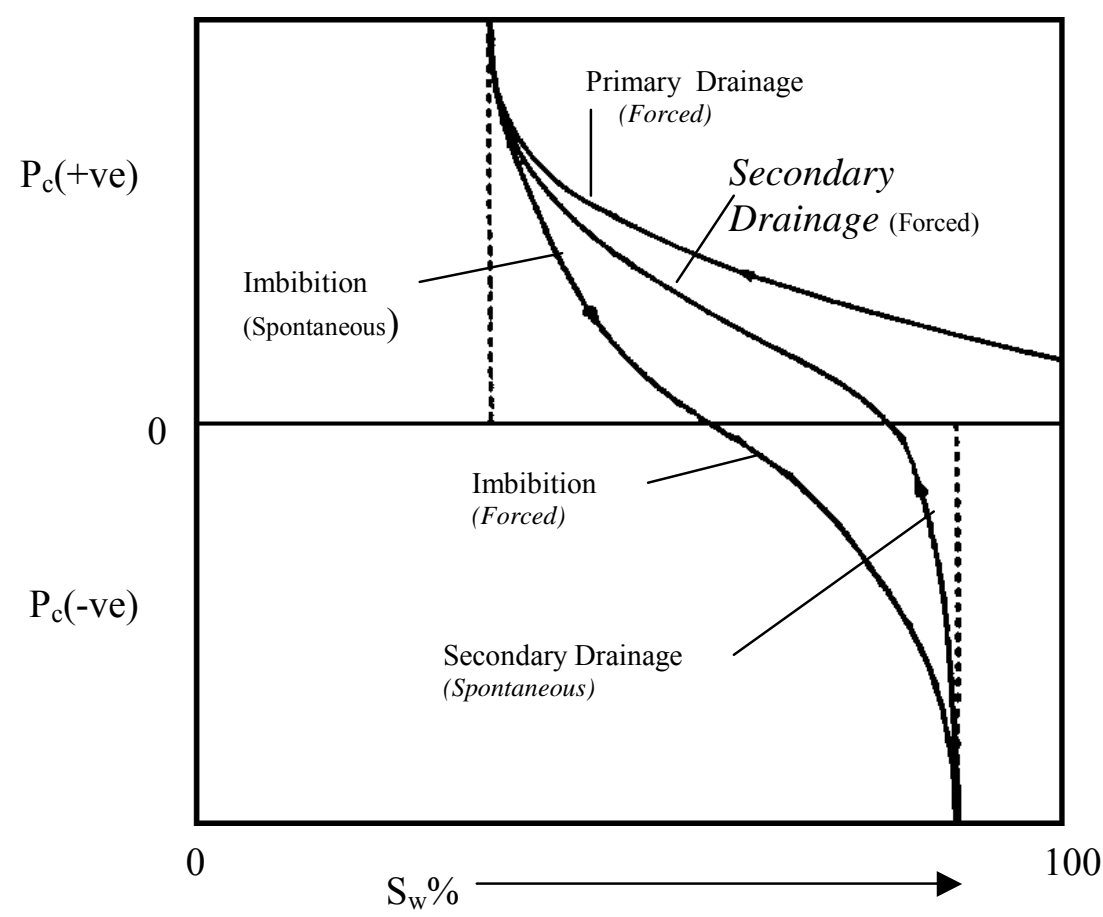

Figure III-1.2. Illustration of spontaneous and forced imbibition and drainage capillary pressure curves. Spontaneous (free) imbibition

The second common approach is to measure the rate of imbibition with free access of brine to the sample face. This type of measurement is the main subject of this review. The rock is immersed in brine and recovery of oil versus time is recorded. The capillary pressure acting during the course of flow is often referred to as the dynamic capillary pressure as distinct from the displacement being controlled in steps, as is usual in many forms of capillary pressure measurement. The change in saturation can be monitored either volumetrically or gravimetrically, or some combination of the two as a check on material balance. Results are commonly presented as fractional recovery of oil initially in place versus either time or log time.

\section{Dynamic Forced imbibition}

In contrast to psuedo-quasistatic imbibition and free imbibition, when oil is displaced by forcing water through a porous medium under a pressure gradient, as in a laboratory waterflood, the process can be referred to as dynamic forced imbibition. If the media is oil-wet, the displacement mechanism of forced imbibition essentially corresponds to drainage (displacement tends to occur in the larger pores first). Imbibition capillary pressure curves, measured under psuedo-quasistatic conditions, and relative permeability relationships, measured under either steady or unsteady state flow conditions, are input to the simulation of two-phase flow as in the prediction of oil recovery by spontaneous imbibition. Dependence of imbibition on the conditions of measurement continues to feature in longstanding debate on appropriate methods 
of measuring the relative permeabilities and capillary pressures and how they apply to various displacement processes.

\section{Scaling of Spontaneous Imbibition}

Imbibition is a complex process, which depends on numerous variables. One area of recent research has been on correlation of spontaneous imbibition rate data (oil recovery versus time). A major objective is prediction of the rate of recovery from fractured reservoirs from laboratory imbibition tests on small rock samples. The test consists of measuring oil recovery versus time.

Mattax and Kyte [9] defined a scaling group:

$t_{D M K}=\frac{t \sqrt{\frac{k}{\phi} \sigma}}{\mu L^{2}}$

This group expresses the ratio of capillary force to viscous resistance, which is a form of inverse capillary number. The group is comparable to the scaling group for cylindrical tubes, Eq. III-1.4, in several respects. The group $\sqrt{\frac{k}{\phi}}$ is proportional to the Leverett microscopic radius.

Application of the scaling group Eq. III-1.10 is subject to the following six conditions:

1) gravity effects can be neglected

2) sample shapes and boundary conditions must be identical

3) oil/water viscosity ratio is duplicated

4) initial fluid distributions are duplicated

5) the relative permeability functions must be the same

6) the capillary pressure functions must be directly proportional

Obviously, laboratory results to will rarely satisfy these conditions with respect to the reservoir. The crux of the scaling problem is to provide a working account of the factors that can affect imbibition through identification of the dominant variables under the various complex conditions that are typical of oil reservoirs. Conditions 1-6 provide a useful starting point in identifying the factors that need to be investigated in order to predict oil recovery.

Mattax and Kyte [9] presented separate correlations for two sets of data, one for two geometrically similar sandstone cores, and the other for four synthetic (alundum) cores of different lengths or permeabilities that were only open at one end face. Cylindrical samples, with only one end open to imbibition, are of special interest because the exchange of water and oil can only occur by linear countercurrent flow. The viscosity ratios were constant for the individual groups. Duplication of the initial fluid distribution (Condition 4) was satisfied by using initially dry samples $($ Swi $=0 \%)$. The results for each set were correlated by $t_{D M K}$. There is strong 
practical interest in developing correlations, which are less restrictive than the Conditions 1-6. Correlations of imbibition data are of special importance to reservoir simulation. Reservoirs were modeled as dual porosity systems given by the fracture and matrix geometry. The computational efficiency of the simulators could be increased many orders of magnitude by including imbibition rate in the form of scaled dimensionless time in transfer functions, of the type introduced for flow between the fractures and the matrix [10].

\section{Very Strongly Water-Wet Media}

Many studies of imbibition have been for strongly water-wet media with zero initial water saturation. This provides a reference condition, which is relatively easy to investigate by experiment. Developments in scaling imbibition data for strongly water-wet conditions will be reviewed first before bringing in the complexities of variation in wettability on rate and extent of imbibition. For very strongly water-wet media, the ratio of gravity to capillary forces is very small for most laboratory studies and Condition 1 is satisfied.

\section{Characteristic length.}

In the development of more versatile transfer functions, the two sets of data of Mattax and Kyte, were correlated by introduction of a characteristic length defined by the distance from the open face to the center of the core sample [11]. However, the Mattax and Kyte [9] data included variation of viscosity ratio, which should also affect imbibition rate. Examination of the results of Hamon and Vidal [12] for a synthetic porous media (Aerolith with initial water saturation in the range 16 to $18 \%$ ) for which viscosity ratio was constant (Condition 3 ), showed that results could be correlated if a characteristic length, $\mathrm{L}_{\mathrm{c}}$, was defined by the distances to the no flow boundaries with respect to counter current flow from all open faces of the rock. [13] With this modified definition of characteristic length, the results of Mattax and Kyte separated into two groups distinguished by difference in viscosity ratio.[15]

\section{Viscosity ratio.}

In a systematic test of the effect of oil/brine viscosity ratio on imbibition, Ma et al [13] measured change in imbibition rate with viscosity ratio. Oil recovery rate was found to be inversely proportional to the geometric mean of the oil and water viscosities.

$$
t_{D}=t \sqrt{\frac{k}{\phi}} \frac{\sigma}{\sqrt{\mu_{o} \mu_{w}}} \frac{1}{L_{c}^{2}}
$$

Tests of viscosity ratio included various combinations of boundary conditions (Fig. III1.3) and viscosity ratios. [14] These results and those of Mattax and Kyte [9] and Hamon and Vidal [12] all fitted this semi-empirical correlation (Fig. III-1.4). [15] The fact that all data was correlated by the characteristic length implies that the basic imbibition mechanism was counter 
current for all of the tested geometries and boundary conditions. [14] Correlation of results for which counter-current flow is believed to be dominant will be reviewed before extending discussion to co-current imbibition.

(a) All faces open

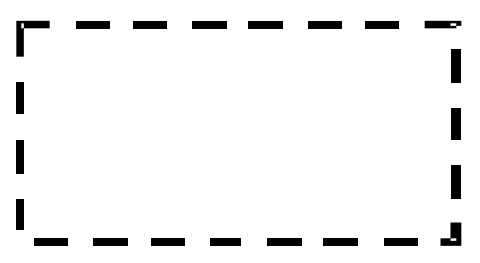

(c) Two ends open

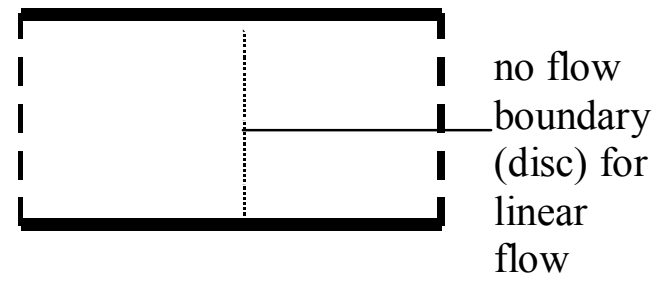

Open face $-\boldsymbol{-}$ (b) One end open

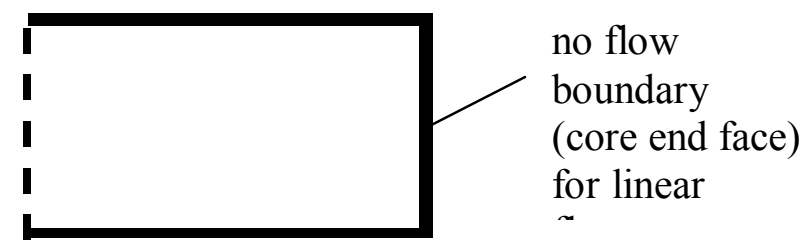

(d) Two ends closed

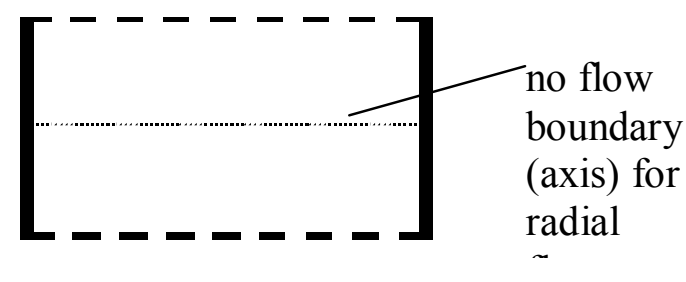

Closed surface

Figure III-1.3. Boundary conditions for core samples used by Mattax and Kyte [9] (a and b), Hamon and Vidal [12] (a to c) and Zhang et al [14] (a to d)

\section{Rock properties}

For the data discussed so far, variations in rock properties were accounted for satisfactorily by $\sqrt{\frac{k}{\phi}}$ for two types of sandstone and two types of synthetic porous media, where $\mathrm{k}$ is the absolute permeability to gas. Use of gas permeability, a routinely measured rock property, to correlate results for two-phase flow, is open to question. The single phase permeability and two-phase flow properties of reservoir rocks often depend on rock-fluid colloidal properties. Also, the rocks may exhibit directional variation in permeability. Nevertheless, the correlation also gave reasonable prediction of results for a variety of chalks and other sandstones [16]. Thus, spontaneous imbibition curves, normalized with respect to final recovery, for media of distinctly different pore size and porosity, and many orders of magnitude difference in permeability, all have approximately the same shape, and are correlated by a microscopic pore-scale dimension based on the parallel bundle of tubes model [7]. 


\section{Mobility ratio}

A remarkable feature of the correlation obtained for chalk and sandstones is that it does not overtly contain the relative permeabilities of the flowing phases. If they were included they would be linked to viscosity through two-phase flow relationships as mobilities $\left(\frac{k_{r o}}{\mu_{o}}\right.$ and $\left.\frac{k_{r w}}{\mu_{w}}\right)$. Mobilities are functions of saturation and are less readily measured than the scaling factors used to define $t_{D}$, particularly for countercurrent flow, which is the operative mechanism for which the correlation was developed. It appears that for counter current flow there are self-compensating effects. A condition of linear countercurrent flow is that

$$
q_{w}=q_{o}
$$

for any cross section along the core. At high oil viscosity, the saturation at which water first enters the core will be low and that for counter flow of oil will be high. For low oil viscosity, counter flow will tend to occur at higher water saturations, with corresponding changes in mobilities.

Application of the scaling group given by Eq. III-1.11 to recovery of gas [17] should be treated with caution. Correlation of data by the geometric mean viscosity of oil and water is empirical. With air as the nonwetting phase, results for imbibition of oil against air were closely correlated for geometrically similar cores (Condition 2) with all other five Conditions held constant [18]. However, unlike VSWW oil/brine results, variation in shape and boundary conditions for gas recovery was not closely correlated by Lc [18]. Another significant difference was that efficiency of gas recovery was significantly higher than for recovery of refined oil[18]. Traditionally, most gas reservoirs are assumed to be very strongly water-wet. However, Wardlaw and McKeller, [19] on the basis of imbibition rate measurements concluded that bitumen deposits remaining after the maturation process cause reduction in water wetness.

Because of the extreme difference in viscosity ratios for air and liquid, imbibition rate will be dominated by the resistance to flow of liquid. Empirical correlation for variation in oil viscosity was given by $\mu_{o}^{\frac{4}{5}} \mu_{\text {air }}^{\frac{1}{5}}$. However the correlation generally showed more spread than for the correlation developed for oil/water systems. The production of gas is marked by very few sites of bubble production corresponding to low gas saturation and, consequently, high water saturation. The rate of gas production is affected to some extent by compression of gas during the course of imbibition [18].

Correlation of simulated imbition results was improved by including the mobility in the definition of dimensionless time. The expression for $t_{D}[20]$ becomes;

$$
t_{D}=t \sqrt{\frac{k}{\phi}} \frac{\sigma}{L_{c}^{2}} \sqrt{\lambda_{r w}^{*} \lambda_{r n w}^{*}} \frac{1}{\sqrt{M^{*}+\frac{1}{\sqrt{M^{*}}}}}
$$


where $\lambda_{r}^{*}$ is a characteristic mobility and $M^{*}$ is a characteristic mobility ratio. In the limit, the gas viscosity was neglected and recovery of gas was scaled by $1 / \mathrm{u}_{\mathrm{o}}$. Experimental results for recovery of gas fell within the range of scaled data obtained for recovery of oil from diatomite.

Whatever scaling is used, there is agreement that a remarkable feature of normalized recovery versus time plots, for very strongly water-wet media, is that they are similar in shape. This feature has provided strong incentive to attempt correlation (alignment of) imbibition data by the product of time and some multiplying factor. In a simulation study of varying the parameters of $t_{D}$, close-to-constant shapes of oil recovery versus time curves were obtained using measured imbibition capillary pressure data and relative permeabilities for VSWW conditions $[21]$.

\section{Initial water saturation}

The condition of zero initial water saturation is frequently adopted because it is the most convenient. However, the presence of an initial connate water content of very low mobility in hydrocarbon reservoirs is a key parameter in reservoir engineering. [22] The effect of initial water saturation is not readily predictable, even qualitatively. High initial water saturation results in reduced capillary pressure but increased mobility of the invading water. Results of Hamon and Vidal [12] (initial water saturations in the range 16\% to $18 \%$ ) correlated with results for zero initial water saturation for sandstones and synthetic media(see Fig III-1.4 ). Further experiments on variation in connate water for sandstone and chalk showed definitive trends but imbibition rates could be somewhat higher or lower than those for zero initial water saturation. The variation either way was well within an order of magnitude. [16]

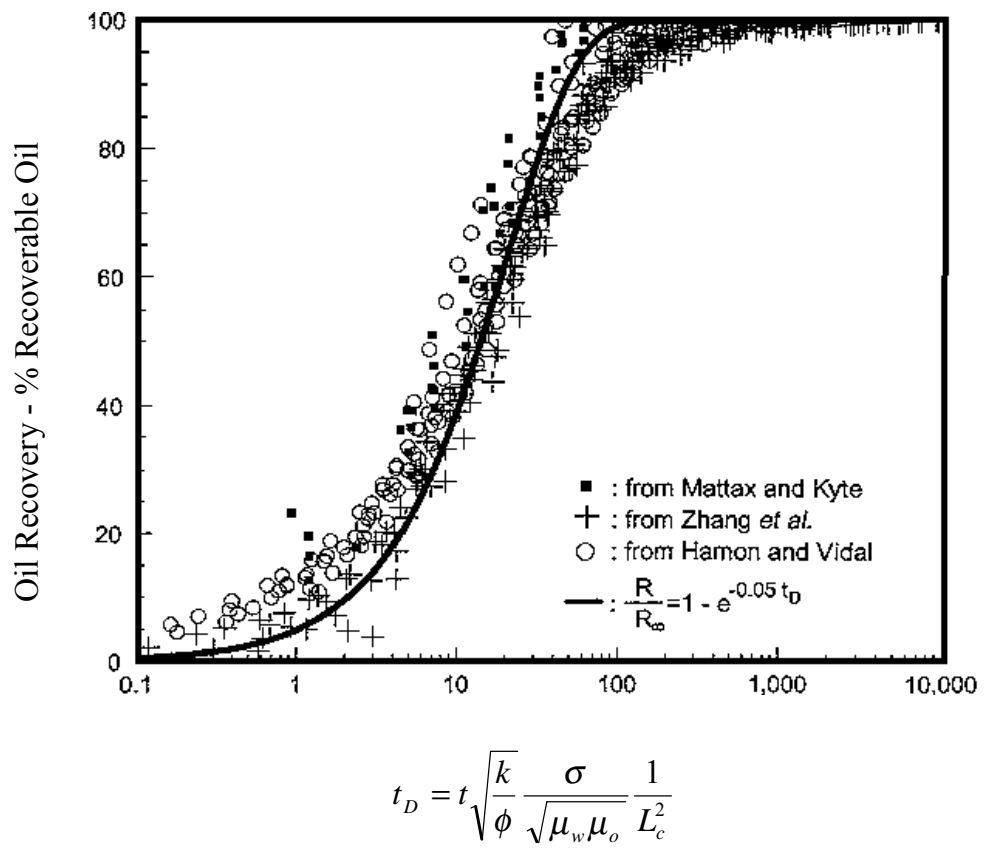

Figure III-1.4. Normalized oil recovery versus dimensionless time for very strongly water-wet imbibition. 


\section{Co-current imbibition}

In the forgoing discussion it was concluded that imbibition occurred by counter current flow. Bourbiaux and Kaladjian [23] showed that countercurrent production of oil was small at the inlet face of the lower end of a $29 \mathrm{~cm}$ long sandstone core when the upper face was exposed to oil. Relative permeabilities were shown to depend on whether flow was co- or counter-current. Although counter-current conditions apply in most imbibition measurements, it has been suggested that cocurrent imbibition may be the dominant process in oil recovery from fractured reservoirs and that the recovery obtained is faster and the displacement efficiency is higher. $[24,25]$ The condition in the reservoir that leads to cocurrent imbibition is that there is gravity segregation of oil and water in the fractures, and only part of the matrix-block surfaces is exposed to water. When part of the rock surface is in contact with oil, it is concluded that oil will flow preferentially towards the boundary in contact with oil. Thus in addition to size, shape, and faces open to imbibition, the overlying phase in contact with a particular open rock face also needs to be specified. Further experiments are needed to test the relative contributions of cocurrent and countercurrent imbibition under various circumstances.

\section{Wettability and Imbibition}

Basic theory for the effect of wetting on imbibition rate and recovery efficiency provides useful background to the factors that determine rate and extent of oil recovery. Rate of imbibition into a cylindrical tube should scale according to the cosine of the contact angle, although, even for such a simple example, issues of static vs. dynamic angles may arise. The cosine of contact angle has often been proposed as a scaling factor in the correlation of capillary pressure and imbibition rate data. [26,27] This approach raises the problem of measurement of contact angles in porous media and expression of their likely variability. Aside from this, a broad range of phenomena involving wettability and its effect on capillary action in porous media, such as the effect of convergent/divergent pore throats and initial water saturation [28], have direct impact on imbibition.

In early studies of the effect of wettability on immiscible displacement, results were often obtained for very strongly water-wet and very strongly oil-wet conditions under the assumption that the results will span all possibilities between these two conditions. It is now recognized that the infinite number of possible wetting states between these two extremes can exhibit a rich variety of behavior.

\section{Wettability control}

Experimental studies of the wetting behavior of the surfaces of polymeric solids and deposited organic films for various liquids against air have provided a large body of reproducible contact angle data. Contact angles were shown to be systematically dependent on the surface tension of the liquid and the surface properties of the polymer. [29] 
Many investigations of wettability and its effect on oil recovery have involved adsorption of known compounds onto the surfaces of porous media. Qualified successes have been reported for this approach, but no single method of wettability control has been widely adopted as a standard technique from one laboratory to another.

It is now common practice to use adsorption from crude oil as a means of inducing changes in wettability. Oil samples must be free of contaminants such as well treatment additives used to mitigate corrosion, scaling, and emulsification. The main disadvantages of this approach are the complexity of crude oils, each being to some extent unique. There is an increasing tendency for investigators to share large samples. The overriding advantage of using crude oils is that the complex mechanisms by which wetting states are established and act on oil recovery mechanisms are much more likely be captured in laboratory studies.

\section{Uniformly wet porous media}

Air/liquid displacement studies have been made for porous media formed from polymeric solids for which contact angles of liquids against air at smooth surfaces are known (this type of wetting will be referred to as uniform wettability). Surface roughness can result in large increase in apparent advancing contact angles and, hence, have a major impact on the interface curvatures, which determine if and how imibibition will occur. Capillary pressure measurements showed that spontaneous imbibition of liquid against air will not occur if the contact angle is more than about $48^{\circ}$ for an initially dry media, or about $62^{\circ}$ if an initial liquid saturation is present [30]. Imbibition rate is slower than predicted from the cosine of the contact angle over these ranges. For uniform wettability, a wide range of intermediate wetting (62 to $133^{\circ}$ ) was observed for which neither phase displaced the other spontaneously.

Control of oil/brine wetting by adsorption of known chemicals has been used in studies of contact angle, relative permeability, and oil recovery. A recent example is the use of hexadecylamine [31] in imbibition studies.

Concentration, and number and conditions of treatment are used to establish variations in wettability. In nearly all of these types of treatment, the reagent solution has access to the rock surface and the general class of wetting alteration will be referred to as uniform. (Obviously there are still many possibilities for local variation such as mineralogy.) Treatment of a dry core by initially saturating the core with crude oil can also be classified as uniform wettability under this operational definition.

The results obtained for imbibition into porous media formed from polymeric solids suggest that spontaneous imbibition only occurs for relatively low contact angles. Of 55 contact angles measured for crude oil/brine/reservoir mineral combinations [32] 41 were greater than 62 degrees. [33] This implies that imbibition has distinct limitations as a possible recovery mechanism in many reservoir situations. However, imbibition measurements for COBR combinations described below suggest that spontaneous imbibition can occur over a broad range of wettability conditions induced by adsorption from crude oil. 


\section{Mixed wettability}

Salathiel [34] reported studies in which wettability was altered by adsorption from crude oil with the distinction that the porous medium contained an initial water saturation. From a combination of observations, it was concluded that those parts of the rock surface overlain by connate water were protected from adsorption. This form of wetting condition was described as mixed and is now the most widely accepted model of reservoir wettability. Mixed wettability (MXW) can be broadly defined to include any degree of wetting alteration at the parts of the rock surface exposed to crude oil. [1]

\section{MIXED WETTABILITY IN TUBES WITH CORNERS}

The basic idea of MXW is illustrated in Fig III-1.5 by a tube of triangular cross section with water in the tube corners.[35] Adsorption occurs on the area of the tube wall overlain by crude oil, but there is no adsorption on the area of solid surface overlain by bulk water. Even if the contact angle is high (MXW has been modeled as a combination of completely water-wet and oil-wet surfaces [36]), water will still spontaneously imbibe into the tube, to the condition where the corner menisci have zero curvature. Detailed analysis of the effect of contact angle on surface curvature and displacement in triangular tubes has been reported [37].

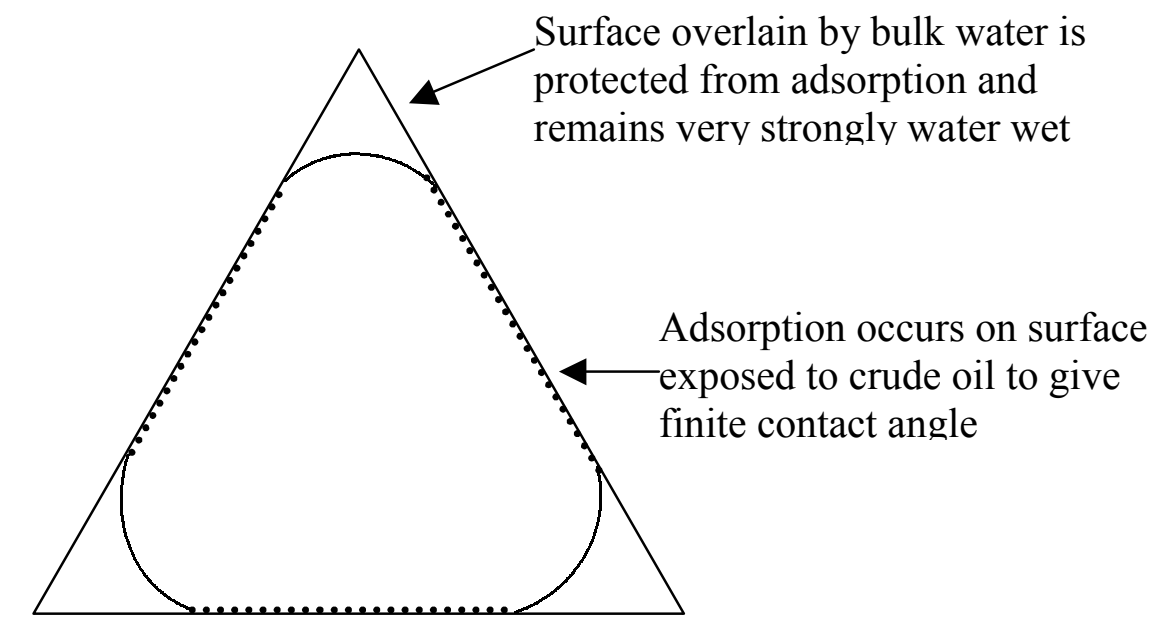

Figure III-1.5. Mixed wettability in a tube of triangular cross-section.

\section{CONTACT ANGLES AFTER ADSORPTION FROM CRUDE OIL}

Wetting change resulting from adsorption of polar components of crude oil onto a smooth solid substrate have been studied extensively through contact angle measurement by the captive bubble or Wilhelmy plate methods. The presence of brine and its composition has been shown to play a crucial role in the adsorption mechanism. Mineral surfaces are initially equilibrated with the brine. [38] The plate is drained and then submersed in oil with temperature and time of treatment being the most commonly varied parameters. This procedure is usually referred to as aging. Next, excess crude oil is removed by washing with a solvent such as toluene or decalin. Contact angles are then measured with a probe oil, usually decane, and distilled water or brine as 
the oleic and aqueous phases. The rationale for this approach was developed from numerous observations on contact angles measured after adsorption from crude oil. A wide range of contact angles has been reported according to the choice of oil and conditions of treatment. Mechanisms of adsorption include acid/base interactions, ion binding, and surface precipitation of crude oils $[39,40]$. Hysteresis is commonly observed, and has been ascribed, on the basis of atomic force microscopy, to heterogeneity with respect to surface coverage by adsorption [41]. For paraffinic crude oils with low asphaltene content, and correspondingly low acid and base numbers, hysteresis decreased with respect to successive cycles of measurement; contact angles measured after adsorption from asphaltic crude oils were distinctly more stable [42]. Selection of crude oils by their wetting behavior predicted from independently measured properties will assist in the development of generic accounts of COBR wetting behavior. A library of crude oils and their properties has been developed. [43]

\section{PREPARATION OF MIXED-WET CORES}

The brine (which originally filled the core) plays a key intermediary role in the mechanism of adsorption. Adsorption of heavy polar components from crude oil does not usually induce a strongly oil-wet state. The combined effects of variation in the composition, amount, and distribution of initial water saturation and the extent of wettability change resulting from adsorption leads to a broad variety of wetting conditions. Factors that can affect wetting changes in chalk have been investigated by Graue and coworkers [44].

Many aspects of the mechanism of adsorption are not well understood. Variation of aging time provides one method of obtaining systematic change in wettability while holding other variables constant (Fig. III-1.6) [1]. In some studies the crude oil is displaced by treatment with a mild solvent, which is in turn displaced by refined oil. Through this procedure, the excess crude oil is removed without incurring precipitation of asphaltenes. An adsorbed film of heavy polar components still remains on the rock surface where it had been exposed to the crude oil during aging $[44,45,46]$. Other convenient methods of wettability control include the variation of aging temperature and initial brine saturation $[44,47,48]$.

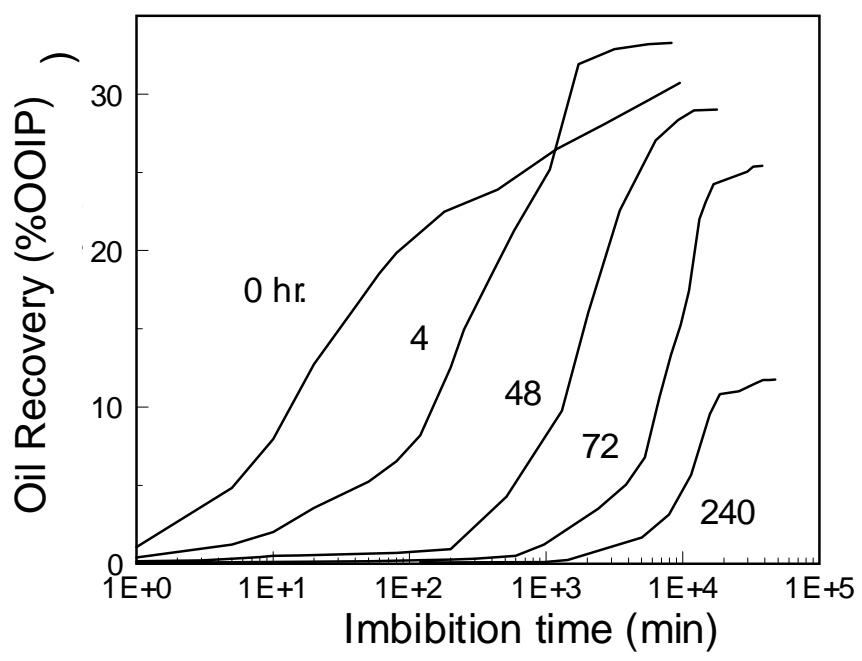

Figure III-1.6. Examples of change in spontaneous imbibition with change in time of aging with an asphaltic crude oil at $15 \%$ initial water saturation [1]. 


\section{RESERVOIR CONDITIONS TESTING}

In displacement tests for oil recovery operations of special economic interest, attempts are made to reproduce the conditions of the reservoir [49]. In restored state tests this includes core cleaning, reestablishing the reservoir brine saturation, and use of live fluids at reservoir pressure, temperature, and overburden pressure. Such tests are technically difficult and very expensive. The aging time should be sufficient to match the adsorption equilibrium reached by the reservoir over geologic time. There is always uncertainty as to whether this is achieved. In practice aging times are usually in the range of one to six weeks. Imbibition tests related to specific reservoirs are often run at reservoir temperature (sometimes with an upper limit of 80 degrees C) with dead crude oil at ambient pressure or even with refined oil [50].

Results of restored state tests were compared with measurements on preserved samples (sometimes referred to as fresh cores). Further comparisons between the two types of measurements are needed [51]. There is strong interest in determining the key parameters in wetting behavior so that laboratory testing can be designed to give meaningful results without being overly complex and costly.

\section{MEASUREMENT OF WETTABILITY}

Although contact angle measurements have bearing, they cannot, for example, reflect the role of the initial water saturation, a key parameter of MXW. Several operational definitions of the wettability of COBR combinations based on displacement tests have been proposed. The Amott test is often used in evaluation of oil recovery by spontaneous imbibition from fractured reservoirs [52]. Amott observed that for cores at an initial water saturation, some fraction of the in place oil is produced by spontaneous imbibition. Additional oil could be recovered by forced imbibition either by centrifuging or, in a later modification, by waterflooding [50]. A wettability index with respect to water, $I_{w}$, was defined by the ratio of the spontaneous increase in water saturation to the total increase.

After achieving residual oil saturation by forced displacement, cores were also tested for spontaneous imbibition of oil followed by measurement of additional oil recovery by forced displacement. The ratio of oil imbibed spontaneously to the total displacement of oil gave a wettability index to oil, $I_{0}$. The difference between the water and oil indices, $I_{w}-I_{0}$, gives an overall wettability index, $\mathrm{I}_{\mathrm{w}-\mathrm{o}}$.

The use of relative displacement efficiency, rather than the rate of spontaneous imbibition, to characterize wettability is not obvious from basic considerations of wetting. For the cylindrical tube model, liquid either fills the tube or it does not enter at all. The Amott index to water is either unity or zero. The rate of filling is directly proportional to the cosine of the contact angle, the fundamental measure of wetting, but in this simple example, the Amott index is unity for all contact angles from zero up to 90 degrees. Similarly, for porous media, the Amott index to water will be unity for any condition of water wetness that gives a residual oil saturation that is robust with respect to further waterflooding. Thus, an Amott index to water of unity does not necessarily mean that the porous media is very strongly water-wet in the sense that spreading conditions or even very low contact angles prevail. There has been an increasing tendency to measure recovery rate during the spontaneous imbibition steps of the Amott test. Imbibition 
curves for strongly water-wet conditions provide a basis for estimating the effect of wettability on the measured imbibition rates $[53,54,55]$.

\section{EXAMPLES OF OIL RECOVERY FOR MIXED WETTABILITY}

Experimental procedures for imbibition tests at MXW conditions are inherently complicated and somewhat arbitrary. Imbibition rates can be several orders of magnitude slower and displacement efficiencies range from barely measurable to significantly higher than obtained for VSWW conditions [54,56]. MXW imbibition curves exhibit considerable variation in shape. Sometimes very little or no imbibition is observed for a period described as the induction time.[1] This phenomena is not understood but is probably related to the time required for water paths to be established and/or for very slow changes towards water wetness that result from exposing the sample to brine. At extremely low imbibition rates, the estimated capillary pressure driving imbibition is of the same order as core-scale buoyancy forces. The mode of expulsion of crude oil from the surface of a core changes significantly with wettability. [57]

Oil recoveries for MXW have been reported for variations in time and temperature of aging, temperature of measurement, initial water saturation, oil and brine compositions, and sample shape, size, and boundary conditions [1,58,59]. Imbibition rate and extent generally decreased with increase in aging time, and aging temperature. MXW can exhibit great sensitivity to initial water saturation. Results presented in Fig III-1.7 show scaled imbibition rates. Results for VSWW conditions vary by much less than an order of magnitude over the range of $10 \%$ to $30 \%$ initial water saturation. The rate of spontaneous imbibition for MXW decreased by up to several orders of magnitude when initial water saturation was varied from only 15 to $30 \%$ [58]. (At very low rates of recovery, the contribution of buoyancy forces comes into play.) Clearly, connate water saturation has a dominant influence on reservoir wetting and hence recovery behavior. This is consistent with field observations [60].

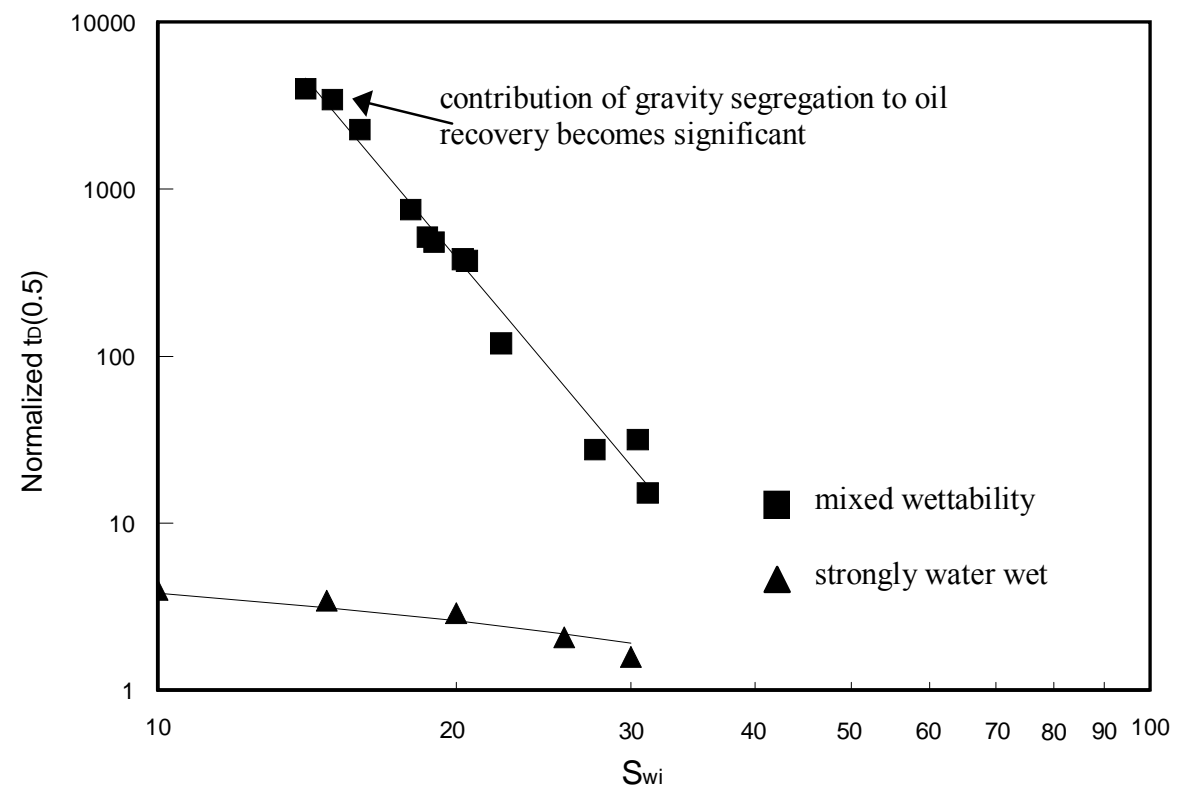

Figure III-1.7. Normalized dimensionless time for $50 \%$ of ultimate recovery versus connate water saturation. Imbibition rate is highly sensitive to initial water saturation for mixed-wet conditions [58]. 
Tests of other variables need to be made with initial water saturations held close to constant. Correlation of results by $\mathrm{L}_{\mathrm{c}}$ for variation in sample size shape and boundary conditions, tested for very narrow ranges of initial water saturation were satisfactory suggesting that countercurrent imbibition was still the dominant recovery mechanism [58].

Brine composition, through its effect on COBR interactions can have significant effect on rate and extent of spontaneous imbibition [48,54,55,61]. In field applications, injected brine is generally of different composition to reservoir connate brine and some mixing will occur during imbibition. Tracer studies show that connate water in unfractured chalk is displaced to form a bank ahead of the injected brine [62]. However, for fractured chalk, 10\% PV retention of connate water was observed [63]. The extent to which banking of connate water reduces the effect of invading brine composition on oil recovery by countercurrent imbibition for MXW is still an open question.

Even more complexities arise in imbibition processes that occur during the thermal recovery of viscous (heavy oil) from fractured reservoirs. Once the injected steam lowers the oil viscosity, recovery of oil results from thermal expansion and imbibition of condensed water from the fractures into the rock matrix. Laboratory experiments and reservoir simulations designed to capture the essential features of this process have been reported. Scaling of the effects of nonisothermal and dynamic (as expressed by flow rate in fractures) conditions and the injection of polymers has also been taken into consideration $[64,65,66,67,68]$.

SCALING OF SPONTANEOUS IMBIBITION FOR MIXED-WET CONDITIONS.

The correlation of imbibition data for VSWW conditions is mainly based on results for zero initial water saturation. Results for crude oil at zero initial water saturation are of much less interest as a reference for MXW imbibition because of the importance of initial water distribution and the key role played by brine in the COBR interactions that control adsorption. VSWW results at zero and finite initial water saturations provide the main basis for assessing the effect of wetting on displacement rate and recovery efficiency.

Because of the variation in overall imbibition curve shapes, the effect of differences in wettability cannot be correlated using a simple wettability factor in the expression for dimensionless time. The main objective in scaling results for MXW is to adjust the results for the factors that appear in the definition of $t_{D}$ without explicitly including a term for wettability. Remaining differences in imbibition rate and displacement efficiency between VSWW results and MXW imbibition can then be ascribed to wettability. Characterization of wettability by spontaneous imbibition tests is of special value in delineating degrees of water wetness that give high values of Amott index to water and are often identified as very strongly water wet.

\section{Large-Scale Laboratory Models and Imbibition Mechanisms}

Investigation of oil recovery by imbibition from large-scale (relative to core plugs) laboratory models is a topic in its own right. Many studies have dealt with recovery from 
strongly water-wet rocks (zero initial water saturation) mounted in a variety of ways with respect to overall geometry and location of fractures. Large-scale models can also demonstrate the effect of buoyancy forces. Firoozabadi and co-workers have obtained recovery data for large models and then matched production data by simulation. In one such study [69], injection conditions were set such that the level of injected water in the fracture increased with time. Variation of water level in the fractures could be taken into account in mass transfer models by multiplying the transfer flux by the square root of the fracture saturation.

Graue and co-workers [70,71,72] applied nuclear tracer techniques to monitoring in situ saturation changes with special attention to recovery from chalk. In one of several studies of the combined effect of fracture geometry and wettability, oil recovery from blocks of strongly waterwet chalk were compared with that for moderately water-wet, and near neutral-wet chalk prepared by treatment with crude oil. Initial water saturations were in the range of 26 to $29 \%$. The blocks were then cut into three pieces and reassembled with artificial fractures of set spacing between the blocks. The presence of fractures and differences in wettability were shown to produce distinct differences in the mechanism of water advance and the displacement efficiency within the individual blocks separated by the fractures.

Baldwin and Spinler [73] used magnetic resonance imaging to monitor in situ increase in water saturation as a function of initial water saturation and wettability. For VSWW and zero initial water saturation, the water saturation fronts were very steep with no further production of oil from the invaded region. At 15\% Swi the invasion was also frontal (Fig. III-1.8a) With increase in initial water saturation, the imbibition front became more diffuse with continued recovery of oil from behind the front. At high initial water saturation (33\%) increase in water saturation was essentially global (Fig. III-1.8b). For a moderately water-wet plug (Amott index of 0.5 ) the mode of saturation increase was also global even though the initial water saturation was only $15 \%$ (Fig III-1.8c). 


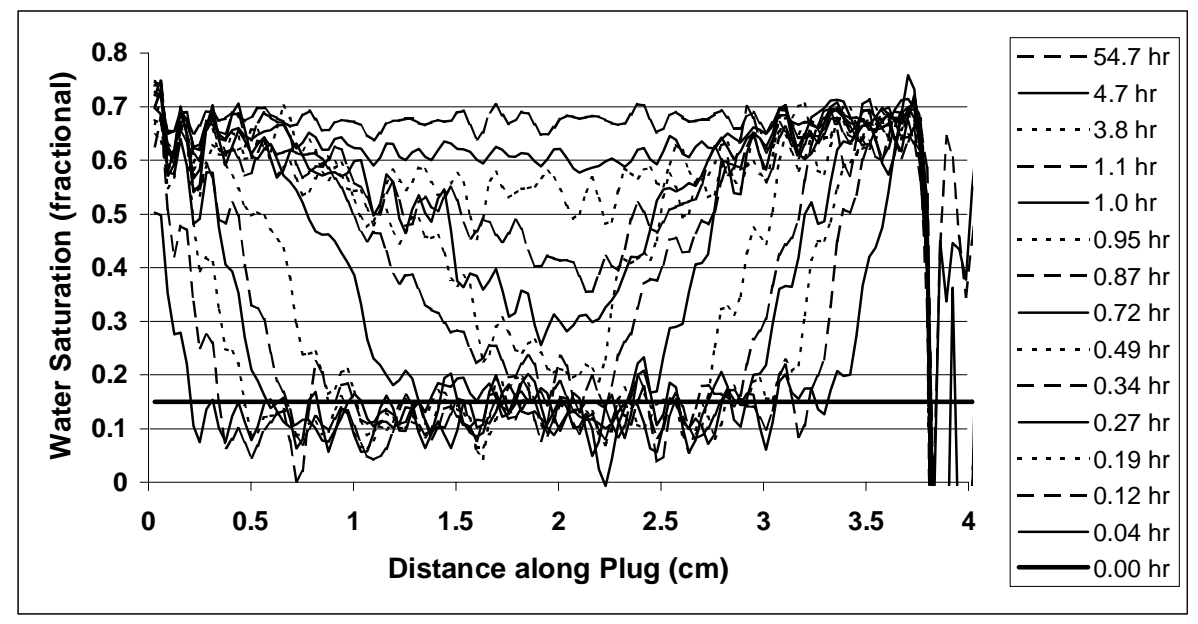

(a) Frontal displacement shown by vertical profiles during imbibition, VSWW,

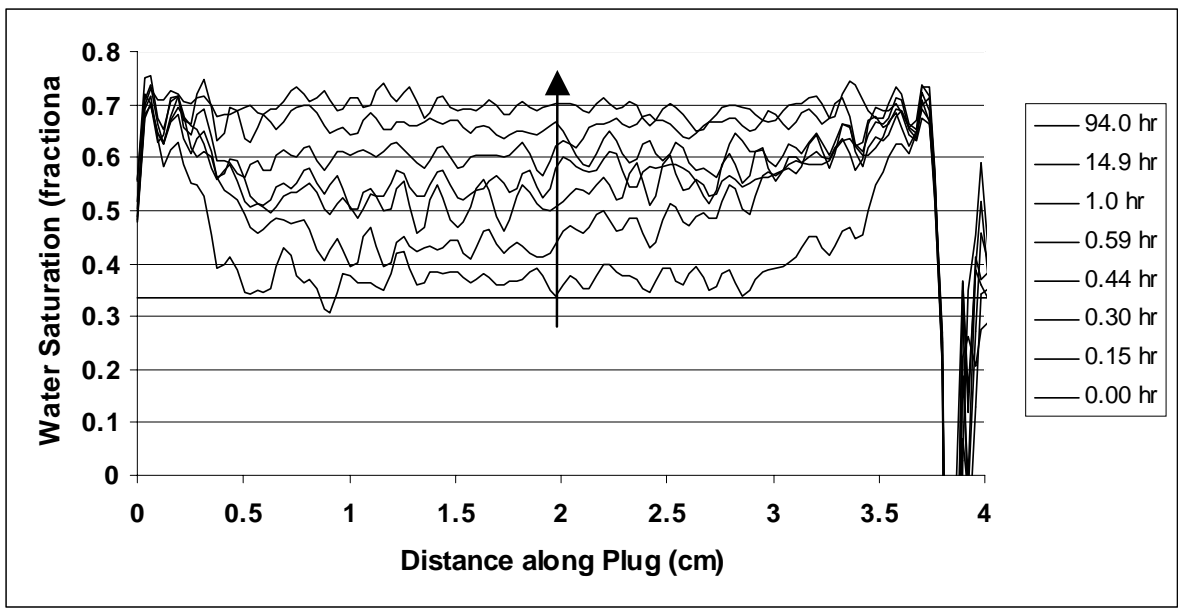

(b) Global displacement shown by horizontal profiles during imbibition, VSWW, Swi=33

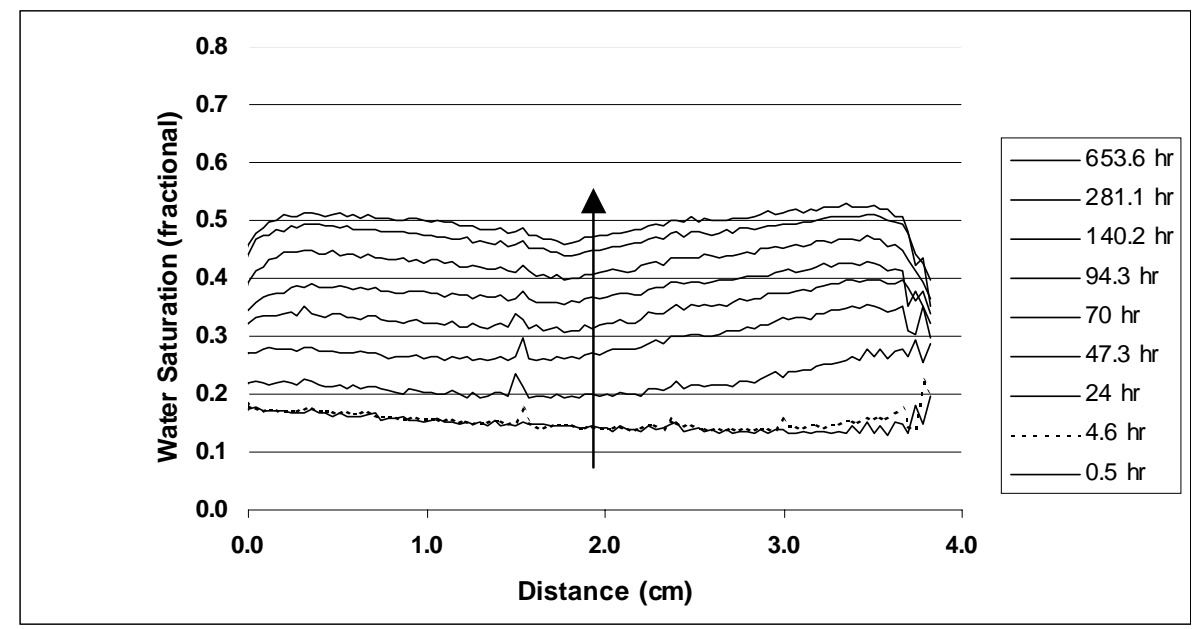

(c) Global displacement shown by horizontal profiles during imbibition, weakly water wet,Swi $=15 \%$

Figure III-1.8. Dependence of mode of imbibition (frontal vs. global) on initial water saturation and wettability. [73]. 


\section{Imbibition of Surfactant Solutions}

Recovery of oil by injection of surfactants solutions was originally aimed at mobilization of oil trapped by capillary action during water floods. The objective was to lower the interfacial tension drastically so that viscous forces, even at field flow rates of about $1 \mathrm{ft} / \mathrm{d}$, were sufficient to mobilize the trapped oil. The oil was commonly assumed to be trapped at very strongly waterwet conditions as individually isolated ganglia. The force for mobilization of a blob was then proportional to the difference between drainage and imbibition capillary pressures [74]. Much research focused on preparing surfactant solutions that minimized the interfacial tension against oil. Interfacial tension reductions of up to six orders of magnitude were reported. Technical and economic problems have curtailed most activity in this area.

Over about the last decade, there has been growing interest in use of surfactants to enhance recovery of oil from fractured reservoirs. [75,76,77,78,79,80,81] In many of these studies emphasis is placed on changing wettability towards increased water wetness so that imbibition is enhanced. In contrast to surfactant flooding, reduction of interfacial tension by the surfactant will decrease the imbibition rate and should be minimized. The reduction of interfacial tension must be more than compensated by $\mathrm{COBR} /$ surfactant interactions, which make the rock sufficiently, water-wet for imbibition to occur at economic rates. Marangoni forces that arise because of interfacial tension gradients may also contribute to the recovery mechanism. Increase in the ratio of gravity to capillary forces and the role of connate water saturation will also come into play [82].

Austad and coworkers $[76,77,78]$ made a series of detailed studies with special emphasis on recovery of acidic crude oils from chalk. Comparison of cationic, anionic, and nonionic surfactants showed cationic to be the most effective, and of these CTAB 12 was the most effective. The surfactant concentration needs to be held above the critical micelle concentration. In the proposed mechanism CTAB forms a complex with adsorbed carboxylates, which then dissolves in the oil phase. Removal of the adsorbed carboxylates from the carbonate surface was shown to render the surface more water-wet, as determined from both enhanced imbibition rates and from contact angle measurements at smooth surfaces.

Increased recovery of crude oil was reported for a field test on a dolomite reservoir. One discouraging laboratory observation was that expulsion of oil, apparently driven partly by surface tension gradients, was followed by re-imbibition of oil into the matrix [79]. The pressing need for improved fundamental understanding of MXW imbibition clearly extends to the use of surfactants to promote oil recovery by imbibition and gravity drainage in fractured reservoirs.

\section{Concluding Remarks}

Research on the role of imbibition in oil recovery spans half a century. The current rate of publication in this area is at an all-time high. Many questions of practical importance have, as yet, far from complete answers. Technical interest is likely to continue for the coming years with emphasis on low permeability fractured reservoirs. Imbibition studies aimed at prediction and improvement of oil recovery are often reservoir specific. The challenge of developing a 
consistent account of the evolving patterns of MXW imbibition behavior is now well recognized and is likely to remain the focus of research for some time to come.

Improved predictive capabilities will come from further parametric studies and advances in basic understanding of how wetting changes induced by crude oil determine the imbibition mechanism. Further advances in classification of crude oils according to their ability to induce wetting changes are expected. In advancing knowledge of COBR interactions, work on sandstones and chalks needs to be expanded to a broader range of rock types.

Many of the experimental observations on oil recovery by imbibition require explanation. Insight into displacement mechanisms is provided by increasingly versatile deterministic network models (84-88). There is growing interest in the development of rules for pore level displacement that apply to multiphase flow. The models include dual occupancy of fluids in individual pores at VSWW, uniform, and MXW. Application of such models to spontaneous imbibition will lead to improved understanding of displacement mechanisms and predictive capability. Advances in understanding pore scale mechanisms are also likely to follow from further observations of spontaneous imbibition in single capillaries [89,90] and laboratory micromodels (two dimensional pore networks).

On a larger scale, continued work on imaging saturation profiles developed during imbibition is essential to development of a more complete understanding of imbibition mechanisms and the superimposed effects of gravity segregation. Simulation of imbibition recovery rate and efficiency, and the saturation profiles which develop during the course of oil recovery, from independently measured relative permeability and capillary data will continue to serve as a rigorous test of the applicability and predictive capability of classical reservoir engineering mechanics.

\section{References}

1. Zhou, X, Morrow, NR and Ma, S: Interrelationship of wettability, initial water saturation, aging time and oil recovery by spontaneous imbibition and waterflooding. SPE Journal, June 2000, 5, 2:199-207. For tabulated summary see: SPE/DOE 35436 presented at the SPE/DOE $10^{\text {th }}$ Symposium on Improved Oil Recovery, April 21-24, 1996, Tulsa OK.

2. Washburn, EW: The dynamics of capillary flow. Phys. Rev., 1921, 17:273-283.

3. Mason, G and Morrow, NR: Capillary behavior of a perfectly wetting liquid in irregular triangular tubes. J. Coll. Inter. Sci., Jan. 1991, 141:262-74.

4. Morrow, NR and Xie, X: Surface energy and imbibition into triangular pores. In Characterization and Measurement of the Hydraulic Properties of Unsaturated Porous Media, Edited by vanGenuchten, Leij \& Wu, the Univ. of CA, Riverside CA; 1999:109-120.

5. Dong, $M$ and Chatzis, I: The imbibition and flow of a wetting liquid along the corners of a square capillary tube. J. Colloid Interface Sci., 1995, 172, 2 
6. Morrow, NR: Physics and thermodynamics of immiscible displacement in porous media. Ind. Eng. Chem. (1970) 62:32-56. Reprinted as a chapter in Flow Through Porous Media, American Chemical Soc. (1970) 104-28.

7. Leverett, MC: Flow of oil-water mixtures through unconsolidated sands. Trans. AIME, 1939, 132:151-169.

8. Mason, G: Porous materials and percolation theory. In Characterisation of Porous Solids edited by K.K. Unger et al., Elsevier, Amsterdam, 1988:323-332.

9. Mattax, CC and Kyte, JR: Imbibition oil recovery from fractured, water-drive reservoir. SPE Journal, June 1962, 177-84.

10. Aronofsky, JS, Masse, L, Natanson, SG: A model for the mechanism of oil recovery from the porous matrix due to water invasion in fractured reservoirs. Trans., AIME, 1958, 213:17-19.

11. Kazemi, H, Gilman, JR and Eisharkawy, AM: Analytical and numerical solution of oil recovery from fractured reservoirs with empirical transfer functions. SPE Reservoir Engineering, May 1992, 7 (2):219-27.

12. Hamon, G and Vidal, J: Scaling-up the capillary imbibition process from laboratory experiments on homogeneous and heterogeneous samples. SPE 15852, presented at the 1986 European Petroleum Conf. of SPE, London.

13. Ma, S, Zhang, $\mathrm{X}$ and Morrow, NR: Influence of fluid viscosity on mass transfer between rock matrix and fractures. $J$. Can. Pet. Tech., (July 1999) 38, (7) 25-30.

14. Zhang, X, Morrow, NR and Ma, S: Experimental verification of a modified scaling group for spontaneous imbibition. SPE Reservoir Evaluation November, 1996, 11 (4) 280-285

15. Ma, S, Morrow, NR and Zhang, X: Generalized scaling of spontaneous imbibition data for strongly water-wet systems. J. Pet. Sci. and Eng., September 1997,614, 1997.

16. Viksund, BG, Morrow, NR, Ma, S, Wang, W, Graue, A: Initial water saturation and oil recovery from chalk and sandstone by spontaneous imbibition. Proceedings of the 1998 International Symposium of Society of Core Analysts, The Hague, September 1998.

17. Kantzas, A, Ding, M, \& Lee, J: Residual gas saturation revisited. SPE 59782, presented at the 2000 SPE/CERI Gas Technology Symposium, Calgary, Alberta, Canada, April 2000

18. Wang, R: Gas recovery from porous media by spontaneous imbibition of liquid. Master's Thesis, Dec. 1999, University of Wyoming

19. Wardlaw, NC and McKellar, M: Wettability and connate water saturation in hydrocarbon reservoirs with bitumen deposits. JPSE, 1998, 20:141-146 
20. Zhou, D, Jia, L, Kamath, J and Kovscek, AR: Scaling of counter-current imbibition processes in low-permeability porous media. presented at the $6^{\text {th }}$ International Symposium on Reservoir Wettability and its effect on oil recovery, Sept. 2000, Socorro, NM.

21. Ruth, D, Morrow, NR, Li, Y, and Buckley, JS: A simulation study of spontaneous imbibition. Proceedings of the International Symposium of the Society of Core Analysts Annual Meeting, Oct. 2000, Abu Dhabi, UAE.

22. Morrow, NR and Melrose, JC: Applications of Capillary Pressure Data to the Determination of Connate Water Saturation," in Interfacial Phenomena in Oil Recovery, Ed. N.R. Morrow, Marcel Dekker (Oct. 1990).

23. Bourbiaux, B and Kalaydjian, FJ: Experimental study of cocurrent and countercurrent flows in natural porous media. SPE Reservoir Engineering, 361; Trans. AIME, , Aug. 1990, 289.

24. Pooladi-Darvish, $M$ and Firoozabadi, A: Cocurrent and countercurrent imbibition in a water-wet matrix block. SPE Journal, March 2000, 5, 1:3-11.

25. Firoozabadi, A: Recovery mechanisms in fractured reservoirs and field performance. $J$. Can. Pet. Tech, Nov. 2000, 39 (11) 13-17.

26. Gupta, A and Civan, F: An improved model for laboratory measurement of matrix to fracture transfer function parameters in immiscible displacement. SPE 28929 presented at the SPE $69^{\text {th }}$ Annual Conference and Exhibition, Sept. 25-28, 1994, New Orleans, LA.

27. Guo, B, Schechter, DS and Baker, RO: An integrated study of imbibition waterflooding in the naturally fractured Spraberry. SPE 39801, presented at the SPE Permian Basin Oil and Gas Recovery Conference, March 25-27 $7^{\text {th }}$, 1998, Midland, TX.

28. Melrose, JC: Wettability as related to capillary action in porous media. SPE Journal, Sept. 1965, 259.

29. Zisman, WA: Relation of the equilibrium contact angle to liquid and solid constitution. Chapter 1 in Contact Angle, Wettability, and Adhesion, Adv. in Chem. Ser. 43, F.M. Fowkes, Symposium Chairman, Washington, D.C., ACS, 1964:1-51.

30. Morrow, NR and McCaffery, FG: "Fluid displacement studies in uniformly wetted porous media. in Wetting, Spreading and Adhesion, Ed. J.F. Padday, Academic Press, New York (1978) 289-319.

31. Kowalewski, E, Holt, T and Torsaeter, O: Wettability alterations due to an oil soluble additive. Proceedings of the $6^{\text {th }}$ International Symposium on Reservoir Wettability and its Effect on Oil Recovery, Sept. 2000, Socorro, NM.

32. Treiber, LE, Archer, DL and Owens, WW: A laboratory evaluation of the wettability of fifty oil-producing reservoirs. SPE Journal, Dec. 1972, 531-540. 
33. Morrow, NR: Capillary pressure correlations for uniformly wetted porous media. $J$. Can. Pet. Tech., 1976, 15, 49.

34. Salathiel, RA: Oil recovery by surface film drainage in mixed-wettability rocks. J. Pet. Tech., Oct. 1973, Trans. AIME, 255:1216-24

35. Buckley, JS, Takamura, K and Morrow, NR: Influence of electrical surface charges on the wetting properties of crude oils. SPEFE, August 1989, 332-340.

36. Kovscek, AR, Wong, $\mathrm{H}$ and Radke, CJ: A pore-level scenario for the development of mixed wettability in oil reservoirs. AIChE Journal, June 1993, 39, 6:1072-1085.

37. Ma, S, Mason, G and Morrow, NR: Effect of contact angle on drainage and imbibition in regular polygonal tubes. PS-1191, Colloids and Surfaces, A: Physicochemical and Engineering Aspects, 1996, 117:273-291.

38. Buckley, JS and Morrow, NR: An overview of crude oil adhesion phenomena. In Physical Chemistry of Colloids and Interfaces in Oil Production, Edited by H. Toulhoat and J. Lecourtier, Éditions Technip, Paris, 1992:39-45.

39. Buckley, JS and Liu, Y: Some mechanisms of crude oil/brine/solid interactions. J. Pet. Sci. Eng., 1998, 20:155-160.

40. Buckley, JS., Liu, Y, and Monsterleet, S: Mechanisms of wetting alteration by crude oils. SPE Journal, March 1998, 3:54-61.

41. Yang, S-Y, Hirasaki, GJ, Basu, S and Vaidya, R: Mechanisms for contact angle hysteresis and advancing contact angles. J. Pet. Sci. and Eng., Dec. 1999, 24, 2-4:63-73.

42. Xie, X, Morrow, NR, Buckley, JS: Contact angles hysterisis and stability of wetting changes induced by absorption from crude oil, $6^{\text {th }}$ International Symposium on Evaluation of Reservoir Wettability and Its Effect on Oil Recovery, Socorro, NM, Sept. 2000.

43. Buckley, JS and Wang, J: Crude oil characterization for prediction of wetting alteration. Proceedings of the $6^{\text {th }}$ International Symposium on Reservoir Wettability and its Effect of Oil Recovery, Sept. 2000, Socorro, NM.

44. Graue, A, Viksund, BG, and Baldwin, BA: Reproducible wettability alteration of lowpermeable outcrop chalk. SPE Reservoir Eng. and Eval., April 1999, 14 (2) 134-140.

45. Rathmell, JJ, Braun, $\mathrm{PH}$, and Perkins, TK: Reservoir waterflood residual oil saturation from laboratory tests. J. Pet. Tech, Feb. 1973, 175-85; Trans., AIME 255.

46. Morrow, NR, Lim, HT, and Ward, JS: Effect of crude oil induced wettability changes on oil recovery. SPE Formation Evaluation, April 1986, 1:89-103.

47. Jadhunandan, $P$ and Morrow, NR: Effect of wettability on waterflood recovery for crude oil/brine/rock systems. SPE Reservoir Engineering, Feb. 1995, 40-46. 
48. Jadhunandan, P and Morrow, NR: Spontaneous imbibition of water by crude oil/brine/rock systems. In Situ, 1991, 15, 4:319-45.

49. Anderson, WG: Wettability literature survey-Part 1: Rock/oil/brine interactions and the effects of core handling on wettability. J. Pet. Tech, Oct. 1986, 38, 11:1125-1144.

50. Cuiec, L: Evaluation of reservoir wettability and its effects on oil recovery. In Interfacial Phenomena in Oil Recovery, edited by NR Morrow, Marcel Dekker, Inc., New York City, 1991:319-375.

51. Cuiec, L: Wettability laboratory evaluation under reservoir conditions: a new apparatus. paper SCA 9529 presented at the 1995 Conference, San Francisco, Sept. 12-13.

52. Amott, E: Observations relating to the wettability of porous rock. Trans. AIME, 1959, 216:156-162.

53. Ma, S, Morrow, NR, Zhou, X and Zhang, X: Characterization of wettability from spontaneous imbibition measurements. J. Can. Pet. Tech., Special Edition 1999, 38, 13:56.

54. Tang, GQ and Morrow, NR: Effect of temperature, salinity and oil composition on wetting behavior and oil recovery by waterflooding. SPE Reservoir Engineering, Nov. 1997, 269-276.

55. Tang, GQ, Morrow, NR: Influence of brine composition and fines migration on crude oil/brine/rock interactions and oil recovery. J. Pet. Sci. and Eng., 1999, 24:99-111.

56. Iffly, R, Rousselet, DD and Vermeulen, JL: Fundamental study of imbibition in fissured oil fields. SPE 4102, presented at the $47^{\text {th }}$ Annual Fall Meeting of the SPE of AIME, Oct. 1972, San Antonio, TX.

57. Zhou, X, Torsæter, O, Xie, X, and Morrow, NR: The effect of crude oil aging time and temperature on the rate of water imbibition and long term recovery by imbibition. SPE Formation Evaluation, December 1995, 10 4:259-265.

58. Xie, X, and Morrow, NR: Oil recovery by spontaneous imbibition from weakly waterwet rocks. Proceedings of the International Symposium of the Society of Core Analysts Annual Meeting, Oct. 2000, Abu Dhabi, UAE (In press Petrophysics, Aug., 2001).

59. Viksund, BG, Hetland, S, Graue, A and Baldwin, BA: Imaging saturation during flow in fractured chalk: Emphasizing recovery mechanisms, capillary continuity and scaling. Proceedings of the 1996 International Symposium of the Society of Core Analysts, Montpellier, France.

60. Hamon, G: Field-wide variations of wettability. SPE 63144, presented at the 2000 SPE Annual Technical Conference and Exhibition, Dallas, TX, Oct. 2000.

61. Morrow, NR, Tang, G, Valat, M and Xie, X: Prospects of improved oil recovery related to Wettability and Brine Composition. J. Pet. Sci. and Eng., June 1998, 20:267-276. 
62. Graue, A, Moe, R and Bognoe, T: Impacts of wettability on oil recovery in fractured carbonate reservoirs. Proceedings of the International Symposium of the Society of Core Analysts, Oct. 2000, Abu Dhabi, UAE.

63. Spinler, EA and Maloney, DR, Movement of connate water during water injection in fractured chalk. Proceeding of the International Symposium of the Society of Core Analysts, Oct. 2000, Abu Dhabi, UAE.

64. Babadagli, T, Al-Bemani, A and Boukadi, F: Analysis of capillary imbibition recovery considering the simultaneous effects of gravity, low IFT, and boundary conditions. SPE 57321, presented at the 1999 SPE Asia Pacific Improved Oil Recovery Conference, Kuala Lumpur, Malaysia, Oct. 1999.

65. Babadagli, T: Temperature effect on heavy-oil recovery by imbibition in fractured reservoirs. J. Pet. Sci. and Eng. 1996, 14:197.

66. Babadagli, T: Heavy-oil recovery from matrix during thermal applications in naturally fractured reservoirs. In Situ, 1996, 20, No. 3:221.

67. Donmez, A: Investigation of the effect of temperature on heavy oil recovery by imbibition mechanism. SPE 37555, presented at the SPE International Thermal Operations \& Heavy Oil Symposium, Bakersfield, CA, Feb. 1997.

68. Chimienti, ME, Illiano, SN, and Najurieta, HL: Influence of temperature and interfacial tension on spontaneous imbibition process. SPE 53668, presented at the 1999 SPE Latin American and Caribbean Petroleum Engineering Conference, Caracas, Venezuela, April 1999.

69. Pooladi-Darvish, $M$ and Firoozabadi, A: Experiments and modeling of water injection in water-wet fractured porous media. J. Can. Pet. Tech., March 2000, 39, 3:31-42.

70. Graue, A, Viksund, BG, Baldwin, BA, Spinler, E: Large scale imaging of impacts of wettability on oil recovery in fractured chalk. SPE Journal, March 1999, 4, 1.

71. Viksund, BG, Eilertsen, T, Graue, A, Baldwin, B and Spinler, E: 2D-imaging of the effects from fractures on oil recovery in larger blocks of chalk. Proceedings of the International Symposium of the Society of Core Analysts, Sept. 1997, Calgary, Canada

72. Graue, A, Bogno, T, Baldwin, BA and Spinlet, EA: Wettability effects on oil recovery mechanisms in fractured reservoirs, APE 56672, presented at 1999 SPEATCE, Houstong, Tx, Oct. 3-6, 1999. In press for SPE RERVOIR ENG EVALE.

73. Baldwin, BA and Spinler, EA: In-situ saturation development during spontaneous imbibition. SCA 9922 proceedings of the SCA International Symposium, Aug. 1999, Golden, CO.

74. Melrose, JC and Brandner, CF: Displacement efficiency for oil recovery by waterflooding. J. Can. Pet. Tech., 1974, 13, 42. 
75. Spinler, EA, Zornes, DR, Tobola, DP and Moradi-Araghi, A: Enhancement of oil recovery using a low concentration of surfactant to improve spontaneous and forced imbibition in chalk. SPE 59290, presented at the 2000 SPE/DOE Improved Oil Recovery Symposium, Tulsa, OK, April 3-5, 2000.

76. Austad, T, Matre, B, Milter, J, Saevereid, A and Oyno, L: Chemical flooding of oil reservoirs. 8. spontaneous oil expulsion from oil- and water-wet low permeable chalk material by imbibition of aqueous surfactant solutions. Colloid and Surface A:

Physicochemical \& Engr. Aspects, June 1998, 137:117-129

77. Austad, T and Miller, J: Spontaneous imbibition of water into low permeable chalk at different wettabilities using surfactants. SPE 37236, presented at the International Symposium of Oil field Chemistry, Houston, TX Feb. 1997.

78. Standnes, DC, and Austad, T: Wettability alteration in low-permeable chalk. Mechanism for wettability alteration from oil-wet to water-wet using surfactants. proceedings from the $6^{\text {th }}$ International Symposium on Reservoir Wettability, Socorro, NM, Sept. 2000.

79. Chen, HL, Lucas, LR, Nogaret, LAD, Yang, HD and Kenyon, DE: Laboratory monitoring of surfactant imbibition using computerized tomography. SPE 59006, presented at the 2000 SPE International Petroleum Conference and Exhibition in Mexico, 1-3 February 2000.

80. Baldwin, BA and Spinler, EA: Enhancement of oil recovery using a low concentration of surfactant to improve spontaneous and forced imbibition in chalk. SPE 59290, presented at the 2000 SPE/DOE Improved Oil Recovery Symposium, Tulsa, OK, April 3-5, 2000.

81. Keijzer, PPM and de Vries, AS: Imbibition of surfactant solutions. SPE 20222, presented at the 1990 SPE/DOE $7^{\text {th }}$ Symposium on Enhanced Oil Recovery, Tulsa, OK, April 22-25, 1990.

82. Schechter, DS, Zhou, D and Orr, FM, Jr: Low IFT drainage and imbibition. Pet. Sci. \& Eng. 1994, 11, 283-300.

83. Spinler, EA and Baldwin, BA, Surfactant induced wettability alteration in porous media. in Surfactant Fundementals and Application in the Petroleum Industry, Edited by LL Schramm, Cambridge University Press, 2000, 159-202.

84. Blunt, MJ: Pore level modeling of the effects of wettability. SPE Journal, Dec. 1997, 2 , 494-510.

85. McDougall, SR and Sorbie, KS: The Impact of Wettability on Waterflooding: PoreScale Simulation. SPE RERVOIR ENG, Aug. 1995, 208-213.

86. Øren, P-E, Bakke, S and Arntzen, OJ: Extending predictive capabilities to network models. SPE Journal, Dec. 1998, 324-336.

87. Patzek, TW and Kristensen, JG: Shape-factor and hydraulic conductance in noncircular capillaries: II. two-phase creeping flow, JCIS, April, 2001, 236, 305-317. 
88. Hui, M-H and Blunt, MJ: Effects of Wettability on three-phase flow in porous media. $J$. Phys. Chem. B, 2000, 104:3833-3845.

89. Kim, E and Whitesides, GM: Imbibition and flow of wetting liquids in noncircular capillaries. J Phys. Chem. B, Feb. 6, 1997, 101, 6:855-863.

90. Spildo, K and Buckley, JS: Wetting alteration in square tubes. J. Pet. Sci. Eng. 2000, 24, 145-154. 


\title{
III-2. Theoretical Studies
}

\section{III-2.1. Simulation Study of Spontaneous Imbibition}

\begin{abstract}
Ruth, D., Morrow, N., Yu, L., and Buckley, J.: “A Simulation Study of Spontaneous Imbibition," paper SCA 0024, presented at the 2000 International Symposium of the Society of Core Analysts, Abu Dhabi, 18-22 Oct.
\end{abstract}

Imbibition is an important phenomenon in many displacement processes. In particular, the transfer of liquids between fissures and the matrix in fractured reservoirs is dominated by imbibition. Re-examination of published results and new experimental data has resulted in a modified version of the Mattax and Kyte time-scaling that includes variations in water-oil viscosity ratio, sample shape, and boundary conditions. The resulting semi-empirical scaling group can be compared with simulations based on the mechanics of two-phase flow with capillary pressure as the driving force. This paper presents the results of a simulation study of one-dimensional, counter-current imbibition in a strongly water-wet sample.

As a first step, the generalized correlation for production was history-matched using core and fluid properties for which imbibition results have been reported. Initial water saturation was assumed to be zero. A logarithmic form was assumed for the capillary pressure curve and Corey equations were assumed for the relative permeability curves. A close match of oil recovery was obtained between the experimental data and the simulated imbibition. The core and fluid properties were systematically modified to test sensitivities. Variations in permeability, porosity, sample length and diameter, and interfacial tension were scaled exactly by the simulation. For oil viscosities in the range covered by the experimental data, agreement was good but not exact. The study was then extended to investigate the effects of varying capillary pressure curve shape, relative permeability, and initial water saturation, properties not explicitly included in the scaling group. For these simulations, the basic shapes of the oil-production-versus-time curves were essentially preserved. Overall agreement with the correlation was satisfactory. The initial water saturation was varied between $0 \%$ and $30 \%$ and the production curves were normalized with respect to the mobile oil saturation. Saturation profiles are presented which illustrate the simulated effect of initial water saturation on the imbibition process.

\section{Introduction}

Displacement of oil by spontaneous imbibition of water can be the dominant recovery mechanism in low permeability, fractured reservoirs that are water-wet (Aronofsky et al., 1958; Warren and Root, 1963; Kazemi, et al., 1992). Spontaneous imbibition is driven by the surface energy of the reservoir. Imbibition is a highly nonlinear process and conditions under which the process can be scaled are still being explored.

Scaling groups developed by Rapoport (1955) were applied by Mattax and Kyte (1962) to the problem of oil recovery by spontaneous imbibition. The conditions under which the scaling group was applicable were very restrictive. Necessary conditions included negligible influence of gravity forces, identical sample shapes, boundary conditions, oil/water viscosity ratio, initial fluid saturations, and directly proportional capillary pressure relationships. Re- 
examination of published results (Mattax and Kyte, 1962; Hamon and Vidal, 1986) and additional experimental results lead to a semi-empirical scaling group (Ma et al. 1997). The following fit to an Aronofsky model will be referred to as the correlation:

$$
\frac{R}{R_{-}}=1-\frac{1}{\left(1+0.04 t_{D}\right)^{1.5}}
$$

where $R$ is the recovery, $R_{\infty}$ is the ultimate recovery, and

$$
t_{D}=t \sqrt{\frac{k}{\phi}} \frac{\sigma}{L_{C}^{2} \sqrt{\mu_{w} \mu_{o}}} .
$$

Here $t$ is time, $k$ is permeability, $\phi$ is porosity, $\sigma$ is interfacial tension, $L_{C}$ is a geometrical scaling factor (in the current paper, simply the length of the sample $L$ ), $\mu_{w}$ is the water viscosity and $\mu_{o}$ is the oil viscosity

The scaled results apply to very strongly water-wet media with zero initial water saturation. Changes in imbibition rate with initial water saturation at strongly water-wet conditions have also been reported (Viksund et al 1998). The resulting semi-empirical scaling groups and relative trends in imbibition rate with changes in initial water saturations provide a body of data that can be tested against simulations using the basic principles of two-phase flow. Blair (1964) stressed the value of using simulation to investigate imbibition phenomena and the need to test results against experiments. The difference with respect to standard simulations of two-phase flow is that capillary pressure rather then an imposed pressure gradient provides the driving force.

The first step in the simulation is to show that the experimental results can be matched using measured or otherwise realistic values of input data. Parameters that are included in the scaling group and other parameters such as the relative permeabilities of each phase can then be varied independently, to identify trends in imbibition behavior. The results are compared with experimental results for ranges of parameters covered by the experimental data.

\section{Formulation of the Numerical Simulator}

The simulator is based on a conventional, explicit, finite-difference scheme. The software program was developed by modifying a commercially available numerical code that is normally used to simulate forced displacement (injection of water to displace oil). The program was already designed to handle potential imbibition at the production face. The modifications, designed to simulate the case of a cylindrical core with one end open, were minor and consisted of setting the injected flow to zero and removing mathematical problems (zero divides) that resulted. Two features of the simulator allow simulation of imbibition processes: (1) The differencing scheme is "upstream." With reference to Fig. III-2.1, this means that the properties at the grid boundaries are taken to be the properties of the cell from which the fluid (either oil or water) is flowing. Because the flow is countercurrent, this means that oil-flow at a grid boundary 
is governed by properties to the left of the boundary while water-flow is governed by properties to the right of the boundary. There is nothing unique about this scheme; it is standard in many numerical simulators. (2) The properties of the imbibition face (the end of the sample that would normally be the production face) are determined by assuming that that face is fully contacted by water, that is, that the capillary pressure is zero. This results in the maximum water relative permeability (the upstream direction for the water at the sample face is outside the sample) and the minimum capillary pressure. The driving force is the difference in the capillary pressure. Because the grid block at the inlet face is originally at zero water saturation, this driving force is maximum at the beginning of the simulation.

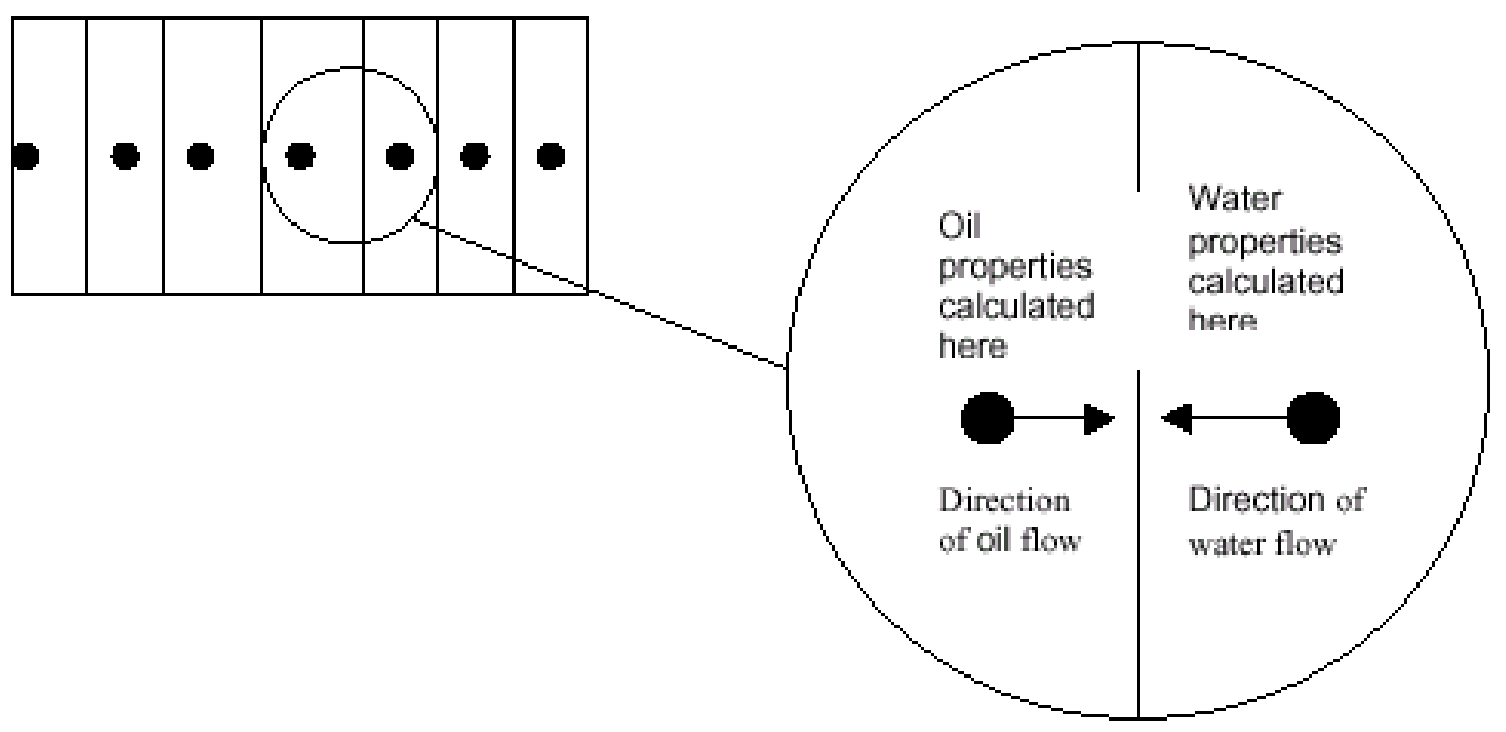

Figure III-2.1. The upstream differencing scheme.

\section{Test Parameter Specifications}

The present study uses a base-case system of parameters as listed in Table III-2.1. The parameters $\phi, k, S_{w r}$, and $S_{o r}$ are typical experimental values for Berea sandstone core. The relative permeabilities were assumed as Corey (1955) functions,

$$
\begin{aligned}
& k_{r o}=k_{r o x}\left(\frac{S_{o}-S_{r o}}{1-S_{r o}}\right)^{\mathrm{n}_{o}} \\
& \text { and } \\
& k_{r w}=k_{r w e}\left(\frac{S_{w}}{1-S_{r o}}\right)^{\mathrm{B}_{w}}
\end{aligned}
$$

with the end-point oil relative permeability set to 1.0. The capillary pressure curve was assumed to be logarithmic in form, and related to the threshold pressure by 


$$
P c=P c_{t}\left[1-B \ln \left(\frac{S_{w}}{1-S_{w}}\right)\right]
$$

with the $S_{w}=1-S_{r o}$ value given by the Leverett J-Function (1941) formulation

$$
P c_{r}=J \cos (\theta) \sqrt{\frac{k}{\phi}}
$$

with $J=0.15$.

Table III-2.1. Test Parameters

\begin{tabular}{|c|c|c|c|c|c|}
\hline Variable & Value & Units & Variable & Value & Units \\
\hline$\phi$ & 0.215 & - & $k$ & 600.0 & $\mathrm{mD}$ \\
\hline$L$ & 5.08 & $\mathrm{~cm}$ & $D$ & 3.18 & $\mathrm{~cm}$ \\
\hline$\mu_{o}$ & 0.97 & $\mathrm{cp}$ & $\mu_{w}$ & 0.97 & $\mathrm{cp}$ \\
\hline$k_{\text {roe }}$ & 1.0 & - & $k_{r w e}$ & 0.04 & - \\
\hline$n_{o}$ & 1.756 & - & $n_{w}$ & 2.018 & - \\
\hline$S_{o r}$ & 0.43 & - & $S_{w i}$ & 0.0 & - \\
\hline$\sigma$ & 50.0 & dynes/cm & $B$ & 5.255 & - \\
\hline$P c_{i}$ & 2.29 & $\mathrm{kPa}$ & & & \\
\hline
\end{tabular}

Base case viscosities for oil and water were both set at $0.97 \mathrm{cp}$. The core dimensions are typical of the laboratory samples. The first step in the study was to predict recovery versus time by applying the test parameter specifications to the correlation. Therefore, relative permeability and capillary pressure curves were chosen by setting $k_{r w e}=0.04$ (Braun and Holland, 1995) and optimizing values of $n_{o}, n_{w}$ and the capillary pressure curve shape $(B)$. The resultant values, included in Table III-2.1, gave the curves shown in Fig. III-2.2. These curves are typical of experimental data for Berea sandstone.

Productions predicted by the simulator for this base case, and by the correlation, are compared in Figure III-2.3a. This figure uses linear scales. Differences in the early time results between the simulation and the correlation for this base case simulation are apparent from the plots of recovery versus $\log t_{D}$ shown in Fig. III-2.3b. 


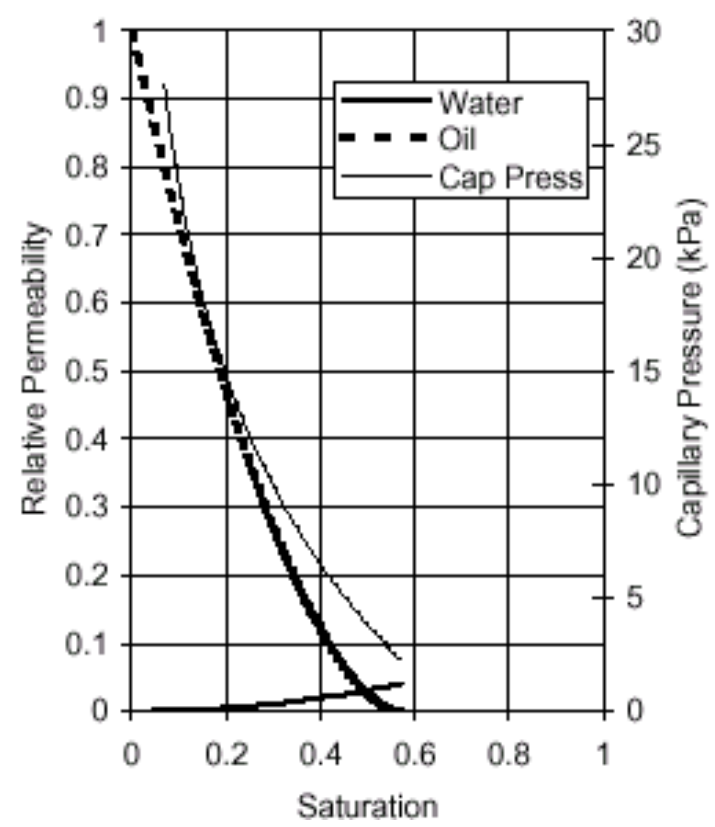

Figure III-2.2. Relative permeabilities and capillary pressure obtained by matching the water-wet correlation with base case parameters.

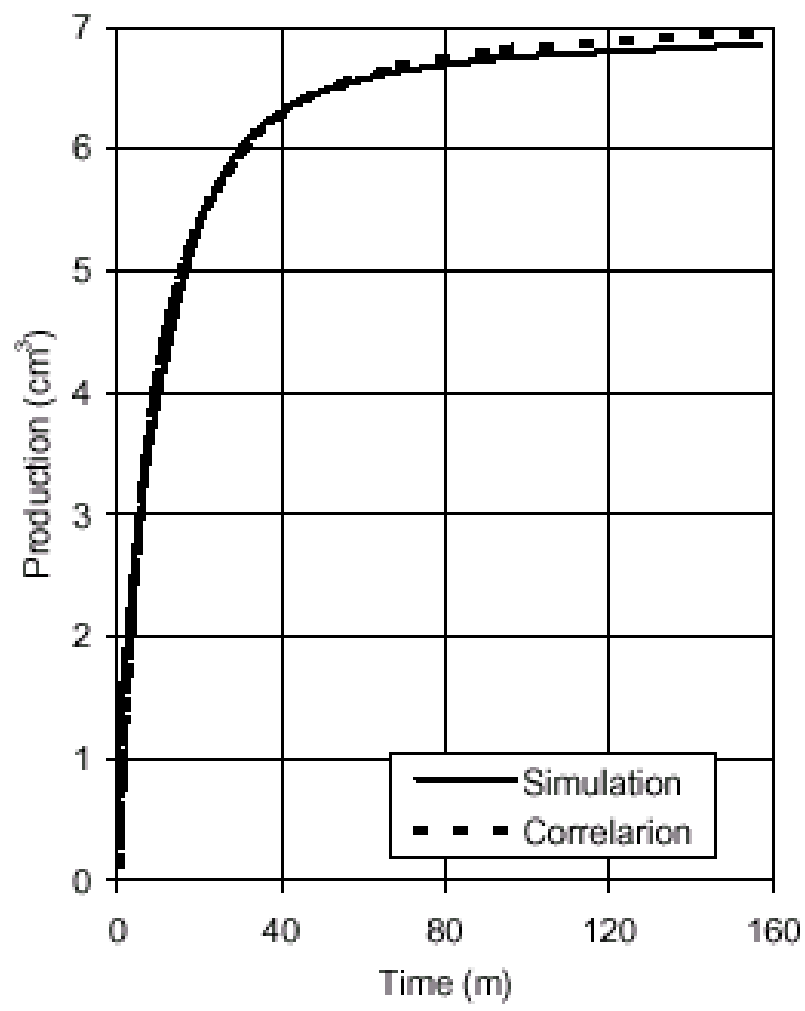

(a) production match

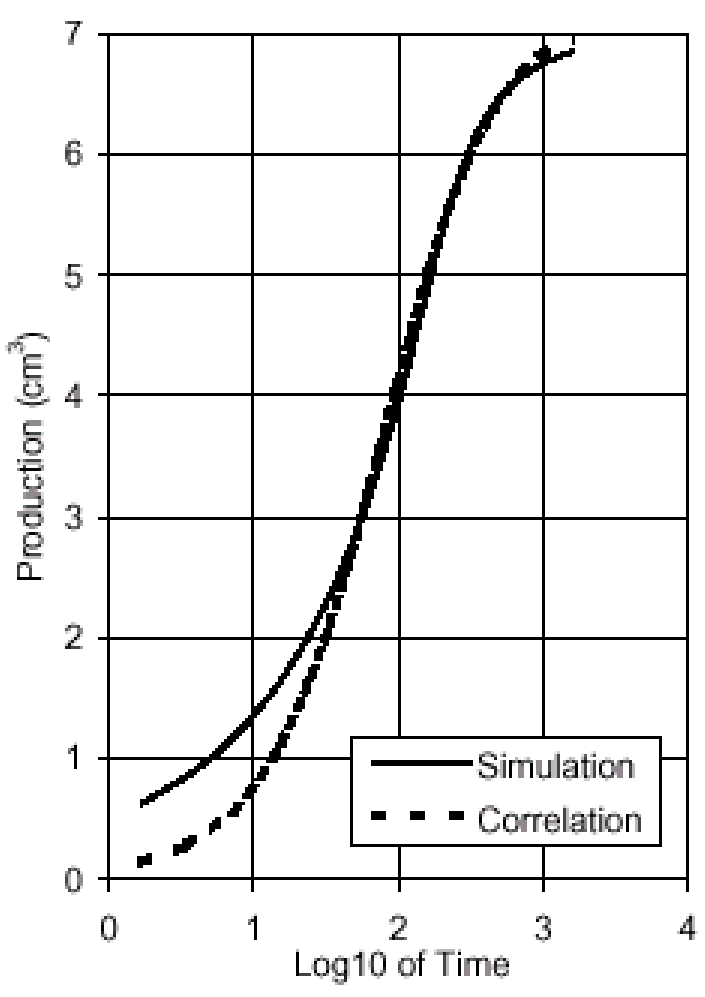

(b) history match

Figure III-2.3. Comparison of base-case simulation to the correlation. 
Inspection of experimental data from which the correlation was developed indicates that the early time differences could be reduced by modifying the one parameter Aronofsky equation (1958) used as a mathematical model of the correlation. An example of an improved oneparameter fit to the correlated recovery for late time data was used by Viksund et al 1998. Obviously an improved fit to the data could be obtained by increasing the number of fitting parameters. However, the early time differences may also be related to the time needed for water to establish imbibition paths within the rock. The simulator assumes that this will occur immediately. Furthermore, collection of oil that is recorded as production may be delayed if the displaced oil clings to the rock surface. However, for most practical purposes, the differences in the early time results are not important. These differences were observed for all simulations. The aim of the present study is to determine which of the mechanisms that influence imbibition are important, and to test the scaling group both within and outside the range of experimental data. Therefore, the sensitivity of the simulated results to various parameters will be presented with respect to the base case.

\section{Testing Parameters in the Scaling Group}

The second stage in the study was to vary systematically the parameters that appear in the scaling group $\left(k, \phi, \sigma, L, \mu_{o}\right.$, and $\left.\mu_{w}\right)$ and determine the amount by which the resulting simulated results deviated from the base-case-simulation. Not surprisingly, several of these parameters had no effect, giving results, which duplicated the base-case. These parameters were $k, \phi, L$, and $\sigma$. The values of $k$ tested ranged from $0.6 \mathrm{mD}$ to $6000 \mathrm{mD}$ and $\phi$ from 0.05 to 0.25 . $L$ and $\sigma$ were each changed by a factor of 2 . These results provided confirmation that the performance of the simulator is as expected.

A feature of the scaling group that is of special interest is the correlation of experimental results by the geometric mean of the oil and water viscosities. In experimental studies, the value of $\mu_{o}$ was varied from 0.97 to $156 \mathrm{cp}$ (Ma et al. 1999). There is close agreement between the base case and the simulated results for variations in $\mu_{o}$ in this range (see Figure III-2.4a). The results are consistent with simulations of imbibition reported by Blair (1964) for input data of significantly different character (for example imbibition capillary pressure that decreased continuously to zero at residual oil, and about one order of magnitude higher end point relative permeabilities to water) from those of the base case. From Fig. III-2.4a it is seen that there is divergence from the base case at later times. However, detailed inspection of the original data (Ma et al. 1999) for recovery of viscous oil did not provide any indication of this trend. When the values of $\mu_{o}$ were varied over 5 orders of magnitude, 0.01 to 1000 , the dimensionless time

predicted for displacement of low viscosity non-wetting phase is significantly longer than for the base case (Fig. III-2.4b). 


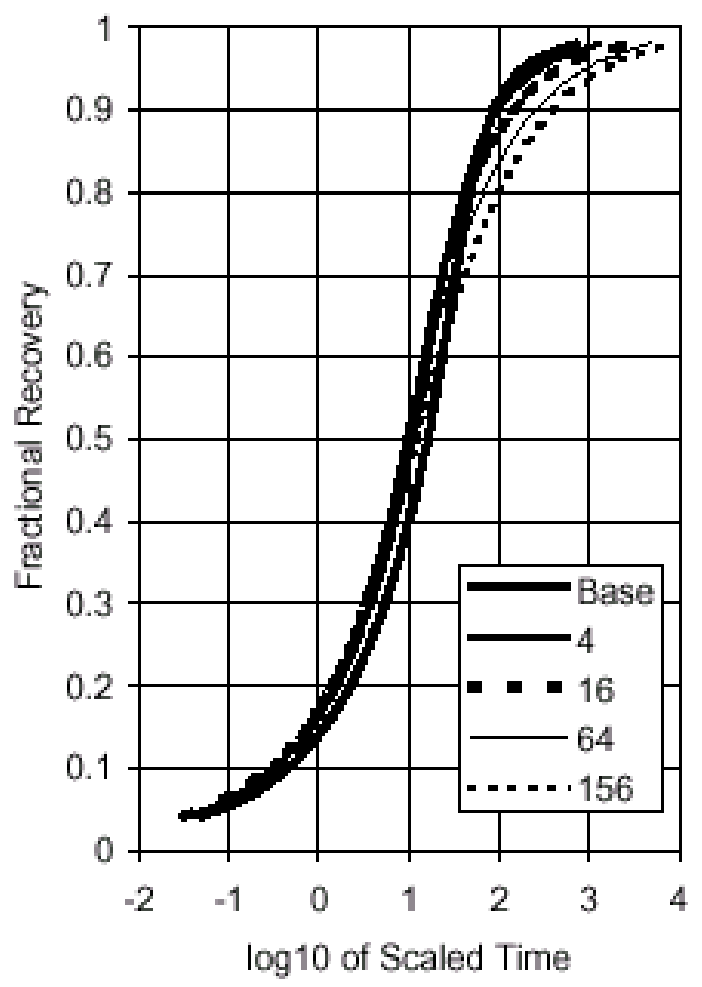

(a) comparison for the range of experimental observations

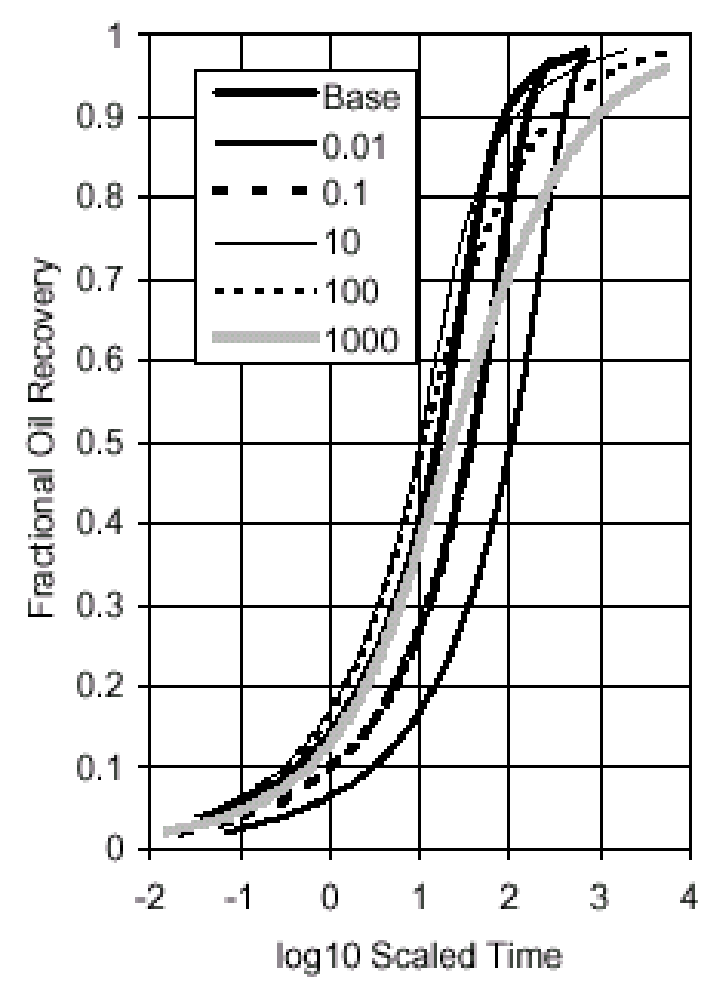

(b) model calculations over a wide range of viscosity ratios

Figure III-2.4. Effect of oil viscosity.

The last parameter tested was $\mu_{w}$. Preliminary experimental data showed little effect of wetting phase viscosity for $\mu_{w} 0.1$ to $15 \mathrm{cp}$. Varying $\mu_{w}$ in the simulation from 0.97 to $15 \mathrm{cp}$ also showed little deviation from the base case.

Experiments on displacement of gas $\left(\mu_{g} \approx 0.02 \mathrm{cp}\right.$ ) by imbibition of oil (oil is now the wetting phase) with viscosities in the range 1 to $162 \mathrm{cp}$ gave dimensionless times for imbibition that were much larger than predicted by the scaling group (Wang, 1999). The results of simulations with $\mu_{\mathrm{o}}$ in the range $1-162 \mathrm{cp}$ and a non-wetting phase of $0.02 \mathrm{cp}$ exhibited the same trends as the experiments (Fig. III-2.5). However, the rate of imbibition will have been affected by compressibility of the gas phase in the experiments and this is not taken into account in the version of the simulator used in the present study. This aspect of fluid properties and imbibition will be the subject of further study. 


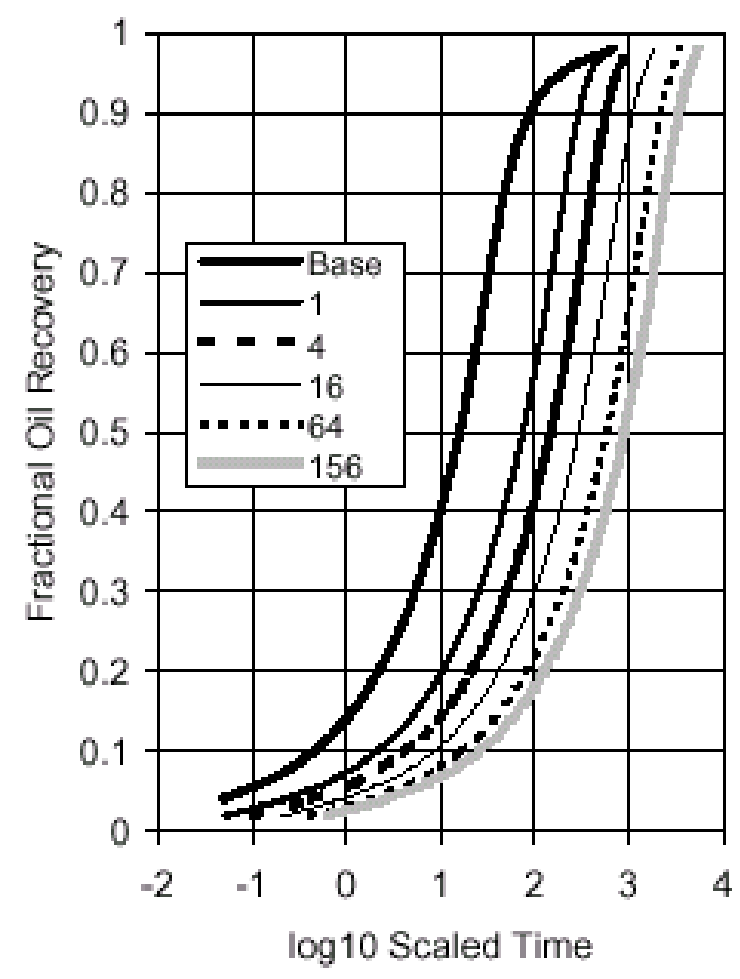

Figure III-2.5. Effect of invading phase viscosity.

\section{Testing Parameters Not Included in the Scaling Group}

A remarkable aspect of the scaling group is that many parameters such as the shape and end-point values of the relative permeabilities and the shape of the capillary pressure curve, that can be expected to affect the displacement processes, are not explicitly included in the correlation. As a test of their effect, variations in the relative permeability and capillary pressure curves were simulated.

\section{Capillary pressure}

Figure III-2.6 shows the effect of varying capillary pressure curve shape as expressed by $B$. Figure III-2.6a shows the capillary pressure curves considered and Fig. III-2.6b the resulting production curves. The values of $B$ used were 2.62, 3.93, 5.26 (base case), 6.55, and 7.86. The capillary pressure curve shape clearly has a systematic effect on the production. This is not surprising because the shape of the curve controls the derivative of $P c$ with respect to length; it is this derivative that determines the flow rate. However, with changes in $B$ of almost a factor of 4 , the change in $t_{D}$ is less than one order of magnitude. 


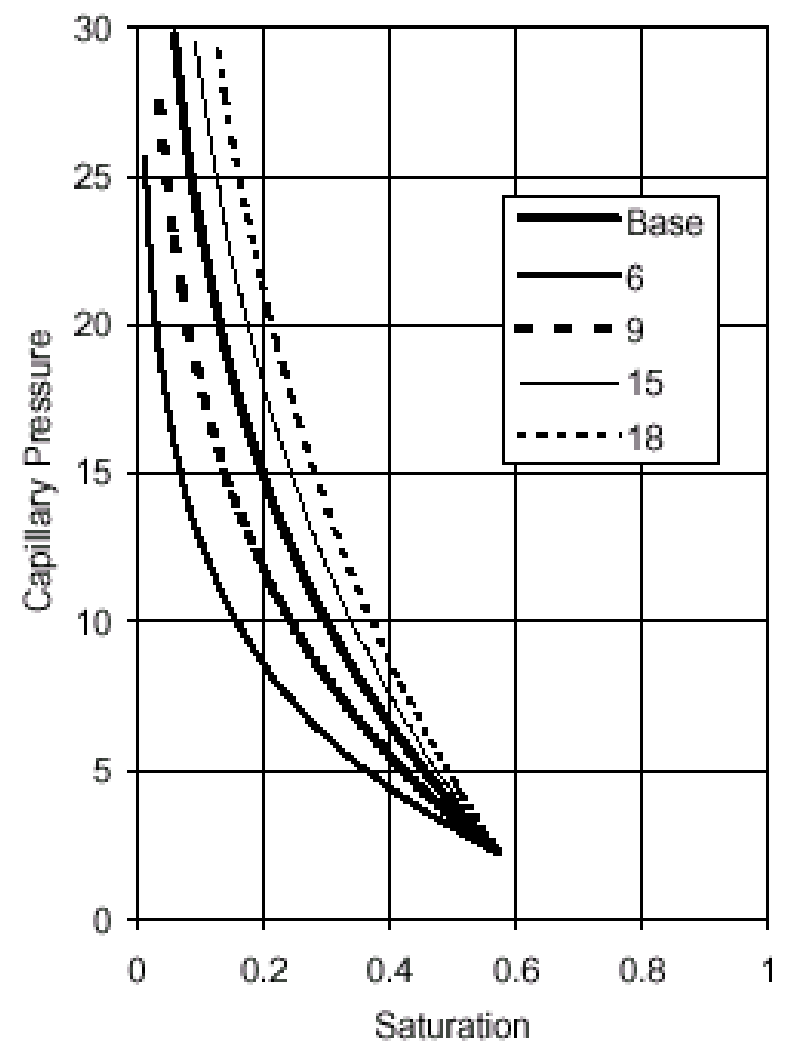

(a) $P c$ curves tested

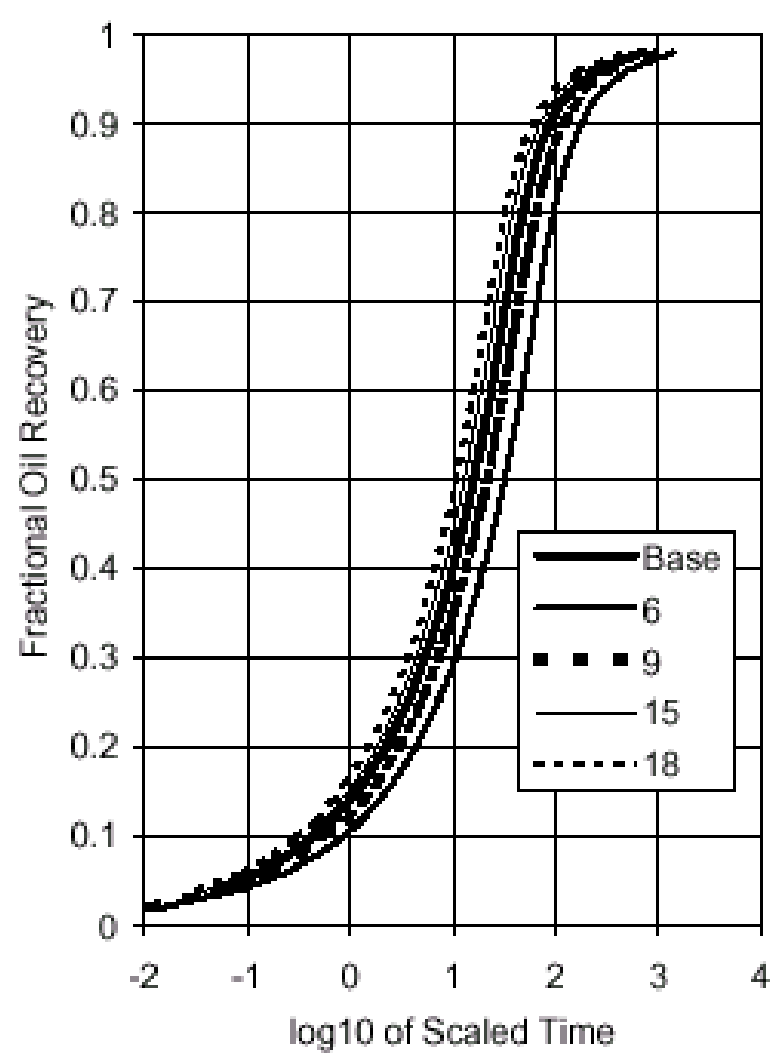

(b) results of varying shape of $P c$ curve

Figure III-2.6. Effect of the shape of $P c$ (with shape varies by varying $B$ ).

\section{Relative permeability}

Figure III-2.7 shows the effect of varying the end-point relative permeability, $\mathrm{k}_{\mathrm{rwe}}$, of the water phase. Here the tested values of $k_{r w e}$ were $0.02,0.04$ (base case), $0.08,0.16$, and 0.32 . The simulated curves deviate from the base curve by about an order of magnitude for a 16-fold variation in $k_{r w e}$. For water-wet rocks such as Berea, the end-point relative permeability to water should remain low. From one half to double the base case of $k_{r w e}=0.04$ the variation from the base case is minor.

Figure III- 2.8 shows the effect of varying the shape of the water relative permeability curve. The values of the exponent $n_{w}$ were 1.1, 1.756 (base case), 3.0, 4.0, and 5.0. For high values of $n_{w}$ the disagreement with the scaling group is apparent. However, the variation is less than an order of magnitude, even for this extreme range of shapes. Values of $n_{w}$ as high as 5.0 are well removed from the shape of water phase relative permeabilities for Berea sandstone. 


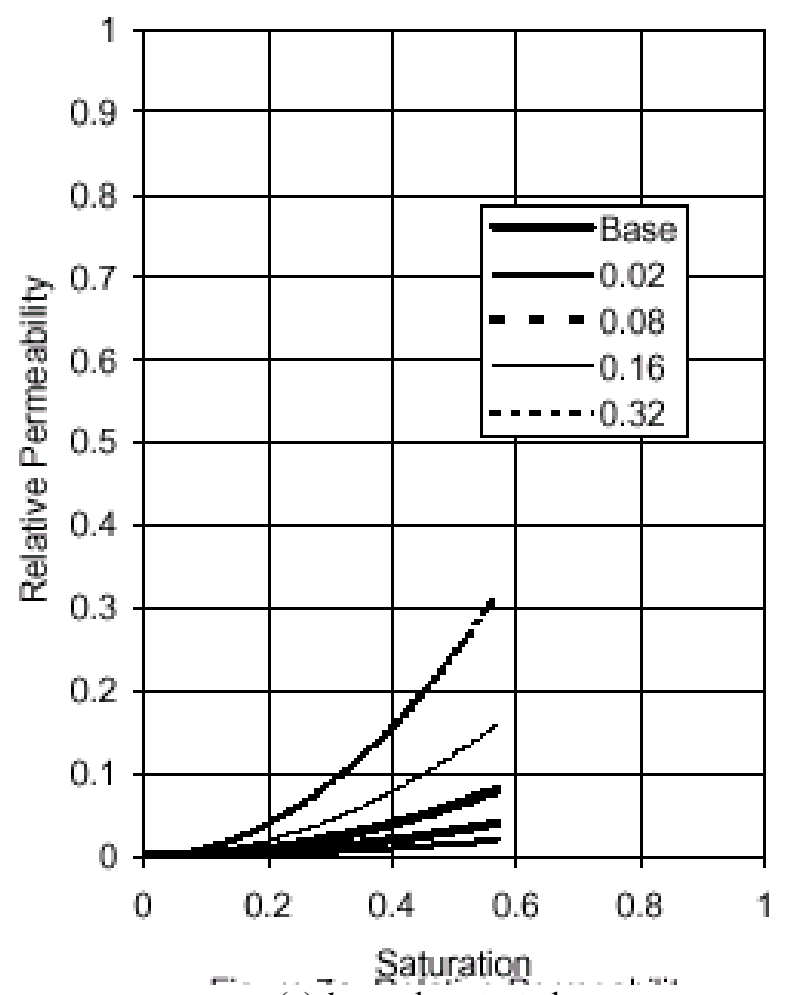

(a) $\vec{k}_{r w e}$ values tested

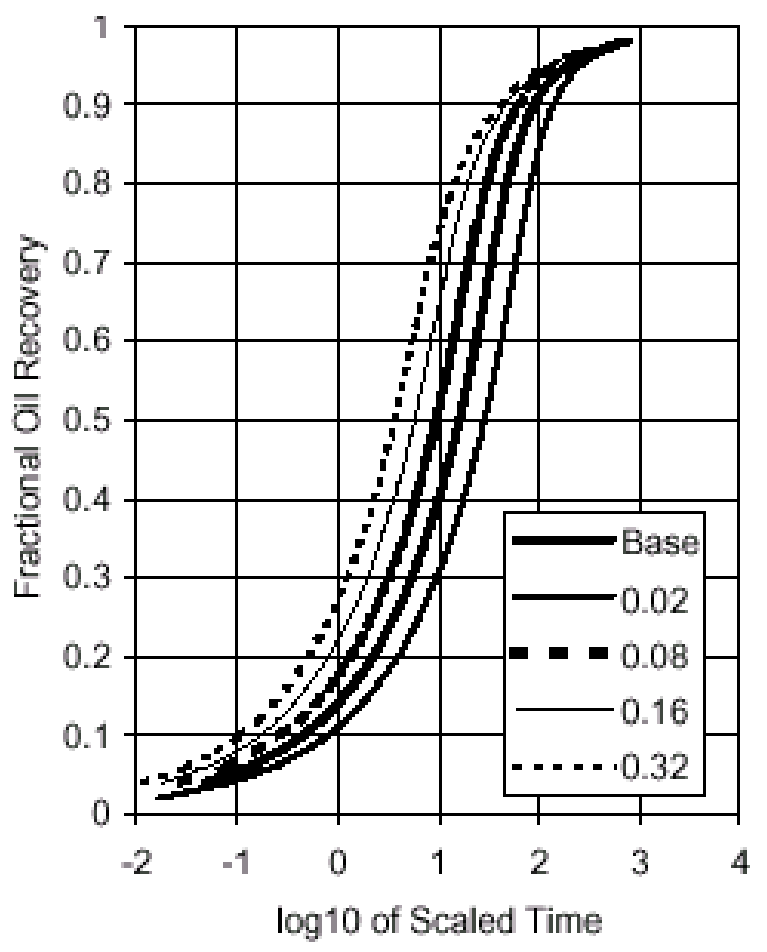

(b) results of varying $k_{r w e}$

Figure III-2.7. Effect of varying the end-point relative permeability to water, $\boldsymbol{k}_{r w e}$

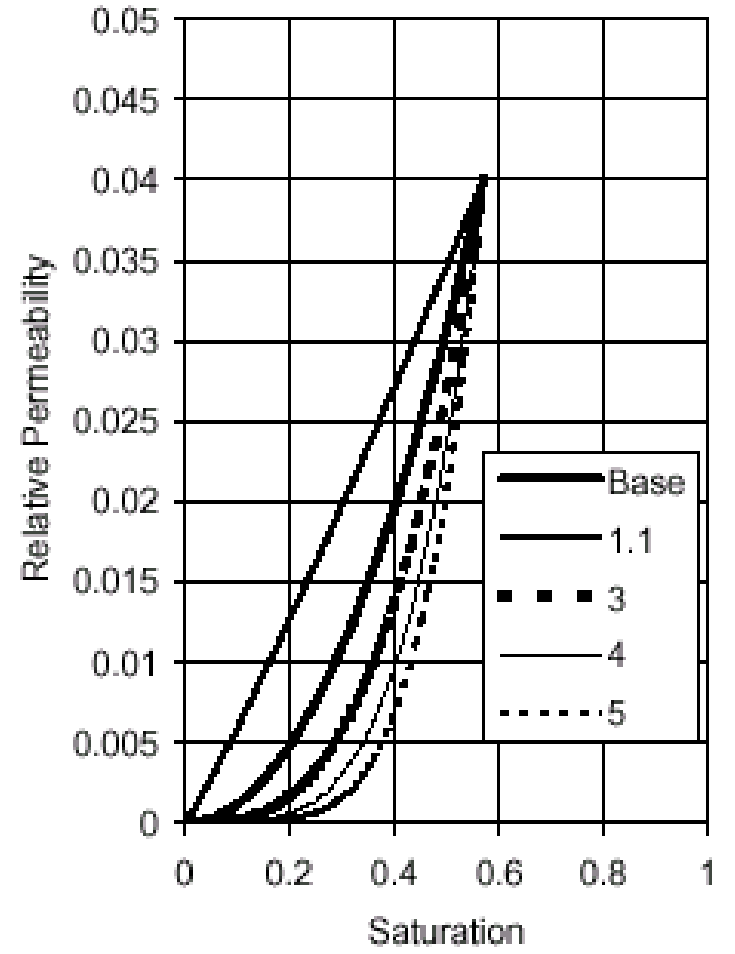

(a) values of $n_{w}$ tested

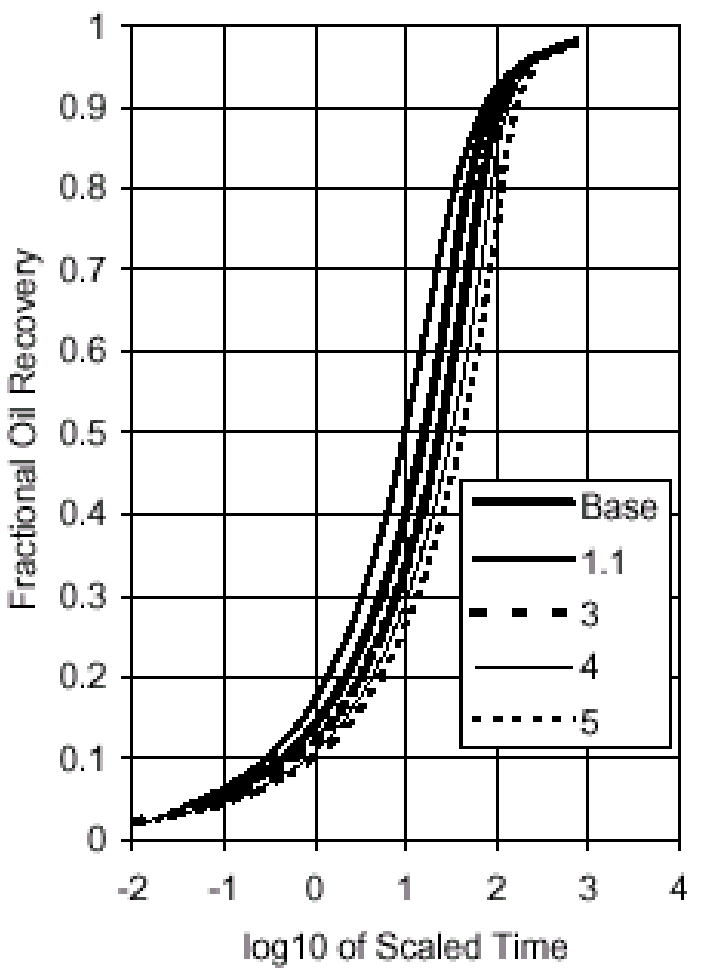

(b) results of varying $n_{w}$

Figure III-2.8. Effect of varying the shape of the water relative permeability curve by varying $n_{w}$. 
Figure III-2.9 shows the effect of varying the shape of the relative permeability curve to oil. The values of $n_{o}$ were 1.1, 2.018 (base case), 3.0, 4.0, and 5.0. The results group around the base case results. There is a systematic increase in $t_{D}$ with $n_{o}$ that is accentuated at higher values of $n$ and later times, and is obviously related to the extremely low permeabilities to oil as the water saturation increases.

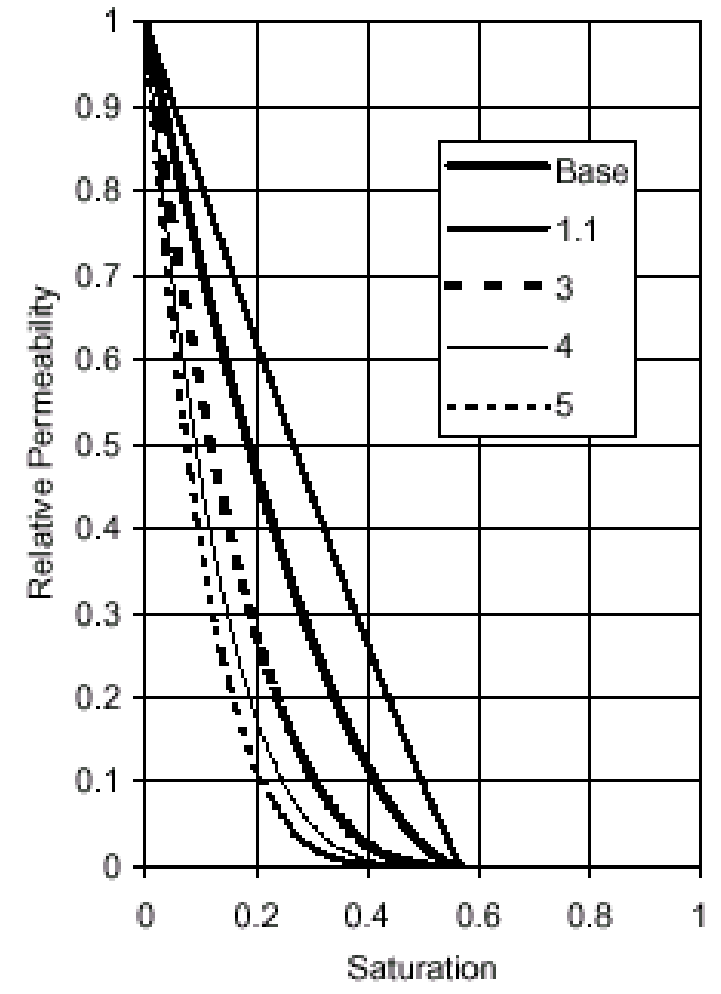

(a) values of $n_{0}$ tested

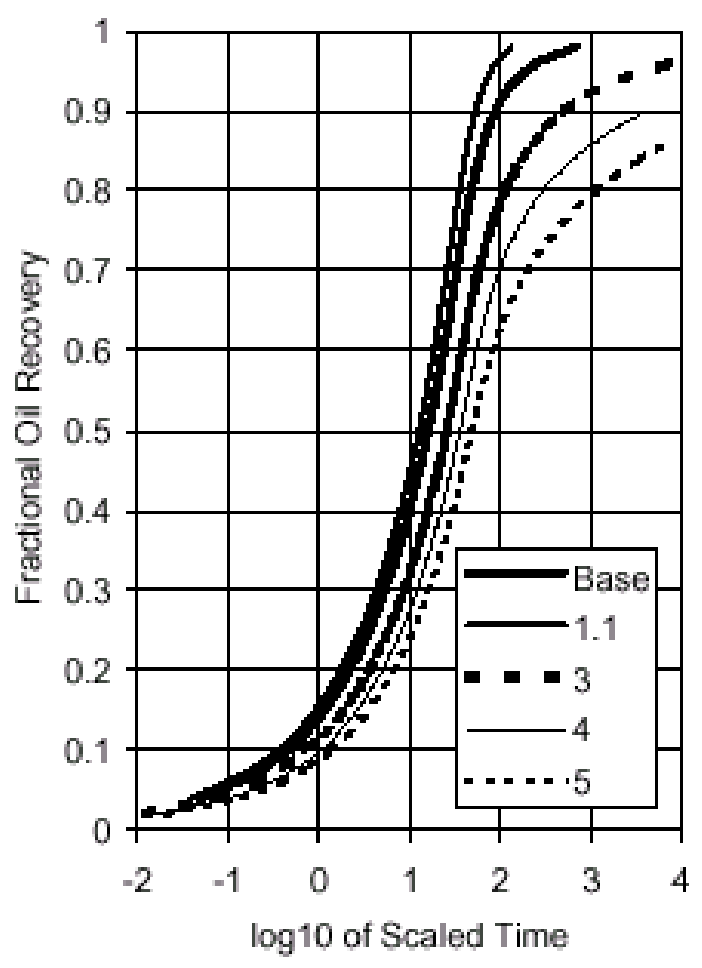

(b) results of varying $n_{o}$

Figure III-2.9. Effect of varying the shape of the water relative permeability curve by varying $n_{v^{*}}$

In summary, there are no major inconsistencies between the correlation derived from experimental data and simulations that result from parametric variations of the base case. Furthermore, many of the changes in one parameter, for example changing one relative permeability without affecting the other, may not be physically reasonable. There may well be compensating effects that contribute to the success of the scaling group such as, for example the mobility conditions for two phase counter-current flow.

\section{Initial water saturation}

The effect of initial water saturation on imbibition rate is of special practical interest and has been investigated by experiment for Berea sandstone and chalk (Viksund et al. 1998). Figure III-2.10 shows the simulated effect of initial water saturation. The saturations considered were $0.0,0.05,0.1,0.2$, and 0.3 . The decrease in dimensionless time for imbibition is qualitatively similar to that measured by Viksund et al (1998) for chalk (very low permeability). The behavior measured for Berea sandstone is more complicated (Viksund et al., 1998). The dimensionless time for imbibition increased by a factor of about 8 with increase in initial saturation up to $7 \%$, stayed close to constant up to $14 \%$, and then decreased. The trend of decrease in $\mathrm{t}_{\mathrm{D}}$ with increase in $S_{w i}$ above $14 \%$ agrees with the trend predicted by simulation, but 
unlike chalk, the dimensionless time for imbibition into Berea sandstone is always longer than that for the correlation developed for $S_{w i}=0$.

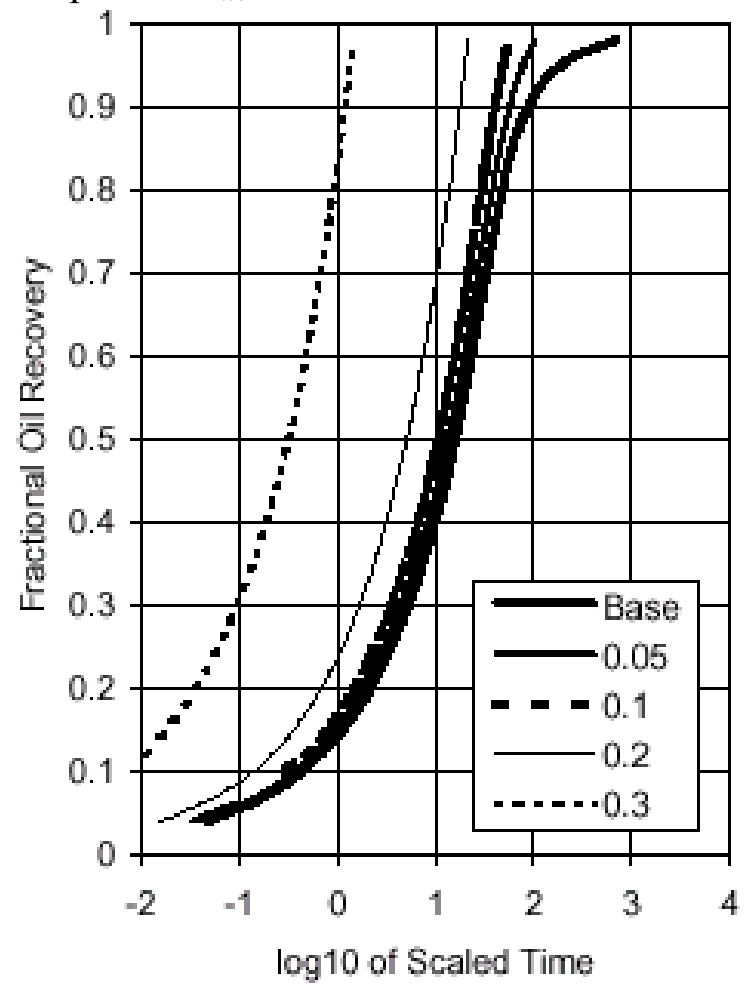

Figure III-2.10. Effect of initial water saturation.

The simulator can also predict water saturation profiles. Figure III-2.11 shows the profiles for the base case. Generally, the water advances from the invading face as a wedge in saturation until it reaches the end of the sample. The saturation in the first grid block at the imbibition-end of the sample obviously cannot go to residual oil because under counter current flow conditions, a significant mobile oil saturation must remain for oil to exit the sample. Figure III-2.12 shows water saturations for the case of an initial water saturation of $10 \%$. The shapes of the curves are very similar to the base case, except that the base line is at $10 \%$ saturation rather then zero. 


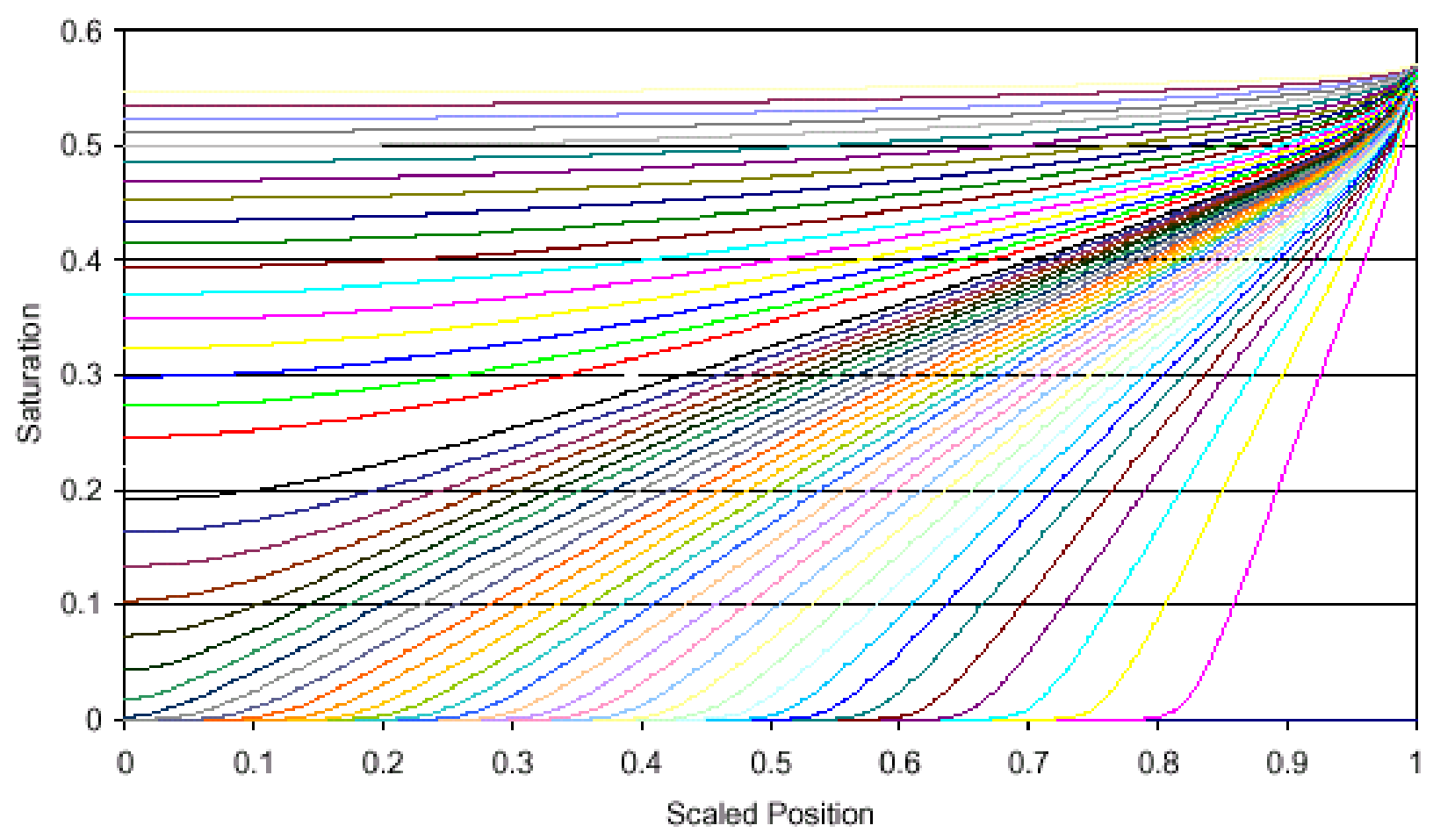

Figure III-2.11. Saturation profiles (base case).

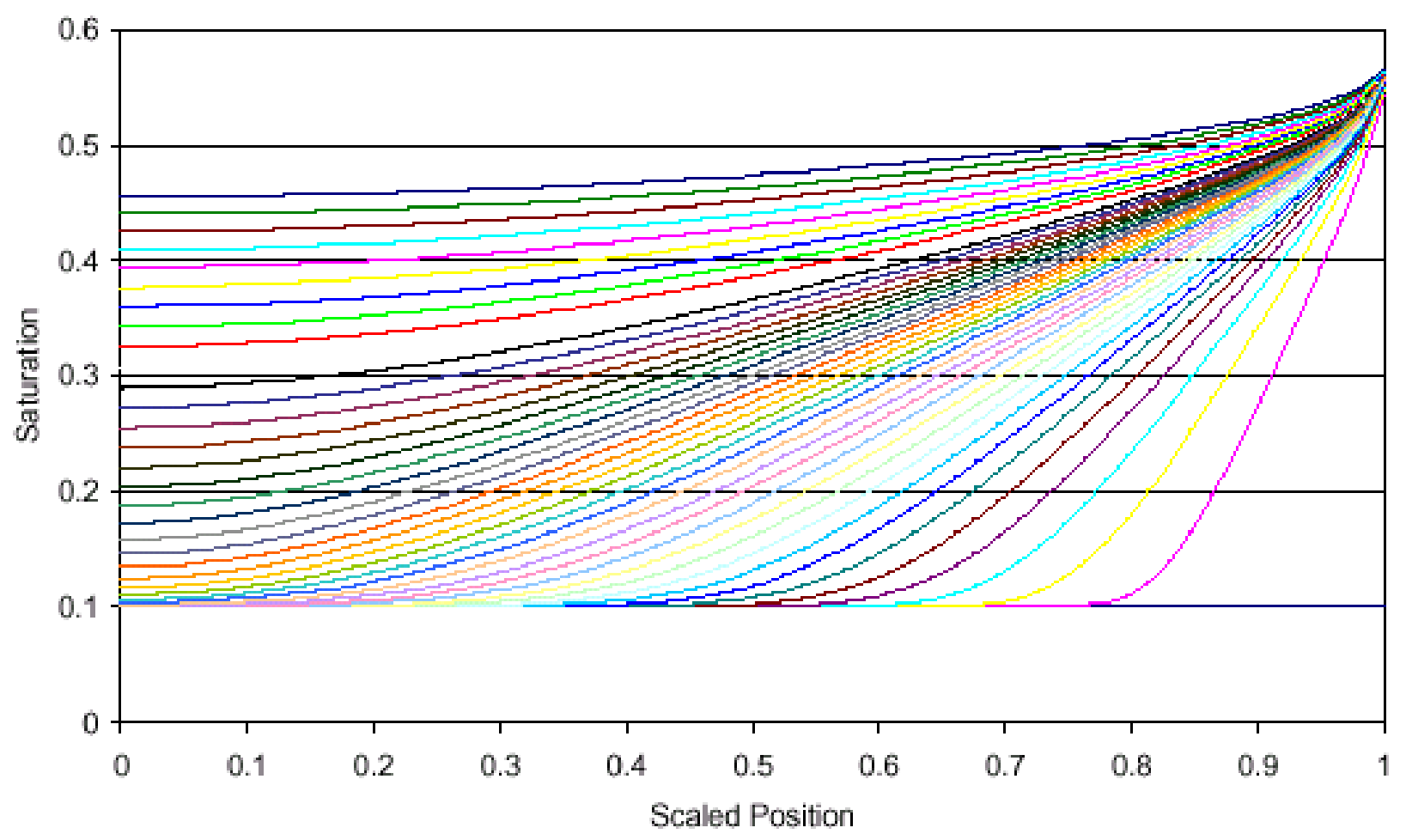

Figure III-2.12. Saturation profiles (10\% initial water saturation). 


\section{Conclusions}

The results of simulations presented in this paper provide useful guidance to further experimental studies. In situ saturation measurement techniques can provide saturation profiles (Graue \& Viksund, 1994) that can be tested against simulation. Experimental micromodel studies of the imbibition mechanisms, and theoretical network modeling (such as those reported by Blunt, 1997 and Dixit, et al 1999) will also contribute to further understanding of the imbibition problem.

Some specific conclusions include:

- The simulation study provides substantial agreement with predictions from a correlation based on experimental data. Within the ranges of parameters used in the experiments, agreement between the model and simulated results is very close.

- Predictions can be made of the effect on imbibition of variation of parameters that were held constant in the original development of the basic scaling groups.

- Over the range of viscosity ratios tested by experiment the correlation based on the geometric mean of the viscosity ratios was in close agreement with the simulated effect of viscosity ratio.

- Trends predicted by simulation for very high viscosity ratios given by using a very low viscosity non-wetting fluid (gas) were qualitatively consistent with experimental results.

- Changes in imbibition rate with initial water saturation predicted by simulation were similar to trends exhibited by experimental results.

\section{References}

Aronofsky, J.S., Masse, L. and Natanson, S.G.: "A Model for the Mechanism of Oil Recovery for the Porous Matrix Due to Water Invasion in Fractured Reservoirs," Trans. AIME, 213, p.17, 1958.

Blair, P.M.: "Calculation of Oil Displacements by Countercurrent Water Imbibition” SPEJ, p. 195-202, Sept. 1964.

Blunt, M.J.: "Pore Level Modeling of the Effects of Wettability," SPEJ, 2, (December), p.494510, 1997. 
Braun, E.M. and Holland, R.F.: "Relative Permeability Hysteresis: Laboratory Measurements and a Conceptual Model," SPE RE, p. 222-8, Aug. 1995.

Corey, A.T: "The Interrelation Between Gas and Oil Relative Permeabilities", Producers Monthly. p. 38-41, Nov 1954.

Dixit, A.B., McDougall, S.R., Sorbie, K.S., and Buckley, J.S.: "Pore Scale Modelling of Wettability Effects and their Influence on Oil Recovery," SPE REE, 2, (February), p.25-36, 1999.

Graue, A. and Viksund, B.G.: "Imaging Immiscible Two Phase Flow in Low Permeability Chalk Emphasis on Recovery Mechanisms and Scaling," EAPG/AAPG Special Conference on Chalk, Copenhagen, Sept. 7-9, 1994.

Hamon, G. and Vidal, J.V.: "Scaling-up the Capillary Imbibition Process from Laboratory Experiments on Homogeneous and Heterogeneous Samples," SPE 15852, the European Petr. Conf., London, Oct. 20-22, 1986.

Kazemi, H. Gilman, J.R., and Eisharkawy, A.M.: "Analytical and Numerical Solution of Oil Recovery from Fractured Reservoirs with Empirical Transfer Function," SPE RE, p. 219-27, May 1992.

Leverett, M.C.: "Capillary Behavior in Porous Solids", Trans. AIME, 142, p. 152, 1941.

Ma, S., Morrow, N.R. and Zhang, X.: "Generalized Scaling of Spontaneous Imbibition Data for Strongly Water-Wet Systems”, JPSE, 18, p. 165-178, 1997.

Ma, S., Zhang, X., and Morrow, N.R.: "Influence of Fluid Viscosity on Mass Transfer Between Rock Matrix and Fractures," JCPT, 38, 7, p. 25-30, July 1999.

Mattax, C.C. and Kyte, J.R.: "Imbibition Oil Recovery From Fractured, Waterdrive Reservoirs," SPEJ, June, p.177, 1962.

Rapoport, L.A.: "Scaling laws for use in design and operation of water-oil flow models" Trans. AIME 204, p. 143-150, 1955.

Viksund, B.G., Morrow, N.R., Ma, S., Wang, W., and Graue, A.: "Initial Water Saturation and Oil Recovery from Chalk and Sandstone by Spontaneous Imbibition", Proceedings of the International Symposium of the Society of Core Analysts, The Hague, September 14-16, 1998.

Wang, R.: “ Gas Recovery from Porous Media by Spontaneous Imbibition of Liquid,” Univ. of Wyoming Masters Thesis, December, 1999.

Warren, J.E. and Root, P.J., "The Behavior of Naturally Fractured Reservoirs", SPEJ, p. 245255, September, 1963. 


\section{III-2.2. Similarity Solution for Linear Counter-Current Spontaneous Imbibition}

Li, Y., Morrow, N.R. and Ruth, D.: “A similarity solution for linear counter-current spontaneous imbibition," ${ }^{\text {th }}$ Internat. Symp. on Reservoir Wettability, Freycinet, Tasmania, 12-14 Mar. 2002.

Recovery of nonwetting phase (NWP) by counter-current spontaneous imbibition of a wetting phase (WP) has been investigated. Experimental results cover a range of viscosity ratios of over seven orders of magnitude. An analytical solution for linear counter-current spontaneous imbibition based on similarity is presented. The mathematical model is based on: (1) Darcy's law for each phase, (2) the imbibition capillary pressures acting as the only driving force, (3) a back pressure given by the drainage interfaces associated with production of NWP at the open face (4) and continuity of counter-current flow. An important consequence of the similarity solution is that the ratio of the WP flow rate at any location to the WP flow rate at the inlet of the sample is a function only of saturation.

\section{Introduction}

Investigation of oil recovery by spontaneous imbibition has been a highly active area of research in multiphase flow in porous media over the past ten years. Developments in this subject were recently reviewed by Morrow and Mason (2001). Many investigations have concerned the recovery of oil from fractured reservoirs. Experimental studies have lead to the development of empirical correlations of imbibition data, which include the effects of sample geometry, boundary conditions and differences in viscosity ratio of the invading WP and the displaced NWP (Ma et al., 1997). The physical basis for these correlations and the possible range of applicability outside the range of observations is not well understood. This paper concerns the mechanism of imbibition and development of a tractable theory.

In subsequent discussion it is convenient to distinguish between three types of imbibition.

(1) Dynamic spontaneous imbibition (DSI), the main subject of this investigation is, by definition, driven only by capillary forces. The contribution of gravity to displacement is assumed to be negligible compared to the action of capillary forces. Two main forms of spontaneous displacement are recognized: counter-current spontaneous imbibition (COUCSI) in which the invading WP flows in the opposite direction to the produced NWP; and co-current spontaneous imbibition (COCSI) in which WP and NWP flow in the same direction. DSI is sometimes also referred to as free imbibition because the WP has unrestricted access at the inlet area(s) of the sample. In studies of DSI, change in saturation is commonly measured as a function of time.

(2) Pseudo-quasistatic spontaneous imbibition. Pseudo-quasistatic displacement is an idealization of the conditions of measurement of drainage and imbibition capillary pressure curves whereby the displacement occurs under balanced pressure (Morrow, 1971). Pseudo-quasistatic spontaneous imbibition refers to controlled increase in the wetting phase saturation. In practice the change in saturation is measured for incremental decrease in capillary pressure and should be unidirectional. The final 
saturation distribution for each measured point on the capillary pressure curve is essentially uniform and independent of the rate of displacement. Change in saturation is commonly measured as a function of capillary pressure.

(3) Forced imbibition is operationally defined as increase in invading phase saturation by external pressures or gravity. Change in saturation is commonly measured as a function of pore volume injected at some specified injection rate or pressure. Buckley and Leverett (1942) provided the most widely applied analysis of two-phase flow. Similarity solutions are obtained whereby the invading saturation profile depends on a combination of relative permeability and viscosity ratio.

Idealized representations of saturation profiles for two extreme modes of saturation change, with constant residual NWP saturation, $S_{n w, r}$, are shown in Fig. III-2.13. They will be referred to as piston-like displacement, PD (Fig.1a) and global displacement, GD (Fig III-2.13b) respectively. PD models are often used to model dynamic displacements in porous media, including DSI. For piston-like counter-current imbibition a leaky piston can be invoked to allow flow of nonwetting phase to the open face.

Pseudo-quasistatic spontaneous imbibition is, in essence, a step-by-step GD. Capillary pressure measurements are essentially equivalent to pseudo-quasistatic displacements. Capillary pressure and corresponding relative permeability measurements are dependent on saturation history.

One aspect of the complexity of modeling DSI in porous media can be illustrated by reference to imbibition into cylindrical capillaries. Displacement depends on the balance between viscous and capillary forces. For displacement of a gas by a liquid in a tube $\left(\mu_{w}\right.$ much greater than $\mu_{n w}$ ) the interface advance is equivalent to PD and distance of advance is proportional to the tube radius and to the square root of time (Washburn, 1921). For a parallel bundle of tubes of different diameter, the displacement is non piston-like and the rate of advance in each tube is proportional to tube radius. However this commonly used model of flow is highly misleading as a model for DSI in porous media. If the tubes are part of a pore network, the influence of capillary forces on local flow must be considered (Li and Wardlaw, 1985).

Handy (1960) modeled COCSI of liquid against gas as PD by combining Darcy's law for flow of the WP, with a definition of imbibition capillary pressure and the equation of continuity. The solution is analogous to the Washburn equation in that when the viscosity of the displaced phase is negligible, the distance advanced by the front is proportional to the square root of time. The solution, although restricted to high WP/NWP viscosity ratios, indicates some important features of frontal advance by linear COCSI; the imbibition time is inversely proportional to permeability and capillary pressure and directly proportional to porosity, (average) saturation, and viscosity of the WP. 


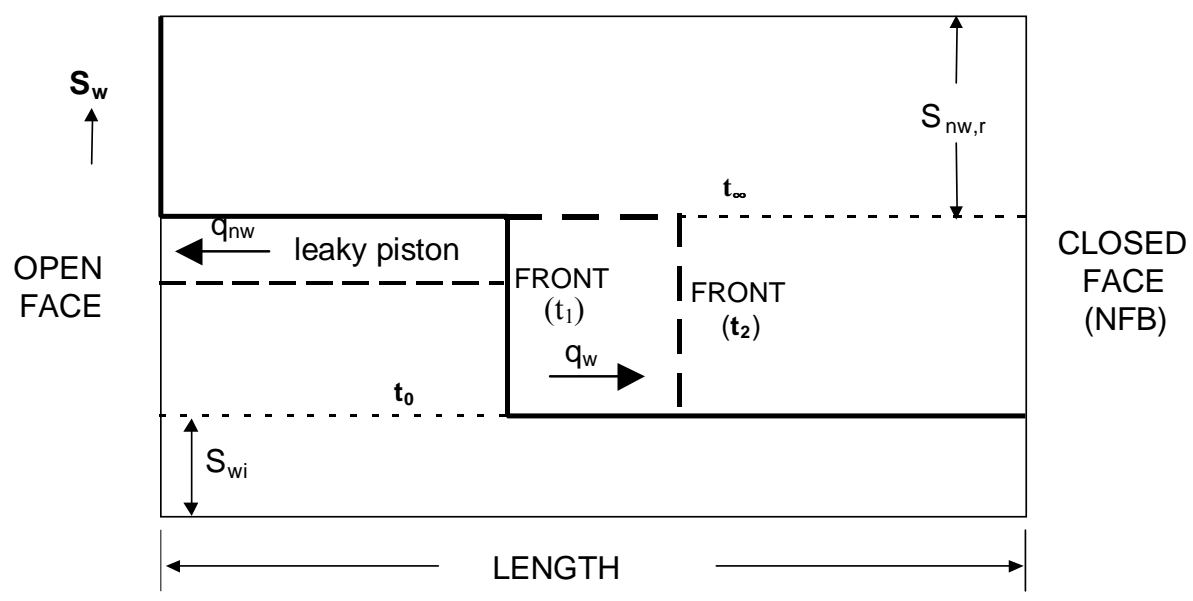

a. Piston-like displacement (PD)

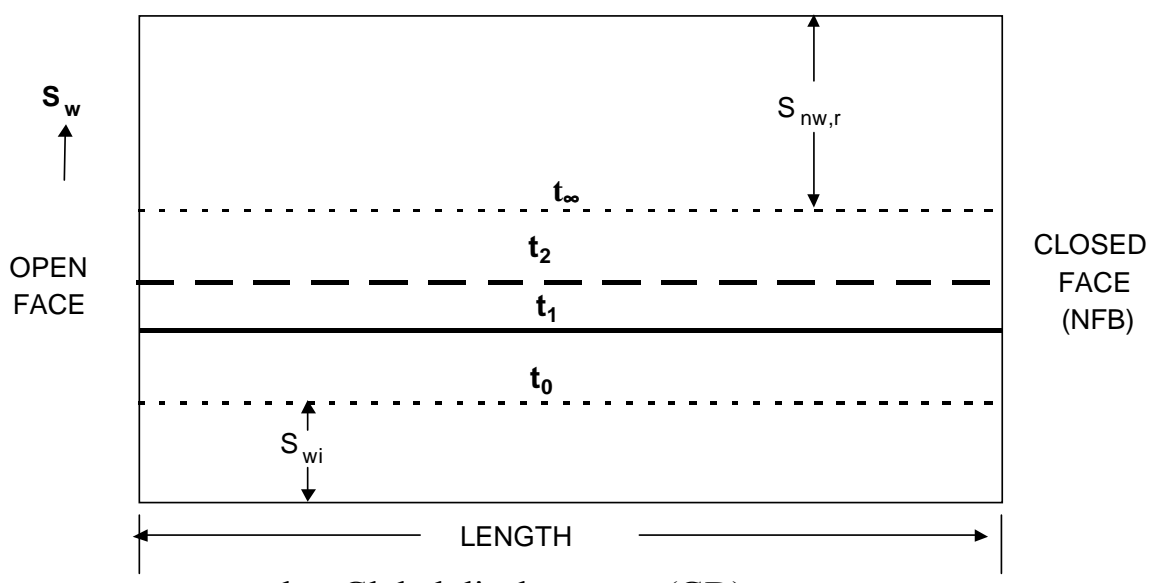

b. Global displacement (GD)

Figure III-2.13. Illustrations of piston-like and global displacement. The heavy solid line represents the saturation profile at an early time $\left(t_{1}\right)$ and the heavy broken line at a later time $\left(t_{2}\right) . S_{w i}$ is the initial WP saturation and $S_{n w, r}$ is the residual NWP saturation. both GD and PD are idealized. For the PD model, the nonwetting phase can leak through the piston. All displacement occurs during the frontal flow period. For the GD model, the gradient is zero and all displacement occurs during the post contact period.

Reis and Cil (1993) proposed a non-PD model of DSI based on the basic equations of Buckley and Leverett except that COUCSI flow is driven by capillary forces and the net flow at any saturation section is zero. A simplified description of the non-PD displacement profile was obtained by assuming that both capillary pressure and NWP saturation gradients are constant. An example of saturation profile and distribution of capillary pressure given by the Ries and Cil model is shown in Fig. III-2.14. In contrast to PD there is continuous variation in saturation between the open face and the imbibition front. Equations were presented for periods described as infinite-acting, prior to contact of the front with the no-flow boundary (NFB) of the sample 
(indicated as a closed face in Fig. III-2.14). The period that follows contact with the boundary was described as the late acting period.

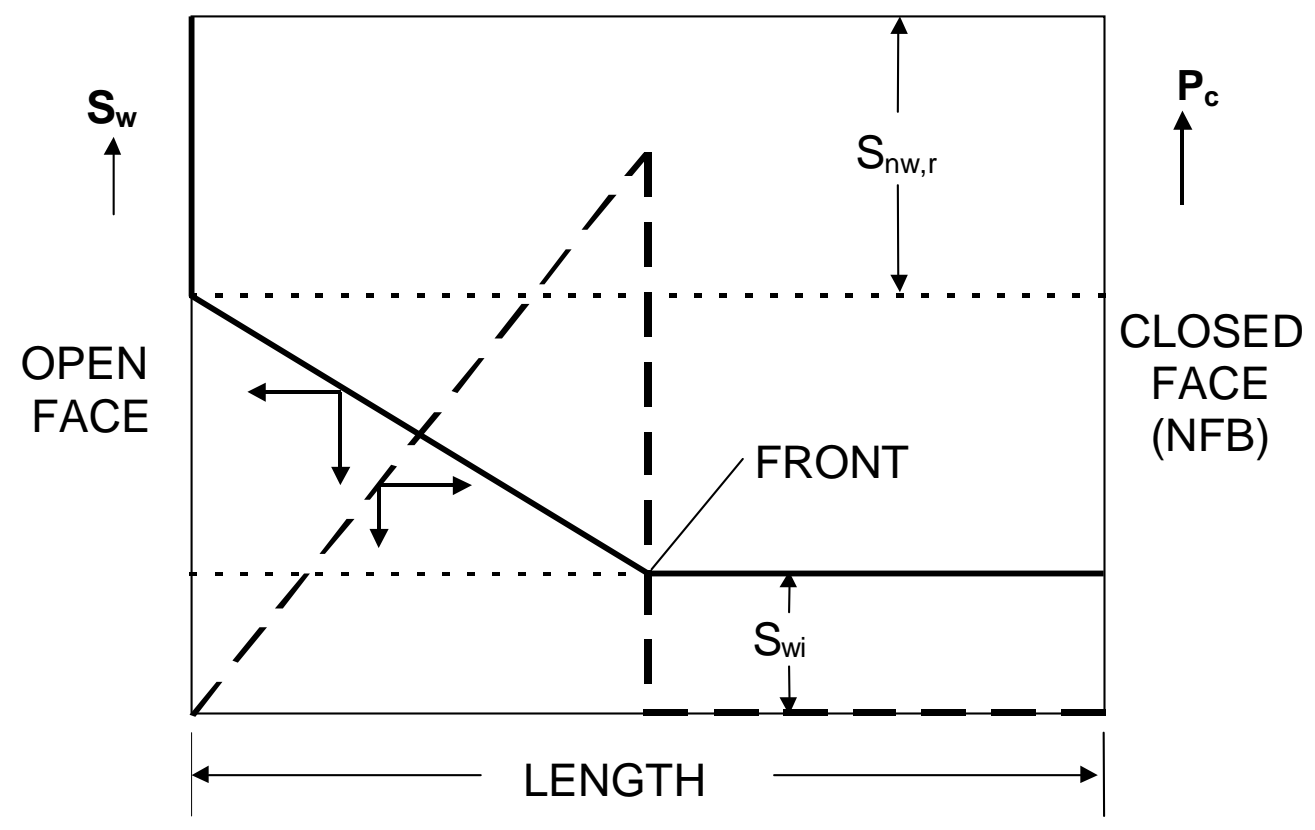

Figure III-2.14. Ries and Cil COUSCI model. The capillary pressure gradient and the saturation gradient between the front and open face are assumed to be linear.

Experimental results on the effect of viscosity ratio on DSI were reported by Ma et al (1997). For displacement of oil by water for two orders of magnitude variation in oil viscosity, imbibition rates were found to scale with the geometric mean of the viscosities. This empirical correlation did not hold for displacement of gas. Detailed results on gas recovery have been reported by Wang (1999). Numerical simulation of imbibition confirmed that scaling by the geometric mean of the viscosities was satisfactory for oil/water but not for gas/liquid (Ruth et al., 2000). Zhou et al (2000a) showed that correlation between results for gas and oil as the nonwetting phase could be improved by incorporating estimates of characteristic mobilities in the definition of dimensionless time.

Experimental studies of DSI have shown that, depending on wetting conditions, increase in saturation can range from invasion of the wetting phase as a distinct front with steep changes in saturation (Fig.III-2.15a) to essentially global increase in saturation with only shallow saturation gradients (Fig. III-2.15b)(Wardlaw and Li, 1988; Baldwin and Spinler, 1999). Such displacement profiles almost span the two extremes of PD and GD shown in Fig. III-2.12. The flow and time period before the invading phase reaches the end face will be referred to as frontal flow and frontal flow period, respectively. For certain crude oil/brine/rock wetting conditions a delay in the onset of imbibition of brine, described as the induction period, is observed (Zhou et al, 2000b). This behavior is probably related to complex pore level wetting and capillary phenomena. For the experimental systems used in the present work, the process of wetting is expected to have only slight effect on the initial imbibition behavior. The flow and time period after first contact of the front with the closed end face (no-flow boundary) will be referred to 
respectively as the post contact flow and the post contact flow period, PCFP. For the extreme cases shown in Fig. III-2.12, all of the displacement occurs during the frontal flow period for PD whereas all flow occurs during the post contact flow period for GD. In practice, the displacement front profile will always fall between these two extremes.

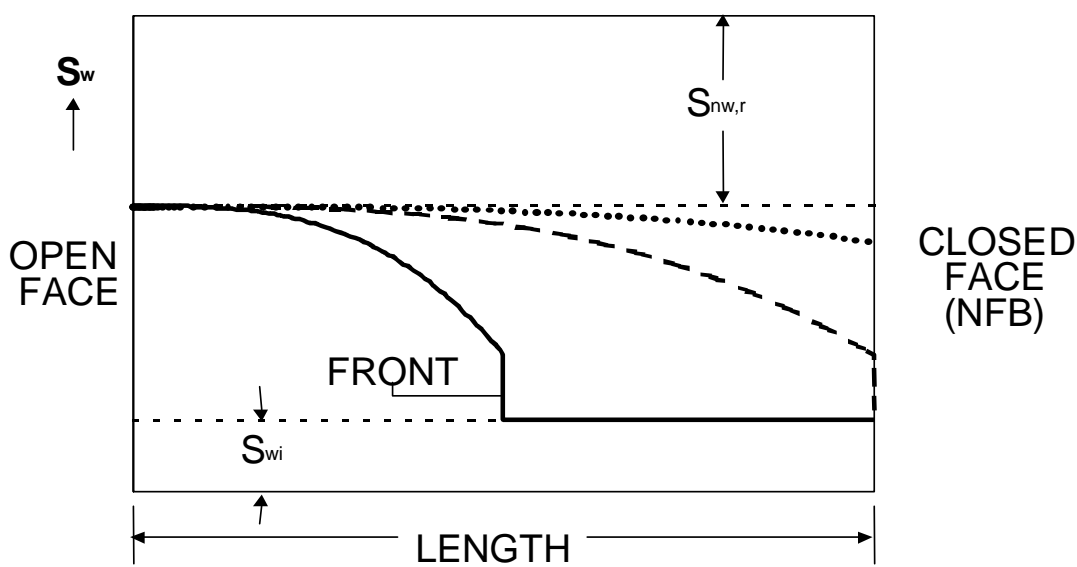

a. Dynamic spontaneous imbibition (DSI) with a truncated saturation profile

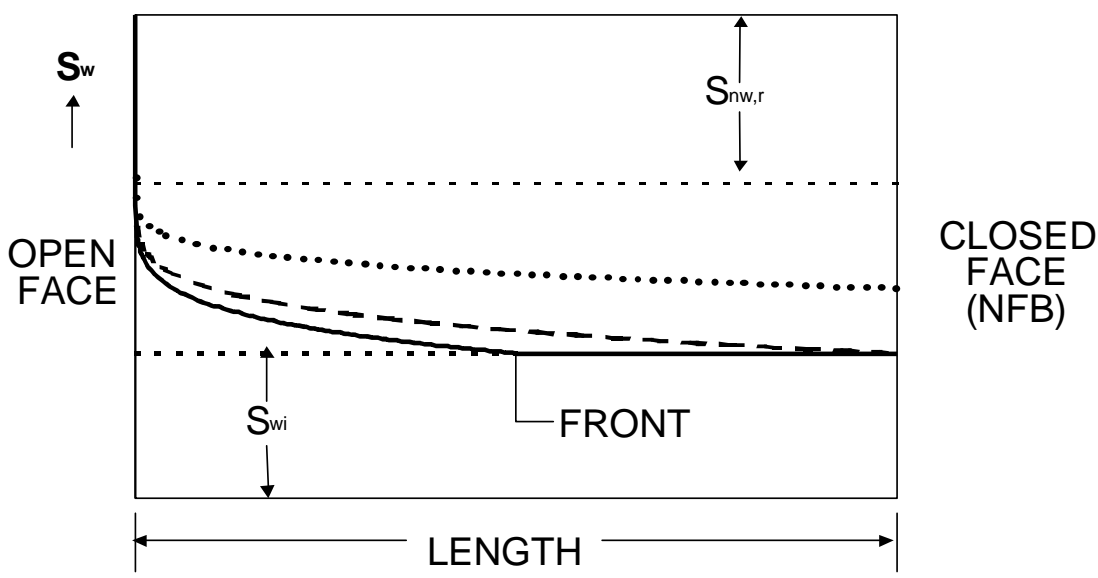

b. DSI with a non-truncated saturation profile

Figure III-2.15. Sketches of truncated and non-truncated saturation profiles. The heavy solid line corresponds to a saturation profile during the frontal flow period. The heavy broken line corresponds to the profile when the front contacts the no-flow boundary. The heavy dotted line shows a profile during the post contact flow period. Production by frontal flow is given by the area swept by the profile up to the time of contact of the front with the no flow boundary. Production during post contact flow period is given by the area under the curve minus the total frontal flow production.

The combined effect of capillary pressure and relative permeabilities can cause variation in the shapes of saturation profiles. Saturation profiles can be sub-classified as truncated and non-truncated as illustrated in Fig. III-2.15. The frontal flow period spans from the onset of imbibition to when the truncated front (or the tip of an non-truncated saturation profile) contacts 
the no flow boundary. The objective of the present study is to develop a COUCSI model that can describe a wide range of possible non-linear saturation profiles.

\section{Mathematical Model}

This investigation considers the case of linear COUCSI given by a homogenous cylindrical geometry with the columnar surface and one end face sealed (Fig.III-2.16a). Imbibition is a result of preferential wettability; fluid movement is sustained by the capillary pressure. The results are directly applicable to any comparable configuration of uniform cross section and also to comparable cases of COUCSI with both ends of the sample open to exchange of wetting and nonwetting phases (Ma et al, 1997) (Fig. III-2.16b). For incompressible liquids, the flow of WP, $q_{w}$, into the sample at the open face and through any cross section, is equal to the counter flow of NWP, $q_{n w}$. Gravity effects are neglected.

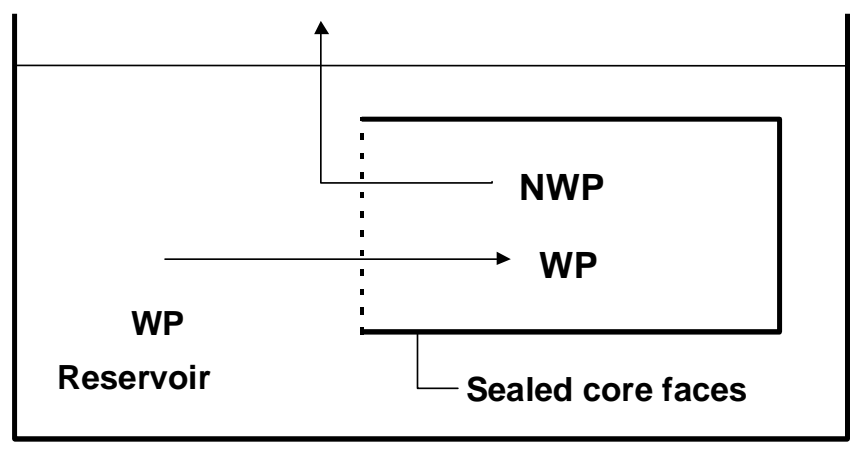

(a) One end open

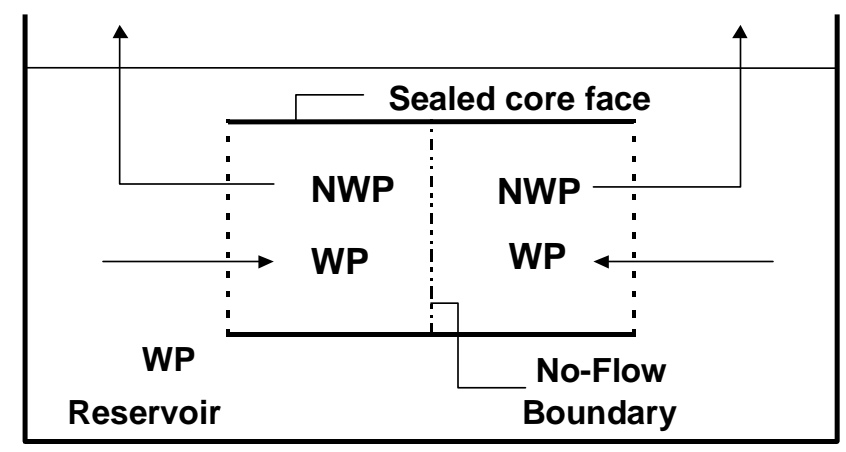

(b) Two ends open

Figure III-2.16. Equivalent conditions for linear counter-current spontaneous imbibition (COUCSI) given by cores with (a) one end open and (b) two ends open. 
Two-phase flow can be described by the generalized Darcy law for each phase:

$q_{w}=-\frac{K k_{r w} A}{\mu_{w}} \frac{\partial P_{w}}{\partial x}$

and

$q_{n w}=-\frac{K k_{r n w} A}{\mu_{n w}} \frac{\partial P_{n w}}{\partial x}$

where $x$ is distance and $t$ is time, $q$ is the flow rate, $P$ is pressure, $A$ is the area of cross section, $K$ is the permeability, $k_{r}$ is relative permeability for counter-current flow, and $\mu$ is viscosity. The subscripts $w$ and $n w$ designate the wetting and the non-wetting phases respectively.

The difference between the nonwetting and wetting phase pressures defines the capillary pressure, $P_{c}$,

$P_{c}=P_{n w}-P_{w}$

Capillary pressure in porous media is a function of saturation (Haines 1929). Counter-current spontaneous imbibition is driven by imbibition capillary pressure.

For counter-current flow,

$q_{w}=-q_{n w}$

Conservation of mass for incompressible flow gives,

$\phi A \frac{\partial S_{w}}{\partial t}+\frac{\partial q_{w}}{\partial x}=0$

and

$\phi A \frac{\partial S_{n w}}{\partial t}+\frac{\partial q_{n w}}{\partial x}=0$

where $\phi$ is porosity, and $S_{w}$ and $S_{n w}$ are the wetting and nonwetting phase saturations respectively. 
Combining equations III-2.7 through III-2.10 results in

$q_{w}=\frac{K k_{r w} k_{r n w} A}{\mu_{w} k_{r n w}+\mu_{n w} k_{r w}} \frac{\partial P_{c}}{\partial x}$

or in terms of the change in saturation

$q_{w}=\frac{K k_{r w} k_{r n w} A}{\mu_{w} k_{r n w}+\mu_{n w} k_{r w}} \frac{\partial P_{c}}{\partial S_{w}} \frac{\partial S_{w}}{\partial x}$

Let

$M_{w}=\frac{K k_{r w} k_{r n w}}{\mu_{w} k_{r n w}+\mu_{n w} k_{r w}} \frac{\partial P_{c}}{\partial S_{w}}$

Substitution of Eq. III-2.14 into Eq. III-2.11 yields

$\phi \frac{\partial S_{w}}{\partial t}+\frac{\partial}{\partial x}\left(M_{w} \frac{\partial S_{w}}{\partial x}\right)=0$

Flow at the inlet/outlet

The open face of the rock is in contact with the wetting phase reservoir. A common assumption under this condition is that the residual nonwetting phase saturation is at its residual value, $S_{n w, r}$, at the boundary. However for COUCSI the nonwetting phase must exit through the face and, at any time, t, the maximum values of $q_{n w}$ (and $q_{w}$ ) are at the face (designated as $q_{n w 0}$ and $q_{w, 0}$ respectively). From direct observation, the number of locations at which NWP is produced is very small compared to the number of pores at the core face (of the order of $10^{4}$ for a rock with $100 \mu$ diameter grain size). For production of gas from core faces (the cores were 3.81 $\mathrm{cm}$ diameter) of Berea sandstone with permeabilities in the range of 0.08 to $1 \mu \mathrm{m}^{2}$ (Darcy) commonly only one site is observed. Production at the open boundary involves added complexities in flow behavior related to the discontinuity and the selectivity of the mechanism that determines the production sites. There will be a tendency, as yet unaccounted for in detail, for the nonwetting phase saturation at the outlet to increase with the viscosity and flow rate of the produced phase. However, in development of the mathematical model it will be assumed that there is an essentially constant wetting phase saturation, $S_{w}($ at $x=0)=S_{w, 0}$, which, for the imbibition experiments reported in the present work, appears to be only slightly less than 1- $S_{n w, \mathrm{r}}$, where $S_{n w, r}$ is the residual nonwetting phase saturation.

Production of NWP at the open face clearly involves a drainage capillary back pressure which opposes NWP production. Although there are local oscillations in capillary pressure, measurements of gas pressure in the uninvaded region of the core show that the effective back 
pressure is essentially constant. Detailed consideration of the physical conditions at the open face will be presented in a subsequent paper.

\section{Similarity solution}

Eq. III-2.16 is satisfied by the similarity condition

$$
\eta=\frac{x}{\sqrt{t}}
$$

This similarity condition leads to an ordinary differential equation:

$-\frac{\phi}{2} \eta \frac{d S_{w}}{d \eta}+\frac{d}{d \eta}\left(M_{w} \frac{d S_{w}}{d \eta}\right)=0$

From Eq. III-2.14 and Eq. III-2.17, we can write

$$
q_{w}=\frac{K k_{r w} k_{r n w} A}{\mu_{w} k_{r n w}+\mu_{n w} k_{r w}} \frac{\partial P_{c}}{\partial S_{w}} \frac{\partial S_{w}}{\partial \eta} \frac{1}{\sqrt{t}}
$$

Furthermore we can define a function, $\gamma$, so that at any particular WP saturation, $S_{w}$,

$\gamma\left(S_{w}, \eta\right)=\frac{K k_{r w} k_{r n w} A}{\mu_{w} k_{r n w}+\mu_{n w} k_{r w}} \frac{\partial P_{c}}{\partial S_{w}} \frac{\partial S_{w}}{\partial \eta}$

It follows that for this similarity solution, $q_{w}$, the flow at any location, $x$ and time, $t$, is characterized by a saturation $S_{w}$.

$q_{w}=\frac{\gamma\left(S_{w}, \eta\right)_{x=x_{S_{w}}}}{\sqrt{t}}$

Flow at the inlet face is then given by,

$q_{w 0}=\frac{\gamma\left(S_{w, 0}, \eta\right)_{x=0}}{\sqrt{t}}$

Division of Eq. III-2.21 by Eq. III-2.22 gives,

$$
\frac{q_{w}}{q_{w 0}}=\frac{\gamma\left(S_{w}, \eta\right)_{x=x_{S_{w}}}}{\gamma\left(S_{w, 0}, \eta\right)_{x=0}}
$$


It follows that the flow rate at any location $x_{S w}$ is simply a function of the flow rate at the inlet and the local saturation, so that

$q_{w}=q_{w 0} F_{w}$

where $F_{w}$, is a function of $\eta\left(S_{w}\right)$ given by the solution to Eq. III-2.18.

Comparison with the Buckley Leverett model of forced imbibition

Several features of the similarity solution for COUCSI bear resemblance to the BuckleyLeverett model of forced imbibition. Gravity is neglected in both models, but whereas capillarity is neglected in the Buckley-Leverett model, it is the driving force for COUCSI. There are also key differences in the form of the equations for flow. For the Buckley-Leverett model, the fractional flow of the WP, $f_{w}$, is defined by

$$
f_{w}=\frac{q_{w}}{q_{w}+q_{n w}}=\frac{1}{1+\frac{k_{r n w}}{k_{r w}} \frac{\mu_{w}}{\mu_{n w}}}
$$

For forced imbibition or COCSI $F_{w}=f_{w}$ and $0 \leq f_{w} \leq 1$. However, for linear COUCSI, $F_{w} \neq f_{w}$ because the NWP flows in opposite direction to the WP, and $q_{w}+q_{n w}$ is always zero. Furthermore $F_{w}$ for COUCSI is not only a function of the relative permeabilities (which may differ because of the different mechanisms of forced imbibition and COUCSI) and viscosities for each phase, but is also a function of capillary pressure, $P_{c}$.

\section{Relationship between Swept Volume and Cumulative Imbibed Volume}

Substitution of Eq. III-2.24 into Eq. III-2.11 gives,

$\phi A \frac{\partial S_{w}}{\partial t}+q_{w 0} \frac{\partial F_{w}}{\partial x}=0$

The interrelationships between $S_{w}, x$ and $t$ can be written in a functional form as $S_{w}=S_{w}(x, t)$ or equivalently in a differential form as:

$$
d S_{w}=\left(\frac{\partial S_{w}}{\partial t}\right)_{x} d t+\left(\frac{\partial S_{w}}{\partial x}\right)_{t} d x
$$

In order to trace a particular saturation to find when a fixed saturation, $S_{w}$, reaches a particular location, $x$, we set $d S_{w}=0$ in Eq. III-2.27, thus 


$$
\left(\frac{d x}{d t}\right)_{S_{w}}=-\frac{\left(\frac{\partial S_{w}}{\partial t}\right)_{x}}{\left(\frac{\partial S_{w}}{\partial x}\right)_{t}}
$$

We can obtain the relationship between $x$ and $t$ for advance of a particular saturation, $S_{w}$, by combining Eq. III-2.26 and Eq. III-2.28:

$$
\left(\frac{d x}{d t}\right)_{S_{w}}=\frac{q_{w 0}}{\phi A} \frac{d F_{w}}{d S_{w}}
$$

The location of a fixed saturation $S_{w}$, corresponding to the cumulative imbibed volume into the core, $Q_{w}$, can be obtained by separating the $x$ and $t$ dependencies in Eq. III-2.29 and integrating from distance 0 to $x_{S w}$ with respect to time from 0 to $t$. The distance, $x_{S w}$, from the inlet at which the saturation is $S_{w}$ is given by,

$x_{S_{w}}=\frac{\int_{0}^{t} q_{w 0} d t}{\phi A} \frac{d F_{w}}{d S_{w}}=\frac{Q_{w}}{\phi A} \frac{d F_{w}}{d S_{w}}$

For a sample of finite length, $L$, the equivalent form is

$$
\frac{x_{S_{W}}}{L}=\frac{Q_{w}}{V_{\phi}} \frac{d F_{w}}{d S_{w}}
$$

where $L$ is the length of the sample, and $V_{\phi}$ is the pore volume. In the linear-flow case, the volume swept by wetting phase up to the distance $x_{S w}$ is given by,

$V_{w}\left(S_{w}\right)=x_{S_{w}} A \phi$

The total pore volume is

$V_{\phi}=L A \phi$

Therefore Eq. III-2.31 can be rewritten as:

$$
\frac{V_{w}}{V_{\phi}}=\frac{Q_{w}}{V_{\phi}} \frac{d F_{w}}{d S_{w}}
$$

\section{Saturation Gradient}

Eq. III-2.24 provides a definition of an imbibition flow ratio 
For any section with saturation $S_{w}, F_{w}$ is a function of $x, t$, (or $S_{w}$ ). Combination of Eq. III-2.25 with expressions for $q_{w}$ and $q_{w 0}$ (Eq. III-2.14), and Eq. III-2.15 for $S_{w}$ and $S_{w, 0}$, gives $F_{w}$ as a function of $x$ and $S_{w}$ at any time $t$ for linear flow:

$$
F_{w}=\frac{\left(M_{w}\right)_{S_{w}}}{\left(M_{w}\right)_{S_{w, 0}}} \frac{\left(\partial S_{w} / \partial x\right)_{S_{w}}}{\left(\partial S_{w} / \partial x\right)_{S_{w, 0}}}
$$

Because $F_{w}$ can be expressed as a function of $S_{w}$ only, then the term $\left(\partial S_{w} / \partial x\right)_{S w} /\left(\partial S_{w} / \partial x\right)_{S w, 0}$ in Eq. III-2.36 must be a constant at any time, $t$. This requirement is satisfied by the similarity of the saturation versus distance profiles (Eq. III-2.31).

Combination of Eqs. III-2.36 and III-2.15 gives:

$$
F_{w}=\frac{\left(\frac{\mu_{n_{w}}}{k_{r n w}}+\frac{\mu_{w}}{k_{r_{w}}}\right)_{S_{w 0}}}{\left(\frac{\mu_{n_{w}}}{k_{r n w}}+\frac{\mu_{w}}{k_{r_{w}}}\right)_{S_{w}}} \frac{\left(\frac{d P_{c}}{d S}\right)_{S_{w}}}{\left(\frac{d P_{c}}{d S}\right)_{S_{w 0}}} \frac{\left(\frac{\partial S}{\partial x}\right)_{S_{w}}}{\left(\frac{\partial S}{\partial x}\right)_{S_{w 0}}}
$$

If the relevant relative permeability and capillary pressure curves are not available, the corresponding $F_{w}$ can still be calculated directly from the measured recovery versus time. (Details of the procedure will be addressed separately.)

\section{Capillary pressure gradient}

Production of NWP at the open face requires that the capillary back pressure which resists formation of NWP bubbles or drops at the open face be overcome. Despite the small oscillation in pressure caused by generation of bubbles or drops, in the present analysis it will be assumed that the capillary back pressure, $P_{c 0}$, is constant. A detailed study of boundary conditions at the open face that provides justification of this assumption will be reported separately.

Under the similarity conditions (Eq. III-2.31), the capillary pressure gradient at any instant and position, $x_{S w}$, can be expressed in terms of the capillary pressure at that position, $P_{c}$ $\left(x_{S w}\right)$ and the capillary pressure at the open face, $P_{c 0}$, as

$$
\frac{d P_{c}}{d x}=\frac{\Omega_{w}\left(P_{c}-P_{c 0}\right)}{x_{S_{w}}}
$$

where $\Omega_{w}$ is a parameter which accounts for non-linearity in the capillary pressure gradient. The physical meaning of $\Omega$ is discussed in relation to PD in Appendix A. $\Omega_{w}$ depends only on the saturation and is independent of the location $x_{S w}$ of any saturation. 
Eq. III-2.38 may be written as

$$
\Omega_{w}=\frac{d\left(P_{c}-P_{c 0}\right)}{d\left(x_{S_{w}} / L\right)} \frac{\left(x_{S_{w}} / L\right)}{\left(P_{c}-P_{c 0}\right)}
$$

Note that in the above equation $\Omega_{w}$ is a function of the gradient of $P_{c}$, which means that the variation in $k_{r w}, k_{r n w}, \mu_{w}$ or $\mu_{n w}$ will affect the value of $\Omega_{w}$ (see Eq. III-2.37). Substitution of the expression for the capillary pressure gradient (Eq. III-2.38) into Eq. III-2.13 results in

$$
q_{w}=\frac{K k_{r w} k_{r n w} A}{\mu_{w} k_{r n w}+\mu_{n w} k_{r w}} \frac{\Omega_{w}\left(P_{c}-P_{c 0}\right)}{x_{S_{w}}}
$$

Eq. III-2.30 gives

$$
Q_{w}=\frac{\phi A x_{S_{w}}}{\left(d F_{w} / d S_{w}\right)}
$$

The flow rate at the entrance to the sample is given by differentiating each side of this equation with respect to time while holding the saturation constant.

$$
q_{w 0}=\frac{\phi A}{\left(d F_{w} / d S_{w}\right)} \frac{d x_{S_{w}}}{d t}
$$

From Eq. III-2.42 and Eq. III-2.24, the flow rate $q_{w}$ at any location is given by,

$$
q_{w}=\frac{\phi A F_{w}}{\left(d F_{w} / d S_{w}\right)} \frac{d x_{S_{w}}}{d t}
$$

Combination of Eqs. III-2.40 and III-2.43, and re-arrangement yields

$$
x_{S_{w}} d x_{S_{w}}=\frac{K k_{r w} k_{r n w}}{\mu_{w} k_{r n w}+\mu_{n w} k_{r w}} \frac{\Omega_{w}\left(P_{c}-P_{c 0}\right)}{\phi} \frac{\left(d F_{w} / d S_{w}\right)}{F_{w}} d t
$$

Integration for constant $S_{w}$ from zero time (the mathematical assumption is made that all saturations are initially present at the inlet to the sample), gives an equation for frontal advance

$$
x_{S_{w}}^{2}=\frac{2 K k_{r w} k_{r n w}}{\mu_{w} k_{r n w}+\mu_{n w} k_{r w}} \frac{\Omega_{w}\left(P_{c}-P_{c 0}\right)}{\phi} \frac{\left(d F_{w} / d S_{w}\right)}{F_{w}} t
$$


Therefore, $x_{S w}$ is related to time by,

$x_{S_{w}}=R_{1} \sqrt{t}$

where $R_{l}$ is a constant for a particular combination of rock and fluids and any particular saturation in the saturation profile

Combination of Eqs. III-2.45 and III-2.31 and rearrangement gives an equation for the recovery-time in terms of the cumulative fraction input $Q_{w} / V_{\phi}$, the core length $L$, and the time $t$

$\left(\frac{Q_{W}}{V_{\phi}}\right)^{2}=\frac{2 K k_{r w} k_{r n w}}{\mu_{w} k_{r n w}+\mu_{n w} k_{r w}} \frac{\Omega_{w}\left(P_{c}-P_{c 0}\right)}{L^{2} \phi} \frac{t}{F_{w}\left(d F_{w} / d S_{w}\right)}$

Increase in saturation is related to time by

$\frac{Q_{w}}{V_{\phi}}=R_{2} \sqrt{t}$

where $R_{2}$ is a constant for the same conditions as for $R_{1}$.

Thus, from the basic flow equations (Eq. III-2.7 to Eq. III-2.13), an analytical solution has been obtained for the rate of linear counter-current spontaneous imbibition for any selected value of $S_{w}$ between the WP saturation at the front, $S_{w, f}$ and the saturation at the inlet, $S_{w, 0}$. The distance of advance of any particular saturation proceeds as the square root of time (Eq. III-2.46). The equation for rate of linear frontal advance (Eq. III-2.48) and the similarity condition (Eq. III2.17) are therefore consistent. Measurement of the distance of advance of the saturation front with time provides a test of this conclusion.

\section{Experiments}

COUCSI tests were conducted in Berea-sandstone cores with diameters of approximately 3.8 $\mathrm{cm}$ and lengths of from 5.55 to $7.89 \mathrm{~cm}$ (Table III-2.2). The porosities, $\phi$, and permeabilities, $K$ of the test cores ranged from $17.2 \%$ to $21.75 \%$ and from 0.061 and $1.324 \mu m^{2}$ respectively. Core surfaces were partially sealed with clear epoxy resin to give the one end open boundary condition. WP/NWP fluid pairs were either water/oil or oil/air. Experimental results covered over seven orders of magnitude variation in viscosity ratio. Comparison of results for the open face being set upward versus downward showed that gravitational effects could be neglected. 
Table III-2.2 Core Dimensions and Rock and Fluid Properties $\left(\rho_{w}=1 \mathrm{~kg} / \mathrm{L}\right)$

\begin{tabular}{|c|c|c|c|c|c|c|c|c|c|}
\hline \multirow{3}{*}{$\begin{array}{l}\text { Group } 1 \\
\text { CORE }\end{array}$} & \multicolumn{9}{|c|}{ Cumulative oil imbibed measured gravimetrically. } \\
\hline & D & L & W & $\Phi$ & K & $\rho_{o}$ & $\mu_{\mathrm{a}}$ & $\mu_{0}$ & $\sigma$ \\
\hline & $\mathrm{cm}$ & $\mathrm{cm}$ & $g$ & $\%$ & $\mu \mathrm{m}^{2}$ & $\mathrm{~kg} / \mathrm{L}$ & Pa.s & Pa.s & $\mathrm{N} / \mathrm{m}$ \\
\hline AO1 & 3.804 & 6.425 & 146.61 & 21.65 & 1.134 & 0.783 & 0.000018 & 0.0038 & 0.02582 \\
\hline $\mathrm{AO} 2$ & 3.804 & 7.463 & 170.89 & 21.75 & 1.032 & 0.783 & 0.000018 & 0.0038 & 0.02582 \\
\hline $\mathrm{AO} 3$ & 3.810 & 7.556 & 175.26 & 21.58 & 0.961 & 0.783 & 0.000018 & 0.0038 & 0.02582 \\
\hline $\mathrm{AO} 4$ & 3.808 & 6.387 & 147.52 & 21.47 & 0.899 & 0.783 & 0.000018 & 0.0038 & 0.02582 \\
\hline AO5 & 3.804 & 6.467 & 153.22 & 20.06 & 0.540 & 0.783 & 0.000018 & 0.0038 & 0.02582 \\
\hline AO6 & 3.815 & 6.332 & 150.47 & 19.77 & 0.486 & 0.783 & 0.000018 & 0.0038 & 0.02582 \\
\hline $\mathrm{AO7}$ & 3.808 & 6.532 & 154.66 & 20.02 & 0.474 & 0.783 & 0.000018 & 0.0038 & 0.02582 \\
\hline AO8 & 3.810 & 6.565 & 155.28 & 19.95 & 0.464 & 0.783 & 0.000018 & 0.0038 & 0.02582 \\
\hline AO9 & 3.802 & 6.574 & 156.05 & 19.82 & 0.423 & 0.783 & 0.000018 & 0.0038 & 0.02582 \\
\hline AO10 & 3.810 & 6.585 & 156.21 & 19.53 & 0.349 & 0.783 & 0.000018 & 0.0038 & 0.02582 \\
\hline AO11 & 3.806 & 5.550 & 132.42 & 19.45 & 0.335 & 0.783 & 0.000018 & 0.0038 & 0.02582 \\
\hline AO12 & 3.792 & 7.879 & 192.56 & 17.36 & 0.089 & 0.783 & 0.000018 & 0.0038 & 0.02582 \\
\hline $\mathrm{AO} 13$ & 3.805 & 6.321 & 149.68 & 19.48 & 0.360 & 0.8736 & 0.000018 & 0.1632 & 0.03146 \\
\hline Group 2 & \multicolumn{9}{|c|}{$\begin{array}{l}\text { Cumulative oil imbibed and air produced are measured } \\
\text { volumetrically. }\end{array}$} \\
\hline \multirow[t]{2}{*}{ CORE } & D & L & W & $\Phi$ & $\mathbf{K}$ & $\rho_{o}$ & $\mu_{a}$ & $\mu_{0}$ & $\sigma$ \\
\hline & $\mathrm{cm}$ & $\mathrm{cm}$ & $g$ & $\%$ & $\mu \mathrm{m}^{2}$ & $\mathrm{~kg} / \mathrm{L}$ & Pa.s & Pa.s & $\mathrm{N} / \mathrm{m}$ \\
\hline AOS1 & 3.803 & 7.722 & 176.53 & 21.70 & 1.324 & 0.783 & 0.000018 & 0.0038 & 0.02582 \\
\hline AOS2 & 3.818 & 7.612 & 177.95 & 20.60 & 0.779 & 0.783 & 0.000018 & 0.0038 & 0.02582 \\
\hline AOS3 & 3.794 & 7.701 & 192.85 & 17.20 & 0.061 & 0.783 & 0.000018 & 0.0038 & 0.02582 \\
\hline AOS4 & 3.808 & 7.712 & 176.59 & 21.60 & 1.292 & 0.8736 & 0.000018 & 0.1632 & 0.03146 \\
\hline Group 3 & \multicolumn{9}{|c|}{ Cumulative oil produced by imbibition measured volumetrically. } \\
\hline \multirow[t]{2}{*}{ CORE } & D & $\mathbf{L}$ & W & $\Phi$ & $\mathbf{K}$ & $\rho_{o}$ & $\mu_{0}$ & $\mu_{w}$ & $\sigma$ \\
\hline & $\mathrm{cm}$ & $\mathrm{cm}$ & g & $\%$ & $\mu \mathrm{m}^{2}$ & $\mathrm{~kg} / \mathrm{L}$ & Pa.s & Pa.s & $\mathrm{N} / \mathrm{m}$ \\
\hline OW1 & 3.808 & 7.720 & 176.53 & 21.60 & 1.241 & 0.783 & 0.00432 & 0.0010 & 0.02117 \\
\hline OW2 & 3.792 & 7.870 & 183.43 & 20.40 & 0.638 & 0.783 & 0.00432 & 0.0010 & 0.02117 \\
\hline OW3 & 3.792 & 7.850 & 183.63 & 20.20 & 0.555 & 0.783 & 0.00432 & 0.0010 & 0.02117 \\
\hline OW4 & 3.792 & 7.261 & 176.26 & 17.60 & 0.092 & 0.783 & 0.00432 & 0.0010 & $0.04000^{*}$ \\
\hline OW5 & 3.793 & 7.381 & 180.53 & 17.60 & 0.087 & 0.783 & 0.00432 & 0.0010 & $0.04000^{*}$ \\
\hline OW6 & 3.787 & 7.885 & 180.49 & 21.60 & 0.959 & 0.8736 & 0.16320 & 0.0010 & 0.04566 \\
\hline OW7 & 3.790 & 7.774 & 181.56 & 20.20 & 0.515 & 0.8736 & 0.16320 & 0.0010 & 0.04566 \\
\hline OW8 & 3.796 & 7.864 & 196.58 & 17.40 & 0.072 & 0.8736 & 0.16320 & 0.0010 & 0.04566 \\
\hline
\end{tabular}

*oil cleaned by exposure to silica gel and alumina

For imbibition of oil against air, the location of a well defined air/oil front could be tracked by direct observation. For oil/water displacement, the location of an advancing water front was determined from sudden rise in electrical conductivity between the wetting phase (brine) and embedded electrodes spaced along the length of the core.

Air/oil imbibition tests were made on 17 samples. The oil uptake $\left(Q_{w} / V_{\phi}\right)$ of 13 samples (Group 1) was calculated from weight gain. For the other 4 experiments (Group 2), the volumes of invading oil and produced air were measured simultaneously so that the effect of gas compressibility, albeit small, could be measured and taken into account. This permitted valid comparisons between imbibition results for incompressible and compressible nonwetting phase 
fluids. Oil/water imbibition was tested for 8 samples (Group3). The oil production $\left(Q_{n w} / V_{\phi}\right)$ was measured volumetrically.

\section{Results And Discussion}

Examples of oil and air recovery versus time data, representative of Berea sandstone of $0.5 \mu \mathrm{m}^{2}$ permeability, are presented in Fig. III-2.17. Comparison of the imbibed volume of oil with the produced volume of gas showed that the gas evolution is delayed because the gas in the core is compressed by the initial inflow of wetting phase, $Q_{w}$, up to about 0.03 to $0.04 V_{\phi}$. After the onset of gas evolution there was no detectable difference between the volumetric uptake of liquid and the production of air (corrected to $1.03-1.04$ times atmospheric pressure) during the course of evolution of air.

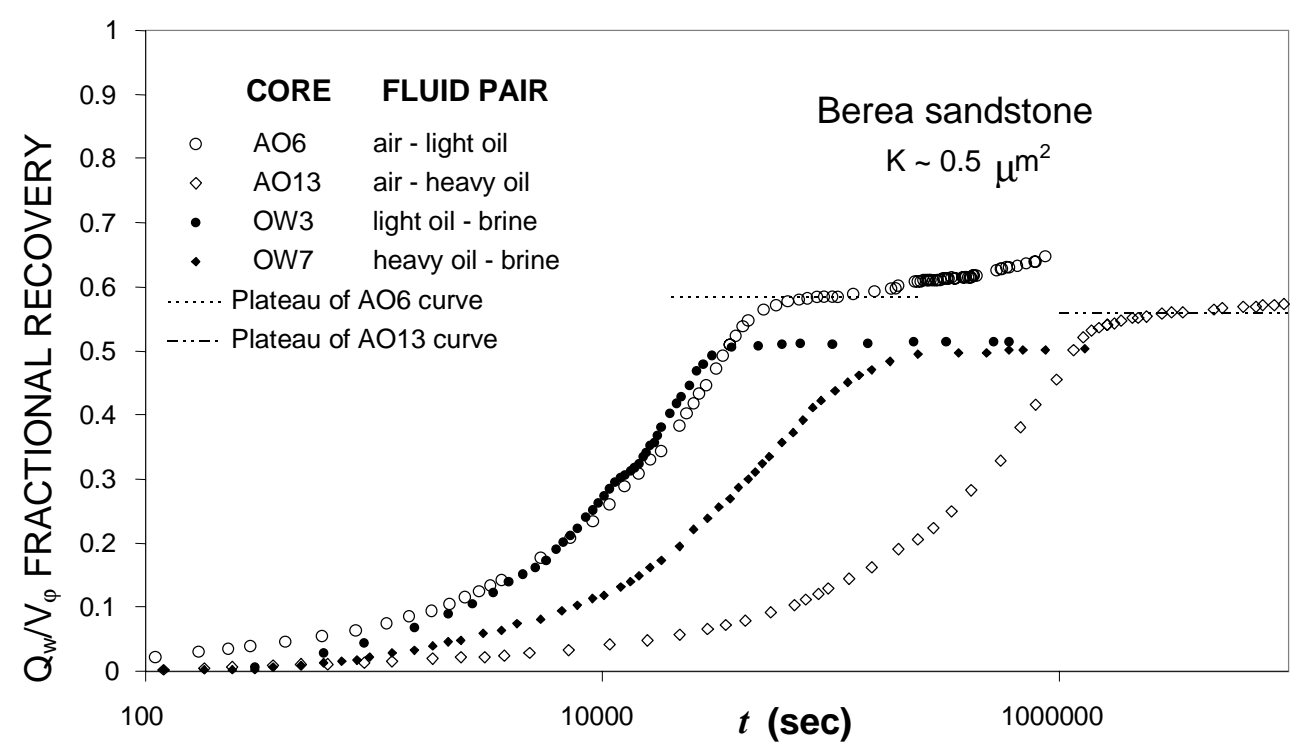

Figure III-2.17. Examples of increase in WP saturation with time for air/oil and oil/brine. In comparison of results, the increase in WP saturation at long times caused by dissolution of trapped air is neglected. The indicated plateau achieved at long times is used to define the maximum increase in WP saturation by DSI.

Increase in WP saturation at long times occurs because of dissolution and diffusion of trapped gas, this can be readily distinguished from recovery by dynamic spontaneous imbibition because of the large difference in time scales for the two processes. After correction for the initial compression of gas and the slow diffusion of trapped gas, valid comparison can be made between the effect of viscosity ratio for gas/liquid and liquid/liquid imbibition. 
The experimental results presented in Fig. III-2.18 show that the recovery of NWP from behind the front is a constant fraction of the volume swept by the front throughout the frontal flow period. For oil/air, the fractional recoveries, $Q_{w} / V_{w}$, increased from 0.42 to 0.525 with increase in permeability from 0.09 to $1.2 \mu \mathrm{m}^{2}$ for the series of Berea sandstones used in the present work.

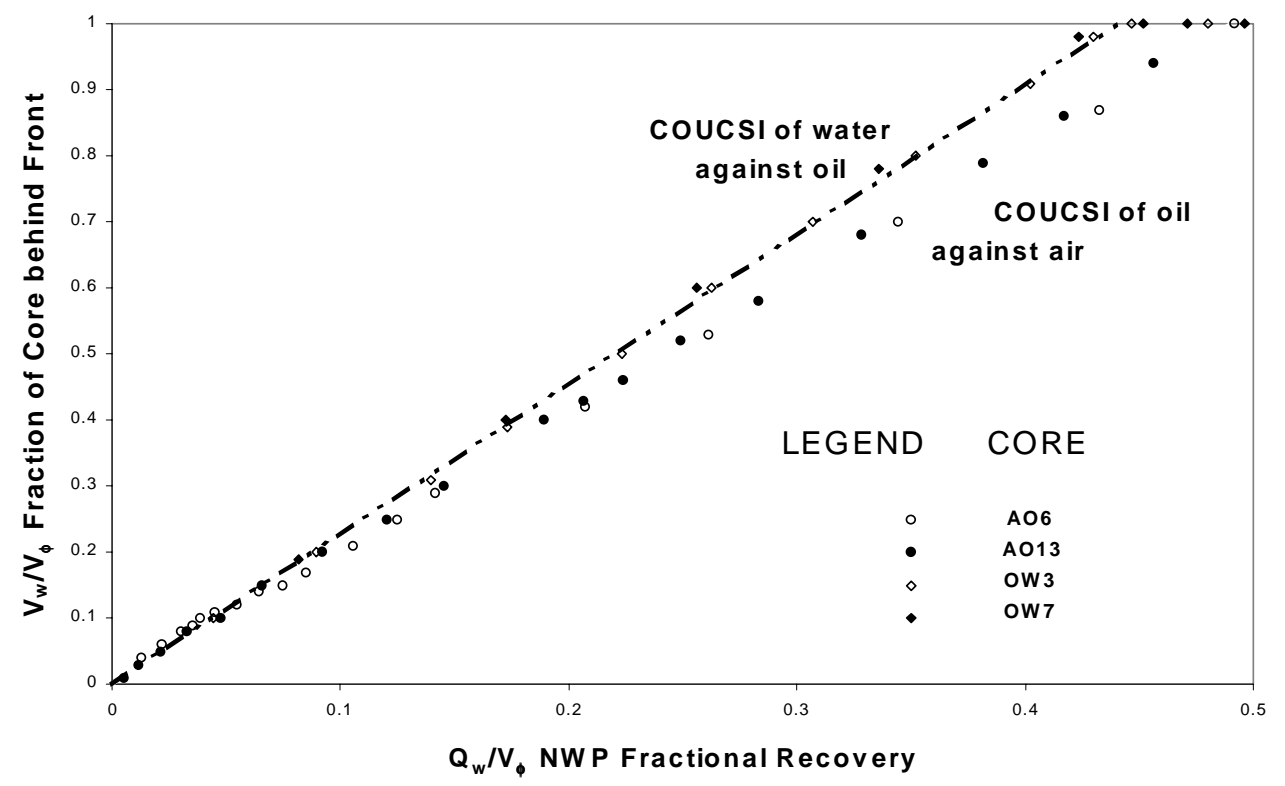

Figure III-2.18. Experimental results representative of Berea sandstone of $0.5 \mu^{2}$ permeability, for which the recovery behind the front is a constant fraction of the swept volume. For Berea sandstone with the same permeability, the fractional recoveries for oil/brine pairs are always less than for air/oil pairs by a constant proportion.

For the same rock and location of the front relative to the total length of the core, the fractional NWP recovery, $Q_{w} / V_{w}$ for oil-brine pairs was always less than for air-oil pairs. From the results shown in Fig. III-2.18, the fractional recoveries for oil/brine pairs are always less than for air/oil pairs by a constant proportion

Confirmation that $Q_{w} / V_{\phi}$ is Proportional to $\sqrt{t}$

The air/oil and oil/water results were first tested as plots of recovery, $Q_{w} / V_{\phi}$ versus $\sqrt{t}$. For frontal flow, the recovery curves all fall very close to straight lines. The tendency at early time for the curves to bend slightly downward is believed to result from the time required to establish the wetting front at the inlet during the initial stage of imbibition (the induction period) even though the systems are all very strongly wetted. When the data are plotted as $Q_{w} / V_{\phi}$ versus $\sqrt{t}-B$ (Fig. III-2.19), all of the frontal flow period imbibition data, except for very early time, 
fall close to a straight line from the origin to the end point of the frontal flow period. After a front reaches the no flow boundary the subsequent post contact flow data fall away from the frontal flow period straight lines.

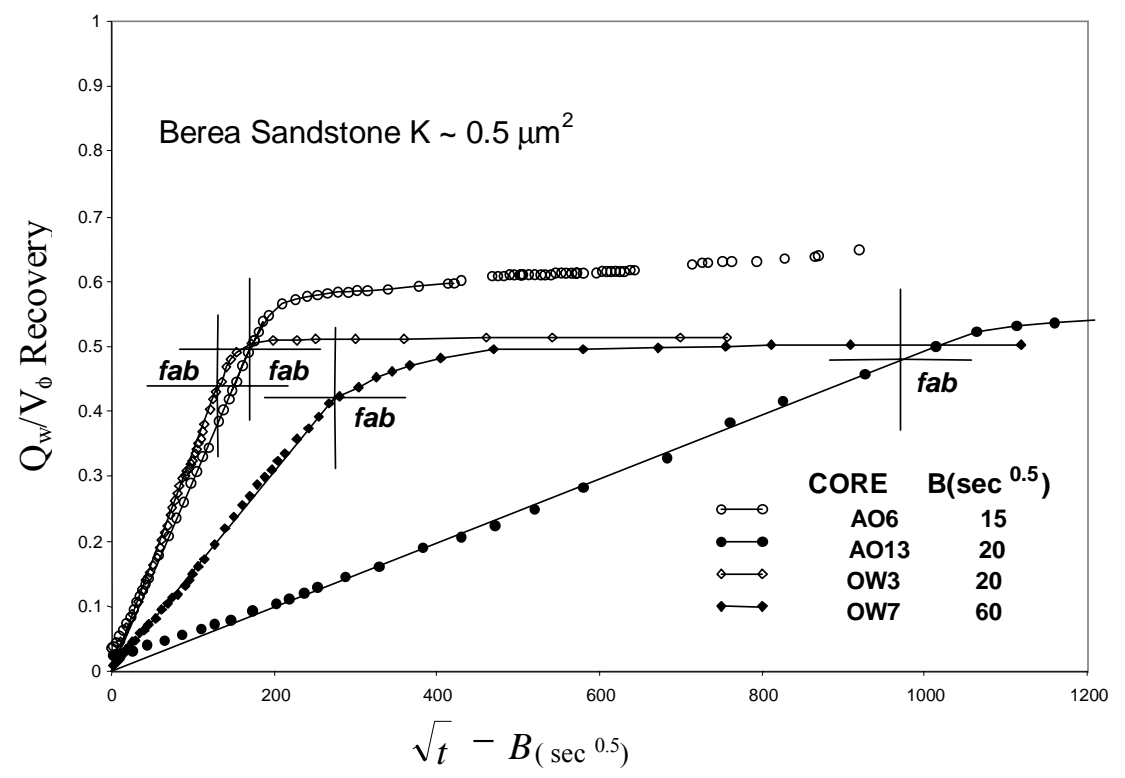

Figure III-2.19. Recovery of NWP versus adjusted $\sqrt{t}$. Apart from the very small correction to $t$ for the development of flow at very early time, the rate of frontal advance for both air-oil and oil-water pairs is proportional to the square root of time until the front is at the boundary $(f a b)$.

Recovery during Frontal Flow and Post Contact Flow

If it is assumed that all saturation sections advance as described by Eq. III-2.47 and a saturation profile is known at any stage of the frontal flow period, the complete recovery curve can be calculated. For example, for the chosen profile with the front at $x_{S w, f},\left(t_{1}\right)=0.5 \mathrm{~L}$ at time, $t_{1}$, as shown in Fig. III-2.20, the recovery at time $t_{1}, Q_{n} / V_{\phi},\left(t_{1}\right)$, given by the area under the saturation profile is 0.25 (if $S_{w i}$ is finite the related area is excluded). The distance of frontal advance either before or after $t_{1}$ is proportional to the square root of time (see Line A, Fig. III2.21). The time at which the front reaches the no flow boundary $(x=L), t_{2}$, is equal to $4 t_{1}$ and the corresponding recovery, $Q_{w} / V_{\phi},\left(t_{2}\right)$, is 0.5 (Fig. III-2.22). After completion of frontal flow, the rate of recovery falls away from the straight line given by the $Q_{w} / V_{\phi}$ vs $\sqrt{t}$ plot as illustrated in Fig. III-2.21. 


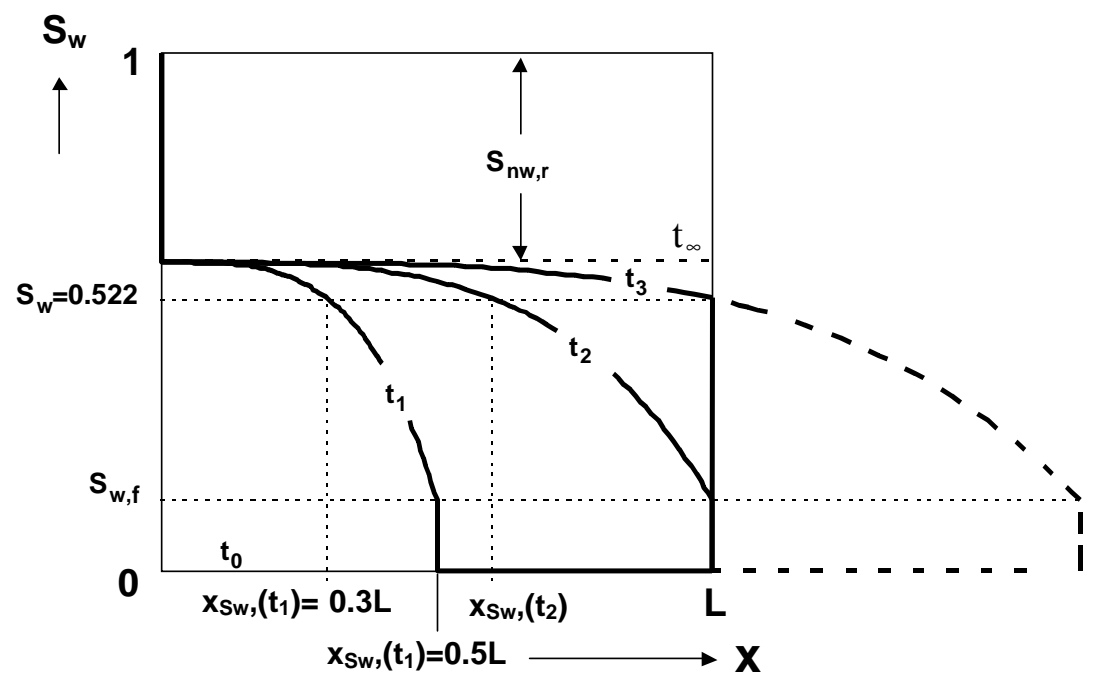

Figure III-2.20. Saturation profiles before, at, and after contact of the saturation front with the no flow boundary. It is assumed that the saturation profile within the core developed as if the core length is infinite. Graphically, when the initial WP saturation, $S_{w i}$ is zero, the recovery for incompressible fluids $Q_{w} / V_{\phi}$ is equal to the ratio of the area under the saturation profile (if $S_{w i}$ is finite the related area is excluded) within the core length, $L$ to the total area, $L \times 1$.

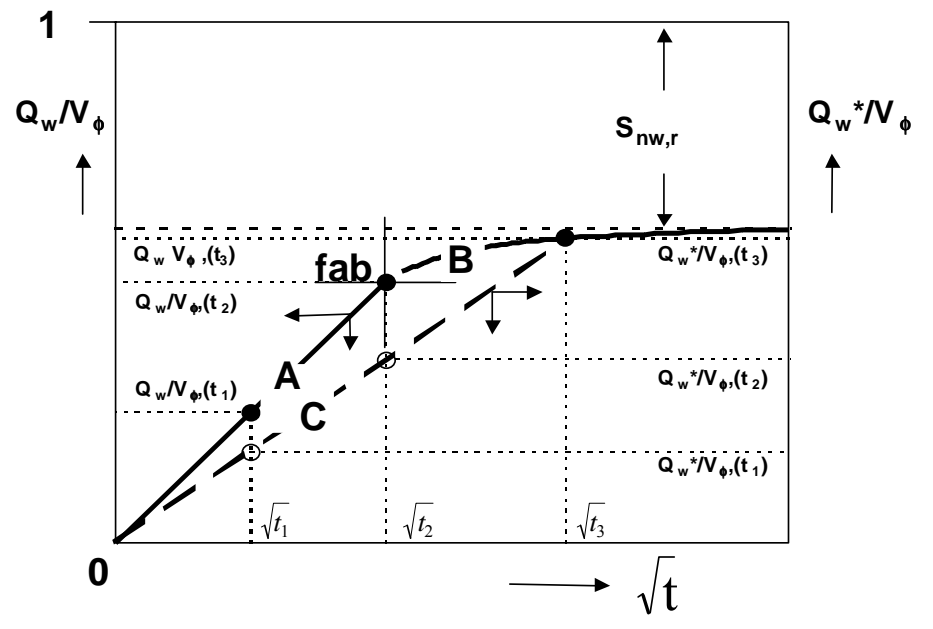

Figure III-2.21. Recovery, $Q_{w} / V_{\phi}$ vs $\sqrt{t}$. Fractional recoveries corresponding to the profiles in Fig.8 are indicated. The frontal flow period part of the recovery curve (straight line A, on the $Q_{w} / V_{\phi} \sim \sqrt{t}$ plot), ends when the front reaches the no flow boundary $\left(t_{2}=t_{f a b}\right)$. Curve $B$ is the post contact flow period part of recovery curve, which is the locus of the arrival of all saturation sections at the no flow boundary.For any section with $S_{w}$ greater than $S_{w, f}$, there exists a comparable linear relation between $\boldsymbol{Q}_{w}{ }^{*} / V_{\boldsymbol{\phi}}$ and $\sqrt{t}$, where $\boldsymbol{Q}_{w} *$ is defined by the volume of imbibed WP left behind the section. Line $C$ is a plot of the increase in saturation with respect to the imbibed wetting phase behind the section with a saturation of 0.522 . 


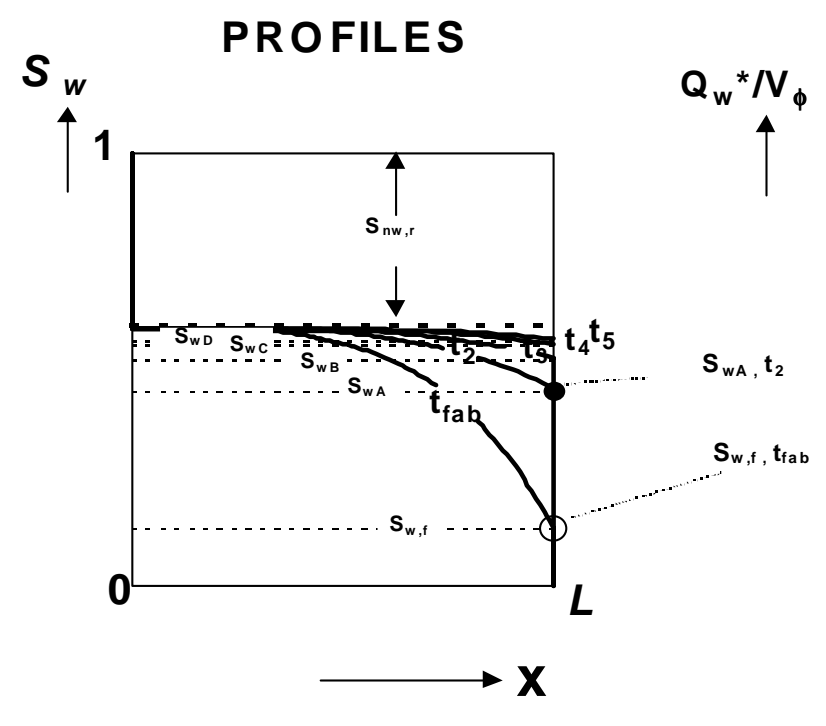

\section{RECOVERY CURVE}

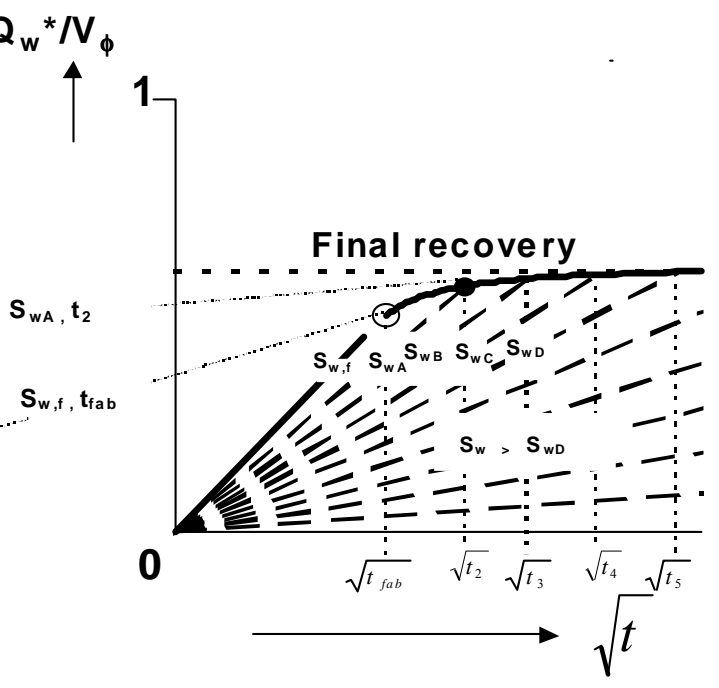

Figure III-2.22. Relations between the saturation profile and recovery after time, $t_{\text {fab }}$, at which the front reaches the no flow boundary. After $t_{f a b}$, the recovery curve is given by the locus of the average saturations, $Q_{w} * / V_{\phi}$ corresponding to the value of $S_{w}$ at the no flow boundary. The relationships between arrival of saturation sections at the no flow boundary and recovery for arrival times $t_{\text {fab }}$ and $t_{2}$ are indicated by the two connecting lines which link the figures.

The solutions to Eqs. III-2.46 and III-2.48 are for infinite length. The presence of a closed boundary or the hypothetical no-flow boundary for the case of two ends open has only small effect on the imbibition mechanism (Li et al., 1986). The continuity condition at the boundary is $q_{w}=q_{n w}=0$. For prediction of recovery versus time from the saturation profile during post contact flow, for example as illustrated by the curve at time, $t_{3}$, in Fig. III-2.20, the saturation is integrated from the inlet up to the distance $L$ of the no-flow boundary.

Consider the movement of the section with saturation of 0.522 up to the time $t_{3}$ that it reaches the no flow boundary (Fig. III-2.20). The section with saturation 0.522 is located at $0.3 \mathrm{~L}$ at time $t_{1}$. The average saturation behind the 0.522 saturation section is always 0.585 until the 0.522 saturation section arrives at the no flow boundary at time $t_{3}=11.111 t_{1}$. Up to this time, the fraction of imbibed WP behind the 0.522 saturation section, $\mathrm{Q}^{*} / V_{\phi}$, is proportional to $\sqrt{t}$ and corresponds to Line $\mathrm{C}$ in Fig. 9. At $t_{3}$, the fractional recovery for the sample as a whole is $0.585=Q_{w} / V_{\phi},\left(t_{3}\right)=Q_{w} * / V_{\phi},\left(t_{3}\right)$. The recovery at $t_{3}$ during post contact flow is indicated in Fig. 9. The locus of the end points for the arrival of saturation sections at the no flow boundary provides the relationship (Curve B in Fig. III-2.21) between recovery and time for the post contact flow period. Plots of $\mathrm{Q}^{*} / V_{\phi}$ versus $\sqrt{t}$ are shown in Fig. III-2.22 for a series of values of saturation greater than $S_{w, f}$ at the no flow boundary.

Detailed information on the development of saturation profiles during the course of COUCSI would provide a further rigorous test of the similarity model. Change in saturation 
profiles with time can be obtained by in situ monitoring of saturation development. Examples of such measurements for spontaneous imbibition have been reported by Baldwin and Spinler (1999).

The solutions dealing with the implicit relations for the saturation sections with $S_{w}>S_{w, f}$ can be used to develop detailed information on the acting relative permeabilities and capillary pressures. A systematic account of interrelationships between relative permeability, capillary pressure, fluid properties, and rock/fluid interactions which determine the shapes of saturation profiles is beyond the scope of the present work and will be treated separately.

\section{Conclusions}

1. A mathematical model of linear COUCSI based on similarity, but with distinct differences from the Buckley-Leverett solution for forced displacement, has been developed which can account for a wide range of modes of imbibition as characterized by the saturation profiles that evolve during imbibition.

2. The capillary driving force for spontaneous imbibition is given by the capillary pressure acting along the saturation profile minus an average capillary back-pressure associated with production of the NWP at the open face.

3. For linear COUCSI, the imbibition flow ratio, $F_{w}$, at any section of the saturation profile is a function of saturation only.

4. The model provides implicit solutions for recovery with respect to any saturation section of the saturation profile.

5. Predictions of the model, in particular, that flow rate and the distance advanced by the front are proportional to the square root of time during frontal flow and that recovery after the frontal flow period is implicitly dependent on the production profile, are supported by COUCSI experiments.

\section{Nomenclature}

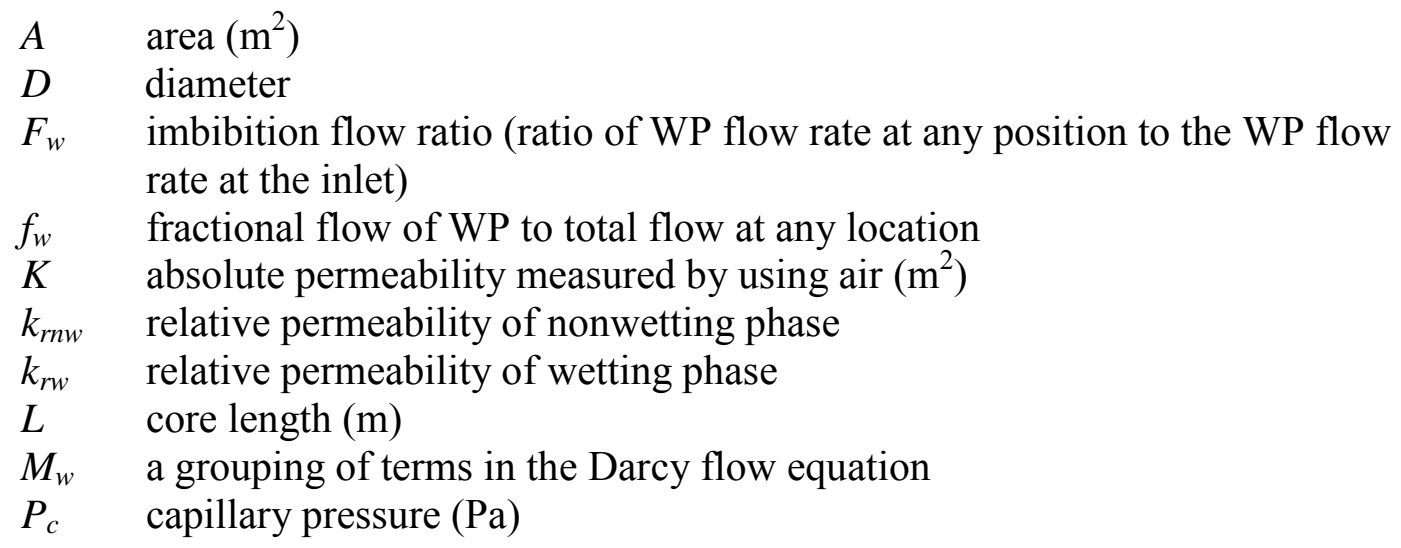


$P_{c 0} \quad$ capillary pressure at outlet $(\mathrm{Pa})$

$P_{n w} \quad$ pressure in nonwetting phase $(\mathrm{Pa})$

$P_{w} \quad$ pressure in wetting phase $(\mathrm{Pa})$

$Q_{n w} \quad$ cumulative output of nonwetting phase $\left(\mathrm{m}^{3}\right)$

$Q_{w} \quad$ cumulative input of wetting phase $\left(\mathrm{m}^{3}\right)$

$Q_{w} * \quad$ cumulative input of wetting phase left behind any saturation section $\left(\mathrm{m}^{3}\right)$

$q_{n w} \quad$ flow rate of nonwetting phase $\left(\mathrm{m}^{3} / \mathrm{s}\right)$

$q_{w} \quad$ flow rate of wetting phase $\left(\mathrm{m}^{3} / \mathrm{s}\right)$

$q_{w, 0} \quad$ flow rate of wetting phase at the inlet $\left(\mathrm{m}^{3} / \mathrm{s}\right)$

$R_{1} \quad$ a constant for a particular combination of rock and fluids and any particular saturation in the saturation profile

$R_{2} \quad$ a constant for the same conditions as for $R_{1}$

$S_{n w} \quad$ saturation of nonwetting phase

$S_{n w, r} \quad$ residual nonwetting phase saturation

$S_{w} \quad$ saturation of wetting phase

$S_{w, f} \quad$ saturation of wetting phase at the front

$S_{w, 0} \quad$ saturation of wetting phase at the inlet

$S_{w i} \quad$ initial wetting phase saturation

$t \quad$ time (sec)

$t_{f a b} \quad$ time at which the front meets the flow boundary (sec)

$V_{\phi} \quad$ pore volume $\left(\mathrm{m}^{3}\right)$

$V_{w} \quad$ volume swept by any specified $S_{w}$ section $\left(\mathrm{m}^{3}\right)$

$x \quad$ distance $(\mathrm{m})$ from inlet

$x_{S w} \quad$ distance (m) from inlet to the $S_{w}$ section

$\Omega_{w} \quad$ a parameter related to the effect of the distribution of permeabilities and imbibition capillary pressures over the saturation profile

$\gamma \quad$ a function only of saturation and $\eta$

$\eta \quad$ a self-similarity variable

$\mu_{n w} \quad$ viscosity of the nonwetting phase (Pa.s)

$\mu_{w} \quad$ viscosity of wetting phase (Pa.s)

$\rho_{o} \quad$ oil density $\left(10^{3} \mathrm{~kg} / \mathrm{m}^{3}\right)$

$\rho_{w} \quad$ water density $\left(10^{3} \mathrm{~kg} / \mathrm{m}^{3}\right)$

$\sigma \quad$ interfacial tension $(\mathrm{N} / \mathrm{m})$

$\phi \quad$ porosity

\section{Acronyms/abbreviations}

\section{Phases}

WP wetting phase

NWP nonwetting phase

\section{Imbibition mode}

DSI dynamic spontaneous imbibition

COCSI co-current spontaneous imbibition

COUCSI counter-current spontaneous imbibition 


\section{Displacement mode}

GD global displacement

PD piston-like displacement

Flow periods and boundaries

fab front at boundary

\section{Acknowledgments}

We are pleased to acknowledge discussions with Dr. Geoffrey Mason, Loughborough University, and Dr. Shaochang Wo, University of Wyoming.

\section{References}

Baldwin, B.A., and Spinler, E.A., 1999, In-situ Saturation Development during Spontaneous Imbibition, Proceedings of the International Society of Core Analysts, SCA-9922, 1-11.

Buckley, S.E., and Leverett, M., 1942, Mechanism of Displacement in Sands, Tran. AIME, 146: 107.

Haines, W.B., 1930, Studies in the Physical Properties of Soil V. The hysteresis effect in capillary properties and the modes of moisture distribution associated therewith, Journ. Agric. Sci. 20, 97-116.

Handy, L.L., 1960, Determination of Effective Capillary Pressures for Porous Media from Imbibition Data, Trans. AIME, 219, 75-80.

Li, Y., Laidlaw, W.C., and Wardlaw, N.C., 1986, Sensitivity of Drainage and Imbibition to Pore Structure as Revealed by Computer Simulation of Displacement Process, Advances in Colloid and Interface Science, 26, 1-68.

Li, Y. and Wardlaw, N.C., 1986, The Influence of Wettability and Critical Pore-Throat Size Ratio on Snap-off, J. Colloid Interface Sci. 109, 461-473.

Ma, S., Morrow, N.R., and Zhang, X., 1997, Generalized Scaling of Spontaneous Imbibition Data for Strongly Water-wet Systems, J Pet Sci Eng, 18, 3/4, 165-178.

Morrow, N.R.: "Irreducible Wetting Phase Saturations in Porous Media," Chem. Eng. Sci., 1971, 25, 1799-1815.

Morrow, N.R,, and Mason, G., 2001, Recovery of Oil by Spontaneous Imbibition, Current Opinion in Colloid \& Interface Science, 6, 321-337

Reis, J.C., and Cil, M., 1993, A Model for Oil Expulsion by Counter-current Water Imbibition in Rocks: One-dimensional Geometry. J. Pet Sci Eng, 10, 97-107

Ruth, D., Morrow, N.R., Li, Y., and Buckley, J.S., 2000, A Simulation Study of Spontaneous Imbibition, Proceedings of the International Symposium of the Society of the Core Analysts Annual Meeting, Oct., Abu Dhabi, UAE, 
Wang, R., 1999, Gas Recovery from Porous Media by Spontaneous Imbibition of Liquid, MS Thesis, University of Wyoming.

Wardlaw, N.C., and Li, Y., 1988, Fluid Topology, Pore Size and Aspect Ratio during Imbibition, Transport in Porous Media, 3, 17-34.

Washburn, E.W., 1921, The Dynamics of Capillary Flow, Phys Rev, 17, 273-283.

Zhou, D., Jia, L., Kamath, J., and Kovscek, A.R., 2000a, Scaling of Counter-current Imbibition Processes in Low-permeability Porous Media, Proceedings of the $6^{\text {th }}$ International Symposium on Reservoir Wettability and its Effect on Oil Recovery, Socorro, NM, Sep. (JPSE in press)

Zhou, X., Morrow, N.R. and Ma, S., 2000b, Interrelationship of Wettability, Initial Water Saturation, Aging Time and Oil Recovery by Spontaneous Imbibition and Waterflooding, SPEJ, 5, (2), June, 199-207. 


\section{Appendix to Section III-2.2}

The physical meaning of the parameter, $\Omega_{w}$, can be illustrated with reference to pistonlike COUCSI (Fig.III-2.13b). For PD the relative permeability to WP and NWP between the inlet and the WP front are constant. All capillary interfaces that drive fluid flow are at the imbibition front. The capillary pressure at the front is $P_{c}$. All interfaces tending to retain NWP within the core are at the open face. At the open face, the capillary pressure is $P_{c 0}$. In this hypothetical case there are no other interfaces that promote flow between the inlet and the front. The pressure gradients between the inlet and the front in the WP and the NWP are constant. Between the inlet and the front

$$
\frac{d P_{n w}}{d x}=\frac{\Delta P_{n w}}{x_{S_{w}}}
$$

and

$$
\frac{d P_{w}}{d x}=\frac{\Delta P_{w}}{x_{S_{w}}}
$$

The pressure balance for the whole system (Fig. III-2.23) is

$$
\Delta P_{n w}-\Delta P_{w}=P_{c}-P_{c 0}
$$

Dividing the two sides of Eq.A3 by $x_{S w}$, and combining Eqs. A1 and A2, we obtain

$$
\frac{d\left(P_{n w}-P_{w}\right)}{d x}=\frac{P_{c}-P_{c 0}}{x_{S_{w}}}
$$

Note that for the highly idealized condition of PD, although the difference in pressure between the NWP and WP is given by $P_{n w}-P_{w}$ at any point $x$ between the inlet and $x_{S w}$, interfaces which drive imbibition are all located at the front.

However, PD does not provide an accurate representation of COUCSI because $k_{r n w}$ increases and $k_{r w}$ decreases as the WP saturation decreases with distance from the open face to the front. The capillary interfaces that drive fluid flow are now distributed between the inlet and the front. Therefore, unlike PD, there is a continuous increase in capillary pressure from the inlet to the front with corresponding variation in $d P d d x$ and decrease in the wetting phase saturation (cf L.H.S. of Eqs.III-2.38 and III-2.52). The calculated rate of advance of a particular saturation is adjusted for the non-piston like flow by the parameter, $\Omega_{w}$ (cf R.H.S. of Eqs.III-2.38 and III2.52). Since $P_{c}$ and $\Omega_{w}$ are functions only of saturation, in contrast to $\mathrm{PD}$, a series of expressions for pressure distribution for different saturations are obtained which depend on the saturation profile between the open face and the location of the saturation $S_{w}$. 


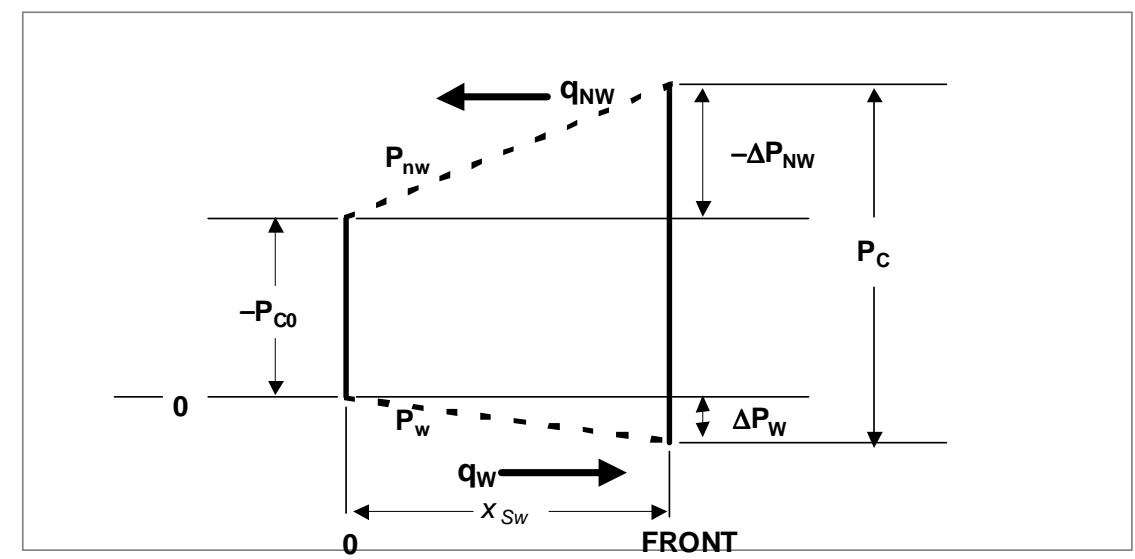

a. Piston like COUCSI model

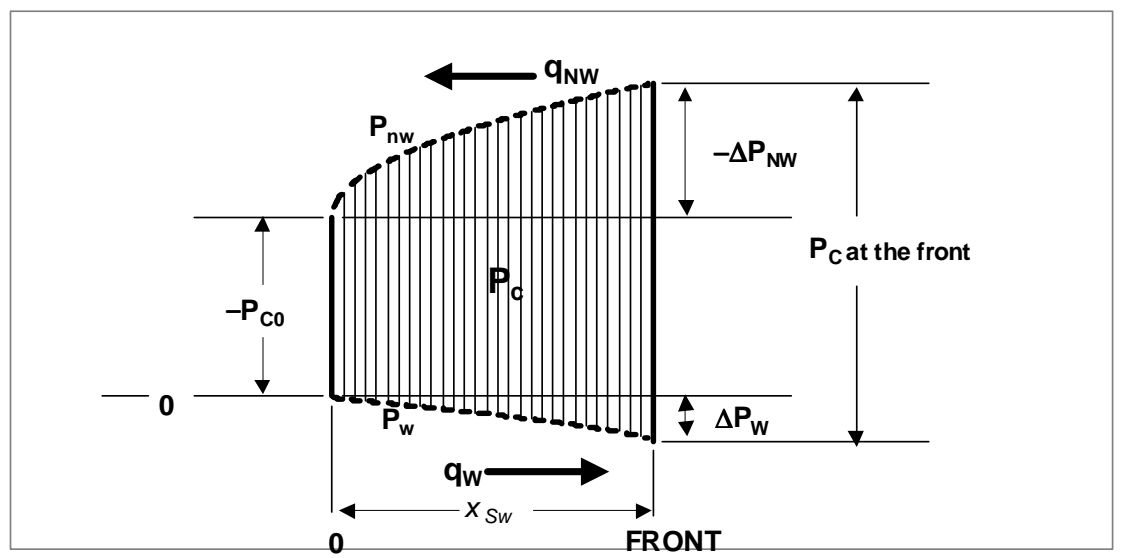

b. Non-Piston like COUCSI model

Figure III-2.23. Pressure distribution and flow in the piston like COUCSI model (a) and in the non-piston like COUCSI model (b). $\boldsymbol{P}_{n w}$ is the pressure in NWP. $\boldsymbol{P}_{w}$ is the pressure in WP. $\boldsymbol{P}_{c}$ is the capillary pressure. $\boldsymbol{P}_{c 0}$ is the average capillary pressure at the open face associated with production of the NWP. $x_{S w}$ is distance from the open face to the front. 


\section{III-3. Experimental Studies}

\section{III-3.1. Test of Characteristic Length for Scaling Air/Oil/Rock Imbibition Rates (VSWW)}

\section{R. Wang, N.R. Morrow, and Y. Li, U. Wyoming}

The scaling equation proposed by Mattax and Kyte (1962) has been widely used to correlate the imbibition measurements for very strongly water-wet oil/brine/rock systems. However, the conditions under which the scaling equation applies are very restrictive. For example, the shape and boundary conditions of the sample should be the same as the prototype, and the wetting and non-wetting phase viscosity ratio should be the same, as well. Those two conditions limit the scope of applicability of the Mattax and Kyte scaling equation. In order to relax the condition pertaining to the sample geometry, a shape factor $\mathrm{F}_{\mathrm{s}}$ was proposed by Kazemi (1992). The data of Mattax and Kyte has been used to test the definition of $F_{s}$. However, this data has errors, and it is possible to misinterpret this data (Ma et al., 1995). A modified definition of characteristic length, $\mathrm{L}_{\mathrm{c}}$, was given by Ma et al. (1997)

$$
L_{c}=\sqrt{V_{b} / \sum_{i=1}^{n} \frac{A_{i}}{l_{A i}}}
$$

where $V_{b}$ is bulk volume of the matrix, $A_{i}$ is the area open to imbibition at the $i$ th direction, $l_{A i}$ is the distance traveled by the imbibition front from the open surface to the no-flow boundary, and $n$ is the total number of surfaces open to imbibition. The position of a no-flow boundary depends on the shape and boundary conditions of the sample, as illustrated in Fig. III-3.1.
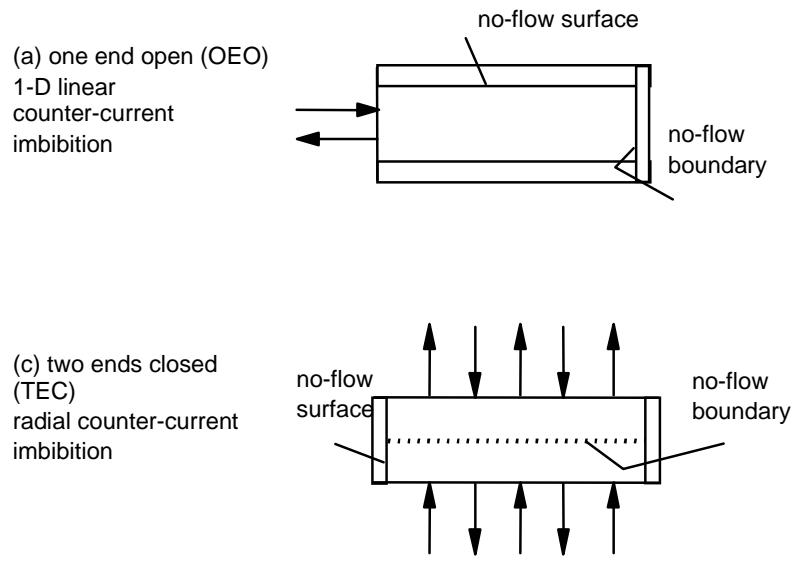

(c) two ends closed (TEC) radial counter-current imbibition
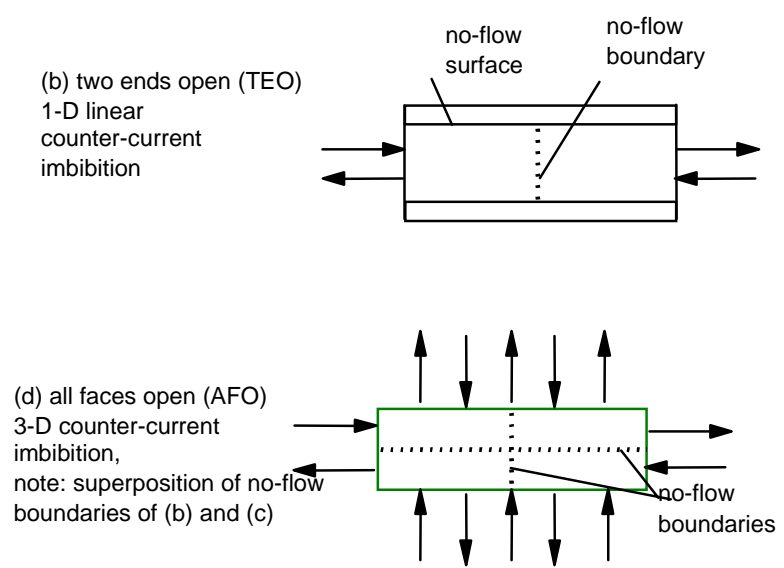

Figure III-3.1. Countercurrent imbibition into cores with varying boundary conditions.

An example of the effectiveness of the characteristic length defined by Eq. III-3.1 in correlating imbibition rates for water displacing mineral oil is shown in Fig. III-3.2 using data from Zhang et al. (1995). The amount of oil displaced is expressed as a fraction of the amount of oil initially in the core. 


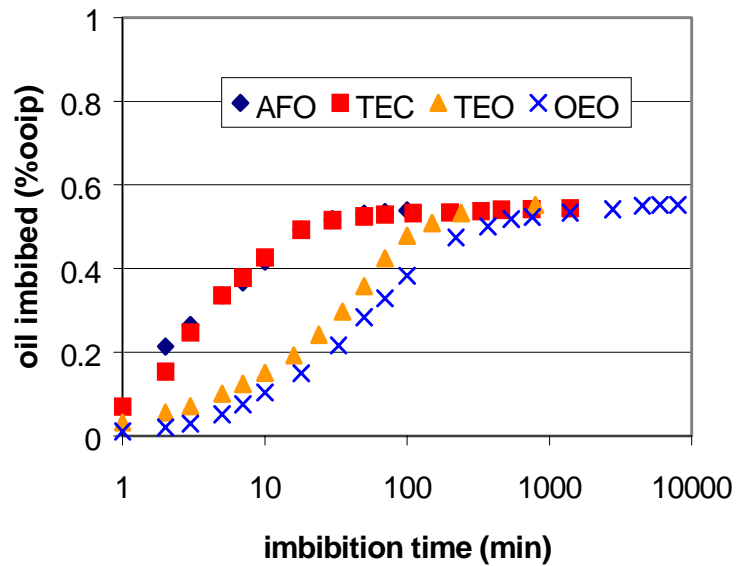

(a)

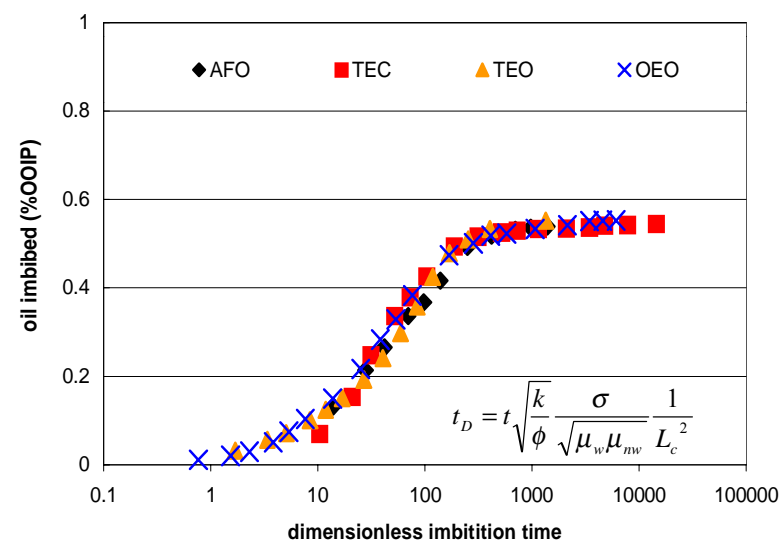

(b)

Figure III-3.2. Imbibition of water into very strongly water-wet cylindrical cores. Boundary conditions are specified using the notation of Fig. III-3.1. Core lengths for these four samples were $L_{\mathrm{AFO}}=1.3059$ $\mathrm{cm} L_{\mathrm{TEC}}=1.3506 \mathrm{~cm}, \mathrm{~L}_{\mathrm{TEO}}=3.8700 \mathrm{~cm}$, and $\mathrm{L}_{\mathrm{OEO}}=4.998 \mathrm{~cm}$; displaced mineral oil had a viscosity of 37.82 cP. (after Zhang et al., 1995).

\section{Results and Discussion}

For tests of imbibition of oil displacing air, cylindrical cores with the four boundary conditions illustrated in Fig. III-3.1 were used. Experimental conditions are summarized in Table III-3.1. Mixtures of mineral oils were used to produce a range of viscosities of the wetting phase.

Three cores with boundary conditions of AFO, TEO and OEO were tested with an $11.8 \mathrm{cP}$ viscosity mineral oil displacing air. The cores all had the same diameter $(3.82 \mathrm{~cm})$ and similar lengths $(7.7$ to $8.95 \mathrm{~cm})$. $\mathrm{L}_{\mathrm{c}}$ ranged from 1.27 to $8.95 \mathrm{~cm}$. The core with all faces open gave the fastest imbibition rate and that with one end open was the slowest, as expected (Fig. III3.4a). Using $\mathrm{L}_{\mathrm{c}}$ to account for the geometrical differences, however, does not completely correlate the differences among these three curves, as shown in Fig. III-3.4b.

In Fig. III-3.5, cores with boundary conditions of TEC and TEO are compared for a 68.1 cP oil displacing air. The rock properties and sizes of the two cores are nearly identical; boundary condition is the only significant difference between these two cases. Figure 5b shows that a wide separation remains between the curves even after the correlation is applied.

Another example is shown in Fig. III-3.6 for four SW series cores. Rock properties and sample sizes were all equal (Table III-3.1); the tests were distinguished only by the four different boundary conditions. In this case the oil used was Soltrol-220 (4.32 cP viscosity). The four imbibition curves are brought closer together by application of the correlation, but there is still distinct separation. 
Table III-3.1. Experimental conditions for a strongly oil-wet oil/air/rock system

\begin{tabular}{|c|c|c|c|c|c|c|c|c|}
\hline Core No. & B.C. & $\mathbf{L}, \mathbf{c m}$ & $\mathbf{d}, \mathbf{c m}$ & $\mathbf{L c}, \mathbf{c m}$ & $\mathbf{k , m d}$ & $\mathbf{p o r} \mathbf{}$ ity, $\boldsymbol{\%}$ & $\mathbf{V i s c o s i t y , ~} \boldsymbol{\mu , \mathbf { c P }}$ & $\boldsymbol{\sigma}, \mathbf{m N} \mathbf{m}$ \\
\hline SW-1 & AFO & 7.56 & 3.82 & 1.26 & 362.8 & 20.5 & 4.32 & 25.82 \\
\hline SW-3 & TEO & 7.12 & 3.82 & 3.56 & 362.8 & 20.5 & 4.32 & 25.82 \\
\hline SW-4 & OEO & 7.67 & 3.82 & 7.67 & 362.8 & 20.5 & 4.32 & 25.82 \\
\hline SW-7 & TEC & 6.72 & 3.82 & 1.34 & 362.8 & 20.5 & 4.32 & 25.82 \\
\hline R2-2 & AFO & 5.13 & 2.63 & 0.87 & 325 & 20.4 & 163.2 & 31.46 \\
\hline R2-3 & AFO & 7.59 & 3.82 & 1.27 & 325 & 20.4 & 163.2 & 31.46 \\
\hline R2-4 & AFO & 8.79 & 4.39 & 1.46 & 325 & 20.4 & 163.2 & 31.46 \\
\hline R2-5 & AFO & 10.2 & 5.03 & 1.68 & 325 & 20.4 & 163.2 & 31.46 \\
\hline R2-6 & OEO & 7.7 & 3.82 & 7.7 & 325 & 20.4 & 163.2 & 31.46 \\
\hline CW-2 & OEO & 8.95 & 3.82 & 8.95 & 311 & 20.6 & 11.8 & 27.21 \\
\hline CW-9 & OEO* & 7.67 & 3.82 & 7.67 & 311 & 20.6 & 163.2 & 31.46 \\
\hline CW-6 & TEO & 7.7 & 3.82 & 3.85 & 311 & 20.6 & 11.9 & 27.21 \\
\hline CW-10 & AFO & 7.83 & 3.82 & 1.27 & 311 & 20.6 & 11.9 & 27.21 \\
\hline CW-13 & TEO & 7.7 & 3.82 & 3.85 & 311 & 20.6 & 68.1 & 29.98 \\
\hline CW-14 & TEC & 7.75 & 3.82 & 1.35 & 311 & 20.6 & 68.1 & 29.98 \\
\hline
\end{tabular}

*In core CW-9 all faces except one were sealed; a teflon tube provided a means for air to escape from the face opposite the open face.

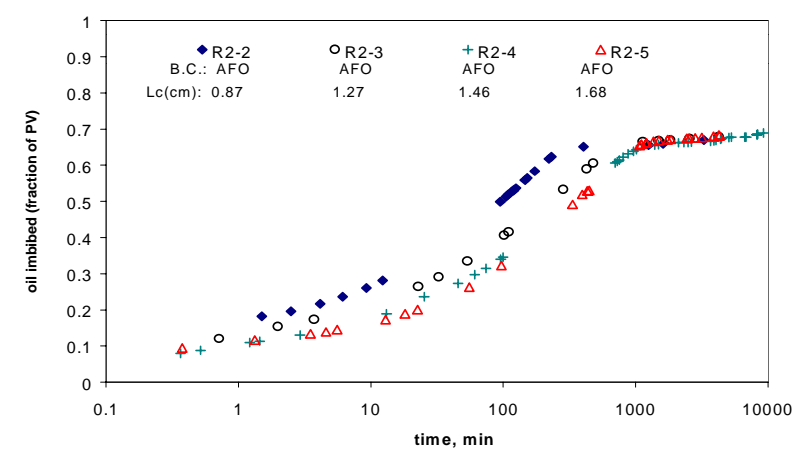

(a) Rate varies with sample size.

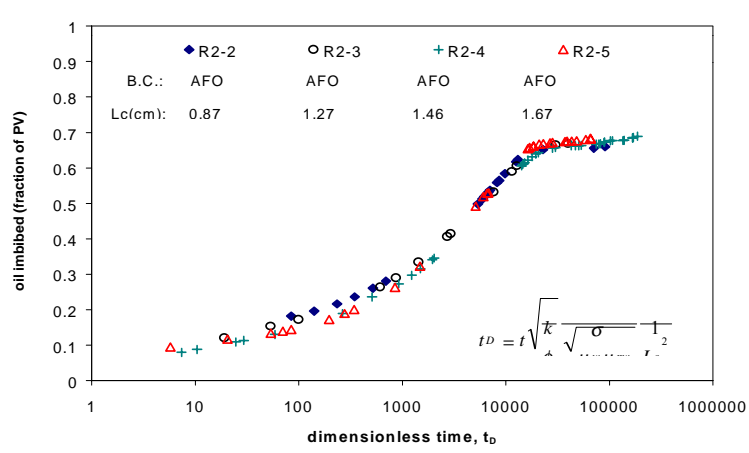

(b) Rates correlated by $\mathrm{L}_{c}$, as defined in Eq. III-3.1.

Figure III-3.3. Rate of imbibition of $163.2 \mathrm{cP}$ mineral oil into air-filled cylindrical cores of Berea sandstone. 


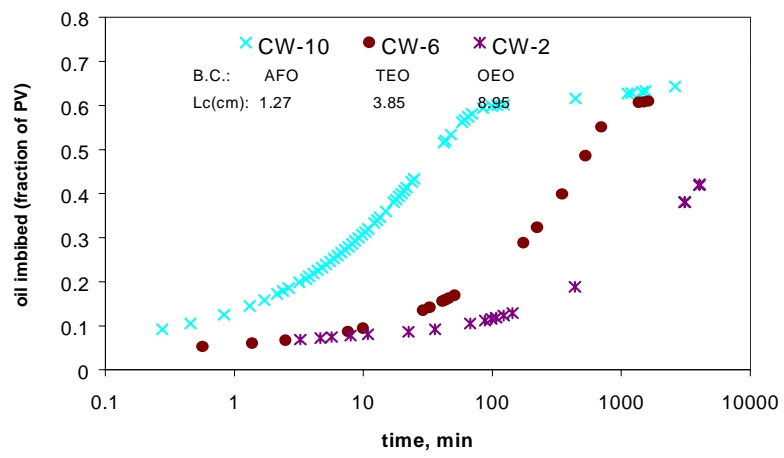

(a) Rate varies with boundary condition.

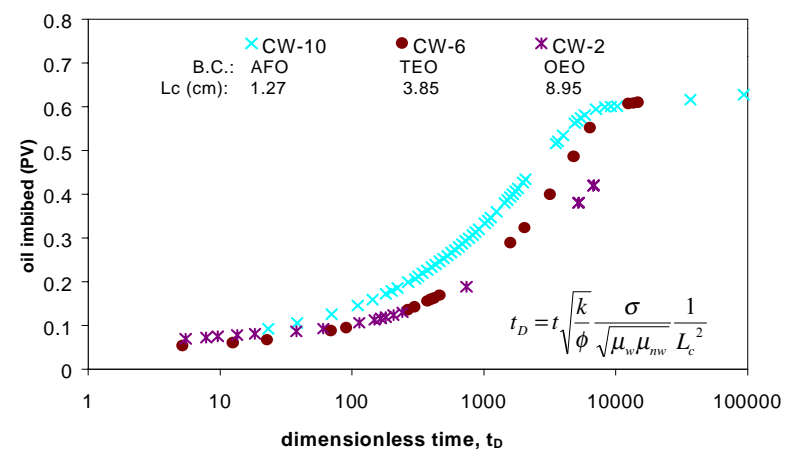

(b) Rates only partially correlated by $\mathrm{L}_{c}$,

Figure III-3.4. Rate of imbibition of $11.8 \mathrm{cP}$ mineral oil into air-filled cylindrical cores of Berea sandstone.

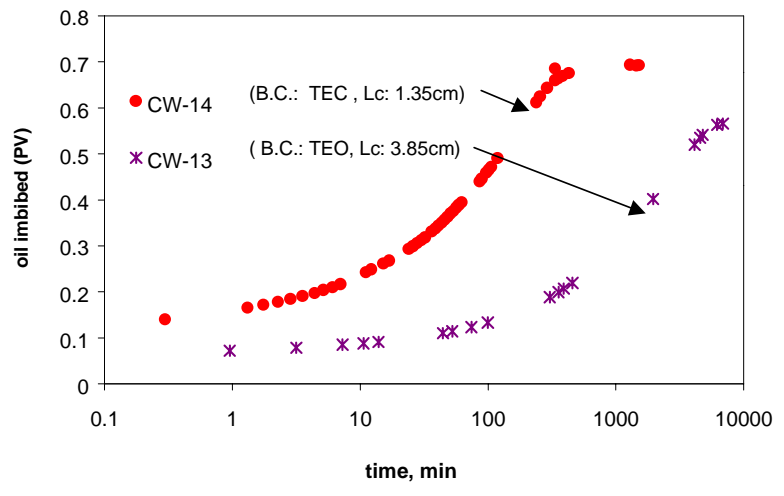

(a) Rate varies with boundary condition.

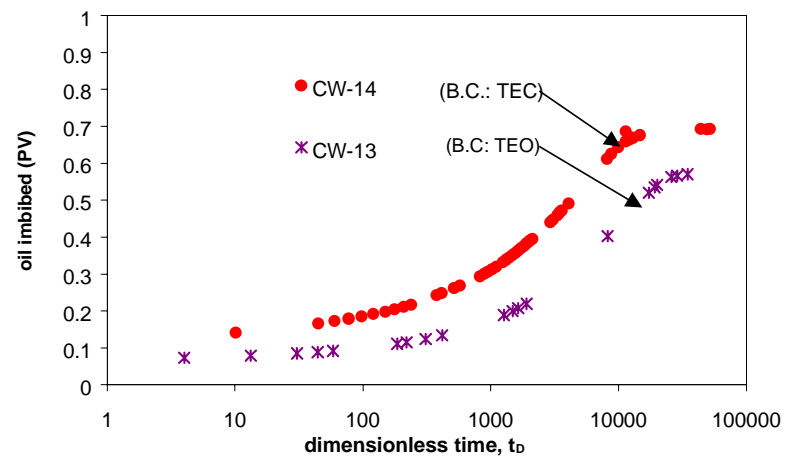

(b) Rates only partially correlated by $\mathrm{L}_{\mathrm{c}}$,

Figure III-3.5. Rate of imbibition of $68.1 \mathrm{cP}$ mineral oil into air-filled cylindrical cores of Berea sandstone.

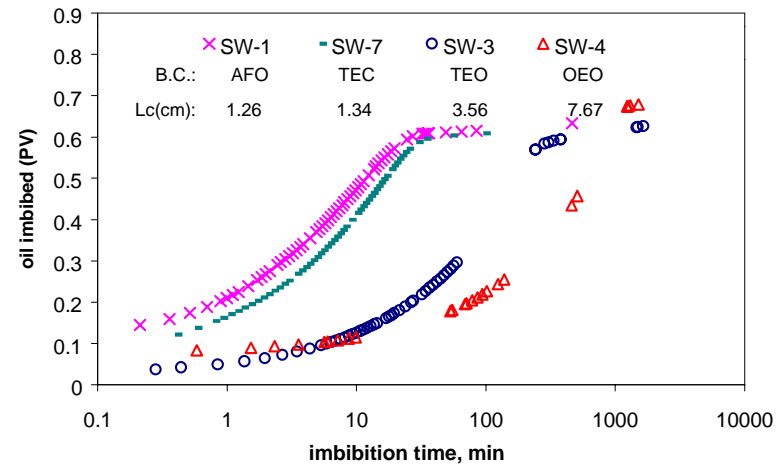

(a) Rate varies with boundary condition.

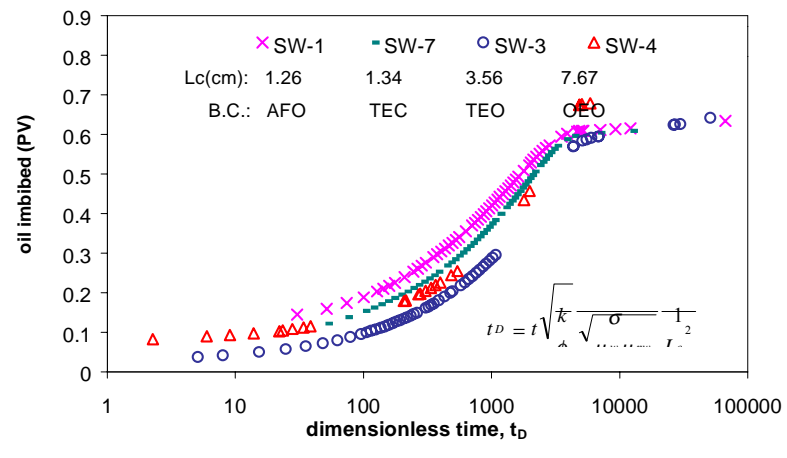

(b) Rates only partially correlated by $\mathrm{L}_{\mathrm{c}}$,

Figure III-3.6. Rate of imbibition of $4.32 \mathrm{cP}$ Soltrol 220 mineral oil into air-filled cylindrical cores of Berea sandstone. 
We next consider why $L_{c}$ is less effective in correlating strongly-wetted oil/air displacement rates than it was for water/oil imbibition tests. Several differences between the oil/air and water/oil systems are evident that may or may not play a role. The viscosity of gas $(0.018 \mathrm{cP})$ is much smaller than any of the liquid viscosities. Densities are more dissimilar in the oil/air case than in the water/oil cases. Finally gas, unlike water or oil, is highly compressible. The possibility that this may affect the mechanism of two-phase flow and entrapment of gas during imbibition was therefore tested.

The effect of compression of the non-wetting phase was tested by preparing two OEO cores, R2-6 and CW-9, with essentially the same rock properties and sizes. A hole was drilled through the sealed end of CW-9 of length equal to about one-third of the core length (about 2.5 $\mathrm{cm})$. A tube was inserted in the hole and sealed around the periphery at the point of entrance (see Fig. III-3.7). This permitted air in the otherwise sealed end to vent, so the pressure of untrapped gas in the core could rise only slightly above atmospheric.

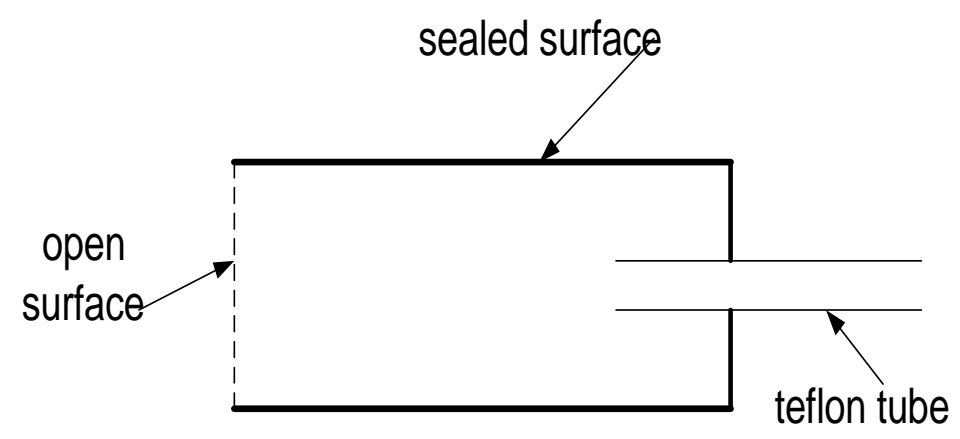

Figure III-3.7. Schematic diagram illustrating vent tube inserted into core CW-9 with OEO boundary conditions.

Figure III-3.8 shows that the imbibition rate for the core CW-9 with the vent is much higher than for the OEO core R2-6. An AFO core of the same properties and size, R2-3, is also shown for comparison. The imbibition rate for the OEO (vented) core is still less than for the AFO core. Correlated results based on $\mathrm{L}_{\mathrm{c}}$ are shown in Fig. 8b. As for results presented in Fig. 6, the OEO core (R2-6) does not correlate with the AFO core (R2-3).

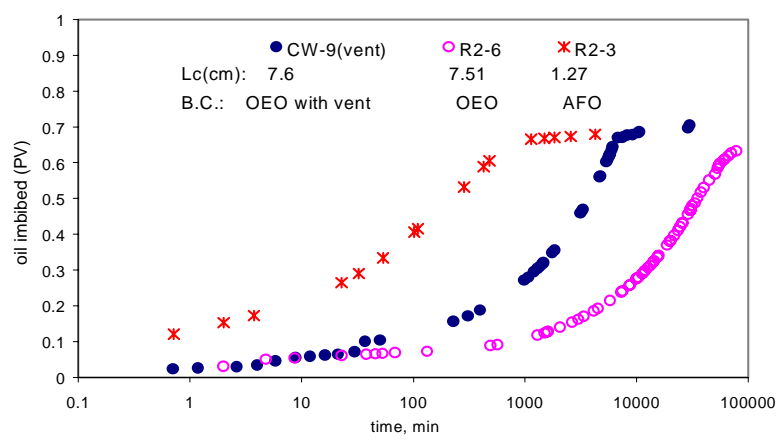

(a) Rate varies with boundary condition.

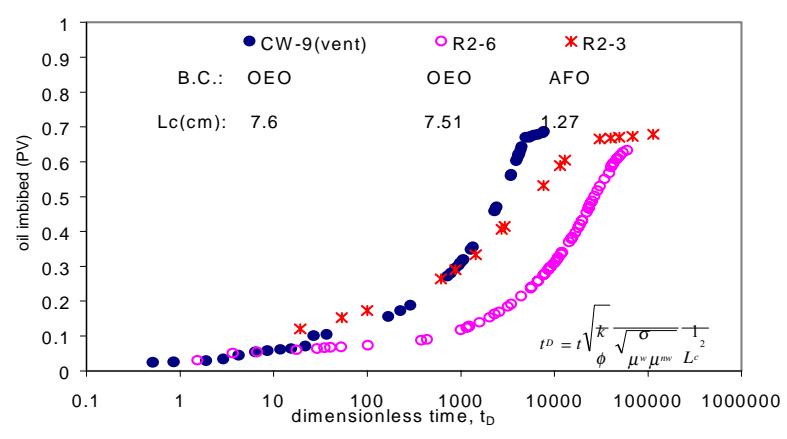

(b) Rates partially correlated by $\mathrm{L}_{\mathrm{c}}$,

Figure III-3.8. Rate of imbibition of $163.2 \mathrm{cP}$ mineral oil into air-filled cylindrical cores of Berea sandstone with and without addition of a vent. 
Three stages can be identified for imbibition into the CW-9 OEO (vented) core, based on the scaled results. Initially the rates for both OEO cores, CW-9 and R2-6, are identical. After about $10 \%$ of the air has been produced, the rate for the vented core, CW-9, rises above that for R2-6 and correlates with the AFO core, R2-3. Beyond $40 \%$ air recovery, the rate in the vented core exceeds that for the AFO core, perhaps reflecting the effect of compression in the unvented cores. Improvements in correlation of imbibition rate, which explicitly account for the effect of gas compression, are under investigation.

\section{Conclusions}

- Correlation of imbibition rates in strongly wetting water/oil systems do not adequately account for the phenomena occurring in oil/air systems.

- Imbibition rates for oil displacing air in cores of different sizes, but with similar geometry, can be correlated using the scaling rules established for water displacing oil in strongly water-wet cores (although the resulting correlation is different than that for water/oil systems).

- Cores with different boundary conditions had rates of imbibition that could not be correlated by the existing scaling rules, apparently because of the effects of gas compressibility.

- Allowing air to escape, instead of its being trapped at elevated pressure, improved correspondence between rates in cores with varying boundary conditions.

\section{References}

Kazemi, H., Gilman, J.R., and Eisharkawy, A.M.: “Analytical and Numerical Solution of Oil Recovery from Fractured Reservoirs with Empirical Transfer Functions," SPERE (May, 1992) 219.

Ma, S., Morrow, N.R., and Zhang, X.: "Generalized Scaling of Spontaneous Imbibition Data for Strongly Water-Wet Systems," J. Petr. Sci. and Eng. (1997) 18, 165-178.

Ma, S., Zhang, X., and Morrow, N.R.: "A Characteristic Length for Scaling of Mass Trnsfer between Rock Matrix and Fractures," paper SPE 30232 (1995).

Mattax, C.C. and Kyte, J. R.: "Imbibition Oil Recovery from Fractured, Waterdrive Reservoirs," SPEJ (June 1962) 177.

Zhang, X., Morrow, N.R., and Ma, S.: "Experimental Verification of a Modified Scaling Group for Spontaneous Imbibition," paper SPE 30762 presented at the 1995 SPE ATCE, Dallas, 2225 Oct. 


\title{
III-3.2. Oil Recovery by Spontaneous Imbibition from Weakly Water-Wet Rocks (MWX)
}

\author{
Xie, X. and Morrow, N.R.: “Oil Recovery by Spontaneous Imbibition from Weakly Water-Wet \\ Rocks," paper SCA 0026, presented at the 2000 International Symposium of the Society of Core \\ Analysts, Abu Dhabi, 18-22 Oct.
}

Spontaneous imbibition is of special importance to oil recovery from fractured reservoirs. Laboratory measurements of volume of liquid imbibed versus time are often used in the prediction of oil recovery. Imbibition measurements also provide a useful approach to the complex problem of characterizing the wetting properties of porous media. Correlation of a large body of data for imbibition of brine into porous media initially saturated with refined oil was achieved through a semi-empirical scaling group which includes permeability, porosity, interfacial tension, oil and brine viscosity, and the size, shape and boundary conditions of the sample.

The objective of the present study was to test this correlation for samples of different geometry for spontaneous imbibition under weakly water-wet conditions established by adsorption from crude oil with initial water saturation ranging from $14 \%$ to $31 \%$. After establishing initial water saturations, the wettability of thirty-two core samples was changed to weakly water-wet by aging in an asphaltic crude oil at elevated temperature. Initial water saturation had a dominant effect on rate of oil recovery. Times for imbibition decreased by about 2 to 4 orders of magnitude with decrease in initial water saturation. Results for cores with the same initial water saturation but of different size and shape (cylindrical, annular, and rectangular) and boundary conditions (given by sealing off part of the rock surface with epoxy resin) were closely correlated. The presence of epoxy resin during aging in crude oil enhanced the decrease in water-wetness attained for cores and crude oil alone. The contribution to oil recovery by gravity segregation at very weakly water-wet conditions is addressed.

\section{Introduction}

Rate and extent of spontaneous imbibition provides a measure of wettability that is inherently dependent on surface energy. However, the mechanism of imbibition is highly complex and many factors besides wettability affect the rate of spontaneous imbibition and displacement efficiency. In previous studies, a semi-empirical scaling group was developed for recovery of oil from very strongly water-wet (VSWW) media (Ma et al., 1997). Dimensionless time, $\mathrm{t}_{\mathrm{D}}$, was defined by,

$$
t_{D}=t \sqrt{\frac{k}{\phi}} \frac{\sigma}{\sqrt{\mu_{o} \mu_{w}}} \frac{1}{L_{c}^{2}}
$$

where $\mathrm{t}$ is the imbibition time; $\sqrt{k / \phi}$ is proportional to a microscopic pore radius, where $\mathrm{k}$ is permeability and $\phi$ is porosity; $\sigma$ is the interfacial tension; $\sqrt{\mu_{o} \mu_{w}}$ is the geometric mean of the oil and brine viscosity. $\mathrm{L}_{\mathrm{c}}$ is a characteristic length that compensates for sample size, shape and boundary conditions, and is defined by $L_{c}=\sqrt{V /\left(\sum_{i=1}^{n} A_{i} / x_{A i}\right)}$, where $V$ is the bulk volume of the 
core, $A_{i}$ is the open area of the $i$ th face, and $x_{A i}$ is the distance from the no-flow boundary to the open face.

This scaling relationship has been shown to correlate imbibition data with variations in porosity, permeability, boundary conditions, and liquid viscosity ratios. The correlation was initially developed mainly for VSWW conditions with zero initial water saturation. Changes in dimensionless time for imbibition with change in water saturation at VSWW conditions have also been investigated (Viksund et al., 1998).

The applicability of the scaling group to wettability conditions other than VSWW needs to be investigated. Most oil reservoirs are considered to have some form of mixed wettability (Salathiel, 1973). Mixed wettability arises because the distribution of connate water controls which parts of the rock surface are exposed to adsorption from crude oil. As the connate water saturation decreases, the exposed area increases and so change in wetting increases. Change in the disjoining pressure of aqueous films with the increase in capillary pressure that accompanies decrease in water saturation may also be a factor in wettability alteration (Melrose, 1982; Kovscek et al., 1993).

Wettability change from VSWW conditions to mixed-wet can be induced by adsorption from crude oil (Jadhunandan and Morrow, 1991, 1995; Zhou et al., 1995, 2000). Changes in wettability can exhibit a wide spectrum of wetting behavior. The changes have been shown to depend on the crude oil type, the rock, aging time, temperature of aging, $\mathrm{T}_{\mathrm{a}}$, and of measurement, $\mathrm{T}_{\mathrm{m}}$, the brine composition and concentration, and the initial water saturation.

Experimental studies have shown that if a sandstone core is aged at ambient temperature,

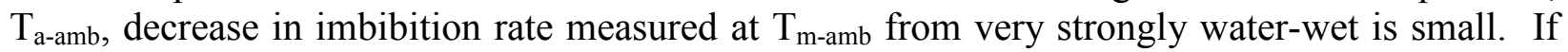
the core is aged at elevated temperature, $T_{a-h i g h}$, the imbibition rate at $T_{m-a m b}$ is reduced by up to several orders of magnitude relative to VSWW conditions. If the imbibition rate, for a core aged at $\mathrm{T}_{\mathrm{a} \text {-high }}$ is measured at $\mathrm{T}_{\mathrm{m} \text {-high }}$, the imbibition rate is much higher than that measured at $\mathrm{T}_{\mathrm{m} \text {-amb }}$ (Tang and Morrow, 1997). The controlling mechanisms for these differences are not well understood. In the present study, the conditions $\mathrm{T}_{\mathrm{a} \text {-high }}$ and $\mathrm{T}_{\mathrm{m} \text {-amb }}$ were chosen to provide weakly water-wet conditions that were readily distinguished from imbibition behavior for VSWW rocks. All conditions were held essentially constant except for initial water saturation, sample geometry, and boundary conditions.

\section{Experimental}

Cores: The rock samples in this study, 32 in all, were cut from the same block of Berea sandstone which had been tested for uniformity of properties. Different shapes of cores were prepared, including 1", 1.5 " and 2" diameter cylindrical plugs of different lengths (some chosen to give cylinders of similar geometry), 2" outside diameter and 1" inside diameter annular plugs and rectangular blocks. Core dimensions and petrophysical properties are listed in Table 3.1. Some of the cores were partially coated with epoxy resin to obtain selected boundary conditions. The air permeabilities of the cores were all about $450 \mathrm{md}$, and the porosities were all close to $20 \%$. 
Brine: Synthetic sea water containing $\mathrm{NaCl}$ (28000 ppm), $\mathrm{KCl}$ (935 ppm), $\mathrm{MgCl}_{2}$ (5365 ppm), $\mathrm{CaCl}_{2}$ (1190 ppm), and $100 \mathrm{ppm}$ of $\mathrm{NaN}_{3}$ as biocide, was used as the brine phase. The total dissolved solids content was $35490 \mathrm{ppm}$. The interfacial tension between A95 crude oil and the synthetic seawater was 25.6 dynes/cm at room temperature measured by the du Nouy ring method.

Crude oil: An asphaltic crude oil, Alaska 95 (A95) from Prudhoe Bay, was used to change the wettability of the cores. The oil was degassed by vacuum treatment. The oil had $6.55 \mathrm{wt} \% \mathrm{of} n-$ heptane asphaltenes and no detectable wax content. The viscosity of the evacuated oil was 40.3 $\mathrm{cp}$ at room temperature $\left(22^{\circ} \mathrm{C}\right)$ and $18.5 \mathrm{cp}$ at $55^{\circ} \mathrm{C}$. The acid and base numbers were 0.24 and 2.2 respectively. The API gravity of the oil was 25.2. This oil was selected because it was known to induce significant change in wettability and would not give problems associated with wax deposition even at ambient temperature.

Core surfaces partially sealed with epoxy resin: Devcon 5 minute epoxy was used to partially seal the core surfaces to give different boundary conditions. The components are bisphenol (a diglycidyl ether resin), and a hardener that contained 2,4,6-tri (dimethylaminomethyl) phenol and polymercaptan curing agent. The partially sealed cores were set at room temperature to dry. The cores were then rinsed with tap water and dried at $110^{\circ} \mathrm{C}$. The effect on core wettability of exposing the crude oil during aging to a block of the hardened epoxy resin was also tested.

Establishing initial water saturation: The core samples were first saturated with the synthetic sea water by vacuum. They were then left in the brine for about 10 days to attain ionic equilibrium. A porous plate apparatus (Soil Moisture ceramic plate extractor) was used to establish the initial water saturation. Contact with the plate was ensured by seating the lower end of a core in a very thin layer of fine cuttings obtained from Berea sandstone. Pressure differences of 10, 25, 40 and 55 psi, were applied sequentially (the capillary pressure in the core was always well below the applied pressure). Initial water saturations established by this procedure (after up to 10 days) ranged from $14-30 \%$ (see Table 3.1 ).

Saturation with crude oil: After establishing $\mathrm{S}_{\mathrm{wi}}$, the cores were saturated with A95 crude oil under vacuum. Any core that was determined, from mass balance, to be not fully saturated, was set under crude oil in a stainless steel aging cell at 800 psi for up to 3 days to ensure full saturation by liquids.

Aging: The cores containing initial water and crude oil were submerged in crude oil in aging cells which were then sealed. All of the cores were aged at $55^{\circ} \mathrm{C}$ for 10 days.

Imbibition test: After aging, the cores were set in glass imbibition cells filled initially with brine. All of the imbibition tests were performed at room temperature. Oil volume produced by imbibition of brine (expressed as percentage of original oil in place - \%OOIP) versus time was recorded. 


\section{Results and Discussion}

Conditions for 32 imbibition tests are listed in Table III-3.2 according to general classes of boundary conditions.

Table III-3.2 Core sample dimensions and properties ( $\left.\mathrm{k}_{\mathrm{g}} \cong 450 \mathrm{md}, \phi \cong 20 \%\right)$.

\begin{tabular}{|c|c|c|c|c|c|c|c|}
\hline Core No. & $\begin{array}{l}\mathrm{L}, \mathbf{c m} \\
\text { or } \mathrm{L}_{1}\end{array}$ & $\begin{array}{l}\text { D, cm } \\
\text { or } L_{2}\end{array}$ & $\begin{array}{l}\text { d, } \text { cm }^{*} \\
\text { or } L_{3}\end{array}$ & $\phi, \%$ & $\mathbf{L}_{\mathrm{c}}$ & $\begin{array}{c}\mathrm{S}_{\mathrm{wi}}, \\
\%\end{array}$ & Boundary conditions \\
\hline \multicolumn{8}{|c|}{ All faces open: } \\
\hline S15-3 & 7.802 & 3.808 & - & 20.47 & 1.2727 & 31.2 & AFO (all faces open) \\
\hline S2-3 & 5.884 & 5.03 & - & 19.91 & 1.5219 & 30.5 & $\mathrm{AFO}$ \\
\hline S15-14 & 7.660 & 3.808 & - & 19.97 & 1.2701 & 27.6 & AFO \\
\hline S15-5 & 7.812 & 3.808 & - & 20.24 & 1.2728 & 22.2 & $\mathrm{AFO}$ \\
\hline S15-16 & 7.836 & 3.808 & - & 20.8 & 1.2730 & 20.5 & $\mathrm{AFO}$ \\
\hline S15-4 & 7.543 & 3.808 & - & 20.14 & 1.2680 & 20.3 & AFO \\
\hline SA2-3 & 5.682 & 5.03 & 3.014 & 20.55 & 0.9500 & 19 & AFO \\
\hline $\mathrm{S} 2-8$ & 4.721 & 5.03 & - & 20.27 & 1.4204 & 18.7 & AFO \\
\hline S2-12 & 4.390 & 5.03 & - & 20.34 & 1.3818 & 18.1 & $\mathrm{AFO}$ \\
\hline $\mathrm{SS} 3 * *$ & $\mathrm{~L}_{1}=3.22$ & $\mathrm{~L}_{2}=4.49$ & $\mathrm{~L}_{3}=5.44$ & 18.84 & 1.1790 & 18 & AFO \\
\hline SA2-4 & 4.293 & 5.03 & 3.014 & 19.84 & 0.9124 & 16 & $\mathrm{AFO}$ \\
\hline S1-7 & 4.890 & 2.618 & - & 20.28 & 0.8656 & 15 & $\mathrm{AFO}$ \\
\hline S2-14 & 3.612 & 5.03 & - & 20.39 & 1.2670 & 14.1 & $\mathrm{AFO}$ \\
\hline S2-10 & 4.520 & 5.03 & - & 20.67 & 1.3976 & 14.9 & $\mathrm{AFO}$ \\
\hline \multicolumn{8}{|c|}{ Partially sealed: } \\
\hline S15-1 & 7.948 & 3.808 & - & 20.03 & 3.9740 & 27.1 & TEO (two ends open) \\
\hline S2-11 & 3.744 & 5.03 & - & 20.3 & 1.0273 & 23.5 & TEO \\
\hline S2-1 & 6.462 & 5.03 & - & 20.47 & 3.2310 & 23 & TEO \\
\hline S15-18 & 6.023 & 3.808 & - & 20 & 3.0115 & 21 & TEO \\
\hline S2-5 & 4.633 & 5.03 & - & 20.51 & 2.3165 & 21 & TEO \\
\hline S1-5 & 4.599 & 2.622 & - & 19.7 & 2.2995 & 20.8 & TEO \\
\hline S2-6 & 4.612 & 5.03 & - & 20.16 & 1.7784 & 20.4 & TEC (two ends closed) \\
\hline S1-3 & 4.760 & 2.622 & - & 19.56 & 2.3800 & 19.7 & TEO \\
\hline S15-6 & 7.451 & 3.808 & - & 20.89 & 1.3463 & 20.3 & TEC \\
\hline SS1 & $\mathrm{L}_{1}=4.30$ & $\mathrm{~L}_{2}=3.00$ & $\mathrm{~L}_{3}=5.42$ & 19 & 2.7115 & 18.5 & $\begin{array}{l}\text { Two adjacent faces sealed } \\
\mathrm{L}_{1} / \mathrm{L}_{3} \& \mathrm{~L}_{2} / \mathrm{L}_{3}\end{array}$ \\
\hline S2-9 & 4.273 & 5.03 & - & 20.7 & 2.1365 & 18.2 & TEO \\
\hline SS4 & $\mathrm{L}_{1}=5.56$ & $\mathrm{~L}_{2}=4.41$ & $\mathrm{~L}_{3}=3.50$ & 19.25 & 1.9534 & 18.1 & $\begin{array}{l}\text { TEO, both } \mathrm{L}_{2} / \mathrm{L}_{3} \text { ends } \\
\text { open }\end{array}$ \\
\hline S15-9 & 6.451 & 3.808 & - & 20.47 & 1.3463 & 17.8 & TEC \\
\hline S1-4 & 4.837 & 2.618 & - & 19.9 & 0.9256 & 17.7 & TEC \\
\hline S1-1 & 5.068 & 2.622 & - & 20.3 & 0.927 & 17.3 & TEC \\
\hline S15-15 & 6.552 & 3.808 & - & 19.9 & 1.3463 & 17 & TEC \\
\hline SA2-1 & 5.823 & 5.03 & 3.014 & 20.1 & 0.504 & 16.8 & TEC \\
\hline S1-8 & 5.143 & 2.618 & - & 19.9 & 2.5715 & 16.8 & TEO \\
\hline
\end{tabular}

*inner diameter of the annular core

** rectangular core with dimensions $\mathrm{L}_{1}, \mathrm{~L}_{2}$ and $\mathrm{L}_{3}$

\#AFO core aged with epoxy resin in crude oil during aging 


\section{All-faces-opens cores (AFO)}

Recovery by spontaneous imbibition versus time for weakly water-wet cores with all faces open are presented in Fig.III-3.9a. Imbibition time increased systematically over about three orders of magnitude with decrease in $\mathrm{S}_{\mathrm{wi}}$ from $31.2 \%$ to $14.1 \%$. (Table III-3.2)

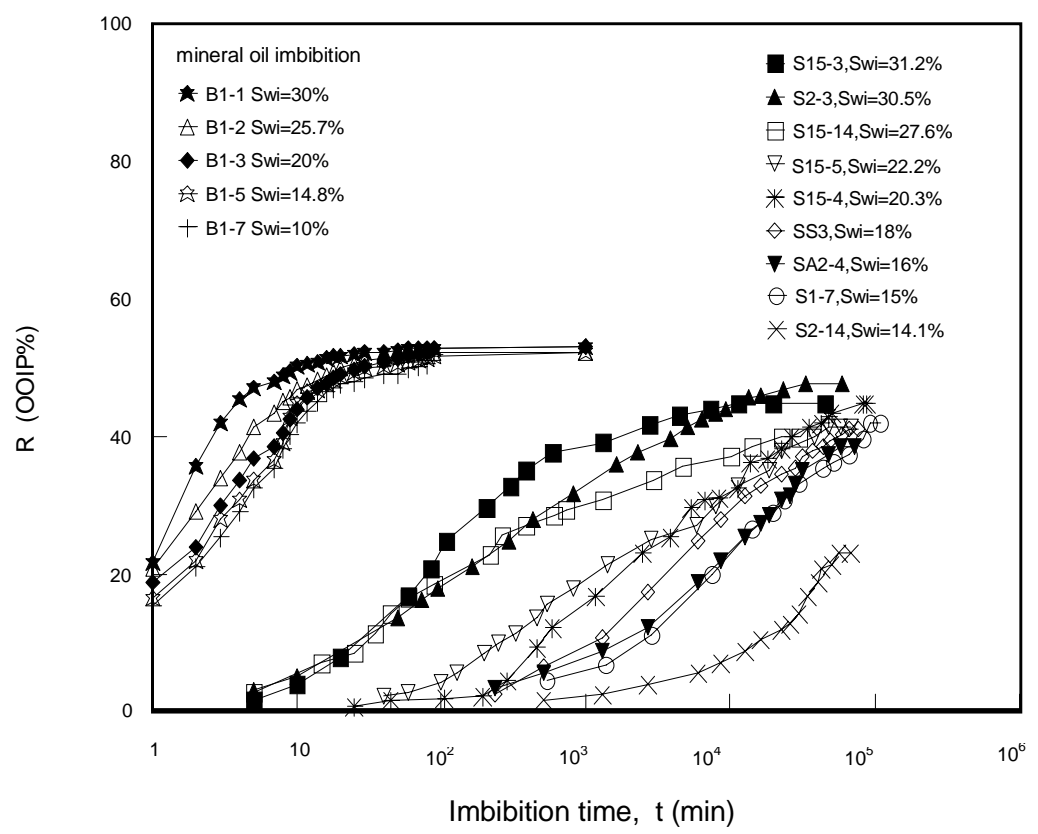

(a) Oil recovery vs. imbibition time

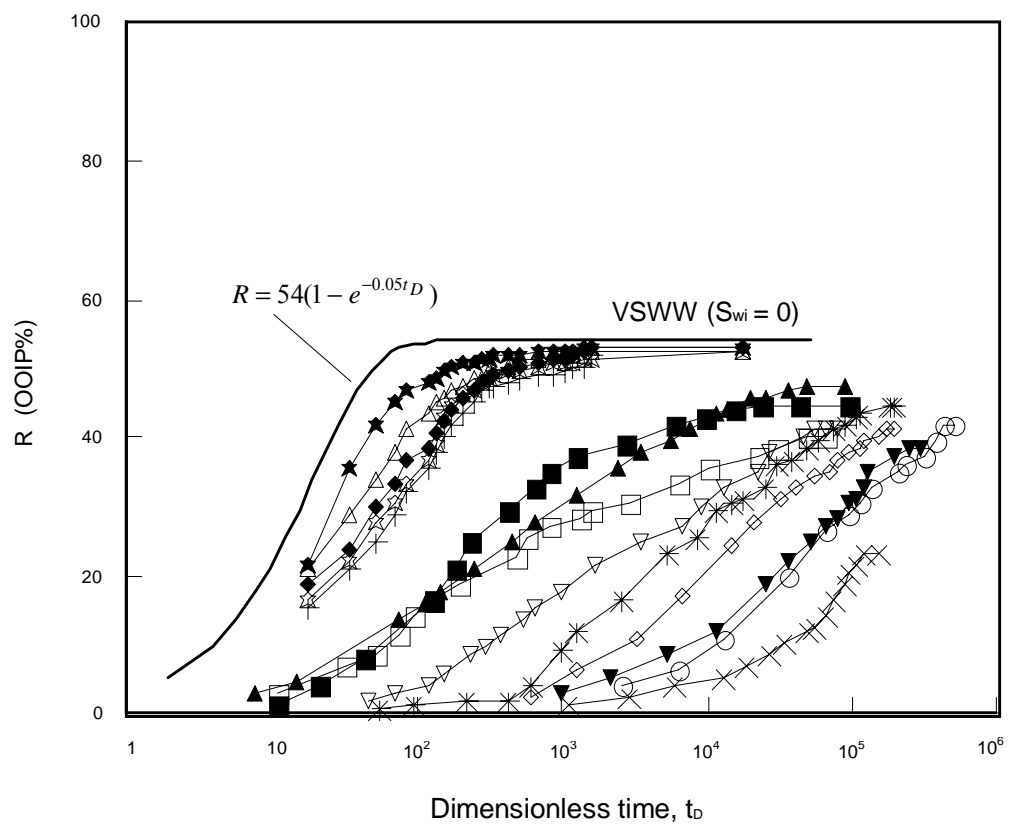

(b) Scaled imbibition curves

Figure III-3.9. Sensitivity of spontaneous imbibition to initial water saturation at weakly water-wet conditions for cores with all faces open. 
Results (Viksund et al, 1998) for recovery of mineral oil from Berea sandstone at very strongly water-wet (VSWW) conditions for $\mathrm{S}_{\mathrm{wi}}$ ranging from $9.6 \%$ to $30 \%$ are included in Fig. III-3.9. Within this range of initial water saturation the imbibition times decrease with increase in $\mathrm{S}_{\mathrm{wi}}$ but by much less than an order of magnitude. These results are shown as plots of recovery versus dimensionless time in Fig. III-3.9b. A general correlation for imbibition at VSWW conditions with zero initial water saturation is also included in Fig. III-3.9b. This correlation is a fit of the model proposed by Aronofsky et al (1958) to a large body of imbibition data (Ma et al., 1997).

For chalk, scaled imbibition curves for $S_{w i}=0$ were in close agreement with results for Berea sandstone, even though the permeability of chalk was over two orders of magnitude less than for sandstone, and the porosity was higher by a factor of about 2 . Increase in $S_{w i}$ in chalk from $0 \%$ to $34 \%$ resulted in increase in imbibition rate (Viksund et al., 1998), but, in contrast to Berea sandstone, scaled imbibition times were all less than for the correlation obtained for $\mathrm{S}_{\mathrm{wi}}=$ 0 . For both chalk and sandstone, the variation in imbibition time with initial water saturation at VSWW conditions was always less than an order of magnitude.

Scaled results for weakly water-wet conditions show a large systematic dependence on initial water saturation. The dominant effect of initial water saturation on the scaled imbibition data, especially for weakly water-wet conditions, requires that, for a meaningful test of scaling by $\mathrm{L}_{\mathrm{c}}$, the initial saturation should be essentially constant. Results for initial water saturation in the range 18.7 to $20.5 \%$ are shown in Fig. III-3.10a. The agreement between results for duplicate tests with cores S15-4 and S15-16 demonstrates that close reproducibility can be obtained. The separation between these results and those for S2-8 and SA2-3 is greatly reduced after scaling by the characteristic length $\mathrm{L}_{\mathrm{c}}$ (Fig. III-3.10b). All other factors in the definition of $t_{D}$ for the results presented in Fig. III-3.10b are very close to constant. 


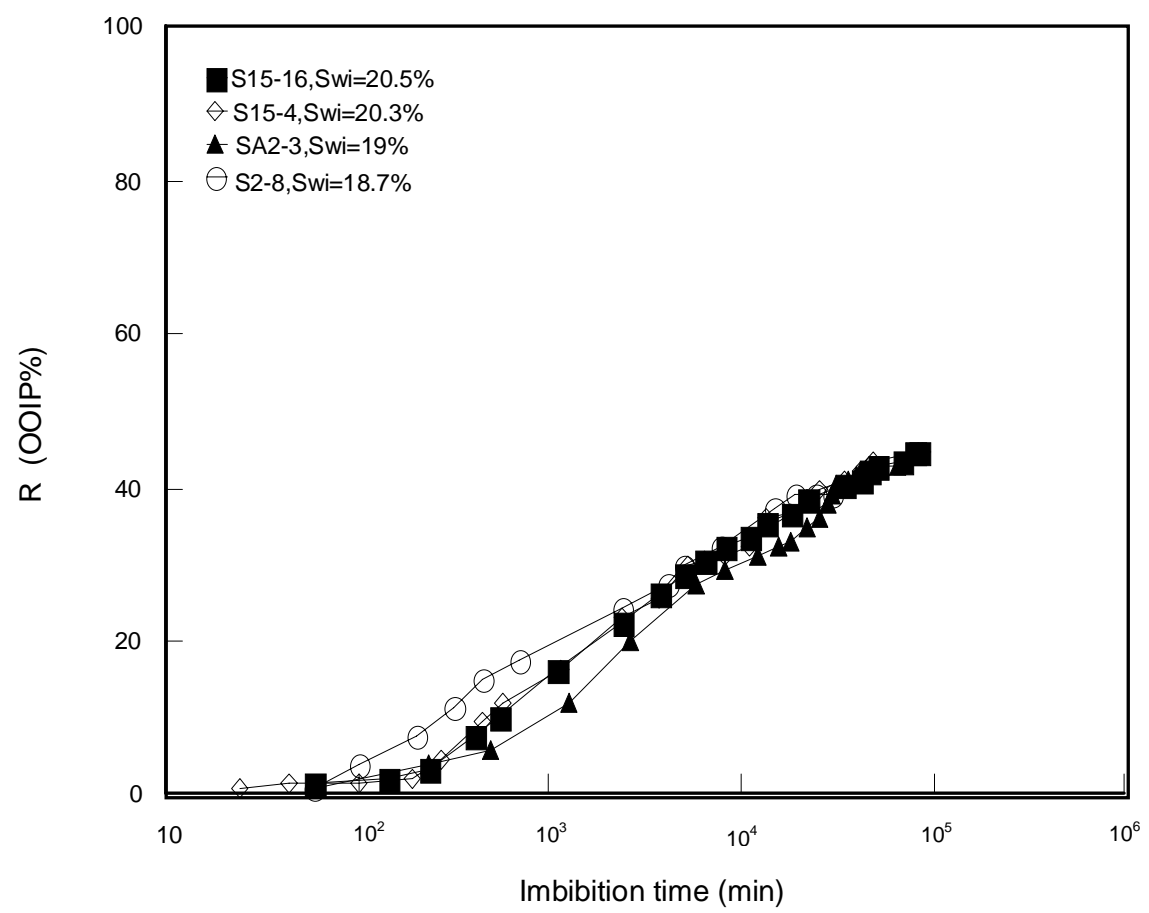

(a) Oil recovery vs. time

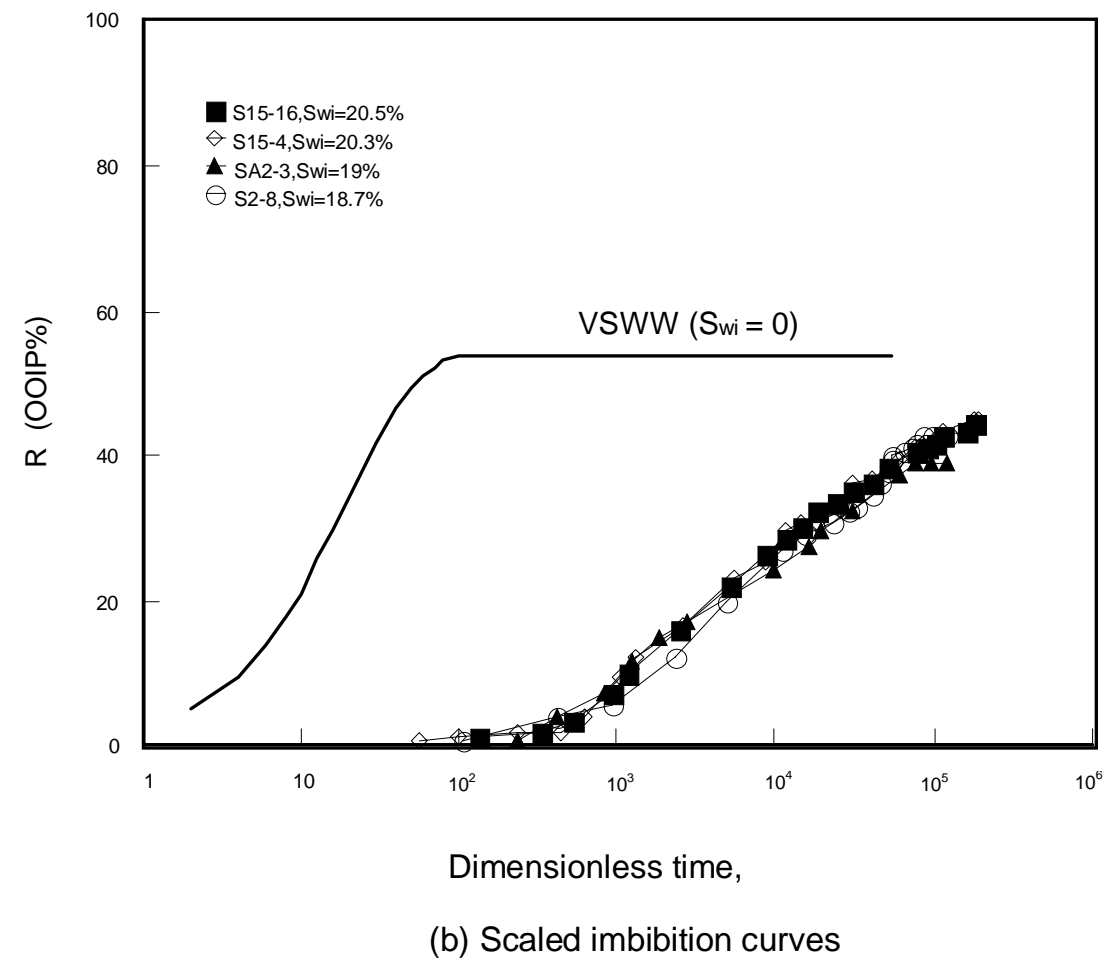

Figure III-3.10. Application of the characteristic length to scaling imbibition data for weakly water-wet cores with all faces open. 
A data set for linear imbibition in cylindrical cores with two-ends-open (TEO) is shown in Fig. III-3.11a. Recovery vs. $t_{D}$ is shown in Fig. III-3.11b. As for AFO cores there is a distinct overall trend; scaled imbibition time increases with decrease in $S_{w i}$.

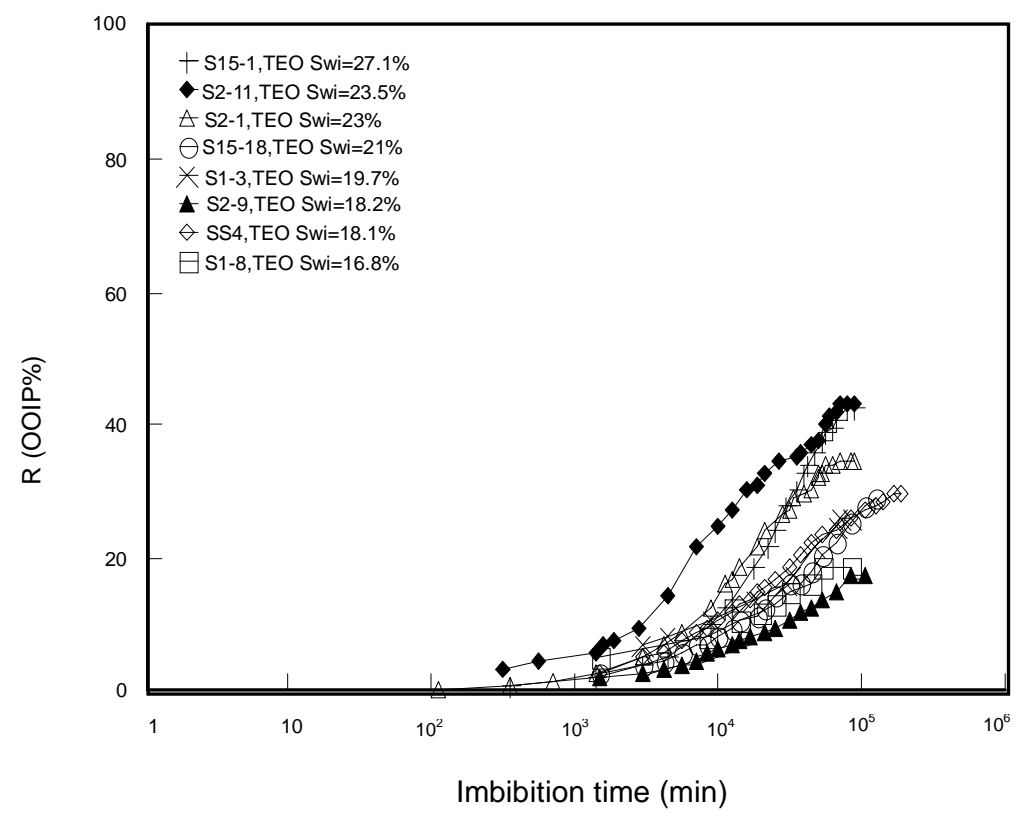

(a) imbibition data for cores with two ends open

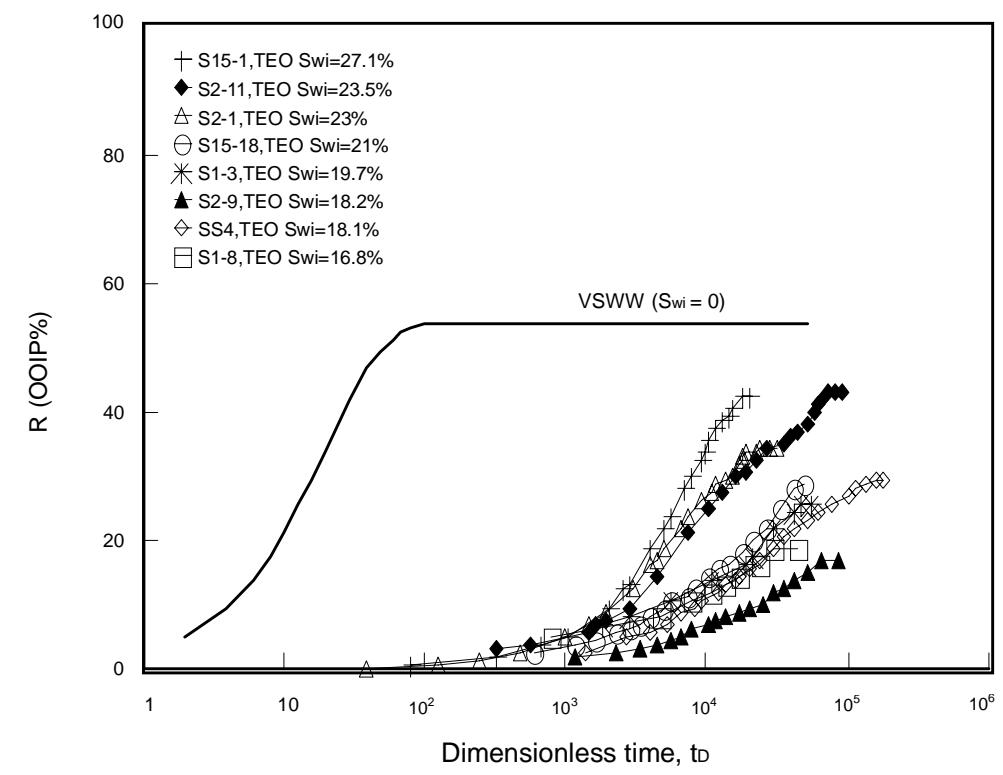

(b) Scaled curves for cores with two ends open

Figure III-3.11. Effect of initial water saturation on imbibition rate and oil recovery for weakly water-wet cores with two ends open. 
The same trend is observed for radial imbibition in cores with two-ends closed (TEC), and other boundary conditions (see Table III-3.2) that involve partially sealed core surfaces (Fig. III-3.12a and III-3.12b). Fig. III-3.12 includes, in addition to data for TEC, results for an annular core with two ends closed, and two rectangular cores with two faces closed (see Table III-3.2). As for the AFO cores, there was an overall trend of increase in imbibition time with decrease in $\mathrm{S}_{\mathrm{wi}}$. This is more systematic for the scaled results (Fig. III-3.12a and III-3.12b).

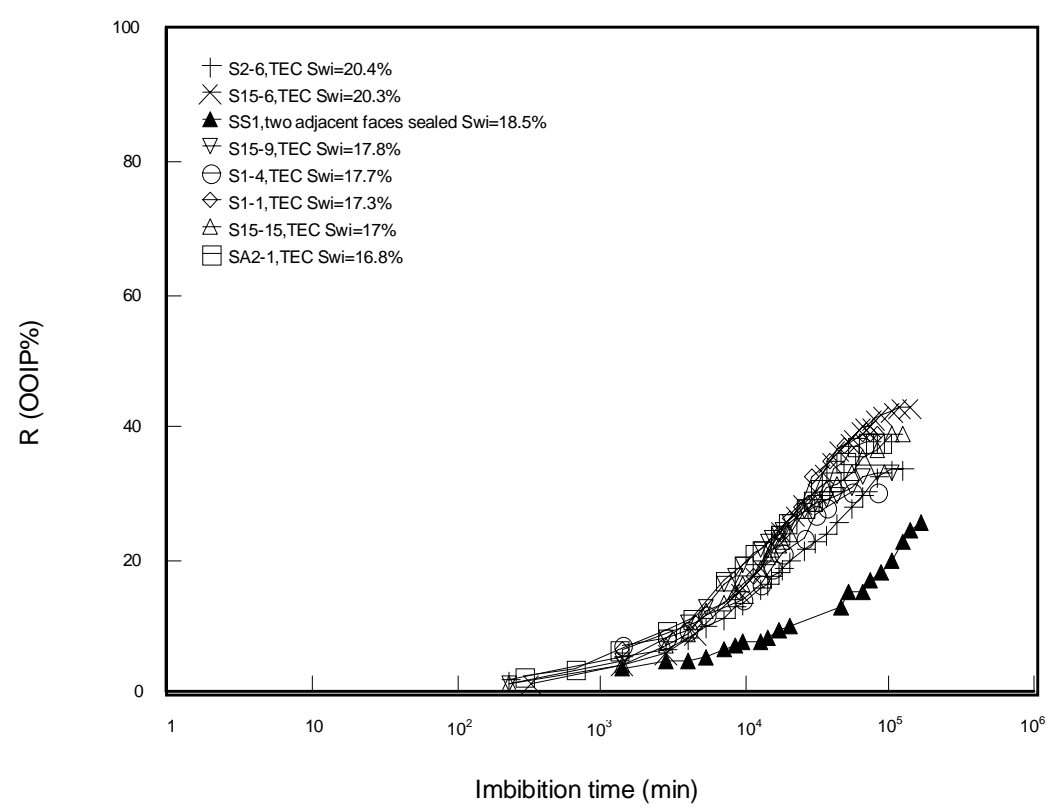

(a) Imbibition data for PS cores

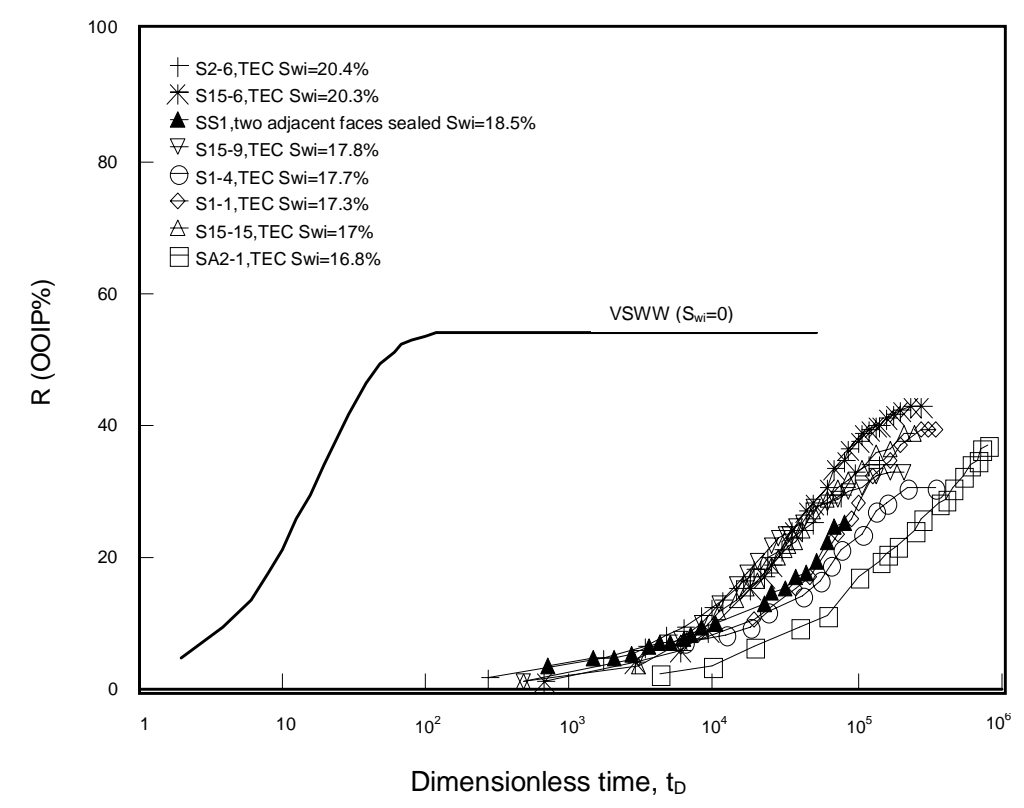

(b) Scaled imbibition curves for PS cores

Figure III-3.12. Effect of initial water saturation on imbibition behavior for weakly water-wet cores with partially sealed surfaces. 
As for the all faces open cores, a more definitive test of scaling is given by comparing results within a close saturation range. Data for cylindrical cores with either TEO or TEC in the saturation range of 19.7 to $21 \%$ are shown in Fig. III-3.13a. The scaling group with $L_{c}{ }^{2}$ ranging from 0.25 to about 16 gave close correlation of the results (Fig. III-3.13b).

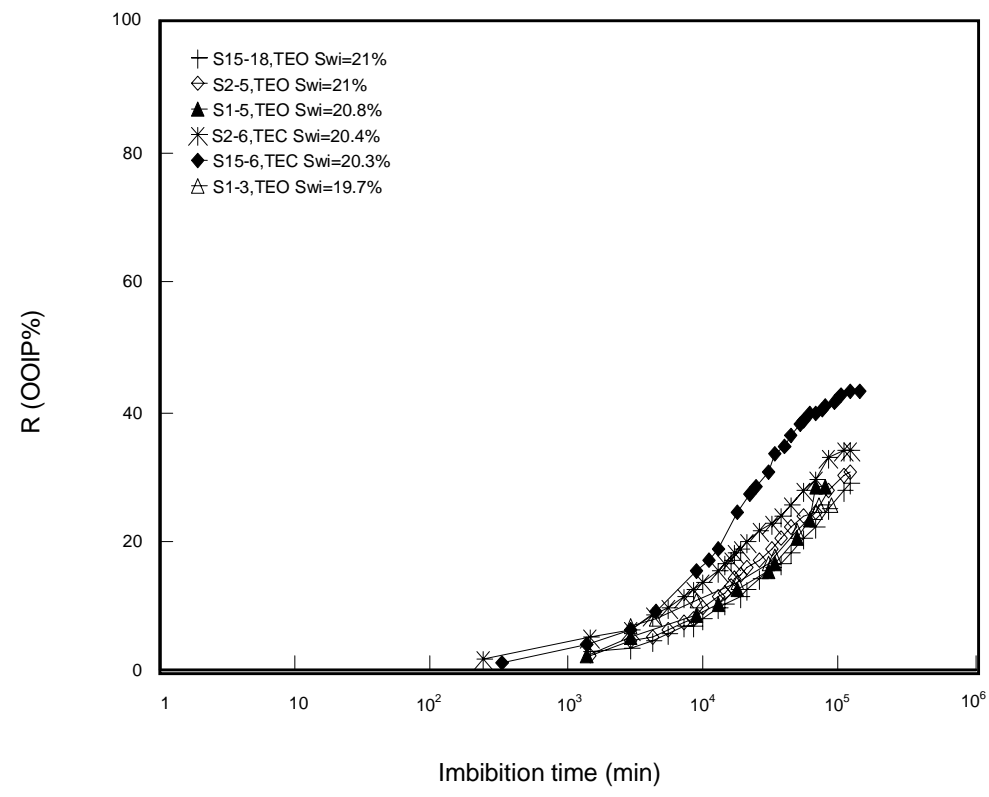

(a) Imbibition curves for Swi $=19.7 \sim 21 \%$

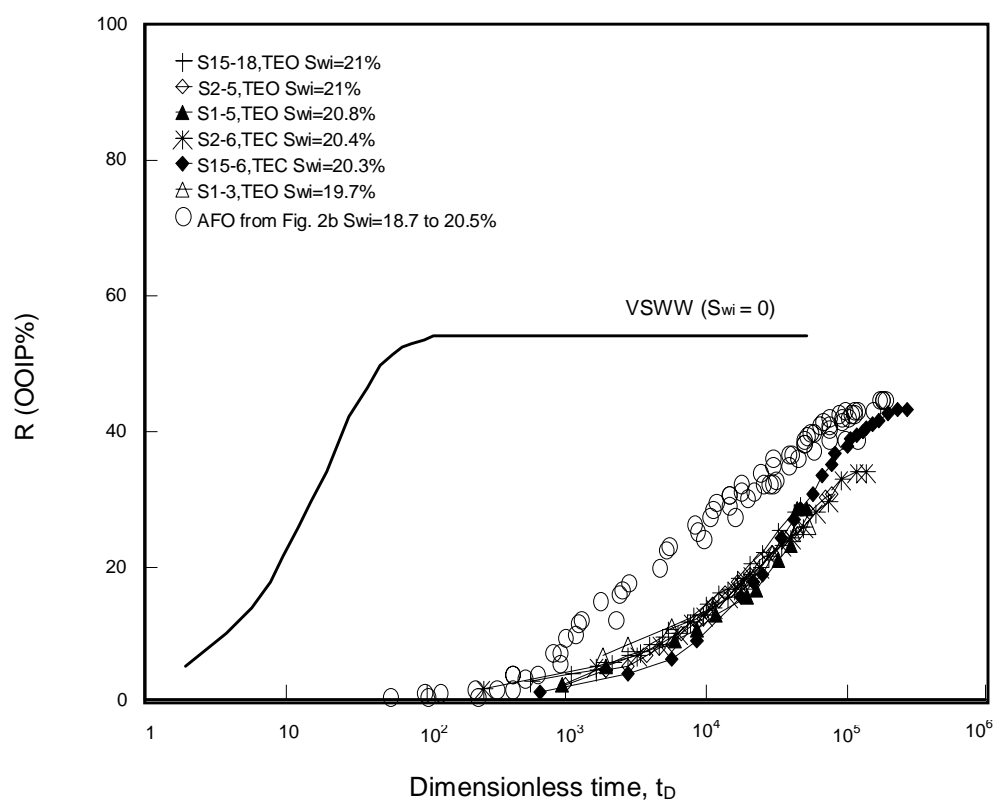

(b) Correlated results for PS cores

Figure III-3.13. Scaling of imbibition data for weakly water-wet cores with partially sealed surfaces and initial saturation in the range $19.7 \sim 21 \%$. 
Results in the saturation range 17.3 to $18.5 \%$ were also tested (Fig. III-3.14a). Although there was some degree of correlation it was less satisfactory for this data set (Fig. III-3.14b).

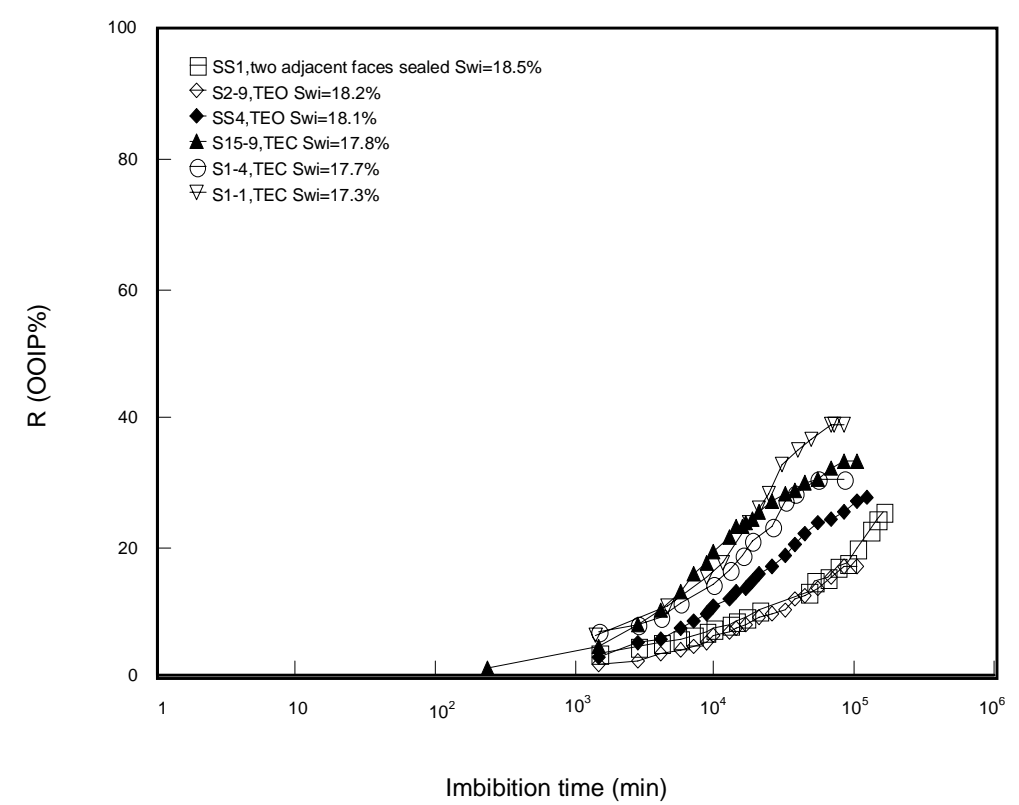

(a) Recovery vs. time

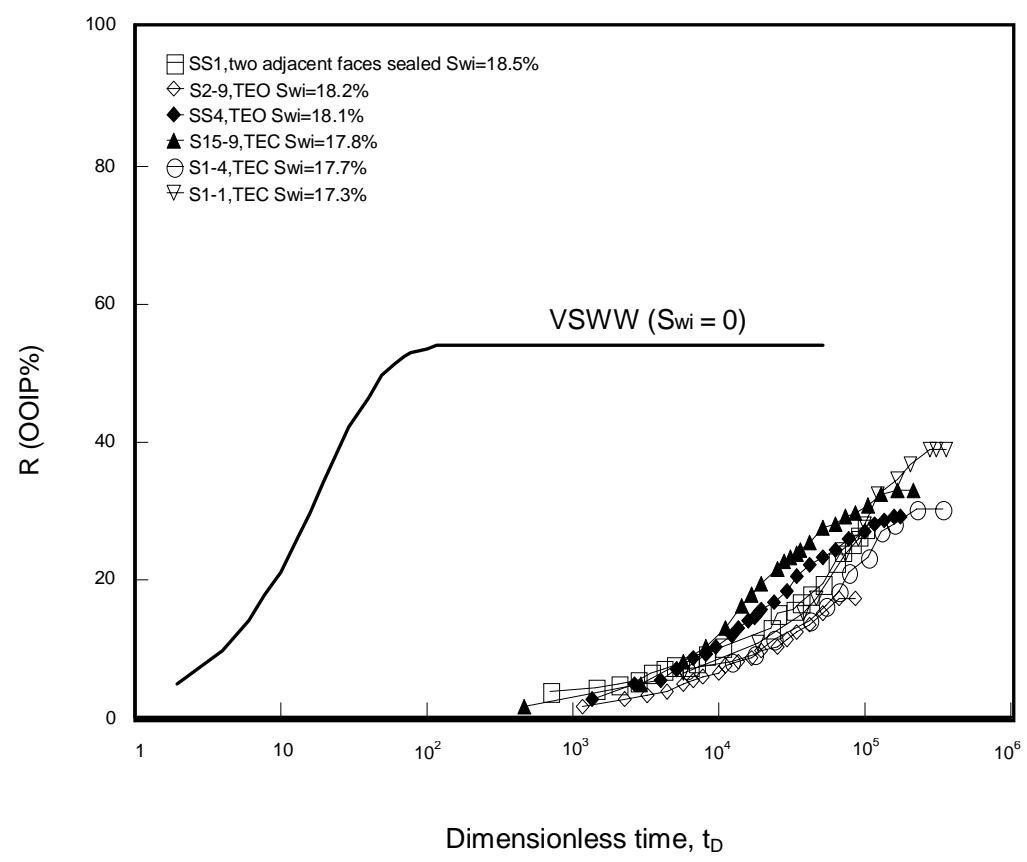

(b) Scaled curves

Figure III-3.14. Scaling of results for partially sealed weakly water-wet cores with partially sealed surfaces and with initial water saturation in the range of 17 to $18.5 \%$. 
The data points for the correlation of AFO cores shown in Fig. III-3.10b with $\mathrm{S}_{\mathrm{wi}}=18.7$ to $20.5 \%$ are included in Fig. III-3.13b. Even though the average $\mathrm{S}_{\mathrm{wi}}$ for the partially sealed (PS) core results is slightly higher than the AFO cores $(20.35 \%$ vs. $19.6 \%)$, the relative values of $t_{D}$ for the AFO cores are clearly less than for the PS cores.

This trend in relative values of $t_{D}$ applies to all of the results. Scaled imbibition times for $50 \%$ recovery, $t_{D}(0.5)$, attained after about 12 weeks of imbibition, are plotted in Fig. III-3.15. Because of very slow continued imbibition, the end point saturations that were used to define $t_{D}(0.5)$ were not clearly defined. However, the results for the AFO cores provide a well defined trend of increase in imbibition time, $t_{D}(0.5)$, with decrease in initial water saturation. The same plot for the PS cores show a comparable trend but with much more scatter; all of the values of $t_{D}(0.5)$ except for S1-8 lie above the trend line for AFO cores. The longer values of $t_{D}$ for the PS cores show that, overall, the change in wettability was significantly larger than for the AFO cores. The difference provides a measure of the additional decrease in water-wetness resulting from the presence of the epoxy resin.

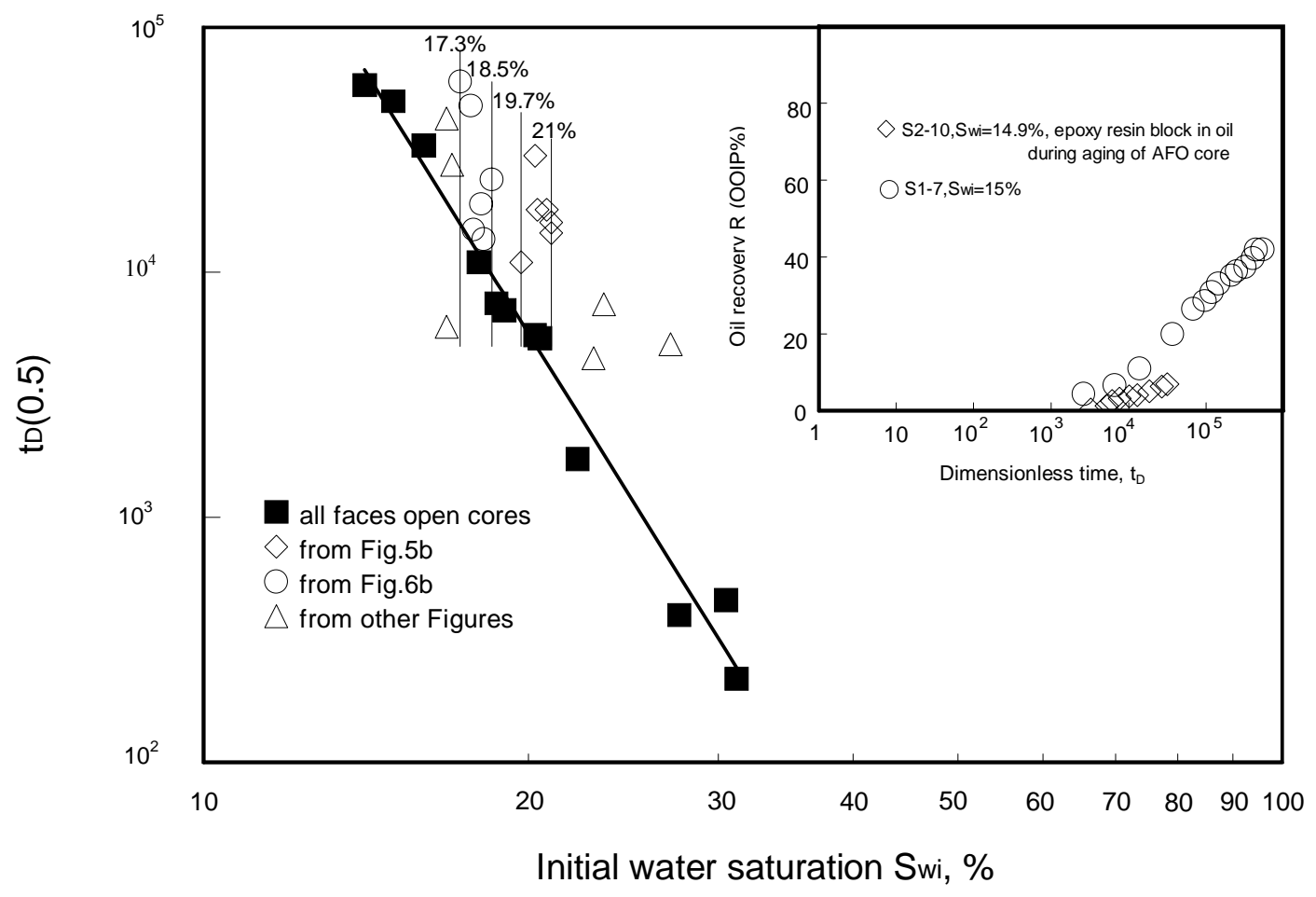

Figure III-3.15. The relationship between Swi and imbibition time for 50\% oil recovery. Insert shows effect of epoxy resin on imbibition by AFO cores.

Use of the Devcon epoxy resin in experiments at ambient temperature with refined oil gave results for PS cores which could be correlated precisely with results for AFO cores (Zhang, et al., 1996). The results of the present work indicate that when cores with partially sealed faces were aged in crude oil, the presence of the epoxy resin caused the cores to undergo a larger 
change in wetting than cores with all faces open. The shift towards less water-wetness may be related to the presence of amine groups in the epoxy resin curing agent.

As a test of the effect of the epoxy resin on wetting, an AFO core was prepared with a block of solidified resin in the crude oil during aging. Unlike the partially sealed cores, the resin was not in direct contact with the core. The initial water saturation was $14.9 \%$. After aging for 10 days at $55^{\circ} \mathrm{C}$, the imbibition rate of the AFO core was consistent with that of the PS cores. Imbibition results are included in Fig. 7 as an insert. These results confirmed that interaction between the resin and the crude oil caused the PS cores to become less water-wet than the cores exposed to crude oil alone. This could be avoided in future work by, for example, the choice of resin, sealing the core with low melting point metal or the use of boundary surfaces such as PTFE heat shrink tubing. However, in practice only AFO cores are used in the majority of imbibition tests. The results obtained for PS cores with epoxy resin are still of interest with respect to the problem of scaling.

Inspection of the scaled results in Fig. III-3.13b and the $t_{D}(0.5)$ values in Fig. III-3.15 shows that in four cases they are in close agreement. The high and low values of $t_{D}(0.5)$, for S15-6 and S1-3 respectively, result from differences in extent of imbibition rather than in curve shape (Fig. 3.5b). The values of $t_{D}(0.5)$ for the PS cores in the saturation range 17.3 to $18.5 \%$ show considerable scatter (Fig. III-3.15) as indicated by the range of deviation from the trend for AFO cores. For these results, the scatter is mainly related to the spread in the scaled results shown in Fig. III-3.14b.

Two key factors in characterizing the imbibition process are the imbibition time, for example the time required for $50 \%$ of final recovery $t_{D}(0.5)$, and the fraction of in place oil recovered, otherwise known as the microscopic displacement efficiency. It is unlikely that a general account can be developed of change in imbibition behavior with wettability because of the number of variables that are involved, their individual complexity, and the complexity of their interactions. Zhou et al.(2000) reported imbibition results for water saturations of $15 \%$, $20 \%$ and $25 \%$. In these tests, the final recovery showed greater sensitivity to initial water saturation and the imbibition time showed less sensitivity than found in the present work. However, there were many differences in the two studies including the crude oil and an aging temperature of $85^{\circ} \mathrm{C}$, compared to aging at $55^{\circ} \mathrm{C}$ in the present work.

\section{Scaling of wettability and gravity}

The values of $t_{D}(0.5)$ for AFO cores shown in Fig. III-3.15 were normalized with respect to $t_{D}(0.5)(=14.5)$ for VSWW with $S_{w i}=0$, to obtain the scaling factor presented in Fig. III-3.16. Normalized values of $t_{D}(0.5)$ for VSWW conditions with an initial water saturation are also presented. The increase in $t_{D}(0.5)$ caused by change in wetting is given by the ratio of scaling factor for the weakly water-wet and strongly water-wet conditions. For example at $20 \% \mathrm{~S}_{\mathrm{wi}}$, as a result of interaction with crude oil, the dimensionless imbibition time is increased from 37.4 to 5,404, a 145 fold increase (Fig. III-3.16). 


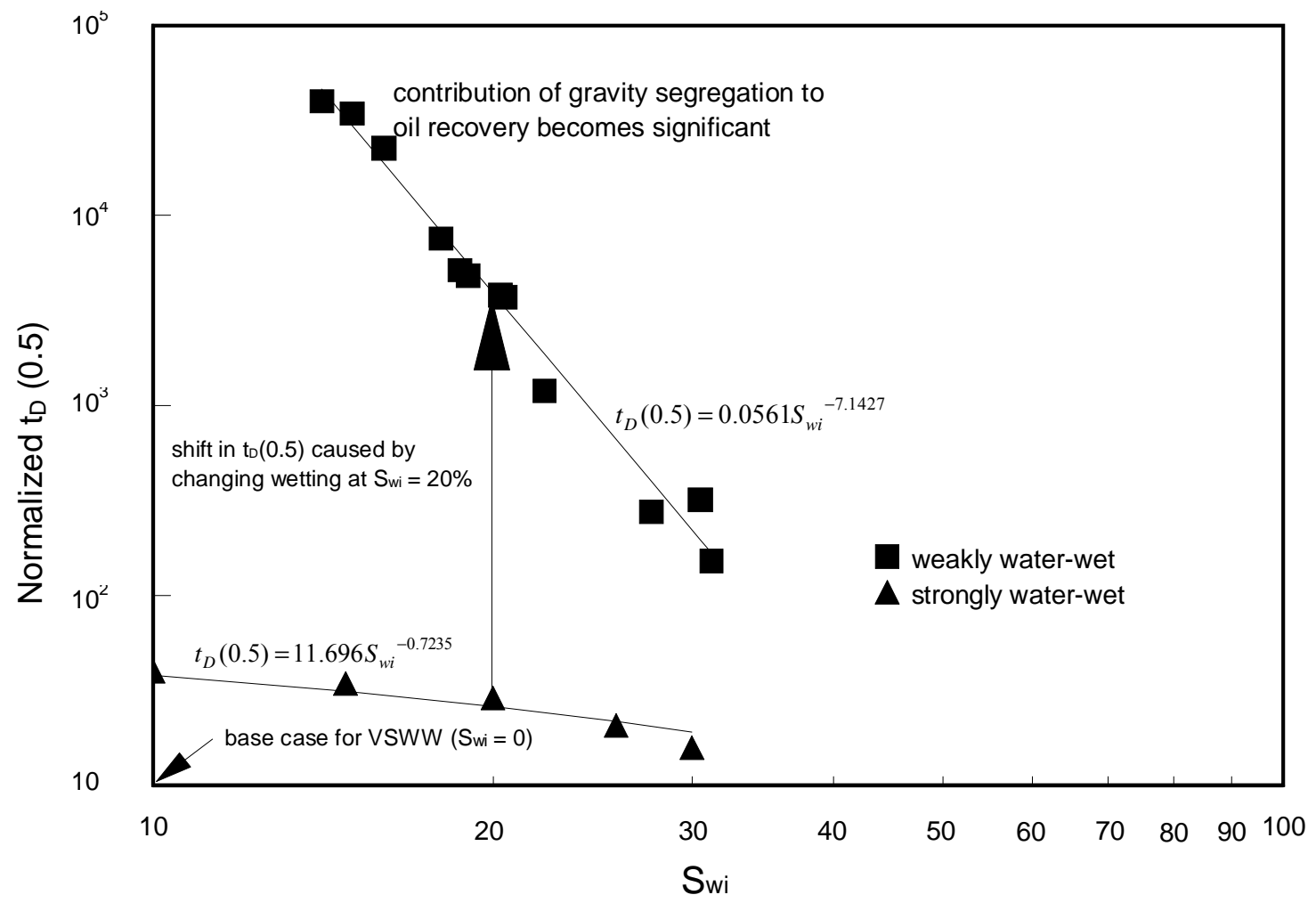

Figure III-3.16. Effect of initial water saturation and change in wettability on imbibition time for 50\% recovery (relative to VSWW with $\mathrm{Swi}=\mathbf{0}$ ).

A condition in the development of scaling laws for imbibition was that the effect of gravity be neglected (Rapopport, 1955; Mattax and Kyte, 1962). If capillary forces are sufficiently small, gravity segregation will make a significant contribution to oil recovery. If gravity is included, the expression for dimensionless time for linear imbibition will have the form

$$
t_{D}(c+g)=t \frac{k / \phi}{L_{c}{ }^{2} \sqrt{\mu_{w} \mu_{o}}}\left(P_{c i} f(\Theta)+\frac{\Delta \rho g L_{c}{ }^{2}}{L_{H}}\right)
$$

where $t_{D}(c+g)$ is the dimensionless time for imbibition that includes capillary and gravity forces, $P_{c i}$ is a representative imbibition capillary pressure and is proportional to $\frac{\sigma}{\sqrt{k / \phi}}, f(\Theta)$ is a wettability factor, and $L_{H}$ is the vertical height of the sample.

For a vertical Berea sandstone core of length $7 \mathrm{~cm}$, with difference in density of oil and brine of $0.1 \mathrm{~g} / \mathrm{ml}$, the buoyancy force that drives gravity segregation is given by $\Delta \rho g L_{H}=29 \mathrm{dynes} / \mathrm{cm}^{2}$.

A representative capillary pressure in Berea sandstone at strongly water-wet conditions is about $23 \times 10^{3}$ dynes $/ \mathrm{cm}^{2}$. If wettability change reduces the pressure by about three orders of magnitude, the relative contribution to oil recovery by gravity forces will become significant. 
If capillary forces become negative and exceed gravity, imbibition will not occur. This condition, which corresponds to an Amott wettability index (Amott, 1959) to water of zero, was not observed in the present work. (Imbibition behavior at water saturations lower that $15 \%$ still need to be tested.) It appears that imbibition is promoted by slow change towards increased water-wetness during the course of the test. Such evidence of slow dynamic changes towards increased water-wetness further complicates interpretation of results with respect to the relative role of surface and gravity forces when imbibition is very slow.

Aronofsky model of imbibition behavior for change in $S_{w i}$

The Aronofsky model (Aronofsky et al, 1958) provides a reasonable one parameter fit for VSWW cores (see Fig. III-3.9b)

$$
\frac{R}{R_{\infty}}=1-e^{-a t_{D}}
$$

where $\mathrm{R}$ is the oil recovery at time $t_{D}, R_{\infty}$ is the final recovery, and $a=0.05$.

The oil recovery at $t_{D}(0.5), \mathrm{R}(0.5)$ was used to determine the constant $a$ for saturations of 15 to $30 \%$ (Fig. III-3.17), where $a=-\ln [1-R(0.5)] / t_{D}(0.5)$. Although this model does not give a close fit to imbibition curve shape for the weakly water-wet conditions, it does provide a clear illustration of the dominant effect of initial water saturation on oil recovery by spontaneous imbibition.

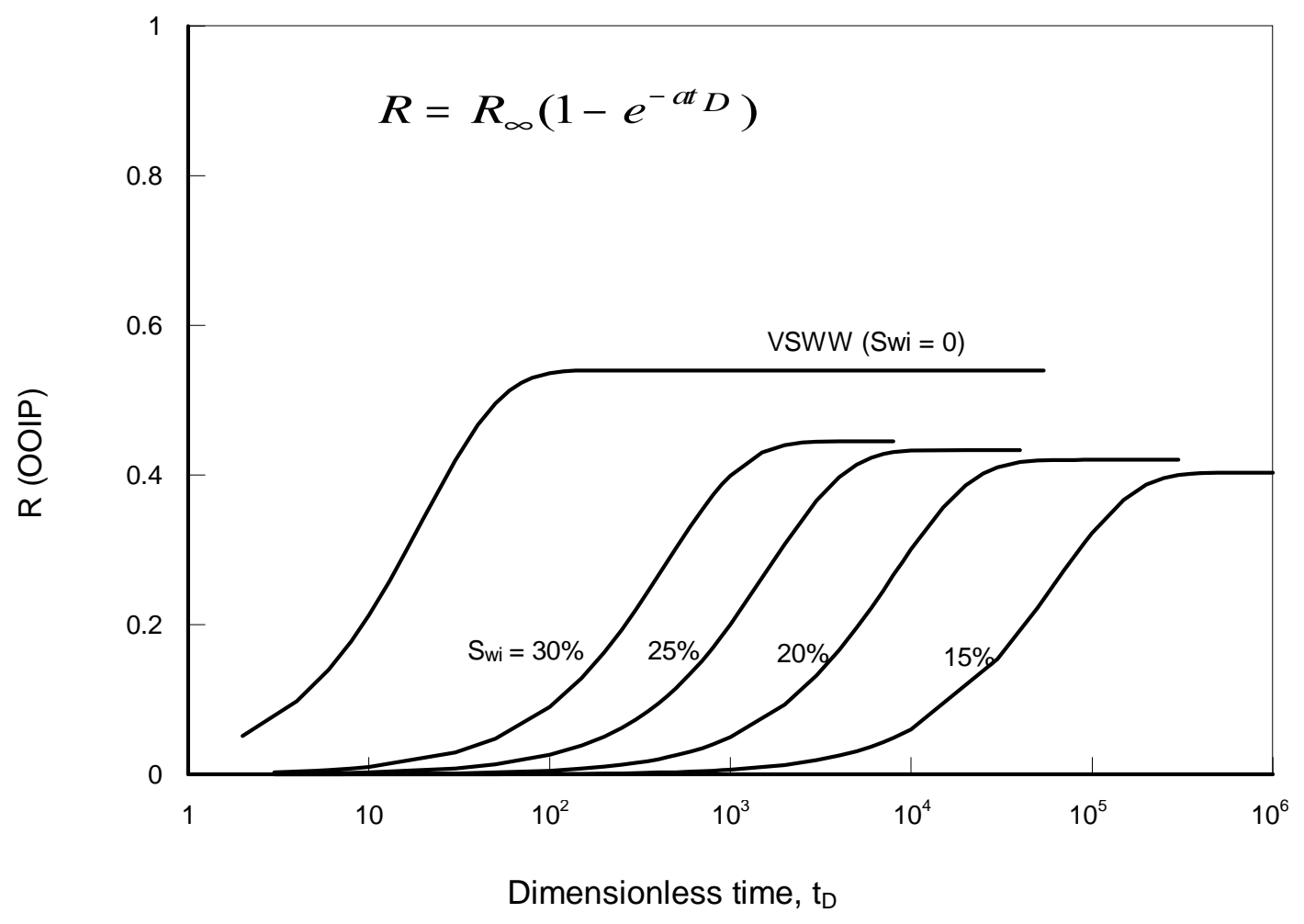

Figure III-3.17. Aronofsky model based on values of tD at 50\% recovery for AFO cores. 


\section{Conclusions}

The following conclusions apply to the conditions of generation of mixed wettability used in the present work. Care must be taken in applying results beyond the range of the experimental data.

- Initial water saturation has a dominant influence on the rate of oil recovery.

- A characteristic length used to scale the effect of sample size, shape, and boundary conditions for strongly water-wet cases gave satisfactory scaling for weakly water-wet conditions.

- Scaled imbibition time decreased systematically with decrease in initial water saturation.

- Aging of sandstone with crude oil in the presence of epoxy resin can cause a significant shift towards reduced water-wetness relative to that induced by crude oil alone.

\section{Nomenclature}

a - constant for Aronofsky model,

$A_{i}$ - open area of ith face of the sample, $\mathrm{cm}^{2}$,

$f(\Theta)$ - wettability factor,

$\mathrm{k}$ - gas permeability, md,

$\mathrm{L}_{\mathrm{c}}$ - characterize length, $\mathrm{cm}$,

$\mathrm{L}_{\mathrm{H}}$ - vertical height of the sample, $\mathrm{cm}$

$\mathrm{P}_{\mathrm{ci}}$ - imbibition capillary pressure,

$\mathrm{R}$ - oil recovery by imbibition, OOIP\%,

$R_{\infty}$ - final oil recovery by imbibition,

OOIP\%,

$\mathrm{t}$ - imbibition time, min,
$t_{D}$ - dimensionless imbibition time, $t_{D}(c+g)-$ dimensionless imbibition time that includes capillary and gravity forces, $\mathrm{x}_{\mathrm{Ai}}$

$\phi$ - porosity, $\%$

$\Delta \rho$ - oil/water density difference, $\mathrm{g} / \mathrm{ml}$.

$\sigma$ - oil-water interfacial tension, dynes/cm.

$\mu_{\mathrm{w}}$ - water viscosity, cp,

$\mu_{\mathrm{o}}$ - oil viscosity, cp.

\section{References}

Amott, E., 1959, Observations relating to the wettability of porous rocks, Trans., AIME 216, 156.

Aronofsky, J.S., Masse, L. and Natanson, S.G., 1958, A model for the mechanism of oil recovery from the porous matrix due to water invasion in fractured reservoirs, Trans. AIME 213, 17-19.

Jadhunandan, P.P. and Morrow, N.R., 1991, Spontaneous imbibition of water by crude oil/brine/rock systems, In Situ 15 (4), 319-345. 
Jadhunandan, P.P. and Morrow, N.R., 1995, Effect of wettability on waterflood recovery by crude oil/brine/rock systems, SPERE, Feb., 40-46.

Kovscek, A.R., Wong, H. and Radke, C.J., 1993, A pore-level scenario for the development of mixed wettability in oil reservoirs, AIChE J., June, 39, No.6, 1072-1085.

Ma, S., Morrow, N.R. and Zhang, X., 1997, Generalized scaling of spontaneous imbibition data for strongly water-wet systems, J. Pet. Sci. \& Eng. 18 165-178.

Mattax, C.C. and Kyte, J.R., 1962, Imbibition oil recovery from fractured, water-drive reservoirs, SPEJ, June, 177-184.

Melrose, J.C., 1982, Interpretation of mixed wettability states in reservoir rocks," paper $S P E$ 10971, SPE Tech. Conf., New Orleans, September.

Rapopport, L.A., 1955, Scaling laws for use in design and operation of water-oil flow models, Trans., AIME (204), 143-150.

Salathiel,R.A., 1973, Oil recovery by surface film drainage in mixed wettability rocks, JPT , Oct., 1216.

Tang G.Q. and Morrow, N.R., 1997, Salinity, temperature, oil composition, and oil recovery by waterflooding, SPE Reservoir Evaluation Nov., 269-276.

Viksund, B.G., Morrow, N.R., Ma, S., Wang, W. and Graue, A., 1998, Initial water saturation and oil recovery from chalk and sandstone by spontaneous imbibition, Proceedings of the International Symposium of Core Analysts, The Hague, The Netherlands, Paper SCA-9814.

Zhang, X., Morrow, N.R. and Ma, S., 1996, Experimental verification of a modified scaling group for spontaneous imbibition, SPE Reservoir Evaluation, November, 280-285.

Zhou, X.M., Torsaeter, O., Xie, X. and Morrow, N.R., 1995, The effect of crude oil aging time and temperature on the rate of water imbibition and long-term recovery by imbibition, $S P E$ Formation Evaluation, December, 259-265.

Zhou, X., Morrow, N.R., and Ma, S., 2000, Interrelationship of wettability, initial water saturation, Aging time, and oil recovery by spontaneous imbibition and waterflooding, SPE Journal, 5, (2), June, 199-207. 


\title{
III-3.3. Scaling of Viscosity Ratio for Oil Recovery by Imbibition from Mixed-Wet Rocks (MWX-F)
}

\author{
Tong, Z., Xie, X., and Morrow, N.R.: "Scaling of Viscosity Ratio for Oil Recovery by Imbibition \\ from Mixed-Wet Rocks, paper SCA-2001-18 presented at the International Symposium, \\ Edinburgh,16-19 Sept.
}

Displacement of oil by spontaneous imbibition from the rock matrix of fractured reservoirs can be a dominant production mechanism. Laboratory tests on reservoir cores are often used to predict oil recovery from the reservoir by scaling results to reservoir conditions. Factors involved in scaling are the rock properties, liquid viscosities, interfacial tensions, core geometry and wettability. Some previous developments in scaling were based on oil recovery from very strongly water-wet rocks (VSWW); results for different viscosity ratios were closely correlated by the geometric mean of the oil and aqueous phase viscosities.

Most reservoirs have mixed wettability (MXW), and, as judged from rate and extent of imbibition, many are weakly water-wet. Results have been obtained for mixed-wet sandstone prepared by adsorption of organic film (F) from an asphaltic crude oil. The crude oil used to induce wettability change was displaced by decalin followed by mineral oil. The mixed wettability states attained by this technique, referred to as MXW-F, depend on the aging temperature, the initial water saturation, and the number of pore volumes of decalin used to displace the crude oil. For MXW-F cores prepared by this technique, imbibition rates were much slower than for strongly water-wet cores and were highly sensitive to initial water saturation. A series of imbibition tests were performed with initial water saturation ranging from $11.0 \%$ to $28.0 \%$. MXW-F imbibition results for recovery of mineral oil, with viscosities ranging from 3.8 to $180.0 \mathrm{cp}$ and initial water saturation close to $21 \%$, were correlated satisfactorily by the geometric mean of the viscosity ratio.

\section{Introduction}

Reservoir wettability and its effect on oil recovery involve a variety of complex issues. In fractured reservoirs, displacement of oil from the rock matrix by spontaneous imbibition may be essential to economic recovery. In heterogeneous reservoirs, spontaneous imbibition into bypassed zones of low permeability may make a significant contribution to oil recovery. The view that most reservoirs are strongly water wet has changed to acceptance that adsorption from crude oil in the presence of connate water results in some form of mixed wettability (MXW). Prediction of oil recovery from laboratory measurements at MXW conditions requires that results be scaled to reservoir conditions.

A large body data for oil recovery by spontaneous imbibition at very strongly water-wet (VSWW) conditions has been correlated (Ma et al., 1997) as original oil in place (OOIP) versus dimensionless time, $\mathrm{t}_{\mathrm{D}}$, defined by, 


$$
t_{D}=t \sqrt{\frac{k}{\phi}} \frac{\sigma}{\sqrt{\mu_{o} \mu_{w}}} \frac{1}{L_{c}{ }^{2}}
$$

where $t$ is time, $k$ is permeability, $\phi$ is porosity, $\sigma$ is the interfacial tension, $\mu_{\mathrm{o}}$ and $\mu_{\mathrm{w}}$ are the oil and brine viscosities. $\mathrm{L}_{\mathrm{c}}$, is a characteristic length that compensates for sample size, shape and boundary conditions. The correlation was initially developed mainly for VSWW conditions with zero initial water saturation. Imbibition with finite initial water saturation has also been investigated (Viksund et al., 1998).

Spontaneous imbibition measurements have been reported for MXW conditions induced by adsorption from crude oil for variation in sample size, shape, boundary conditions, and initial water saturation. For the tested MXW conditions, rate of recovery of the crude oil by imbibition decreased by several orders of magnitude relative to VSWW results. The characteristic length $\mathrm{L}_{\mathrm{c}}$ provided satisfactory correlation of the MXW data (Xie and Morrow, 2000).

Tests of scaling oil/water viscosity ratios at MXW conditions are also needed. However, variation of viscosity ratio at fixed MXW conditions generated by adsorption from crude oil is problematic. Crude oils of different viscosities will generally have different wetting properties. There are limitations to modification of the composition of a crude oil to give variation in viscosity because of likely variation in wetting properties. The chosen approach was to induce wettability change by adsorption from an asphaltic crude oil. After exposure to crude oil, a core was flushed with a solvent that was in turn displaced by a mineral oil. This approach (Morrow et al., 1986, Graue et al., 1999) allows imbibition tests to be performed at MXW-F conditions prepared by a set procedure up to the introduction of mineral oil according to the required viscosity. The designation $\mathrm{F}$ indicates behavior is determined by the presence of a film in the absence of crude oil.

\section{Experimental}

Cores: Berea sandstone blocks, nominally of 200 md permeability, were supplied by Cleveland Quarries as being of the highest available permeability. Core plugs of approximately $3.8 \mathrm{~cm}$ diameter and $7.6 \mathrm{~cm}$ length were cut from the blocks. The air permeabilities of the cores ranged from about 80 to $100 \mathrm{md}$, and the porosities were all close to $18.5 \%$ (see Tables III-3.3 and III3.4). This rock will be identified by an average permeability of $90 \mathrm{md}$ as Berea 90.

Table III-3.3. VSWW imbibition $\left(\mathrm{S}_{\mathrm{wi}}=0 \%\right.$, saturated by vacuum)

\begin{tabular}{|c|c|c|c|c|c|c|}
\hline Core $\#$ & $\boldsymbol{k}_{\mathbf{g}}, \mathbf{m d}$ & $\mathbf{\phi}, \boldsymbol{\%}$ & $\mathbf{L}_{\boldsymbol{c}}, \mathbf{c m}$ & $\boldsymbol{\mu}_{\mathbf{o}}, \mathbf{c p}^{*}$ & Density* $\mathbf{g} / \mathbf{m l}$ & Fig. No. \\
\hline 2B3 & 86.6 & 18.49 & 1.2707 & 3.8 & 0.7819 & III-3.18a, III-3.18b \\
\hline 2B4 & 85.2 & 18.44 & 1.2682 & 3.8 & 0.7819 & III-3.18a, III-3.18b \\
\hline 2B10 & 81.5 & 18.36 & 1.2698 & 40.7 & 0.8462 & III-3.18a, III-3.18b \\
\hline 2B12 & 81.8 & 18.37 & 1.2667 & 40.7 & & III-3.18a, III-3.18b \\
\hline 2B20 & 84.6 & 18.40 & 1.2633 & 3.8 & 0.7819 & III-3.18a, III-3.18b \\
\hline 4B19 & 106.6 & 18.76 & 1.2606 & 3.8 & 0.7819 & III-3.18a, III-3.18b \\
\hline 4B20 & 93.0 & 18.67 & 1.2608 & 3.8 & 0.7819 & III-3.18a, III-3.18b \\
\hline
\end{tabular}

* measured at ambient conditions. 
Table III-3.4. MXW imbibition

\begin{tabular}{|c|c|c|c|c|c|c|c|c|c|}
\hline Core \# & $k_{\mathrm{g}}, \mathrm{md}$ & $\phi, \%$ & $\mathbf{L}_{\mathrm{c}}, \mathbf{c m}$ & $\begin{array}{l}\mathbf{T}_{\mathrm{a}}, \\
{ }^{\circ} \mathbf{C}\end{array}$ & $\begin{array}{c}\mu, \\
\mathbf{c p}^{*}\end{array}$ & $\begin{array}{l}\text { Density } \\
\text { g/ml * }\end{array}$ & $\begin{array}{c}\mathrm{S}_{\mathrm{wi}}, \\
\%\end{array}$ & $\begin{array}{c}\text { Decalin } \\
\text { (pv) }\end{array}$ & Fig. No. \\
\hline 1B13 & 100.4 & 18.51 & 1.2669 & 75 & 3.8 & 0.7819 & 21.8 & 20 & III-3.19, III-3.20, III-3.22a \\
\hline 1B14 & 87.1 & 18.04 & 1.2665 & 75 & 3.8 & 0.7819 & 21.6 & 20 & III-3.19, III-3.22a \\
\hline 1B17 & 86.7 & 18.16 & 1.2652 & 75 & 36.7 & 0.8385 & 21.0 & 20 & III-3.22a \\
\hline 1B18 & 105.2 & 18.57 & 1.2708 & 75 & 3.8 & 0.7819 & 19.4 & 10 & III-3.21a \\
\hline 1B20 & 98.6 & 18.37 & 1.2731 & 75 & 16.0 & 0.8234 & 21.3 & 20 & III-3.22a \\
\hline 1B21 & 106.2 & 18.43 & 1.2657 & 75 & 8.2 & 0.8072 & 21.2 & 20 & III-3.22a \\
\hline 1B22 & 97.6 & 18.35 & 1.2741 & 75 & 3.8 & 0.7819 & 21.7 & 10 & III-3.21a \\
\hline 1B24 & 89 & 18.24 & 1.2691 & 75 & 3.8 & 0.7819 & 24.2 & 10 & III-3.21a \\
\hline $1 \mathrm{~B} 25$ & 98.2 & 18.47 & 1.2662 & 75 & 3.8 & 0.7819 & 27.3 & 10 & III-3.20, III-3.21a \\
\hline 1B26 & 85.6 & 17.74 & 1.271 & 95 & 3.8 & 0.7819 & 11.0 & 5 & III-3.21b \\
\hline 1B28 & 100.3 & 18.21 & 1.2663 & 95 & 3.8 & 0.7819 & 15.8 & 5 & III-3.21b \\
\hline 1B29 & 93.1 & 18.46 & 1.2692 & 95 & 3.8 & 0.7819 & 12.5 & 5 & III-3.21b \\
\hline 1B32 & 100.1 & 18.94 & 1.2646 & 95 & 8.4 & 0.8088 & 21.1 & 5 & III-3.22b \\
\hline 1B33 & 91.7 & 18.7 & 1.2670 & 95 & 18.0 & 0.8255 & 21.3 & 5 & III-3.19, III-3.22b \\
\hline 1B34 & 98.1 & 18.97 & 1.2698 & 95 & 3.8 & 0.7819 & 24.8 & 5 & III-3.21b \\
\hline $2 \mathrm{~B} 2$ & 79.4 & 18.53 & 1.2619 & 95 & 39.5 & 0.8425 & 20.8 & 5 & III- $3.22 \mathrm{~b}$ \\
\hline 2B9 & 88.1 & 18.55 & 1.2684 & 95 & 18.0 & 0.8255 & 21.3 & 5 & III-3.19, III-3.22b \\
\hline $2 \mathrm{~B} 14$ & 82.2 & 18.48 & 1.2687 & 95 & 3.8 & 0.7819 & 28.0 & 5 & III-3.21b \\
\hline 2B18 & 80.1 & 18.49 & 1.2660 & 95 & 66.0 & 0.8544 & 21.4 & 5 & III- $3.22 \mathrm{~b}$ \\
\hline $2 \mathrm{~B} 25$ & 89.3 & 18.74 & 1.2637 & 95 & 3.8 & 0.7819 & 19.0 & 5 & III-3.21b \\
\hline 2B27 & 106.4 & 18.56 & 1.2646 & 95 & 3.8 & 0.7819 & 21.7 & 5 & III- $3.21 b$, III- $3.22 b$ \\
\hline $2 \mathrm{~B} 28$ & 83.9 & 17.85 & 1.2745 & 75 & 3.8 & 0.7819 & 27.0 & 5 & III-3.20 \\
\hline 4B5 & 86.6 & 18.11 & 1.2646 & 75 & 18.0 & 0.8255 & 21.7 & 5 & III-3.22c \\
\hline $4 \mathrm{~B} 6$ & 85.6 & 18.10 & 1.2663 & 75 & 3.8 & 0.7819 & 21.2 & 5 & III-3.20, III-3.22c \\
\hline 4B7 & 83.8 & 18.13 & 1.2665 & 75 & 106.8 & 0.8620 & 21.4 & 5 & III-3.22c \\
\hline 4B9 & 101.2 & 18.18 & 1.2631 & 75 & 106.8 & 0.8620 & 21.7 & 5 & III-3.22c \\
\hline 4B13 & 100.0 & 18.47 & 1.2609 & 75 & 180.0 & 0.8727 & 21.4 & 5 & III- $3.22 \mathrm{c}$ \\
\hline $4 \mathrm{~B} 16$ & 107.4 & 19.17 & 1.2592 & 75 & 3.8 & 0.7819 & 21.3 & 5 & III-3.22c \\
\hline
\end{tabular}

* measured at ambient conditions.

Crude oil: A degassed asphaltic crude oil of $0.9086 \mathrm{~g} / \mathrm{ml}$ density, Alaska 95 (A95) from Prudhoe Bay, was used to change the wettability of the cores. The oil had $6.55 \mathrm{wt} \%$ of n-heptane asphaltenes and no dissolved wax (Xie, 1996). The viscosity of the evacuated oil was $70.9 \mathrm{cp}$ at room temperature $\left(22^{\circ} \mathrm{C}\right)$. The acid and base numbers were 0.24 and 2.2 respectively. This oil was selected because it was known to induce significant change in wettability by deposition of stable films (Xie et al 2002) and problems associated with wax deposition would not be encountered, even at ambient temperature.

Decalin: Decalin, decahydronaphthalene $\left(\mathrm{C}_{10} \mathrm{H}_{18}\right)$, has a density of $0.8816 \mathrm{~g} / \mathrm{ml}$ and viscosity of $2.5 \mathrm{cp}$ at $22^{\circ} \mathrm{C}$. When decalin is used to displace the crude oil from the aged core samples, the bulk crude oil is displaced, but most of the adsorbed polar components, which alter the wettability are retained on the rock surface.

Mineral Oil: Mineral oils with different viscosities were prepared by mixing Soltrol 220 mineral oil $(3.8 \mathrm{cp})$ and white mineral oil $(180.0 \mathrm{cp})$ in ratios selected to give intermediate viscosities as required. The mineral oils were equilibrated with silica gel and alumina and then filtered. 
Densities and viscosities of the two oils and their mixtures are presented in Table III-3.3 and III3.4 .

Brine: Synthetic reservoir brine of density $1.025 \mathrm{~g} / 1$ was prepared: $\mathrm{NaCl} 21.3 \mathrm{~g} / \mathrm{L}, \mathrm{KCl}$ $0.10 \mathrm{~g} / \mathrm{L}, \mathrm{CaCl}_{2} 0.61 \mathrm{~g} / \mathrm{L}, \mathrm{MgCl}_{2} 0.20 \mathrm{~g} / \mathrm{L} . \mathrm{NaN}_{3}(0.10 \mathrm{~g} / \mathrm{L})$ was added as a biocide.

Establishing initial water saturation: The core samples were first saturated with reservoir brine by vacuum and allowed to soak for at least 10 days to attain ionic equilibrium. Initial water saturation, $\mathrm{S}_{\mathrm{wi}}$, higher than $19 \%$ was established by displacing reservoir brine with up to $25 \mathrm{PV}$ of A95 crude oil at room temperature. $\mathrm{S}_{\mathrm{wi}}$ lower than $19 \%$ was established by means of a porous plate apparatus (soil moisture ceramic plate extractor). Pressures from 15 up to 130 psi, were applied in increments of 5 psi for the lower range (less than 80 psi) and 10 psi for the higher range. The desaturation process took 1 month to reach the lowest tested water saturation of $11 \%$. The cores were next saturated with A95 crude oil under vacuum. Full saturation of each core was checked by mass balance.

Aging: The cores containing initial water and crude oil were submerged in crude oil in sealed aging cells. Cores were aged at either $75^{\circ} \mathrm{C}$ or $95^{\circ} \mathrm{C}$ for 10 days.

Replacement of crude oil with mineral oil: After aging, the core was mounted in a core holder. The temperature was then raised to $60^{\circ} \mathrm{C}$ and the crude oil was displaced by 5 to $20 \mathrm{PV}$ of decalin. The objective of the solvent flush was to remove the bulk crude oil from the core but leave the polar components adsorbed on the rock surfaces. This procedure avoids precipitation of asphaltenes and other possible effects on adsorbed components that might result from direct displacement with mineral oil. The decalin was then displaced by $5 \mathrm{PV}$ of the selected mineral oil at room temperature.

Spontaneous imbibition: After displacement with mineral oil, the cores were set in glass imbibition cells filled initially with brine. All of the imbibition tests were performed at room temperature. Oil volume produced by imbibition of brine (expressed as percentage of original oil in place - \%OOIP) versus time was recorded.

\section{Results and Discussion}

\section{VSWW Reproducibility and representative results}

Results for recovery of 3.8 and $40.7 \mathrm{cp}$ mineral oil from Berea 90 with zero initial water saturation are shown in Fig. III-3.18a for seven core samples. The fractional recovery of original oil in place was about $46 \%$ for all cores. Plots of normalized recovery versus dimensionless time, shown in Fig. III-3.18b, fall into two slightly separated groups. A representative curve for Berea $90, \mathrm{~S}_{\mathrm{wi}}=0 \%$, was obtained by curve fitting the later recovery times, for the group with shorter overall $\mathrm{t}_{\mathrm{D}}$, to a model of the form $R / R_{\infty}=1-e^{-\alpha t_{D}}$ (Aronofsky et al, 1958). $\mathrm{R}$ is the oil recovery 
at dimensionless time $t_{D}, R_{\infty}$ is the final recovery, and $\alpha=0.014$. This curve is indicated in subsequent plots as Berea $90, \mathrm{~S}_{\mathrm{wi}}=0 \%$.
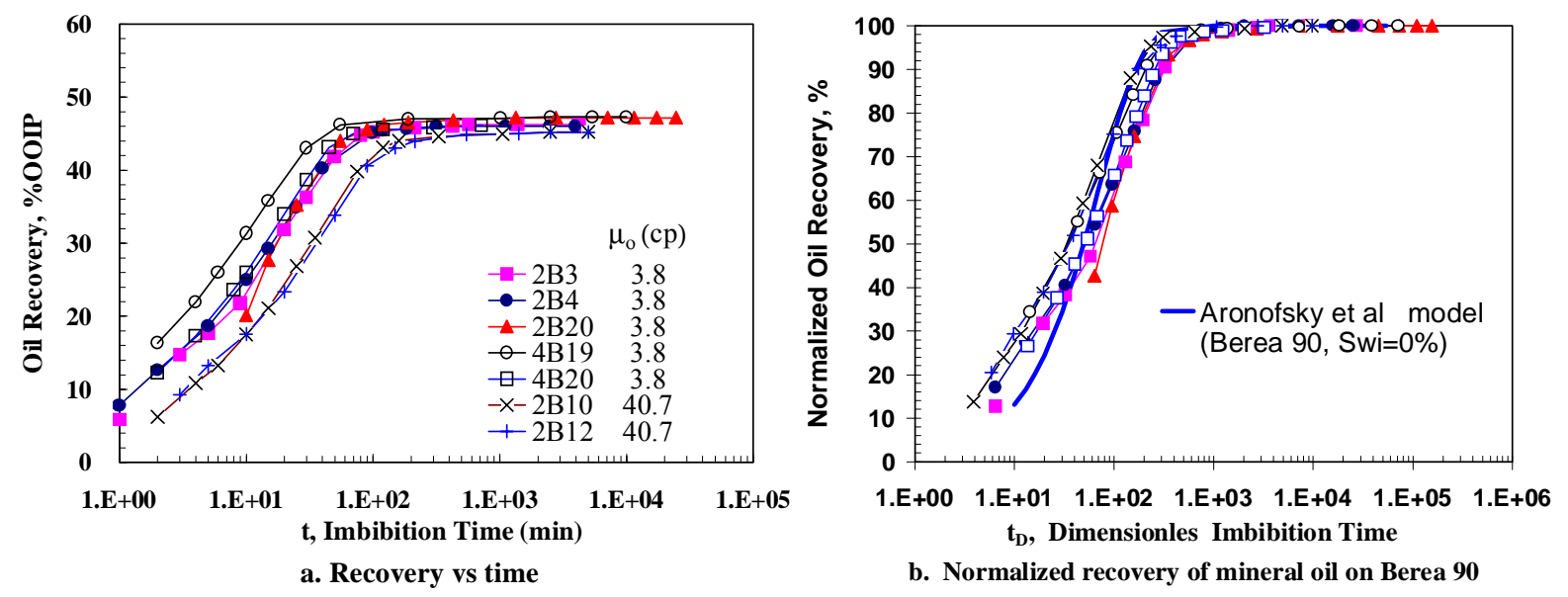

b. Normalized recovery of mineral oil on Berea 90
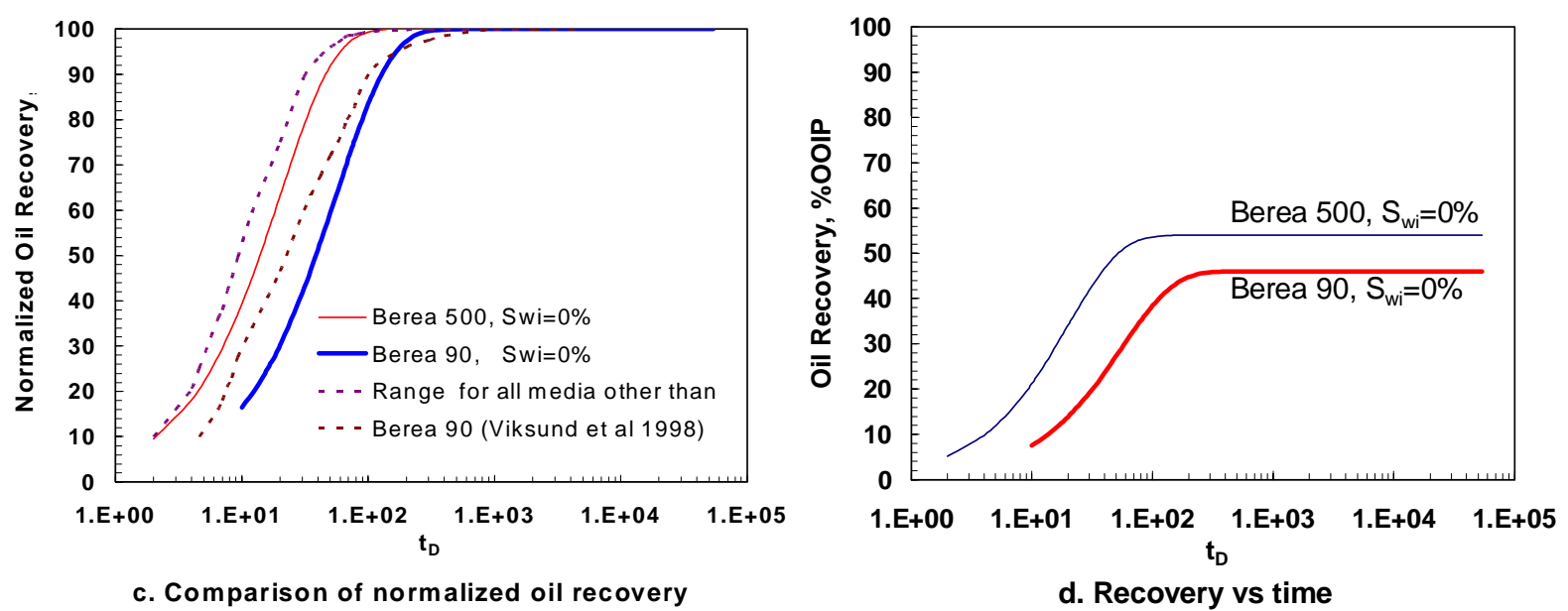

Figure III-3.18. Comparison of recovery of mineral oil by spontaneous imbibition from Berea 90 and other porous media.

For Berea sandstone used in previous studies (nominally of $500 \mathrm{md}$ permeability and referred to as Berea 500), close correlation of results for variation in viscosity ratio and boundary conditions was obtained by Zhang et al. (1996). Berea 500 has served as a model rock for many past studies but was not available for the present work. The correlation obtained for Berea 500, which was for zero initial water saturation, was found to be representative of other types of very strongly water-wet porous media with permeabilities ranging from a few millidarcies to several darcies (Viksund et al, 1998). The upper and lower ranges of $t_{D}$ covered by these rocks are included in Fig. III-3.18c. Dimensionless times for imbibition by Berea 90 are significantly longer than for the correlated results represented by Berea 500. One possible reason for the increased imbibition times is that Berea $90, \mathrm{~S}_{\mathrm{wi}}=0 \%$, was somewhat less than VSWW. Possible causes, such as distinct differences in mineralogy and pore structure and the state of hydration of various minerals at the time of saturation with oil, are being investigated. Preliminary measurements show that the imbibition rate for Berea 90 is increased when an initial water saturation is present; however decrease was found for Berea 500 (Viksund et al, 1998). 
At present, provision of a representative curve for Berea 90 that, without doubt, corresponds to VSWW conditions is somewhat problematic. However, for the MXW-F Berea 90 cores prepared in this study, imbibition rates were several orders of magnitude slower that for the as-supplied Berea 90. In presenting correlated results for MXW-F cores, the typical curves for Berea 500 and Berea 90 shown in Fig. III-3.18d are included for comparison.

\section{Wettability Alteration and Reproducibility of $M X W$ spontaneous imbibition:}

In the course of investigating the wettability alteration procedure, the amount of decalin used to displace the crude oil was tested at 5, 10, and $20 \mathrm{PV}$ for a range of initial water saturation. Examples of imbibition curves for MXW-F are shown in Figs. III-3.19 and III-3.20 for the indicated conditions of initial water saturation $S_{w i}$, aging temperature, $T_{a}, P V$ decalin displacement, and mineral oil viscosity. Examples of close reproducibility of results for duplicate tests are included in Fig. III-3.19.

Increase in PV flush from 5 to $10 \mathrm{PV}$ had minor effect on imbibition rate (1B25 and 2B28 in Fig. III-3.20) for $27 \% \mathrm{~S}_{\mathrm{wi}}$. The large effect shown in Fig. III-3.20 for a 5 versus 20 PV flush (1B13 and 4B6) was probably related to the lower initial water saturation of $21 \%$, more than the larger (20 PV) flush volume. The increased effect on imbibition of initial water saturation is consistent with the concept of mixed wettability. As connate water saturation is decreased, the area of rock surface exposed to adsorption increases and so the relative effect on wettability that results from increasing the decalin flush PV also increases (Fig. III-3.20).

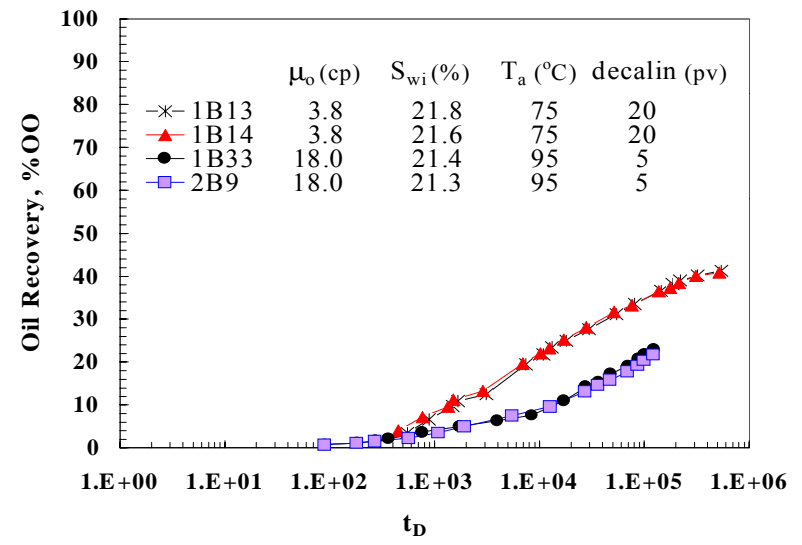

Figure III-3.19. Reproducibility of recovery of mineral oil by spontaneous imbibition.

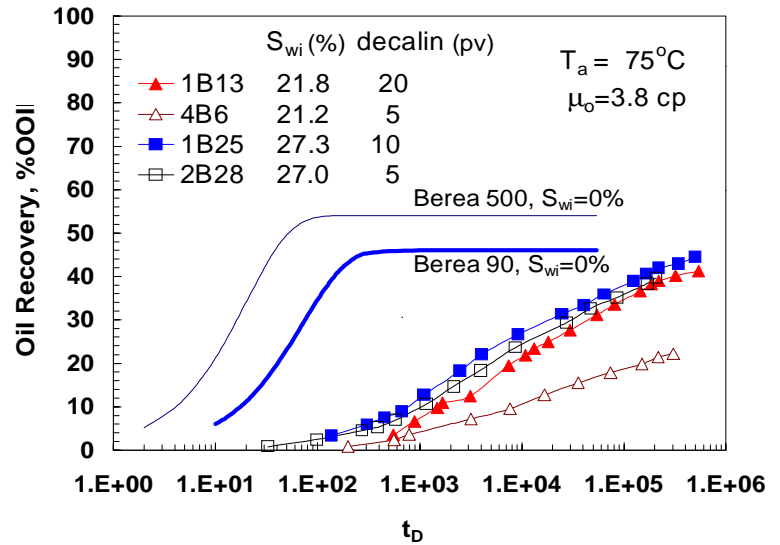

Figure III-3.20. Sensitivity of MXW-F imbibition to decalin flush volume.

\section{Initial water saturation}

Initial water saturation has been shown to be a dominant variable in the generation of MXW as shown by its effect on rate and extent of recovery of crude oil by spontaneous imbibition (Xie and Morrow, 2000). Two sets of MXW-F data obtained for variation in initial 
water saturation with other factors held constant are shown in Fig. III-3.21. For the set presented in Fig. III-3.21a(i) the aging temperature was $75^{\circ} \mathrm{C}$; the cores were flushed with $10 \mathrm{PV}$ of decalin. Initial water saturation was varied from 19.4 to $27.3 \%$. Scaled imbibition rate decreased systematically with decrease in initial water saturation (Fig. III-3.21a(ii)). For the second data set, the aging temperature was $95^{\circ} \mathrm{C}$; the cores were flushed with $5 \mathrm{PV}$ of decalin. Seven values of initial water saturation, ranging from 11.0 to $28.0 \%$, were tested (see Fig. III-3.21b(i)). Again the results showed strong and systematic dependence on initial water saturation (4b(ii)). Tests of scaling of other variables such as viscosity ratio at mixed-wet conditions therefore requires that the initial water saturation, at the time the core is exposed to crude oil, be held essentially constant.
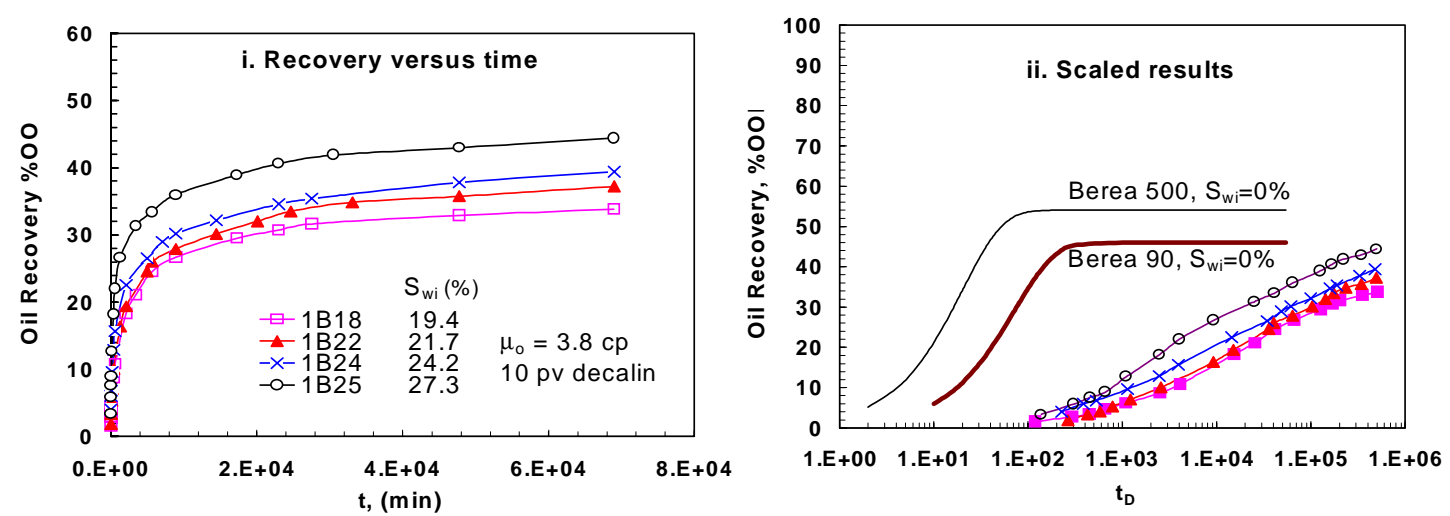

a. $\mathrm{S}_{\mathrm{wi}}$ from $19.9 \%$ to $27.3 \%$ and $\mathrm{T}_{\mathrm{a}}=75^{\circ} \mathrm{C}$
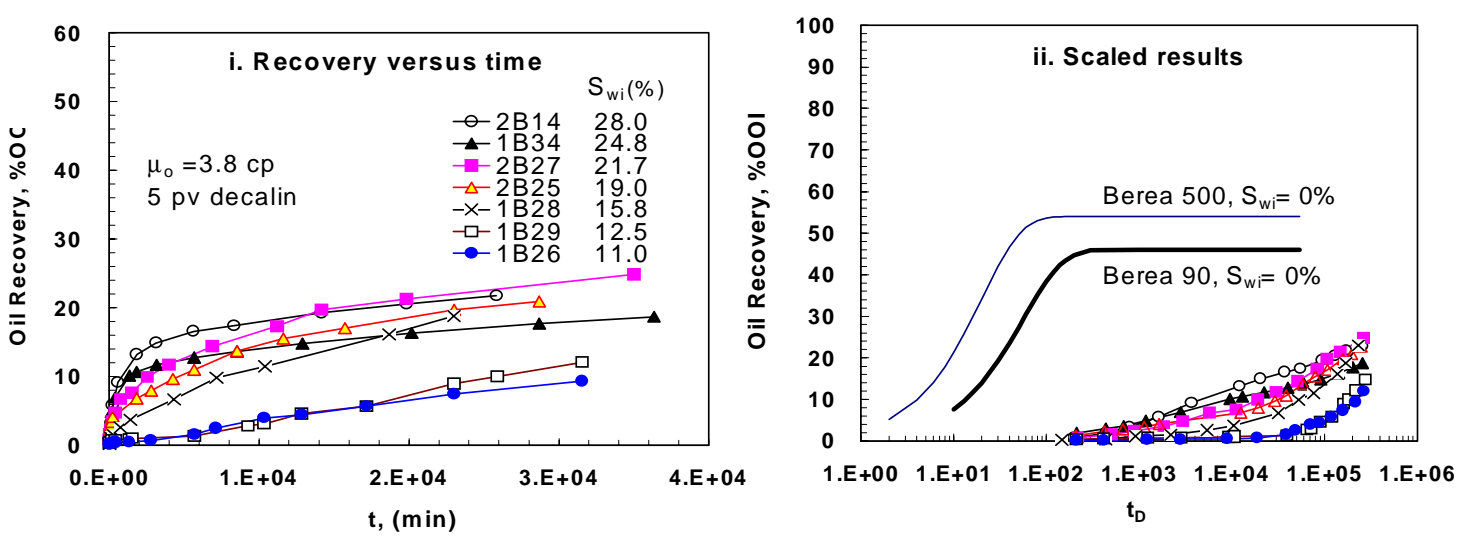

b. $\mathrm{S}_{\mathrm{wi}}$ from $11.0 \%$ to $28.0 \%$ and $\mathrm{T}_{\mathrm{a}}=95^{\circ} \mathrm{C}$

Figure III-3.21. Effect of initial water saturation on oil recovery by spontaneous imbibition.

\section{Viscosity scaling}

Imbibition recovery curves are shown in Fig. III-3.22a(i) for recovery of 3.8, 8.2, 16.0, 18.2 , and $36.7 \mathrm{cp}$ mineral oil with initial water saturations all close to $21.5 \%$. The cores were flushed with 20 PV of decalin. Results for the 8.2, 16.0, and $36.7 \mathrm{cp}$ oils were closely correlated. For the $3.8 \mathrm{cp}$ oil, the dimensionless times were somewhat less than for the more viscous oils. 
Results are shown in Fig. III-3.22b(i) for an aging temperature of $95^{\circ} \mathrm{C}$ and $5 \mathrm{PV}$ decalin flush. Five oil viscosities, ranging from 3.8 to $66.0 \mathrm{cp}$, were tested. The results were closely correlated by dimensionless time; there was continued slow recovery of oil with time except for the $66.0 \mathrm{cp}$ oil which approached a plateau in recovery of about $13 \%$.

A third data set was obtained for cores aged at $75^{\circ} \mathrm{C}$. In these tests, the crude oil was displaced by 5 PV decalin followed by $5 \mathrm{PV}$ of the mineral oil of selected viscosity. Results for mineral oil viscosities of 3.8, 18.0, 106.8, and $180.0 \mathrm{cp}$ are shown in Fig. III-3.22c(i). The scaled results gave the close correlation shown in Fig. III-3.22c(ii).

For each data set of the present study, core preparation conditions are held the same until the introduction of mineral oil. Differences in wetting properties could arise from differences in the chemical properties of the refined oils. However ability to scale results for the tested mixedwet conditions by the geometric mean of the viscosities, an empirical function originally identified from VSWW studies, suggests that this is minor. The observed difference between correlated results for strongly water-wet and mixed-wet conditions can therefore be ascribed to the effect of wettability on imbibition rate.

\section{Conclusions}

1. Dimensionless times for imbibition into Berea 90 sandstone, nominally of 90 md permeability, were longer than previously observed for a wide range of very strongly water wet porous media.

2. Aging of sandstone in crude oil followed by displacement of crude oil with decalin gave closely reproducible spontaneous imbibition, and hence MXW-F wetting states, for duplicate core plugs.

3. Water wetness decreased with decrease in initial water saturation during aging and increased with the volume of decalin used to displace crude oil after aging.

4. Recovery of mineral oil of different viscosities from MXW-F cores was correlated satisfactorily by the square root of the geometric mean of the water and oil viscosities. 

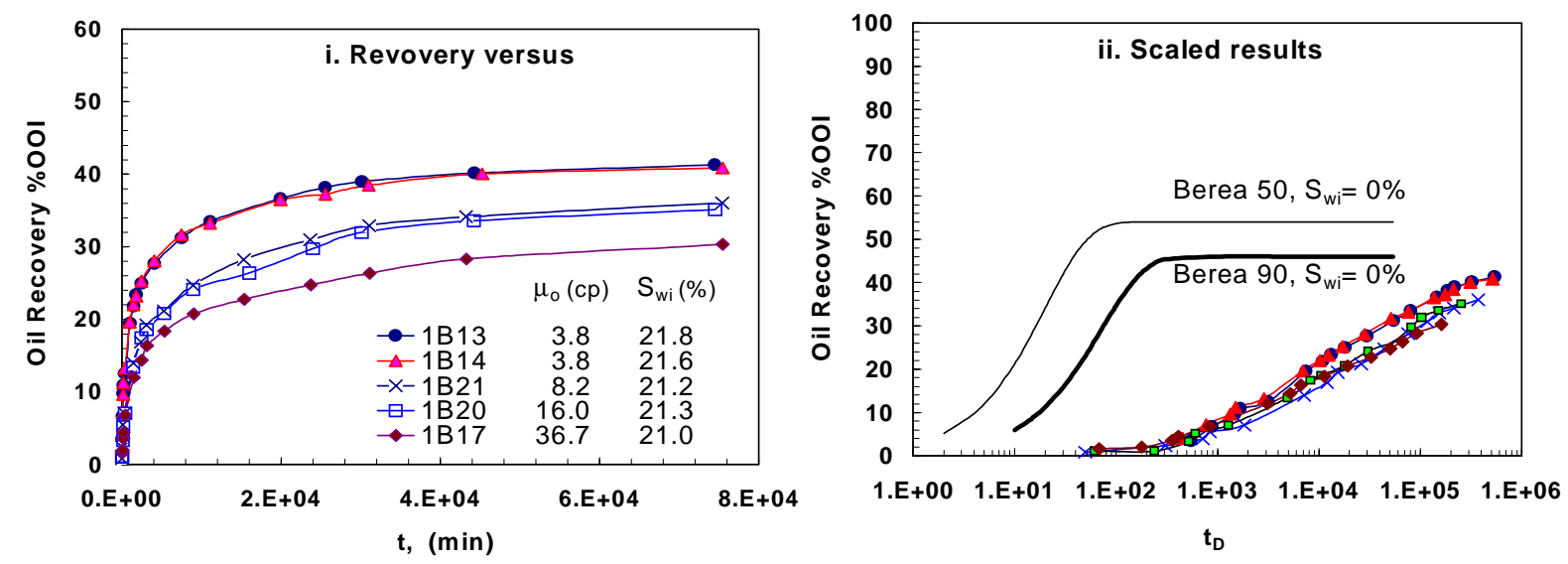

a. Viscosity from $3.8 \mathrm{cp}$ to $36.7 \mathrm{cp}, \mathrm{T}_{\mathrm{a}}=75^{\circ} \mathrm{C}$ and $20 \mathrm{pv}$ decalin
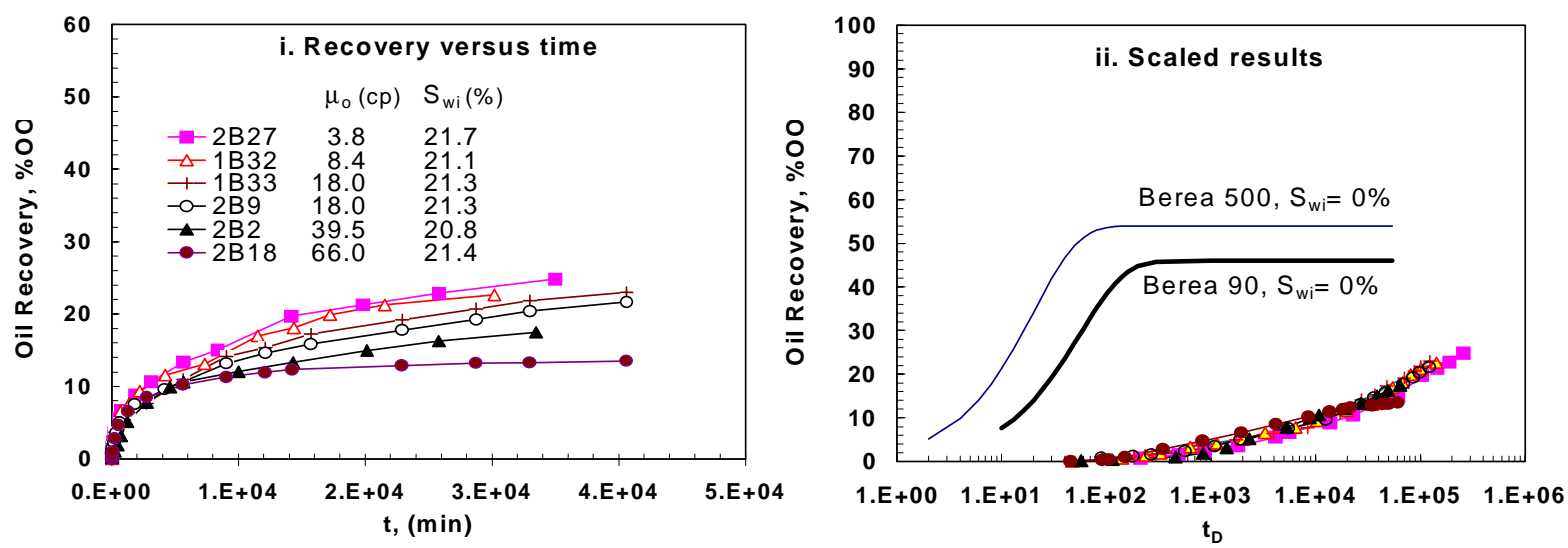

b. Viscosity from $3.8 \mathrm{cp}$ to $66.0 \mathrm{cp}, \mathrm{T}_{\mathrm{a}}=95^{\circ} \mathrm{C}$ and $5 \mathrm{pv}$ decalin
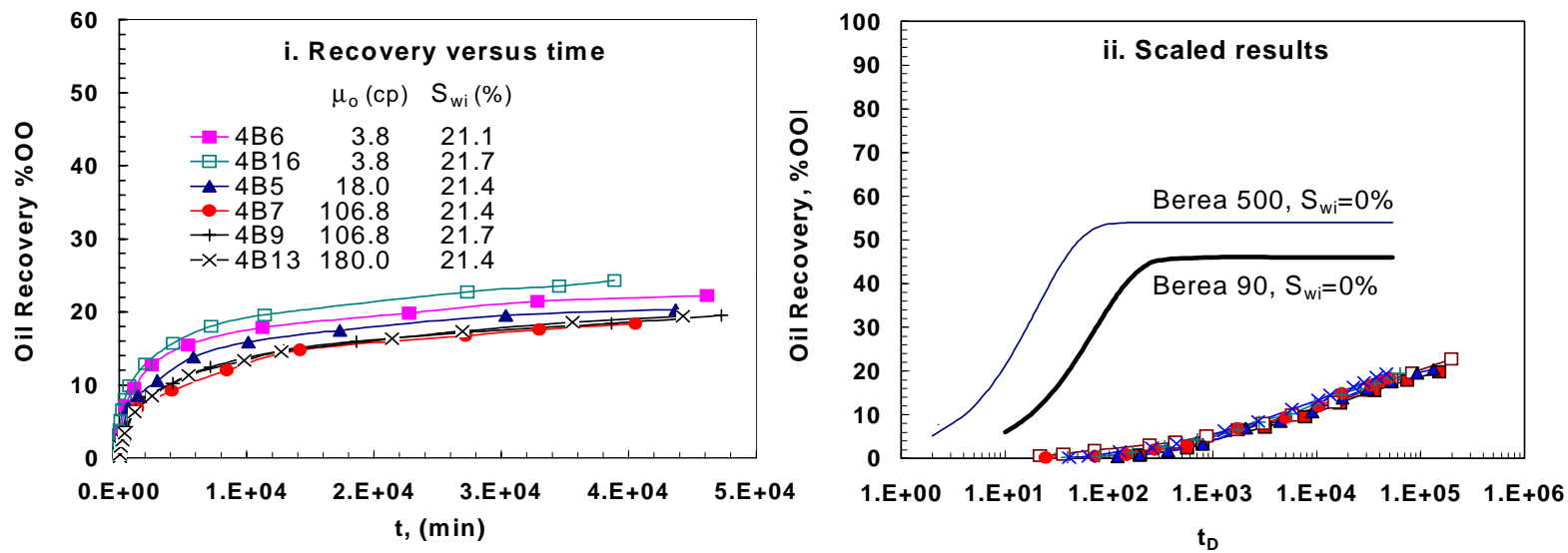

c. Viscosity from $3.8 \mathrm{cp}$ to $180.0 \mathrm{cp}, \mathrm{T}_{\mathrm{a}}=75^{\circ} \mathrm{C}$ and $5 \mathrm{pv}$ decalin

Figure III-3.22. Recovery by imbibition from mixed-wet cores for different oil viscosities. 


\section{Nomenclature}

$A_{i}$ open area of ith face of the sample, $\mathrm{cm}^{2}$

$k$ gas permeability, md,

$\mathrm{L}_{\mathrm{c}}$ characteriistic length, cm,

$\mathrm{R}$ oil recovery, \%OOIP

$R_{\infty}$ final oil recovery, $\%$ OOIP

$S_{w i}$ initial water saturation, $\%$,

$\mathrm{t}$ imbibition time, min.

$t_{D}$ dimensionless imbibition time

$\mathrm{T}_{\mathrm{a}}$ aging temperature

$\mathrm{T}_{\mathrm{m}}$ imbibition test temperature

$\mathrm{T}_{\mathrm{f}}$ decalin flush temperature $\alpha$ constant for Aronofsky model

$\phi$ porosity, $\%$

$\sigma$ oil-water interfacial tension, dynes $/ \mathrm{cm}$.

$\mu_{\mathrm{w}}$ water viscosity, cp,

$\mu_{\mathrm{o}}$ oil viscosity, $\mathrm{cp}$.

\section{References}

Aronofsky, J.S., Masse, L. and Natanson, S.G., 1958, A model for the mechanism of oil recovery from the porous matrix due to water invasion in fractured reservoirs, Transactions of American Institute of Mining, Engineers, v. 213, p. 17-19.

Chatzis, I., Morrow, N.R., and Lim, H.T., 1983, Magnitude and detailed structure of residual oil saturation, Society of Petroleum Engineers Journal, v. 35, April, p. 311-326.

Graue, A. Viksund, B.G. and Baldwin, B.A., 1999, Reproducible wettability alteration of lowpermeable outcrop chalk, Society of Petroleum Engineers Reservoir Engineering and Evaluation, v.2, 2, April, p. 134-140.

Ma, S., Morrow, N.R. and Zhang, X., 1997, Generalized scaling of spontaneous imbibition data for strongly water-wet systems, Journal of Petroleum Science and Engineering, v. 18, p. 165178.

Morrow, N.R., Lim, H.T., and Ward, J.S., 1986, Effect of crude oil induced wettability changes on oil recovery, Society of Petroleum Engineers Formation Evaluation, v. 1, April, p. 89-103.

Viksund, B.G., Morrow, N.R., Ma, S., Wang, W. and Graue, A., 1998, Initial water saturation and oil recovery from chalk and sandstone by spontaneous imbibition, Paper SCA-9814, in the International Symposium of The Society of Core Analysts, Proceedings, The Hague, The Netherlands.

Wardlaw, N.C. and Cassan, J.P., 1978, Estimation of recovery efficiency by visual observation of pore systems in reservoir rocks, Bulletin of Canadian Petroleum Geology, v. 26, December, p. 572-585.

Xie, X., 1996, Application of the dynamic Wilhelmy plate technique to investigation of oil/brine/quartz wetting alteration by adsorption from crude oil, $\mathrm{Ph}$. D. dissertation, University of Wyoming. 
Xie, X. and Morrow, N.R., 2000, Oil recovery by spontaneous imbibition from weakly water-wet rocks, Paper SCA-2000-26 Proceedings of International Symposium of the Society of Core Analysts, Abu Dhabi, United Arab Emirates.

Xie, X., Morrow, N.R., Buckley, J.S., 2002, Contact Angle Hysteresis and Stability of Wetting Changes Induced by Absorption from Crude Oil, J. Petrol. Sci. Eng. (2002) 33, 147-159.

Zhang, X., Morrow, N.R. and Ma, S., 1996, Experimental verification of a modified scaling group for spontaneous imbibition, Society of Petroleum Engineers Reservoir Engineering, v. 11, no. 4, November, p. 280-285. 


\section{III-3.4. Spontaneous Imbibition for Mixed-Wettability States in Sandstones Induced by Adsorption from Crude Oil (MWX-F)}

Tong, Z., Xie, X., and Morrow, N.R.: "Spontaneous imbibition for mixed-wettability states in sandstones induced by adsorption from crude oil," $7^{\text {th }}$ Internat. Symp. on Reservoir Wettability, Freycinet, Tasmania, 12-14 Mar. 2002.

Wettability control by methods that are of practical relevance to oil recovery is an ongoing problem in wettability research. The preparation of mixed-wettability cores by adsorption from an asphaltic crude oil at elevated temperature and pressure has been investigated. After aging, crude oil was displaced by flow of decalin, an intermediate solvent that was compatible with the crude oil with respect to asphaltene precipitation but not likely to cause undue desorption of polar components of crude oil from pore walls. The decalin was in turn removed by flow of refined oil.

In tests of the stability of the induced wetting states obtained by this approach, imbibition measurements were repeated for up to six cycles of imbibition and drainage. In most cases, wettability changed slightly towards water wetness after the first cycle, but there was comparatively little or no change for further cycles of spontaneous imbibition and forced drainage. Factors in preparation of mixed-wet cores such as the volume of decalin used to flush crude oil from the core and the temperature of flushing were investigated during the course of development of the experimental procedures. Once established, the induced wetting states were stable with respect to further flushing with decalin and also to flushing with toluene, a stronger solvent for asphaltenes.

In experiments on displacement of brine by high viscosity refined oils, the initial water saturation of mixed-wet cores could be readily reduced to much lower values than the water saturation that was present at the time of aging. However, in contrast to the drastic reduction in imbibition rate for cores aged at low water saturation, low initial water saturation obtained by this means had very little effect on subsequent spontaneous imbibition. This observation is consistent with the concept of mixed wettability postulated by Salathiel (1973) that the distribution of water at the time of aging controls the distribution of adsorbed components of crude oil.

\section{Introduction}

Wettability control is a critical factor in laboratory investigation of reservoir wettability and its effect on oil recovery. The development of mixed wettability (MXW) depends on the distribution of connate water at rock surfaces. Areas of rock surface not overlain by bulk water are subject to wettability alteration by adsorption of polar components from crude oil (Salathiel, 1973). Thus, as the connate water saturation decreases, the exposed area increases and a larger change in wetting results (Xie and Morrow, 2000). In addition to initial water saturation, laboratory techniques for the preparation of MXW cores involve numerous choices. Special consideration must be given to avoiding the presence of organic solids through formation of wax or precipitation of asphaltenes. Recently the effect of viscosity ratio on rate of oil recovery by 
spontaneous imbibition was investigated for sandstones prepared by aging the cores in an asphaltic crude oil (Tong, et al., 2001). The cores were then flushed with a solvent, decalin, with the objective of displacing the crude oil but leaving in place most of the adsorbed polar components as an organic film on the rock surface (Morrow, et al., 1985; Graue, 1999; Yang, et al., 1999). The solvent was then replaced with refined oil of various viscosities. Mixed wet cores prepared by this approach will be identified as MXW-F. Key features of preparation of MXW-F cores are listed in Table III-3.5.

Table III-3.5: Factors in MXW-F core preparation.

\begin{tabular}{|l|}
\hline crude oil \\
\hline brine: composition \\
\hline rock \\
\hline initial water saturation prior to aging \\
\hline aging: in high pressure aging cell (aging time $\mathrm{t}_{\mathrm{a}}$ and aging temperature $\mathrm{T}_{\mathrm{a}}$ ) \\
\hline transfer from aging cell to core holder (interim temperature reduction) \\
\hline displacement of crude oil with solvent: temperature $\mathrm{T}_{\mathrm{f}}$ and pore volume $\mathrm{PV}$ \\
\hline mineral oil: cleaned by exposure to silica gel/alumina \\
\hline displacement of solvent with mineral oil: viscosity, $\mathrm{PV}$, temperature, and rate \\
\hline
\end{tabular}

The presence of the adsorbed organic film can cause several orders of magnitude reduction in imbibition rate (Tong, et al., 2001). The stability and properties of the organic film in MXW-F cores present important considerations with respect to wettability control. With mineral oil rather than crude as the oil phase, ambiguity as to possible causes of change in wettability by surface phenomena such as desorption or some form of physical or chemical rearrangement or detachment is greatly reduced because the supply of polar organic material is limited to that initially retained at the rock surface. In the present study we report on:

(1) aspects of preparation of MXW-F cores

(2) the reproducibility of cyclic imbibition/drainage tests

(3) the stability of wetting after further exposure to solvents

(4) the effect of reduction in initial water saturation with respect to the value at the time of aging

(5) the stability of wetting for time periods of up to almost one year.

\section{Experimental}

\section{Material}

CORES

Cores cut from Berea sandstone blocks were nominally $3.8 \mathrm{~cm}$ in diameter and $7.6 \mathrm{~cm}$ in length. The air permeabilities of the cores ranged from 80 to $106 \mathrm{md}$, and the porosities were all within $18.8 \pm 0.3 \%$. Core properties are listed in Table III-3.6. 
Table III-3.6. Physical properties of the cores and the displaced oil

\begin{tabular}{|c|c|c|c|c|c|c|c|}
\hline Core No. & $\mathrm{D}, \mathrm{cm}$ & $\mathrm{L}, \mathbf{c m}$ & $\mathbf{k}_{\mathrm{g}}$, md & $\phi, \%$ & $\mathbf{S}_{\mathrm{wi}}, \%$ & $\mu_{0}, \mathbf{c p}$ & $\mathbf{L}_{\mathbf{c}}, \mathbf{c m}$ \\
\hline 1B32 & \multirow{6}{*}{3.8} & 7.7 & 100.1 & 18.9 & 21.1 & 8.4 & 1.27 \\
\hline $1 \mathrm{~B} 33$ & & 7.9 & 91.7 & 18.7 & 21.3 & 18 & 1.76 \\
\hline $2 \mathrm{~B} 2$ & & 7.7 & 79.4 & 18.5 & 20.8 & 39.5 & 1.26 \\
\hline 2B9 & & 7.9 & 88.1 & 18.6 & 21.3 & 18 & 1.27 \\
\hline 2B18 & & 7.8 & 80.1 & 18.5 & 21.4 & 66 & 1.27 \\
\hline 2B27 & & 7.8 & 106.4 & 18.6 & 21.7 & 3.8 & 1.27 \\
\hline 4B22 & \multirow{8}{*}{3.8} & 7.9 & 95.6 & 19.0 & 25.0 & \multirow{8}{*}{3.8} & 1.57 \\
\hline $4 \mathrm{~B} 23$ & & 7.7 & 98.7 & 19.1 & 24.5 & & 1.55 \\
\hline $4 \mathrm{~B} 25$ & & 8.0 & 92.9 & 19.0 & 25.3 & & 1.57 \\
\hline 4B26 & & 7.9 & 91.6 & 18.9 & 24.8 & & 1.57 \\
\hline 4B27 & & 7.8 & 97.7 & 19.1 & 25.0 & & 1.57 \\
\hline 4B28 & & 7.7 & 95.1 & 19.1 & 24.9 & & 1.56 \\
\hline 4B29 & & 8.0 & 93.3 & 18.9 & 25.2 & & 1.57 \\
\hline 4B31 & & 8.0 & 95.0 & 18.9 & 24.8 & & 1.57 \\
\hline
\end{tabular}

\section{CRUDE OIL}

An asphaltic crude oil of 25.2 API gravity, Alaska 95 (A95) from Prudhoe Bay, was used to change the wettability of the cores. The oil was degassed by vacuum treatment. The oil had $6.55 \mathrm{wt} \%$ of $\mathrm{n}$-heptane asphaltenes and no detectable wax content. The viscosity of the evacuated oil was $70.9 \mathrm{cp}$ at room temperature $\left(22^{\circ} \mathrm{C}\right)$. The acid and base numbers were 0.24 and 2.2 respectively. This oil was selected because it was known to induce significant change in wettability and problems associated with wax deposition would not be encountered. Because of limited supply, some of the crude oil was reused; this oil is indicated as A95*.

\section{MINERAL OIL}

Mineral oils with different viscosities were prepared by mixing Soltrol 220 mineral oil (3.8 cp) and white mineral oil $(180.0 \mathrm{cp})$ in ratios selected to give intermediate viscosities as required. The mineral oils were cleaned by exposure to silica gel and alumina. Viscosities of the mixtures are given in Table 2.

BRINE

Synthetic reservoir brine of 22,310 ppm TDS was prepared of composition: $\mathrm{NaCl}$ $21.3 \mathrm{~g} / \mathrm{L}, \mathrm{KCl} 0.10 \mathrm{~g} / \mathrm{L}, \mathrm{CaCl}_{2} 0.61 \mathrm{~g} / \mathrm{L}, \mathrm{MgCl}_{2} 0.20 \mathrm{~g} / \mathrm{L} . \mathrm{NaN}_{3}(0.10 \mathrm{~g} / \mathrm{L})$ was added as a biocide.

\section{Procedure}

ESTABLISHING INITIAL WATER SATURATION PRIOR TO AGING

The core samples were first saturated with reservoir brine by vacuum and allowed to soak for at least 10 days to attain ionic equilibrium. Initial water saturation, $S_{w i}$, in the range of 21 to 
$25 \%$ (see Table III-3.6) was established by displacing reservoir brine with $\mathrm{A} 95$ crude oil at $75^{\circ} \mathrm{C}$ at a pressure drop of about 300 psi.

\section{AGING AND REPLACEMENT OF CRUDE OIL WITH MINERAL OIL}

The cores containing initial water and crude oil were submerged in crude oil in sealed aging cells. Cores were aged at 75 or $95^{\circ} \mathrm{C}\left(\mathrm{T}_{a}\right)$ for 10 days $\left(\mathrm{t}_{a}\right)$. After aging, the core was mounted in a core holder and the crude oil was displaced by decalin (5 PV, except where the effect of PV decalin was investigated) at $3 \mathrm{ft} /$ day at $60^{\circ} \mathrm{C}$. Decalin was displaced by $3 \mathrm{PV}$ of mineral oil of the selected viscosity at $60^{\circ} \mathrm{C}$, followed by $2 \mathrm{PV}$ of mineral oil at room temperature. The objective of the solvent flush was to remove the bulk crude oil from the core but leave, as expected from previous experience, the polar components adsorbed on the rock surface. This procedure was designed to avoid precipitation of asphaltenes and associated effects that might result from direct displacement of crude oil by the mineral oil.

\section{SPONTANEOUS IMBIBITION}

The MXW-F cores containing initial water saturation and mineral oil of the selected viscosity were set in glass imbibition cells filled initially with brine. Oil volume produced by imbibition of brine, expressed as percentage of original oil in place (\%OOIP), versus time was recorded. All of the imbibition tests were performed at room temperature.

\section{SUBSEQUENT SPONTANEOUS IMBIBITION}

After the initial imbibition tests, the cores were mounted in core holders and imbibed water was displaced by injection of mineral oil at 0.1 to $4.0 \mathrm{ml} / \mathrm{min}$ according to the viscosity and the $S_{\mathrm{wi}}$ at ambient temperature. After most of the displaceable brine had been produced, the water production was monitored. Displacement was continued until the original water saturation had been reached. In all cases, at least 1 to $2 \mathrm{PV}$ of mineral oil was injected. For displacement by mineral oils of higher viscosity, water saturation fell to well below the target value even before $1 \mathrm{PV}$ of mineral oil had been injected at a pressure drop of well below half of that originally used to establish initial water saturation with crude oil. Subsequent imbibition tests were run at these reduced values. The imbibition tests were repeated for up to six cycles of drainage and imbibition in all. For the last two cycles additional flushing at ambient temperature with solvent (5 PV of decalin or toluene at $0.2 \mathrm{ml} / \mathrm{min}$ ) was included as a further test of stability of the MWX-F wetting state. Test conditions are summarized in Table III-3.7. 
Table III-3.7. Conditions for cycles of imbibition and flushing

\begin{tabular}{|c|c|c|c|c|c|c|c|}
\hline Core No. & \#Cycle & $\mathrm{S}_{\mathrm{wi}}, \%$ & $\begin{array}{l}\text { Mineral } \\
\text { oil/solvent } \\
\text { displacements }\end{array}$ & Core No. & \#Cycle & $\mathrm{S}_{\mathrm{wi}}, \%$ & $\begin{array}{l}\text { Mineral } \\
\text { oil/solvent } \\
\text { displacements }\end{array}$ \\
\hline \multirow{6}{*}{ 2B27 } & $1 *$ & 21.7 & \multirow{4}{*}{$\begin{array}{l}\text { mineral oil } \\
\left(\mu_{0}=3.8 \mathrm{cp}\right)\end{array}$} & \multirow{6}{*}{$2 B 9$} & $1 *$ & 21.3 & \multirow{4}{*}{$\begin{array}{l}\text { mineral oil } \\
\left(\mu_{0}=18 \mathrm{cp}\right)\end{array}$} \\
\hline & 2 & 19.2 & & & 2 & 19.9 & \\
\hline & 3 & 18.9 & & & 3 & 19.8 & \\
\hline & 4 & 18.9 & & & 4 & 19.8 & \\
\hline & 5 & 18.5 & \multirow{2}{*}{$\begin{array}{l}5 \mathrm{PV} \text { of toluene } \\
\text { followed by } \\
\text { mineral oil }\left(\mu_{\mathrm{o}}=\right. \\
3.8 \mathrm{cp})\end{array}$} & & 5 & 19.4 & $\begin{array}{l}5 \text { PV of decalin } \\
\text { followed by mineral oil } \\
\left(\mu_{0}=18 \mathrm{cp}\right)\end{array}$ \\
\hline & 6 & 18.8 & & & 6 & 17.6 & $\begin{array}{l}5 \quad P V \text { of toluene } \\
\text { followed by mineral oil } \\
\left(\mu_{0}=18 \mathrm{cp}\right)\end{array}$ \\
\hline \multirow{6}{*}{$1 \mathrm{B32}$} & $1 *$ & 21.1 & \multirow{4}{*}{$\begin{array}{l}\text { mineral oil } \\
\left(\mu_{0}=8.4 \mathrm{cp}\right)\end{array}$} & \multirow{6}{*}{$2 B 2$} & $1 *$ & 20.8 & \multirow{4}{*}{$\begin{array}{l}\text { mineral oil } \\
\left(\mu_{\mathrm{o}}=39.4 \mathrm{cp}\right)\end{array}$} \\
\hline & 2 & 21.4 & & & 2 & 13.6 & \\
\hline & 3 & 21.4 & & & 3 & 12.3 & \\
\hline & 4 & 21.4 & & & 4 & 12.3 & \\
\hline & 5 & 21.4 & \multirow{2}{*}{$\begin{array}{l}5 \text { PV of toluene } \\
\text { followed by } \\
\text { mineral oil }\left(\mu_{\mathrm{o}}=\right. \\
8.4 \mathrm{cp})\end{array}$} & & 5 & 7.5 & $\begin{array}{l}5 \quad \text { PV of decalin } \\
\text { followed by mineral oil } \\
\left(\mu_{0}=39.4 \mathrm{cp}\right)\end{array}$ \\
\hline & 6 & 21.4 & & & 6 & 7.5 & $\begin{array}{l}5 \quad \text { PV of toluene } \\
\text { followed by mineral oil } \\
\left(\mu_{0}=39.4 \mathrm{cp}\right)\end{array}$ \\
\hline \multirow[t]{6}{*}{$1 \mathrm{B33}$} & $1 *$ & 21.3 & \multirow{4}{*}{$\begin{array}{l}\text { mineral oil } \\
\left(\mu_{o}=18 \mathrm{cp}\right)\end{array}$} & \multirow[t]{6}{*}{$2 B 18$} & $1 *$ & 21.4 & \multirow{4}{*}{$\begin{array}{l}\text { mineral oil } \\
\left(\mu_{0}=66 \mathrm{cp}\right)\end{array}$} \\
\hline & 2 & 21.2 & & & 2 & 9.7 & \\
\hline & 3 & 20.7 & & & 3 & 9.7 & \\
\hline & 4 & 20.7 & & & 4 & 9.7 & \\
\hline & 5 & 20.7 & $\begin{array}{l}5 \mathrm{PV} \text { of toluene } \\
\text { followed by } \\
\text { mineral oil }\left(\mu_{\mathrm{o}}=\right. \\
18 \mathrm{cp})\end{array}$ & & 5 & 9.7 & \multirow[t]{2}{*}{$\begin{array}{l}5 \quad \text { PV of toluene } \\
\text { followed by mineral oil } \\
\left(\mu_{0}=66 \mathrm{cp}\right)\end{array}$} \\
\hline & 6 & 20.9 & $\begin{array}{l}5 \text { PV of decalin } \\
\text { followed by } \\
\text { mineral oil }\left(\mu_{0}=\right. \\
18 \mathrm{cp})\end{array}$ & & 6 & 9.7 & \\
\hline
\end{tabular}

* After aging the crude oil was initially displaced with $5 \mathrm{PV}$ of decalin and then flushed with mineral oil.

\section{Results and Discussion}

The effect of solvent flush temperature and volume used to displace crude oil

Preparation of MXW-F cores involves several steps beyond choice of the crude oil/brine/rock (COBR) combination and the aging conditions (Table III-3.5). The first step is the removal of crude oil by flushing the core with a solvent. Decalin was chosen because it was not likely to precipitate asphaltenes as may be the case with alkanes or direct displacement with mineral oil. Decalin does not act as a strong solvent for adsorbed polar films and can be expected to remove somewhat less of the adsorbed species than an aromatic solvent such as toluene.

The crude oil A95* was used in core preparation for the results described in this section. The wetting induced for $75^{\circ} \mathrm{C}$ aging and $60^{\circ} \mathrm{C}$ flushing indicated greater water wetness than 
obtained in a comparable test with A95 oil, as might be expected for an oil sample from which some of the polar components had already been depleted by adsorption.

Imbibition results for recovery of $3.8 \mathrm{cp}$ mineral oil for solvent displacement temperatures of $22,45,60$ and $75^{\circ} \mathrm{C}$ and essentially constant $S_{w i}$ are shown in Fig. III-3.23. Raising the flush temperature to $45^{\circ} \mathrm{C}$ caused a distinct increase in water-wetness. Results for the $60^{\circ} \mathrm{C}$ flush showed only slight increase in imbibition rate over that for $45^{\circ} \mathrm{C}$. The choice of flush temperature also involves a compromise between avoiding wax precipitation and causing the formation of a gas phase during experimentation. A $60^{\circ} \mathrm{C}$ flush temperature was adopted.
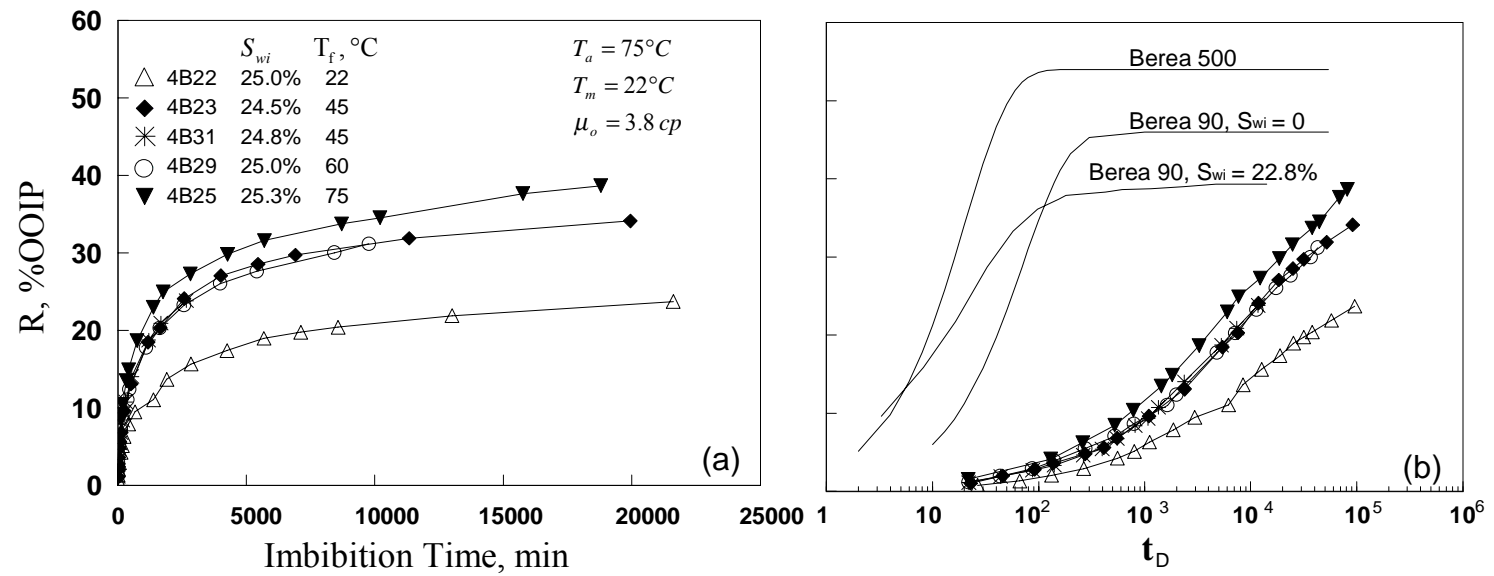

Figure III-3.23. The effect of temperature of $5 \mathrm{PV}$ decalin flush on oil recovery.

For very strongly water-wet systems, recovery of refined oil of different viscosities by spontaneous imbibition was correlated satisfactorily by normalized oil recovery versus dimensionless time, $\mathrm{t}_{\mathrm{D}}$, where

$$
t_{D}=t \sqrt{\frac{k}{\phi}} \frac{\sigma}{\sqrt{\mu_{o} \mu_{w}}} \frac{1}{L_{c}^{2}} .
$$

$t$ is time, $k$ is gas permeability, $\phi$ is porosity, $\sigma$ is the interfacial tension, $\mu_{\mathrm{o}}$ and $\mu_{\mathrm{w}}$ are the oil and brine viscosities, $\mathrm{L}_{\mathrm{c}}$ is a characteristic length that compensates for sample size, shape, and boundary conditions (Ma et al., 1997). A correlation developed for a wide range of rock types is indicated in Fig.III-3.23b as Berea 500. This designation is used because, although many types of porous media were tested, a significant proportion of the data used to define the correlation was obtained for Berea sandstone with nominal permeability to brine of $500 \mathrm{md}$.

However, dimensionless times for Berea 90 as received, the available model rock used in this study, were consistently less than observed for other rock types. A representative curve for zero $\mathrm{S}_{\mathrm{wi}}$ is shown in Fig. III-3.23b. For imbibition by Berea 90 containing an initial water saturation, higher imbibition rates and increased trapping of oil were observed; the curve 
included in Fig. III-3.23b was closely reproduced for duplicate core plugs. Possible causes of the differences between results for Berea 500 and Berea 90 both with and without a $S_{w i}$ are being investigated.

In a previous test of MXW-F cores prepared using Berea 90, results for recovery of mineral oils, with viscosity ranging from 3.8 to $66.0 \mathrm{cp}$, were all closely correlated (Tong et al., 2001). If the effect of fluid viscosity and other factors can be correlated, differences in imbibition rates can be ascribed to wettability.

The MXW-F results for different flush temperature are plotted in Fig. III-3.23b as oil recovery versus dimensionless time. The decrease in imbibition rate by several orders of magnitude relative to the three reference curves for non-treated cores included in Fig. III-3.23b clearly shows the effect of adsorption on wettability.

The sensitivity of wettability to the volume of solvent, as indicated by spontaneous imbibition, is shown in Fig. III-3.24. In this test, four cores with about the same initial water saturation $\left(\mathrm{S}_{\mathrm{wi}} \approx 25 \%\right)$ were flushed with either $2.5,5,10$, or 20 pore volumes $(\mathrm{PV})$ of decalin. Decalin was then replaced by mineral oil with a viscosity of $3.8 \mathrm{cp}$ at $0.2 \mathrm{ml} / \mathrm{min}$. The imbibition tests indicated modest increase in water-wetness with increase in solvent PV. However the difference between 2.5 to $5 \mathrm{PV}$ was quite small and $5 \mathrm{PV}$ was adopted for subsequent tests. Scaled results are plotted in Fig. III-3.24b.
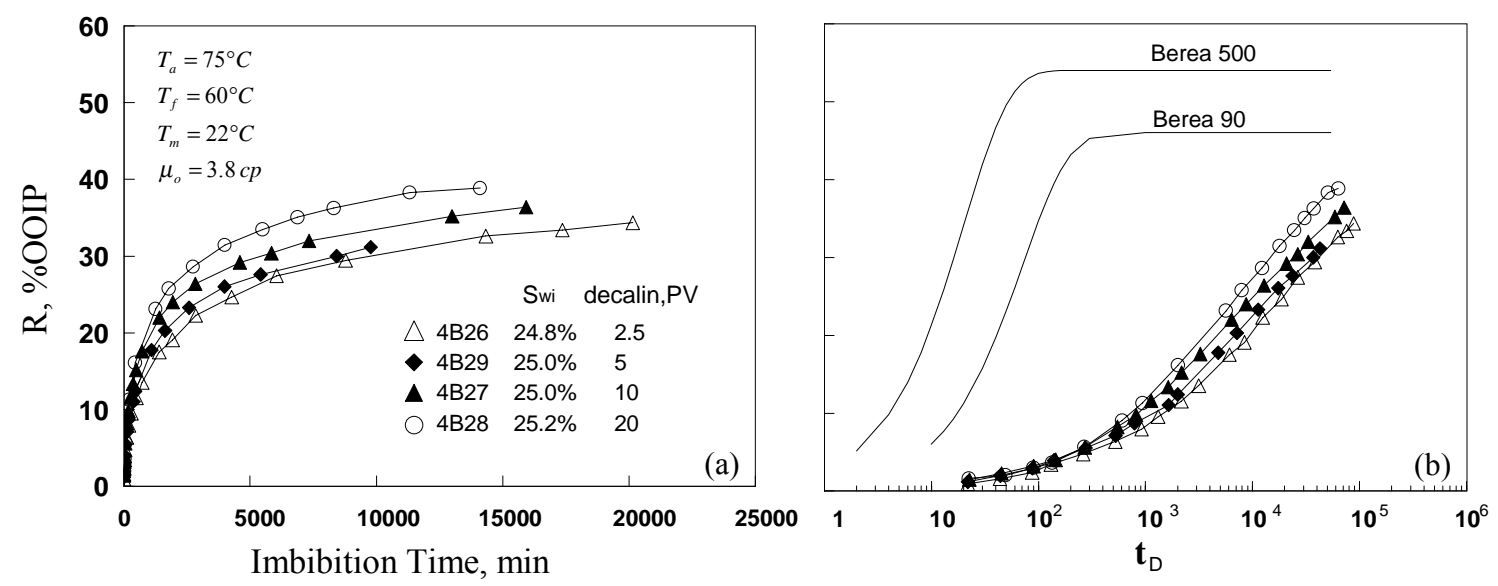

Figure III-3.24. The effect of flush volume (PV) of decalin on oil recovery.

Wettability change caused by multiple cycles of spontaneous imbibition and forced displacement

REPRODUCIBILITY

Reproducibility of wettability alteration as judged from imbibition data for the first cycle was satisfactory (Tong et al, 2001). Additional comparison of such results is presented in Figs.III-3.25a and III-3.25b for an $18 \mathrm{cp}$ mineral oil. 


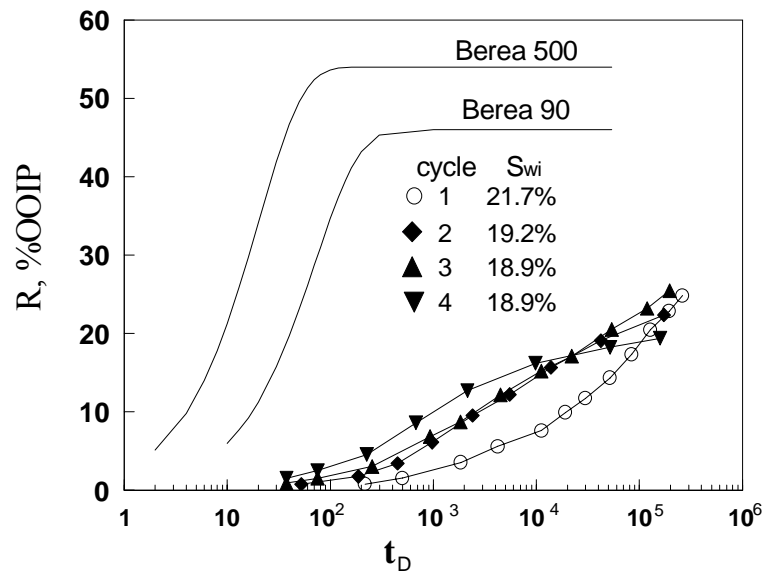

a. Core \#2B27, $\mu_{0}=3.8 \mathrm{cp}$

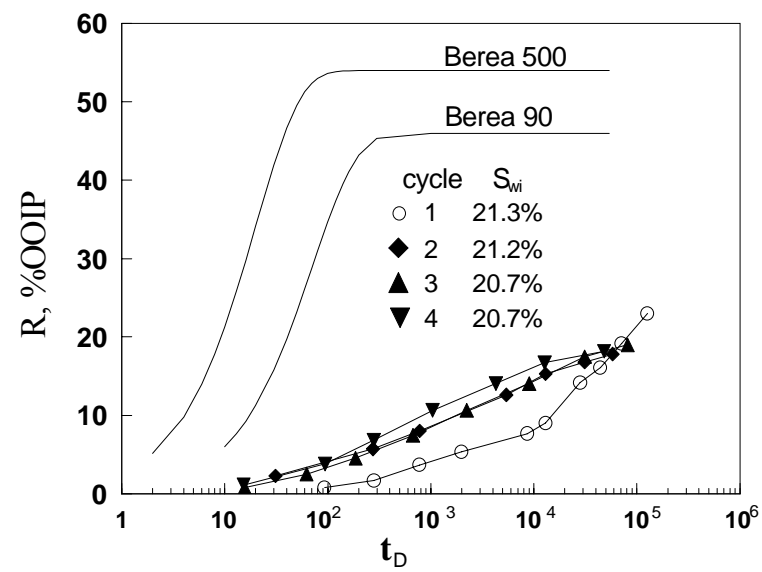

c. Core \#1B33, $\mu_{\mathrm{o}}=18.0 \mathrm{cp}$

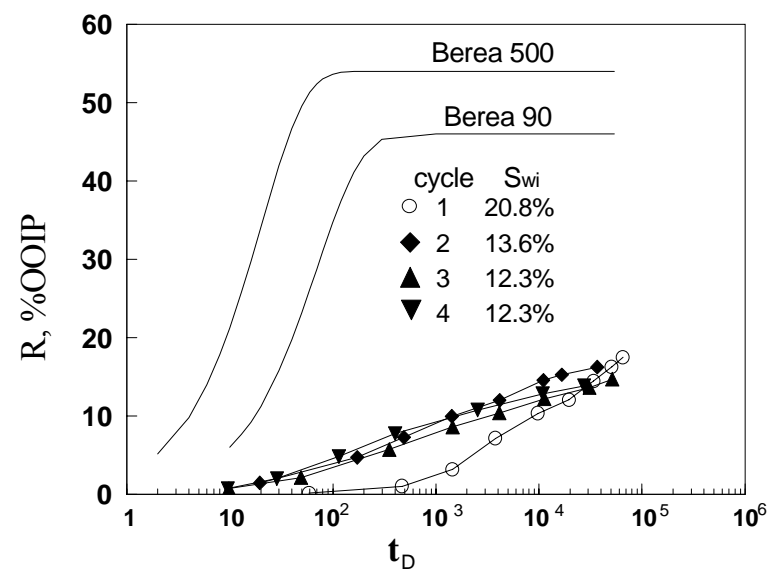

e. Core $\# 2 B 2, \mu_{0}=39.4 \mathrm{cp}$

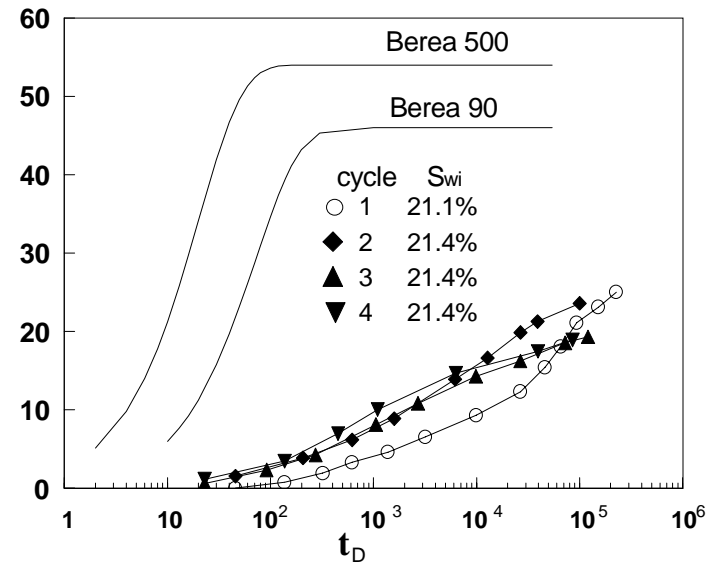

b. Core \#1B32, $\mu_{0}=8.4 \mathrm{cp}$

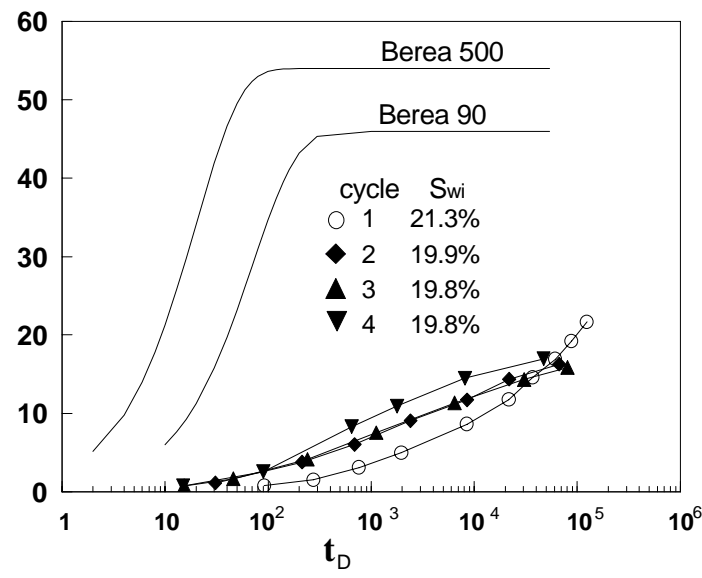

d. Core $\# 2 B 9, \mu_{0}=18.0 \mathrm{cp}$

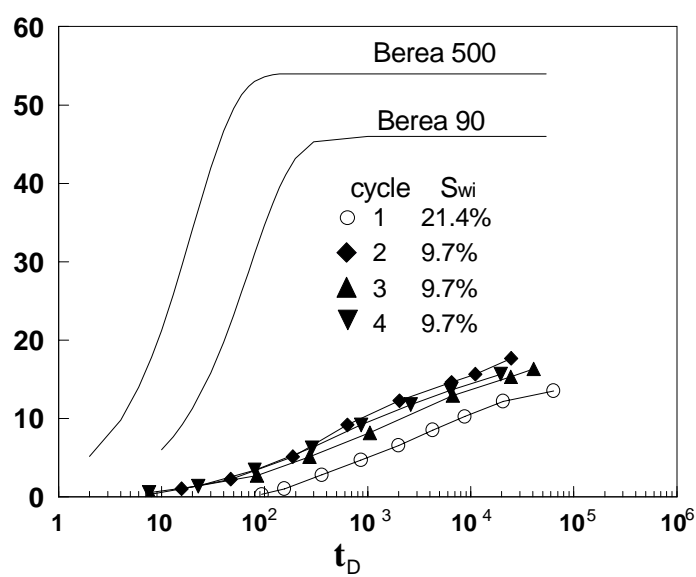

f. Core $\# 2 B 18, \mu_{0}=66 \mathrm{cp}$

Figure III-3.25. The stability of wetting. $T_{a}=95^{\circ} \mathrm{C}, T_{f}=60^{\circ} \mathrm{C}, T_{m}=22^{\circ} \mathrm{C}$. 


\section{IMBIBITION AND DRAINAGE CYCLES}

To test the stability of the induced MXW-F wetting states, multiple cycles of imbibition measurements were performed for mineral oils with viscosities of 3.8, 8.4, 18.0, 39.5 and $66 \mathrm{cp}$. After the primary imbibition measurements, mineral oil was injected to reestablish initial water saturation $\mathrm{S}_{\mathrm{wi}}$ (see Table III-3.7). Imbibition rate curves were then measured for further cycles of drainage and imbibition.

Wettability changed towards water-wetness after the first cycle, but there was comparatively little or no change for further measurements of spontaneous imbibition. These results also provide useful indication of the temporal stability of the adsorbed films, the total period of experimentation being 9 months.

The data in Fig.III-3.25 are re-plotted in Fig. III-3.26 for comparison of correlated data for each cycle of displacement. For all 6 cores, the first cycle imbibition curves were closely correlated (Fig. III-3.26a). The curves for the second cycle all showed increased imbibition rate compared to the first cycle but were all closely correlated (Fig. III-3.26b). Results for cycle 3 were also closely correlated and in very close agreement with cycle 2 results (Fig. III-3.26c). Similarly imbibition curves for cycle 4 were in good agreement with those for cycles 2 and 3 (Fig. III-3.26d). All of these results indicate that the choice of mineral oil introduced after the primary aging procedure did not have significant effect on the wettability of the cores.

Research on wettability and oil recovery often involves the choice of whether to use the same cores repeatedly or to use duplicate core plugs. Individual core samples, especially for reservoir cores and for comparative tests on MXW or MXW-F cores have the advantage that problems of variation in mineralogy and pore structure are minimized if not completely avoided. However, there is no assurance that the wetting condition can be reproduced from one test to the next. The advantage of duplicate core plugs is that comparative studies can be made on cores that have the same preparation history. Duplicate cores are usually preferable if the rock is homogeneous and available in quantity.

The forgoing imbibition tests suggest that for MXW-F wetting, comparison of a selected change in displacement conditions should be made between second and subsequent cycles of displacement rather than the first and second cycle. The observed shift towards water wetness for the first and second cycle may be related to the behavior of the adsorbed organic films. Atomic force microscopy studies on films laid down on mica, a molecularly smooth aluminumsilicate substrate, show that when water is overlying, the film may blister and peel away from the solid (Buckley and Lord, 2002). The behavior of the film depends on the robustness of adhesion to the solid and the lateral strength of the film. Films deposited in rocks may be partially unstable as a result of the decrease in capillary pressure and the movement of interfaces through pores that accompany spontaneous imbibition. The most significant rearrangements of the film, for example by detachment and folding, are most likely to occur during the first cycle of spontaneous imbibition and drainage by viscous displacement. This would explain the small but consistent shift towards water wetness between the first and second imbibition. 


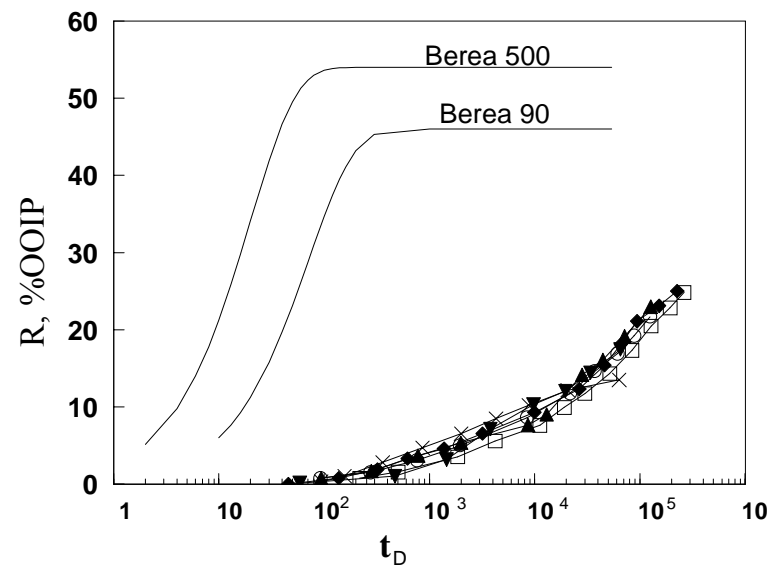

a. first cycle

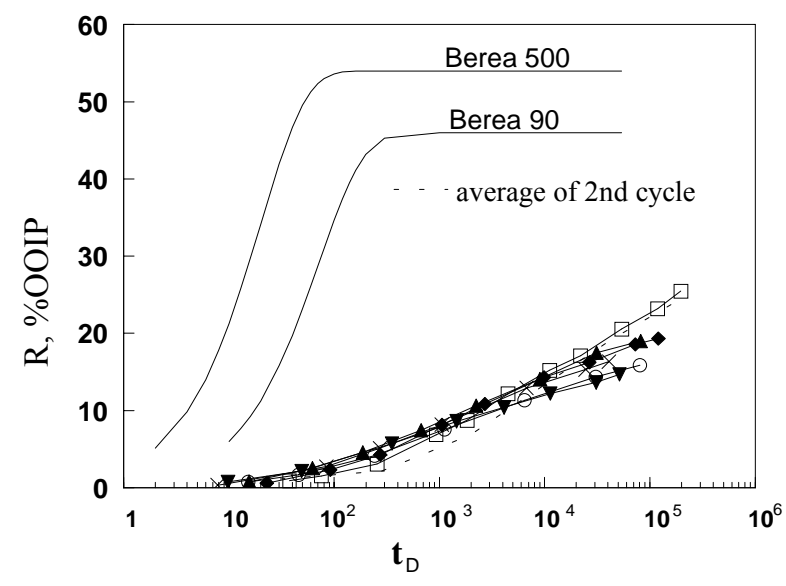

c. third cycle

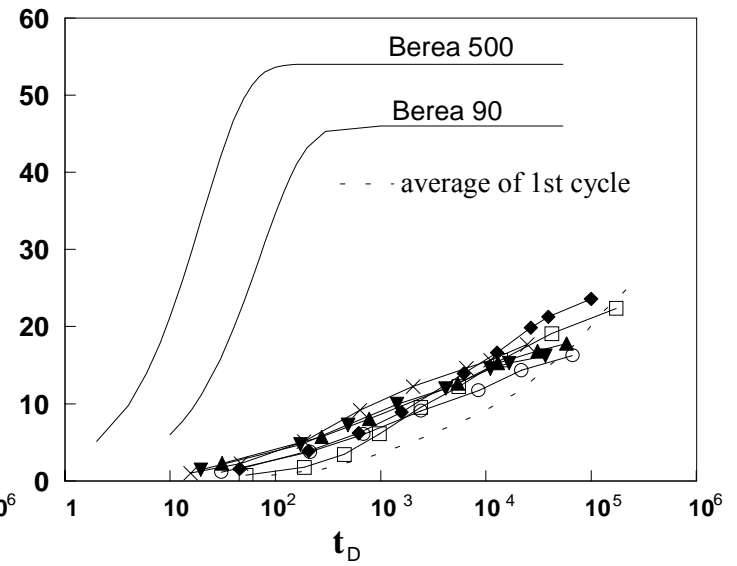

b. second cycle

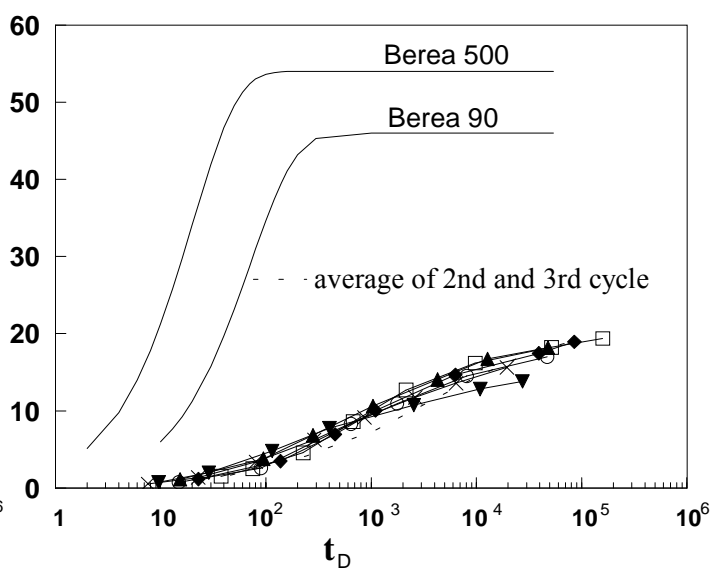

d. fourth cycle

Figure III-3.26. Sequential imbibition curves for mixed-wettability with $\mu_{0}$ of 3.8, 8.4, 18, 39.4, and $66 \mathrm{cp}$. Results for individual cores are indicated as follows: $\square 2 \mathrm{~B} 27, \bullet 1 \mathrm{B32}, \triangle 1 \mathrm{B33}, \mathrm{O} 2 \mathrm{~B} 9,-2 \mathrm{~B} 2$, *2B18.

Stability of induced wetting states to flushing with solvent at ambient temperature

After four cycles of imbibition and drainage, mineral oil was displaced from the cores by either decalin, the solvent used originally to flush crude oil from the cores, or toluene a stronger solvent for the asphaltenes than decalin. Imbibition data measured after replacing the solvent with mineral oil, cycles 5 and 6, are shown in Fig. III-3.27. Imbibition rates after flushing the MXW-F core with solvent were slightly faster than the average of cycles 2,3 and 4 in most cases for early times. At later imbibition times there was a closer overall match with the average imbibition rates. This indicates that the adsorbed layer of crude oil components was stable even to exposure to toluene. 


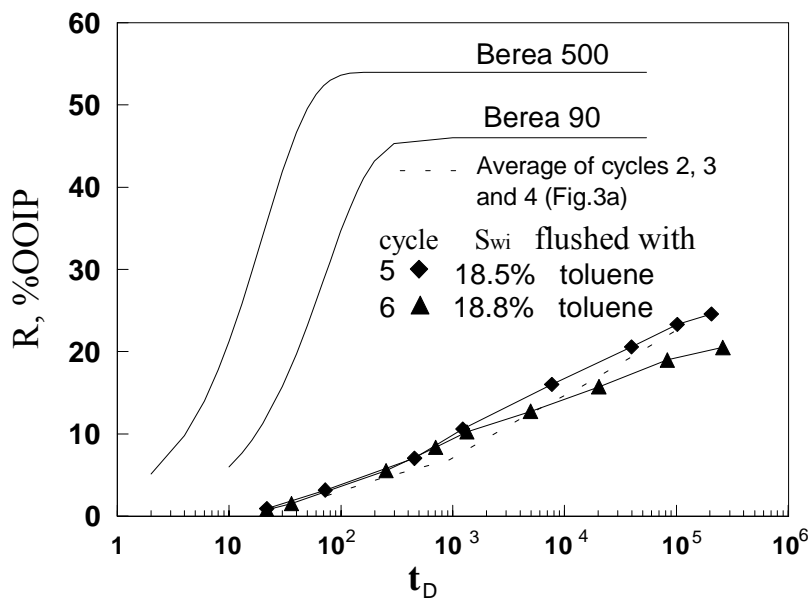

a. Core \#2B27, $\mu_{\mathrm{o}}=3.8 \mathrm{cp}$

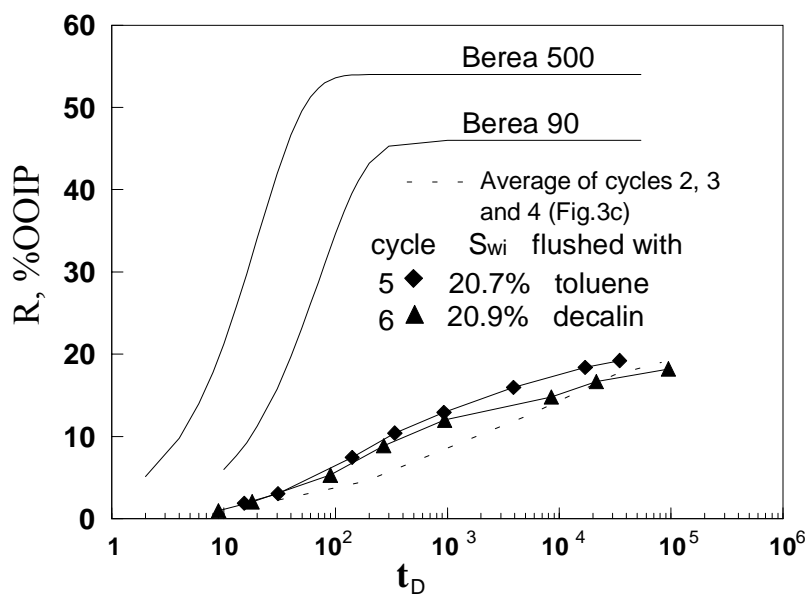

c. Core \#1B33, $\mu_{0}=18.0 \mathrm{cp}$

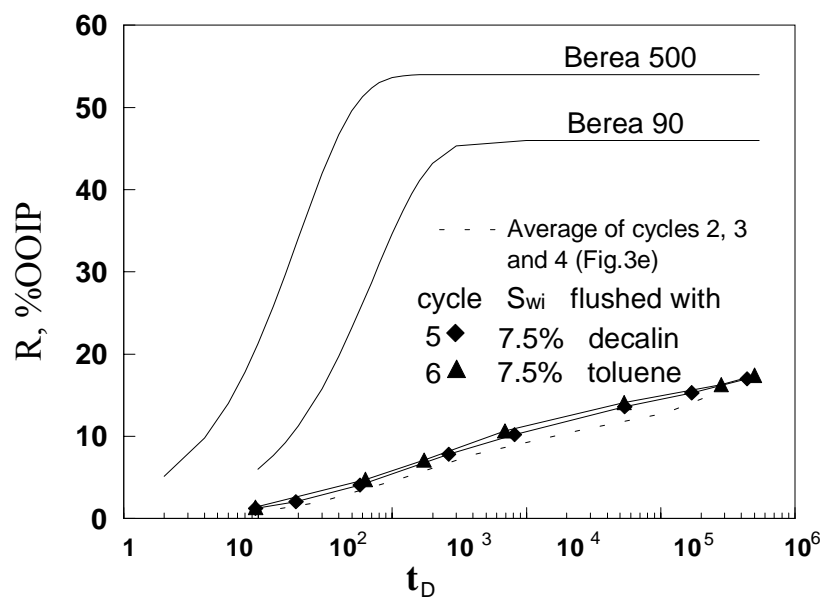

e. Core \#2B2, $\mu_{\mathrm{o}}=39.4 \mathrm{cp}$

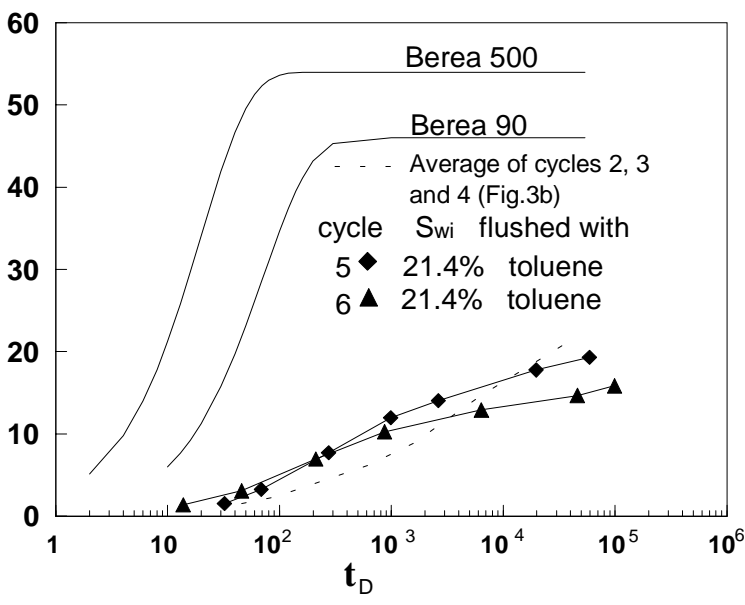

b. Core $\# 1 B 32, \mu_{o}=8.4 \mathrm{cp}$

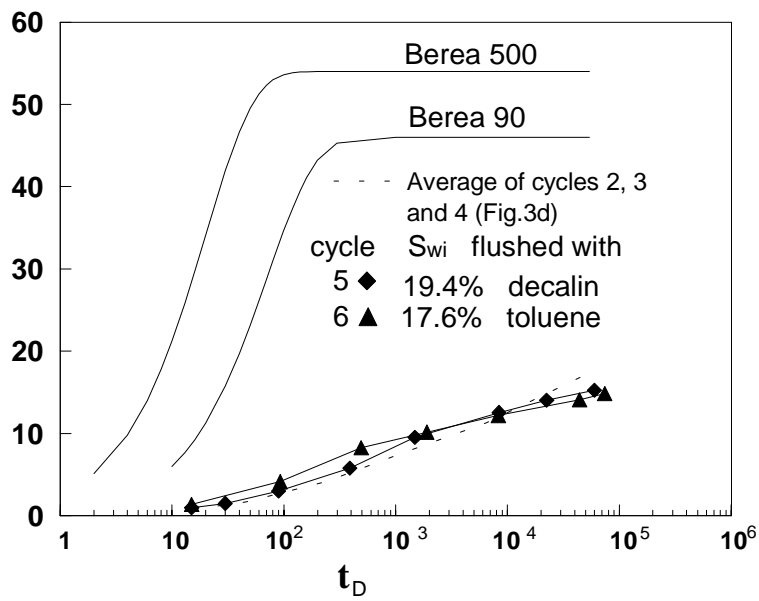

d. Core \#2B9, $\mu_{\mathrm{o}}=18.0 \mathrm{cp}$

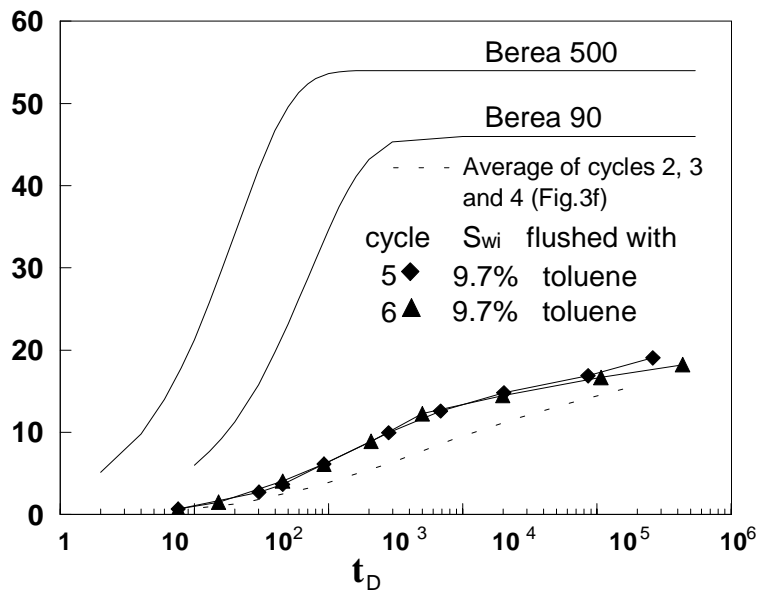

f. Core \#2B18, $\mu_{\mathrm{o}}=66 \mathrm{cp}$

Figure III-3.27. The stability of wetting. $T_{a}=95^{\circ} \mathrm{C}, T_{f}=60^{\circ} \mathrm{C}, T_{m}=22^{\circ} \mathrm{C}$ 


\section{Mixed wettability and initial water saturation}

In the course of establishing initial water saturations it was observed that, for MXW-F cores displaced with viscous mineral oils (39.5 and $66 \mathrm{cp}$ ), $\mathrm{S}_{\mathrm{wi}}$ was readily decreased by the second and subsequent drainage with mineral oil. For cores \#2B2 $\left(\mu_{\mathrm{o}}=39.4 \mathrm{cp}\right)$ and \#2B18 $\left(\mu_{\mathrm{o}}\right.$ $=66 \mathrm{cp}$ ) the connate water saturation $\mathrm{S}_{\mathrm{wi}}$ decreased respectively from $20.8 \%$ and $21.4 \%$ by about $50 \%$ for subsequent cycles (see Table III-3.7). This was unexpected because, in previous comparisons of primary and secondary drainage including MXW cores, water retention was higher for secondary drainage. Three examples are compared in a review article by Morrow and Melrose (1991).

Achievement of low connate water saturation, often typical of oil reservoirs above the transition zone, is sometimes only attained after drainage at high capillary pressure for periods of many months. Because of this added time and expense for reservoir conditions displacement tests, further exploration of this approach to achieving low initial water saturations for other crude oil/brine/ rock combinations is justified.

Although $\mathrm{S}_{\mathrm{wi}}$ was markedly decreased by secondary drainage with viscous mineral oil, the basic MXW-F wettability pattern can be expected to be maintained because there was no further exposure of the rock to crude oil. Imbibition rates were closely reproduced for subsequent imbibition and displacement cycles, indicating that the imbibition mechanism was essentially determined by the basic wettability pattern of the cores and the effect of subsequent variation in initial water saturation was minor. Examples of marked reduction in drainage capillary pressures that result from re-aging cores with crude oil for sequential reduction in initial water saturation at the time of aging have been reported by Malsalmeh (2000).

Examples (Tong et al., 2001) of recovery of mineral oil by imbibition for duplicate MXW-F core plugs are included in Fig.III-3.28. Reduction in the initial water saturation at which cores are exposed to crude oil during the aging period caused a large reduction in rate of spontaneous imbibiton. These observations are consistent with the concept of mixed wettability postulated by Salathiel (1973) that the distribution of connate water at the time of aging controls the distribution of adsorbed components of crude oil. 


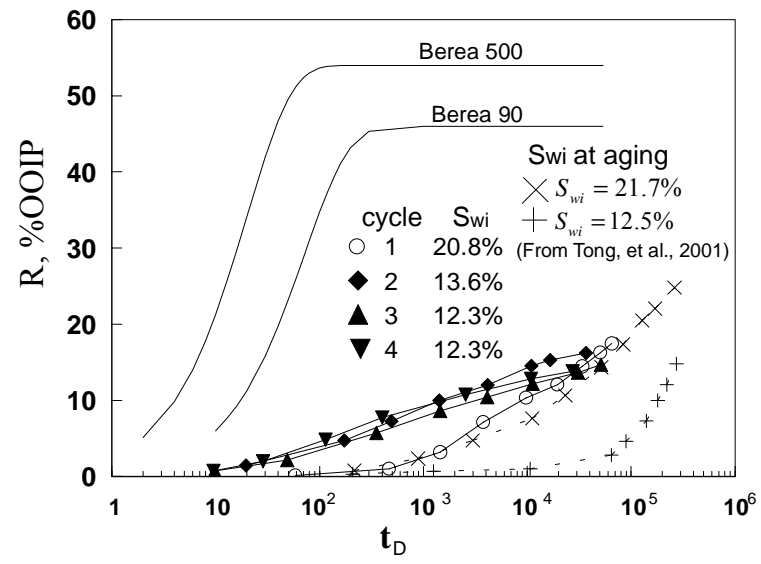

a. Core \#2B2, $\mu_{\mathrm{o}}=39.4 \mathrm{cp}$

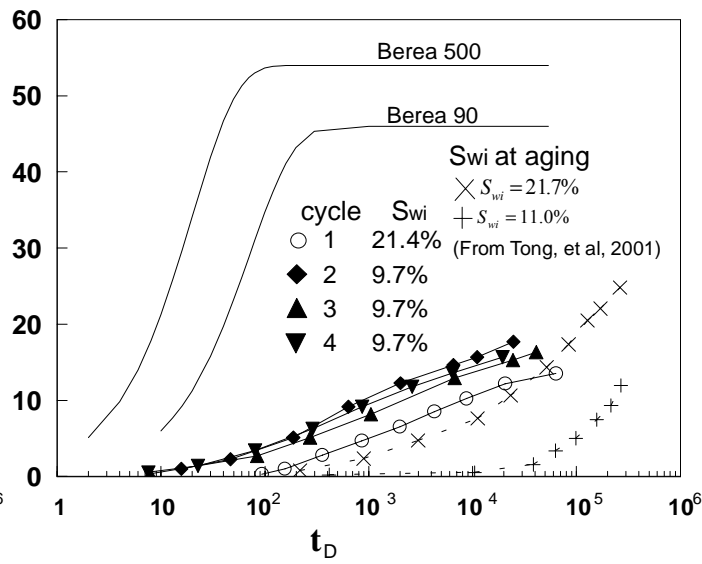

b. Core $\# 2 B 18, \mu_{o}=66 \mathrm{cp}$

Figure III-3.28. Comparison of effect on spontaneous imbibition of initial water saturation at time of aging and reduction in initial water saturation after mixed wettability had been established.

\section{Conclusions}

1. In the preparation of MXW-F cores that were weakly water wet, water wetness increased with the temperature of displacement of crude oil by solvent after aging, and with the volume of solvent used to displace the crude oil.

2. Comparison of primary spontaneous imbibition curves with subsequent measurement showed a shift towards increased water wetness.

3. Reproducibility of secondary and subsequently measured spontaneous imbibition indicated that a stable wetting condition had been achieved by adsorption from an asphaltic crude oil.

4. These wetting states were independent of the viscosity of the mineral oil used in the imbibition tests.

5. Unexpectedly large reductions in initial water saturation were achieved by flow of viscous mineral oil for MXW-F cores. Imbibition recovery was essentially unaffected by reduction in initial water saturation of MXW-F cores. 


\section{Nomenclature}

$D$ core diameter, $\mathrm{cm}$

$k$ gas permeability, md

$L$ core length, $\mathrm{cm}$

$L_{c}$ characteristic length, $\mathrm{cm}$

$R$ oil recovery, \%OOIP

$S_{w i}$ initial water saturation, \%

$t$ imbibition time, min

$t_{D}$ dimensionless imbibition time
$T_{a} \quad$ aging temperature

$T_{m}$ imbibition test temperature

$T_{f}$ decalin flush temperature

$\phi$ porosity, $\%$

$\sigma$ oil-water interfacial tension, dynes $/ \mathrm{cm}$.

$\mu_{w}$ water viscosity, cp,

$\mu_{o} \quad$ oil viscosity, cp.

\section{References}

Buckley, J.S. and Lord, D., 2002. Wettability and morphology of mica surfaces after exposure to crude oil. To be presented in International Symposium on Reservoir Wettability, Tasmania, Australia.

Graue, A. Viksund, B.G. and Baldwin, B.A., 1999. Reproducible wettability alteration of lowpermeable outcrop chalk. SPEREE, 2, (2), April 134-140.

Ma, S., Morrow, N.R. and Zhang, X., 1997. Generalized scaling of spontaneous imbibition data for strongly water-wet systems. J. Pet. Sci. \& Eng. 18, 165-178.

Malsalmeh, S.K., 2002. Studying the effect of wettability heterogeneity on the capillary pressure curves using the centrifuge technique. J. Petrol. Sci. Eng. (2002) 33, 29-38.

Morrow, N.R., Lim, H.T., and Ward, J.S., 1986. Effect of Crude Oil Induced Wettability Changes on Oil Recovery. SPE Formation Evaluation, April, 1, 89-103.

Morrow, N.R. and Melrose, J.C., 1991. Applications of Capillary Pressure Data to the Determination of Connate Water Saturation. Interfacial Phenomena in Oil Recovery, Ed. N.R. Morrow, Marcel Dekker, 257-287.

Salathiel,R.A., 1973. Oil recovery by surface film drainage in mixed wettability rocks. JPT, Oct., $1216-1224$.

Tong, Z., Xie, X. and Morrow, N.R., 2001. Scaling of viscosity ratio for oil recovery by imbibition from mixed-wet rocks. Proceedings of International Symposium of the Society of Core Analysts, Edinburgh, paper SCA-0121.

Xie, X. and Morrow, N.R., 2000. Oil recovery by spontaneous imbibition from weakly waterwet rocks. Proceedings of International Symposium of the Society of Core Analysts, Abu Dhabi, United Arab Emirates, Paper SCA-0026.

Yang, S.Y., Hirasaki, G.J., Basu, S. and Vaidya, R., 1999. Mechanisms for contact angle hysterersis and advancing contact angles. JPSE, Dec., 4, No.2-4, 63-73. 


\section{III-3.5. Crude Oil Composition and the Stability of Mixed Wettability in Sandstones (MWX-F)}

Tong, Z., Xie, X., and Morrow, N.R.: "Crude Oil Composition and the Stability of Mixed Wettability in Sandstones," paper SCA 2002-31 presented at the International Symposium, Monterey, 22-25 Sept.

The effect of crude oil composition on wettability alteration at smooth quartz surfaces is compared to alteration of the wetting properties of sandstones. In both types of study, after adsorption at elevated temperature, the mineral surface was washed or flushed with a solvent to leave an adsorbed organic film. Cores prepared in this way have mixed wettability and are referred to as MXW-F cores. Wetting studies were then performed using an aqueous phase and mineral oil as the oleic phase. Previously reported contact angle measurements at smooth quartz surfaces for ten crude oils showed that asphaltic crudes gave stable advancing and receding contact angles whereas for paraffinic oils wetting was unstable. Instability was evidenced by decrease in contact angle hysteresis for repeated measurements of advancing and receding contact angles.

In the present work, two of the ten crude oils, one asphaltic (Minnelusa) and one paraffinic (Gullfaks), were used to prepare sandstone MXW-F cores. Changes in wettability were assessed by rate and extent of spontaneous imbibition for recovery of mineral oils of different viscosities. Results for the MXW-F cores treated with Minnelusa oil were consistent with previously reported MXW-F results for a Prudhoe Bay (A95) crude oil of comparable acid and base numbers and asphaltene content. For A95 crude oil, imbibition rates were slightly lower for the first measured imbibition curve than for those measured subsequently. For Minnelusa oil, the first and subsequently measured imbibition curves were in closer agreement. Close reproducibility of sequential spontaneous imbibition measurements provides a measure of stability for induced MXW-F wetting states. Scaling of recovery of mineral oils by the geometric mean viscosity of oil and water provided further indication of reproducible wetting states for the two asphaltic crude oils. For the Gullfaks oil, erratic variation in imbibition behavior of MXW-F cores treated with Gullfaks oil indicated that established wetting states were not reproducible.

\section{Introduction}

It is widely accepted that the wettability of reservoir rocks controls the oil/water distribution and can have a major impact on oil recovery, especially in fractured reservoirs. Crude oil composition is a controlling factor in the wettability of reservoir rock (Akhlaq et al., 1996). Al-Maamari and Buckley (2000) raised the question of the stability of wettability of reservoirs changes during the course of oil production. If so, how and why does it change? The stability of wettability states generated under laboratory conditions is also a key question with respect to wettability research. The complexity of wetting states that result from crude oil/brine interactions with mineral surfaces is well recognized. Wettability states induced will be described as reproducible if the results given by duplicate experiments (i.e. separate cores from a homogeneous rock) are essentially the same. Wettability states that give reproducible results for repeated cycles of measurement will be described as stable. 
The effect of crude oil composition on wettability was also studied on smooth surfaces (Buckley et al., 1997; Liu and Buckley, 2000). Xie et al. (2002) investigated the wetting behavior of quartz surfaces after aging in 10 different crude oils at elevated temperature. The plates were lightly rinsed with toluene to leave an adsorbed organic film on the mineral surface. Contact angles of treated plate/brine/decane were measured by dynamic Wilhelmy plate technique. Contact angle measurements for the 10 crude oils showed that asphaltic crudes gave stable wetting as judged from cyclic measurements of advancing and receding contact angles. The wetting states obtained by adsorption from paraffinic oils were unstable as evidenced by systematic decrease in contact angle hysteresis. The acid and base numbers and asphaltene contents of the related oils are included in Fig.III-3.29a (Xie, et al., 2002). Examples of reproducible contact angle data for three crude oils of relevance to the present work are presented in Fig.III-3.29b, Fig.III-3.29c, and Fig.III-3.29d. The contact angle data shown in Fig.III-3.29b and Fig.III-3.29c indicate stable wetting states. The results in Fig. III-3.29d indicate a series of unstable wetting states.
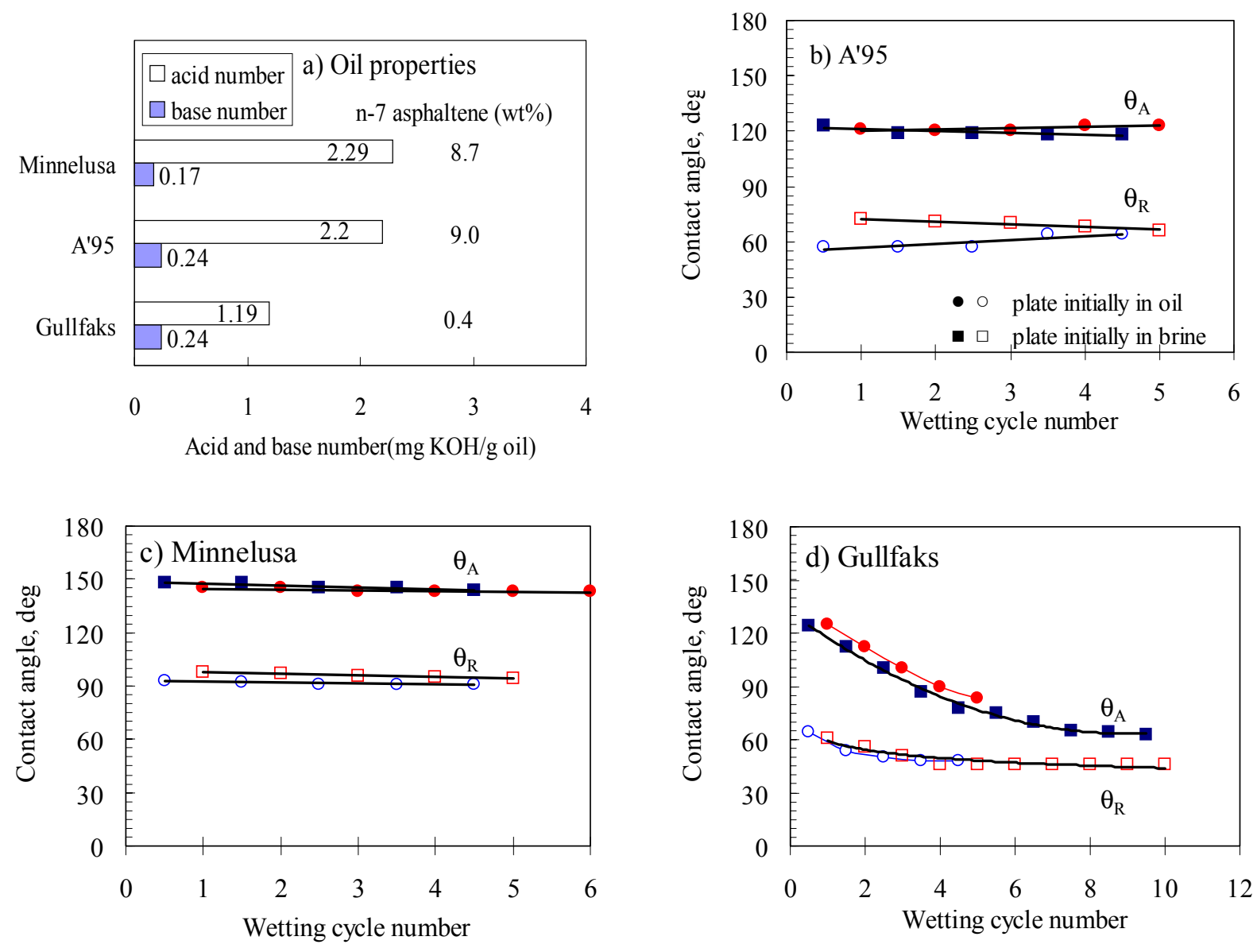

Figure III-3.29. Properties of crude oil and contact angles after deposition of organic films on smooth quartz surfaces (Xie, et al, 2002). Advancing $\left(\theta_{\mathrm{A}}\right)$ and receding $\left(\theta_{\mathrm{R}}\right)$ results shown in (b) and (c) obtained from asphaltic crude oil are characterized as reproducible and stable. Results in (d) obtained from non- asphaltic crude oil are reproducible but unstable. 
The stability of wetting states obtained after alteration of strongly water-wet sandstone cores by an asphaltic crude oil from Prudhoe Bay, A95, was investigated by Tong et al. (2002). Reproducible mixed-wetting was achieved by aging the Berea sandstone cores, at initial water saturation, $\mathrm{S}_{\mathrm{wi}}$, in A95 crude oil at elevated temperature, removal of crude oil by flushing with decalin and then replacing the decalin by mineral oil. The objective of displacing the crude oil with decalin is to remove the bulk oil but to leave in place an organic film of the adsorbed polar components on the rock surface. This procedure is used to avoid precipitation of asphaltenes and associated effects that might result from direct displacement of crude oil by mineral oil (Morrow, et al., 1986; Graue, 1999; Yang, et al., 1999; Tong, et al., 2002). Mixed wettability cores prepared in this way are referred to as having MXW-F wettability.

In the present work, the reproducibility and stability of MXW-F wetting induced in sandstone by an asphaltic crude oil (Minnelusa) and a paraffinic crude oil (Gullfaks) are compared with the results for A95, an asphaltic oil of comparable base numbers, asphaltene content, and viscosity to that of Minnelusa crude oil. Factors investigated included variation in the viscosity of the mineral oil and the effect of further aging of cores with crude oil.

\section{Experimental}

\section{Material}

Cores. Cores cut from Berea sandstone blocks were nominally $3.8 \mathrm{~cm}$ in diameter and $7.6 \mathrm{~cm}$ in length. The air permeabilities of the cores ranged from 80 to $106 \mathrm{md}$, and the porosities were all within $18.3 \pm 0.3 \%$. Core properties are listed in Tables III-3.8 and III-3.9.

Table III-3.8. Minnelusa crude oil system

\begin{tabular}{|l|l|l|l|l|l|l|l|l|l|}
\hline $\begin{array}{l}\text { Core } \\
\#\end{array}$ & $\begin{array}{l}\mathbf{K}_{\mathbf{g}}, \\
\mathbf{m d}\end{array}$ & $\boldsymbol{\phi}, \boldsymbol{\%}$ & $\mathbf{L}_{\mathbf{c}}, \mathbf{c m}$ & $\boldsymbol{\mu}_{\mathbf{o}}, \mathbf{c p}$ & $\boldsymbol{\rho}_{\mathbf{o}}, \mathbf{g} / \mathbf{m l}$ & $\begin{array}{l}\mathbf{S}_{\mathbf{w i}}, \\
\mathbf{\%}\end{array}$ & 2nd cycle* & 3rd cycle* & 4th cycle* \\
\hline 5B3 & 97.9 & 18.0 & 1.26 & 3.8 & 0.7819 & 25.0 & $3.8 \mathrm{cp}, 23.3 \mathrm{PV}$ & $170 \mathrm{cp}, 2.5 \mathrm{PV}$ & - \\
\hline 5B32 & 94.3 & 18.5 & 1.26 & 3.8 & 0.7819 & 25.7 & - & - & - \\
\hline 5B5 & 105.6 & 18.4 & 1.27 & 40.0 & 0.8456 & 24.5 & $40.0 \mathrm{cp}, 5.5 \mathrm{PV}$ & $40.0 \mathrm{cp}, 5.1 \mathrm{PV}$ & $170 \mathrm{cp}, 2 \mathrm{PV}$ \\
\hline 5B1 & 87.0 & 17.7 & 1.26 & 83.8 & 0.8168 & 24.9 & $84 \mathrm{cp}, 7.5 \mathrm{PV}$ & $84 \mathrm{cp}, 7.0 \mathrm{PV}$ & $84 \mathrm{cp}, 6 \mathrm{PV}$ \\
\hline 5B7 & 104.9 & 18.3 & 1.26 & 83.8 & 0.8168 & 24.6 & $84 \mathrm{cp}, 5.7 \mathrm{PV}$ & $84 \mathrm{cp}, 6.6 \mathrm{PV}$ & $84 \mathrm{cp}, 5.7 \mathrm{PV}$ \\
\hline 5B4 & 102.6 & 18.4 & 1.27 & 172.6 & 0.8745 & 24.9 & $173 \mathrm{cp}, 6.9 \mathrm{PV}$ & $173 \mathrm{cp}, 6.6 \mathrm{PV}$ & $173 \mathrm{cp}, 5.5 \mathrm{PV}$ \\
\hline 5B11 & 79.1 & 17.6 & 1.27 & 172.6 & 0.8745 & 24.8 & $173 \mathrm{cp}, 4.4 \mathrm{PV}$ & $173 \mathrm{cp}, 4.0 \mathrm{PV}$ & $173 \mathrm{cp}, 4.0 \mathrm{PV}$ \\
\hline
\end{tabular}

*The viscosity and pore volume of the mineral oil used to establish $\mathrm{S}_{\mathrm{wi}}$. 
Table III-3.9. Gullfaks crude oil system

\begin{tabular}{|l|l|l|l|l|l|l|l|l|}
\hline Core\# & $\mathbf{K}_{\mathbf{g}}, \mathbf{m d}$ & $\mathbf{\phi}, \boldsymbol{\%}$ & $\mathbf{L}_{\mathbf{c}}, \mathbf{c m}$ & $\boldsymbol{\mu}_{\mathbf{0}}, \mathbf{c p}$ & $\boldsymbol{\rho}_{\mathbf{o}}, \mathbf{g} / \mathbf{m l}$ & $\mathbf{S}_{\mathbf{w i}}, \boldsymbol{\%}$ & 2nd cycle* $^{*}$ & 3rd, cycle* \\
\hline 5B13 & 92.1 & 18.2 & 1.26 & 3.8 & 0.7819 & 25.8 & $170 \mathrm{cp}, 11 \mathrm{PV}$ & $170 \mathrm{cp}, 7.8$ PV \\
\hline 5B21 & 79.4 & 17.7 & 1.26 & 3.8 & 0.7819 & 25.9 & - & - \\
\hline 5B28 & 99.5 & 18.7 & 1.27 & 3.8 & 0.7819 & 25.8 & - & - \\
\hline 5B20 & 88.0 & 18 & 1.26 & 9.3 & 0.8079 & 25.6 & - & - \\
\hline 5B15 & 89.1 & 18.2 & 1.26 & 17.4 & 0.8240 & 25.2 & - & - \\
\hline 5B18 & 79.5 & 17.7 & 1.26 & 19.8 & 0.8280 & 25.8 & $170 \mathrm{cp}, 15$ PV & $170 \mathrm{cp}, 9.4$ PV \\
\hline 5B23 & 81.3 & 17.7 & 1.26 & 19.8 & 0.8280 & 25.8 & - & - \\
\hline 5B25 & 106.1 & 18.6 & 1.27 & 19.8 & 0.8280 & 26.2 & - & - \\
\hline 5B16 & 90.8 & 18.1 & 1.26 & 38.7 & 0.8420 & 25.7 & $170 \mathrm{cp}, 1.8$ PV & $170 \mathrm{cp}, 3.3$ PV \\
\hline 5B30 & 109.9 & 18.8 & 1.26 & 38.7 & 0.8420 & 25.9 & - & - \\
\hline 5B17 & 88.9 & 18 & 1.27 & 83.8 & 0.8168 & 26.0 & - & - \\
\hline 5B29 & 97.7 & 18.5 & 1.26 & 83.8 & 0.8168 & 25.9 & - & - \\
\hline 5B14 & 78.7 & 17.7 & 1.26 & 172.6 & 0.8745 & 25.8 & - & - \\
\hline
\end{tabular}

* The viscosity and pore volume of the mineral oil used to establish $\mathrm{S}_{\mathrm{wi}}$.

Crude oil. Two crude oils were used in this study. One was an asphaltic crude oil from the Gibbs field (Minnelusa formation) of Wyoming, designated as Minnelusa oil. The other was from the North Sea Gullfaks field, designated as Gullfaks oil. Selected properties of these oils and that of A95 (Tong et al., 2002) are listed in Table III-3.10.

Table III-3.10. Selected properties of crude oil samples

\begin{tabular}{|c|c|c|c|c|c|c|}
\hline Oil & Type & $\begin{array}{l}\text { Density, } \\
\text { g/ml }\end{array}$ & $\begin{array}{l}\mu_{0} \text { at } 22^{\circ} \mathrm{C}, \\
\mathrm{cp}\end{array}$ & $\begin{array}{l}\text { n-C7 asphalt., } \\
\text { wt \% }\end{array}$ & $\begin{array}{l}\text { Acid \#, } \\
\text { mg KOH/g oil }\end{array}$ & $\begin{array}{l}\text { Base \#, } \\
\text { mg KOH/g oil }\end{array}$ \\
\hline Minnelusa & asphaltic & 0.9062 & 77.2 & 9.0 & 0.17 & 2.29 \\
\hline A95 & asphaltic & 0.9086 & 70.9 & 8.7 & 0.24 & 2.20 \\
\hline Gullfaks & paraffinic & 0.8803 & 18.6 & 0.4 & 0.24 & 1.19 \\
\hline
\end{tabular}

Mineral oil. Mineral oils with different viscosities were prepared by mixing Soltrol 220 mineral oil $(3.8 \mathrm{cp})$ and white mineral oil $(180.0 \mathrm{cp})$ in ratios selected to give intermediate viscosities as required. The mineral oils were cleaned by exposure to silica gel and alumina. Viscosities of the mixtures are given in Tables III-3.8 and III-3.9.

\section{Procedure}

\section{ESTABLISHING INITIAL WATER SATURATION PRIOR TO AGING}

Initial water saturations were established with synthetic reservoir brine or sea water (Table III-3.11). $\mathrm{NaN}_{3}(0.10 \mathrm{~g} / \mathrm{L})$ was added as a biocide in the brine. The core samples were first saturated with brine and soaked for at least 10 days to attain ionic equilibrium. $\mathrm{S}_{\mathrm{wi}}$ of about $25 \%$ was established by displacing reservoir brine with Minnelusa crude oil at $45^{\circ} \mathrm{C}$ or Gullfaks crude oil at $40^{\circ} \mathrm{C}$ at $0.2 \mathrm{ml} / \mathrm{min}$ to $5.0 \mathrm{ml} / \mathrm{min}$ (about 0.72 to $18.75 \mathrm{PV} / \mathrm{hr}$ ) (see Tables III-3.8 and III-3.9). 
Table III-3.11. Synthetic brine composition.

\begin{tabular}{|l|l|l|l|l|l|l|l|l|l|}
\hline Brine & $\begin{array}{l}\mathbf{N a C l} \\
(\mathbf{g} / \mathbf{L})\end{array}$ & $\begin{array}{l}\mathbf{K C l} \\
(\mathbf{g} / \mathbf{L})\end{array}$ & $\begin{array}{l}\mathbf{C a C l}_{\mathbf{2}} \\
(\mathbf{g} / \mathbf{L})\end{array}$ & $\begin{array}{l}\mathbf{M g C l}_{\mathbf{2}} \\
(\mathbf{g} / \mathbf{L})\end{array}$ & $\begin{array}{l}\mathbf{M g S O}_{4} \\
(\mathbf{g} / \mathbf{L})\end{array}$ & $\begin{array}{l}\mathbf{N a}_{2} \mathbf{S O}_{4} \\
(\mathbf{g} / \mathbf{L})\end{array}$ & $\begin{array}{l}\mathbf{N a N}_{\mathbf{3}} \\
(\mathbf{g} / \mathbf{L})\end{array}$ & $\mathbf{p H}$ & $\begin{array}{l}\mathbf{T D S} \\
(\mathbf{m g} / \mathbf{L})\end{array}$ \\
\hline Minnelusa & 29.8 & 0 & 2.1 & 0 & 0.394 & 5.903 & 0.1 & 6.8 & 38297 \\
\hline Gullfaks & 28 & 0.935 & 1.19 & 5.365 & 0 & 0 & 0.1 & 6.6 & 35590 \\
\hline
\end{tabular}

AgING AND REPLACEMENT OF CRUde OIL WITH MinERAL OIL

The cores containing crude oil at $\mathrm{S}_{\mathrm{wi}}$ were submerged in crude oil in sealed pressure vessels for 10 days at $75^{\circ} \mathrm{C}\left(\mathrm{T}_{a}\right)$. After aging, the crude oil was displaced by $5 \mathrm{PV}$ of decalin at 3 $\mathrm{ft} /$ day (about $0.72 \mathrm{PV} / \mathrm{hr}$ ). The displacement temperature was $50^{\circ} \mathrm{C}$ for Minnelusa treated cores and $45^{\circ} \mathrm{C}$ for Gullfaks treated cores. Decalin was then displaced with $5 \mathrm{PV}$ of mineral oil of selected viscosity at ambient temperature.

\section{FIRST AND SUBSEQUENT SPONTANEOUS IMBIBITION}

The core samples containing initial water saturation and mineral oil of the selected viscosity were set in glass imbibition cells filled with synthetic brine. Oil volume produced by imbibition of brine, expressed as percentage of original oil in place (\%OOIP), versus time was recorded. All of the imbibition tests were performed at ambient temperature.

For sequential imbibition tests, brine was displaced by mineral oil at room temperature to re-establish initial water saturation. The displacement rate was 0.1 to $6.0 \mathrm{ml} / \mathrm{min}$ (about 0.36 to 22.5 PV/hr) according to the viscosity of the mineral oil and the $\mathrm{S}_{\mathrm{wi}}$. The water production was monitored during the displacement until the original water saturation had been reestablished. For some low viscosity mineral oils (less than $80 \mathrm{cp}$, see Tables III-3.8 and III-3.9), the desired initial water saturation was achieved by flushing the core sample with $170 \mathrm{cp}$ white mineral oil followed by flushing with mineral oil of the desired viscosity for the imbibition test. Up to four sequential imbibition tests were run for each core. After the third imbibition, two cores were displaced first with $5 \mathrm{PV}$ of decalin and then with 5 to $12 \mathrm{PV}$ of Minnelusa crude oil, followed by aging in Minnelusa oil for 10 days at $75^{\circ} \mathrm{C}$. A fourth imbibition test was then run after decalin/mineral oil displacement.

\section{Results and Discussion}

Wettability of MXW-F cores was assessed by comparing results scaled by the following group (Ma, et al., 1997):

$$
t_{D}=t \sqrt{\frac{k}{\phi}} \frac{\sigma}{\sqrt{\mu_{o} \mu_{w}}} \frac{1}{L_{c}^{2}}
$$

where $t_{D}$ is dimensionless time, $t$ is time, $k$ is permeability, $\phi$ is porosity, $\sigma$ is the interfacial tension, $\mu_{\mathrm{o}}$ and $\mu_{\mathrm{w}}$ are the oil and brine viscosities. $\mathrm{L}_{\mathrm{c}}$ is a characteristic length that compensates for sample size, shape and boundary conditions. 


\section{Core Samples Aged with Minnelusa Crude Oil}

\section{FIRST SPONTANEOUS IMBIBITION}

For Minnelusa MXW-F cores, mineral oil with viscosities of 3.8, 41.4, 83.8 and $172.6 \mathrm{cp}$ were used in the imbibition tests. The first imbibition curves for recovery vs time are shown in Fig. III-3.30a. The imbibition rate curves were correlated satisfactorily by Eq. III-3.7 as shown in Fig. III-3.30b. This indicates that, as found for A95 MXW-F cores, the viscosity of the mineral oil did not affect the wettability. The average of the correlated first imbibition data for MXW-F (A95) cores (Tong, et al., 2002) falls close to the Minnelusa MXW-F data set. This correspondence is probably related to the similarity in chemical characteristics of the two oils (see Fig. III-3.29a). The higher advancing contact angles for Minnelusa vs A95 (cf., Figs. III$3.29 \mathrm{~b}$ and III-3.29c) measured on smooth quartz are only weakly reflected by the imbibition results.

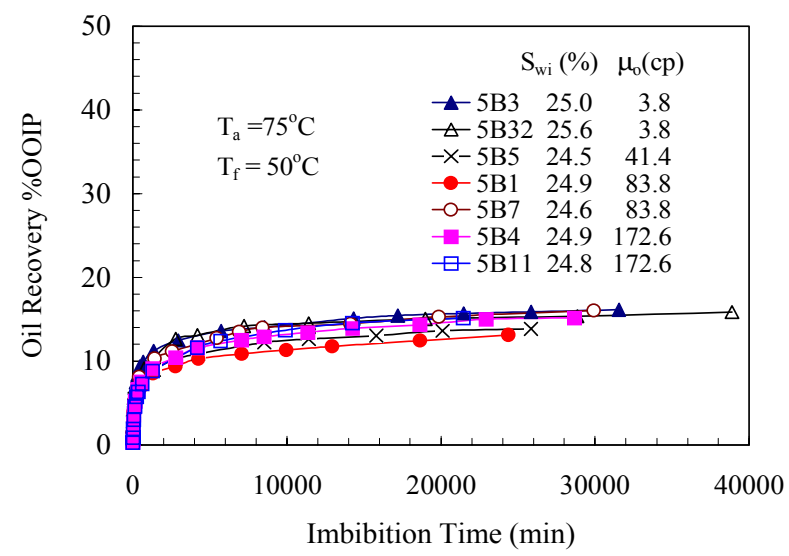

a) Recovery of MXW-F cores vs time

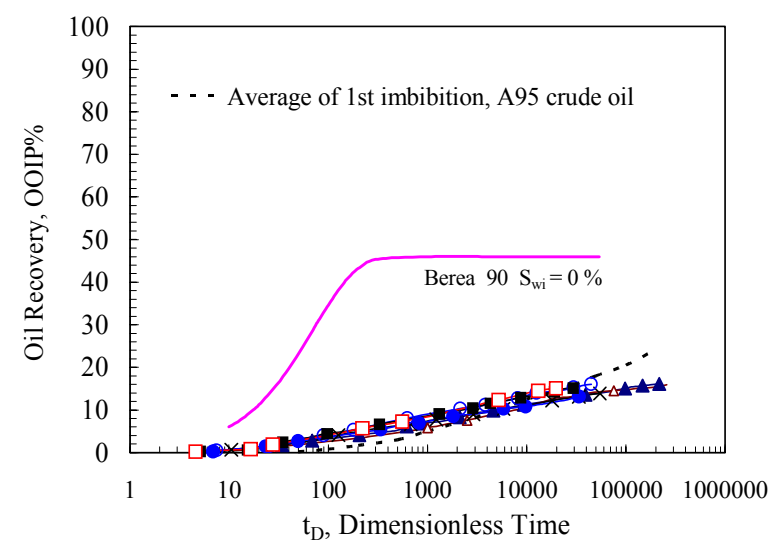

b) Scaled results of MXW-F cores

Figure III-3.30. First imbibition recovery and scaled results for MXW-F (Minnelusa) cores.

\section{SUBSEQUENT SPONTANEOUS IMBIBITION}

The scaled results for second spontaneous imbibition by MXW-F (Minnelusa) cores are generally close to and slightly higher than the average of the first imbibition curves (dashed line in Fig. III-3.31a). For core 5B3, imbibition rate fell below that measured for first imbibition; this does not fit the usual pattern of behavior for first and second imbibition. Scaled rate of recovery for $172.6 \mathrm{cp}$ oil (see core 5B4) was somewhat higher than the average given by the semiempirical scaling group (Eq.III-3.7). third imbibition results, except for core 5B5, also fell very close to the averages for the first and second cycles (Fig. III-3.31b). Results for fourth imbibition tests for Core 5B7 were also very close to the average for the previous measurements (Fig. III3.31b). 


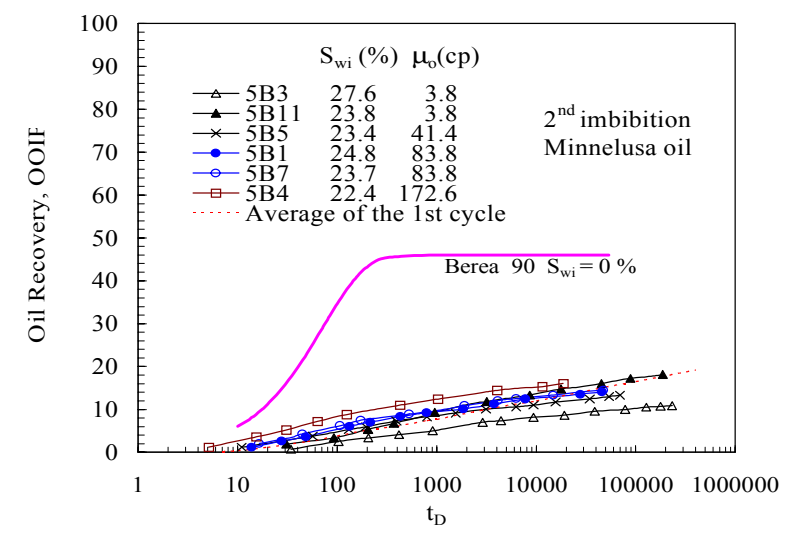

a) Scaled results of mineral oil recovery for MXW-F cores

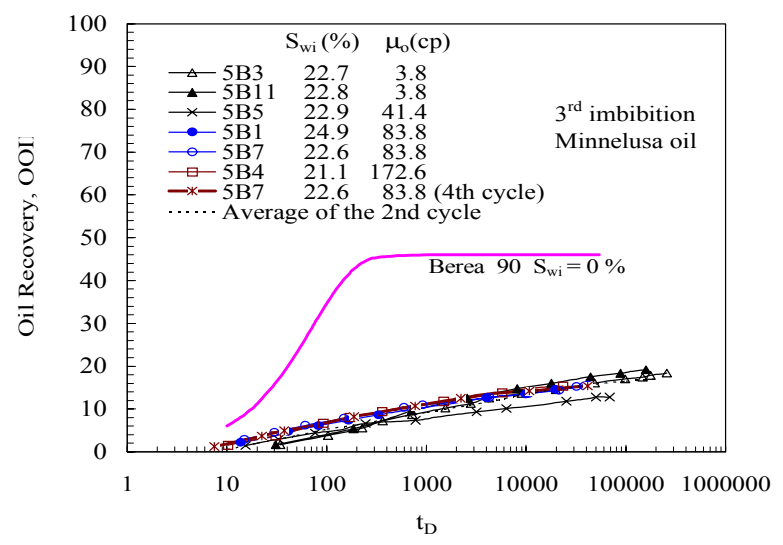

b) Scaled results of mineral oil recovery for MXW-F cores

Figure III-3.31. Scaled subsequent spontaneous imbibition results of MXW-F (Minnelusa) cores.

Two examples of sequential imbibition measurements, drawn from the results presented in Figs. III-3.30 and III-3.31, are shown in Fig. III-3.32. Core 5B11 exhibited stable wetting with close correlation of the first, second and third imbibition recovery curves, even when the mineral oil viscosity was changed from $172.6 \mathrm{cp}$ for the first cycle to $3.8 \mathrm{cp}$ for the following second, third cycles (Fig. III-3.32a). Stable wetting was also indicated by reproducibility of recovery of $40 \mathrm{cp}$ mineral oil from core 5B5 (Fig. III-3.32b). Thus, in general, the sequential imbibition results were in close agreement showing that the wettability of the MXW-F Minnelusa cores was stable. (The A95 MXW-F cores showed shift between first and second imbibition but were subsequently stable.)
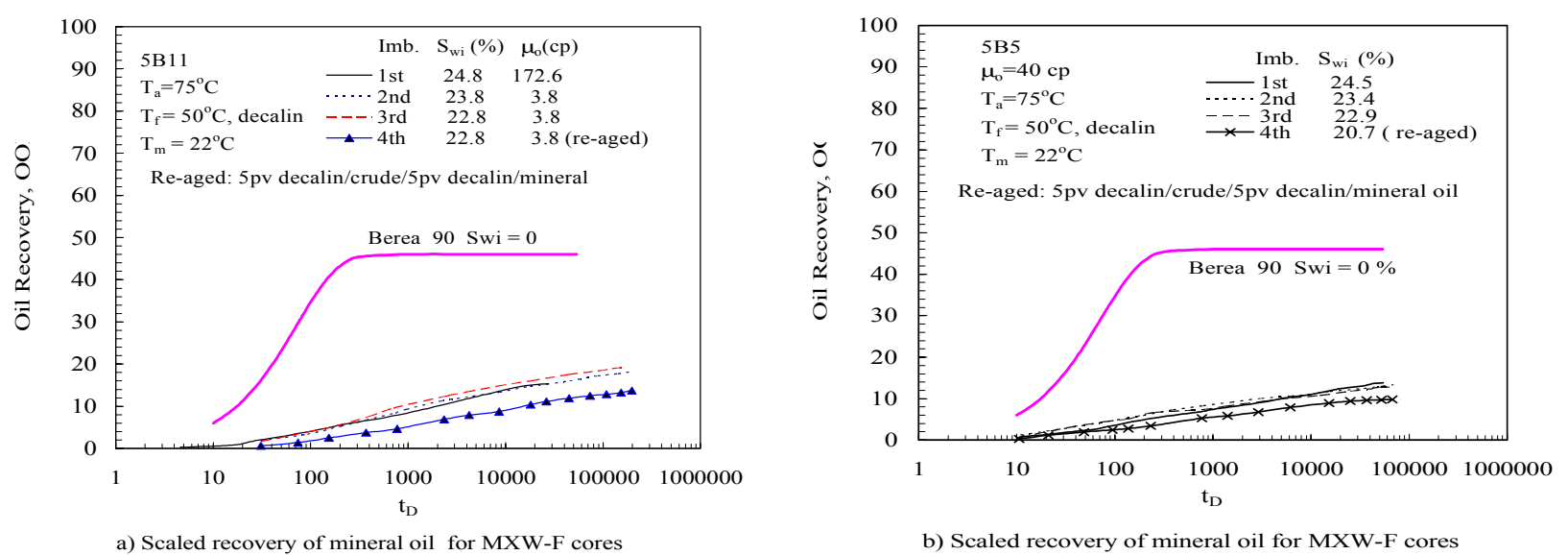

Figure III-3.32. The effect of Minnelusa oil re-aging on spontaneous imbibition.

REAGING WITH MINNELUSA CRUDE OIL

After the third imbibition test on these cores, $S_{\text {wi }}$ was re-established with $170 \mathrm{cp}$ mineral oil. The mineral oil was then displaced from the cores by flooding with $5 \mathrm{PV}$ of decalin followed by 5 PV of Minnelusa crude oil. The cores were then re-aged in the Minnelusa oil for 10 days at 
$75^{\circ} \mathrm{C}$. After aging, the crude oil was displaced by $5 \mathrm{PV}$ decalin which was in turn displaced by $3.8 \mathrm{cp}$ mineral oil for 5B11 and $40 \mathrm{cp}$ mineral oil for core 5B5. In both cases imbibition rates decreased slightly as a result of further aging (Figs. III-3.32a and III-3.32b), indicating less water-wetness and further adsorption of polar components at the rock pore surfaces.

\section{Core Samples Aged with Gullfaks Crude Oil}

\section{REPRODUCIBILITY OF FIRST SPONTANEOUS IMBIBITION}

Initial water saturations established with Gullfaks crude oil were all close to $26 \%$. In all cases the rate and extent of recovery by imbibition was higher than for either Minnelusa or A95 oil. However, for MXW-F (Gullfaks) cores problems were experienced with reproducibility of first imbibition curves for duplicate core plugs. Scaled results for 3 duplicate tests with $3.8 \mathrm{cp}$ mineral oil, 4 with $19.8 \mathrm{cp}$ mineral oil and 1 with $9.3 \mathrm{cp}$ mineral oil are presented in Fig. III3.33a. Recoveries after about 27 days of imbibition were comparable, but 3 of the recovery curves are well separated from the other 5 with respect to rate. Scaled results for duplicate core plugs using 38.7 and $83.8 \mathrm{cp}$ mineral oil and 1 curve for $172.6 \mathrm{cp}$ mineral oil are presented in Fig. III-3.33b. The MXW-F (Gullfaks) cores that gave similar slow rates of imbibition will be referred to as Group 1 (5B13, 5B15 and 5B18 three cores, see Fig. III-3.33a) and the remainder as Group 2 ( 9 cores, see Figs. III-3.33a and III-3.33b). Examination of rock properties, and the batches and dates of core preparation and testing gave no indication as to why the results fell into 2 groups. For the 9 plugs which exhibit higher recovery rates the scaled results are shown in Figs. III-3.33a and III-3.33b, recoveries at long times are comparable, but there is still considerable scatter in the rates of imbibition.
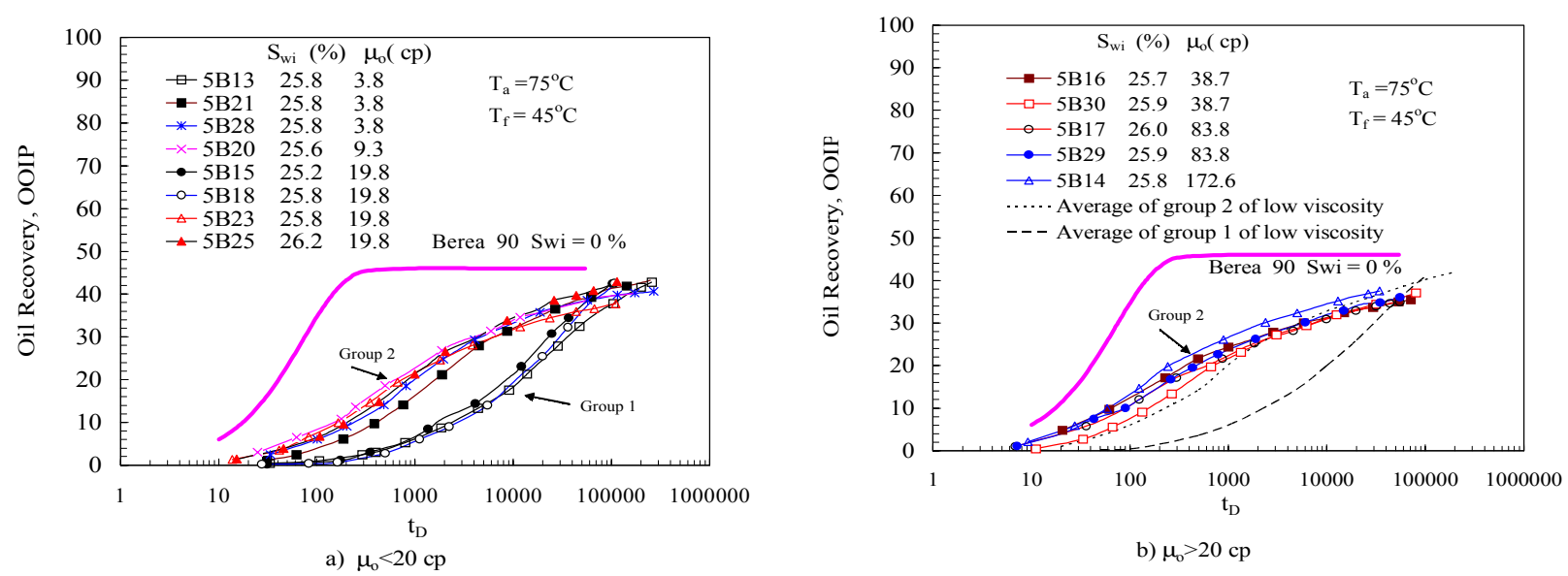

Figure III-3.33. Scaled first imbibition results for MXW-F (Gullfaks) cores.

STABILITY OF MXW-F (GULLFAKS) WETTING

The stability of wetting was tested for two cores from each of Groups 1 and 2 through measurement of three sequential spontaneous imbibition curves. Scaled results for the two Group 1 cores $(5 \mathrm{~B} 13,5 \mathrm{~B} 18)$ are shown in Figs. III-3.34a and III-3.34b. Results for recovery of $3.8 \mathrm{cp}$ and $19.8 \mathrm{cp}$ mineral oil were very similar in form. For the second and third cycle imbibition, 
rates of recovery were higher, but final recoveries were lower. Both cores exhibited the same rate of recovery at early time but decreased recovery for the third imbibition.

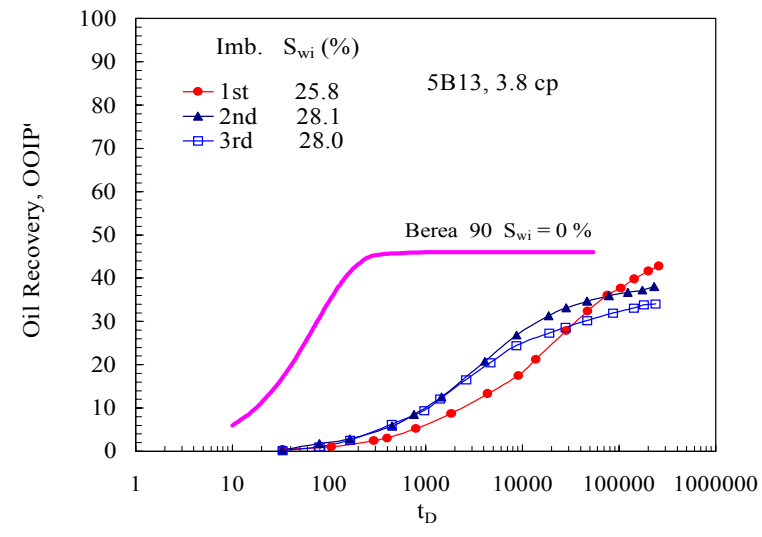

a) Scaled recovery of mineral oil for MXW-F cores

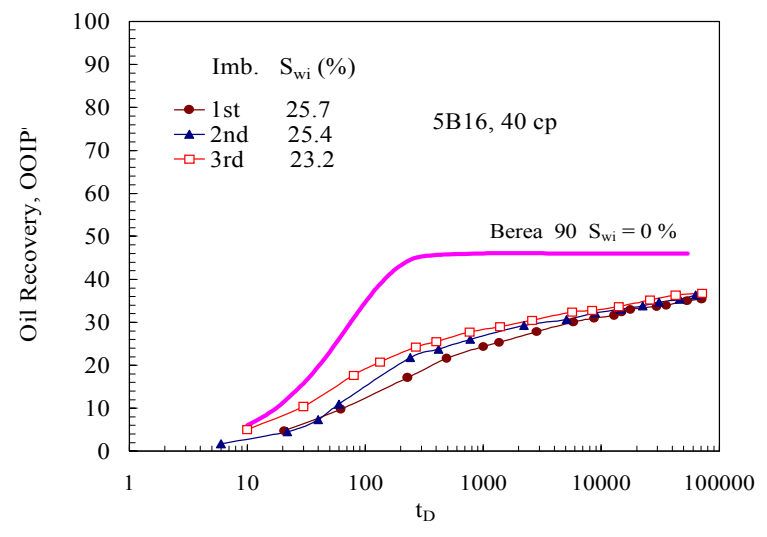

c) Scaled recovery of mineral oil for MXW-F cores

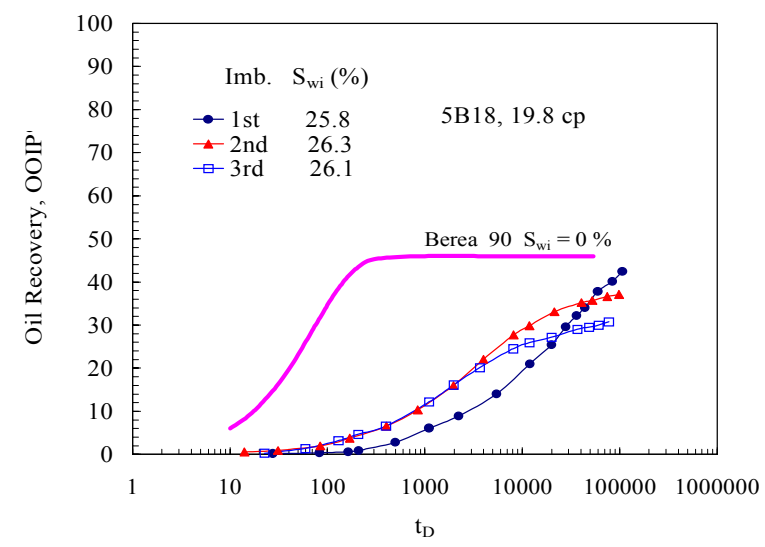

b) Scaled recovery of mineral oil for MXW-F cores

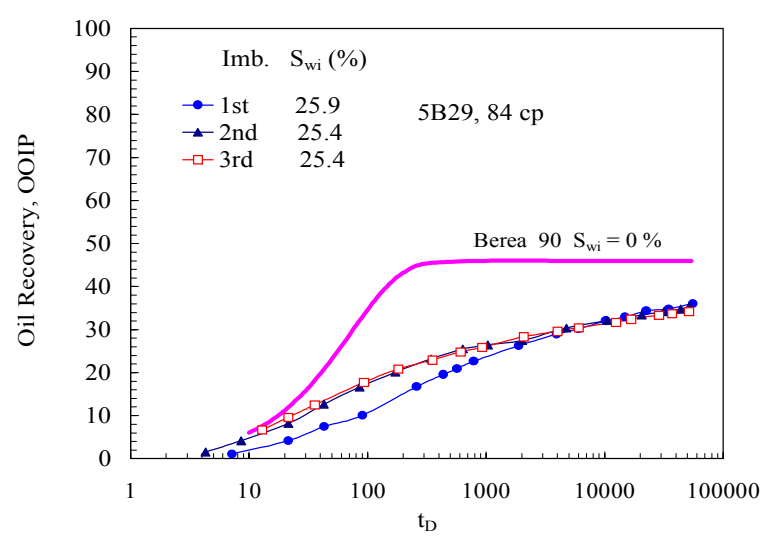

d) Scaled recovery of mineral oil for MXW-F cores

Figure III-3.34. Sequential spontaneous imbibition tests for MXW-F (Gullfaks) cores.

Imbibition curves for the two Group 2 cores 5B16 and 5B29 are plotted in Figs. III-3.34c and III-3.34d, respectively. Rates of imbibition increased for the second and third imbibition but there was essentially no change in final recovery. The second and third imbibition were closely reproduced for Core 5B29, indicating attainment of a stable wetting state. Overall, although the MXW-F (Gullfaks) wetting states were not reproducible (i.e. varied between duplicate cores). Particular achieved wetting states, as indicated by imbibition measurements, showed partial reproducibility between second and third imbibition (Figs. III-3.34a-d).

\section{Comparison of Spontaneous Imbibition with Contact Angles}

As for contact angle behavior at smooth quartz surfaces (Fig. III-3.29), the MXW-F (Gullfaks) wetting states are clearly more water-wet than those induced by the two asphaltic crude oils with relatively high base numbers. Reproducibility of sequentially measured contact angles was observed for the asphaltic oils (Figs. III-3.29b and III-3.29c). Minnelusa oil gave the most stable wetting behavior as indicated by sequential imbibition measurements (Figs. III-3.30, 
III-3.31, and III-3.32) and A95 oil results indicated stable wetting after completion of the first imbibition (Tong, et al., 2002). During the course of imbibition, a slight amount of dark material accumulated at the mineral oil/brine interface in the imbibition cells for A95 oil but not for Minnelusa. Also, the mode of precipitation of solids in response to addition of alkane for A95 (Wang, 2000) was different to that for the Minnelusa oil (Wang, 2002).

Compared to results for the cores treated with asphlatic crude oils, less change in wetting was induced by Gullfaks crude oil. However, the sequential decrease in advancing contact angles (see Fig. III-3.29d) was not matched by a clearly sequential increase in water wetness for consecutive spontaneous imbibition tests. In addition to the difference in brine composition, there are many other reasons why contact angle measurements at smooth surfaces are not likely to provide reliable prediction of core displacement behavior. These include differences in mineralogy and pore morphology and the response of adsorbed organic films to movement of oil-water interfaces and the differences in time (less than one day for contact angle measurements) versus many months for sequential imbibition tests.

\section{Conclusions}

1) In interpretation of the imbibition behavior of MXW-F cores, distinction needs to be made between reproducibility of wetting for duplicate core plugs, and the stability of wetting for consecutive imbibition tests.

2) Reproducible and stable wetting was exhibited by films deposited from Minnelusa oil. Crude oils which provide stable films are particularly useful for oil recovery research.

3) Imbibition results for recovery of mineral oils of different viscosity from MXW (Minnelusa cores) were scaled by the geometric mean of the oil and water viscosities.

4) A paraffinic oil (Gullfaks) which gave reproducible but unstable contact angle behavior, gave poorly reproducible MXW-F wetting, but any particular achieved wettability was to some degree stable.

5) From imbibition measurements, the paraffinic oil (Gullfaks) caused less change in wetting than the asphaltic Minnelusa crude oil and a previously studied asphaltic oil. This observation is qualitatively consistent with advancing contact angle measurements on smooth quartz after deposition of organic films from these oils. However, such contact angle measurements are not reliable for prediction of imbibition rates. 


\section{Nomenclature}

$k$ gas permeability, md

$L_{c}$ characteristic length, $\mathrm{cm}$

$S_{w i}$ initial water saturation, \%

$t$ imbibition time, min

$t_{D}$ dimensionless imbibition time
$T_{a} \quad$ aging temperature

$T_{m}$ imbibition test temperature

$T_{f}$ decalin flush temperature

$\phi$ porosity, $\%$

$\sigma$ oil-water interfacial tension, dynes $/ \mathrm{cm}$.

$\mu_{w}$ water viscosity, cp,

$\mu_{o} \quad$ oil viscosity, cp.

\section{Acknowledgement}

We thank Eric Robertson of Idaho National Engineering and Environmental Laboratory and Kyle True of True Oil Company for providing Minnelusa crude oil and also, Statoil for providing Gullfaks crude oil.

\section{References}

Akhlaq, M.S., Kessel, D. and Dornow, W., "Separation and Characterization of Wetting Crude Oil Compounds," J. Colloid Interface Sci. (1996) 180, 309-314.

Al-Maamari, R.S.H. and Buckley, J.S., "Asphaltene Precipitation and Alteration of Wetting: Can Wettability Change during Oil Production?" paper SPE 59292 presented at the 2000 SPE/DOE IOR Symp., Tulsa, 3-5 April.

Buckley, J.S., Liu, Y., Xie, X. and Morrow, N.R., "Asphaltene and Crude Oil Wetting - the Effect of Oil Composition," SPEJ, (June 1997), 107-119.

Graue, A. Viksund, B.G. and Baldwin, B.A., "Reproducible Wettability Alteration of Lowpermeable Outcrop Chalk", SPEREE, (1999) April.

Liu, L. and Buckley, J.S., “Alteration of Wetting of Mica Surfaces,” J. Pet. Sci. Eng. (2000) 24 , 75-83.

Ma, S., Morrow, N.R. and Zhang, X., "Generalized Scaling of Spontaneous Imbibition Data for Strongly Water-Wet Systems”, J. Pet. Sci. \& Eng. (1997), 18, 165-178.

Morrow, N.R., Lim, H.T., and Ward, J.S., "Effect of Crude Oil Induced Wettability Changes on Oil Recovery" SPE Formation Evaluation, (1986) April, 1, 89-103.

Tong, Z.X., Xie, X. and Morrow, N.R., "Spontaneous Imbibition For Mixed-Wettability States In Sandstones Induced By Adsorption From Crude Oil", presented at the $7^{\text {th }}$ International Symposium on Reservoir Wettability, Tasmania, Australia (March 2002).

Tong, Z., Xie, X. and Morrow, N.R., "Scaling of Viscosity Ratio for Oil Recovery by Imbibition from Mixed-Wet Rocks," Proceedings of International Symposium of the Society of Core Analysts, Edinburgh, paper SCA-0121 (2001) Petrophysics, in press. 
Wang, J., "Predicting Asphaltene Flocculation in Crude Oils", Ph.D. dissertation, (2000) New Mexico Institute of Mining \& Technology, Socorro, NM.

Wang, J., Personal communication, April 2002.

Xie, X., Morrow, N.R. and Buckley, J.S., "Contact Angles Hysteresis and Stability of Wetting Changes Induced By Adsorption from Crude Oil”, J. Pet. Sci. Eng., (2002) 33, 1-3, 147-159.

Yang, S.Y., Hirasaki, G.J., Basu, S. and Vaidya, R., "Mechanisms for Contact Angle Hysteresis and Advancing Contact Angles", J. Pet. Sci. Eng., (1999), Dec., 4, No.2-4, 63-73. 